This document was too large to scan as a whole document, therefore it required breaking into smaller sections.

Document number: SD-FF-CSWD-OGI

Section 1 of 2

Title: Soduim Storage facility Loader Angie Neagrame

Date: 6/12/97 Revision: A000

Originator: Cones Q

Co: Bu Ne

Recipient:

Co:

References: $\angle D T-619594$ 


\section{Sodium Storage Facility Ladder Logic Diagrams}

\section{Derek D. Jones}

B \& W Hanford Company, Richland, WA 99352

U.S. Department of Energy Contract DE-AC06-96RL13200

$\begin{array}{llll}\text { EDT/ECN: } & 619594 & \text { UC: } 541 & \\ \text { Org Code: } & 18240 & \text { Charge Code: } & \text { E29146/B1/82 } \\ \text { B\&R Code: } & \text { EX7002000 } & \text { Tota1 Pages: } 797\end{array}$

Key Words: Sodium, SSF, Trace Heat, PLC, Computer

Abstract: This document provides the computer technician or software engineer with a way to study the algorithms that are actually used by the SSF PLC.

TRADEMARK DISCLAIMER. Reference herein to any specific commercial product, process, or service by trade name, trademark, manufacturer, or otherwise, does not necessarily constitute or imply its endorsement, recommendation, or favoring by the United States Government or any agency thereof or its contractors or subcontractors.

Printed in the United States of America. To obtain copies of this document, contact: Document Control Services, P.0. Box 950, Mailstop H6-08, Richland WA 99352, Phone (509) 372-2420; Fax (509) 376-4989.
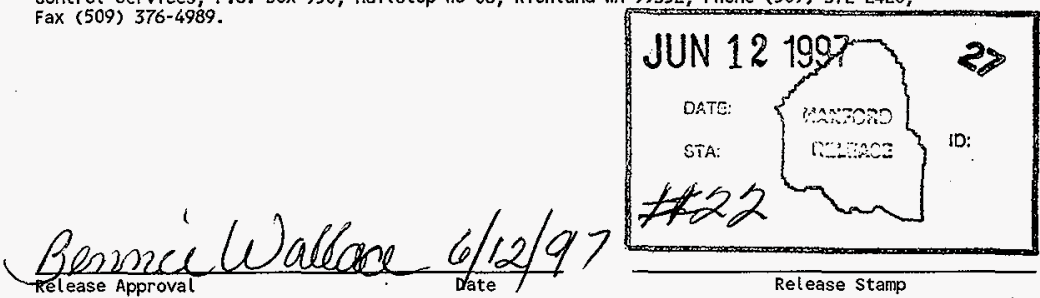

\section{Approved for Public Release}


Processor and Datacops Program Listing

Unit 1)

Processor File: SODIUM1A.ACH
October 23, 1996

Page 1 Rung $2: 0$

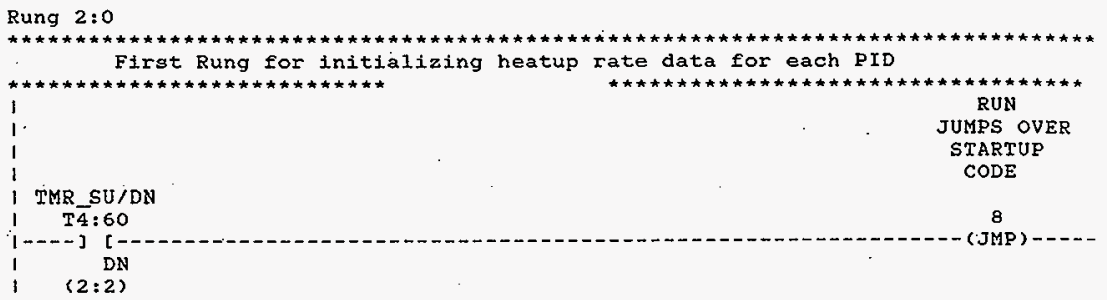

Rung 2:2

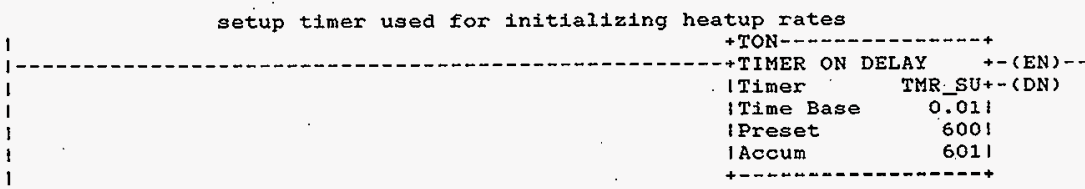

Rung $2: 3$

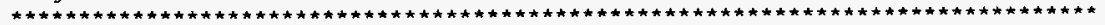

HNF-SD-FF-CSWD-61 Rev. 0 
Processor and Data(OPS Unit 1)

Rung $2: 4$

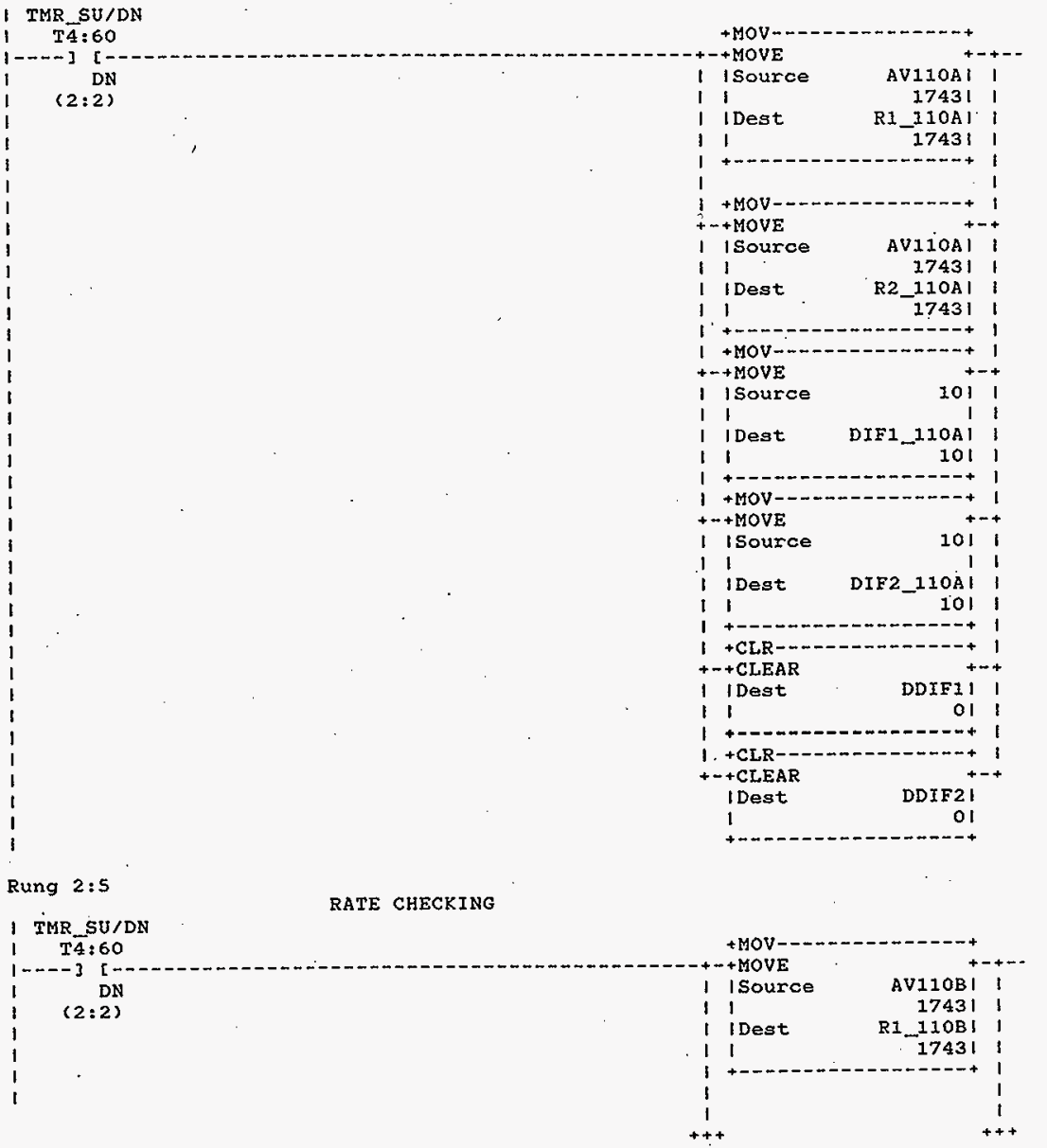

HNF-SD-FF-CSWD-61 Rev. 0 
Processor and Data(ops Unit 1)

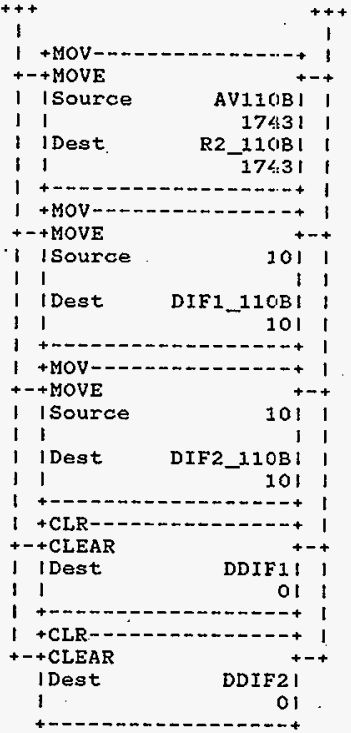

Rung $2: 6$

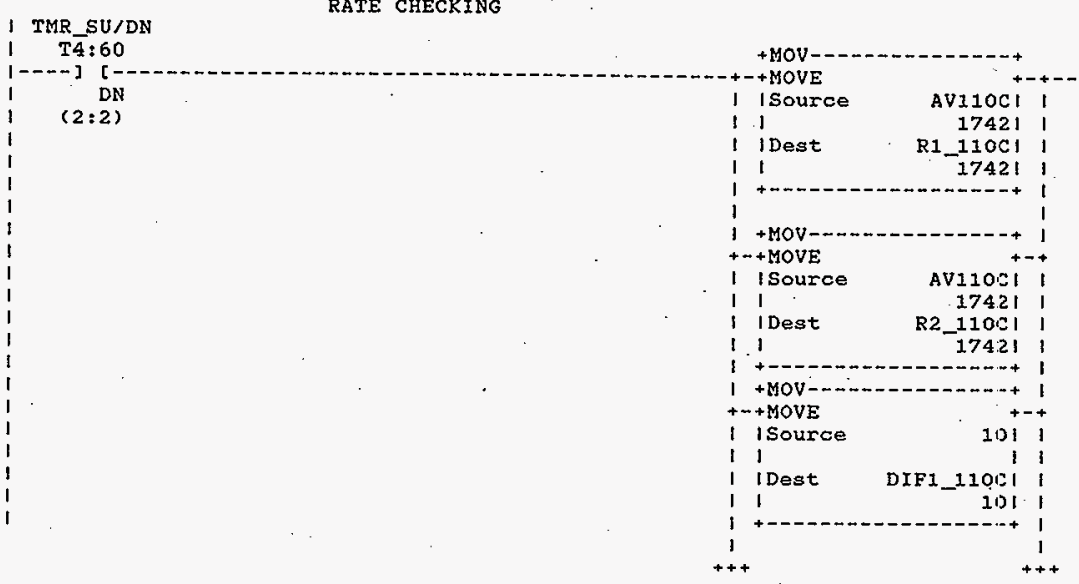


C.

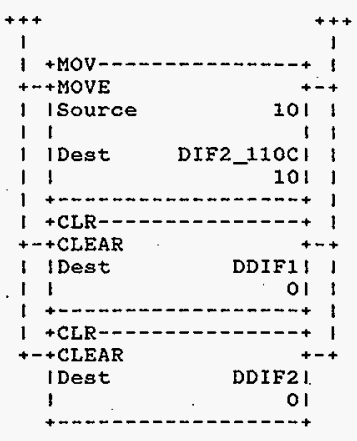

Rung $2: 7$

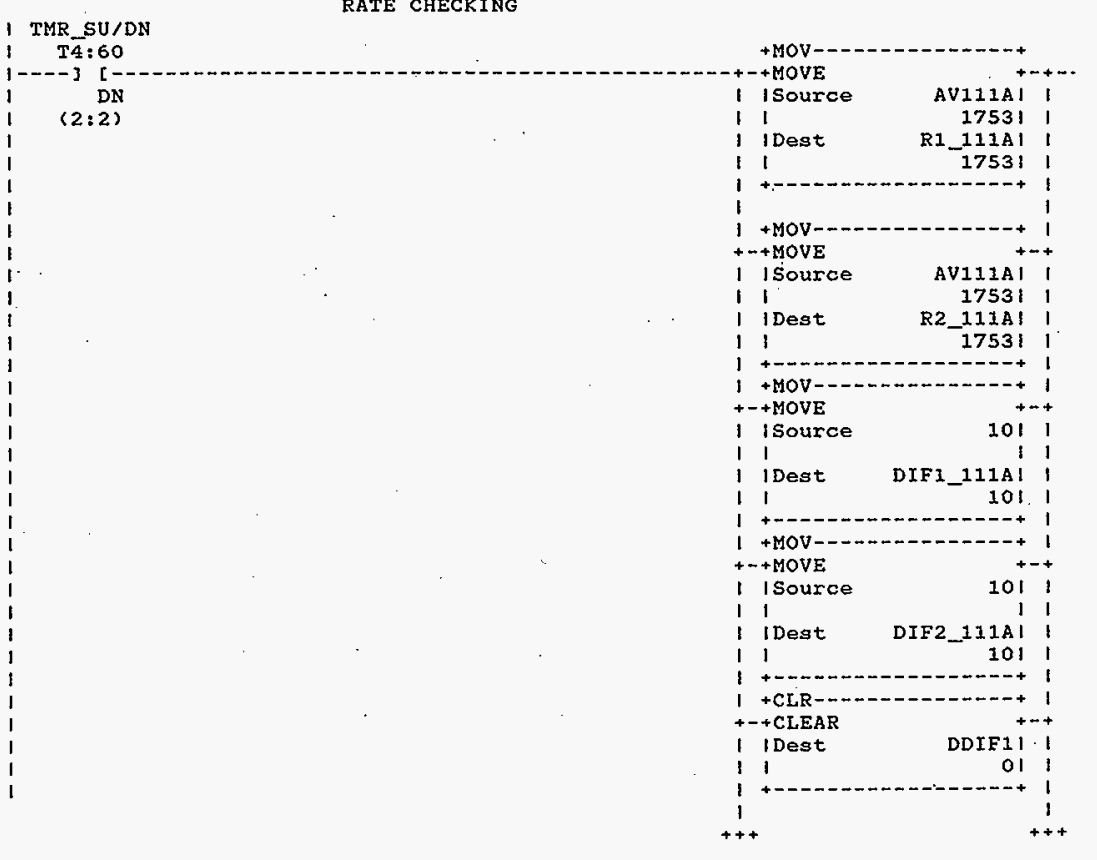


Processor and Data(OPS Unit 1)

$$
\begin{aligned}
& 1 \\
& 1 \\
& 1
\end{aligned}
$$
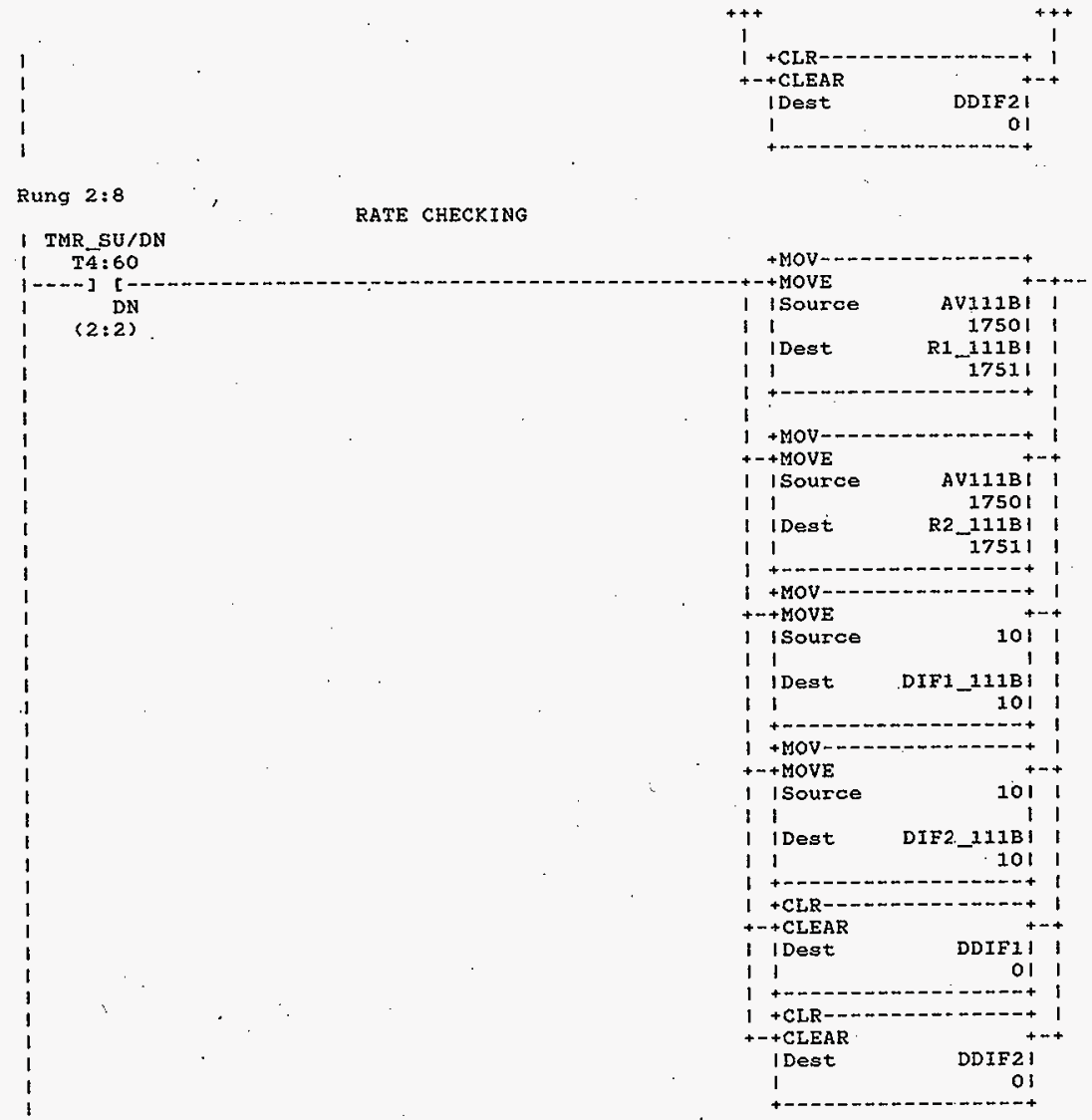

HNF-SD-FF-CSWD-61 Fiev. 0

Page 5 
Processor and Data(OPS Unit 1)

\section{Rung 2:9}

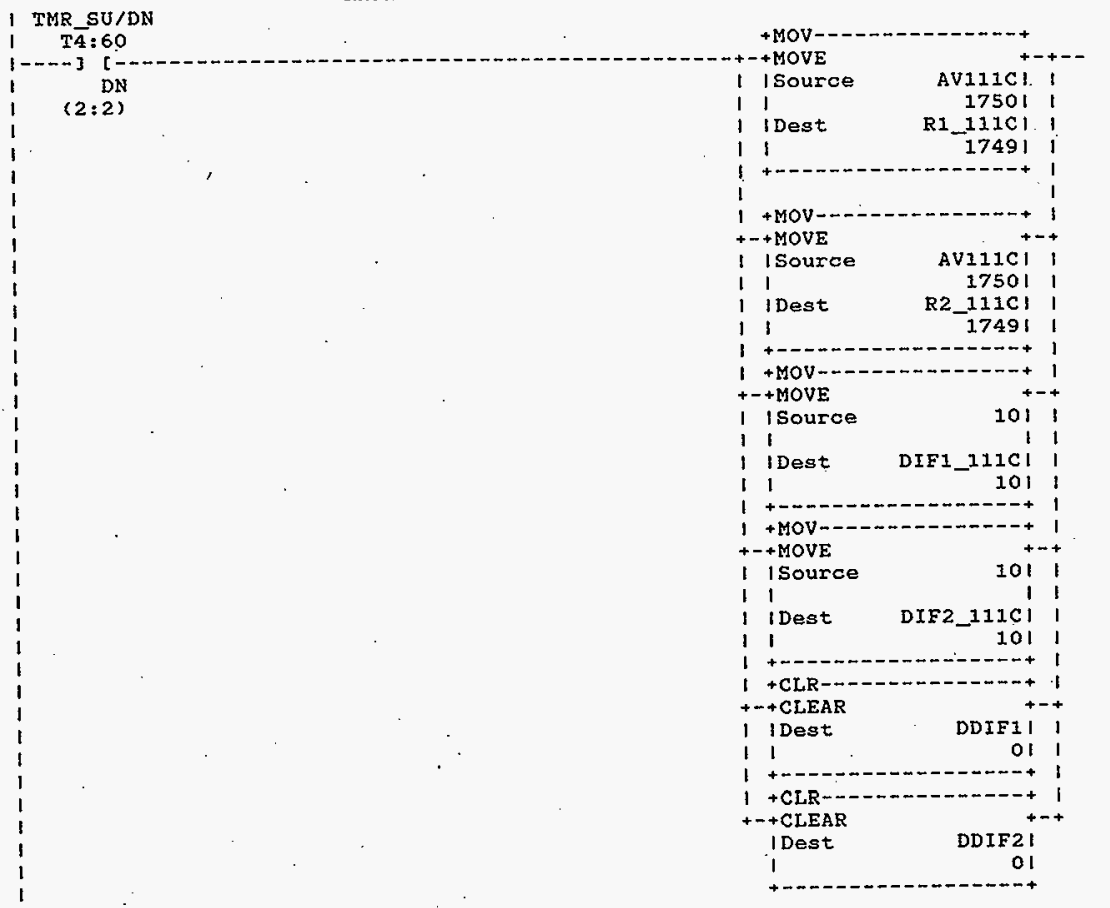

Rung 2:10

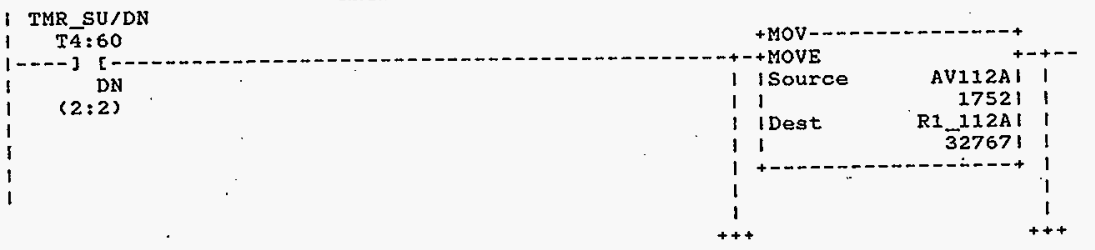


Processor and Data(OPS Unit 1 )

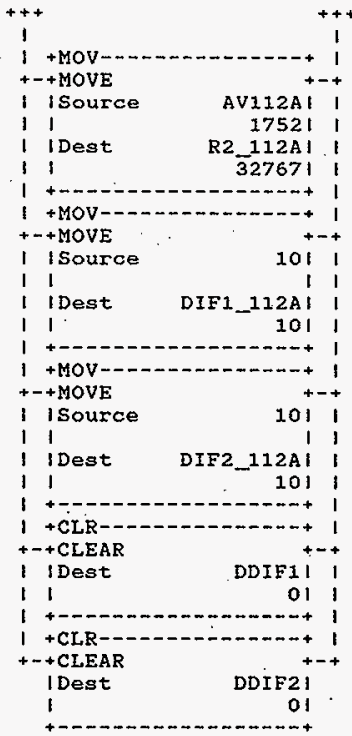

Rung 2:11

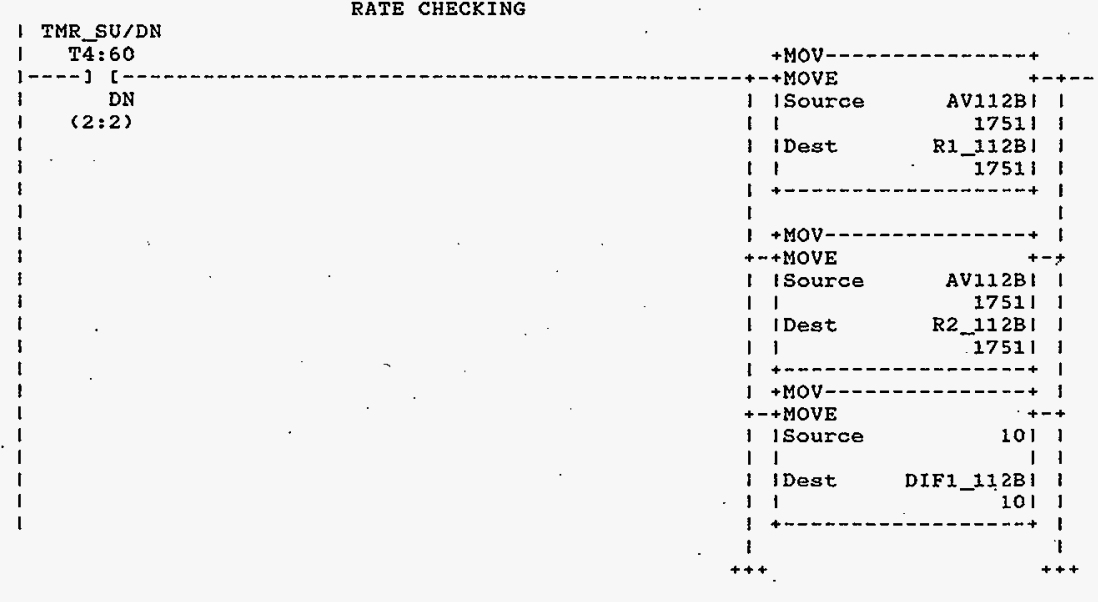

HNF-SD-FF-CSWD-61 Fiev. 0 


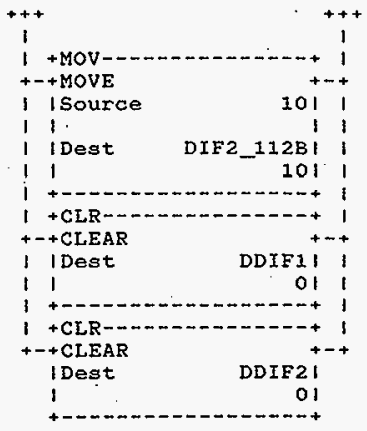

Rung 2:12

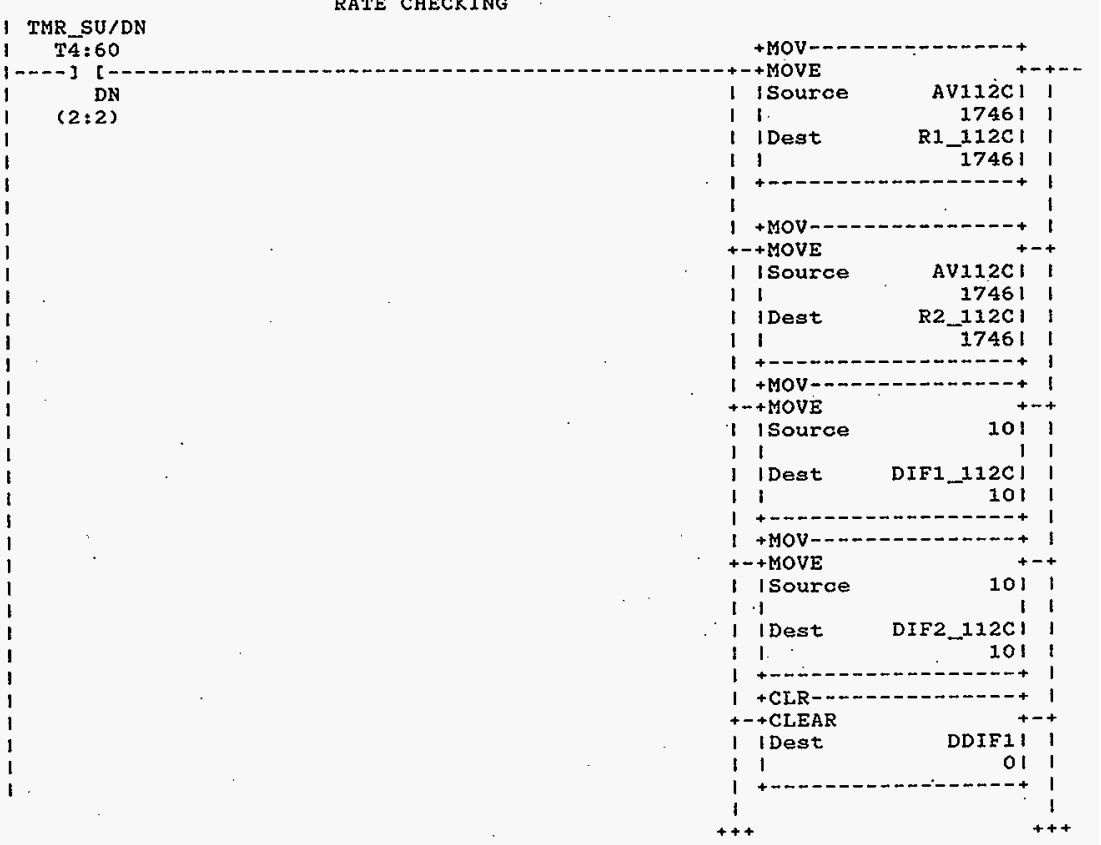

HNF-SD-FF-CSWD-61 Rev. 0 


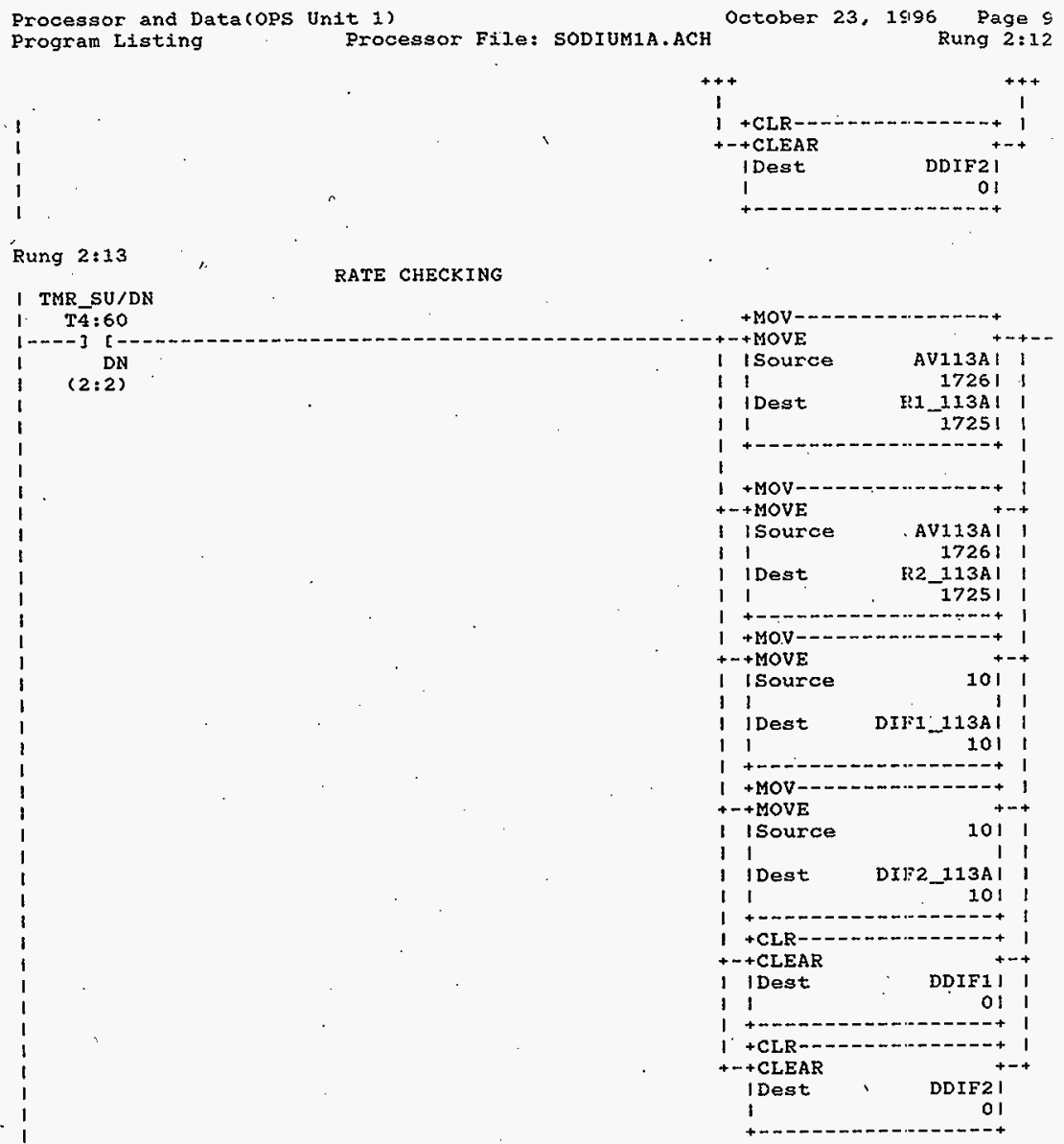


Processor and Data(OPS Unit 1 )

October 23, 1996 : Page $1 \mathrm{C}$ Program Listing

Rung $2: 14$

1 TMR_SU/DN

RATE CHECKING

$1 \mathrm{~T} \overline{4}: 60$

DN

+ MOVE

I Source

11

1 Dest

1

1

pest

173211

I + MOV-

+-+ MOVE

1 isource

11

i Dest

I

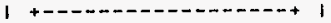

$1+$ MOV-D.----........+ 1

+-+ MOVE

1 isource

11

1011

11

11

Dest

DIF1 113BI

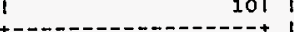

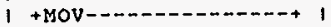

+-+ MOVE

1 ISource

11

1011

IDest DIF2_113Bi I

11

1011

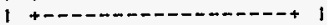

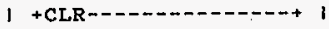

++ +CIEAR

1 IDest

DDIE1) 1

11

of 1

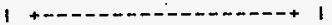

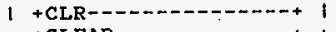

+-+ CLEAR

I Dest

I

Rung 2:15

1 TMR SU/DN

I T4:60

1----] [

1 DN

$(2: 2)$

RATE CHECKING

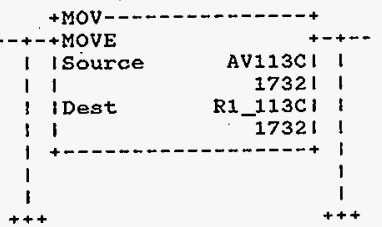

HNF-SD-FF-CSWD-61 Rev. 0 


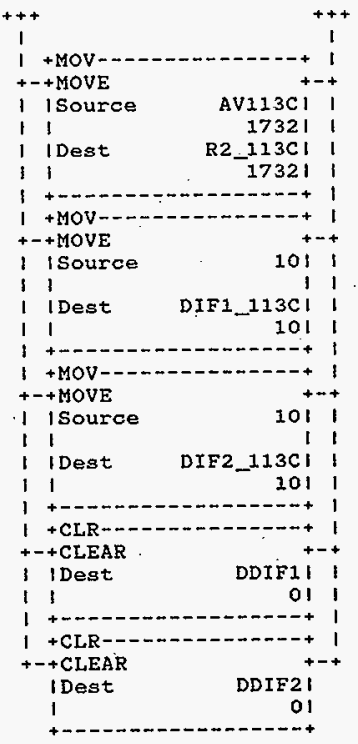

Rung $2: 16$

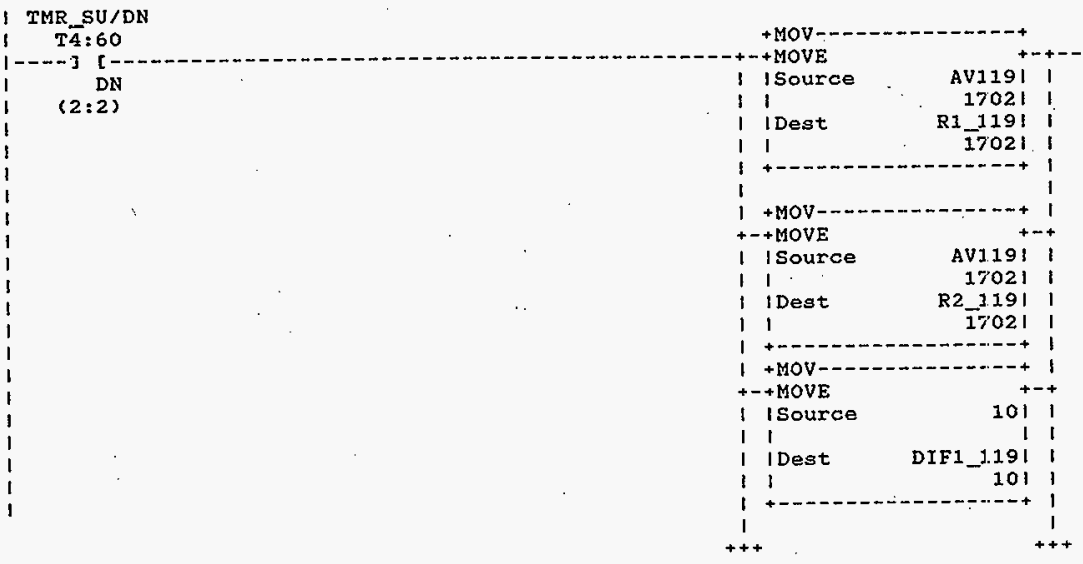

HNF-SD-FF-CSWD-61 Rev. 0 
Processor and Data(OPS Unit 1 )

October 23, 1996 Page 1:

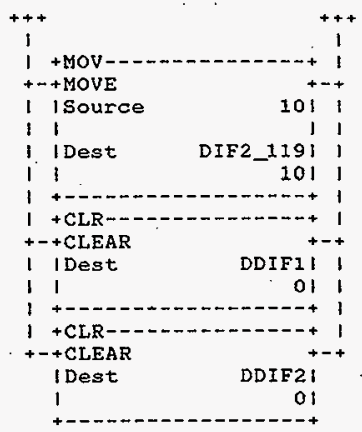

Rung 2:17

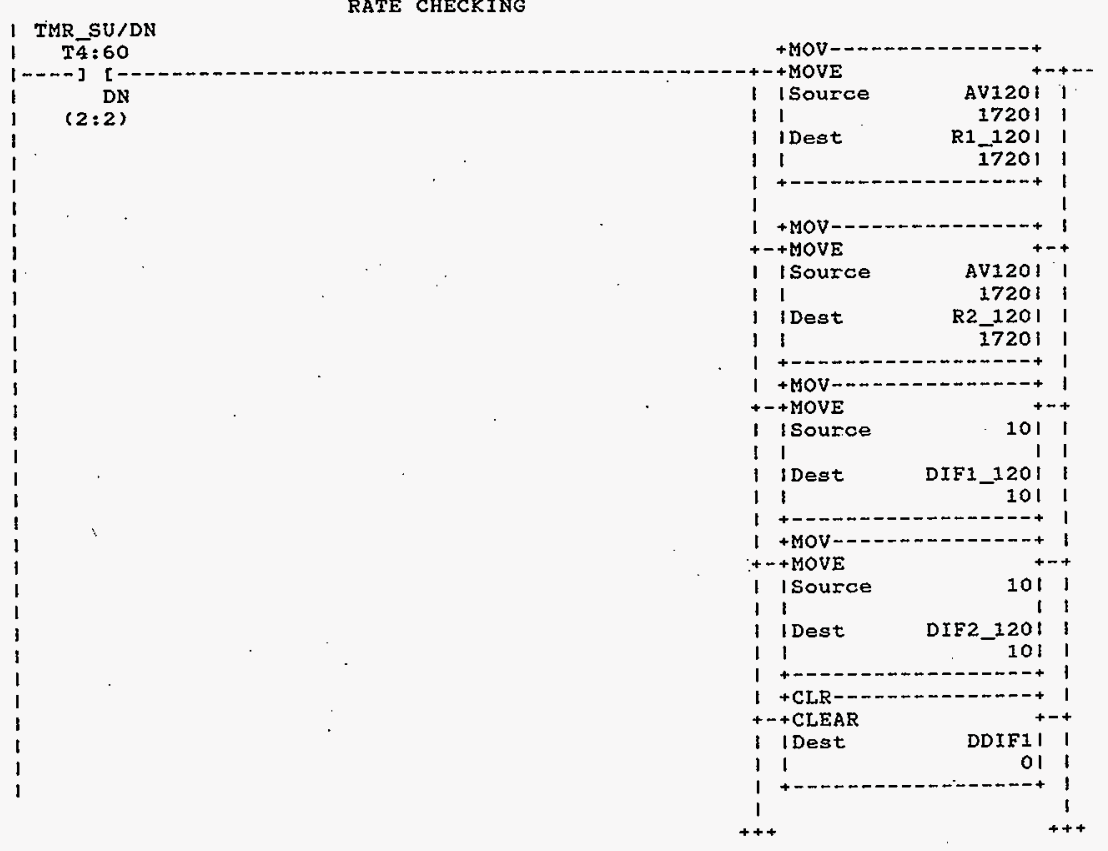


1
1
1
1

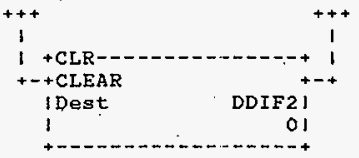

Rung 2:18

1 TMR SU/DN

RATE CHECKING

$T \overline{4}: 60$

1...- ] [-

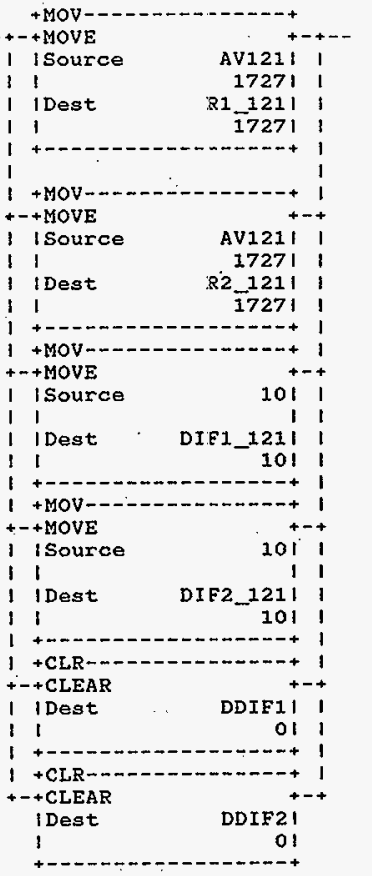


Processor and Data(OPS Unit 1)

Program Listing

Rung $2: 19$

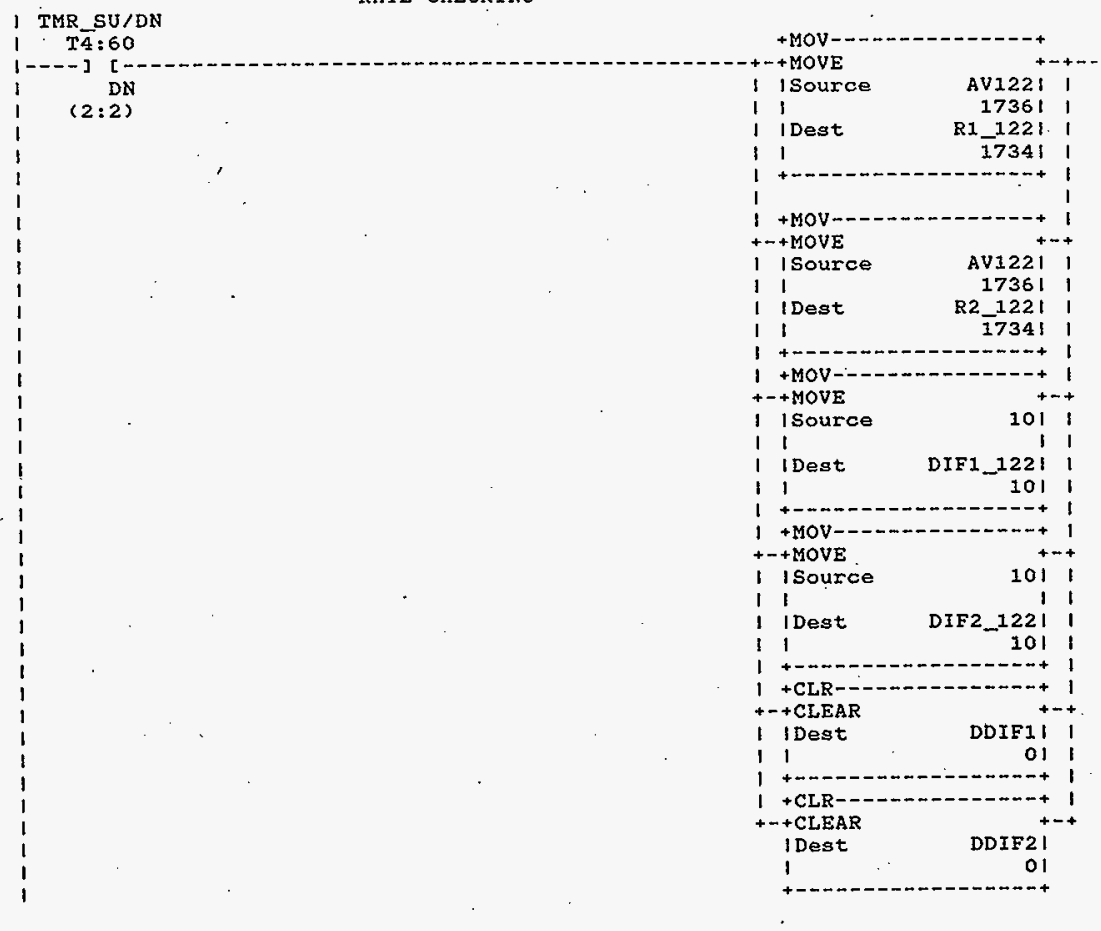

Rung $2: 20$

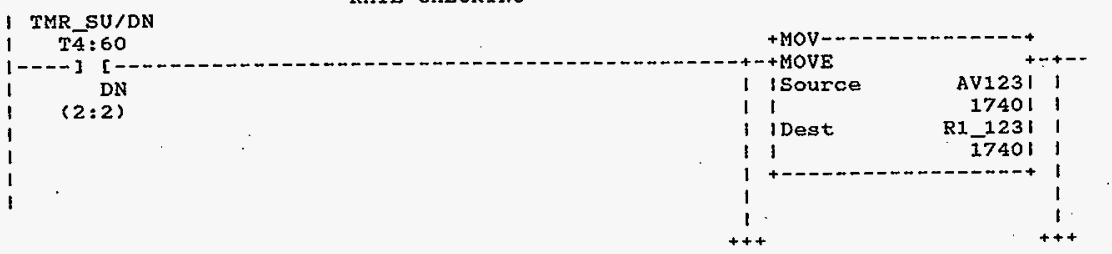




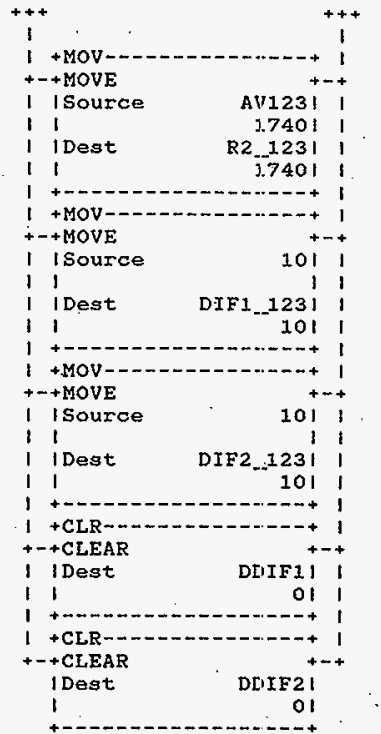

Rung $2: 21$

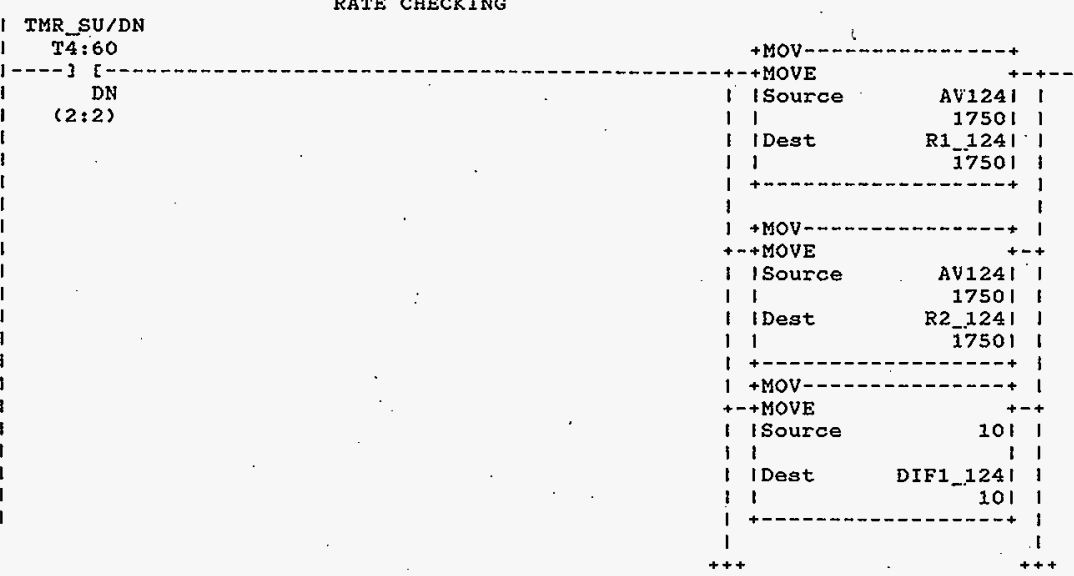

HNF-SD-FF-CSWD-61 Rev. 0 
Processor and Data(OPS Unit 1)

$$
\begin{aligned}
& 1 \\
& 1 \\
& 1 \\
& 1 \\
& 1 \\
& 1 \\
& 1 \\
& 1 \\
& 1 \\
& 1 \\
& 1 \\
& 1 \\
& 1 \\
& 1 \\
& 1 \\
& 1
\end{aligned}
$$

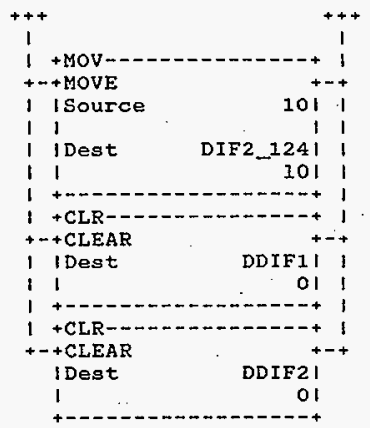

Rung $2: 22$

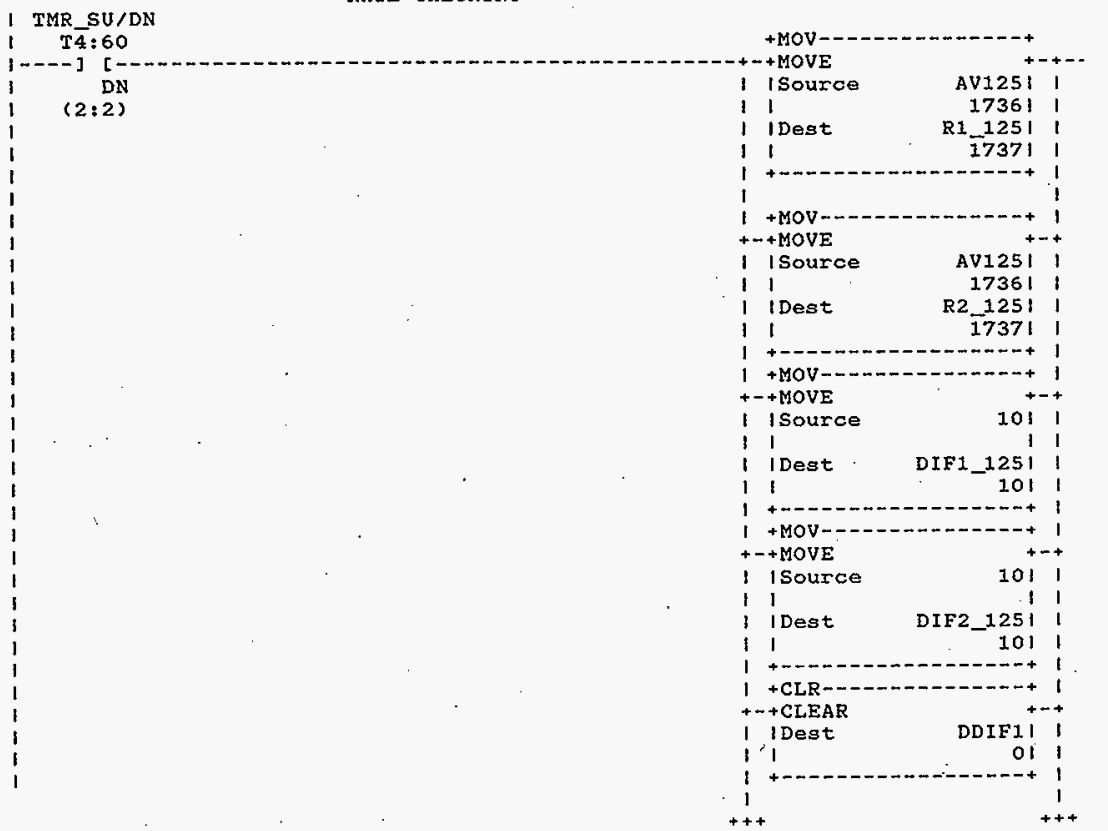

HNF-SD-FF-CSWD-61 Rev. 0 
Processor and Data(OPS Unit 1 ) program Listing
Processor File: SODIUM1A.ACH
October 23,1996

Page 17

Rung $2: 22$

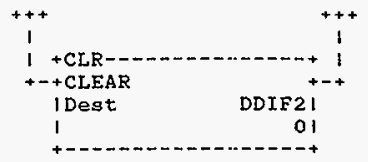

Rung $2: 23$

1 TMR_SU/DN

$\mathrm{T} 4: 60$

$1-14: 60$

DN

(2:2)

RATE CHECKING

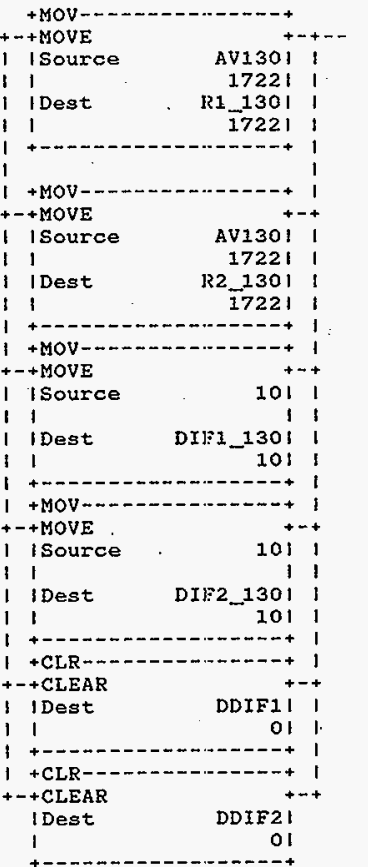


Processor and Data(ops Untt 1 )

Rung $2: 24$

RATE CHECKING

TMR_SU/DN

$T \overline{4}: 60$

DN

$(2: 2)$

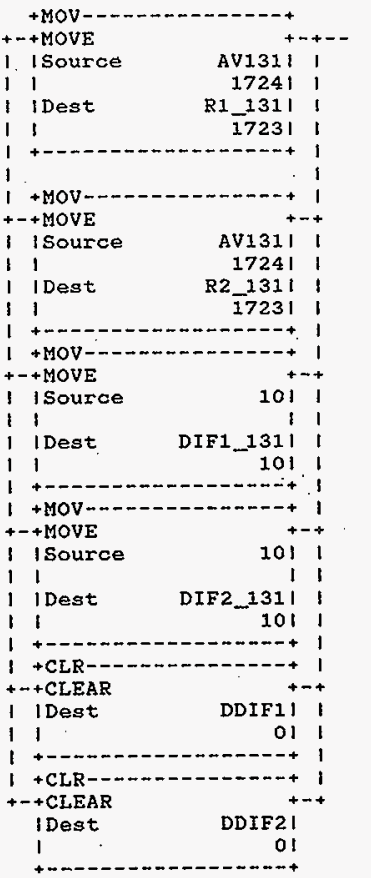

Rung $2: 25$

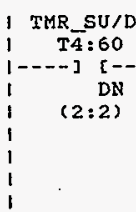

RATE CHECKING 


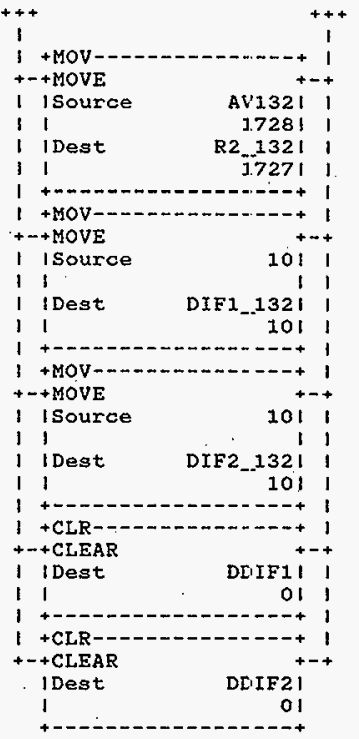

Rung 2:26

\section{TMR $S U / D N$}

RATE CHECKING

T4:60

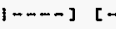

$(2,2)$

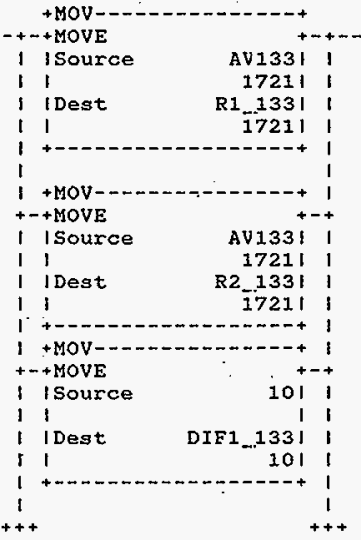

HNF-SD-FF-CSWD-61 Rev. 0 


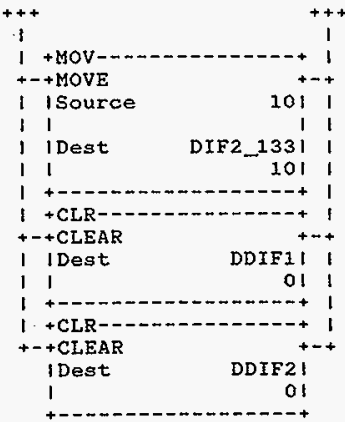

Rung 2:27

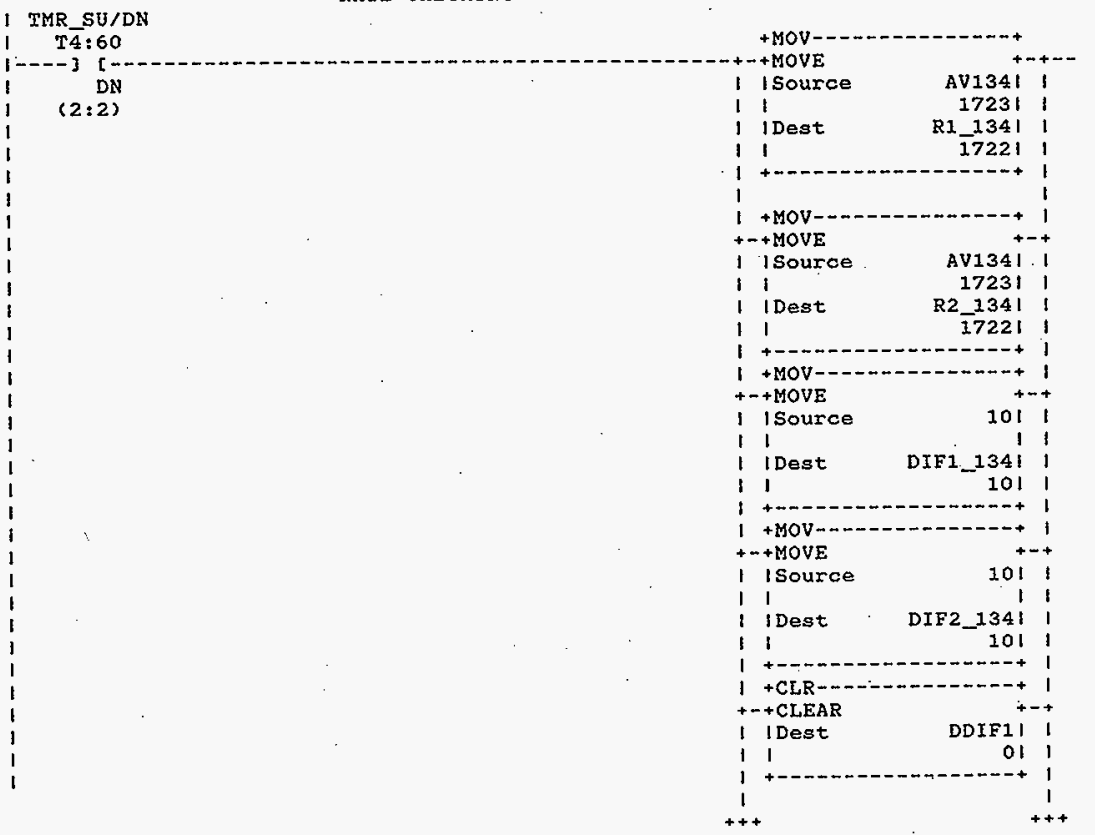


Processor and Data(OPS Unit i)

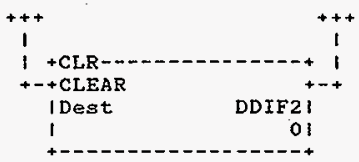

\section{Rung $2: 28$}

RATE CHECKING

\section{TMR_SU/DN}

$T 4: 60$

$1-m-2][$

$(2: 2)$

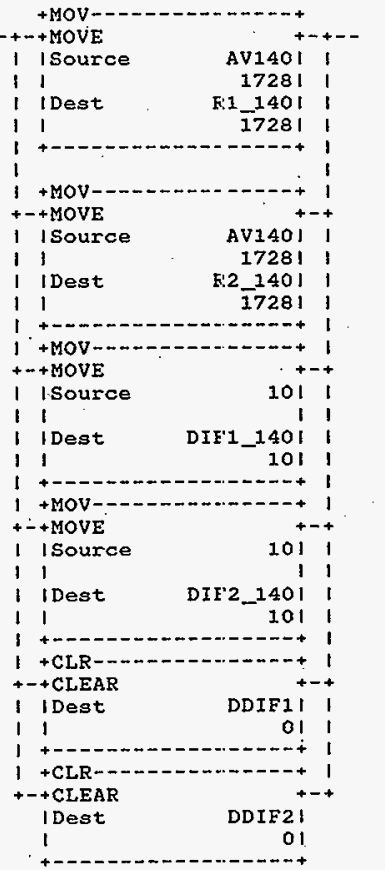

HNF-SD-FF-CSWD-61. Rev. 0 
Processor and Data(OPS Unit 1)

October 23, 1996

Page $2 \hat{2}$

Program Listing

Processor File: SODIUMzA.ACH

Rung 2:29

I TMR_SU/DN

$T 4: 60$

RATE CHECKING

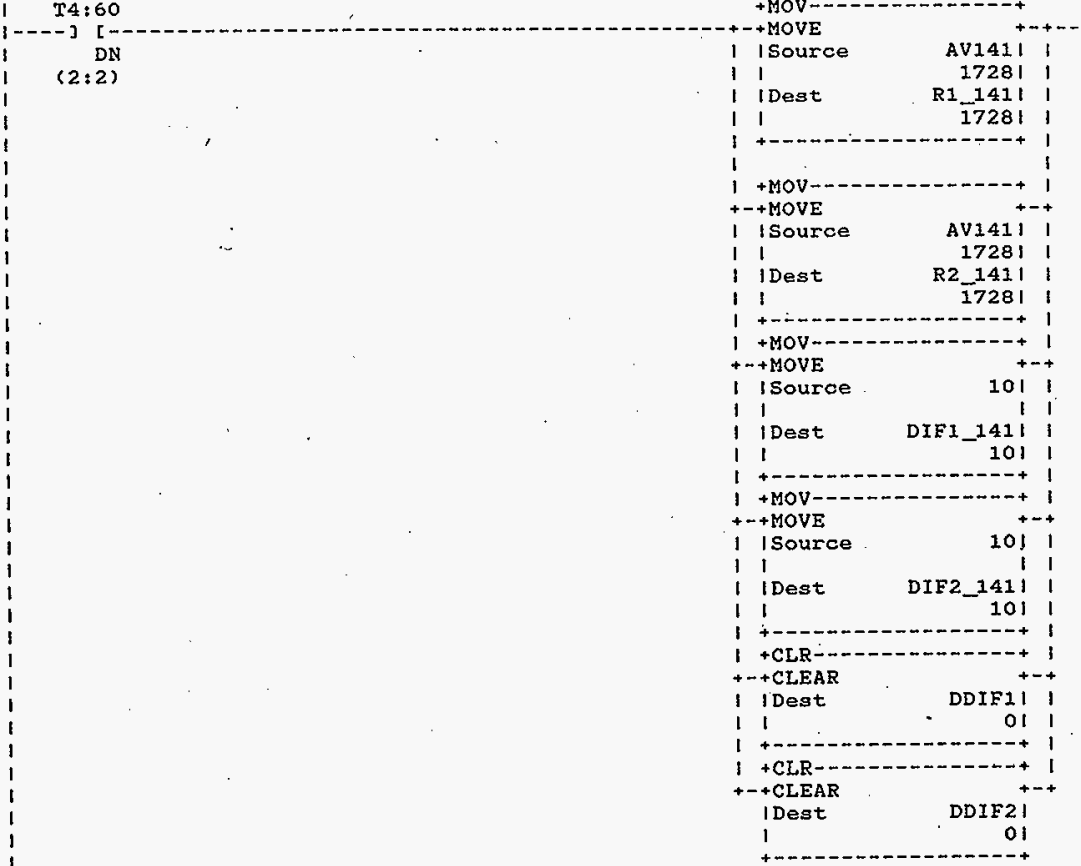

Rung $2: 30$

1 TMR_SU/DN

$1 \mathrm{~T} \overline{4}: 60$

RATE CHECKING

$1----]$ :

$(2: 2)$

HNF-SD-FF-CSWD-61 Rev. 0

Page 22 


$$
\begin{aligned}
& 1 \\
& 1 \\
& 1 \\
& 1 \\
& 1 \\
& 1 \\
& 1 \\
& 1 \\
& 1 \\
& 1 \\
& 1 \\
& 1 \\
& 1 \\
& 1 \\
& 1 \\
& 1 \\
& 1 \\
& 1 \\
& 1 \\
& 1 \\
& 1 \\
& 1 \\
& 1 \\
& 1 \\
& 1 \\
& 1 \\
& 1 \\
& 1 \\
& 1
\end{aligned}
$$

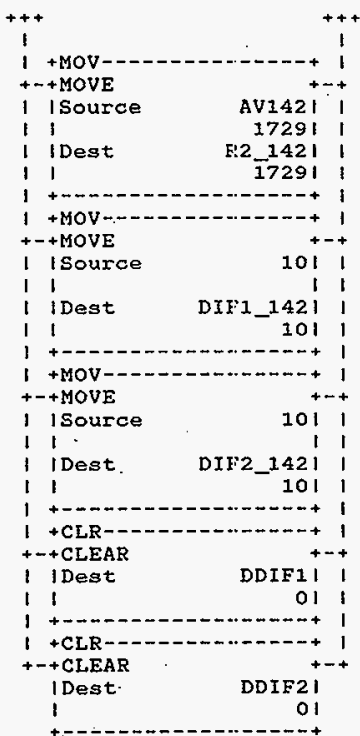

Rung 2:31

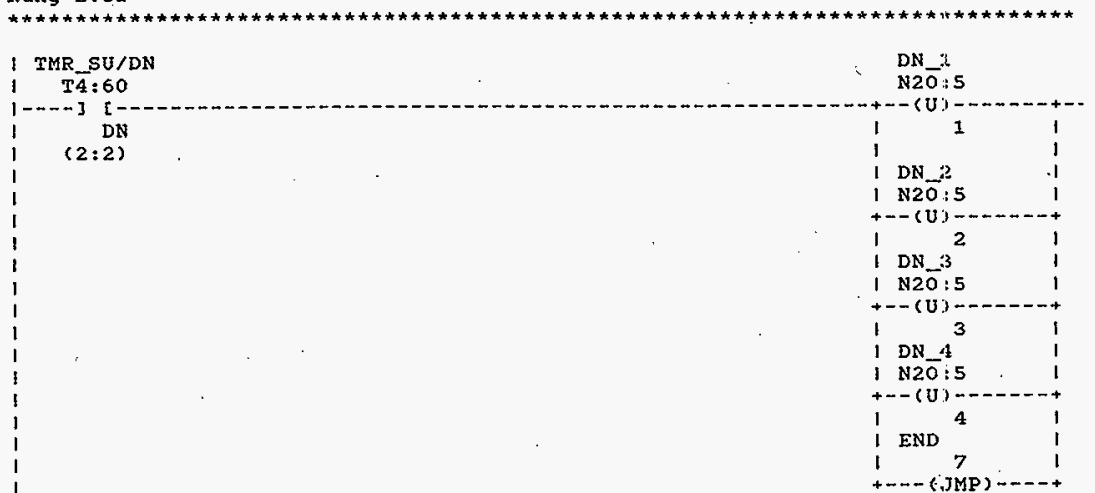




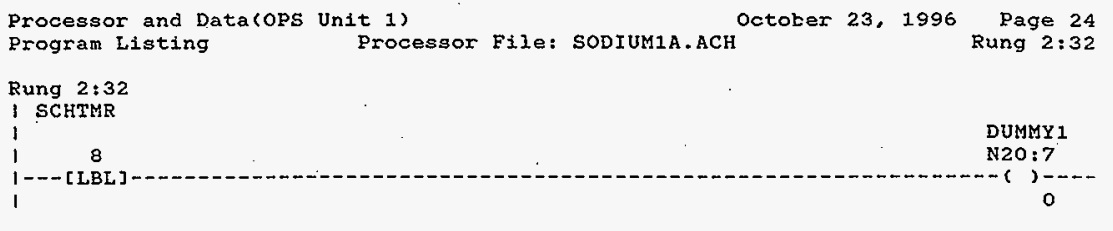

Rung $2: 33$
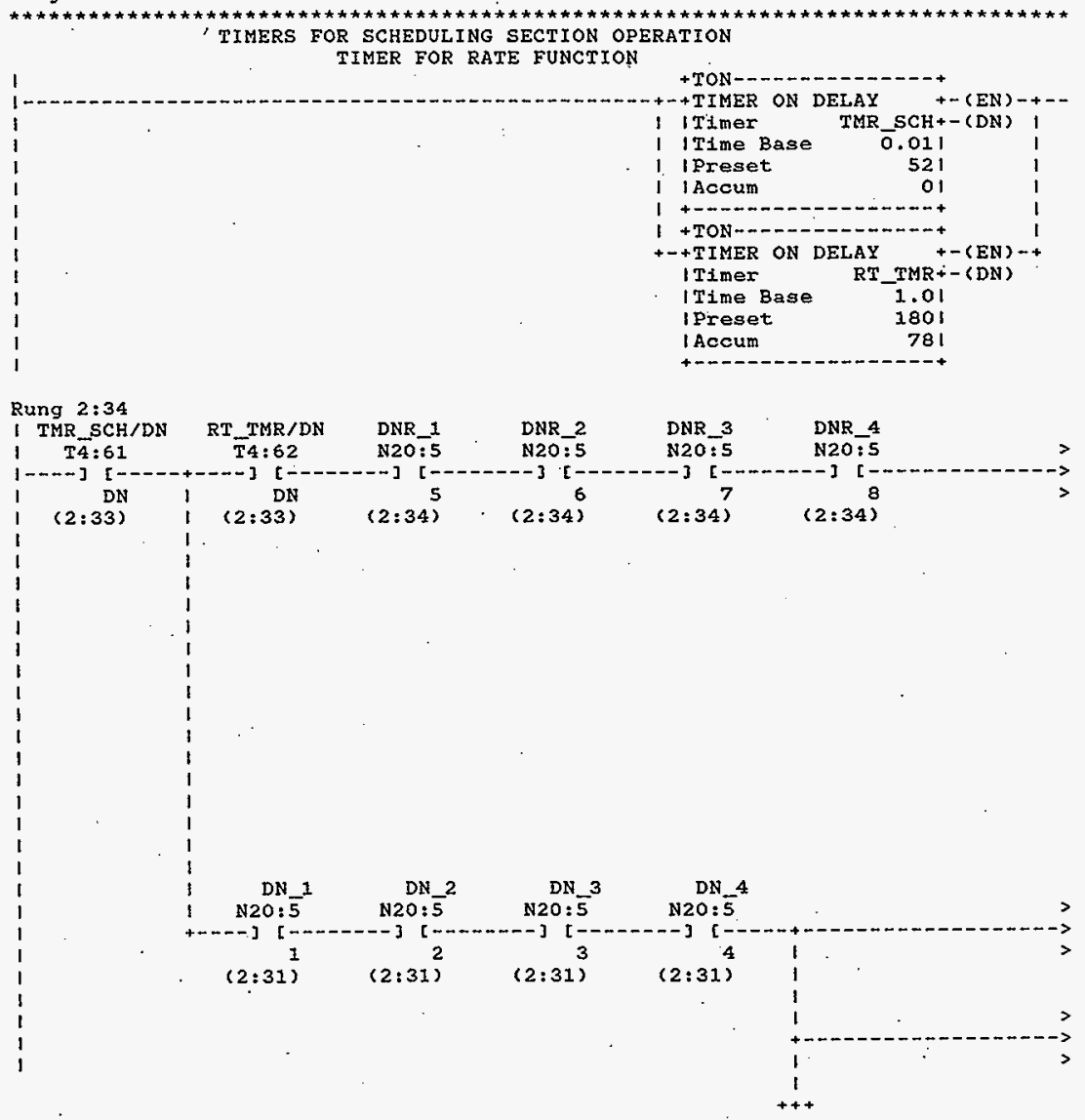
Processor and Data(OPS Unit 1)

Progran Listing
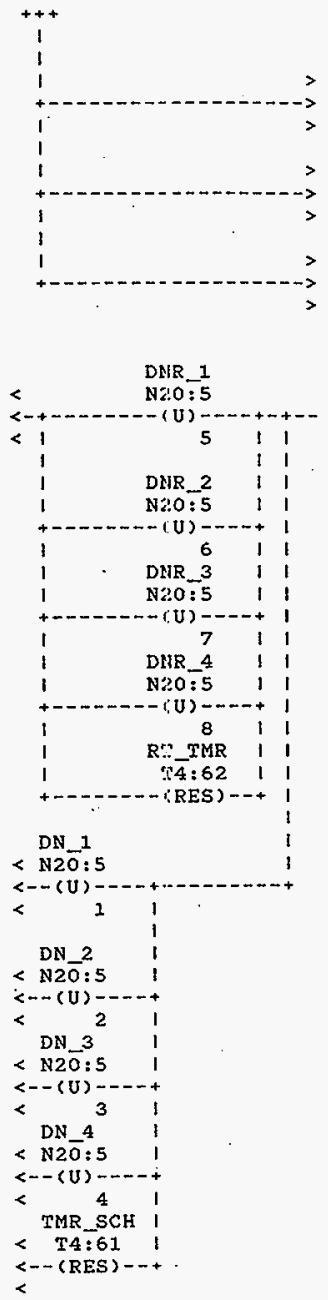

HNF-SD-FF-CSWD-61 Rev. 0 
Rung $2: 35$

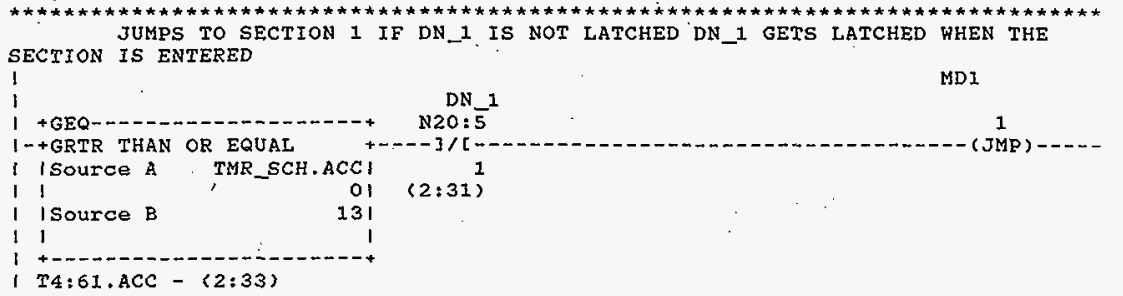

Rung 2:36

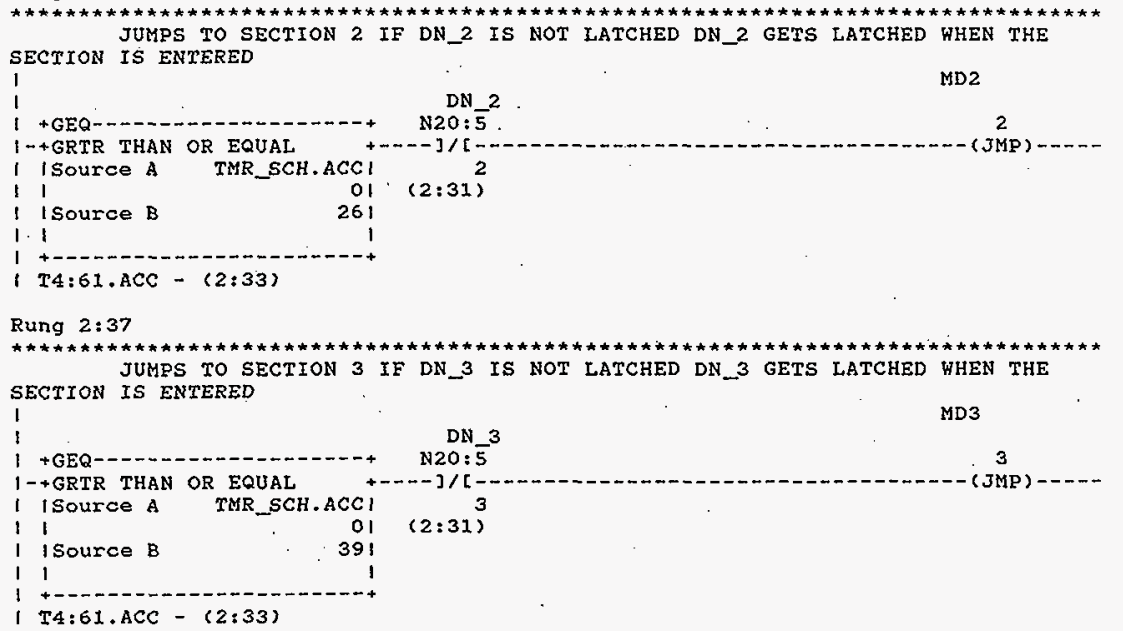

Rung 2:38

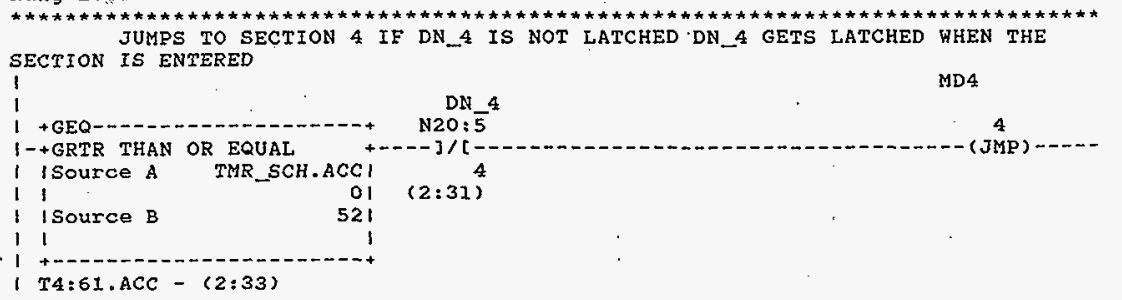


Processor and Data(ops Unit 1 )

Rung $2: 39$

$$
\text { I }
$$

\section{Rung 2:40}

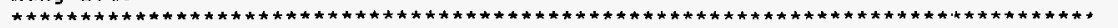

I MD1 BEGIN SECTION 1

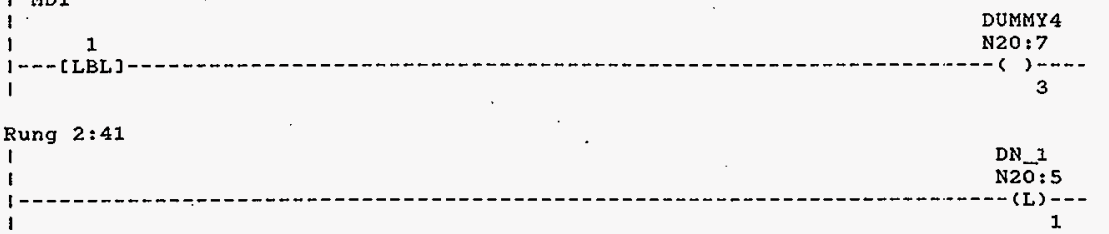

Rung 2:42

Read each $\mathrm{T} / \mathrm{C}$ and store each $\mathrm{T} / \mathrm{C}$ 's value in its own memory register 1 $1-$

$+A D D$
$-+A D D$ 1

isource B

1

I Dest

1

Rung $2: 43$

$1+$ GRT--

1-+GREATER THAN

I ISource A C1IOAI

I I 17421

| Isource B 16383 I

1 I

1 +...--

I N7:1-(2:42)

Ring 2:44

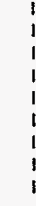

NiT END

TC110A1I

7421

10001

C110A1I

17421
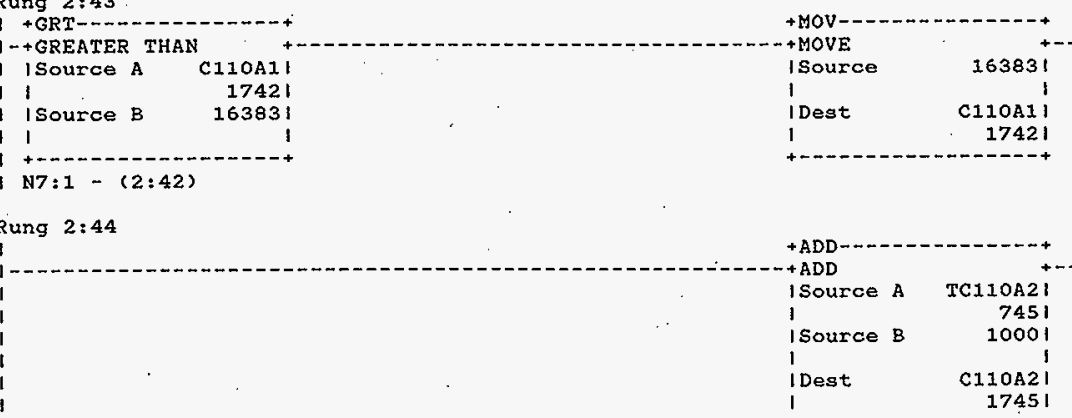

HNF-SD-FF-CSWD-61 Rev. 0 
Processor and Data(OPS Unit 1) Program Listing

Rung 2:45

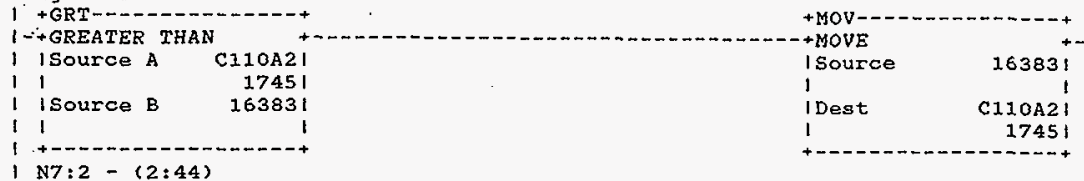

Rung $2: 46$
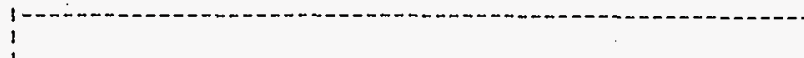

$+A D D$

ISource A TCI10A3|

I

15ource B

7441

1

I Dest

1000

C130A31

$$
1
$$

Rung $2: 47$
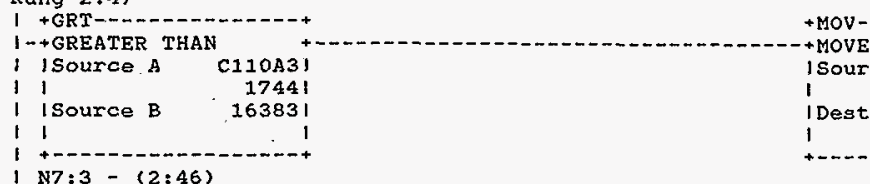

Rung $2: 48$

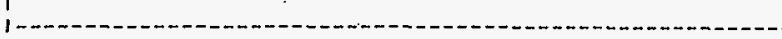

$+A D D$

ISource A TC110A4|

741

ISource B

1000

i Dest

C110A4

1

17411

Rung 2:49

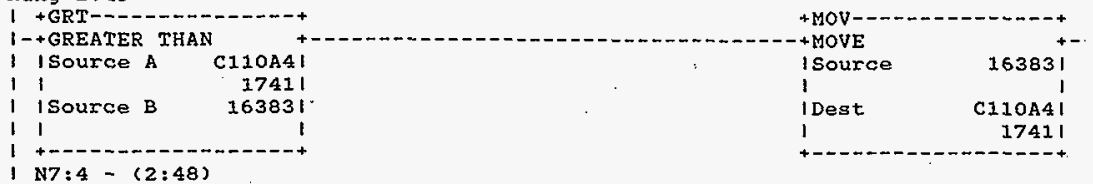


Processor and Data(OPS Unit 1)

Program Listing

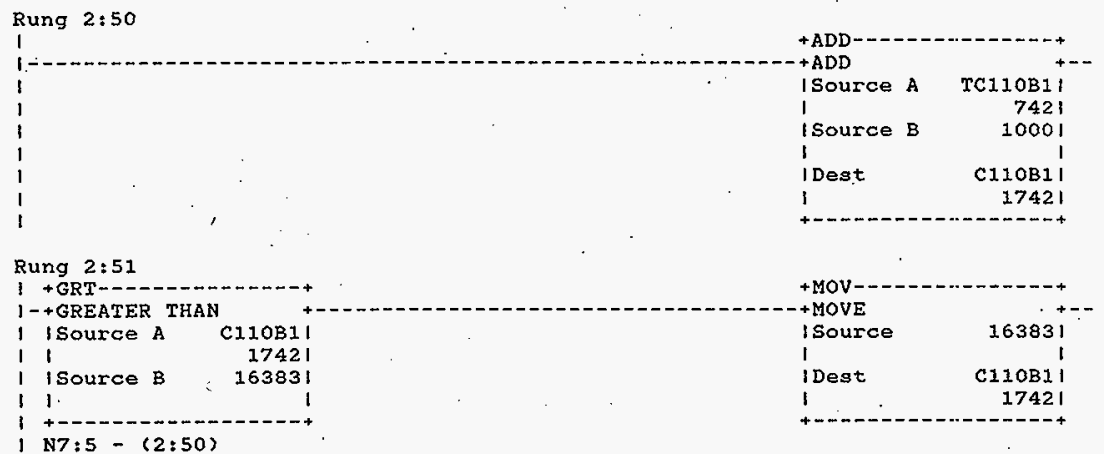

Rung 2:52

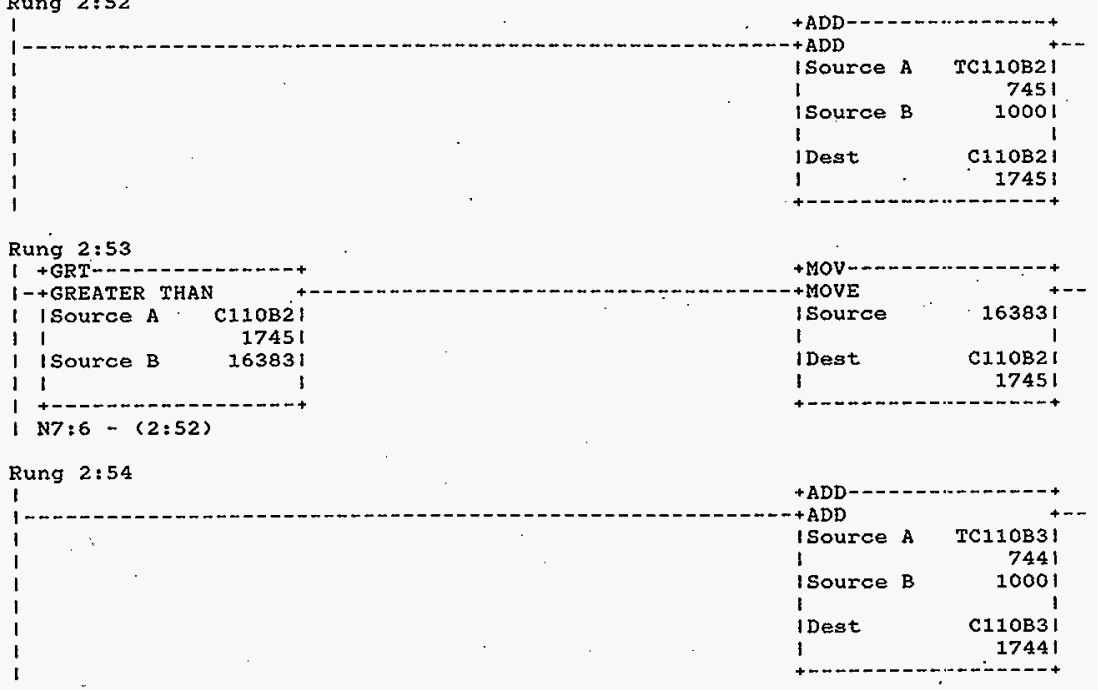

3. 
Processor and Data(OPS Unit 1)

October 23, 1996

Page $3 C$

Program Listing

Processor File: SODIUMzA.ACH

Rung $2: 55$

\section{Rung $2: 55$}

$1+$ GRT-.-....-.-.

I-+GREATER THAN

I ISource A C110B3I

I 17441

I ISource B 163831

11

163831

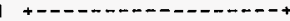

I N7:7-(2:54)

Rung 2:56

,$$
1-
$$$$
\text { i }
$$$$
\text { i }
$$$$
1
$$$$
1
$$$$
\text { , }
$$

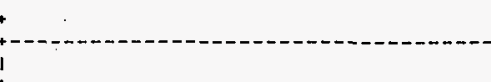

$+$

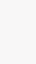
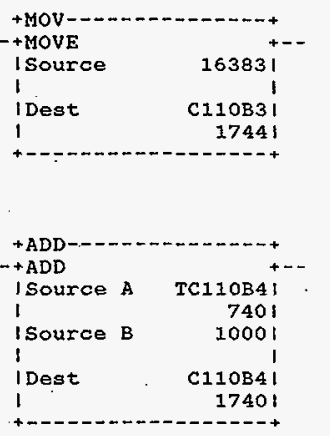

Rung $2: 57$

I +GRT------D.-------

I-+GREATER THAN

I ISource A C11OB4I

11 17401

| |Source B 16383 |

I. 1 ।

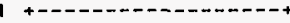

1 N7:8 - (2:56)

Rung 2:58

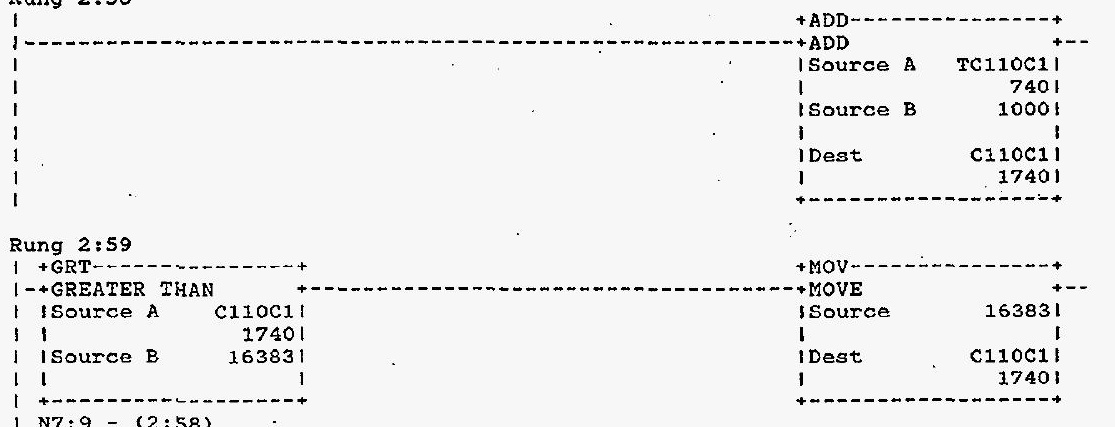

HNF-SD-FF-CSWD-61 Rev. 0

Page 30 
Processor and Data(OPS Unit 1)

Rung 2:60

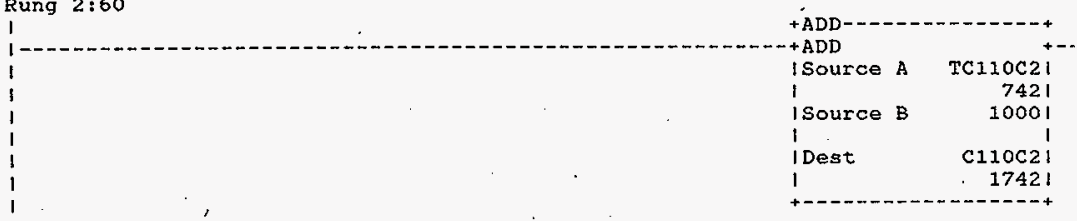

Rung $2: 61$

1 +GRT--D-..--.---- +

1-+GREATER THAN

I ISource A C110C2!

1 I $\cdot 17421$

| Source B $16383 \mid$

11 I

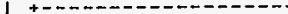

I N7:10-(2:60)

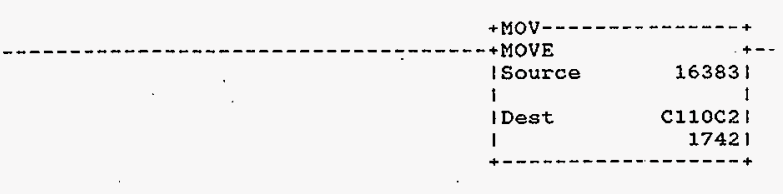

Rung 2:62

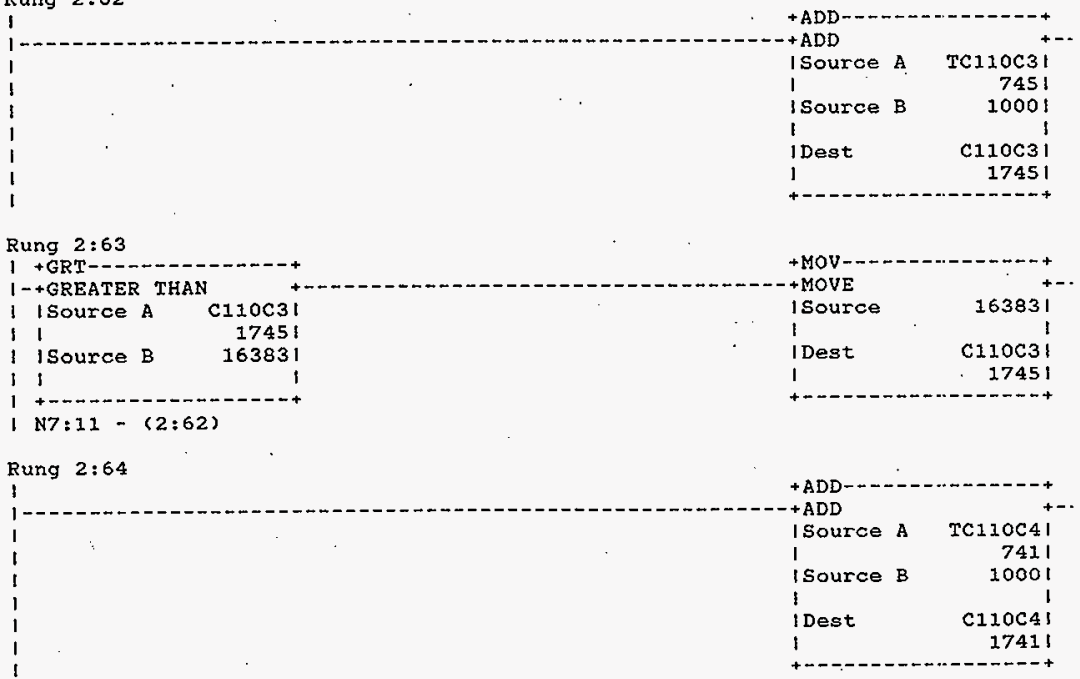


Processor and Data(OPS Unit I) Program Listing

Processor File: SODIUM1A.ACH

\section{Rung 2:65}

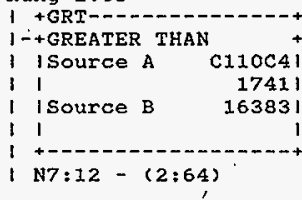

Rung $2: 66$

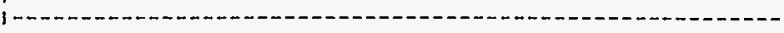

1

$+A D D$

ISOurce A

TC111AII

I Source B

7521

i Dest

10001

Rung 2:67

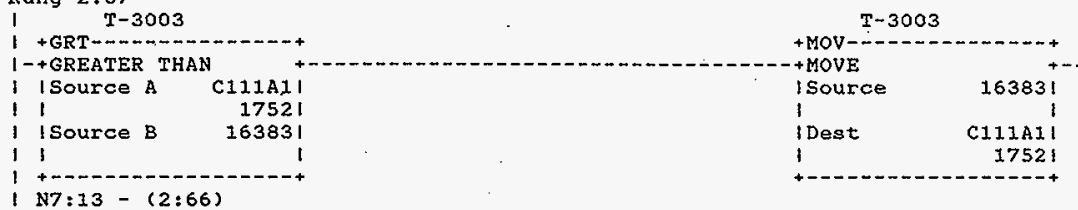

Rung $2: 68$

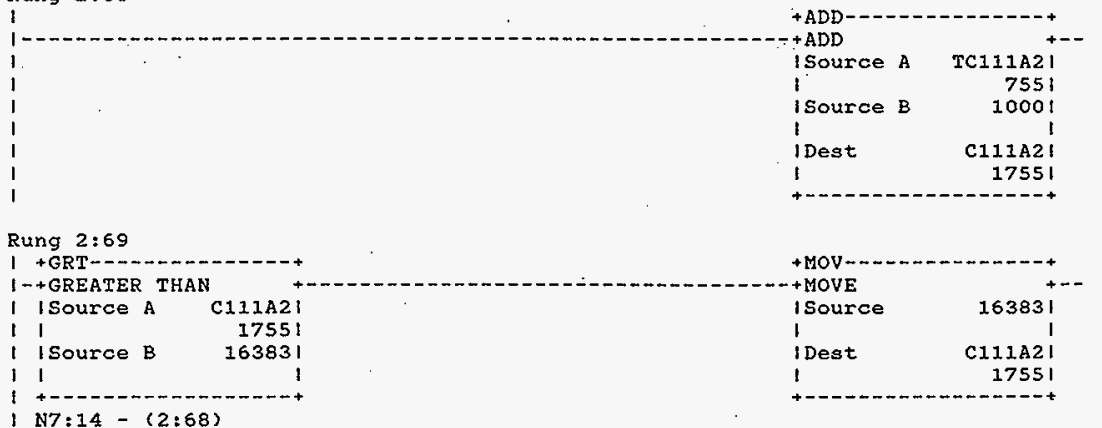

HNF-SD-FF-CSWD-61 Rev. 0 


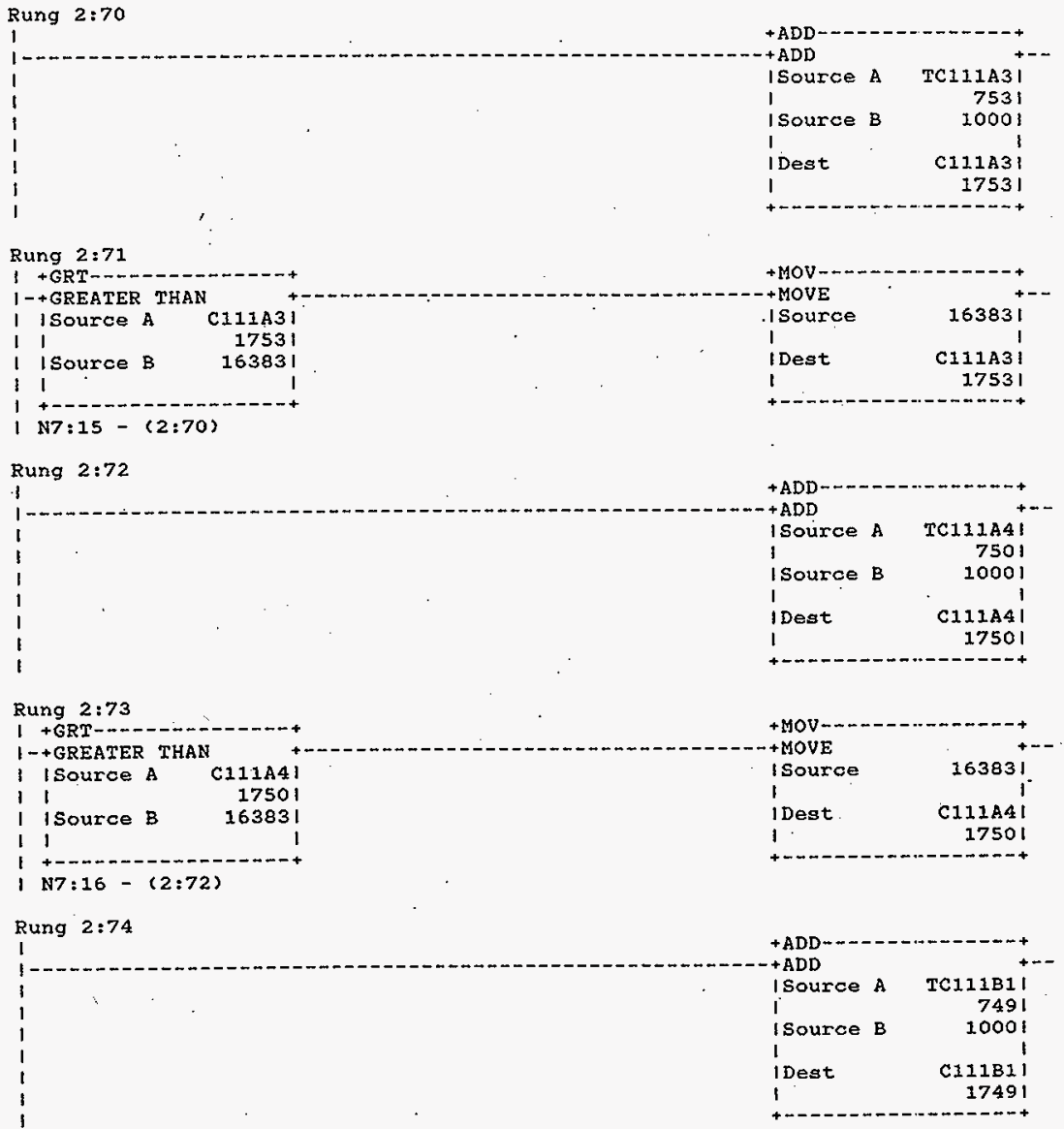


Processor and Data(OPS Unit 1)

Rung 2:75

I +GRT--

1 -+GREATER THAN

I ISOUICe A CIIIBI!

I 1 17491

I ISource B 16383|

i 1

163831

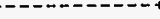

.

IN7:17-(2:74)

Rung 2:76

I

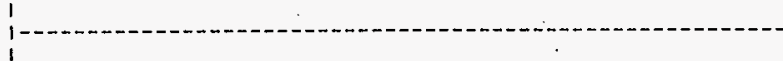

$+\mathrm{ADD}-$

$+A D D$

| Source A TC111B2|

I Source B

I

I Dest

10001

I

C111B21

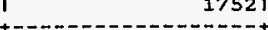

Rung $2: 77$

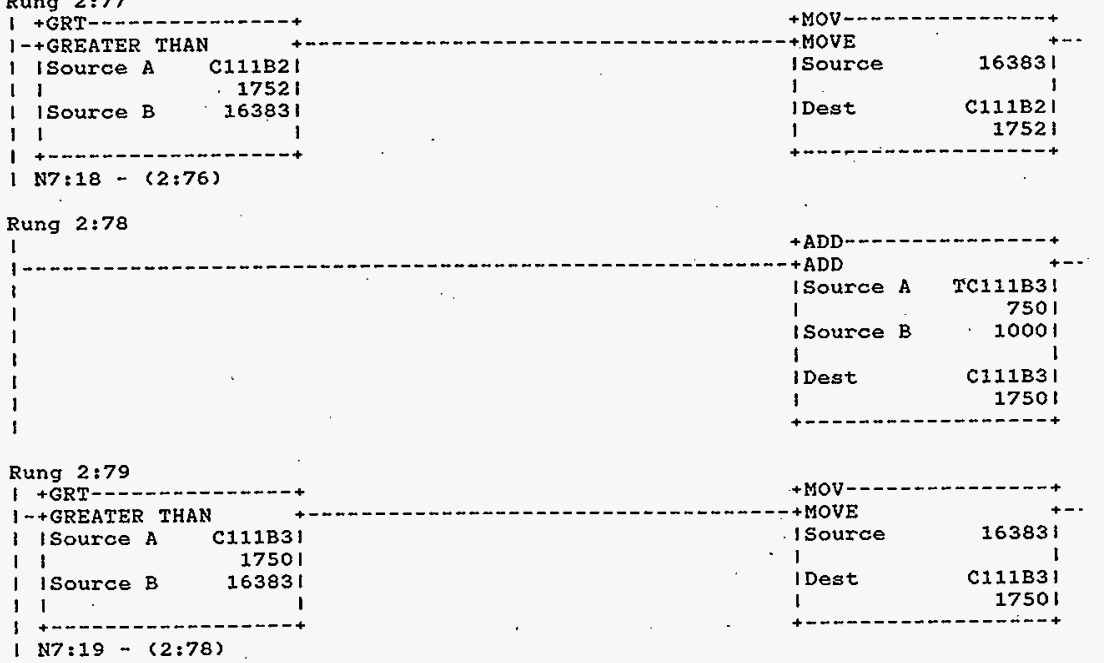

HNF-SD-FF-CSWD-61 Rev. 0 
Processor and Data(OPS Undt 1 )

October 23, 1996

Page 3 ! Program Listing

Rung 2:80

2.

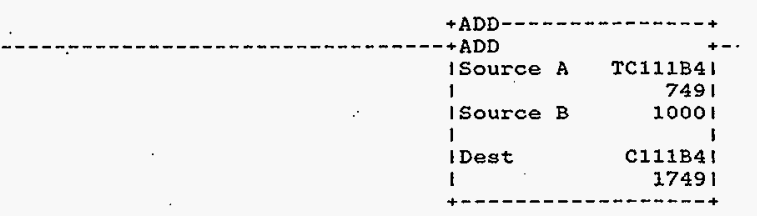

Rung 2:81

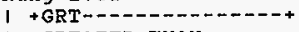

1-+GREATER THAN

1 ISource A

11

17491

ISource B 163831

11

16383

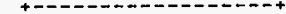

( N7:20-(2:80)

Rung $2: 82$

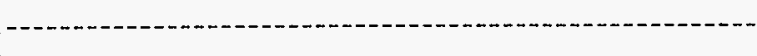

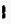

$+$

$+\mathrm{MOV}-$

MOVE

I Source

163831

i Dest

C111B4:

1

17491

1749

$+A D D$

I Source

A

TC1

I Source B

I Dest

1

C111C1I

17471

Rung $2: 83$

1 +GRT--

I-+GREATER THAN

| |Source A C11IC11.

i i 1747 |

| ISource B 163831

11

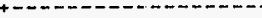

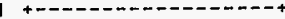

I N7:21-(2:82)

Rung $2: 84$

$$
\begin{aligned}
& 1 \\
& 1 \\
& 1 \\
& 1 \\
& 1
\end{aligned}
$$

HNF-SD-FF-CSWD-61 Rev. 0 
Processor and Datalops Unit 1) Program Listing Processor File: SODIUM1A.ACH

October $23,1996 \quad$ page $3 \xi$

Rung 2:85

$1+$ GRT---D...-----

$1-+$ GREATER THAN

I ISource A C111C2

I isource B 16383 I

Rung $2: 85$

$\begin{array}{ll}1 & 1 \\ 1 & 1\end{array}$

I $N 7: 22-(2: 84)$

Rung $2: 86$
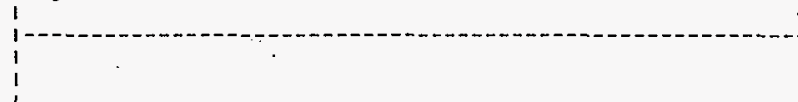

(1)

$$
1
$$

t.

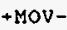

17521

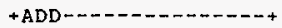

+ ADD

|Source A TC111C31

I 7491

Isource B 1000!

1

I Dest

C111C3!

I

17491

Rung $2: 87$

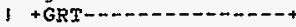

1-+GREATER THAN

I ISource A C111C31

I I 17491

I 1Source B 163831

1 1 1

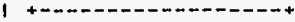

I N7:23-(2:86)

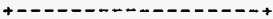

\section{Rung 2:88}$$
\text { I }
$$

1

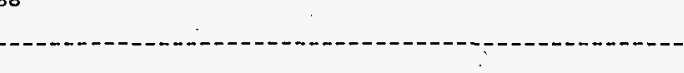

$+A D D$

ADD

+ MOV-
+ MOVE

isource

1

I Dest

I

$+$

I Source A

ISource B

(11C4I

7511

i

I Degt

10001

I

C111C4:

Rung 2:89

$1+$ GRT-_............

1-+GREATER THAN

I ISource A

C111C4I

11

17511

I ISource B 16383 |

11

1

+
+
1
+

+ MOV-

MOVE

isource

1

I Dest

$-0 .+$

I N7:24-(2:88)

HNF-SD-FF-CSWD-61 Rev. 0

Page 36 
Processor and Data(ops Unit 1)

Rung $2: 90$

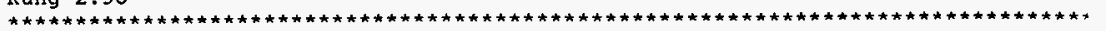

For each T/C read itg STATUS BITS to detect T/C problems HARDWARE ALARMS FOR TANK T-3002

\section{T/C ENABLE}

$I: 3$

HA11OA1

$1: 3$
]$/[$

N7: 240

1
$-+-0-0]$

I OPEN $T / C$

$I: 3$

I

+.-- ] [-..-.

1

I IO IIMIT

1 I:3

+-.-- ] [-.---i

$1 \quad 77$

I HI LIMIT

I I: 3

+--- $1-\cdots+\cdots$

$178 \quad 1$

I CNFG ERROR

i. 1

11 I:3

$1+\ldots-0]$

79

Rung 2:91

I

T/C ENABLE

HA110A2

N7 : 240

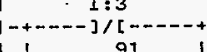

OPEN T/C

$I: 3$

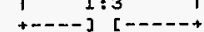

192

1 LO LIMIT

$1 \quad I: 3$

+.-- ] [

193

i HI IIMIT

1 I 3

+--.-] [--..-.

194

I CNFG ERROR ।

i. 1

I

$I: 3$

$+\cdots-3$ [ $1-2--+$

95 
Processor and Data(OPs Unit 1)

Rung 2:92

I/C ENABLE

I.

$1 \quad \mathrm{I}: 3$

$I: 3$

HA110A3

1
$1-+----] /[-$

OPEN $\mathrm{T} / \mathrm{C}$

$1 \quad I: 3$

1108

1 I LO LIMIT

$11 \quad 1: 3$

+----] [-m.-- +

1109

I HI LIMIT

1 I I:3

$1+---0]$ [----

I I CNFG ERROR ।

I)

I $1 \quad I: 3$

$1+----][----n+$

1111

Rung 2:93

$1 \mathrm{~T} / \mathrm{C}$ ENABLE

1

1 I:

$1-+\cdots-7 / 2-123$

1 OPEN $T / C$

I $\quad I: 3$

$+\ldots-0][-\ldots+$

12124

1 LO LIMIT

I $I: 3$

.... ] [ [-...

1125

) HI LIMIT

$1 \quad I: 3$

+----] [-n.--+

I 126 ।

CNEG ERROR I

11

I $I: 3$

127

Rung 2:94

I $T / C$ ENABLE

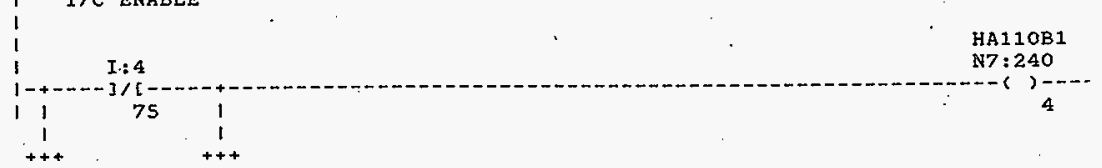




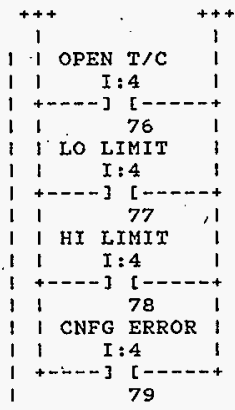

\section{Rung 2:95}

1 T/C ENABLE

$1 \quad I: 4$

++
1
1
+
1
1
1
+
1
1
+
1
1
1
+

$1-+---3 / 2-$

l I OPEN $\mathrm{T} / \mathrm{C}$

$11 \quad x: 4$

$1+---]$ [-..--

$1192 \quad 1$

1 IO LIMIT 1

$11 \quad \mathrm{I}: 4$ ।

$1+----]$ [

| 1 HI IIMIT |

| 1 HI IIMIT

$1+\cdots--][---\cdots+$

11 ।

I I CNEG ERROR |

11 I I:4

$1+----]$ [--.--t

195

Rung 2:96

1 T/C ENABEE

$1 \quad I:$

HA $110 B 3$

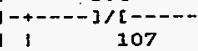

I OPEN T/C

1 I I:4

$1+\ldots-n][---\cdots+$

। 108

1 LO LIMIT

11 I:4

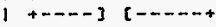

11109

I

$+++$

$109 \quad 1$ 
Processor and Data (OPS Unit 1 )
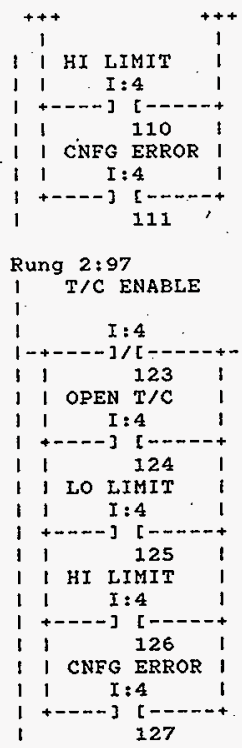

\section{Rung 2:98}

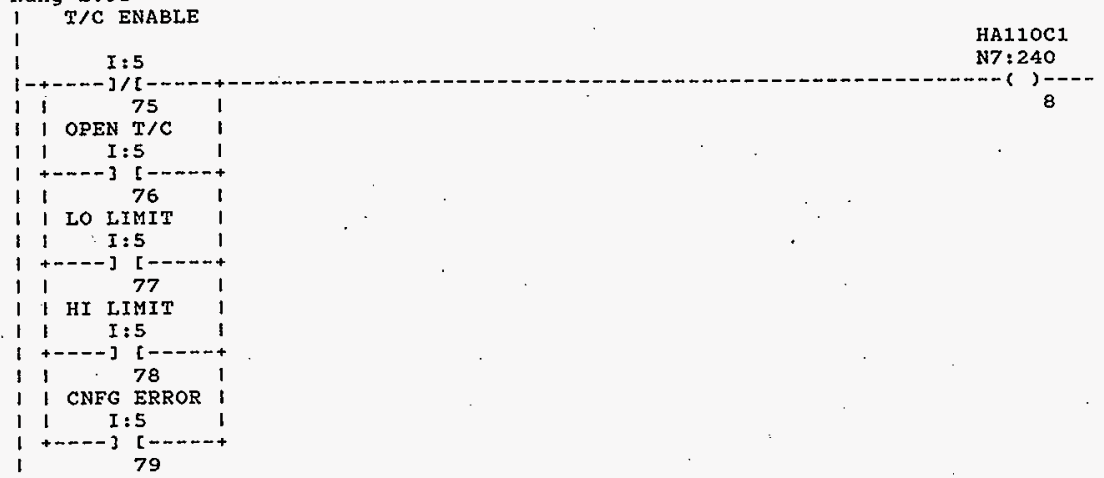


Processor and Data(OPS Unit 1)

Rung 2:99

1. $T / C$ ENABLE

$1 \quad I: 5$

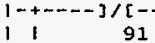

1 I OPEN T/C

1 I 5

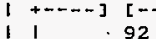

1 I LO LIMIT

I I I:5

$1+\ldots . .].[-\ldots+$

1193

1 HI LIMIT

1 I I:5

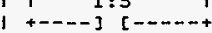

1194

I I CNFG ERROR

$1 \quad \mathrm{X}: 5$

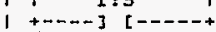

I 95

\section{Rung 2:100}

T/C ENABLE

$x: 5$

107

OPEN $\mathrm{X} / \mathrm{C}$

$\mathrm{I}: 5$

$1+-100$

100

1 LO LIMIT

1 1 $1: 5$

$1+\cdots--3$ t

11.109

I HI LIMIT

1 I:5

$1+\cdots-.-0][----+$

11110

I CNFG ERROR

l I I:5

1 +-.-- ] [-.....

III

Rung 2:101

I T/C ENABLE

I. $I: 5$

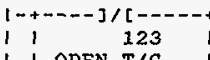

1 I OPEN $T / C$

1 I:5

$1+\cdots . .-1]$

I I 124

124

$\begin{array}{ll}\text { HA110C2 } & \\ & \text { N7:240 }\end{array}$

HAIIOC3

N7: 240

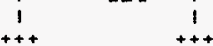

HA110C4

N7 $: 240$ 
Processor and Data(OPS Unit 1)

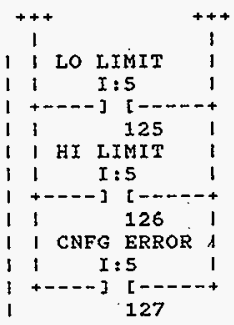

Rung 2:102

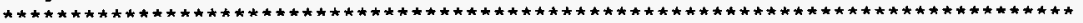
for each T/C read its STATUS BITS to detect T/C problems

T/C ENABLE HARDHARE AIARMS FOR TANK T-3003

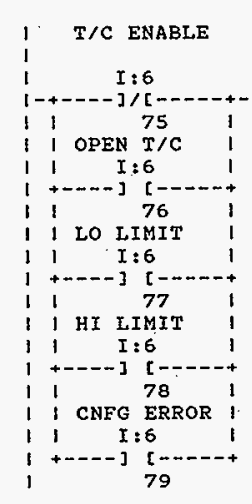

HA111A1

N7: 240

(1)

Rung 2:103

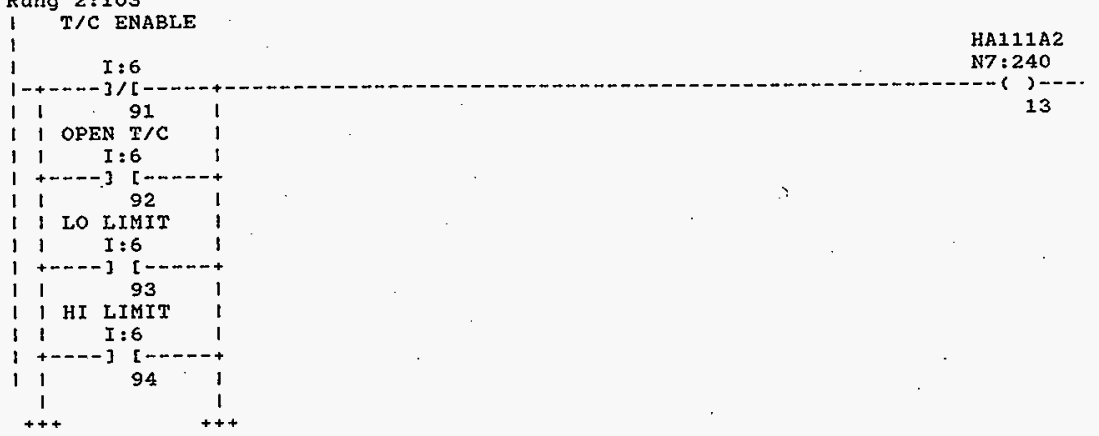


Processor and Data(OPS Unit 1$)$

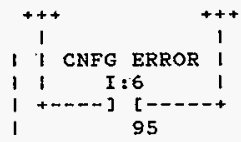

Rung 2:104

1 T/C ENABLE

1 TIC ENABLE,

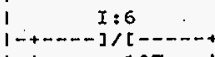

$107 \quad 107$

OPEN $T / C$

$I: 6$

$+\ldots-n][---n+$

1108

1 LO IIMIT

$1 \quad I: 6$

+--- ] [-- ....

$1 \quad 109$

I HI LIMIT

1 I: 6

$1+----][-\ldots-n+$

1110

1 CNFG ERROR ।

1 I: 6

1 +--.-3 [-....+

111

Rung 2:105

I T/C ENABLE

I $I: 6$

HA111A4

N7: 240

11.23

1 OPEN $T / C$

$11 \quad I: 6$

+--n] [--.--t

12124

1 LO LIMIT

$11 \quad I: 6$

$1+\ldots-.+3[----.+$

I. 1125

1 HI LIMIT

1 I:I:6

$1+\cdots-m]$ [--n-

11126

I CNFG ERROR

1 I $I: 6$

+----] [--.--

$1 \quad 127$ 
Processor and Data(ops Unit 1)

Program Listing

Rung 2:106

$1 \quad T / C$ ENABLE

1 HA111B1

$1 \quad I: 7 \quad$ N7:241

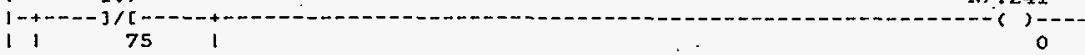

1 OPEN $T / C$

$11 \quad I: 7 \quad 1$

$1+----3[---\infty+$

$1176 \quad 1$

1 I LO LIMIT

$1+----][----+$

$1177 \quad 1$

1) HI LIMIT

$11 \quad I: 7$

$1+-\cdots-7$ [ $1-\cdots+-$.

i I CNFG ERROR

1 I:7

$1+---7\left[\begin{array}{c}1 \\ 1\end{array}\right.$

79

Rung 2:107

1 T/C ENABLE

$1 \quad I: 7$

HA1 $11 \mathrm{~B} 2$

$1-+----7 /[$

1 1. OPFN 91

11 I:7

$1+\ldots+\ldots]$

I 92

1 LO IIMIT

$11 \quad I: 7$

$1+\cdots--][-\cdots--+$

$193 \cdot 1$

1 HI IIMIT

$11 \quad I: 7$

$1+\cdots--3$ [-.--.+

I $94 \quad 1$

I I CNFG ERROR ।

$11 \quad I: 7$

$1+-\infty-\infty][-\ldots-n+$

I 95

Rung 2:108

1 T/C ENABLE

1 I :

I : 7

HA111B3

$110+--3 /[--\ldots+\cdots$

N7:241

1 OPEN T/C

$11 \quad 1: 7$

$1+---7$ [--...

11108

1

$++$

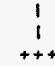

N7: 241 


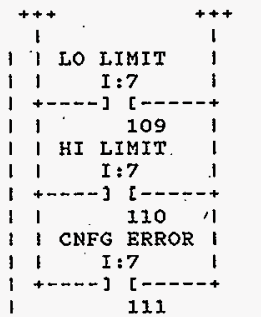

Rung $2: 109$

1 T/C ENABLE

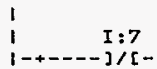

HA111B4

$7 / 2-$

N7: 241

11123

OPEN $\mathrm{T} / \mathrm{C}$

$I: 7$

1 +---$]$ [

11124

I 1 . LO LIMIT

$11 \quad I: 7$

$1+---3$ [-.--

11125

1 HI IIMIT

11 I:7

1 +--n] [--n-m+

1 126

1 I CNFG ERROR I

I 1 I $: 7$

I +-..-.] [----n+

127

Rung 2:110

1 T/C ENABLE

$: 8$

HA111CI

N7 $: 241$

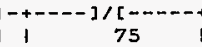

OPEN $T / C$

$I: 8$

\begin{tabular}{lll}
1 & $+---]$ & {$[--\cdots+$} \\
\hline & 76
\end{tabular}

1 LO LIMIT

$1 \quad I: 8$

+---- ] [---- +

$1 \quad 77$

I HI LIMIT

1 I: 8

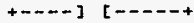

I 78

I CNFG ERROR

I $I: 8$

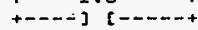

79

HNF-SD-FF-CSWD-61 Rev. 0

Page $4 s$ 
Processor and Data Cops Unit 1) Program Listing

Rung 2:111

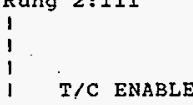

T/C ENABLE

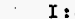

$\mathrm{I}: \mathrm{8}$

HAI $11 \mathrm{C2}$

$1-+\ldots+3 / 2$

91

OPEN $T / C$

$I: 8$

+---- ]

1192

I LO LIMIT

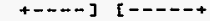

1193

1 HI LIMIT

11 I:8

$1+---\infty$ ] [ $\ldots \ldots+--+$

11

1 CNFG ERROR

I I I:8

$1+\ldots . .7[-\ldots+$

I 95

Rung 2:112

I T/C ENABLE

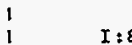

$\begin{array}{ll}1 & I: 8 \\ 1-+-\infty & 1 /[---\infty\end{array}$

I I 107

I 1 I:8

1 +----] [ $[-\ldots+$

11108

1 LO. LIMIT

$11 \quad I: 8$

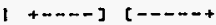

11209

1 I HI IIMIT

$11 \quad I: 8$

$1+-\ldots]$ [-..-.

11110

I I CNFG ERROR

11 I $1: 8 \quad 1$

I $+----3[11$

Rung 2:113

$1 \quad I: 8$

$\mathrm{HA} 111 \mathrm{C} 4$

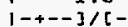

N7:241

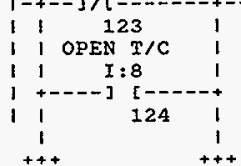

HNF-SD-FF-CSWD-61 Rev. 0 
Processor and Data(OPS Unit 1)

October 23, 1996; Page 4:

Program Listing

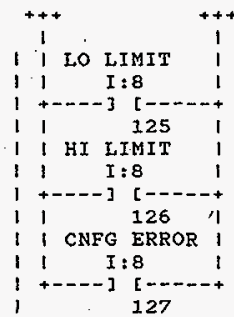

Rung 2:114

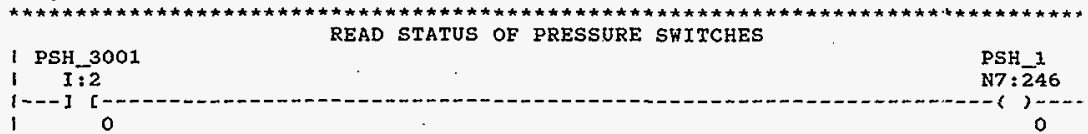

$1=-1$ [ 0

Rung $2: 115$

I PSH 3002

$1 \quad I: 2$

$1-\cdots-1$ [

PSH 2

N7: 246

1. 1

1

Rung 2:116

I PSH_3003

1 I:2

$1--0$ [

N7: $\overline{2} 46$

Rung 2:117

I PSH_3004

$1 \quad 1: 2$

$1---]$ [.

PSH 4

3

Rung 2:118

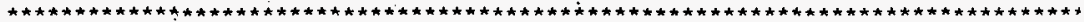
SET DEVIATION SHITCH FOR SECTION I AND JUMP TO SECTION 1 AVERAGING ROUTINE 1 $1:$ : N20:6

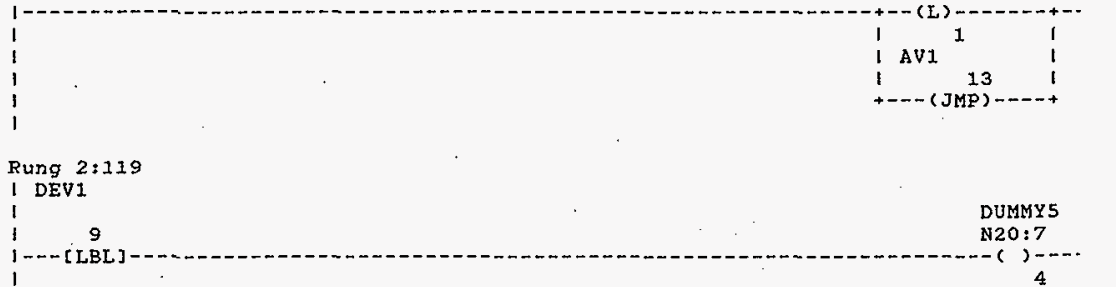

HNF-SD-FF-CSWD-61 Rev. 0 
Rung $2: 120$

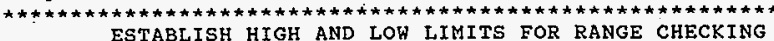

IF NO HARDWARE ALARMS EXIST CHECK T/C VALUES FOR OUT-OF-RANGE CONDITION

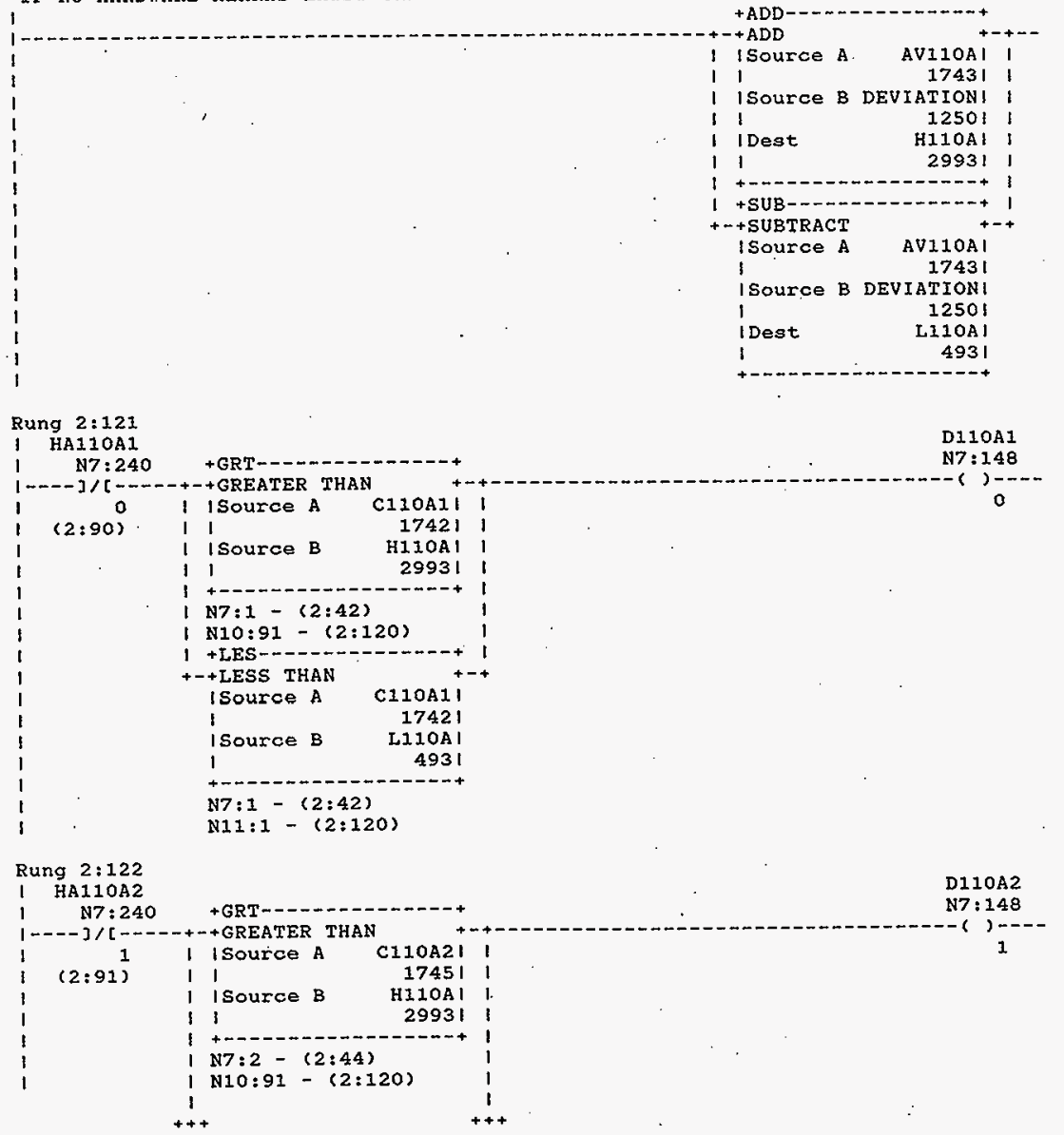

HNF-SD-FF-CSWD-61 Rev. 0 
Processor and Data(OPS Unit 1)

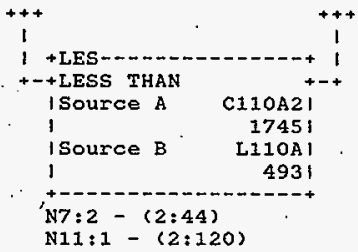

Rung 2:123

I HA110A3

( N7:240

N11:1-(2:120)
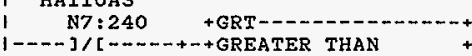

Rung 2:124

I HA110A4 I N7:240 1 ----3/[----+-+GREATER THAN

I 3 I ISource A C110A4I

$(2: 93)$

I. I

17411

I Isource B HiloAI

11

29931

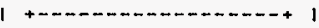

N7:4-(2:48)

(N10:91-(2:120)

1 +LES- - $-\ldots \ldots+\ldots+\ldots+1$

+-+ LESS THAN

ISource A C11OA41

I

17411

| Source B LI10A I

I 4931

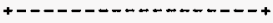

N7:4-(2:48)

N11:1 - (2:120) 
Processor and Data(OPS Unit 1)

October 23, 1996 Page 5C Program Listing

Rung $2: 125$

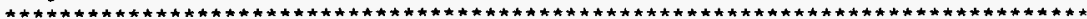
ESTABLISH HIGH AND LOW LIMITS FOR RANGE CHECKING

IF NO HARDWARE ALARMS EXIST CHECK T/C VALUES FOR OUT-OF-RANGE CONDITION

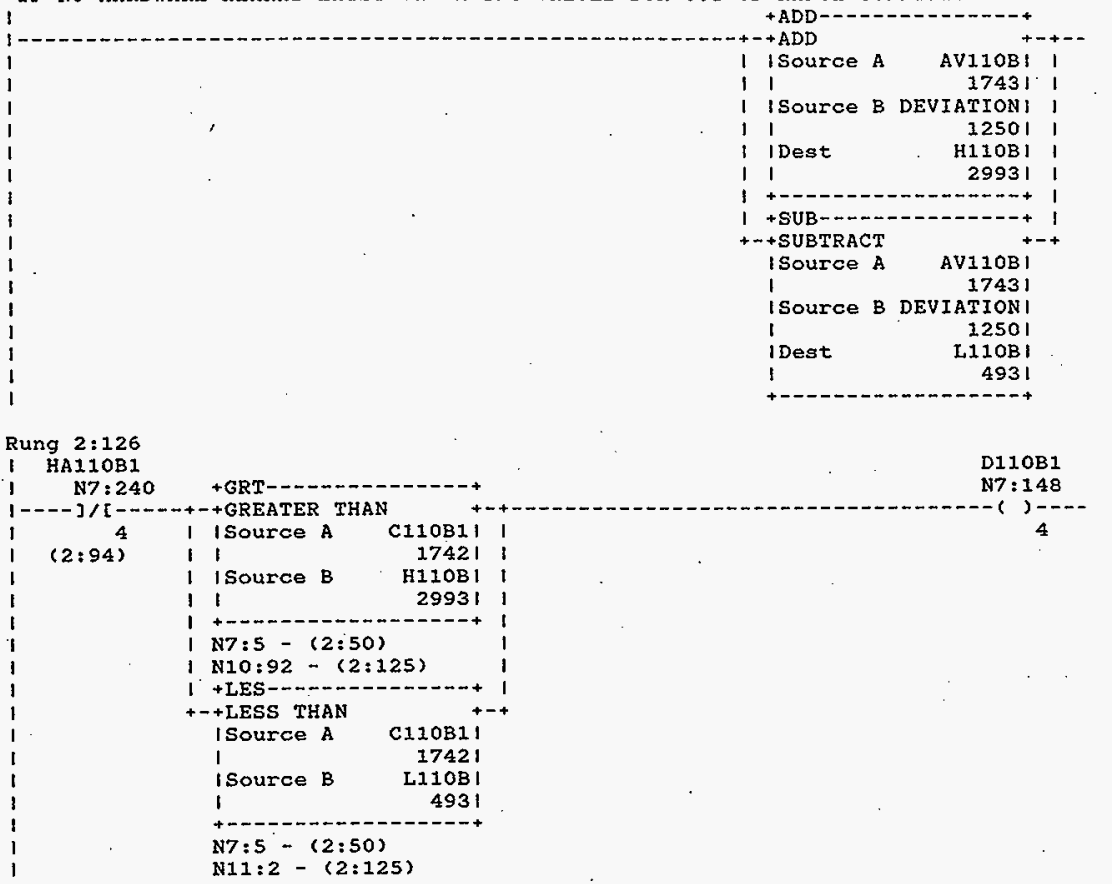

Rung 2:127

1 HA110B2

$1 \quad$ N7:240

$++A D D$

|Source A AV110B! I

I Dest

$1+$ SUB -

++ SUBTRACT

Source A

isource

IDest

4931

D110B1

N7: 148

D110B2 N7: 148

+GRT--

( ) - -

\begin{tabular}{|c|c|c|c|c|c|}
\hline 5 & i & ISource & A & C110B2I & I \\
\hline \multirow[t]{8}{*}{$(2: 95)$} & 1 & 1 & & 17451 & 1 \\
\hline & 1 & ISource & B & $\mathrm{H} 110 \mathrm{~B} 1$ & I \\
\hline & I & 1 & & 29931 & 1 \\
\hline & I & $+\cdots----$ & $-\infty$ & $---:---+$ & I \\
\hline & 1 & N7: $6-$ & <2: & & 1 \\
\hline & 1 & $\mathrm{~N} 2 \mathrm{O}: 92$ & $-c$ & 125) & I \\
\hline & 1 & & & & 1 \\
\hline & ++ & & & & \\
\hline
\end{tabular}

HNF-SD-FF-CSWD-61 Rev. 0 


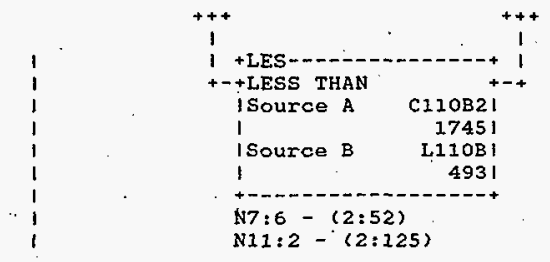

Rung $2: 128$

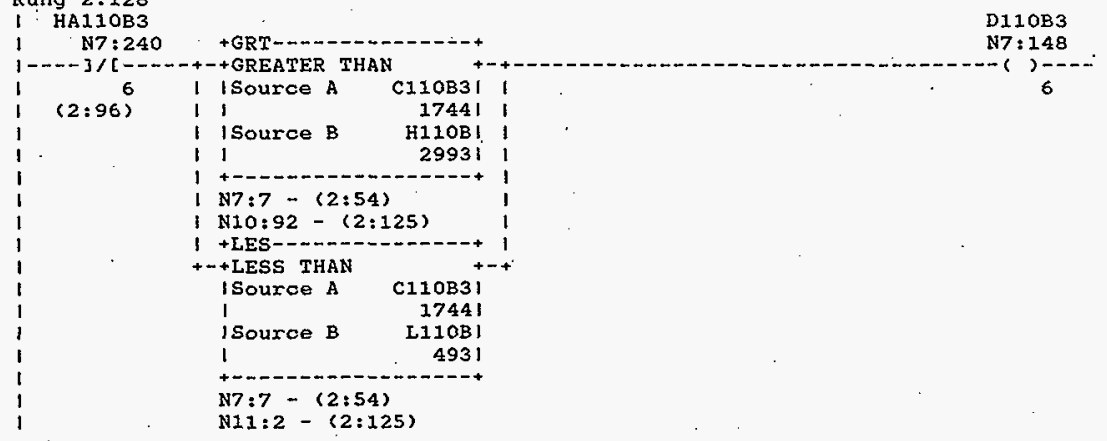

Rung 2:I29

HAIIOB4
N7:240


Processor and Data(OPS Unit 1)

Rung 2:130

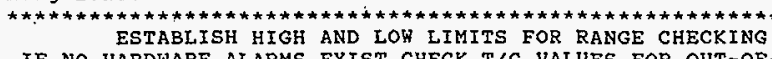

IF NO HARDWARE ALARMS EXIST CHECK T/C VALUES FOR OUT-OF-RANGE CONDITION

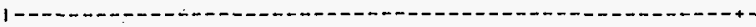

1 .

$+A D D-$

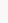

(1)

I ISource A AVI10C' I

11 .

1 isource B DEVIATION |

I I

1 IDest

11

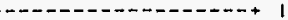

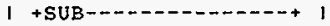

+-+ SUBTRACT

ISource A AVIIOC!

1

ISOurce B DEVIATIONI

IDest

1.2501

1

[110C!

4921

Rung $2: \pm 31$

I HA110C1

$1 \quad \mathrm{N7}: 24 \mathrm{O}$

$1----] /[\ldots-\cdots$

$\begin{array}{ll}1 & 8 \\ 1 & (2: 98)\end{array}$

1

1

1.

1

1

i

I

i

I

Rung 2:132

I HAI10C2

I N7:240

+GRT-- - - - - - -

I 9 l ISource A C1IOC2।

i) (2:99) 1 I 1742!

1 isource $B$ H110C!

i 11 2992।

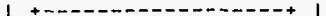

( N7:10-(2:60)

I $N 10: 93-(2: 130)$

1

$+++$

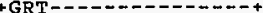

C110C11

110C1

+

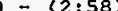

+ LES---n-----n---n+

ISource A C110C11

17401

4921

N7:9- $2: 58)$

N11:3 - $(2: 130)$
D110C1

N7: 148

8
D110C2

N7: 148

HNF-SD-FF-CSWD-61 Rev. 0

Page 52 
Processor and Data(OPS Unit I)

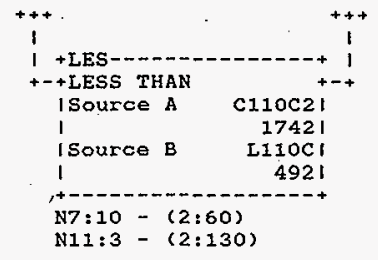

Rung $2: 133$

1 HA110C3

$$
\text { I } \quad \text { H7:240 }
$$

N7:240 +GRT---- $24-0----+$

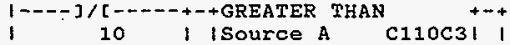

$(2: 100)$

11

17451

I isource B H110Cl

- 1

2992 J ।

$1+--0-0-0 m-0-0-0-0+1$

I $N 7: 11-(2: 62)$

I N10:93 - (2:130)

1 +LES--- -...---------+ 1

$+\cdots+$ LESS THAN

ISource A

I

c110c31

ISource B. L110CI

14921

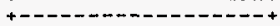

$N 7: 11-(2: 62)$

N11:3-(2:130)

Rung 2:134

1 HA110C4

I N7:240$$
\text { 111:3- }(2: 130)
$$

I I source A

C110C4i

17411

I Source B HiloC)

1

29921

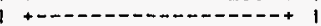

N7:12-(2:64)

N10:93 - (2:130)

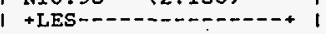

+-+ LESS THAN

isource A C110C4 I

I 17411

isource B L110CI

1

4921

N7:12-(2:64)

$\mathrm{N} 11: 3-(2: 130)$ 
Processor and Data(OPS Unit 1)

Rung $2: 135$

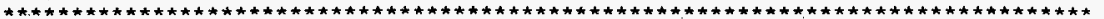
ESTABLISH HIGH AND LOW IIMITS FOR RANGE CHECKING

IF NO HARDHARE ALARMS EXIST CHECK T/C VALUES FOR OUT-OF-RANGE CONDITION

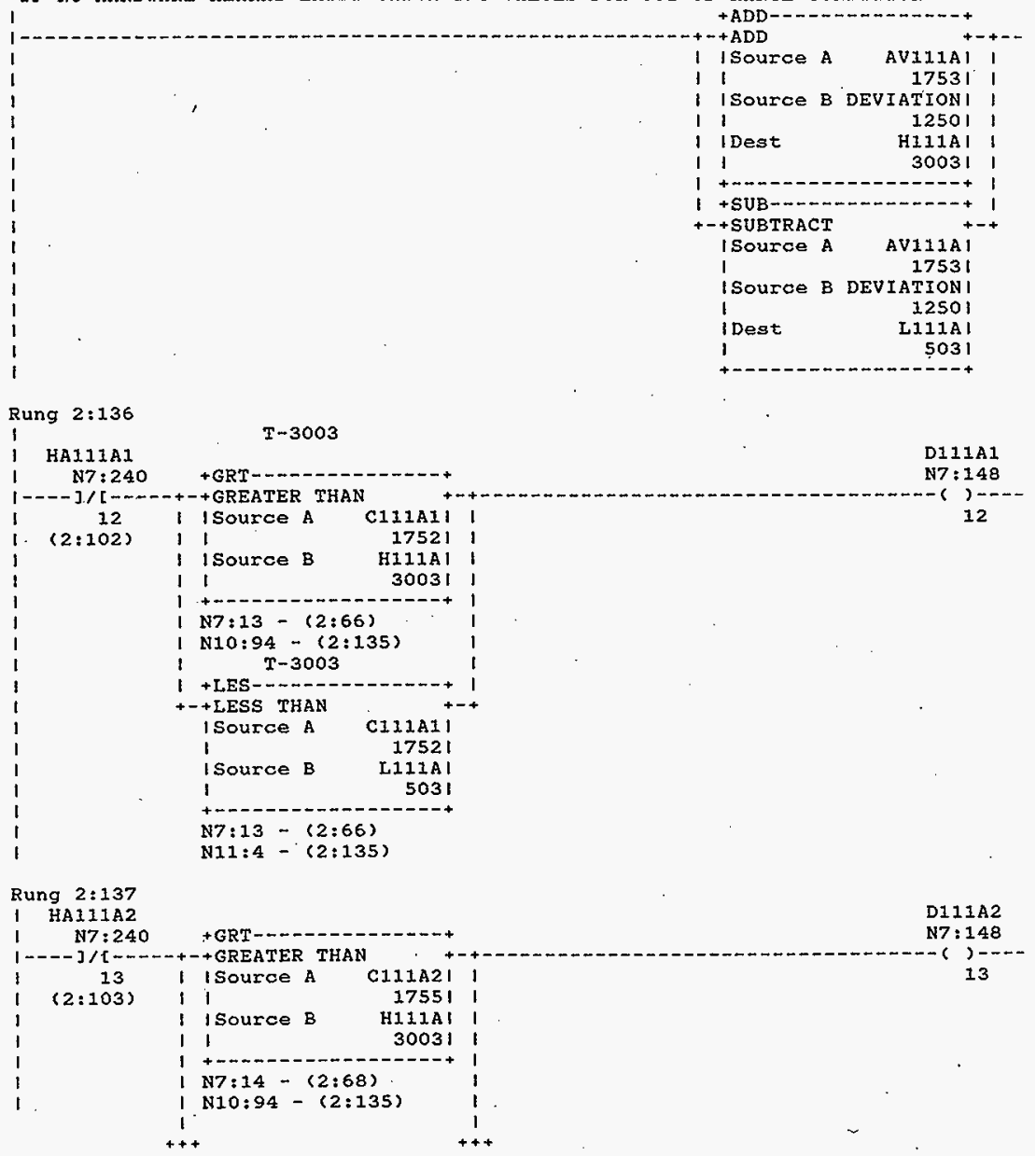


Processor and Data(OPS Unit 1) Program Listing

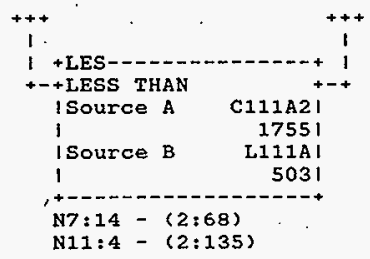

Rung $2: 138$

I HAI11A3

I N7:240

1.-.- $] /[----$

14

$(2: 104)$

+GRT-DO-----------+

D111A3

+ +GREATER THAN

C111A31 ।

i 17531 I

| ISource B H111A| |

11

3003 ! !

(1)

N7:15-(2:70)

1 N10:94 - (2:135)

$1+$ tES--_-

+-+ LESS THAN +-+

ISource A C111A3I

1

17531

ISource B. LIIIAI

1 5031

N7:15-(2:70)

N11:4-(2:135)

Rung 2:139

I HA111A4

1 N7:240

1----3/[--.++-+GREATER THAN

15

I I Source A

(2:105) 11

I isource.B H11.1Al

1 i 30031

$1+----n-m-n----n+1$

( $N 7: 16-(2: 72)$

I N10:94-(2:135)

+LES---_-

+-+ LESS THAN

isource A

C111A4I

1

17501

| Source B L111A|

I

5031

N7:16-(2:72)

N11:4 $-(2: 135)$

HNF-SD-FF-CSWD-61 Rev. 0 
Processor and Data(OPS Unit 1) Program Listing

Rung 2:140

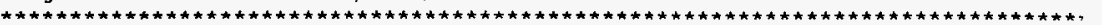
ESTABLISH HIGH AND LOW LIMITS FOR RANGE CHECKING

IF NO HARDWARE ALARMS EXIST CHECK T/C VALUES -FOR OUT-OF-RANGE CONDITION

1 $+A D D------------+$ $+A D D$

ISource A AV111BI I
1

1. ISOurce B DEVIATIONI I

I 1 1250! 1

1 IDest H111BI I

I 1 30001 ।

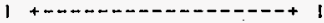

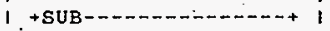

+-+ SUBTRACT

isource A AV111B।

$1 \quad 17501$

ISource B DEVIATION I

i

12501

I Dest

L111B।

1

5001

Rung $2: 141$

I HAI11BI.

N7: 241

$+$

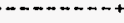

D113B1

1-..- ]/[--.-++ GREATER THAN

O I I Source A

C111B1।

N7: 149

(2:106)

i I Source B

17491 ।

H112B।

11

30001

I $7: 17-(2: 74)$

I N10:95-(2:140)

1 +LES--......-.-.+

+-+ LESS THAN

ISource A C111B1I

1

17491

| Source B L111B |

1

5001

N7:17-(2:74)

N11:5- $(2: 140)$

Rung 2:142

HA111B2

1 N7:241 +GRT---

D111B2

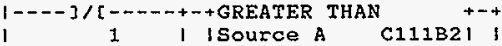

N7 $: 149$

$(2: 107)$

N7:18-(2:76)

N10:95-(2:140)

I

$++$

$-2--+1$

0

$-($ ) $-\cdots$

1

HNF-SD-FF-CSWD-61 Rev. 0 
Processor and Data(ops Unit 1)

October $23,199^{\circ}$ page 57

Program Listing

Processor File: SODIUMIA.ACH

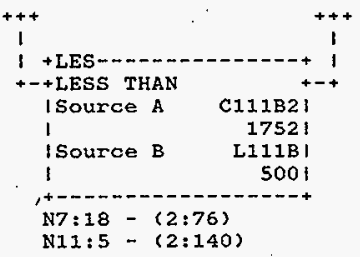

Rung $2: 143$

I HA111B3

$$
\text { ( } \quad \text { N7:241 }
$$

(..-- ]/[---+-+GREATER THAN +

(2:10 2 )

I I Source A

C111B3: ;

175011

I isource B H111Bi i

I 130001 I

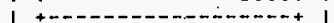

- $N 7: 19-(2: 78)$

( N10:95 - (2:140)

$1+$ LES----........-

++ LESS THAN

|Source A C111B3|

$1 \quad 17501$

isource B L111B

15001

N7:19-(2:78)

N11:5-(2:140)

Rung $2: 144$

I HAI11B4

I N7:241

1----]/[----+-+GREATER THAN

I 3

3

I ISource A

11

I Source B

11

( N7:20-(2:80)

( N10:95-(2:140)

1 + LES--.-_-......... +

+-+ LESS THAN

I Source A

1

C111B4I

$111 \mathrm{~B} 41.1$

1749 ।

H1118I ।

300011

Isource B

17491

B L111BI

5001

N7:20-(2:80)

N11:5- (2:140)

HNF-SD-FF-CSWD- 61 Rev. 0 
Processor and Date(Ops Unit 1)

Program Listing

Processor File: SODIUM1A.ACH

October 23,1996

Page 58

Rung 2:145

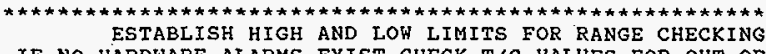

IF NO HARDWARE ALARMS EXIST CHECK T/C VALUES FOR OUT-OF-RANGE CONDITION

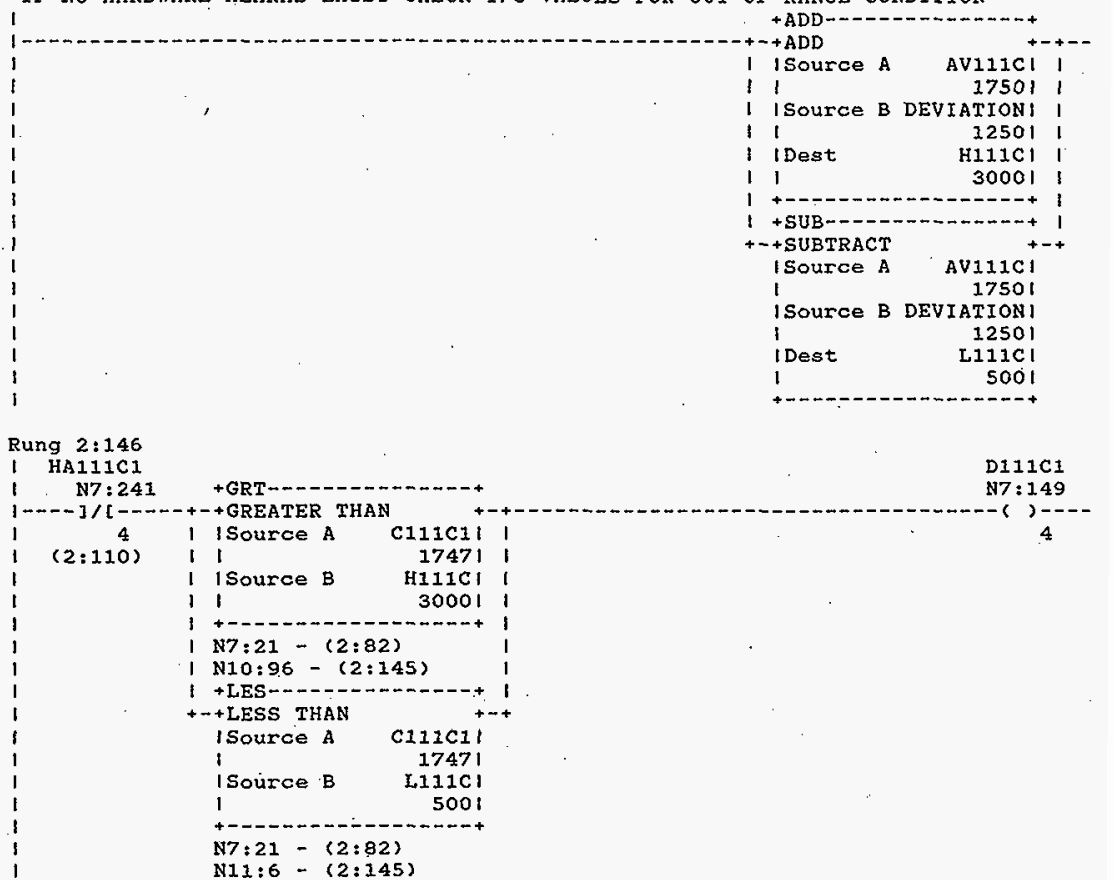

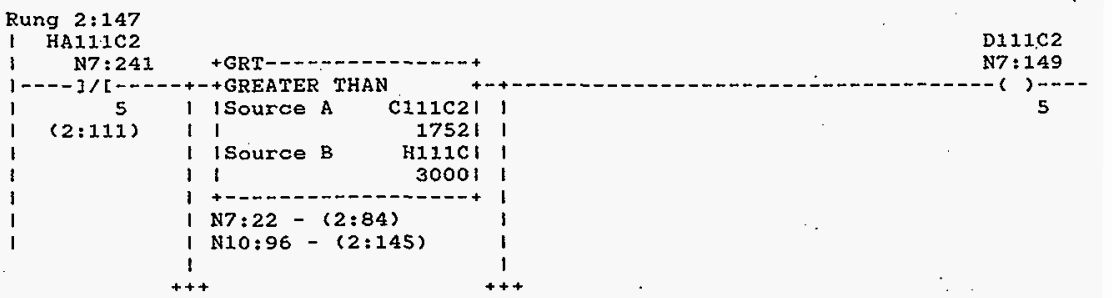

HNF-SD-FF-CSWD-61 Rev. 0 
Processor and Data(OPS Unit i) Program Listing

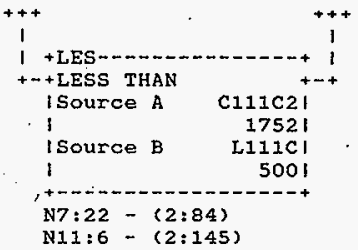

Rung $2: 148$

1 HA111C3

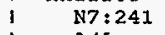

.

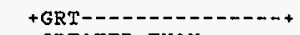

D $111 \mathrm{C} 3$

Rung 2:149

I HA111C4

Rung $2: 150$

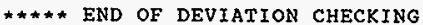


Processor and Data(OPS Unit 1)

Rung 2:151

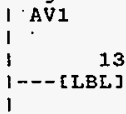

DUMMY 6 N2O:7

Rung $2: 152$

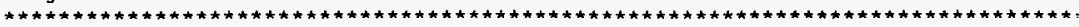
CLEAR COUNT AND TOTAL. IF NO HARDWARE, NO RANGE ALARMS AND NO THERMOCOUPLES HAVE BEEN TAKEN OUT (TE_OUTXXX) THEN AVERAGE THE T/C

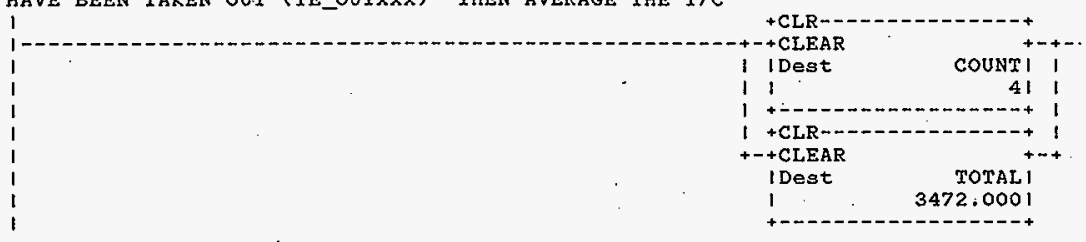

Rung $2: 153$

\begin{tabular}{|c|c|c|c|c|c|c|c|}
\hline HA110A1 & & D110A1 & OUT110A1 & & & & \\
\hline $\begin{array}{r}1 \quad N 7: 240 \\
1-\ldots-[--1\end{array}$ & & $\begin{array}{r}N 7: 148 \\
---] /[----\end{array}$ & $\begin{array}{r}N 7: 246 \\
---] /[-\ldots-\end{array}$ & - & $\begin{array}{l}+\mathrm{ADD}-\cdots- \\
-+\mathrm{ADD}\end{array}$ & 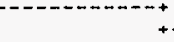 & -+- \\
\hline i $\quad 0$ & 1 & 0 & 14 & 1 & ISource & 11 & 1 \\
\hline$(2: 90)$ & I & $(2: 121)$ & $i$ & I & & I & $t$ \\
\hline - & 1 & . & I & 1 & isource & COUNTI & 1 \\
\hline 1 & I & & 1 & 1 & & 41 & 1 \\
\hline 1 & 1 & & 1 & 1 & IDest & COUNTI & 1 \\
\hline I & I & & i & i & & 41 & 1 \\
\hline$i$ & I & & 1 & 1 & +----- & -----+ & 1 \\
\hline 1 & 1 & & 1 & 1 & & & 1 \\
\hline 1 & 1 & DEV_I & 1 & 1 & & & 1 \\
\hline 1 & 1 & $\mathrm{~N} 20: 6$ & 1 & 1 & $+A D D-\cdots$ & ------4 & I \\
\hline 1 & +- & $---][\ldots$ & & +1 & $-+\mathrm{ADD}$ & & + \\
\hline 1 & & & & & isource & C110A11 & \\
\hline I & & $(2: 118)$ & & & i & $1742 t$ & \\
\hline i & & & & & ISource & TOTAL 1 & \\
\hline i & & & & & 1 & 3472.0001 & \\
\hline 1 & & & & & I Dest & TOTAL & \\
\hline i & & & & & 1 & 3472.0001 & \\
\hline : & & & & & $+-\ldots-n-\infty$ & 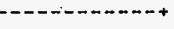 & \\
\hline 1 & & & & & & & \\
\hline Rung $2: 154$ & & & & & & & \\
\hline 1 HA110A2 & & D110A2 & OUT110A2 & & & & \\
\hline i N7:240 & & N7 $: 148$ & N7: 246 & & $+A D D---$ & $-1-2-1-2.0-2+$ & \\
\hline $1----] /[-\cdots$ & -+- & $--n] /[--$ & & & $-+A D D$ & & \\
\hline$i \quad 1$ & 1 & 1 & 15 & 1 & isource & 11 & 1 \\
\hline$(2: 91)$ & i & $(2: 122)$ & i & $i$ & & I & 1 \\
\hline - & 1 & & 1 & i & ISource & COUNTI & 1 \\
\hline 1 & 1 & & i & 1 & I & 41 & i \\
\hline I & 1 & & 1 & 1 & i Dest & COUNTI & 1 \\
\hline . & i & . & 1 & 1 & 1 & 41 & 1 \\
\hline 1 & 1 & & 1 & 1 & $+\cdots+-$ & 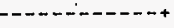 & 1 \\
\hline 1 & ! & & $i$ & i & & $\cdot$ & 1 \\
\hline & 1 & & 1 & 1 & & & 1 \\
\hline & +++ & & +++ & & & & $t++$ \\
\hline
\end{tabular}


Processor and Datacops Unit 1)

October 23, 1996, Page 61
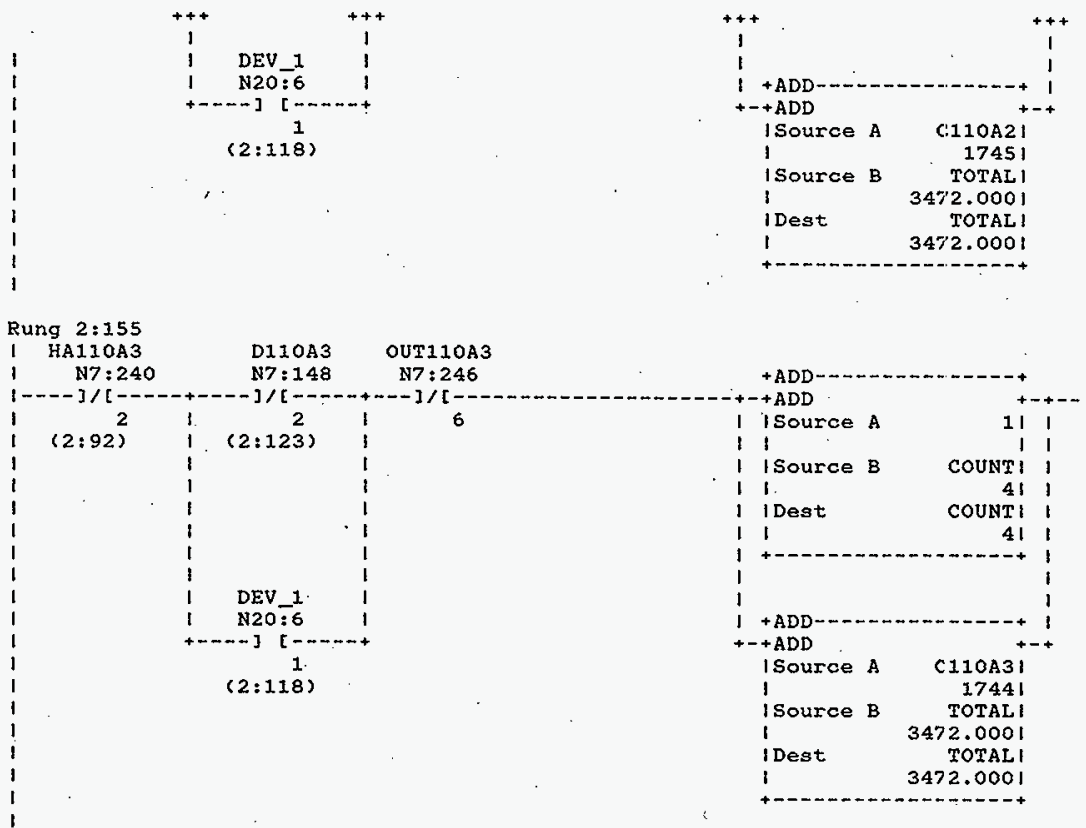

Rung 2:156

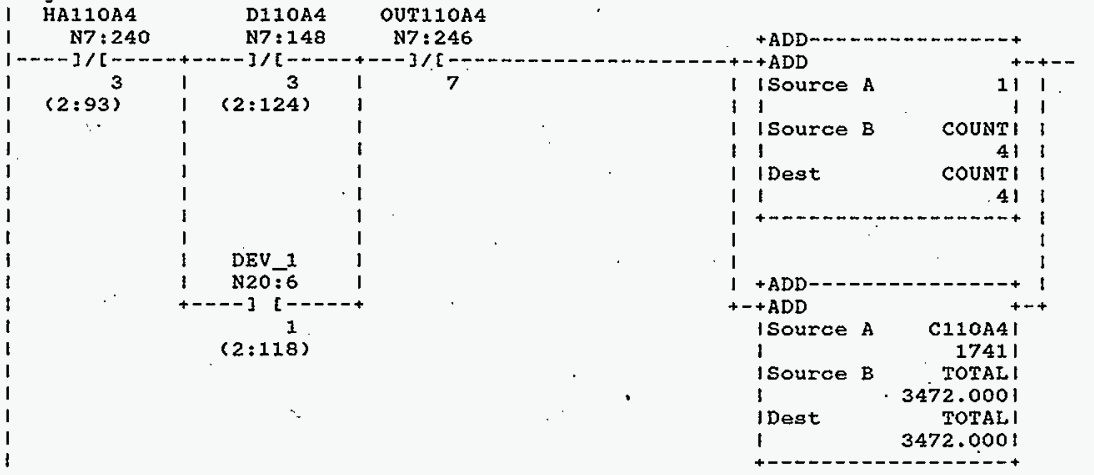


Processor and Data (OPS Unit 1) Program Listing

\section{Rung 2:157}

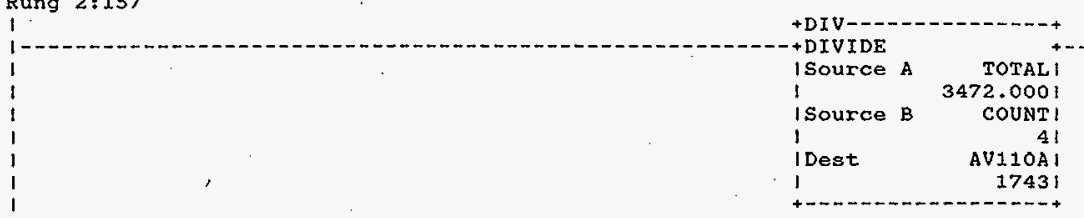

Rung $2: 158$

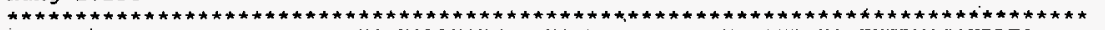
CLEAR COUNT AND TOTAL. IF NO HARDHARE, NO RANGE ALARMS AND NO THERMOCOUPLES HAVE BEEN TAKEN OUT (TE_OUT $\times \times \times$ ) THEN AVERAGE THE T/C

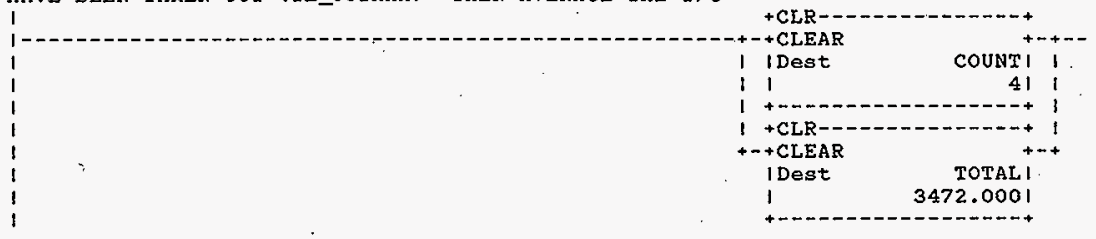

Rung $2: 159$

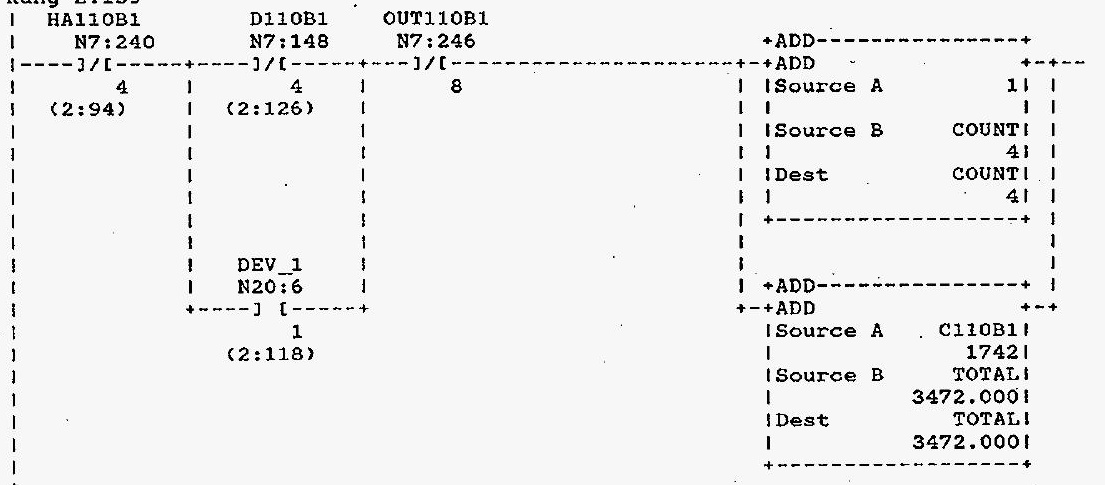

HNF-SD-FF-CSWD-61 Rev. 0 
Processor and Data(Ops Unit 1 )

- October 23, 1996,

Page 65 Program Listing

Processor File: SODIUM1A.ACH

Rung $2: 16 \mathrm{C}$

Rung $2: 160$
I. HA110B2

I N7:240

D110B2 OUT110B2

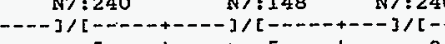

$(2: 95)$

( $12: 127$ )

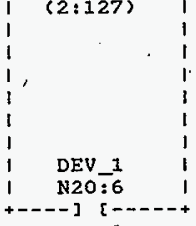

1

$(2: 118)$

Rung 2:161

1 HA110B3

N7: 240

D110B3

OUT110B3

N7: 148

N7: 246

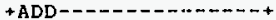

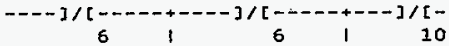

$(2: 96)$

$(2: 128)$

10

DEV

N20:6

] [--- -

(2:118)

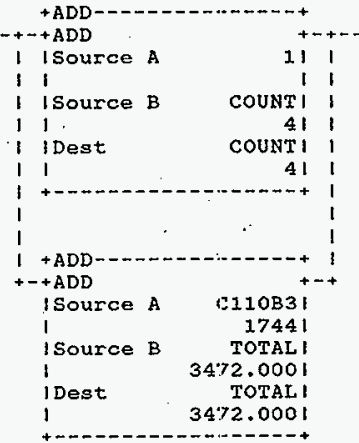

HNF-SD-FF-CSWD-6I Rev. 0

Page 63 
Processor and Data(OPS Unit 1 )

October 23, 1996 Page 64

Program Listing

Processor File: SODIUMIA.ACH

Rung 2:162

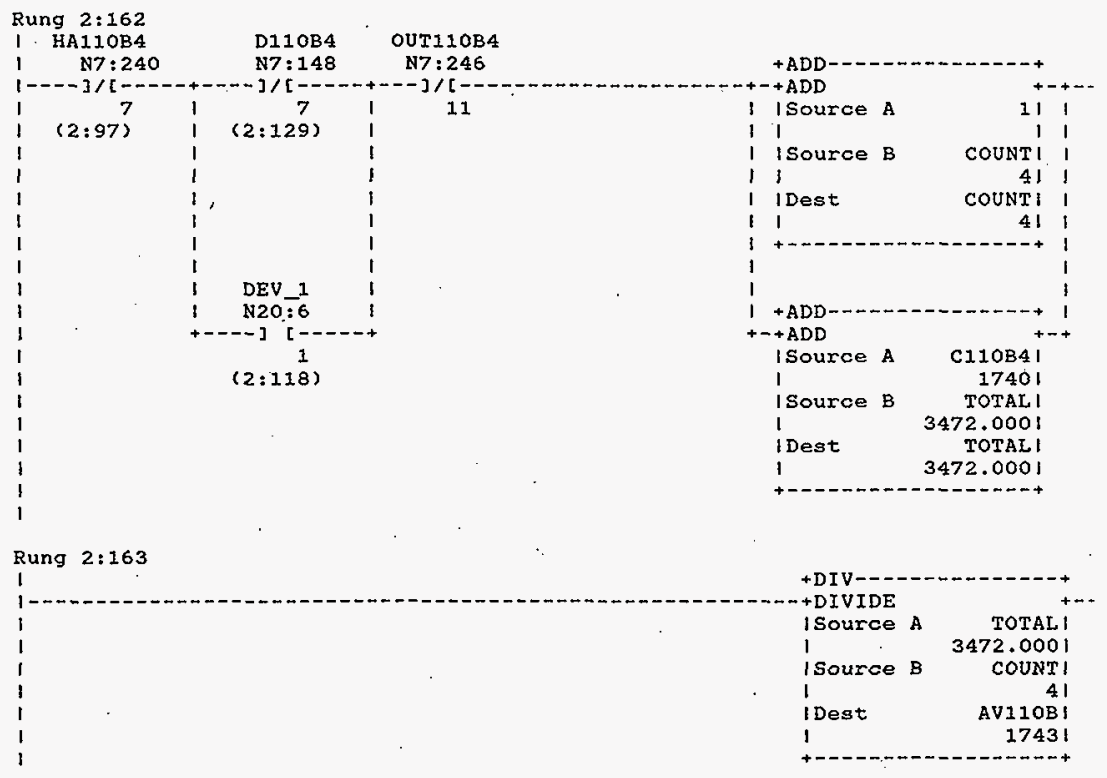

Rung $2: 164$

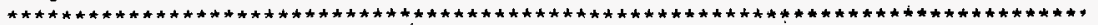
CEEAR COUNT AND TOTAL: IF NO HARDWARE, NO RANGE ALARMS AND NO THERMOCOUPLES HAVE BEEN TAKEN OUT (TE_OUTXXX) THEN AVERAGE THE T/C

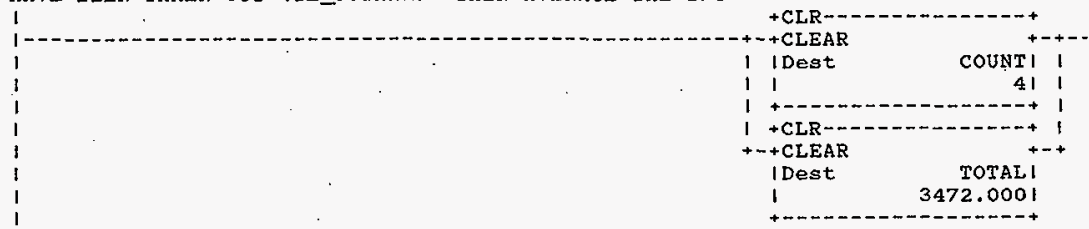

HNF-SD-FF-CSWD-61 Rev. 0 . 
Processor and Data (OPS Unit 1)

October 23, 1996 Page 65 Program Listing

Rung 2:165

. $2: 165$

HA110C1

N7: 240

D110C1 ' OUT110C1

N7:148 N7:246

$\begin{array}{rrrrr}8 & 1 & 8 & 1\end{array}$

$(2: 131)$

1

1,

i

1 DEV_1

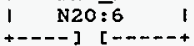

(2:118)

+ ADD $-\div-1----\cdots++$ 
Processor and Data (ops Unit 1 )

\section{Rung 2:167}

I. HAI10C3 N7: 240

$\begin{array}{cr}\text { D110C3 } & \text { OUT110C3 } \\ \text { N7:148 } & \text { N7:246 }\end{array}$

$$
10
$$

$(2: 100)$

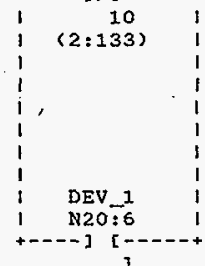

(2:118)

] $/[-$

$---$

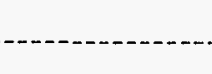


Processor and Data(OPS Unit 1)

Rung $2: 170$

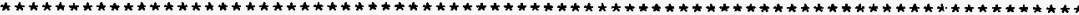
CLEAR COUNT AND TOTAL. IF NO HARDHARE, NO RANGE ALARMS AND NO THERMOCOUPLES HAVE BEEN TAKEN OUT (TE_OUTXXX) THEN AVERAGE THE T/C$$
\text { , }
$$

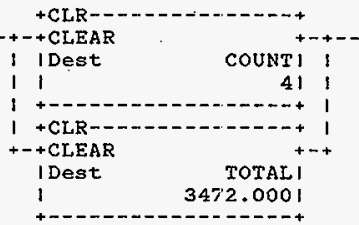

\section{Rung $2: 171$}

i HA111A1

I N7:240

1.--- ] $7: 240$

$$
1 \quad 12
$$

$(2: 102)$

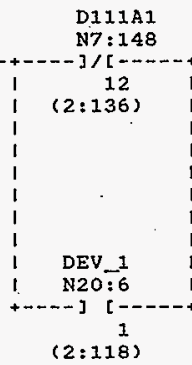

OUT111A1

N7:247

$+A D D$
$+++A D D$

I ISource

I I

I ISource B

11

1 I Dest

I 1

42.000$$
1
$$

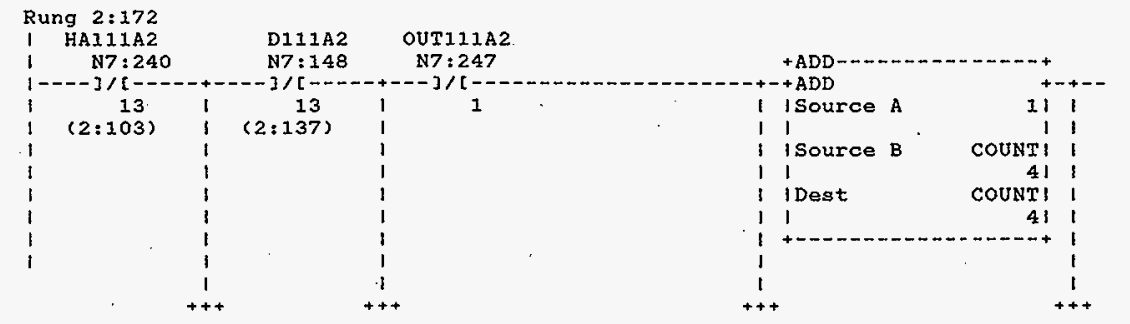

HNF-SD-FF-CSWD-61 Rev. 0 
Processor and Data(OPS Unit 1)
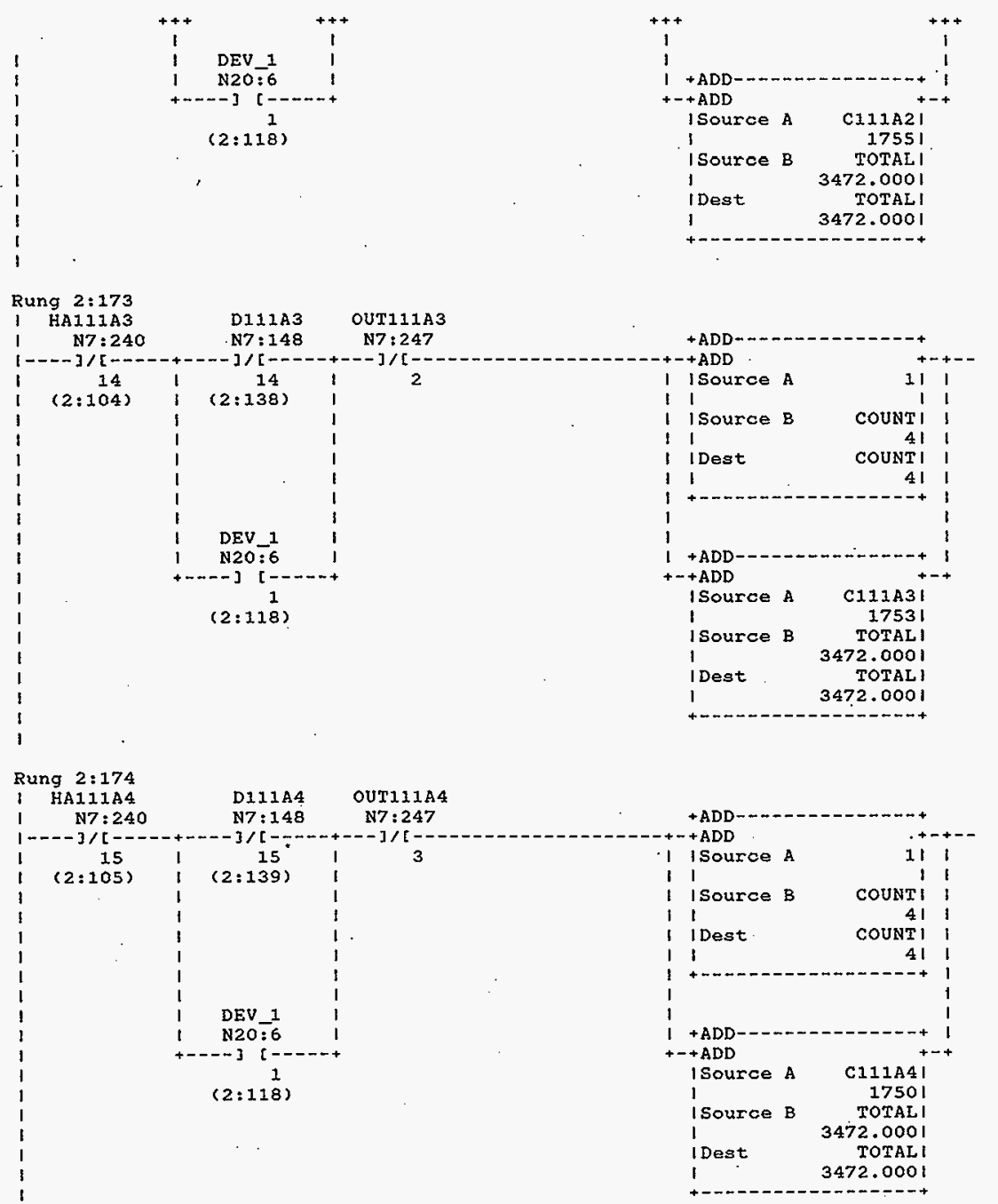

HNF-SD-FF-CSWD-61 Rev. 0 
Processor and Data(OPS Unit 1 )

Program Listing

Processor File: SODIUM1A.ACH

October 23, 1996 Page 65

Rung 2:175

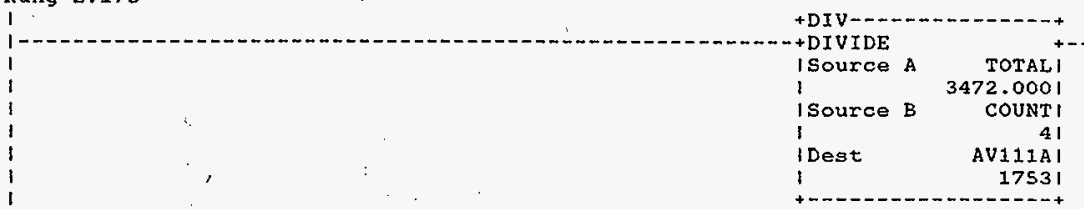

Rung 2:176

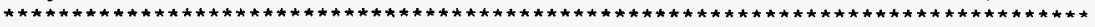
CLEAR COUNT AND TOTAL. IF NO HARDWARE, NO RANGE ALARMS AND NO THERMOCOUPLES HAVE BEEN TAKEN OUT (TE_OUTXXX) THEN AVERAGE THE T/C$$
\text { 1 }
$$
I

I 3472.0001

\section{Rung 2:177}

1 111181

1 N7:24I

N7:149 N7:247

1

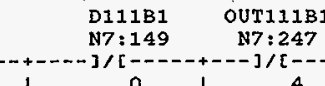

7

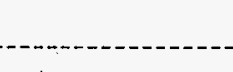

(2:106)

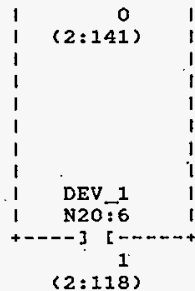

4

$+A D D$
$-+A D D$
I SOU

11

11

I 1

I IDest

1

1

i

+ ADD

$+-+A D D$

I Source A

1

isource $B$

I

i Dest

I

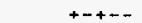

COUNTI I

41 i

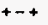


Rung 2:178
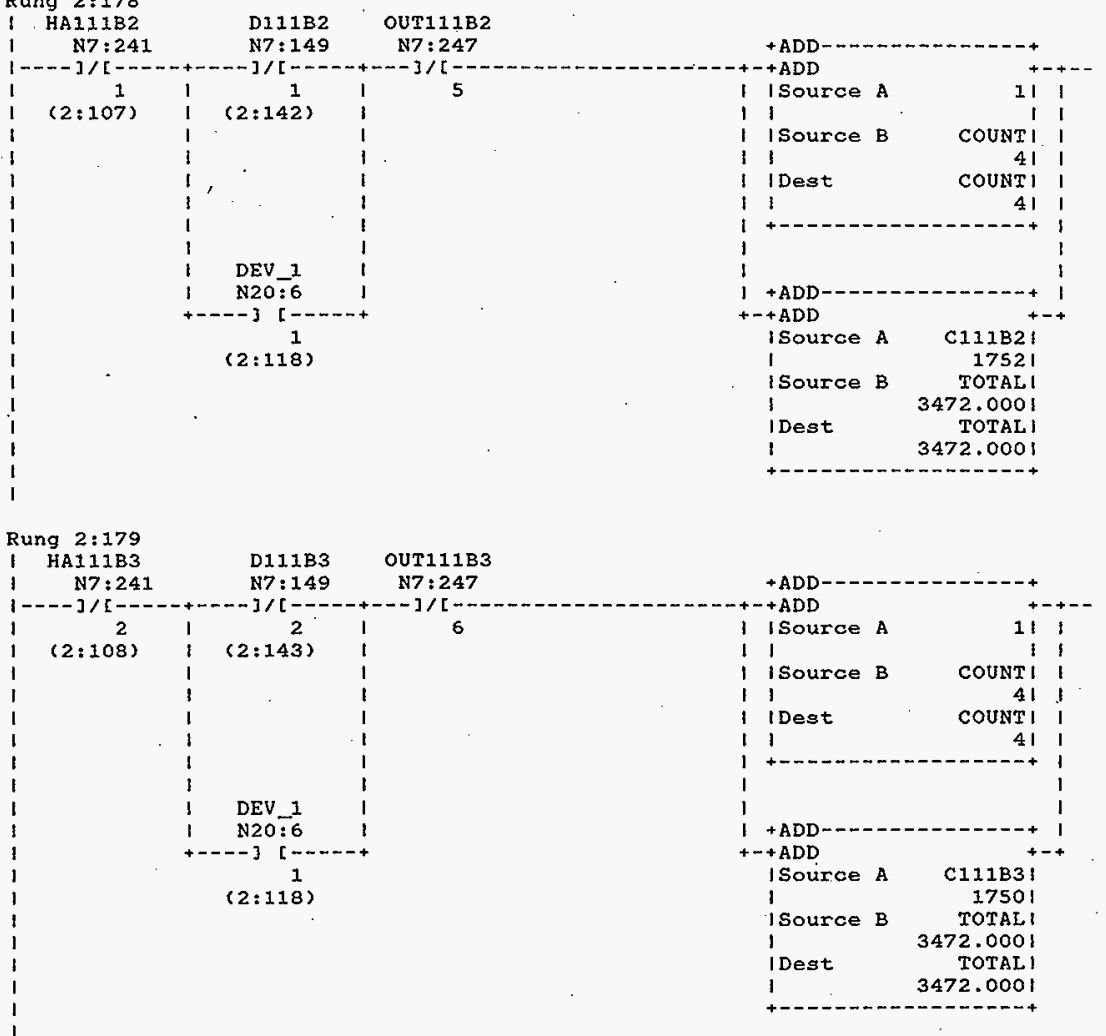
Processor and Data(OPS Unit I)

October 23, 1996 Page 72

program Listing

Processor File: SODIUMIA.ACH

Rung $2: 180$
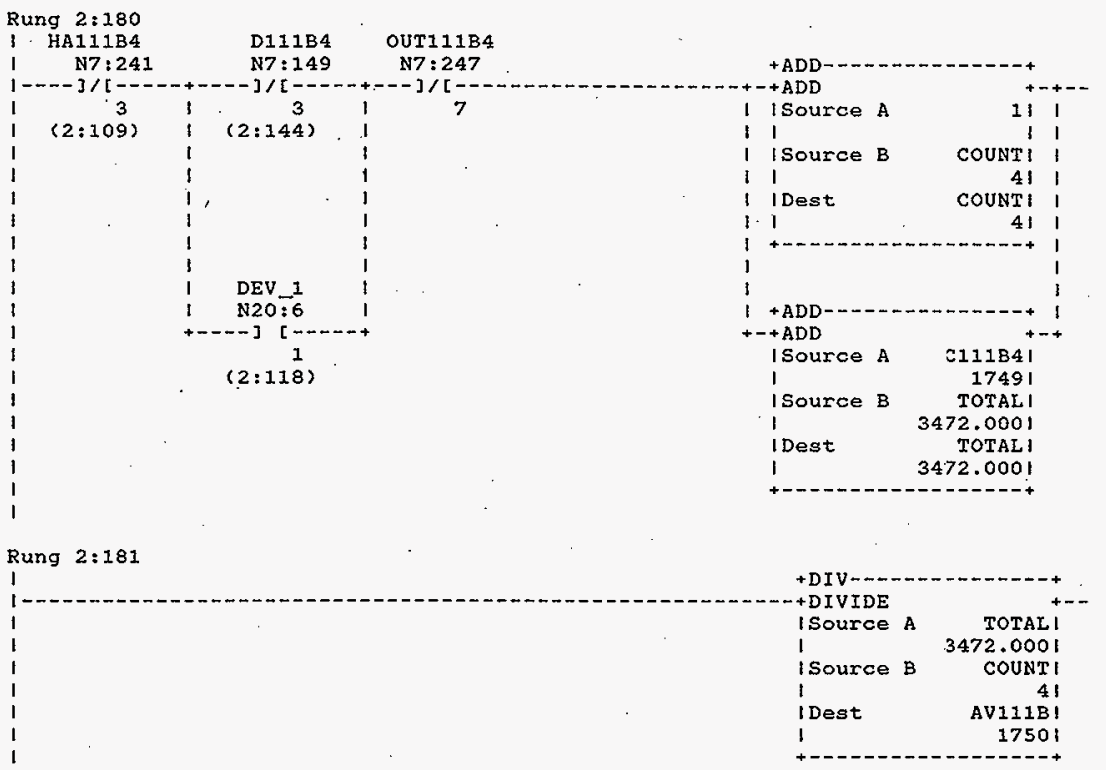

Rung 2:182

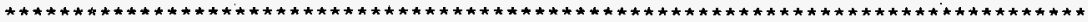
CLEAR COUNT AND TOTAL. IF NO HARDWARE, NO RANGE ALARMS AND NO THERMOCOUPLES HAVE BEEN TAKEN OUT (TE_OUTXXX) THEN AVERAGE THE T/C

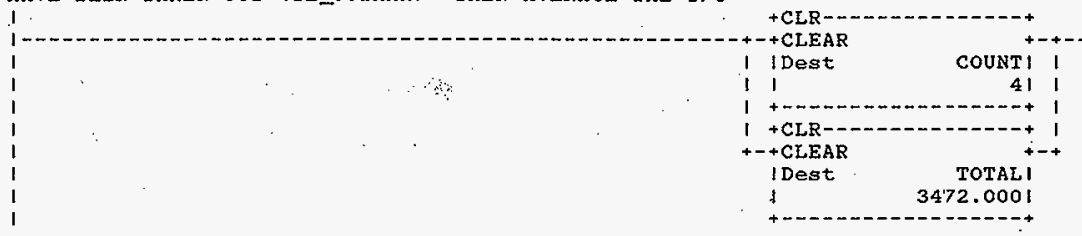

HNF-SD-FF-CSWD-61 Rev. 0 
Processor and Data (ops Unit 1 )

Program Listing

Processor File: SODIUMIA.ACH

October 23, 1996 Page 72

Rung $2: 183$
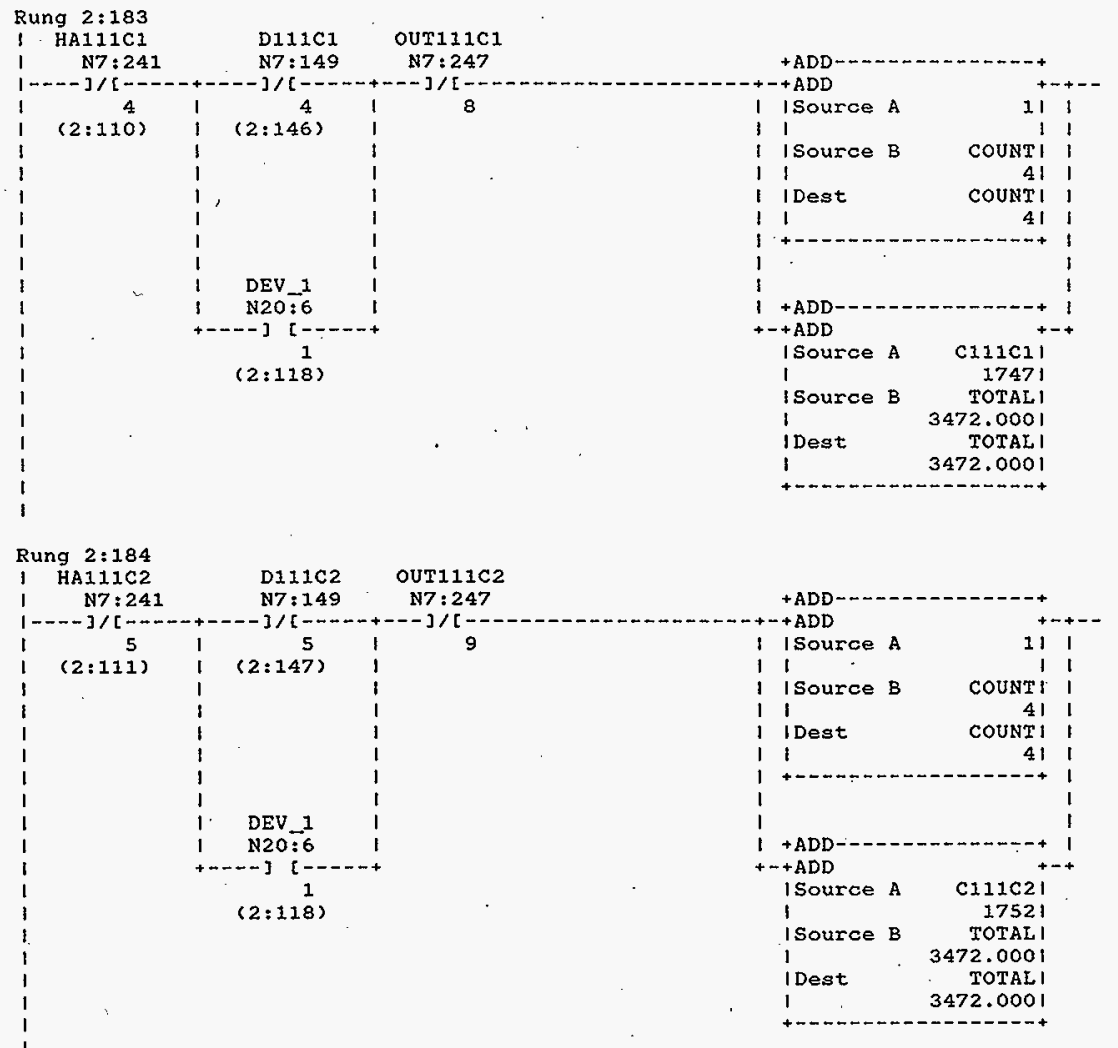
Processor and Data(Ops Unit 1)
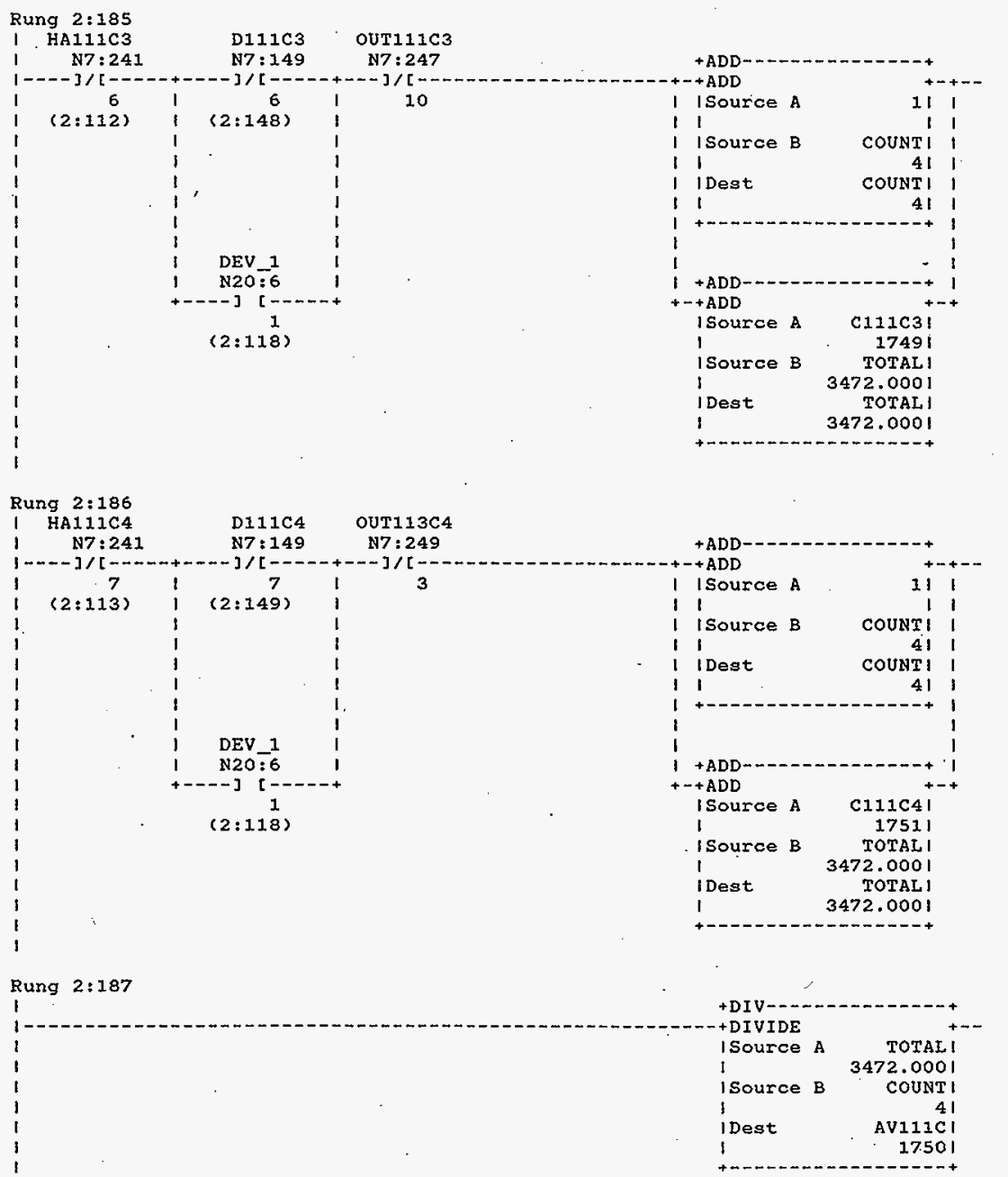
Processor and Data(OPS Unit 1)

Rung 2:188

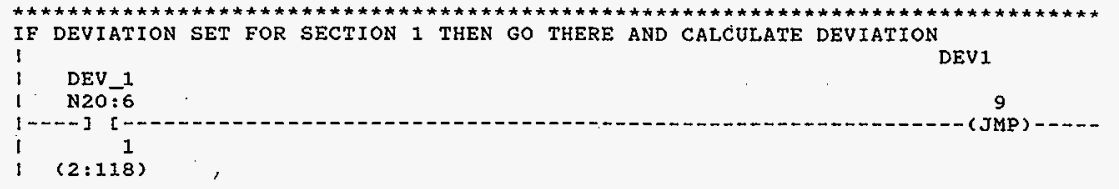

Rung 2:189

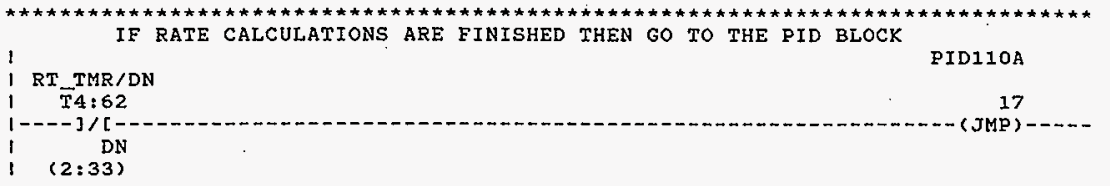

Rung 2:190

RATE CHECKING

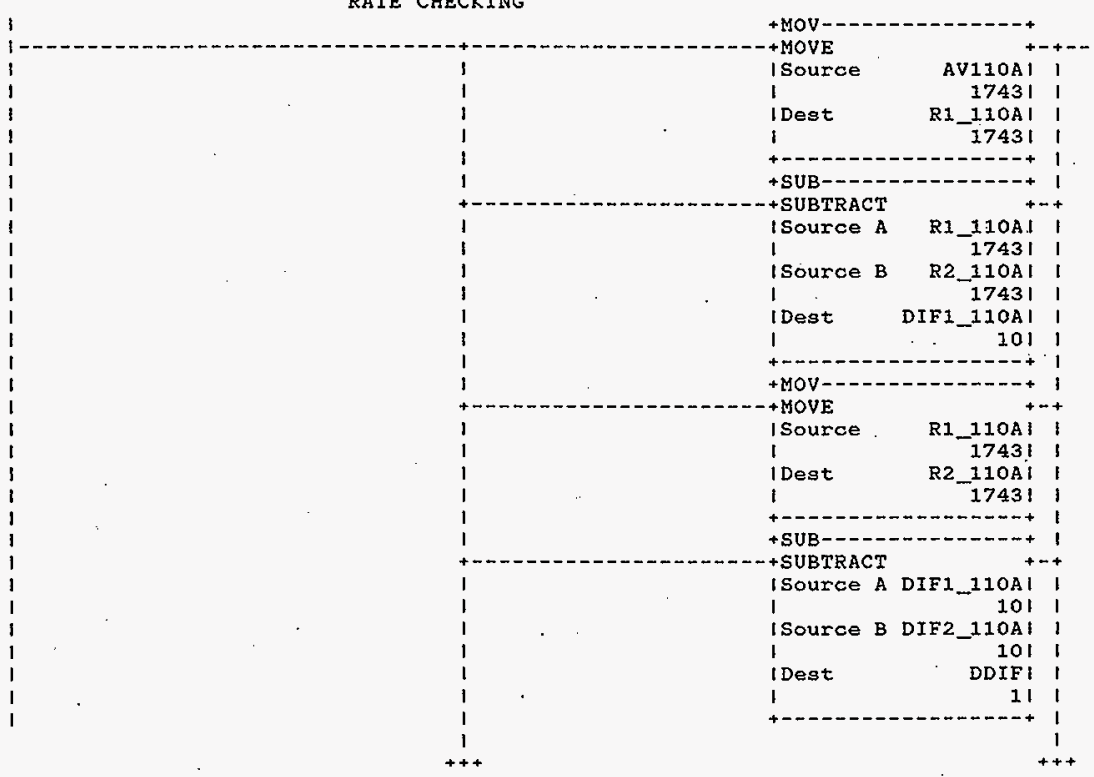

HNF-SD-FF-CSWD-61 Rev. 0 


$$
\begin{aligned}
& 1 \\
& 1 \\
& 1 \\
& 1 \\
& 1 \\
& 1 \\
& 1 \\
& 1 \\
& 1 \\
& 1 \\
& 1 \\
& 1 \\
& 1 \\
& 1 \\
& 1 \\
& 1 \\
& 1 \\
& 1 \\
& 1 \\
& 1 \\
& 1 \\
& 1 \\
& 1 \\
& 1 \\
& 1 \\
& 1 \\
& 1 \\
& 1 \\
& 1 \\
& 1 \\
& 1 \\
& 1 \\
& 1 \\
& 1 \\
& 1 \\
& 1 \\
& 1 \\
& 1 \\
& 1 \\
& 1 \\
& 1 \\
& 1 \\
& 1 \\
& 1
\end{aligned}
$$

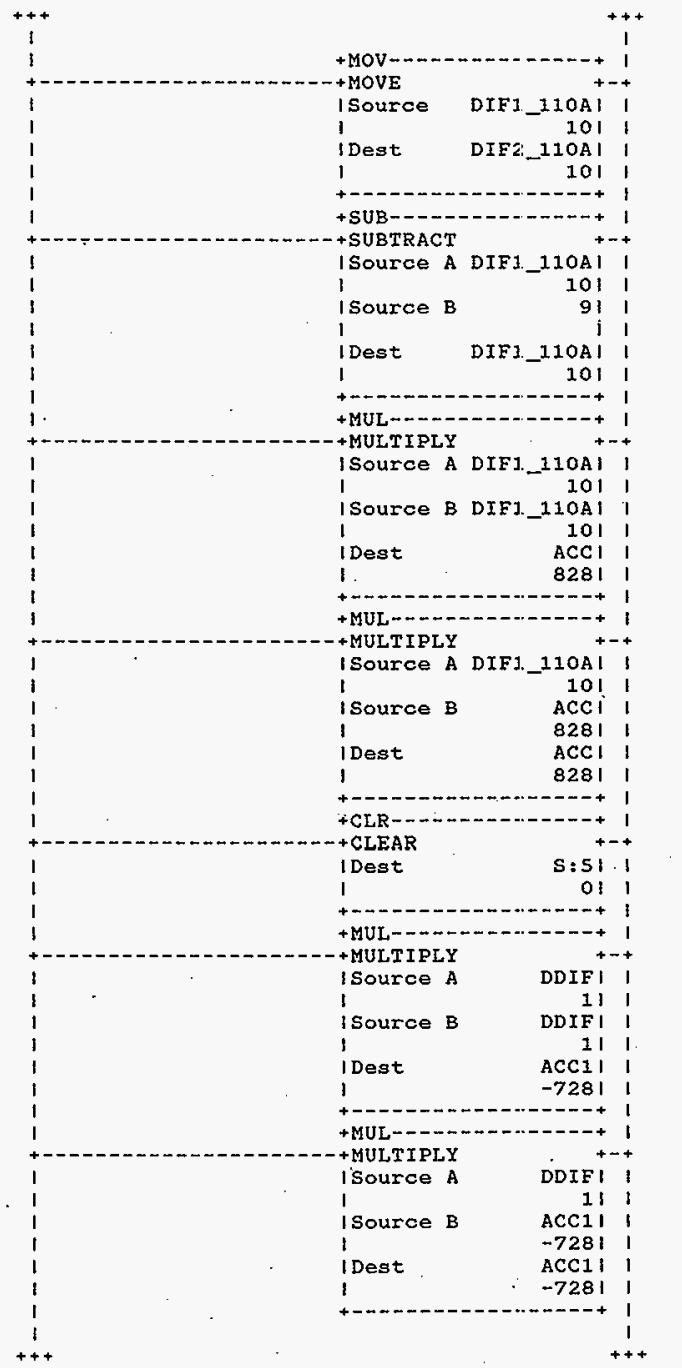

HNF-SD-FF-CSWD-fil Rev. 0 
Processor and Data(OPS Unit 1)
Processor File: SODIUM1A.ACH

Detober 23,1996 page 76

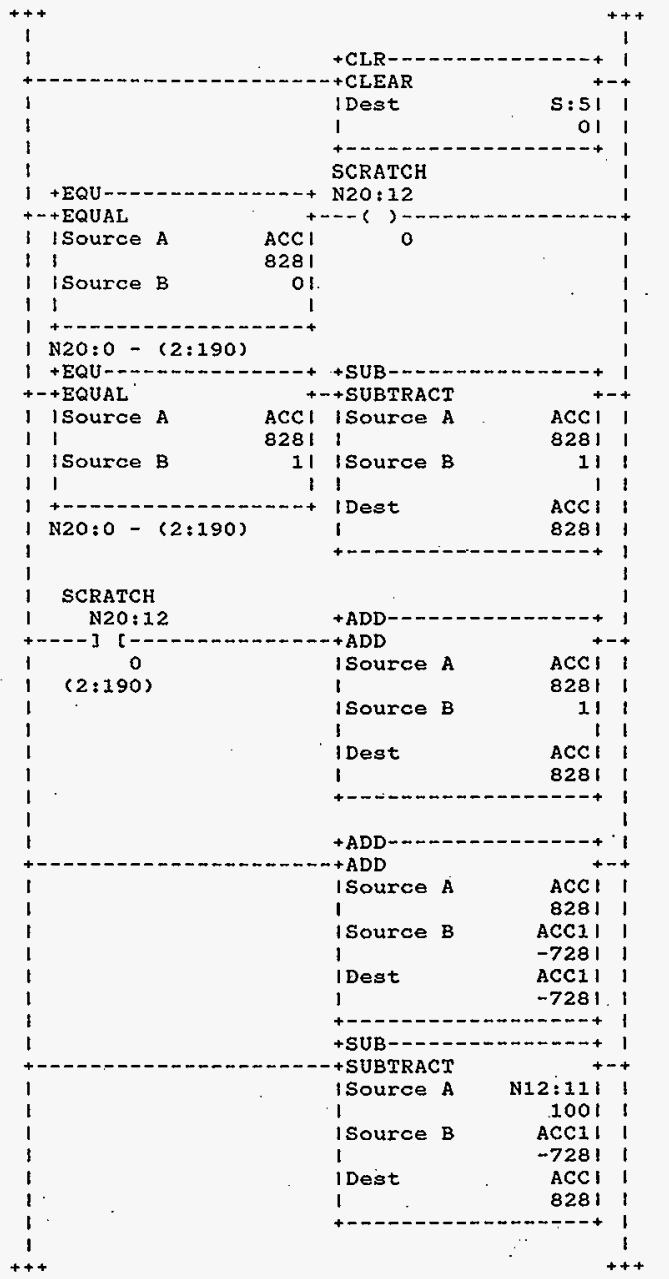


Processor and Data(OPS Unit 1)

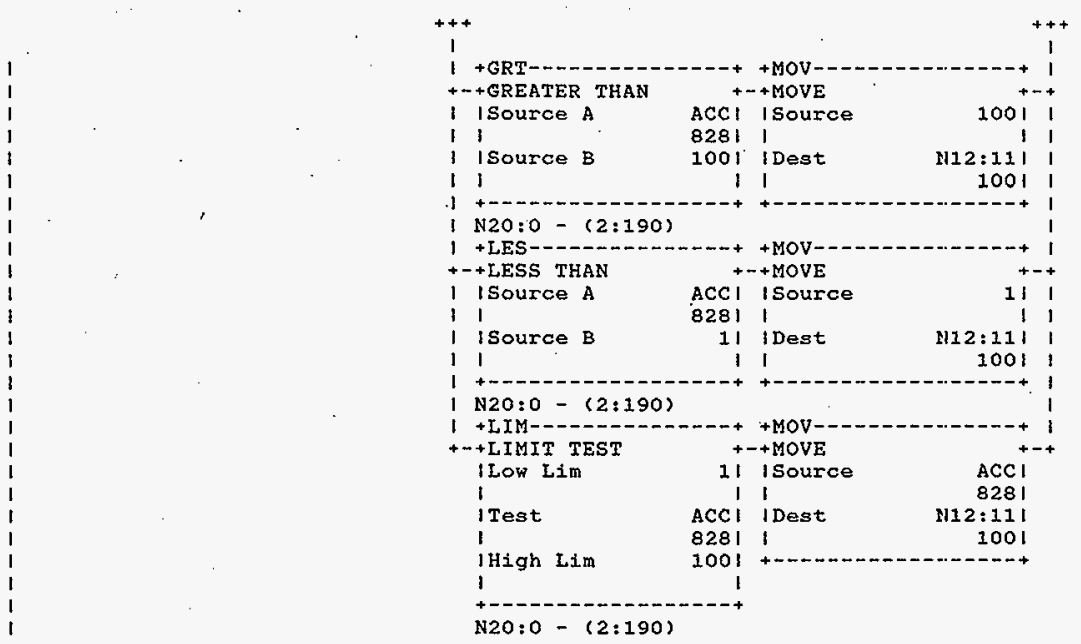

Rung 2:191

1 PID1IOA

1

DUMMY16

17

N20:7

1- - [LBL]

Rung 2:192

\section{DETECT FROM SCADA PID ON/OFF}

IF PID OFE

THEN ZERO PH FOR ZERO OUTPUT AT SCR

AND JUMP AROUND THE PID BLOCK

1 ON $110 \mathrm{~A}$

I $\bar{N} 7: 239$

$1---7 /[-3$

$(2: 820)$

$$
\text { i }
$$

.

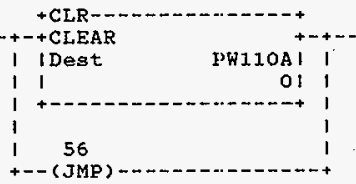

Rung $2: 193$

$\left\{\begin{array}{l}\begin{array}{l}\text { AM11OA } \\ N 7: 118\end{array} \\ \begin{array}{c}0 \\ (3: 23)\end{array}\end{array}\right.$

HNF-SD-FF-CSWD-61 Rev. 0 
Rung 2:194

DETECT SETPOINT VALUE FROM SCADA PUT VALUE IN PID BLOCK

I

+MOV-
PUT

Rung 2:195

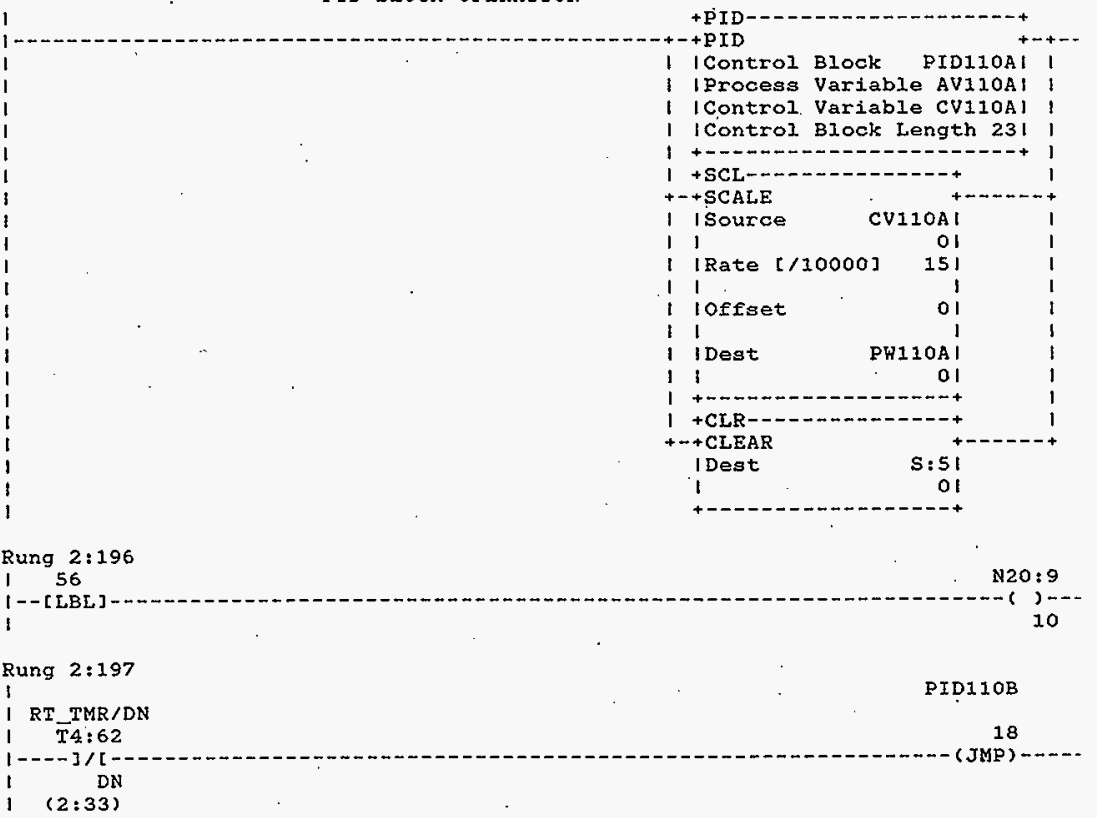


Procegsor and Data(OPS Unit 1)

October 23, 199E, Page 79

Program Listing

Processor File: SODIUM1A.ACH

Rung $2: 198$

RATE CHECKING

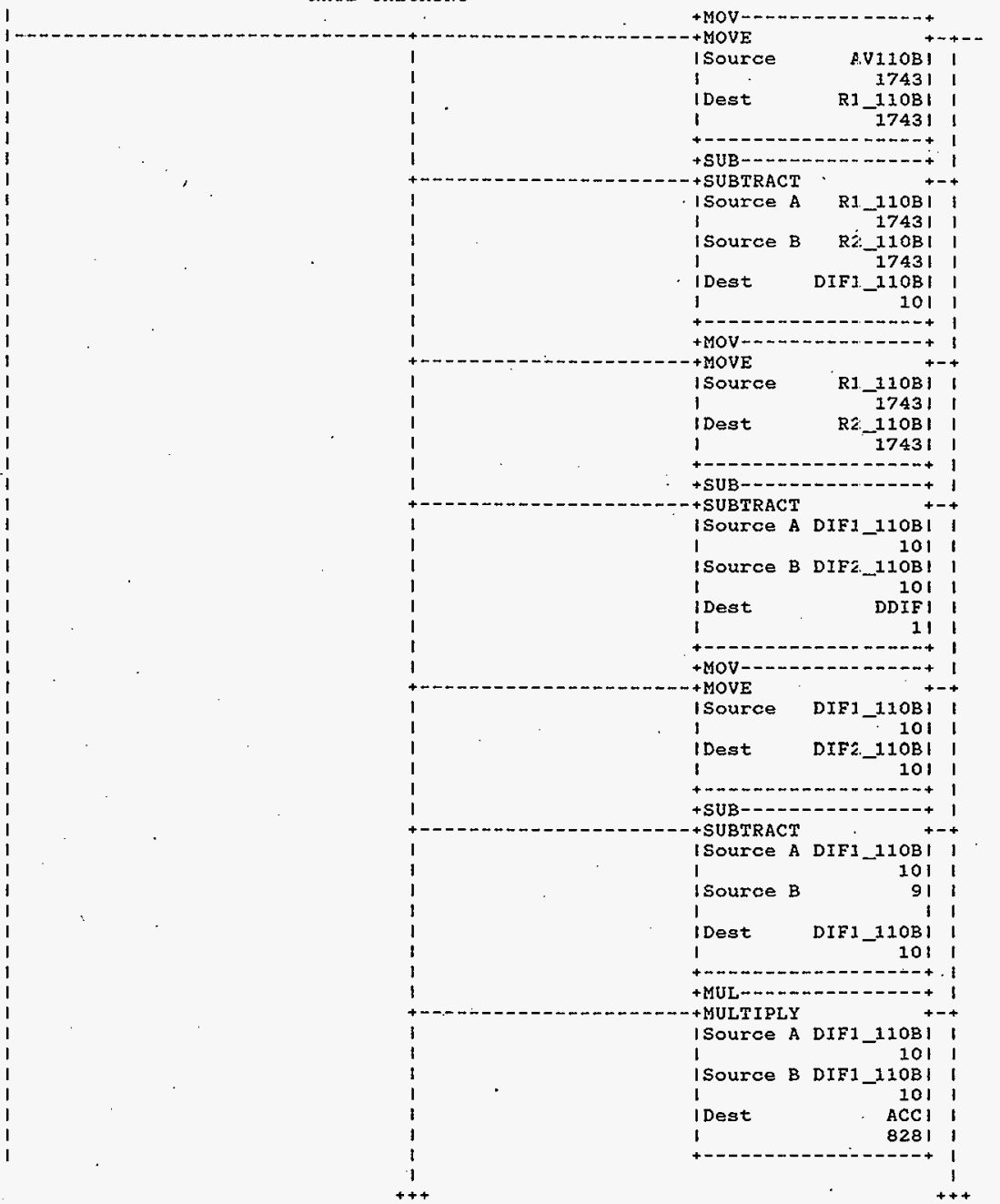

HNF-SD-FF-CSWD-Gil Rev. 0 
Processor and Data(OPS Unit 1)

October 23, 1996 Page 80

Program Listing

Processor File: SODIUM1A.ACH

Rung $2: 198$

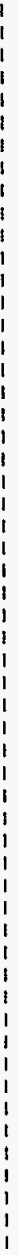

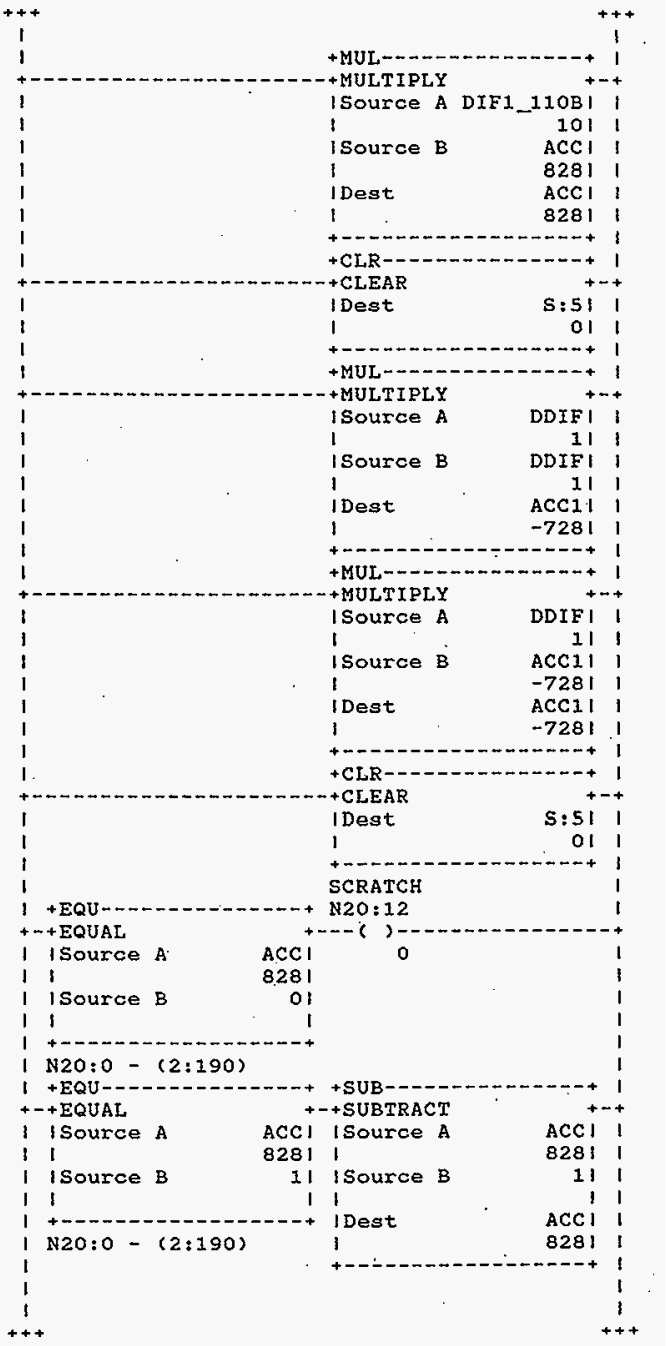

HNF-SD-FF-CSWD-61 Rev. 0 
Processor and Data(ops Unit 1)

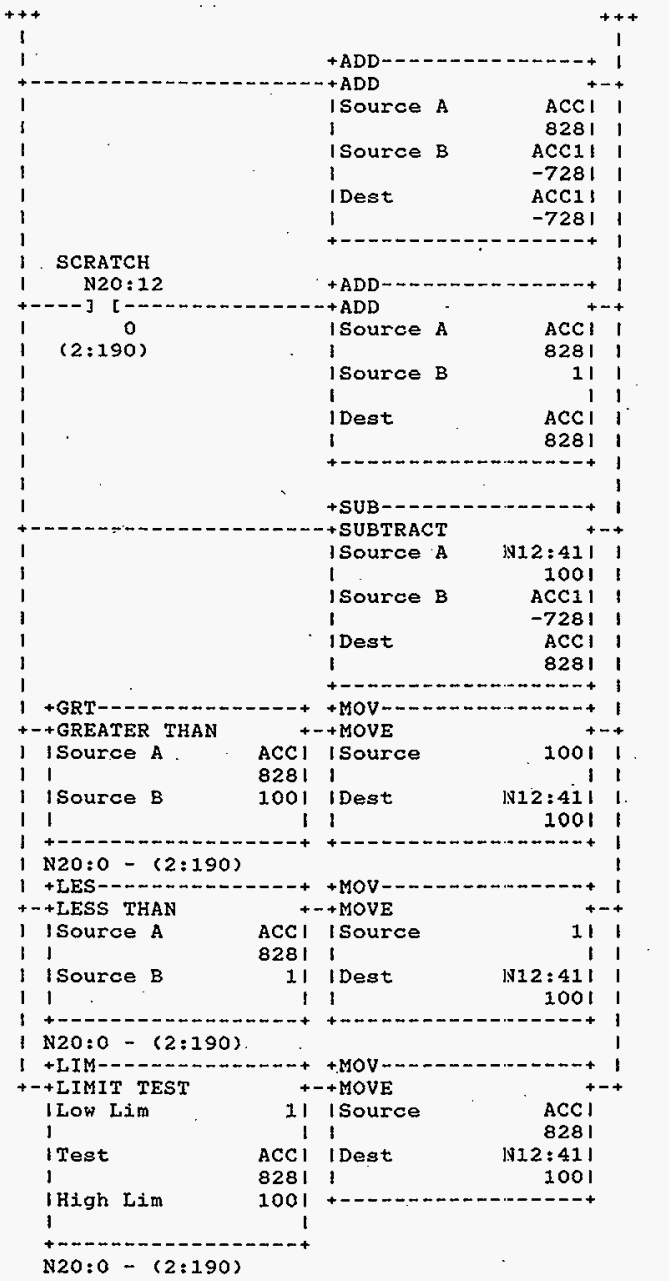


Rung 2:199

I PID110B

18

DUMMY17

$1---[$ LBL $]$

N2O: 8

1

18

Rung $2: 200$

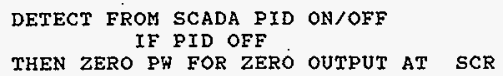

Rung $2: 201$

DETECT AUTO/MANUAL FROM SCADA

SET APPROPRIATE MODE IN PID BLOCK

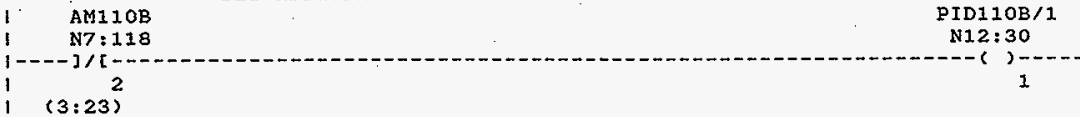

Rung 2:202

DETECT SETPOINT VALUE FROM SCADA PUT VALUE IN PID BLOCK

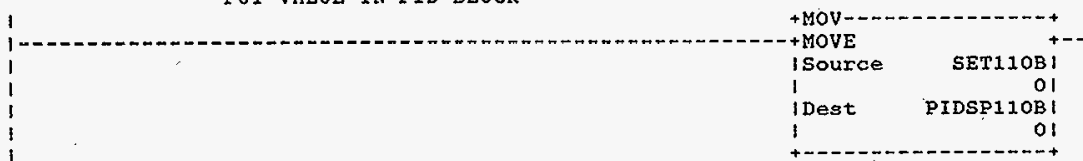

Rung 2:203

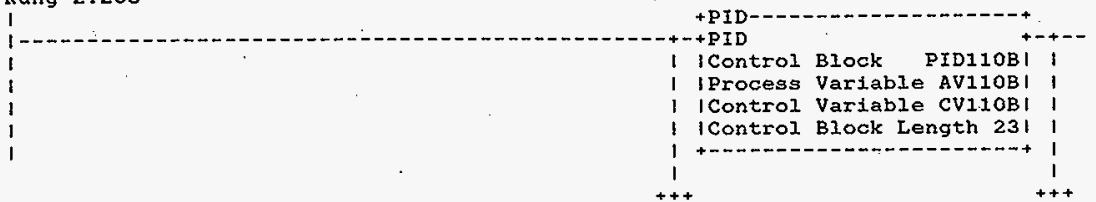

HNF-SD-FF-CSWD-6! Rev, 0 
Processor and Data(OPS Unit 1)

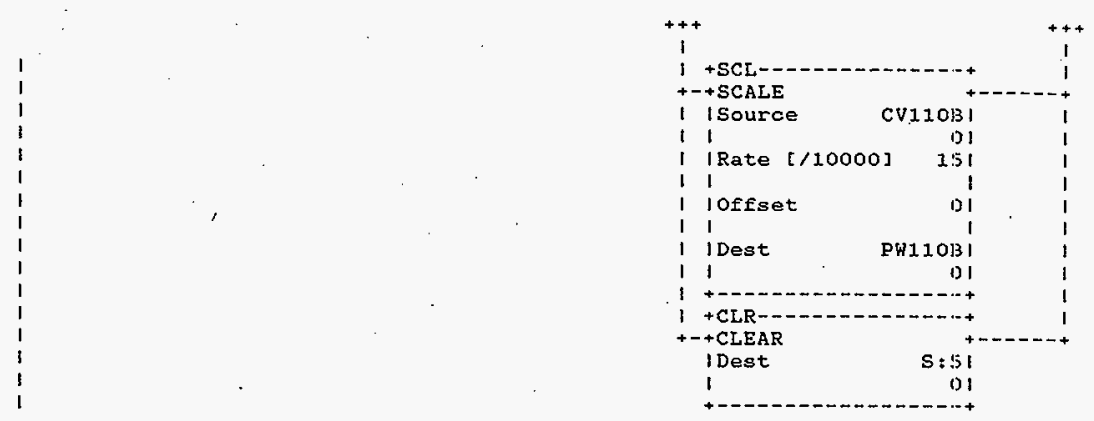

Rung 2:204

157

I - [ LBL ]

N20: 9

Rung 2:205

I

RT.TMR/DN

P:LD110C

T4:62

19

1..-- ] $] /[-$

(JMP)

$(2: 33)$

Rung 2:206

RATE CHECKING

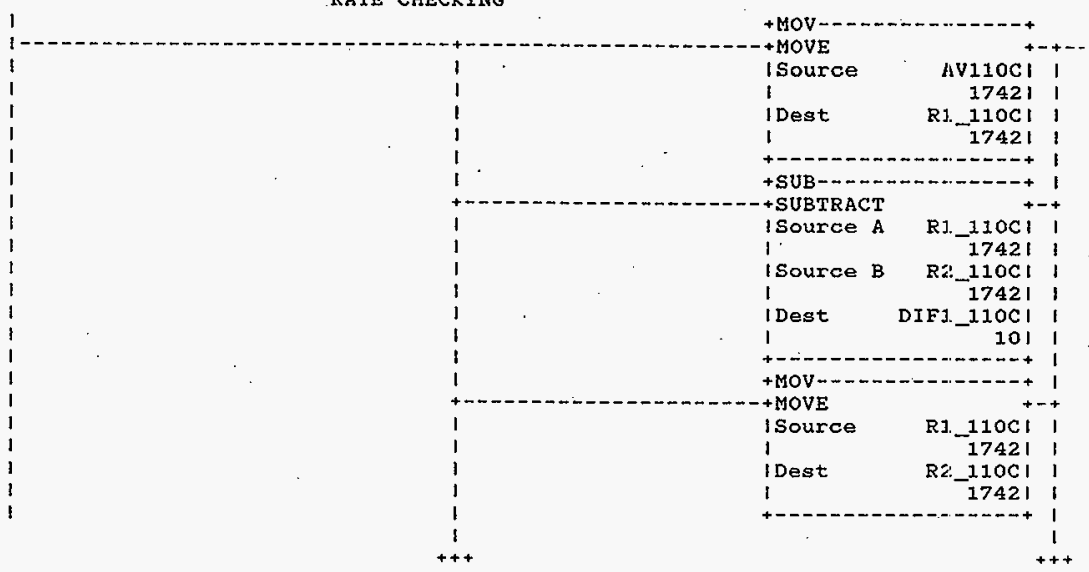

HNF-SD-FF-CSWD-61 Rev. 0 


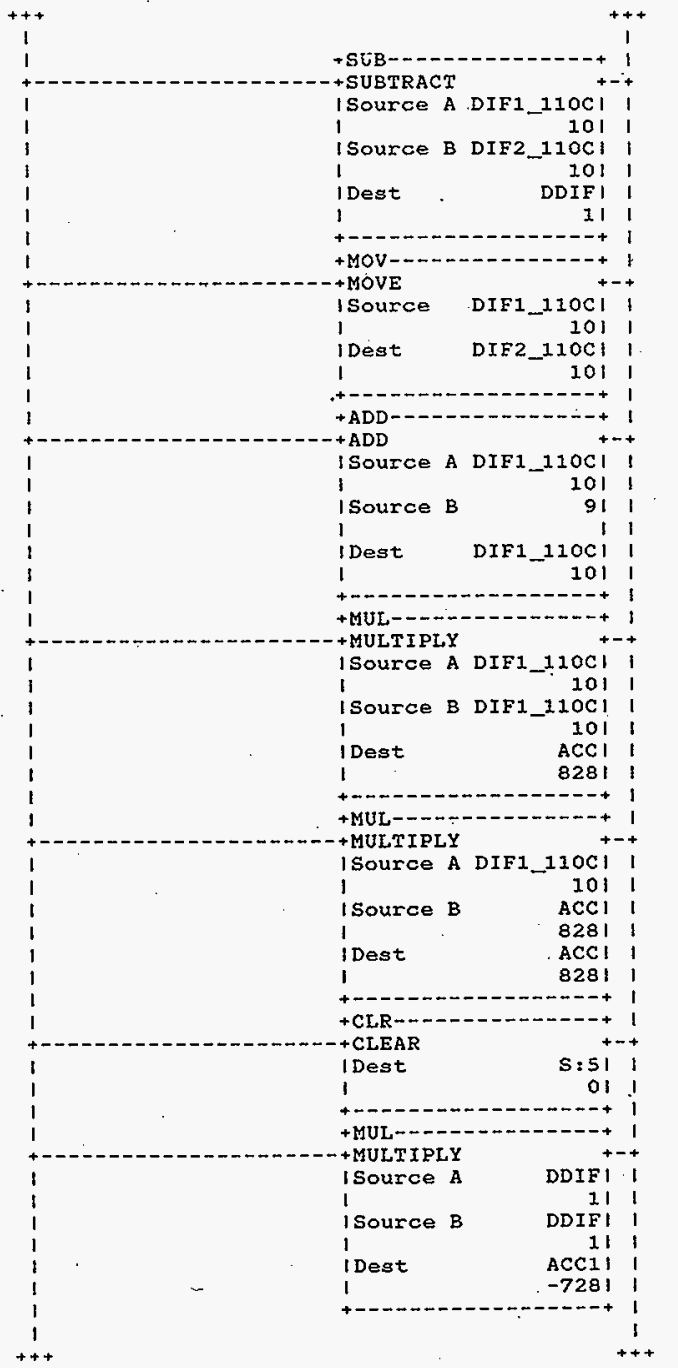


Processor and Data(OPS Unit i)

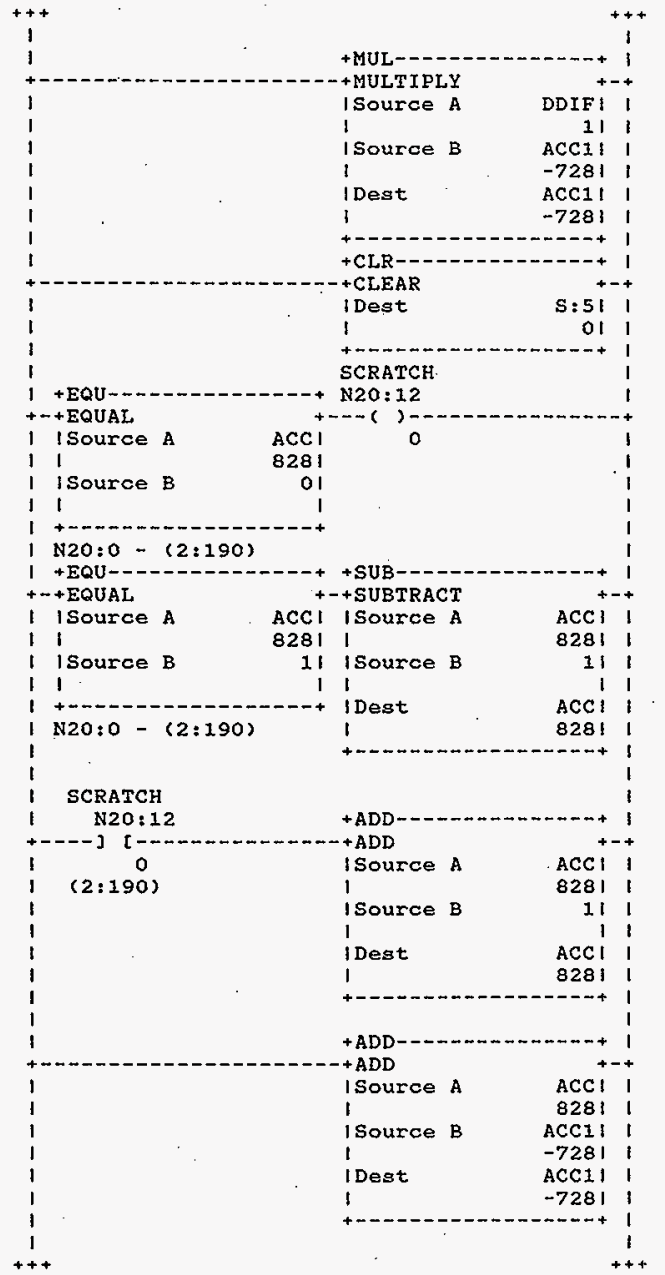

HNF-SD-FF-CSWD-61 Rev. 0 
Processor and Data(OPS Unit 1$)$

October 23, 1996 Page 86 Program Listing Processor File: SODIUMIA.ACH

Rung $2: 206$

$$
\begin{aligned}
& 1 \\
& 1 \\
& 1 \\
& 1 \\
& 1 \\
& 1 \\
& 1 \\
& 1 \\
& 1 \\
& 1 \\
& 1 \\
& 1 \\
& 1 \\
& 1 \\
& 1 \\
& 1 \\
& 1 \\
& 1 \\
& 1 \\
& 1 \\
& 1 \\
& 1 \\
& 1 \\
& 1 \\
& 1 \\
& 1 \\
& 1 \\
& 1 \\
& 1
\end{aligned}
$$

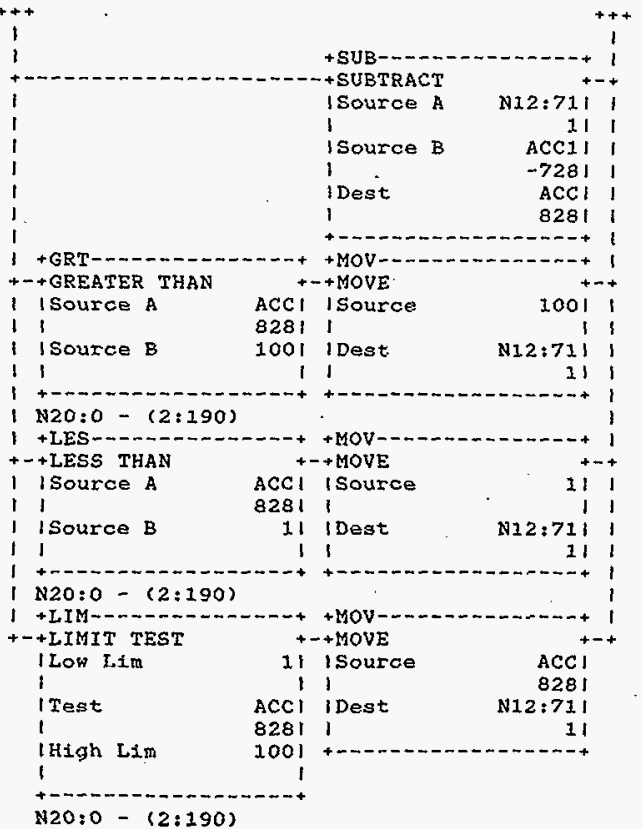

Rung $2: 207$

I PID110C

1. 19

DUMMX18

N20:8

$1---[$ LBL

Rung $2: 208$

DETECT FROM SCADA PID ON/OFF

IF PID OFF

THEN ZERO PH FOR ZERO OUTPUT AT SCR

ON_I10C AND JUMP AROUND THE PID BLOCK

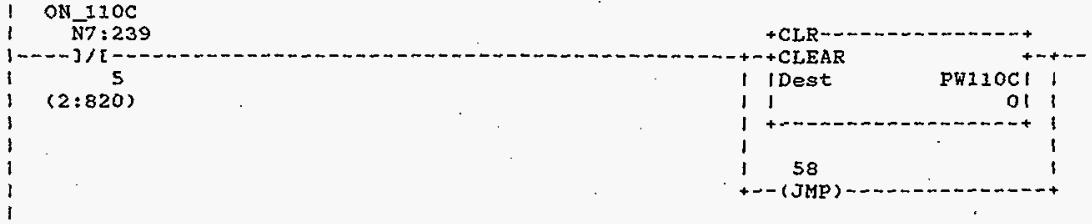

HNF-SD-FF-CSWD-61 Rev. 0 
Processor and Data(OPS Unit 1)

Program Listing

Processor File: SODIUMiA.ACH

October 23, 1996 Page 8:

Rung 2:209

DETECT AUTO/MANUAL FROM SCADA

SET APPROPRIATE MODE IN PID BLOCK

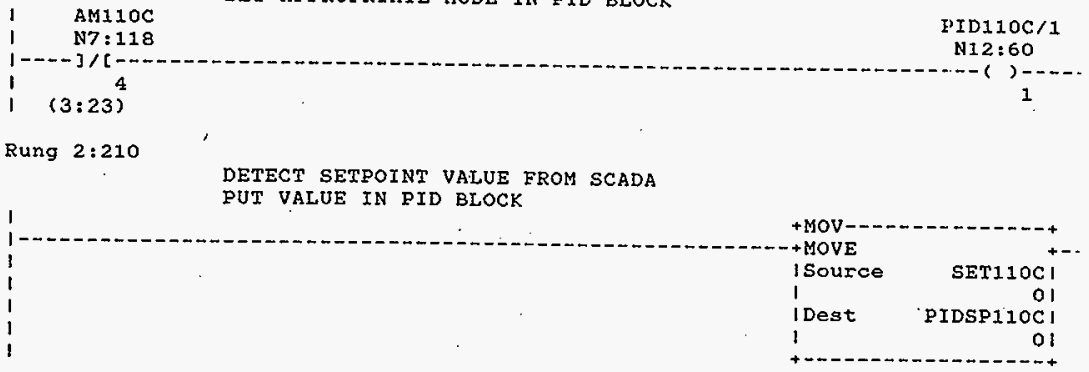

Rung $2: 211$

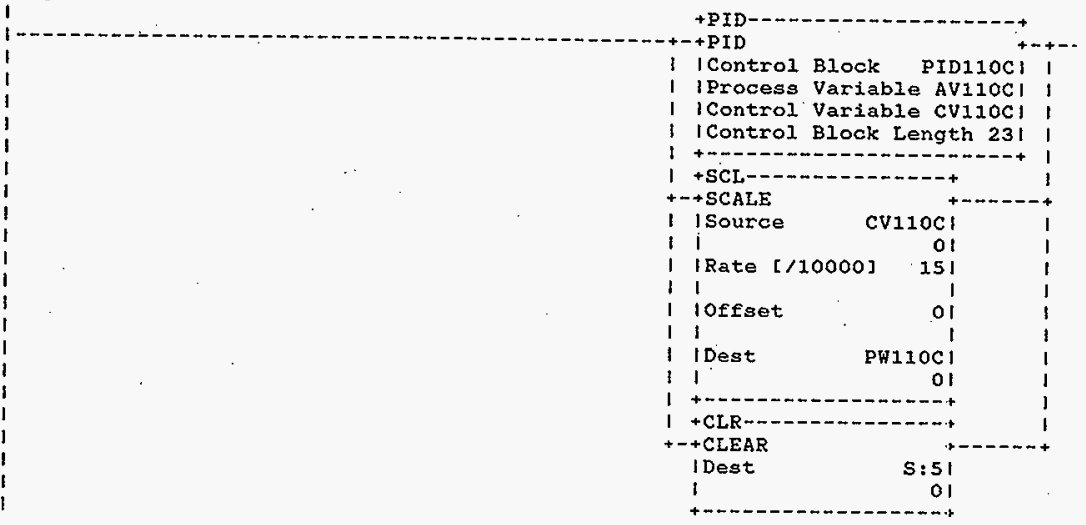

Rung 2:212

I 58

I $--[$ [

N20:9

$-6,-$.

Rung $2: 213$

I

RT TMR/DN

PII)111A

I T4:62

$1---3 /[-$

(2:33) 
Processor and Data(OPS Unit 1 ) Program Listing
October 23, 1996 Page $8 \varepsilon$

Rung $2: 214$

\section{RATE CHECKING}

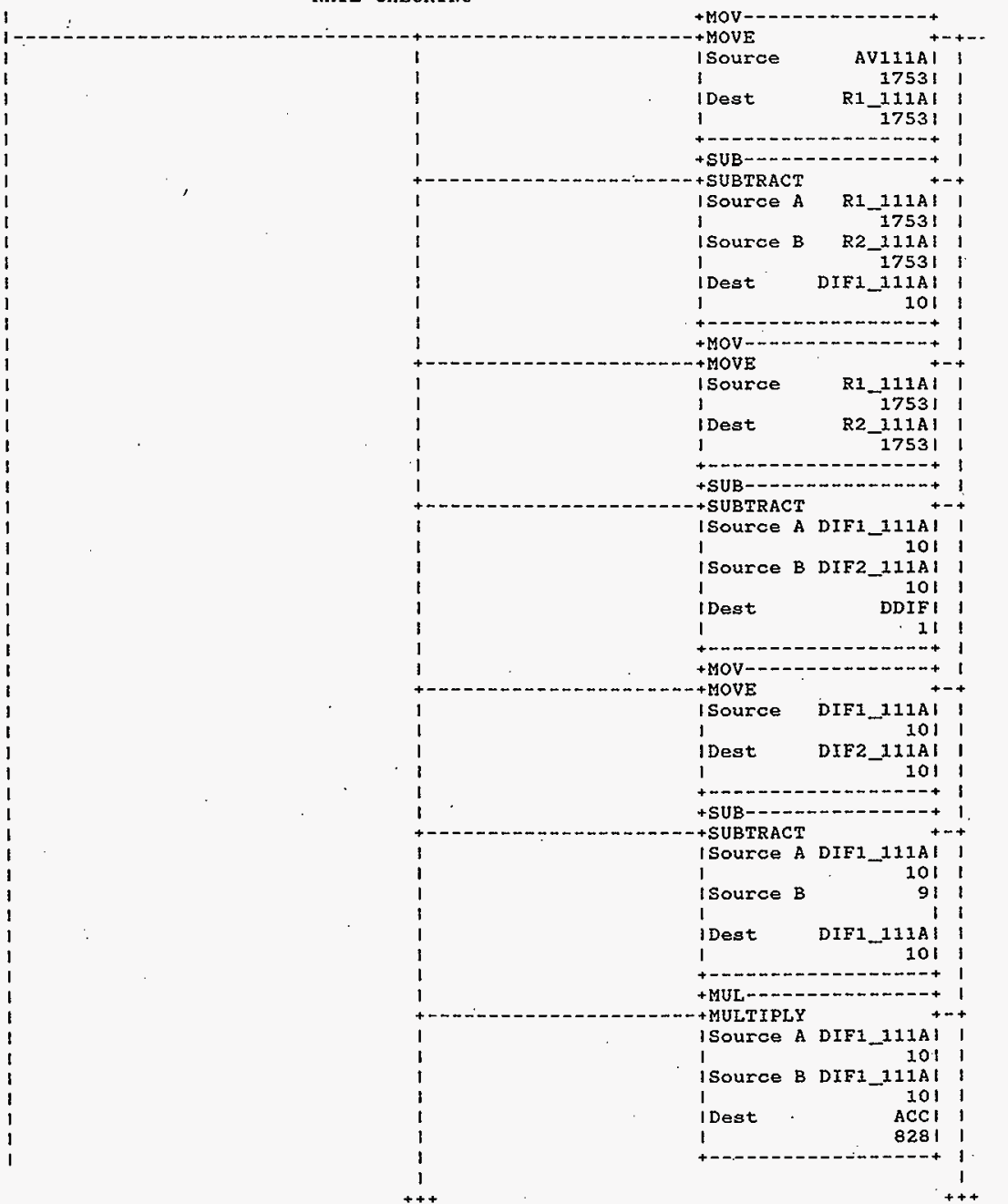




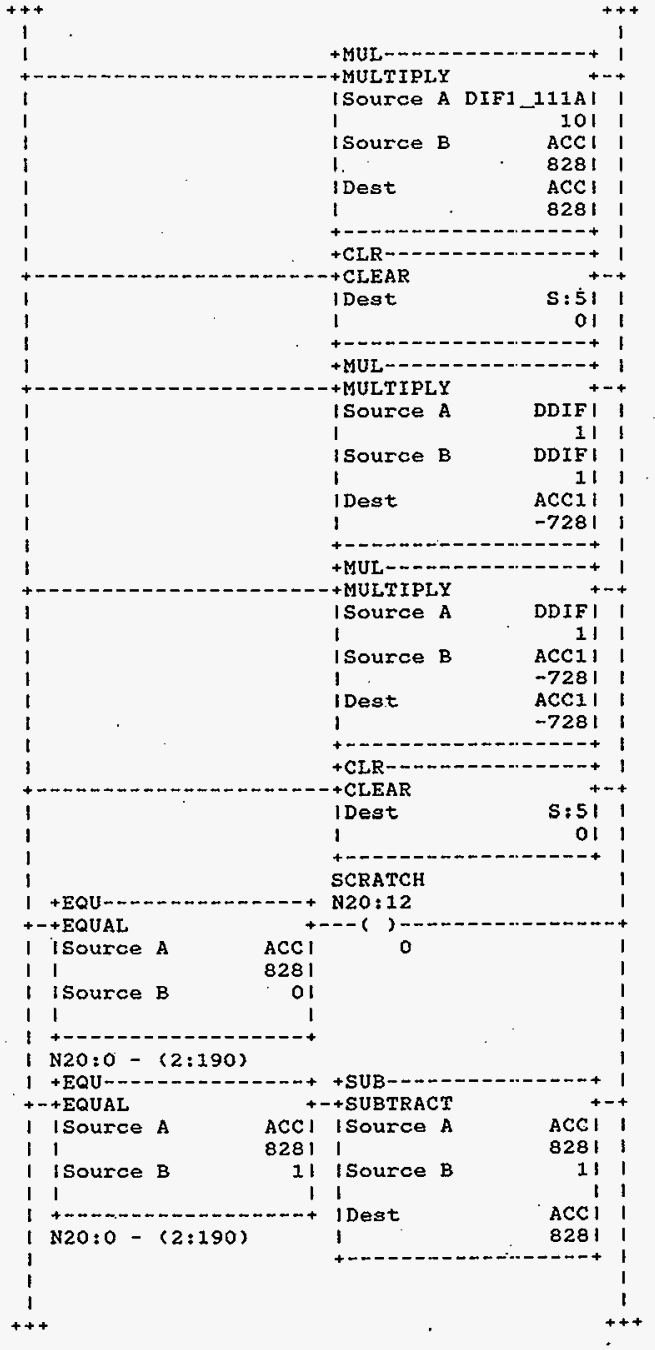

HNF-SD-FF-CSWD-151 Rev. 0 
Processor and Data(OPS Unit 1) Program Listing
October 23,1996 Page 96

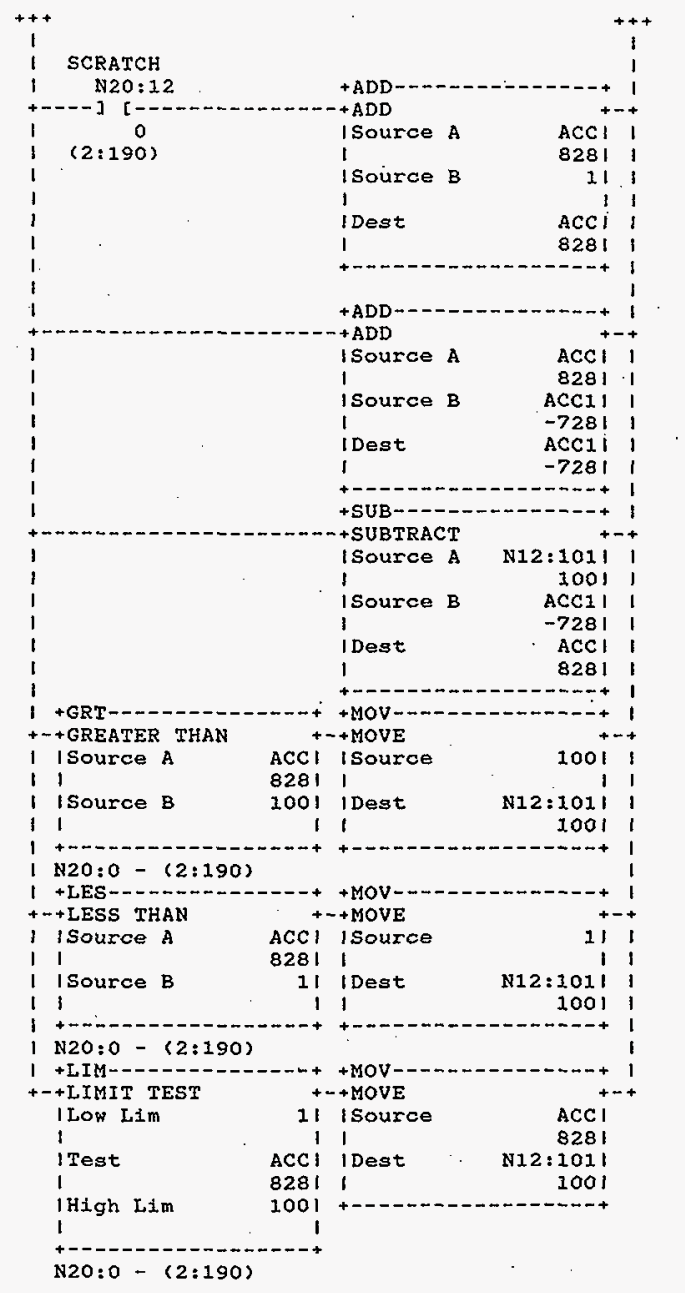



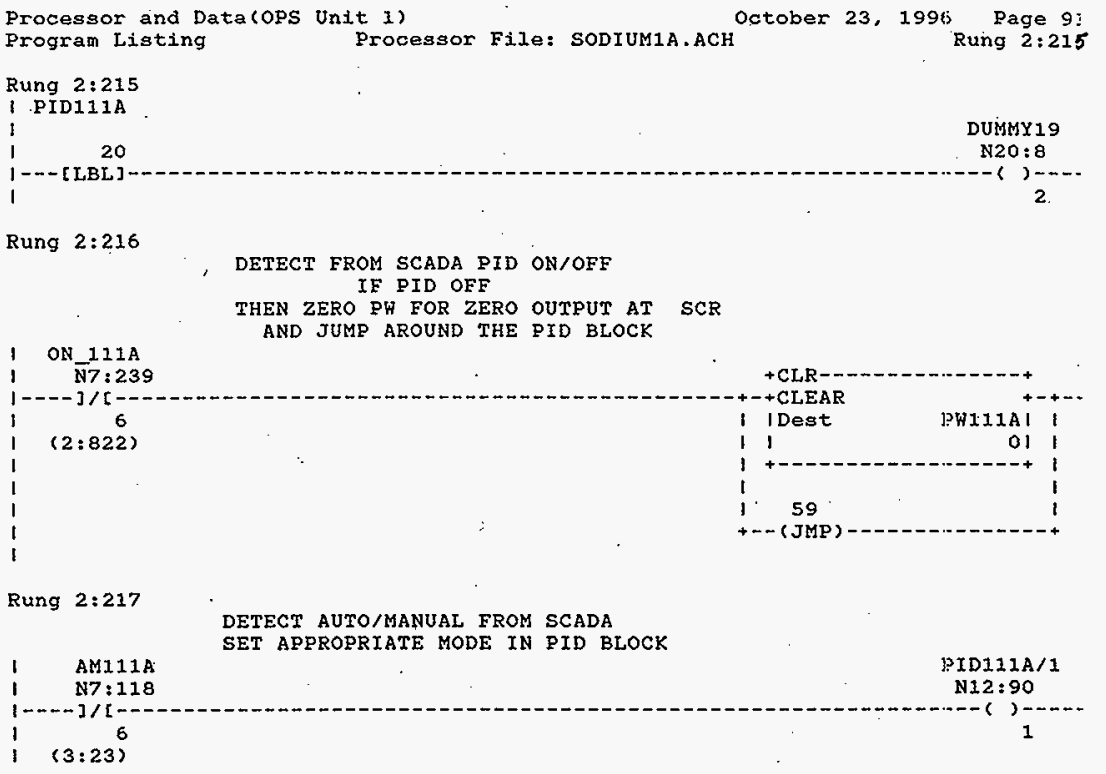

Rung 2:218 DETECT SETPOINT VALUE FROM SCADA

PUT VALUE IN PID BLOCK

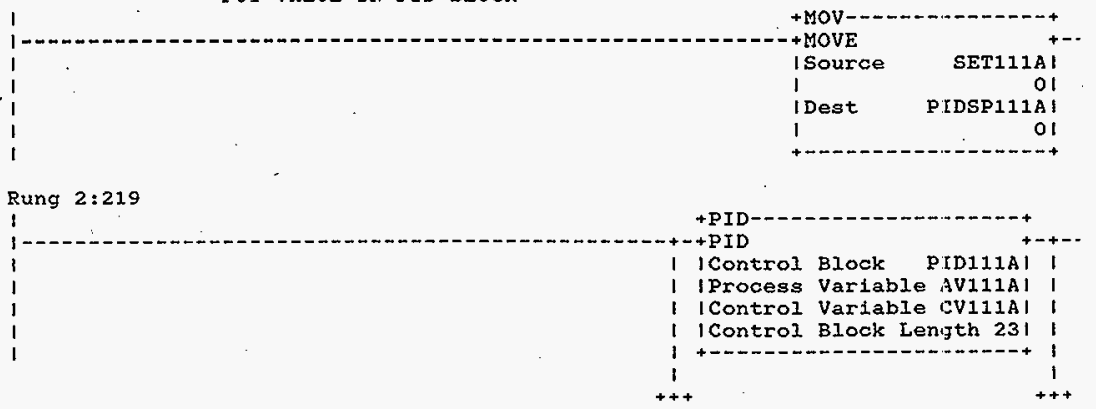

HNF-SD-FF-CSWD-61 R.ev. 0 


$$
\begin{aligned}
& 1 \\
& 1 \\
& 1 \\
& 1 \\
& 1 \\
& 1 \\
& 1 \\
& 1 \\
& 1 \\
& 1 \\
& 1 \\
& 1 \\
& 1 \\
& 1 \\
& 1 \\
& 1
\end{aligned}
$$

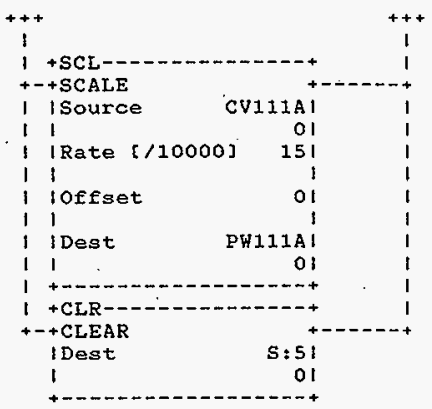

Rung 2:220

Rung $2: 221$

I

(RT_TMR/DN

( T4:62

1. - - ] ] [

i. (2:33)

Rung 2:222

RATE CHECKING

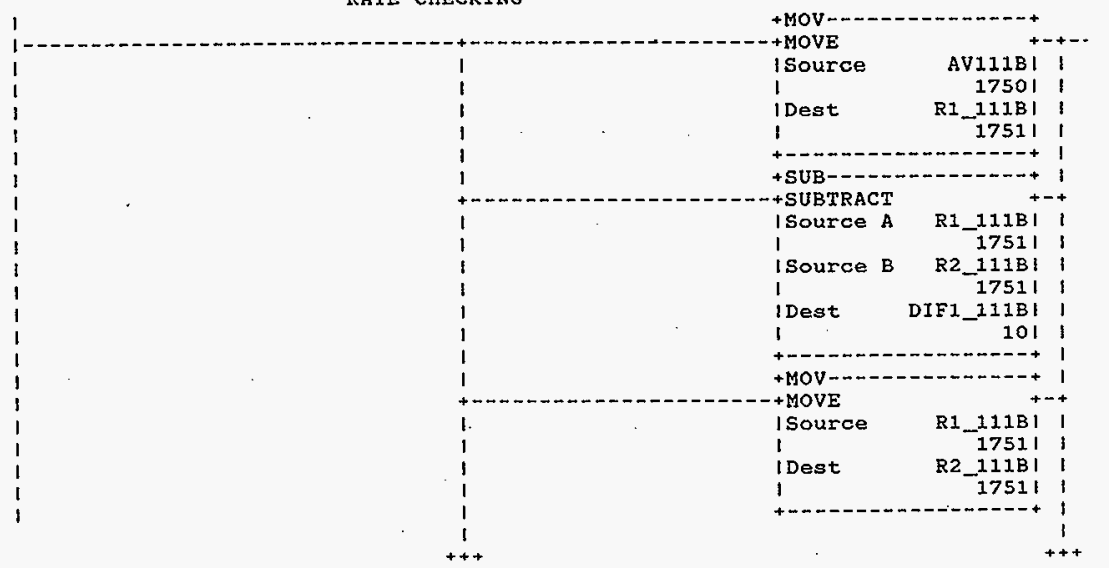

HNF-SD-FF-CSWD-61 Rev. 0

Page 92 


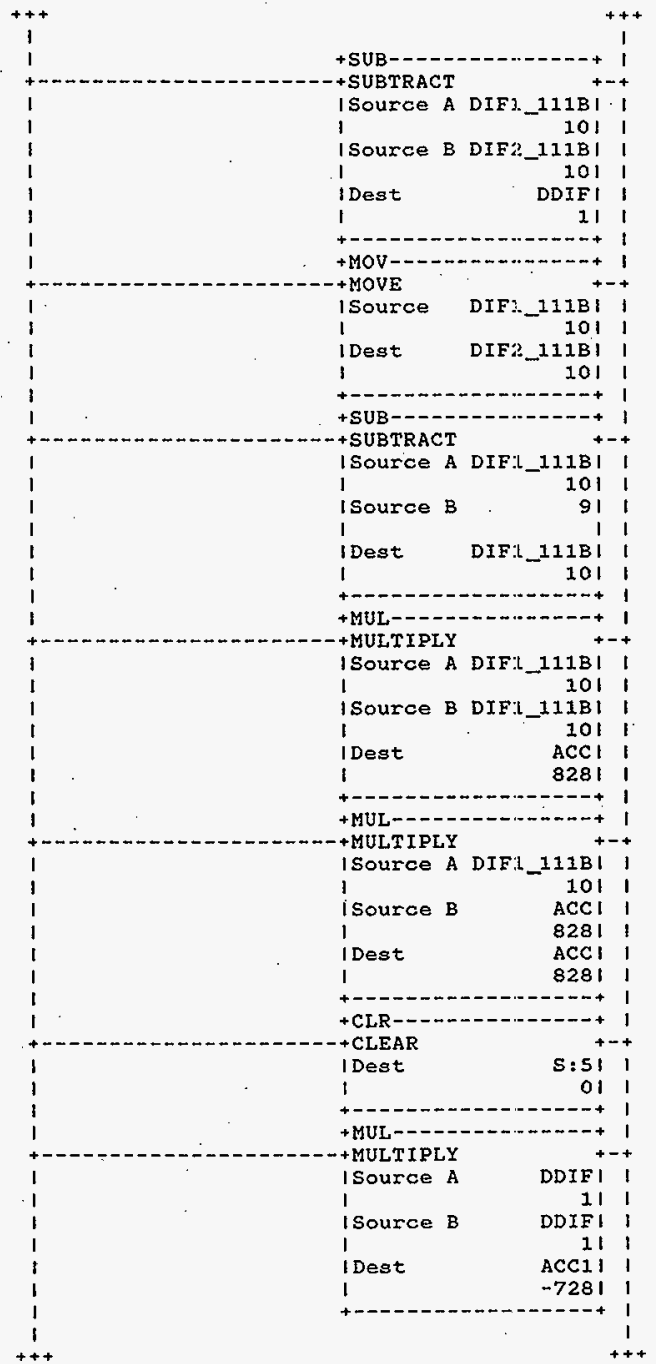

HNF-SD-FF-CSWD-61 Rev. 0

Page 9,3 


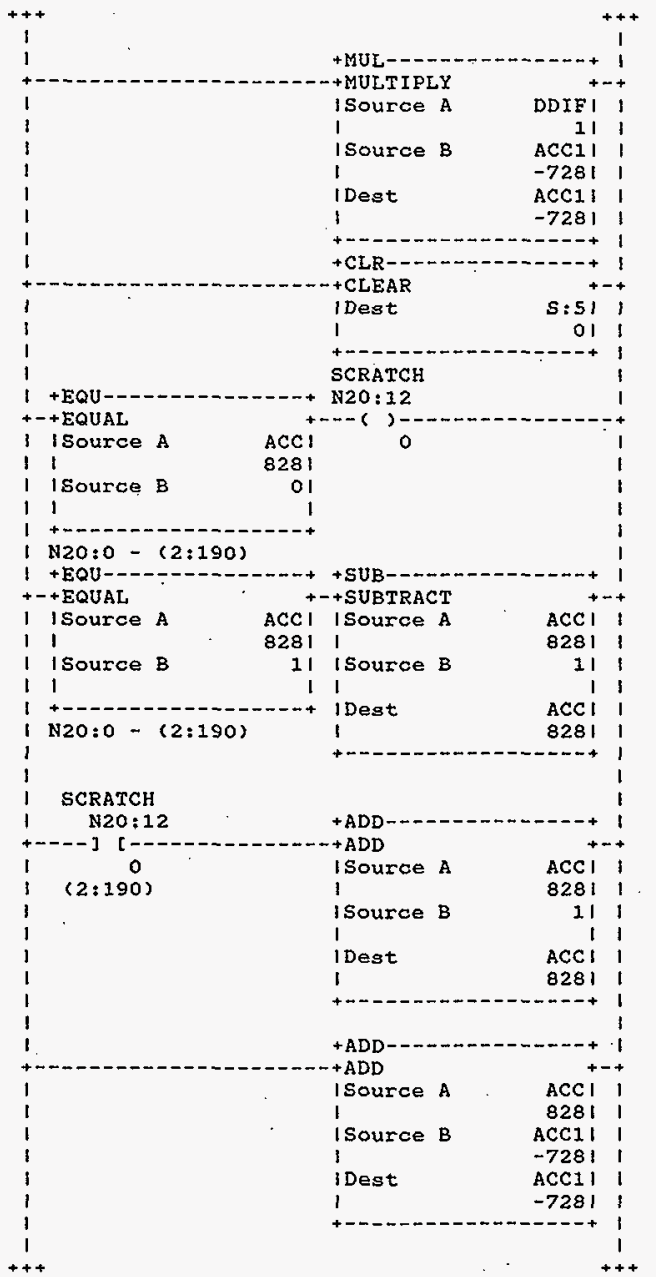


Processor and Data(OPS Unit 1)

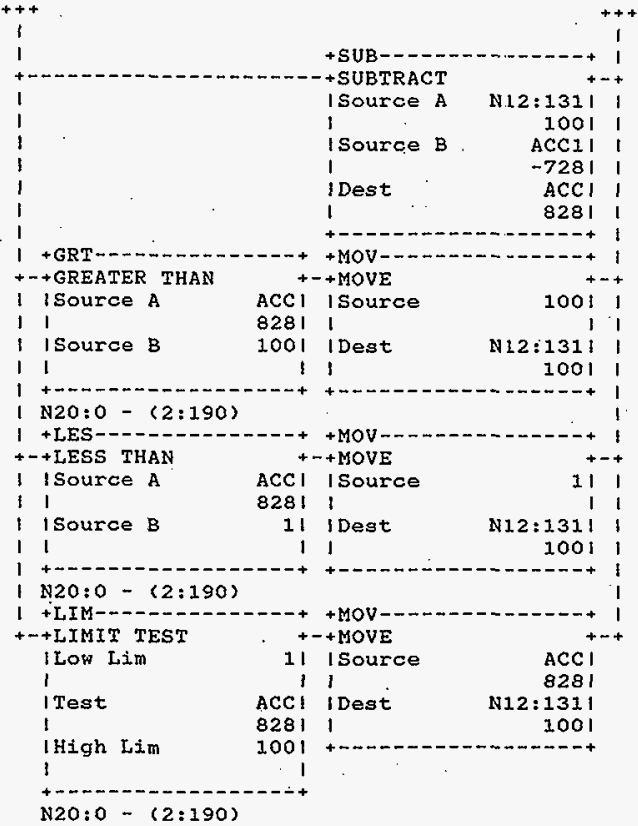

Rung $2: 223$

! PID111B

1

i 21

DUMMY 20

$1---[\operatorname{LBL}]$

N20: 8

Rung 2:224

DETECT FROM SCADA PID ON/OFF

IF PID OFF

THEN ZERO PW. FOR ZERO OUTPUT AT SCR AND JUMP AROUND THE PID BLOCK

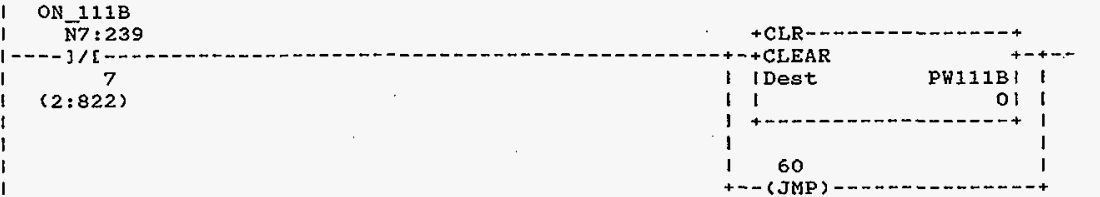

HNF-SD-FF-CSWD-61 Rev. 0 
Processor and Data(OPS Unit 1)

Program Listing

Prodessor File: SODIUMiA.ACH

Rung 2:225

AM111

DETECT AUTO/MANUAL FROM SCADA

SET APPROPRIATE MODE IN PID BLOCK

N7 : 118

PIDI11B/1

N12:120

$1----3 / 6--$

8

$(3: 23)$

Rung 2:226

DETECT SETPOINT VALUE FROM SCADA

PUT VALUE IN PID BLOCK

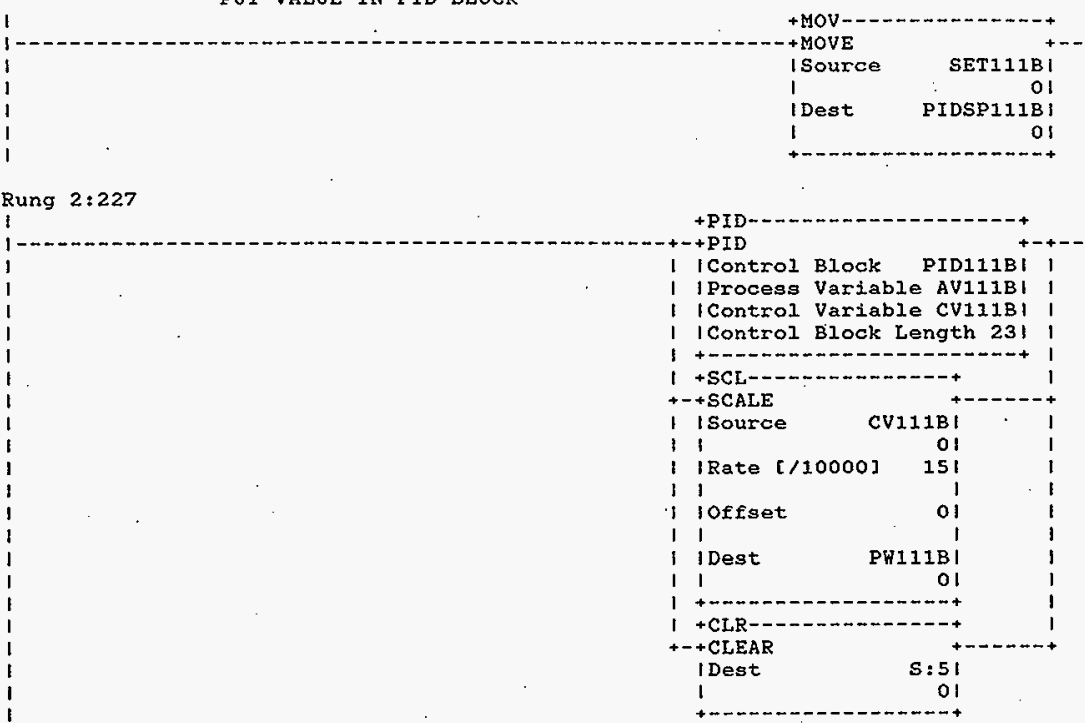

Rung $2: 228$

$$
160
$$

N20:9

I- - [ LBL ]

Rung 2:229

$$
\begin{array}{lc}
\text { I } & \text { RT TMR } / D N \\
1 & T: 62 \\
1 & (2: 33)
\end{array}
$$


Processor and Data(OPS Unit 1) Program listing

Rung 2:230

1 RT TMR/DN

I T4:62

$1-\cdots+2 \quad[-$

DNR 1

$1(2: 33)$

$0: 5$

Rung $2: 231$

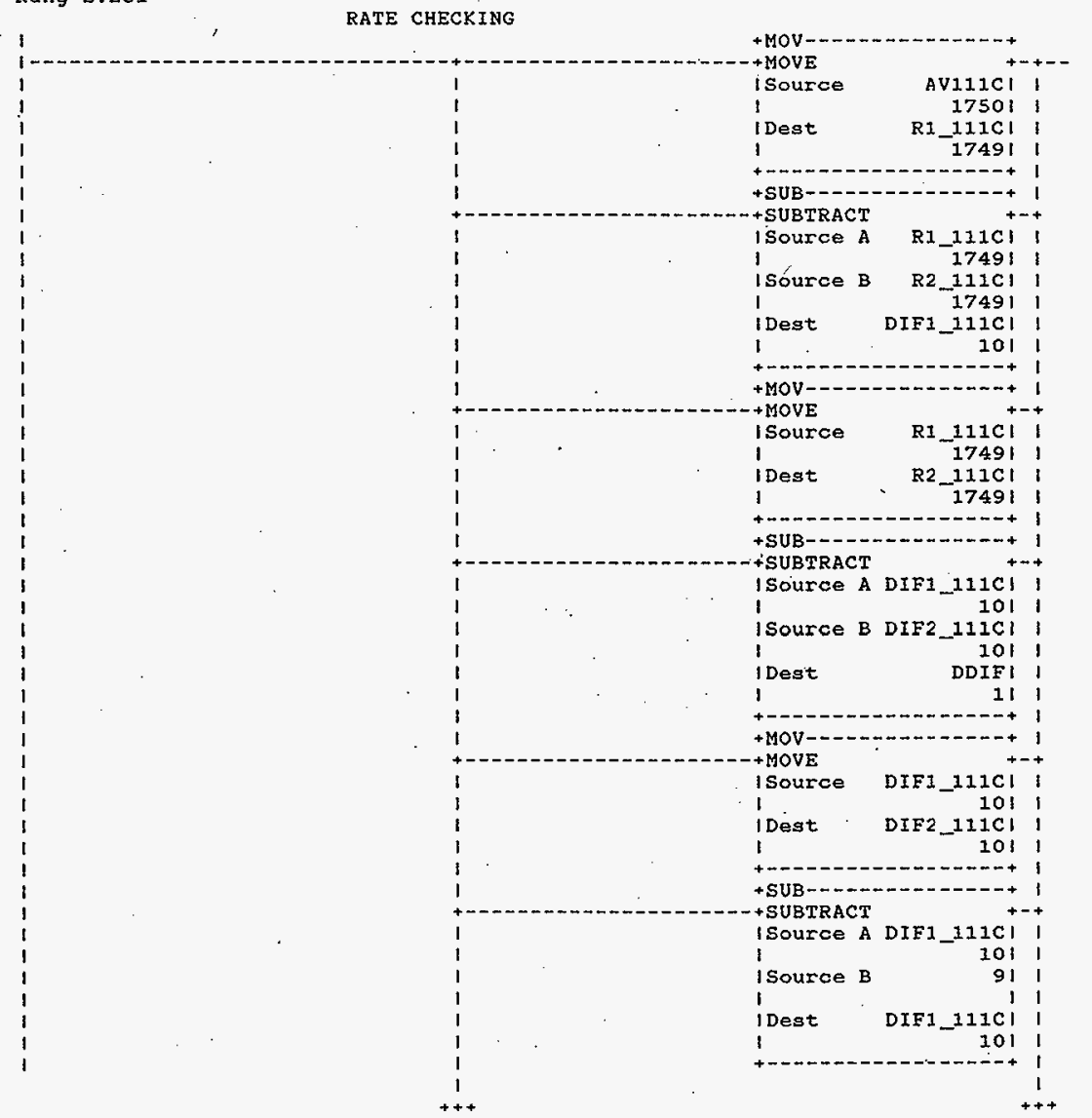

HNF-SD-FF-CSWD-61 Rev. 0 
Processor and Data (ops Unit 1) Program Listing
Processor File: SODIUM1A.ACH

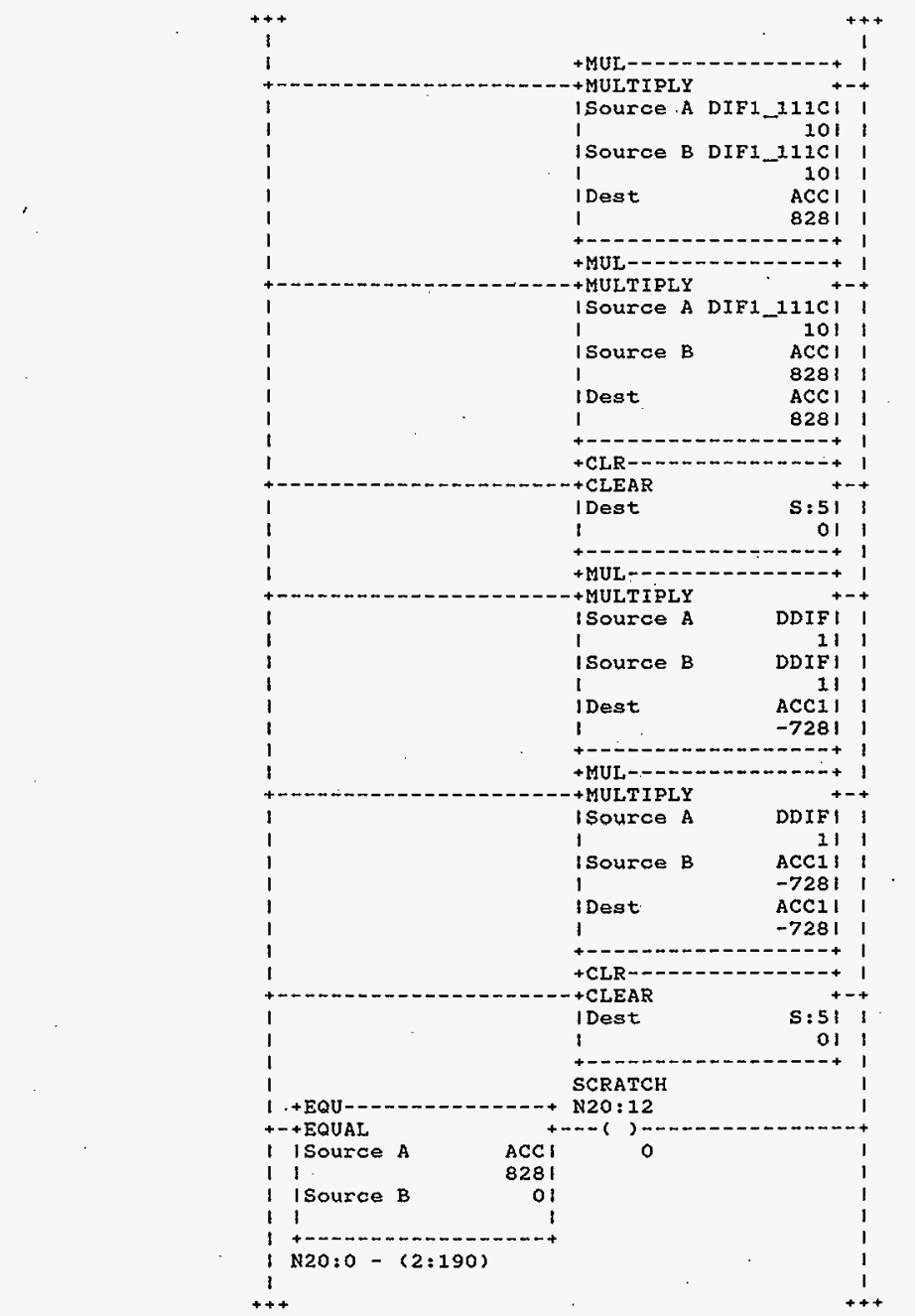

October 23, 1996 Page 98 Rung $2: 231$

$$
\begin{aligned}
& 1 \\
& 1 \\
& 1 \\
& 1 \\
& 1 \\
& 1 \\
& 1 \\
& 1 \\
& 1 \\
& 1 \\
& 1 \\
& 1 \\
& 1 \\
& 1 \\
& 1 \\
& 1 \\
& 1 \\
& 1 \\
& 1 \\
& 1 \\
& 1 \\
& 1 \\
& 1 \\
& 1 \\
& 1 \\
& 1 \\
& 1 \\
& 1 \\
& 1 \\
& 1 \\
& 1 \\
& 1 \\
& 1 \\
& 1 \\
& 1 \\
& 1 \\
& 1 \\
& 1 \\
& 1 \\
& 1 \\
& 1 \\
& 1 \\
& 1 \\
& 1 \\
& 1 \\
& 1
\end{aligned}
$$

HNF-SD-FF.CSWD-61 Rev. 0 
Processor and Data(OPS Unit 1)

1
1
1
1
1
1
1
1
1
1
1
1
1
1
1
1
1
1
1
1
1
1
1
1
1
1
1
1
1
1
1
1
1
1
1
1
1
1
1
1

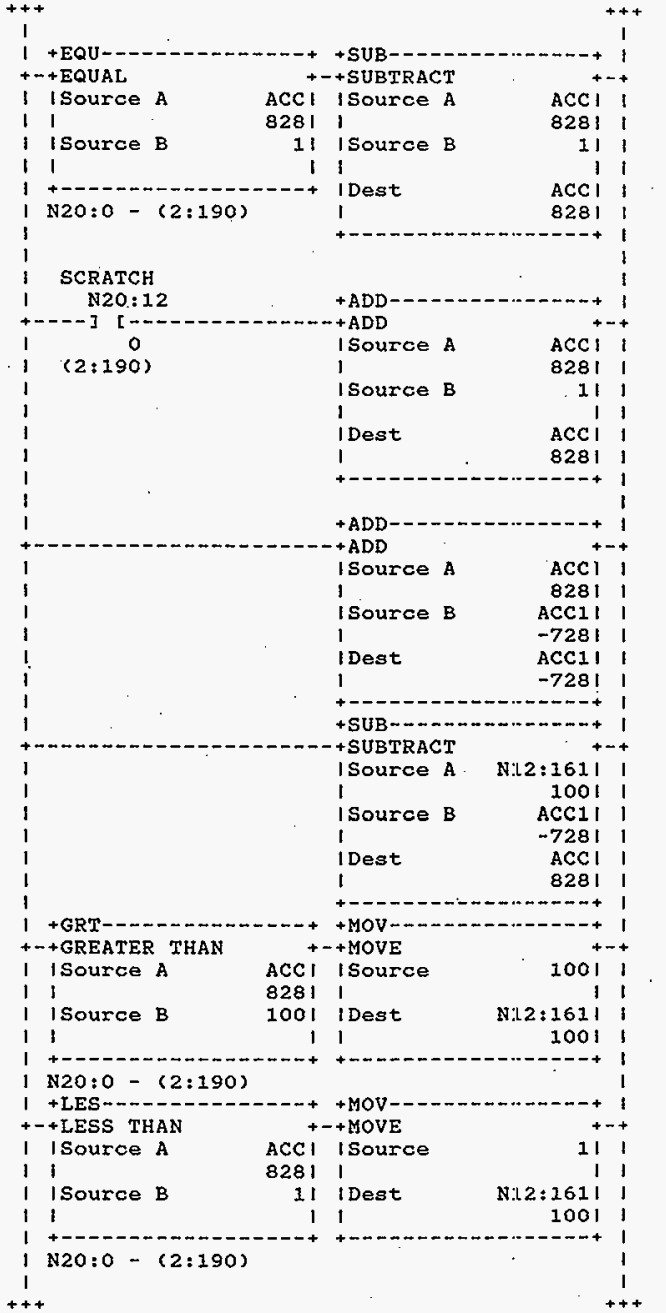

HNF-SD-FF-CSWD-61 Rey. 0 


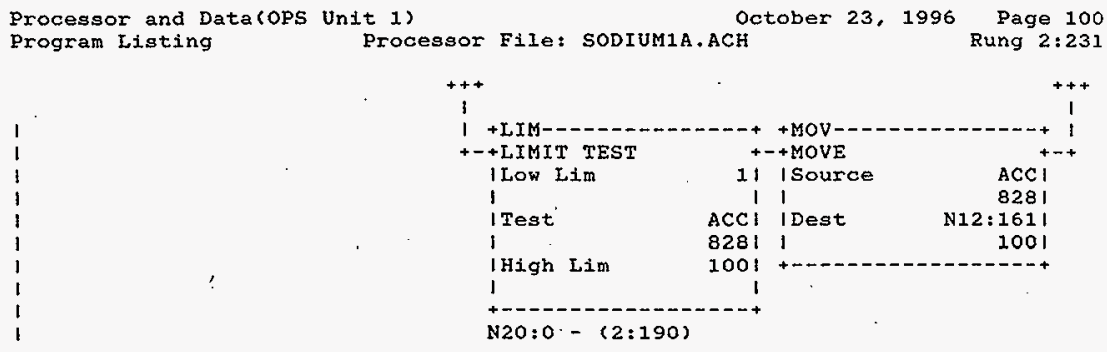

Rung $2: 232$

I PIDIIIC

I 22

DUMMY 21

$1-\cdots[$ LBL $]-$

N20:8 3

Rung $2: 233$

\section{DETECT FROM SCADA PID ON/OFF}

IF PID OFF

THEN ZERO PW FOR ZERO OUTPUT AT SCR AND JUMP AROUND THE PID BLOCK
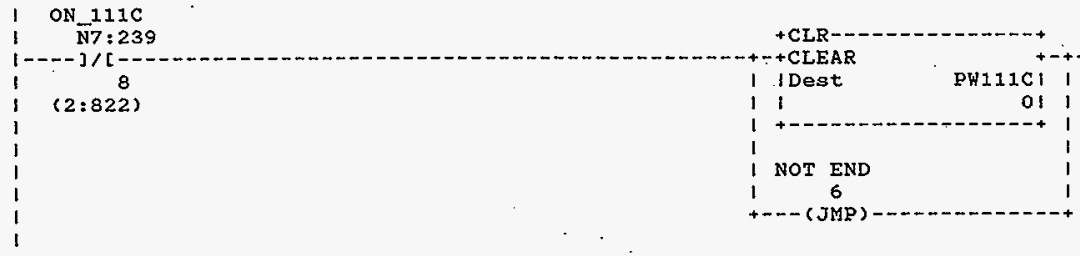

Rung $2: 234$

DETECT AUTO/MANUAL FROM SCADA

SET APPROPRTATE MODE IN PID BLOCK

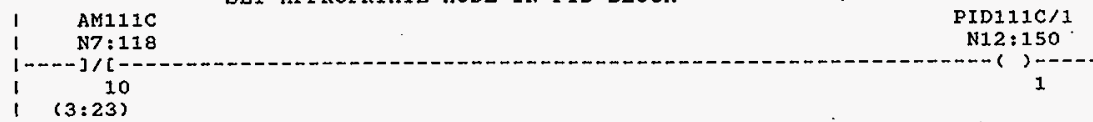

Rung $2: 235$

DETECT SETPOINT VALUE FROM SCADA PUT VALUE IN PID BLOCK

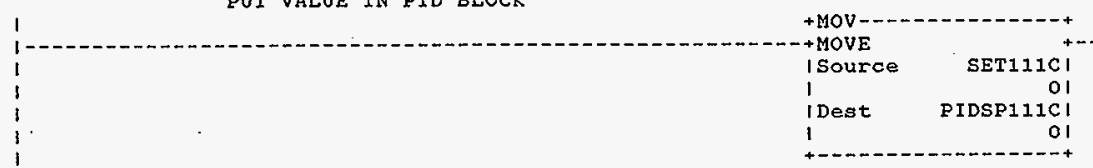

HNF-SD-FF-CSWD-61 Rev. 0 
Rung $2: 236$

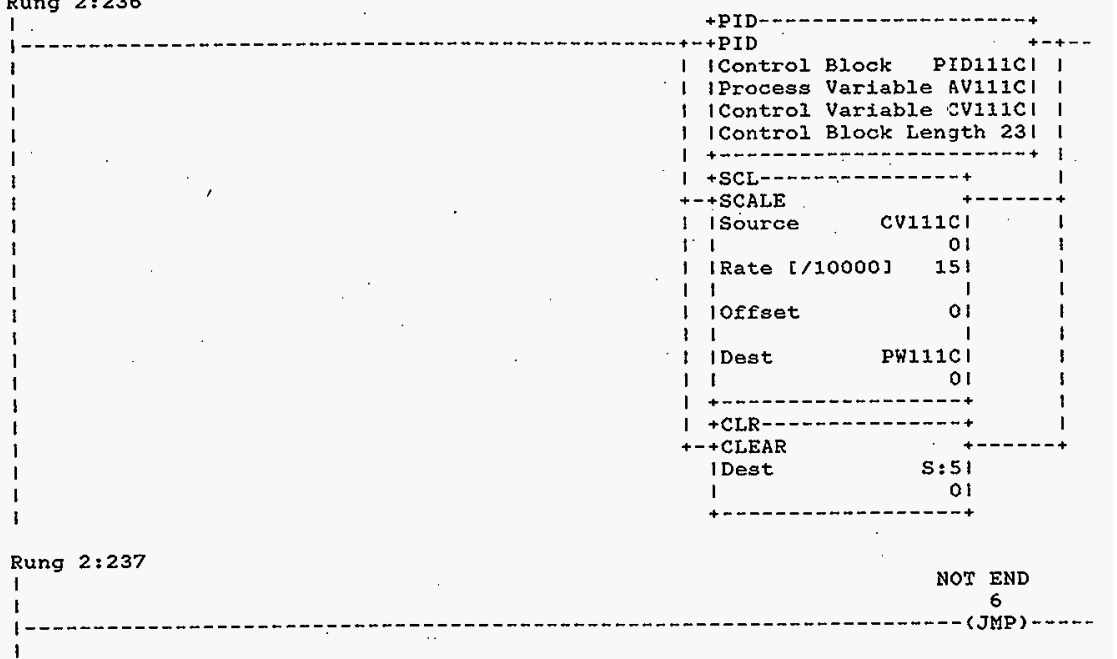

Rung 2:238

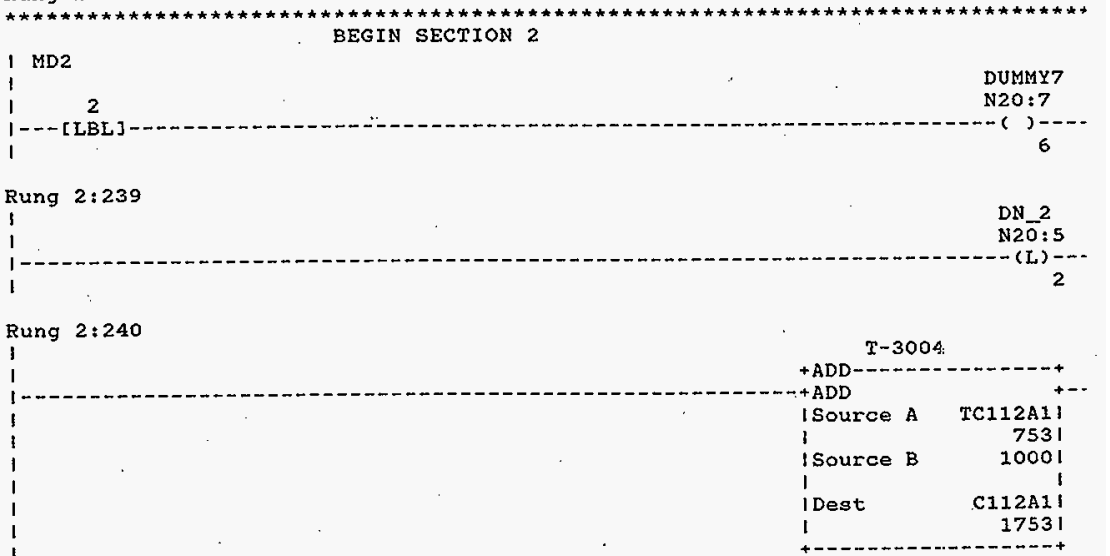

HNF-SD-FF-CSWD-61 Kev. 0 
Processor and Data(OPS Unit 1 )

Rung $2: 241$

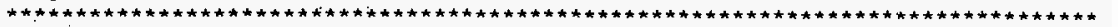
Read each T/C and store each T/C's value in its own memory register

1 T-3004

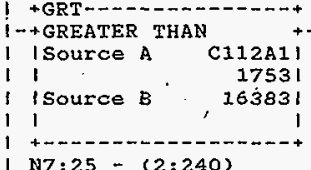
T-3004

Rung $2: 242$

1

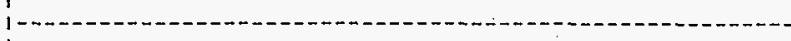

$1+\ldots$

I

31

$+$

$\cdot$

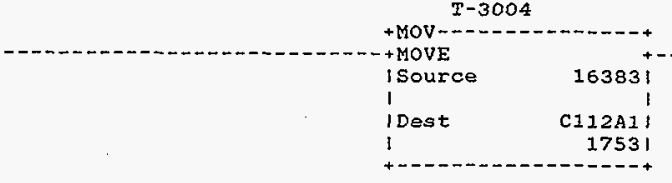

1

Rung 2:243

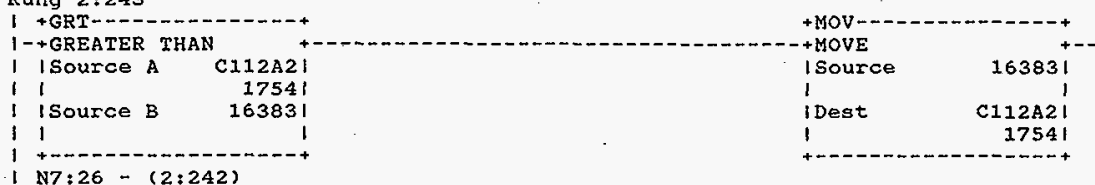

Rung $2: 244$

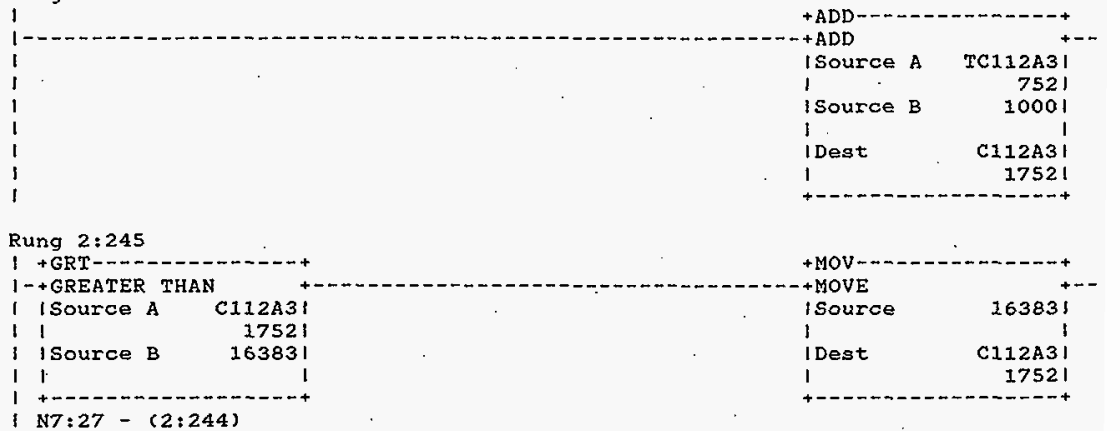

HNF-SD-FF-CSWD-61 Rev. 0 
Processor and Data(OPS Unit 1)

October 23,1996

Page 103

Program Listing

Processor File: SODIUM1A.ACH

Rung 2:246

Rung 2:246
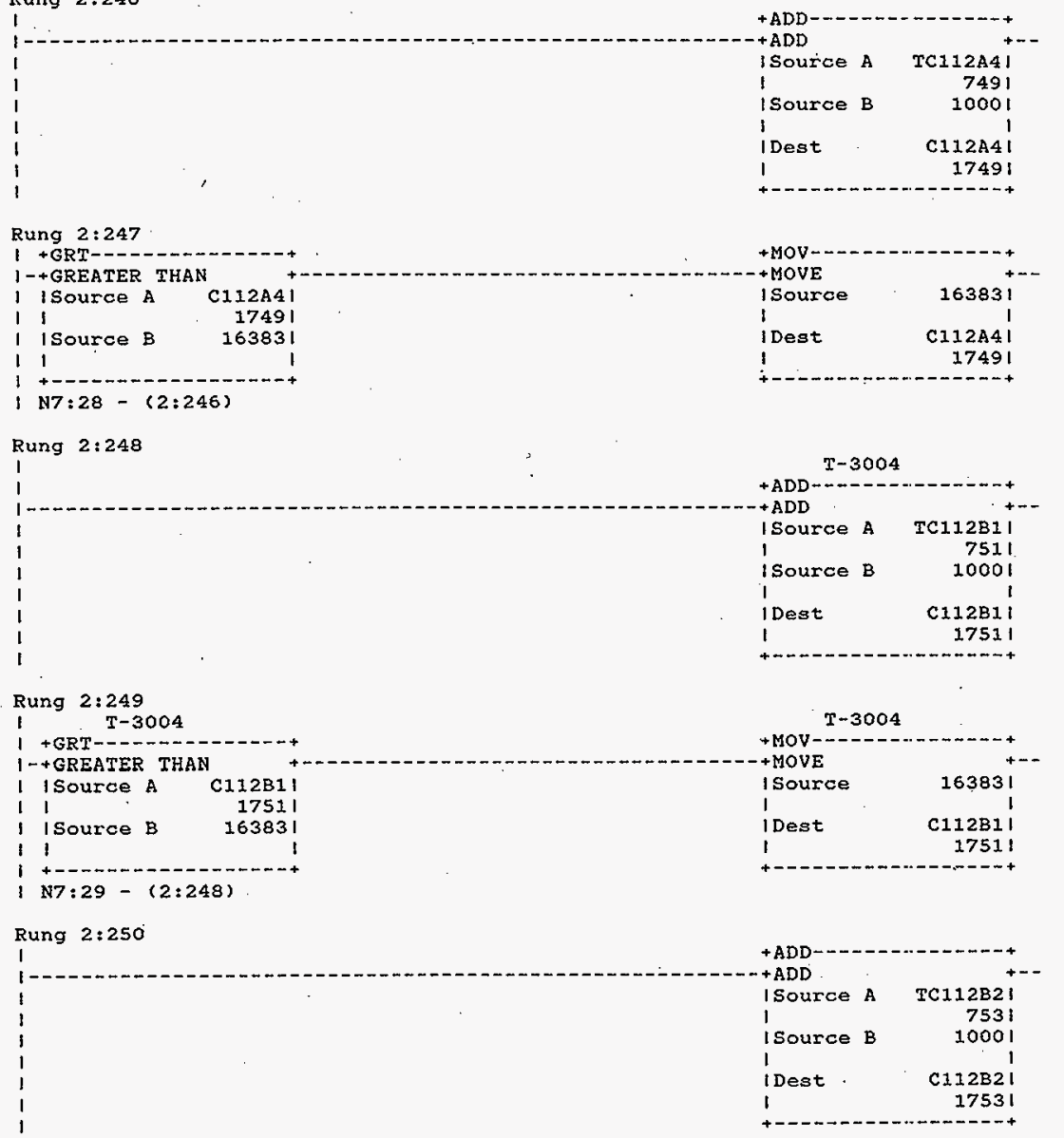

HNF-SD-FF-CSWD-61 Rev. 0 


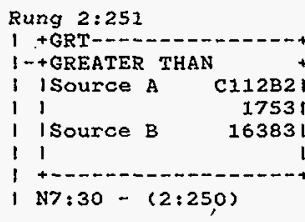

Rung $2: 252$

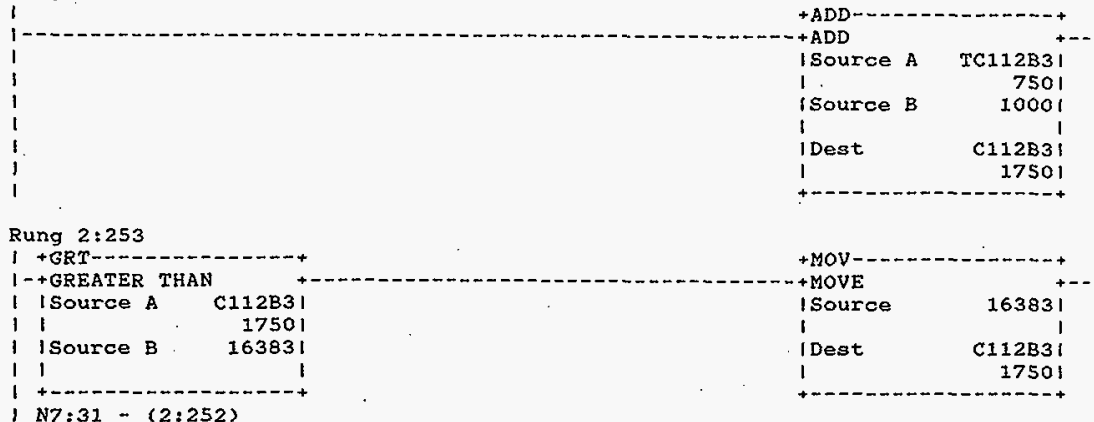

Rung $2: 254$

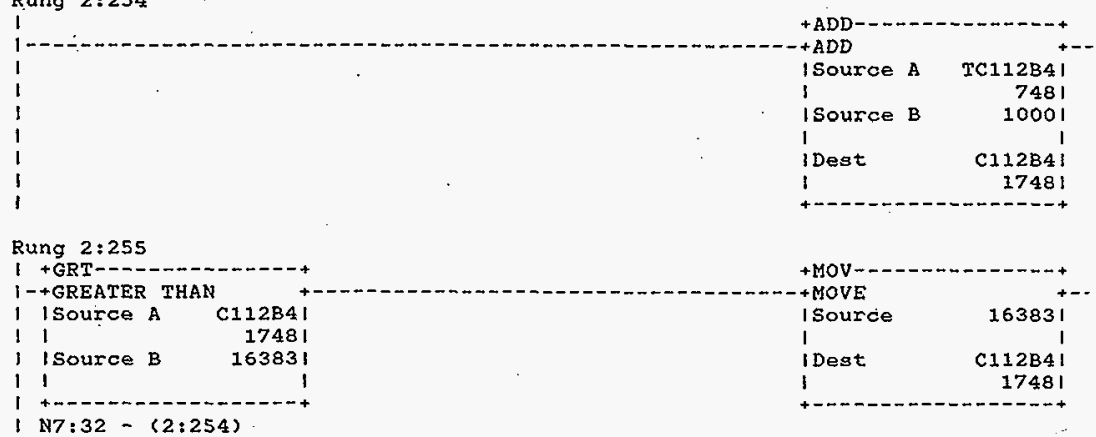


Rung 2:256

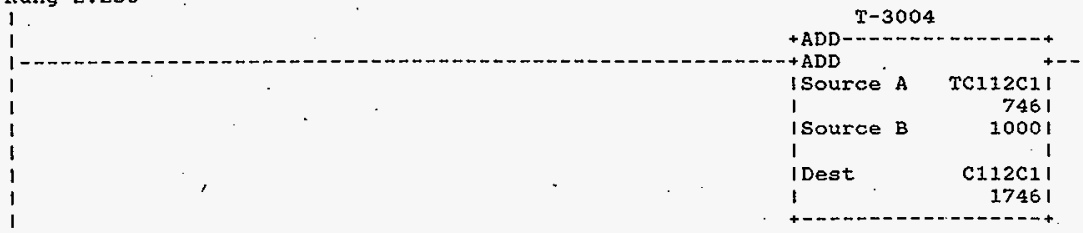

Rung $2: 257$

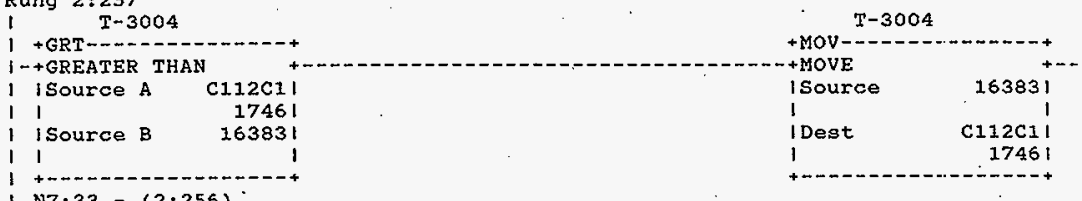

( N7:33-(2:256)

Rung $2: 258$

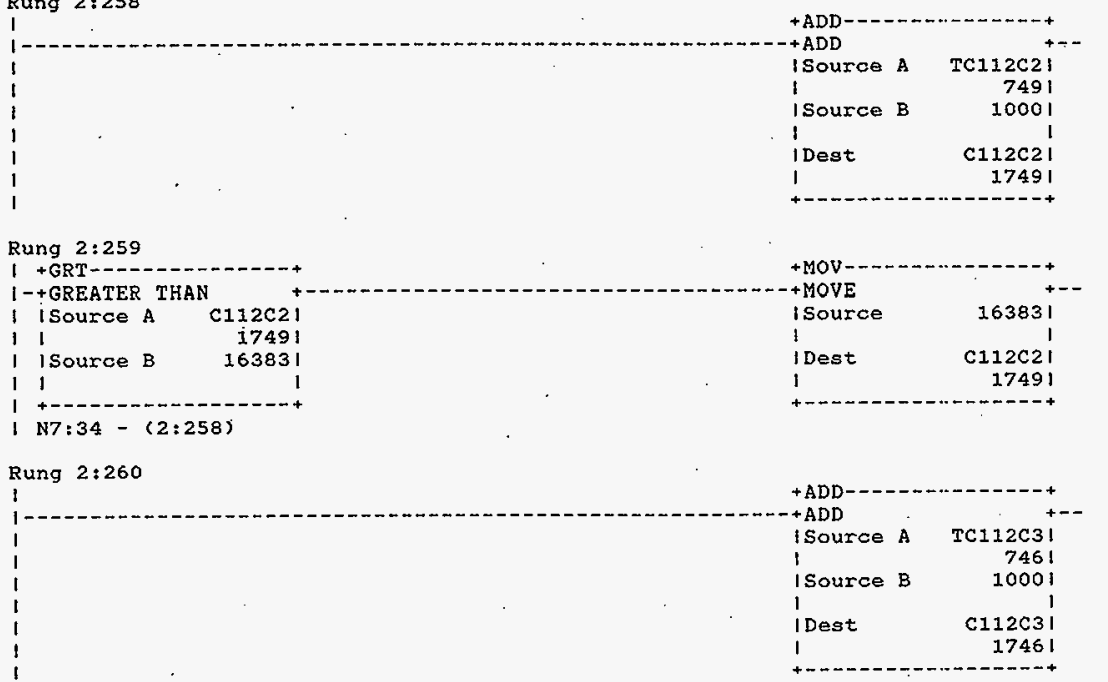

HNF-SD-FF-CSWD-61 Rev. 0 
Processor and Data(OPS Unit 1) Program Listing
October 23, 1996 Processor File: SoDIUM1A.ACH
Page 106

Rung 2:261

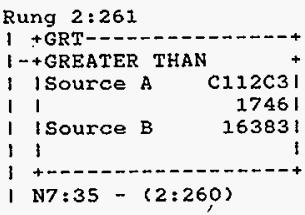


Processor and Data(OPS Unit 1)

October 23, 1996 Page 107

Program Listing

Processor File: SODIUMIA.ACH

kung $2: 26 \mathrm{E}$

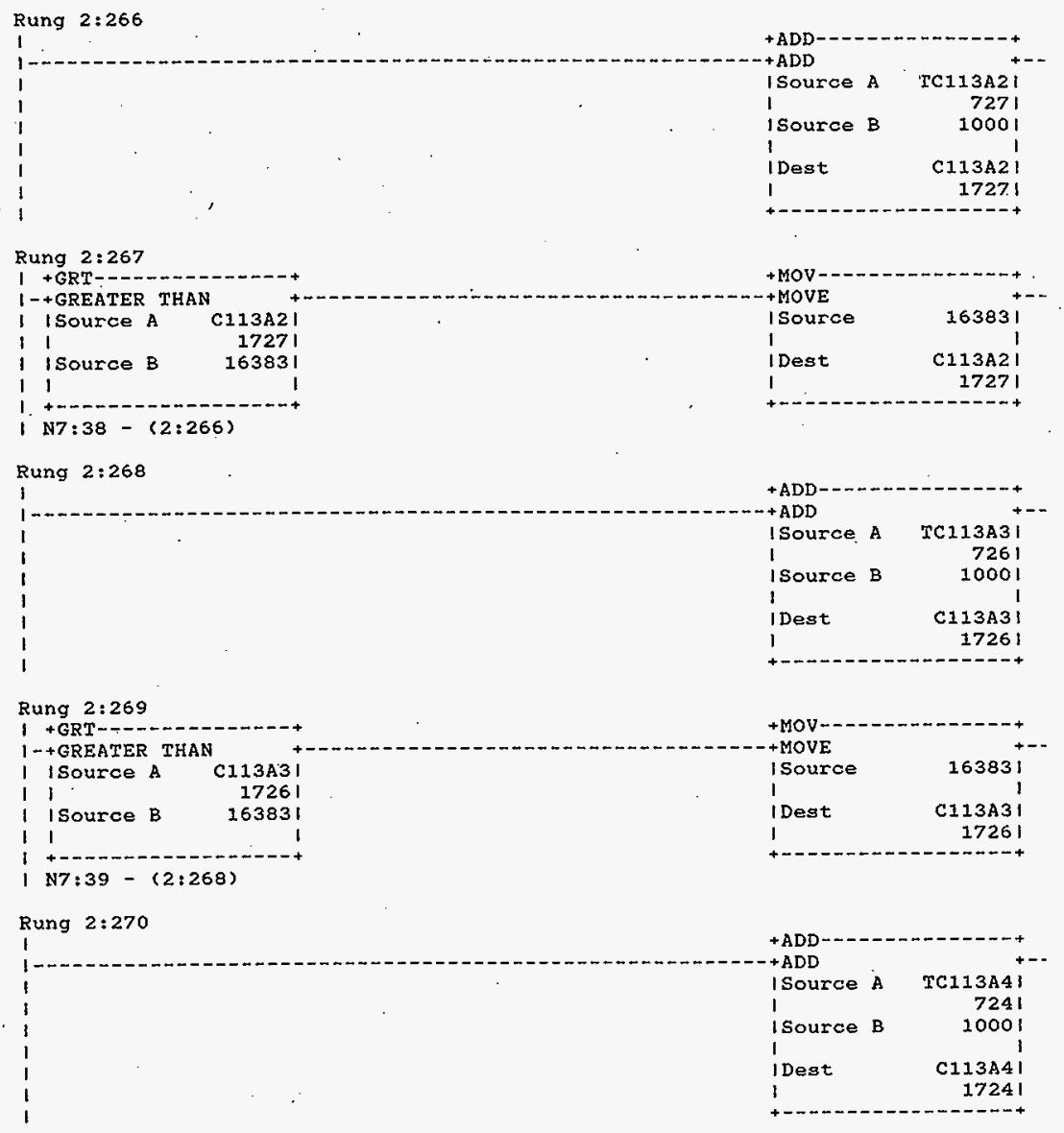

HNF-SD-FF-CSWD-6! Rev. 0 
Processor and Data(OPS Unit 1) program Listing
Processor File: SODIUMlA.ACH

October 23, 1996 Page 108

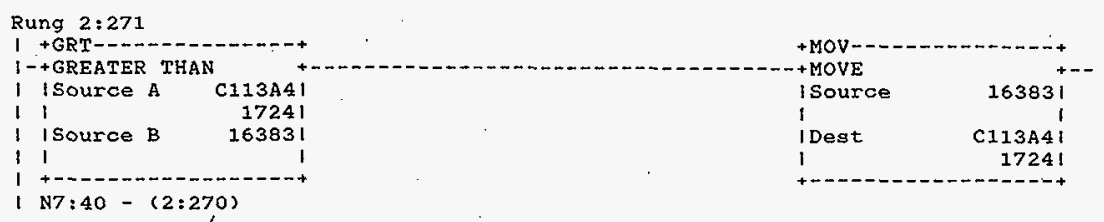

Rung $2: 272$

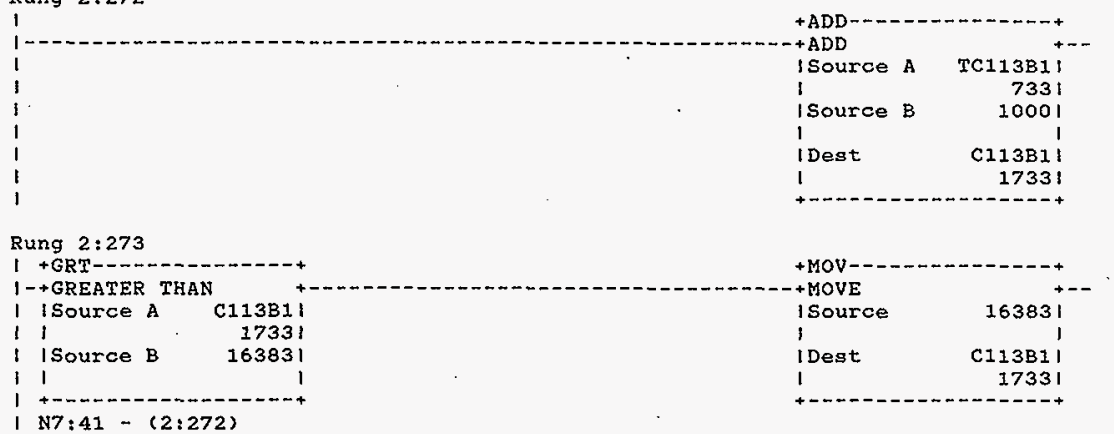

Rung $2: 274$

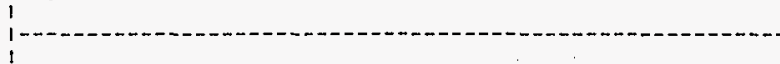$$
1
$$

$+\mathrm{ADD}--\ldots+----\ldots+.++$

$+\mathrm{ADD}$

isource A TC113B2!

1

I Source B

7351

1

I Dest

1

10001

C113B2

17351

Rung $2: 275$

I + GRT-----.---...-

1-+GREATER THAN

I I Source A

11

1735

1 I 16383 !

1 +-

I $N 7: 42-(2: 274)$ 
Processor and Data(OPS Unit 1 )

October 23, 1996 Program Listing

Rung 2:276

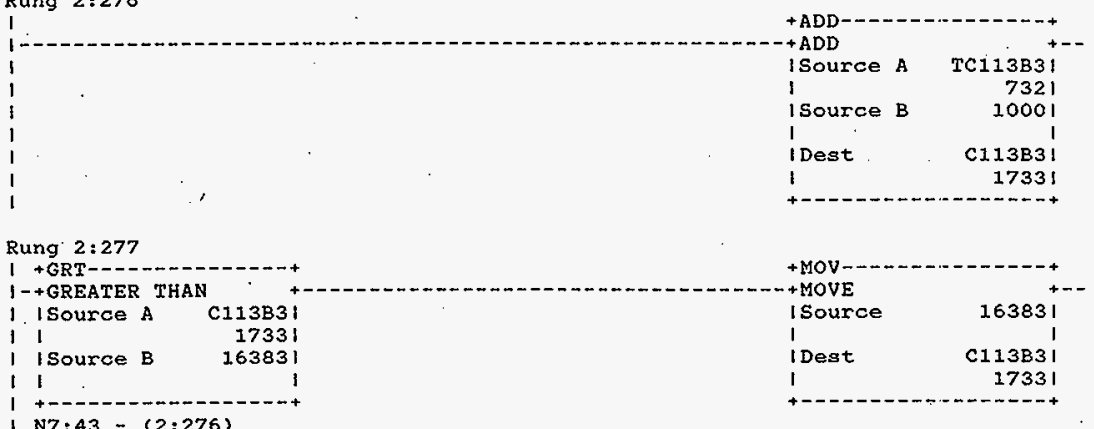

(N7:43-(2:276)

Rung 2:278

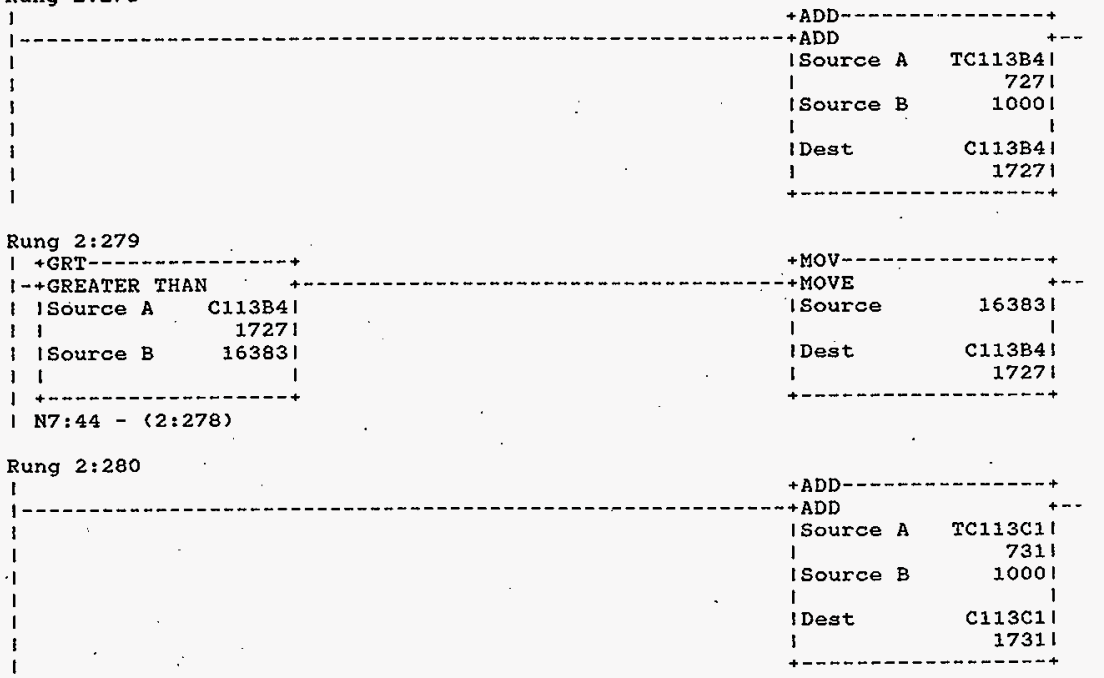


Processor and Data(OPS Unit 1)

Program Listing
Processor File: SODIUM1A.ACH
October 23, 1996 Page 110 Rung $2: 281$

\section{Rung $2: 281$}

1 + GRT------

1-+GREATER THAN +

I JSource A CI13CII

I I 1731 I

I ISource B 16383 |

11 ।

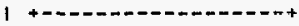

( $17: 45-(2: 28,0)$

Rung 2:282

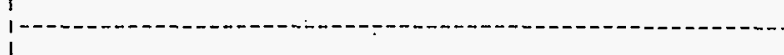

$$
1
$$$$
1
$$

$$
\text { I }
$$

Rung $2: 283$

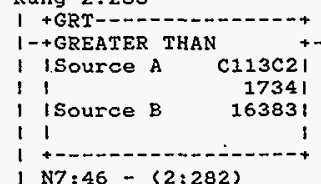

$$
1 \text { N7:46-(2:282) }
$$

Rung $2: 284$

1 1

$$
1
$$

\section{Rung 2:285}

$1+$ GRT-.--

1-+GREATER THAN TH

1 ISource A C113C31
1 I

I isource B 16383|

11 i

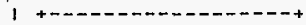

I N7:47-(2:284)

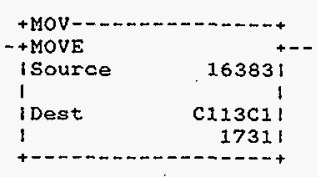

$+\mathrm{ADD}------------+$

$+\mathrm{ADD}$

ISource A TC113C2।

1

7341

Isource B 10001

I

I Dest

C1 $13 \mathrm{C21}$

1

17341

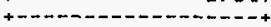

\section{$+\mathrm{MOV}-$}

+ MOVE

isource

1

I Dest

1

$+$

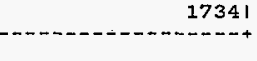

$+A D D$

ISource A

I

I Source B

I

i Dest

1

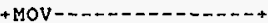

+ MOVE

I Source

16383

I

I Dest

1

$+-$
TCI13C3I

7321

10001

C113C 3 !

17321 


\section{Rung 2:286}

1.

$$
1-
$$

!

\section{Rung $2: 287$}

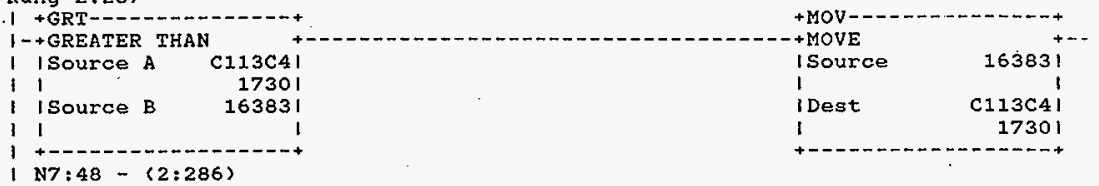

Rung $2: 288$

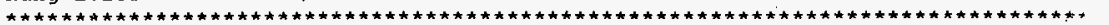

For each T/C read its STATUS BITS to detect T/C problems HARDWARE ALARMS FOR TANK T-3002

\section{T/C ENABLE}

HAI12A1

$I: 9$

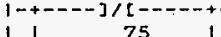

N7 : 241

1 OPEN $T / C$

$11 \quad I: 9$

1 +-...] [----

I 1 I: $9^{76}$

$1+\cdots][--0 .-n+$

1177

) HI LIMIT

$11 \quad I: 9$

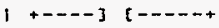

$1178 \quad 1$

11

I CNFG ERROR

I) CNEG ERROR I

1 $1 \quad$ I $1: 9 \quad$

i 79

HNF-SD-FF-CSWD-61 Rev. 0 
Processor and Data(ops Unit 1)

Program Listing

Rung 2:289

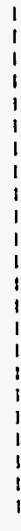

\section{T/C ENABLE}

$I: 9$

HA112A2

N7 : 241

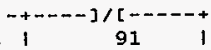

OPEN $\mathrm{T} / \mathrm{C}$ $I: 9$

+- - - ] $[--n+$

1) 92

1. I LO LIMIT

1 I 1 9

$1+---][0 . .-+$

1193

I I HI EIMIT

I I: 9

+----] [-----

$194 \quad$ ।

I CNEG ERROR I

I I:9

1.--- ] [--

95

Rung $2: 290$

1

1

I T/C ENABLE

$1 \quad I: 9$

HA112A3

$1,1,107$

N7: 241

1 OPEN T/C

$1 \quad I: 9$

+---- ] [-.-. +

1108

1 LO LIMIT

$1.1: 9$

+----] [--.-.

1109

i HI LIMIT

$1 \quad I: 9$

+--n- $]$

$1 \quad 210$

I CNFG ERROR

I I:9 ।

+--n] [----n+

111 
Processor and Data(OPS Unit 1)

Rung 2:291

I

1

1 T/C ENABLE

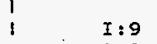

HA112A4

$1-+---7 / 20$

1 OPEN $\mathrm{T} / \mathrm{C}$

N7 : 241

1 I: 9

+---- $]$ [---- +

I 124

I LO LIMIT

I $I: 9$

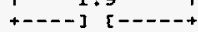

I. 125

1 HI LIMIT

1 I:9

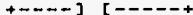

I 126

I CNFG ERROR ।

I I: 9

+.-.- ] [--.-.

127

Rung 2:292

1

T/C ENABLE

HA112B1

$I: 10$

N7: 241

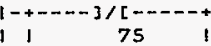

I OPEN $T / C$

11

1.--- ] [---n+

176

I LO LIMIT

I I:10

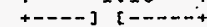

$1 \quad 77$

1 HI IIMIT

1

$+$

$I: 10$

I CNFG ERROR

11

$1+\cdots+-\pi[----+$

79 
Processor and Data(OPS Unit 1)

Program Listing

Proce

Rung $2: 293$

1 .

\section{T/C ENABLE}

$I: 10$

$1-+----7 /[-$

I I OPEN $\mathrm{T} / \mathrm{C}$. I

I $I: 10$ i

$1+\cdots+\cdots][-\cdots+\cdots$

1192

1 LO LIMIT

$11 \quad I: 10$

1 1 1

I HI LIMIT i

I I I:10 1

$1+--\infty-3$ [ $1--\cdots \cdots+$

I I CNFG ERROR

I I:IO I

+...- $][\ldots+\cdots+$

Rung 2:294

I

T/C ENABLE

$I=10$

$\begin{array}{ccc}1 & 1 \\ 1-+\infty & 108 & 107\end{array}$

OPEN T/C

I. I: 10

+----] $[--\ldots-4$

LO LIMIT

I I:10

+-n] [-...-

1109

1 HI LIMIT

$I: 10$

11

$1+\cdots+\cdots][-\cdots \cdots+$

11

1 CNFG ERROR

I 1

$1+\cdots-1$ [

i 111
October 23, 1996 File: SODIUMIA.ACH
Page 114

Rung $2: 293$
HAI 12B2

N7 $7: 241$

13

HA112B3

N7 : 241

14 
Rung 2:295

1 .

T/C ENABTE

$1+\ldots+\ldots+1 /[10$

HA1 $12 B 4$

1

123

$T / C$

N7 : 241

1 OPEN $T / C, 1$

$1+\ldots+10$

$---+$

I 10 IMIT

1 I: 10

$1+\cdots][\ldots+\ldots+$

I 125

1 HI IIMIT

I) $I: 10$

1 +----] [

1 11261

I I CNEG ERROR 1

I 1 I:10

I +-.. ] [-..-

I. 127

Rung 2:296

1

I

T/C ENABLE

1 I:11

HA $112 \mathrm{Cl}$

$\begin{array}{ll}1 & 1 \\ 1 & 0\end{array}$

1 OPEN T/C

I I I:11

$1+\ldots-\infty][\ldots-\ldots+$

I 166

1 LO LIMIT

I 1 I:1I

I +---o] [-n-.-

1177

I I HI LIMIT

I 1 I:11 ।

$1+---][--\ldots+$

I I CNFG ERROR ।

1 I I:11

$1+---]$ ] $79-\cdots+$

Rung $2: 297$

i

T/C ENABLE

i $I: 11$

HA $112 \mathrm{C} 2$

N7: 242

11

1

91

$+++$

N7 : 24.2 
Processor and Datalops Unit 1) Program Listing

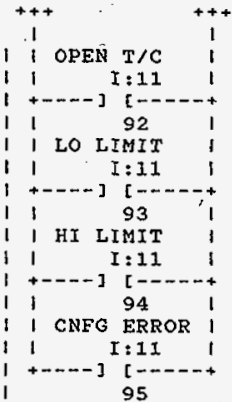

Rung $2: 298$

t

I/C ENABLE

$I: 11$

HA112C3

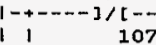

I OPEN $T / C$

1 I:11

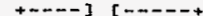

1

LO EIMIT

1

+----3 [n-.--+

1109

HI LIMI

I I:11

+ -..- 3 [ $\ldots-\cdots+$

$1110 \quad 1$

1 CNFG ERROR

1 I:11

+-..-] [ $[--. .+$

112

Rung 2:299

1.

T/C ENABLE

I:11

HA1 $12 \mathrm{C} 4$

N7 $: 242$

1
$-+\cdots--7 / 2$
123

OPEN $T / C$

$\begin{array}{lll}1 & 1 & 11\end{array}$

$1 \quad 124$

1

$+++$

$+$

HNF-SD-FF-CSWD-61 Rev. 0 


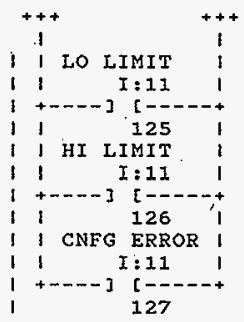

Rung 2:300

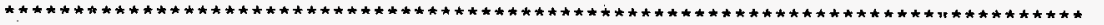
For each T/C read its STATUS BITS to detect T/C problems HARDWARE ALARMS FOR TANK T-3002

\section{T/C ENABLE}

$1-+\ldots-3 / 2: 12$

HA113A1

1 OPEN $\mathrm{T} / \mathrm{C}$

1 I:I2

$+\ldots--7[1---n+$

176

I LO.LIMIT

$1 \quad I: 12$

$+---0][----+$

1.77

1 HI LIMIT

1 I $I: 12$

$1+--\cdots]$ [-----+

I 78 ।

I I CNFG ERROR I

11 I:12

$1+----7$ [

I 79

Rung 2:301

I

T/C ENABLE

$I: 12$

HA1 3 A2

]$/[-$

N7: 242

I 191

1 I OPEN $T / C$

1 I $I: 12$

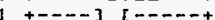

11.92

1

$.92 \quad 1$ 


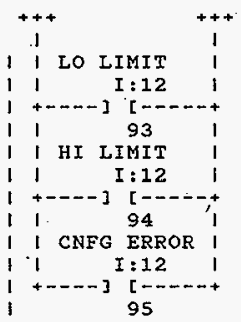

Rung 2:302

I

T/C ENABLE

HA113A3

$I: 12$

N7 : 242

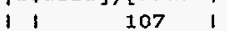

11 OPEN $T / C$

$11 \quad I: 12$

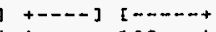

11108

LO IIMIT

$1 \quad I: 12$

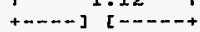

$1 \quad 109 \quad 1$

I HI LIMIT |

1 I:12 ।

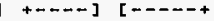

I I 1101

I I CNFG ERROR I

$\begin{array}{lll}1 & I: 12 \quad \mid\end{array}$

1111

Rung 2:303

1

$T / C$ ENABLE

i $\quad I: 12$

HA113A4

N7 : 242

। 123 ।

OPEN T/C

$I: 12$

$1+\cdots-\ldots][-\cdots--+$

I $1124 \quad 1$

I I LO IIMIT

11

$1+---7$ [ $-\ldots-n+$

$11 \geq 25$

1

$+++$

HNF-SD-FF-CSWD-61 Rev. 0 


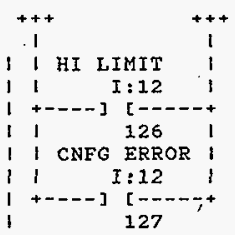

Rung 2:304

T/C ENABLE

$I: 13$

HA113B1

]$/[-2$

N7 : 242

$$
1175
$$

1 OPEN $T / C$

I:13

+--.+] [-----+

176

1 LO IIMIT

$1 \quad I: 13$

+.-.-3 [-....+

I 77

1 HI LIMIT

11

$I: 13$

$1+\cdots--7$ [

1.78

I CNFG ERROR ।

I I:I3 ।

1 +.-.- ] [--....

79

Rung 2:305

$$
1
$$

T/C ENABLE

HA1 13B2

I $: 13$

N7 $: 242$

$\begin{array}{ccc}1 & 92\end{array}$

1 OPEN T/C

1) I:13

$1+----]$ [ [-----

$11+92$

1. LO LIMIT

$1 \quad I: 13$

+----] [---..

193

I HI LIMIT

I $: 13$

+--n] [-n--.+

1194

I I CNFG ERROR ।

l. 1 I:13

$1+-\cdots-\cdots[----\cdots+$

95

HNF-SD-FF-CSWD-61 Rev. 0 
Processor and Data (OPS Unit 1)

\section{Program Listing}

Processor File:

SODIUMIA.ACH

Rung 2:306

1 .

T/C ENABLE

$$
x: 13
$$

]$/[-$

$$
11107 \quad 1
$$

1 OPEN T/C .

11 I:13

$+---][---\cdots+$

$1 \pm 08$

I LO LIMIT

1 I:13

+- n] $[\ldots-n+$

I 109 -

I HI LIMIT I

I I:13 I

+---- $]$ [-----+

I $110 \quad 1$

I CNFG ERROR I

I I:13 I

+- - ] [--.--

$1.1 \pm 1$

Rung $2: 307$

I

\section{T/C ENABLE}

$I: 13$

$-+---] /[--$

1 OPEN T/C

$1 \quad I: 13$

+---1 [ $1-----+$

12124

1 LO LIMIT

1 I:13

+---1 1

1125

I HI LIMIT

1

$+---][--\infty-\infty$

I 126

1 CNFG ERROR I

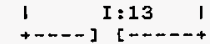

127

Rung 2:308

I

I

T/C ENABLE

$1 \quad I: 14$

October 23,1996

N7 : 242

10

HA $113 \mathrm{~B} 4$

$N 7: 242$

11

HA $113 \mathrm{Cl}$

N7 : 242

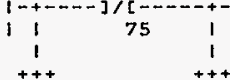




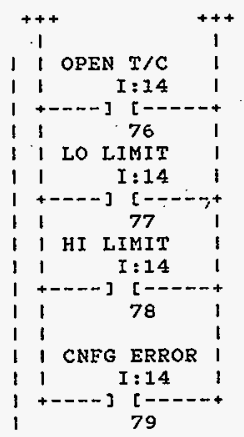

Rung 2:309

I

T/C ENABLE

$I: 14$

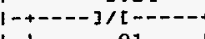

$191 \quad 1$

I OPEN T/C I

I I:14 if

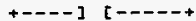

192

1 LO LIMIT

1

+...- $][\ldots-\ldots+$

193

1 HI LIMIT

11

I: 14

+----3 [--.--

194

I CNEG ERROR ।

I I I:14

I +----] [-----

I 95

Rung $2: 310$

1

T/C ENABLE

HA $113 \mathrm{C} 3$

$I: 14$

N7 7242

$1-+---n] /[-0$

107

1 OPEN $T / C$

I I I:14

$1+----3$ [---n+

1

1

108

$++$ 
Processor and Data(OPS Unit 1 )

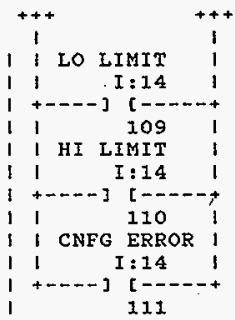

Rung 2:311

\section{T/C ENABLE}

$I: 14$

HA $113 \mathrm{C} 4$

$1+1,123$

OPEN $T / C$

$\begin{array}{lll}1 & 0.14 \\ 1 & 1 & 14\end{array}$

$1 \quad 124$

1 LO EIMIT

1 I:14

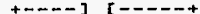

$1 \quad 125 \quad 1$

1 HI LIMIT

1 I $\quad \mathrm{I}: 14$

$1+---7$ [

1126

I CNFG ERROR ।

1 I $I: 14$ ।

i 127

Rung 2:312

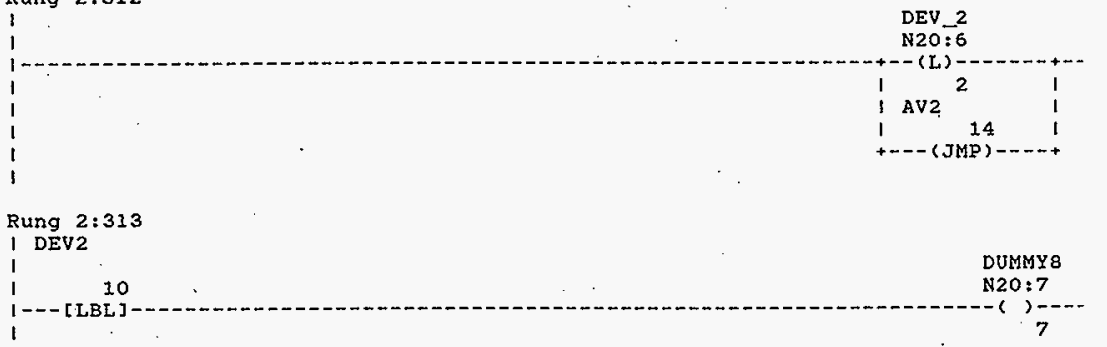

HNF-SD-FF-CSWD-61 Rev. 0 
Rung $2: 314$ ESTABLISH HIGH AND LOW LIMITS FOR RANGE CHECKING

IF NO HARDWARE ALARMS EXIST CHECK T/C VALUES FOR OUT-OF-RANGE CONDITION

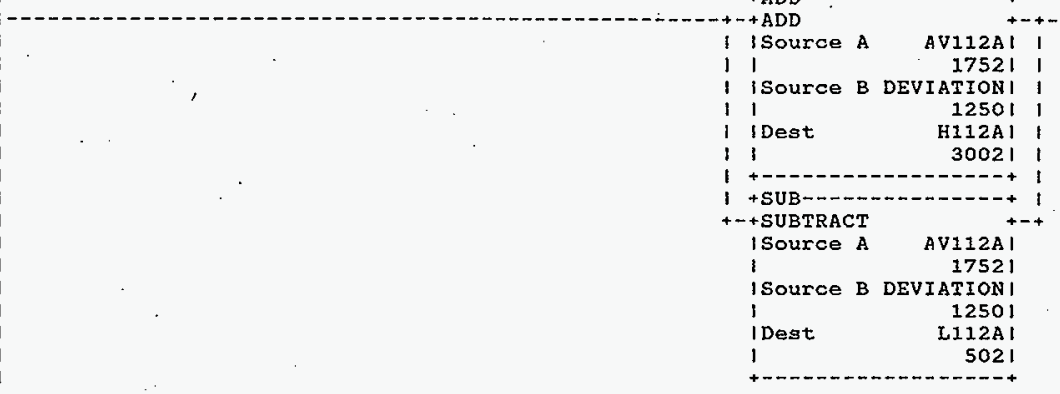

Rung $2: 315$

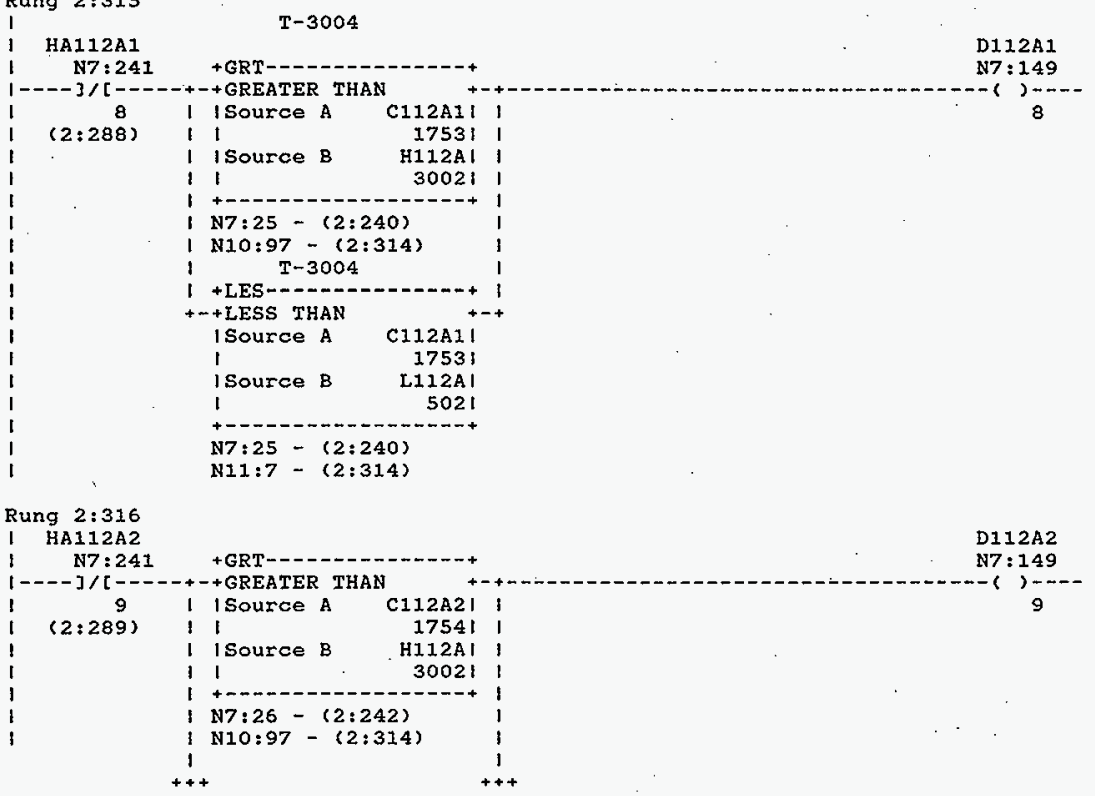

HNF-SD-FF-CSWD-61 R.ev. 0

Page $/ 23$ 
Processor and Data (OPS Unit 1 )

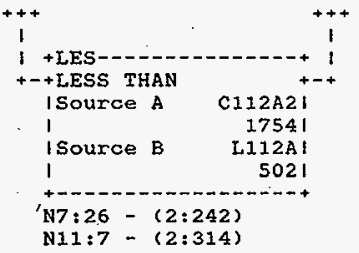

Rung $2: 317$

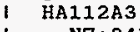

N7 $: 241$

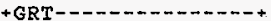

D112A3

1----]/[----++-+GREATER THAN

10
$(2: 290)$

I Isource A

C112A3!

N7: 149

17521

1 ISource B H112A: |

11

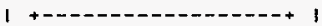

( N7:27-(2:244)

( N10:97-(2:314)

$1+$ LES-----n.......+

+-+ LESS THAN

ISource A

C112A31

ISource B L112A I

1

5021

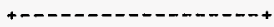

N7:27- (2:244)

$N 11: 7-(2: 314)$

Rung $2: 318$

I HAII2A4

I N7:241

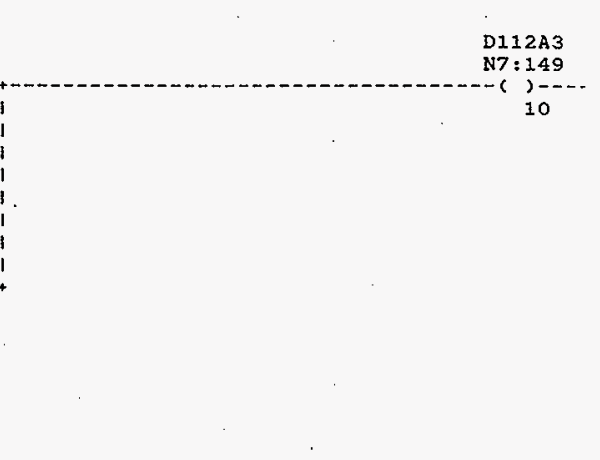

11

+GREATER THAN

$(2: 291)$

i 1

ISource A

isource B

C112A41 I

1749 I

H112A1 I

11

300211

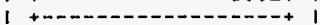

( N7:28-(2:246)

( $\mathrm{N} 10: 97-(2: 314)$

i +

+-+ LESS THAN

Isource A CI12A4I

1749 I

|source B Li12A|

5021

N7:28-(2:246)

N11:7- (2:314)

D112A4

N7: 149 
Processor and Data(OPS Unit 1)

Program Listing

Rung 2:319

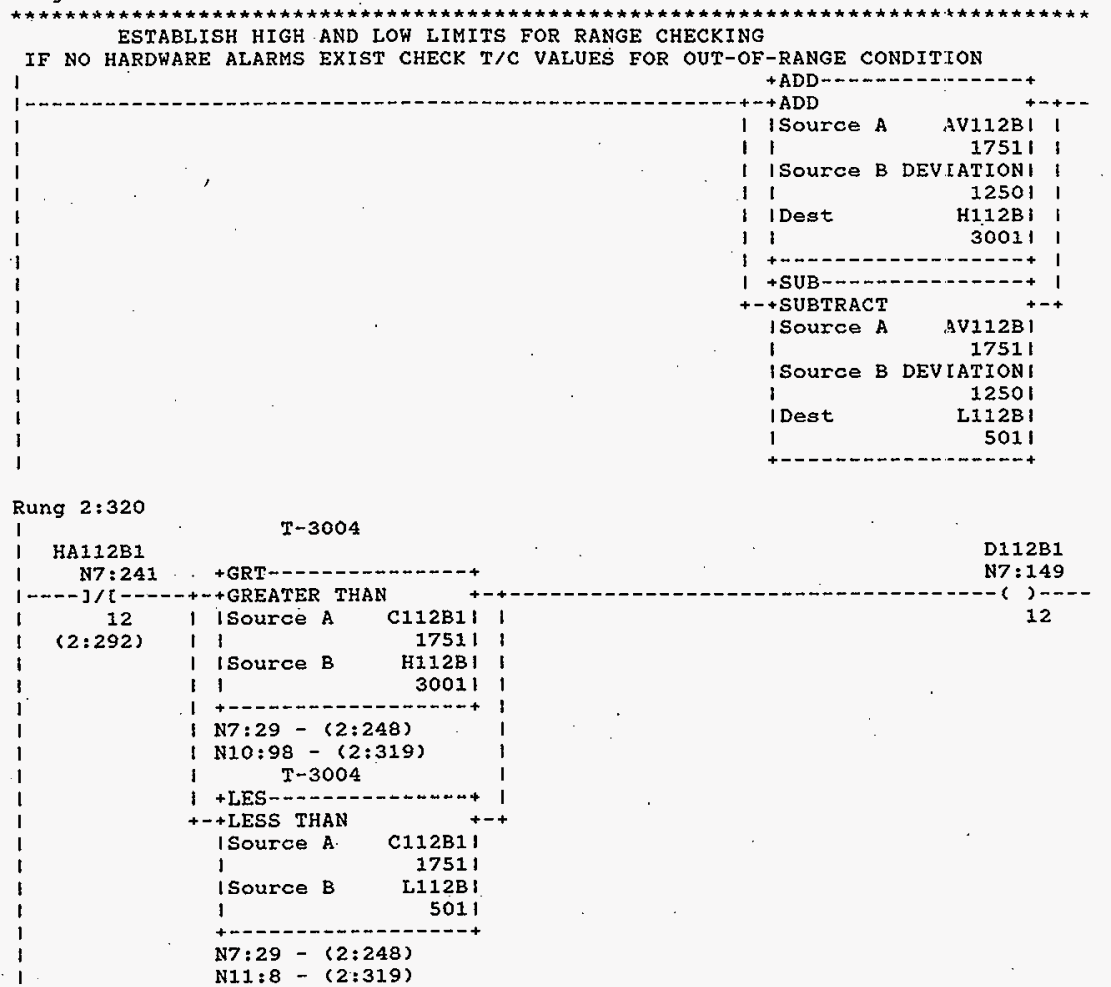

Rung 2:321

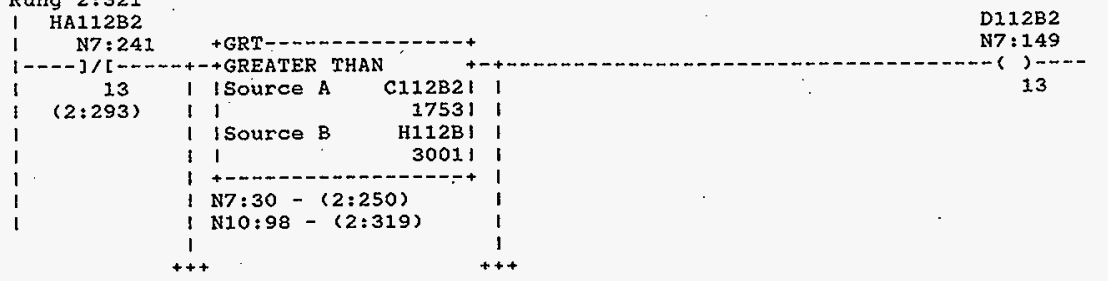

HNF-SD-FF-CSWD-61 Rev. 0 


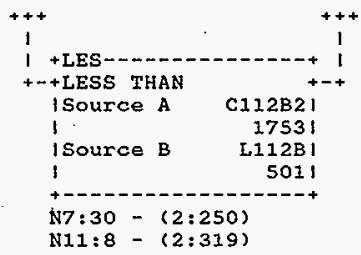

Rung $2: 322$

1 HA112B3

$1 \quad$ N7 $: 241$

$1----] /[---$

14

$(2: 294)$

I

+GRT-_.....-.

D112B3

+-+ GREATER THAN

ISource A

C112B31

N7: 1.49

i l Source 8

I I 30011

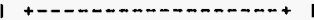

( N7:31-(2:252)

I N10:98-(2:319)

$1+$ LES-- - - - - - - - - + 1

+-+ LESS THAN +-+

ISource A C112B3|

I

17501

|Source B L112B |

1

5011

$N 7: 31-(2: 252)$
$N 11: 8-(2: 319)$

Rung $2: 323$

I HA1I2B4

ำ $7: 241$

+GREATER THAN

D112B4

$3 /[--$

I ISource A

C112B4I

N7: 149

$(2: 295)$

11

17481

I ISource B H112AI I

11

30021

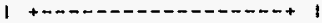

(N7:32-(2:254)

( N10:97-(2:314)

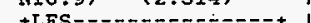

+-+ LESS THAN

ISource A C112B4!

1

ISource B $2112 B$,

1

5011

$N 7: 32-(2: 254)$

$N 11: 8-(2: 319)$ 
Rung $2: 324$

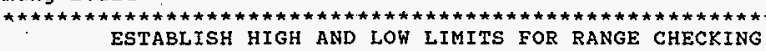

IF NO HARDWARE ALARMS EXIST CHECK T/C VALUES FOR OUT-OF-RANGE CONDITION

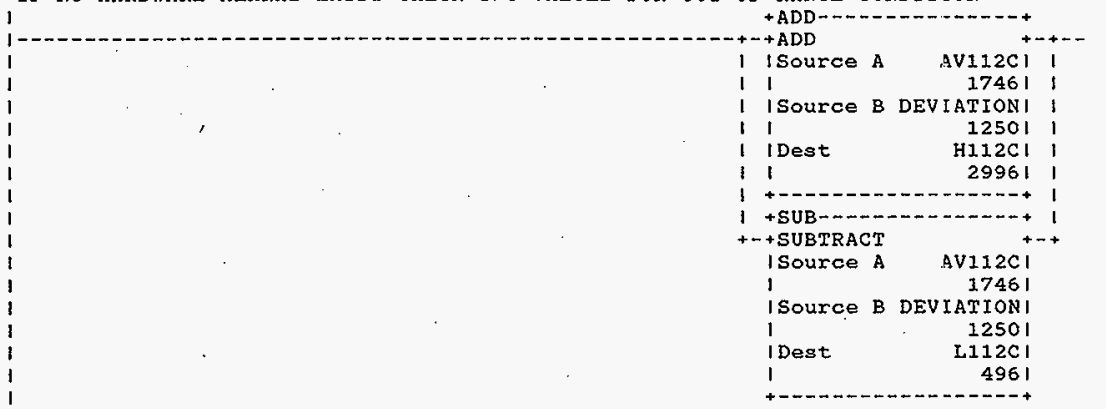

Rung $2: 325$

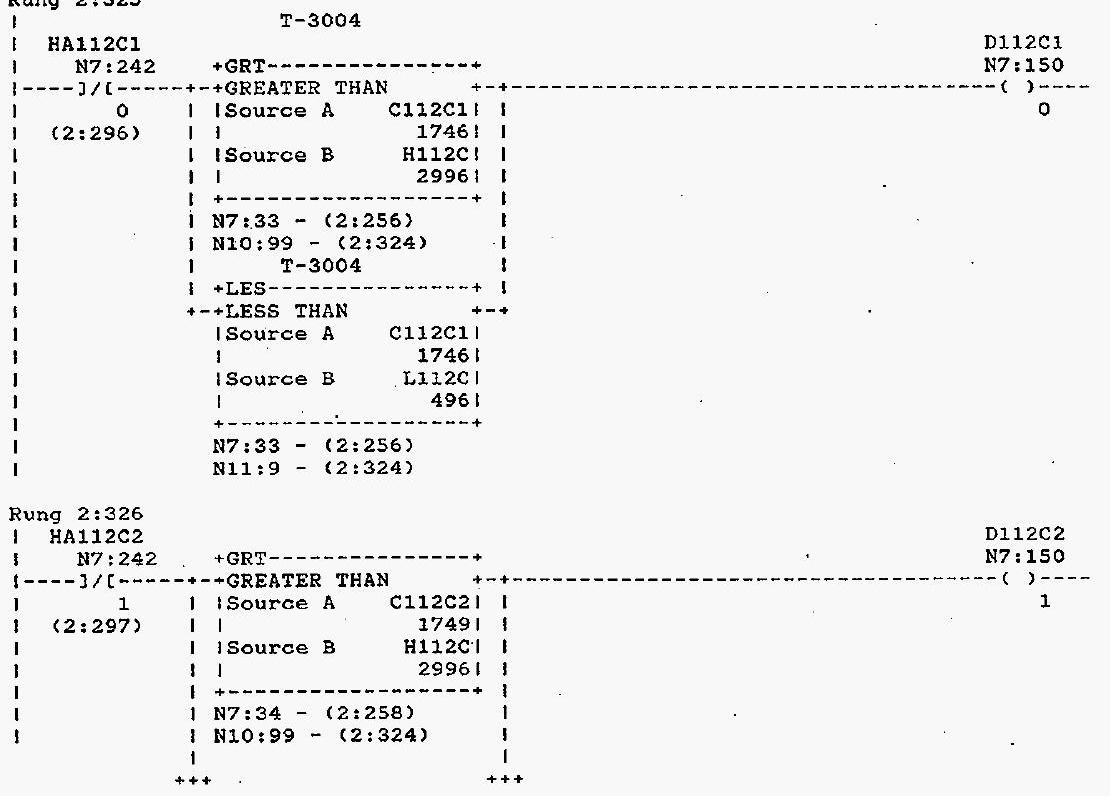

HNF-SD-FF-CSWD-61 Rev. 0 
Processor and Data(ops Unit 1 )

Program Listing

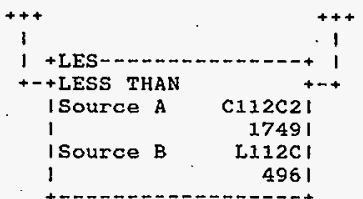

N7:34-(2:258)

$N 11: 9-(2: 324)$

Rung $2: 327$

1 HAII2C3

N7:242 +GRT-

,

$0112 \mathrm{C3}$

+GRTARATER THAN

(2:298)

I I Source A

C112C3I I

274611

I Isource B H112CI I

I 129961 I

$1+----0-0-0-0.0 n+1$

I $N: 35-(2: 260)$

(N10:99 - (2:324)

$1+$ LES----.--...... + 1

+-+ LESS THAN

Isource A C112C3]

$1 \quad 17461$

ISource B L,112C I

1

4961

N7:35 - (2:260)

N11:9-(2:324)

Rung 2:328

I HA112C4

N7 : 242

+GRT------------ - - - - +

D1 $12 \mathrm{C} 4$

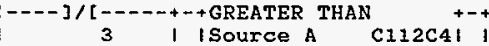

(2:299)

11

174311

1 isource B H112Cl

I 29961 |

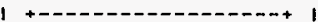

l N7:36-(2:262)

( N10:99-(2:324)

1 + LES----------n...

+-+LESS THAN

I Source A C112C4I

1

17431

| Source B L112C I

1

4961

N7:36-(2:262)

$N 11: 9-(2: 324)$ 
Processor and Data(OPS Unit I)

Rung 2:329

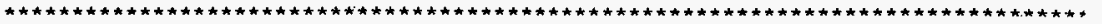
ESTABLISH HIGH AND LOW LIMITS FOR RANGE CHECKING

IF NO HARDWARE ALARMS EXIST CHECK T/C VALUES FOR OUT-OF-RANGE CONDITION

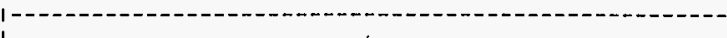

$$
1
$$

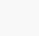

1 isource

1 I

1 isource

I I

I Dest

I I

$1+$

I + SUB

$+\cdots+$ SUBTRACT

ISource A

AVII3AI ,

17261

ISource B DEVIATION I

1250 I

IDest LII3AI

1

4761

Rung $2: 330$

I HA113AI

$T-3001$

D113A1

N7: 242

I----]/[----+-+GREATER THAN

। $12: 300)$

$1,(2: 300)$

I ISource A

C1

C113A11 I.

N7: 150

I I Source B r H113A1 |

1 1 2976I

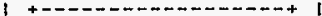

( N7:37-(2:264)

I N10:100-(2:329)

I $T-3001$

$1+$ LES- $-\ldots . . .+-\ldots . .+1$

+++ LESS THAN

ISource A C113AII

I

17251

|source B LI33A|

I

4761

N7:37-(2:264)

$N 11: 10-(2: 329)$

Rung 2:331

i HA113A2

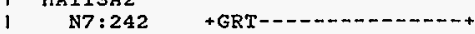

D113A2

$1-\ldots-3 /[---+-+$ GREATER THAN

I 5 I ISource A C113A2I I

N7 : 150

(2:301)

11

17271

$\mid$ |Source B HII3A| |

11 29761 1

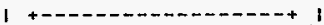

1 N7:38-(2:266)

( N10:100-(2:329)

1

$+++$

HNF-SD-FF-CSWD-61 Rev. 0

Page 129 


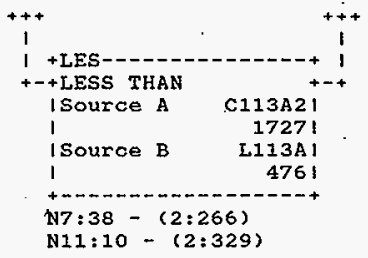

Rung 2:332

I HA113A3

$1 \quad$ N7:242

$1---1 /[-\cdots+++$ GREATER THAN

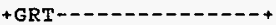

D113A3

6 I ISource A C113A3! I

(2:302) i $\mid$ 1726 i

I ISource B. M113A| I

i 1 29761 I

$1+--------------+1$

1 N7:39-(2:268)

N10:100 - (2:329)

1 +LES--------------.+ 1

+-+ LESS THAN +-+

ISource A C113A3I

I 1726 I

isource B I.113A I

1

4761

N7:39-(2:268)

$N 11: 10-(2: 329)$

N7: 150

6

D113A4

Rung $2: 333$

1 HA113A4

$1 \quad \mathrm{~N} 7: 242$

$--+$

----]/[----+-+GREATER THAN.

I. $7^{\circ}$ I ISource A C113A4I I

$(2: 303)$

17241

I isource B H113Al i

II 297611

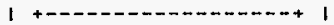

( N7:40-(2:270)

( N10:100-(2:329)

$1+$ LES------------ + 1

+-+ LESS THAN

ISource A C113A4I.

I 17241

isource B LII3Al

4761

N7:40-(2:270)

N11:10-(2:329) 
Processor and Data(OPS Unit 1 )

Rung $2: 334$

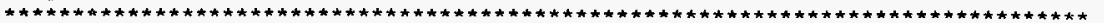
ESTABLISH HIGH AND LOH LIMITS FOR RANGE CHECKING

IF NO HARDHARE ALARMS EXIST CHECK T/C VALUES FOR OUT-OF-RANGE CONDITION

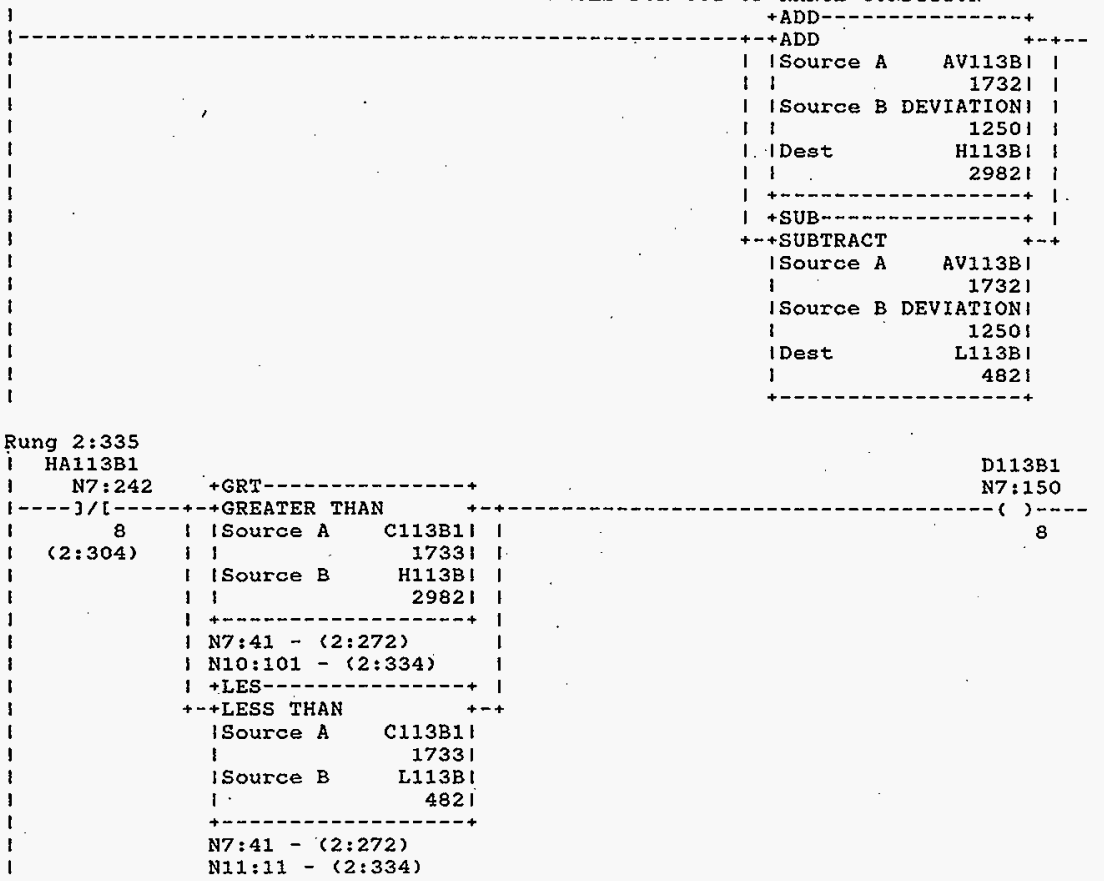

Rung $2: 336$

I HA113B2

N7:242 +GRT-2 +

$\mathrm{D} 113 \mathrm{~B} 2$

$\begin{array}{ccc}1 & 9 & \text { I ISOUrCe A }\end{array}$

(2:305) i $1735 \mid$

I Isource B HII3Bi |

1. 1 29821

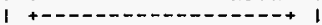

I N7:42-(2:274)

I N10:101-(2:334)

1

$+++$ 


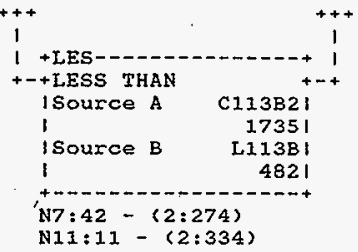

Rung $2: 337$

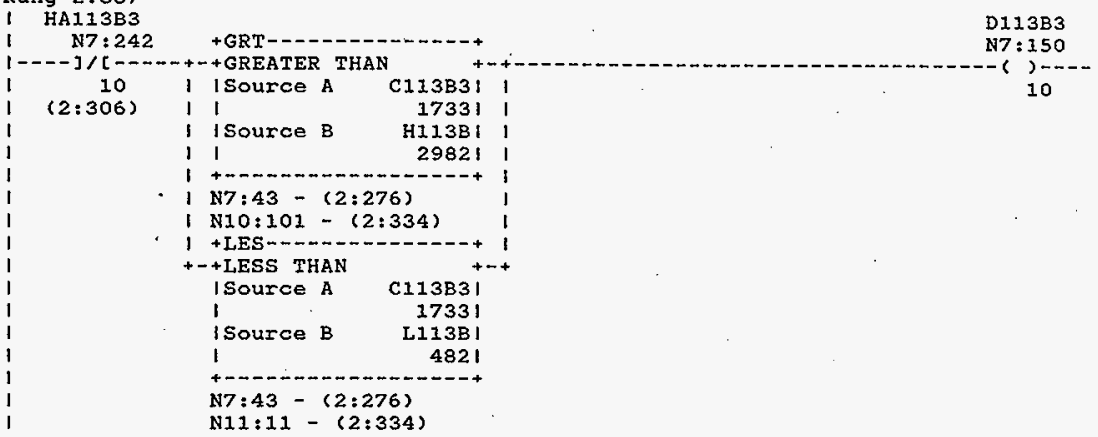

Rung 2:338

1 HA1 1384

1 N7:242 +GRT-- 1 +

1 11 I ISource A CI13B4I ।

(2:307) I । 1727।

D113B4

N7 : 150

I isource B H113B

11

11

29821

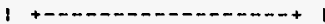

I N7:44-(2:278)

( N10:101-(2:334)

1 +LES- -

+-+ LESS THAN

I Source A C113B41

$1 \quad 1727$ I

15ource B LI13B |

1

4821

N7:44-(2:278)

N11:11-(2:334) 
Rung $2: 339$

ESTABLISH HIGH AND LOW LIMTTS FOR RANGE CHECKING

IF NO HARDWARE ALARMS EXIST CHECK T/C VALUES FOR OUT-OF-RANGE CONDITION

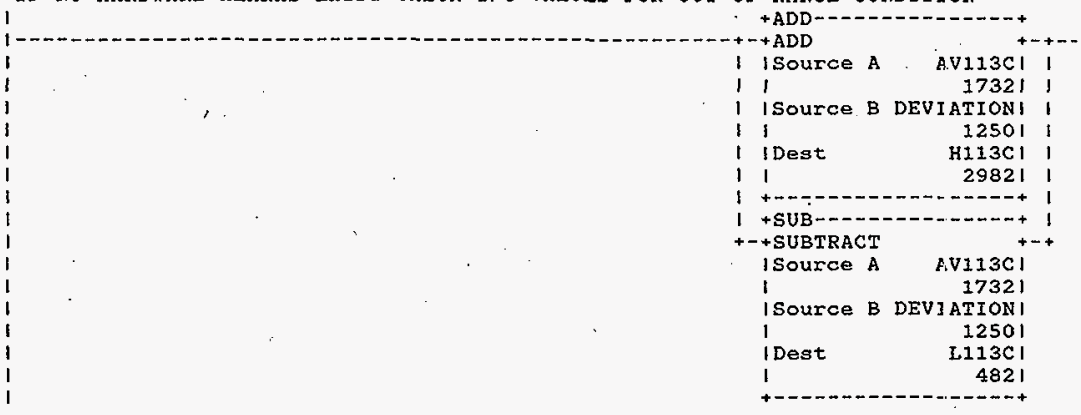

Rung $2: 340$

HA1 $13 \mathrm{C1}$

N7: 242

]$/[-$

12

$(2: 308)$

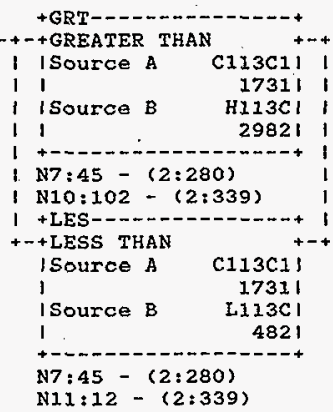

C11

11

1 isource B

11

1731

H113Cl ।

298211

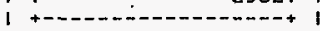

1. N7:45-(2:280)

( N10:102-(2:339)

$1+$ LES--- - - - ----n+ 1

+-+ LESS THAN

ISource A C113C1'

1

17311

| Source B $\mathrm{L} 113 \mathrm{C}$ |

1 4821

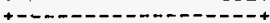

N7:45-(2:280)

N11:12-(2:339)$$
---+
$$

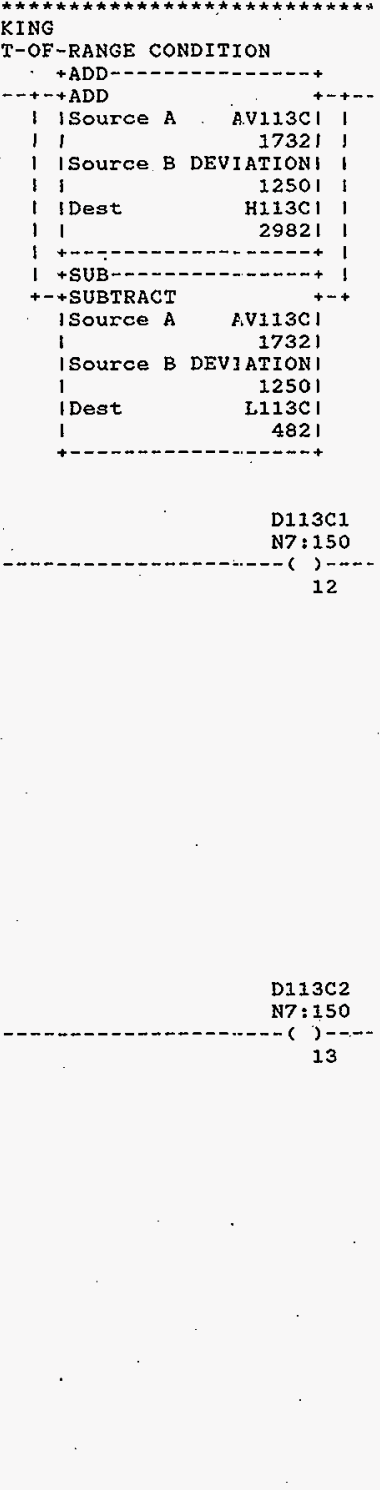

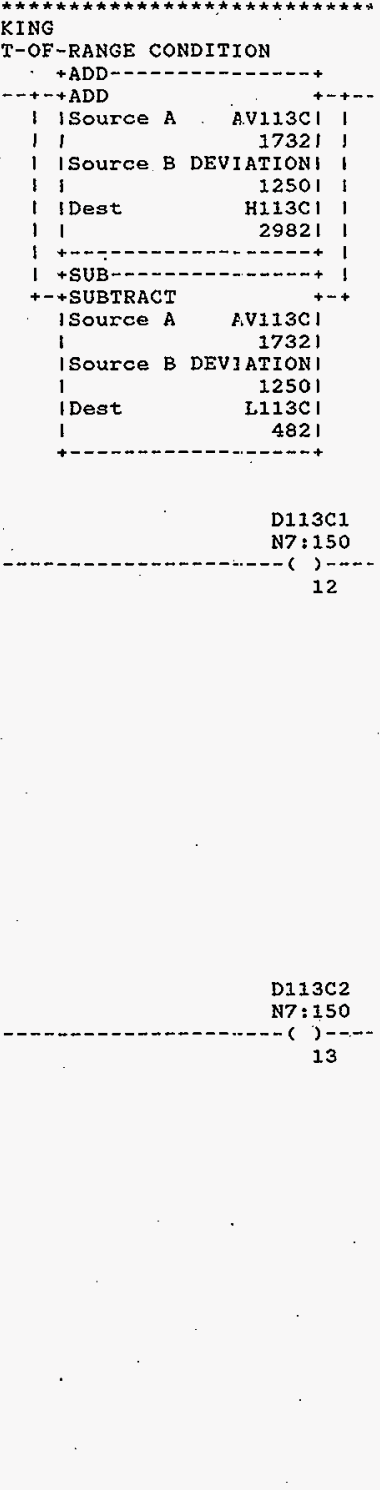

Rung $2: 341$

1 HAII3C2

$1 \quad$ N7:242

$$
\text { 11:12 }-(2: 339)
$$

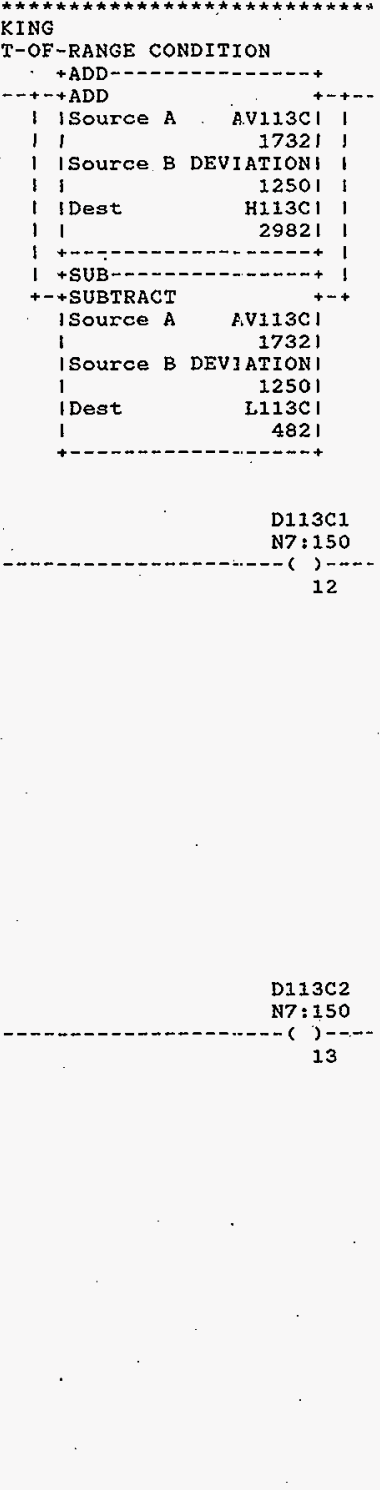

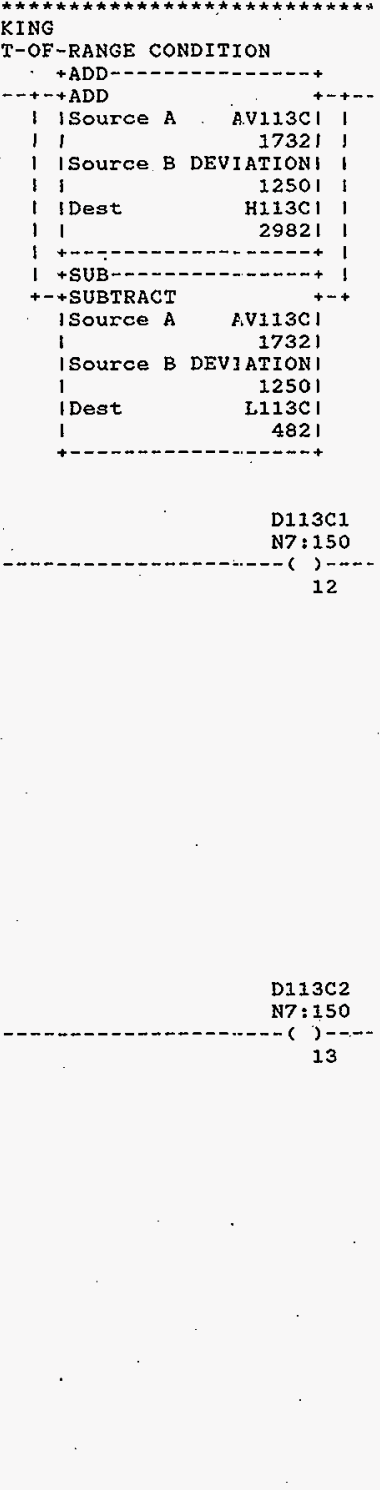

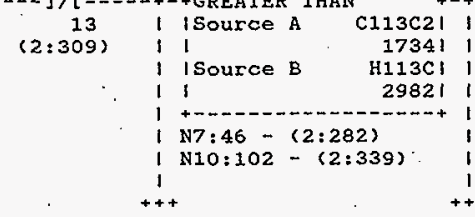




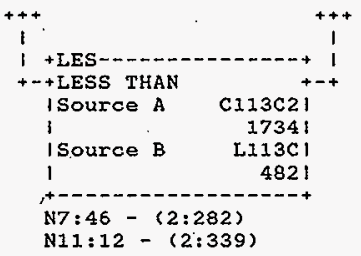

Rung $2: 342$

HA113C3

N7 $: 242$

+GRT--.------------+

D113C3

$1-\ldots-3 /[\ldots \ldots+4$

14

GREATER THAN

N7: 150

(2:310)

I I Source A

C113631 1

17321

I isource B Hil3CI

11 29821

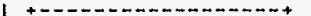

( N7:47 - (2:284)

I N10:102 - (2:339)

$1+$ LES----_...

+- LESS THAN +-+

ISource A C113C31

i 17321

1Source B $[213 \mathrm{CI}$

$1 \quad 4821$

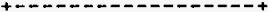

N7:47 - (2:284)

N11:12-(2:339)

\section{Rung 2:343}

I HAI $13 \mathrm{C} 4$

N7 $: 242$

1 N7:242 +GRT--- ]/[----++GREATER THAN

D113C4

1 isource A C113C41

$(2: 311)$

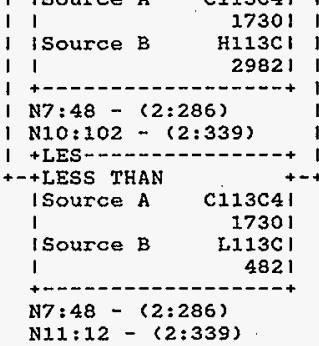

N7:150

14

-()$-\cdots$

15

I Source B H113Cl

N7:48 - $(2: 286)$

I N10:102 - (2:339)

ISource A C113C4I

[113CI

$+----1-0-10-0----+$

Rung $2: 344$

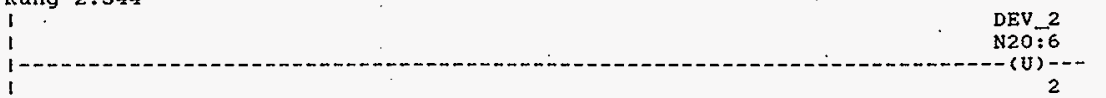

HNF-SD-FF-CSWD-61 Rev. 0 
Processor and Data(OPS Unit 1 )

October 23, 1996 Page 135 program Listing

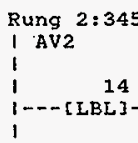

DUMMY 9

N20:7

Rung $2: 346$

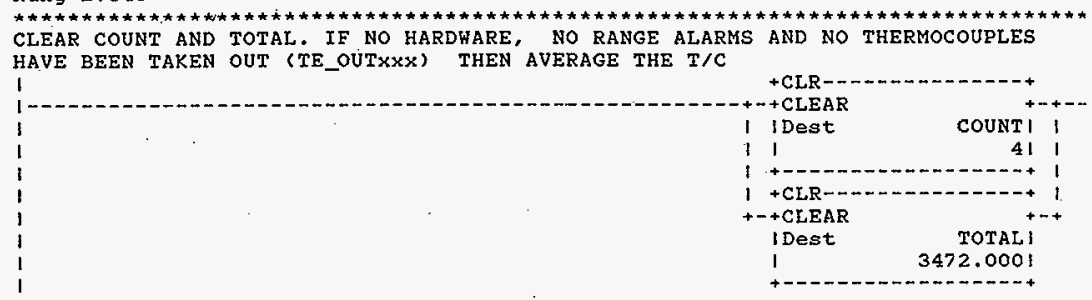

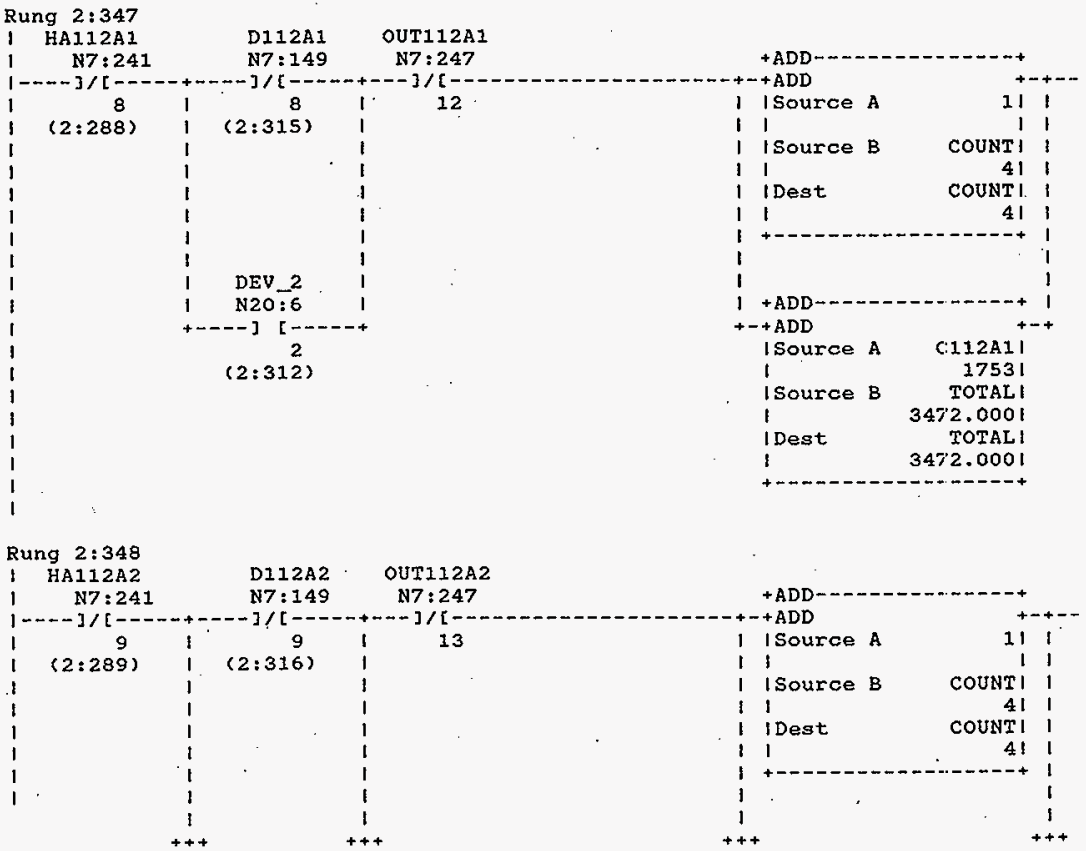




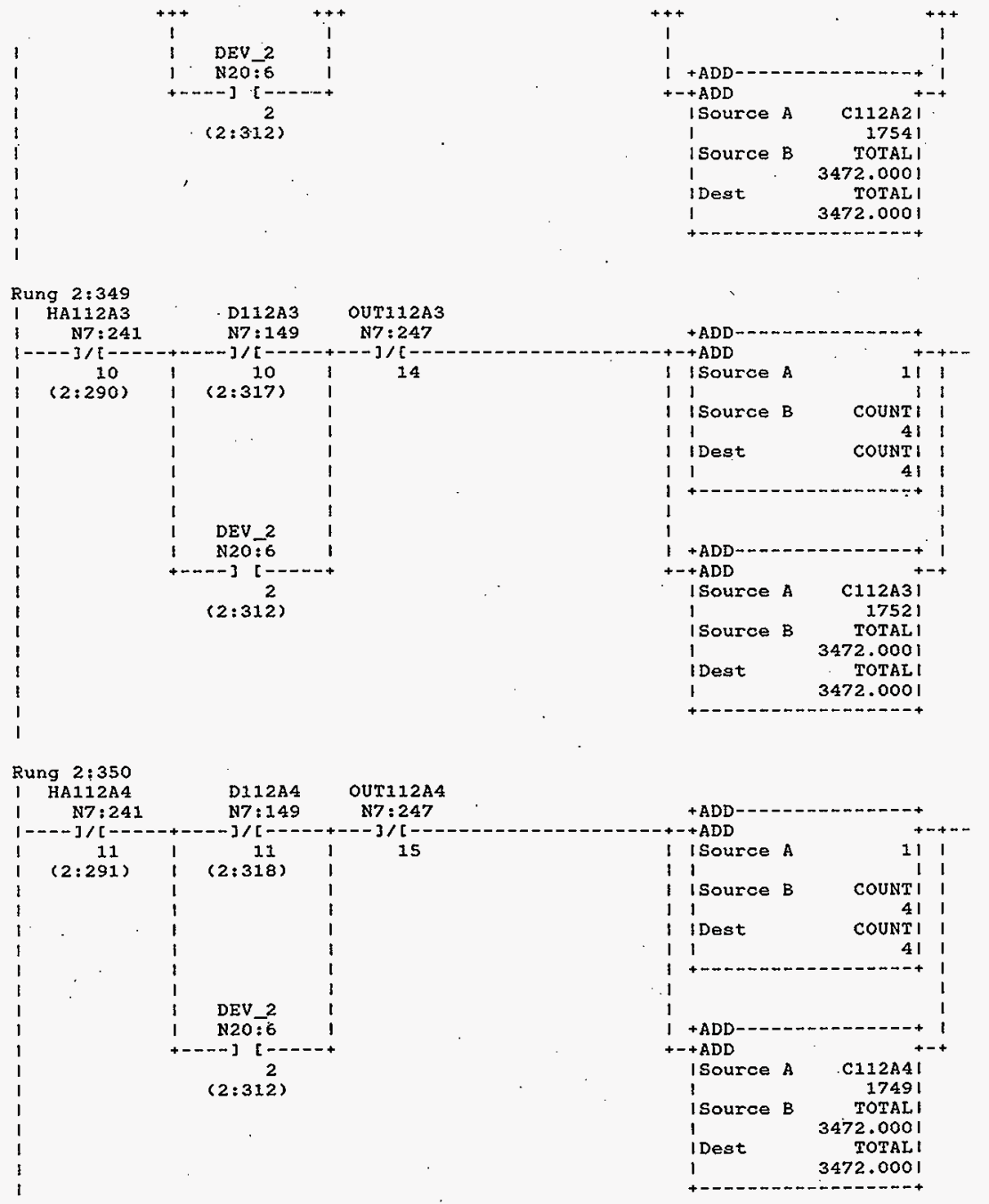


Processor and Data(OPS Unit 1)

Rung 2:351

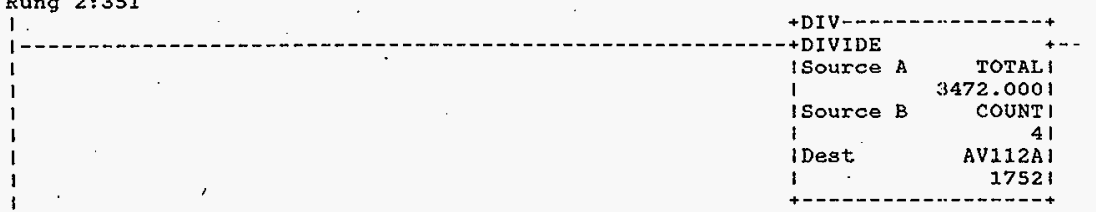

Rung. 2:352

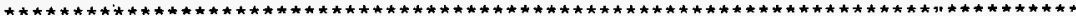
CLEAR COUNT AND TOTAL. IF NO HARDHARE, NO RANGE ALARMS AND NO THERMOCOUPLES HAVE BEEN TAKEN OUT (TE_OUT $\times X \times$ ) THEN AVERAGE THE T/C

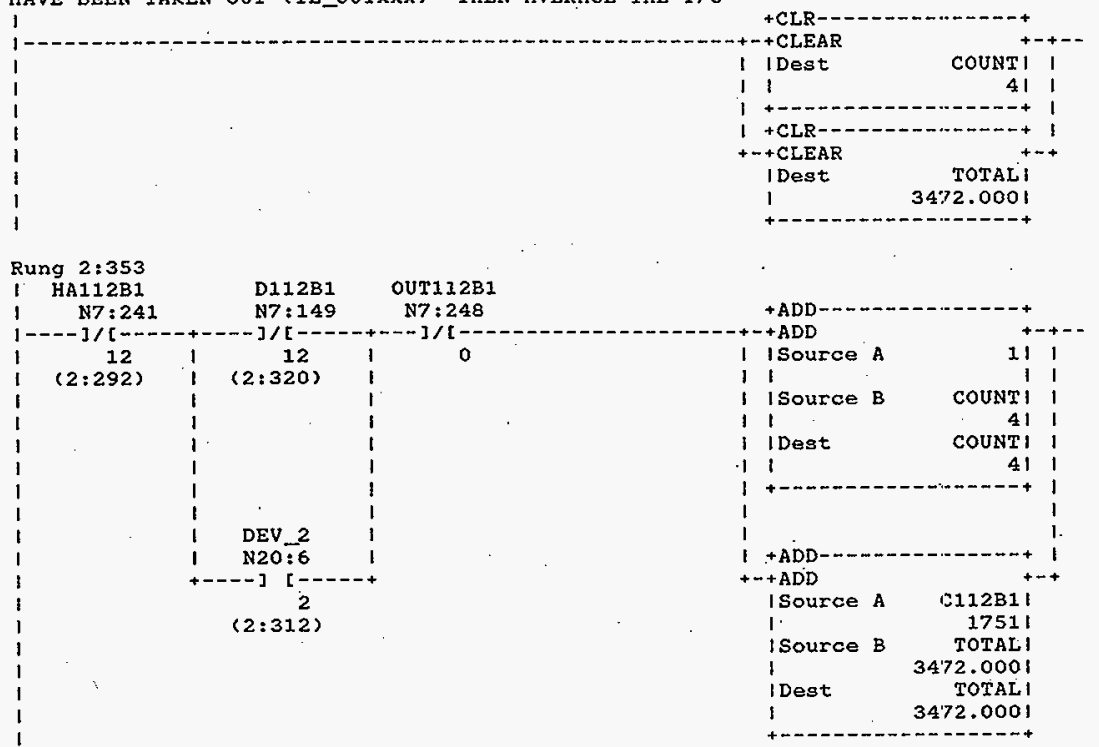


Processor and Data(ops Unit 1 ) Program Listing

\section{Rung 2:354}

I. HA $112 \mathrm{B2}$

$1 \quad$ N7:241

processor File: SODIUMIA.ACH

Rung 2:354

$$
1013
$$

( $(2: 293)$

i

i

i

1

1
1
1
1
1
1
1
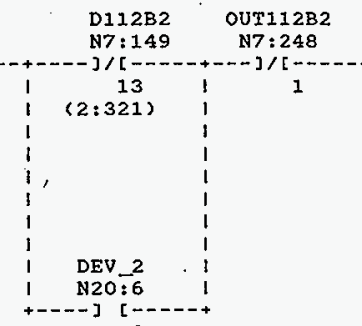

2

(2:312)

1

Rung 2:355

I HA112B3

N7: 241

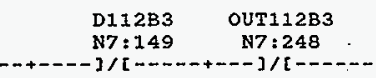

D112B3

]$<[\ldots \ldots$

$+\mathrm{ADD}$

I ISource A

11

I Source B

11

I I Dest

11

1

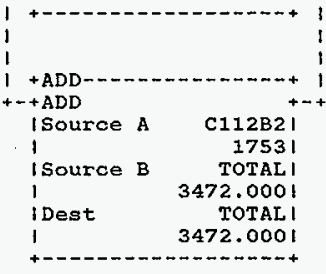

$$
\begin{array}{c|c|c|}
14 & 14 & 1
\end{array}
$$

$(2: 294)$
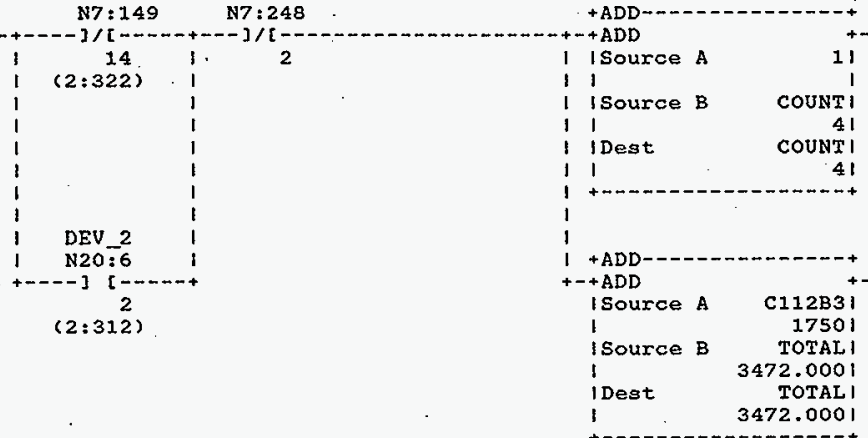

HNF-SD-FF-CSWD-61 Rev. 0

Page 138 
Processor and Data(OPS Unit 1 ) Program Listing
October 23, 1996

Processor File: SODIUMIA.ACH
Page 135 Rung $2: 356$

Rung 2:356

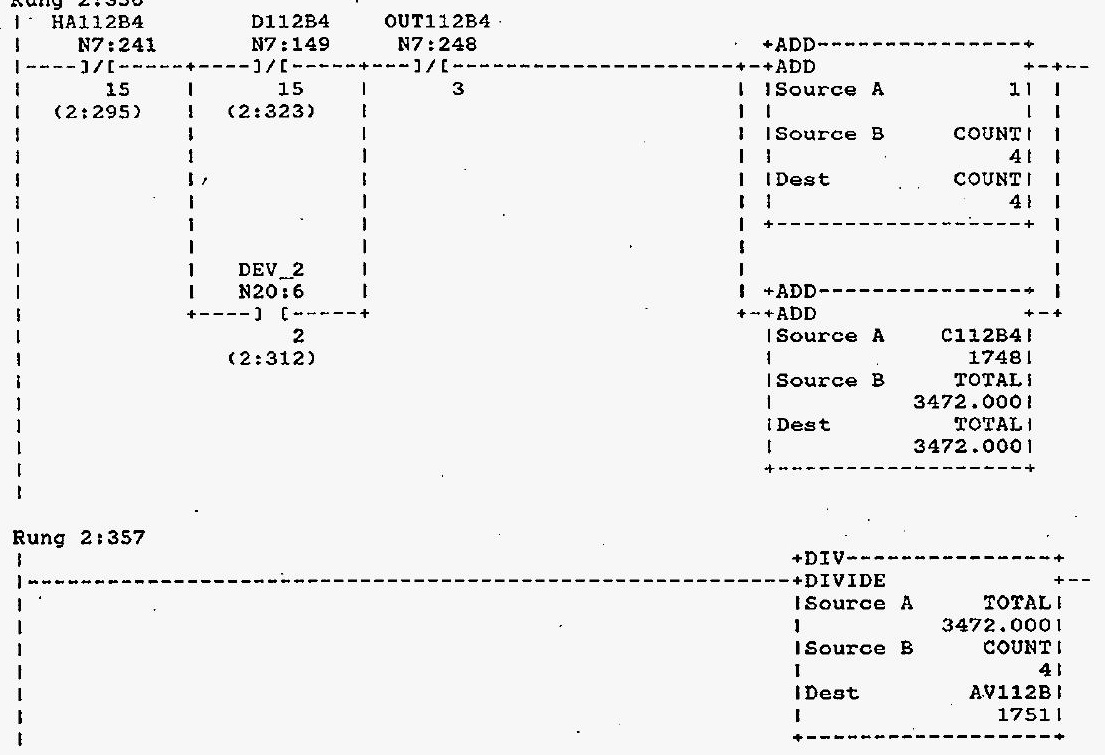

Rung $2: 358$

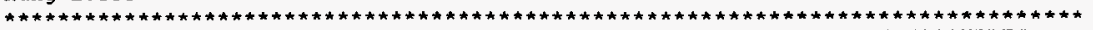
CLEAR COUNT AND TOTAL. IF NO HARDHARE, NO RANGE ALARMS AND NO THERMOCOUPLES HAVE BEEN TAKEN OUT (TE_OUTXXX) THEN AVERAGE THE T/C

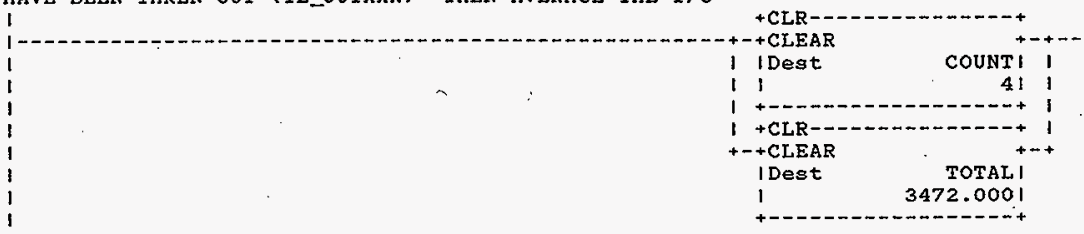

HNF-SD-FF-CSWD-61 Rev. 0 
Processor and Data(OPS Unit 1)

Rung 2:359

i. HA112C1

i $\quad$ 7:242 $1----] /[\ldots-\cdots$ 0

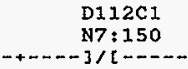

OUT112C1

$(2: 296)$

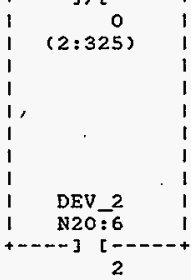

$(2: 312)$
N7 $: 248$

4

$--$

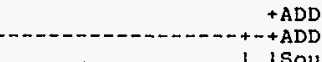

11

1 isource B

11

1 i Dest

11

1

1

$1+A D D$
$+-+A D D$

I Source A 1

I Source B 1

I Dest

1

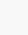
Rung 2:355

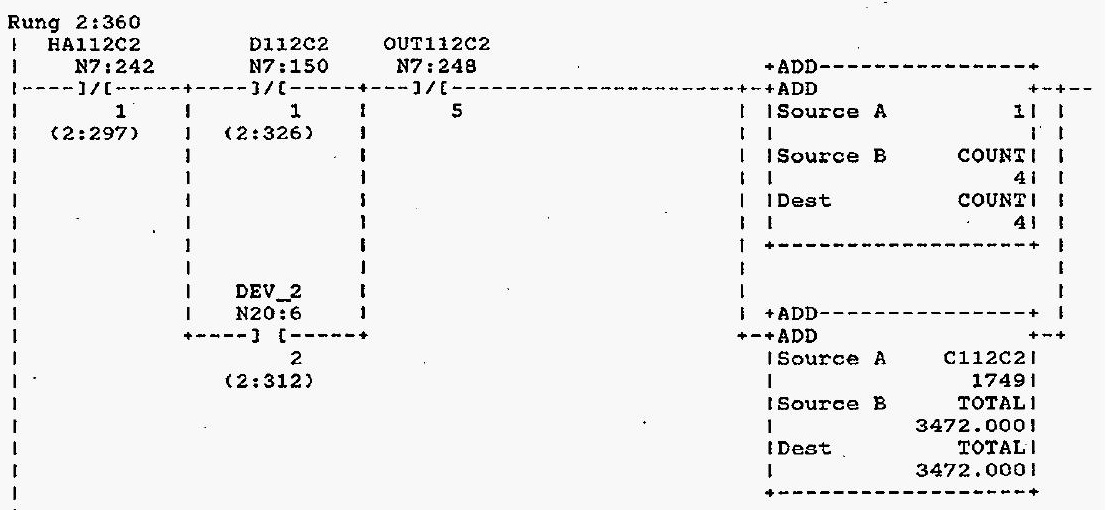

HNF-SD-FF-CSWD-61 Rev. 0 
Processor and Data(OPS Untt 1)

October 23, 1996

Page 141 Program Listing

Processor File: SODIUMIA.ACH

\section{Rung 2:36I}

l. HA112C3

I $\quad$ N7:242

D112C3 - OUT112C3

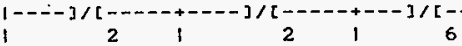

(2:298)

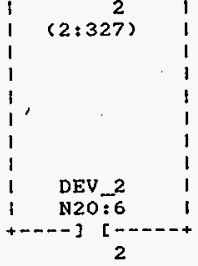

$(2: 312)$

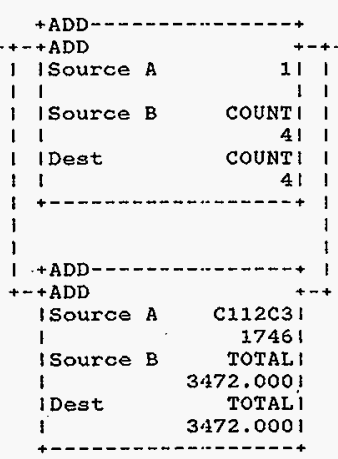

Rung 2:362

2.362

I
IA112C4
N7:242

$1----7 /[\ldots+\cdots$

(2:299)

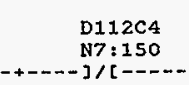

OUT112C4

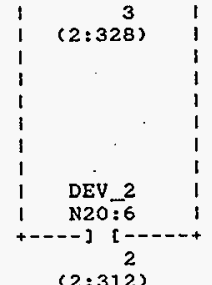

N7: 248

(2:312)

Rung $2: 363$

+DIV----
$---+D I V I D E$

I Source A

I

isource $B$

1

i Dest

1

TOTAL I

3472 . 0001

COUNTI

AV112CI

1746 I

HNF-SD-FF-CSWD-61 R.ev. 0

Page 141 
Processor and Data(ops Unit 1)

Program isisting

Processor File: SODIUM1A.ACH

Rung 2:364

CLEAR COUNT AND TOTAL. IF NO HARDHARE, NO RANGE ALARMS AND NO THERMOCOUPLES HAVE BEEN TAKEN OUT (TE OUTXXX) THEN AVERAGE THE T/C
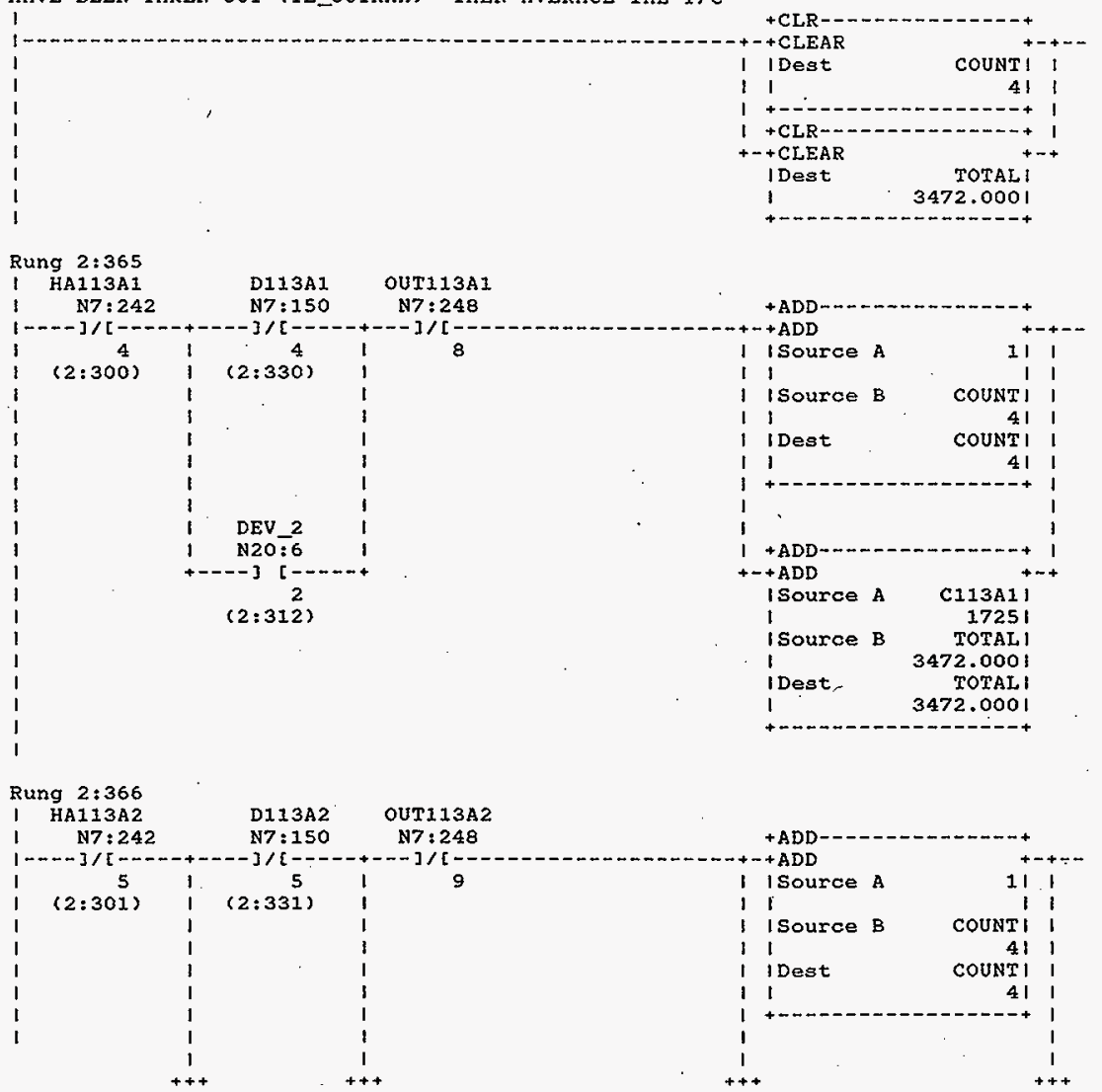

HNF-SD-FF-CSWD-61 Rev. 0 
Processor and Data(OPS Unit 1) Progran Listing
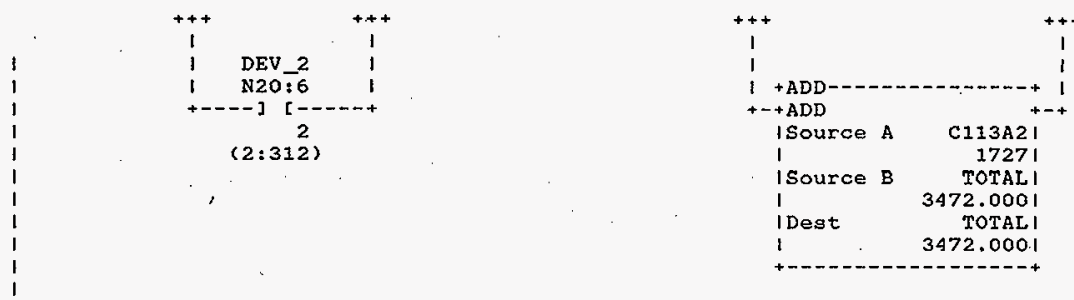

Rung $2: 367$

I HA113A3

N7: 242

D113A3 OUT113A3

N7:150 N7:248

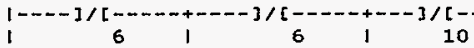

(2:302)

$(2: 332)$

$1(2: 332)$

1

1

$1 \quad N 20: 6 \quad 1$
$+---3 \quad[\ldots \ldots \ldots+$

$(2: 312)$

$+\mathrm{ADD}$

I ISource A

1

I Source B

11

I Dest

1

$+$

1

$1+\mathrm{ADD}-2-2-0-0-0-0-1+1$

++ ADD

ISource A CI13A3I

I 1726 I

|Source B TOTALI

$1 \quad 3472.0001$

IDest TOTALI

1 34.72.0001

Rung $2: 368$

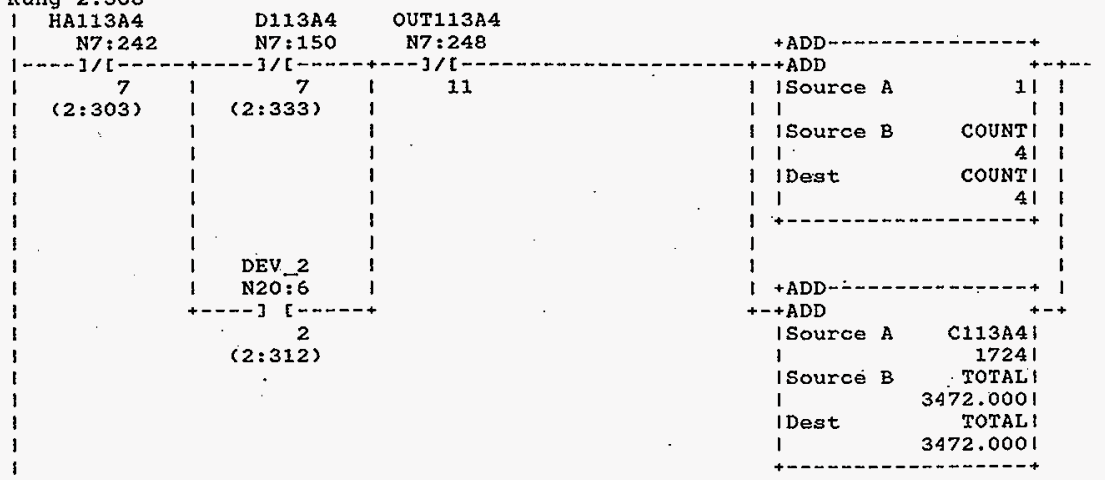

HNF-SD-FF-CSWD-61 Rev. 0

Page 143 
Rung $2: 369$

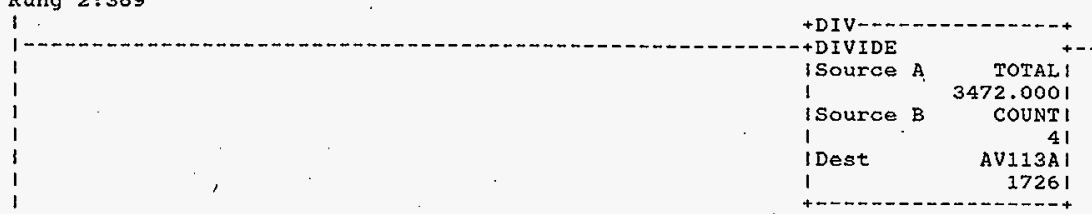

\section{Rung $2: 370$}

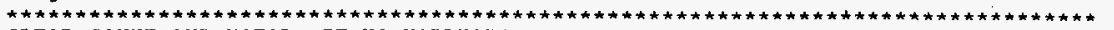
CIEAR COUNT AND TOTAL. IF NO HARDWARE, NO RANGE ALARMS AND NO THERMOCOUPLES HAVE BEEN TAKEN OUT (TE_OUTXXX) THEN AVERAGE THE T/C

1

1

I

I

Rung 2:371

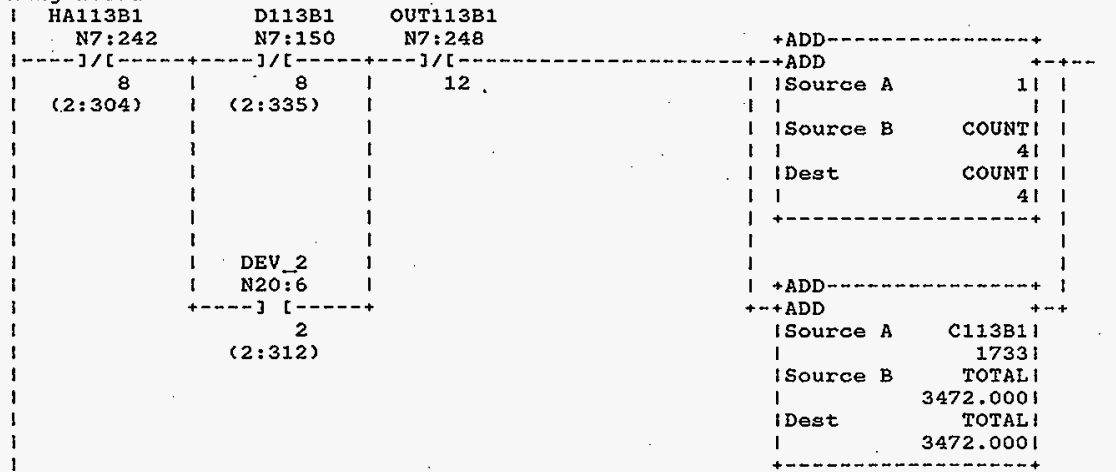


Processor and Data(ops Unit 1) Program Listing

Processor File: SODIUM1A.ACH

Rung 2:372

1. HA113B2

N7 $7: 242$

DI13B2 OUT1138ं2

N7:150 N7:248

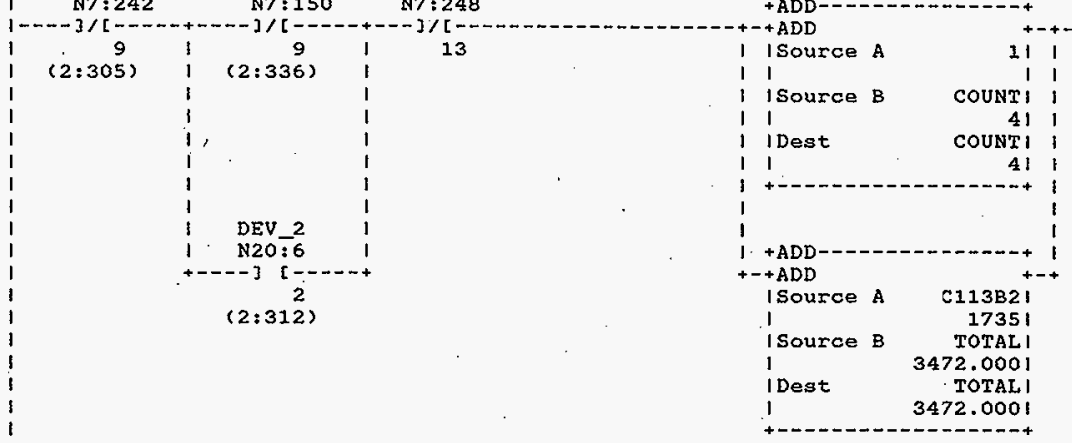

Rung $2: 373$

I HA113B3

I N7:242

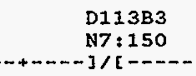

oUT113B3

N7: 248

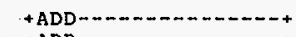

$\ldots+\cdots,[-$

(2:306)

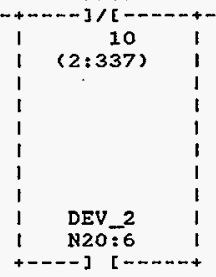

14

ISou

Rung $2: 372$

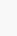

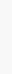$$
\text { i }
$$

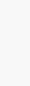

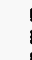$$
(2: 312)
$$ 
Processor and Data(OPS Unit I)

Program Listing

Rung 2:374

1. HA113B4

Processor File: SODIUMA.ACH
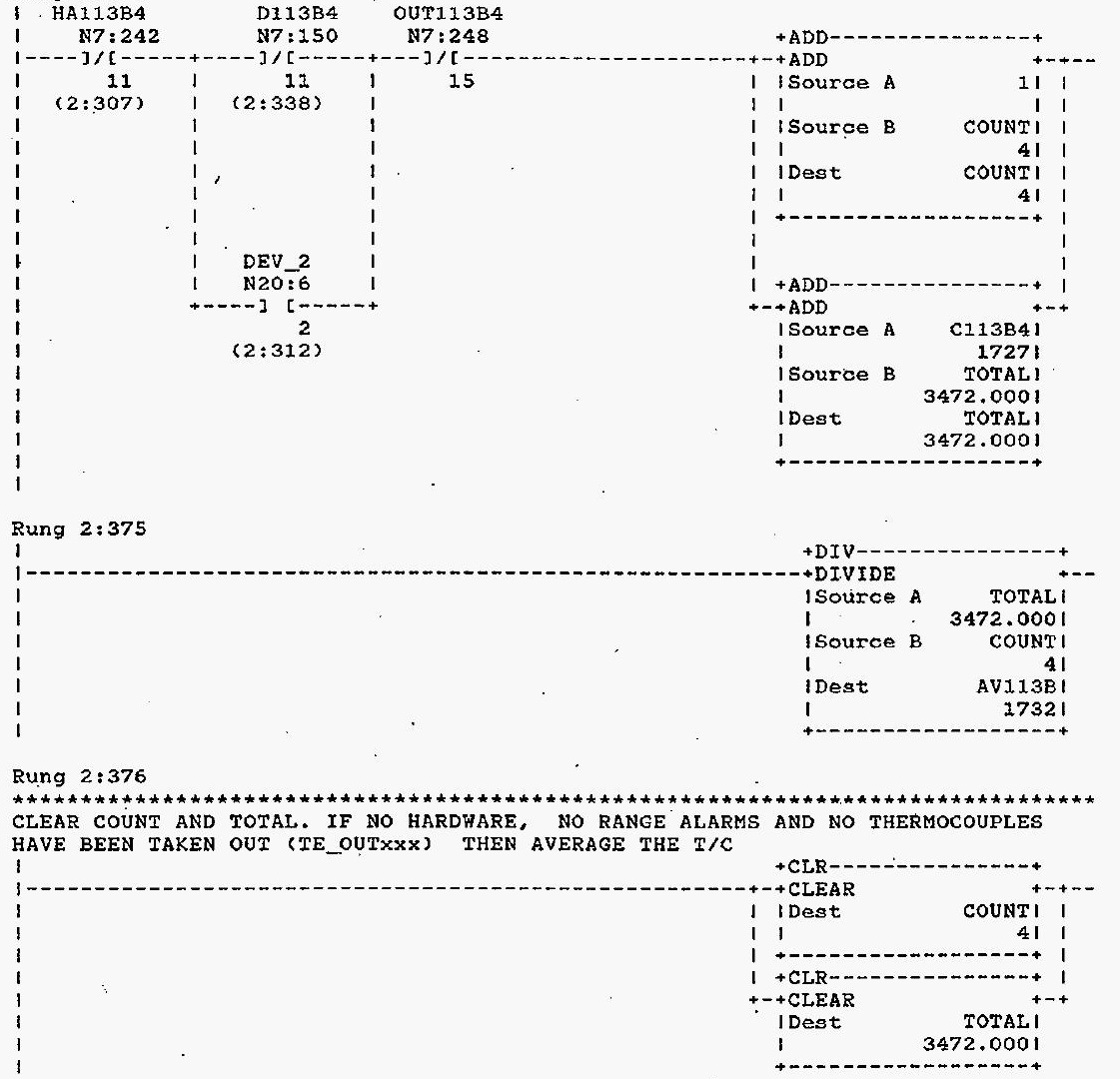

HNF-SD-FF-CSWD-61 Rev. 0 
Processor and Data(ops Unit 1)

Program Listing

Processor File: SODIUMAA.ACH

October 23, 1996

Page 147

Rung 2:377

Rung $2: 377$

1. HA113C1

$1 \quad N 7: 242$

D113C1

OUT113C1

N7:150 N7:249

$(2.308)$

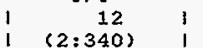

$\rightarrow>0-$

o

l $(2: 340)$

1

1,

1

$\mathrm{DEV} 2$
$\mathrm{~N} 20: 6$

+-..- 1

$(2: 312)$

0

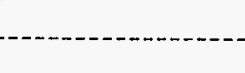
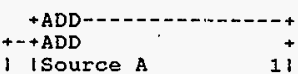

11

I isource B

11

1 IDest

1

$1+$

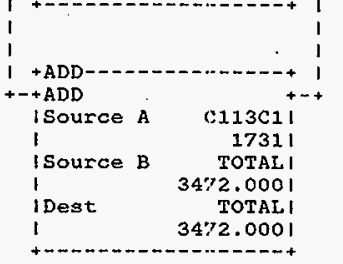

Rung $2: 378$

I HA113C2

N7 $: 242$

D113C2

OUT1I3C2

N7: 150

N7: 249

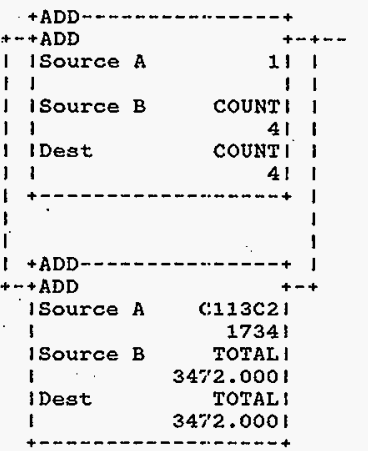

HNF-SD-FF-CSWD-6I Rev. 0

Page 147 
Processor and Data(OPS Unit 1) Program Listing

\section{Rung 2:379}

.

I N7:242

$1--\cdots] /[\ldots \ldots+-4+$

$(2: 310)$

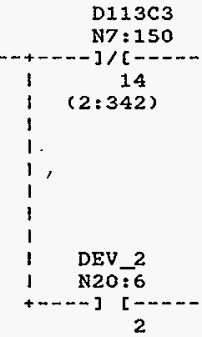

OUT113C3

N7 : 249

$+-+\mathrm{ADD}$

ISource A

1

I ISource B

11

I I Dest

11

+.

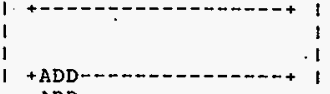

(2:312)
$1+A D D$
$+-+A D D$

ISource A
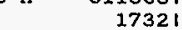

ISource B

I Dest

1

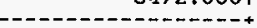

TOTALI

\section{Rung 2:380}

I HA $113 \mathrm{C} 4$

1 N7:242

1----]/[-

(2: 311 )

2

D113C4

OUT113C4

N7:150 N7:249

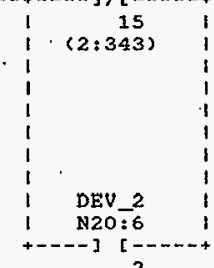

$-3 /[$

$+\mathrm{ADD}$

$(2: 311)$

$(2: 312)$

Rung 2:381

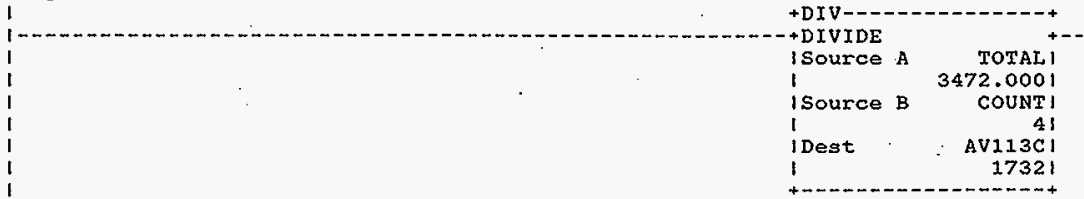

HNF-SD-FF-CSWD-61 Rev. 0 
Processor and Data(ops Unit 1)

Rung $2: 382$

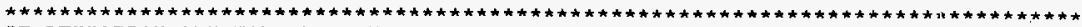
IF DEVIATION SET FOR SECTION 2 THEN GO THERE AND CAECULATE DEVIATION

I

$1 \quad D E V \_2$

DEV 2

I N20:6

1----] [-

I (2:312)

Rung 2:383

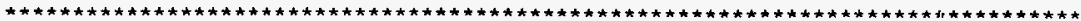
IF RATE CALCULATIONS ARE FINISHED THEN GO.TO THE PID BLOCK

I RT TMR/DN

I $\bar{T} 4: 62$

PJ:D112A

1 DN

I (2:33)

Rung 2:384

RATE CHECKING

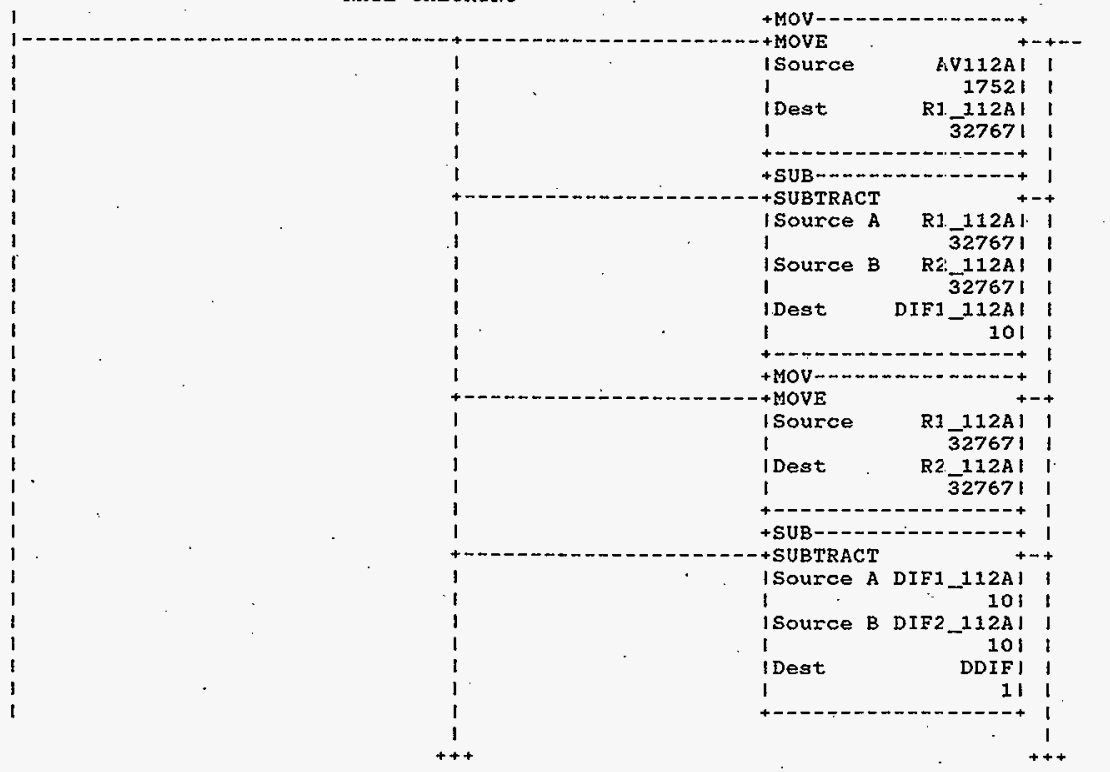

HNF-SD-FF-CSWD-61 Rev. 0 


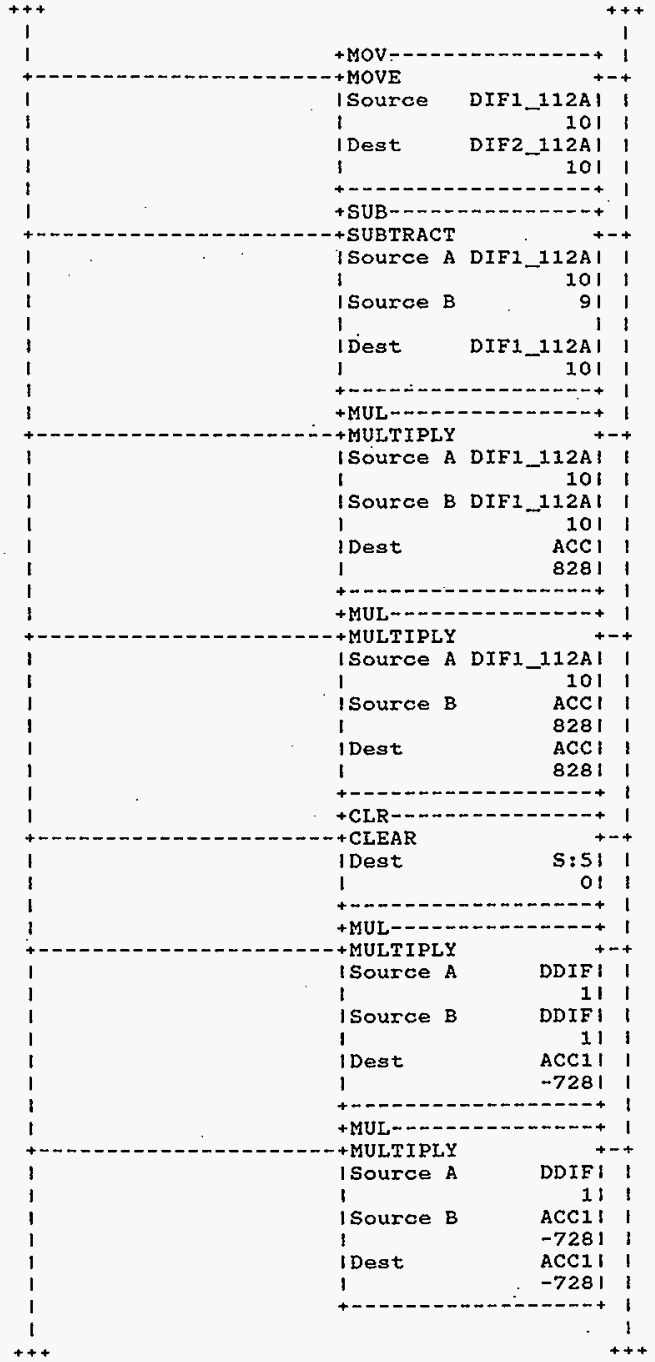

HNF-SD-FF-CSWD-61 Rev. 0 


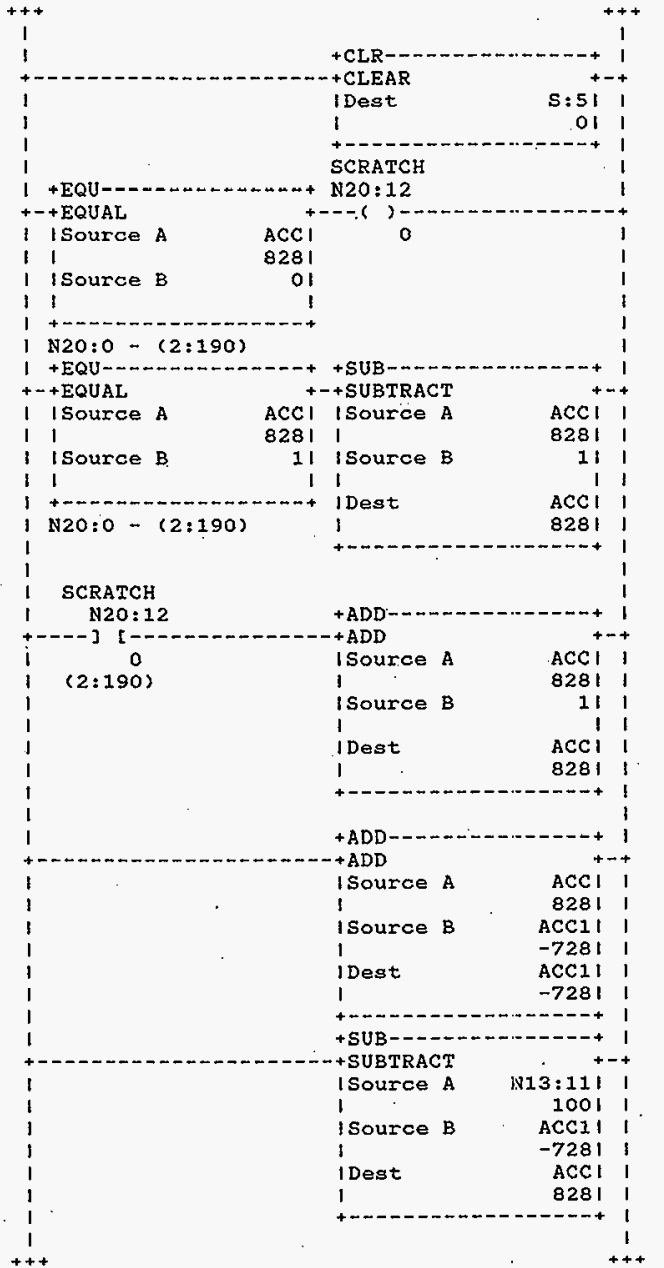

HNF-SD-FF-CSWD-61 Rev. 0 


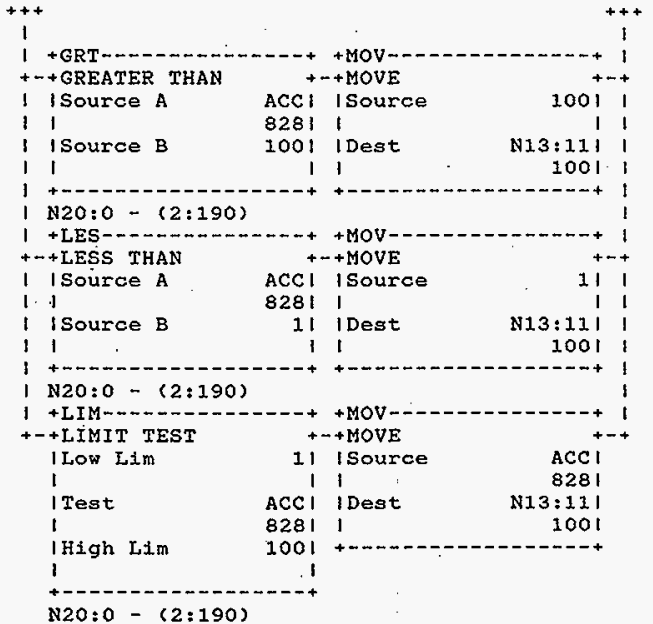

Rung 2:385

1 PID112A

DUMMY22

N20:8

1-- [LBL]

Rung 2:386

DETECT FROM SCADA PID ON/OFF IF PID OFF THEN ZERO PH FOR ZERO OUTPUT AT SCR AND JUMP AROUND THE PID BLOCK

I ON_112A $(2: 824)$

\section{AND JUMP AROUND THE PID BLOCK}


Processor and Data(ops Unit 1 ) Program Listing.

Rung 2:388

DETECT SETPOINT VALUE FROM SCADA PUT VALUE IN PID BLOCK

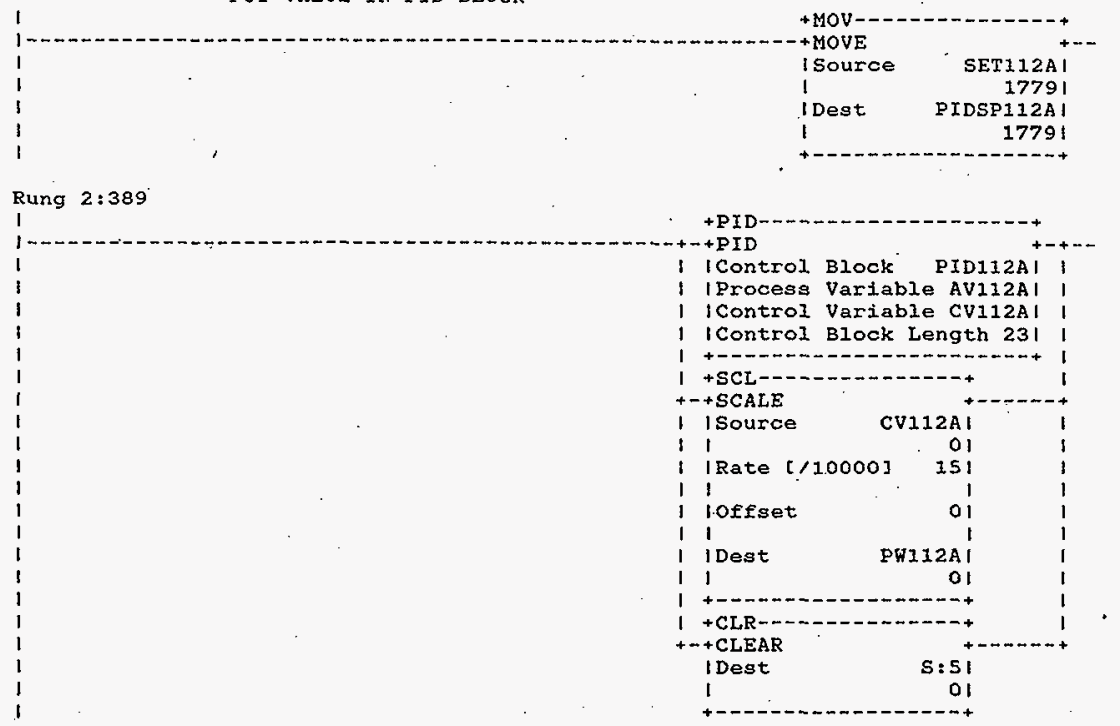

Rung $2: 390$

182

N9: 20

$1--[$ LBL ]

Rung 2:391

1

( RT_TMR/DN

i T4:62

FID112B

$1---m /[-$

l $(2: 33)$

Rung 2:392

RATE CHECKING

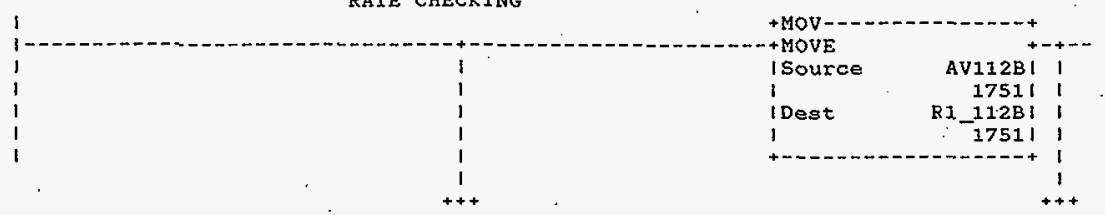

HNF-SD-FF-CSWD-61 Rev. 0 
Processor and Datalops Unit 1)

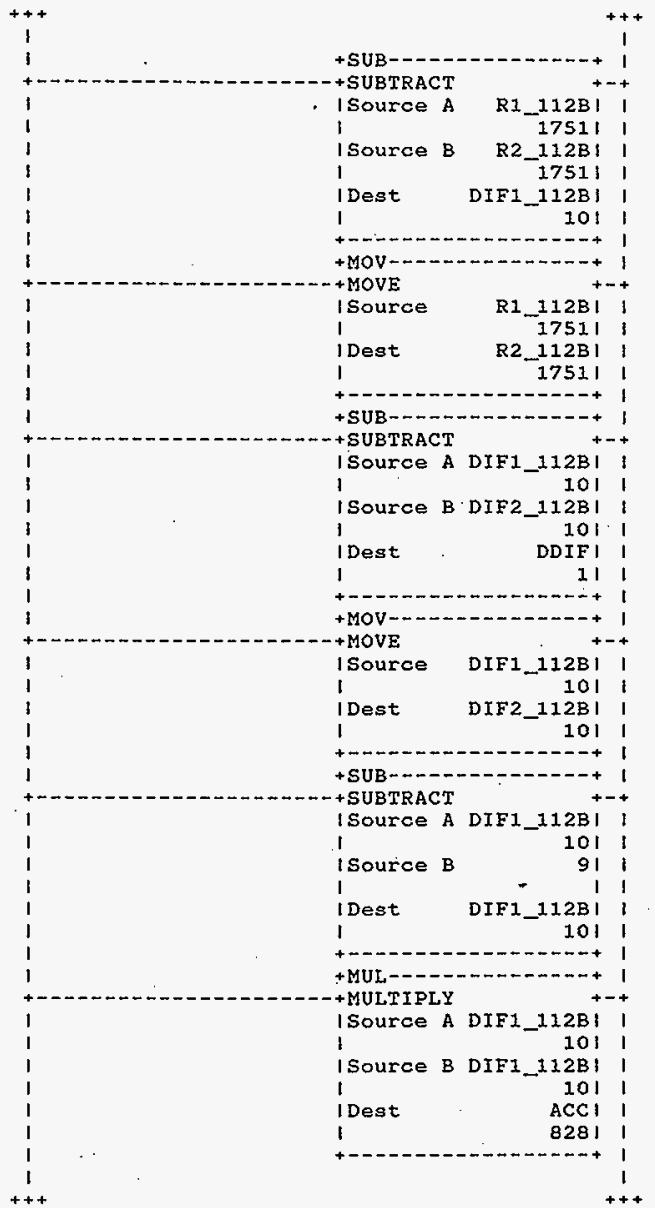

HNF-SD-FF-CSWD-61 Rev. 0 


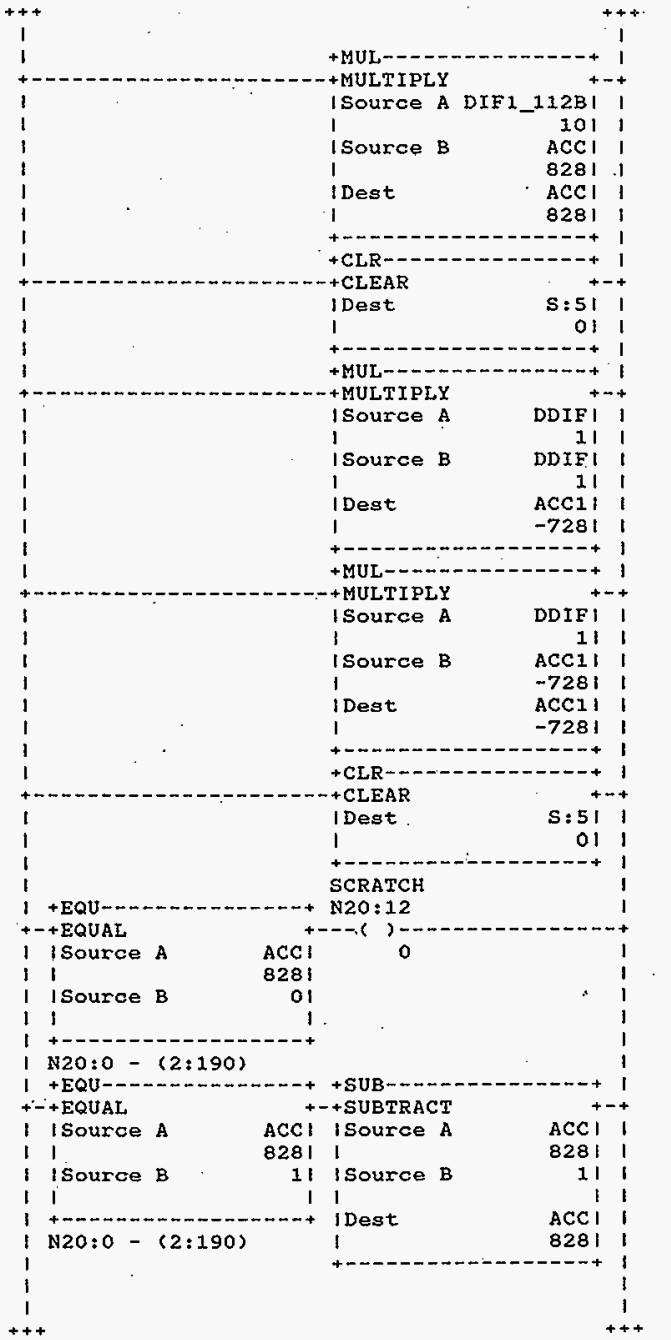




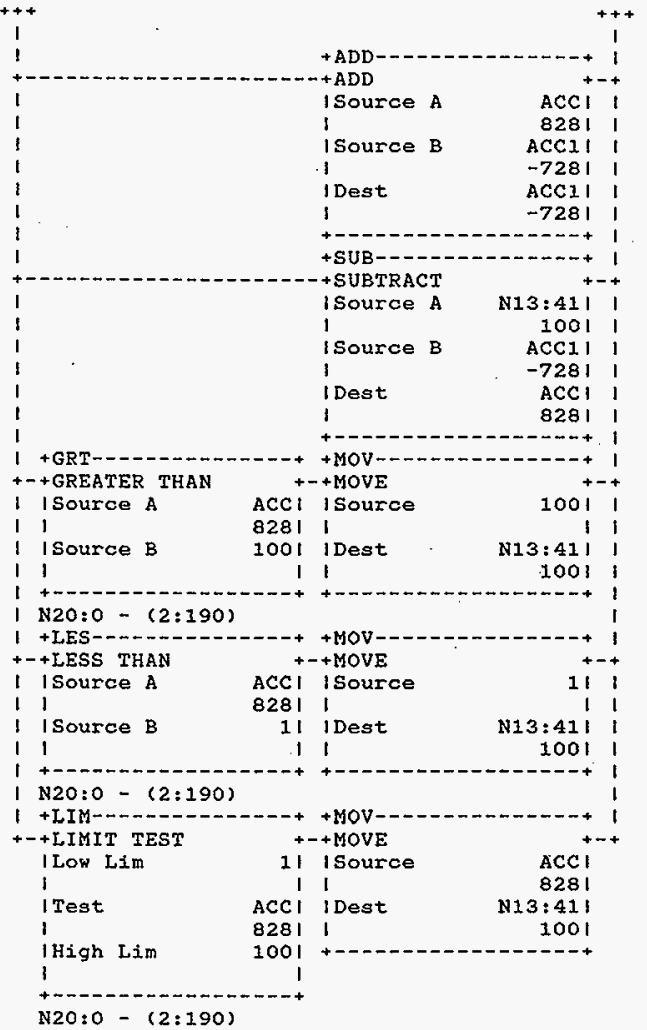

Rung $2: 393$ 
Processor and Data(OPS Unit 1 )

Rung 2:394

$$
\begin{aligned}
& \text { DETECT FROM SCADA PID ON/OFF } \\
& \text { IF PID OEF } \\
& \text { THEN ZERO PW FOR ZERO OUTPUT AT SCR }
\end{aligned}
$$
AND JUMP AROUND THE PID BLOCK
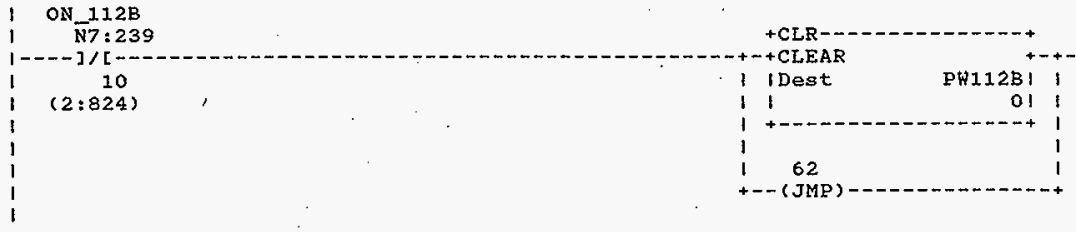

Rung 2:395

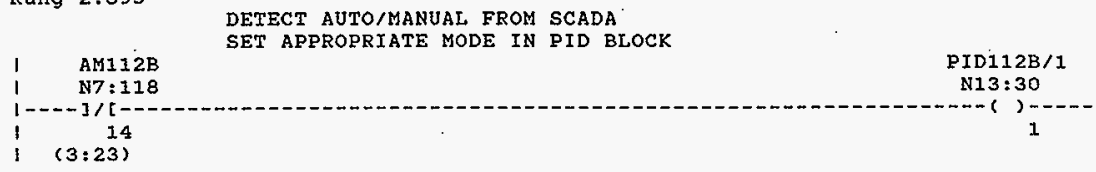

Rung $2: 396$

DETECT SETPOINT VALUE EROM SCADA PUT VALUE IN PID BLOCK

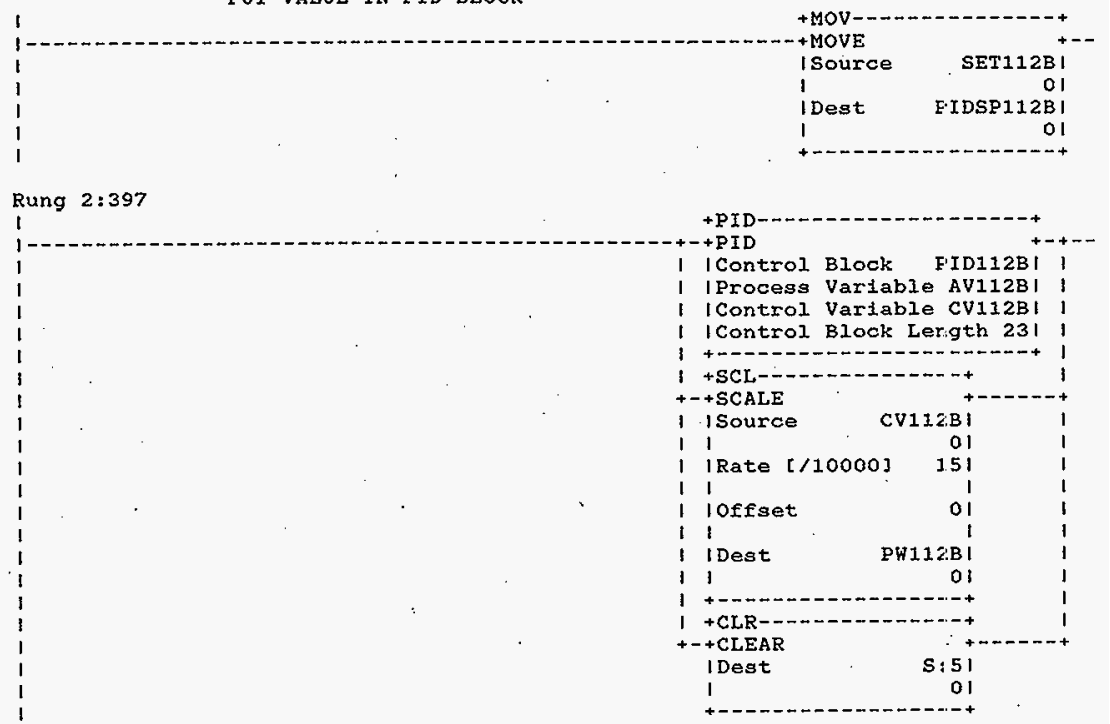

HNF-SD-FF-CSWD-61 Rev. 0 
Processor and Data(OPS Unit 1)

Rung 2:398

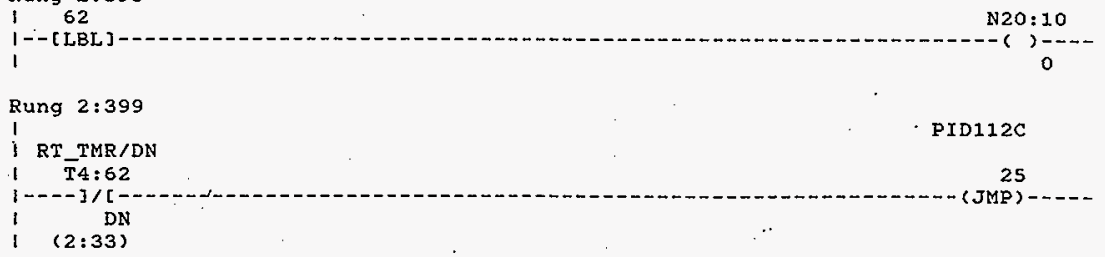

Rung 2:400

RATE CHECKING

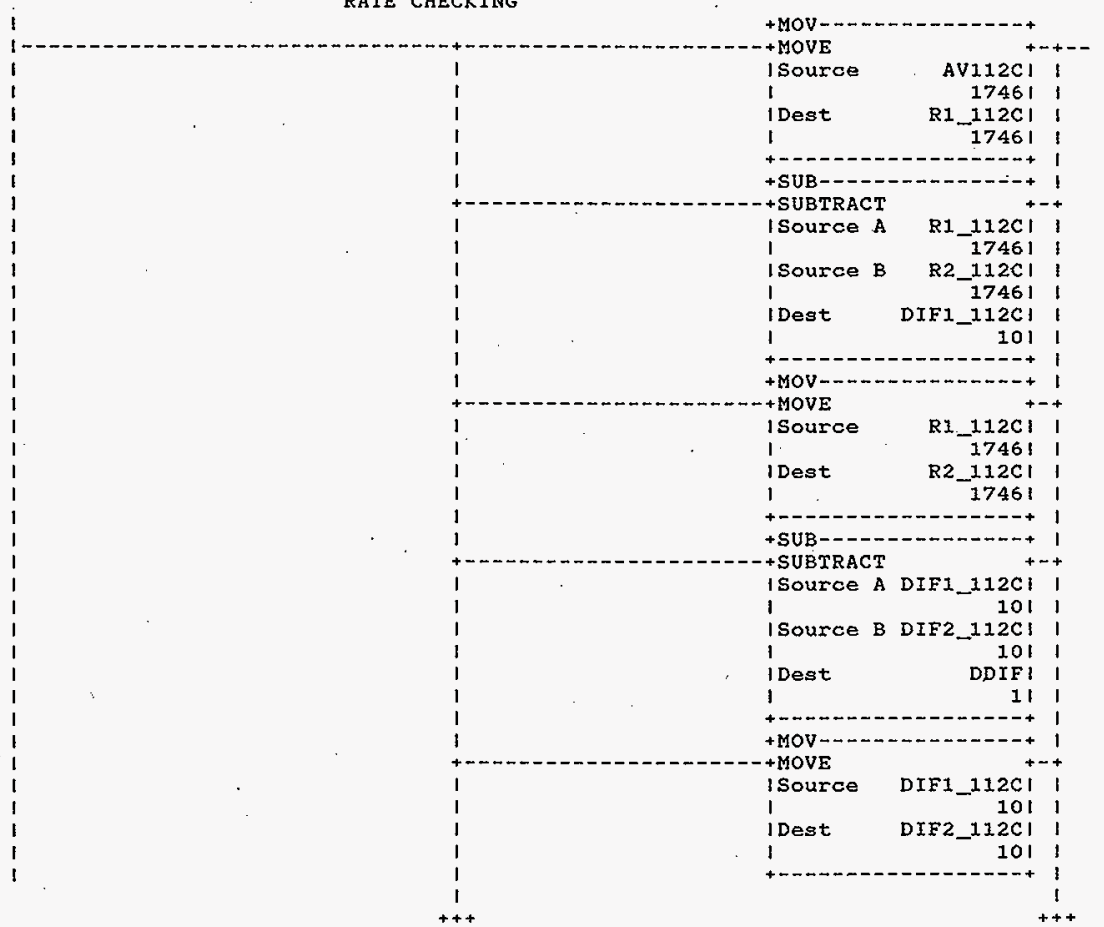

HNF-SD-FF-CSWD-61 Rev. 0 
Processor and Data (OPS Unit 1) Program Listing

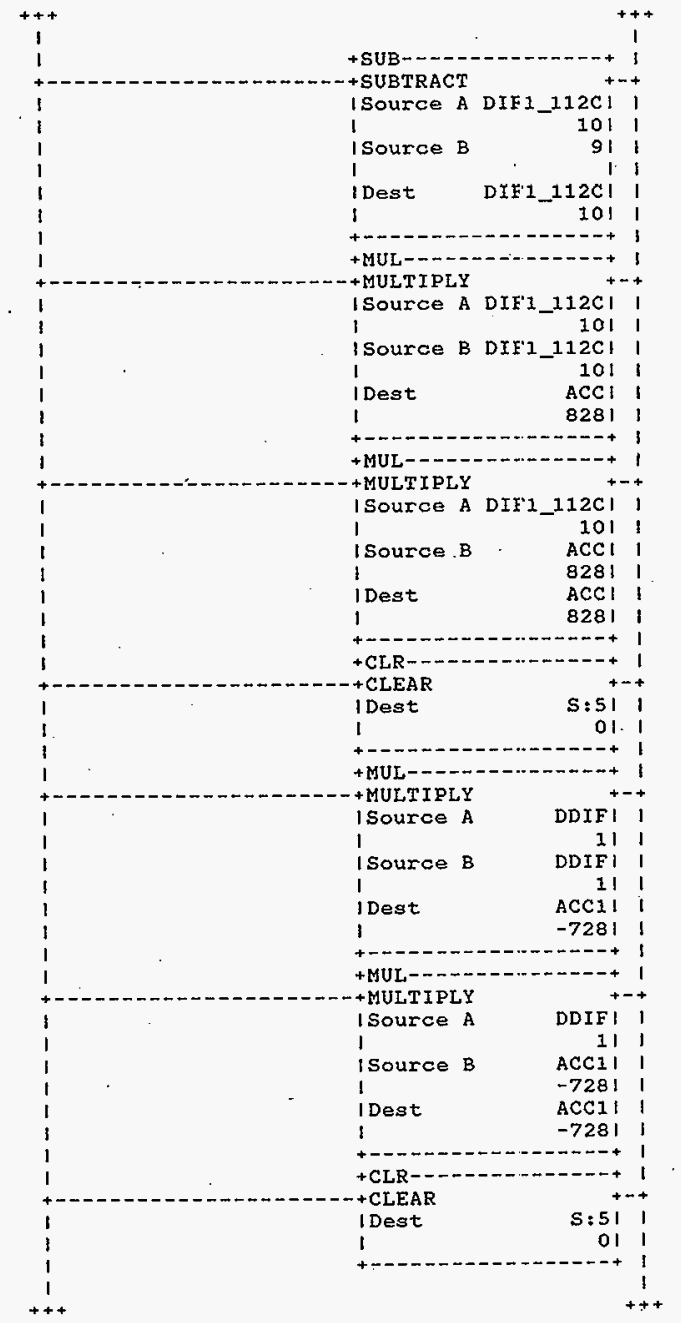

HNF-SD-FF-CSWD-6I Rev. 0 


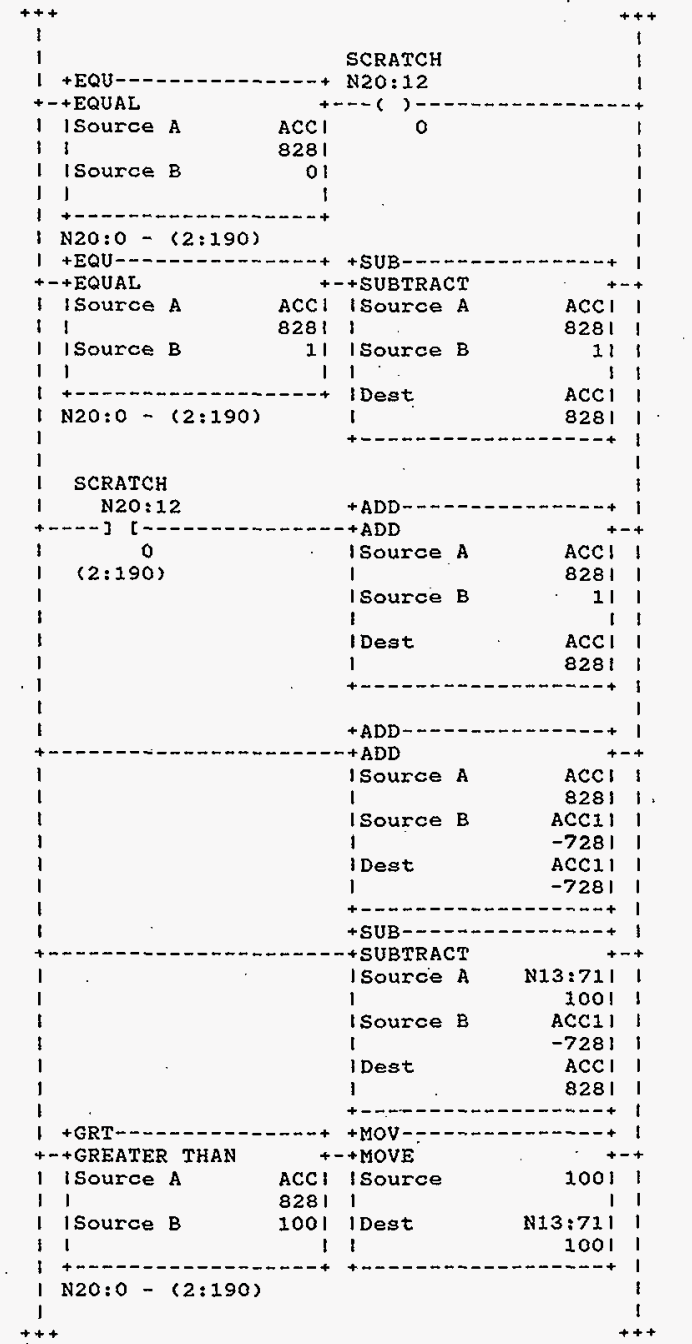

HNF-SD-FF-CSWD-61 Rev. 0 


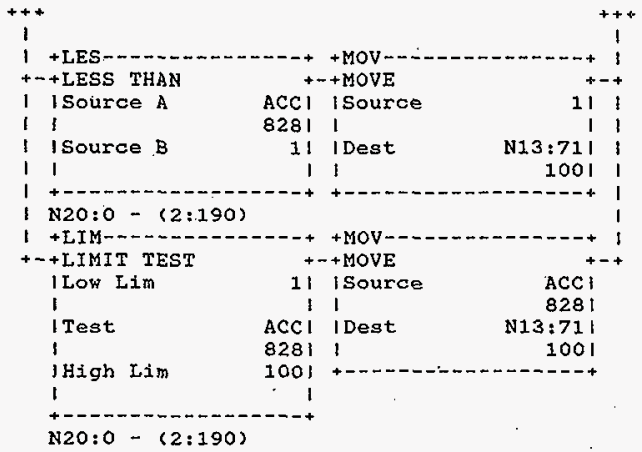

Rung 2:401

1 PID112C

1

125

25

DUMMY 24

:--[LBL]

N20: 8

Rung $2: 402$

DETECT FROM SCADA PID ON/OFF

IF PID OEF

THEN ZERO PW FOR ZERO OUTPUT AT SCR AND JUMP AROUND THE PID BLOCK

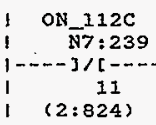

DETECT AUTO/MANUAL FROM SCADA

Rung $2: 403$

SET APPROPRIATE MODE IN PID BLOCK

$\begin{array}{lc}\text { I } & \text { AM112C } \\ 1 & N 7: 119 \\ 1 & 0 \\ 1 & (3: 24)\end{array}$

PID $12 \mathrm{C} / 1$ N13:60

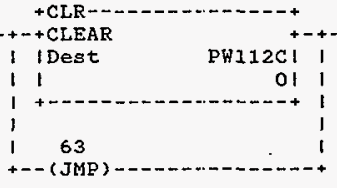

HNF-SD-FF-CSWD 61 Rev. 0 
Processor and Data(OPS Unit 1)

Rung 2:404

DETECT SETPOINT VALUE FROM SCADA PUT VALUE IN PID BLOCK
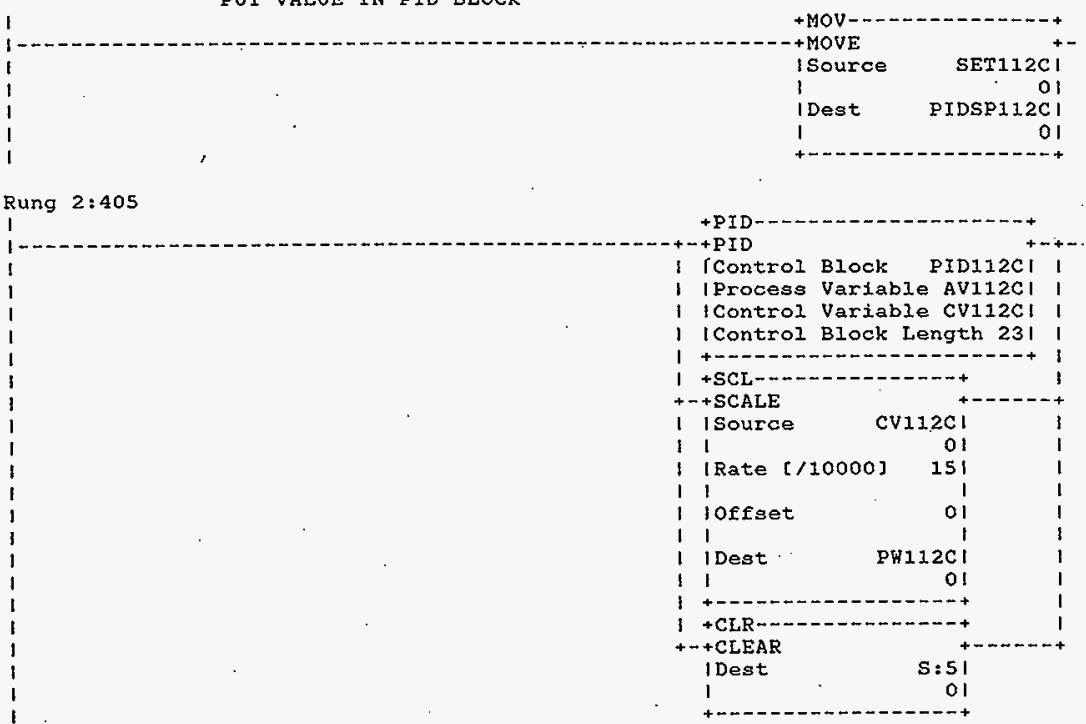

Rung 2:406

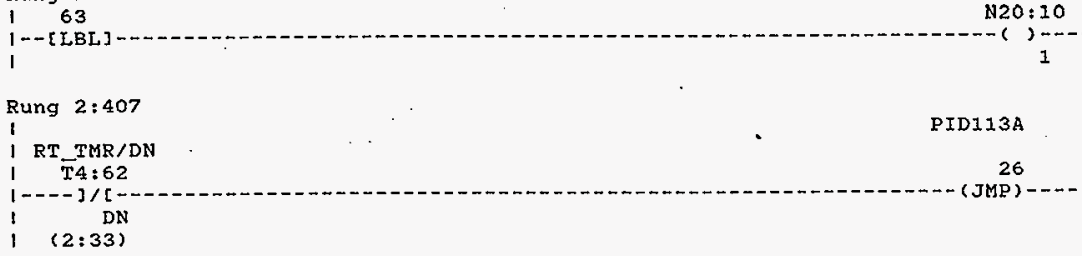

Rung 2:408

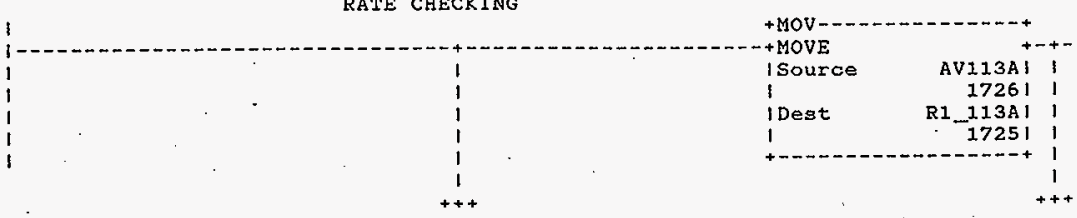

HNF-SD-FF-CSWD-61 Rev. 0 

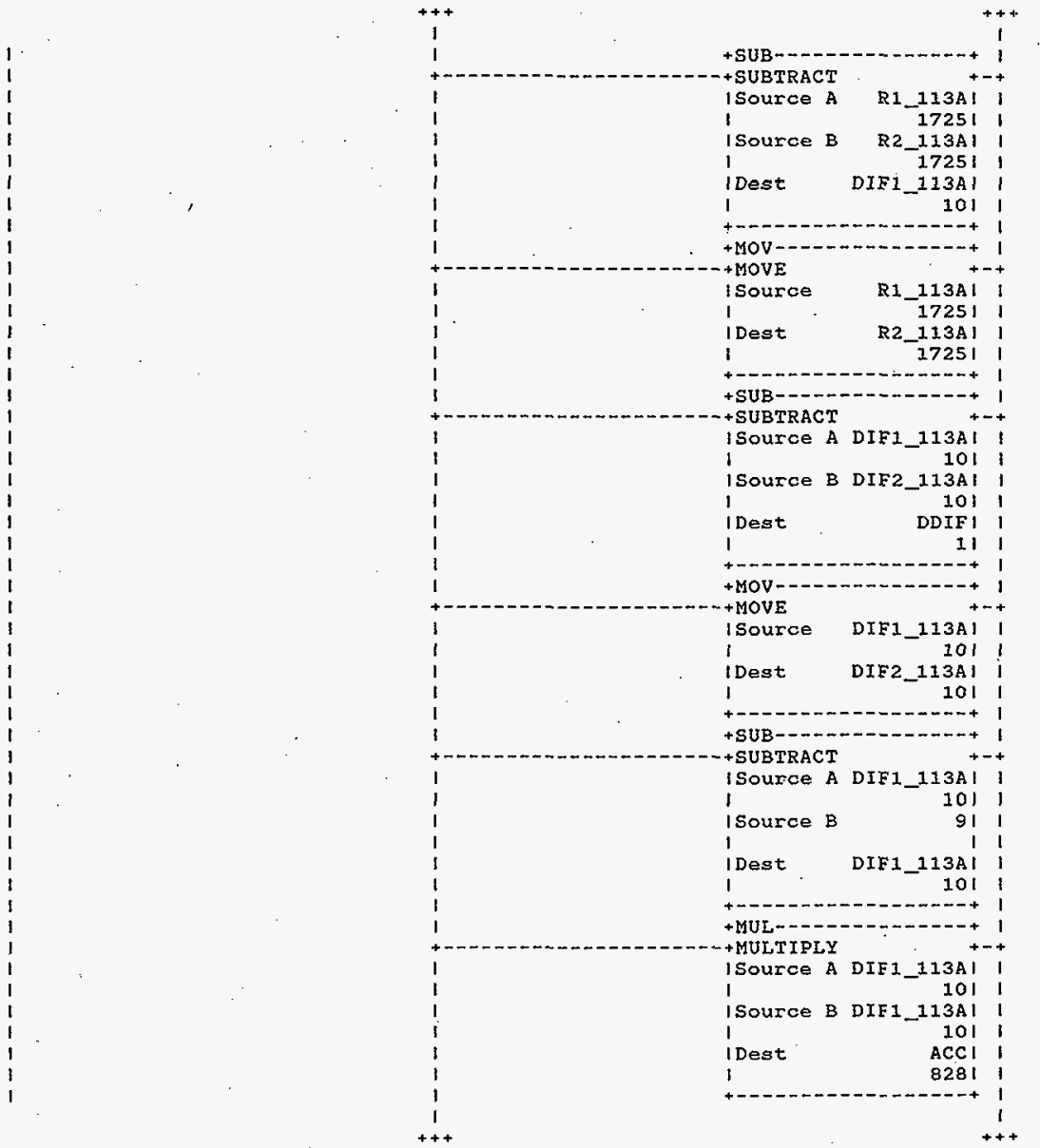


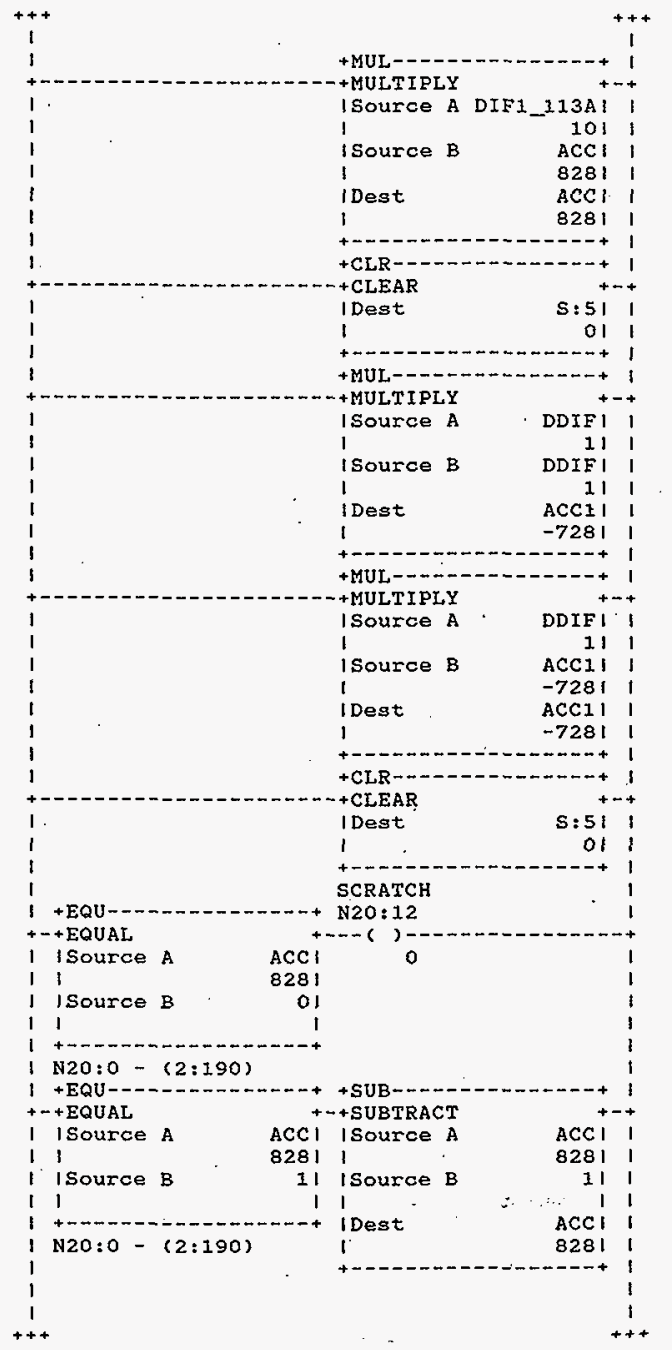

HNF-SD-FF-CSWD-61 Rev. 0 
Processor and Data(OPs Unit 1) Program Listing processor File: SODIUMiA. ACH

October 23, 1996

Page 16: Rung $2: 40 \varepsilon$

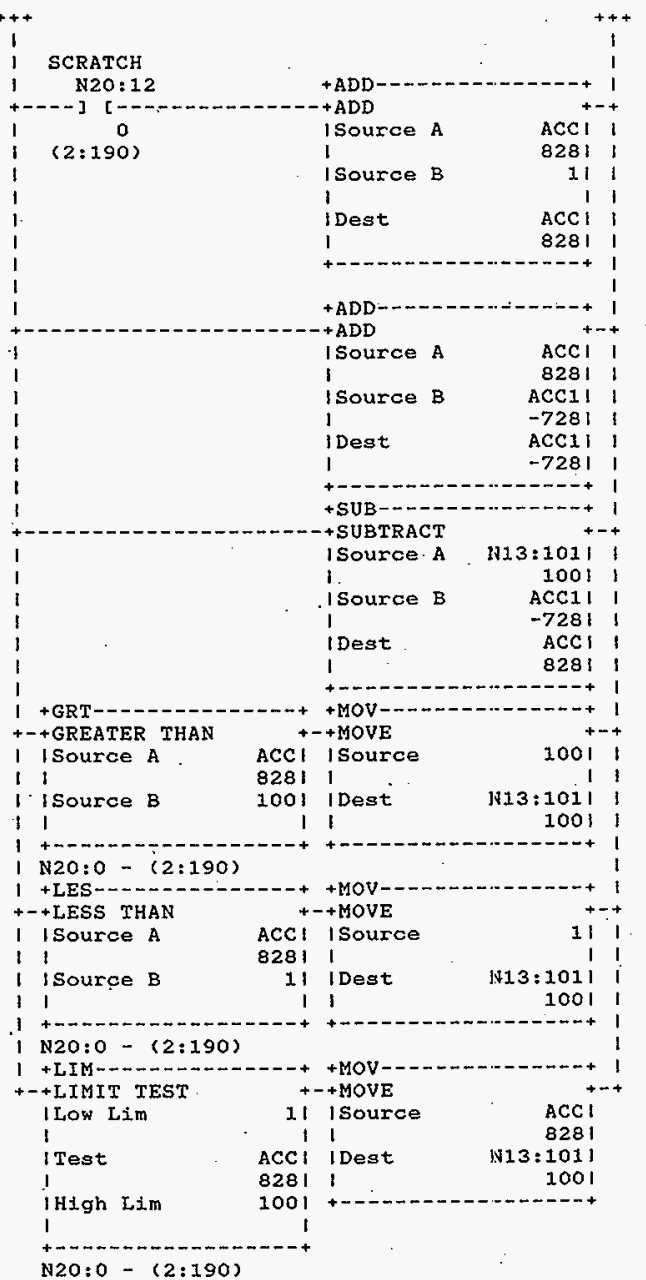


Processor and Data (OPS Unit 1)

Rung 2:409

I PID113A$$
\text { , }
$$$$
126
$$$$
\text { 1-- }[\text { [BL] }
$$$$
26
$$

Rung $2: 410$
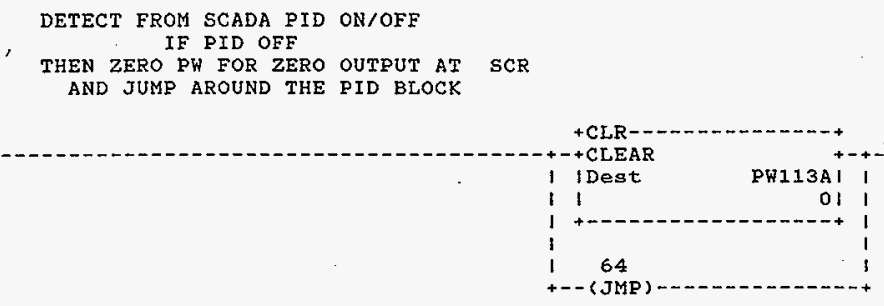

Rung $2: 411$

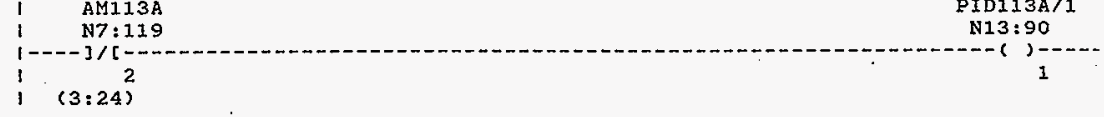

Rung 2:412

DETECT SETPOINT VALUE FROM SCADA PUT VALUE IN PID BLOCK

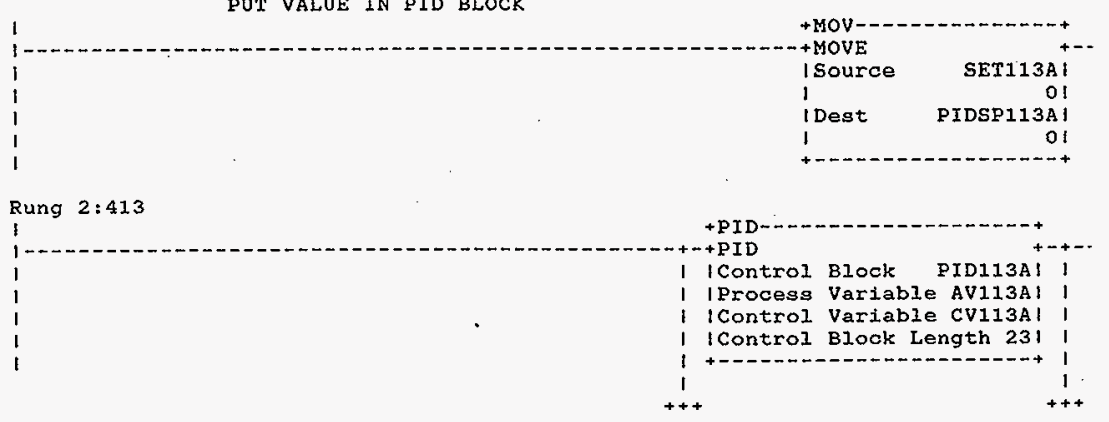

HNF-SD-FF-CSWD-61 Rev. 0 
Processor and Data(OPS Unit 1)

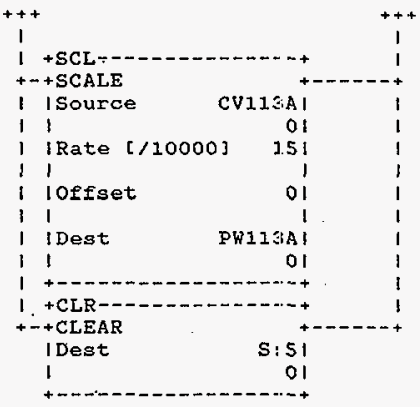

Rung 2:414

\section{Rung 2:415}

I

I RT_TMR/DN

$$
1 \text { T4:62 }
$$

$1-\cdots+1 /[-\cdots$

\section{(2:33)}

Rung 2:416

\section{RATE CHECKING}

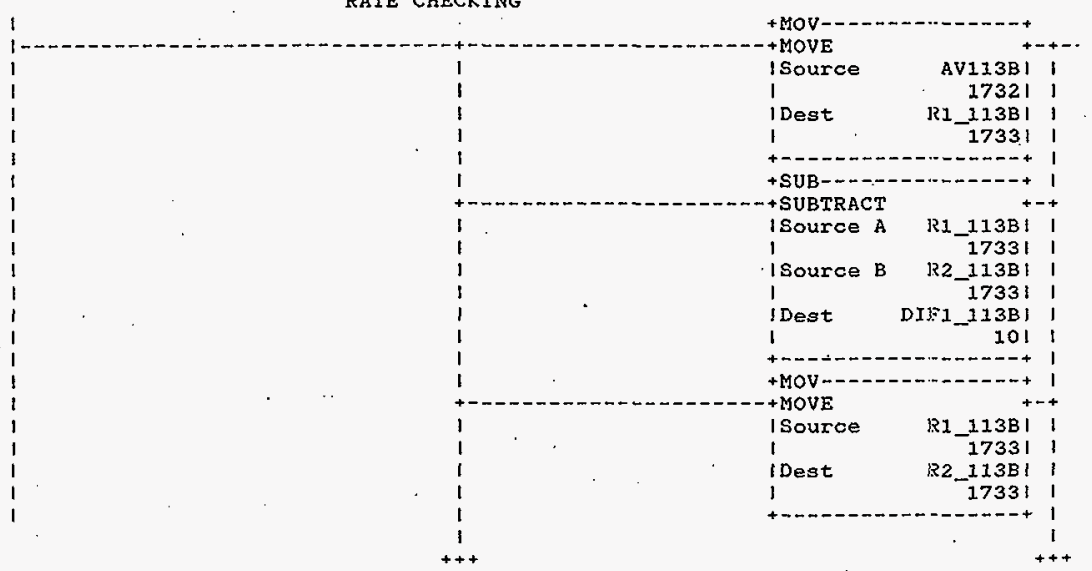

HNF-SD-FF-CSWD-61 R.ev. 0 
Processor and Data (OPS Unit 1)

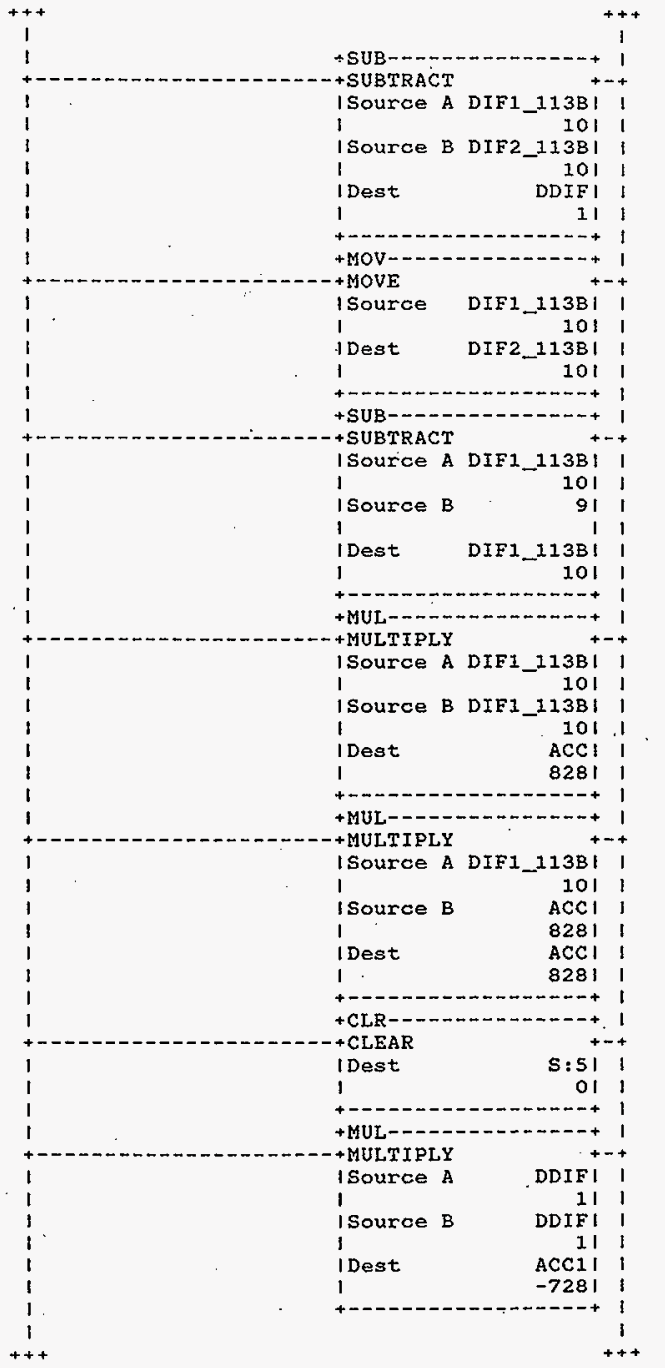

HNF-SD-FF-CSWD-61 Rev. 0 
Processor and Data (OPS Unit 1) Program Iisting
Processor Eile: SODIUMIA.ACH

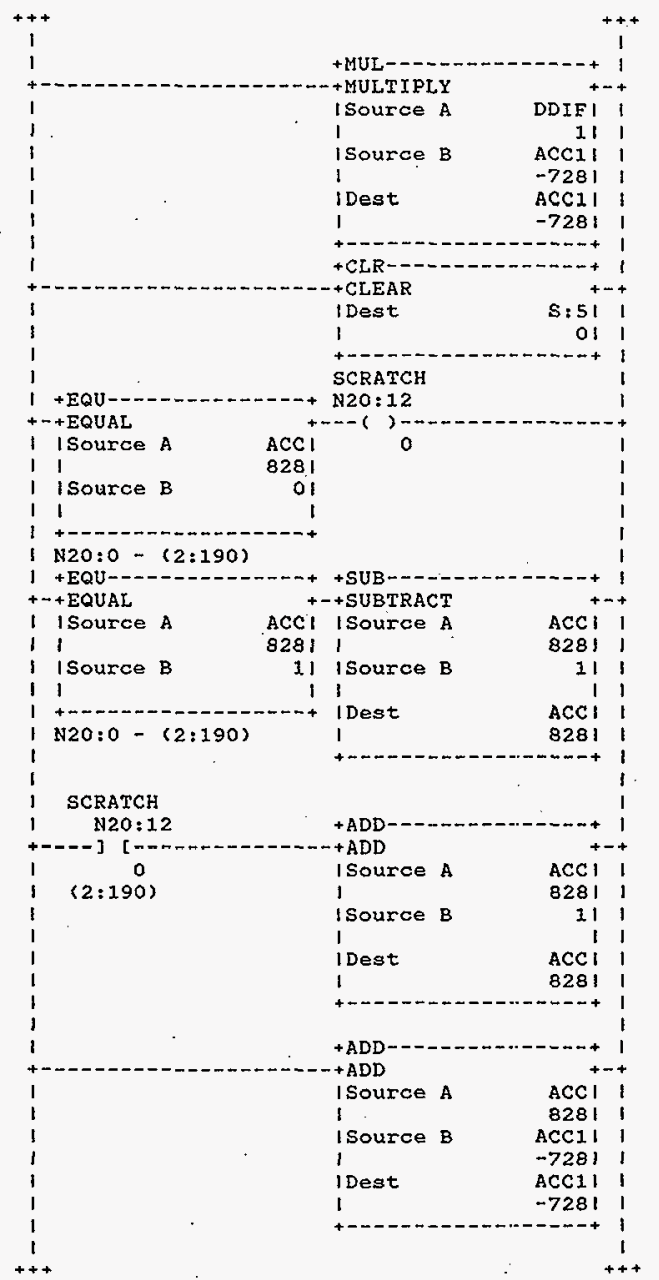

October 23, 1996 Page 165 Rung $2: 416$

1
1
1
1
1
1
1
1
1
1
1
1
1
1
1
1
1
1
1
1
1
1
1
1
1
1
1
1
1
1
1
1
1
1
1
1
1
1
1
1
1
1
1
1
1
1
1
1
1
1
1
1
1

HNF-SD-FF-CSWD-61 Rev. 0

Page 169 


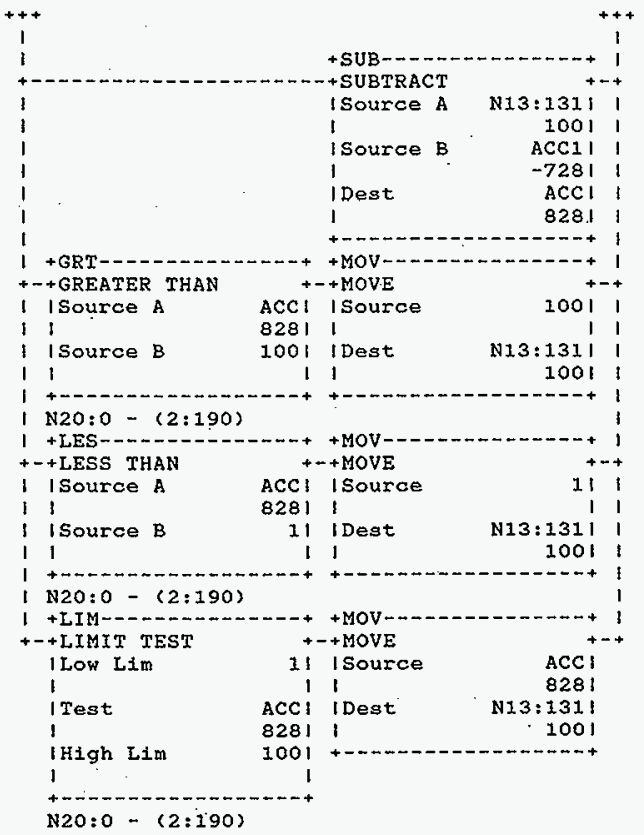

Rung 2:417

I PIDI13B

DUMMY 26

1

Rung $2: 418$

\section{DETECT FROM SCADA PID ON/OFF IF PID OFF \\ THEN ZERO PW FOR ZERO OUTPUT AT SCR AND JUMP AROUND THE PID BLOCK}

ON $113 \mathrm{~B}$
$\mathrm{~N} 7: 239$
$1.11,13$
1
1
1
1

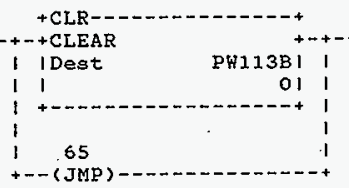

HNF-SD-FF-CSWD-61 Rev. 0 
Processor and Data(OPS Untt 1) Program Listing

Processor File: SODIUMIA.ACH

Rung 2:419
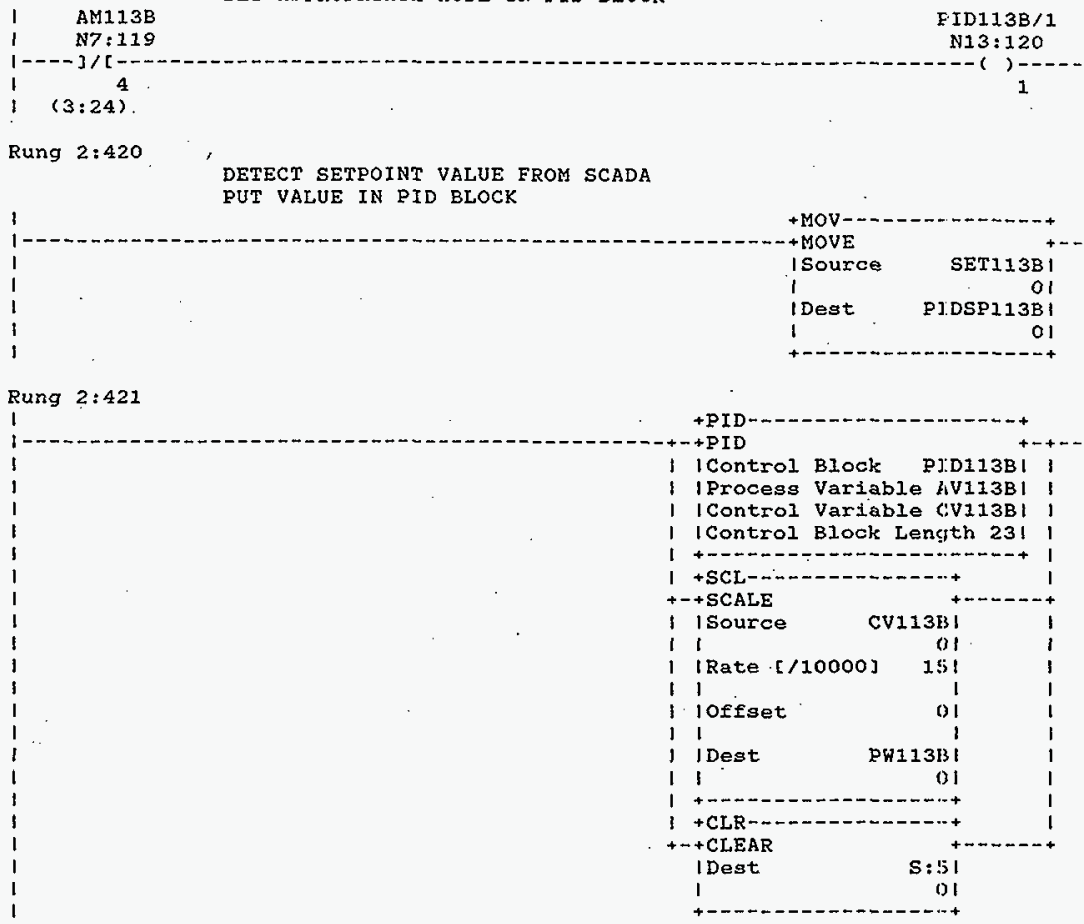

Rung 2:422

165

$1--[$ LBL $]-$

N20:10

DETECT AUTO/MANUAL FROM SCADA

SET APPROPRIATE MODE IN PID BLOCK

Rung 2:419

$1 \quad N 7: 119$

DETECT SETPOINT VALUE FROM SCADA

PUT VALUE IN PID BLOCK

Rung $2: 423$

1

I RT_TMR/DN

1 T4:62

PID113C

$1-\cdots-7 /[-$

28

DN

I (2:33)

FNF-SD-FF-CSWD-61 Rev. 0 
Processor and Data(OPS Unit 1)

Rung 2:424

I RT TMR/DN

T4 : 62

DNR_ 2

$1-\ldots] \quad[-$

N20:5

1 DN

(2:33)

Rung 2:425

\section{RATE CHECKING}

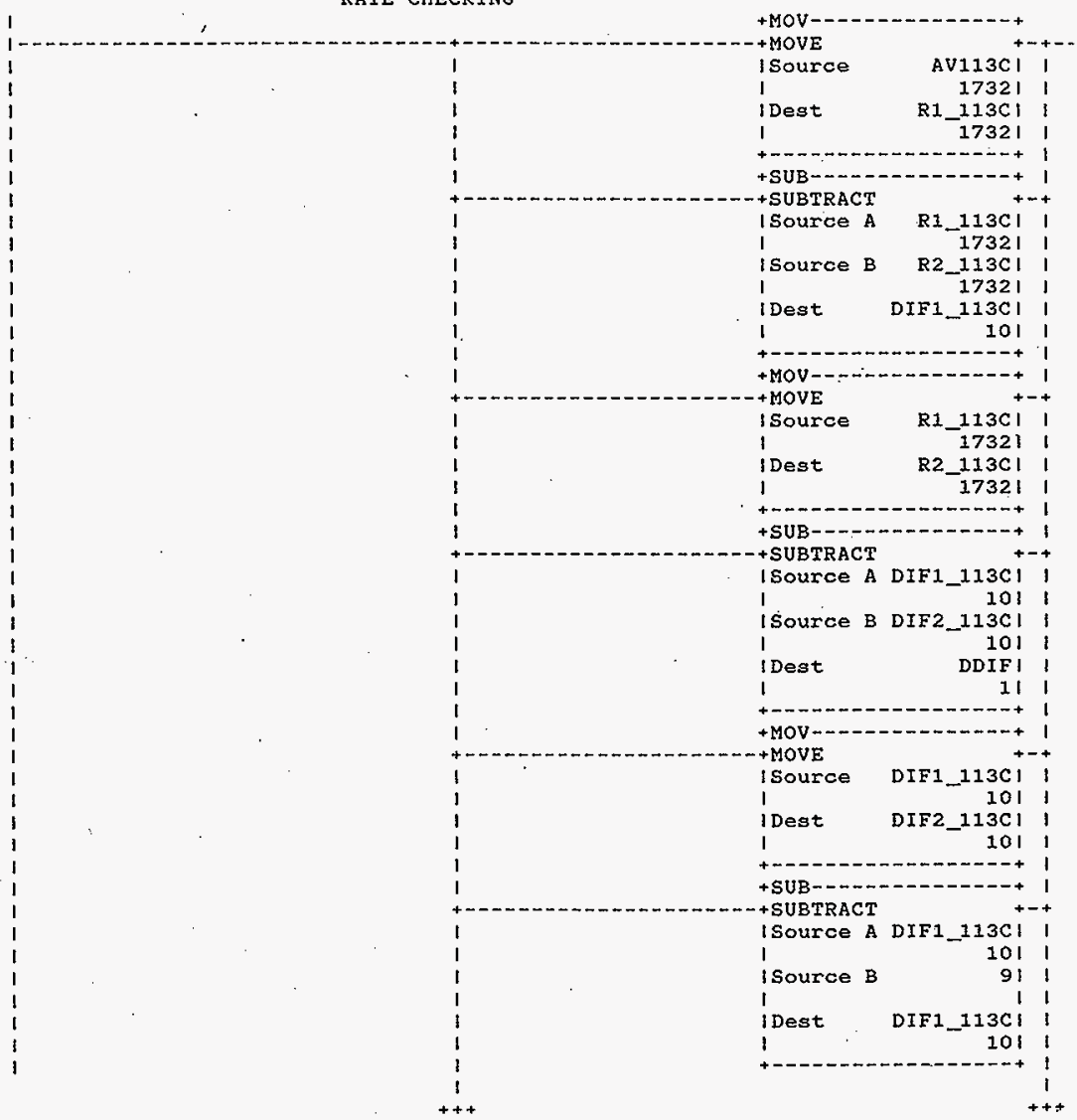

HNF-SD-FF-CSWD-61 Rev. 0

Page 172 


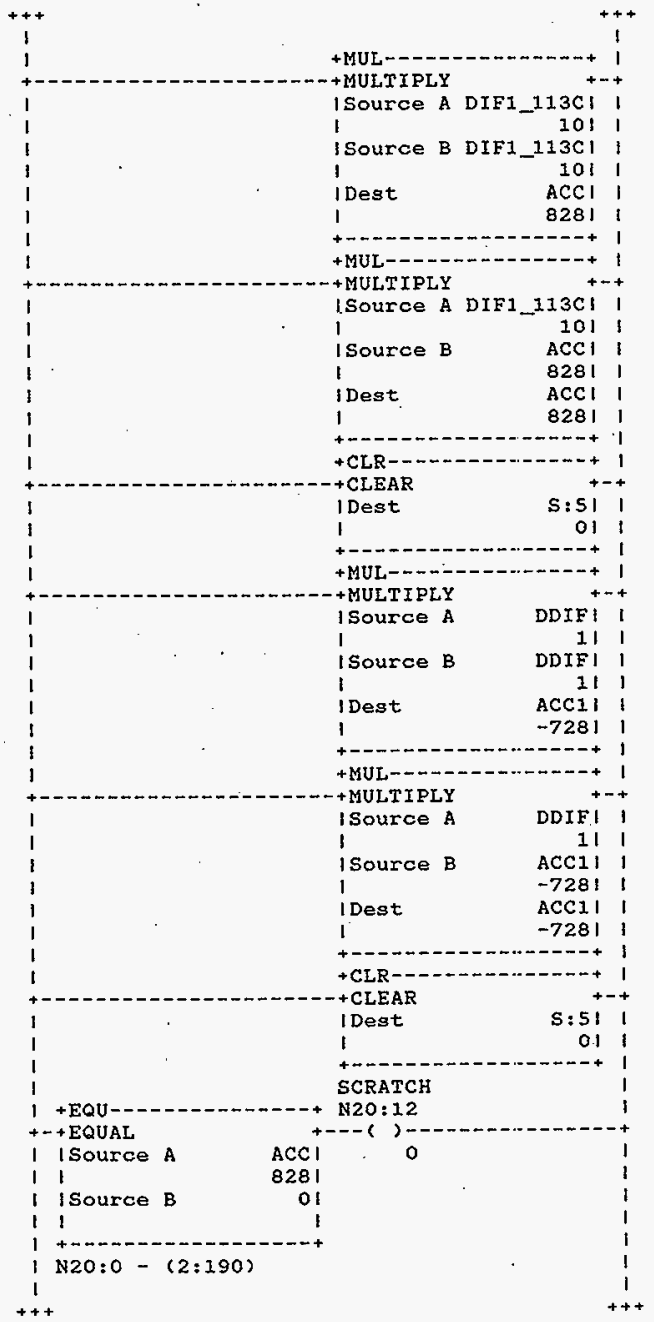

HNF-SD-FF-CSWD-61 Rev. 0 


$$
\begin{aligned}
& 1 \\
& 1 \\
& 1 \\
& 1 \\
& 1 \\
& 1 \\
& 1 \\
& 1 \\
& 1 \\
& 1 \\
& 1 \\
& 1 \\
& 1 \\
& 1 \\
& 1 \\
& 1 \\
& 1 \\
& 1 \\
& 1 \\
& 1 \\
& 1 \\
& 1 \\
& 1 \\
& 1 \\
& 1 \\
& 1 \\
& 1 \\
& 1 \\
& 1 \\
& 1 \\
& 1 \\
& 1 \\
& 1 \\
& 1 \\
& 1 \\
& 1 \\
& 1 \\
& 1 \\
& 1 \\
& 1 \\
& 1
\end{aligned}
$$

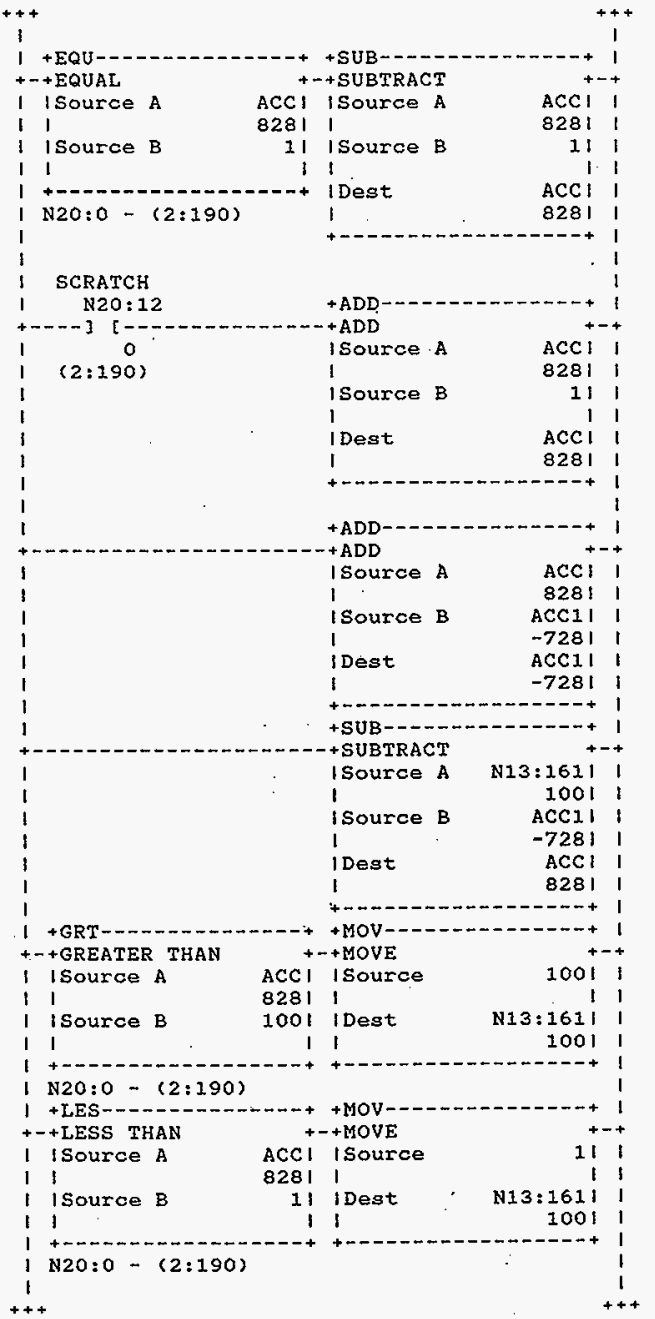

HNF-SD-FF-CSWD-61 Rev. 0

Page 174 

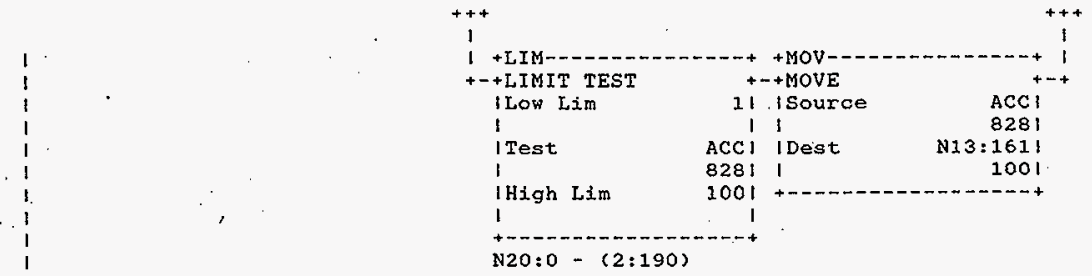

Rung 2:426

I PID113C

DUMMY 27

28

N20:8

$1---[$ [EL ]

N20:8

Rung $2: 427$

\section{DETECT FROM SCADA PID ON/OFF \\ IF PID OFF \\ THEN ZERO PH EOR ZERO OUTPUT AT SCR AND JUMP AROUND THE PID BLOCK}

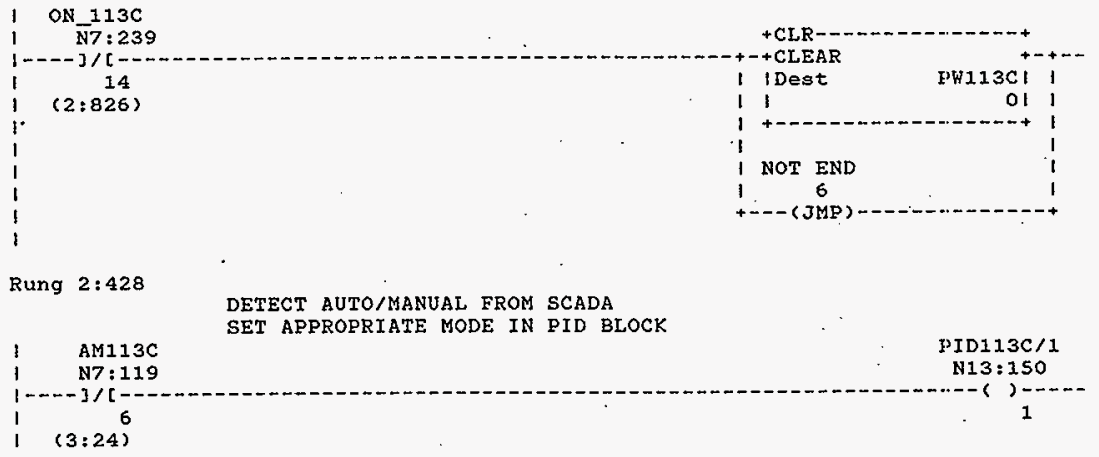

Rung 2:429

DETECT SETPOINT VALUE FROM SCADA PUT VALUE IN PID BLOCK

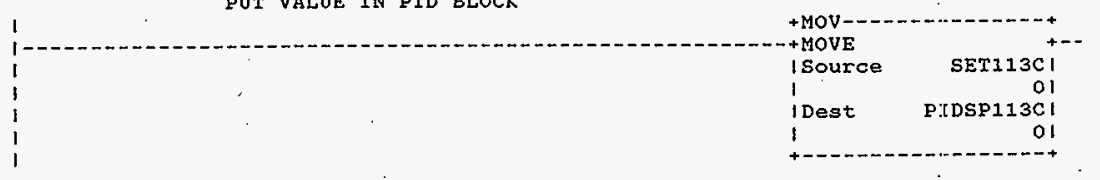

HNF-SD-FF-CSWD-61 Fiev. 0 

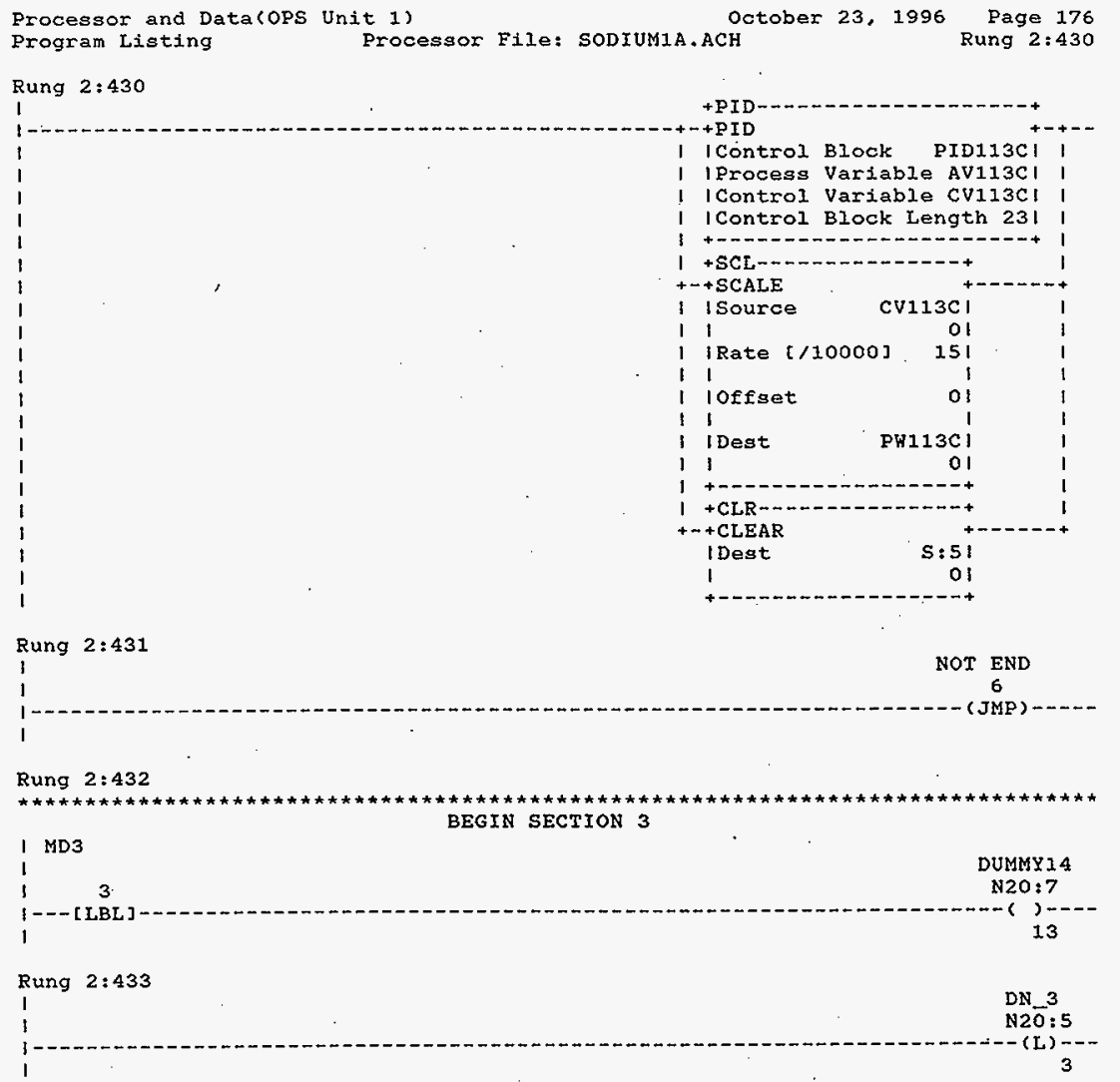

Rung 2:434

SECTION 3 SUBROUTINE 7

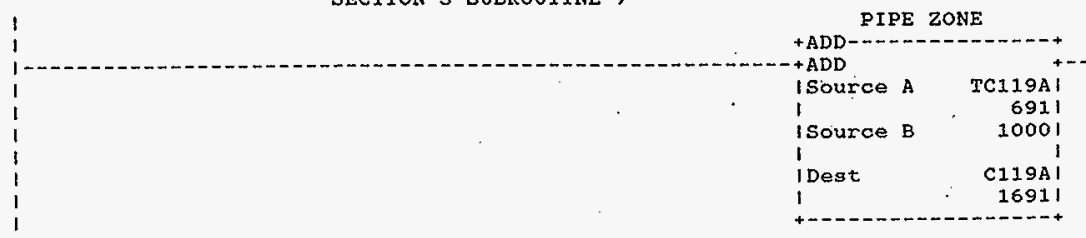

HNF-SD-FF-CSWD-61 Rev. 0 
Processor and Data(OPS Unit 1)

October 23, 1996 Page 177

Program Listing

Processor File: SODIUM1A.ACH

Rung $2: 435$

\section{Rung 2:435}

1 PIPE ZONE

PIPE ZONE

1 +GRT-

I-+GREATER THAN

1 ISource A C119AI

$11 \quad 16911$

I isource B 16383 |

$\begin{array}{ll}1 & 1\end{array}$

( N7:49-(2:434)

\section{Rung 2:436}

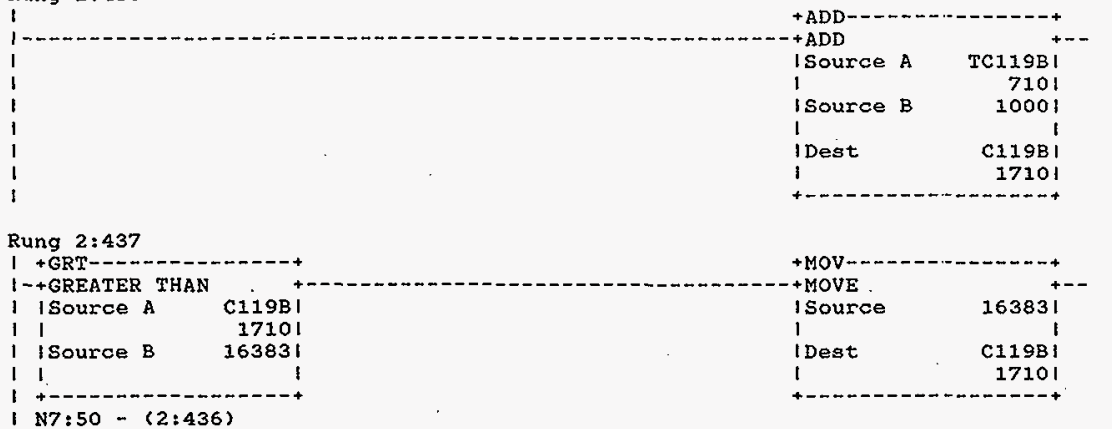

Rung 2:438

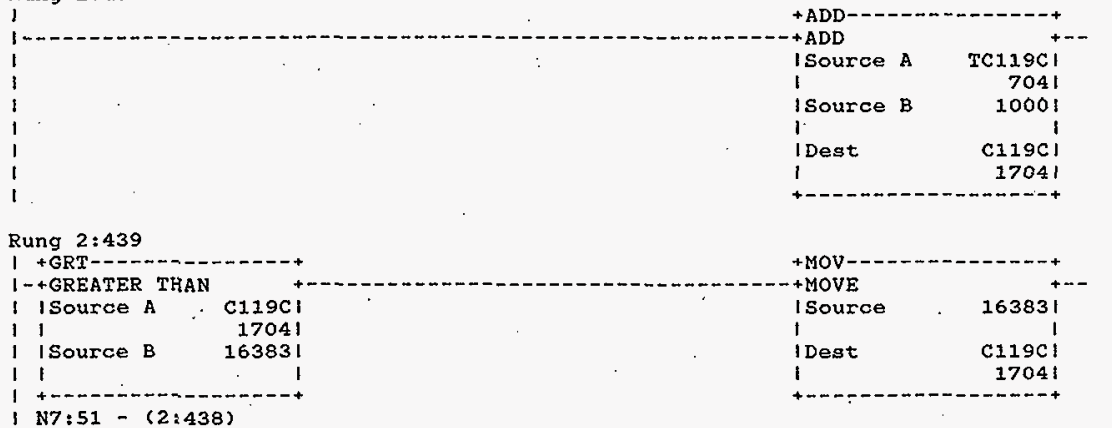

HNF-SD-FF-CSWD-61 Rev. 0 
Processor and Data(OPS Unit 1)

Program Listing

Processor File: SODIUM1A, ACH

October 23, 1996 Page 178

\section{Rung $2: 440$}

1

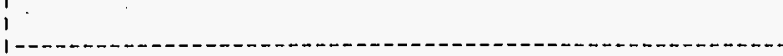

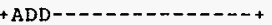

ISource A TCl20A|

1

I Source B 1000

I

I Dest C120A:

I

1709 !

Rung $2: 441$

1

1 + GRT-...-

I-+GREATER THAN

$\begin{array}{ll}\text { I ISource A } & \text { C120A1 } \\ 1 & 17091\end{array}$

1 15ource B 16383 I

11 i 1

I $7: 52-(2: 440)$

Rung 2:442

I

1

$+\mathrm{ADD}$

+MOV--.- - - - - - - - - +

+MOVE

16383

I Dest

C120A

1

1709 :

$+\mathrm{ADD}$

ISource A TC120B

I 722

I Source B 1000 |

1

Dest C120B

1

17221

Rung 2:443

1 + GRT--_...----.-..-

1-+GREATER THAN

I ISource A C12OB

1 i 17221

I Isource B 16383 |

11

+
+
1
1
+

+MOV-D-- - - - - - - - -

+ MOVE

163831

I Dest

с120B

I

17221

$17: 53-(2: 442)$

Rung $2: 444$

1

$+\mathrm{ADD}$

$A D D$

ISource A TC120CI

7301

I source B

$1000 !$

i Dest

c120C!

I

$1730 !$

HNF-SD-FF-CSWD-61 Rev. 0

Page 178 
Processor and Data(OPS Unit 1 )

Rung 2:445
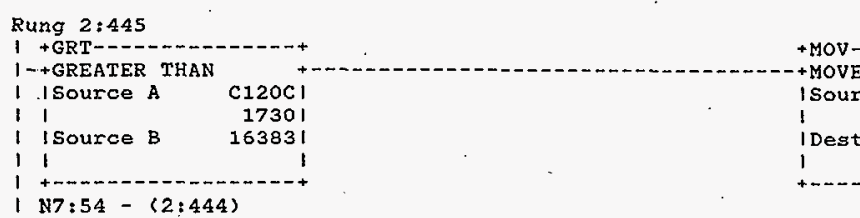

0
1
1

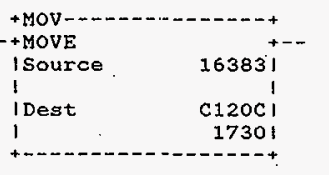

Rung $2: 447$

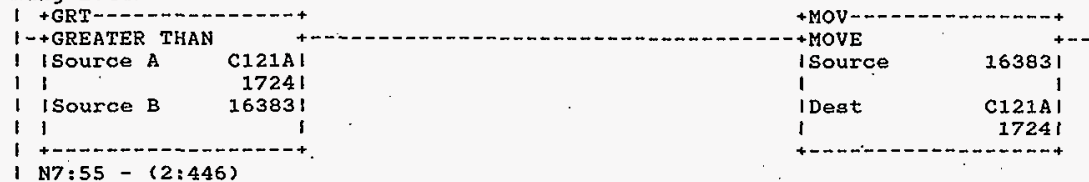

Rung $2: 448$

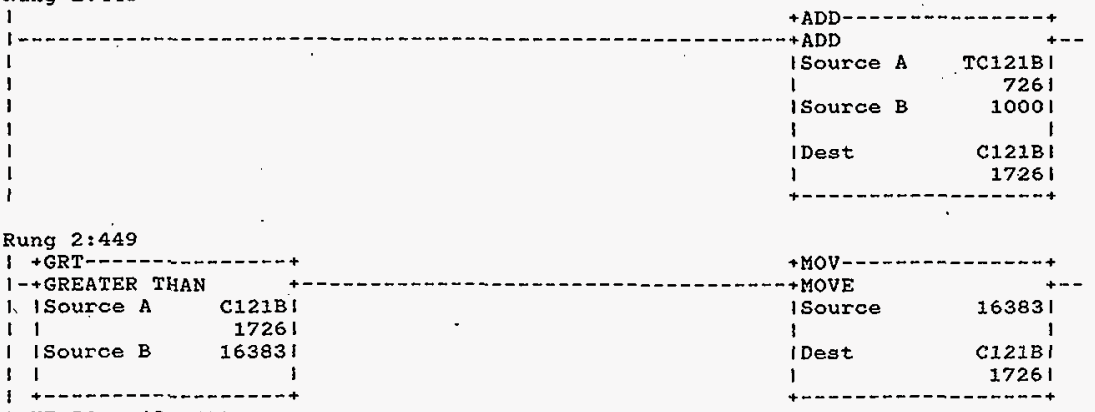

I N7:56-(2:448) 


$$
\text { Rung 2:450 }
$$

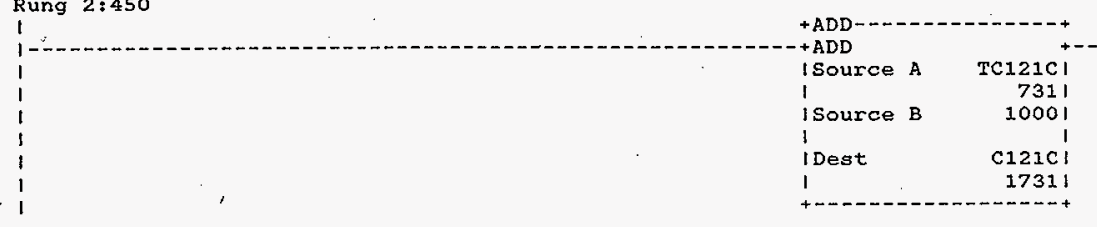

Rung $2: 451$

\begin{tabular}{|c|c|c|c|}
\hline \multicolumn{2}{|c|}{ I + GRT---1--- } & \multicolumn{2}{|c|}{+ MOV $---------5+\cdots+-+$} \\
\hline I-+GREATER & & - MOVE & $+\cdots$ \\
\hline 1 isource A & $\mathrm{C} 121 \mathrm{Cl}$ & I Source & 163831 \\
\hline 1 & 17311 & 1 & 1 \\
\hline isource B & 163831 & I Dest & $\mathrm{C} 121 \mathrm{CI}$ \\
\hline 1 & 1 & I & 17311 \\
\hline$+2+\cdots----$ & -----+ & $+\cdots---$ & $-\infty-\infty+\infty$ \\
\hline
\end{tabular}

Rung 2:452

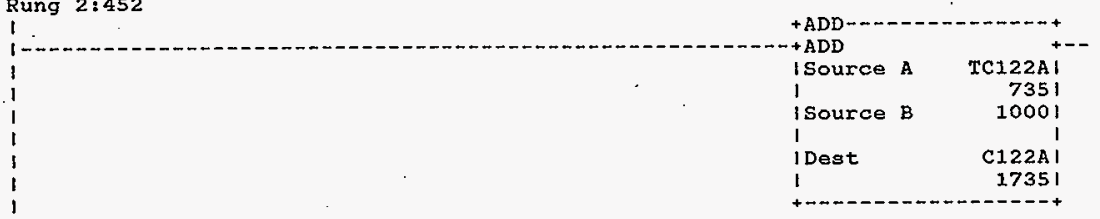

Rung $2: 453$

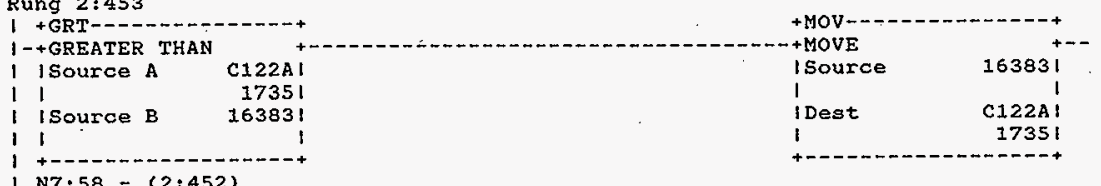

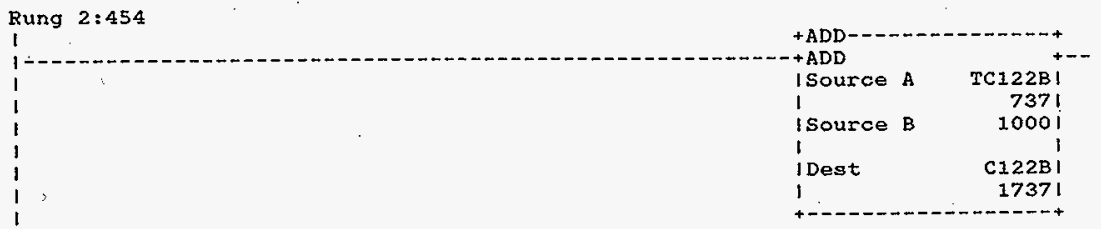

HNF-SD-FF-CSWD-61 Rev. 0 
Processor and Datalops Unit 1) Program Listing

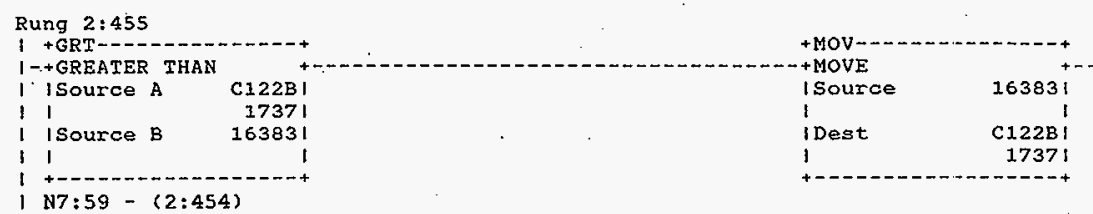

Rung $2: 456$

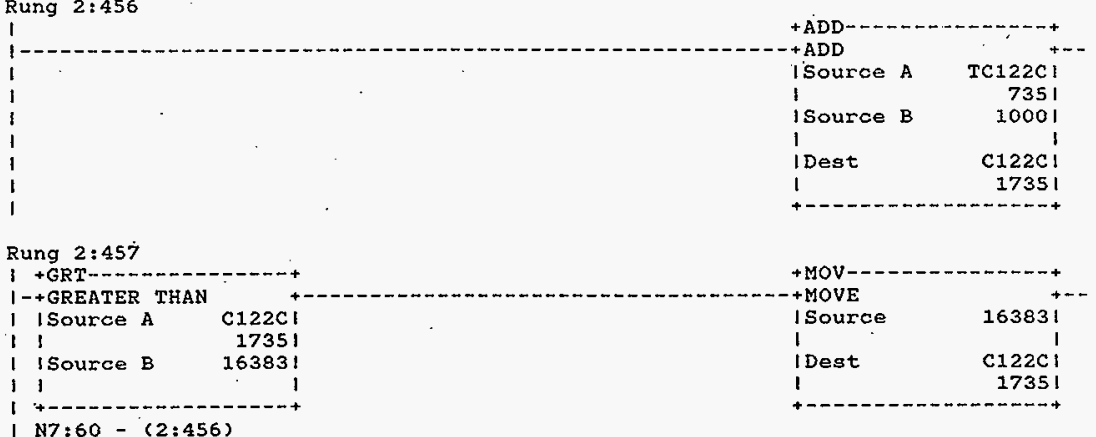

I N7:60-(2:456)

Rung $2: 458$

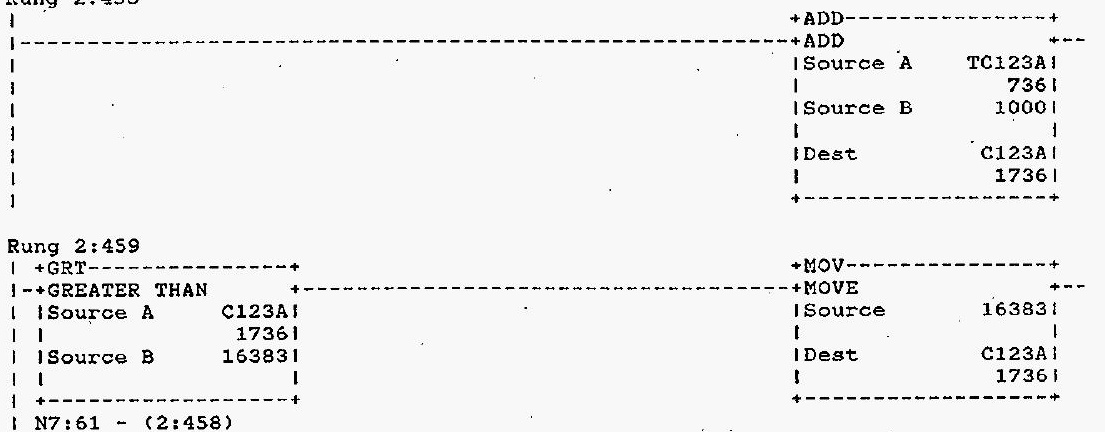




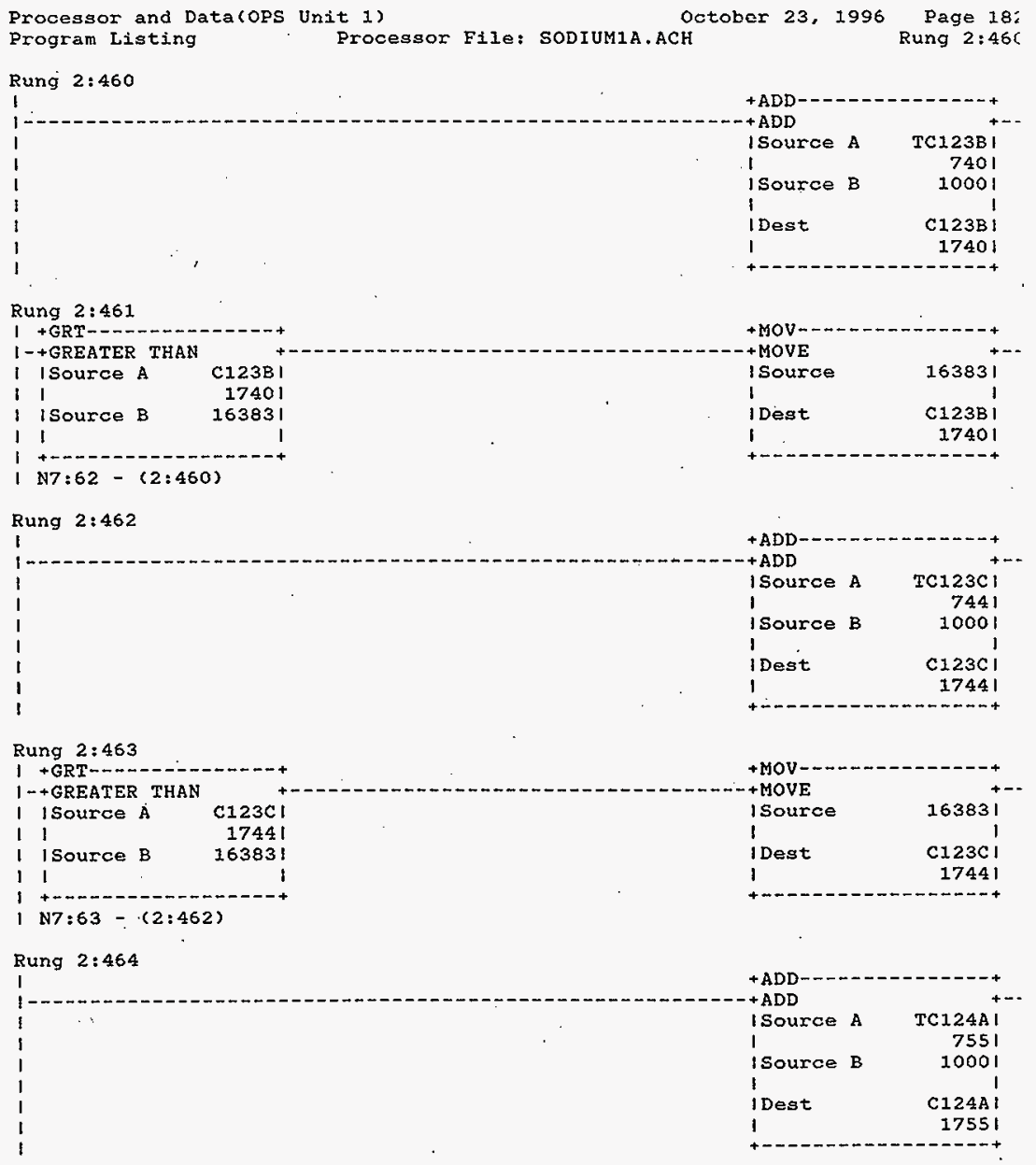

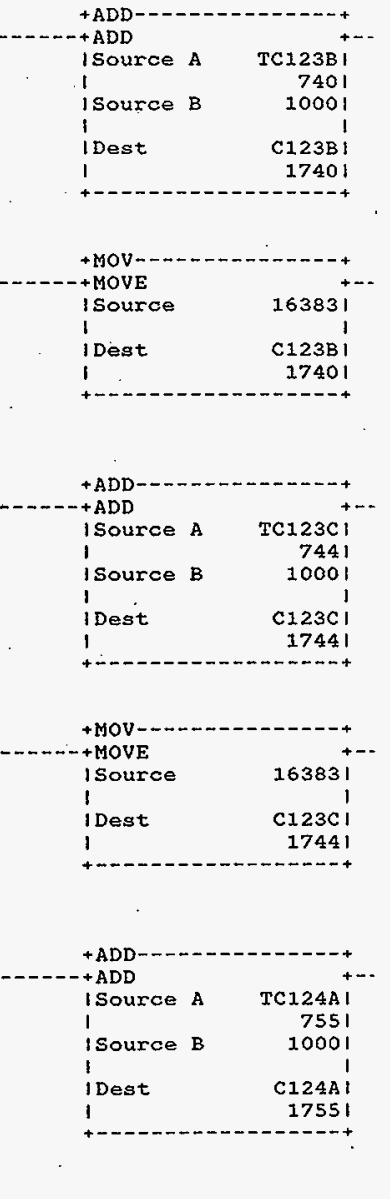

Rung $2: 461$

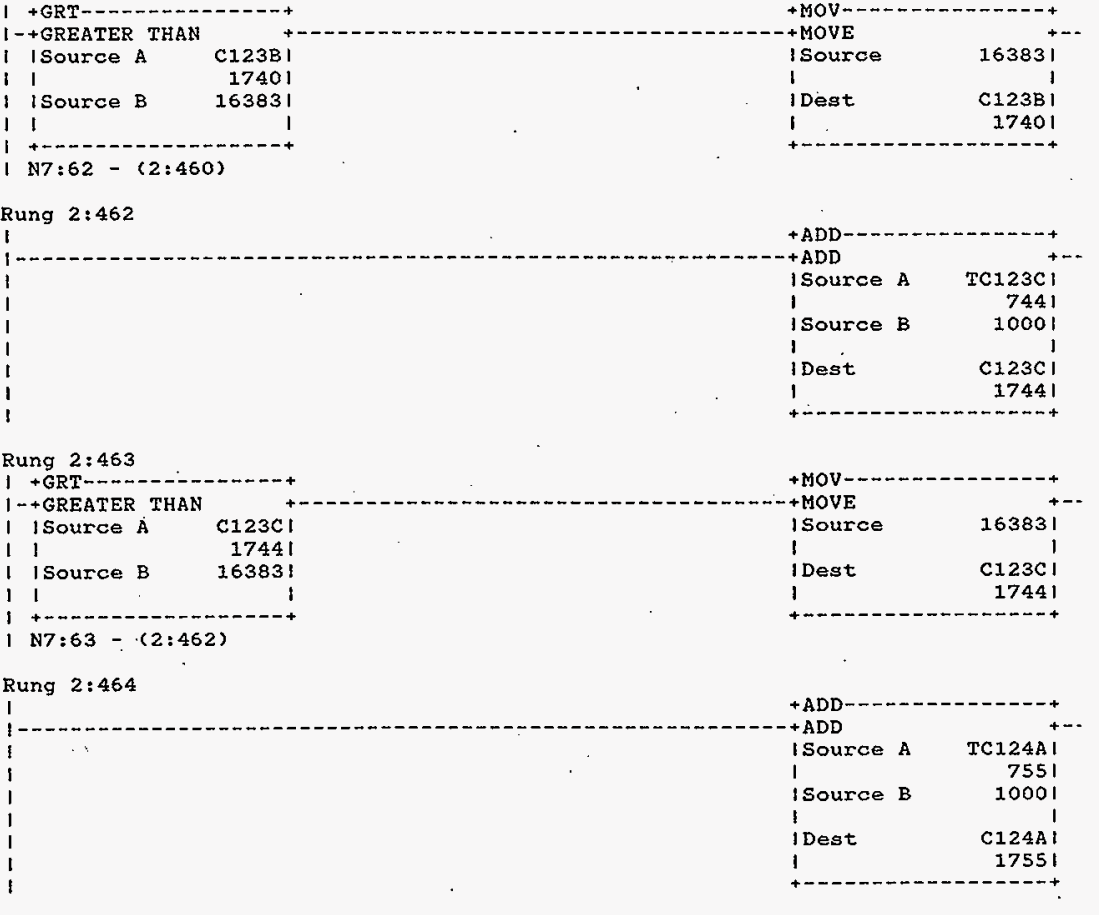

HNF-SD-FF-CSWD-61 Rev. 0 
Processor and Data(OPS Unit 1)

Program Listing

Processor File: SODIUM1A.ACH

October 23, 1996 Page 183

Rung $2: 465$

$1+$ GRT---..---....---

$1-+$ GREATER THAN

I ISource A CI24A|

I 17551

I ISource B 16383 |

1

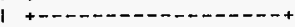

i $N 7: 64-(2: 464)$

Rung $2: 466$

i 1 ing 2.0466

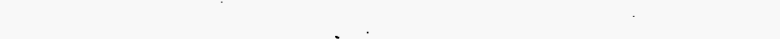

I

i.

1

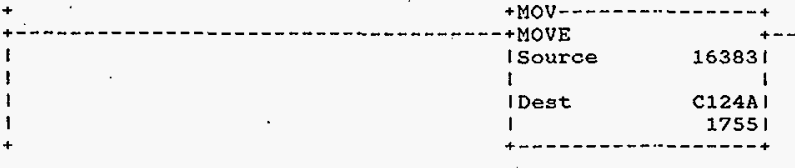

Rung $2: 467$

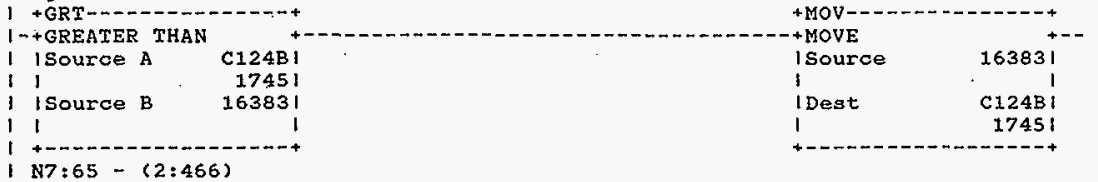

( N7:65-(2:466)

Rung 2:468

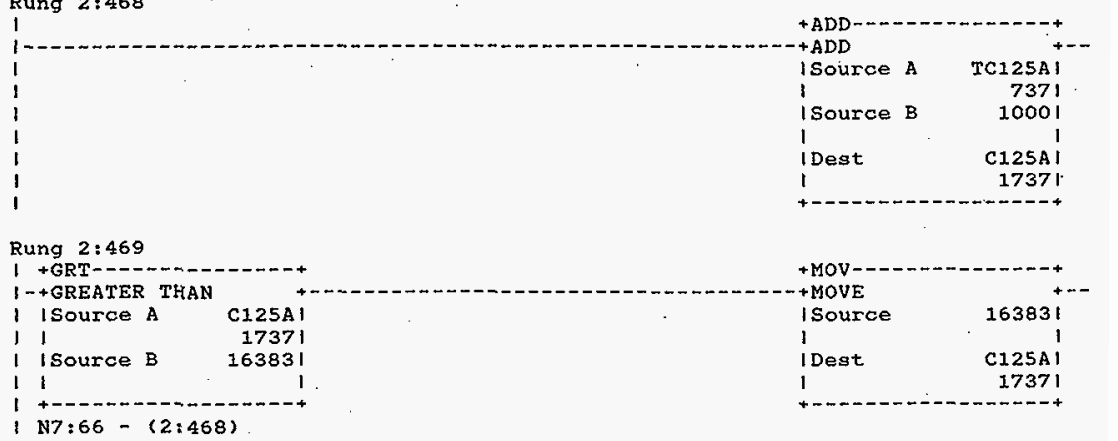

HNF-SD-FF-CSWD-61 Rev. 0 


\section{Rung 2:470}

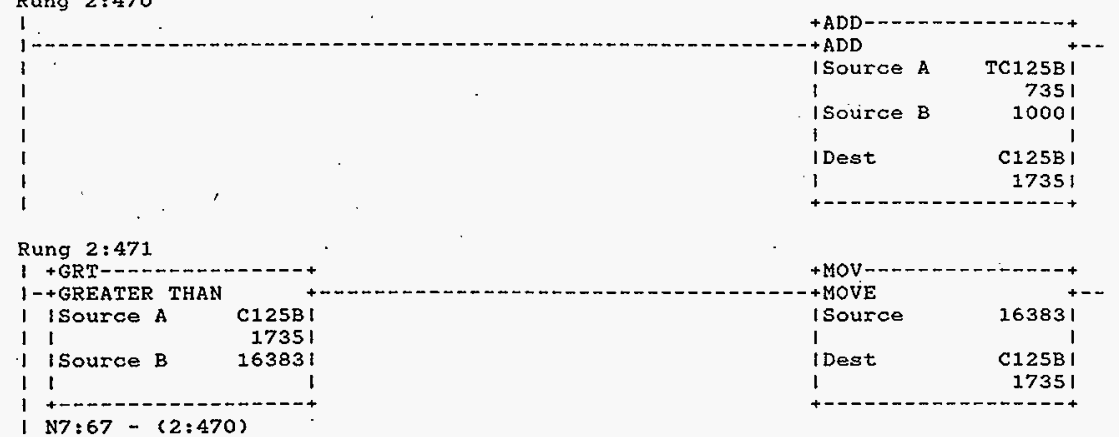

Rung $2: 472$

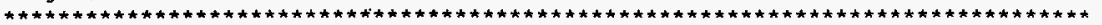
For each T/C read its STATUS BITS to detect. T/C problems HARDWARE ALARMS FOR TANK T-3002

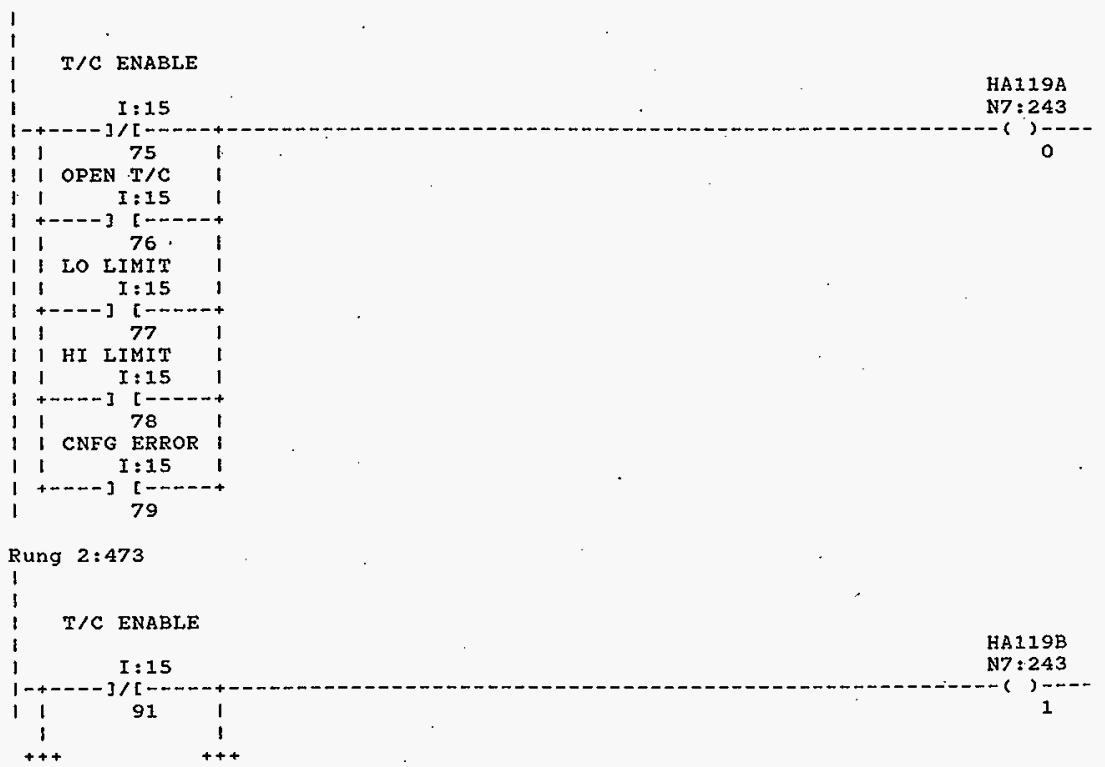




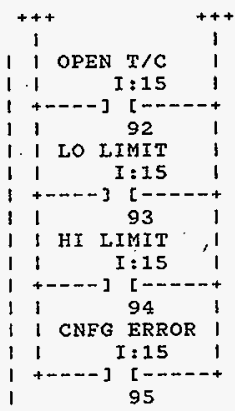

Rung 2:474

T/C ENABLE

$I: 15$

$1: 1,107$

1 OPEN T/C

I) I:15 ।

$1+\cdots--][\ldots . .-+$

l 1108

1 LO L.TMIT

11

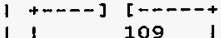

11109

I I. HI LIMIT

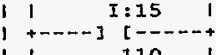

11

1 CNFG ERROR

11

I I:15 |

$1+\ldots]$ [-..-..+

111

Rung $2: 475$

T/C ENABLE

$I: 15$

$1 / 10.712$

1 I OPEN T/C

I I I:15

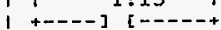

$11 \quad 124 \quad .1$ 1 $++$ 
Processor and Data(OPS Unit 1) Program Listing

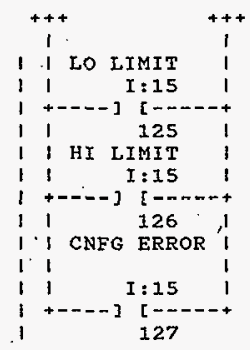

\section{Rung 2:476 \\ 1 \\ 1 \\ 1 T/C ENABLE}

I . HA12OB

$1 \quad I: 16 \quad$ N7:243

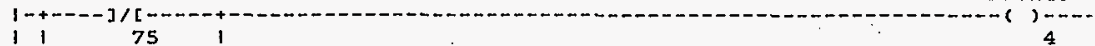

1 OPEN T/C I

1 I I: $16 \quad 1$

$1+--\cdots]$ i $1----+$

1176

1 LO LIMIT

1 I:16

$1+\cdots--][-.--+$

$11 \quad 77$. 1

| 1 HI LIMIT

1 I I:16

$1+----][-----+$

1178 ,

I I CNFG ERROR I

11

11

$1+----1$ I: $1-----$ +

179

Rung 2:477

$$
\text { i }
$$

1

$T / C$ ENABLE

$I: 16$

1191

1 I OPEN T/C

1 I $I: 16$

$1+-\infty-\infty][---n+$

i 92

$++$

921

HNF-SD-FF-CSWD-61 Rev. 0

Page $/ 86$ 


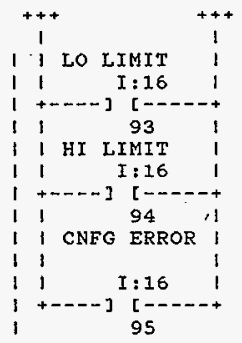

Rung $2: 478$

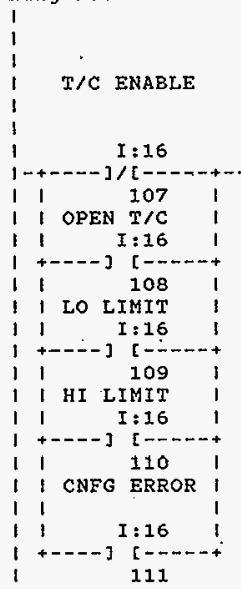

Rung $2: 479$

1

i

1.

T/C ENABIE

$I: 16$

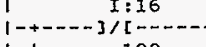

11123

OPEN $\mathrm{T} / \mathrm{C}$

1 I:16

) +---1 ] $[\ldots \ldots--+$

$11 \quad 124$

$++$ 


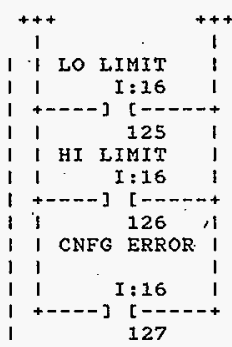

Rung 2:480

1

T/C ENABLE

$I: 17$

HA121C
N7: 243
$-\left(\begin{array}{c}) \\ 8\end{array}\right.$

HA121C
N7: 243
$-\left(\begin{array}{c}) \\ 8\end{array}\right.$

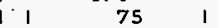

OPEN $T / C$

$I: 17$

+----] $[-\ldots-\cdots+$

1176

1 น० LIMIT

1 I $\quad 17$

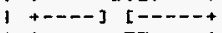

$11.77 \quad 1$

I HI LIMIT

I 1 I: 17

1 +---n] [--....

I I CNFG ERROR

11

11

I : 17

+--- ] [ [--.-.

79

Rung 2:48

I

T/C ENABLE

\section{T/C ENABLE}

$1-+---] 1 /[\ldots \ldots$

$191 \quad 1$

1 I OPEN T/C

$1+\cdots-n$ [--.--

I 1192

1

$++$ 


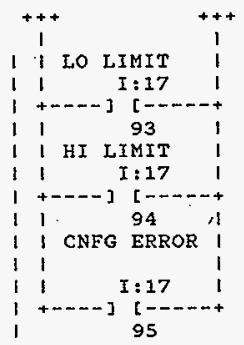

Rung $2: 482$

1

$T / C$ ENABLE

1 LO LIMIT

I $I: 17$

+- -...] $[-\ldots \ldots+$

$1 \quad 1091$

I HI LIMIT

$1 \quad 1: 17$

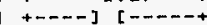

11110

I CNFG ERROR 1

11

$\begin{array}{lll}1 & 1 & 17\end{array}$

I 111

Rung 2:483

1 .

T/C ENABLE

$I: 17$

HA122C

N7: 243

$\begin{array}{ll}1 & I: 17 \\ 1-+-.] & 1 /[-1\end{array}$

1 OPEN $T / C$

I:17

11

] +---- 3 [-----+

11

1

$++$

124

HNF-SD-FF-CSWD-61 Rev. 0 
Processor and Data(OPS Unit 1 )

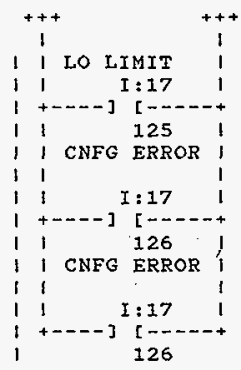

\section{Rung 2:484}

$$
\text { I }
$$

T/C ENABLE

$I: 18$

HA123A

N7: 243

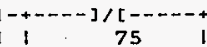

I OPEN $T / C$

I $\quad I: 18$

$+\ldots-1$ is

176

1 LO LIMIT

1 I:18

+----] [ --....

1

HI LIMIT

1 I: 18

+.-- ] $3:-2+$

178

CNFG ERROR

1

11

$1+\cdots--7[18 \ldots+$

I 79

Rung 2:485

1

i

T/C ENABLE

$1-+--3 / 2:-$

HA1 23B

N7 : 243

91

1 OPEN T/C

$1 \quad \mathrm{I}: 18$

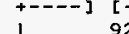

11

$++$

92

ton...

$+++++$

HNF-SD-FF-CSWD-61 Rev. 0 
Processor and Data(OPS Unit 1)

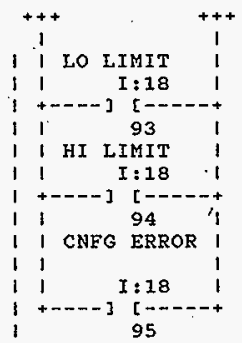

Rung $2: 486$

1

1 T/C ENABLE

I. HA123C

1 $1: 18 \quad$ N7:243

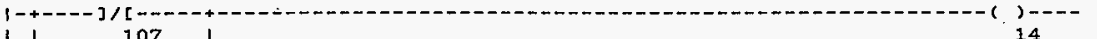

107 ।

OPEN $\mathrm{T} / \mathrm{C}$

I I:18 I

+----] [---:- +

I 108

1 LO LIMIT 1

1 I:18

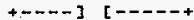

1091

I HI LIMIT

I I: $18 \quad 1$

$+\cdots+-][---\cdots+$

I 1101

I CNFG ERROR

11

$1 \mathrm{I} I: 18$ ।

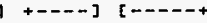

I 111

Rung 2:487

I

I

T/C ENABLE

$I: 18$

HA124A

1

I I I I

N7 : 243

I. OPEN $T / C$

I I I:18 ।

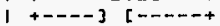

I 124

1

$++$

124

$++$

HNF-SD-FF-CSWD-61 Fiev. 0 
Processor and Data(OPS Unit 1) Program Listing

October 23, 1996

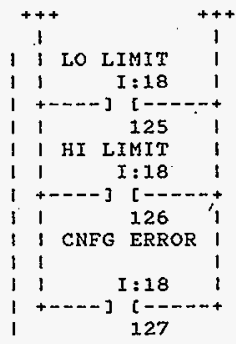

Rung 2:488

1

T/C ENABLE

$10+1: 19$

HA $124 B$

N7 : 244

$1175 \quad 1$

OPEN $\mathrm{T} / \mathrm{C}$

$I: 19$

+---- ] [ $[-\cdots+$

1176

I I LO LIMIT

1 I I:I9

$1+\ldots-\infty][----+$

1177

I) HI LIMIT

11 I: $19 \quad 1$

1 +----] [-----

$\begin{array}{llll}1 & 1 & 78 & \text { I } \\ 1 & \text { CNFG ERROR }\end{array}$

I I

11

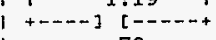

79

\section{Rung 2:489}

I

T/C ENABLE

$I: 19$

HA125A

$\begin{array}{ccc}1 & 1 & 2 \\ 1 & 91\end{array}$

N7 $: 244$

I I OPEN T/C

1 I $I: 19$

$1+\cdots-.+3 \mathrm{t}---\cdots+$

I 192

1

$++$

921

HNF-SD-FF-CSWD-61 Rev. 0 


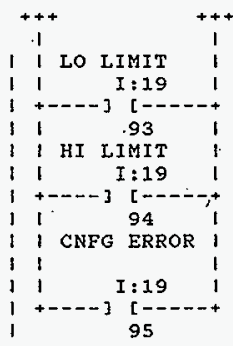

\section{Rung 2:490}

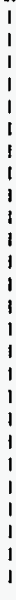

$T / C$ ENABLE

I $: 19$

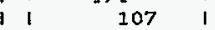

1 OPEN T/C

1 I:19

$1+\ldots--n][\ldots+\cdots$

$11108 \quad 1$

| $\mid$ LO LIMIT

1 I $I: 19$

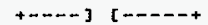

I 109

1 HI LIMIT

I. I:I9

$1+$ +

11 1 1201

I I CNFG ERROR

I 1

11

+---1 ] $[-\cdots---+$

I 111

Rung 2:491

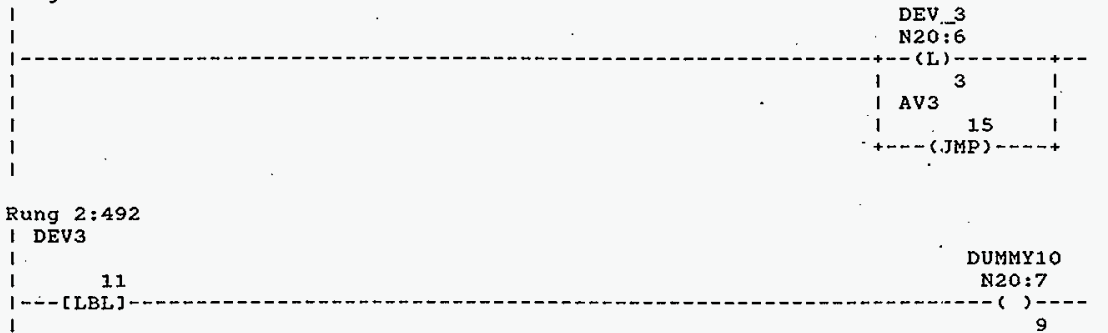

HNF-SD-FF-CSWD-61 Rev. 0 
Rung 2:493

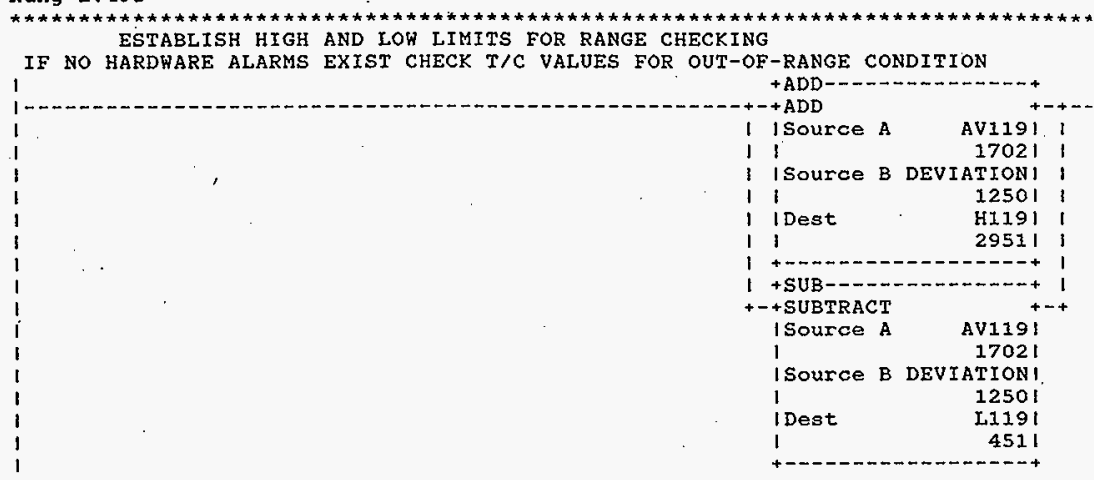

Rung 2:494

PIPE ZONE

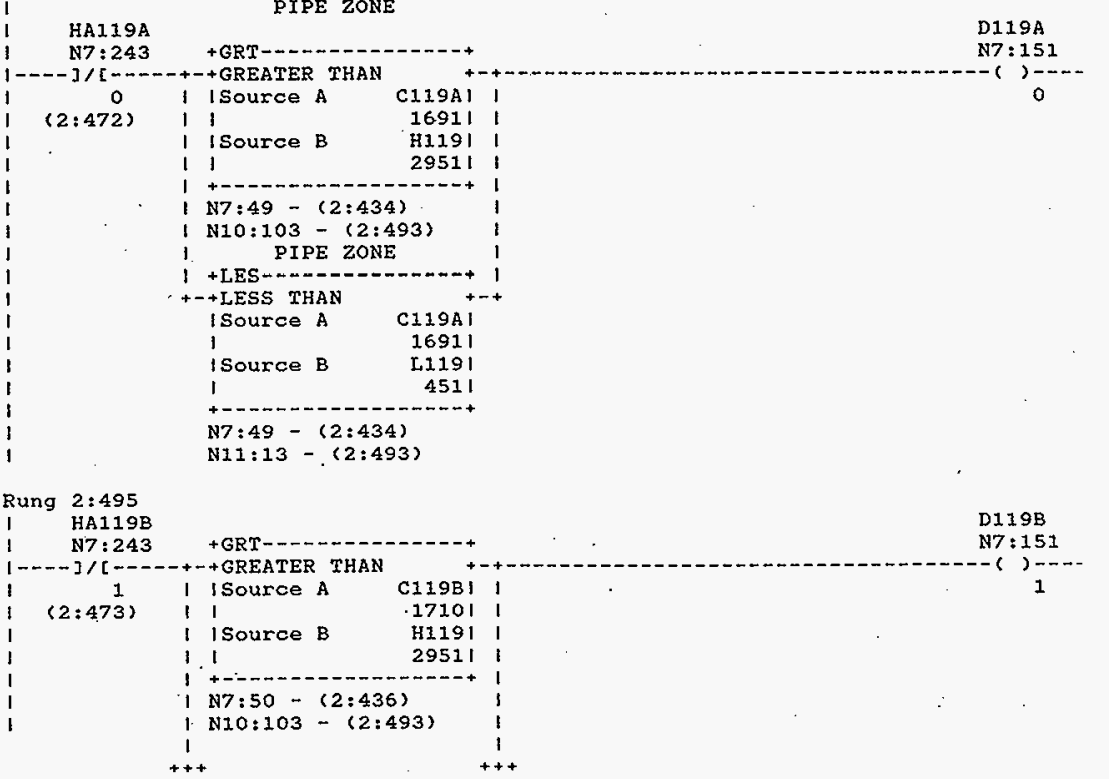



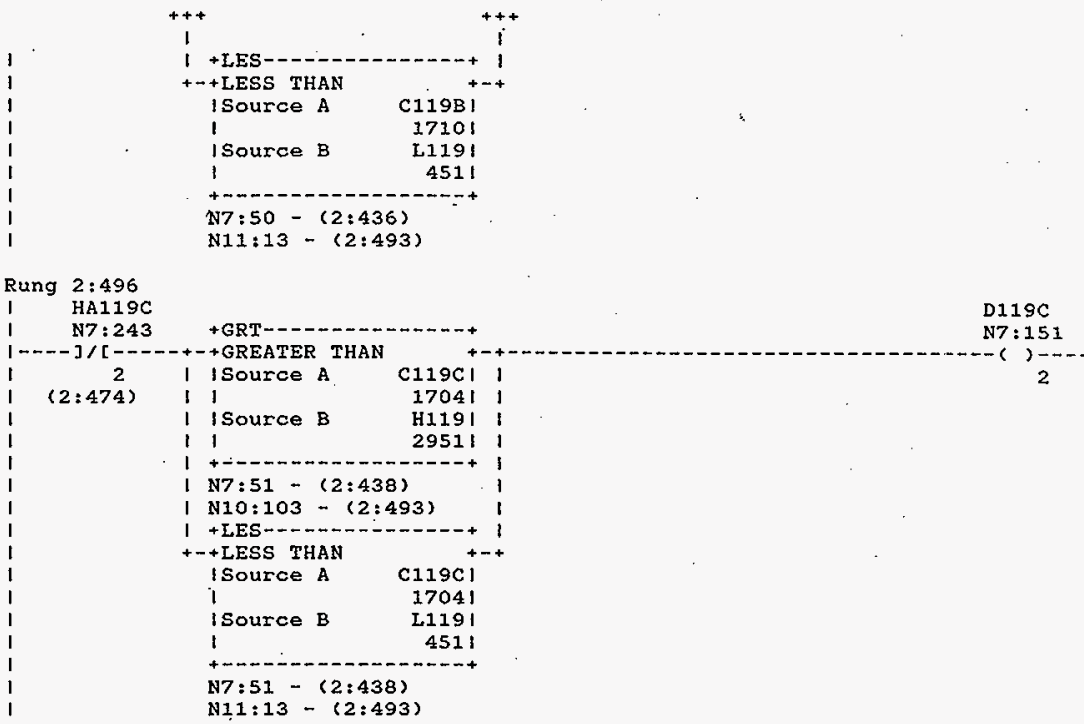

Rung $2: 497$

ESTABLISH HIGH AND LOW LIMITS FOR RANGE CHECKING

IF NO HARDWARE ALARMS EXIST CHECK T/C VALUES FOR OUT-OF-RANGE CONDITILN

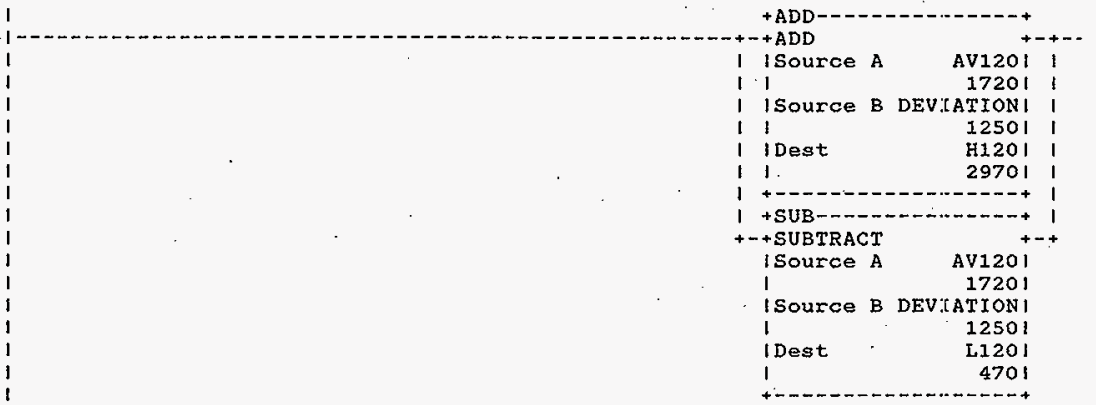

HNF-SD-FF-CSWD-61 Rev. 0 


\section{Rung 2:498}
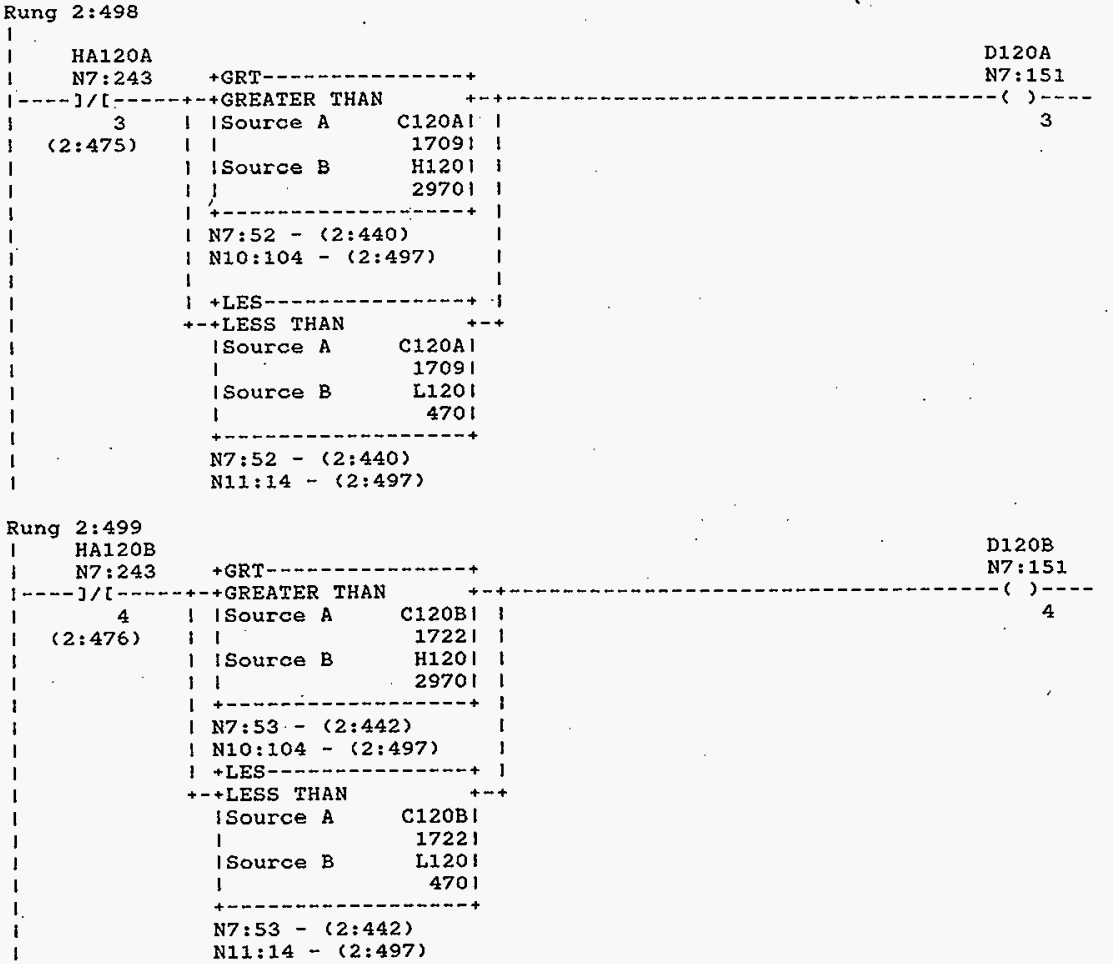

Rung 2:500

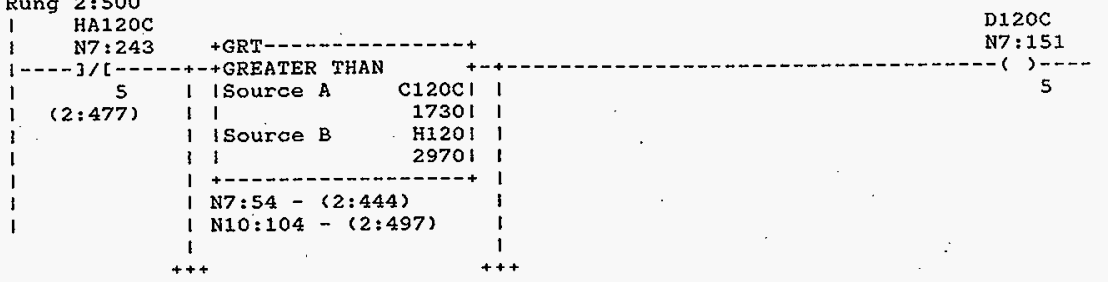

HNF-SD-FF-CSWD-61 Rev. 0 
Processor and Data (ops Unit 1)

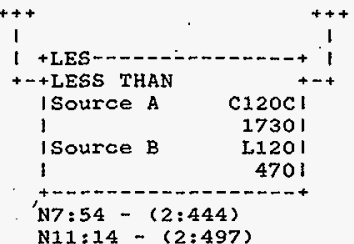

Rung 2:501

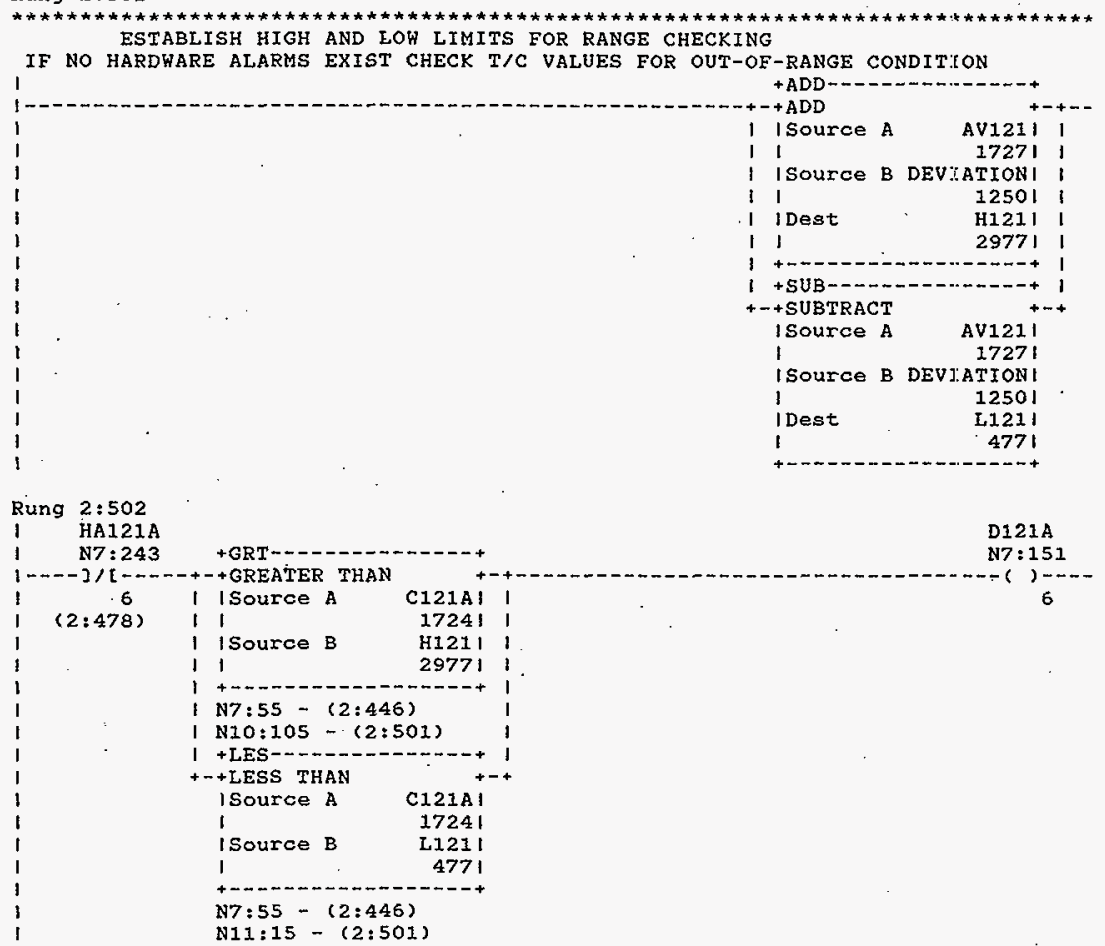

HNF-SD-FF-CSWD-61 Rev. 0 
Procesgor and Data(OPS Unit 1)

\section{Rung 2:503}
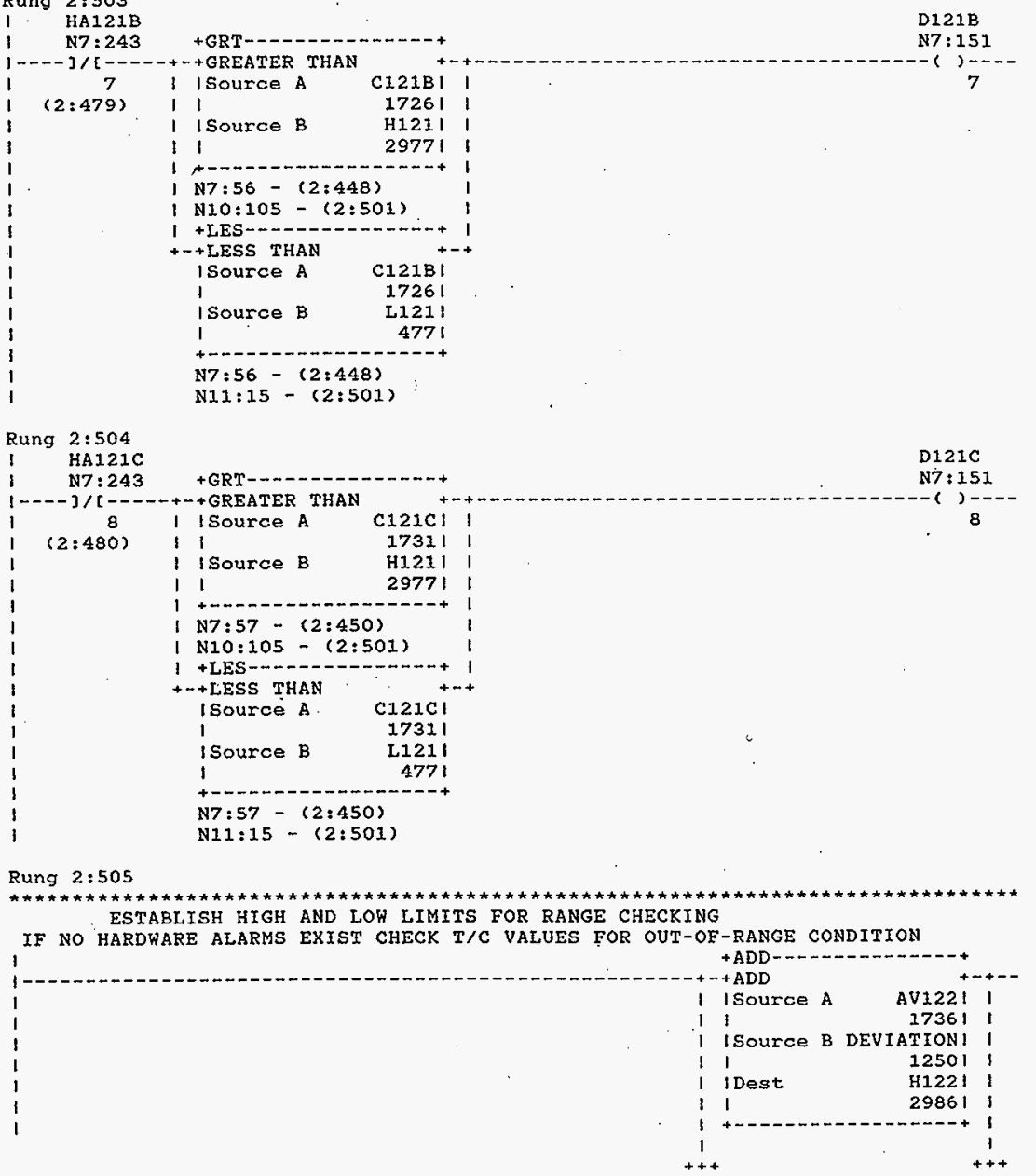

HNF-SD-FF-CSWD-61 Rev. 0 
Rung 2:506

I. HA122A $\mathrm{N7}: 243$ .

Rung 2:507

I HA122B 


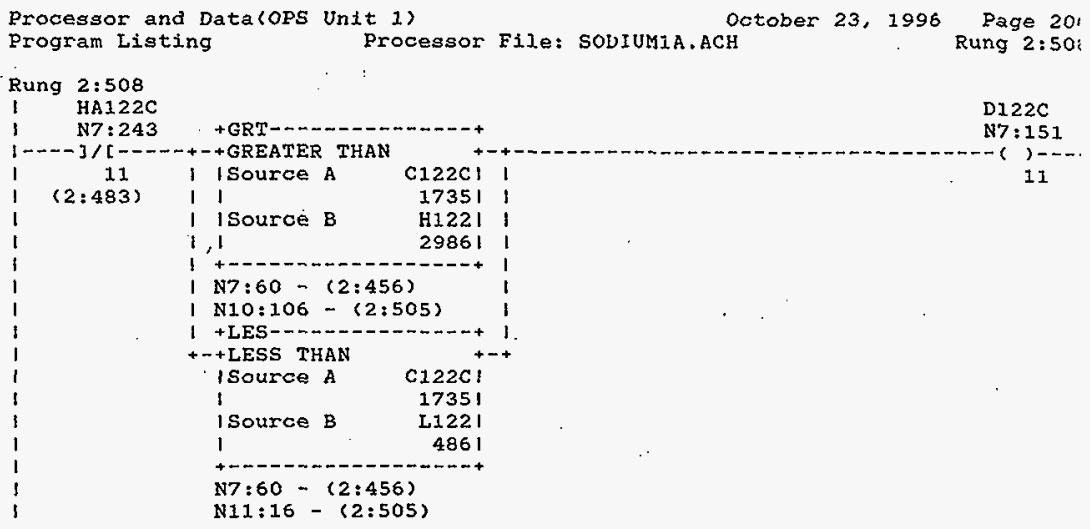

Rung 2:509

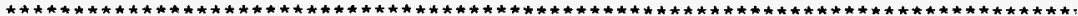
ESTABLISH HIGH AND LOW LIMITS FOR RANGE CHECKING

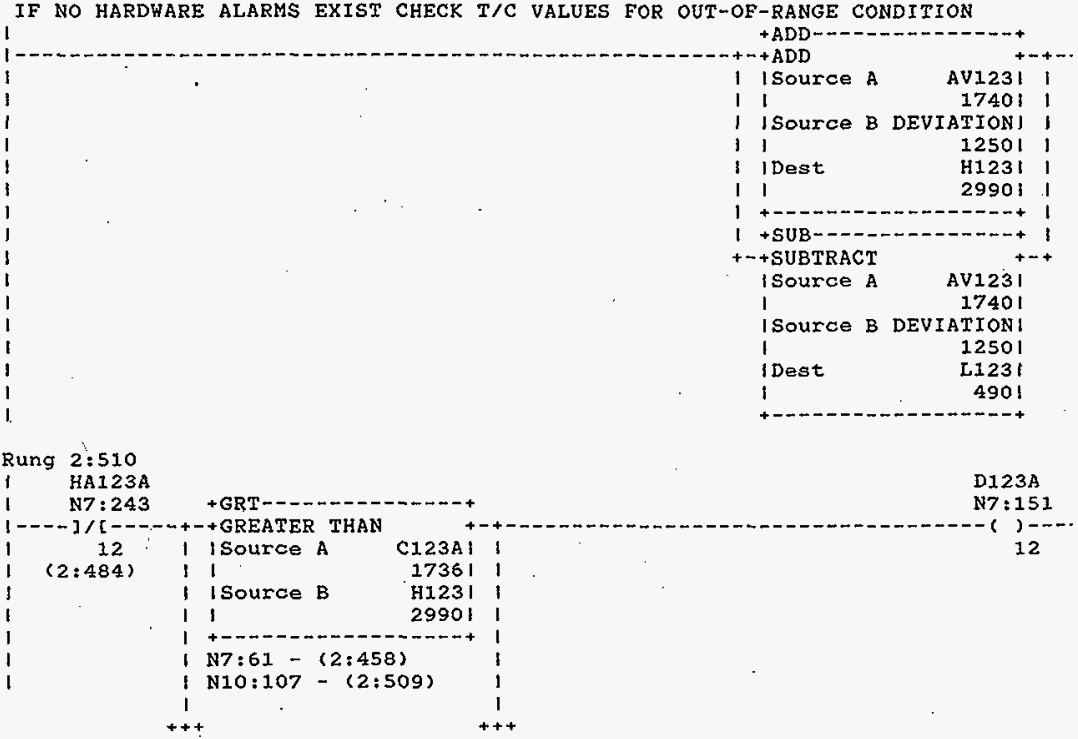


Processor and Data(OPS Unit 1)

October 23, 1996

Page 201 Program Listing

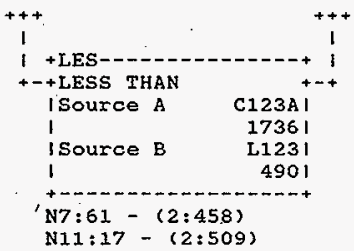

Rung 2:511

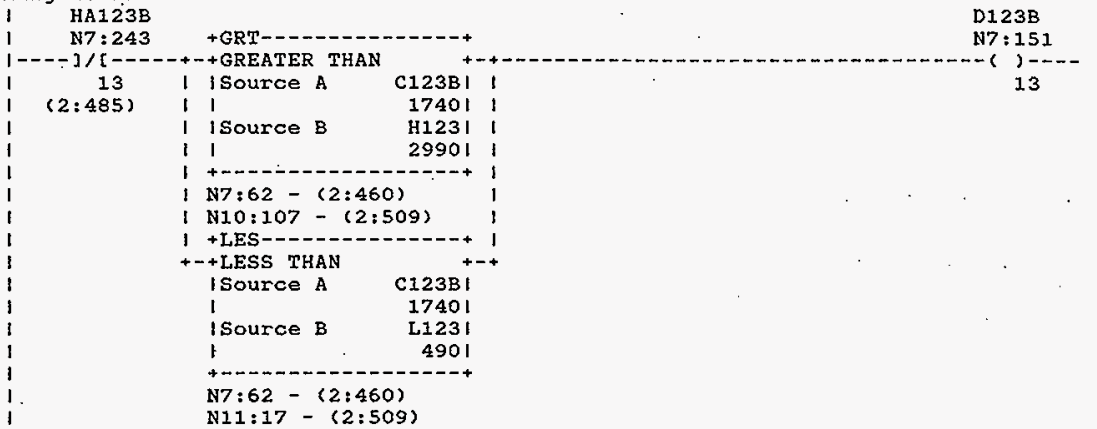

Rung 2:512

1 HA123C

1. $\quad$ N7:243

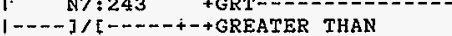

D123C

14

I ISource A

C123Cl

N7: 151

$(2: 486)$

Isource B

299011

N7:63-(2:462)

I N10:107 - (2:509)

1 +LES---- - - - - - . - + 1

+-+LESS THAN

| Source A C123C

I 1744I

| Source B L123|

I

4901

N7:63 - (2:462)

N11:17-(2:509) 
Rung 2:513

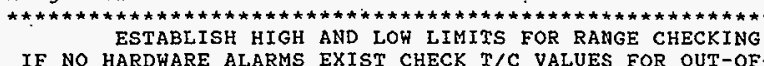

IF NO HARDHARE ALARMS EXIST CHECK T/C VALUES FOR OUT-OF-RANGE CONDITION

+ ADD - - - - - - - - - - + +

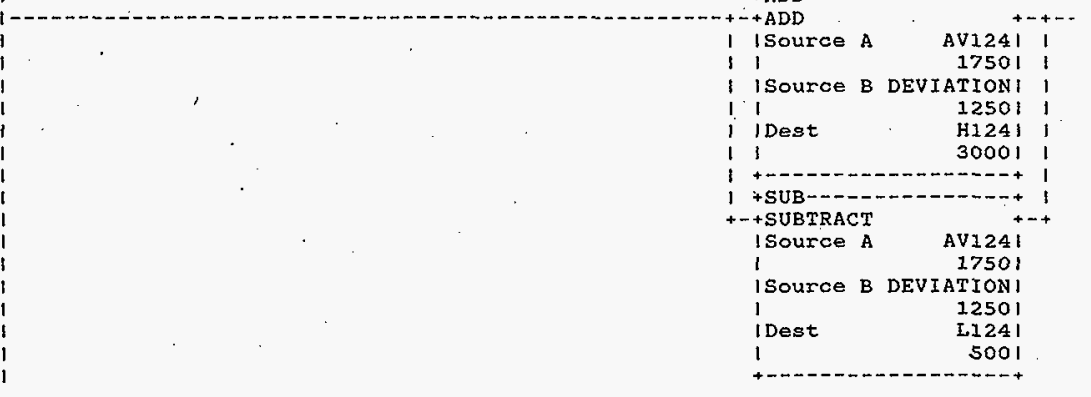

Rung $2: 514$

i HA124A

N7: 243 $1----] /[---+++$ GREATER THAN

115

15

I I Source A

(2:487) I ।

I ISource B

11$$
\begin{aligned}
& \text { N7:64-(2:464) } \\
& \text { N10:108- } 2: 513\rangle
\end{aligned}
$$

Rung $2: 515$

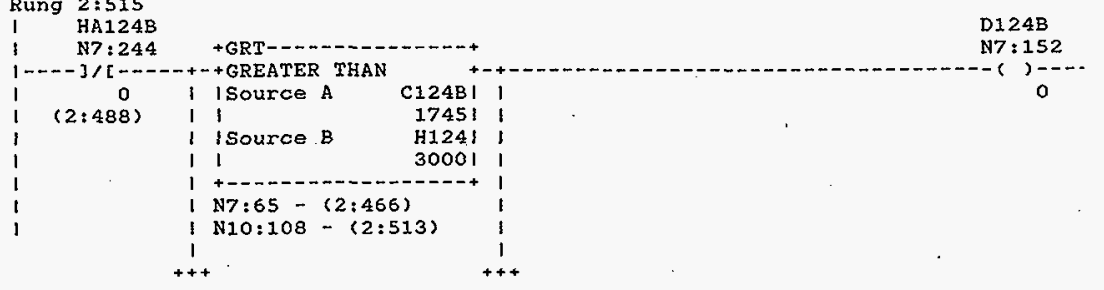

HNF-SD-FF-CSWD-61 Rev. 0 
Processor and Data(OPS Unit 1)

October 23, 1996

Page 20 Program Listing

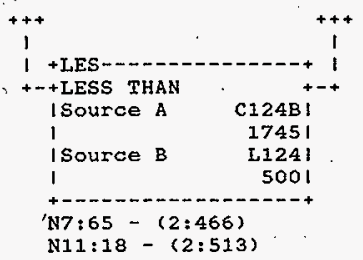

Rung $2: 516$

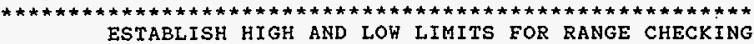

IF NO HARDWARE ALARMS EXIST CHECK T/C VALUES FOR OUT-OF-RANGE CONDITION

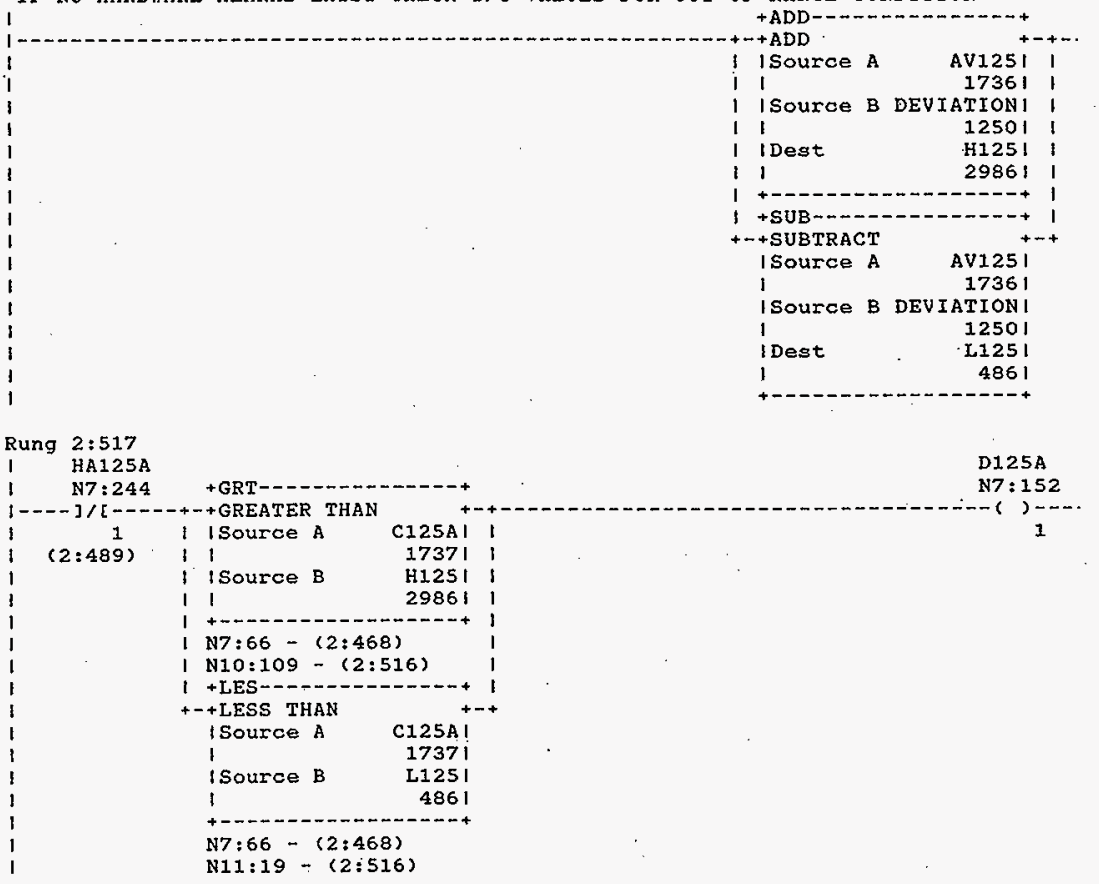

HNF-SD-FF-CSWD-61 Rev. 0 
Processor and Data(OPS Unit 1)

Rung 2:518

i. HA125B

N7 : 244

$1---] /[---+-+$ GREATER THAN

$(2: 490)$

|Source A C125B|

$1735 ;$ i

$\begin{array}{llll}1 & \text { ISource B } & \text { H125 I } \\ \mid & 1 & 2986 \mid\end{array}$

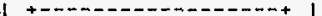

I'N7:67-(2:470)

I N10:109-(2:516)

$1+$ LES-. - -

+-+ LESS THAN +-+

ISource A C125BI

I 1735 !

isource B L 125 |

$1 \quad 4861$

N7:67-(2:470)

N11:19-(2:516)

Rung $2: 519$

I

I

DEV_3

N20:6

Rung $2: 520$

I AV3

I

$1 \quad 15$

DUMMY11

[ - - [ LBL]

$\mathrm{N} 2 \mathrm{O}: 7$

Rung 2:521

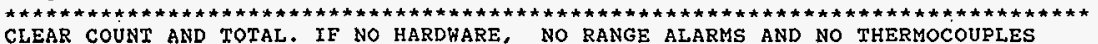
HAVE BEEN TAKEN OUT (TE_OUT $X X X$ ) THEN AVERAGE THE T/C

$$
\begin{aligned}
& 1 \\
& 1 \\
& 1 \\
& 1 \\
& 1 \\
& 1 \\
& 1 \\
& 1
\end{aligned}
$$
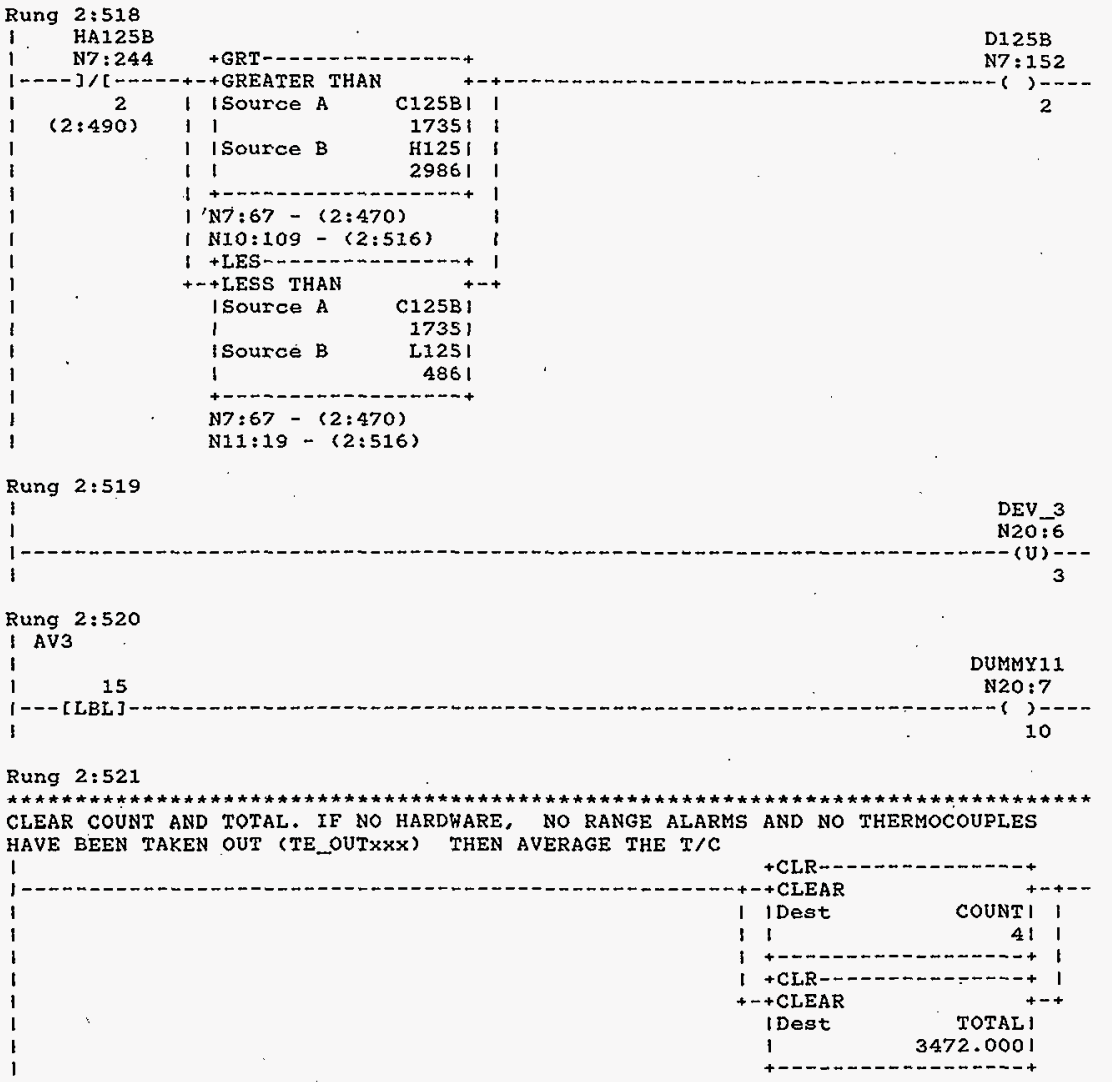
Processor and Data(Ops Unit 1)

\section{Rung 2:522}

1.

i. N7:243

D119A OUT119A

N7:151 N7:249

(2:472)

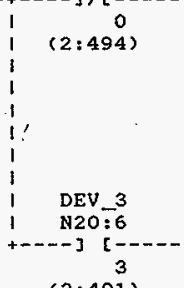

$--3 /[-$

4

$(2: 491)$

.

4

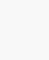

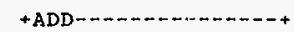

1 isource A

1 I

isource B

11

I IDest

1

1

i

$1+A D D$

$+-+A D D$

ISource A C119AI

1

ISource B

16911

I

TOTAL 1

IDest

3472.0001

1

TOTALI

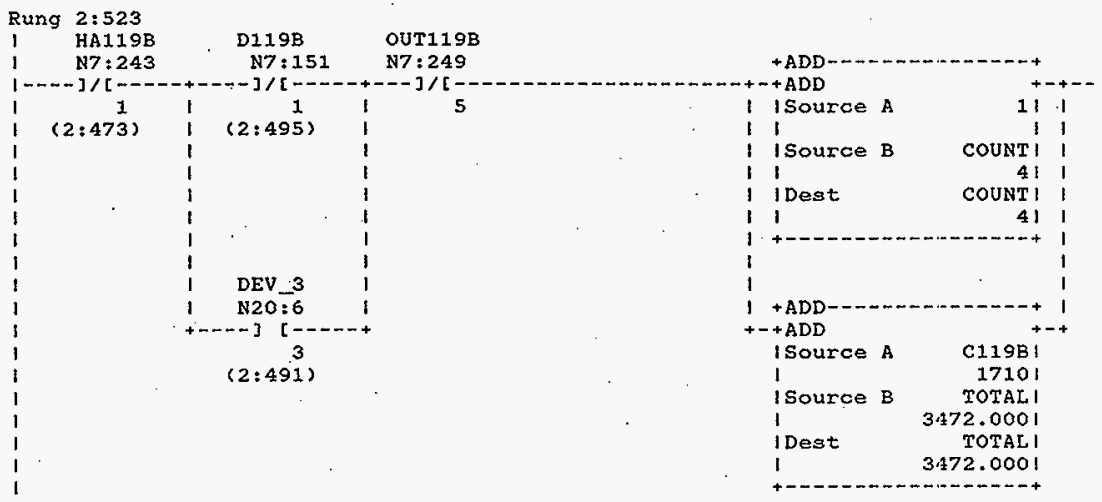

HNF-SD-FF-CSWD-61 Rev. 0 
Processor and Data(OPS Untt 1)

Program Listing

Processor File: SODIUM1A.ACH

Rung $2: 524$

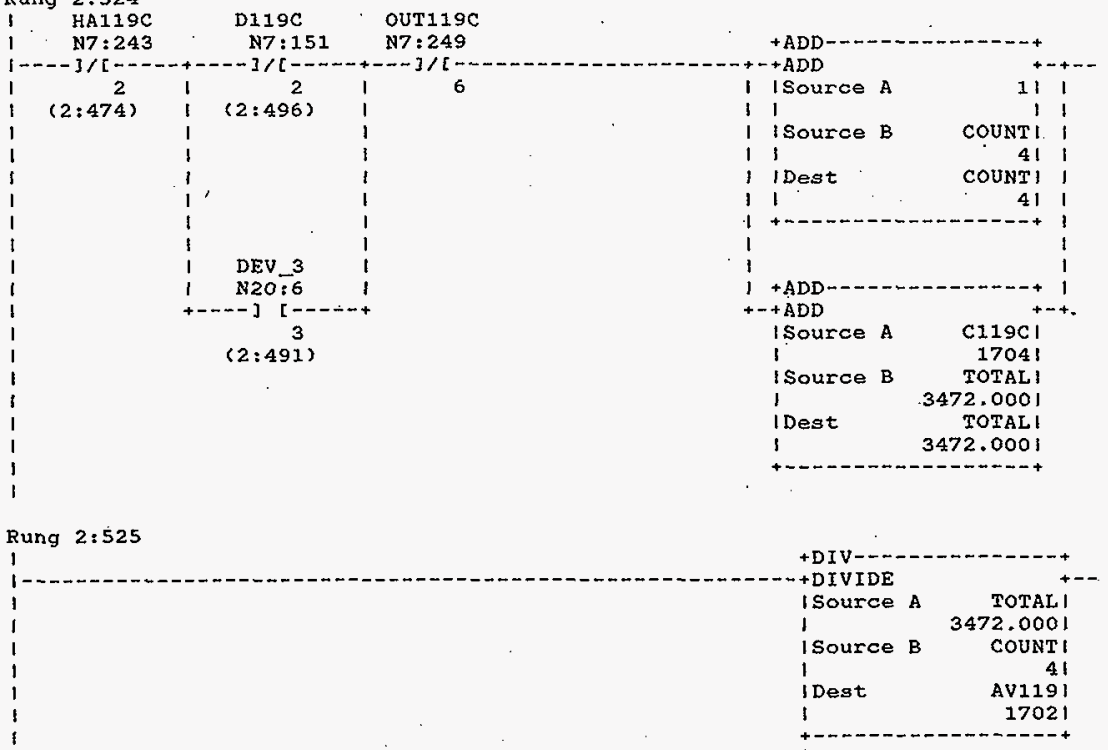

Rung $2: 526$

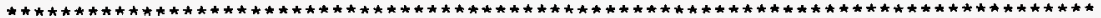
CLEAR COUNT AND TOTAL. IF NO HARDWARE, NO RANGE ALARMS AND NO THERMOCOUPLES HAVE BEEN TAKEN OUT (TE OUTXXX) THEN AVERAGE THE T/C

$$
\text { I. }
$$

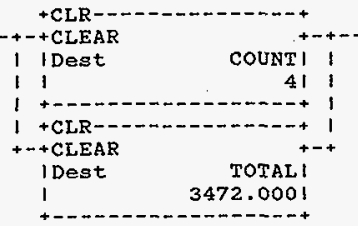

HNF-SD-FF-CSWD-61 Rev. 0 
Processor and Data(OPS Unit 1 )

Program Listing
October 23, 1996

Processor File: SODIUMIA.ACH
Page 207

Rung 2:52\%

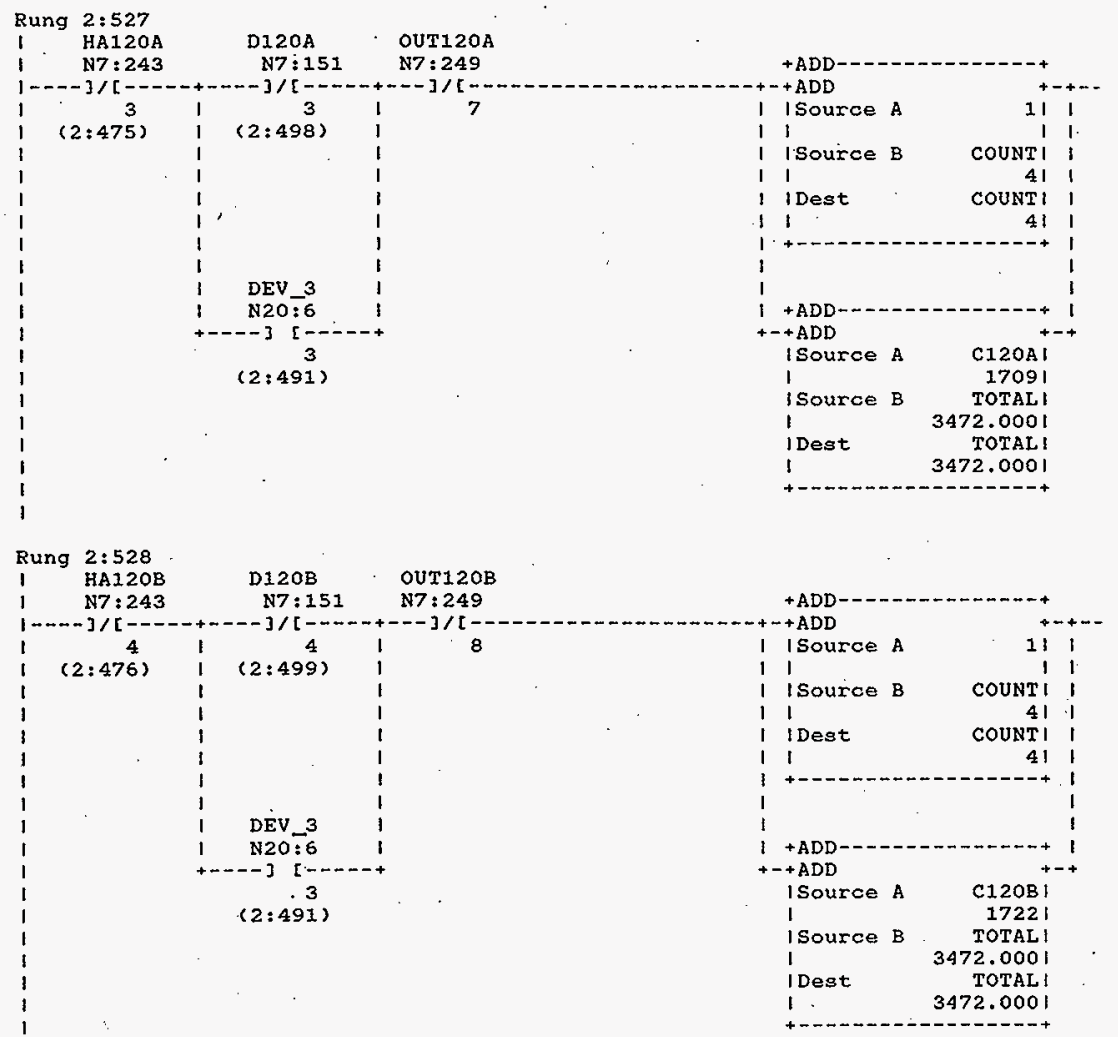

HNF-SD-FF-CSWD-61 Rev. 0 
Processor and Data(ops Unit i)

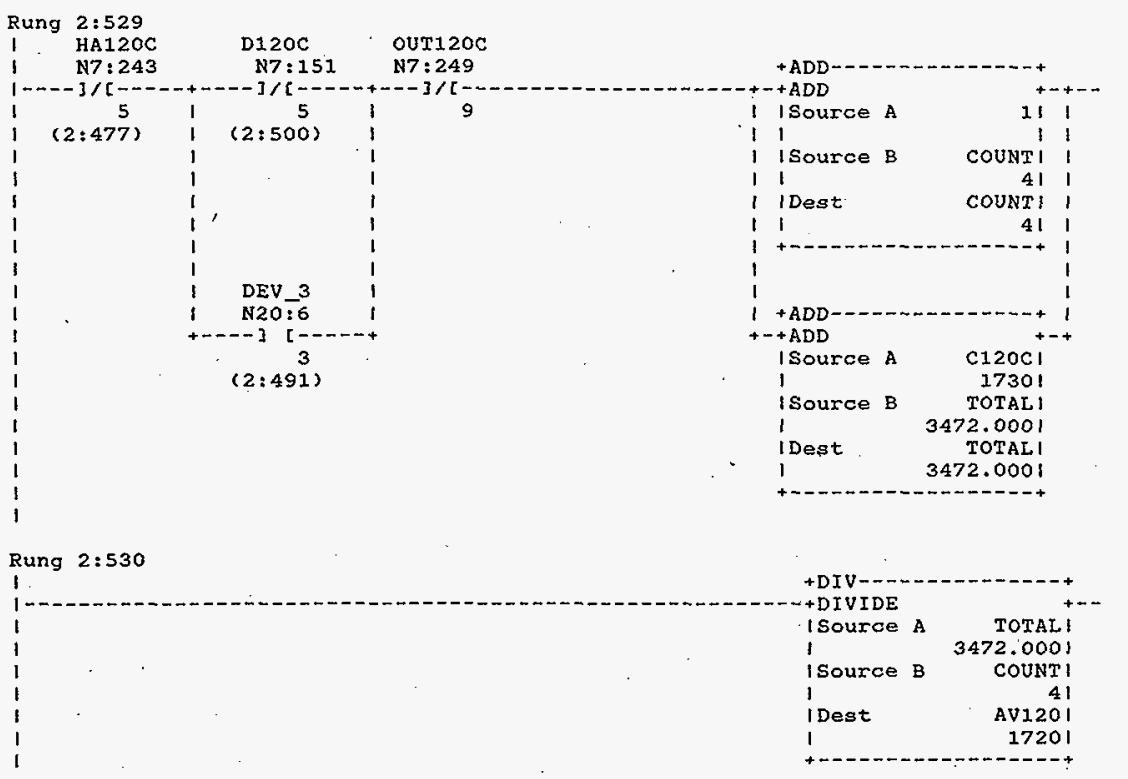

Rung $2: 531$

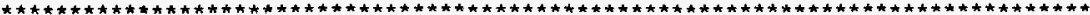
CLEAR COUNT AND TOTAL. IF NO HARDWARE, NO RANGE ALARMS AND NO THERMOCOUPLES HAVE BEEN TAKEN OUT (TE_OUTXXX) THEN AVERAGE THE T/C

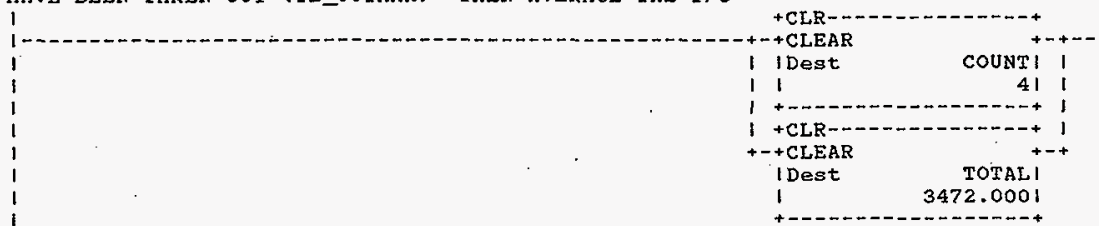


Frocessor and Datacops Unit 1) Program Listing

Rung $2: 532$

1 HA121A

i. N7 $: 243$

Processor File: SODIUMAA.ACH
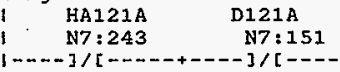

OUT121A

N7: 249

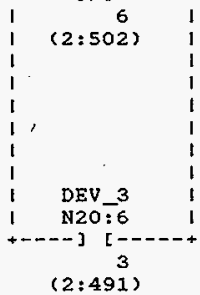

10

0

.
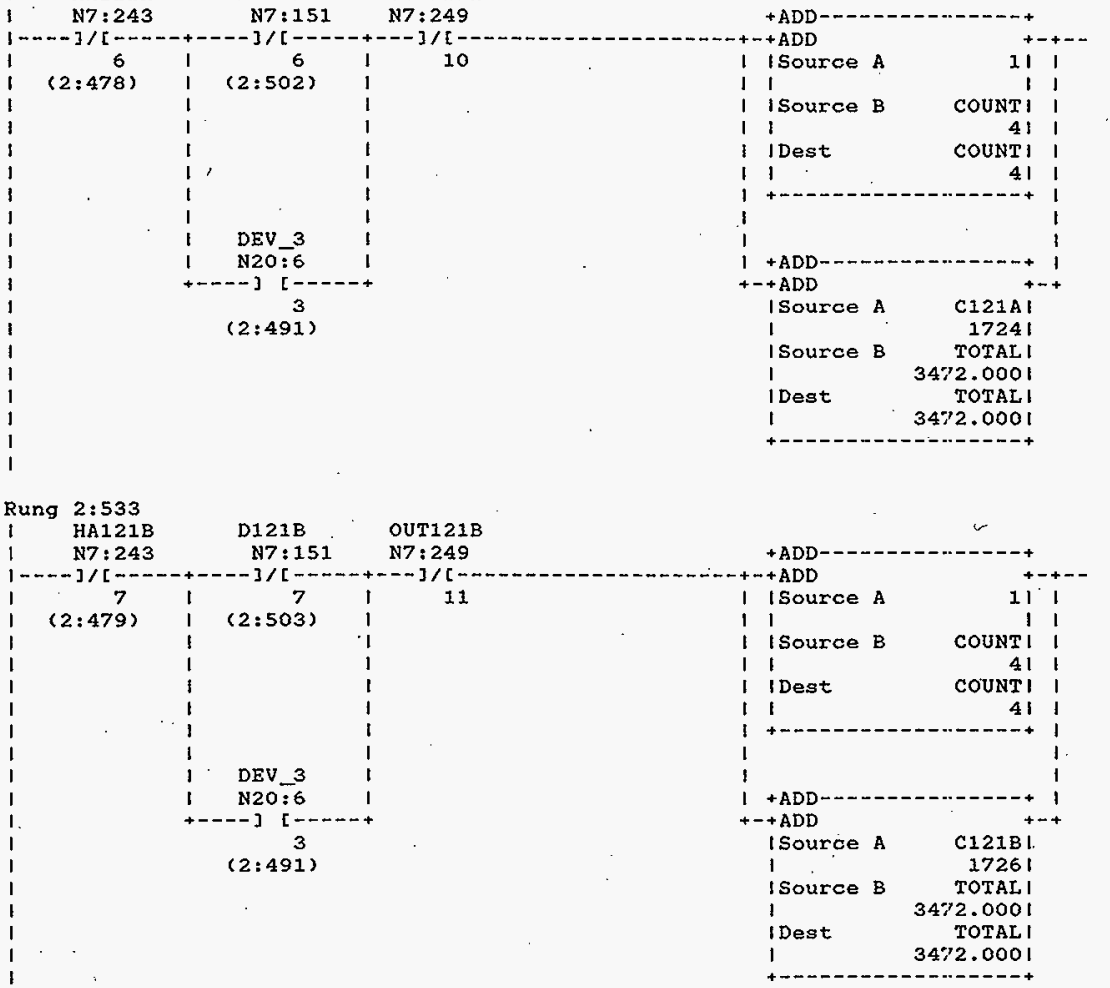

HNF-SD-FF-CSWD-61 Rev. 0 
Processor and Datalops Unit 1 ) Program Listing

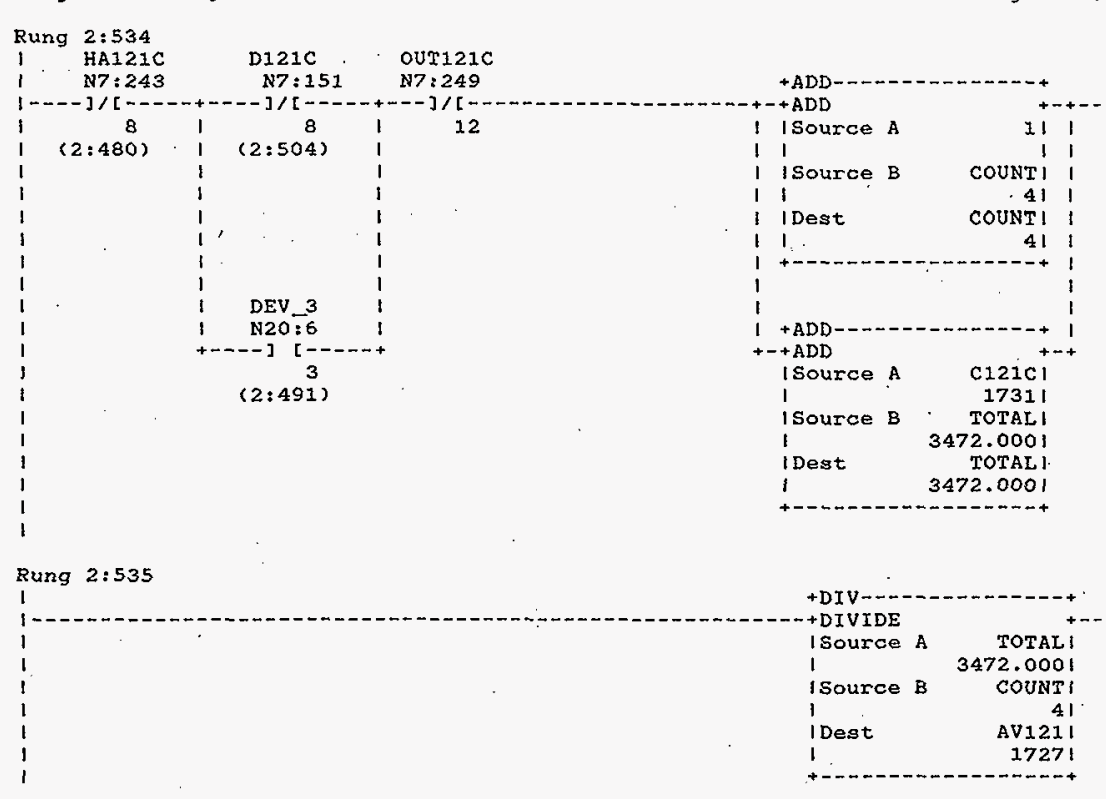

Rung 2:536

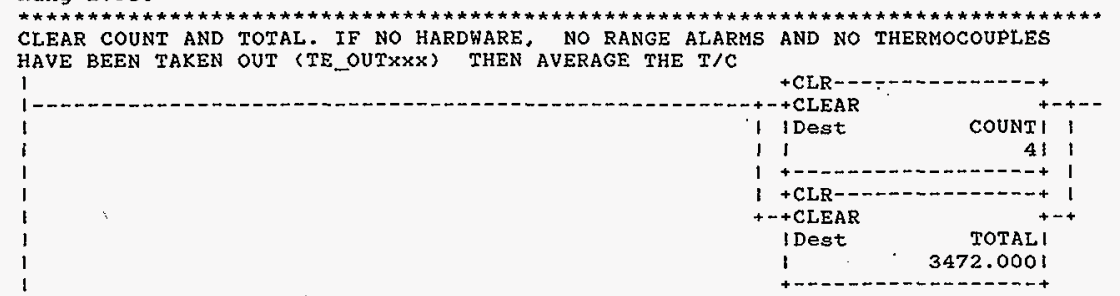

HNF-SD-FF-CSWD-61 Rev. 0 HAVE BEEN TAKEN OUT (TE OUTXXX) THEN AVERAGE THE T/C 
Rung 2:537

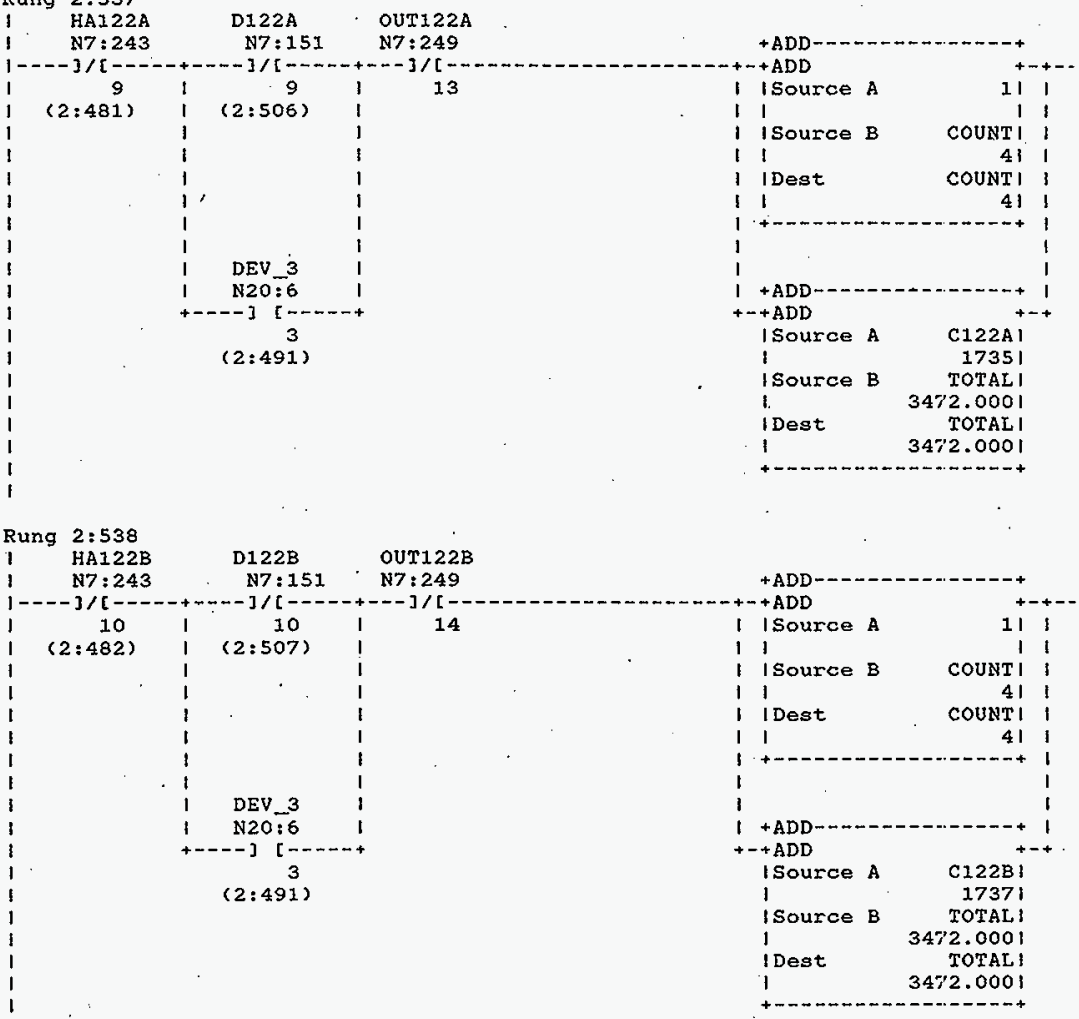


Processor and Data(OPS Unit 1) Program Listing
October 23, 1996

Processor File: SODIUM1A.ACH
Page 212

Rung 2:539

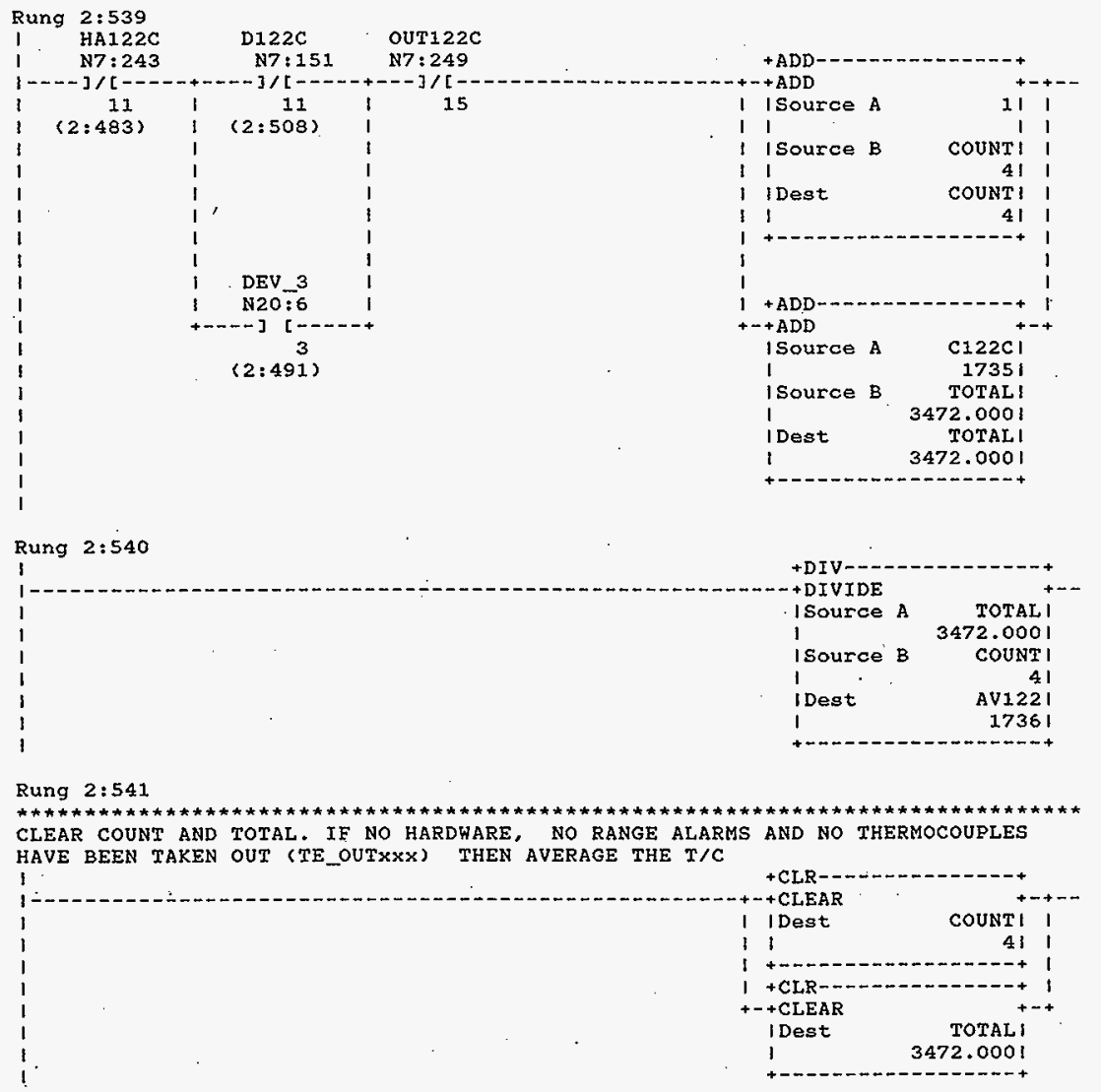

HNF-SD-FF-CSWD-61 Rev. 0 
Processor and Data(OPS Unit 1 )

October 23, 1996

Page $21:$

Program Listing

Processor File: SODIUM1A.ACH

Rung 2:542
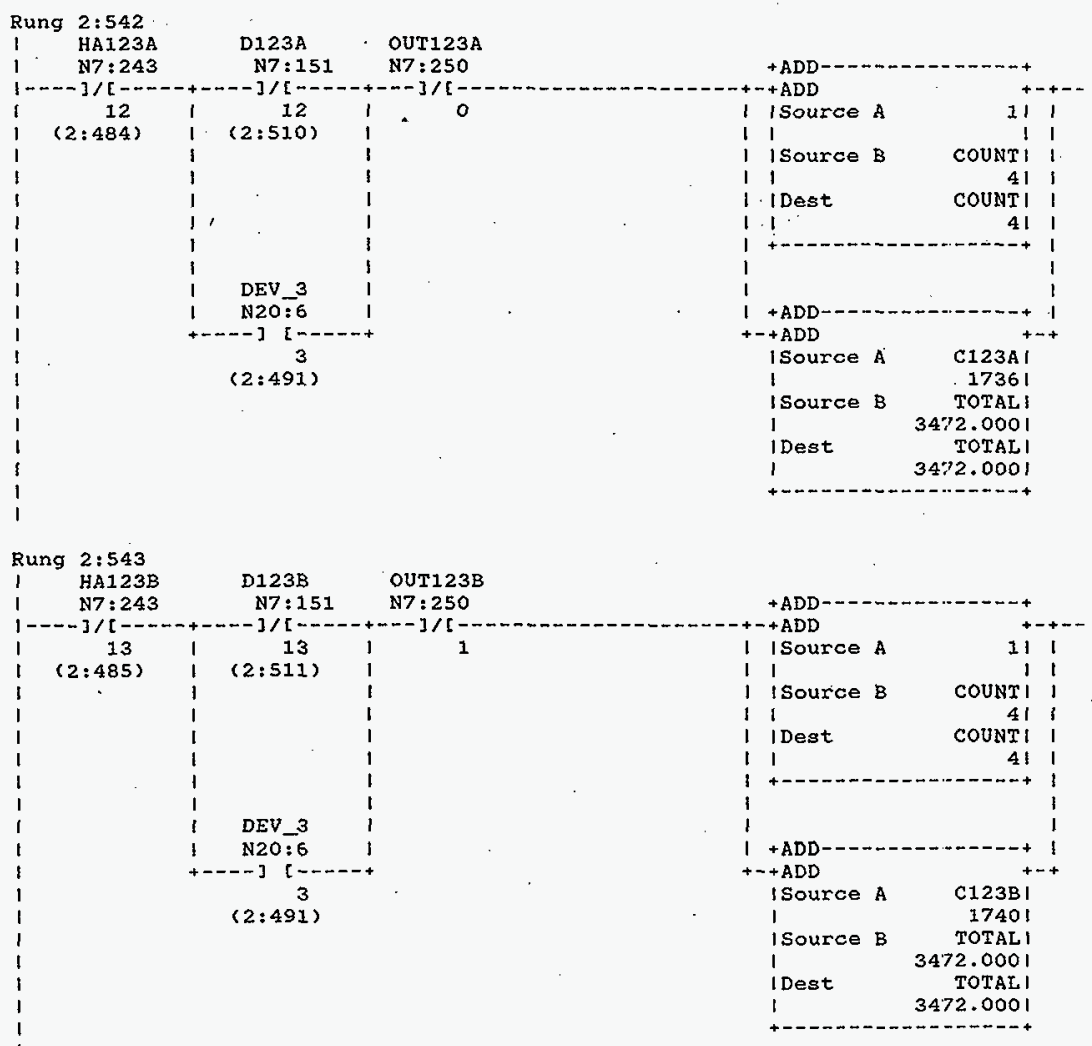

HNF-SD-FF-CSWD-61 Rev. 0

Page $2: 3$ 

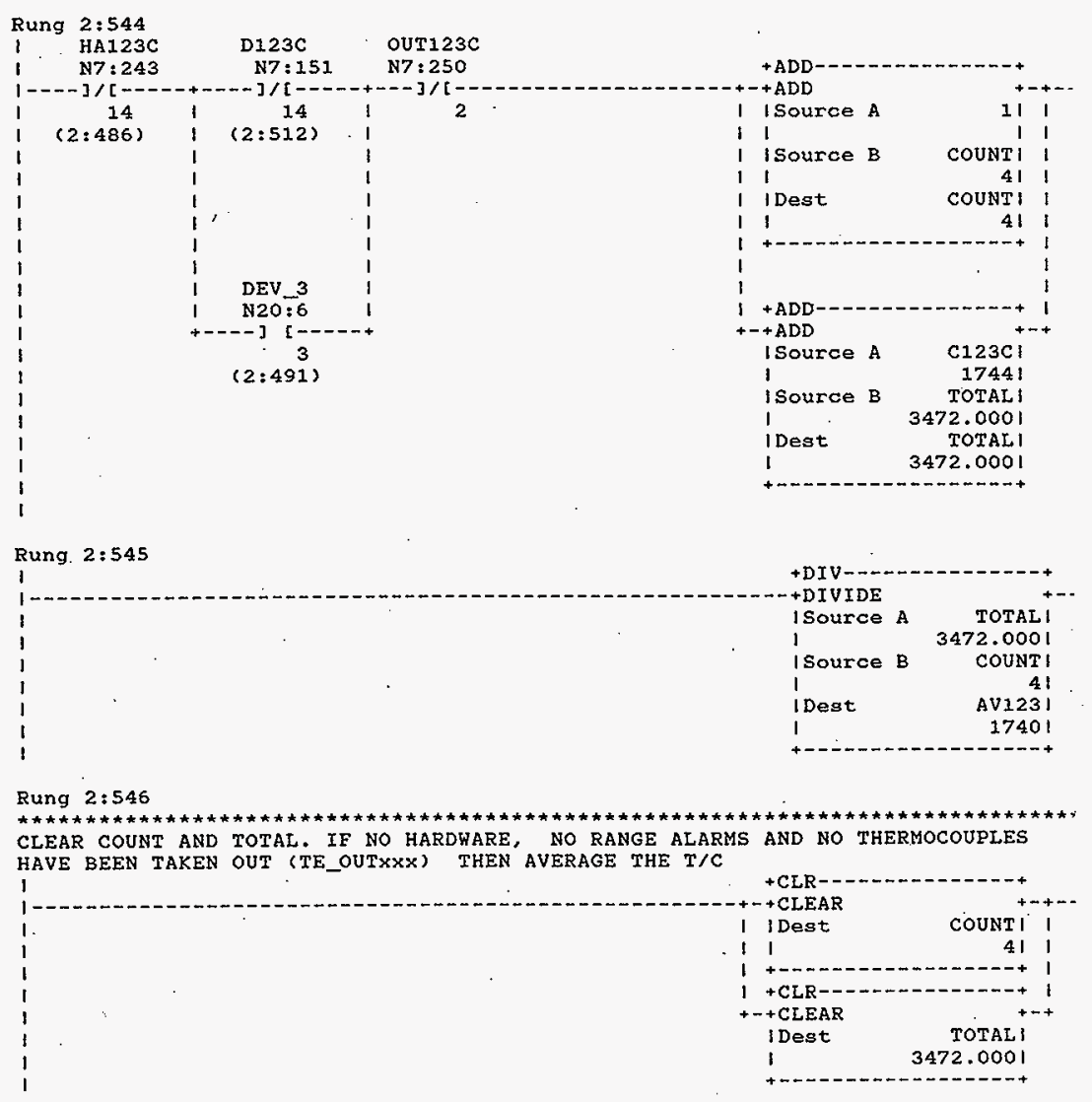

HNF-SD-FF-CSWD-61 Rev. 0 
Processor and Data(OPS Unit 1) Program Listing
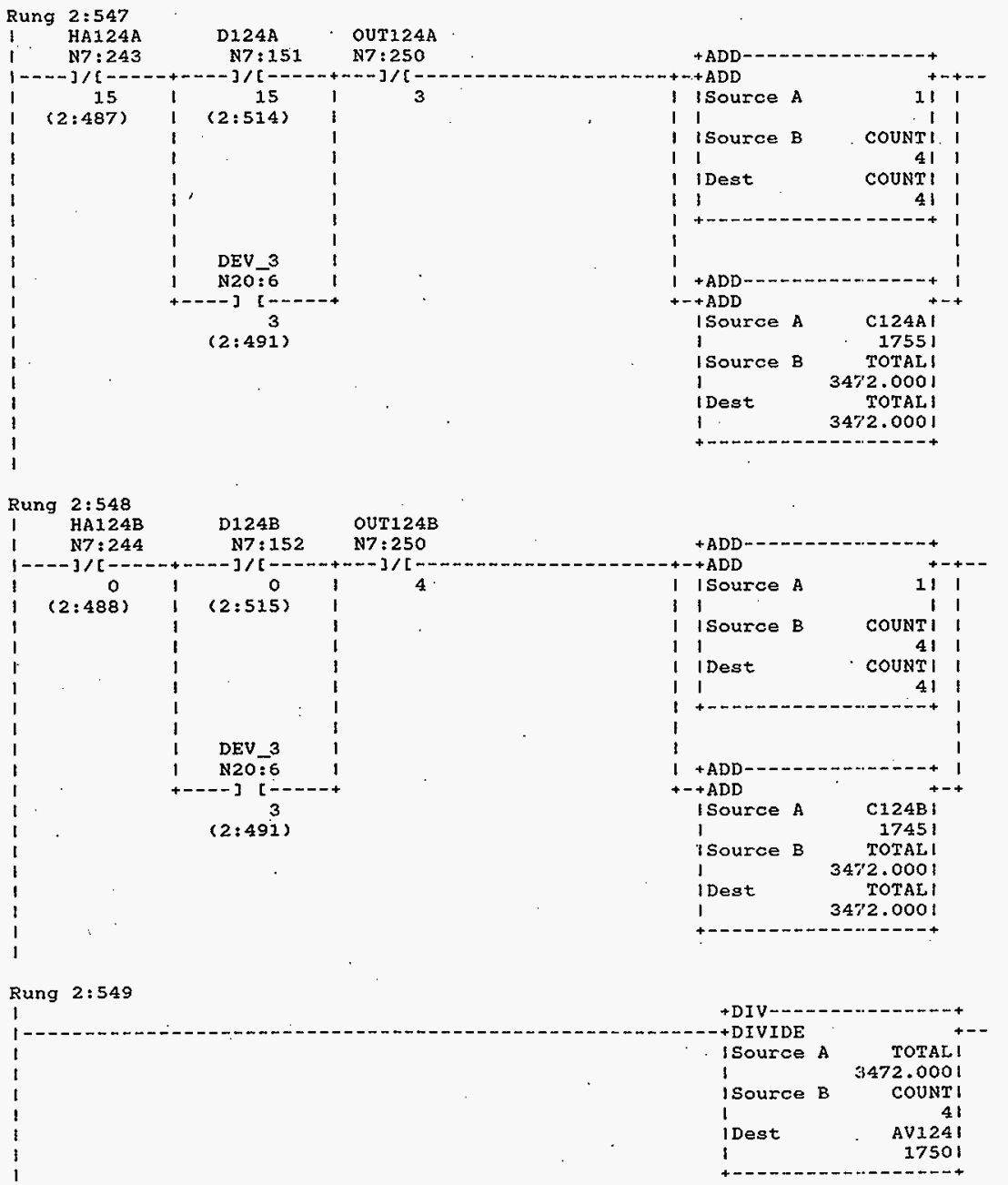

HNF-SD-FF-CSWD-61 Rev. 0 
Rung 2:550

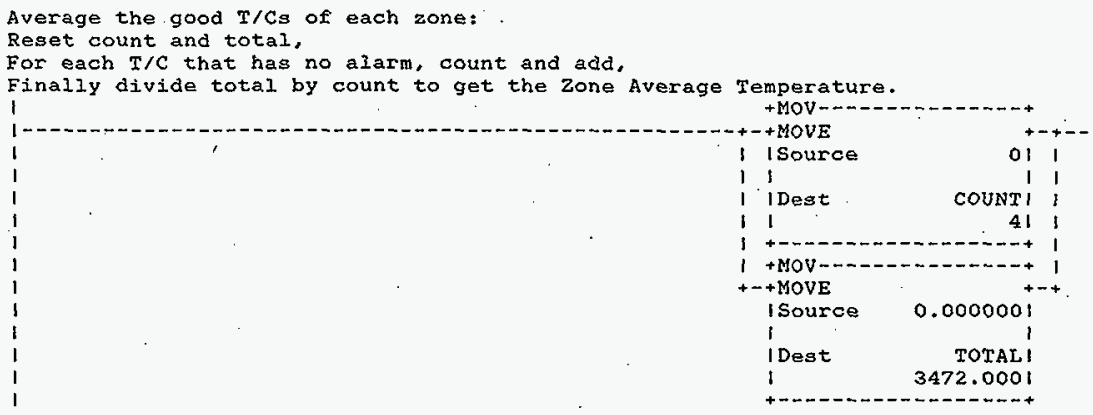

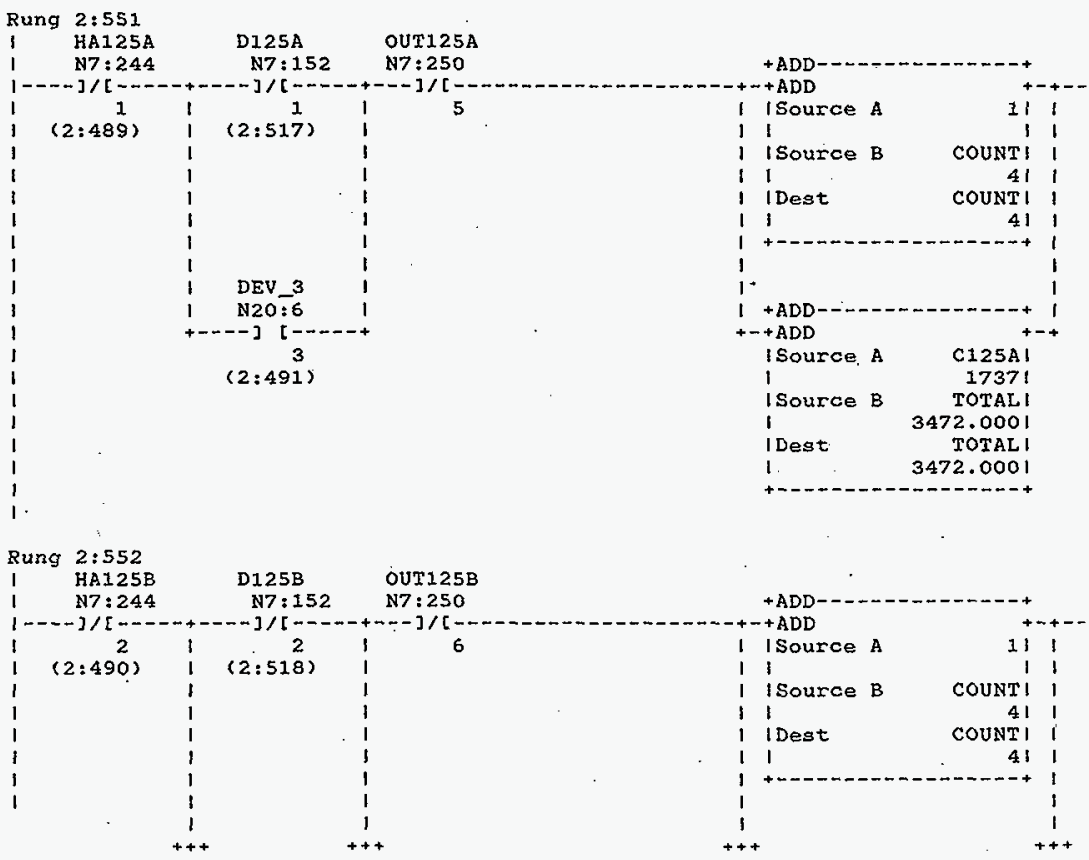


Processor and Data(ops Unit 1 )

October 23,1996

Page 21:

Program Listing

Processor File: SODIUM1A.ACH

Rung 2:55:

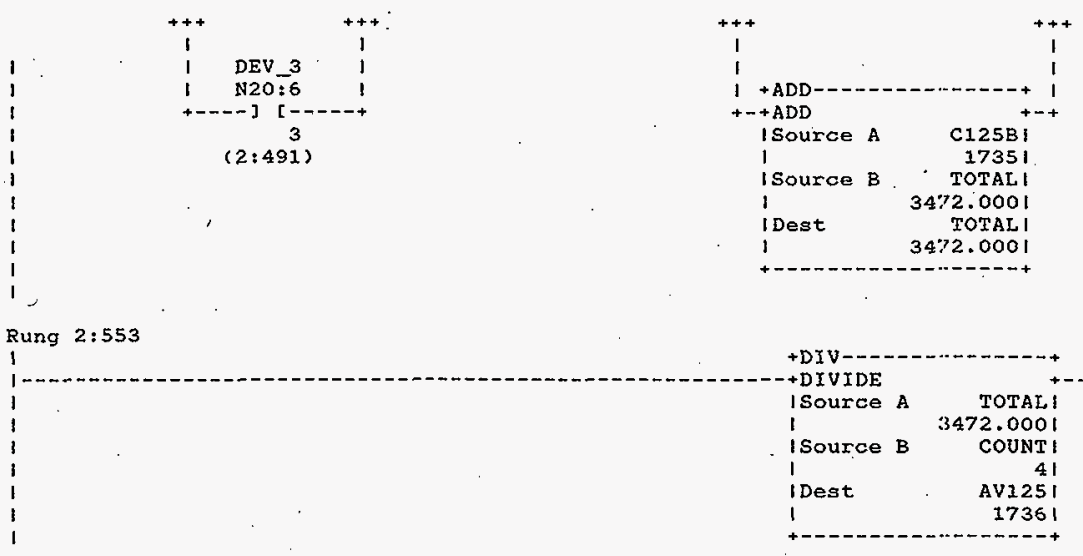

Rung $2: 554$

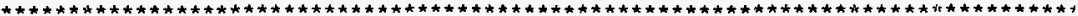
IF DEVIATION SET FOR SECTION 3 THEN GO THERE AND CALCULATE DEVIATYON

1 DEV 3 N20:6

$1---3 \quad 3$

(2:491)

Rung $2: 555$

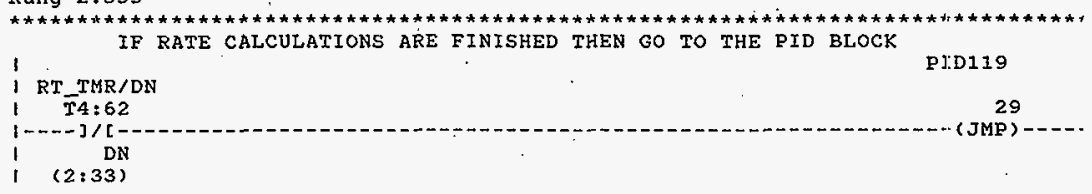

Rung 2:556

RATE CHECKING

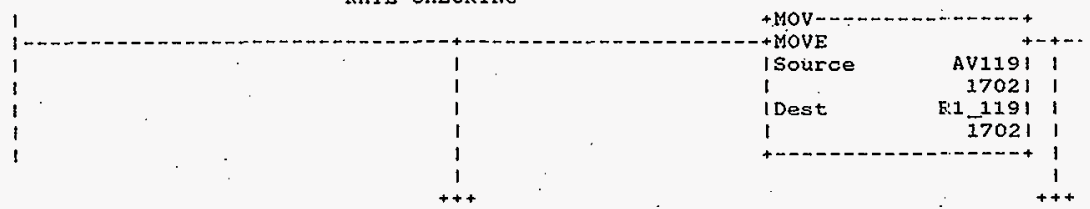

HNF-SD-FF-CSWD-61 Rev. 0 


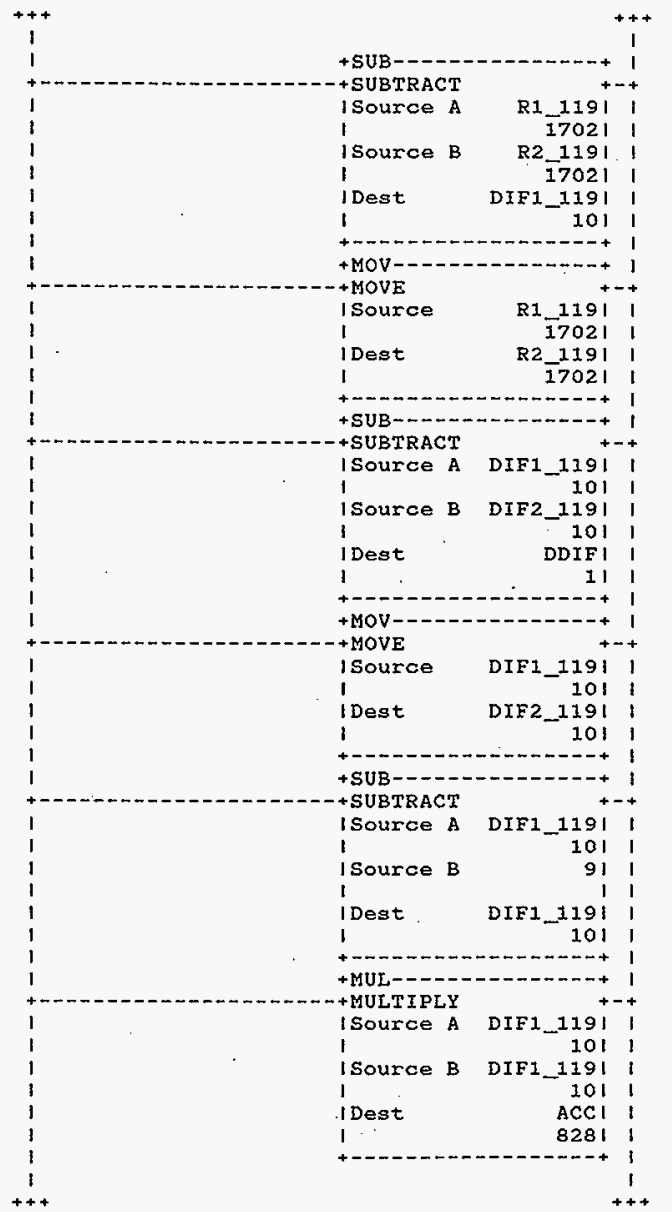

HNF-SD-FF-CSWD-61 Rev. 0 
Processor and Data(OPS Unit 1) Frogram Listing
Processor File: SODIUM1A.ACH

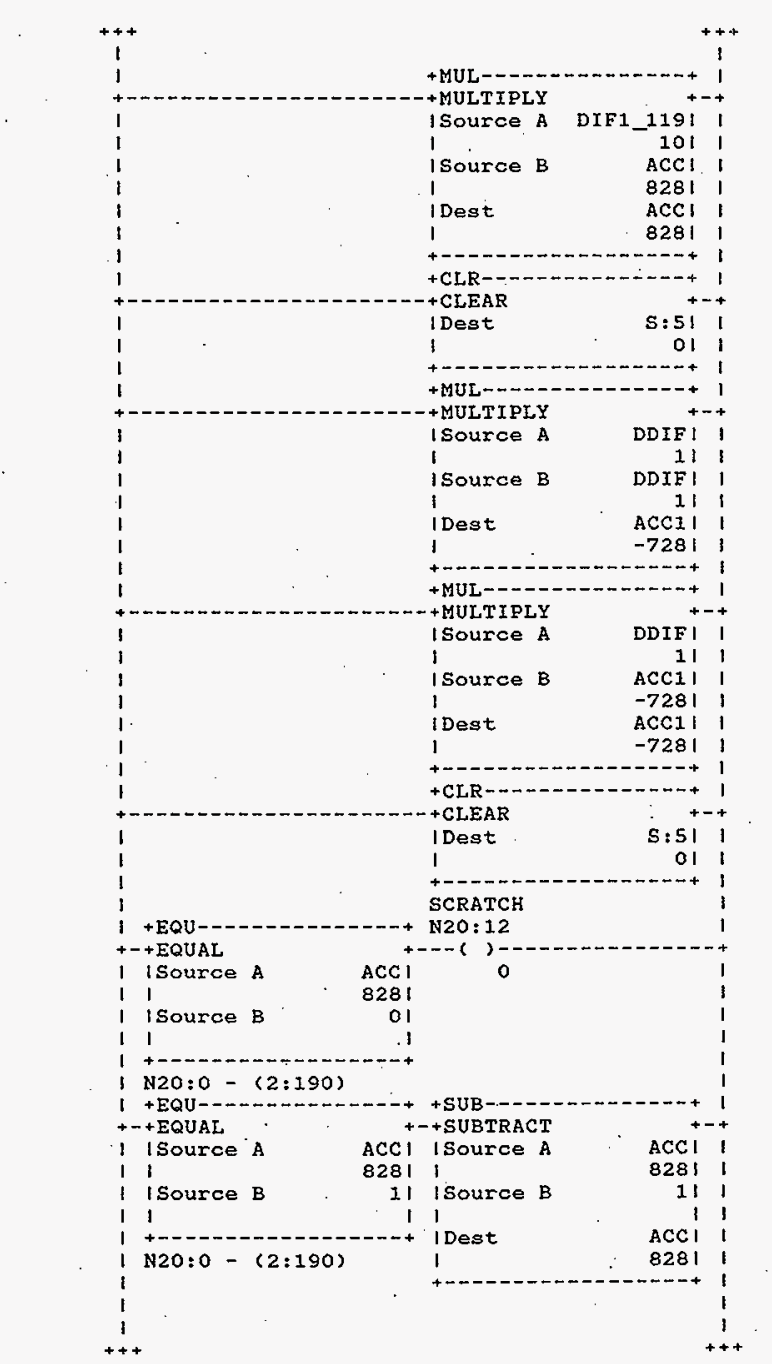

October 23, 1996. Page 215 Rung 2:556

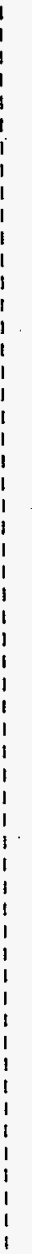

HNF-SD-FF-CSWD-61 Rev. 0 
Processor and Data(OPS Unit 1)

October 23,1396

Page 226

Program Listing

Processor File: SODIUMIA.ACH

Rung $2: 556$

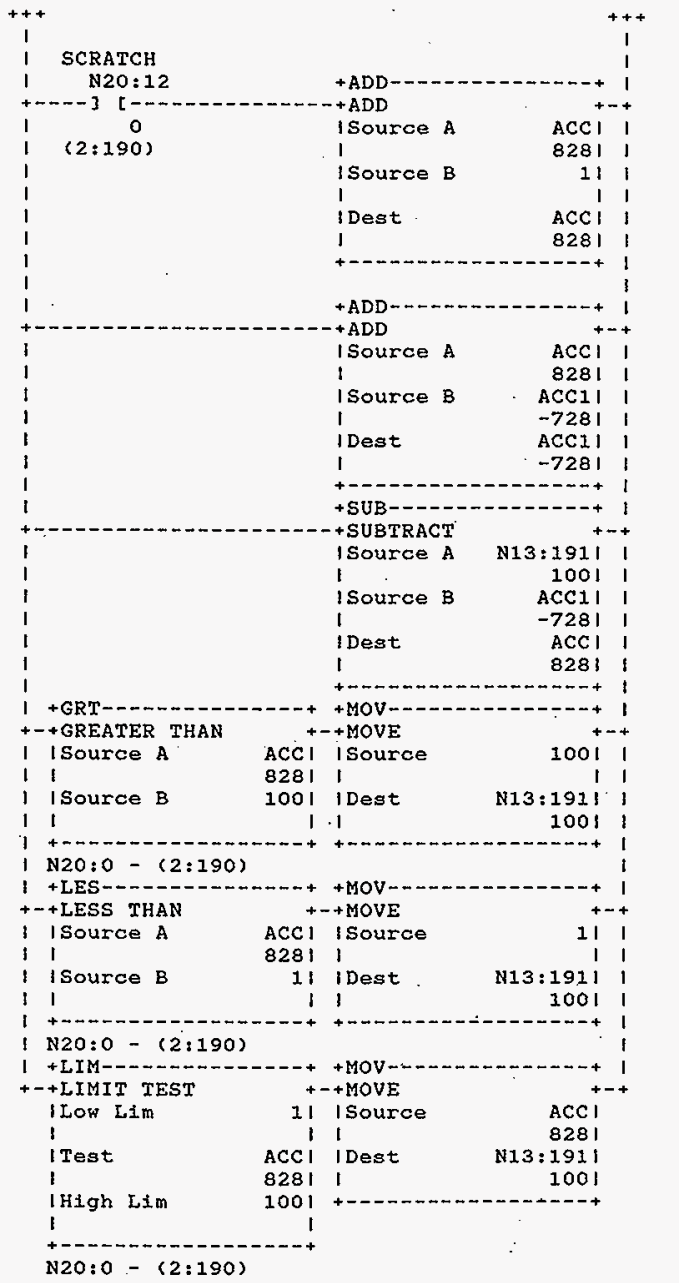

HNF-SD-FF-CSWD-61 Rev. 0 


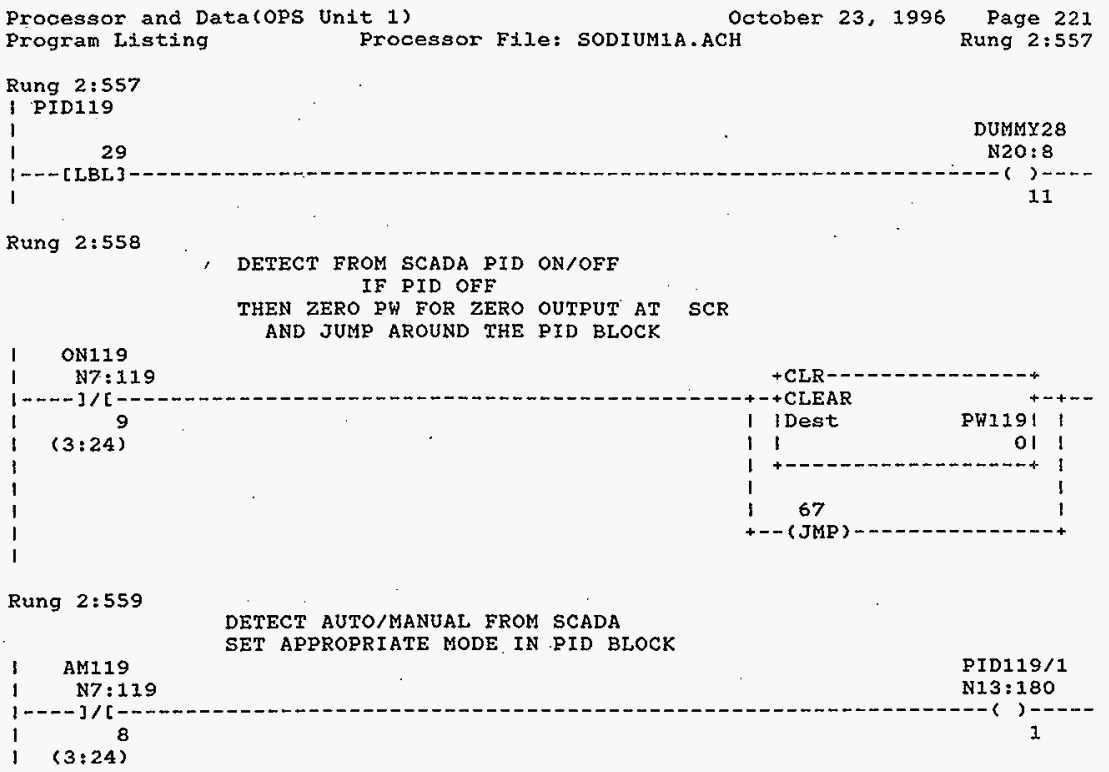

Rung $2: 560$

DETECT SETPOINT VALUE FROM SCADA PUT VALUE IN PID BLOCK

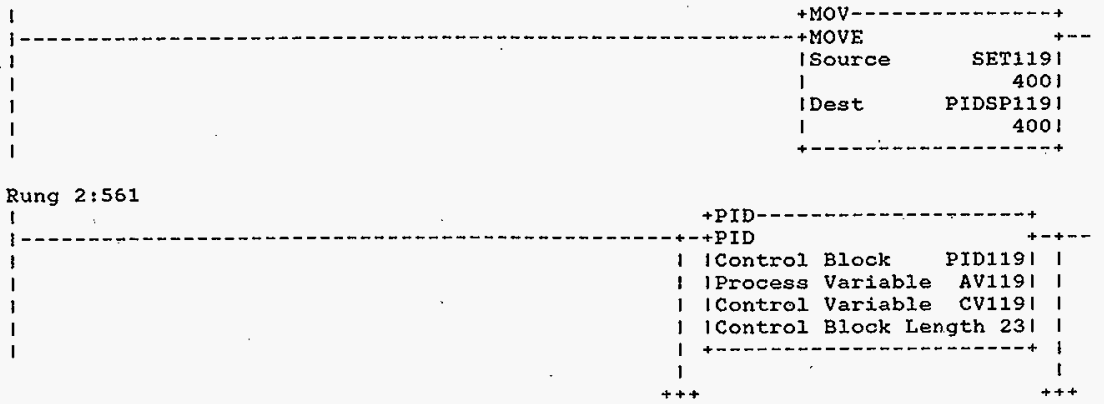


Processor and Data(OPS Unit i)

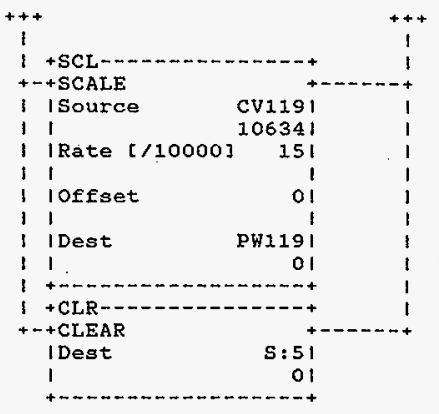

Rung 2:562

167

Rung $2: 563$

1

RT TMR/DN

PID120

$1 \quad T 4: 62$

$1----3 /[-$

(JMP)

$$
\text { (2:33) }
$$

Rung 2:564

RATE CHECKING

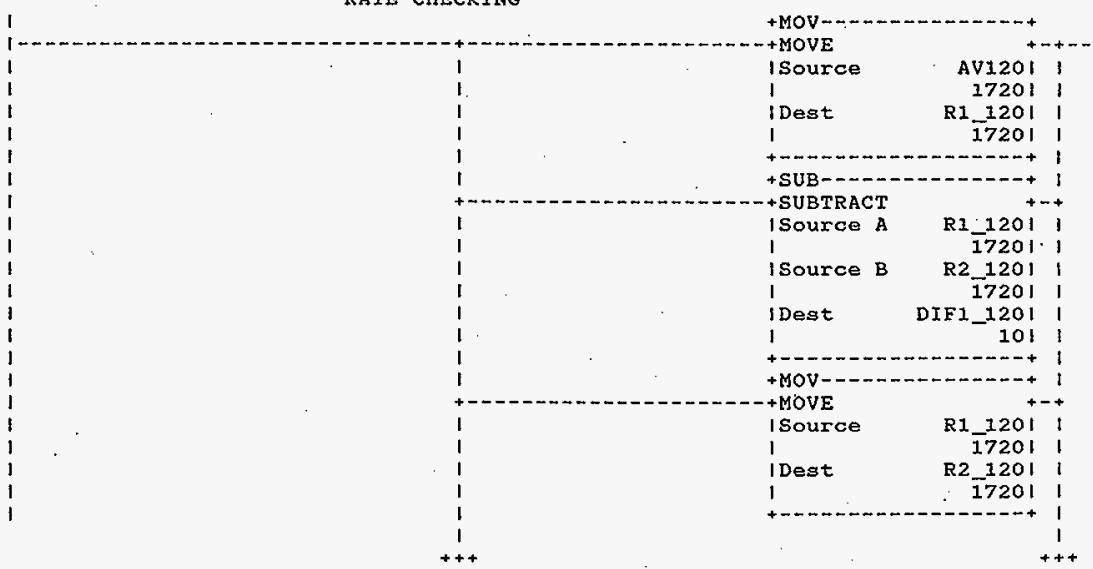




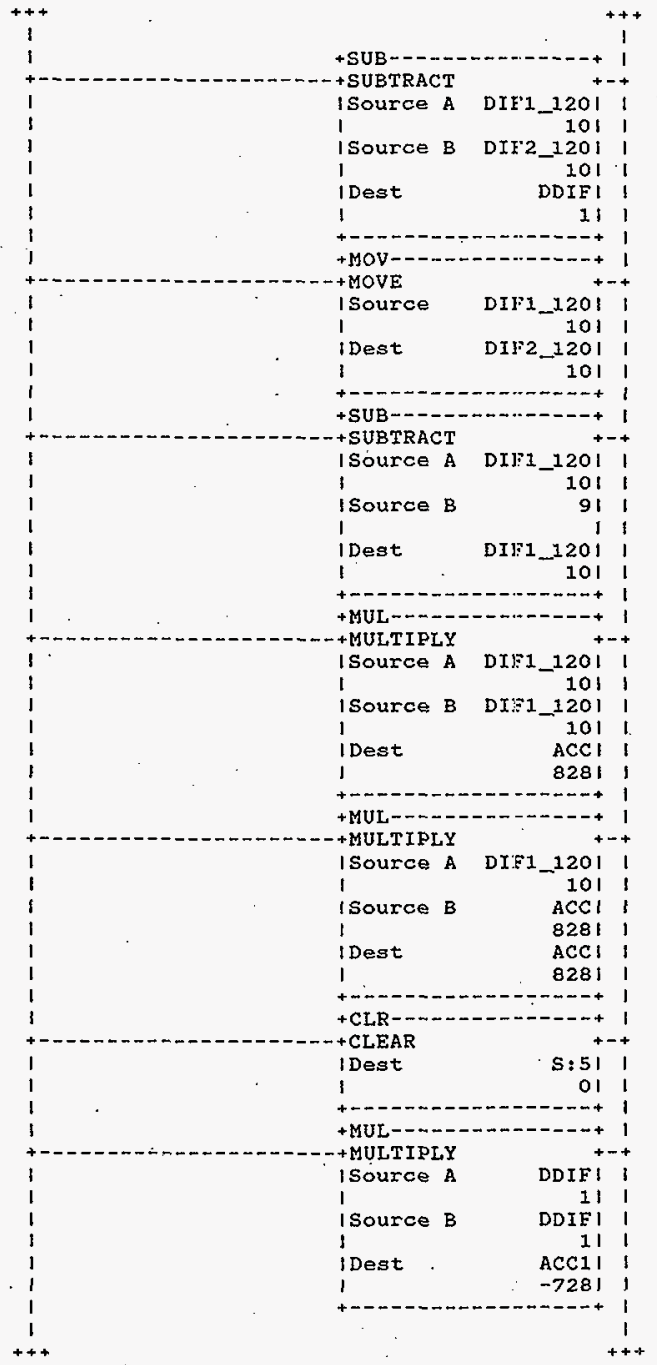

HNF-SD-FF-CSWD-61 Rev. 0 
Processor and Data(OPS Unit 1)

October 23, 1996

Page 224

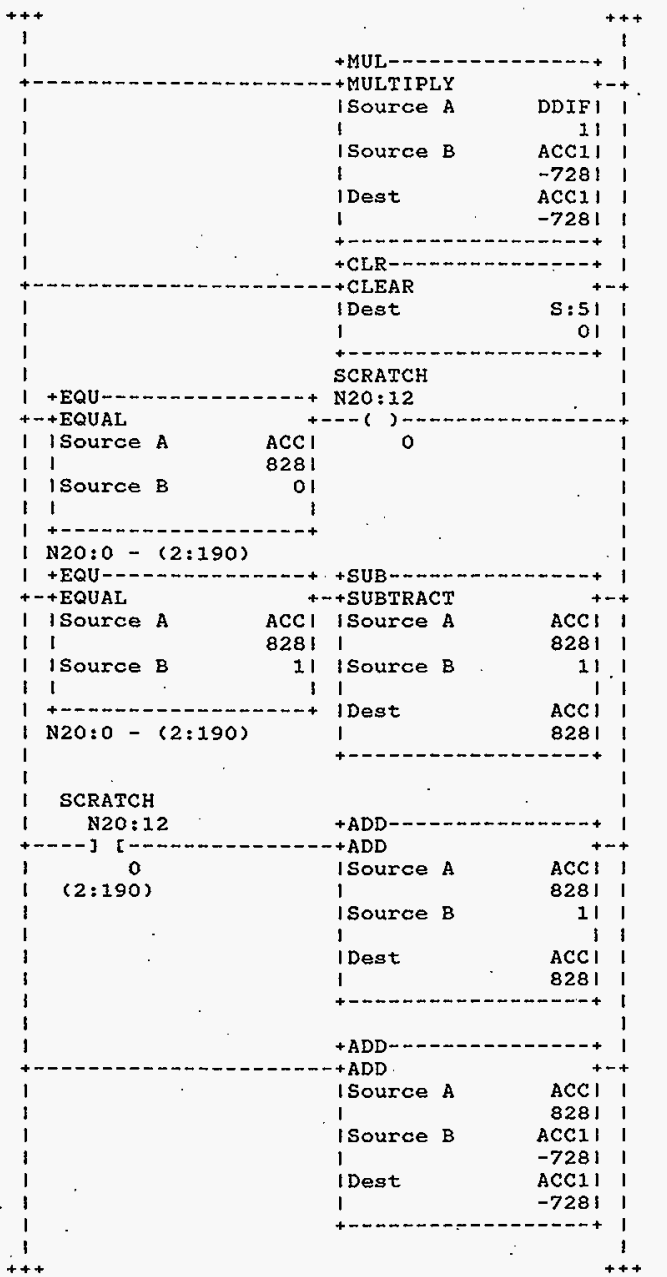

HNF-SD-FF-CSWD 61 Rev. 0 
Processor and Data(OPS Unit 1)

October 23, 1996 Page 225

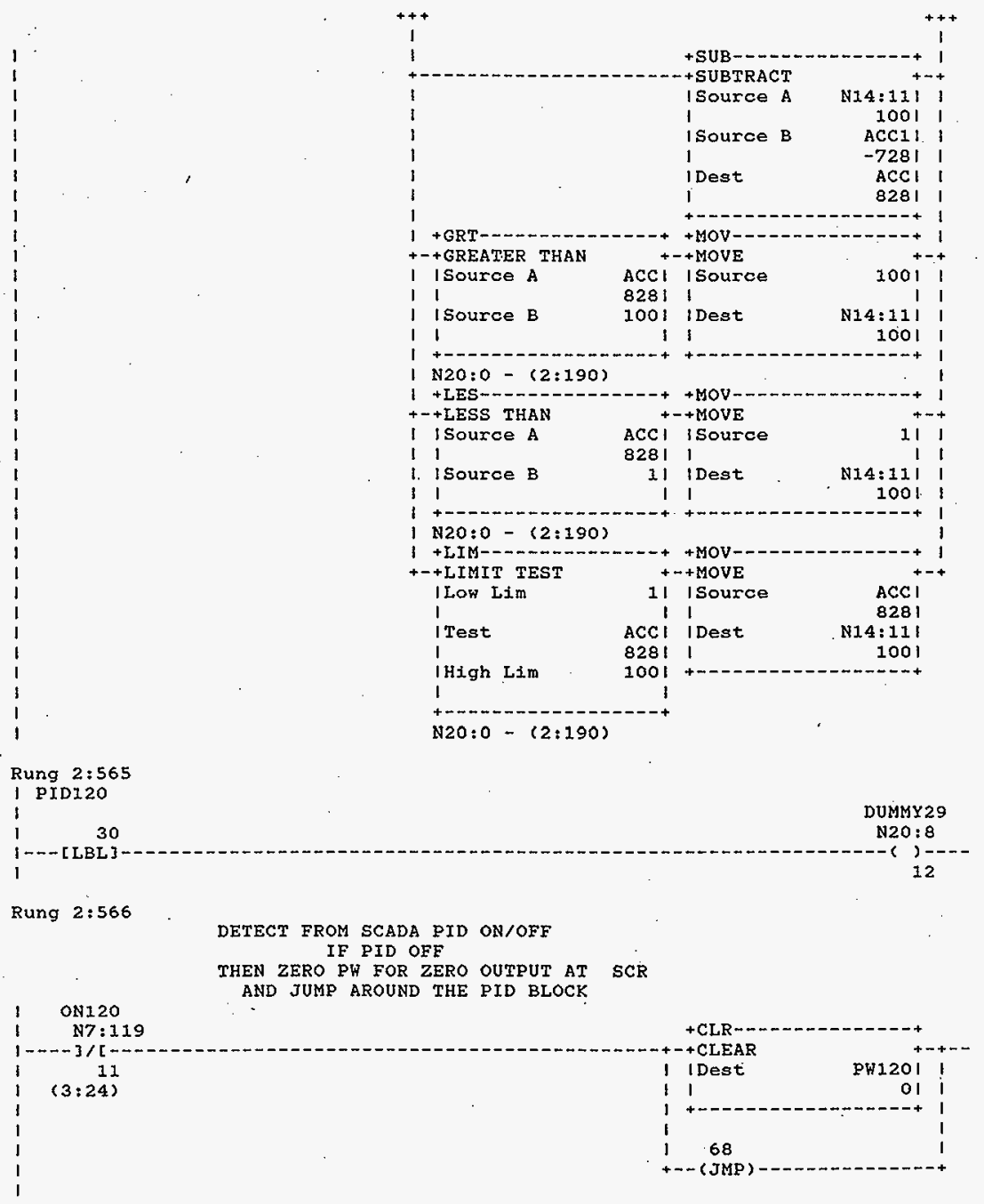

HNF-SD-FF-CSWD-61 Kiev. 0 
Processor and Data(OPS Unit 1)

Rung 2:567

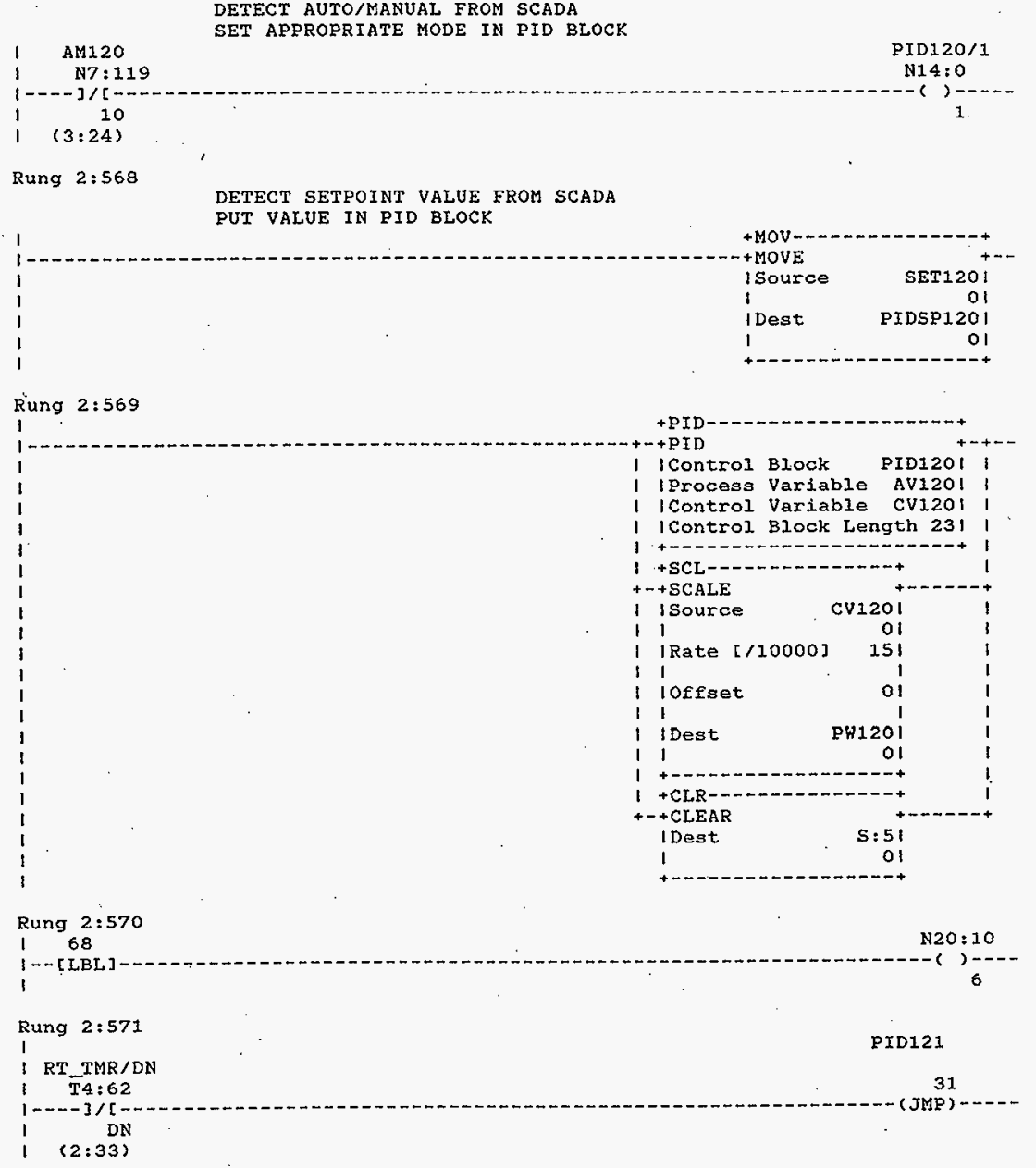


Processor and Data(ops Unit 1 ) Program Listing

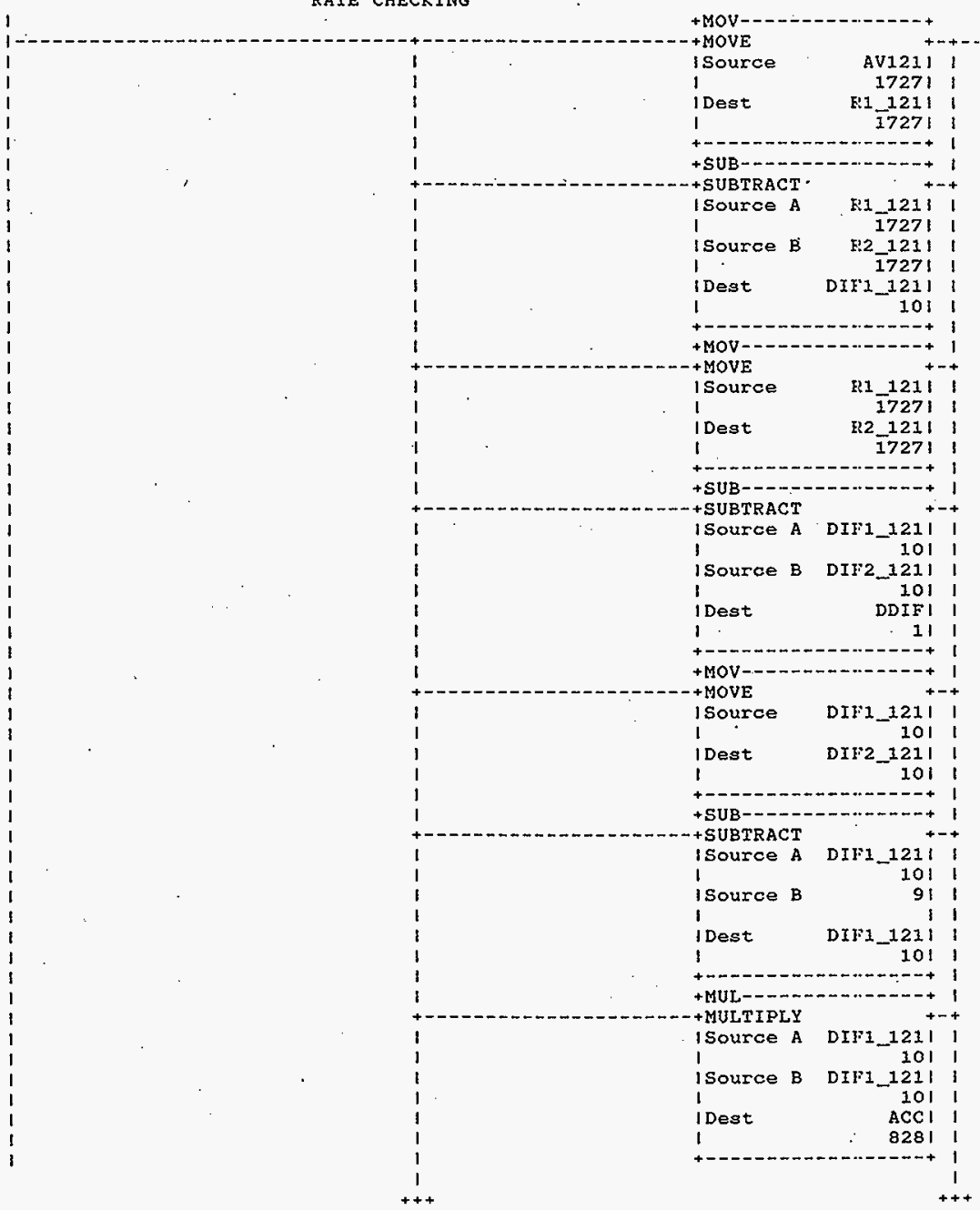




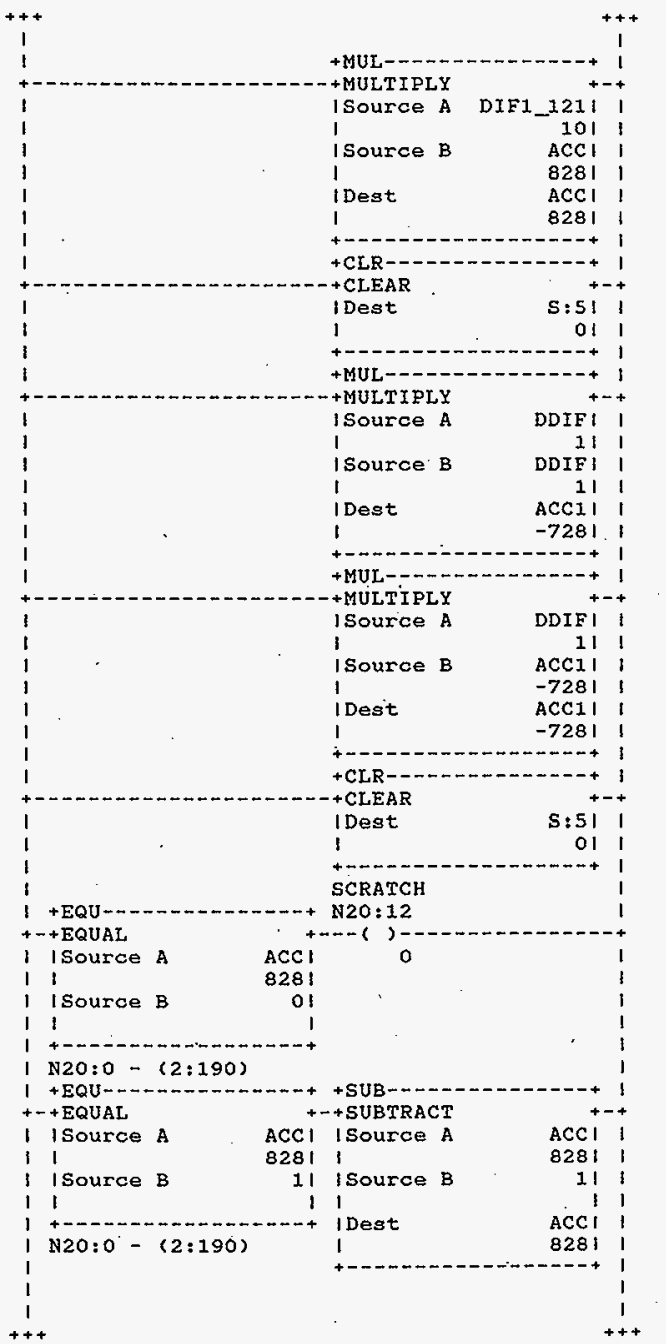




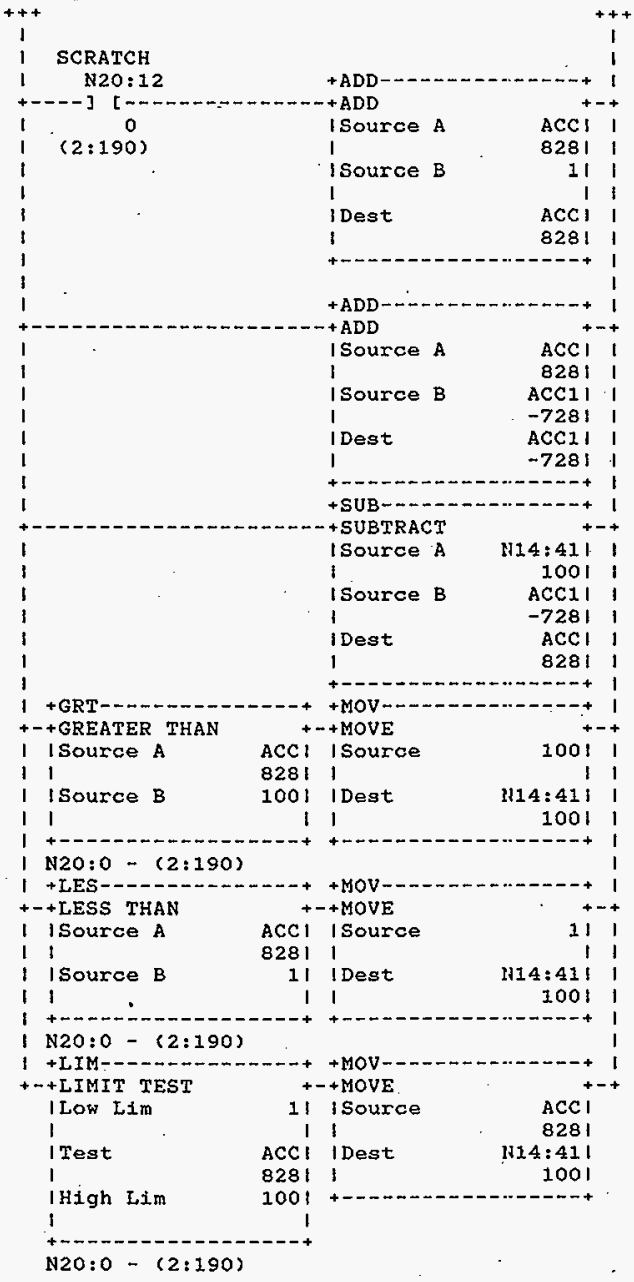




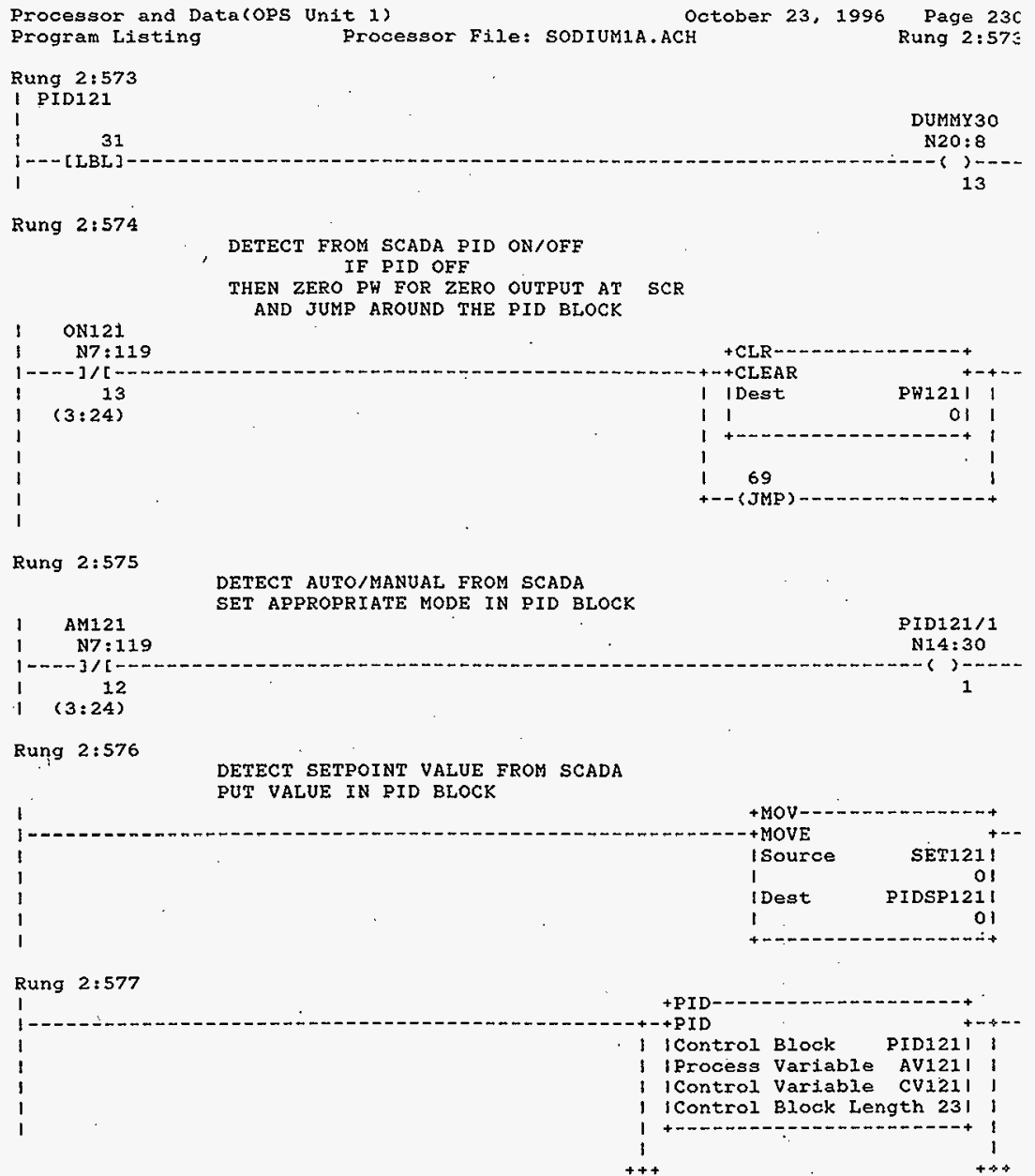

HNF-SD-FF-CSWD-61 Rev. 0 
processor and Data(OPS Unit 1)

October 23, 1996 Page 231

Program Listing

Processor File: SODIUM1A.ACH

Rung 2:577
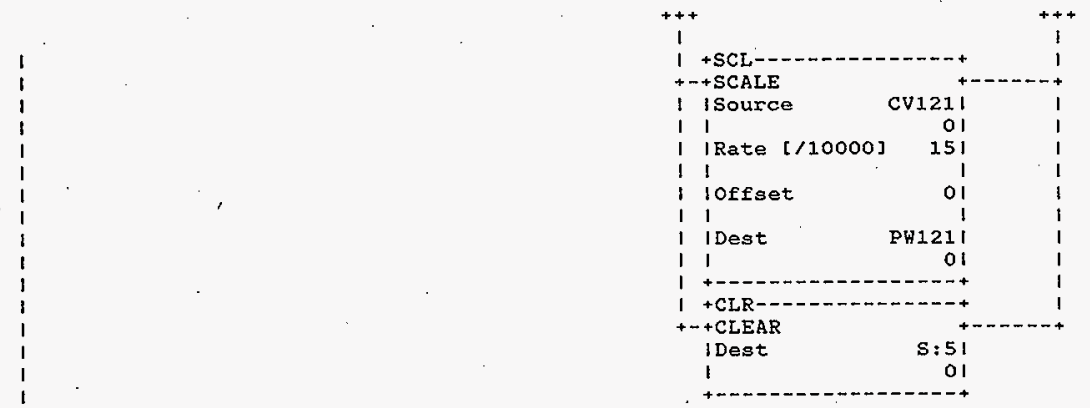

Rung 2:578

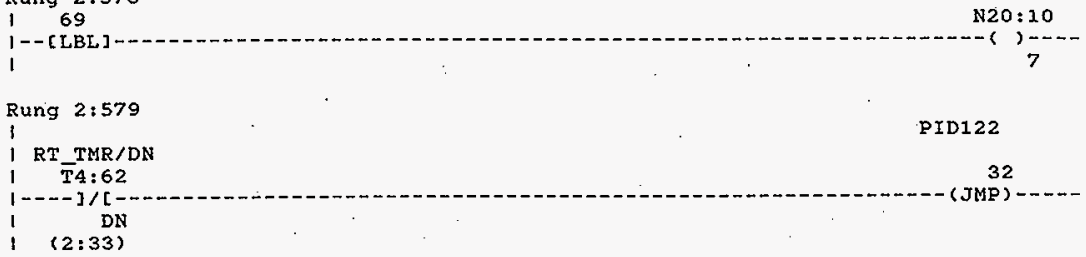

Rung $2: 580$

RATE CHECKING

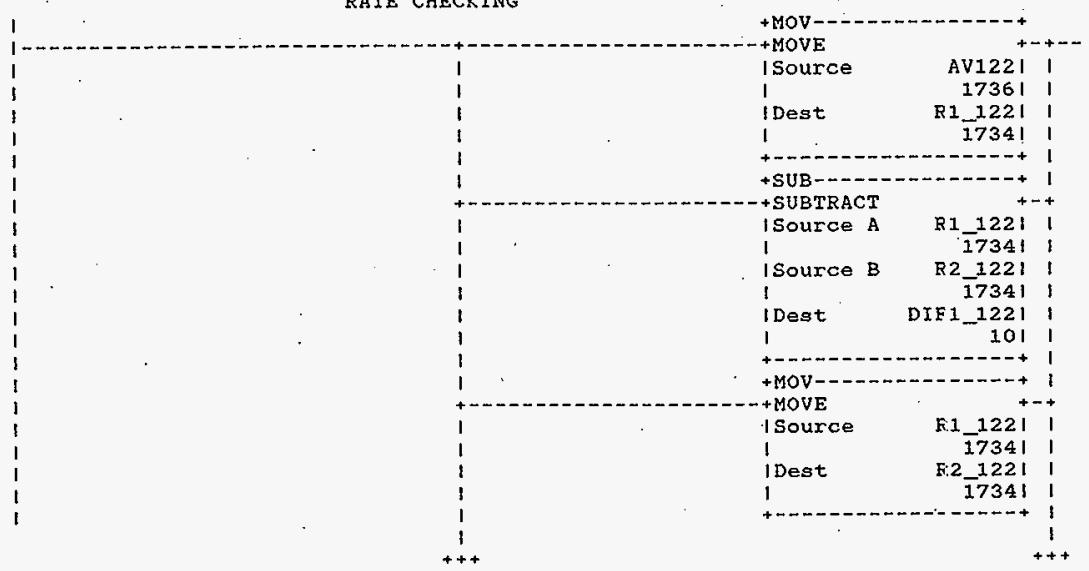

HNF-SD-FF-CSWD-61 Rev. 0

Page 231 


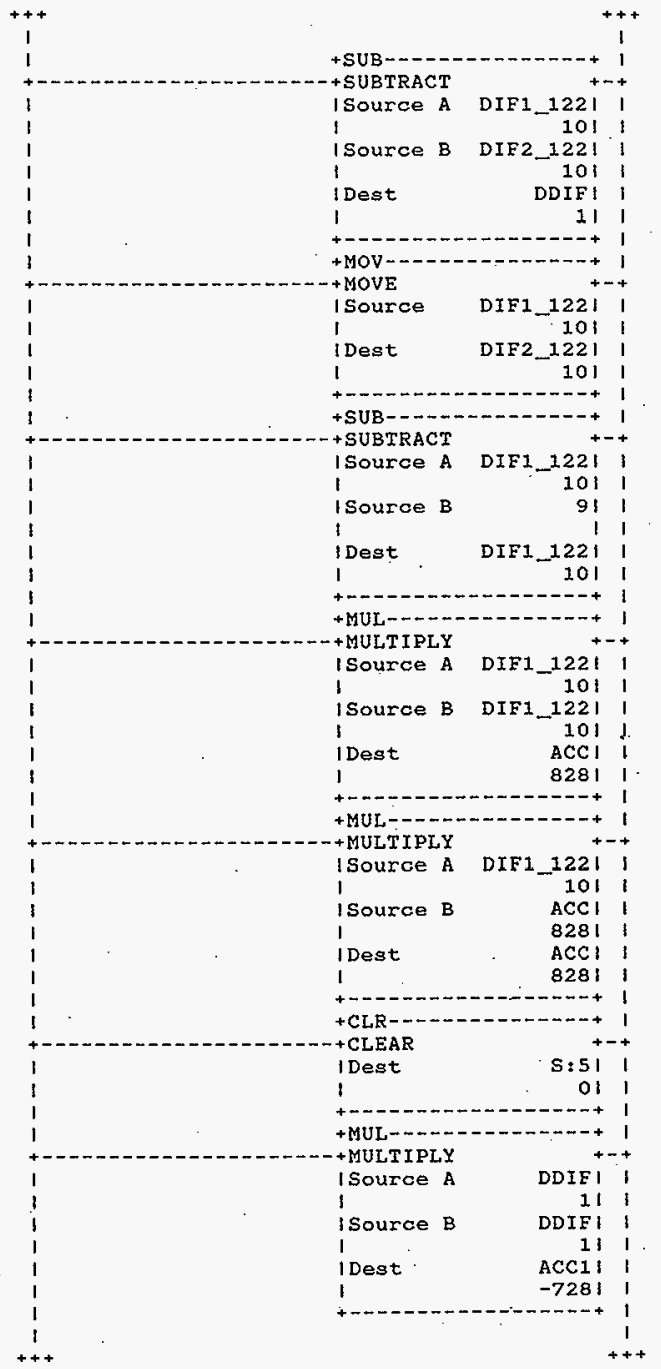

HNF-SD-FF-CSWD-61 Rev. 0 


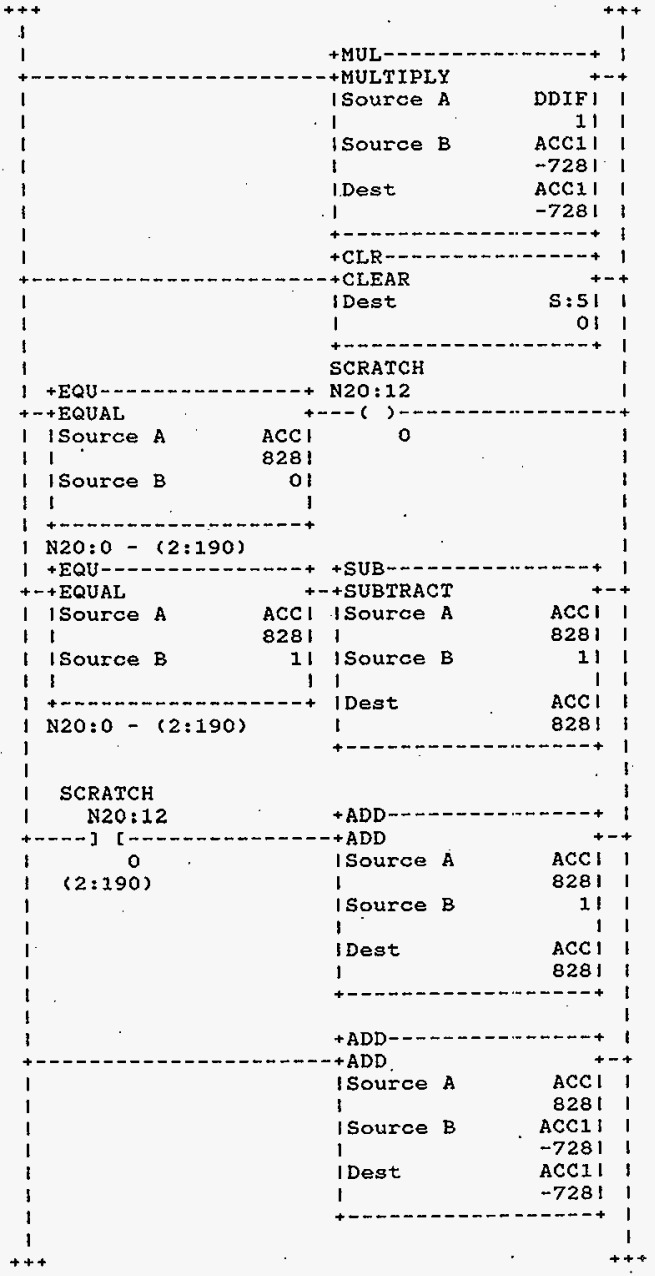

HNF-SD-FF-CSWD-61 Re:v. 0 


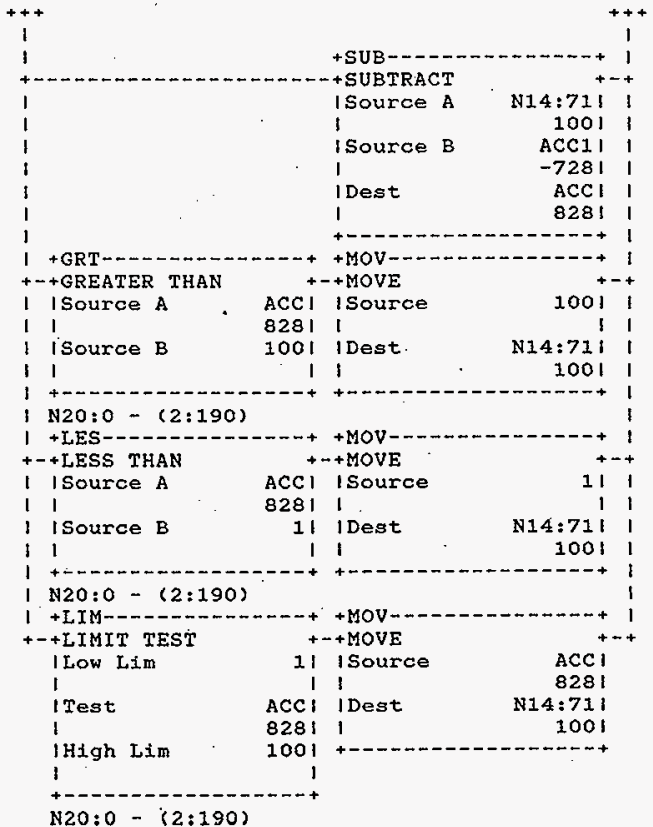

Rung 2:581

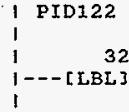

DUMMY 31 $\mathrm{N} 2 \mathrm{O}: 8$ 32

Rung 2:582

\section{DETECT FROM SCADA PID ON/OFE \\ IF PID OFF}

\section{THEN ZERO PW FOR ZERO OUTPUT AT SCR} AND JUMP AROUND THE PID BLOCK
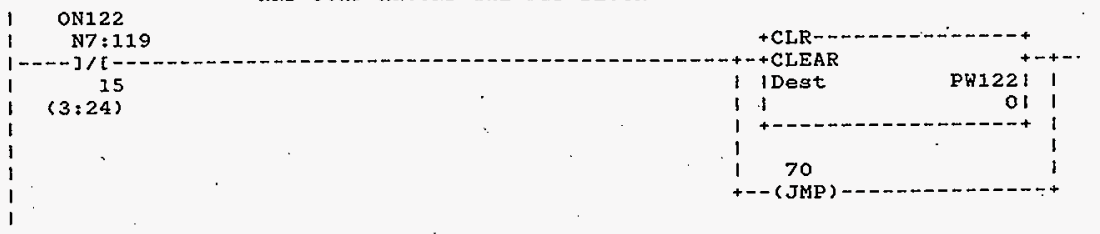
Processor and Data(OPS Unit 1)

Rung $2: 583$

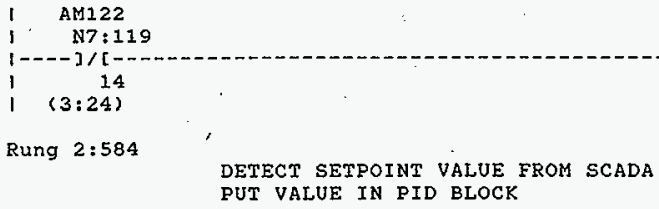

Rung 2:587

I RT TMR/DN

I T4:62 
Rung 2:588

\section{RATE CHECKING}

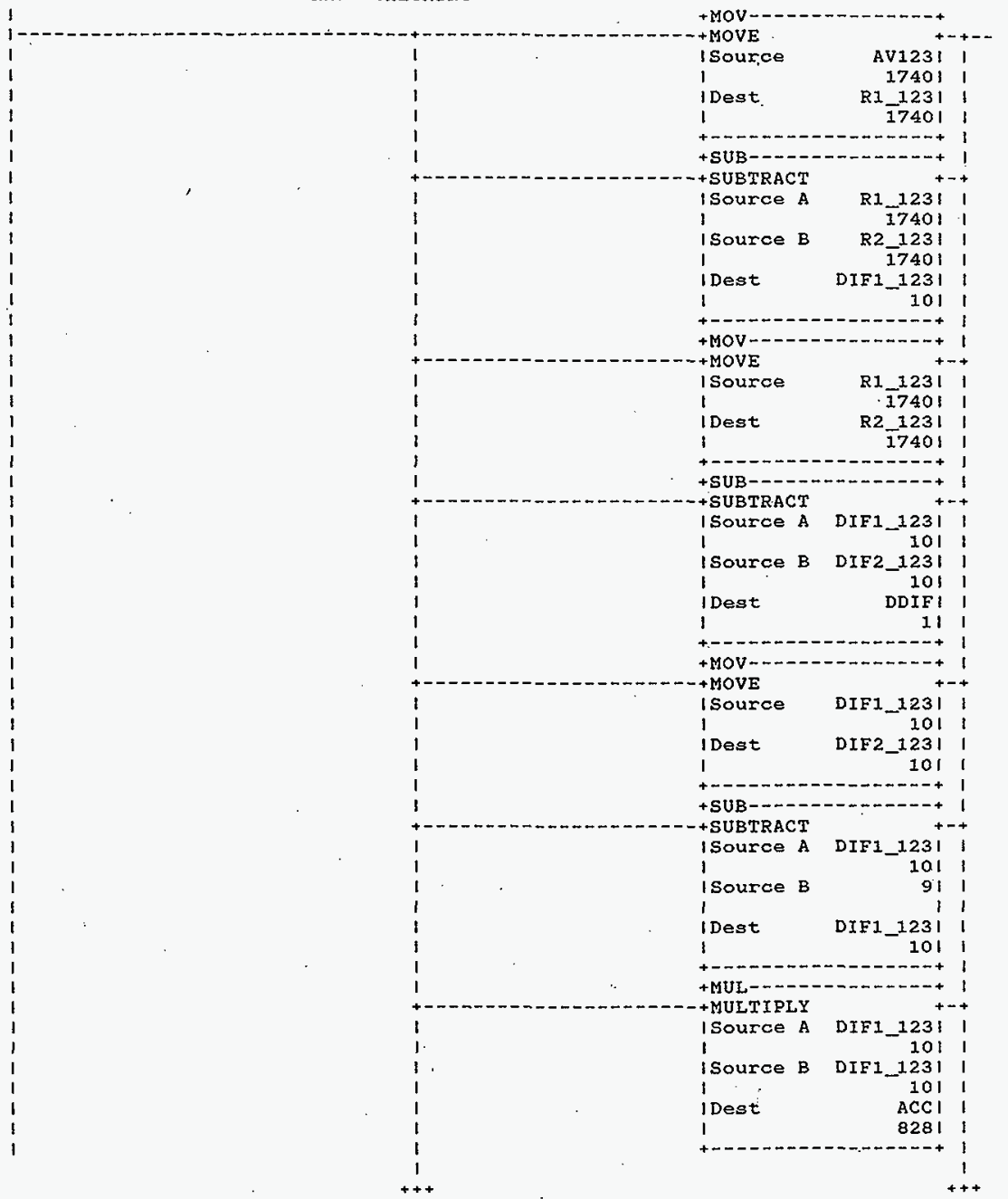

HNF-SD-FF-CSWD-61 Rev. 0 


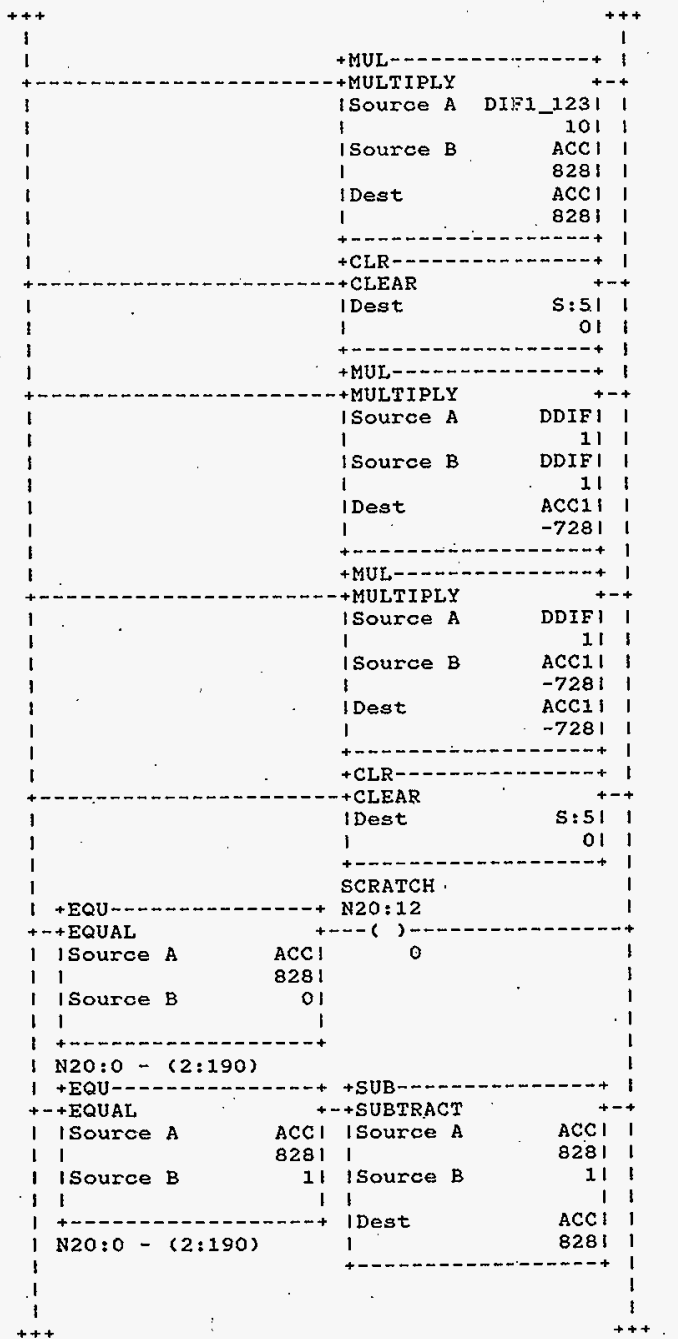

IINF-SD-FF-CSWD-6! Rev. 0 


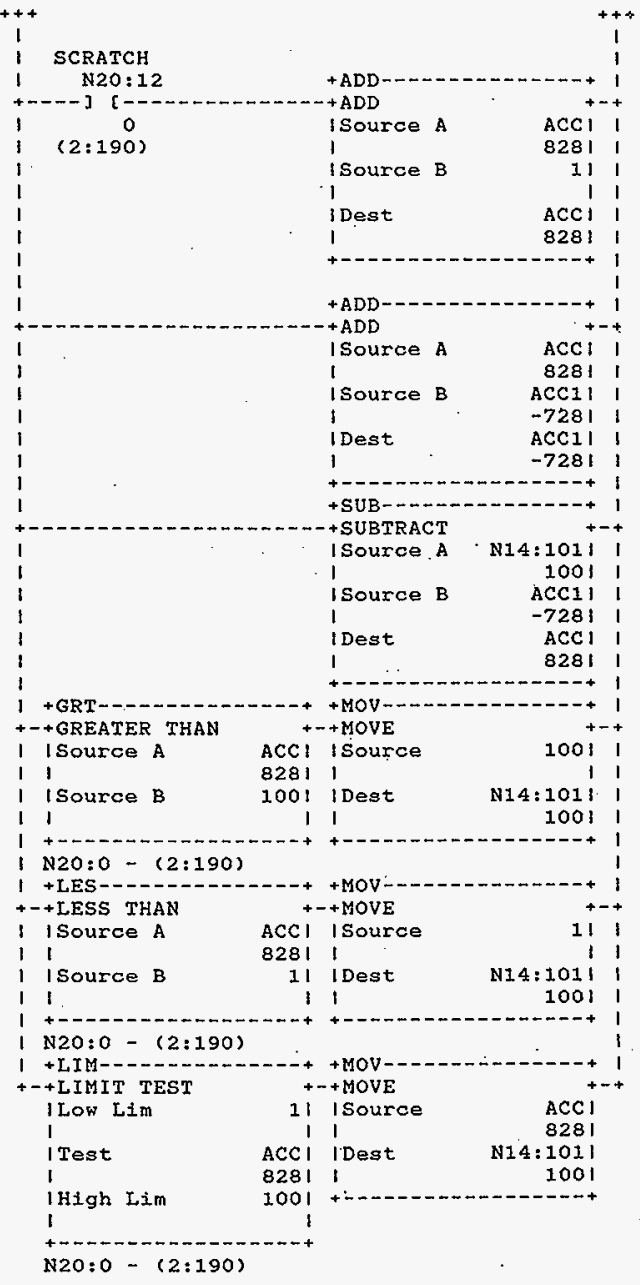


Processor and Data(OPS Unit 1 )

Rung 2:589

I PID 123

I

133

DUMMY 32

$1---[L B L,-$

N20: 8

1

Rung 2:590

DETECT FROM SCADA PID ON/OFF
IF PID OEF
THEN ZERO PW FOR ZERO OUTPUT AT SCR

AND JUMP AROUND THE PID BLOCK

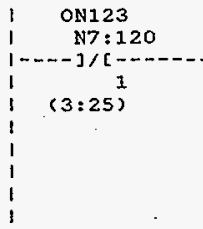

ON123

Riung 2:591

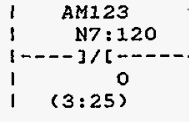

DETECT AUTO/MANUAL FROM SCADA

SET APPROPRIATE MODE IN PID BLOCK

Rung 2:592

DETECT SETPOINT VALUE FROM SCADA

PUT VALUE IN PID BLOCK

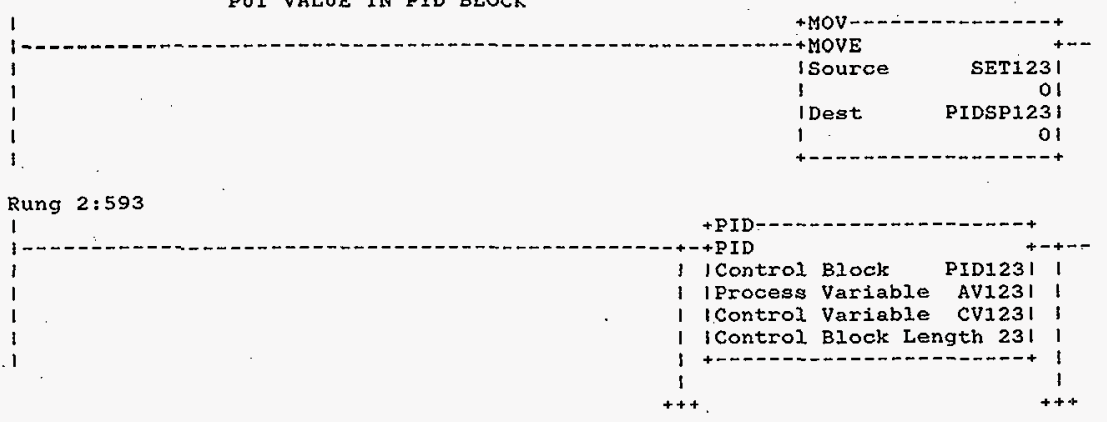




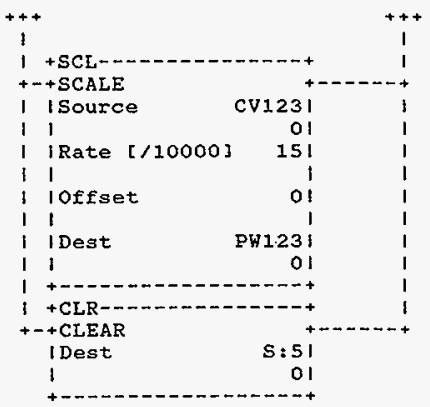

Rung 2:594

171

Rung 2:595

1

I RT_TMR/DN

I T4:62

$1-\cdots+\cdots] /[-$

$(2: 33)$

Rung 2:596

RATE CHECKING

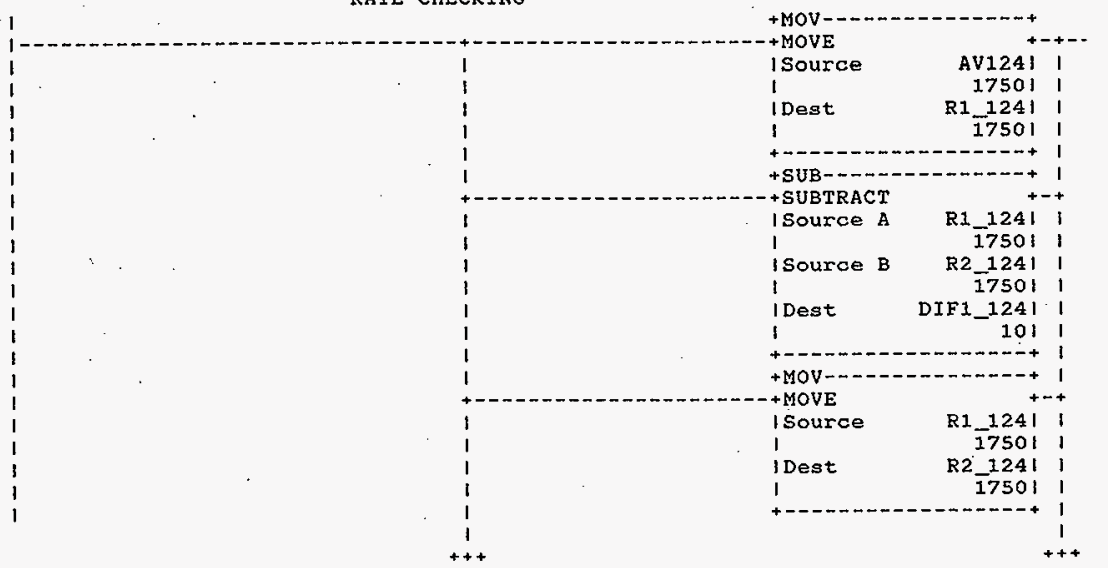

HNF-SD-FF-CSWD-61 Rev. 0 


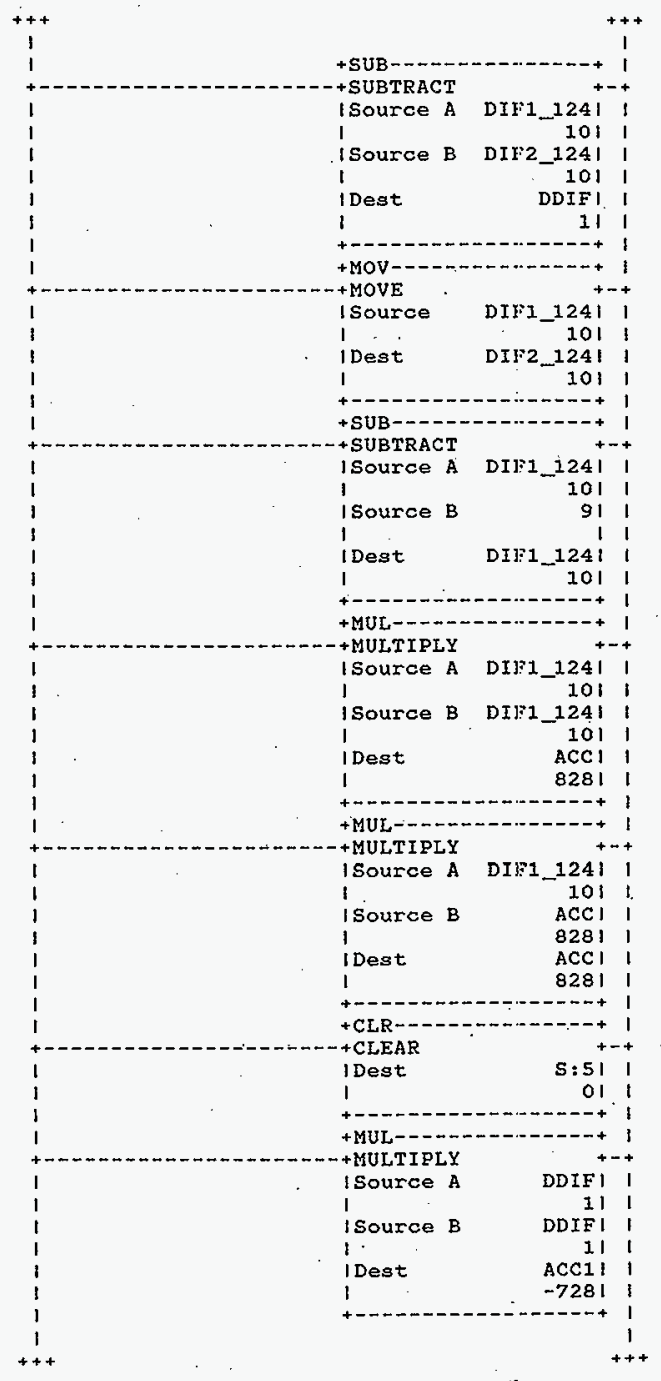

HNF-SD-FF-CSWD-61 Rev, 0 


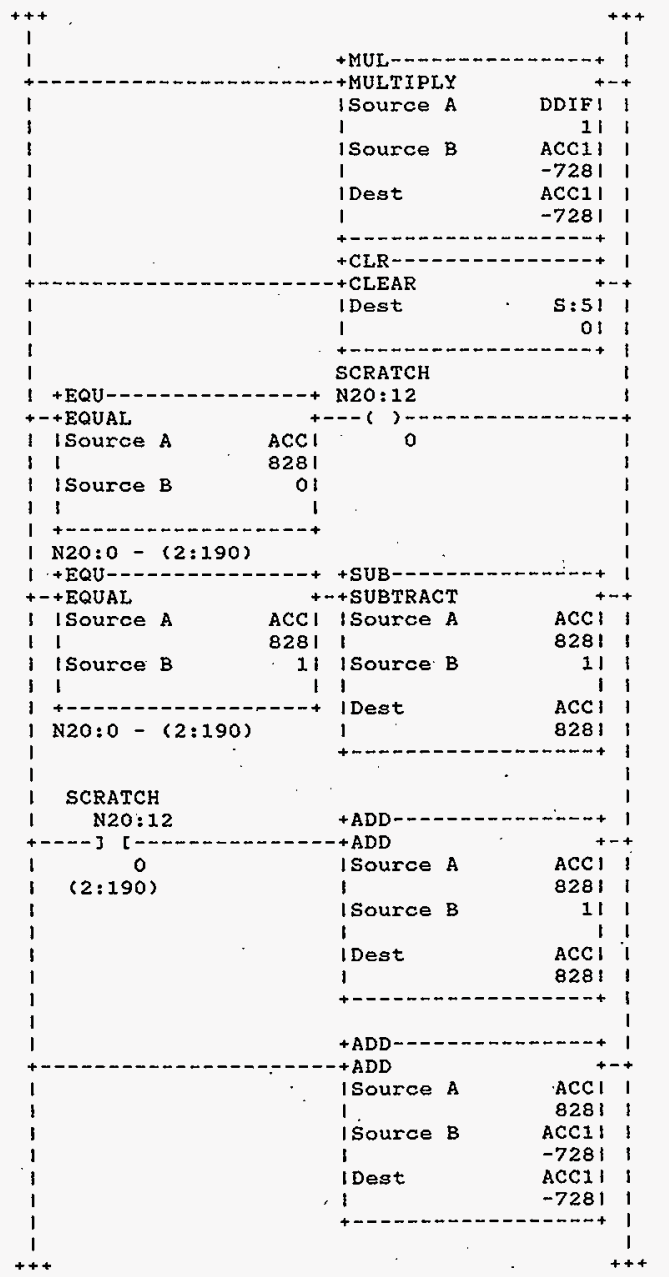

HNF-SD-FF-CSWD-61 Rev. 0 


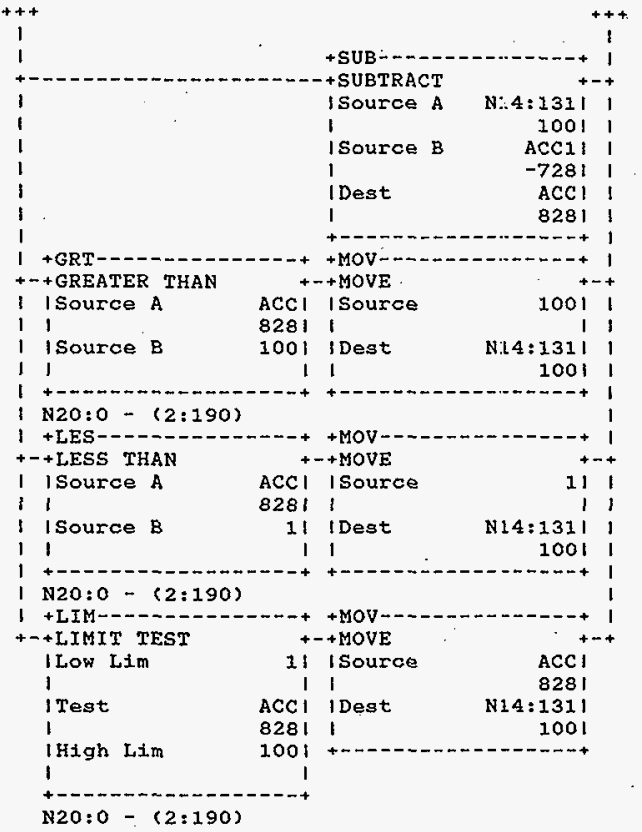

Rung 2: 597

i PID124

i

134

DUммх 33

$1--[$ LBL $]$

N20:9

Rung 2:598

\section{DETECT FROM SCADA PID ON/OFF \\ IE PID OFF \\ THEN ZERO PW FOR ZERO OUTPUT AT SCR AND JUMP AROUND THE PID BLOCK}
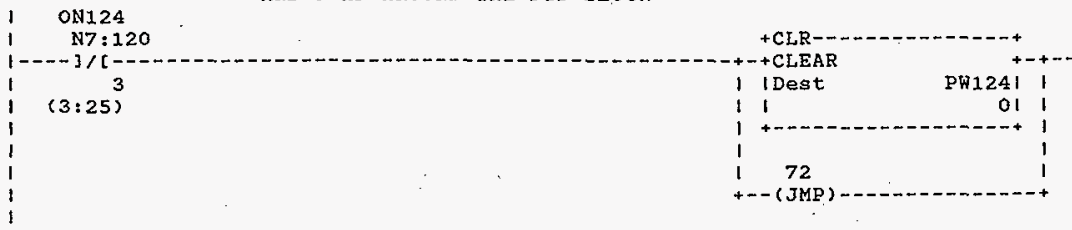

HNF-SD-FF-CSWD-61 Rev. 0 
Processor and Data(ops Unit 1)

Rung 2:599

DETECT AUTO/MANUAL FROM SCADA

SET APPROPRIATE MODE IN PID BLOCK

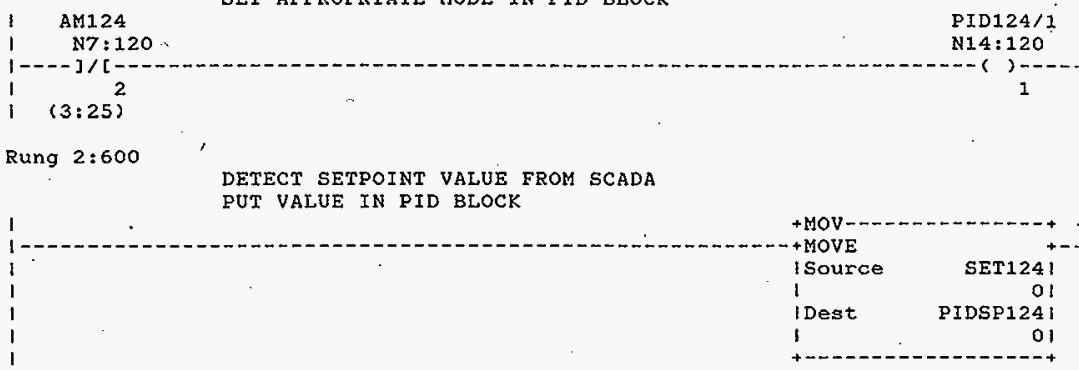

Rung 2:601

!

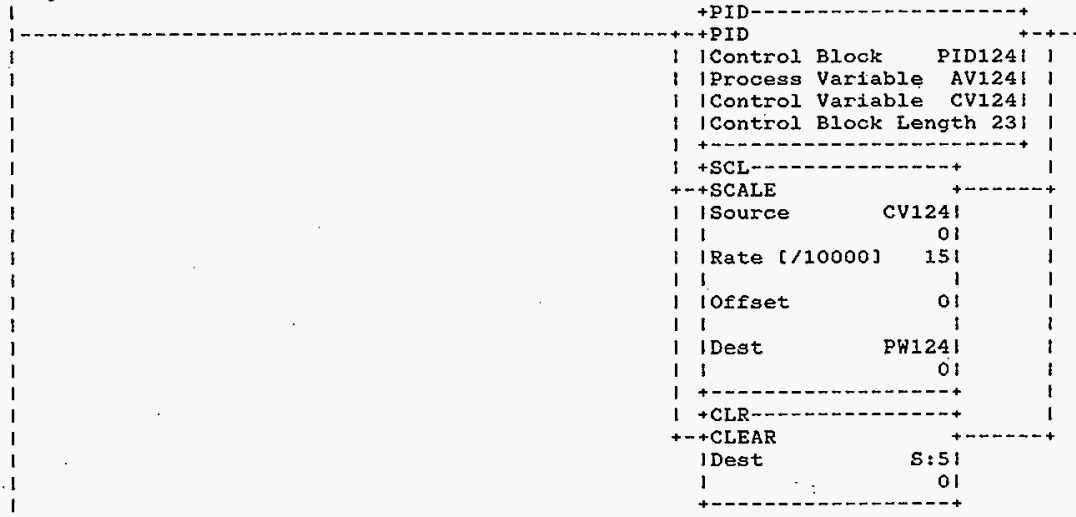

Rung 2:602

172

N20:10

$1--[$ LBL

Rung $2: 603$

1

RT $T M R / D N$

PID125

$\bar{T} 4: 62$

35

$1----] /[-$

$1(2: 33)$

HNF-SD-FF-CSWD-61 Rev. 0 
Rung 2:604

1 . RT TMR/DN

DNR_3

$\mathrm{T} 4: 62$

N20:5

$1----][-$

$(2: 33)$

Rung 2:605

RATE CHECKING

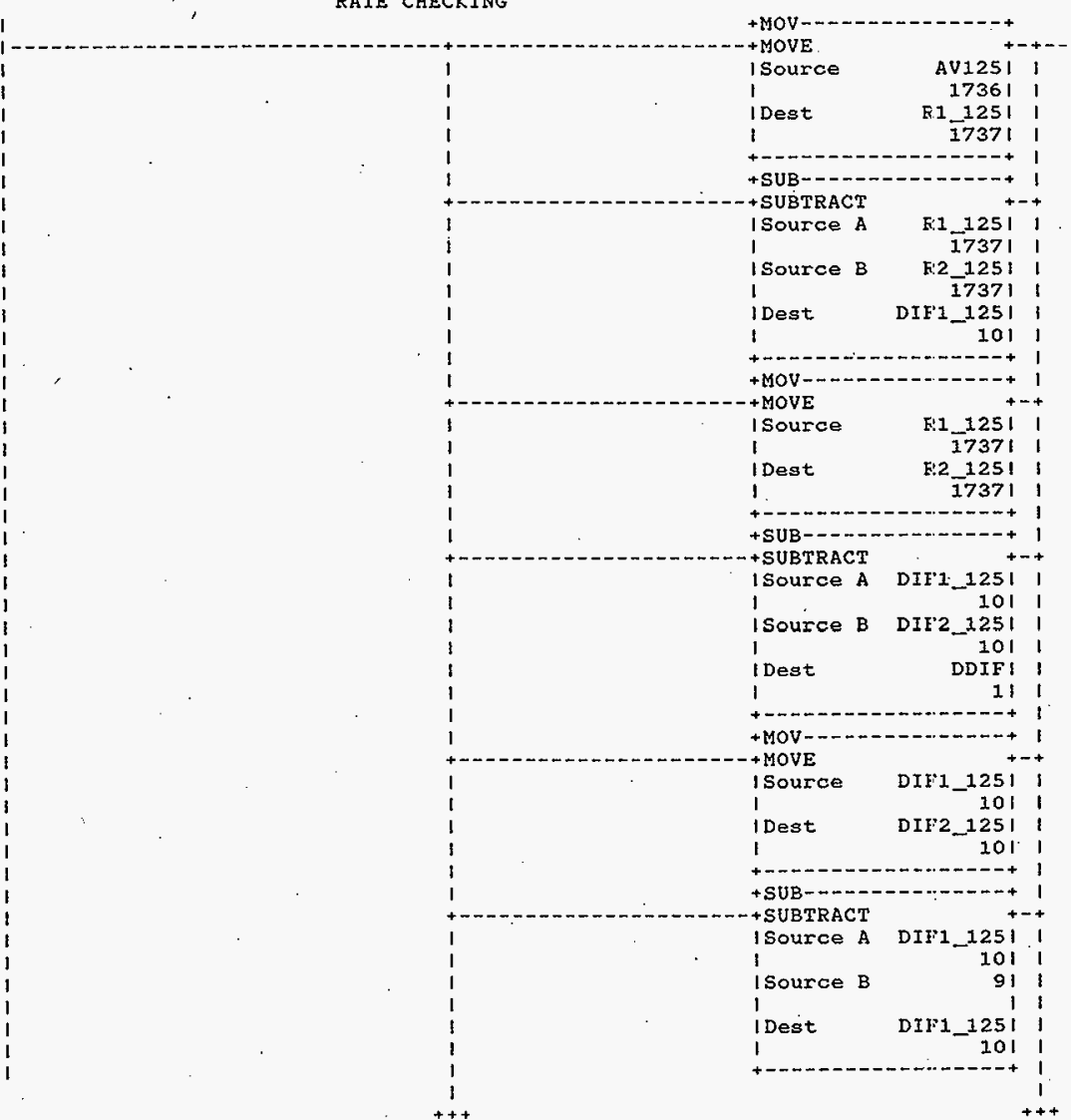

HNF-SD-FF-CSWD-61 Rev. 0 


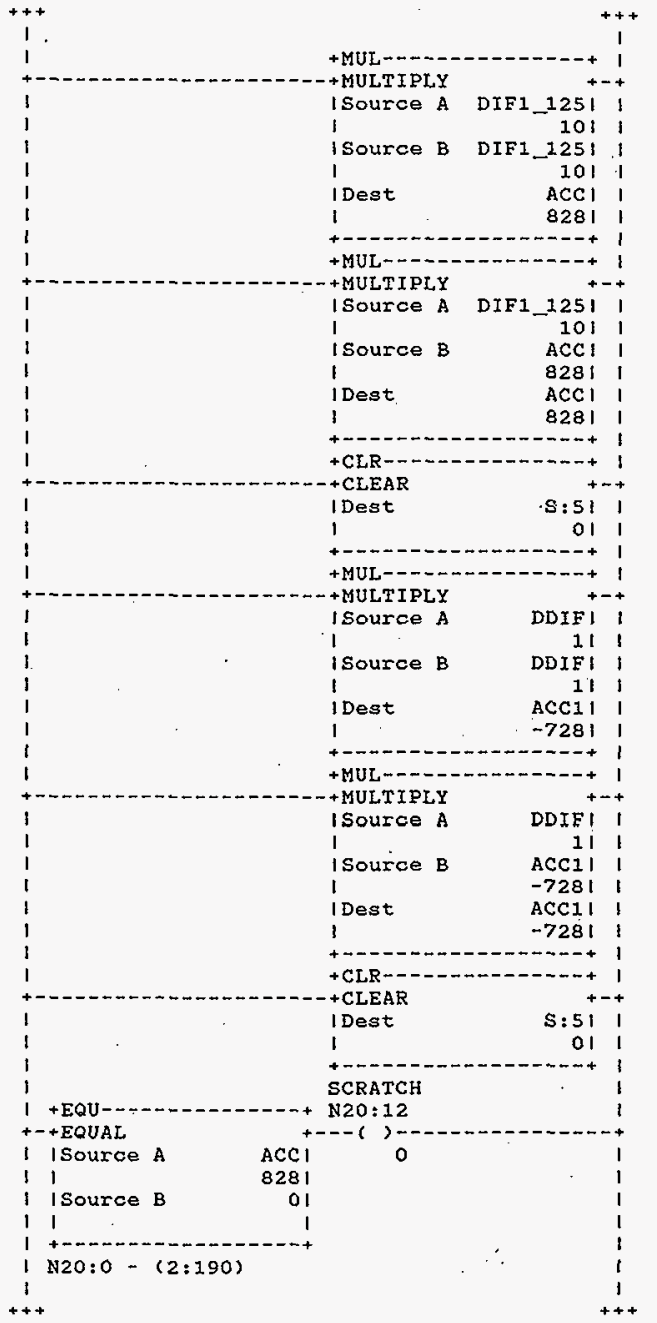

HNF-SD-FF-CSWD-61 Rev. 0 


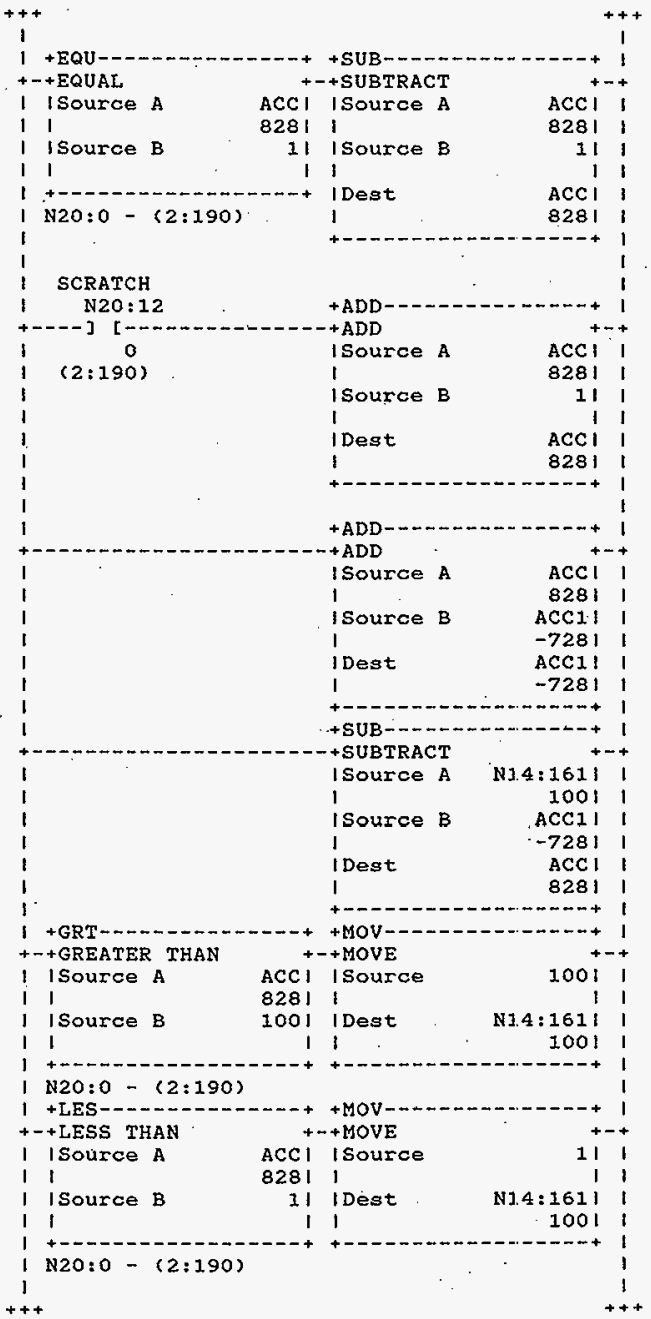

HNF-SD-FF-CSWD-61 Rev. 0 


$$
\begin{aligned}
& 1 \\
& 1 \\
& 1 \\
& 1 \\
& 1 \\
& 1 \\
& 1 \\
& 1 \\
& 1 \\
& 1
\end{aligned}
$$

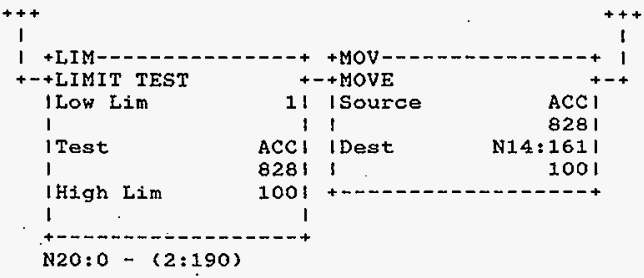

Rung 2:606

I PID125

I 35

DUMMY 34

N20:9

Rung 2:607

DETECT FROM SCADA PID ON/OFF

IF PID OFF

THEN ZERO PW FOR ZERO OUTPUT AT SCR

AND JUMP AROUND THE PID BLOCK

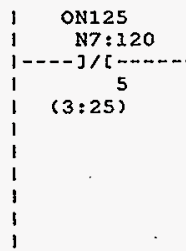

\section{AND JUMP AROUND THE PID BLOCK}

Rung 2:608

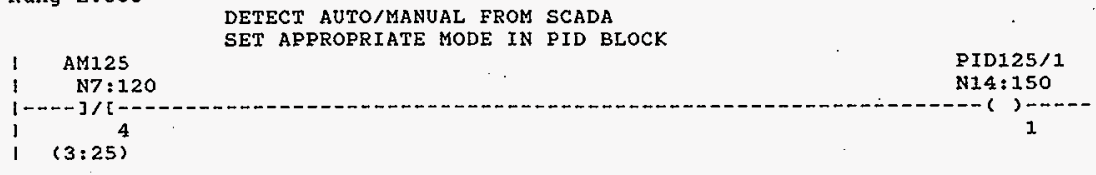

Rung 2:609

DETECT SETPOINT VALUE FROM SCADA

PUT VALUE IN PID BLOCK

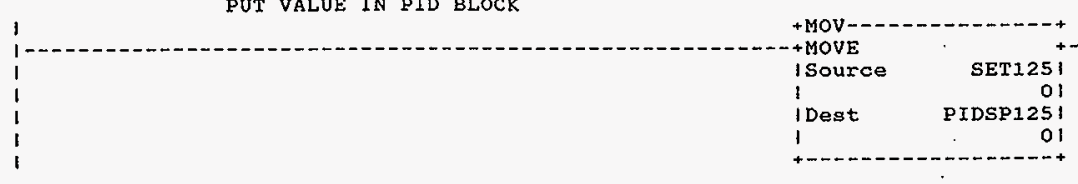

HNF-SD-FF-CSWD-61 Rev. 0 


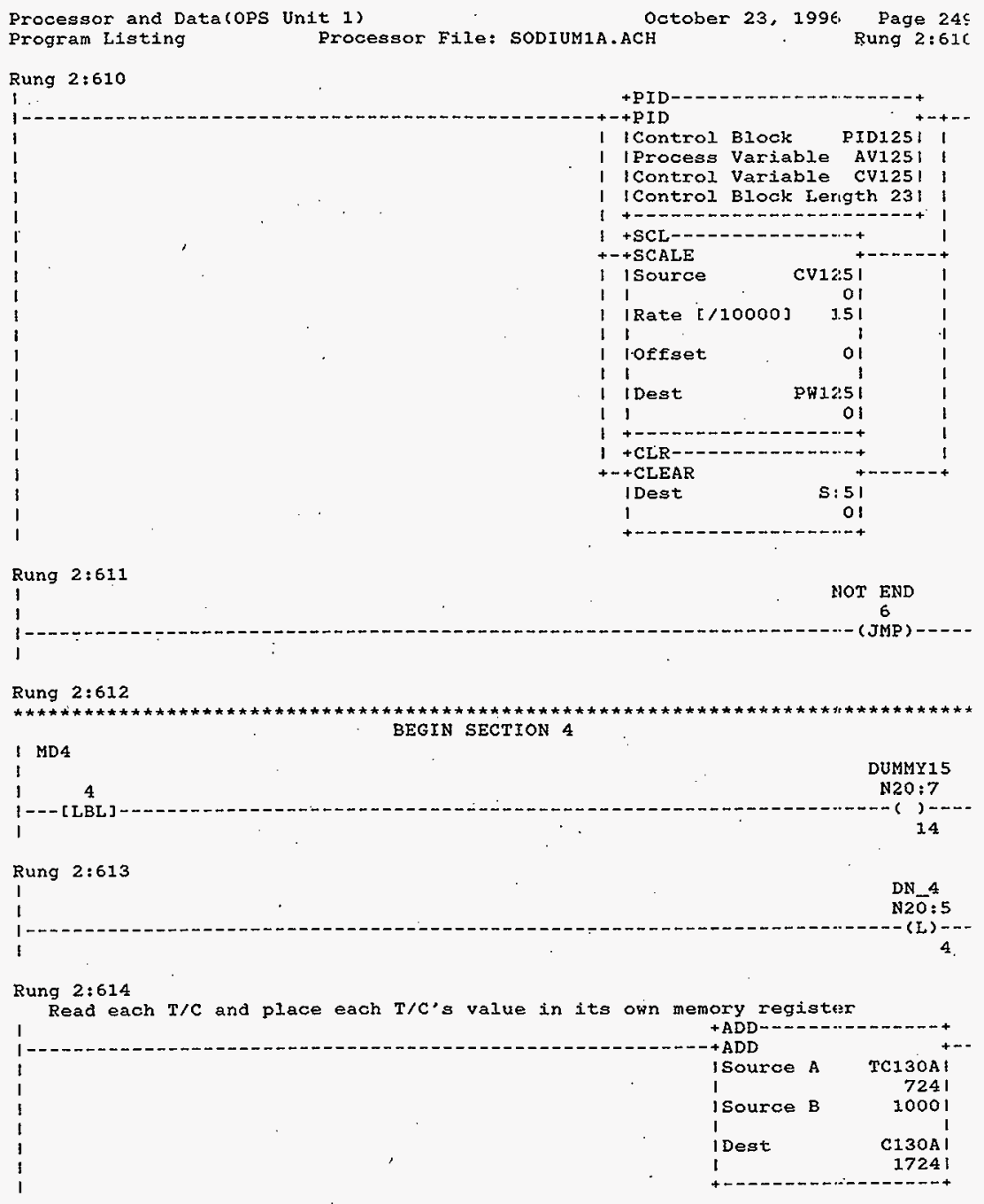

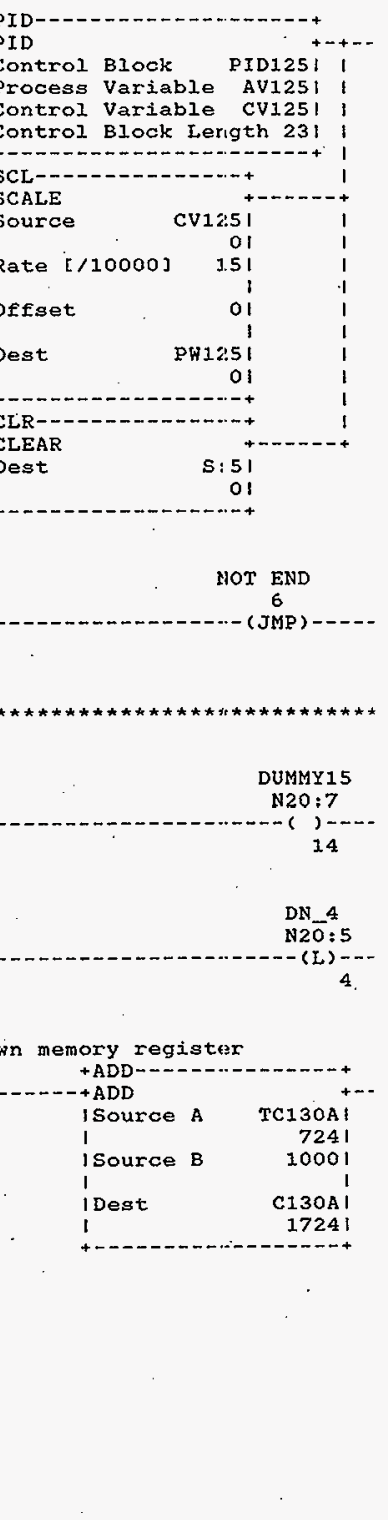

Rung $2: 612$

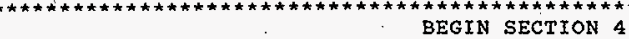

1 MD4

14

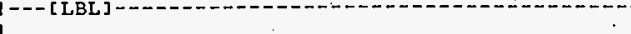


Processor and Data(OPS Unit 1) Program Ligting

\section{Rung 2:615}

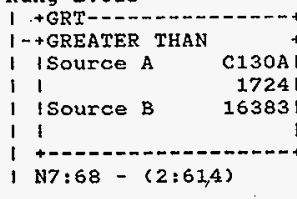

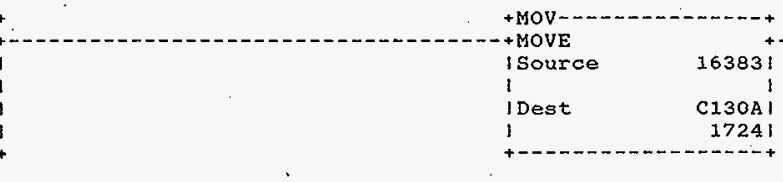

Rung 2:616

$1+$ + ADD

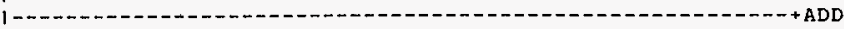

1 ISource A TC130B

I .

$$
\text { (1) }
$$

HNF-SD-FF-CSWD-61 Rev. 0 
Rung $2: 620$

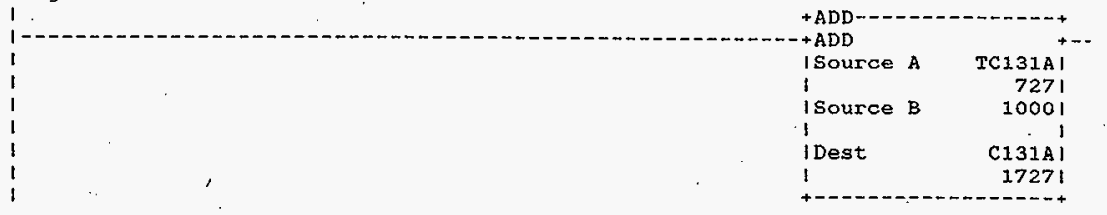

Rung $2: 621$

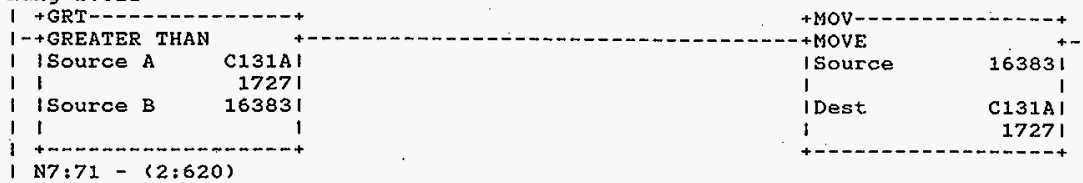

Rung $2: 622^{\circ}$

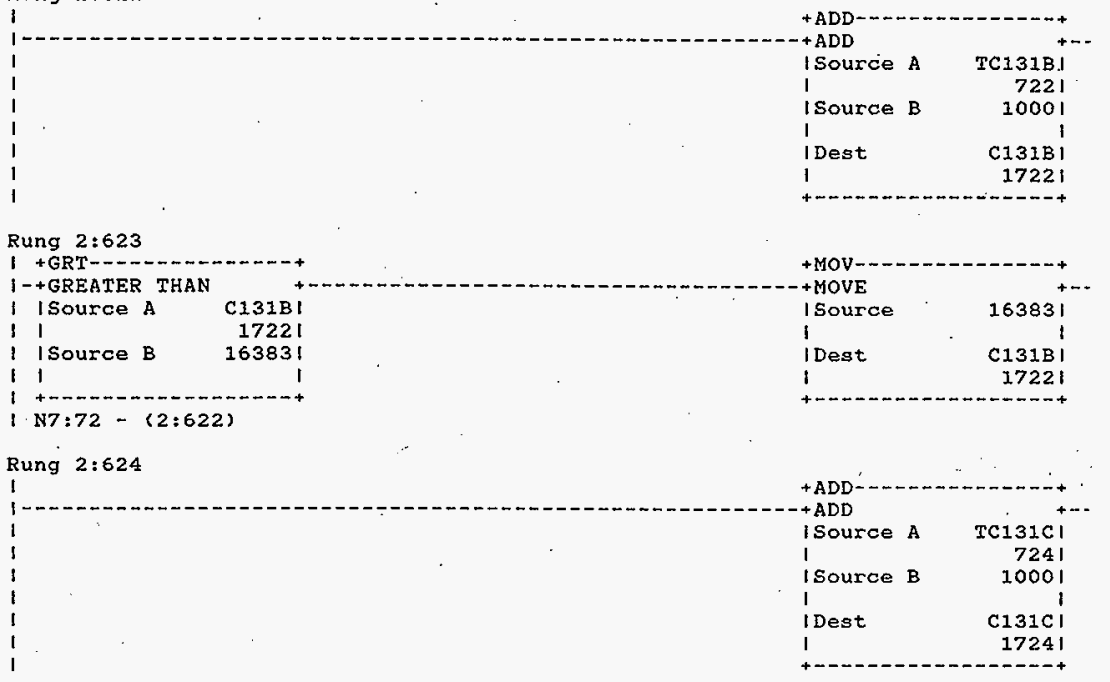

HNF-SD-FF-CSWD-61 Rev. 0 
Processor and Data(OPS Unit 1 )

Rung 2:625

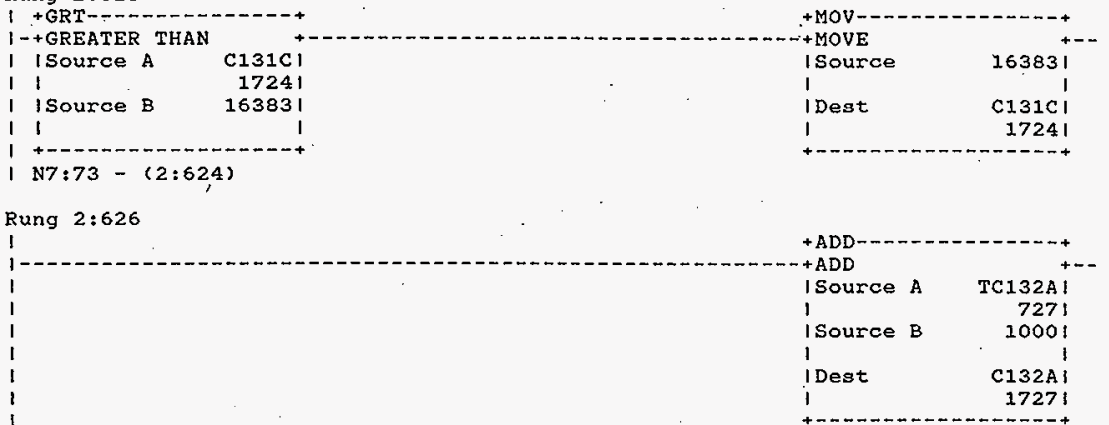

Rung $2: 627$

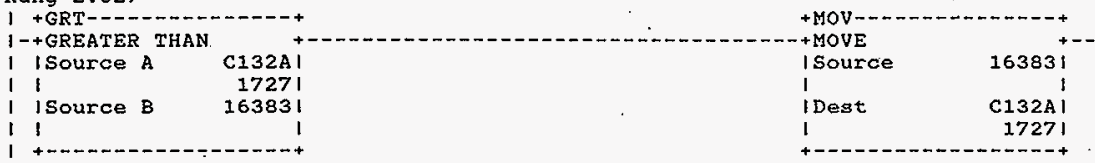

( $17: 74-(2: 626)$

Rung 2:628

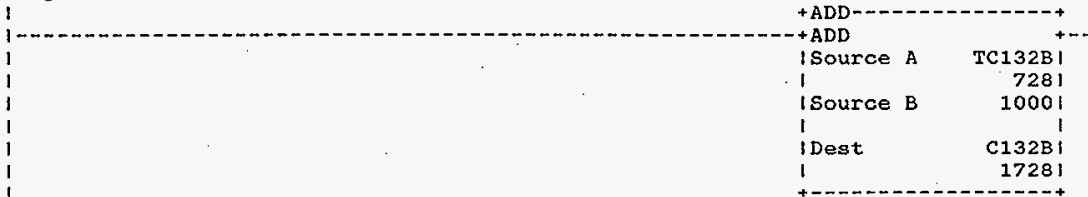

Rung 2:629

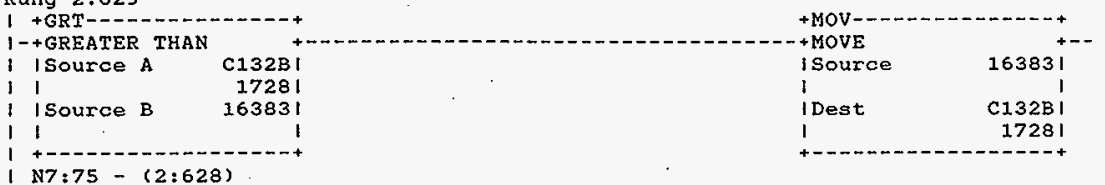


Processor and Data(OPS Unit 1)

Rung $2: 630$

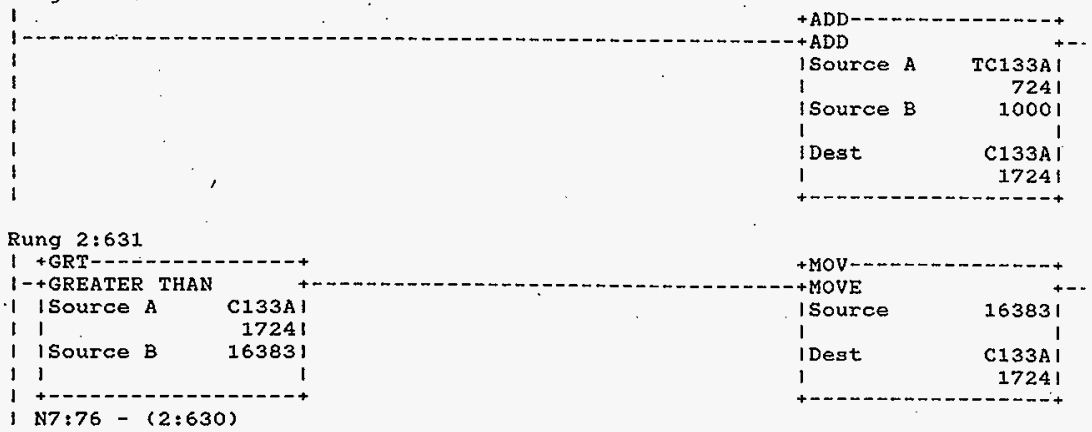

I N7:76-(2:630)

Rung 2:632

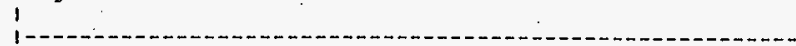

$+\mathrm{ADD}$

$+A D D$

isource A

TC133B !

1

ISource B

7171

I

10001

I Dest

C133B I

I

Rung $2: 633$

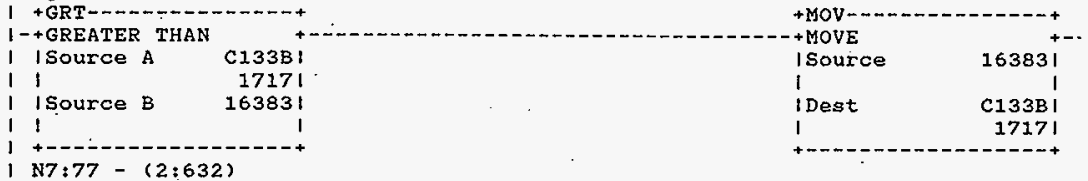

Rung $2: 634$

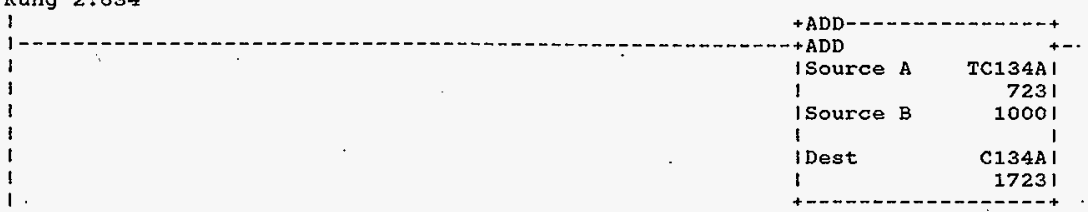


Processor and Data(Óg Unit 1)

Rung $2: 635$

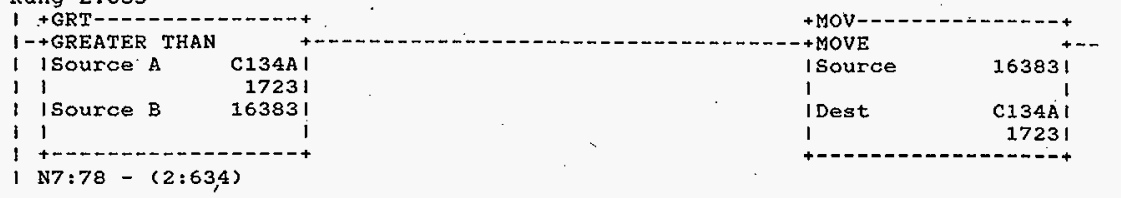

Rung 2:636

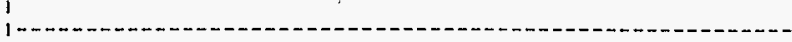

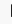

I

I

I

I

$+\mathrm{ADD}$

ISource A TC134B |

$1 \quad 7221$

Isource B

1000

I

I Dest

C134B I

I

17221

Rung 2:637

| +GRT--_._...-.-.+

I-+GREATER THAN
I ISource A CI $34 \mathrm{~B}$

I isource A $\quad$ C134B

| ISource B 16383 I

11

! I

$+-----\cdots----n-\cdots+$

N7:79-(2:636)

Rung $2: 638$

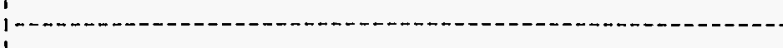

1.

1

I

I

1

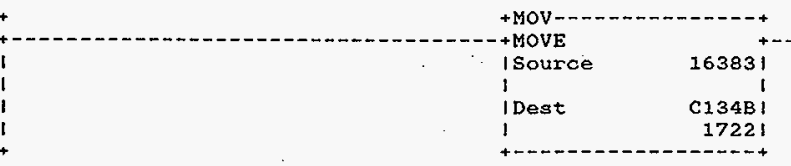

Rung 2:639

\begin{tabular}{|c|c|c|c|}
\hline \multicolumn{4}{|c|}{ I + GRT-- } \\
\hline I-+GREATER & + & - +MOVE & +- \\
\hline I I Source & C140AI & I Source & 163831 \\
\hline 1 & 1726 & 1 & i \\
\hline I Source $B$ & 163831 & I Dest & C140AI \\
\hline 1 & 1 & 1 & 17261 \\
\hline+------- & -----+ & +------ & $--m-\infty+$ \\
\hline
\end{tabular}

HNF-SD-FF-CSWD-61 Rev. 0 
Rung 2:640

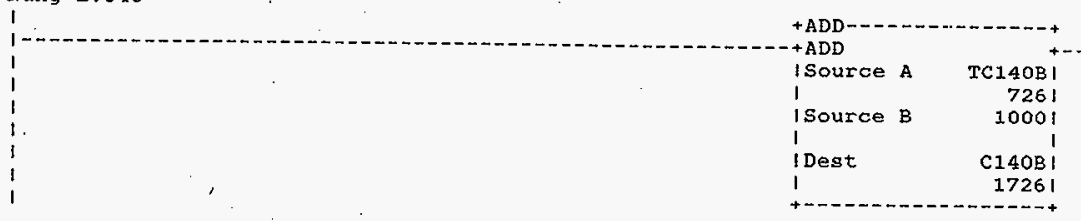

Rung $2: 641$

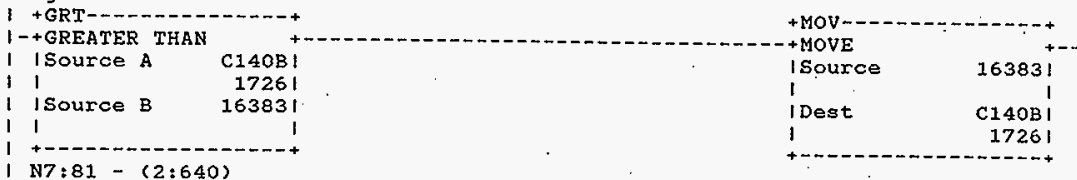

Rung $2: 642$

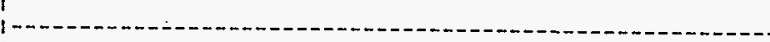

$+A D D$

isource A TC140C1

1

I Source B

7321

1

I Dest

10001

I

C $140 \mathrm{Cl}$

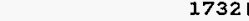

Rung 2:643

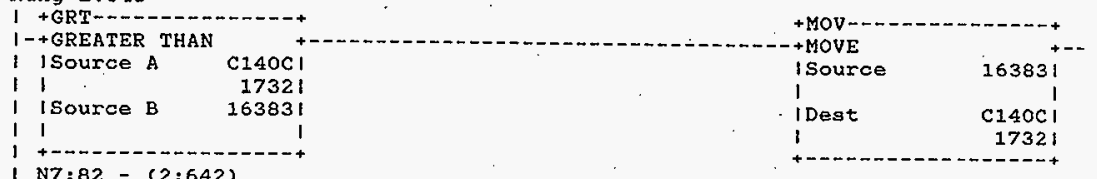

Rung 2:644

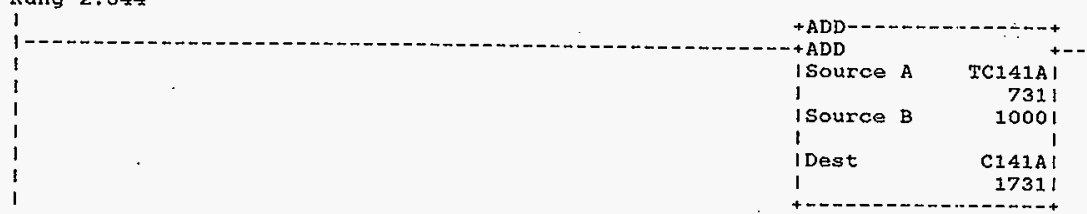

HNF-SD-FF-CSWD-61 Rev, D 


\section{Rung 2:645}

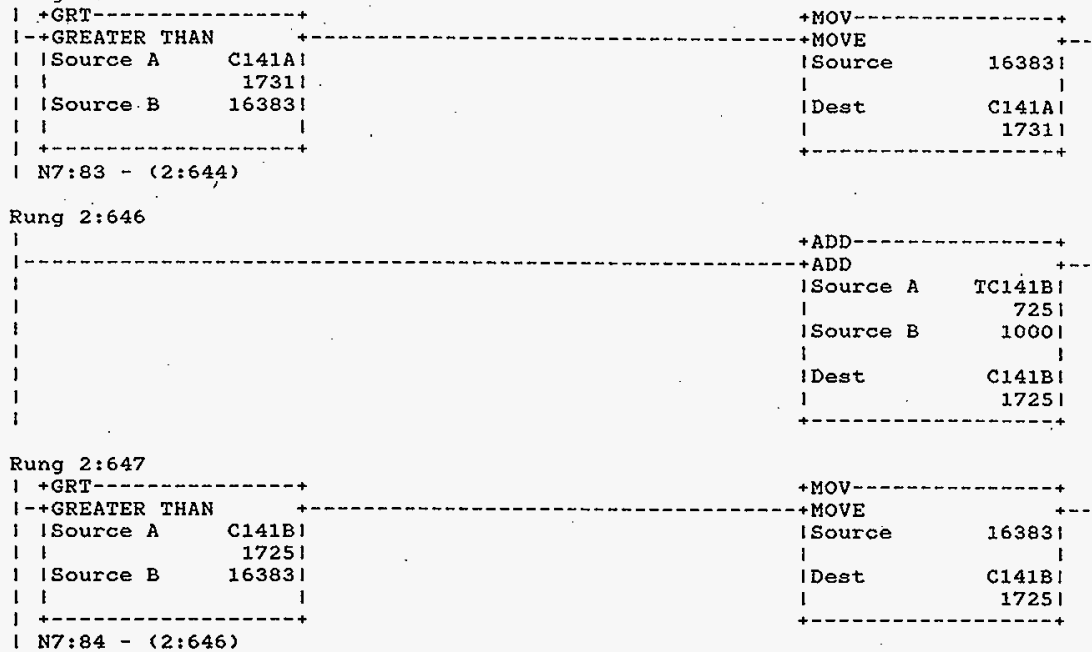

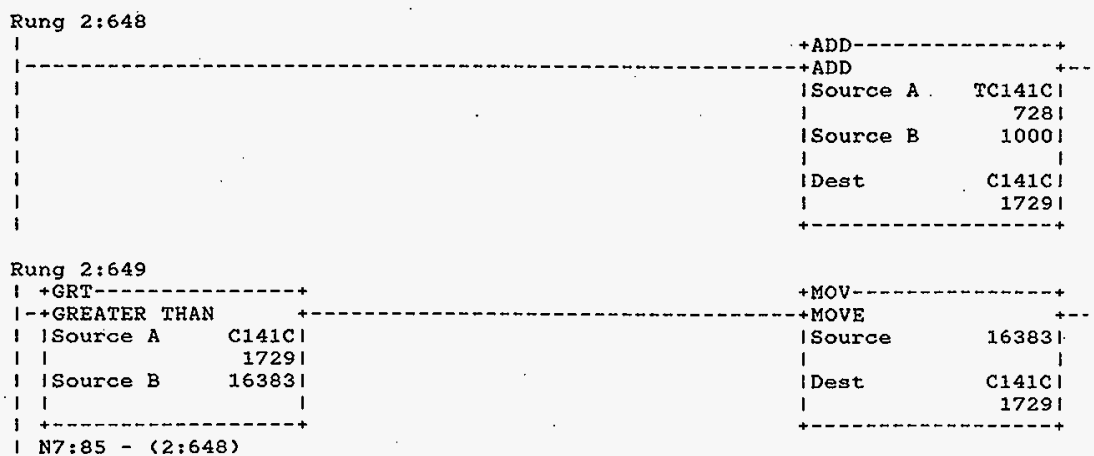


Rung 2:650

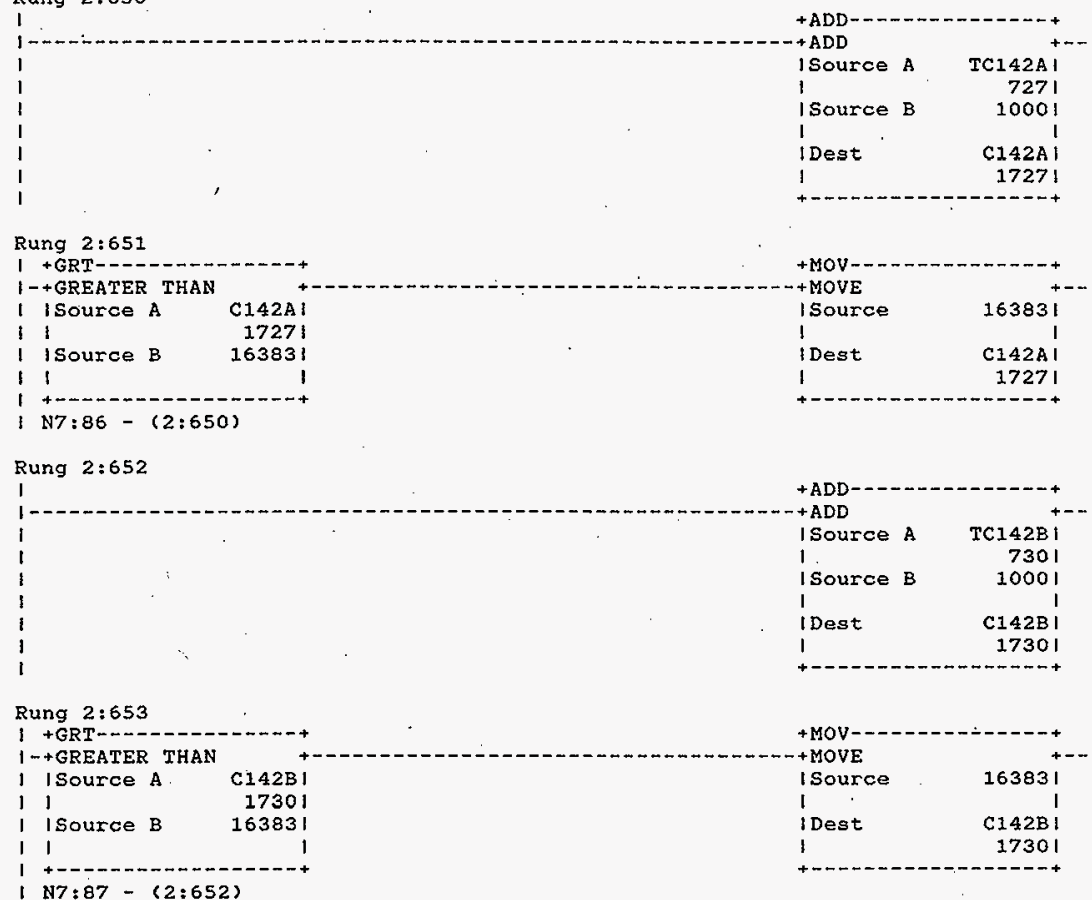

Rung $2: 654$

For each T/C read Its STATUS BITS to detect T/C problems HARDWARE ALARMS FOR TANK T-3002

$\begin{array}{llll}1 & \\ 1 \\ 1 \\ 1\end{array}$


Processor and Data(OPS Unit i) Program Listing

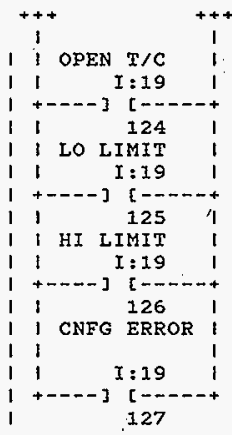

Rung 2:655

I

I/C ENABLE

1 OPEN T/C

I I I:20

$1+\ldots+\cdots][\ldots+\ldots+$

1176

1) LO LIMIT

11 I:20

$1+\ldots-\cdots[-\ldots+$

1177

I I HI LIMIT

1. 1 I: 20

$1+----][----+$

$1178 \quad 1$

I CNFG ERROR I

I 1

11

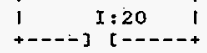

79

Rung 2:656

1

T/C ENABLE

1 $I: 20$

N7: 244

11

91 


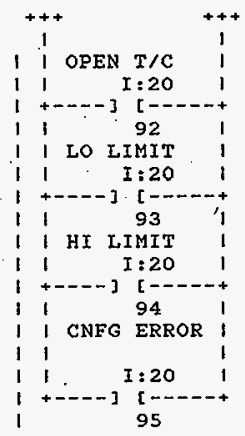

\section{Rung 2:657}

1

\section{T/C ENABLE}

$$
I: 20
$$

HA131A

\section{Rung 2:658}

I

T/C ENABLE

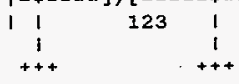

HNF-SD-FF-CSWD-61 Rev. 0 


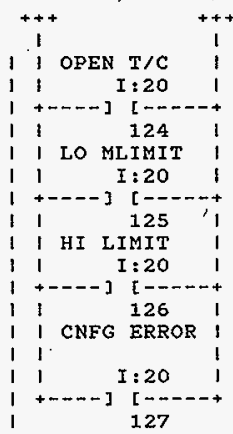

Rung 2:659

T/C ENABLE

$I: 21$

HA13IC

N7: 244

$1-+\cdots-2] / 2-12$

75

OPEN $\mathrm{T} / \mathrm{C}$

$\begin{array}{lll}1 & 1 \\ 1 & 1: 21 & 1\end{array}$

1176

1 LO LIMIT

l $1 \quad I: 21$

$1+--n-7[-2,-+$

1177

I I HI IIMIT

I I I:2I ।

$1+----][--\cdots+$

$11 \quad 79 \quad 1$

1 CNFG ERROR ।

1) 1

1) I $\quad$ I $21 \quad$ ।

$1+\cdots--3$ [ $[-\cdots--+$

179

Rung 2:660

I

$T / C$ ENABLE

$I: 21$

HA $132 \mathrm{~A}$

N7: 244

$1-+\cdots--7 /[----+$

$\begin{array}{ccc}1 & 91 & 1 \\ +++ & & 1 \\ +++.\end{array}$

HNF-SD-FF-CSWD-61 Rev, 0 
Procegsor and Data(OPS Unit 1)

Program Listing

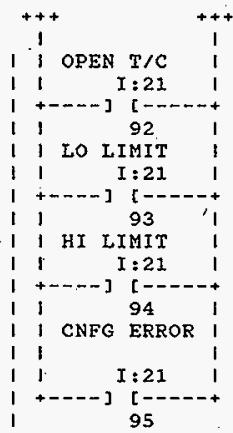

Rung $2: 661$

I

T/C ENABLE

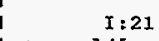

HA132B

$I: 21$

N7 $: 244$

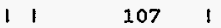

10

i OPEN T/C

1 I $I: 21$

$1+----3$ t-----+

11108

1 I LO LIMIT

1

$1+\ldots][----+$

I 109

i I HI LIMIT

1

I:21 ।

1 1110 ,

I I CNFG ERROR I

I

I 1 I +21 I

111

Rung $2: 662$

I

I

$T / C$ ENABLE

$I: 21$

HA 133 A

$\begin{array}{lll}1 & 1\end{array}$

N7: 244

$123 \quad \mathrm{I}$

$++$ 
Processor and Data(OPS Unit 1)

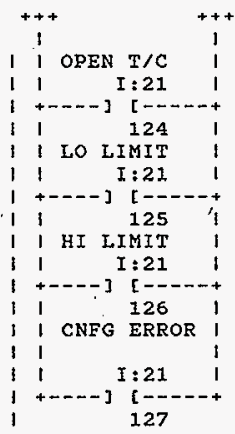

Rung 2:663

I

T/C ENABLE

HA1 33 B

$I: 22$

N7 $: 244$

75

12

$\begin{array}{lll}1 & 75 \\ 1 & \text { OPEN T/C }\end{array}$

1 I $\quad$ I:22

$1+\ldots-\ldots]$ [-.-.+

1176

1 LO IIMIT

$1 \quad I: 22$

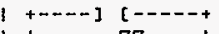

$11 \quad 771$

1 HI LIMIT

1 I I:22 ।

$1+\cdots-\infty[\cdots+\cdots$

| 1 CNFG ERROR

11

$\begin{array}{lll}1 & 1 & I: 22\end{array}$

++
1
1
+
1
1
1
+
1
1
+
1
1
1
+

I

Rung 2:664

1

1

T/C ENABLE

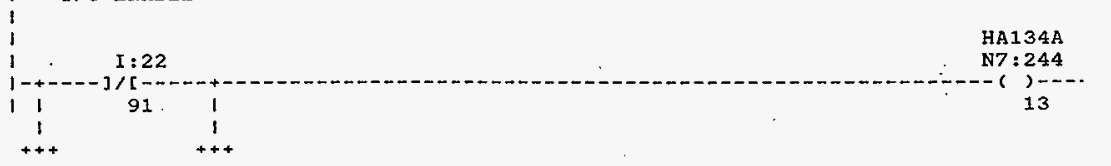


Processor and Dáta(OPS Unit 1) Program Listing

Processor
October 23, 1996 File: SODIUM1A.ACH
Page 263 Rung 2:664

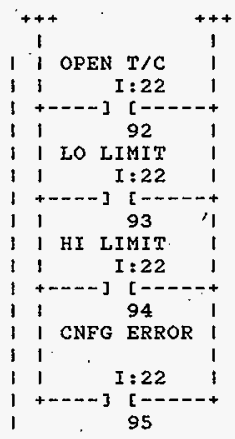

Rung $2: 665$

I

T/C ENABLE

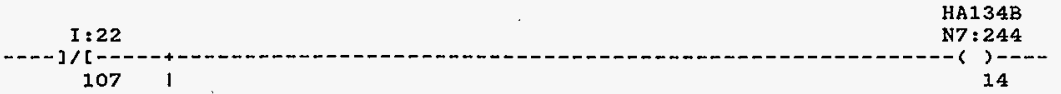

Rung 2:666

I

I

I T/C ENABLE

1 I 1 ENABLE

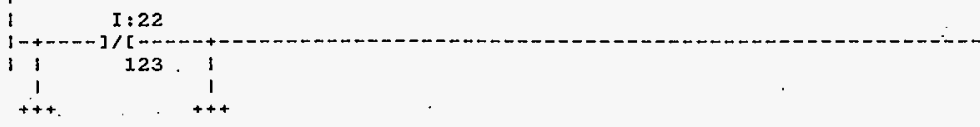




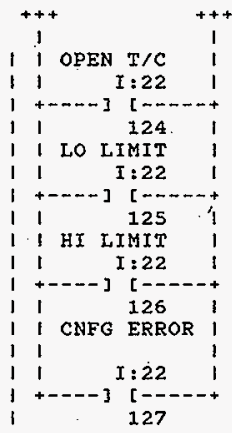

\section{Rung $2: 667$}

1

T/C ENABLE

11

$91 \quad$ i 


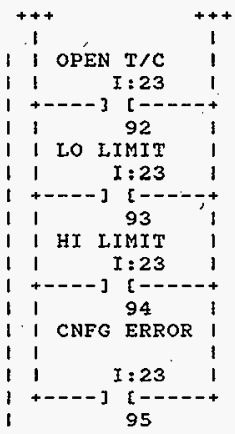

\section{Rung 2:669}

$$
\text { I }
$$

T/C ENABLE

HA141A N7 $: 245$

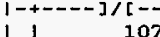

$(2)$

1 OPEN T/C

$1 \quad I: 23$

$+-\cdots--] \quad[-\cdots-m+$

1.108

1 LO LIMIT

$1 \quad I: 23$

$1+-n-7][-\ldots-n+$

11109

I I HI LIMIT

i. 1

I +

I 1

I I

I 1

I 1

I

$I: 23$

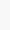


Processor and Data(OPS Unit 1 ) Program Listing
October 23, 1996 Page $26 t$ Processor File: SODIUM1A.ACH

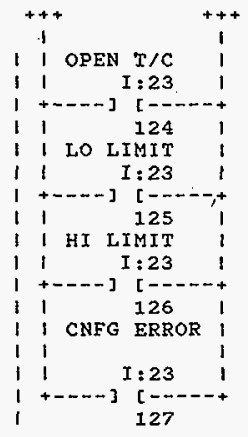

Rung $2: 671$

I

1

I T/C ENABLE

$$
1
$$

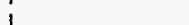

$1-+\ldots-n=24$

HA $141 C$

N7 : 245

I 75

1 OPEN T/C

1

+.--] [ $[-\cdots+$

176

1 LO LIMIT

1 I:24

+..- $]$ [ $[-. . .+$

1

1 HT ITMIT

1 I: 24

+--- $][-\ldots .-2+$

178

CNFG ERROR

11

I 1

1. + +---7 ] $[2,24$

1 79

Rung 2:672

1

T/C ENABLE

I I: 24

HA 142A

N7 $: 245$

I)

1

91

$+++$

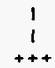

HNF-SD-FF-CSWD-61 Rev. 0

Page 266 
Processor and Data(OPS Unit 1)

October 23, 1996

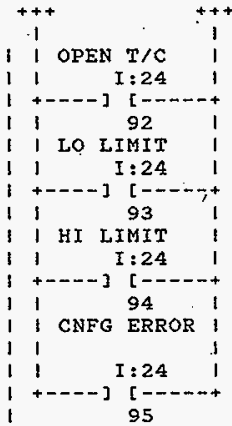

\section{Rung $2: 673$}

1

\section{T/C ENABLE}

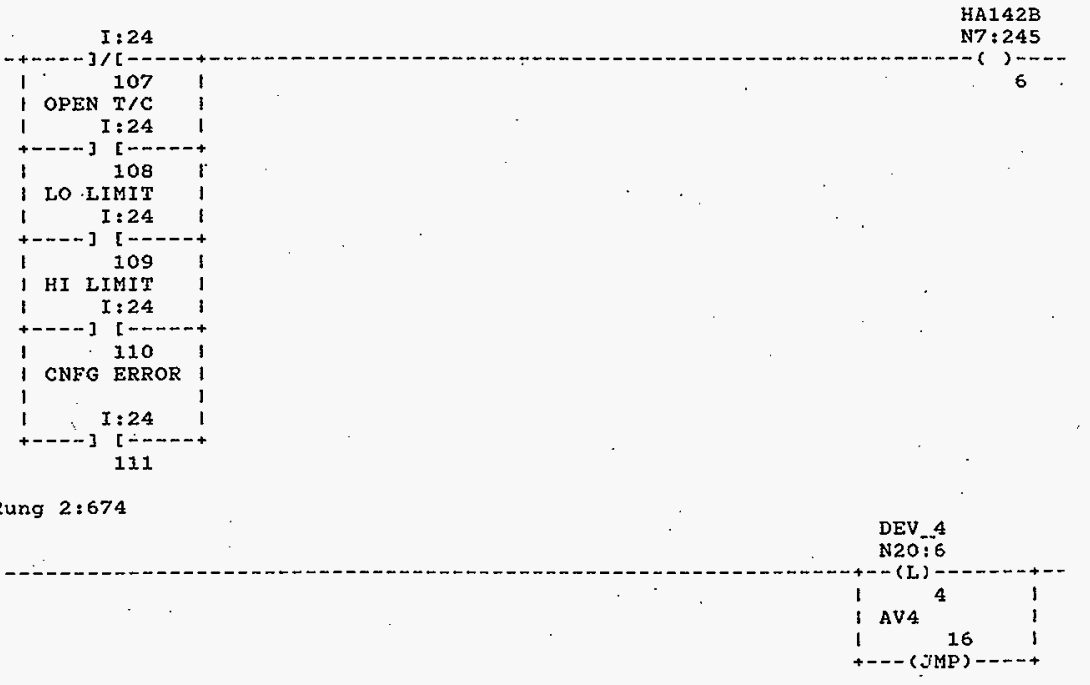

HNF-SD-FF-CSWD-61 R.v. 0 
Processor and Data(OPS Unit 1)

Rung 2:675

1. DEVA

12

DUNMY 12

N20:7

1

Rung 2:676

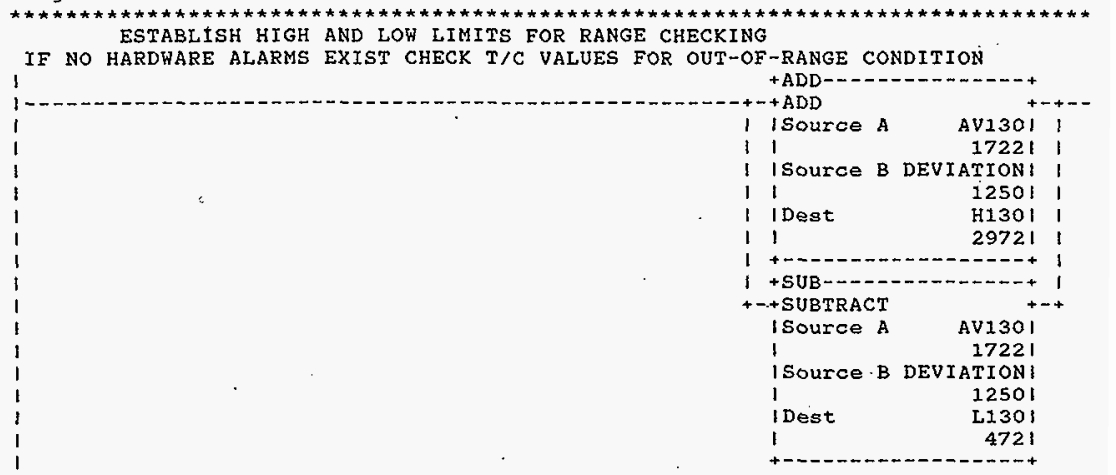

Rung 2:677

HA130A
N7:244


Processor and Data(OPS Unit 1).

October 23, 1996 Page 26؛ Program Listing

Processor File: SODIUM1A.ACH
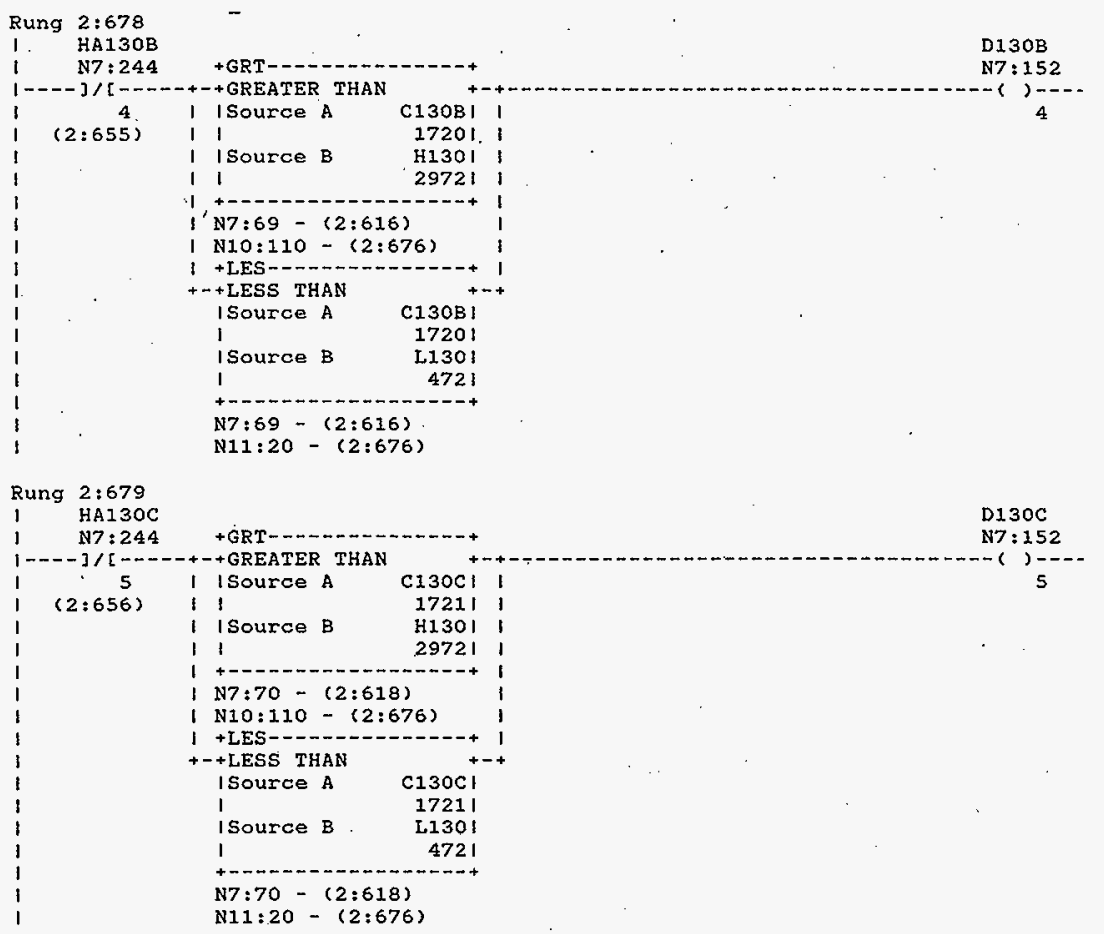

Rung 2:680

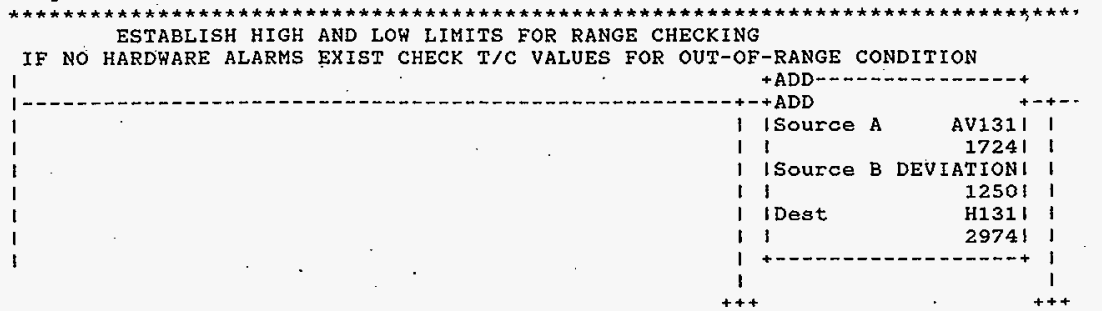

HNF-SD-FF-CSWD-61 Rev. 0 
Processor and Data(OPS Unit 1$)$
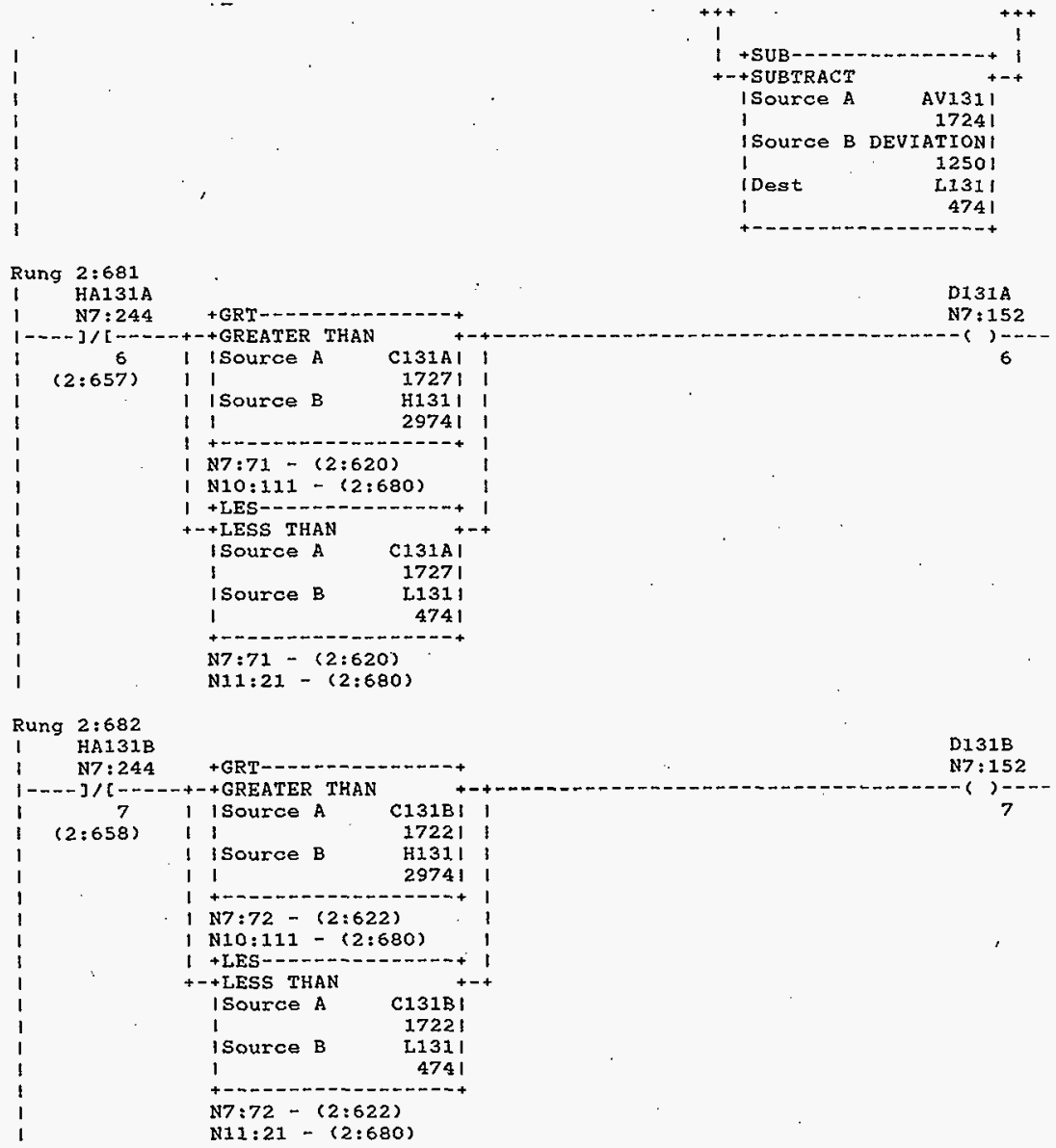

HNF-SD-FF-CSWD-61 Rev. 0 
Processor and Data(OPS Unit 1 )

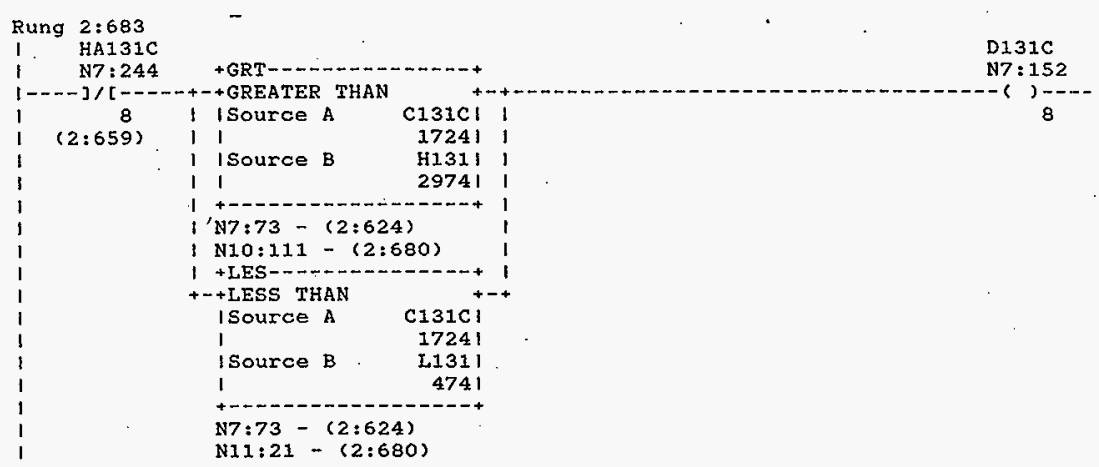

Rung 2:684

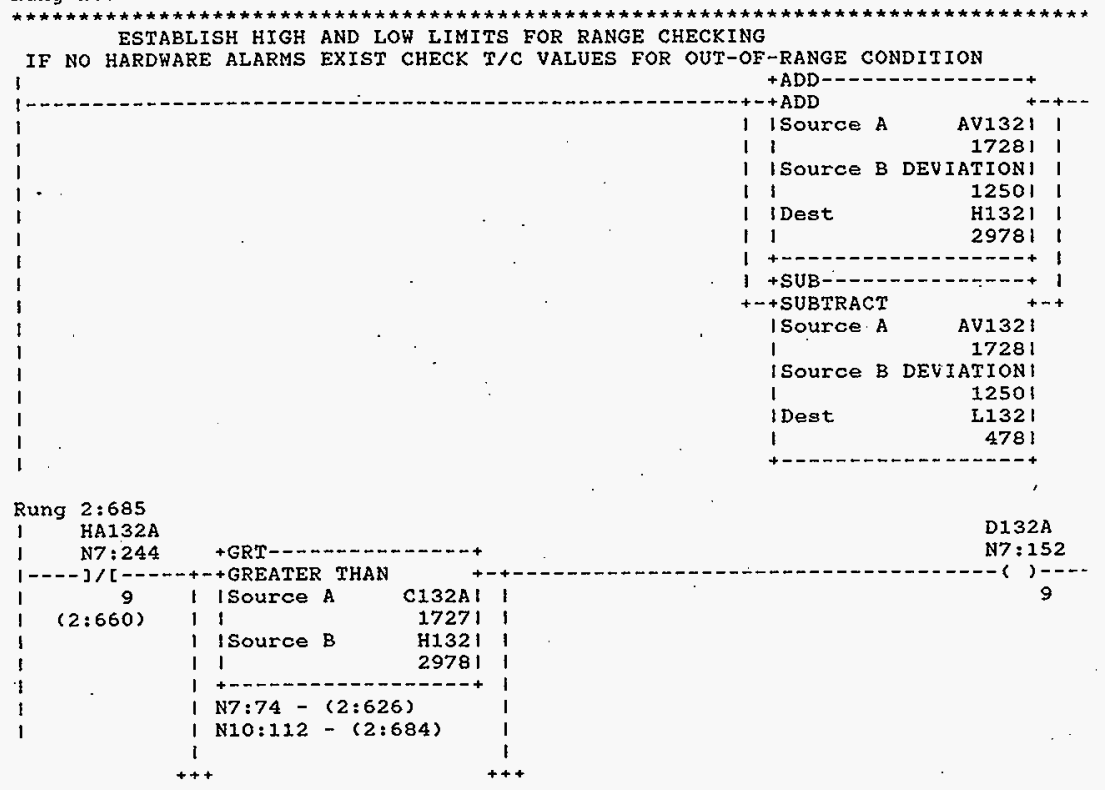

HNF-SD-FF-CSWD-61 Rev. 0 
Processor and Data(Ops Unit 1 )
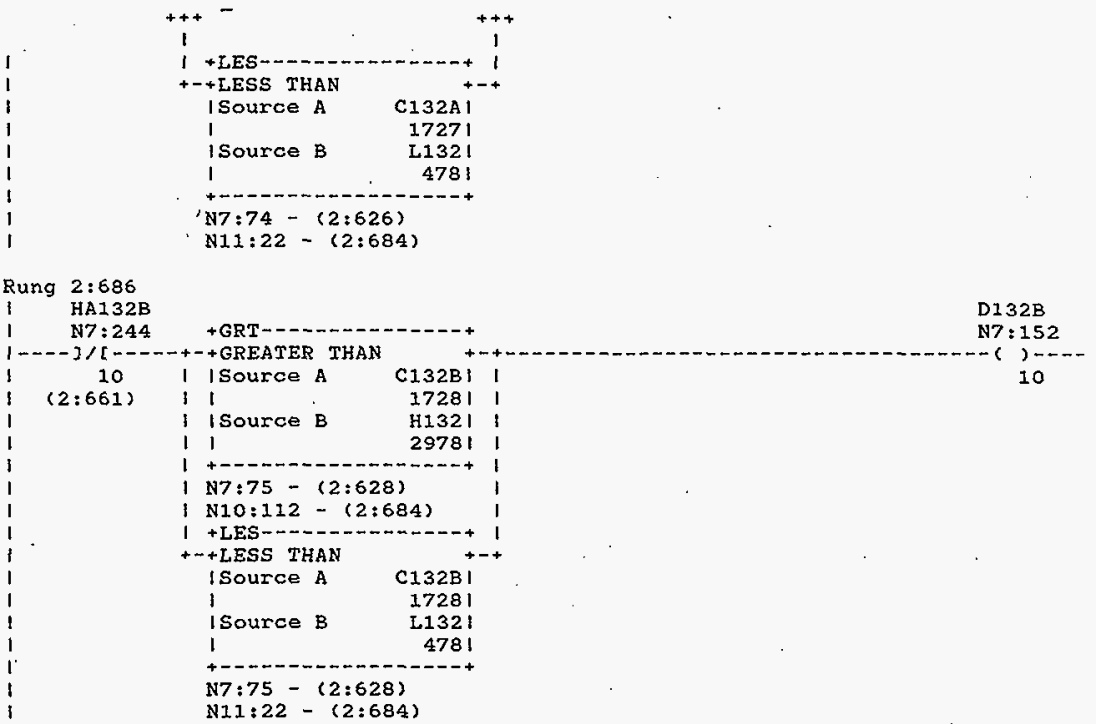

Rung 2:687

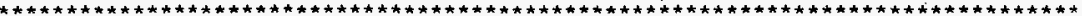
ESTABLISH HIGH AND LOW IIMITS FOR RANGE CHECKING

IF NO HARDWARE ALARMS EXIST CHECK T/C VALUES FOR OUT-OF-RANGE CONDITION

$+A D D-\cdots+-----\cdots+--$

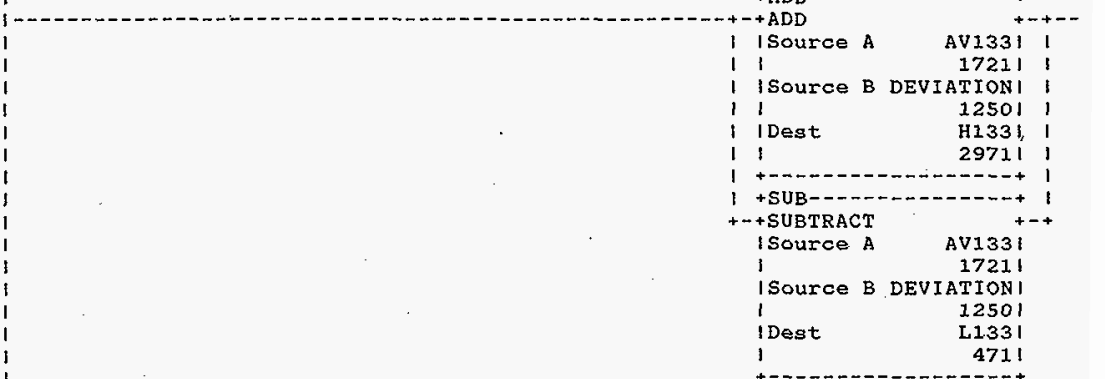

HNF-SD-FF-CSWD-61 Rev. 0 
Processor and Data(OPS Unit 1)

October 23, 1996

Page 27:

Program Llsting

processor File: SODIUM1A.ACH

Rung $2: 68$

Rung 2:688

l. HA133A $-$

$1 \quad$ N7: 244 +GRT-- - - - - - - - - - +

D133A

1---]/[--.+++GREATER THAN

111

$(2: 662)$

I ISource A C133A| I

17241 i

| ISource B H1331 |

11

29711 i

$1+\cdots---\cdots-\cdots-\cdots-\cdots+1$

I N7:76-(2:630)

I N10:113-(2:687)

$1+10: 113-(2: 687)$

++ +LESS THAN +-+

ISource A C133A I

I 17241

isource B L1331

14711

+ - - - - -

N7:76-(2:630)

$N 11: 23-\{2: 687\}$

Rung $2: 689$

1 HA133B

N7: 244

1 $-\cdots--] /[-\ldots$

12

-+GREATER THAN

I ISOUrce A

$(2: 663)$

I 1

1 ISource B

13381

H1331 I

11

297111

$1+--\cdots+-------------+.1$

I N7:77-(2:632)

1 N10:113-(2:687)

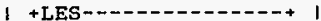

+-+ LESS THAN +-+

isource A C133B I

I 17171

| Source B L133|

1 4711

N7:77-(2:632)

N11:23-(2:687)

Rung 2:690

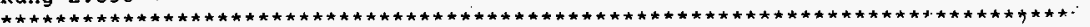
ESTABLISH HIGH AND LOW LIMITS FOR RANGE CHECKING

IF NO HARDWARE ALARMS EXIST CHECK T/C VALUES FOR OUT-OF-RANGE CONDI I!ION

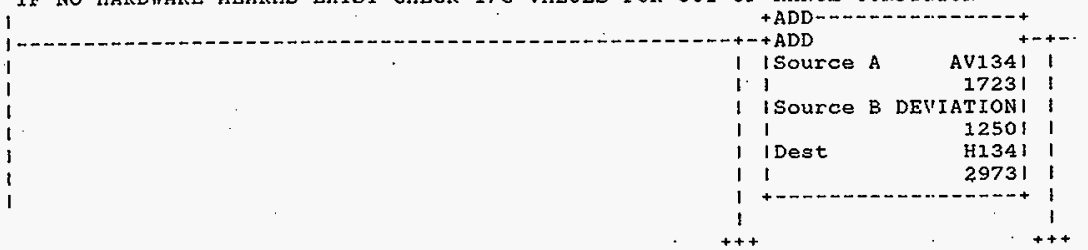

HNF-SD-FF-CSWD-61. Rev. 0 

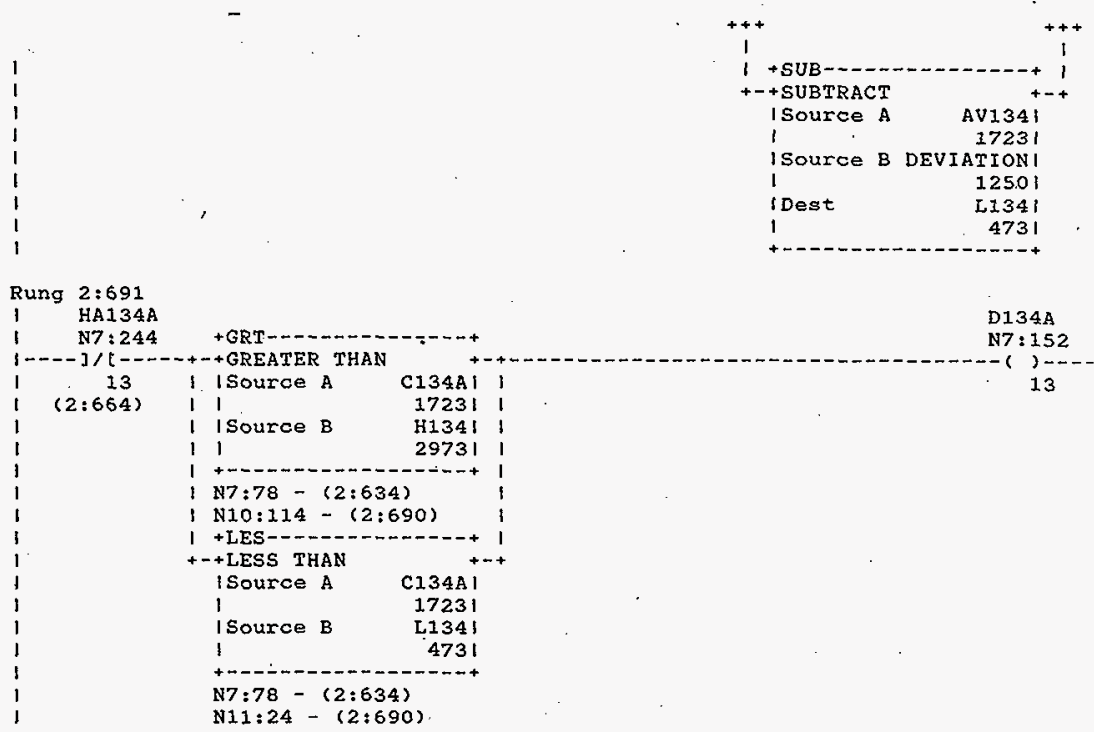

Rung $2: 692$

HA134B
1
N7:244


Rung $2: 693$ ESTABLISH HIGH AND LON LIMITS FOR RANGE CHECKING IF NO HARDHARE ALARMS EXIST CHECK I/C VALUES FOR OUT-OF-RANGE CONDITION 


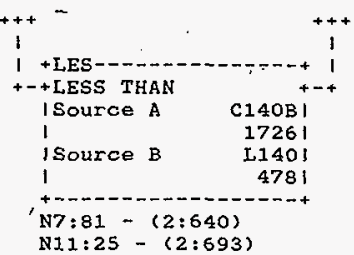

Rung 2:696

i $\quad$ HA140C<smiles>C[Te][Te]I</smiles>

D14OC

+-+ GREATER THAN

1

1

$(2: 668)$

C140CI

173211 i isou

H140I।

297811

11

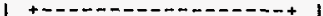

1 N7:82-(2:642)

1 N10:115 - (2:693)

1 +LES----..-----..+

+-+LESS THAN

ISource A

1

1

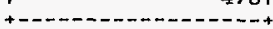

$N 7: 82-(2: 642)$

$N 11: 25-(2: 693)$

Rung 2:697

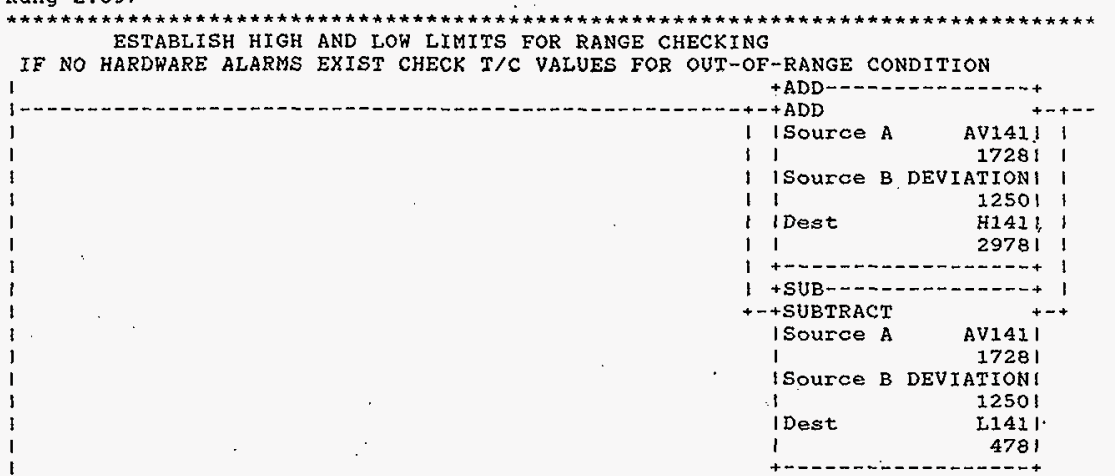

HNF-SD-FF-CSWD-61 Rev. 0 
Processor and Data(OPS Unit 1) Program Listing

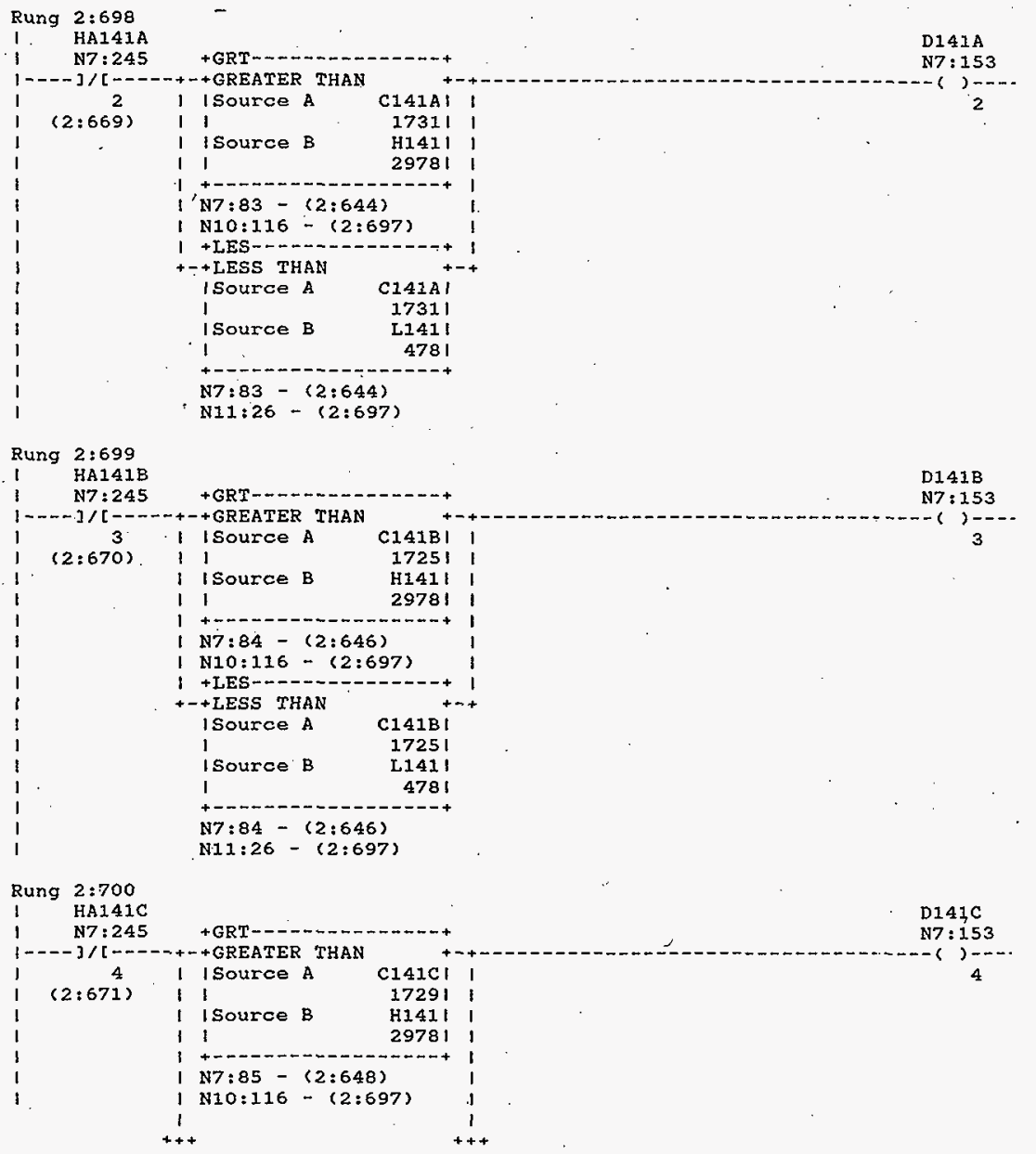


Processor and Data(OPS Unit 1)

Octobar 23, 1996

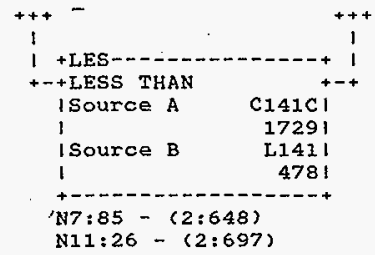

Rung 2:701

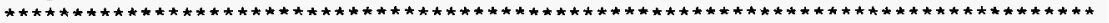
ESTABLISH HIGH AND LOW LIMITS FOR RANGE CHECKING

IF NO HARDWARE ALARMS EXIST CHECK T/C VALUES FOR OUT-OF-RANGE CONDITION

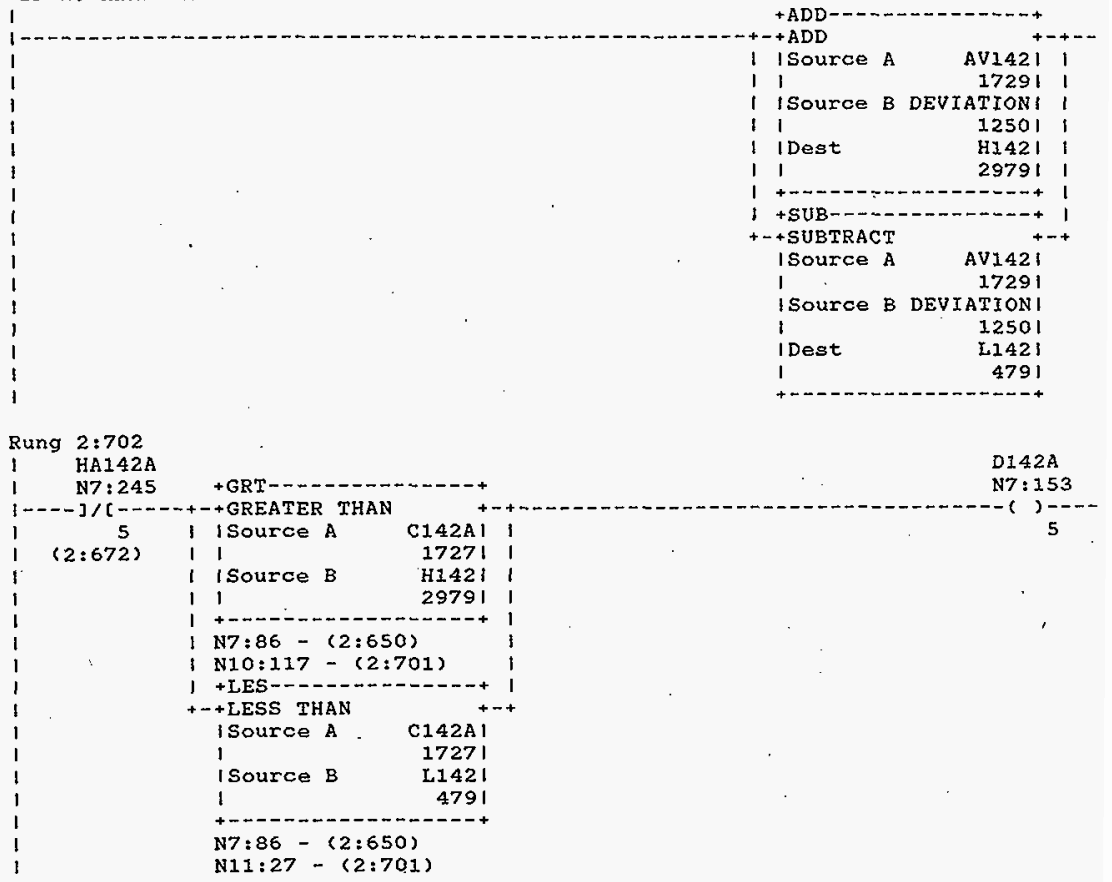


Processor and Data(OPS Unit 1)

Rung 2:703

1. $H A 242 \mathrm{~B}$ $-$

N7 $: 245$

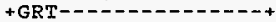

D142B

$1----] /[---+++$ GREATER THAN

( $2: 673$ )

1 ISource A

C142BI I

$11 \quad 173011$

| ISource B H142I I

11

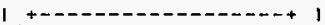

I'N7:87--(2:652)

I N10:117-(2:701)

$1+$ TES-

+-+ LESS THAN +-+

|Source A C142B|

I 1730 I

|Source B LI421

1 4791

N7:87-(2:652)

N11:27-(2:701)

Rung 2:704

1 DEV_4

1

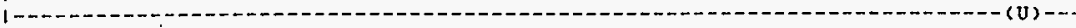

1 . 4

Rung $2: 705$

I AV4

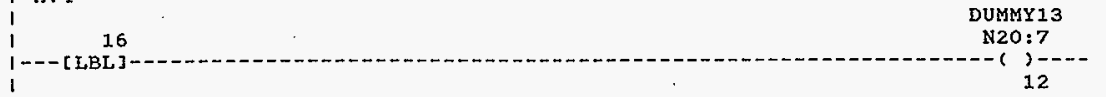

Rung $2: 706$

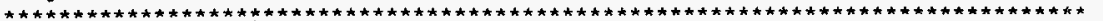
CLEAR COUNT AND TOTAL, IF NO HARDWARE, NO RANGE ALARMS AND NO THERMOCOUPLES HAVE BEEN TAKEN OUT (TE_OUT $\times \times X$ ) THEN AVERAGE THE T/C

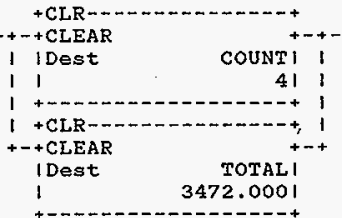


Processor and Data(OPS Unit 1)

\section{Rung 2:707}
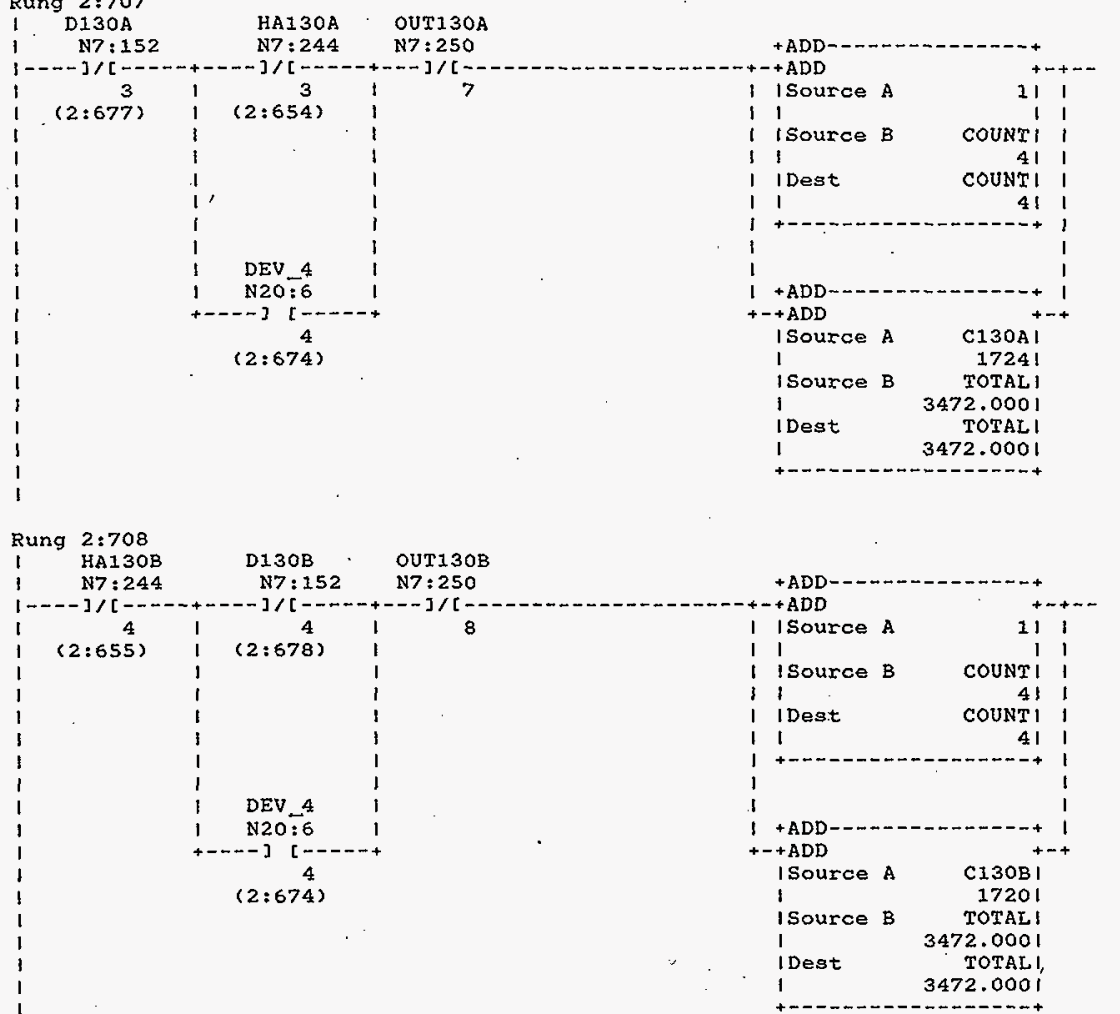


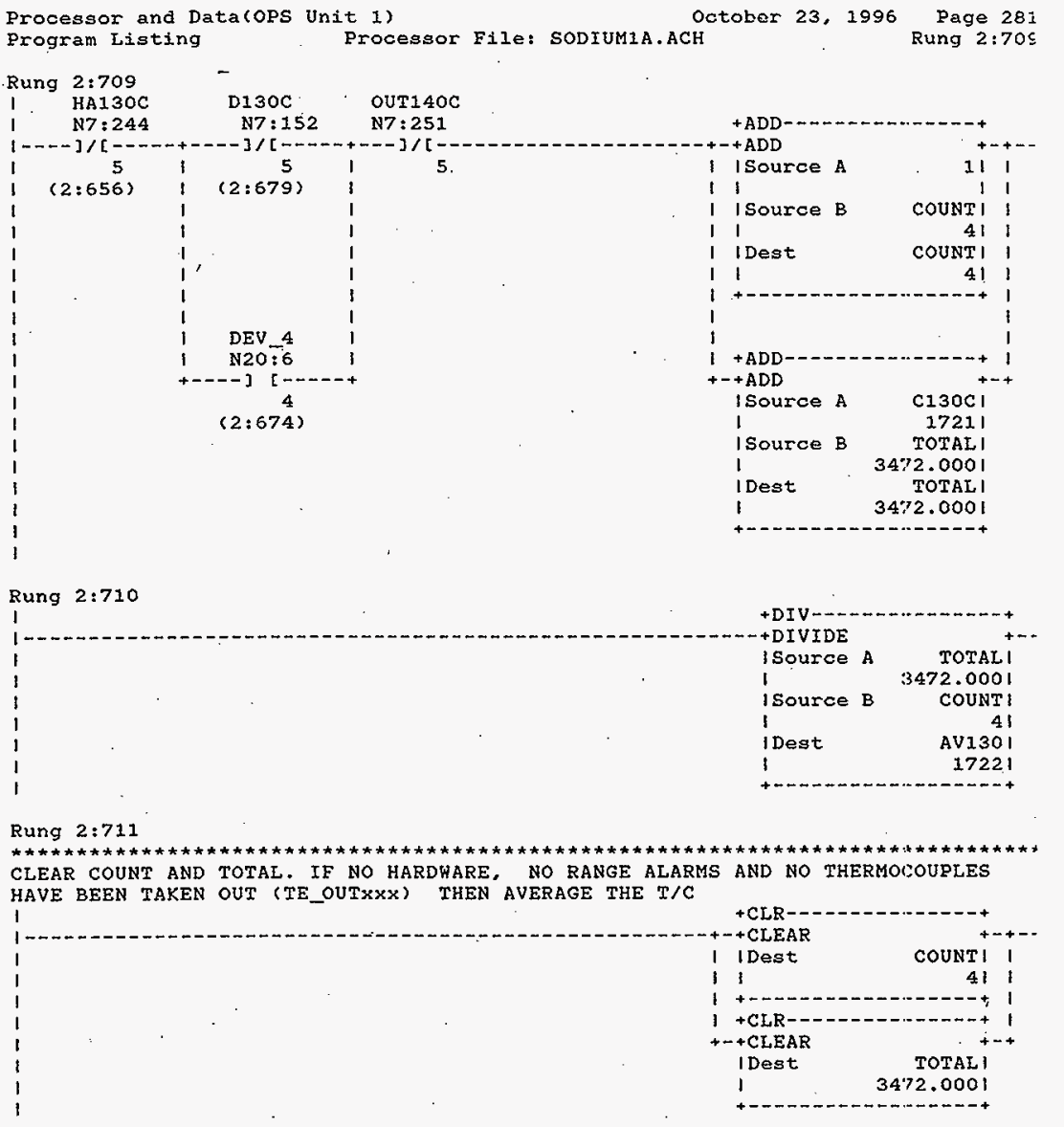

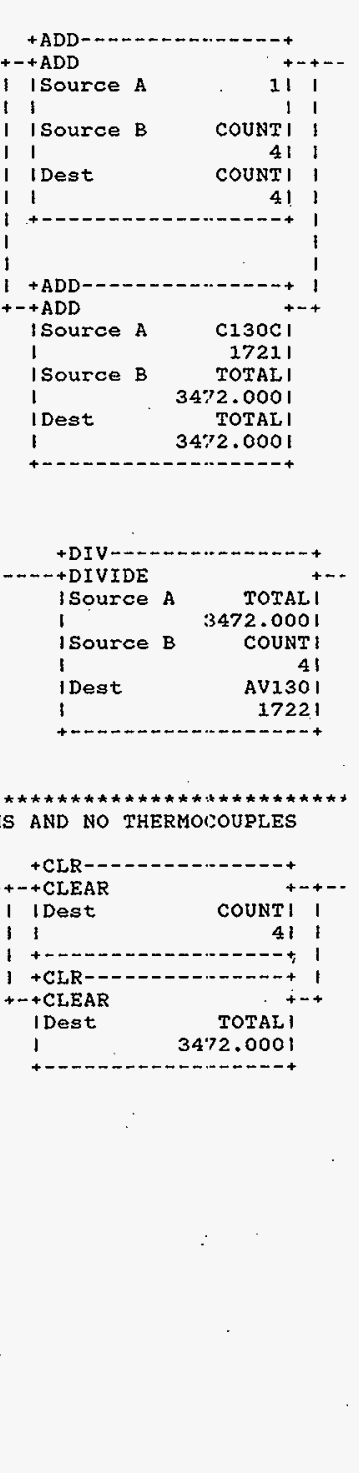


Rung $2: 712$

I HA131A D131A OUT131A

I. N7:244 N7:152 N7:250

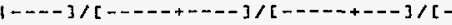

$(2: 657)$

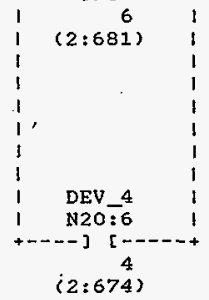

10

10

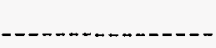
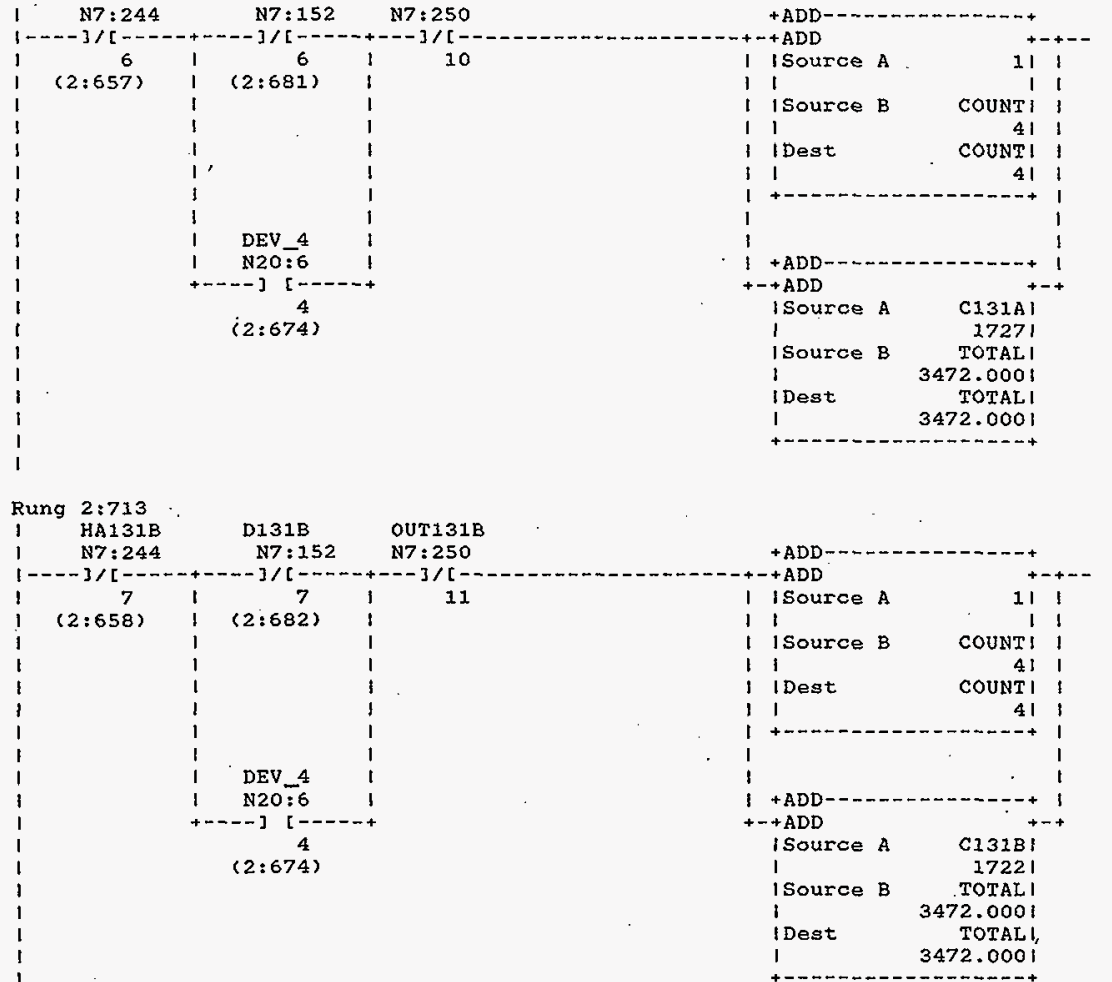
Processor and Data (OPS Unit I) Program Listing

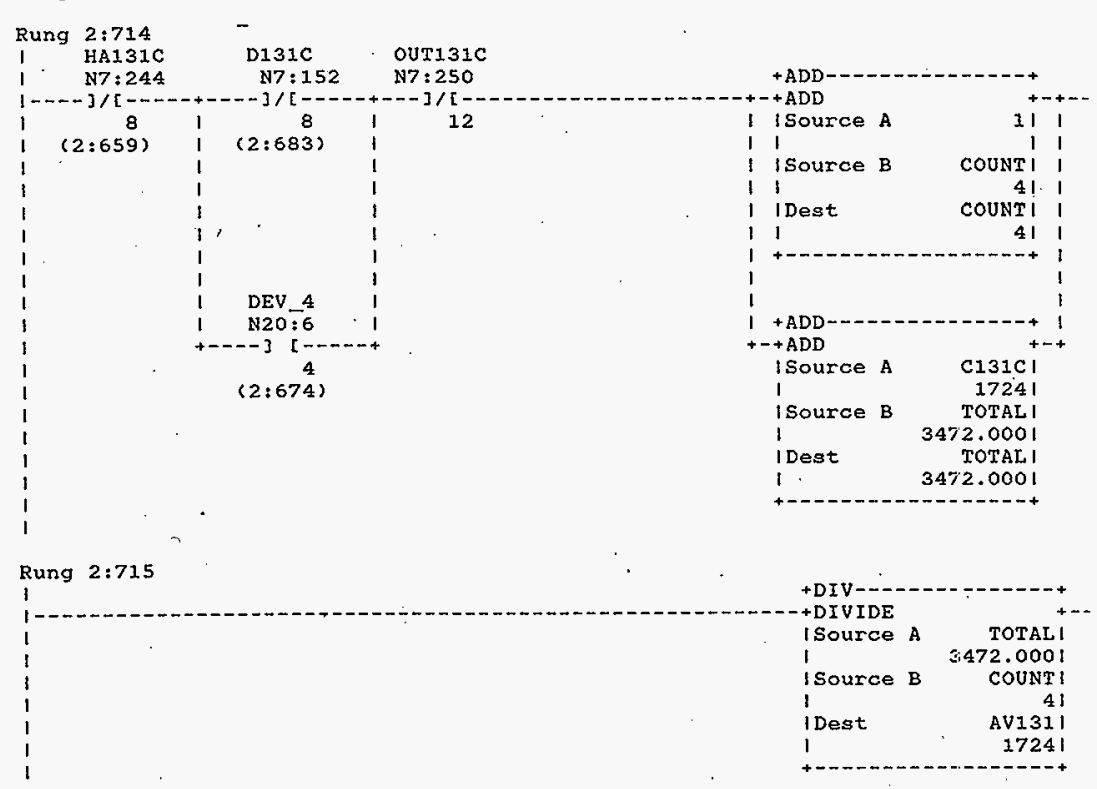

Rung 2:716

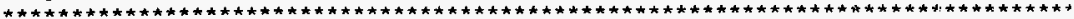
CLEAR COUNT AND TOTAL. IF NO HARDHARE, NO RANGE ALARMS AND NO THERMOC:OUPLES HAVE BEEN TAKEN OUT (TE_OUTXXX) THEN AVERAGE THE T/C

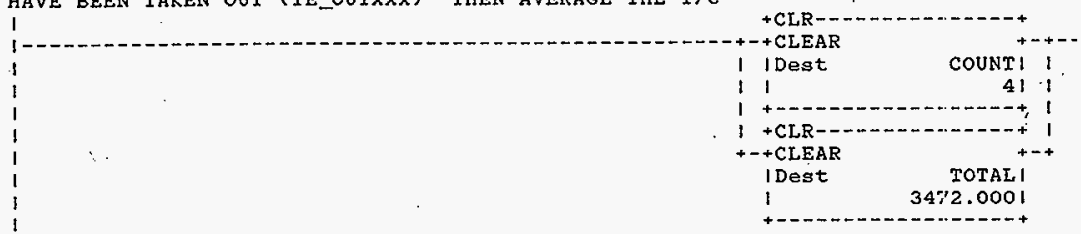


Processor and Data(ops intt 1 )

Program Listing

Processor File: SODIUM1A.ACH

october 23,1996

Page 284

Program 28717
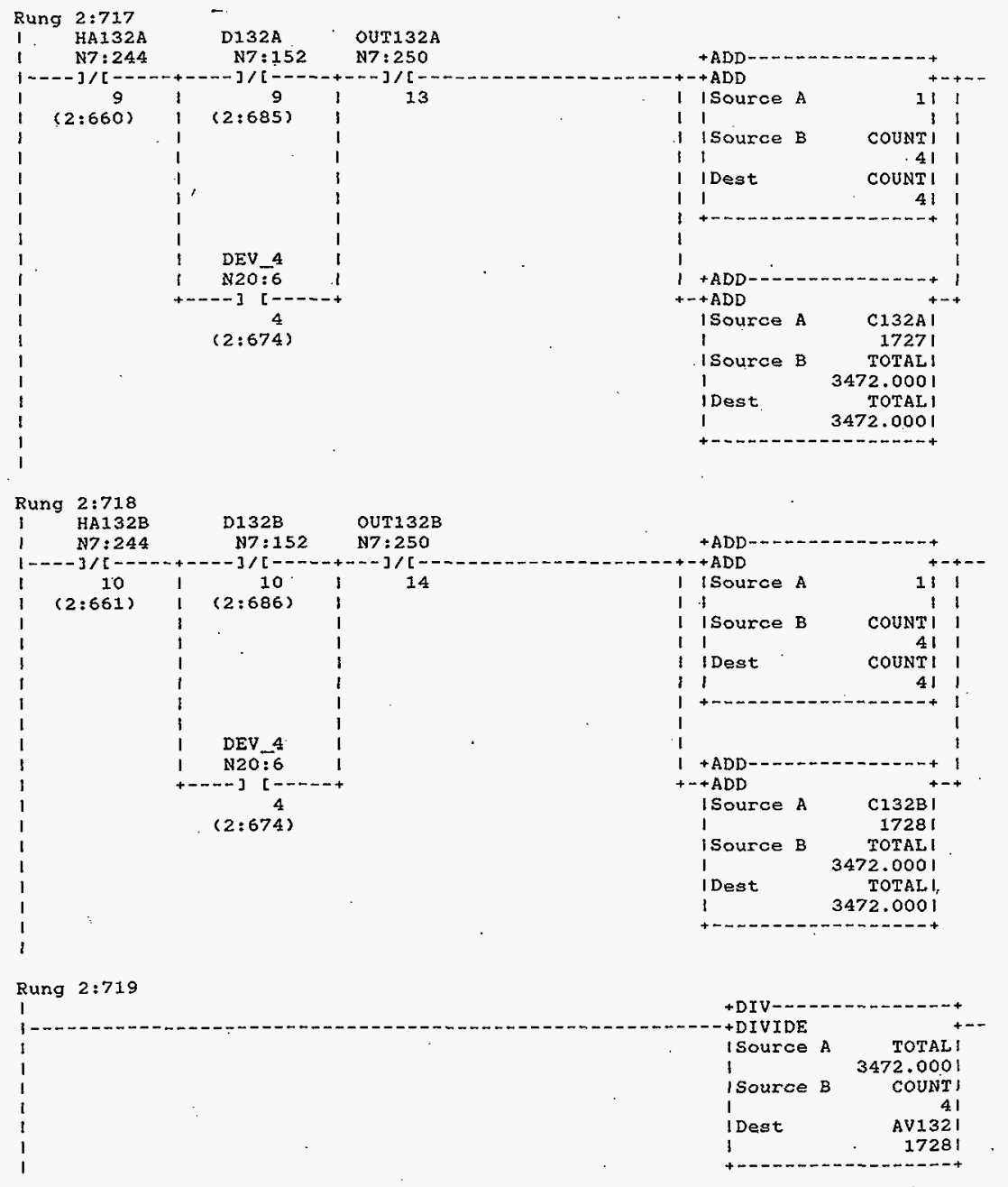

INF-SD-FF-CSWD-61 Rev. 0 


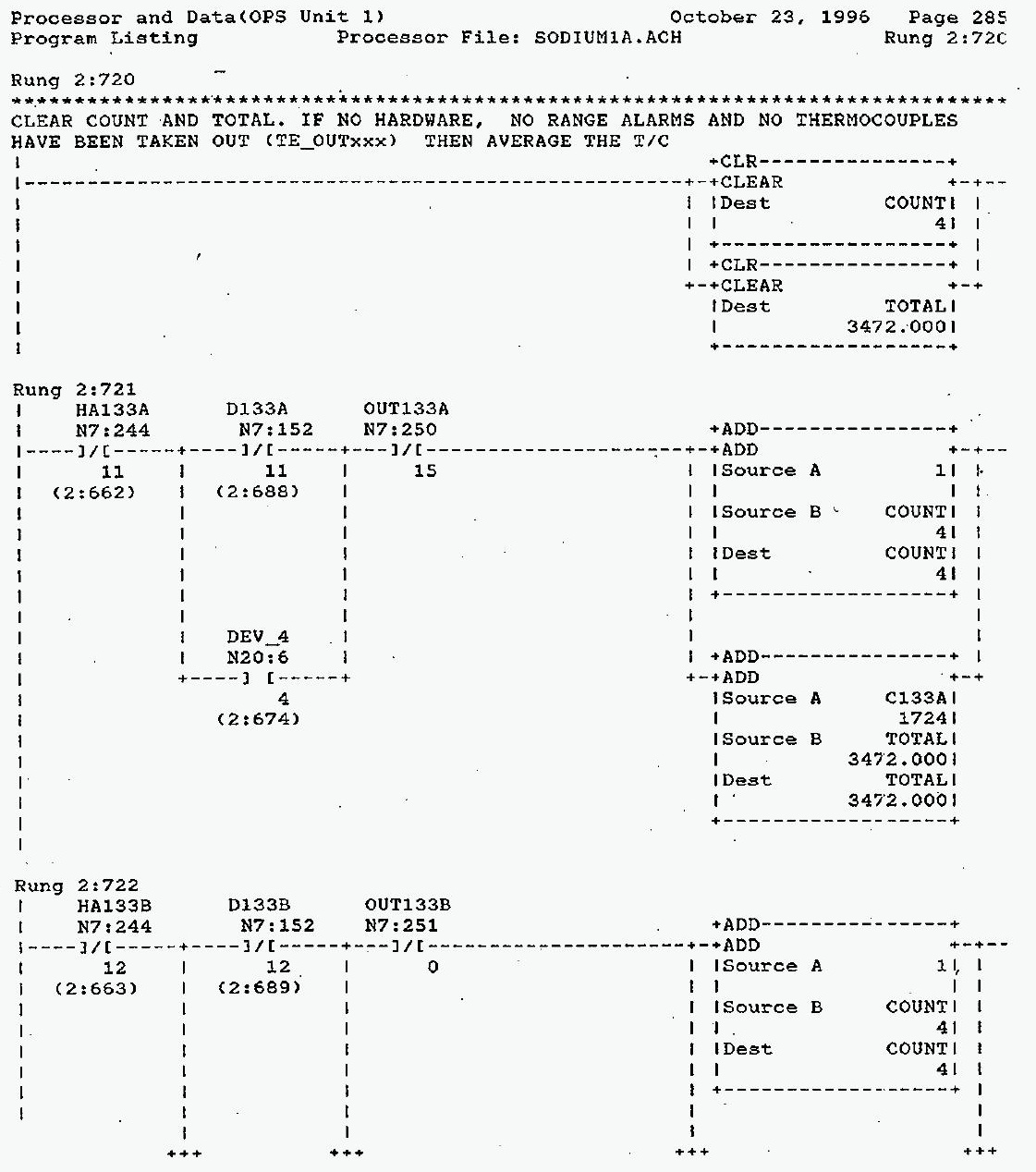

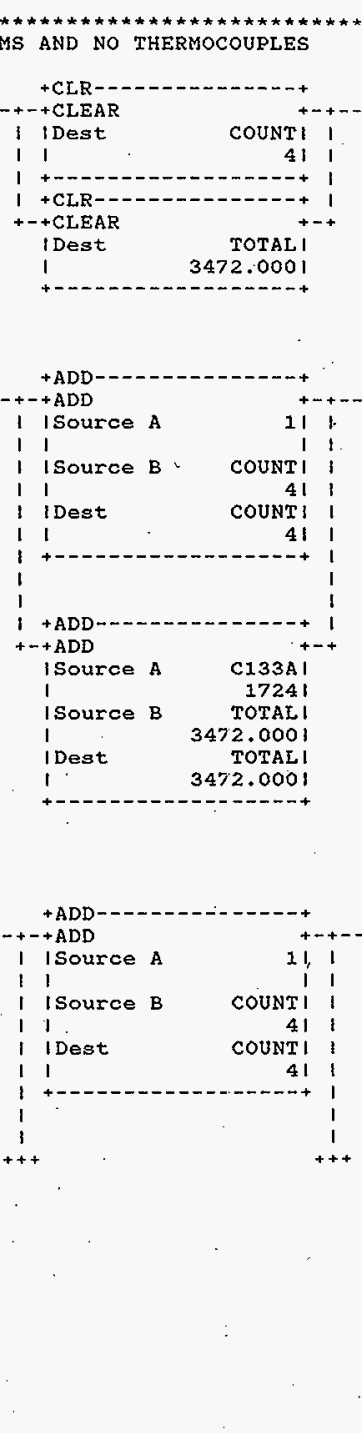


Processor and Data (OPS Unit 1 )

$\begin{array}{cccc} & +++ & +++ \\ 1 & 1 & \text { DEV } 4 & 1 \\ 1 & 1 & N 20: 6 & 1 \\ 1 & +\infty & 4 \\ 1 & & \\ 1 & & \\ 1 & & \end{array}$

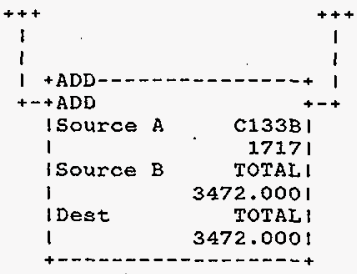

Rung $2: 723$

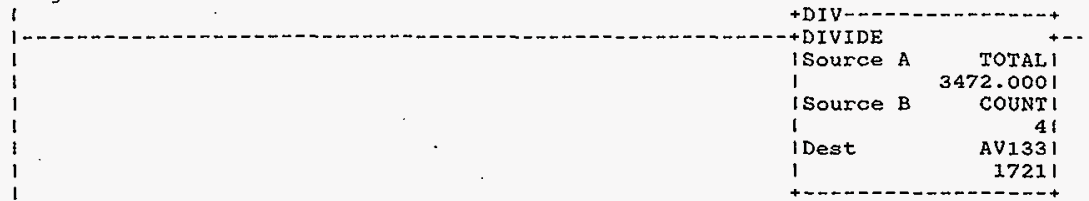

Rung $2: 724$

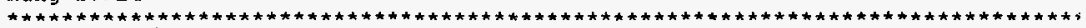
CLEAR COUNT AND TOTAR. IF NO HARDWARE, NO RANGE ALARMS AND NO THERMOCOUPLES HAVE BEEN TAKEN OUT (TE_OUT $X X X)$ THEN AVERAGE THE T/C

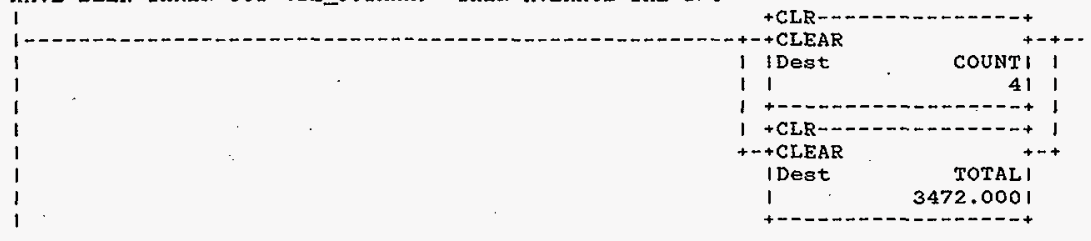

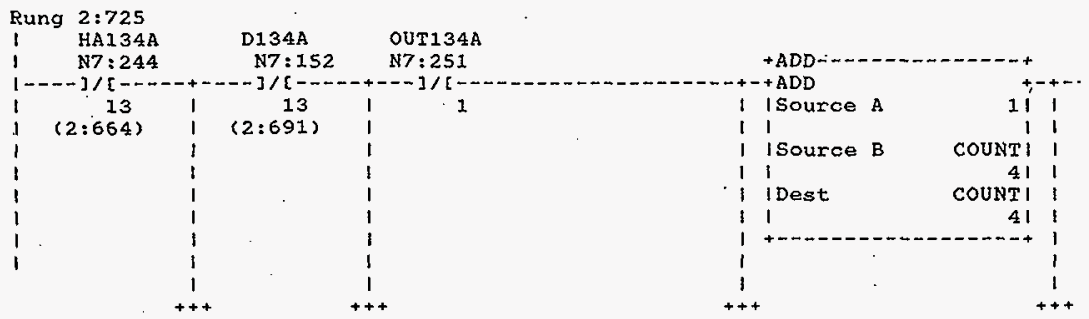


Processor and Data(OPS Unit 1)

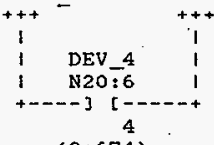

$(2: 674)$
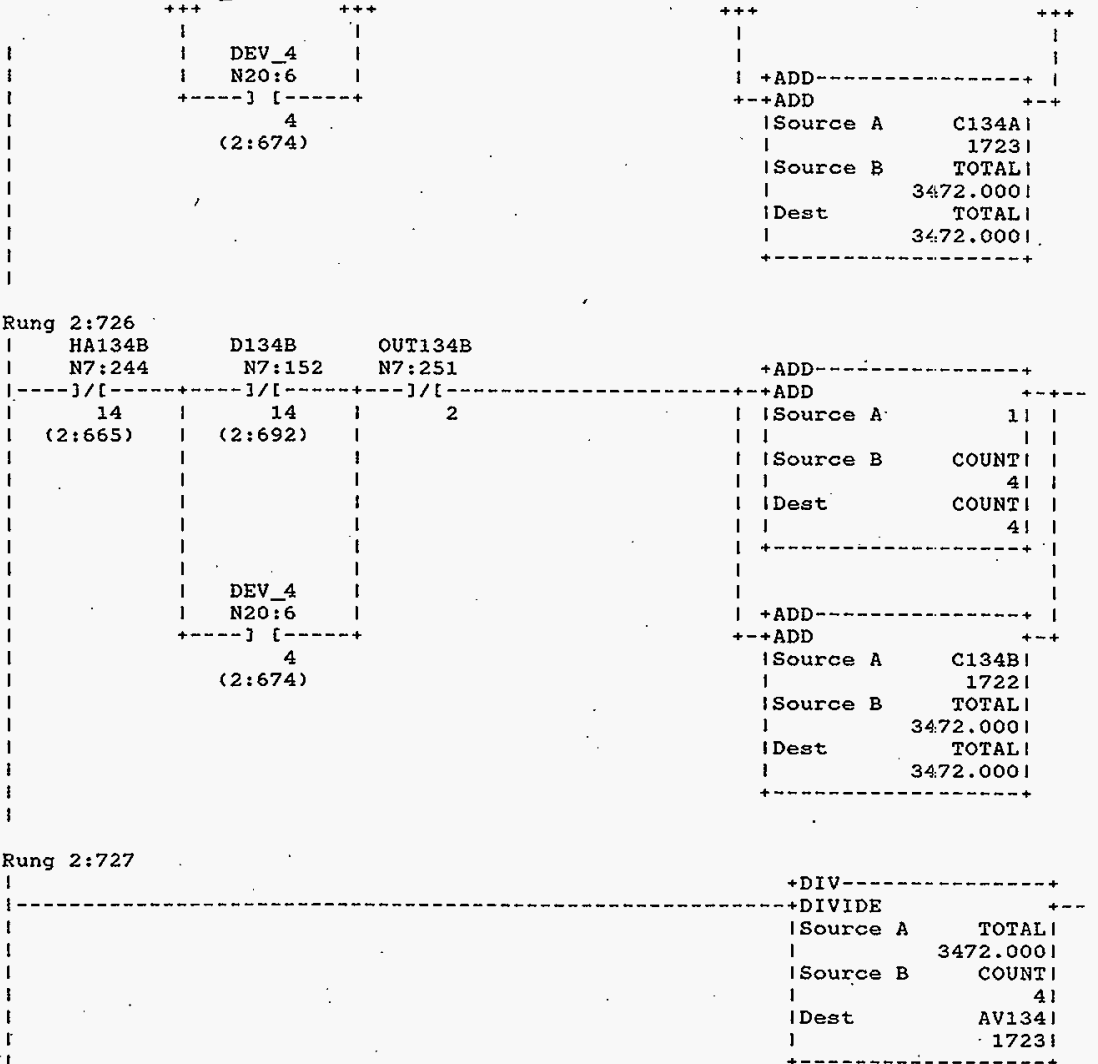

Rung $2: 728$

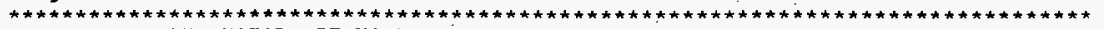
CLEAR COUNT AND TOTAL. IF NO HARDWARE, NO RANGE ALARMS AND NO THERMCCOUPLES HAVE BEEN TAKEN OUT (TE_OUTXXX) THEN AVERAGE THE T/C

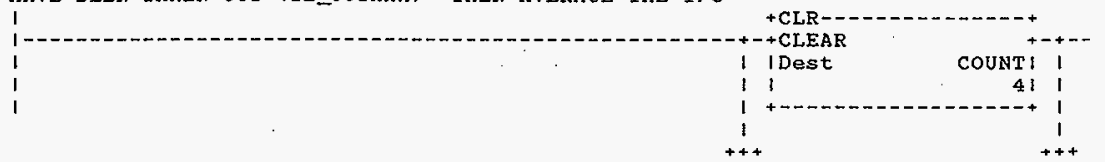

HNF-SD-FF-CSWD-61 Rev. 0 
Processor and Data(OPS Unit 1 ) Program Listing

Processor File: SODIUM1A.ACH

October 23, 1996

Page 28 s

Rung $2: 72 \varepsilon$

1
1
1
1
1
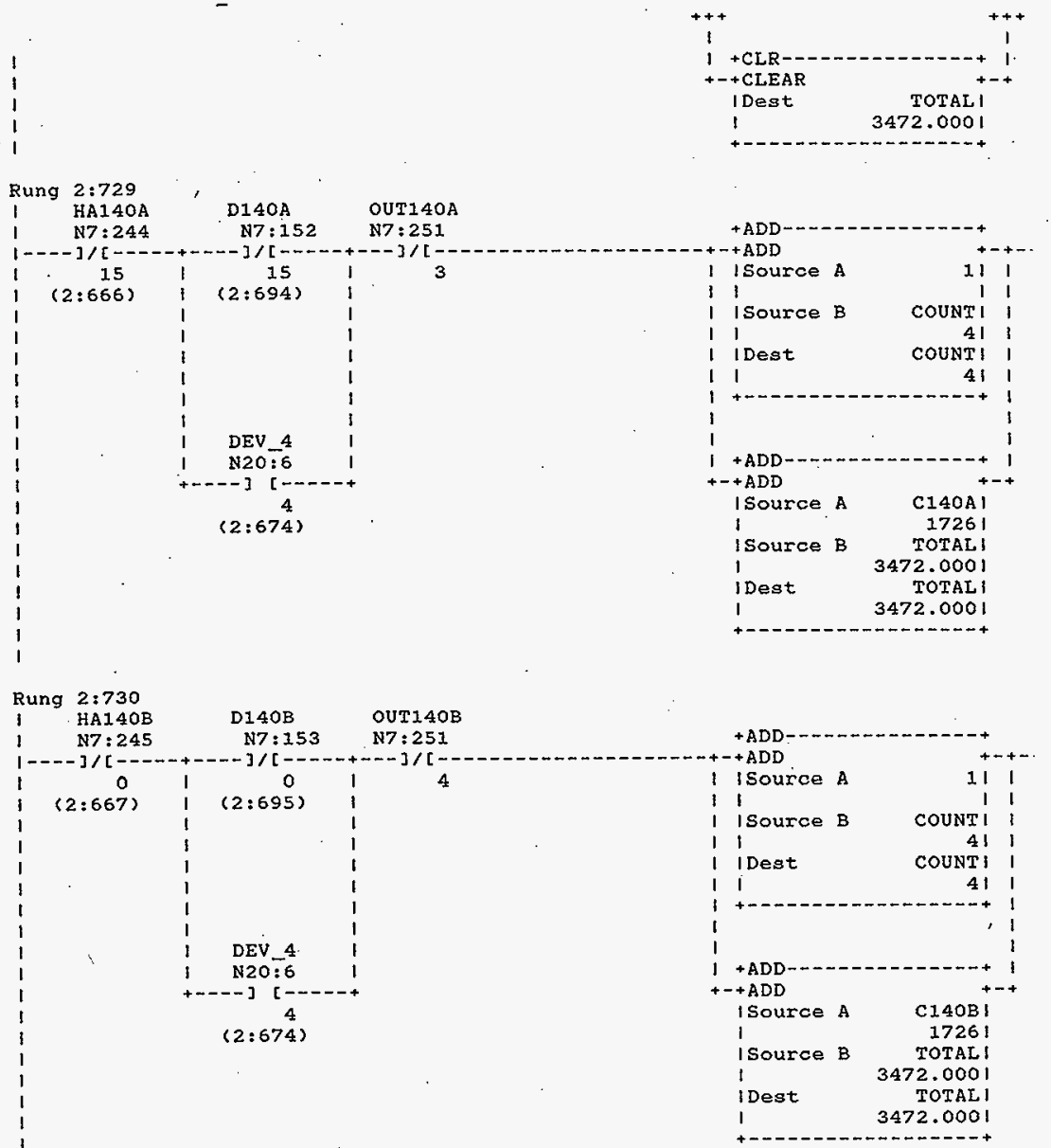

HNF-SD-FF-CSWD-61 Rev. 0 
Processor and Data COPS Unit 1

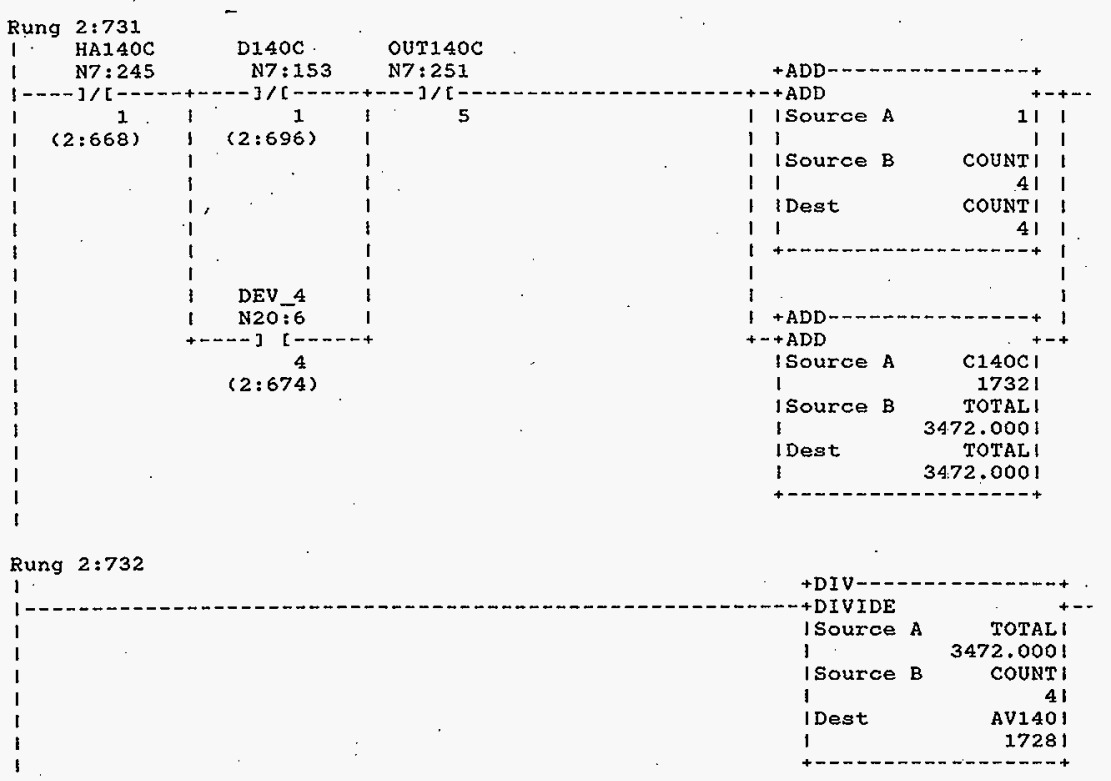

Rung $2: 733$

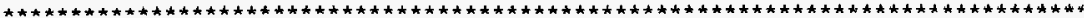
CLEAR COUNT AND TOTAL. IF NO HARDHARE, NO RANGE ALARMS AND NO THERMCICOUPLES HAVE BEEN TAKEN OUT (TE_OUTXXX) THEN AVERAGE THE T/C

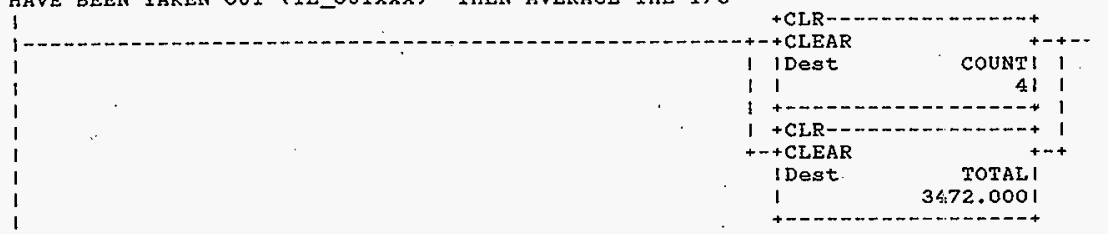


Processor and Data(OPS Unit 1) Program Listing

Process

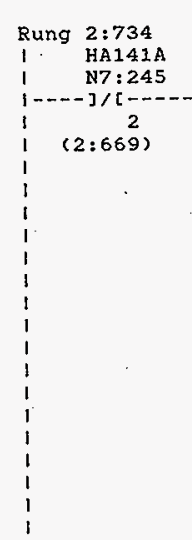

October 23, 1996 File: SODIUMIA.ACH
Page 290

Rung $2: 734$

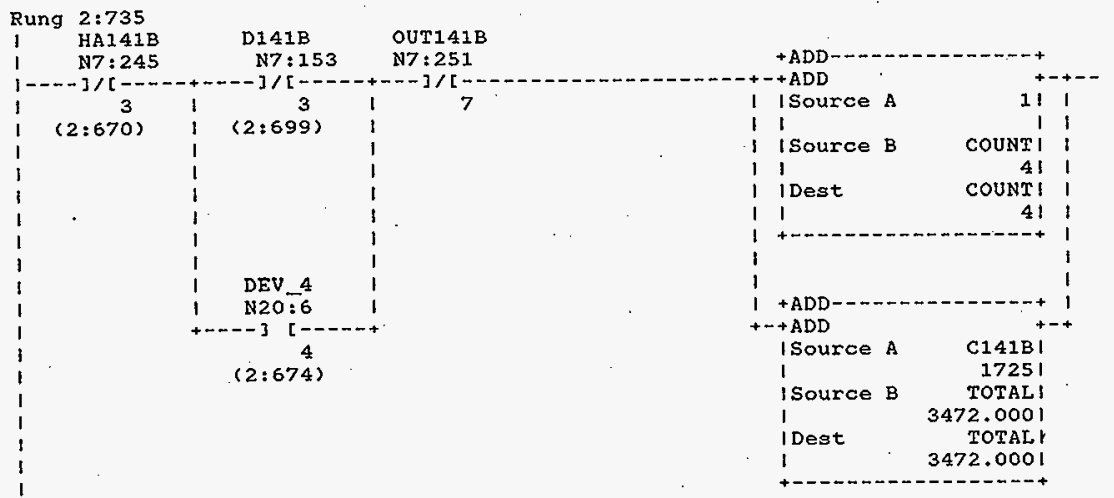

HNF-SD-FF-CSWD-61 Rev. 0 


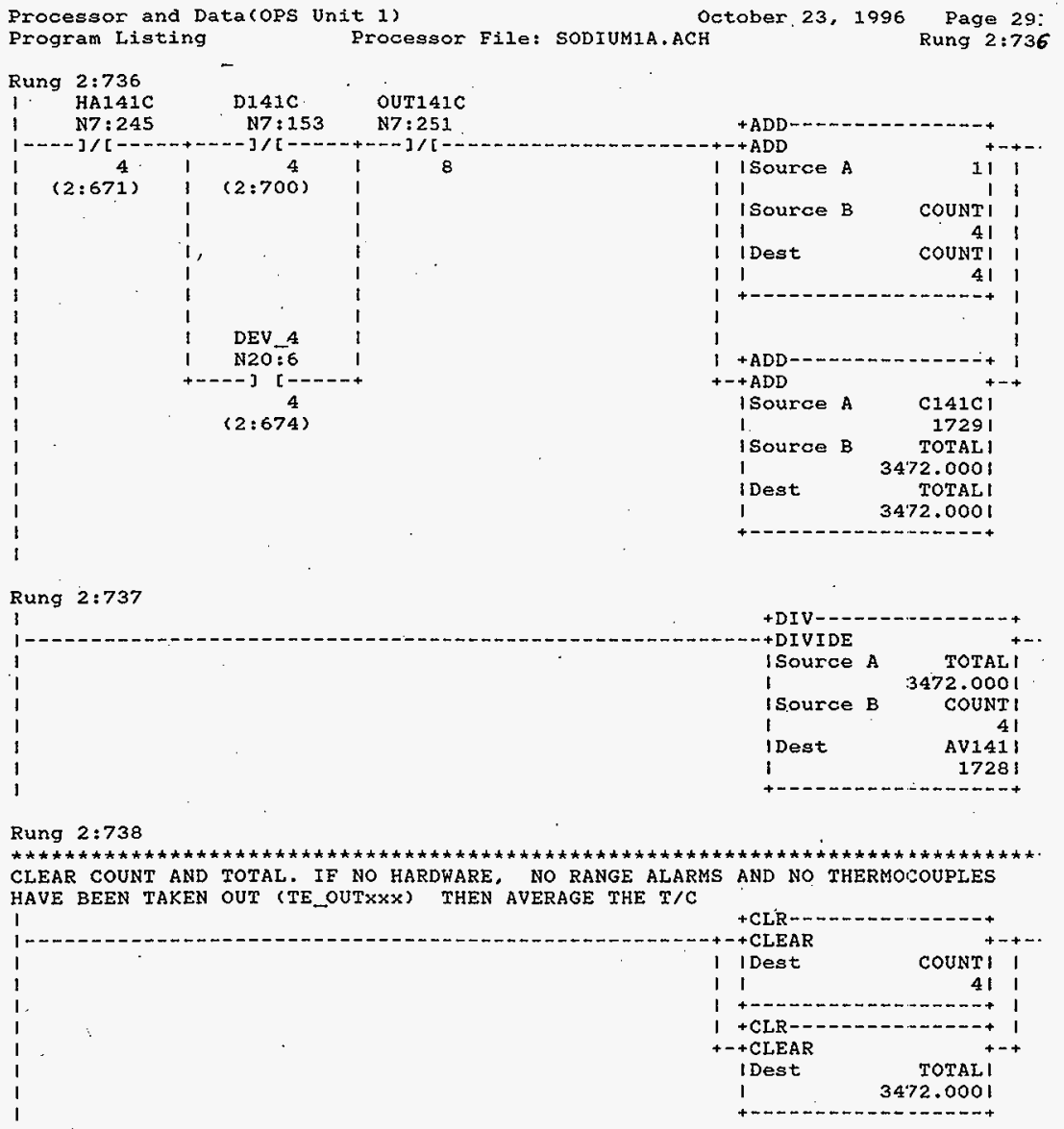

+ACD-1
+ADD 
Processor and Data(OPS Unit 1)
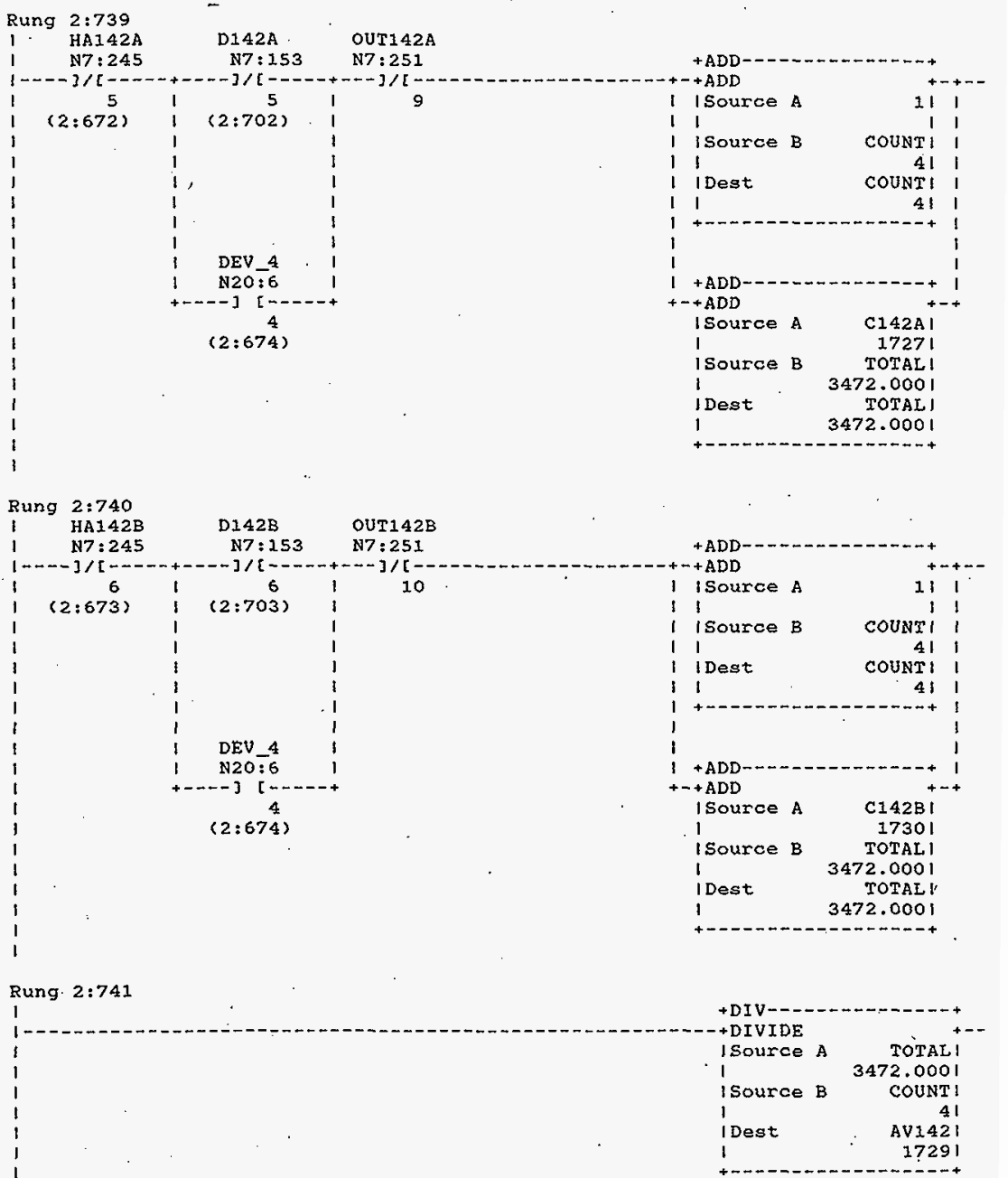
Processor and Data(ops Unit 1 )

Program Listing

Processor File: SODIUM1A.ACH

October 23,1996

Page 293

Rung 2:742

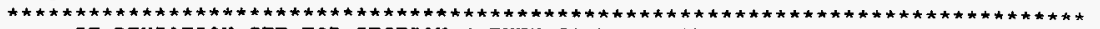
IF DEVIATION SET FOR SECTION 4 THEN GO THERE AND CALCULATE DEVIATION

I

$\mathrm{DEV}-4$

DEV 4

(1) N20:6

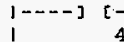

I $(2: 674)$

Rung $2: 743$

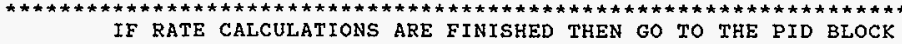

I I RT_TMR/DN

I $\overline{\mathrm{T}} 4: 62$

I--.- ]/ [-

1 DN

I $(2: 33)$

Rung 2:744

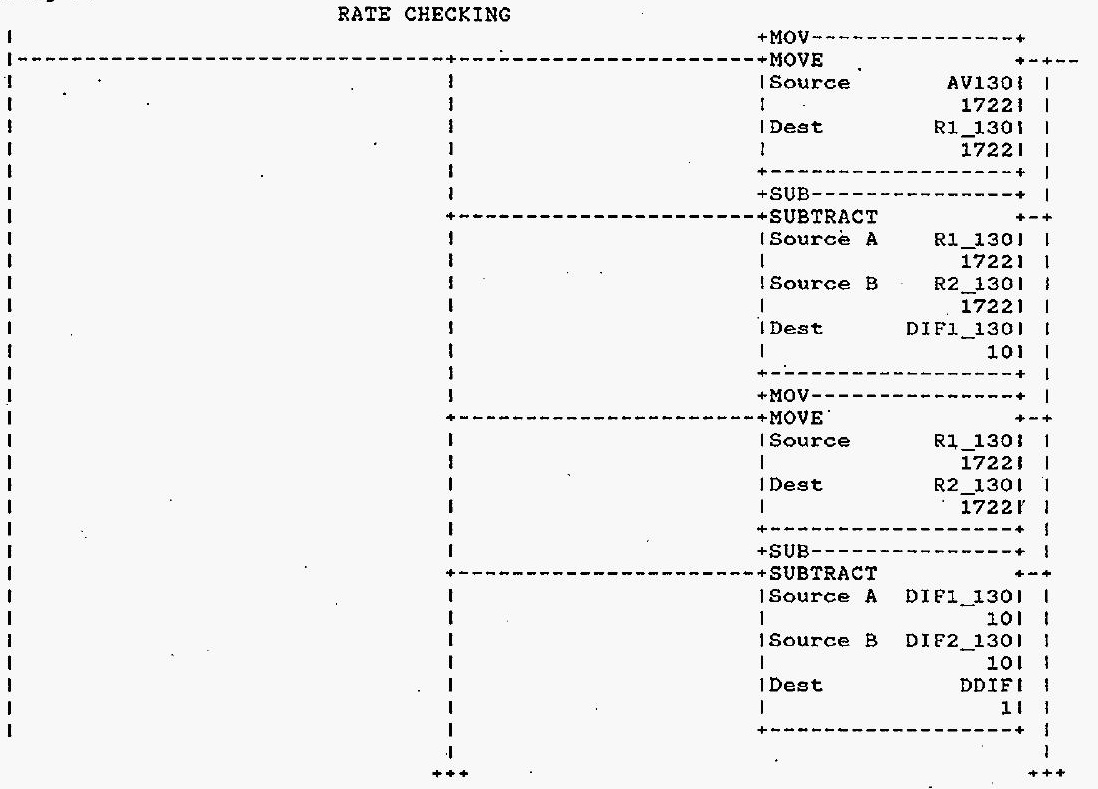

HNF-SD-FF-CSWD-61 Rev. 0 


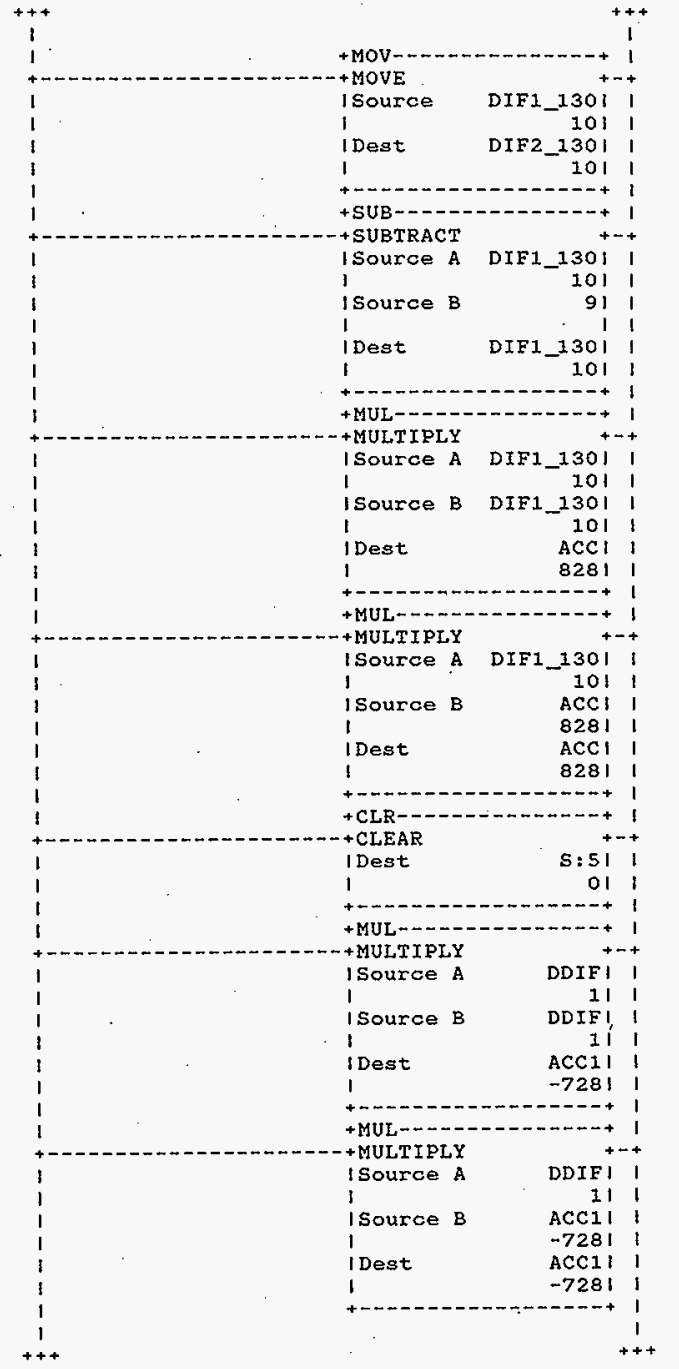



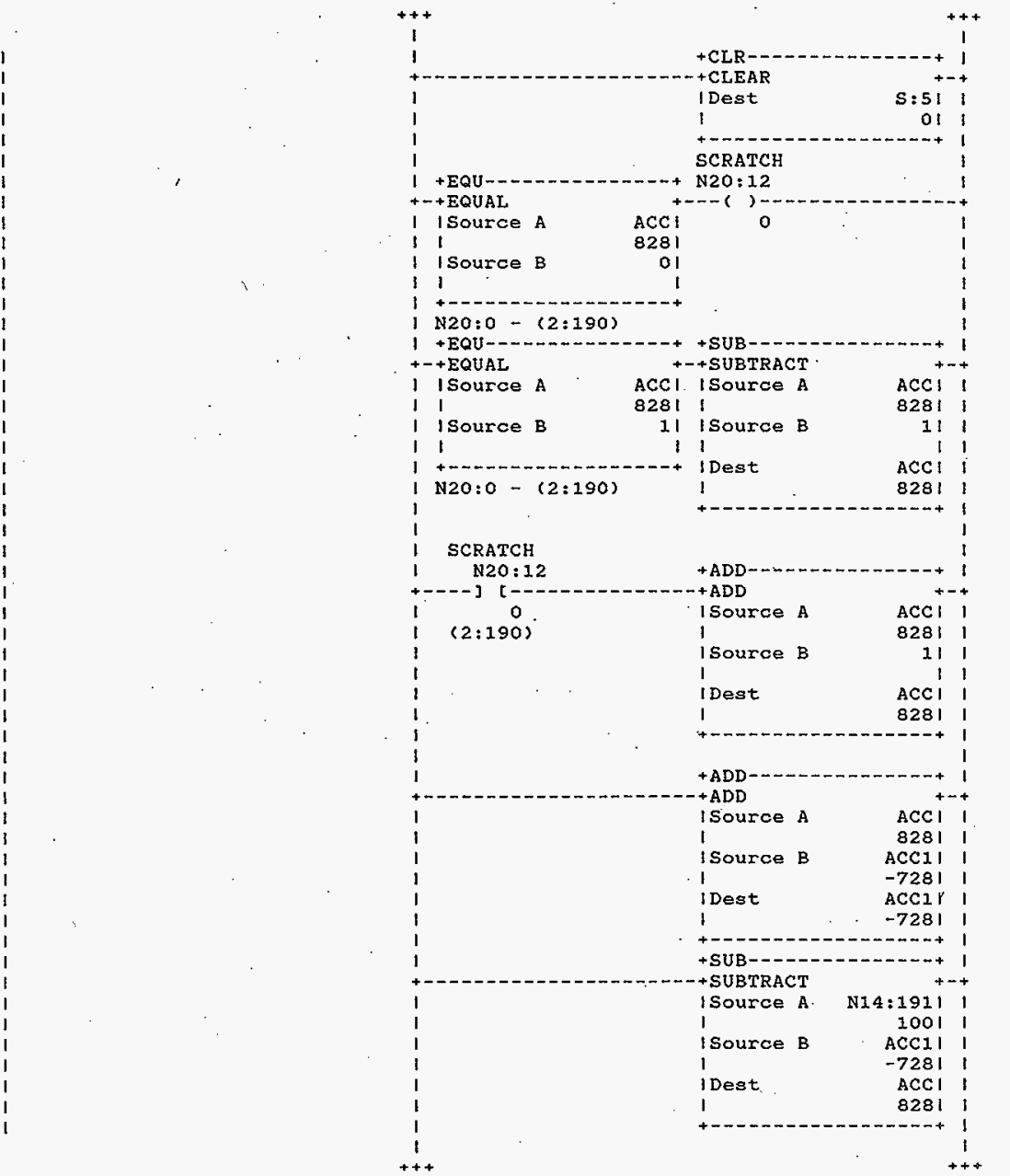

FNF-SD-FF-CSWD-61 Fiev. 0 


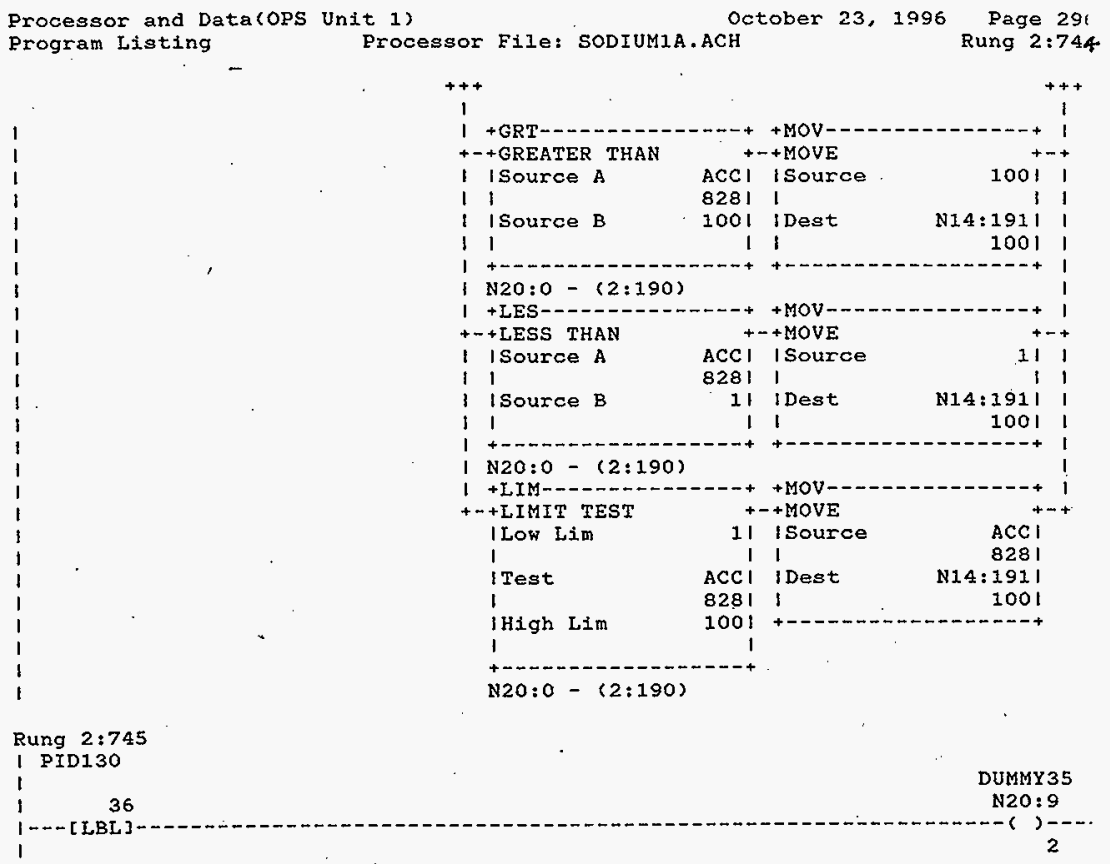

Rung 2:746

DETECT FROM SCADA PID ON/OFF

IF PID OFF

THEN ZERO PW FOR ZERO OUTPUT AT SCR AND JUMP AROUND THE PID BLOCK

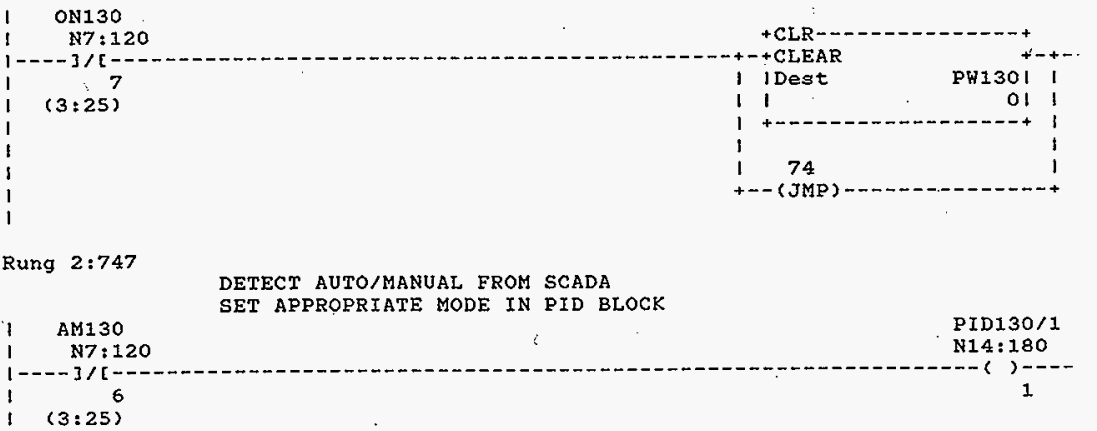

HNF-SD-FF-CSWD-61 Rev. 0 
Processor and Data(ops Unit 1)

October 23, 1996

Page 297

Program Listing

Processor File: SODIUM1A.ACH

Rung $2: 749$

Rung $2: 748$

DETECT SETPOINT VALUE FROM SCADA PUT VALUE IN PID BLOCK
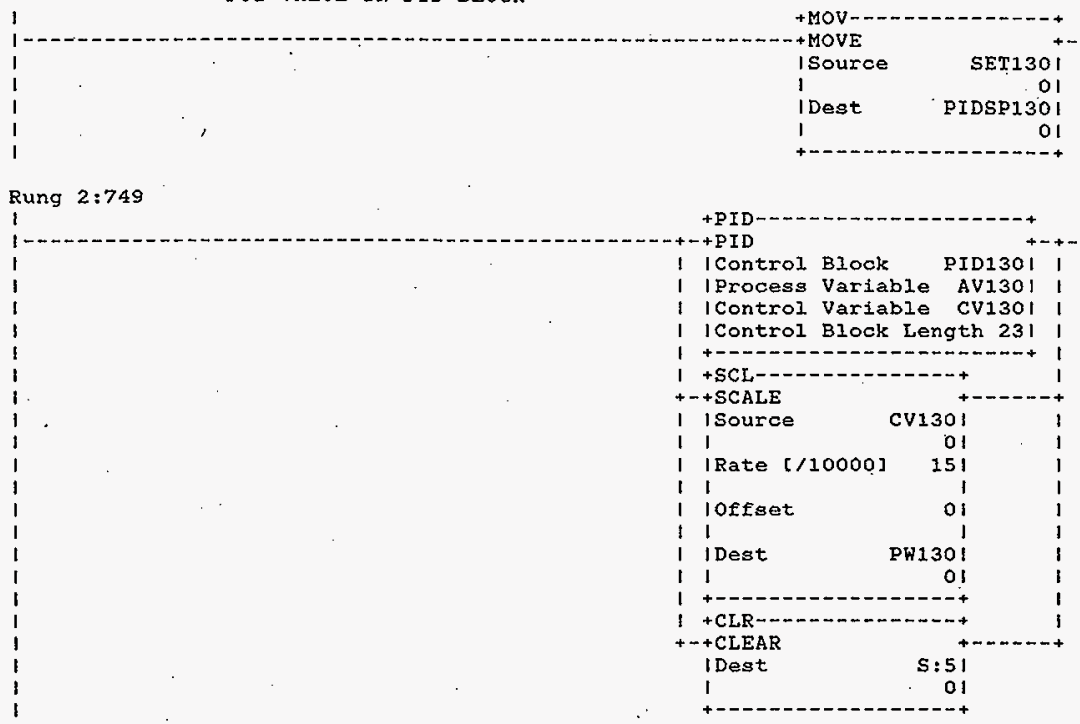

Rung 2:750

$$
174
$$

Rung 2:751

1

I RT TMR/DN

PID131

$1 \mathrm{~T} 4: 62$

$1-\cdots-\cdots /[-$

I $(2: 33)$

Rung 2:752

RATE CHECKING

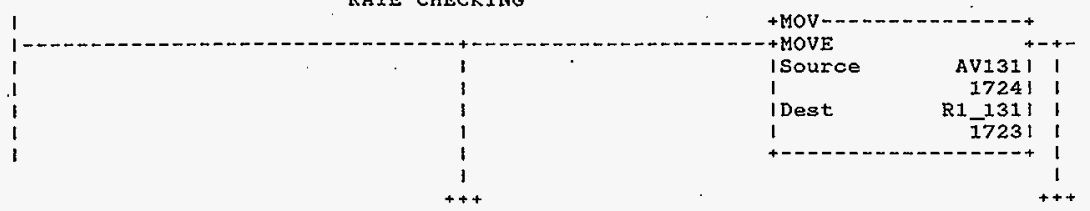

HNF-SD-FF-CSWD-61 Rev. 0 
Processor and Data(OPS Unit 1)

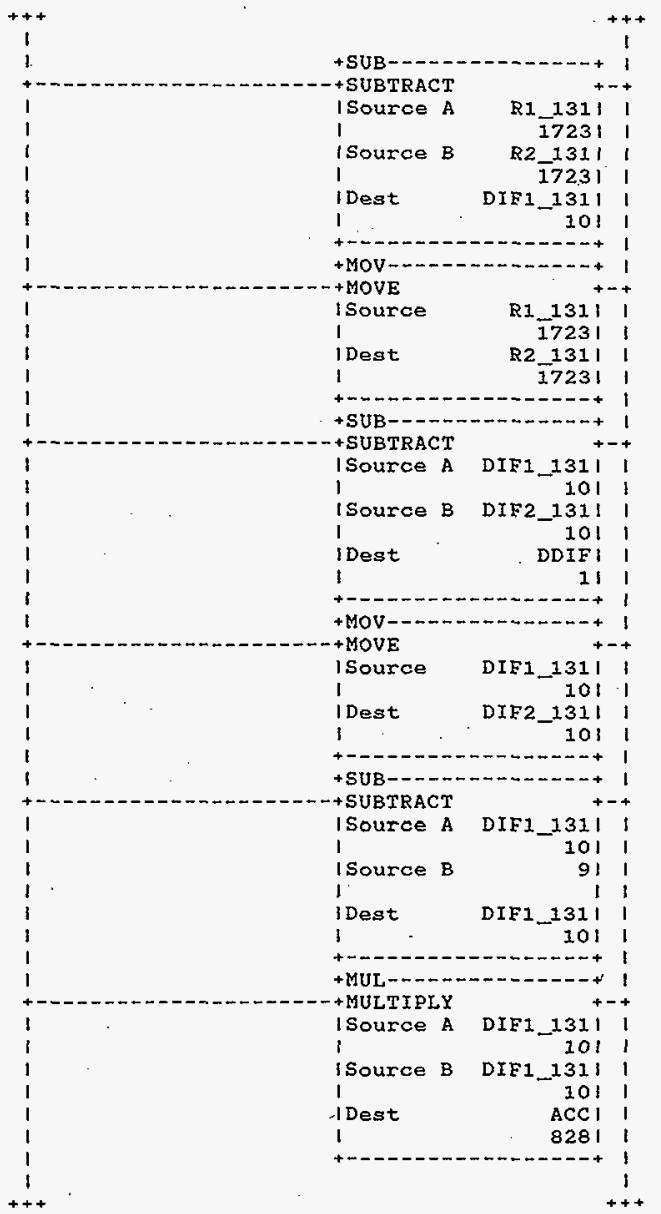


Processor and Data (OPS Unit 1 )

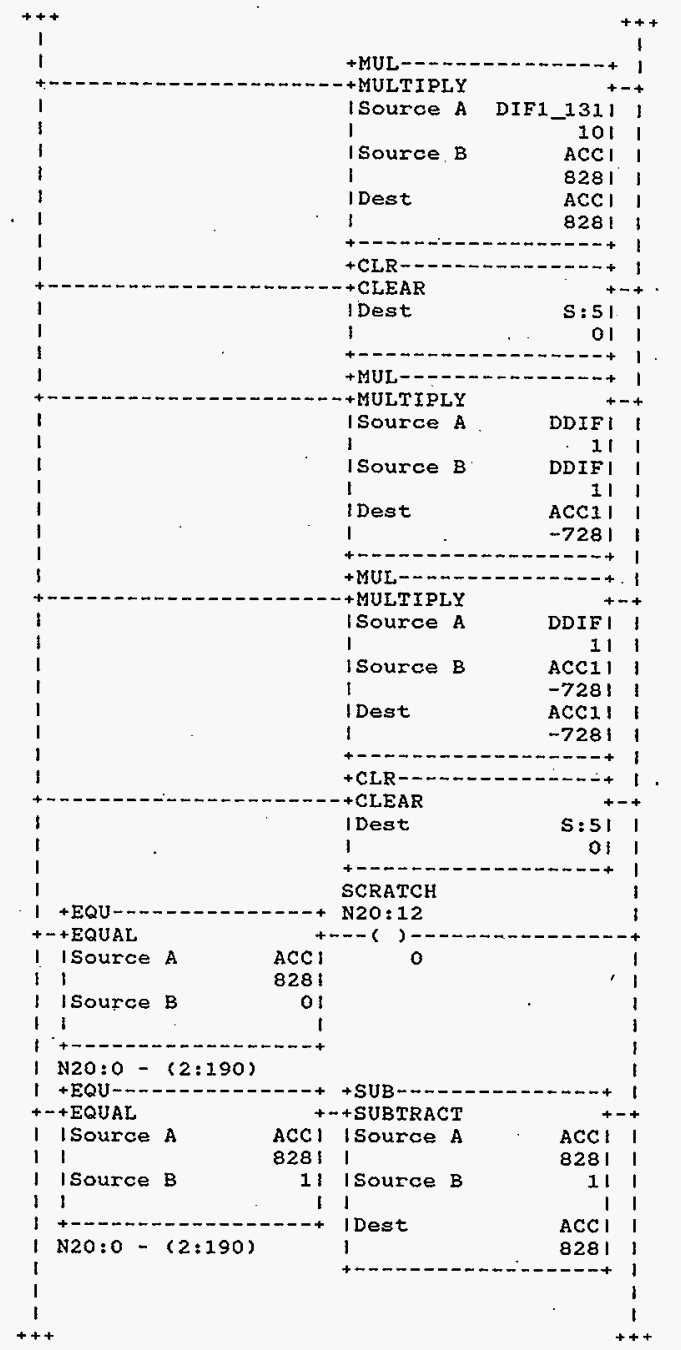




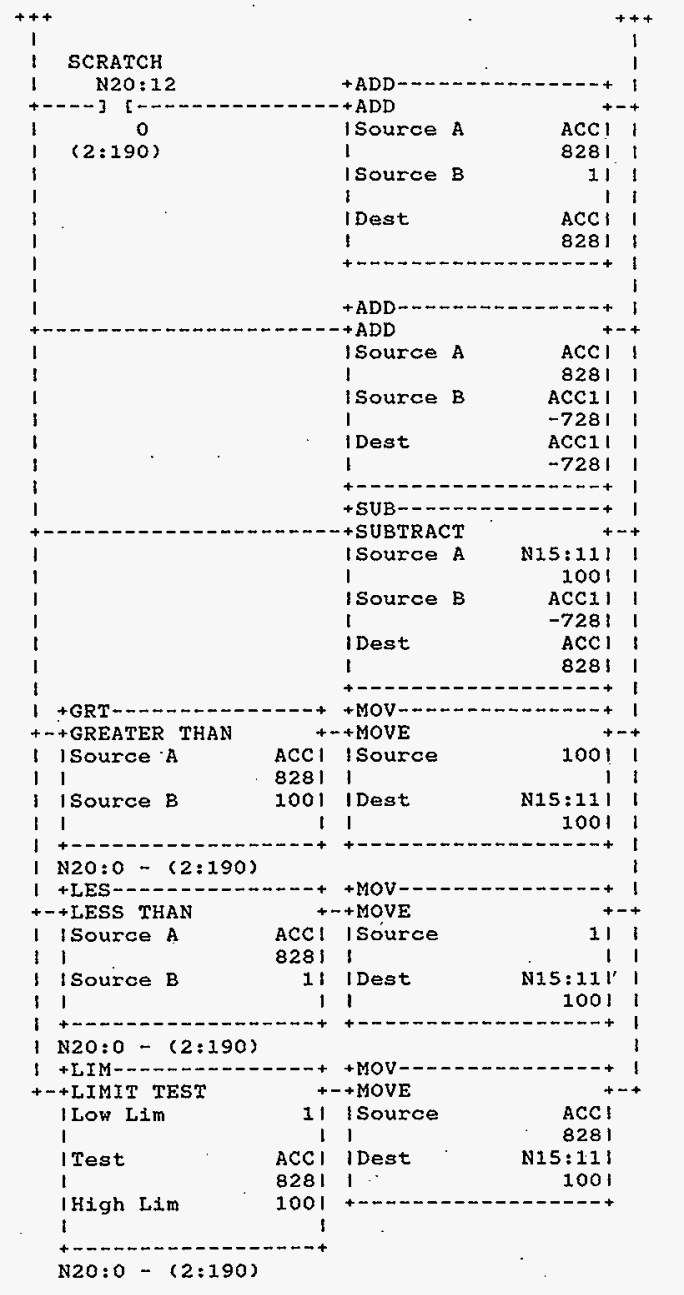


Processor and Data(ops Unit 1)

Rung $2: 753$

1 PIDI31

1. DUMMY36

$137 \quad$ N20:9

1

Rung $2: 754$

DETECT FROM SCADA PID ON/OFF

IF PID OFF

THEN ZERO PW FOR ZERO OUTPUT AT SCR

AND JUMP AROUND THE PID BLOCK

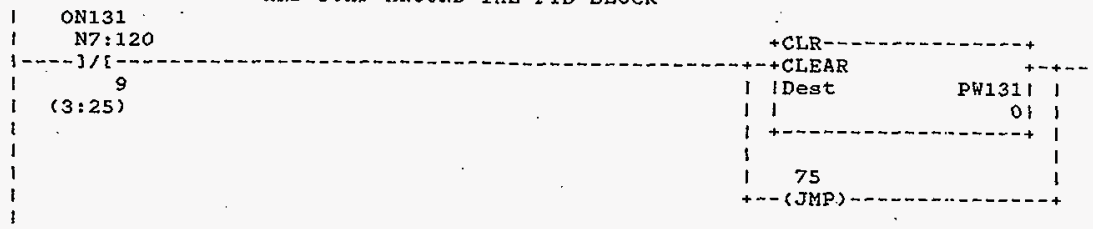

Rung 2:755

DETECT AUTO/MANUAL FROM SCADA

SET APPROPRIATE MODE IN PID BLOCK

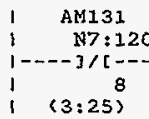

PID131/1

N15:0

$--$

Rung $2: 756$

DETECT SETPOINT VALUE FROM SCADA PUT VALUE IN PID BLOCK

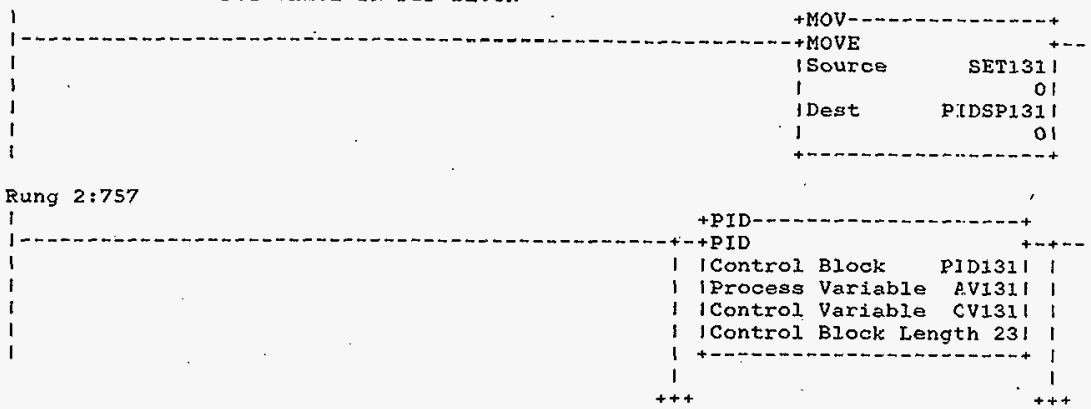

HNF-SD-FF-CSWD-61 Rev. 0 
Processor and Data(OPS Unit 1)

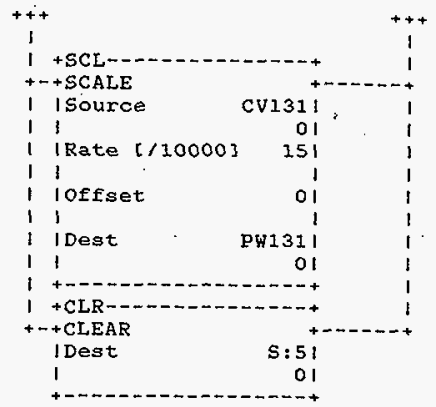

Rung 2:758

Rung $2: 759$

I

I RT TMR/DN

PID132

$$
1 \text { T4:62 }
$$

$1-\cdots /[--1$

I (2:33)

Rung 2:760

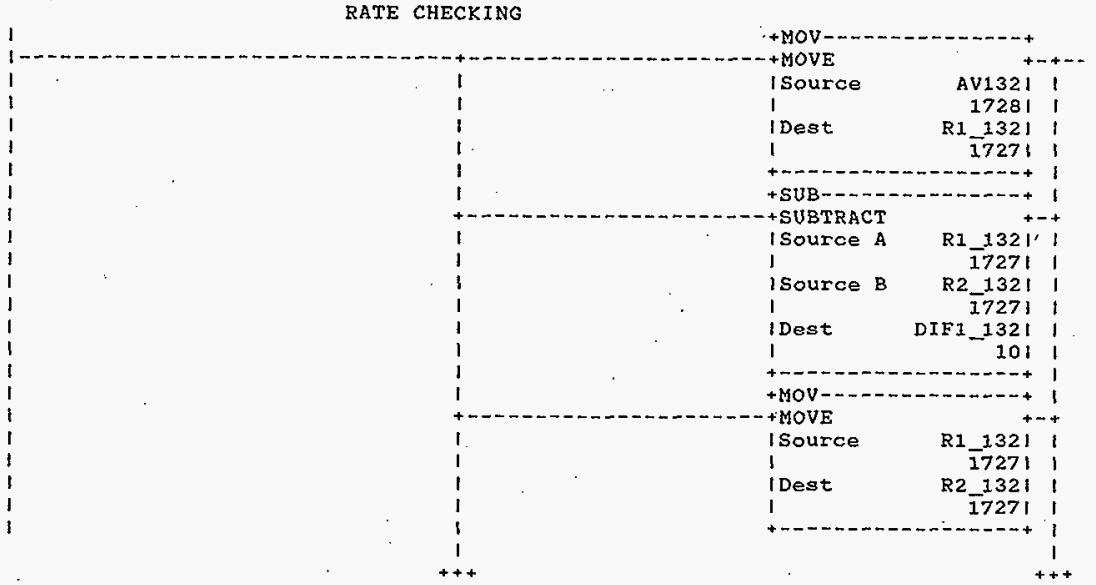

HNF-SD-FF-CSWD-61 Rev. 0 


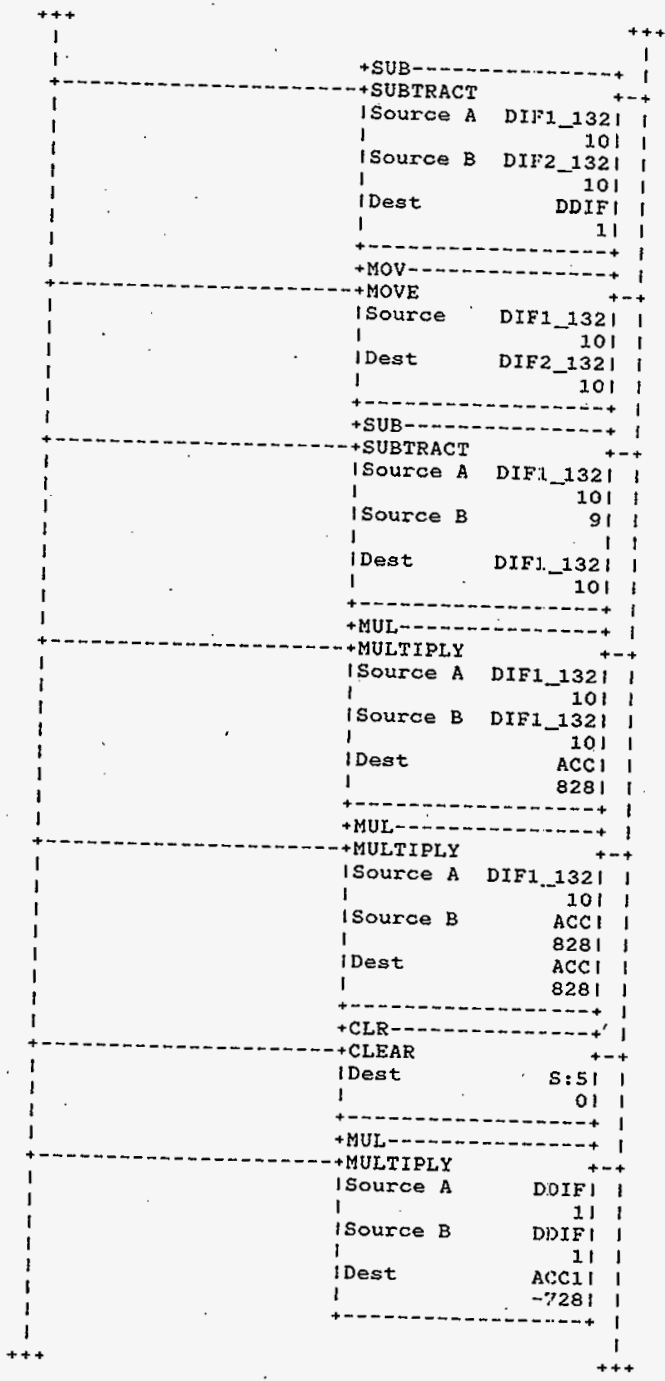

HNF-SD-FF-CSWD-61 Rev. 0 
Processor and Data(ops Unit 1 )
Program Listing

Processor File: SODIUMIA.ACH October 23, 1996 Page 30.

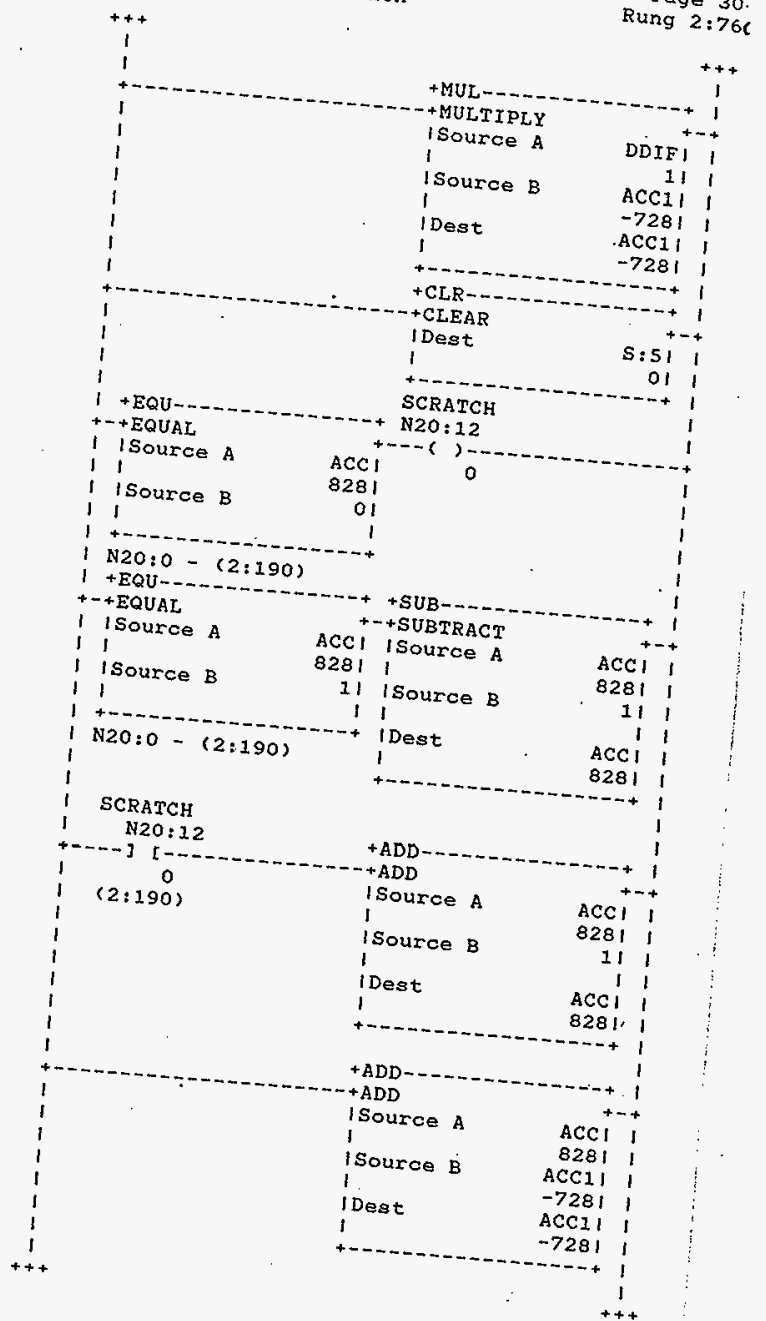

HNF-SD-FF-CSWD-6! Rev. 0

Page 304 


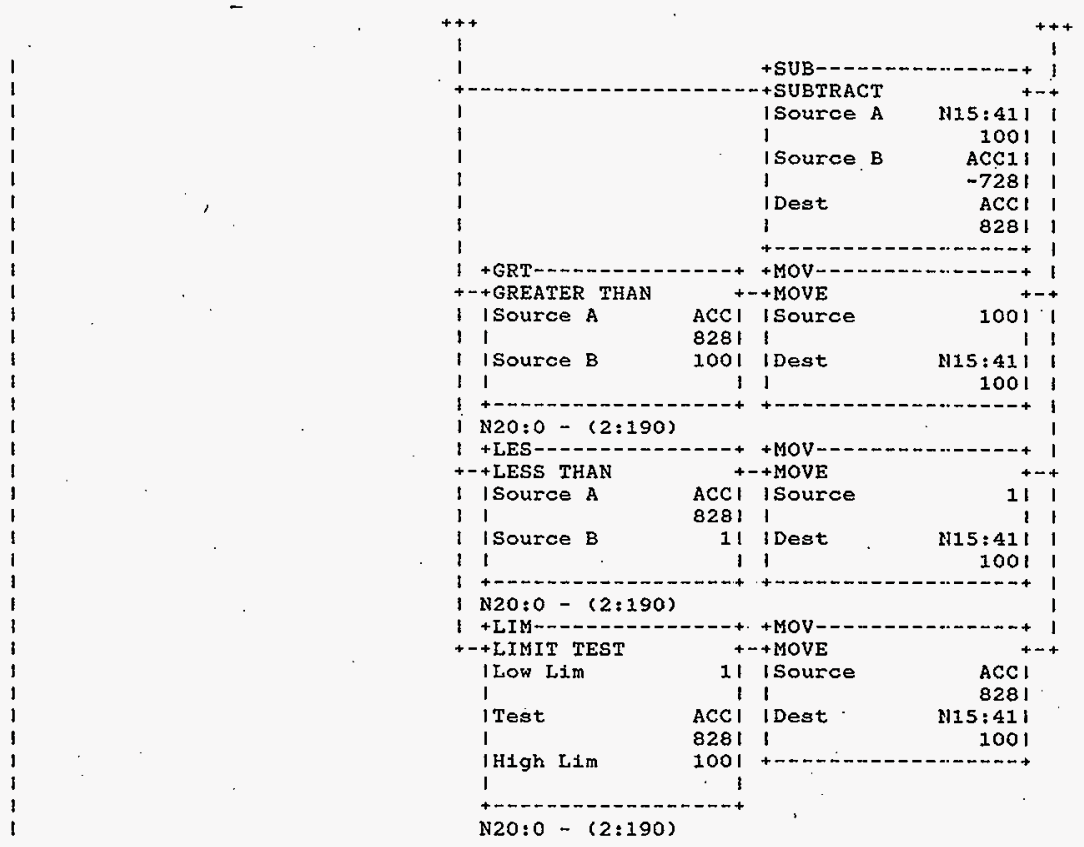

Rung $2: 761$

PIDI32

$\begin{array}{lll}1 & \text {. } & \text { DUMMY37 }\end{array}$

$38 \quad 38 \quad$ N20:9

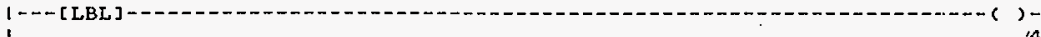

Rung 2:762 DETECT FROM SCADA PID ON/OFF

IF PID OFF

THEN ZERO PW FOR ZERO OUTPUT AT SCR AND JUMP AROUND THE PID BLOCK

\section{ON 132 \\ N7: 120}

11

$(3: 25)$

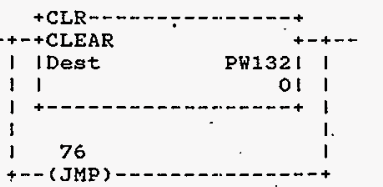

HNF-SD-FF-CSWD-61 Rev. 0 
Processor and Data(OPS Unit 1)

Rung 2:763

DETECT AUTO/MANUAL FROM SCADA

SET APRROPRIATE MODE IN PID BLOCK

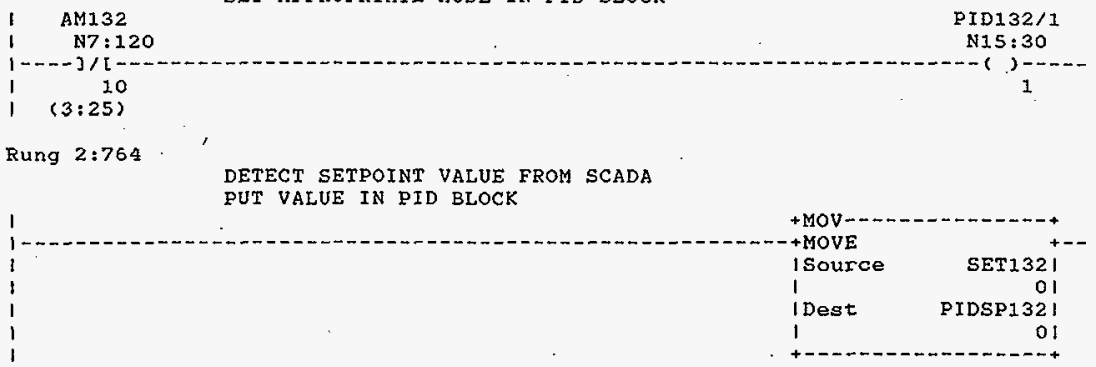

Rung 2:765

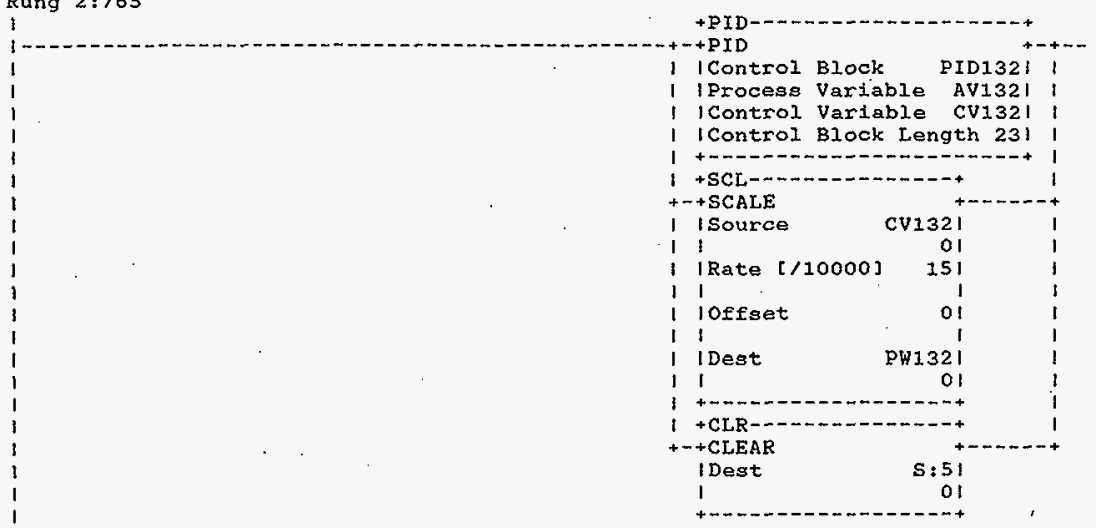

Rung $2: 766$

176

N2O: $10^{\circ}$

$1--[L B L]$

Rung 2:767

I

RT TMR/DN

P1D133

T4:62

39

$1---7 / \mathrm{C}-$

I (2:33)

HNF-SD-FF-CSWD-61 Rev. 0 
Processor and Data (OPS Unit 1)

October 23, 1996

Page 307

Program Listing

Processor File: SODIUM1A.ACH

Rung $2: 768$

Rung 2:768

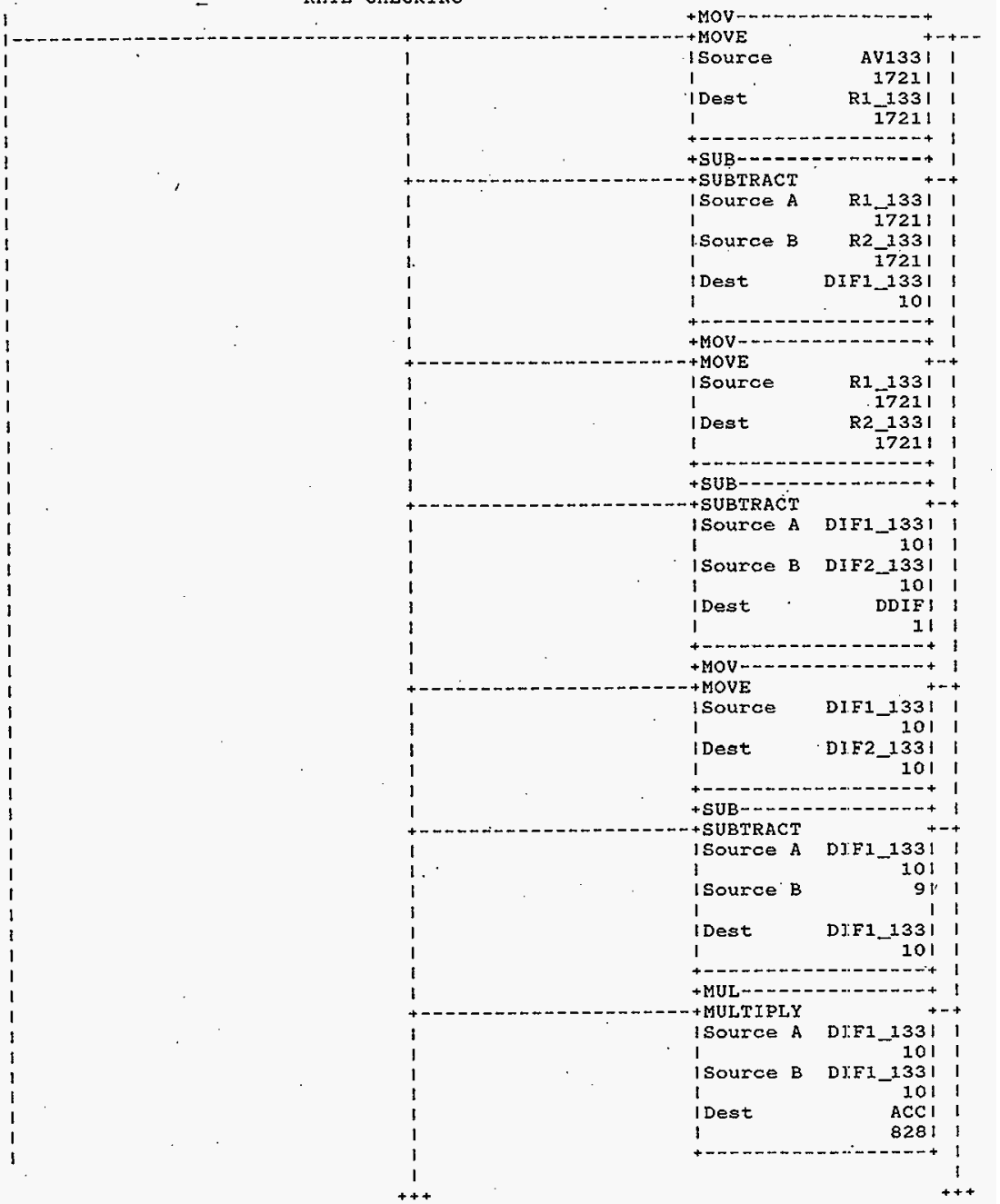

HNF-SD-FF-CSWD-61 ReV. 0

Page 307 

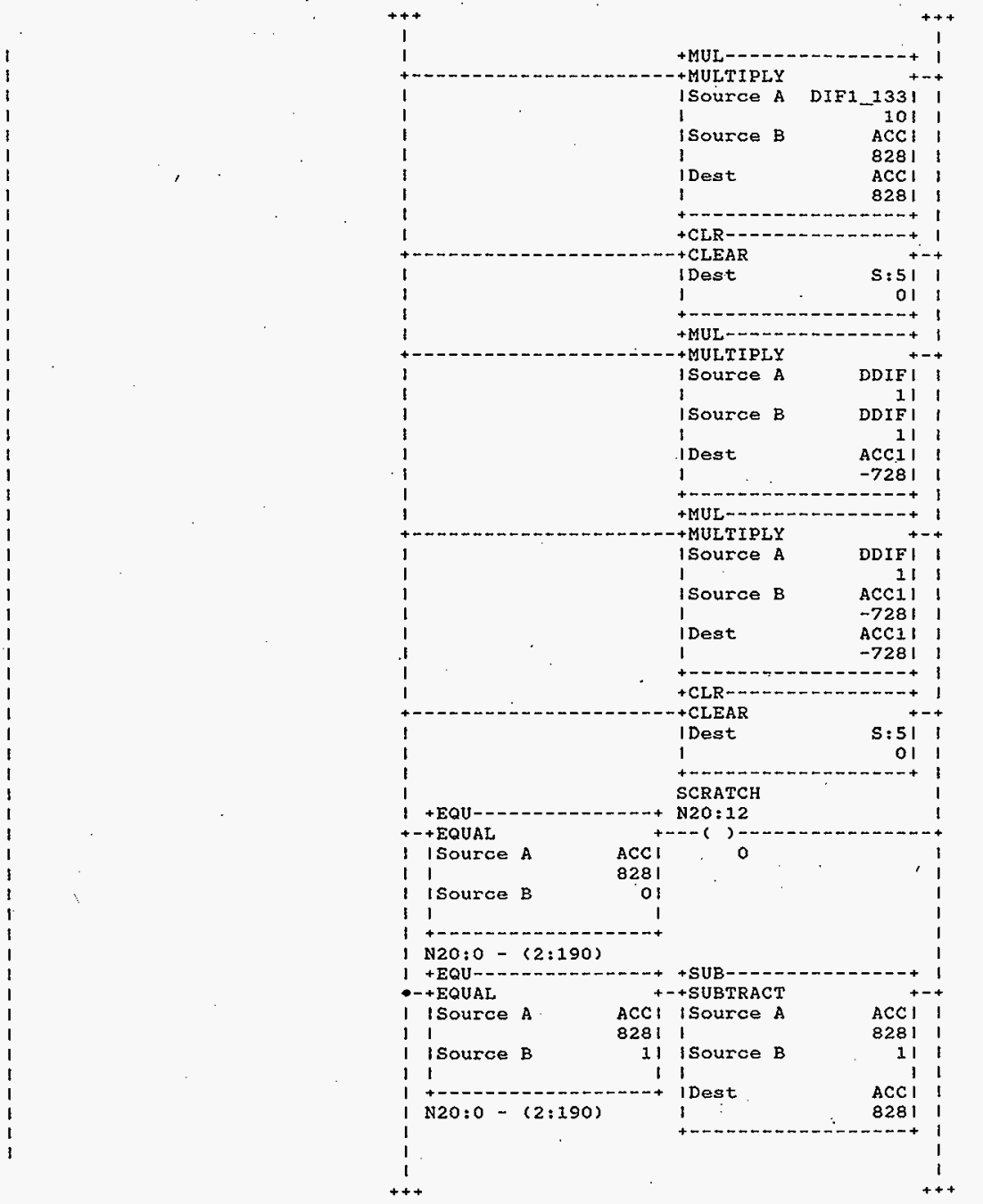

HNF-SD-FF-CSWD-61 Rev. 0 
Processor and Data(OPS Unit 1)

October 23,1996

Page 30

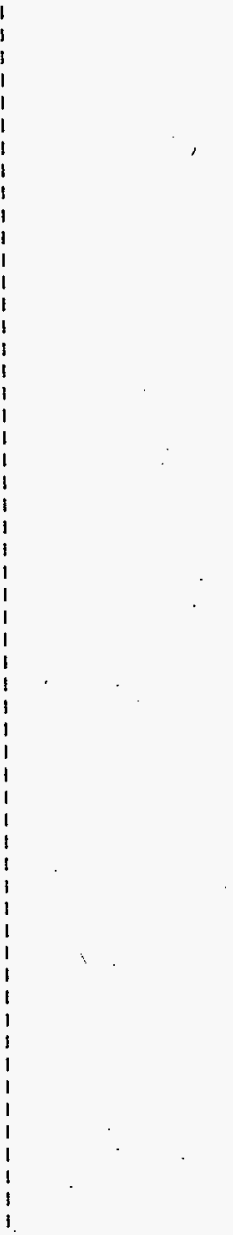

SCRATCH

$\begin{array}{lcr}1 & N 20: 12 & +A D D \\ 1 & 0 & \text { I Sou }\end{array}$

$(2: 190)$

I Source A

isource B

1

I Dest

1

$+---------------+$

$+\mathrm{ADD}$

I Source A

I

I Source B

i Dest

1

$+\mathrm{SUB}---0-0--0-0--0+1$

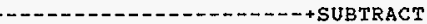

ISource A

I

isource B

N15:71।

1001

I

Dest

ACC1 1

I

ACC 1

$+$

8281

+-+GRTEATER THAN

I I Source A

11

I Isource B

$+\mathrm{MOV}$

$--+1$

11

+-+ MOVE

ACCI isource

82811

1001 IDest

I

N15:711

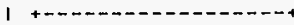

( N20:0 - (2:190)

I +LES--- -

+-+LESS THAN

1 I Source A

11

I ISource B

1001

11

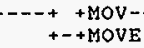

+-+ MOVE

8281

1.1 iDest

11

N15:711 1001

(N20:0-(2:190)

1 +LIM-D-D

+-+ IMIT TES

I Low Lim

1

I Test

1

High LIm

1

N20:0.-(2:190)

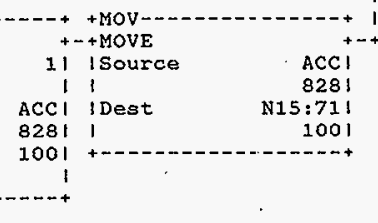

HNF-SD-FF-CSWD-61 Rev. 0

Page 309 
Processor and Data(OPS Unit 1)

Rung $2: 769$

1 PID 133

1 39

39

DUMMY 38

$1---[$ I.BL $]$

N20:9

I

Rung 2:770

, DETECT FROM SCADA PID ON/OFF

IF PID OFF

THEN ZERO PW FOR ZERO OUTPUT AT. SCR AND JUMP AROUND THE PID BLOCK

ON133

N7 : 120

$1----] /[-$

l. $(3: 25)$

$$
\text { (1) }
$$

Rung 2:77I

$\begin{array}{lc}1 & \text { AM133 } \\ 1 & \text { N7:120 } \\ 1 & --3 /[-12 \\ 1 & 12 \\ 1 & (3: 25)\end{array}$

DETECT AUTO/MANUAL FROM SCADA

SET APPROPRIATE MODE IN PID BLOCK

Rung $2: 772$

DETECT SETPOINT VALUE FROM SCADA

PUT VALUE IN PID BLOCK

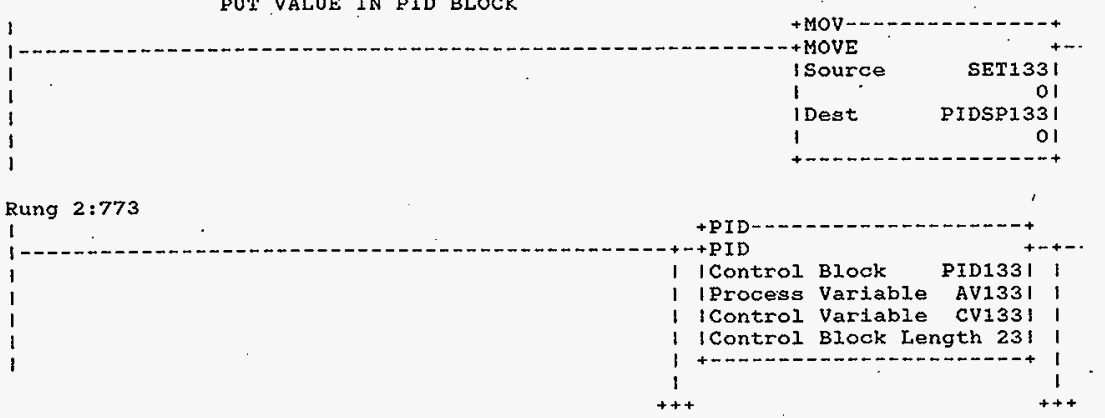

HNF-SD-FF-CSWD-61 Rev. 0 . 


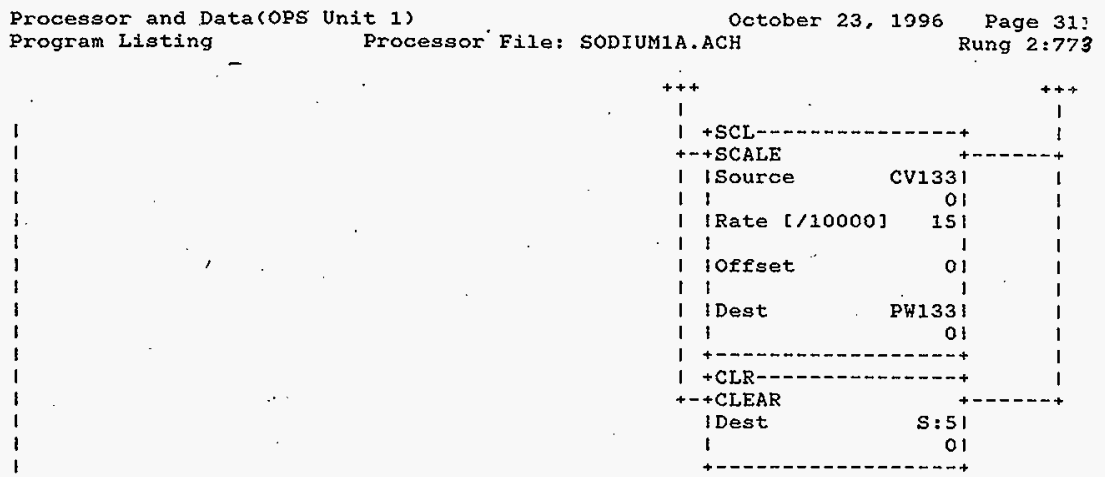

Rung $2: 774$

$1 \quad 77$

N2O: 10

i $--[$ LBL $]$

Rung 2:775

1

RT TMR/DN

PID134

$\mathrm{T} 4: 62$

$1---] /[-$

$(2: 33)$

(JMP)

Rung 2:776

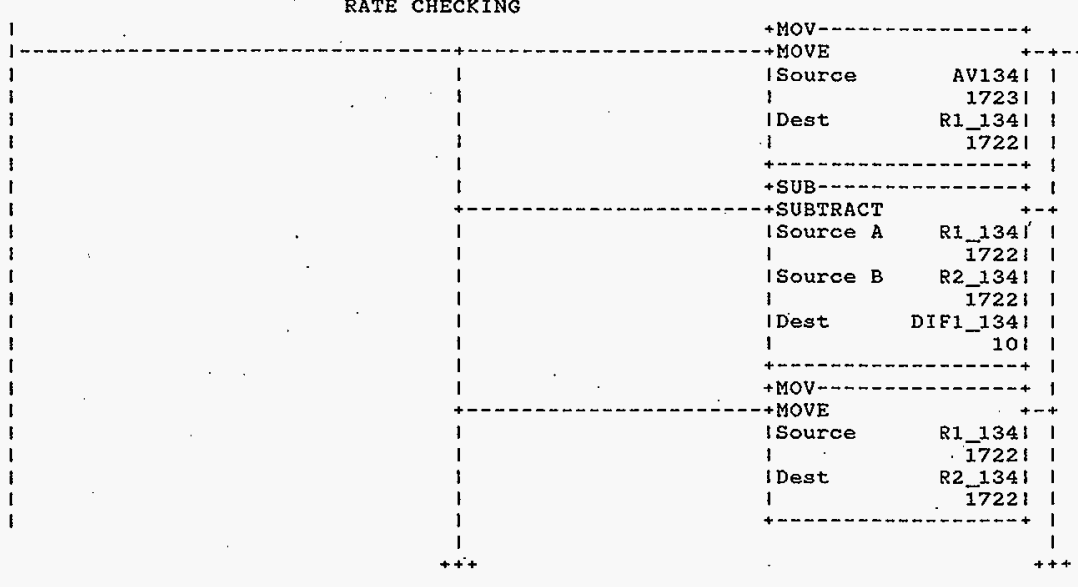

HNF-SD-FF-CSWD-61 Rev. 0

Page $\xi 1 /$ 

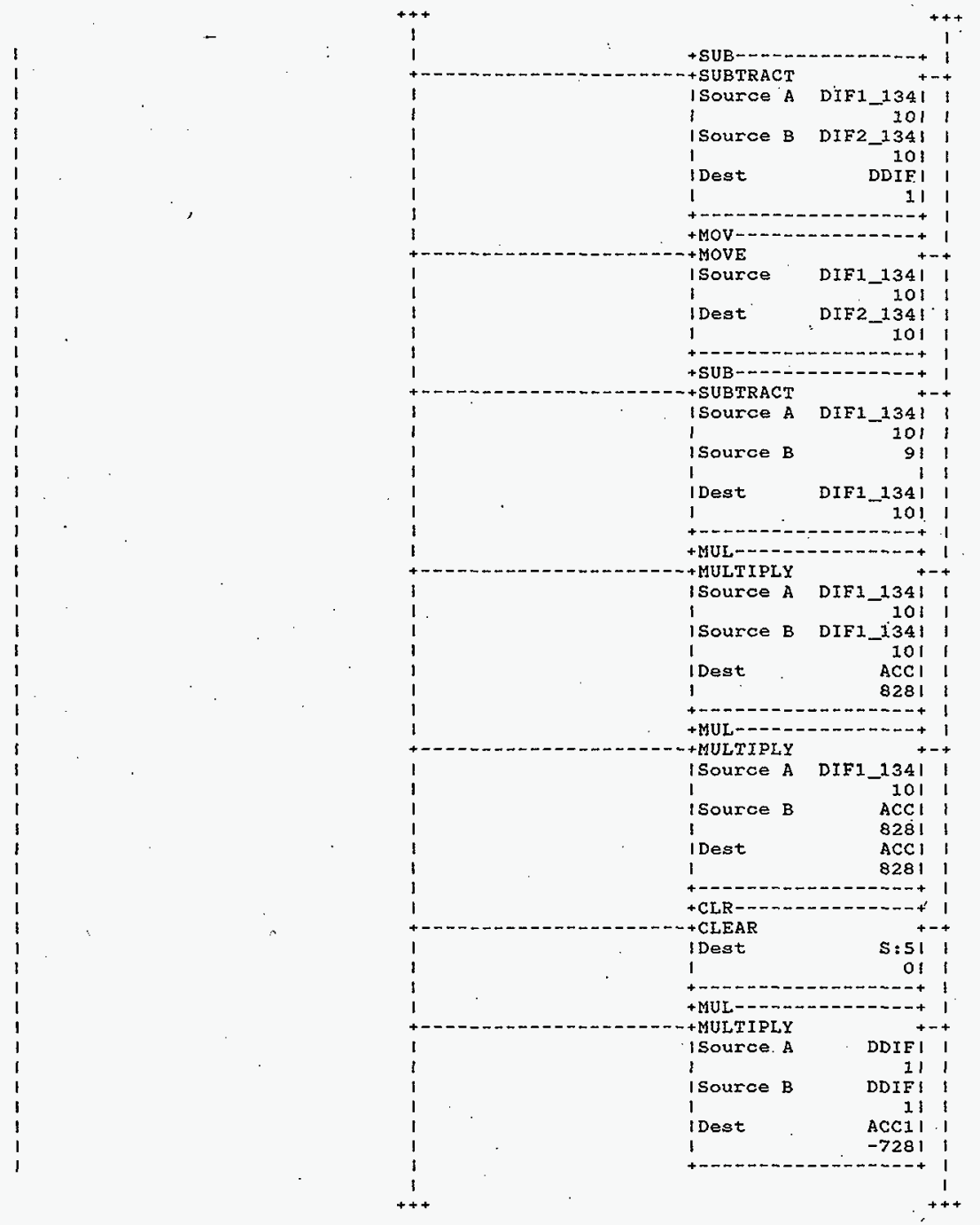


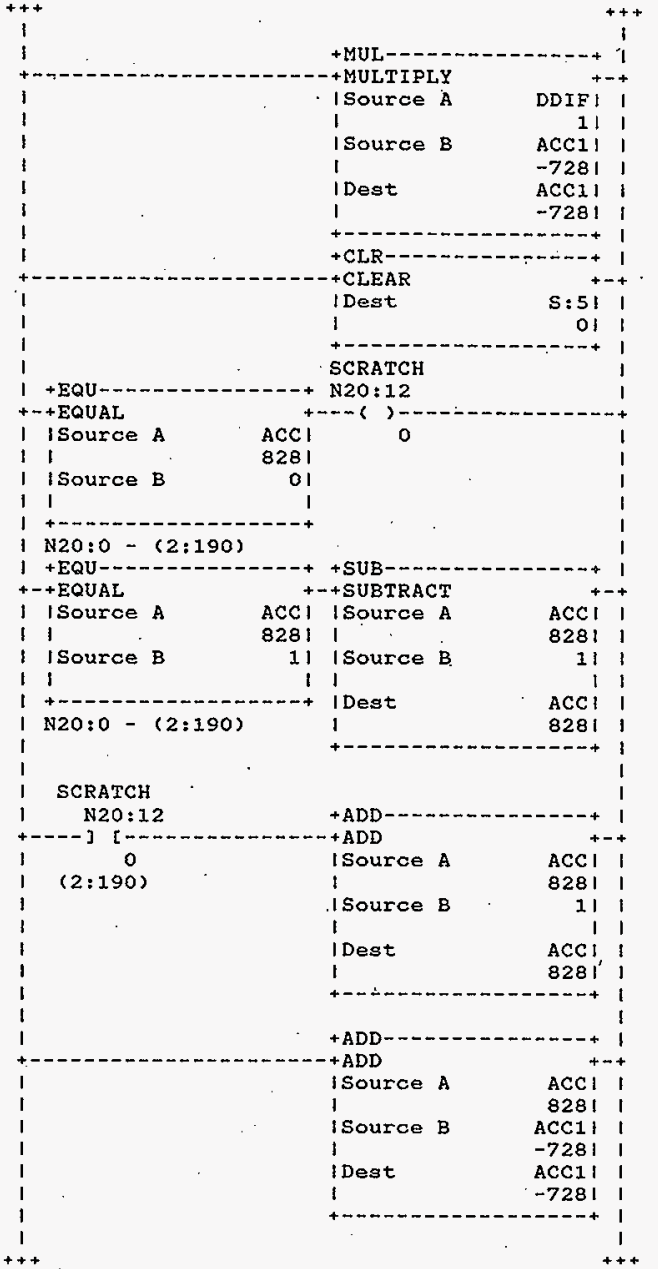


Processor and Data(OPS Unit 1) Program Listing

October 23, 1096 Processor File: SODIUM1A.ACH

Page 314 Rung $2: 776$

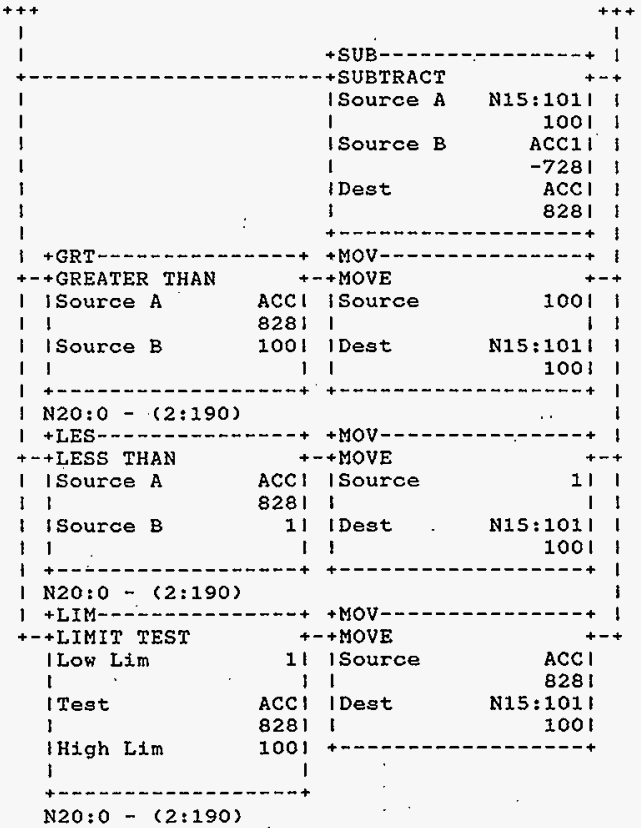

Rung $2: 777$

I PID 134

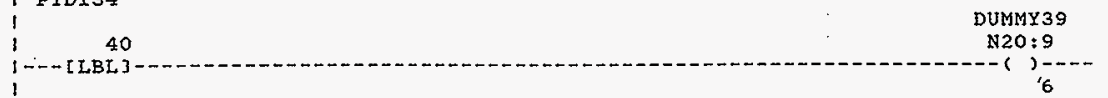

Rung $2: 778$

DETECT FROM SCADA PID ON/OFF

IF PID OEE

THEN ZERO PW FOR ZERO OUTPUT AT SCR AND JUMP AROUND THE PID BLOCK

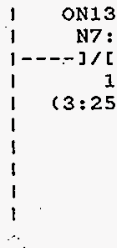

HNF-SD-FF-CSWD-61 Rev. 0 
Processor and Data(OPS Unit 1) Program Listing

DETECT. AUTO/MANUAL FROM SCADA

SET APPROPRIATE MODE IN PID BLOCK

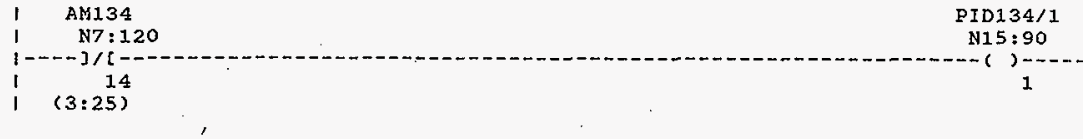

Rung 2:780

DETECT SETPOINT VALUE FROM SCADA PUT VALUE IN PID BLOCK
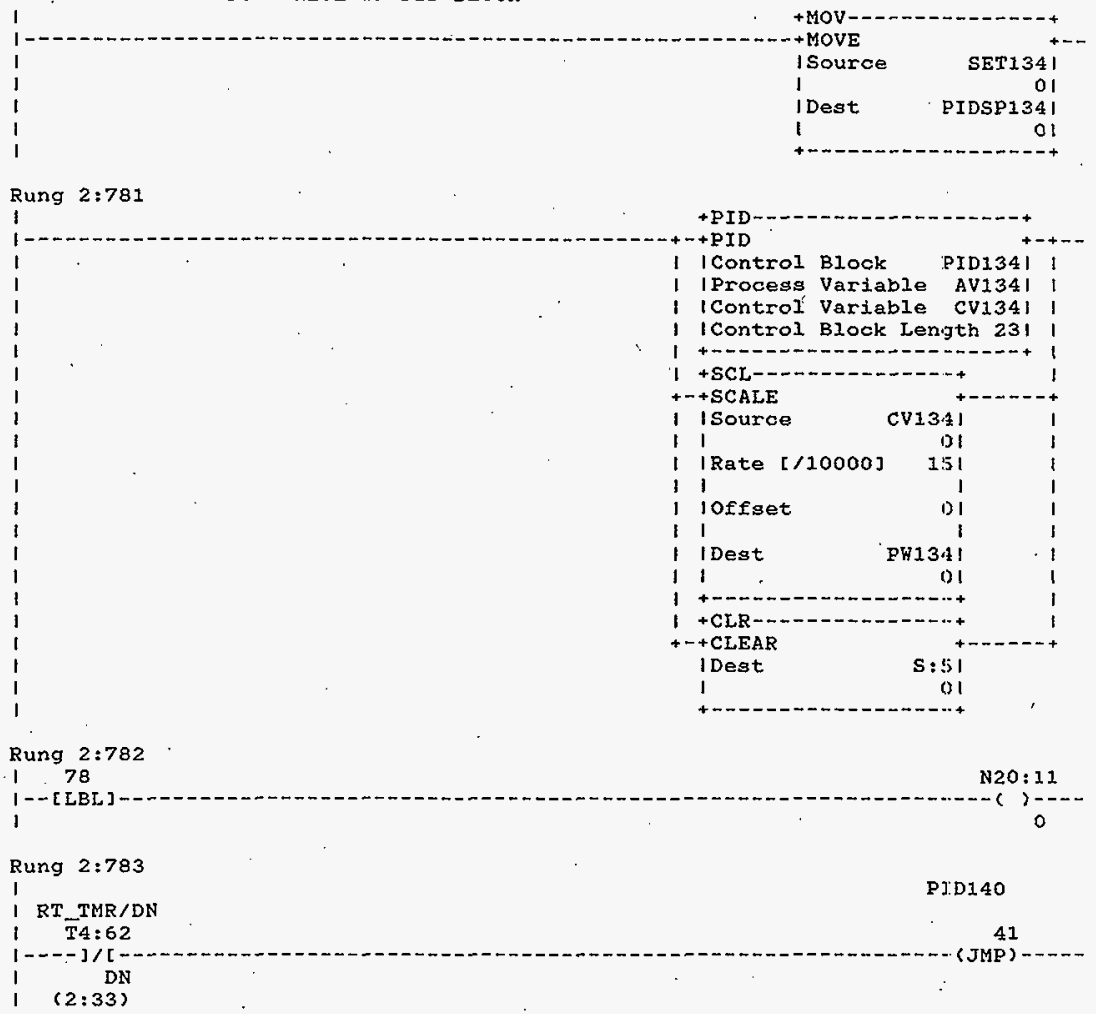

HNF-SD-FF-CSWD-61 Rev. 0 
Processor and Data(OPS Unit 1)

october 23, 1996 Page 316

Program Listing

Processor File: SODIUMIA.ACH

Rung $2: 784$

Rung 2:784

RATE CHECKING

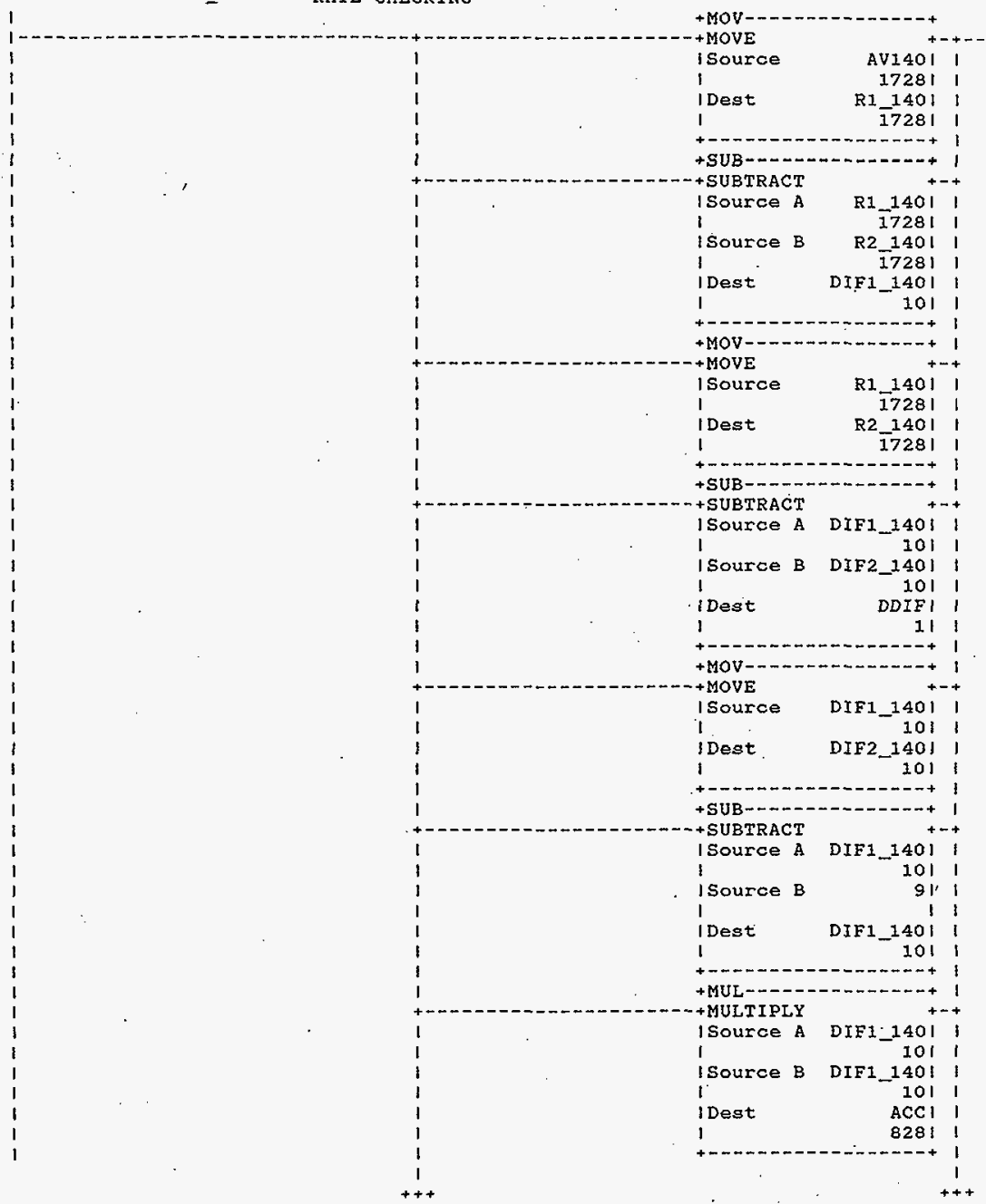

HNF-SD-FF-CSWD-61 Rev. 0 


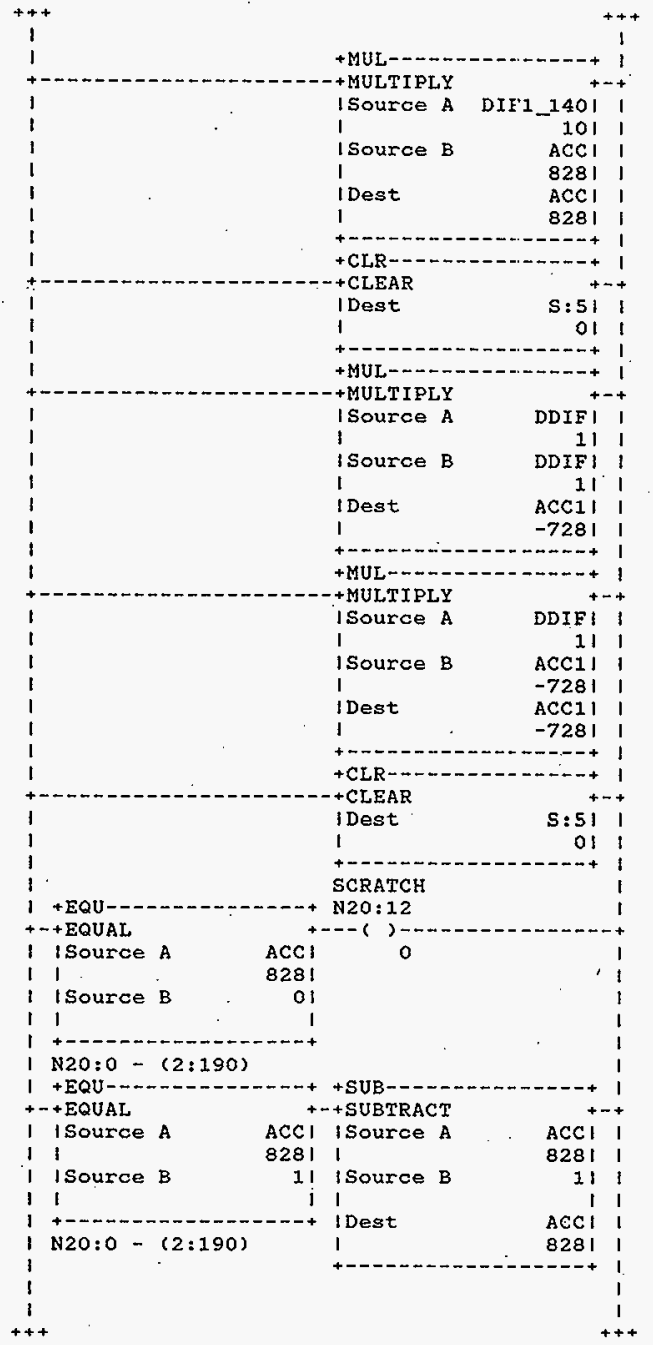

HNF-SD-FF-CSWD-61. Rev. 0 
Processor and Data(OPS Unit 1$)^{\circ}$
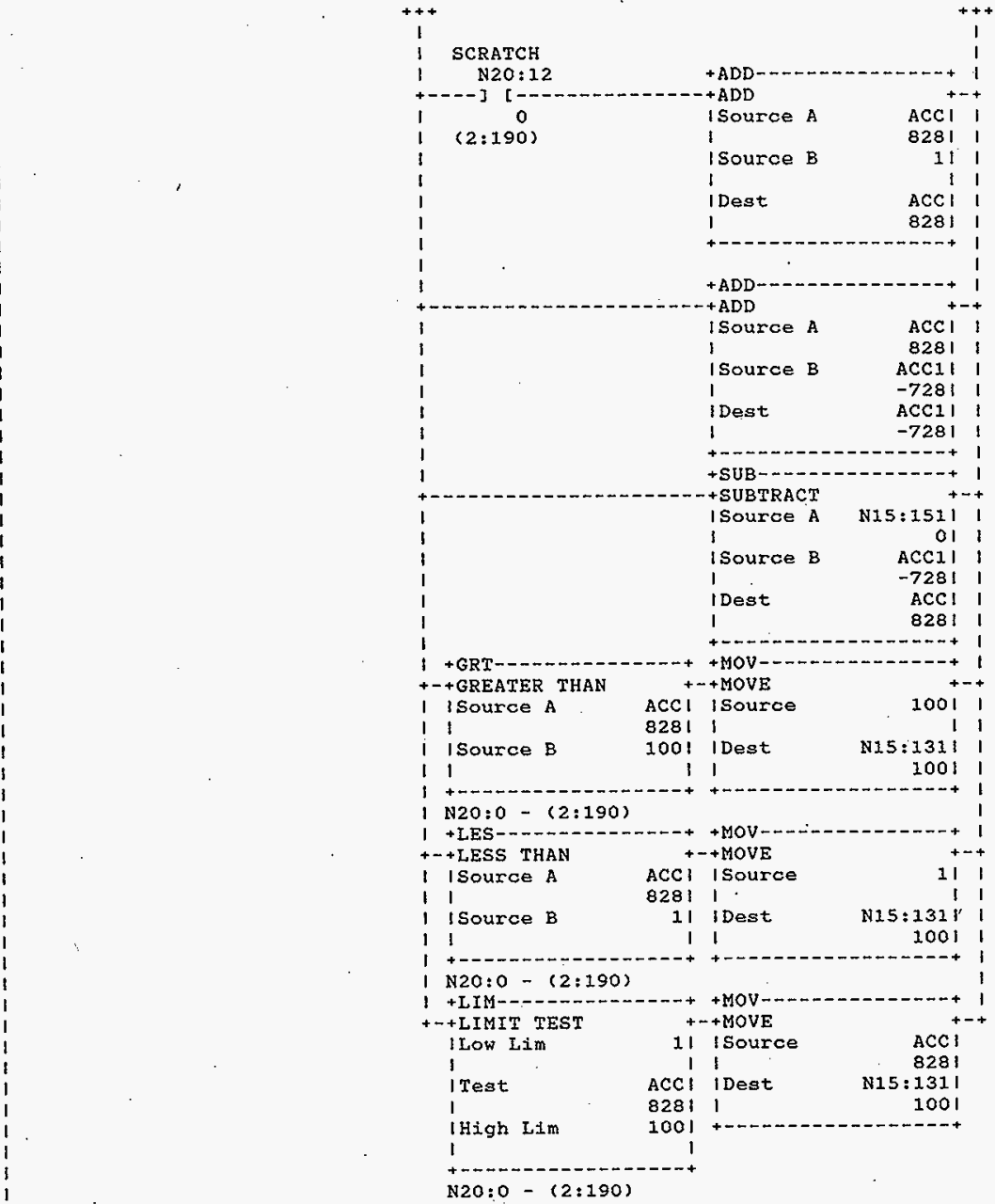

HNF-SD-FF-CSWD-61 Rev. 0 
Processor and Data(OPS Unit 1)

Rung $2: 785$

I.PID140

I

141

DUMMY 40

1--- [ LBL] N2O: 9

1

Rung 2:786

\section{DETECT FROM SCADA PID ON/OFF IF PID OFF}

THEN ZERO PH FOR ZERO OUTPUT AT SCR AND JUMP AROUND THE PID BLOCK

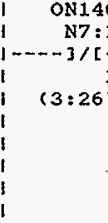

Rung $2: 787$

i. $\quad$ N7:121

SET APPROPRIATE MODE IN PID BLOCK

$1-\cdots-3 /[-1$

i. $(3: 26)$

Rung $2: 788$

DETECT SETPOINT VALUE FRON SCADA

PUT VALUE IN PID BLOCK

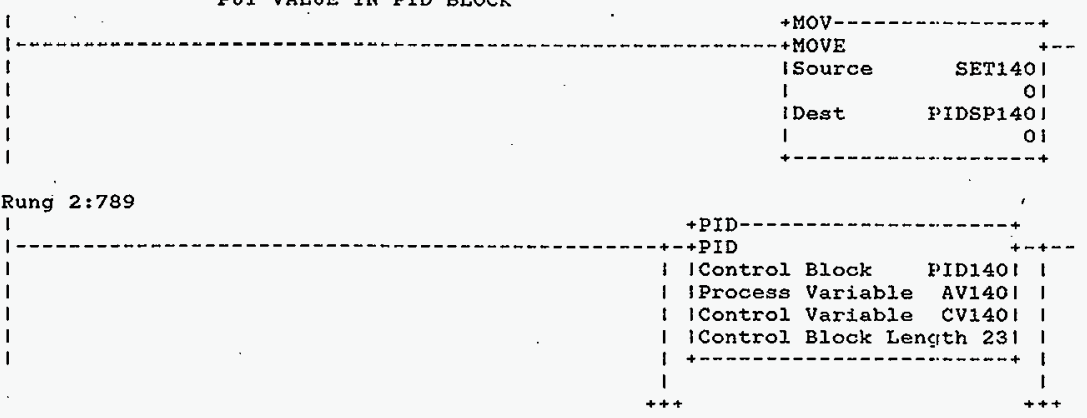

HNF-SD-FF-CSWD-61 Rev. 0 


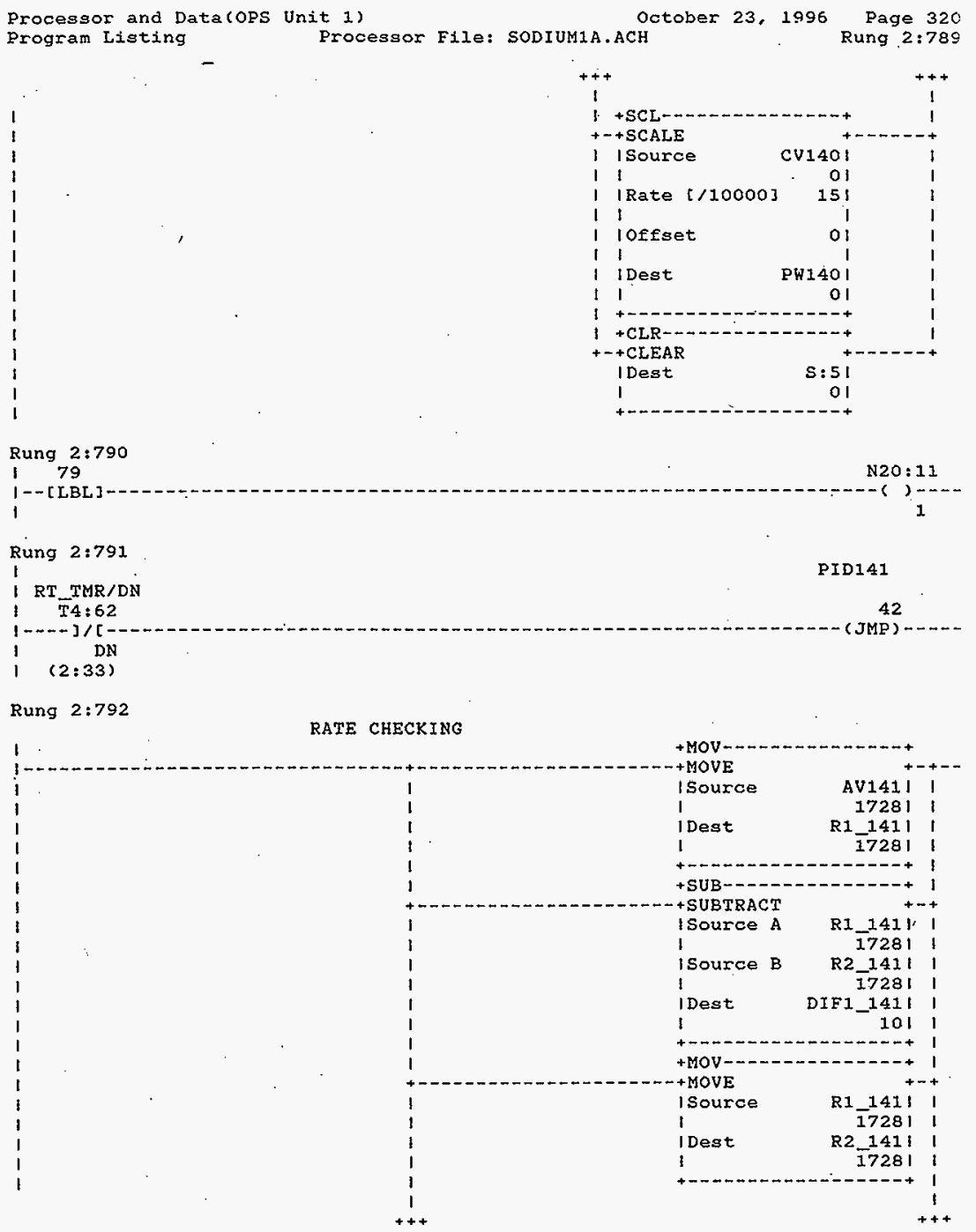

HNF-SD-FF-CSWD-61 Rev. 0 


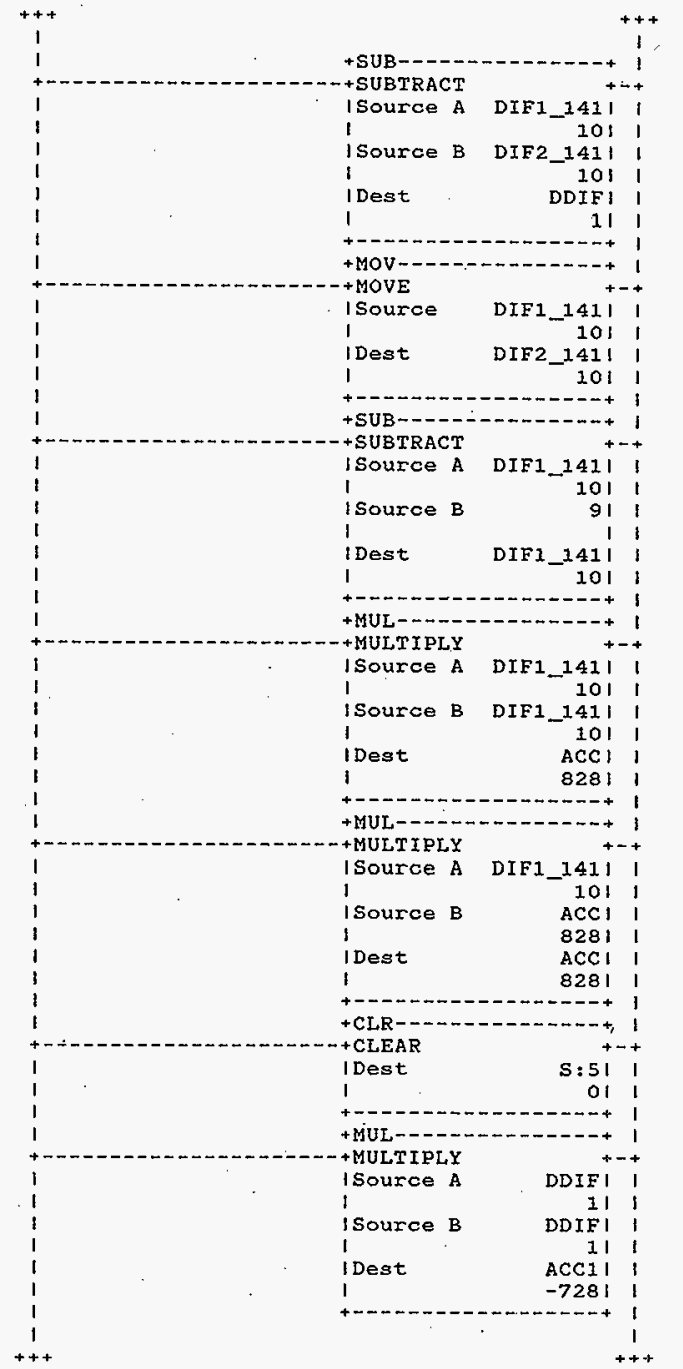




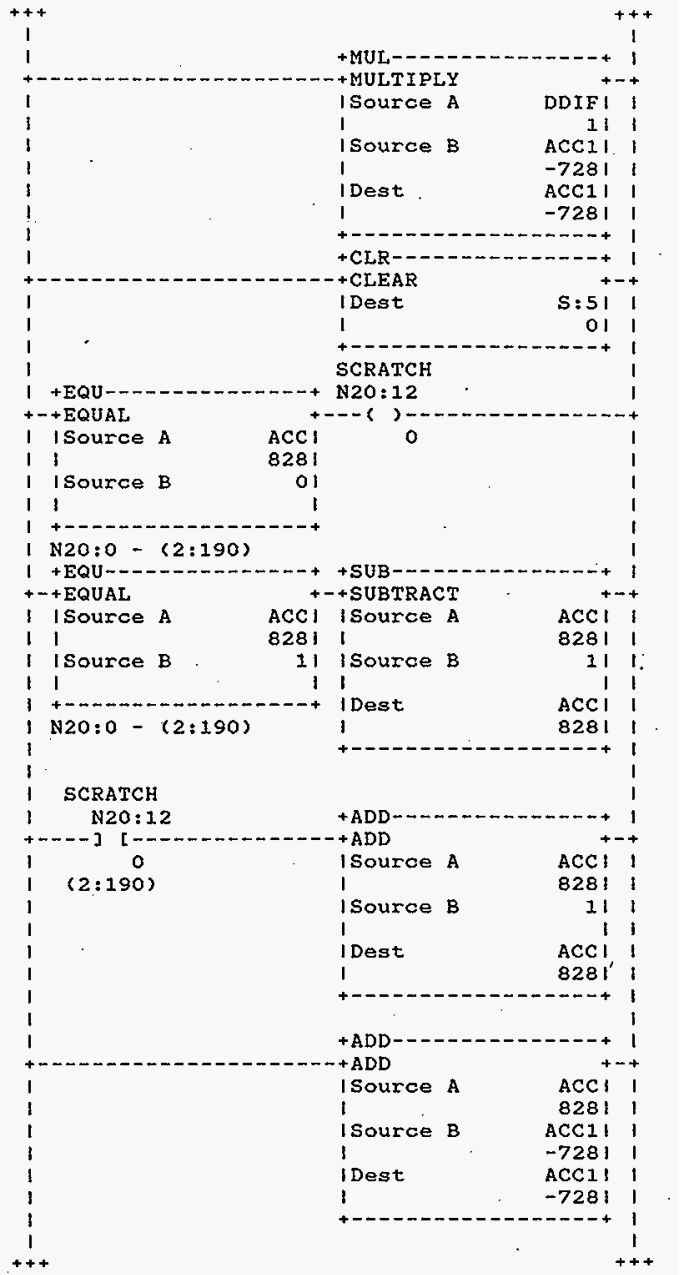


Processor and Data (OPS Unit 1)

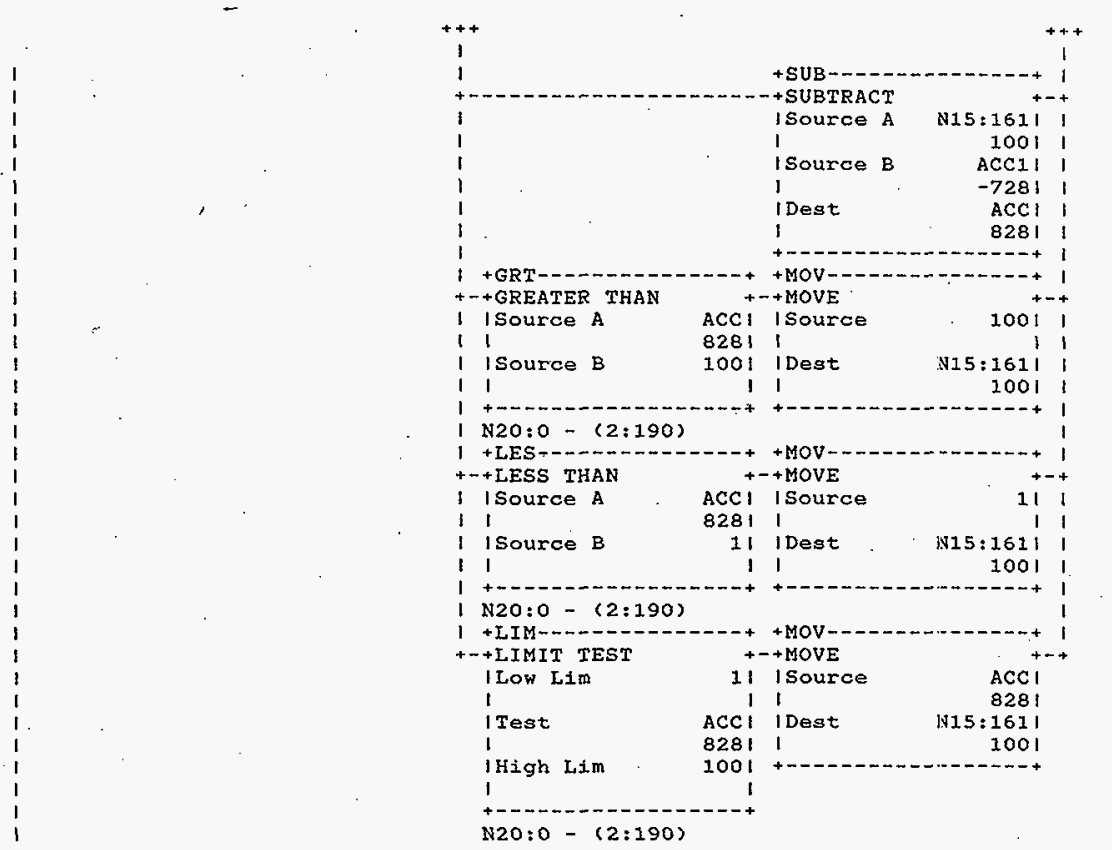

Rung $2: 793$

I PID141

I. 42

DUMMY4I

42

N20:9

I - - [ [ LBL]

Rung 2:794

\section{DETECT FROM SCADA PID ON/OFF \\ IF PID OFF \\ THEN ZERO PW FOR ZERO OUTPUT AT SCR}

AND JUMP AROUND THE PID BLOCK

ON141
N7:121


DETECT AUTO/MANUAL FROM SCADA

SET APPROPRIATE MODE IN PID BLOCK

AM 141
I $\quad$ N $: 121$
1
I $(3: 26)$

Rung 2:796

\section{DETECT SETPOINT VALUE FROM SCADA} RUT VALUE IN PID BLOCK

+ MOVE

I Source

I

I Dest

I
PIDI $41 / 1$

N15:150

1

\section{Rung 2:797}

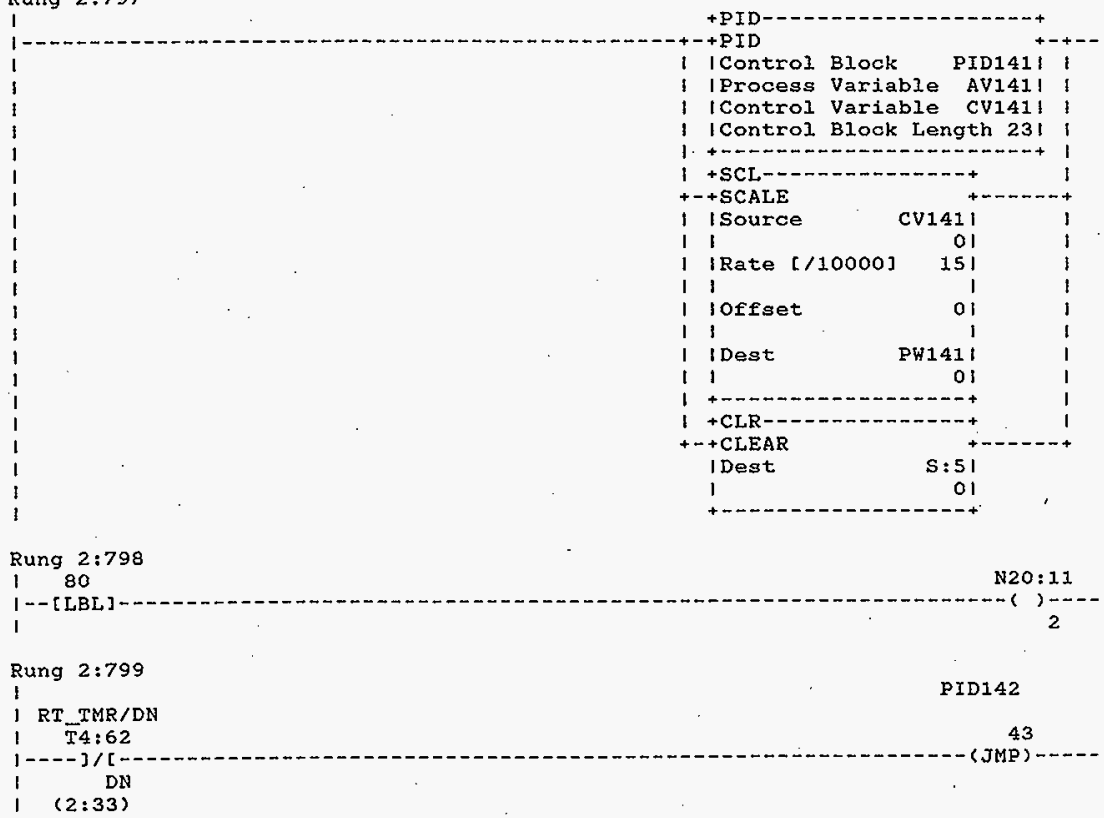

HNF-SD-FF-CSWD-61 Rev. 0 
Processor and Data(OPs Unit 1)

Rung 2:800

I RT_TMR/DN -

( T) T:62

DNR 4

I - - - ] [

$\mathrm{N} 20: 5$

$(2: 33)$

Rung 2:801

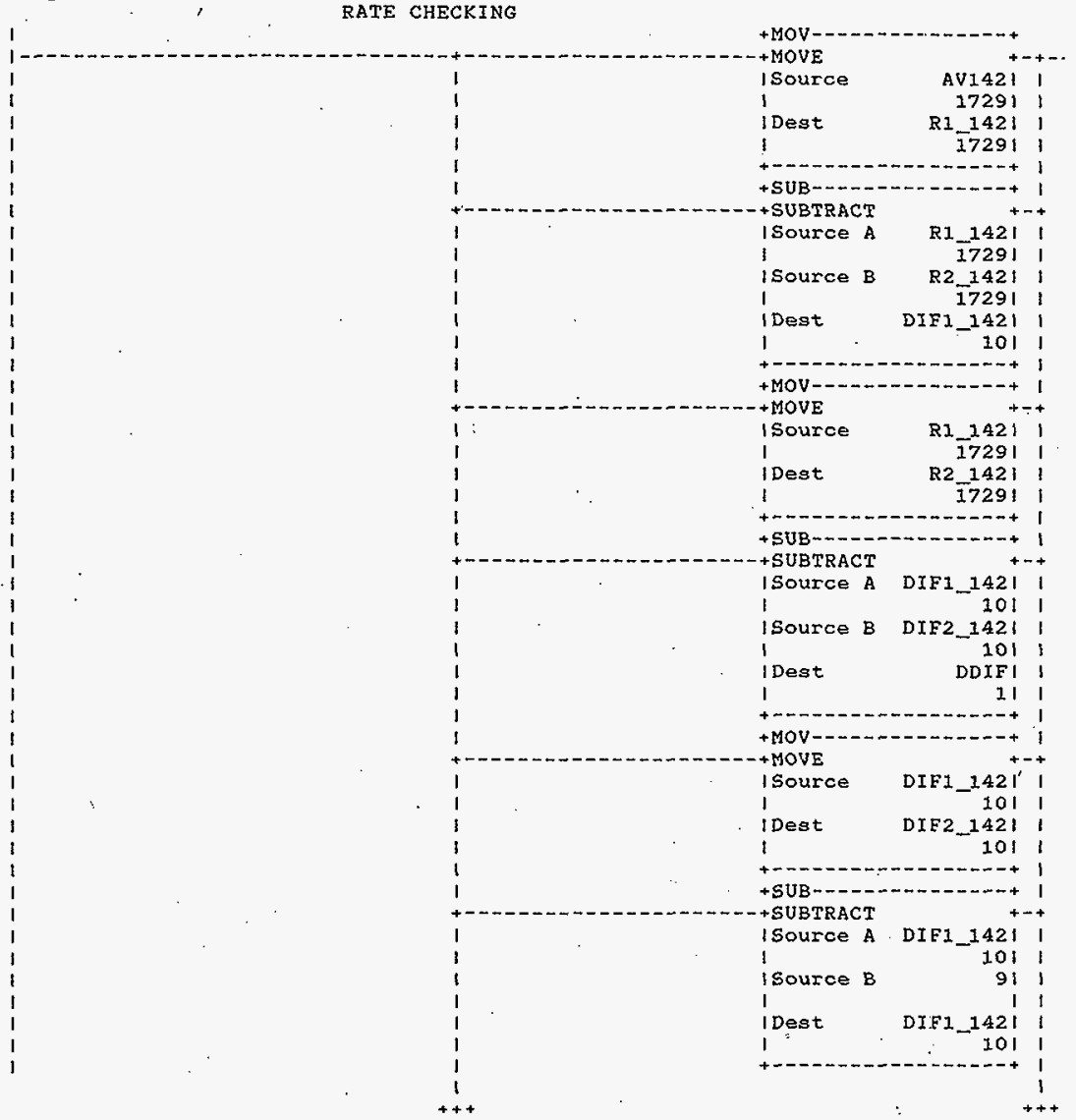

HNF-SD-FF-CSWD-61 Rev. 0 
Processor and Data(OPS Unit 1)

October 23, 1396

Page 326

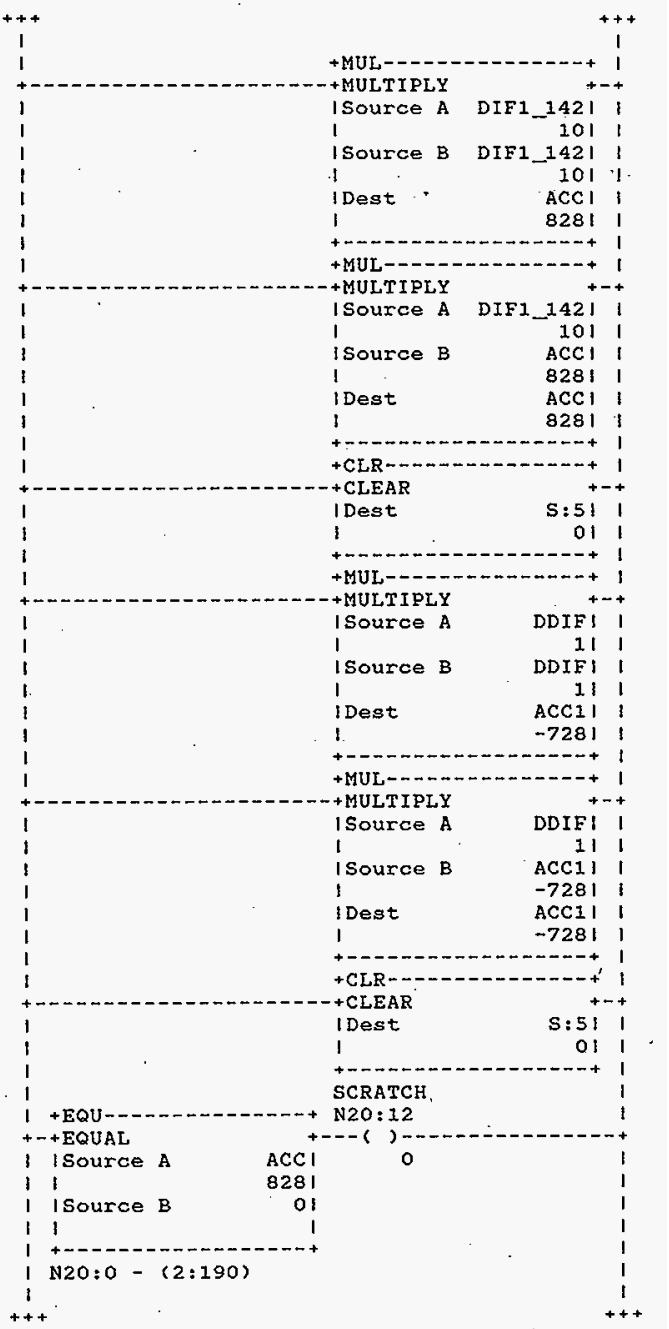

HNF-SD-FF-CSWD-61 Rev. 0 


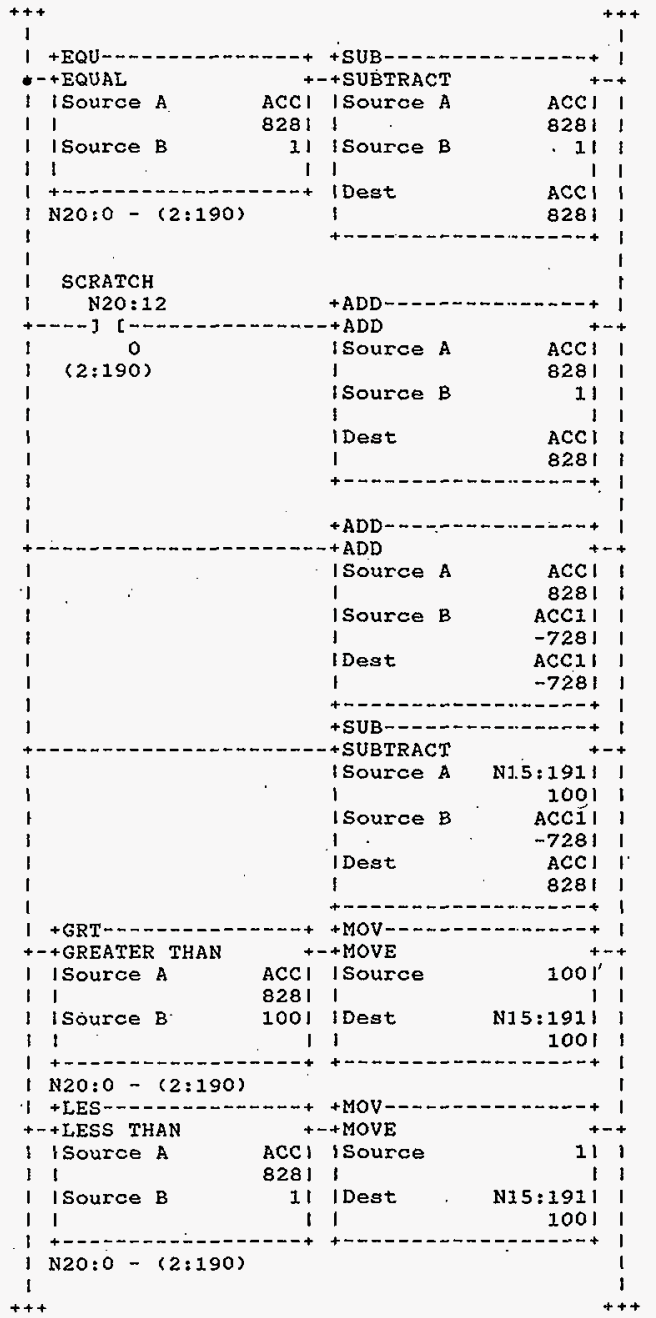




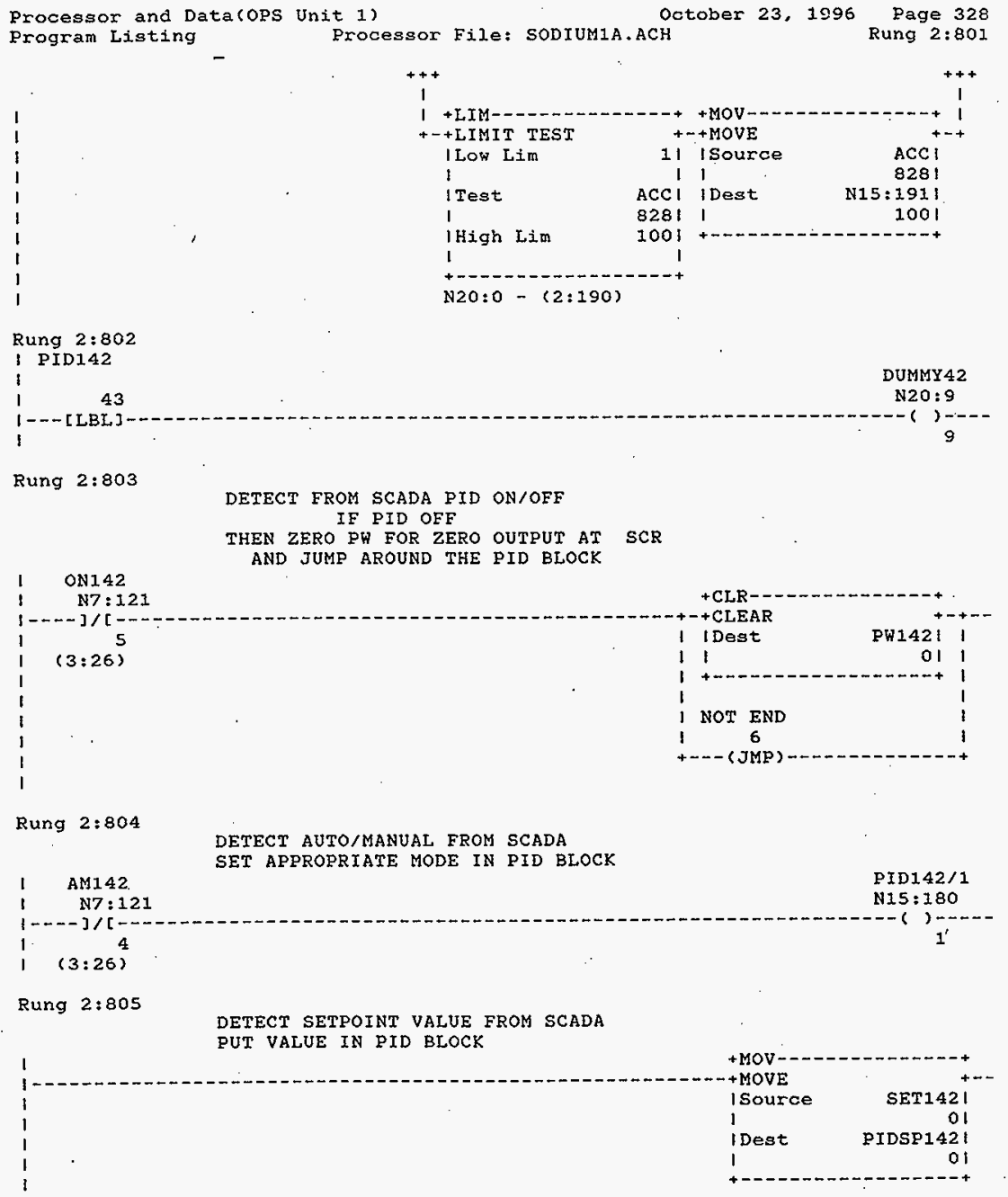

HNF-SD-FF-CSWD-61 Rev. 0 
Processor and Data(OPS Unit 1)

October 23, 1996

\section{Rung 2:806}

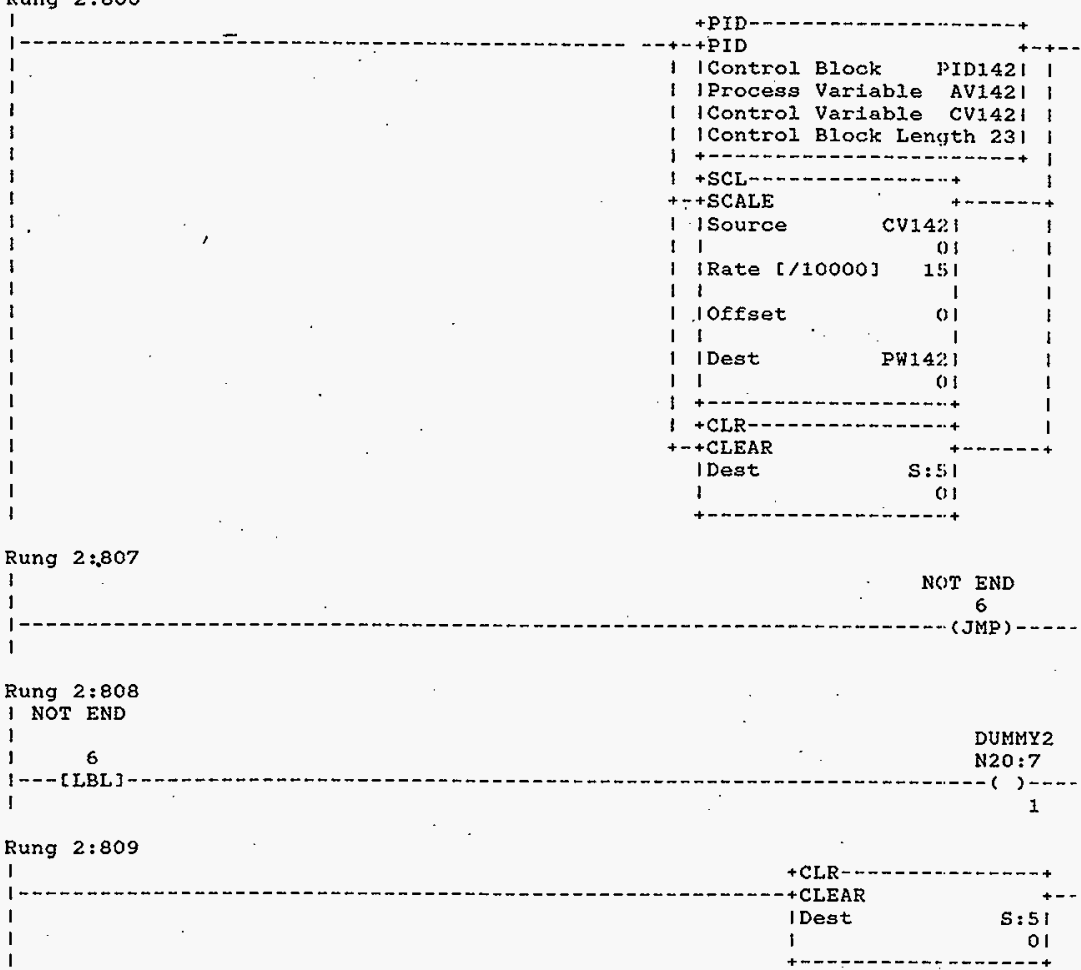

Rung 2:810

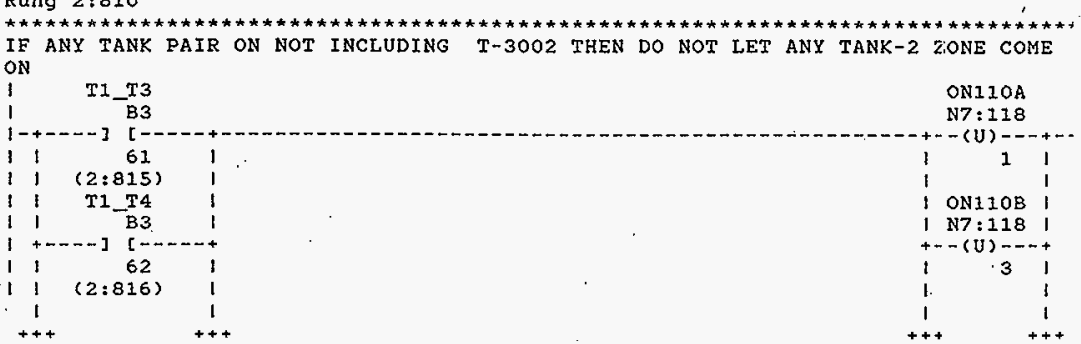


Processor and Data(OPS Unit 1)
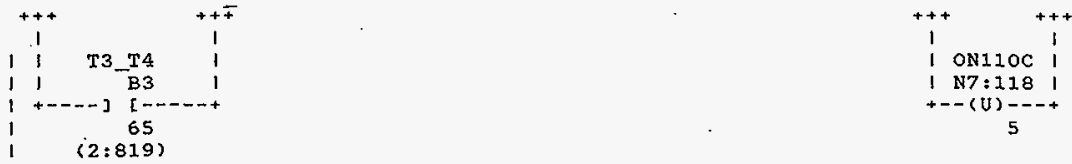

(2:819)

Rung $2: 811$

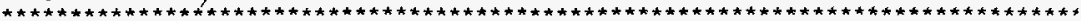

IF ANY TANK PAIR ON NOT INCLUDING T-3003 THEN DO NOT LET ANY TANK-3 ZONES COME ON

$\begin{array}{lccccc}1 & T 1, T 2 & & \text { ON111A } \\ \text { N37:118 } \\ 1\end{array}$

Rụng $2: 812$

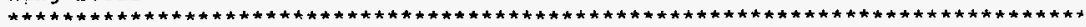
IF ANY TANK PAIR ON NOT INCLUDING T-3004 THEN DO NOT LET ANY TANK-4 ZONES COME ON

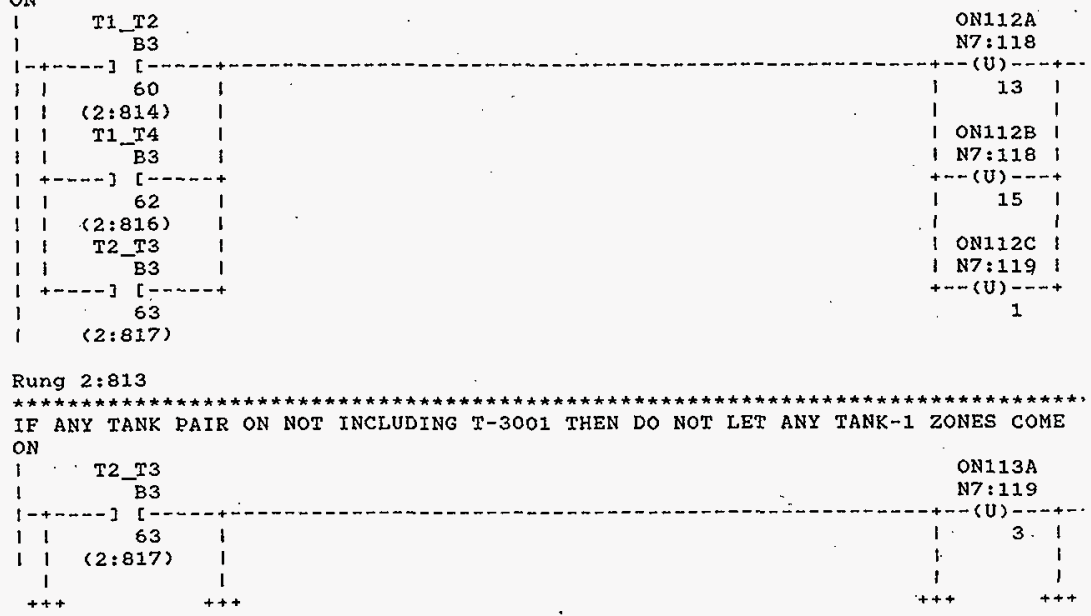



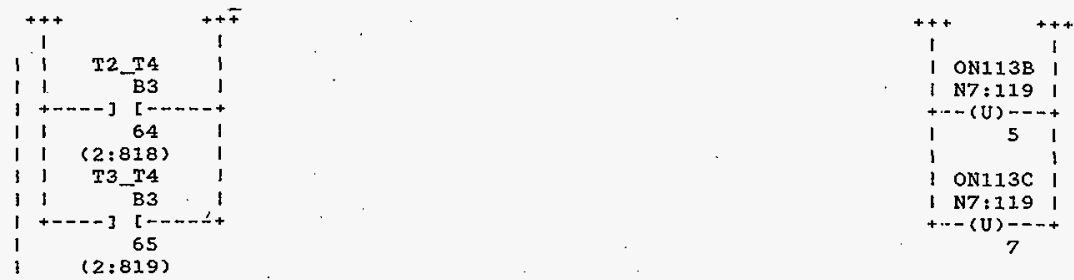

Rung $2: 814$

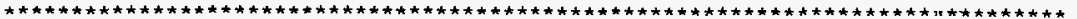
IF TANK-1 AND TANK-2 ARE ENABLED THEN SET THE PAIR_ON BIT

I TNK T-3001 I TNK T-3002

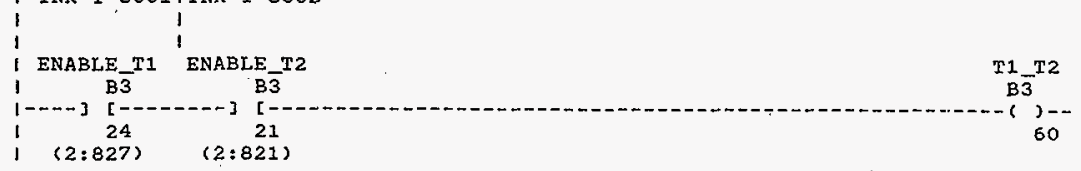

Rung 2:815

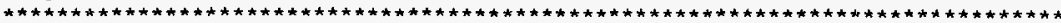
IF T-3001 AND T-3003 ARE ENABLED THEN SET THE PAIR_ON BIT

I TNK T-3001 I TNK T-3003

I ENABLE_T1 ENABLE_T3 T1_T3

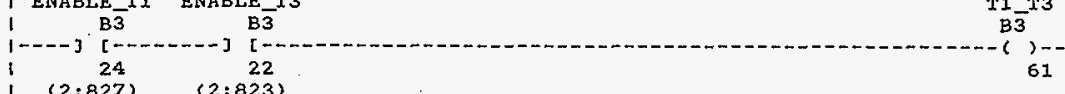

Rung $2: 816$

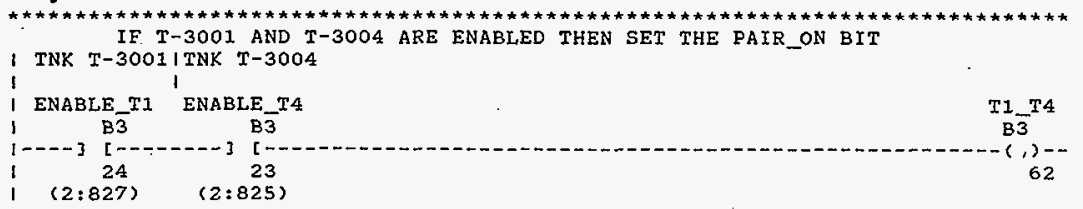

\section{Rung $2: 817$}

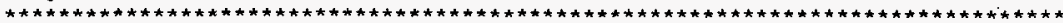
IF T-3003 AND T-3002 ARE ENABLED THEN SET THE PAIR_ON BIT

I TNK T-3003ITNK T-3002

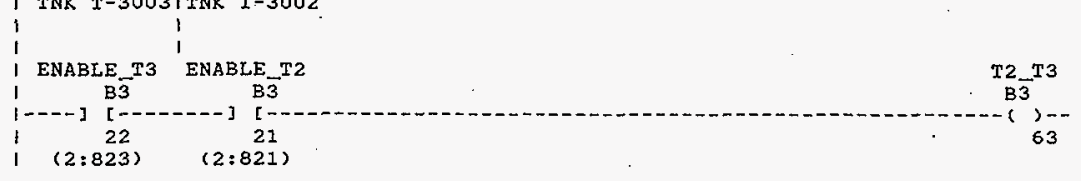

HNF-SD-FF-CSWD-61 Rev. 0 
Processor and Data(ops Unit 1)

Rung $2: 818$

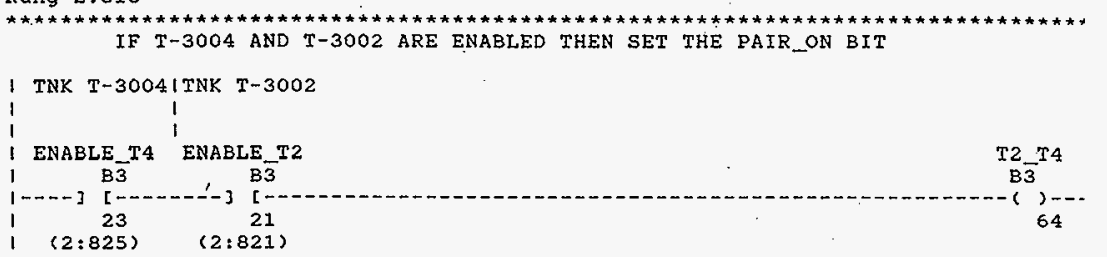

Rung $2: 819$

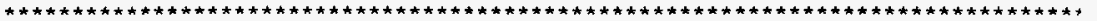
IF T-3003 AND T-3004 ARE ENABLED THEN SET THE PAIR_ON BIT

I TNK T-3003ITNK T-3004

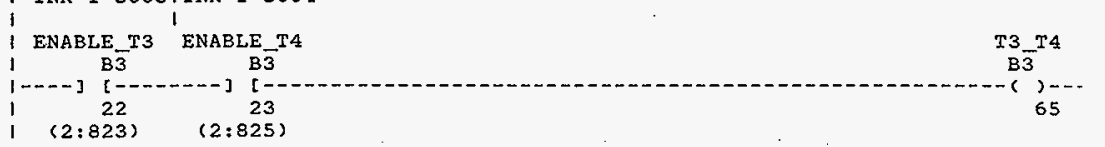

Rung $2: 820$

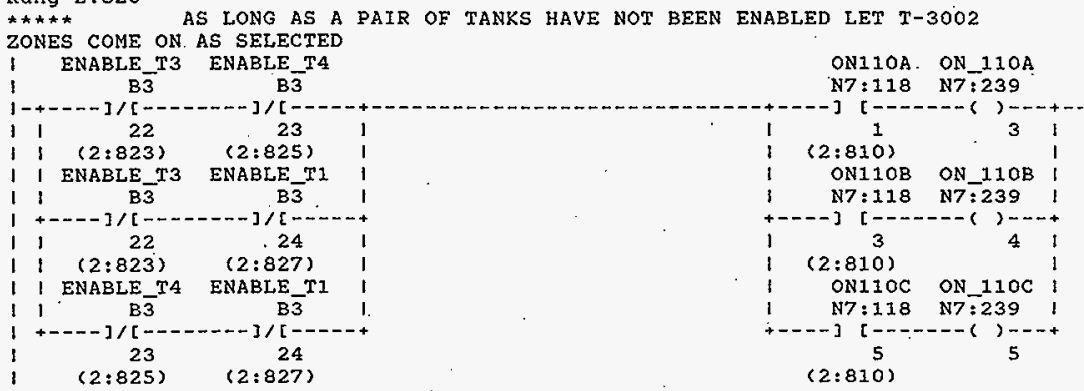

Rung 2:821

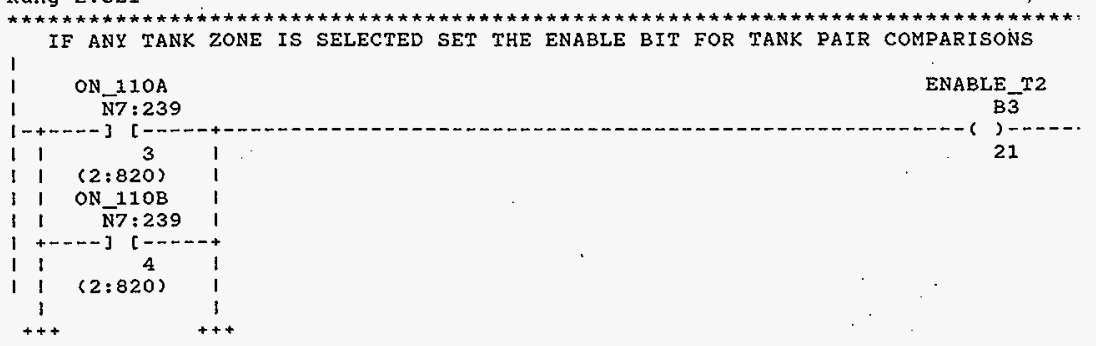


Processor and Data(OPS Unit 1 )

Rung $2: 822$

$* * * * *$ AŚ LONG AS A PAIR OF TANKS HAVE NOT BEEN ENABLED LET T-3DO3 ZONES COME ON AS SELECTED

1

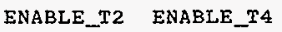

(2:825)

ENABLE $T$ ?

$\mathrm{B} 3$

Rung 2:823

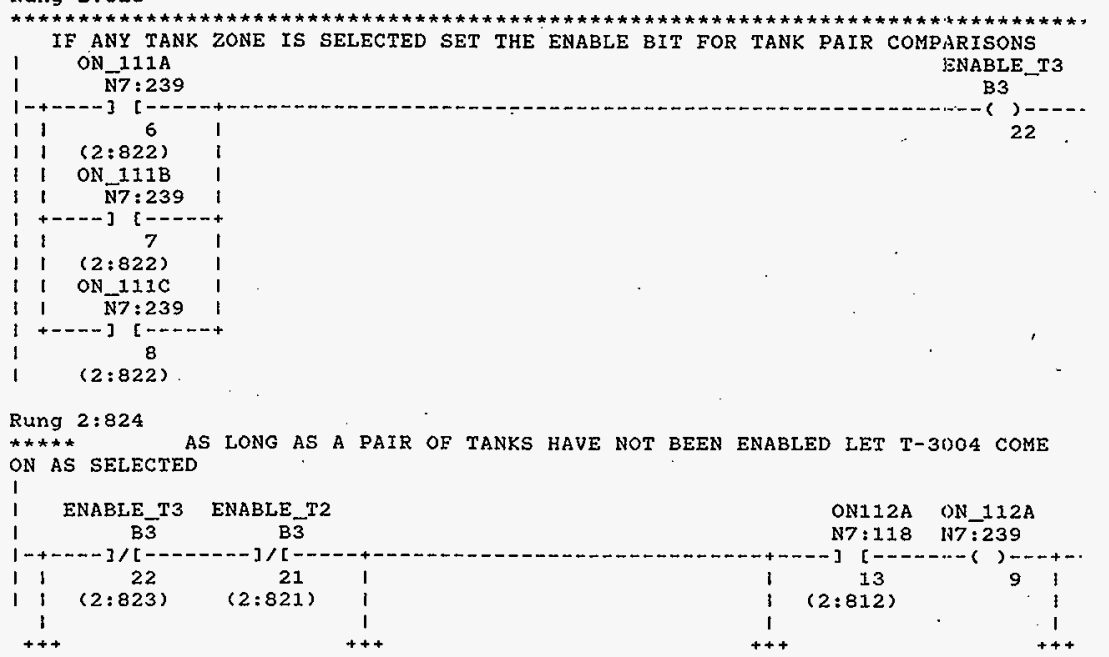

ON11IA DN_111A N7:118 iN7:239

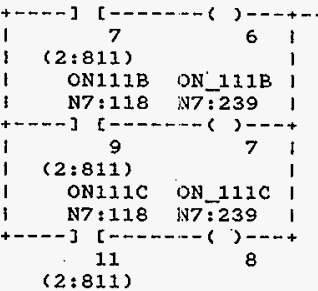


Processor and Data(OPS Unit 1)

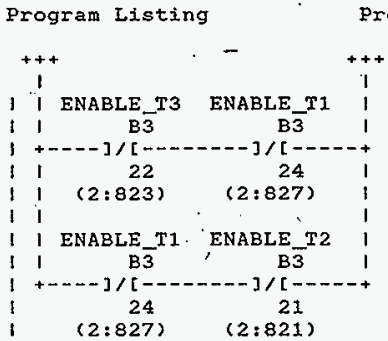

October 23,1996 Page 334 Processor File: SODIUM1A.ACH Rung $2: 824$

$(2: 827) \quad(2: 821)$

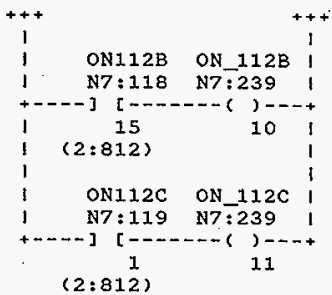

Rung $2: 825$

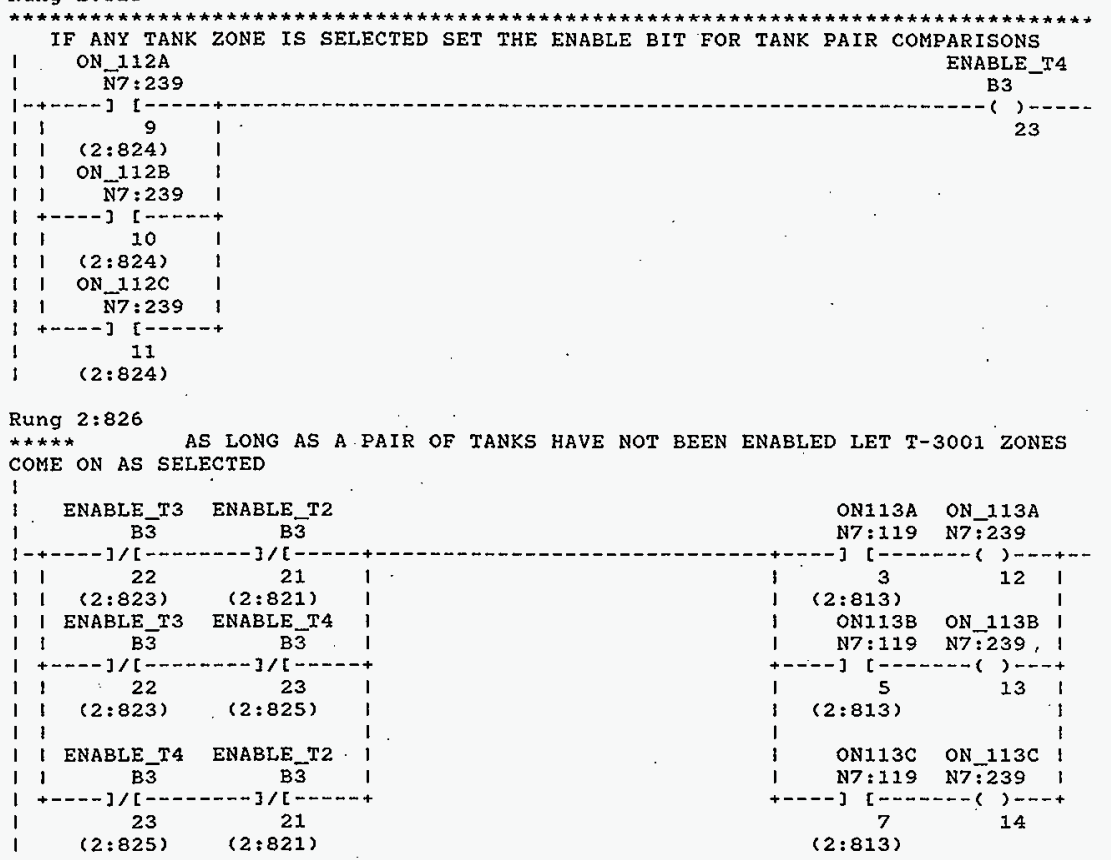

HNF-SD-FF-CSWD-61 Rev. 0

Page 334 


\section{Rung 2:827}

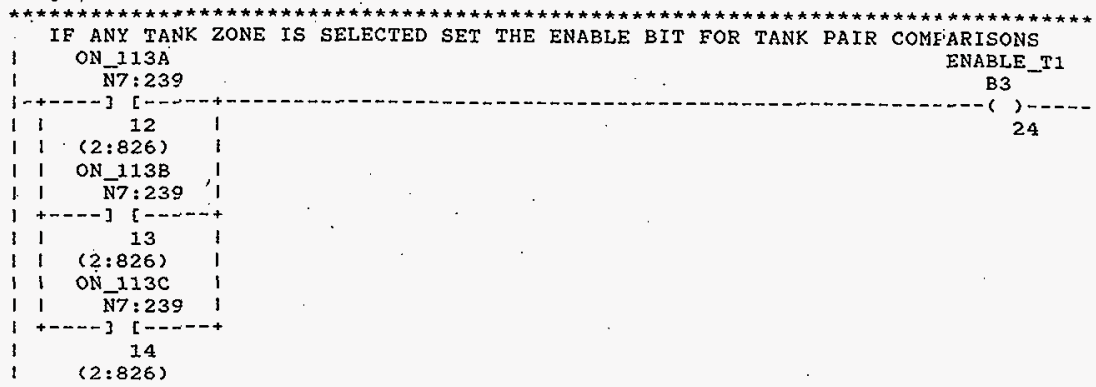

Rung $2: 828$

There is one timer for each zone. Each timer is reset when it reaches $200 \mathrm{~ms}$. The zone's SCR is turned on when the timer is less than the zone's pulse width (PW). Note: no more than two tanks can be enabled at the same time. I ON_110A 1. $\bar{N} 7: 239$ 1 $1-+-1]$ [

)

ITImer Time Base I Preset I Accum

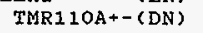


Processor and Data(OPS Unit 1)

October 23, 1996 Page 336 Program Listing

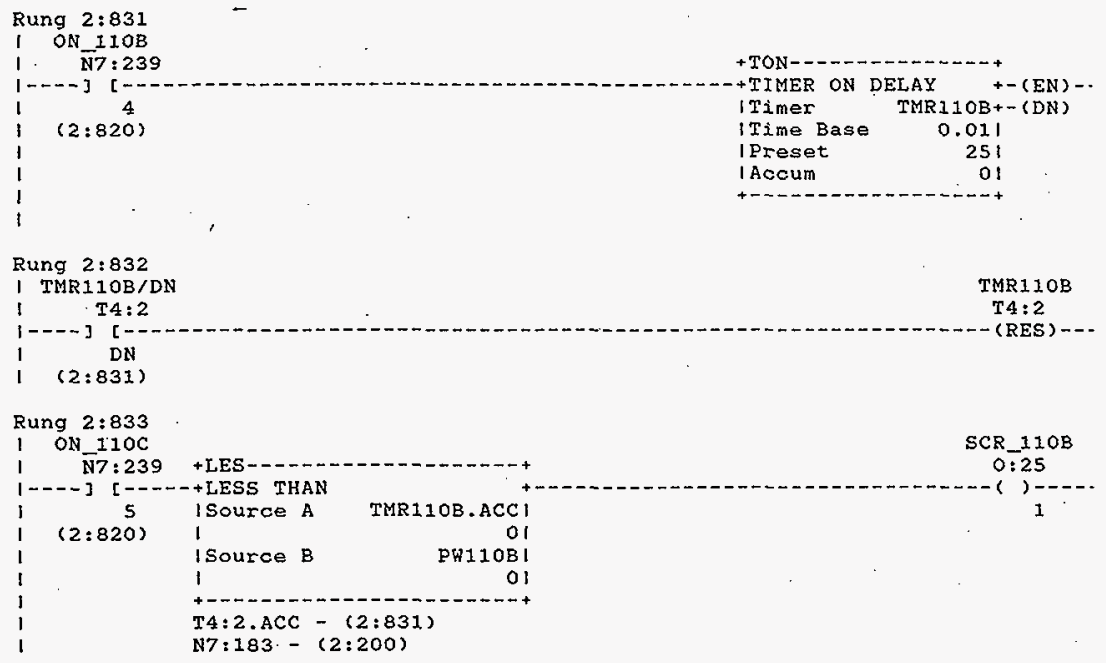

Rung $2: 834$

I ON 1100

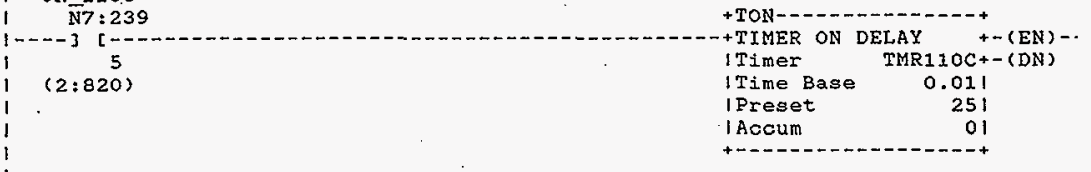

Rung 2:835

1 TMR $110 \mathrm{C} / \mathrm{DN}$

$1 \mathrm{~T} 4: 3$

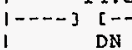

( (2:834)

Rung $2: 836$

I ON $110 \mathrm{C}$

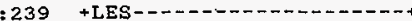

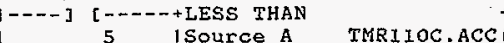

$(2: 820)$

ISource A TMRIIOC.ACC!

PH1 $10 \mathrm{CI}$

ISource B

TMR 1 10C

T4:3

(RES) - -

$T 4: 3 . A C C-(2: 834)$

N7:184 - (2:208) 
Processor and Data(ops Unit 1)

October 23,1996 Page 337 Program Listing
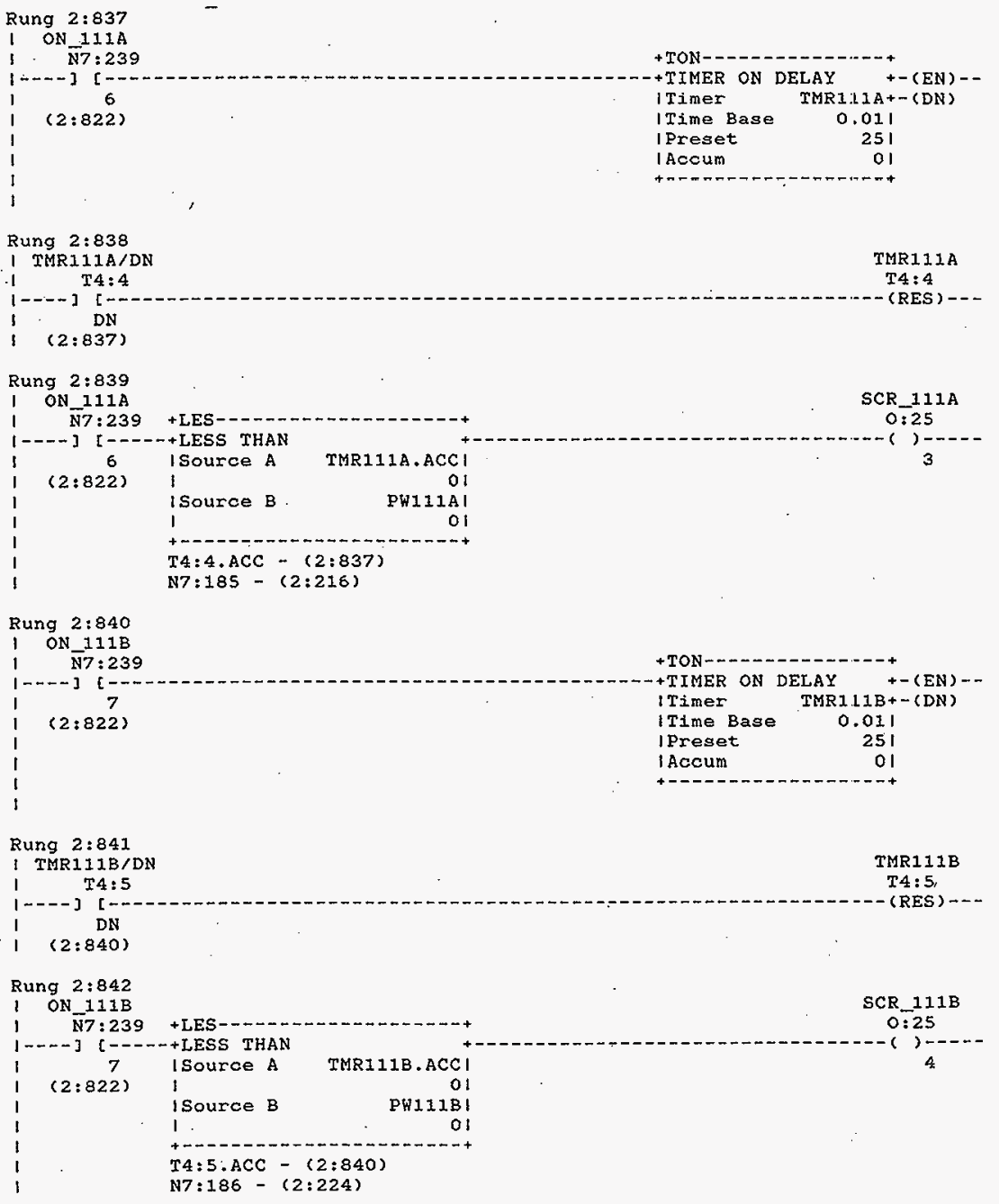

HNF-SD-FF-CSWD-61 Rev. 0 
Processor and Data(OPS Unit 1) Program Listing Processor File: SODIUMIA,ACH

Rung 2:843 -

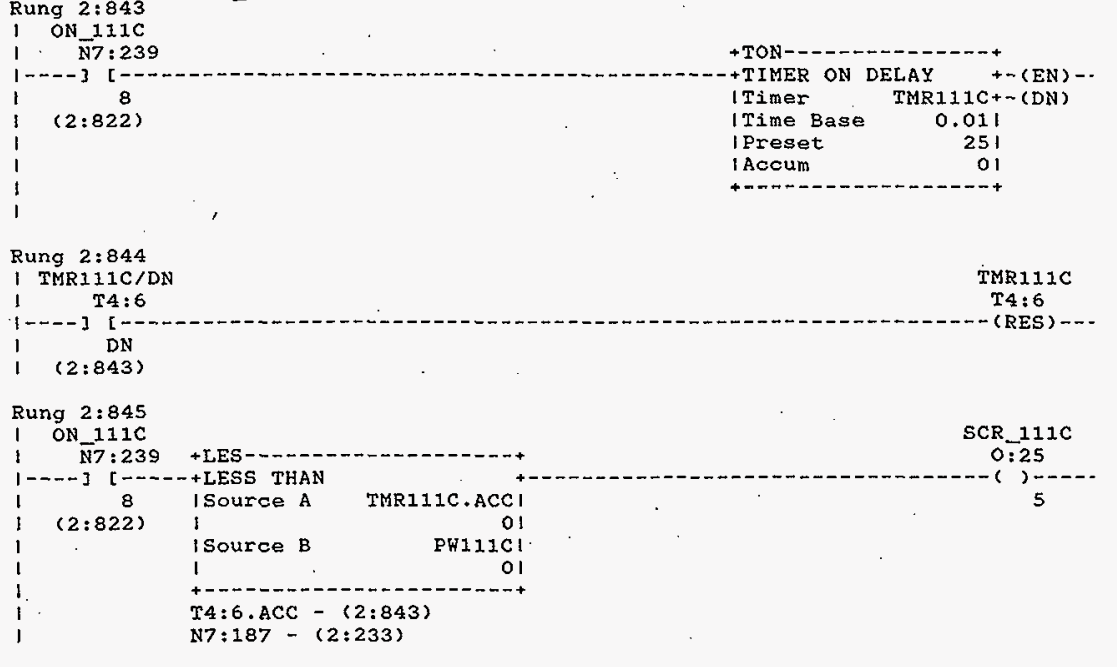

Rung $2: 846$

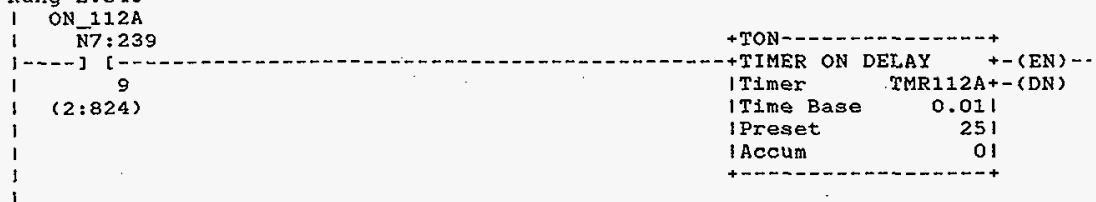

Rung 2:847
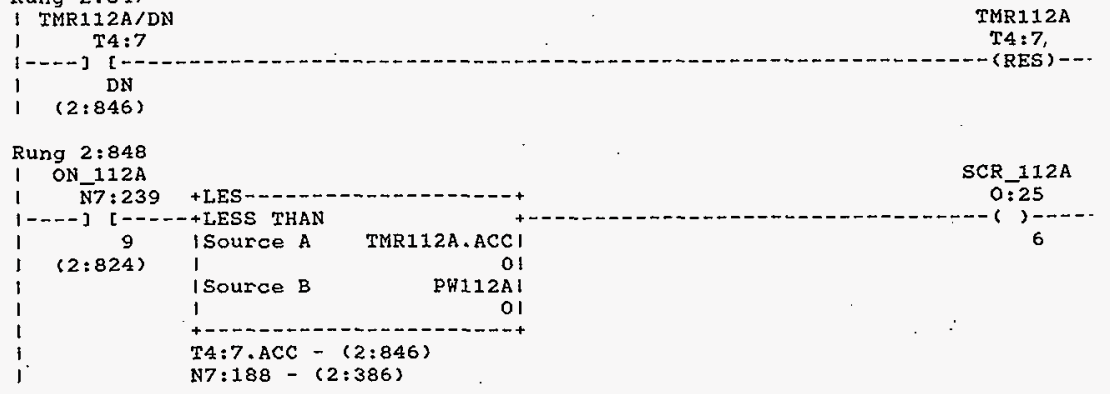

HNF-SD-FF-CSWD-61 Rev. 0 
Processor and Data(OPS Unit I)

Program Listing

October 23,1996

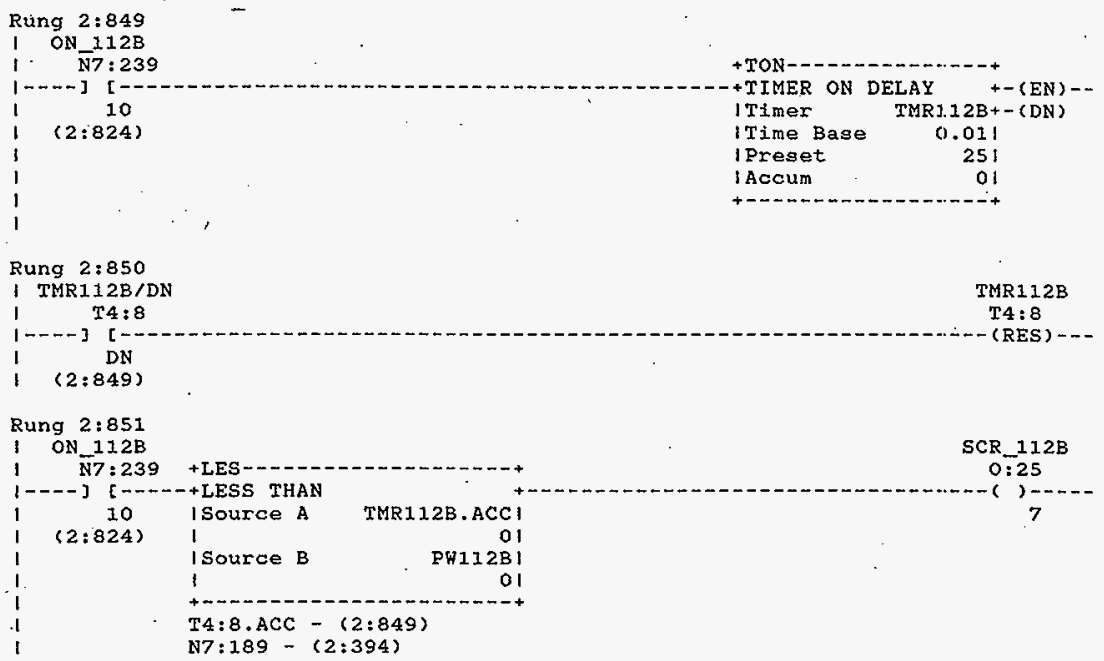

Rung 2:852

$10 N 112 \mathrm{C}$

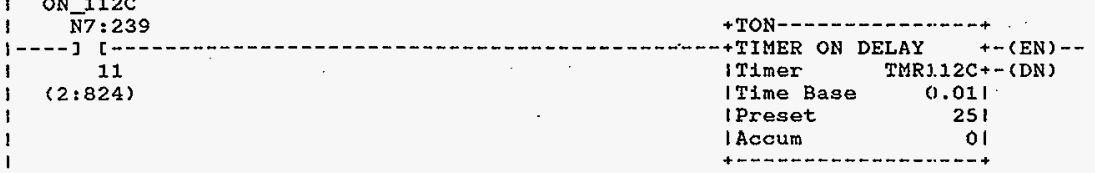

Rung $2: 853$

I TMR112C/DN

TMR112C

T4:9

T4: 9 ,

$1----7$ [

(RES) - - -

(2:852)

Rung $2: 854$

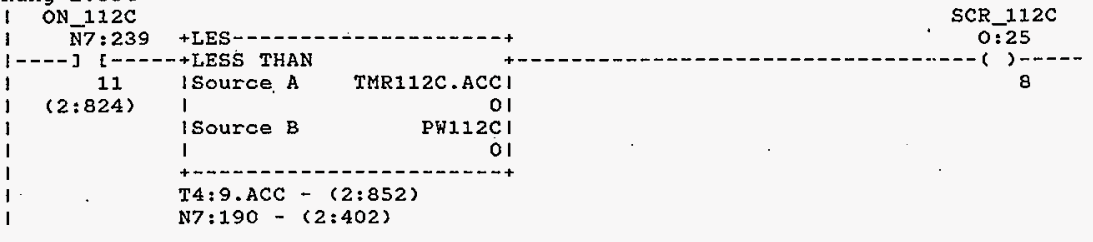

HNF-SD-FF-CSWD-61 Rev. 0 
Processor and Data(ops Unit 1)
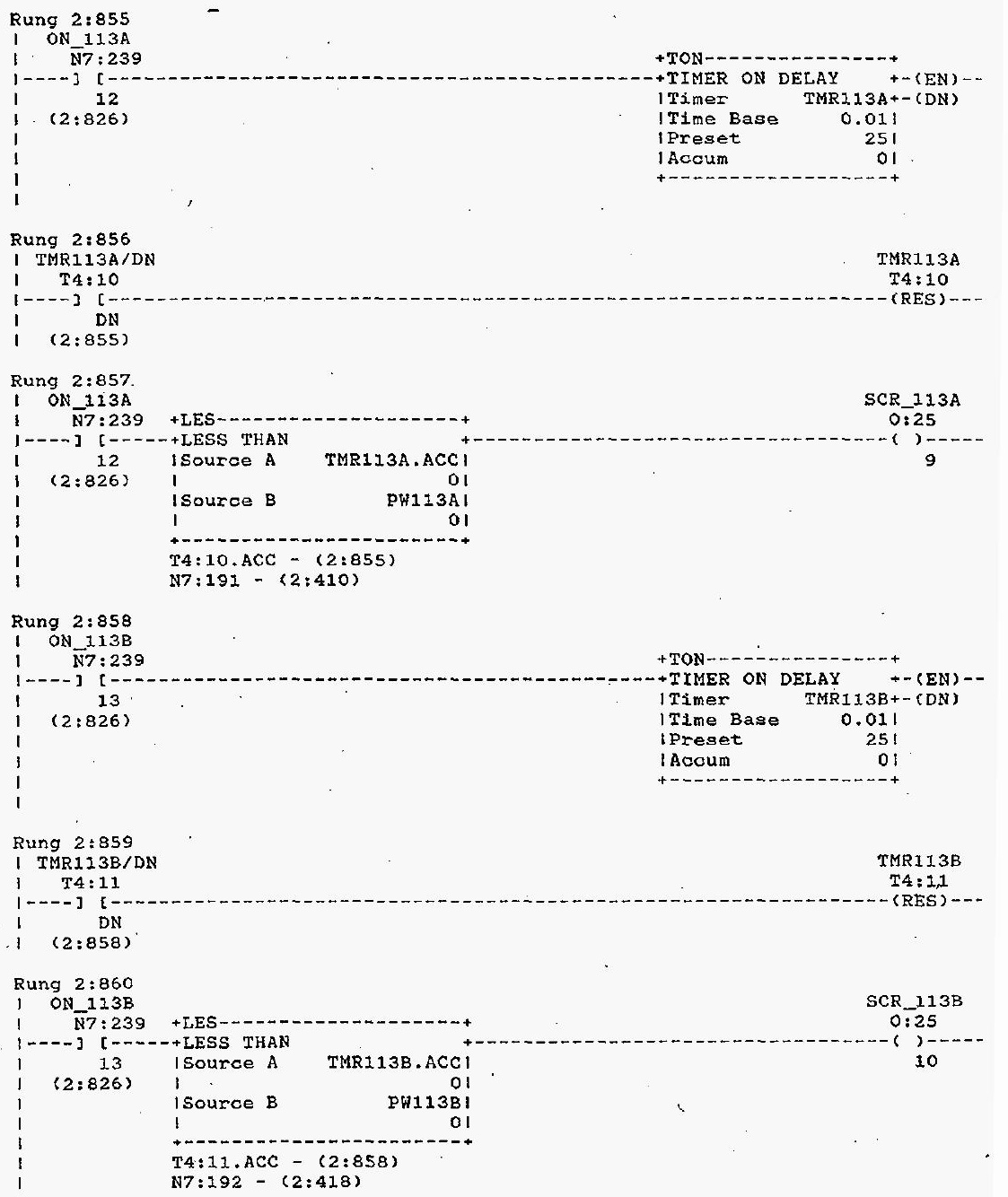
Processor and Data(ops Unit 1)

Rung $2: 861$
1 ON $113 \mathrm{C}$

$$
\text { i. ON_113C }
$$$$
1,-n]
$$$$
114
$$$$
\text { (2:826) }
$$

Rung $2: 862$

1 TMR113C/DN

$T 4: 12$

+ TON-- - - - - - - - +

(....

+ TIMER ON DELAY

I Timer

ITime Base

IPreset

I Accum
TMR] 13C+-(EN)
0. 011 251

Rung 2:864

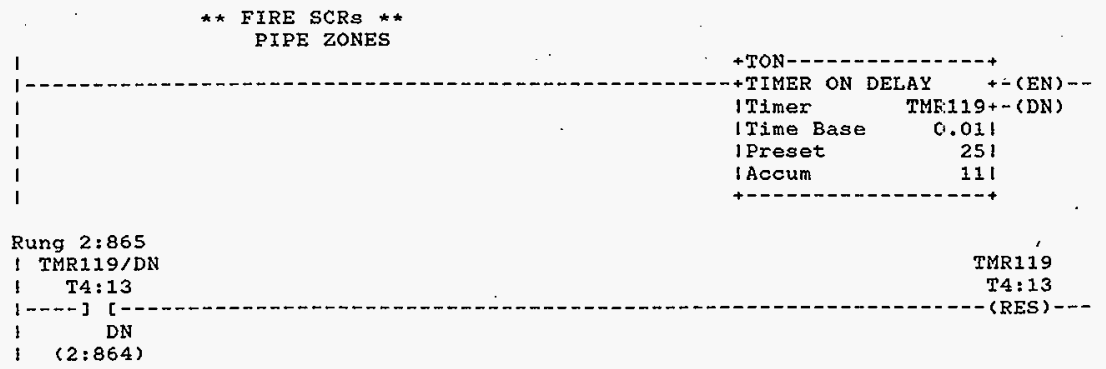


Processor and Data (OPS Unit 1)

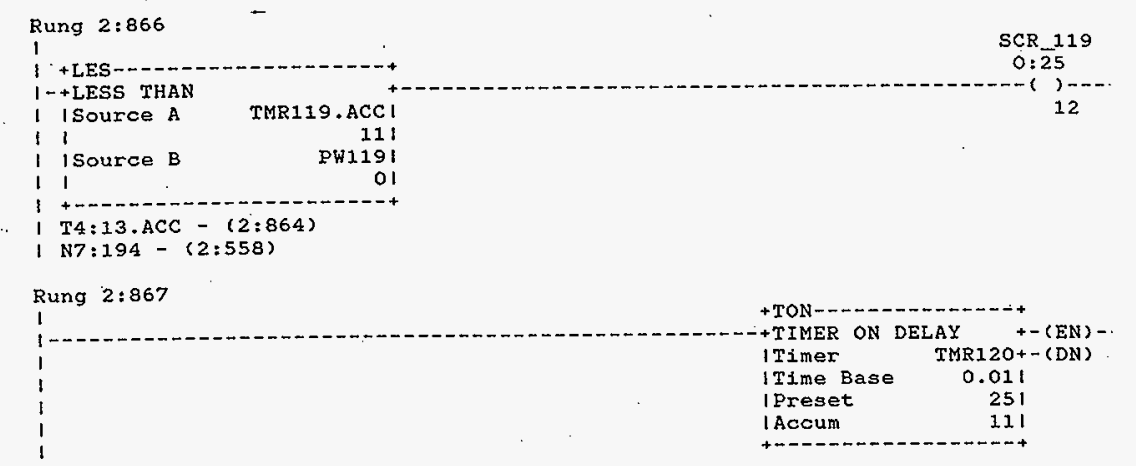

\section{Rung $2: 868$}

I TMR120/DN

$\mathrm{T} 4: 14$

$1-\cdots+3$ [

i. (2:867)

Rung 2:869

1

1-+LES- LESS THAN

11

isource B

TMR120.ACCI

111

PW1201

11

01

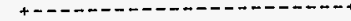

( T4:14.ACC - (2:867)

(N7:195-(2:566)

Rung 2:870

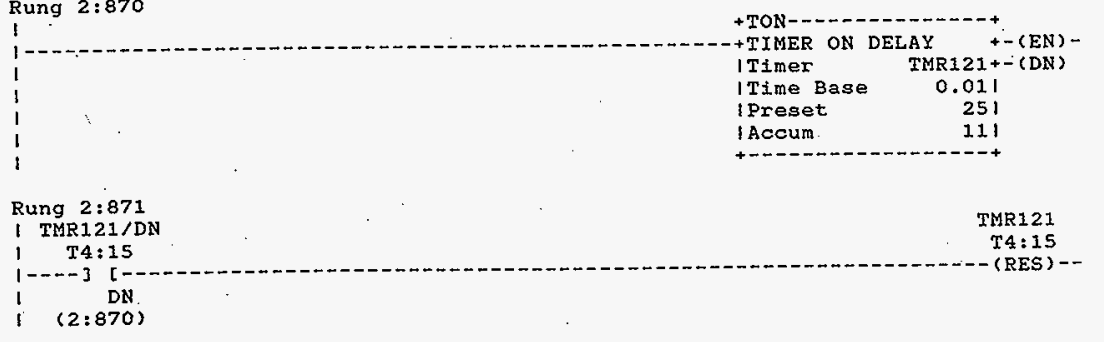

SCR_120

$0: 25$ 
Processor and Data(OPS Unit 1) Program Listing

Processor File: SODIUM1A.ACH

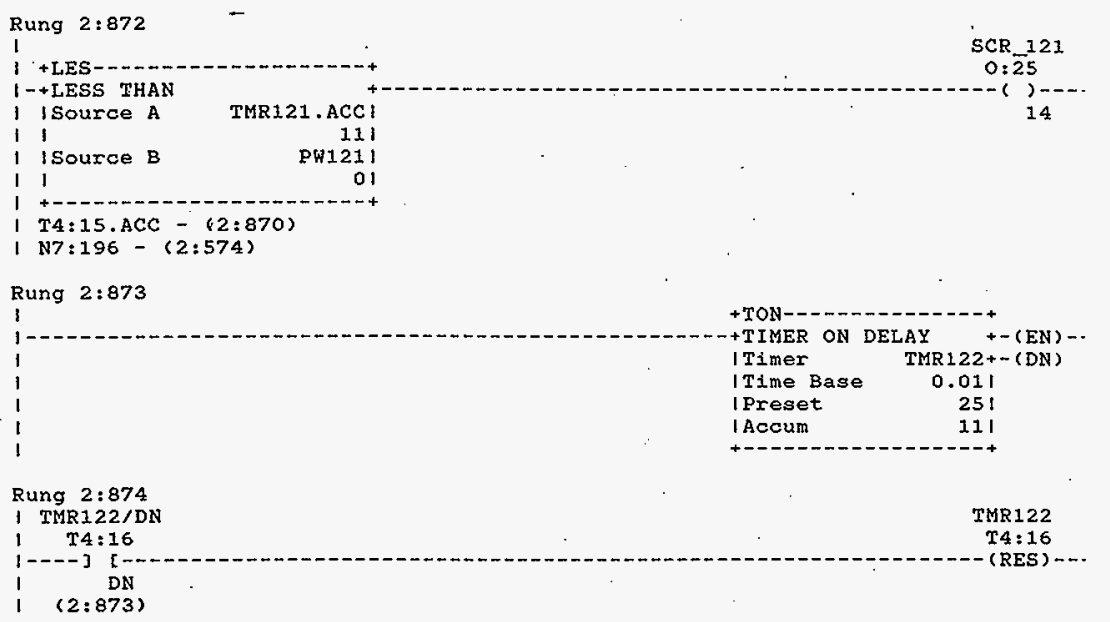

Rung 2:875

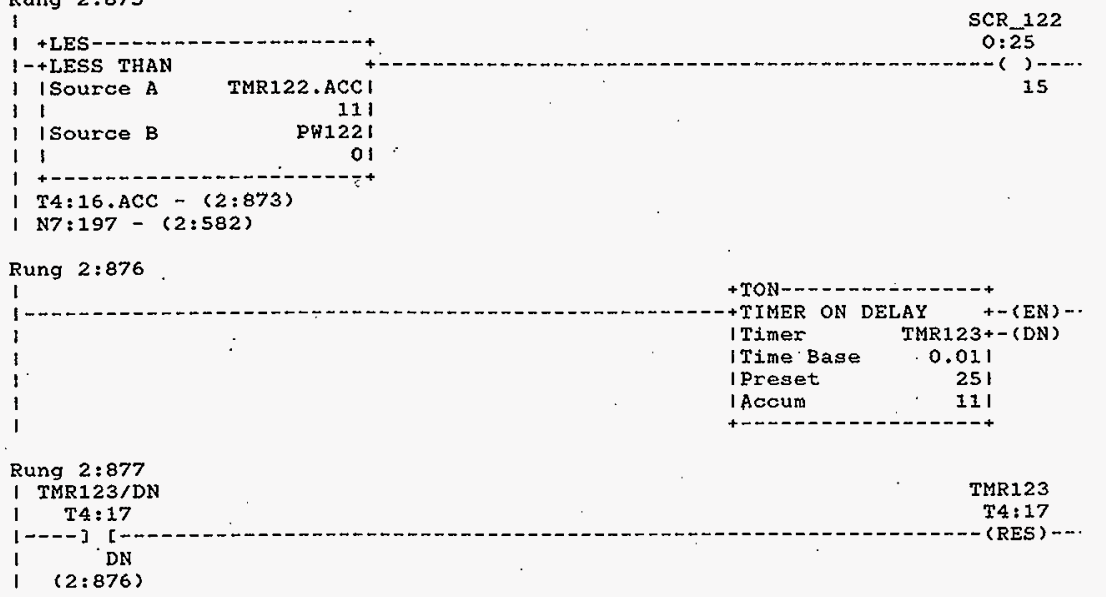

HNF-SD-FF-CSWD-61 Rev. 0 
Processor and Data(ops Unit 1)

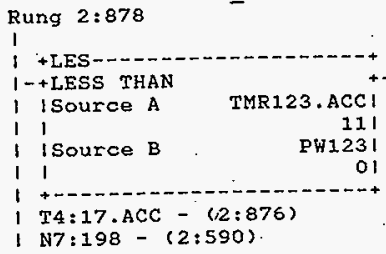


Processor and Datalops Unit 1 ) Program Listing

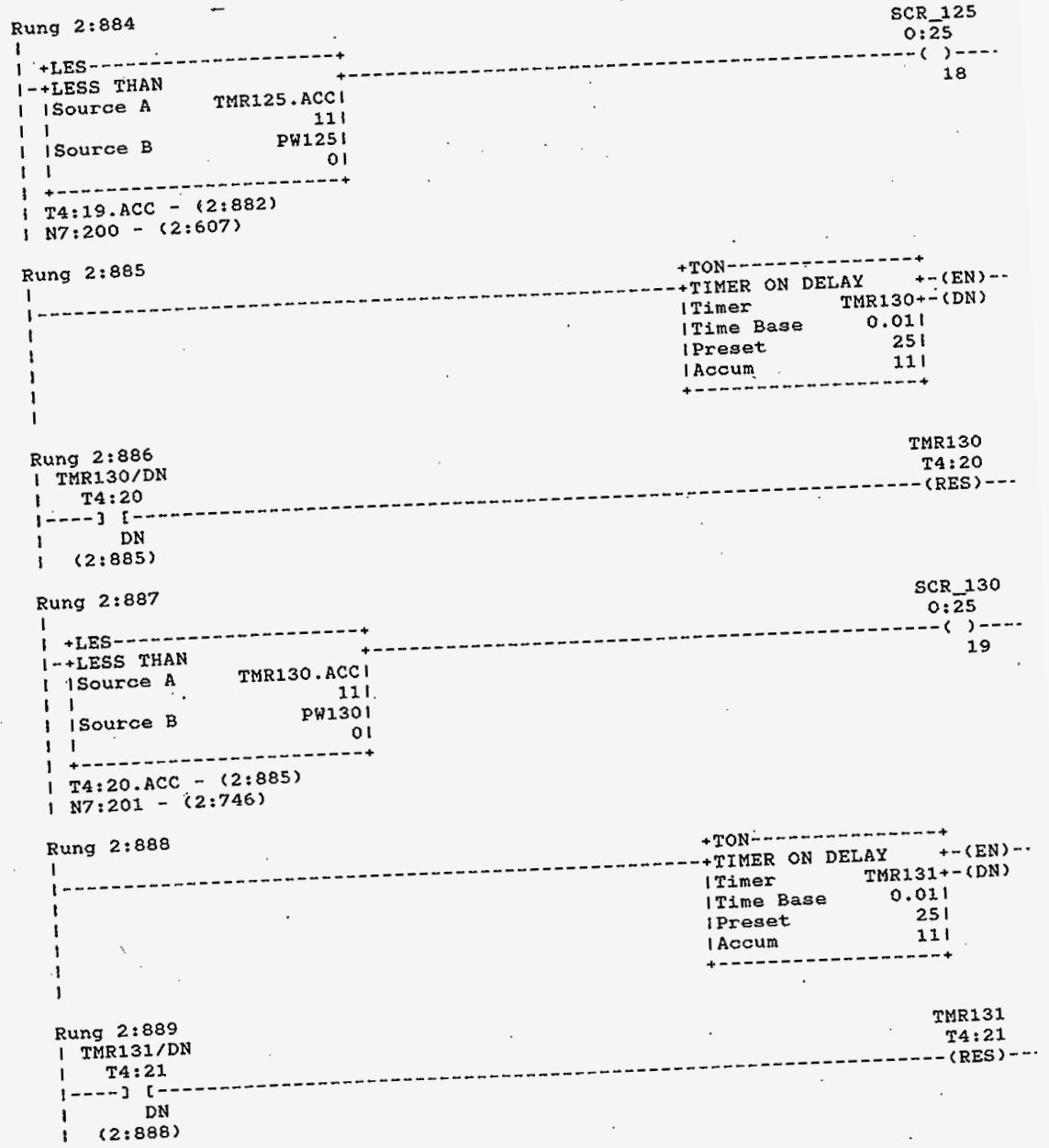

HNF-SD-FF-CSWD-61 Riv. 0 
Processor and Data (OPS Unit 1 )

October 23, 1996

Page 346

Program Listing

Processor File: SODIUM1A.ACH

Rung $2: 890$

Rung $2: 890$

-

1

$1+$ LES-

$1-+$ LESS THAN

I ISource A TMR131.ACCI

111

1

PW1311

1 o!

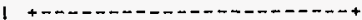

1 T4:21.ACC - (2:888)

I N7:202-(2:754)

Rung 2:891

$$
1-
$$

i

Rung 2:892

1 TMR $132 / \mathrm{DN}$

I T4:22

i- $-\cdots-][-$

1 DN

(1) $2: 893$

Rung 2:893

1

1 +LES-......-

+TON-..-- - - - - - . - +

$1-+$ LESS THAN

1 ISource A TMR132.ACCI

1 I

1 isource B PW1321

1 l 1 !

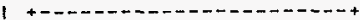

1 T4:22.ACC - (2:891)

I N7:203-(2:762)

Rung 2:894$$
1
$$ 



HNF-SD-FF-CSWD-61 Rev. 0 


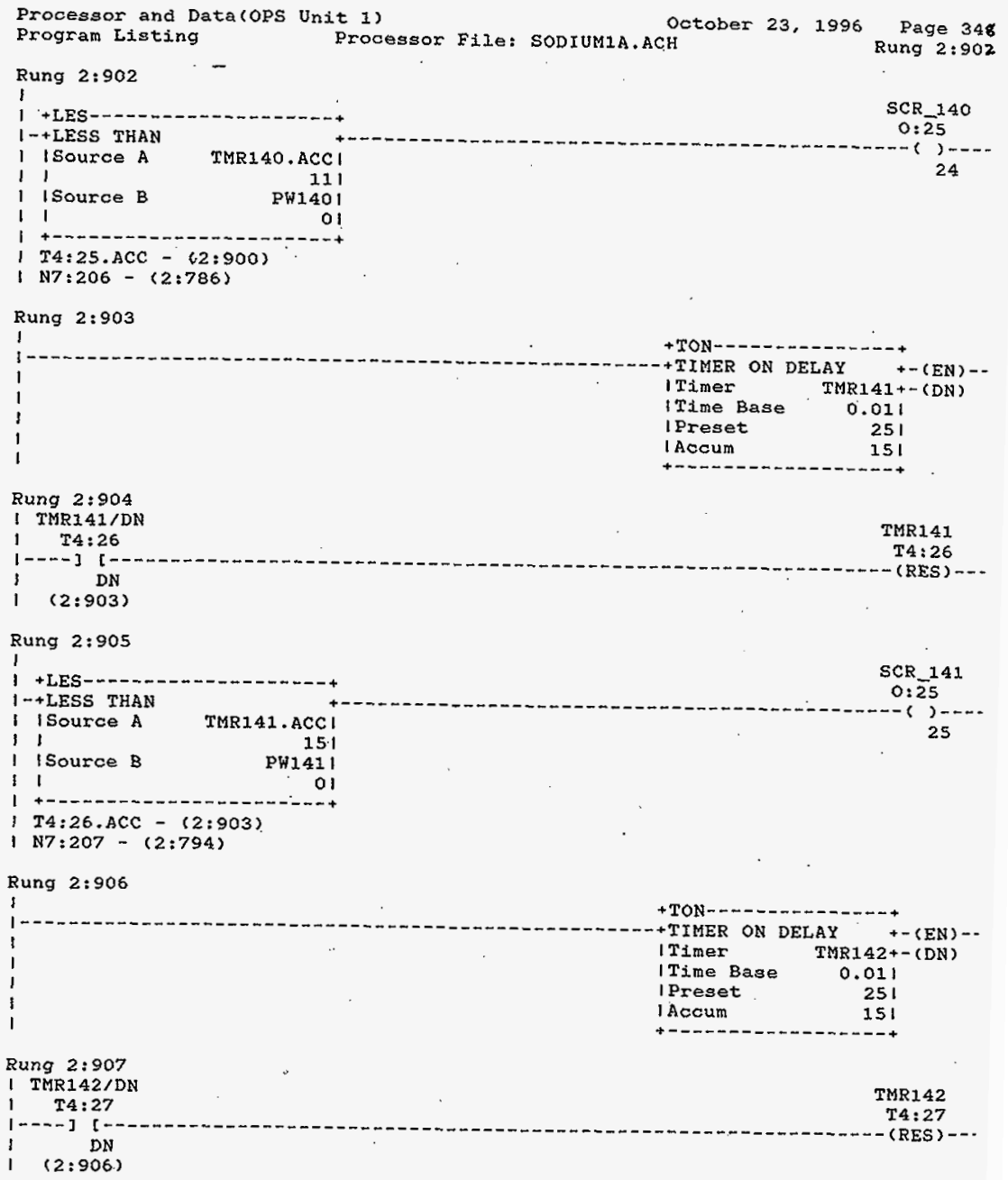

HNF-SD-FF-CSWD-61 Rev. 0 
Processor and Data(OPS Unit 1)

Rung 2:908

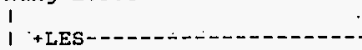

1-+LESS THAN

I Isource A

TMR142.ACCI

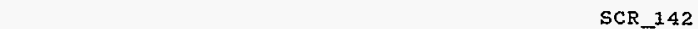

ISource B PW142I

11

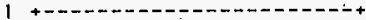

I T4:27.ACC - (2:906)

( $\mathrm{N} 7: 208-(2: 803)$

Rung 2:909

( END

1

17

7

DUMMY 3

I--- [LBL

N20:7

\section{Rung 2:910}

I

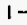

I

1.

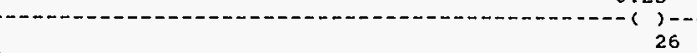

Rung 2:\$11

I

$$
\text { i }
$$

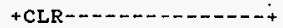
+ CLEAR I Dest

I

$S: 51$

01

I

HNF-SD-FF-CSWD-61 Rey. 0 
Rung $3: 0$

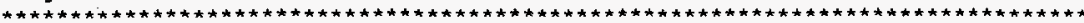

Initialization Subroutine, runs once when the PLC is started.

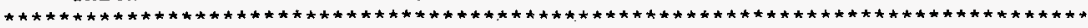
write configuration number (2369d), (100001000001b) to N7:0 (CONFIGURE) for use below, CONFIGURE T/C MODULES

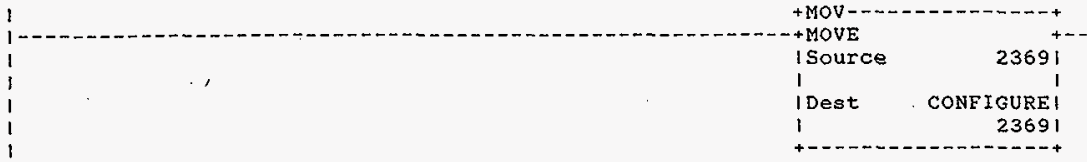

Rung $3: 1$

** CONFIGURE T/C MODULES **

Use Fill File to copy the configuration to each channel of each T/C module

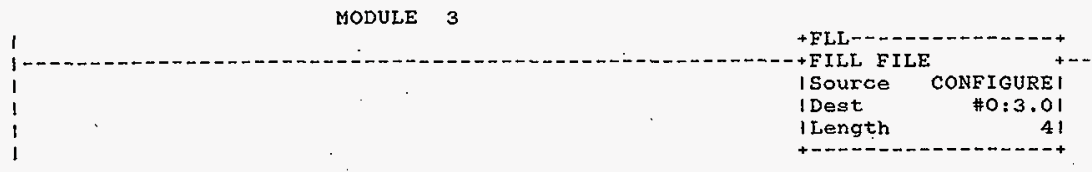

Rung $3: 2$

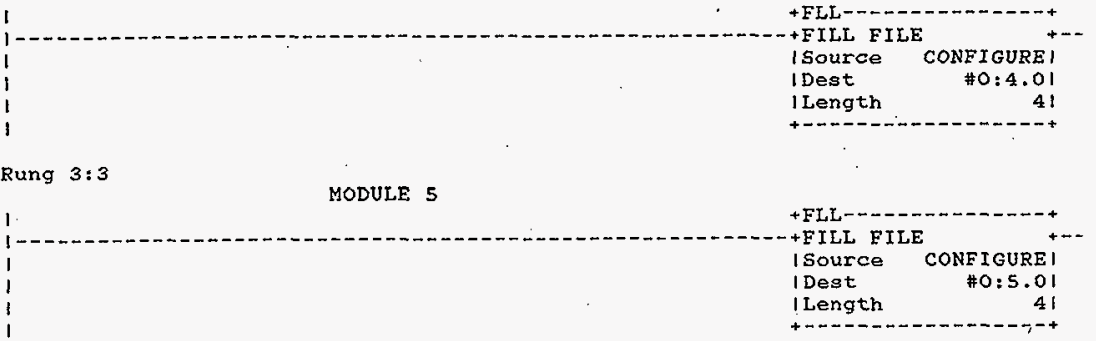

Rung $3: 4$

MODULE 6

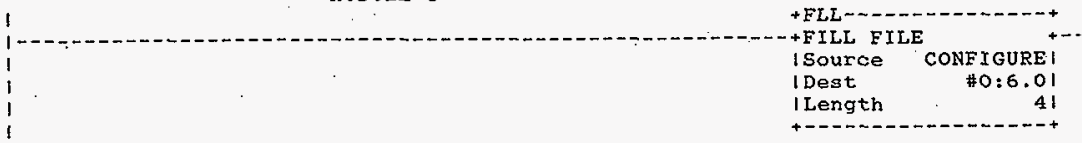

HNF-SD-FF-CSWD-61 Rev. 0 
Processor and Data(ops Unit 1 )

MODULE 7

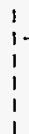

+FIL- FIL FILE

ISource

I Dest

I Length

CONF IGURE। $\# 0: 7.01$

I

Rung $3: 6$$$
\text { I }
$$$$
\text { I }
$$$$
1
$$

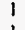

Rung $3: 7$

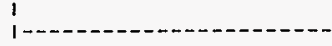

MODULE 9

MODULE 8

I

I

Rung $3: 8$

1$$
\text { i }
$$

1

1

;

Rung $3: 9$

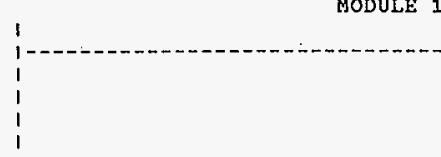

\section{Rung 3:10}

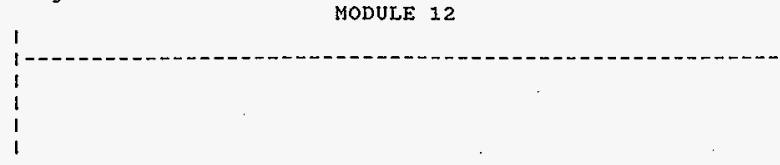

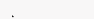

MODULE 10

+FLL- - - - - - - - - - - - +

+EILL FILE

I Source

CONFIGURE।

I Dest

$\# 0: 9.01$

I Length

41

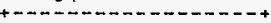

+ FLL

+ FILI FILE

I Source

IDest

CONEIGURE।

I Length

\#0:10.01

MODULE 11

+FLL- -

I Source CONFIGURE |

Dest \#0:11.01

I Length

41

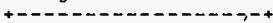

FLLLIS FILE
Source CONFIGURE।
Dest
Length

+FILI FILE

I SOUICE CONFIGURE|

IDest \#0:I2.01

I Length 4 I

+
4

+FILI FILE

HNF-SD-FF-CSWD-61 Rev. 0 
Processor and Data(OPS Unit 1)

October 23, 1996

MODULE 13

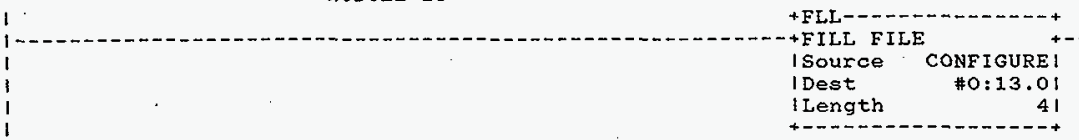

\section{Rung $3: 12$,}

MODULE 14

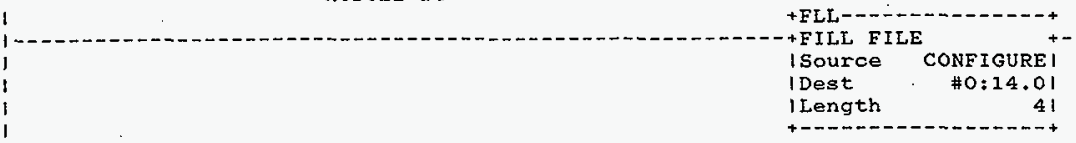

Rung $3: 13$

MODULE 15

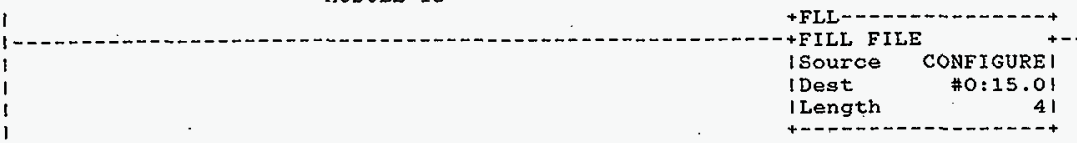

Rung $3: 14$

MODULE 16

+FILL FILE

isource

I Dest

CONFIGURE।

ILength 4 !

\#0:16.01

Rung $3: 15$

MODULE 17

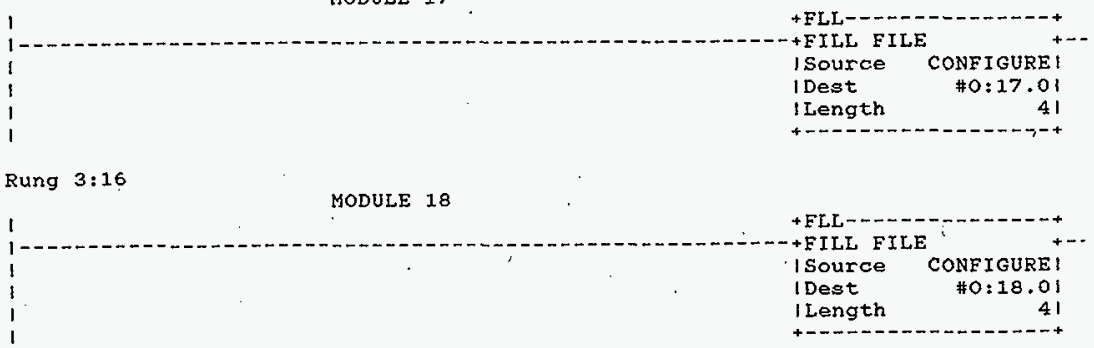

HNF-SD-FF-CSWD-61 Rev. 0

Page 352 
Processor and Data(OPS Unit 1)

Program Listing

Processor File: SODIUM1A.ACH

MODULE 19
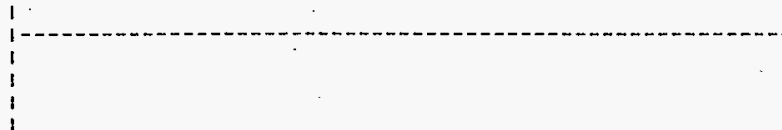

I

MODULE 20

MODULE 20

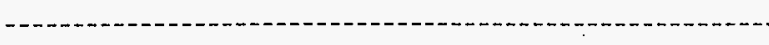

1

Rung $3: 19$$$
1
$$

MODULE 21

Rung $3: 20$

MODULE 22

1

1

i

I Source

IDest

CONFIGURE!

IIength

\#0:22.01

ILength

Rung $3: 21$

MODULE 23

$$
\begin{aligned}
& 1 \\
& 1 \\
& 1 \\
& 1
\end{aligned}
$$

+FLL-_- - - - - - - - - - -

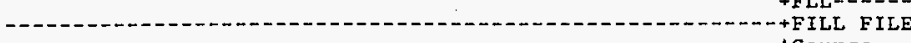

ISource

C.ONEIGURE।

I Dest

\#0:23.01

I Length

41

Rung $3: 22$

$$
\begin{aligned}
& 1 \\
& 1 \\
& 1 \\
& 1 \\
& 1
\end{aligned}
$$

MODULE 24

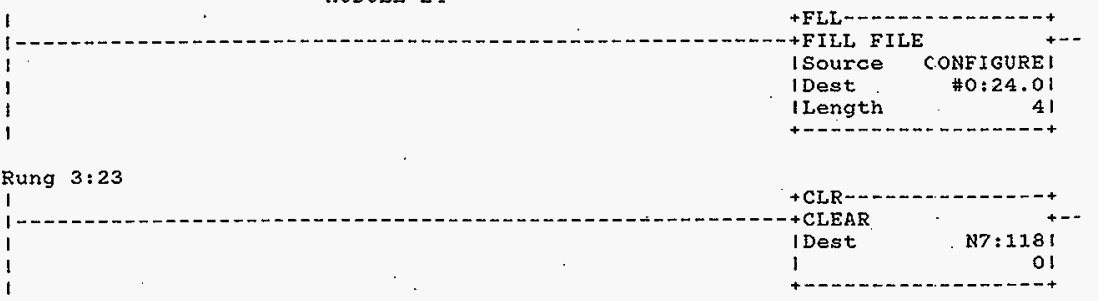

HNF-SD-FF-CSWD-61 Rev. 0

Page 353 
Processor and Data(OPS Unit 1)

October 23, 1996

Program Listing

Processor File: SODIUM1A.ACH
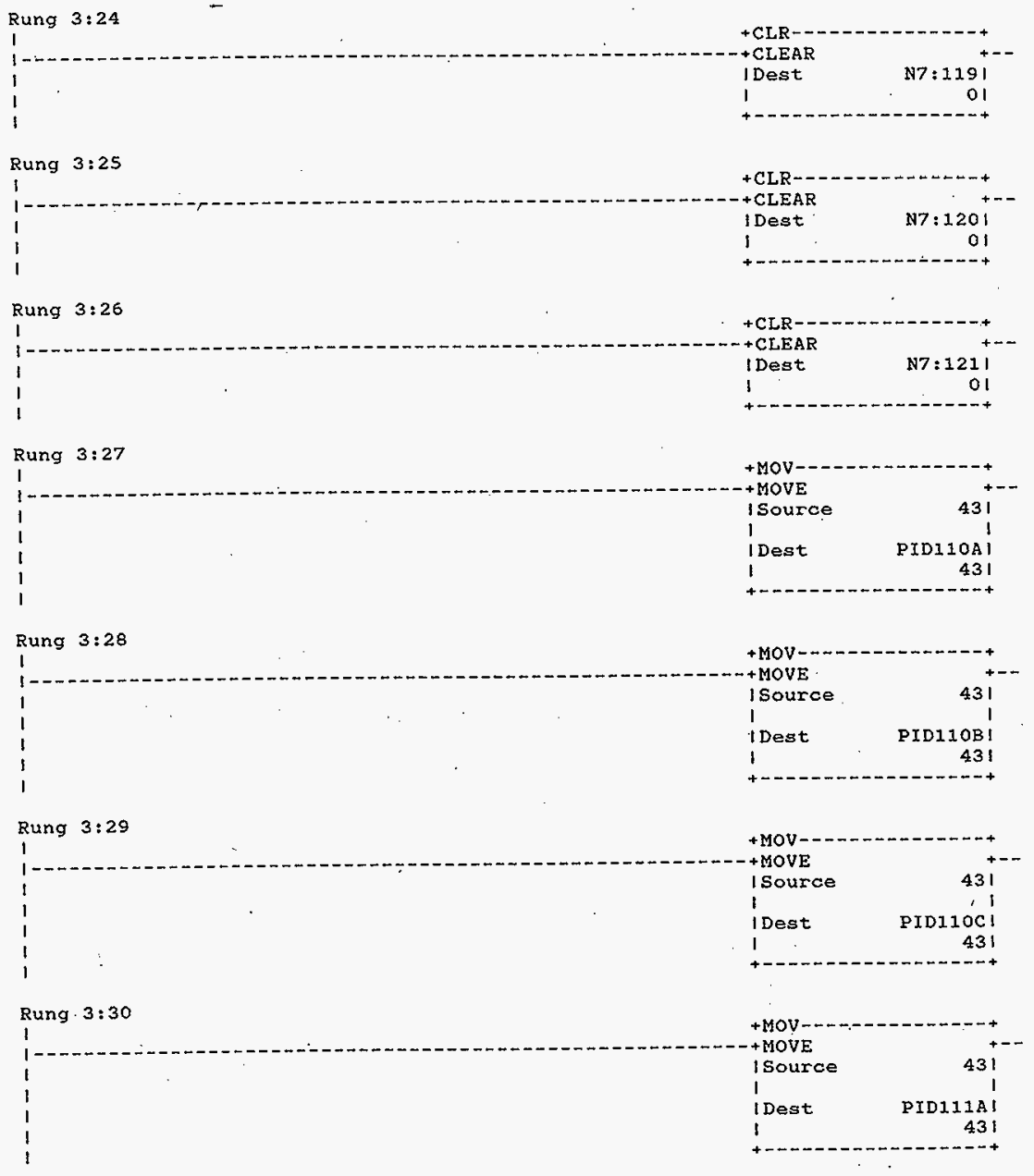

HNF-SD-FF-CSWD-61 Rev. 0 
Processor and Data(OPS Unit 1 )
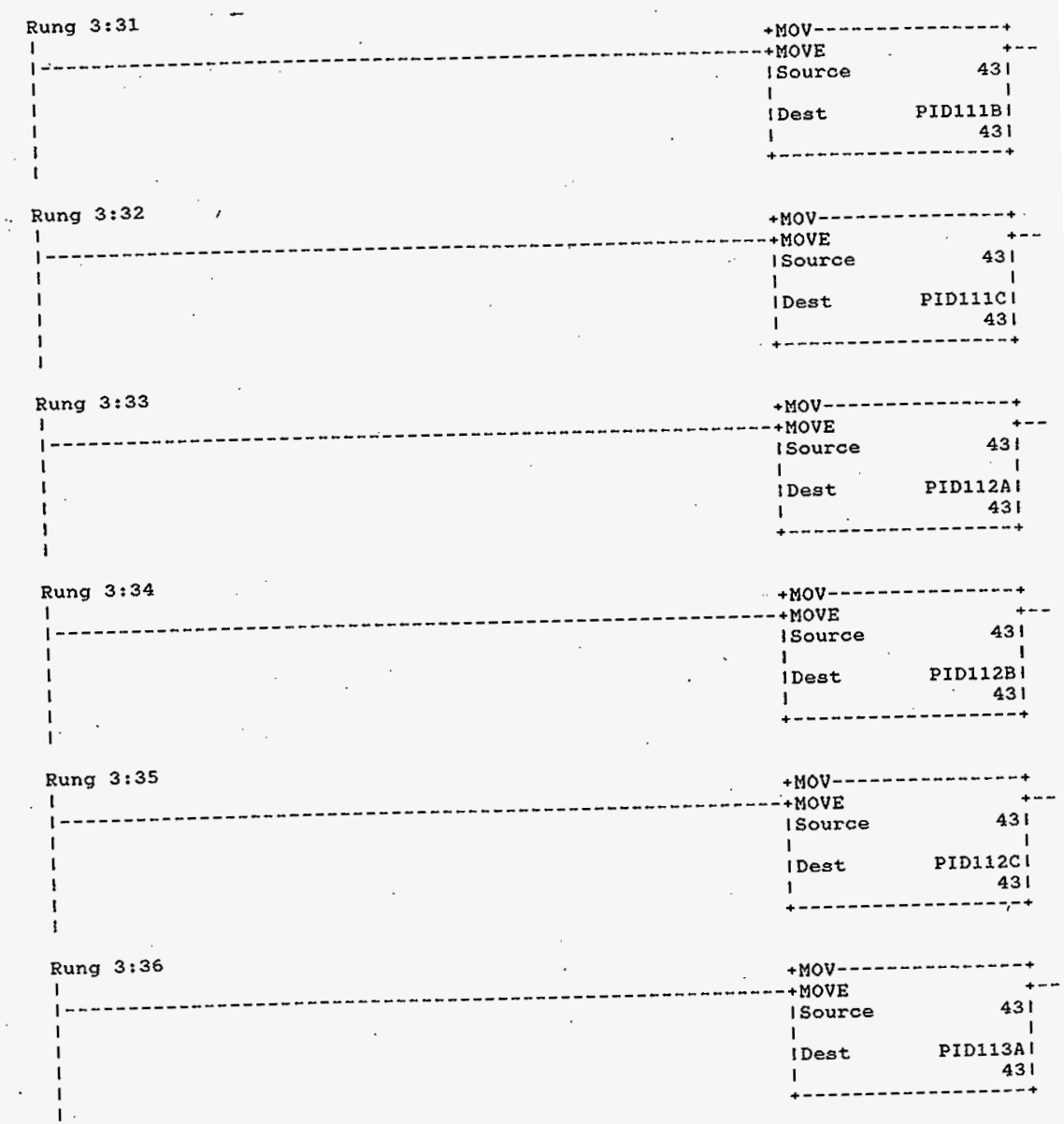

HNF-SD-FF-CSWD-61 Rev, 0 
Processor and Data(ops Unit 1)

Rung $3: 37$

1

!

I

i

i $-$

Rung $3: 38$

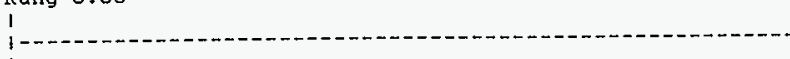

I

I

i
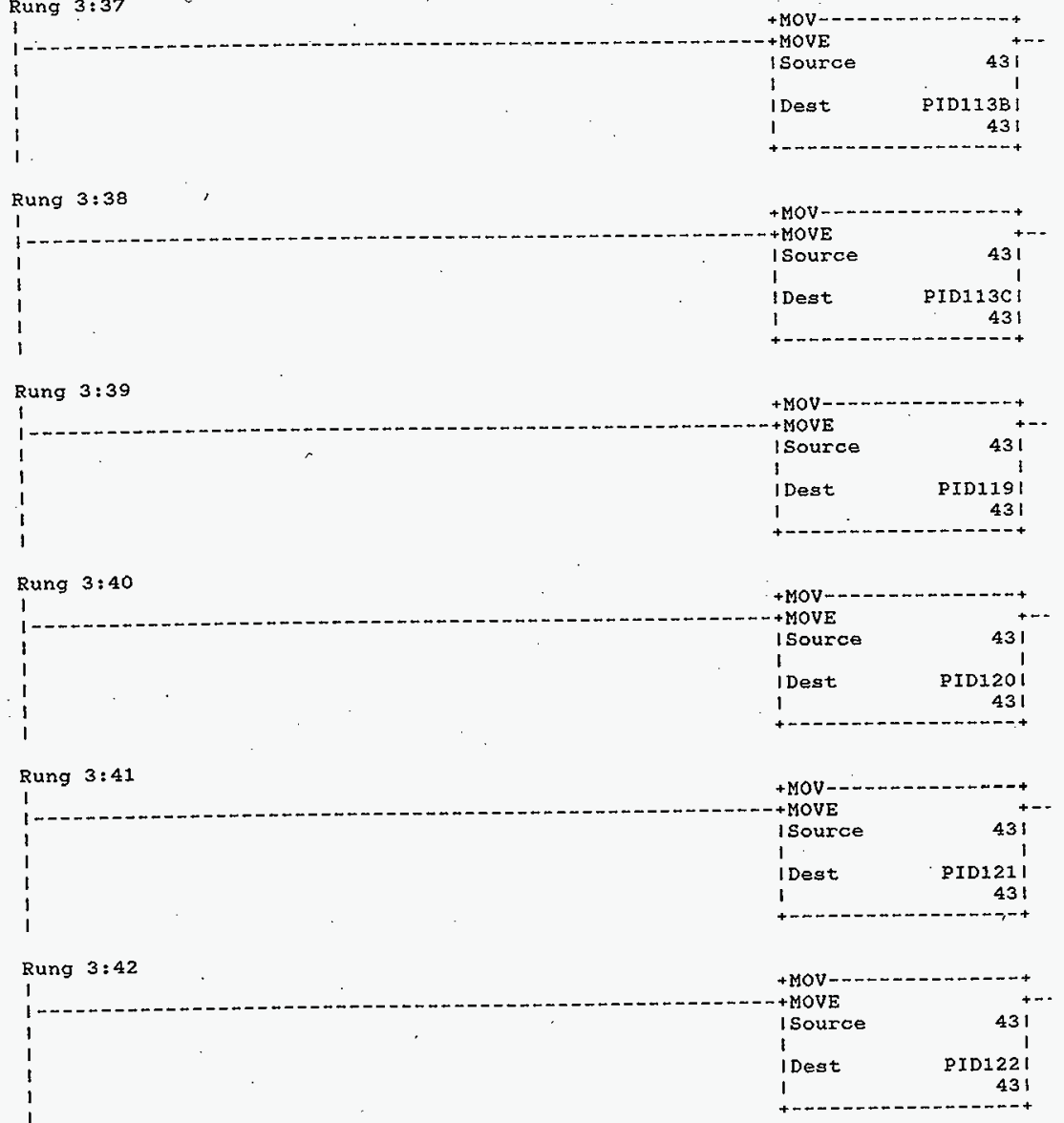

HNF-SD-FF-CSWD-61 Rev. 0

Page 356 
Procesgor and Data (OPS Unit 1) Program Listing

\section{Rung $3: 43$}
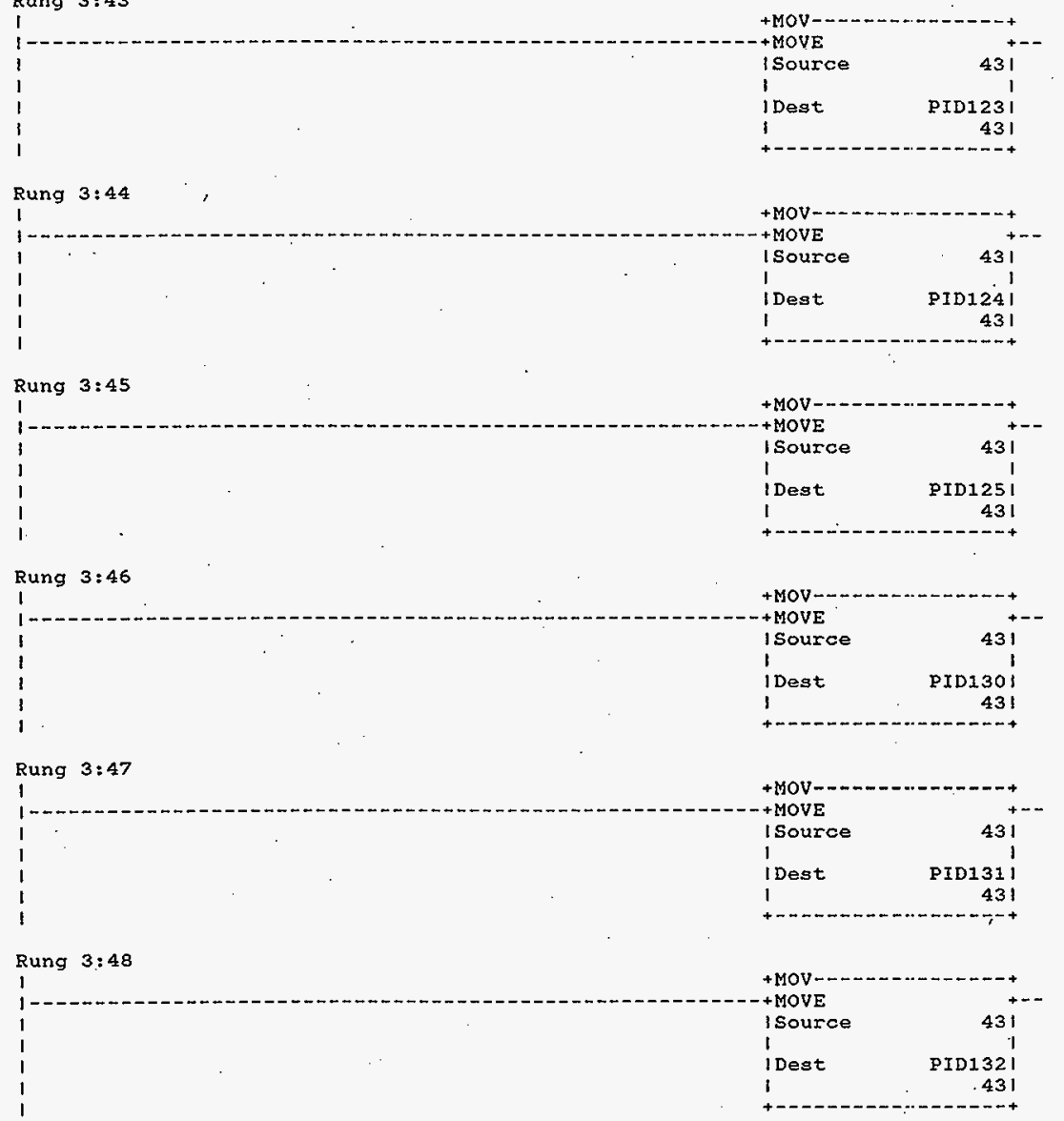
Processor and Data(OPS Unit 1)

October 23, 1996 Program Listing

Processor File: SODIUMAA.ACH

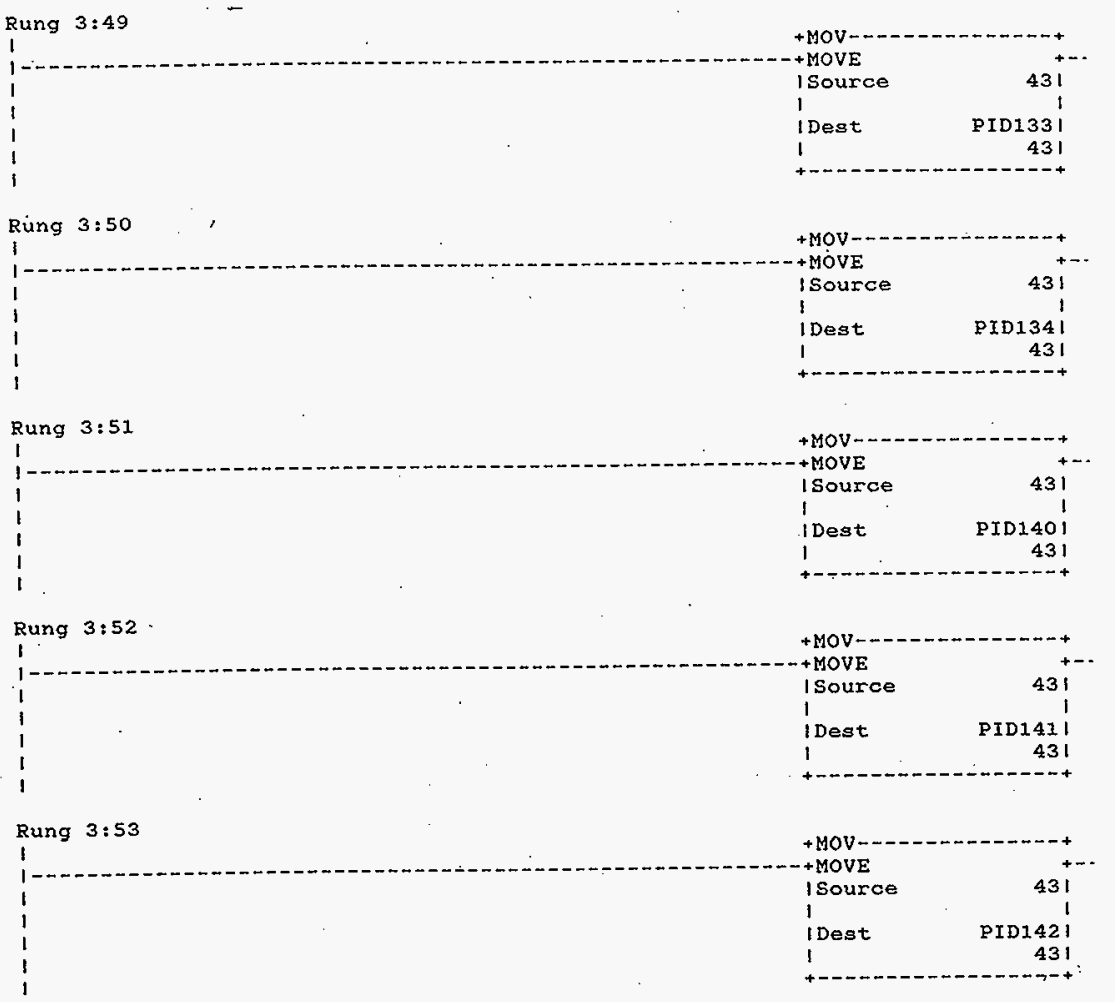

Rung $3: 54$

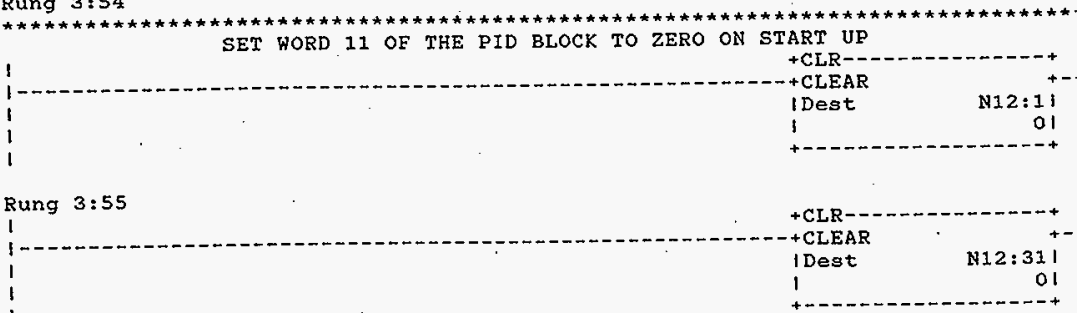

HNF-SD-FF-CSWD-61 Rev. 0

Page 358 


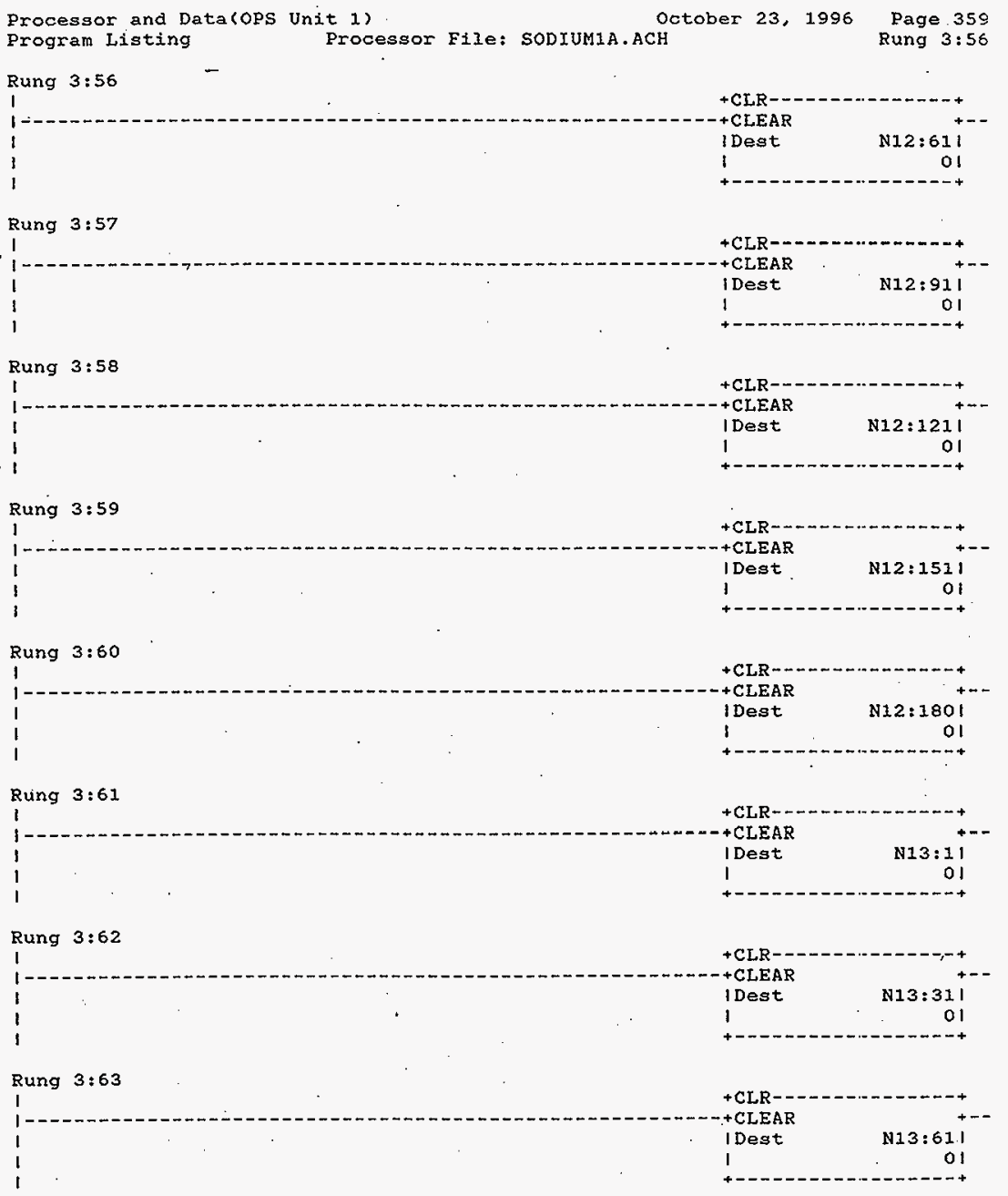

HNF-SD-FF-CSWD-61 Rev. 0 
Processor and Data(OPS Unit 1)

Program Iisting

Processor File: SODIUMiA.ACH

October 23,1996

Page 360

Rung $3: 64$

Rung $3: 64$

1 $\leftarrow$

1

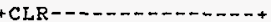

+ CLEAR

N13:91

1

Rung 3:65

1

I

Rung $3: 66$

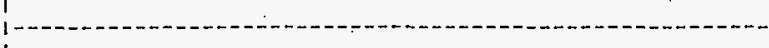

I

I

Rung $3: 67$

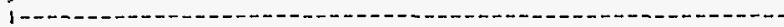

1

1

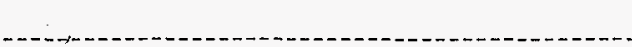

+ CLR-- $---n-----+$

+ CLEAR

N13:1211

I

Rung $3: 68$

(1)

1

1

$+\mathrm{CLR}-------------+$

+CLEAR

N13:151

I

01

\section{Rung $3: 69$}

I

$$
\text { i }
$$

1

1

Rung $3: 70$

1

1

, 1

1

1

Rung $3: 71$

I-

1

1

+CLEAR

IDest

+CLR--

I Dest

I

N13:1811

+--- - - - - - -

+ CLR -

I Dest

N14:11

1

01

$+C L R--$

I Dest

N14:31 I

1

01

+CLEAR

i Dest

$14: 61$

HNF-SD-FF-CSWD-61 Rev. 0

Page 360 
Processor and Data(OPS Unit 1) Program Listing

Processor File: SODIUMiA.ACH

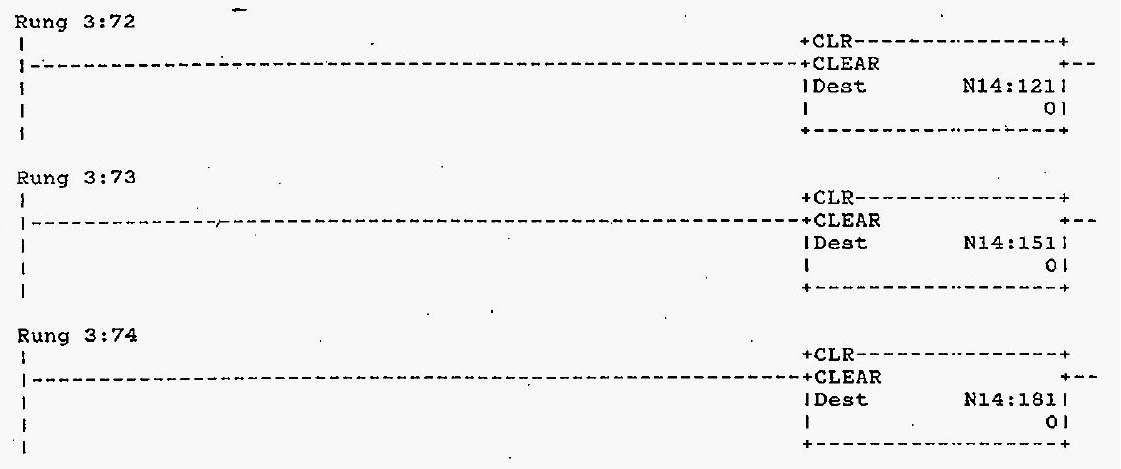

Rung $3: 75$

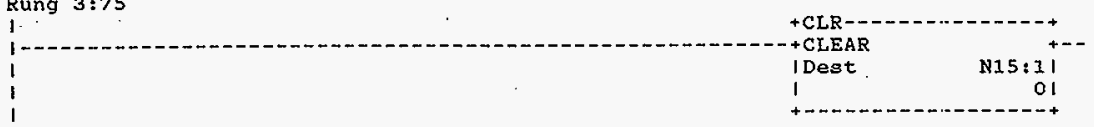

Rung $3: 76$

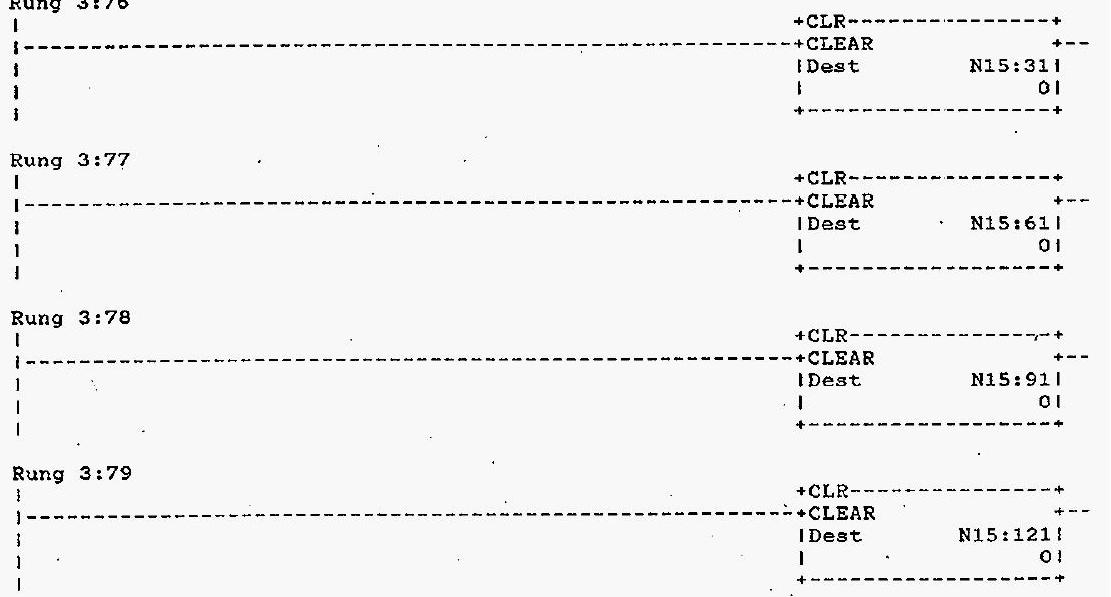

HNF-SD-FF-CSWD-61 Rev. 0

Page $3 \dot{b l}$ 


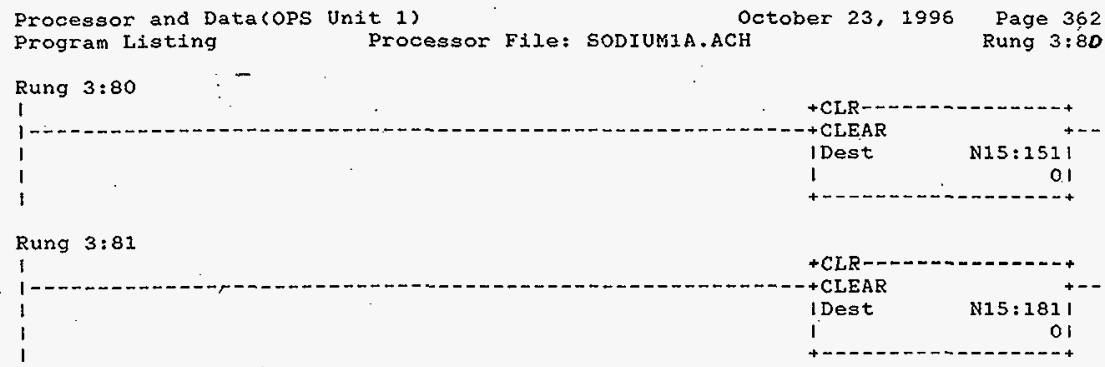

Rung $3: 82$

I

I

HNF-SD-FF-CSWD-61 Rev. 0

Page 362 


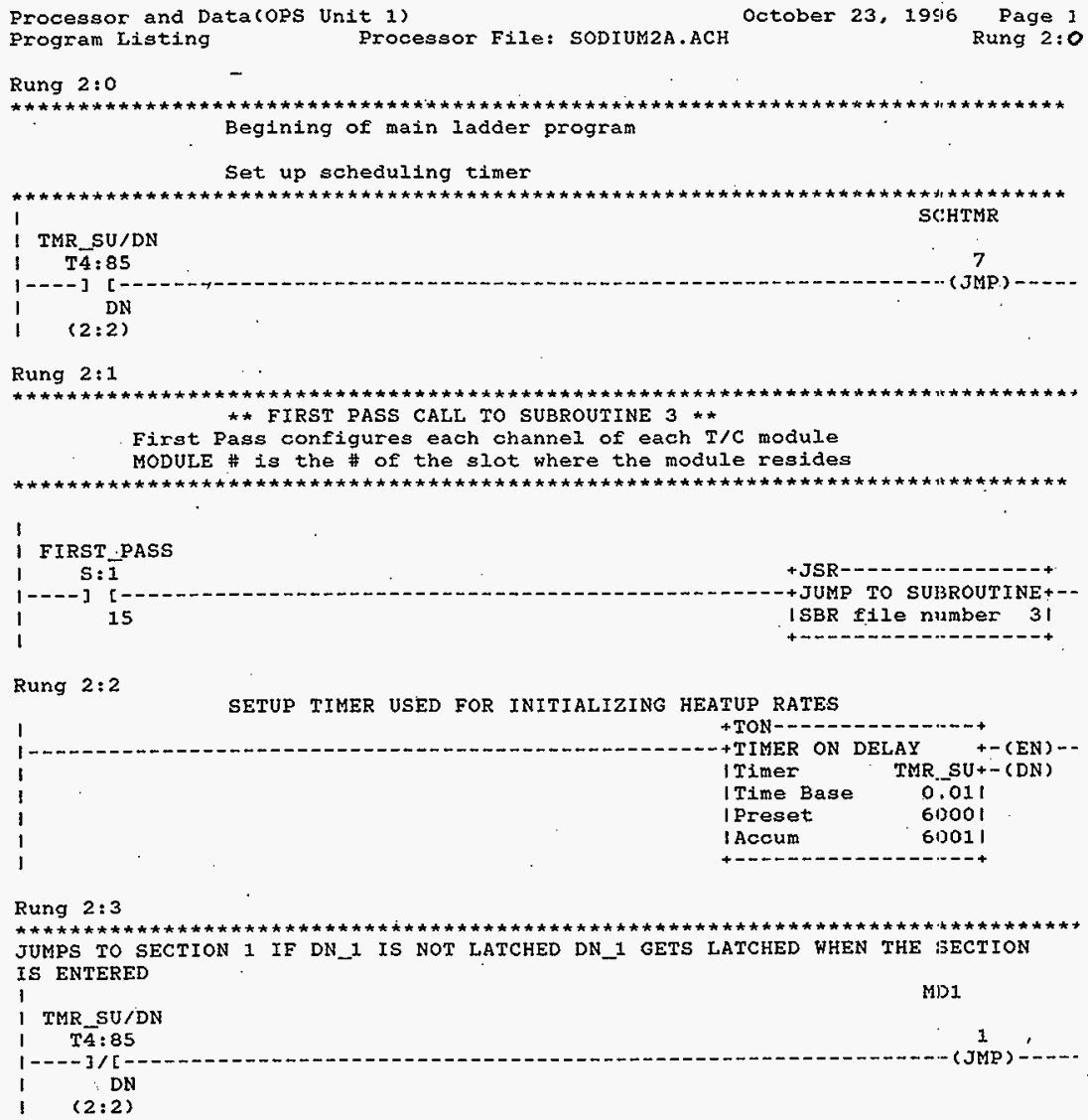

MD1.

HNF-SD-FF-CSWD-61 Rev. 0 
Processor and Data(OPS Unit i)

Rung 2:4

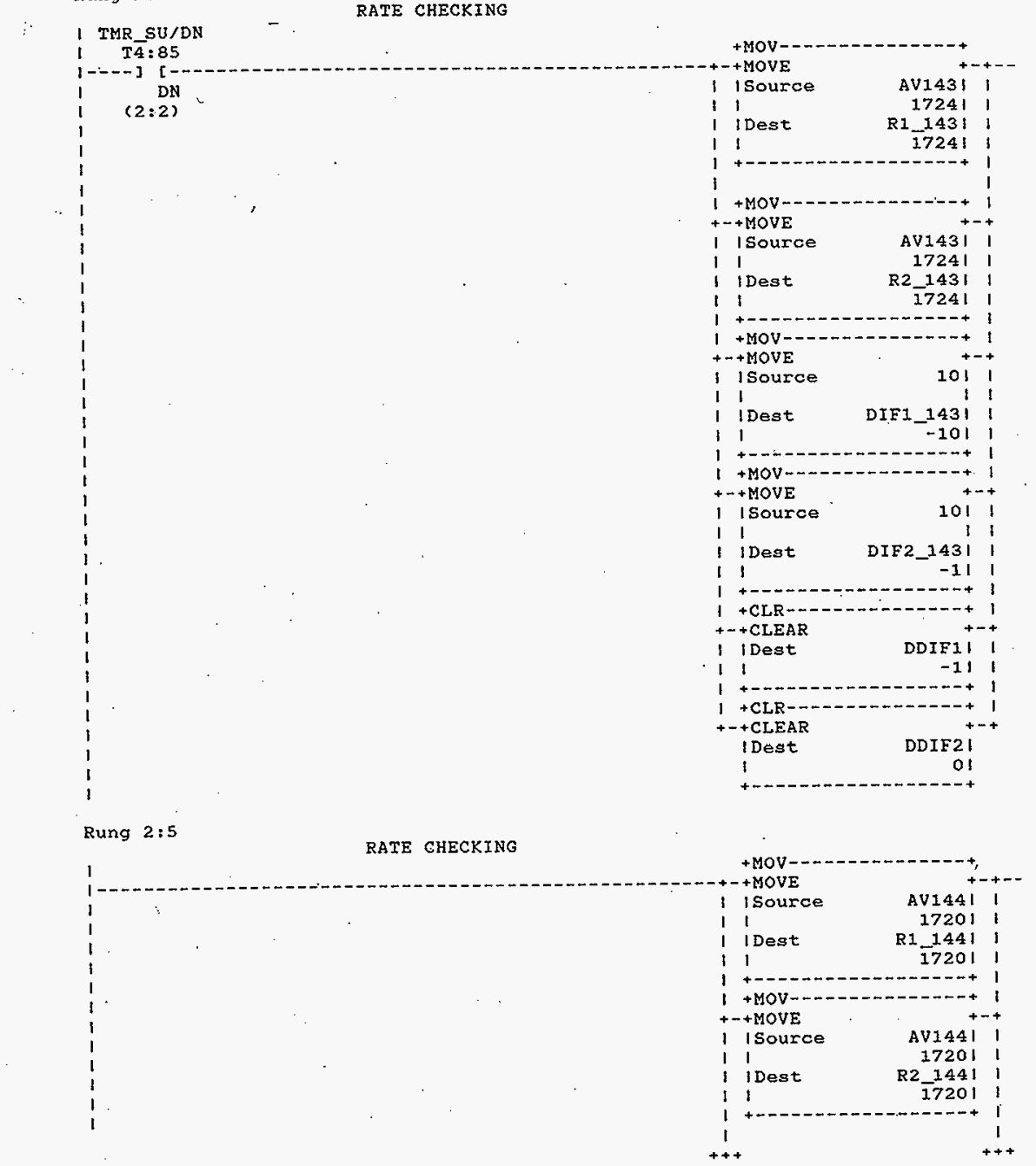

RATE CHECKING

HNF-SD-FF-CSWD-61 Rev. 0 
Processor and Data(OPS Unit 1 )

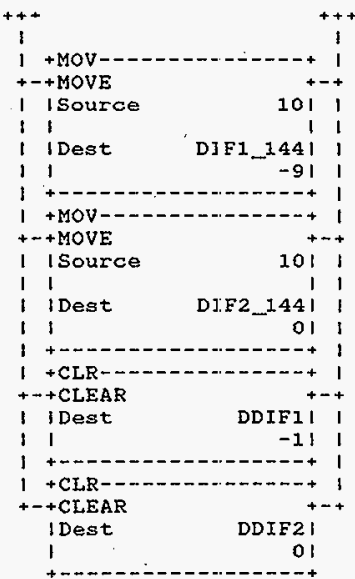

Rung $2: 6$

RATE CHECKING

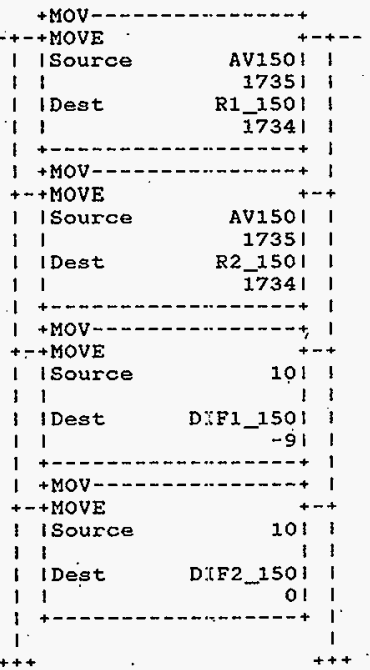

HNF-SD-FF-CSWD-6। Rev. 0 


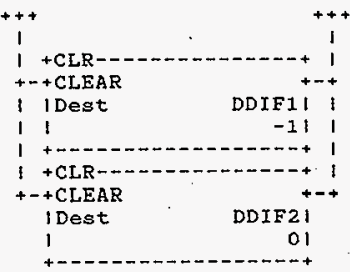

\section{Rung 2:7}

RATE CHECKXNG

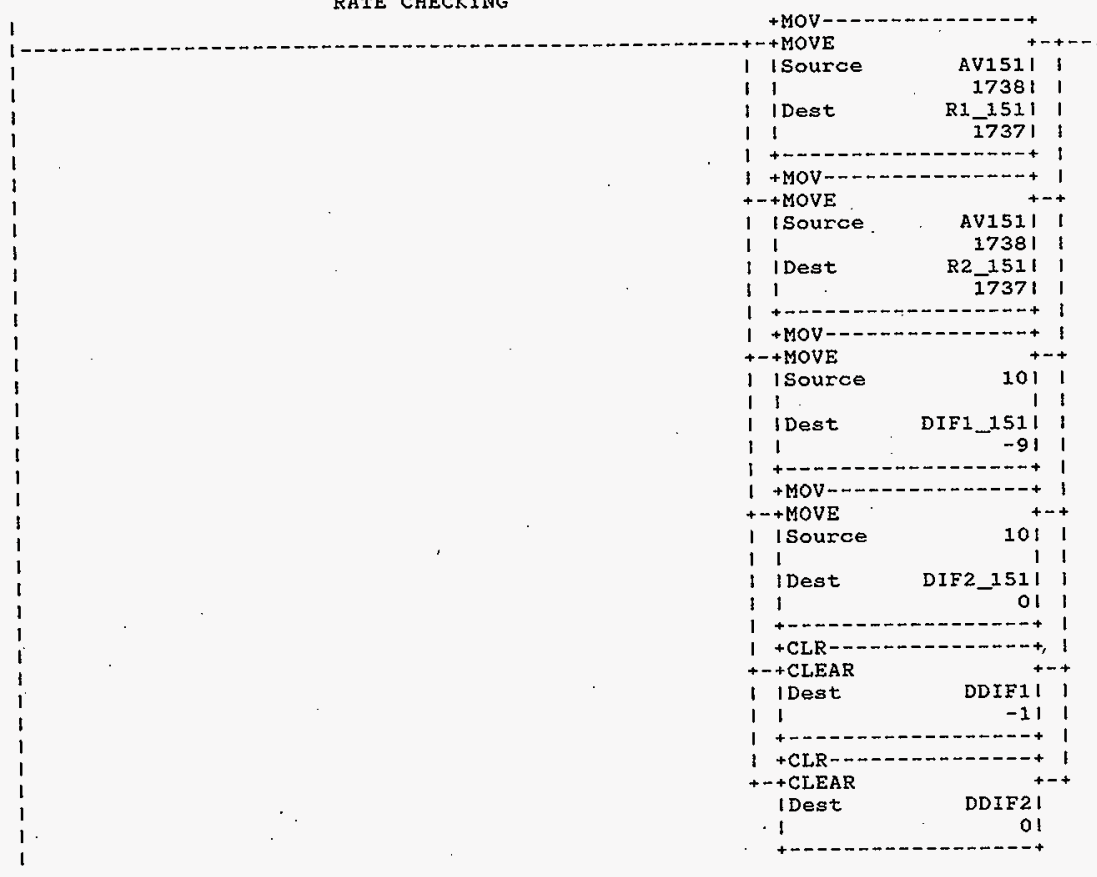

HNF-SD-FF-CSWD-61 Rev. 0 
Processor and Data(OPS Unit 1) Program Listing

Processor File: SODIUM $2 A \cdot A C H$

October 23,1996

Page 5 Rung $2: 8$
RATE CHECKING

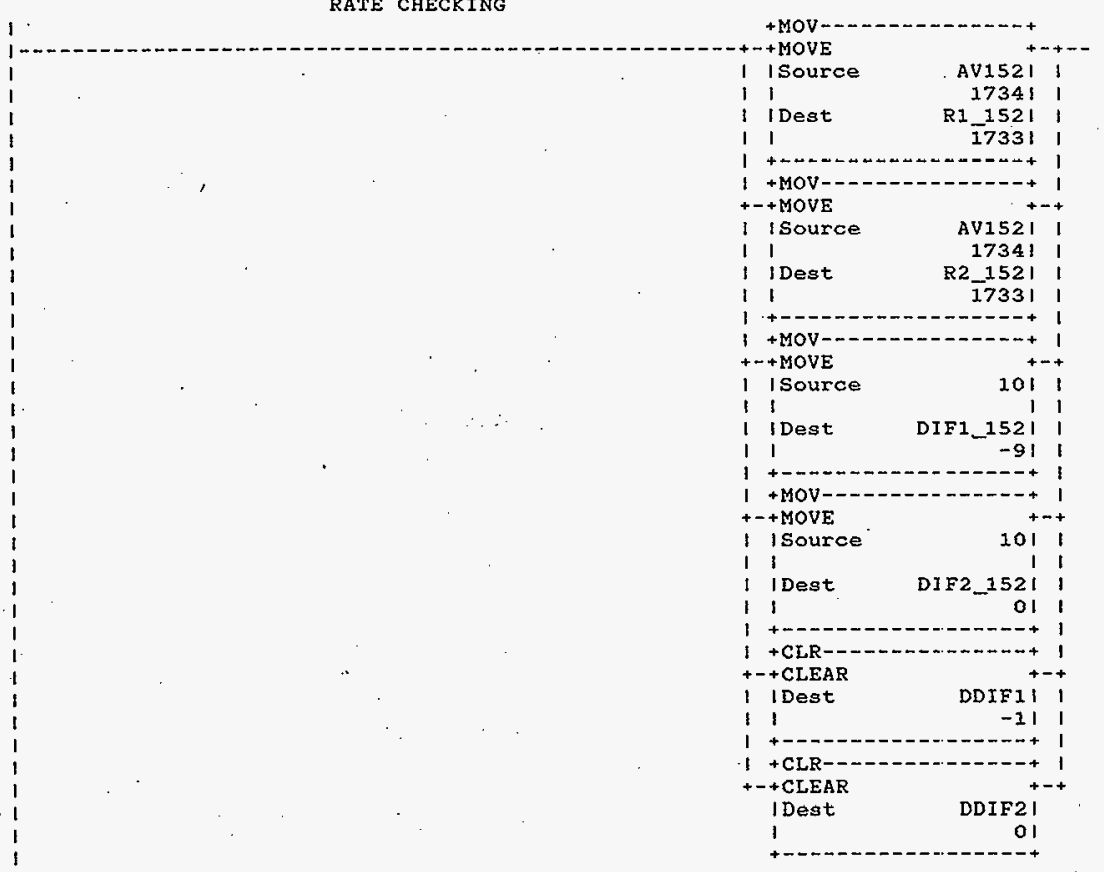

Rung $2: 9$

RATE CHECKING

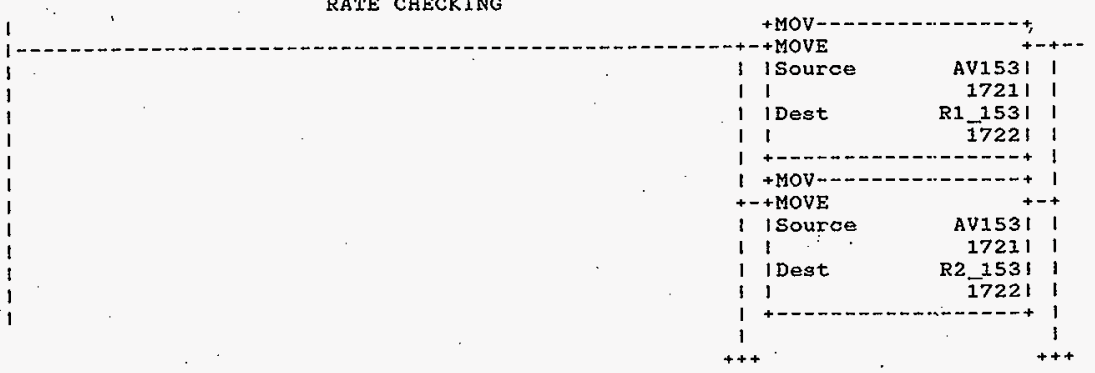

HNF-SD-FF-CSWD-61 Rev. 0 


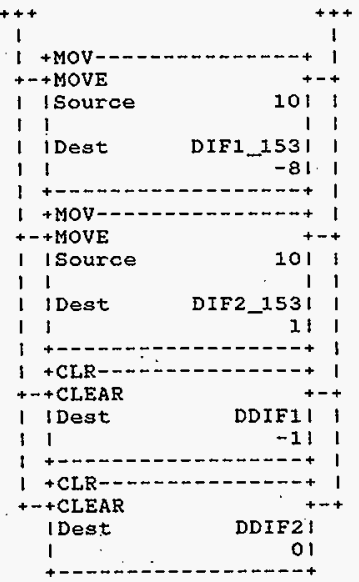

Rung 2:10

RATE CHECKING

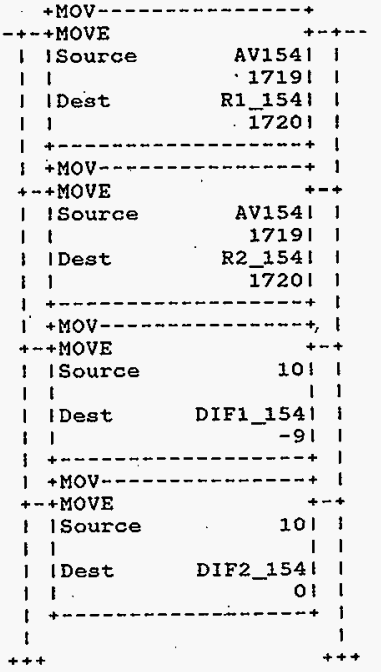

HNF-SD-FF-CSWD-61 Rev. 0 


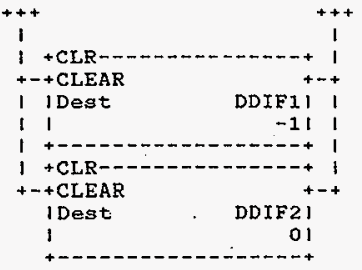

Rung $2: 11$

RATE CHECKING

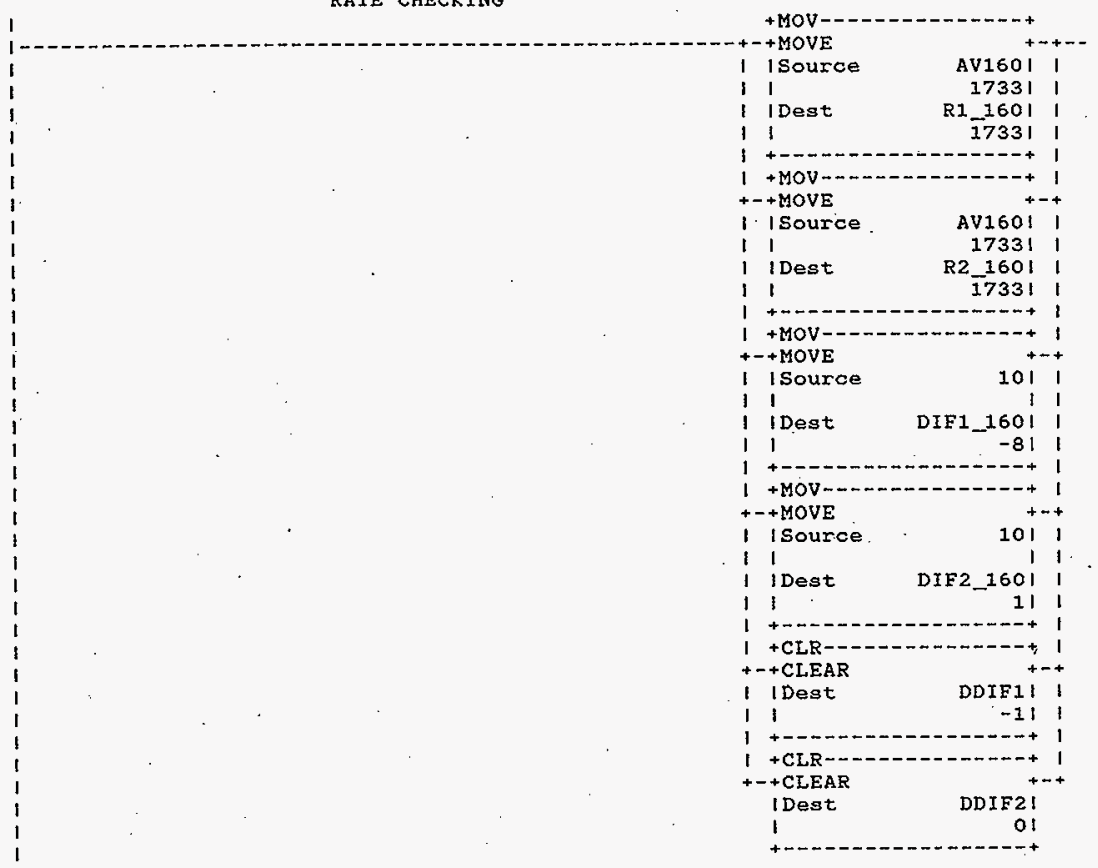


Processor and Data(OPS Unit 1)

\section{Rung 2:12} RATE CHECKING

$$
\begin{aligned}
& 1 \\
& 1 \\
& 1 \\
& 1 \\
& 1 \\
& 1 \\
& 1 \\
& 1 \\
& 1 \\
& 1 \\
& 1 \\
& 1 \\
& 1 \\
& 1 \\
& 1 \\
& 1 \\
& 1 \\
& 1 \\
& 1 \\
& 1 \\
& 1 \\
& 1 \\
& 1 \\
& 1 \\
& 1 \\
& 1 \\
& 1 \\
& 1 \\
& 1 \\
& 1 \\
& 1 \\
& 1 \\
& 1
\end{aligned}
$$

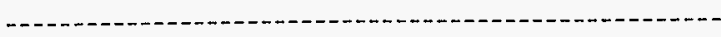




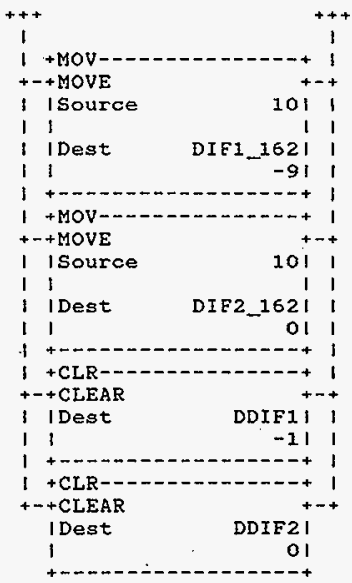

Rung $2: 14$

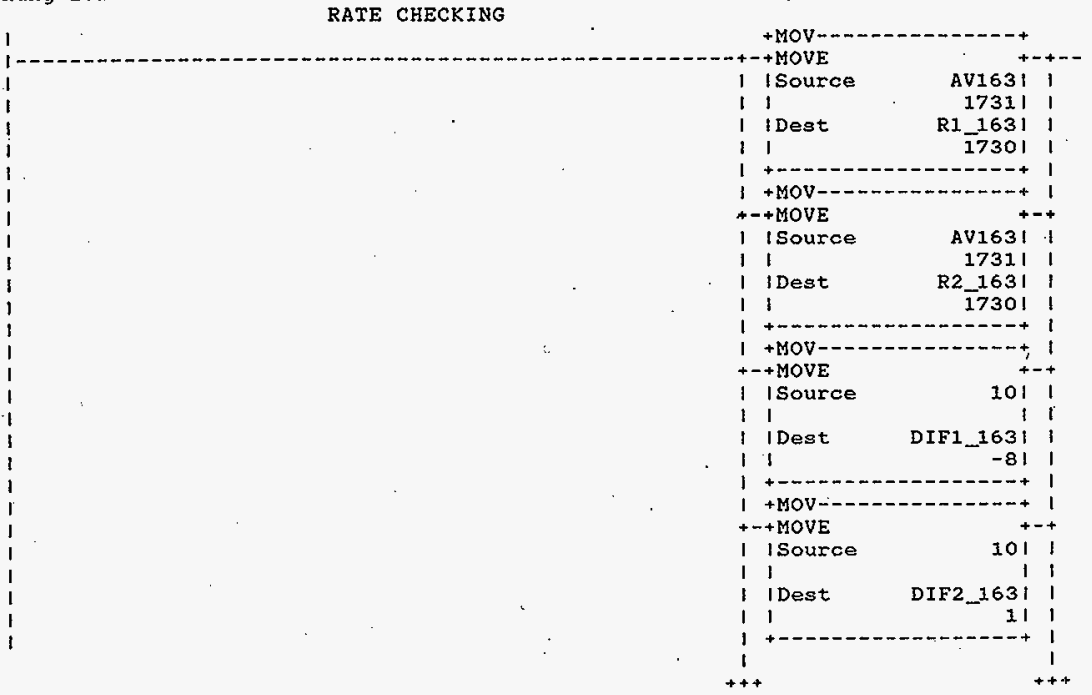

HNF-SD-FF-CSWD-151 Rev. 0 


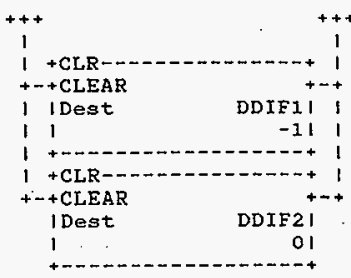

\section{Rung $2: 15$}

RATE CHECKING
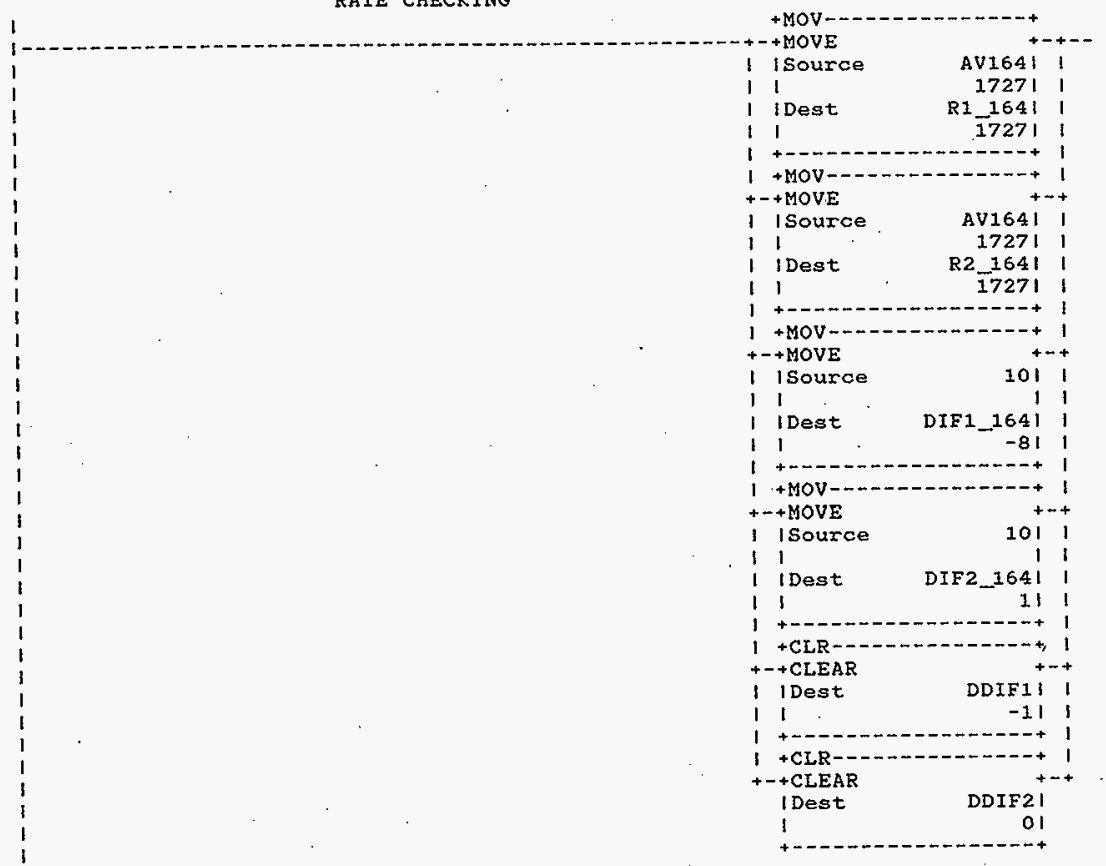

HNF-SD-FF-CSWD-61 Rev. 0 
Processor and Data(OPS Unit 1)

October 23, 1996

Page $I 3$

Program Listing

Rung $2: 16$

RATE CHECKING

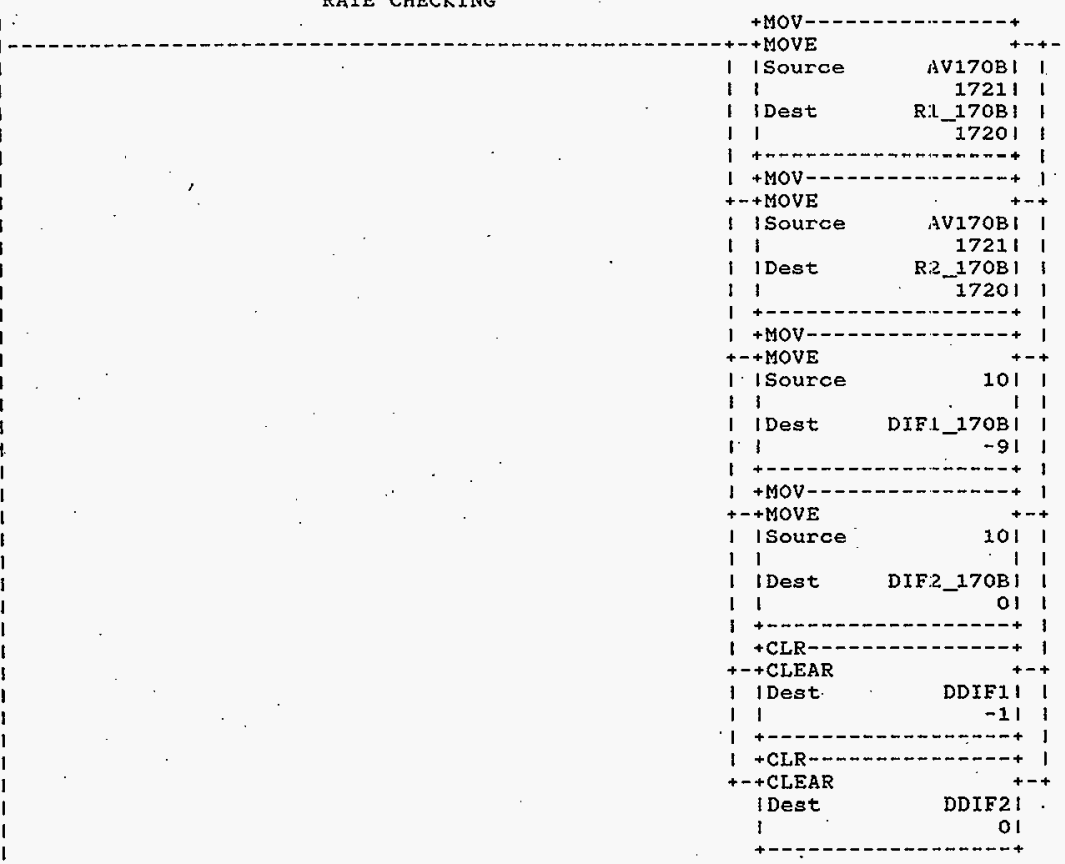

Rung $2: 17$

RATE CHECKING

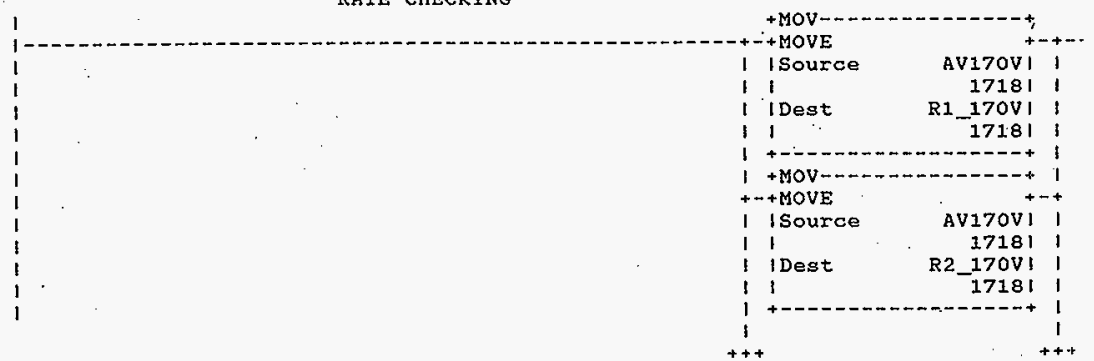

HNF-SD-FF-CSWD-61 Rev. 0 
Processor and Data(OPS Unit 1)

October 23, 1996 Page 12

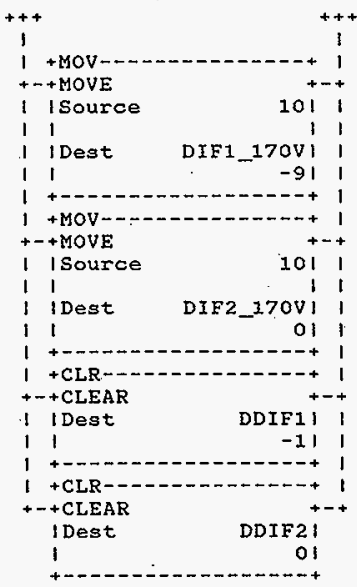

Rung 2:18

RATE CHECKING

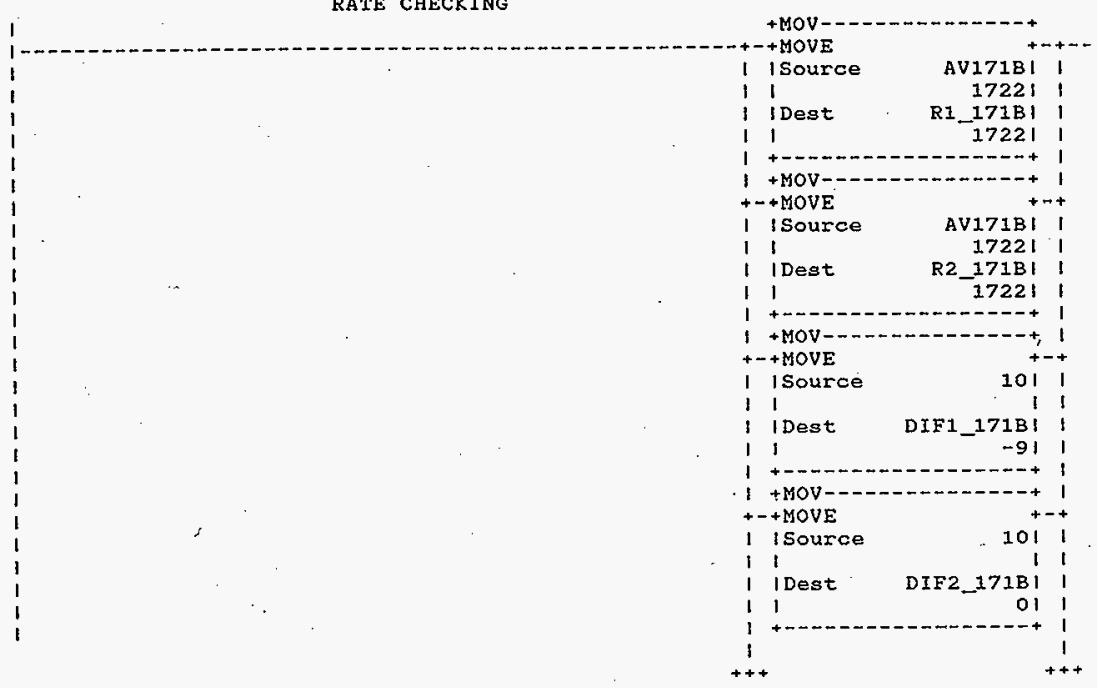

HNF-SD-FF-CSWD-61 Rev. 0 
1
1
1
1
1
1
1

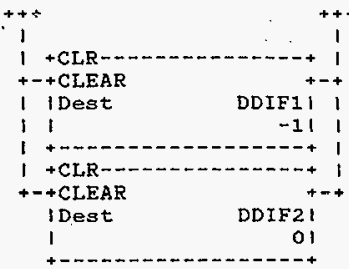

Rung $2: 19$

RATE CHECKING
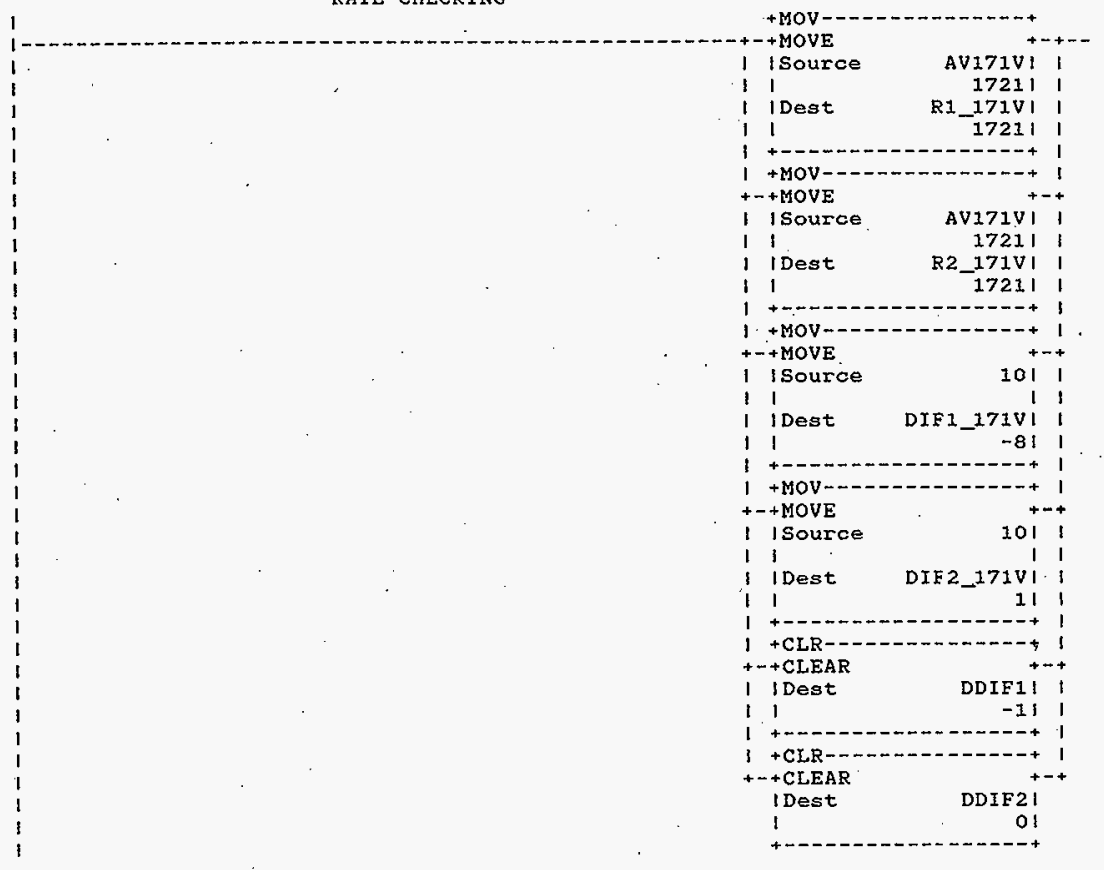

HNF-SD-FF-CSWD-61 Rev. 0 
Procesgor and Data(OPS Unit 1 )

October 23, 1996

Page $1 \leqslant$ Program Listing

\section{Rung 2:20}

RATE CHECKING

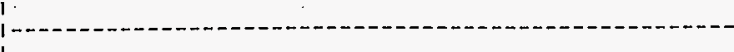

1

11

1 IDest

AV172BI

Source

17331

11

R1 172B I

1

17331

I +MOV-D- - - - ------+1

+-+ MOVE

1 I source

1

I IDest

1

AV1728I

27331 ।

$1+$

$1+$

+ MOV

I Isource

1

I Dest

$172 \mathrm{BI}$

11

$+$

17331

$1+$

++ MOVE

I ISource

11

1 I Dest

11

DIF2_172B I

1

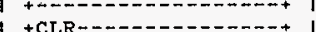

++ CLEAR

I I Dest

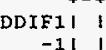

11

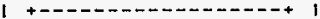

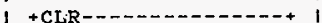

+- +CLEAR

IDest

1

Rung 2:21

RATE CHECKING

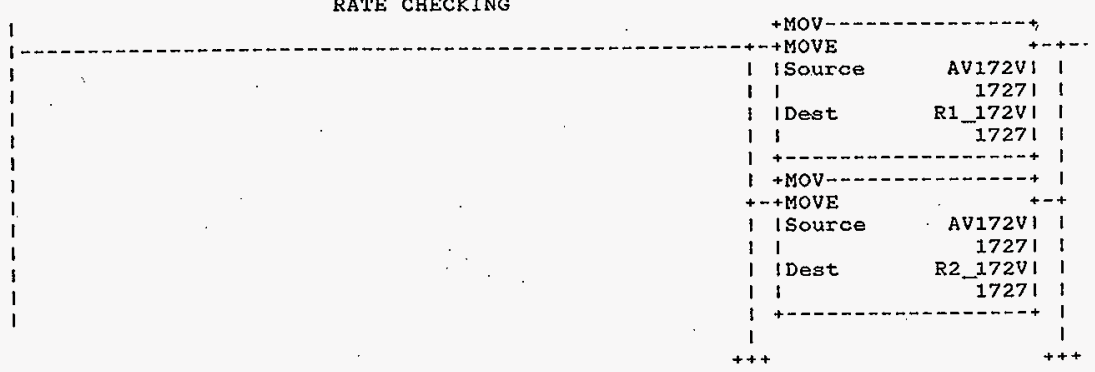

HNF-SD-FF-CSWD-61 Rev. 0 


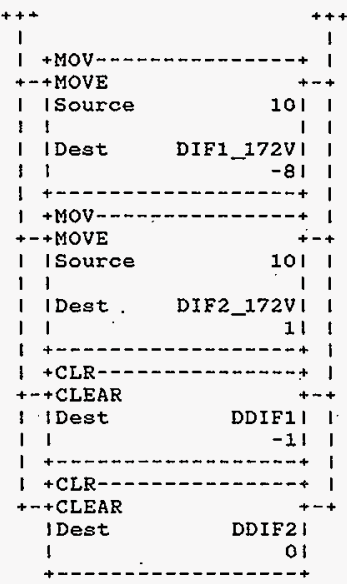

Rung $2: 22$

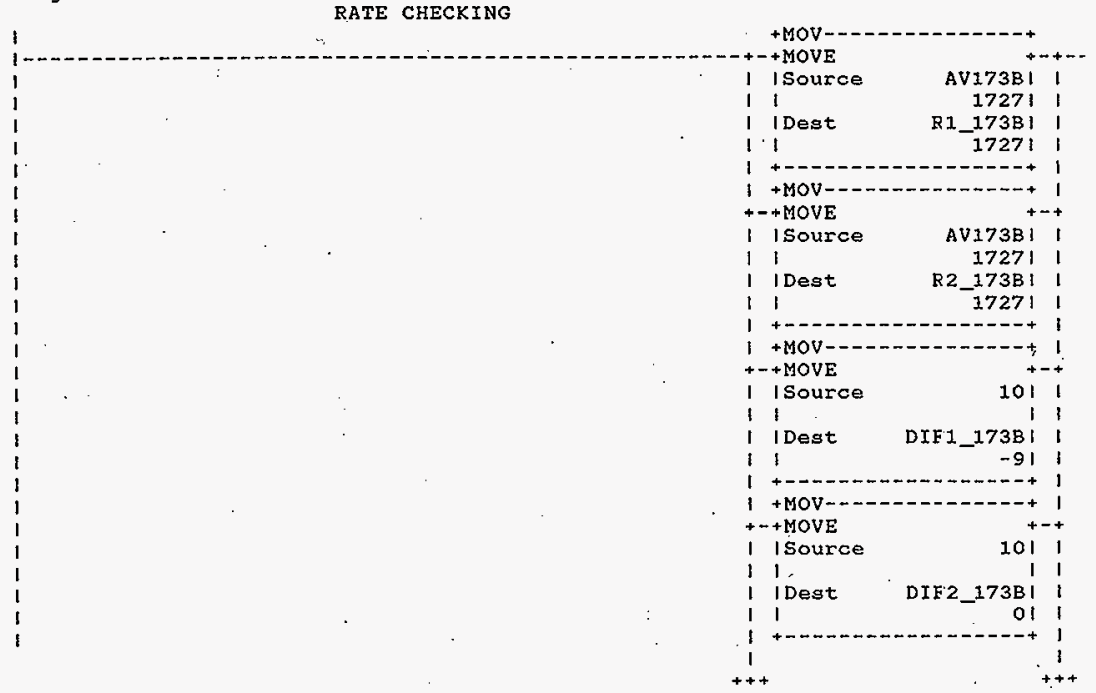

HNF-SD-FF-CSWD-61 Rev. 0 

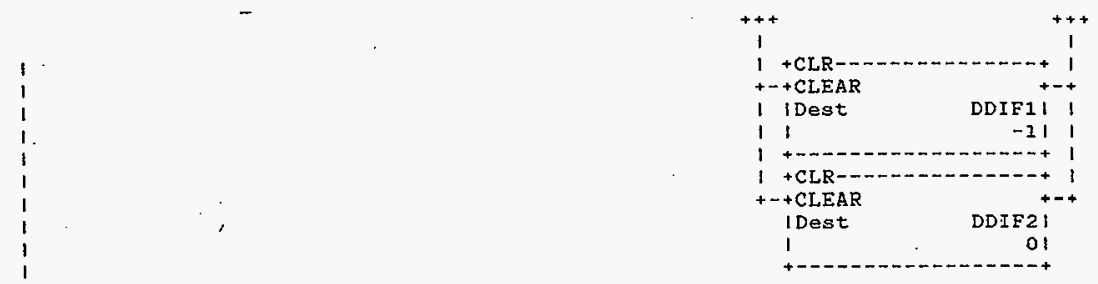

Rung $2: 23$

RATE CHECKING

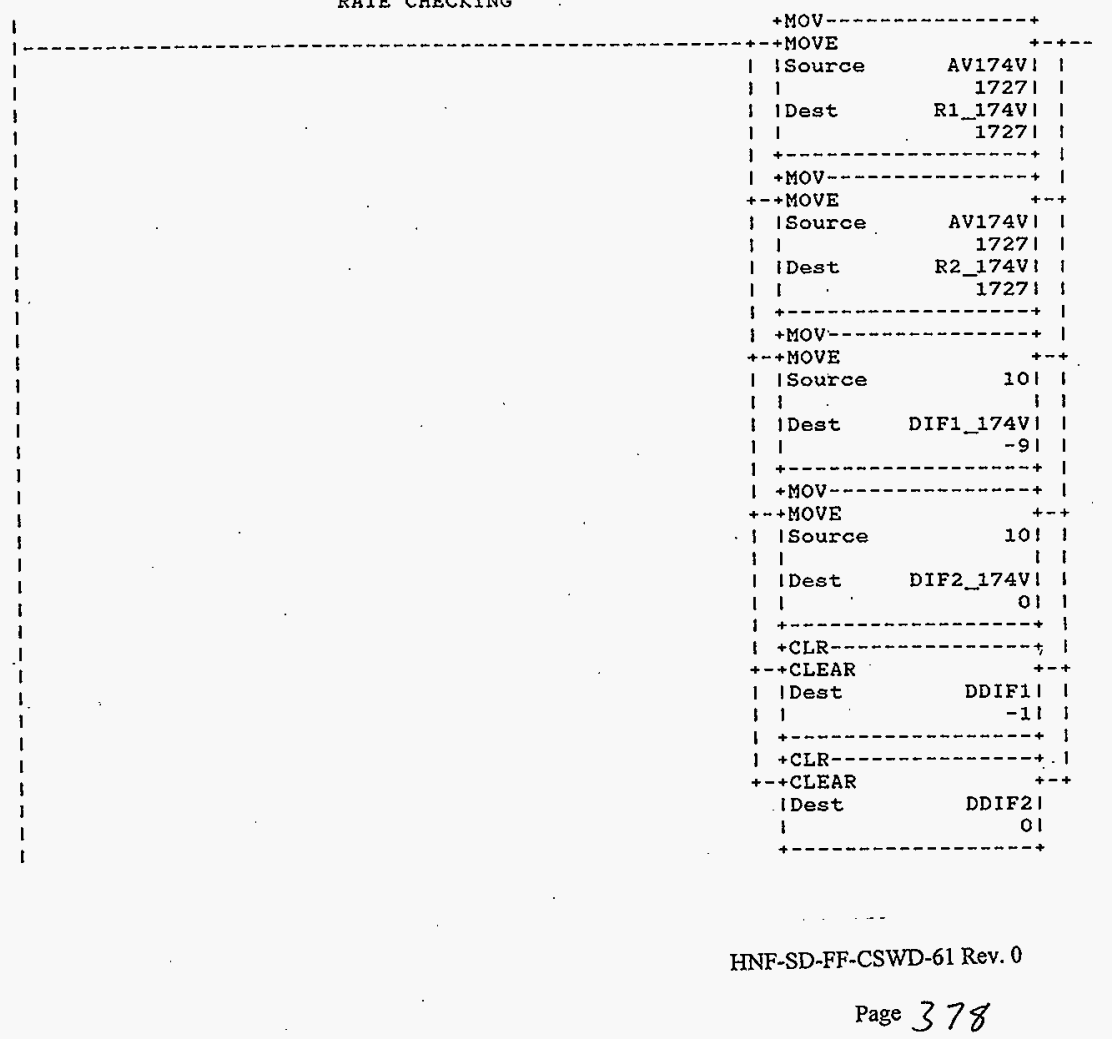




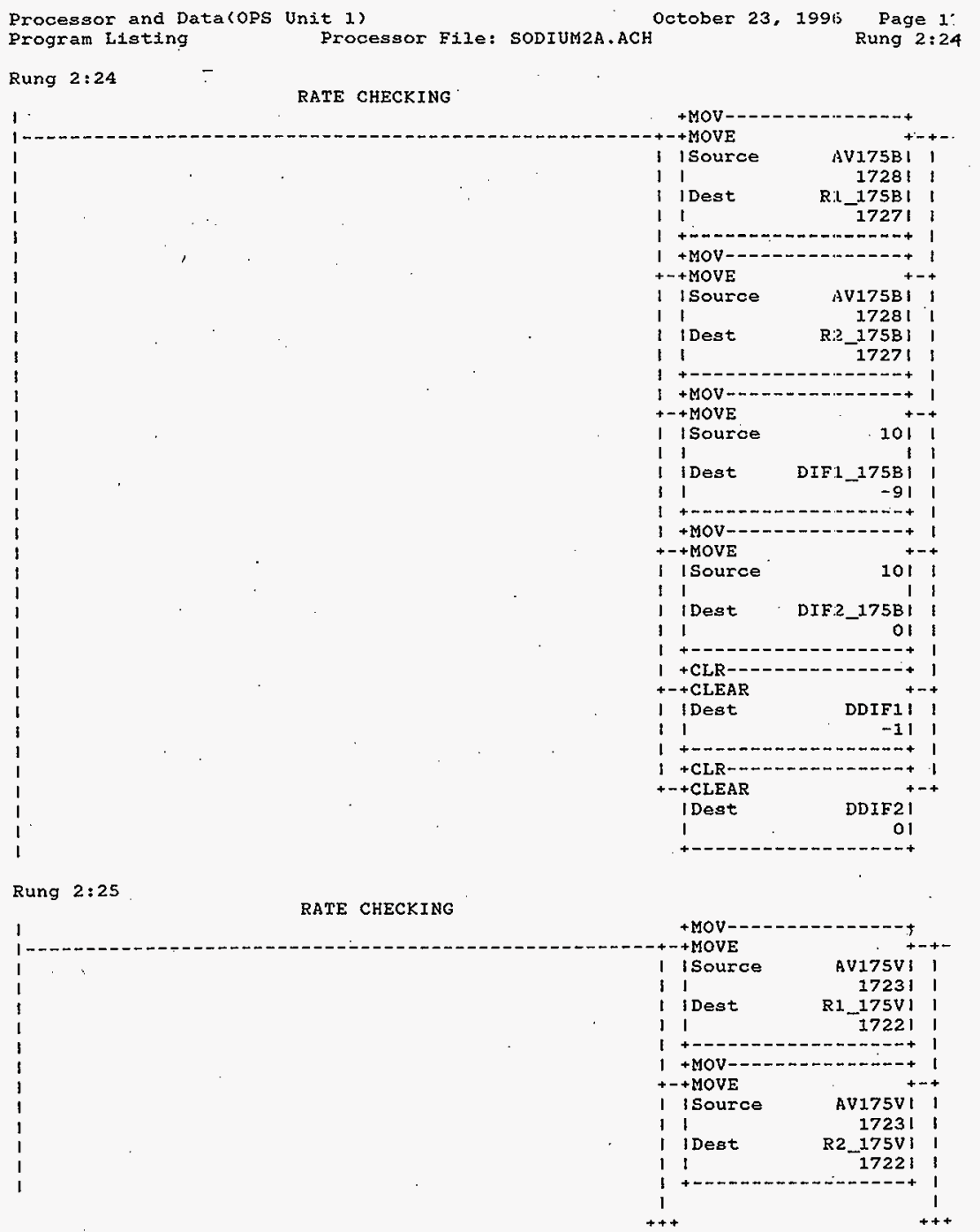

HNF-SD-FF-CSWD-61 Rev. 0

Page 379 
Rung 2:26

RATE CHECKING
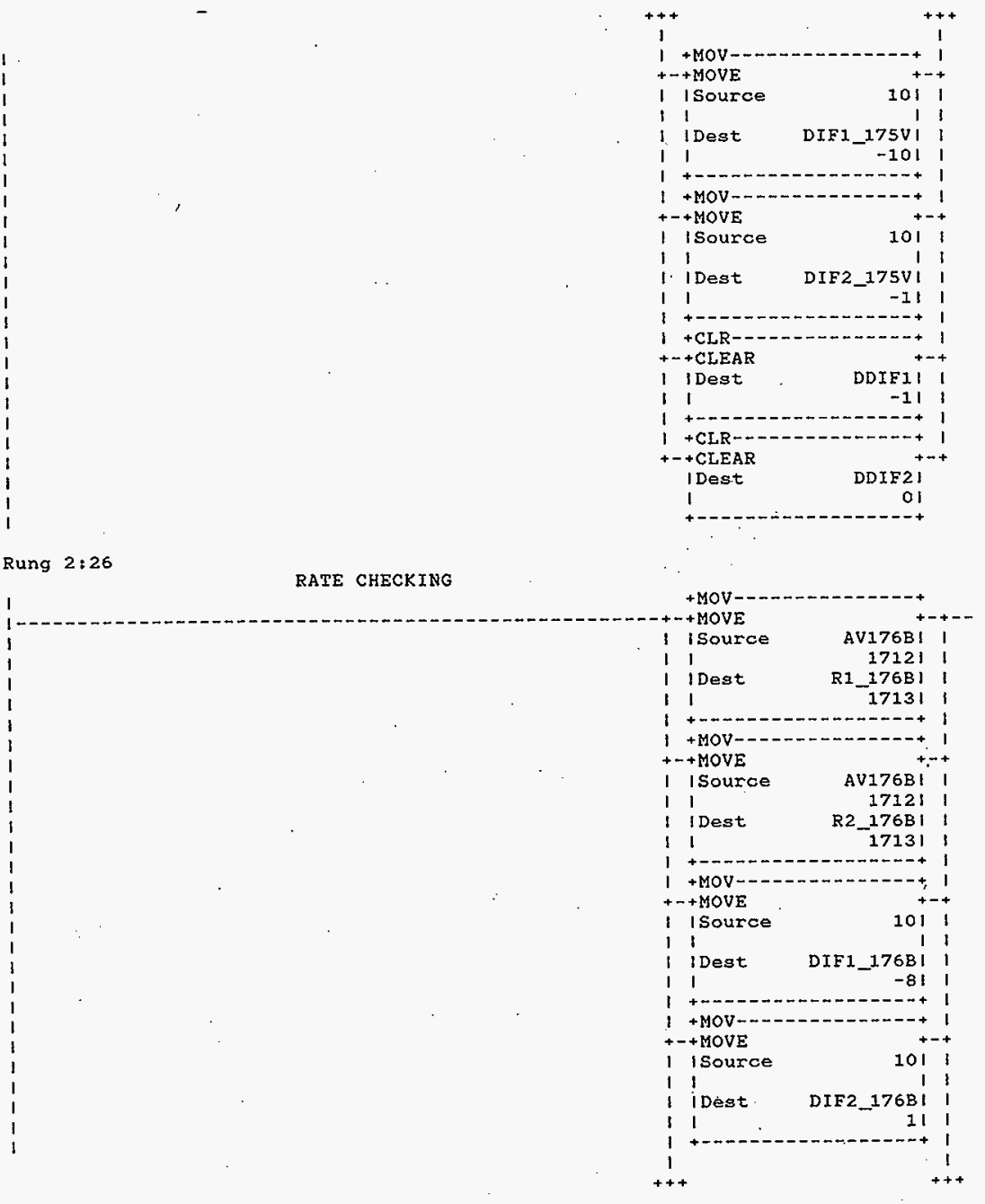

HNF-SD-FF-CSWD-61 Rev. 0 


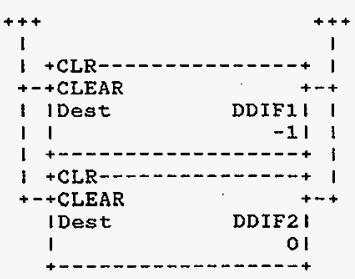

\section{Rung $2: 27$}

\section{RATE CHECKING}

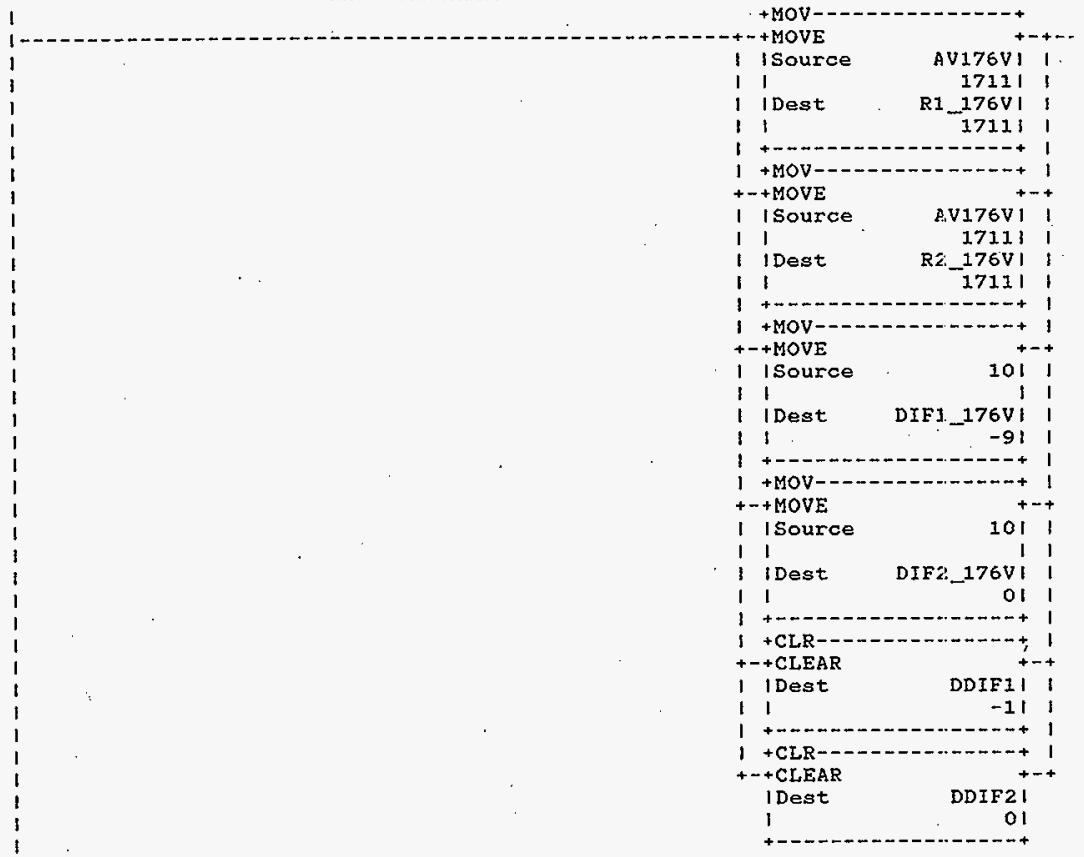

HNF-SD-FF-CSWD-61 R.ev. 0

page 3 iा 


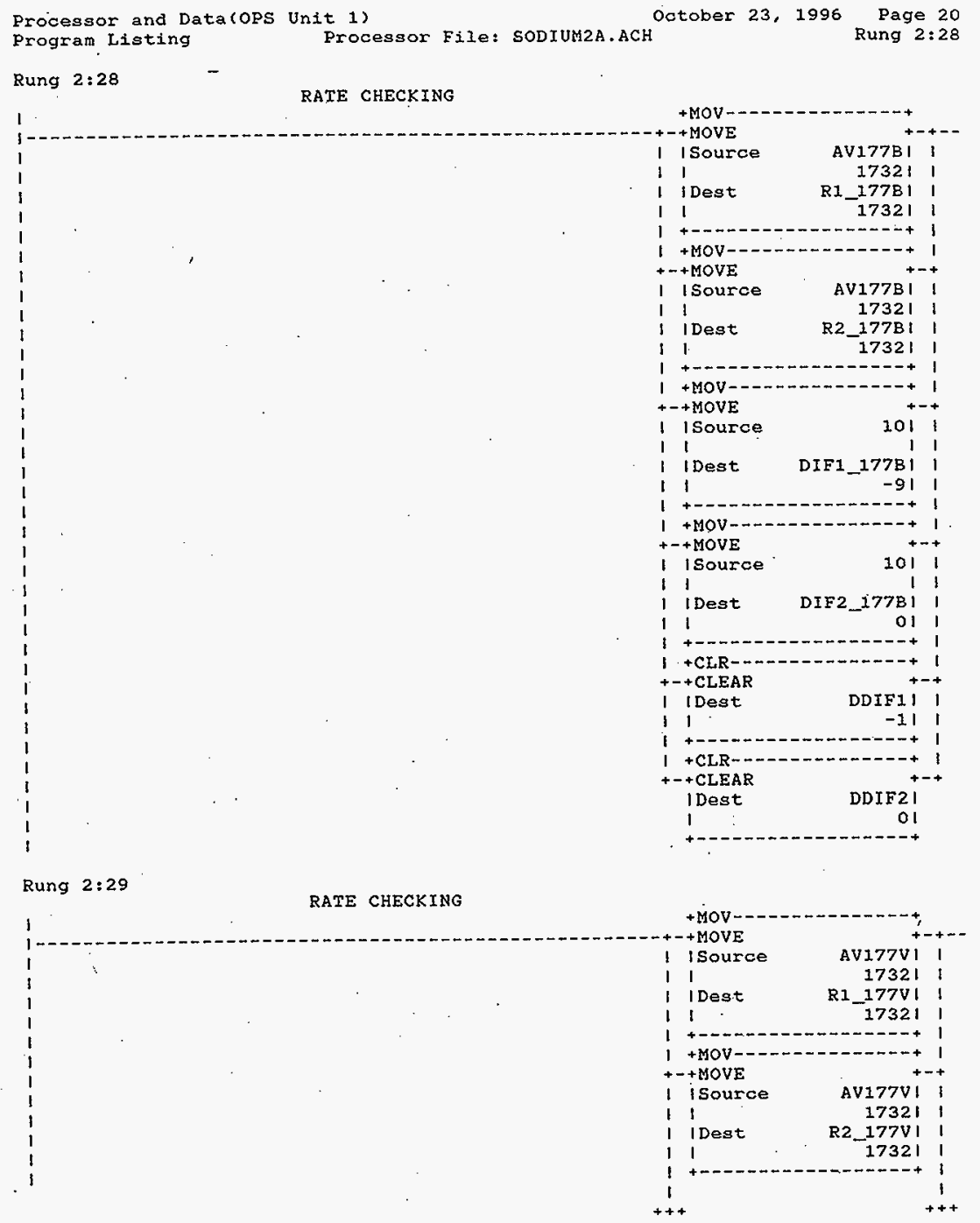

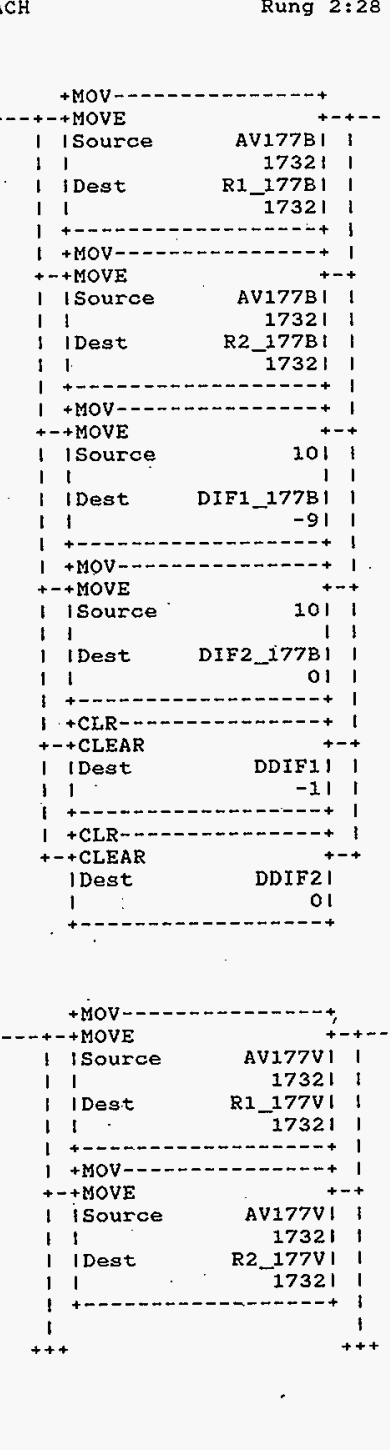

Rung $2: 28$

RATE CHECKING 


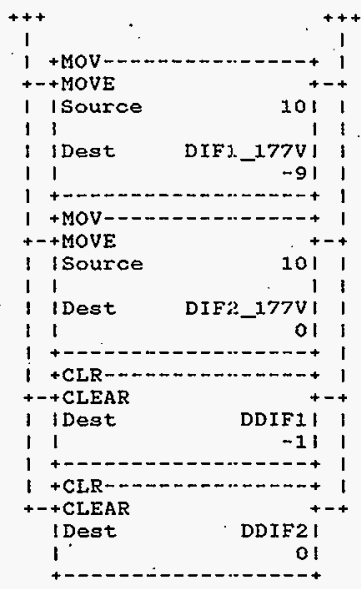

Rung $2: 30$

RATE CHECKING

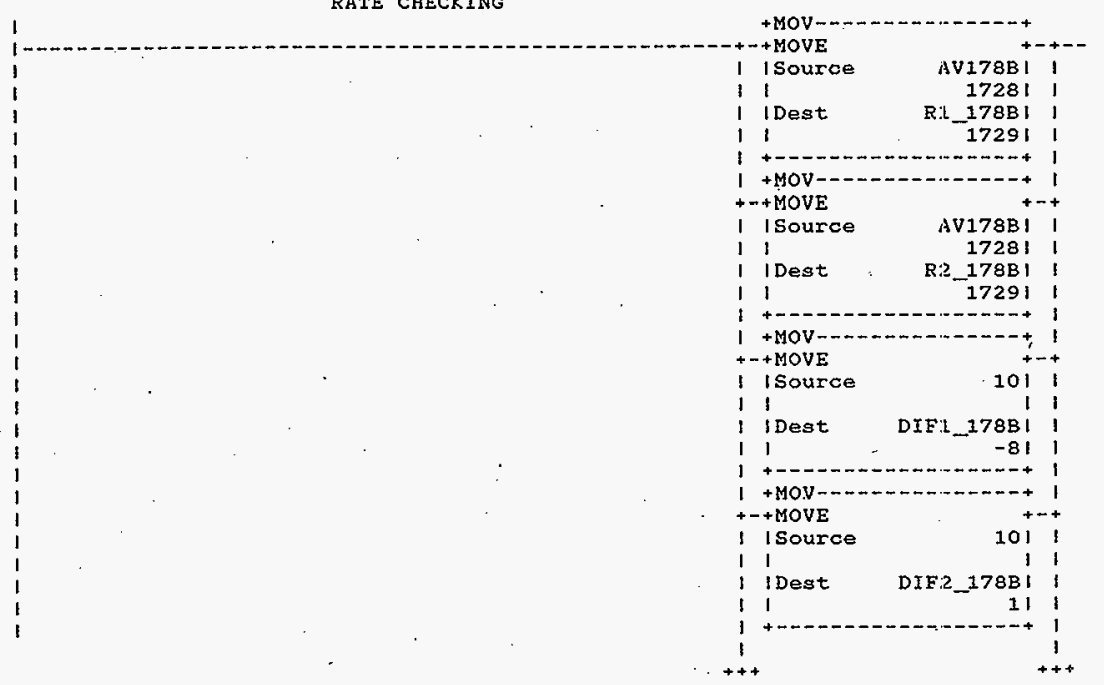

HNF-SD-FF-CSWD-61 Rev. 0 

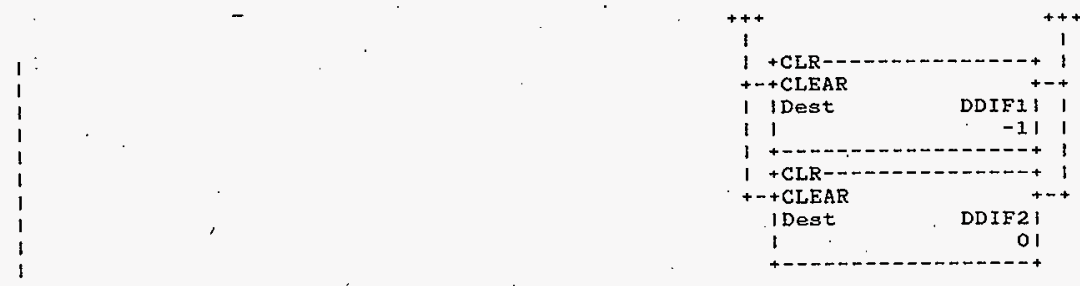

\section{Rung 2:31}

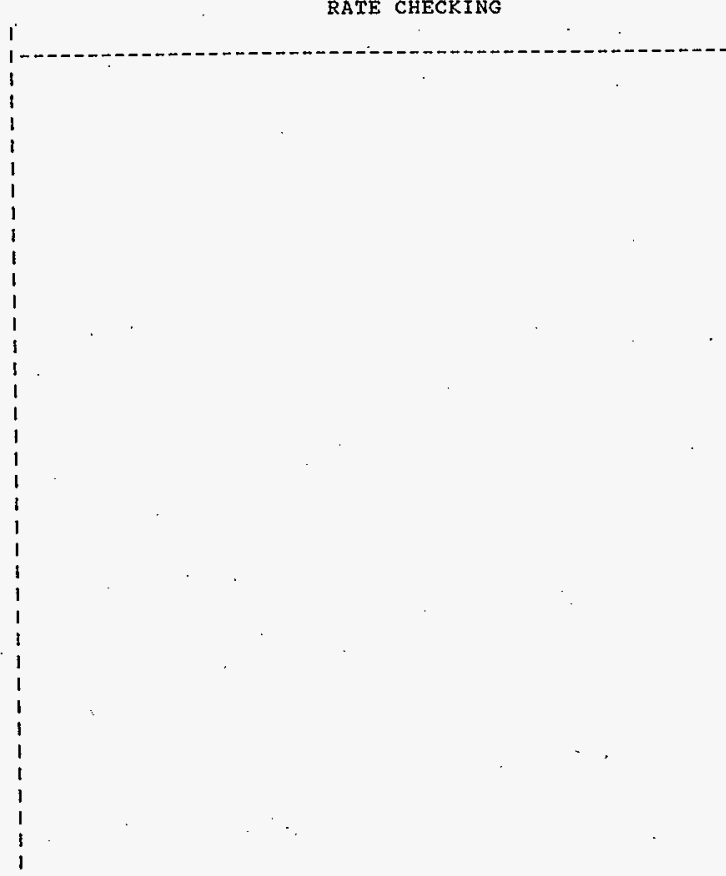

RATE CHECKING

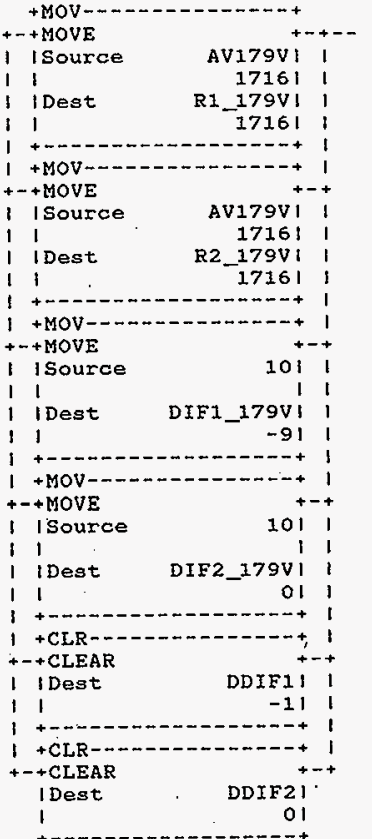

HNF-SD-FF-CSWD-61 Rev. 0

Page 384 
Processor and Data(OPS Unit 1)

October 23, 1996

Page 23

Program Listing

Processor File: SODIUM2A.ACH

Rung 2:32

Rung $2: 32$

RATE CHECKING

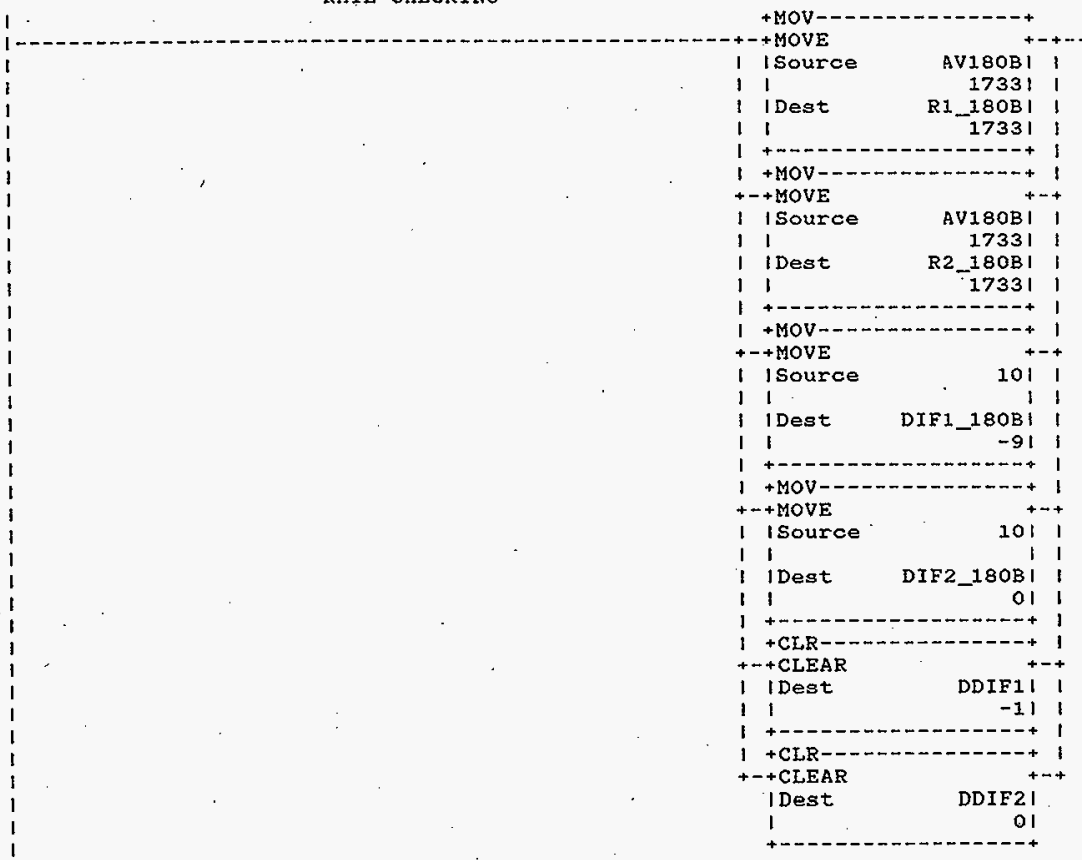

Rung $2: 33$

RATE CHECKING

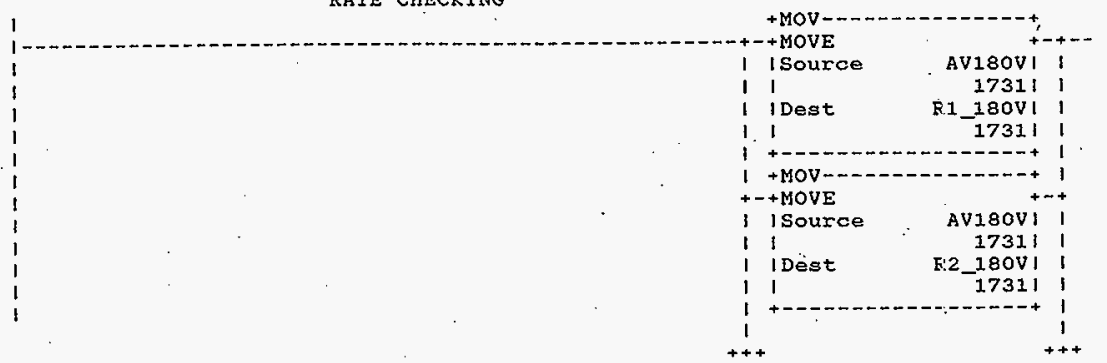

HNF-SD-FF-CSWD-61 Rev. 0

Page 385 


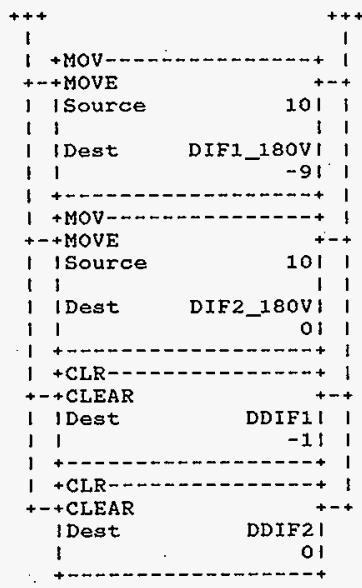

Rung 2:34

RATE CHECKING

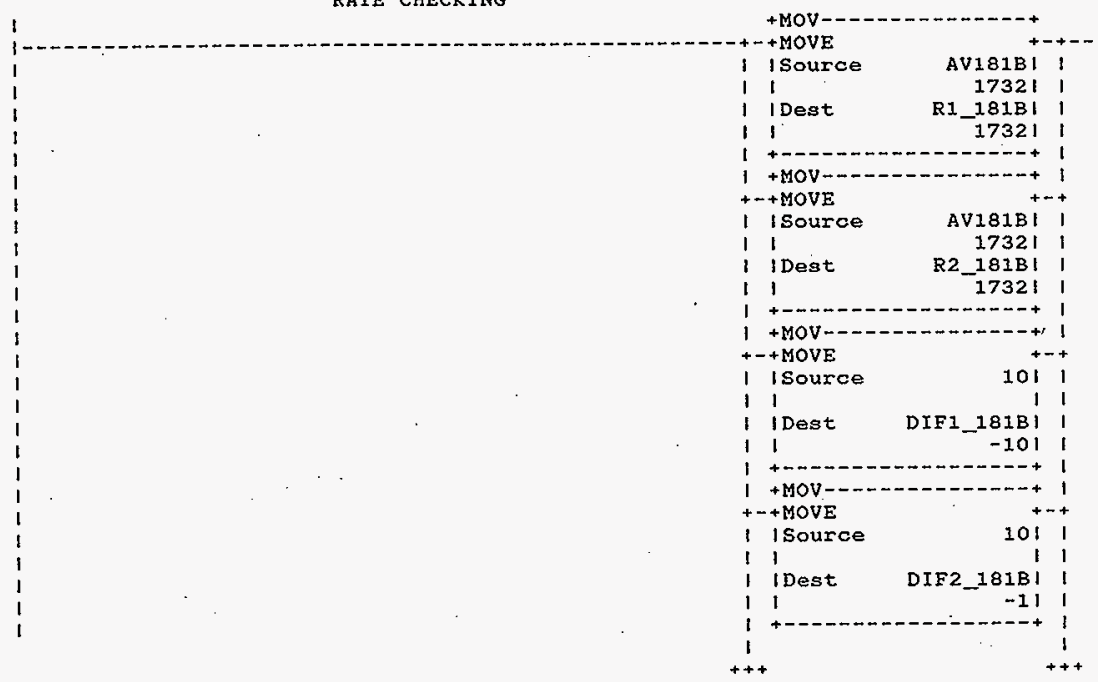

HNF-SD-FF-CSWD-61 Rev. 0 


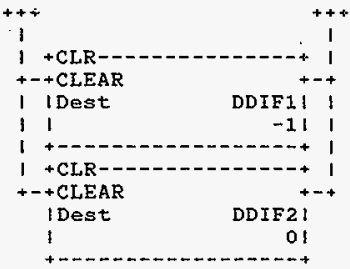

Rung 2:35

\section{RATE CHECKING}

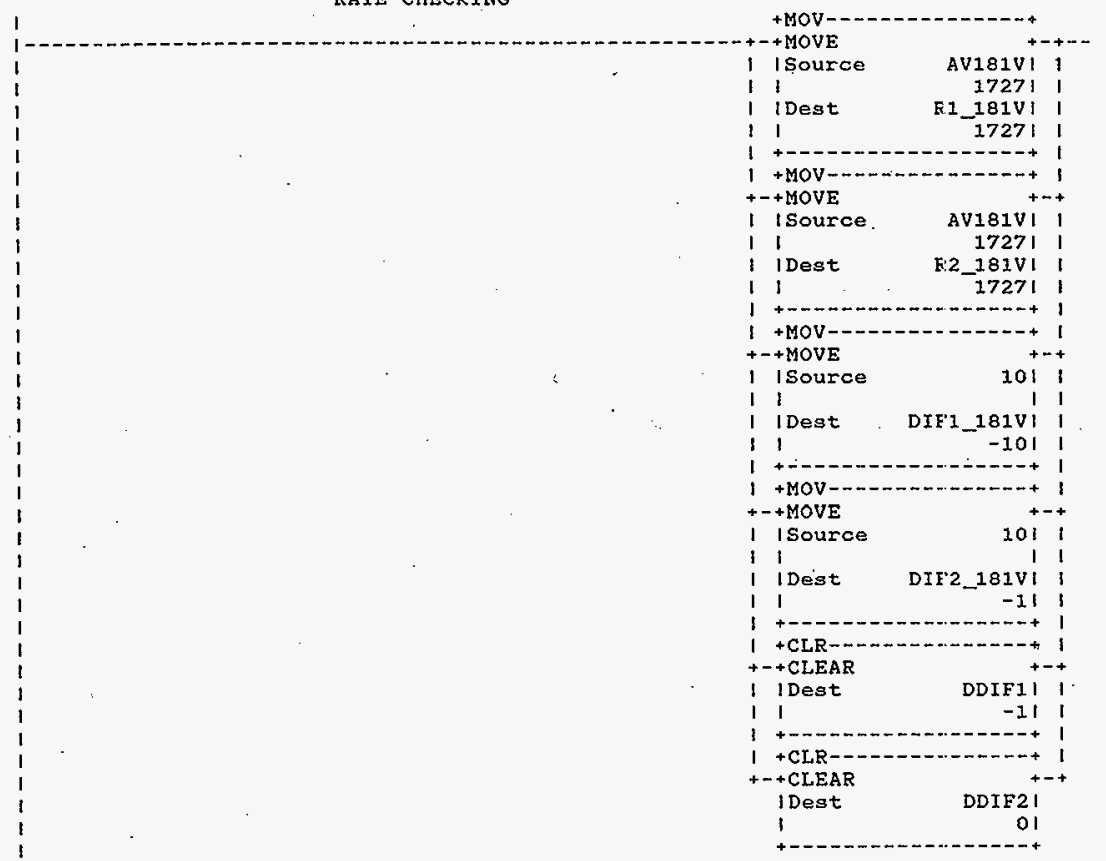

HNF-SD-FF-CSWD-61 Rev. 0 
Processor and Data(OPS Unit 1 )

Rung 2:36

RATE CHECKING

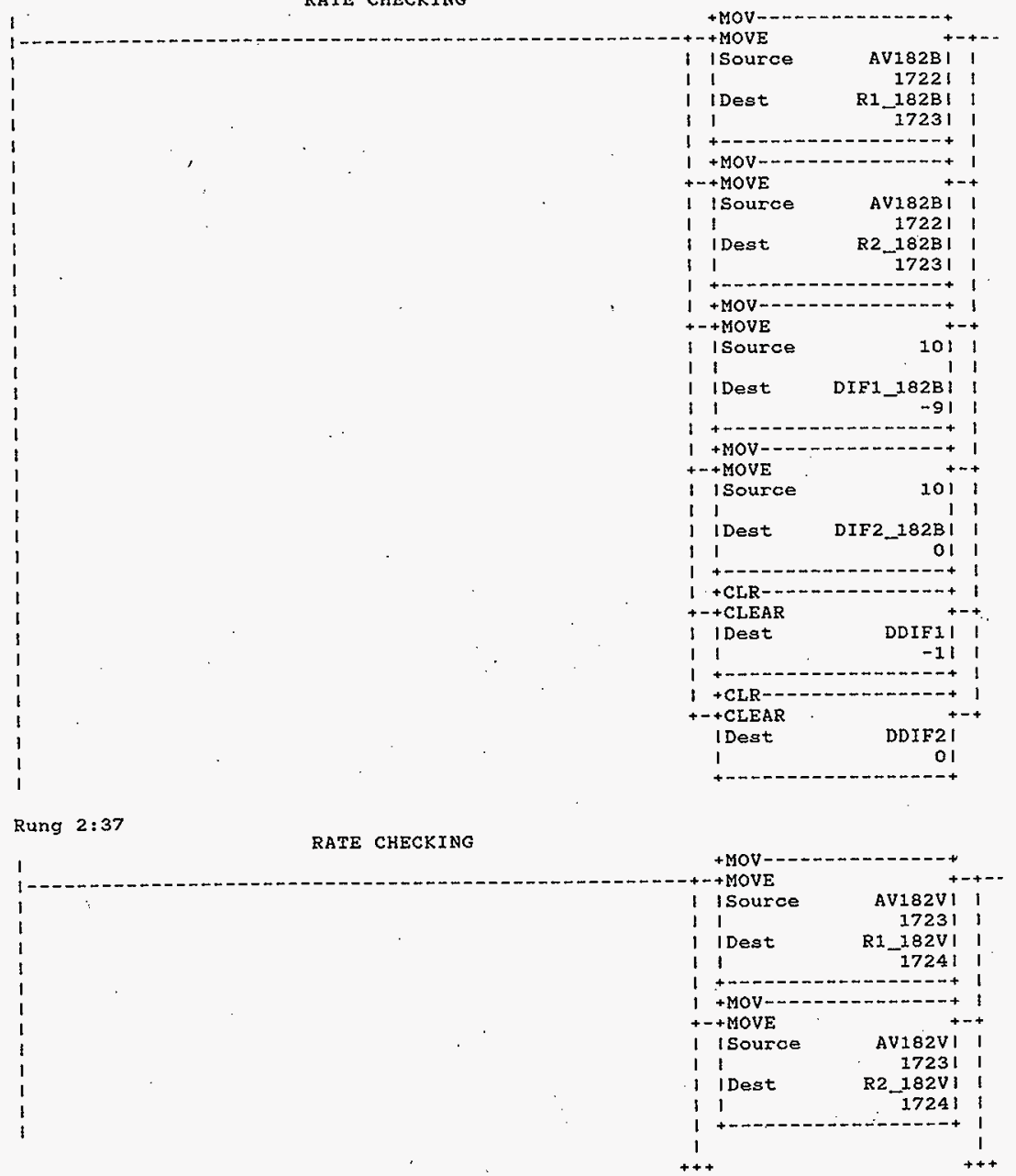

HNF-SD-FF-CSWD-61 Rev. 0

Page 388 


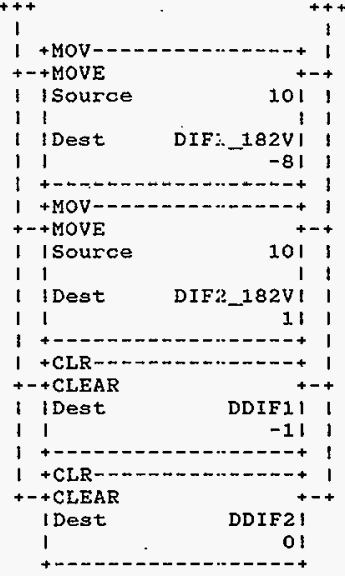

Rung $2: 38$

\section{RATE CHECKING}

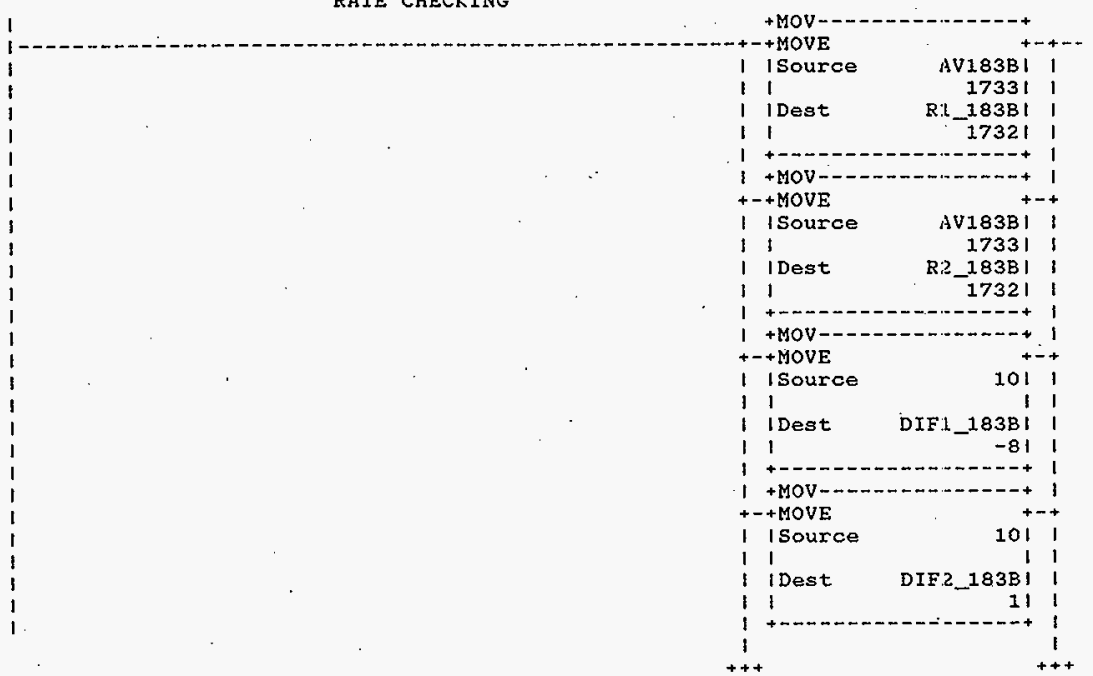

HNF-SD-FF-CSWD-61 Rev. 0

Page 389 
Processor and Data (OPS Unit 1) Program Listing
Octoher 23, 1996

Procesgor File: SODIUM2A.ACH
Page $2 \varepsilon$

Rung $2: 38$

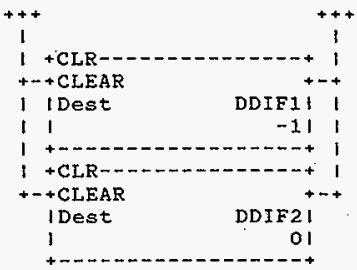

Rung 2:39

RATE CHECKING

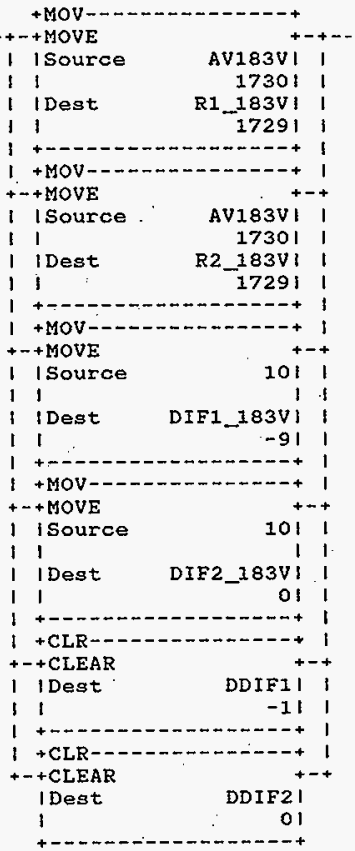

HNF-SD-FF-CSWD-61 Rev. 0

Page 390 
Rung 2:40

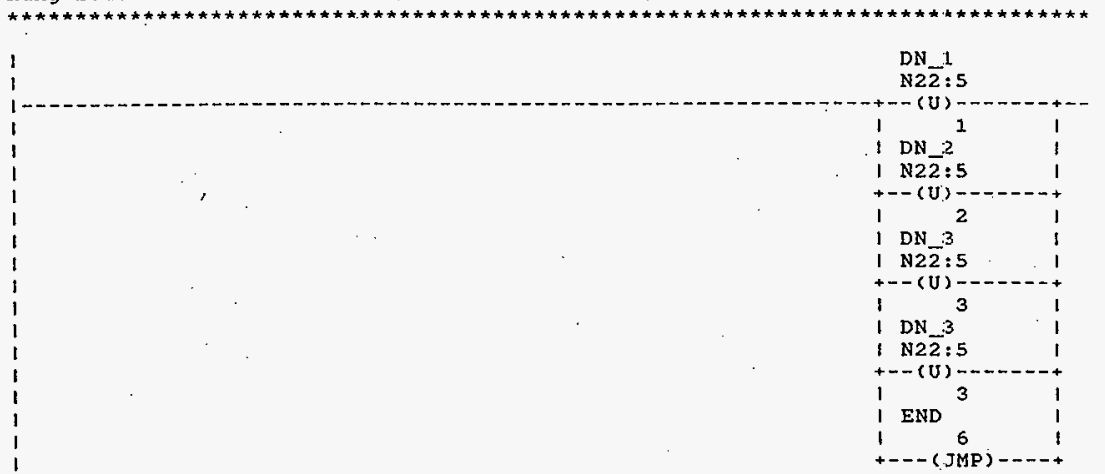

Rung 2:41

I SCHTMR

17

DUMMY1

$i-n-[$ LBL]

N22:7

Rung $2: 42$

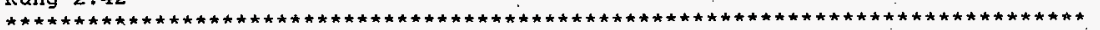

TIMERS FOR SCHEDULING SECTION OPERATION

TIMER FOR RATE FUNCTION

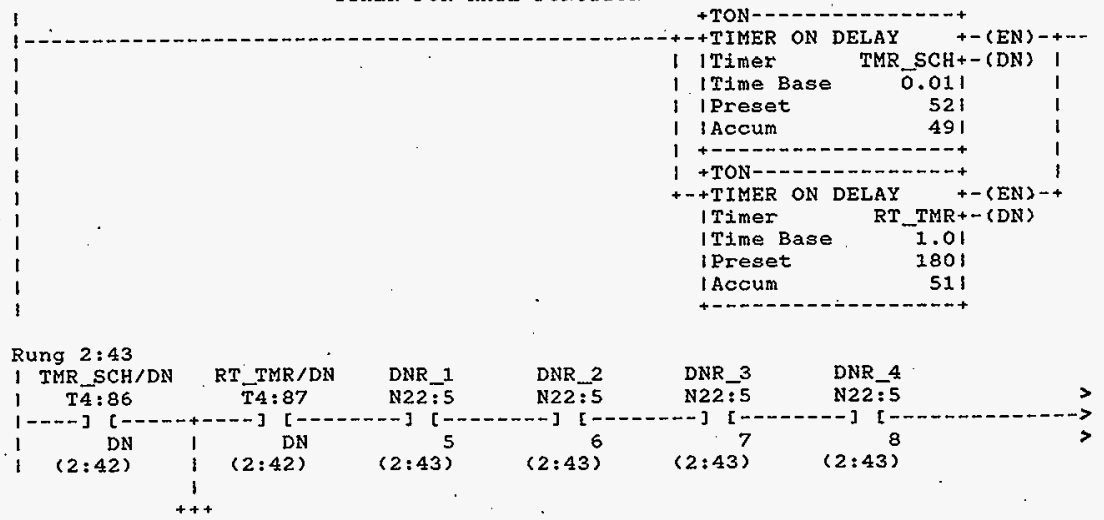

HNF-SD-FF-CSWD-61 Rev. 0

Page 391 
- Processor and Data(OPS Unit 1)

October 23, 2996

Program Listing

Processor File: SODIUM2A.ACH

Page 30 Rung $2: 43$

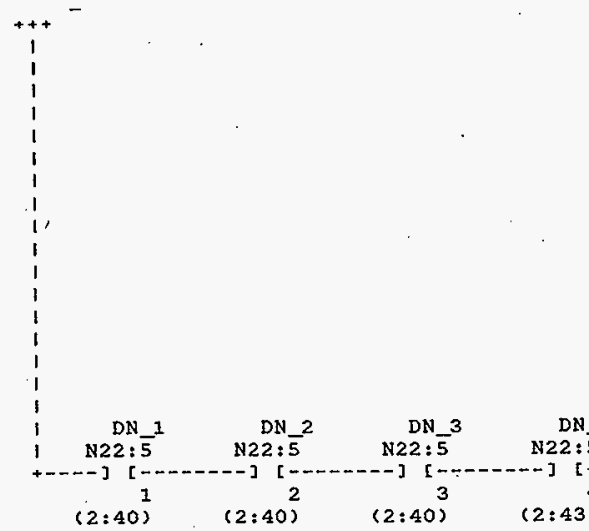

DN_4

$22: 5$

$(2: 43)$

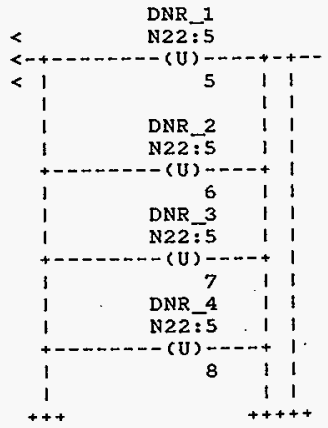

HNF-SD-FF-CSWD-61 Rev. 0 


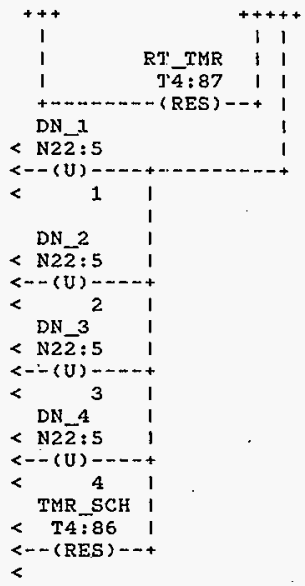

\section{Rung $2: 44$}

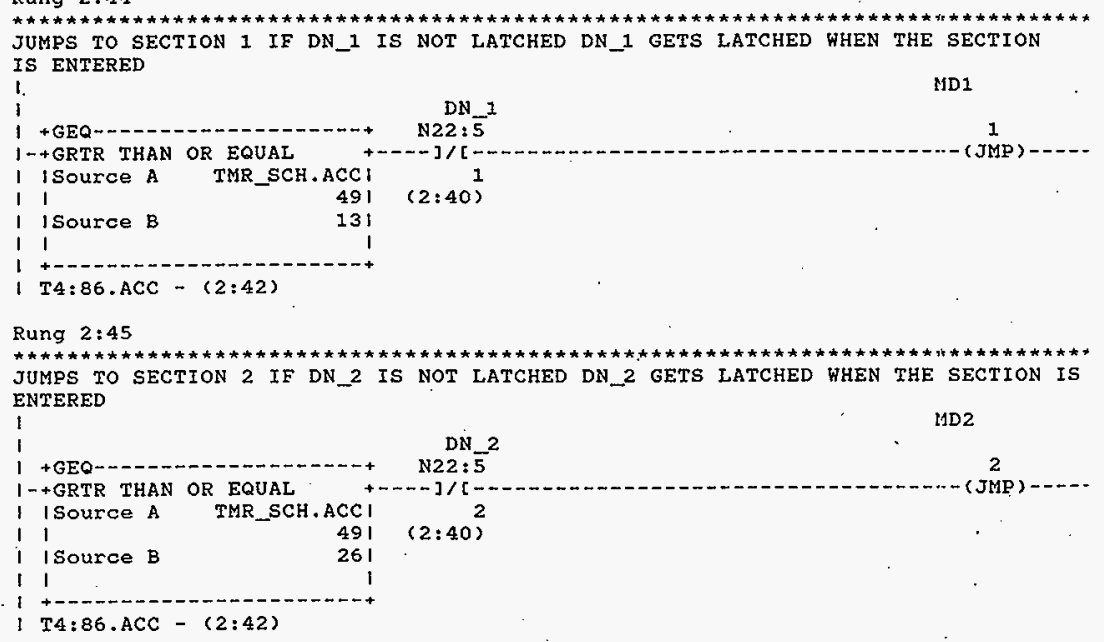

HNF-SD-FF-CSWD-61 Rev. 0 
Processor and Data(OPs Unit 1)

October 23, 1996 Page 32

Program listing

Processor File: SODIUM2A.ACH

Rung 2:46

Rung $2: 46$

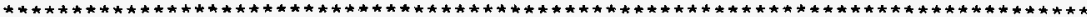
JUMPS TO SECTION 3 IF DN_3 IS NOT LATCHED DN_3 GETS LATCHED WHEN THE SECTION IS ENTERED

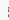

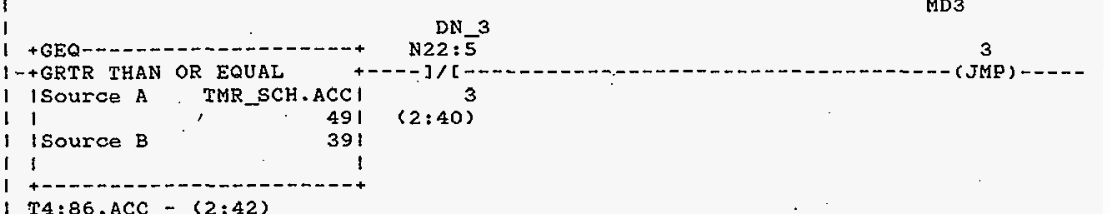

$T 4: 86 . A C C-(2: 42)$

Rung $2: 47$

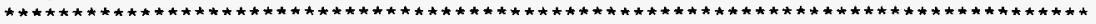
JUMPS TO SECTION 3 IF DN_3 IS NOT LATCHED DN_3 GETS LATCHED WHEN THE SECTION IS ENTERED

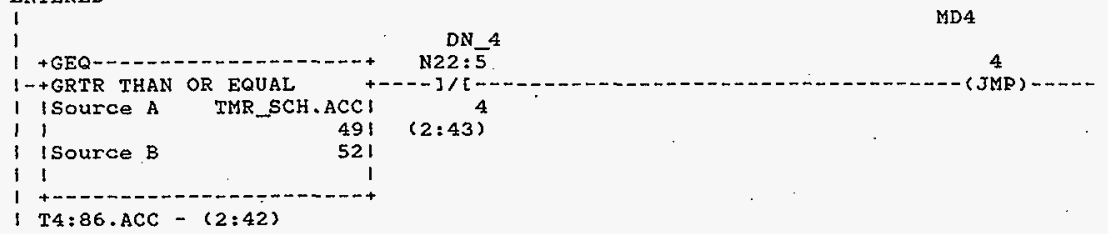

Rung $2: 48$

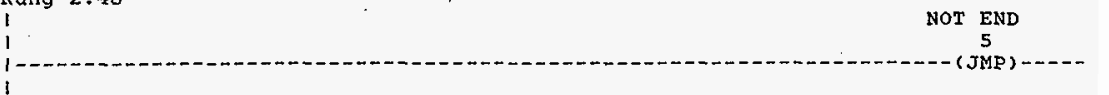

Rung $2: 49$

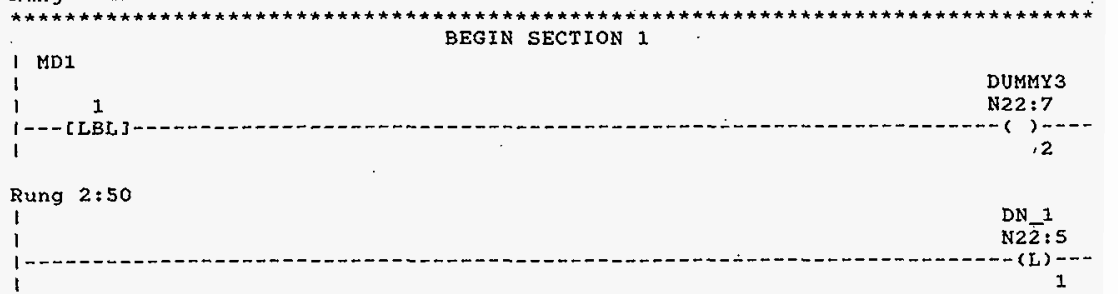

HNF-SD-FF-CSWD-61 Rev. 0

Page 394 


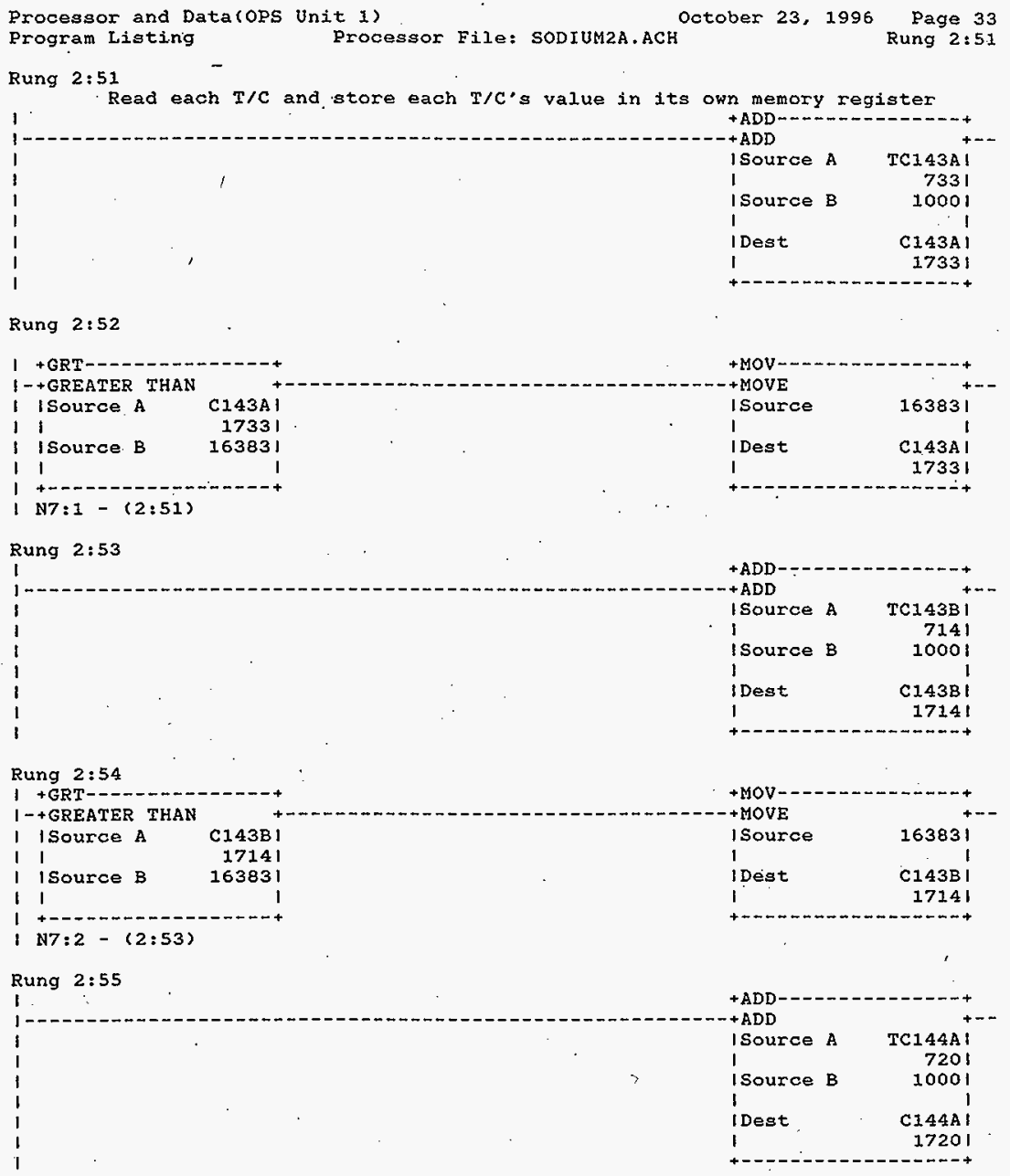

HNF-SD-FF-CSWD-61 Rev. 0

Page 395 
Processor and Data(OPS Unit 1)

October 23, 1996 Page 34

Program Listing

Processor File: SODIUM2A.ACH

Rung $2: 56$

Rung $2: 56$

1 +GRT-

$1-+$ GREATER THAN

I Isource A C144A

11 17201

I isource B 16383

11

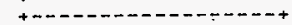

I N7:3-(2:55)

Rung 2:57

1

$$
\text { i }
$$$$
\text { I' }
$$$$
\text { I' }
$$$$
1
$$

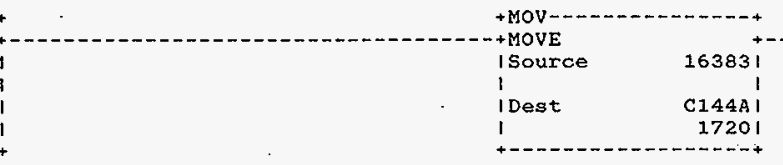

Rung 2:58

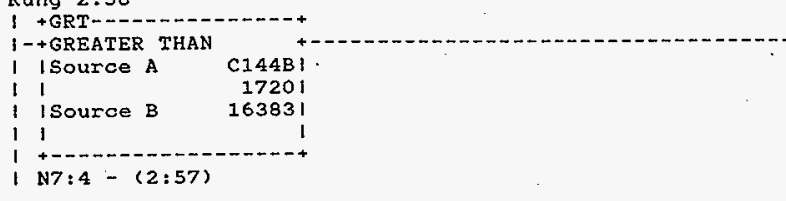

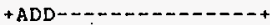

$+A D D$

isource A TCI44B

7201

isource B

10001

I Dest

C144B I

1

17201

Rung 2:59$$
\text { (1) }
$$

+MÓV--- - - -

+MOVE

I Source

$16383 !$

I Dest

C144B

I

17201
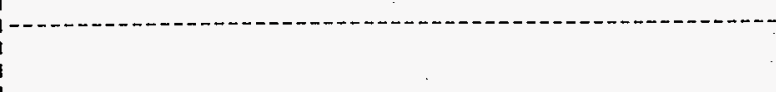

$+A D D$

ISource A TC150A

ISource B 1000 I

1

I Dest

C150AI

I

17311

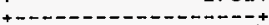

Rung $2: 60$

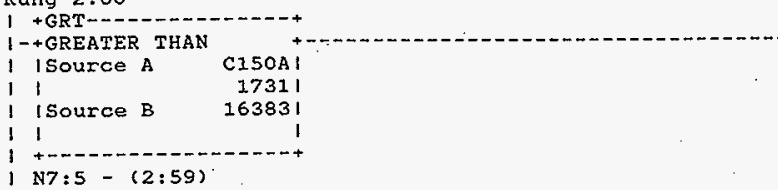

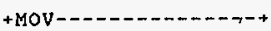

+ MOVE

163831

I

IDest

C150AI

I

17311

HNF-SD-FF-CSWD-61 Rev. 0

Page 396 


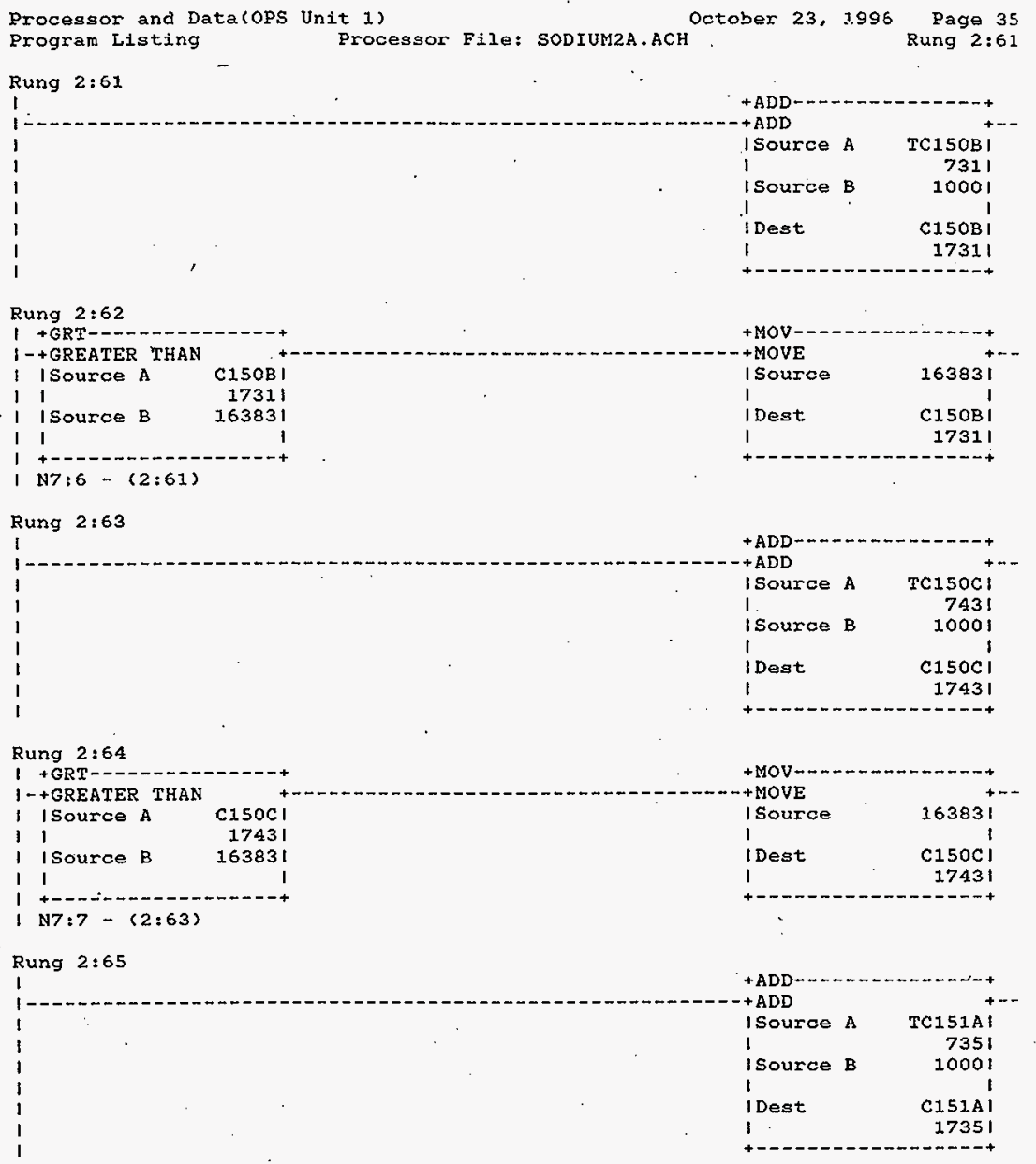

HNF-SD-FF-CSWD-61 Rev'. 0

Page 397 
Processor and Data(OPS Unit 1)

Octoher 23, 1996 Page 36

Program Listing

Processor File: SODIUM2A.ACH

Rung $2: 66$

Rung $2: 68$

1 +GRT---..--..---

1 -+GREATER THAN

- Isource a c151Al

i 1 17351

I 1Source B 16383 I

11

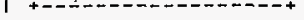

1 N7:8-(2:65)

Rung $2: 67$

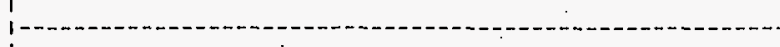

i.

1

,

I

Rung 2:68

1 + GRT-_..-

$1 \rightarrow$-GREATER THAN

I I Source A C151BI

I 1 17361

I Isource B 163831

11

I $N 7: 9-(2: 67)$

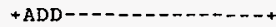

$+A D D$

|Source A TC151B|

$736 !$

I Source B

10001

i Dest

C. $151 \mathrm{~B}$

I

1736 !

\section{Rung 2:69}

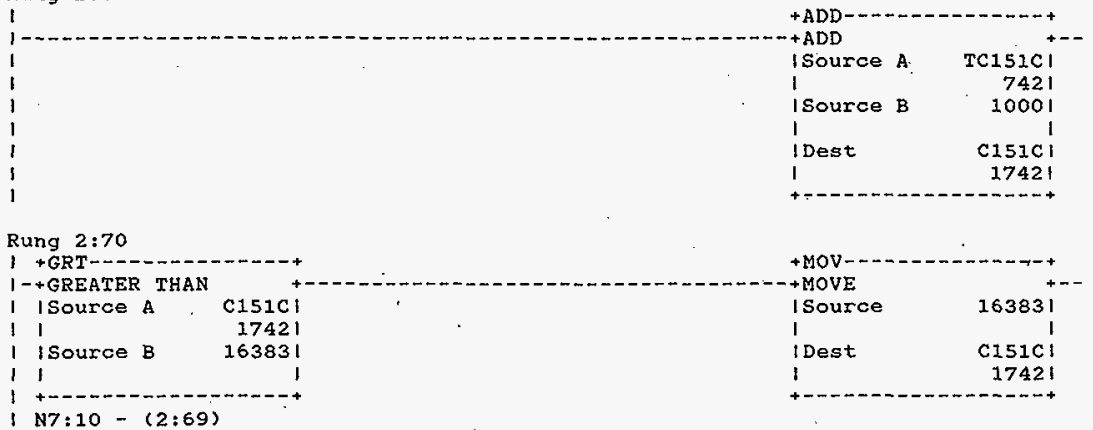

HNF-SD-FF-CSWD-61 Rev. 0

Page 398 
Processor and Data (OPS Unit 1)

october 23, 1996 Page 3\%

Program Listing

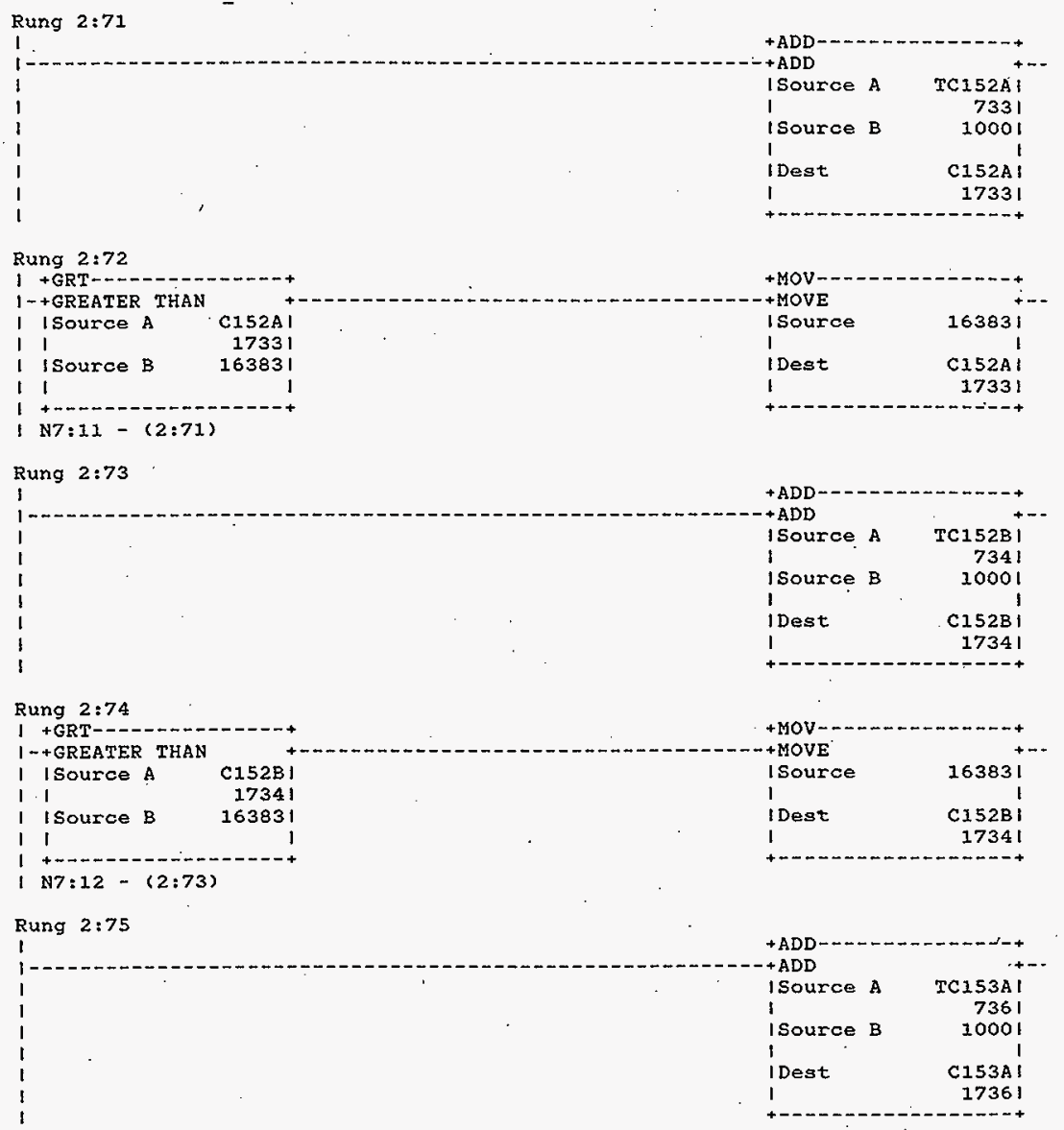

HNF-SD-FF-CSWD-61 Rev. 0 
Processor and Data(OPS Unit 1 )

Rung $2: 76$

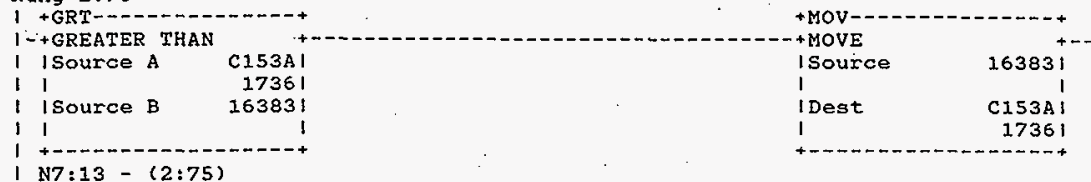

\section{Rung 2:77}$$
\text { 5) }
$$

!

I

I

!

$-$
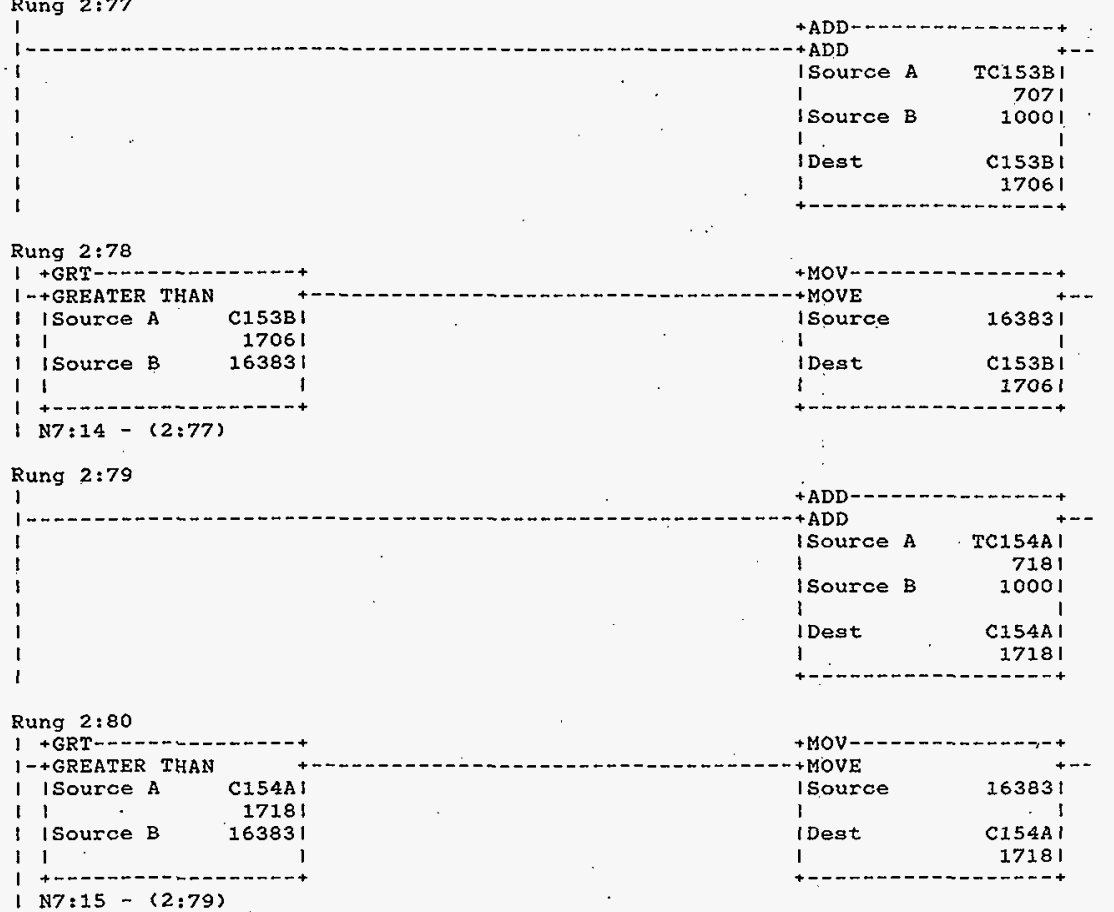

HNF-SD-FF-CSWD-61 Rev. 0

Page 400 


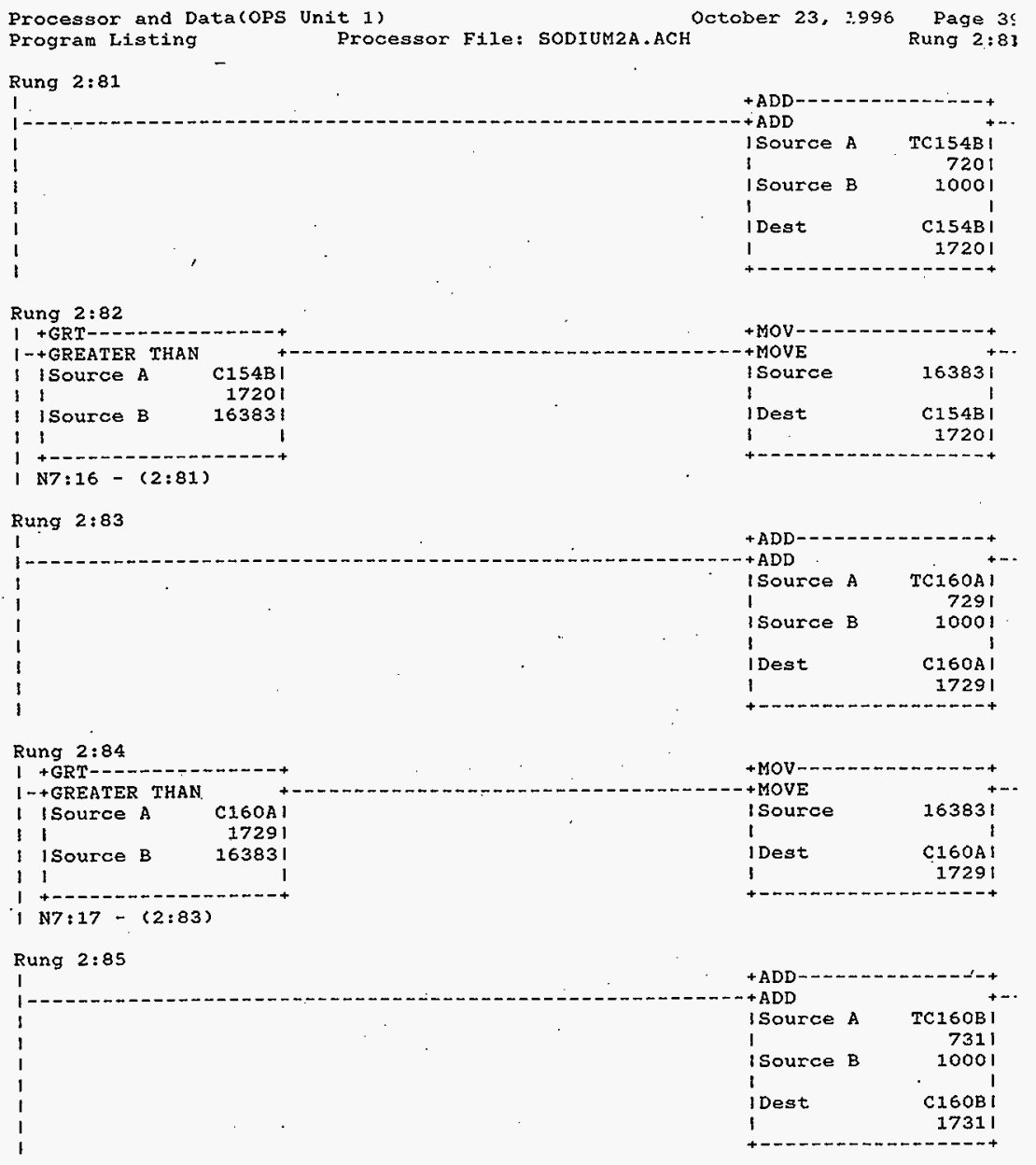

HNF-SD-FF-CSWD-61 Rev. 0 
Processor and Datacops Unit 1

October 23, 1996

Page $4 \mathrm{C}$

Program Listing

Processor File: SODIUM $2 \mathrm{~A} . \mathrm{ACH}$

Rung 2:86
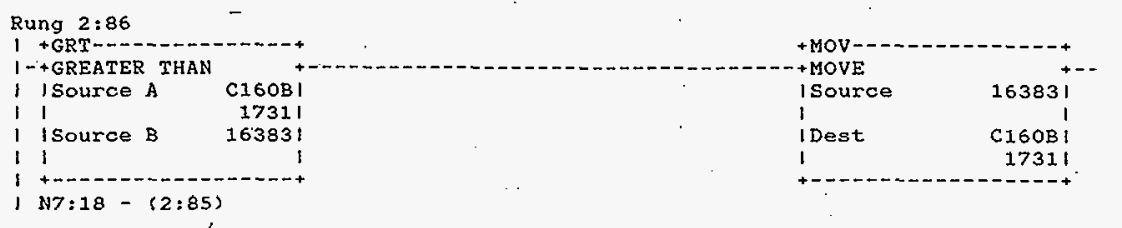

\section{Rung 2:87}

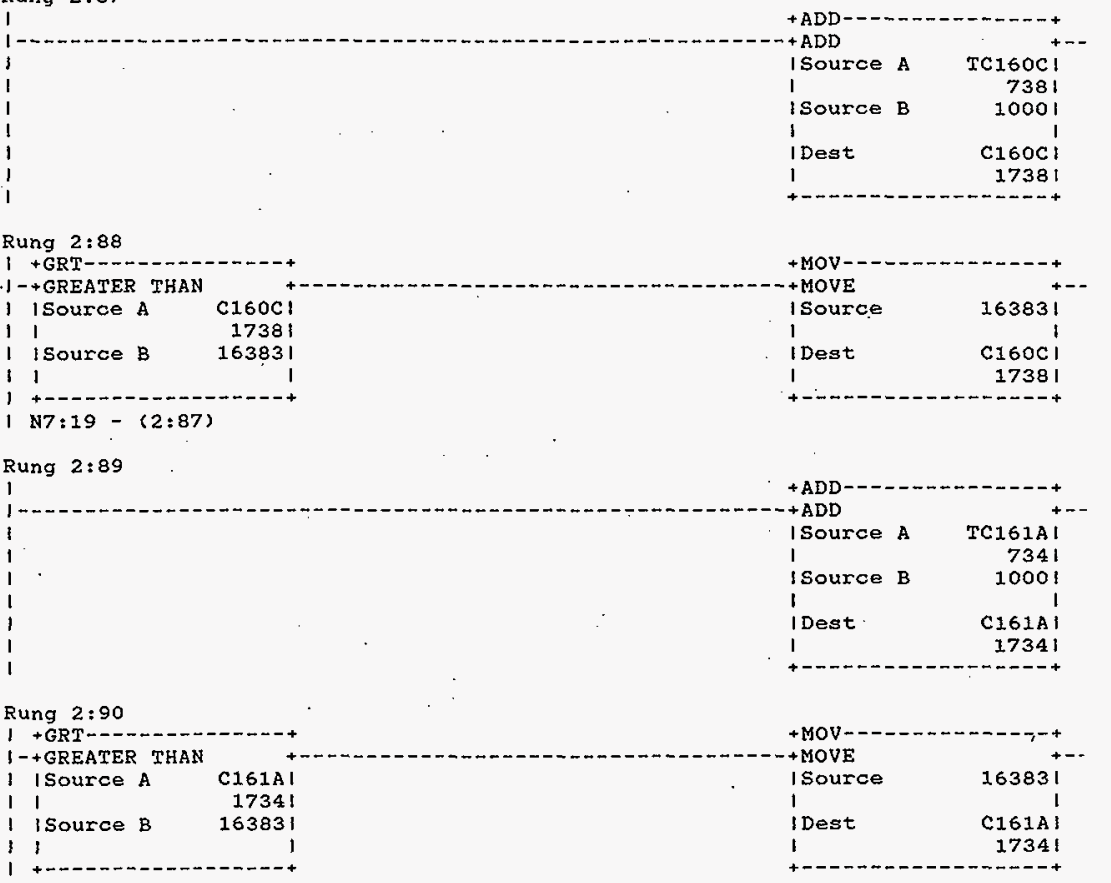

HNF-SD-FF-CSWD-61 Rev. 0 


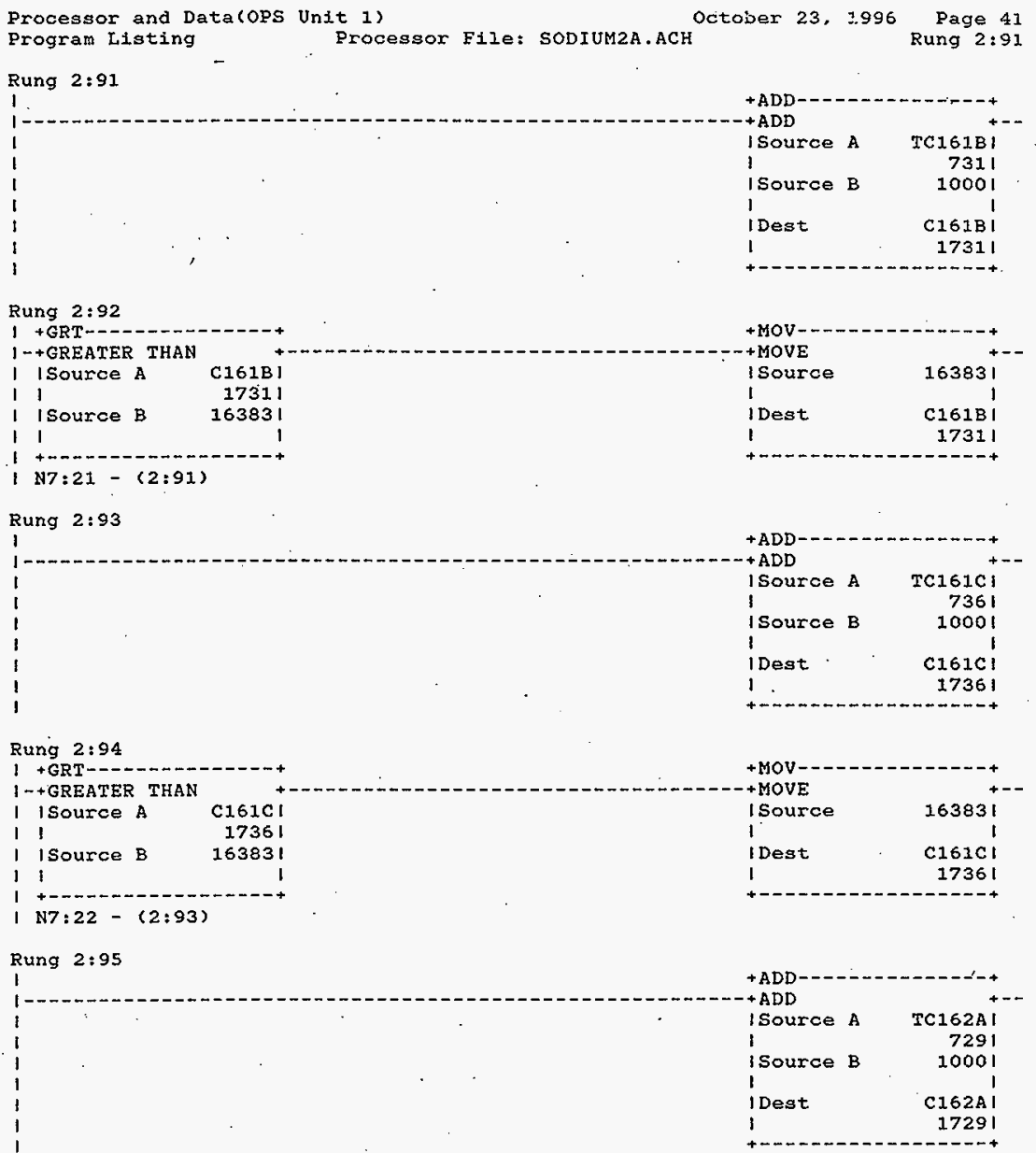

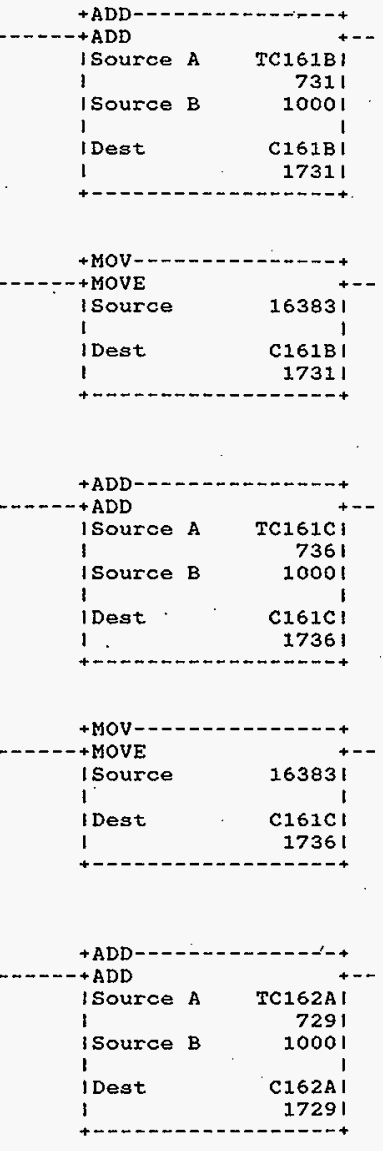

Rung 2:91

1. 
Processor and Datalops Unit 1

Program Listing

Processor File: SODIUN2A.ACH

October 23, 1996

Page 42

Rung $2: 96$

$1+$ GRT-.-...-......-

$1-+$ GREATER THAN

I ISource A C162A I

1117291

I Isource B 163831

1 1 1

1 N7:23-(2:95)

Rung 2:97

.


Processor and Data(OPS Unit 1) Program Listing

\section{Rung 2:101}

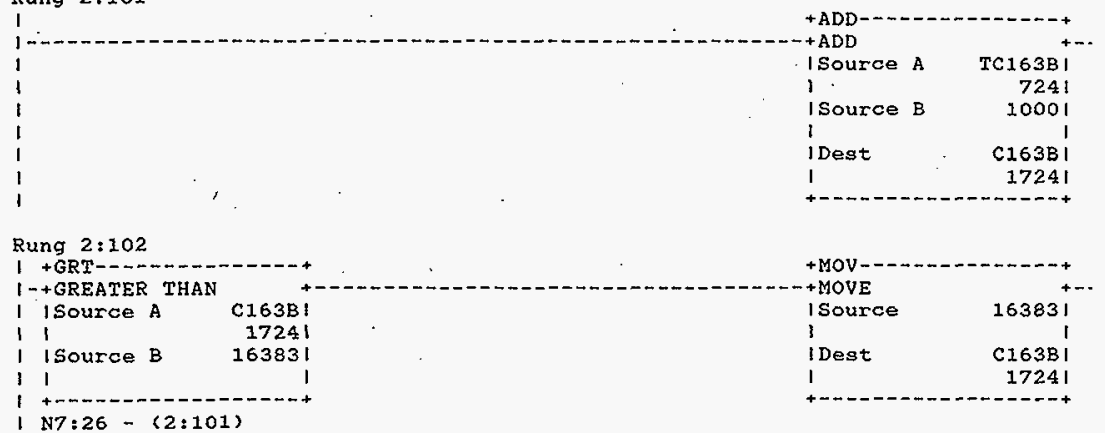

\section{Rung $2: 103$}

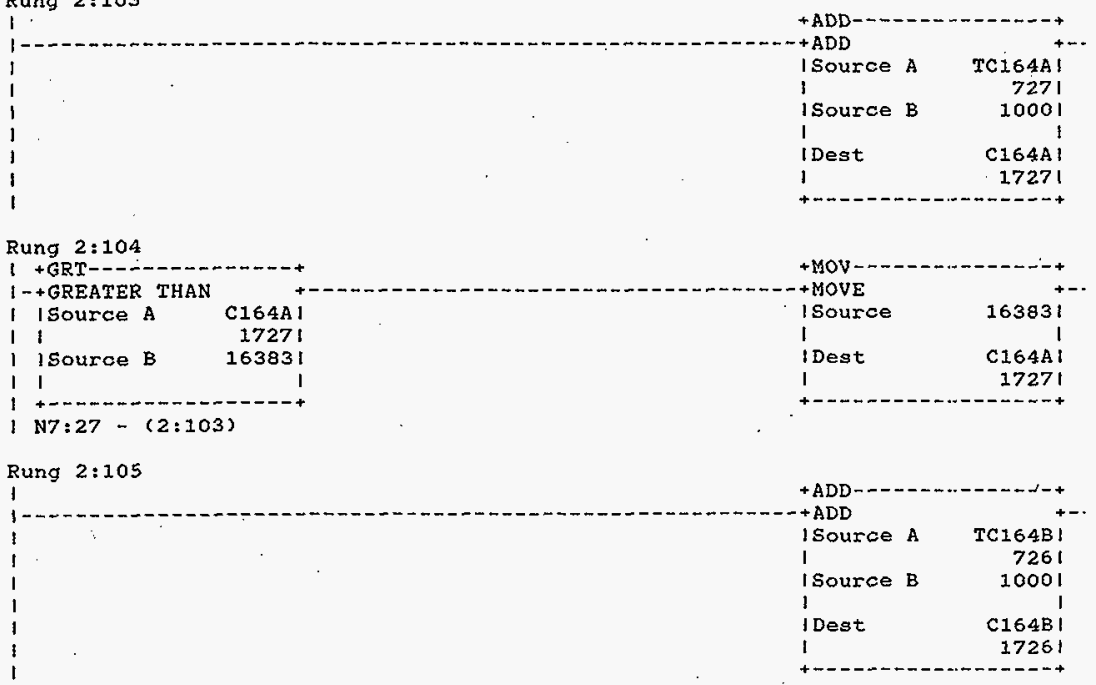

HNF-SD-FF-CSWD-61 Rev. 0 
Processor and Data(OPS Unit 1$)$ Program Listing October 23, 1996 Page 44
Rung $2: 106$

Rung 2:106

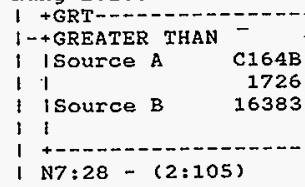

Processor File: SODIUM2A, ACH

Rung $2: 106$

kuing $2: 107$

For each T/C read its STATUS BITS to detect $T / C$ problems $T / C$ ENABLE

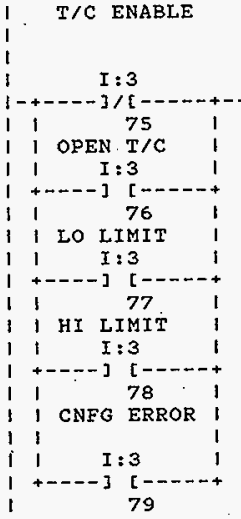

HA143A

N7: 224

Rung 2:108

I

1

I T/C ENABLE

I:3

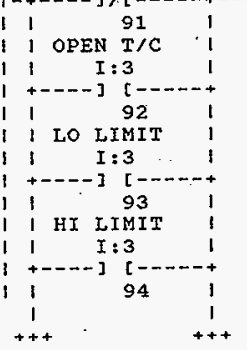

HNF-SD-FF-CSWD-61 Rev. 0 
Processor and Data(ops Unit 1)
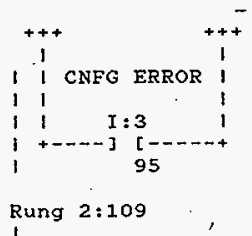

Rung $2: 110$

1

$T / C$ ENABLE

$I: 3$

HNF-SD-FF-CSWD-61 Rev, 0 
Processor and Data(ODS Unit 1 )
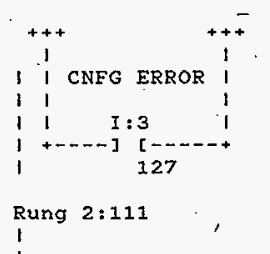

\section{I}

T/C ENABLE

$\mathrm{I}: 4$

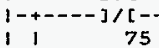

I OPEN T/C

1 I $I: 4$

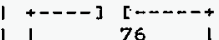

I LO IINIT

I I:4

$1+---1][-\ldots+\cdots+$

$11 \quad 77$

। 1 HI LIMIT

I $I: 4$

$1+----7$ [

1178

I CNFG ERROR

1 I I:4

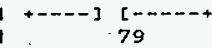

\section{Rung 2:112}

I

T/C ENABLE

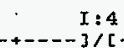

HA $150 B$

1 OPEN $\mathrm{T} / \mathrm{C}$

1 I: 4

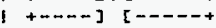

192

1 LO LIMIT

$1 \quad I: 4$

$1+\ldots--][-\ldots-\ldots+$

1193

1 HI LIMIT

11 I:4

$1+----][$

1194

I I CNFG ERROR

$11 \quad I: 4$

$1+\ldots-\cdots]$

1 95

HNF-SD-FF-CSWD-61 Rev. 0 
Processor and Data(ops Unit 1)

\section{Rung 2:113}

$$
\begin{aligned}
& 1 \\
& 1 \\
& 1 \\
& 1 \\
& 1 \\
& 1 \\
& 1
\end{aligned}
$$

HNF-SD-FF-CSWD-61 Rev. 0 
Processor and Data(OPS Unit 1)

Rung 2:115

1
1
1
1
1

$T / C$ ENABLE

$I: 5$

HA151B

$3 /[$

1 OPEN 75

1 I I:5

$1+-n-7[-\cdots+-+$

1176

I I LO LIMIT

1 I I:5

$1+\ldots-\ldots][\ldots-\ldots+$

$11 \quad 77 \quad 1$

1 HI LIMIT

1 I I:5

$1+----][---n+$

1178 ।

I I CNFG ERROR I

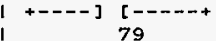

Rung 2:116

I

T/C ENABLE

$I: 5$

OPEN $T 1$. $I: 5$

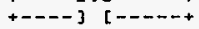

192

1 LO LIMIT

1 I:5

$+----][---\cdots+$

193

I HI LIMIT

I I:5

$1+---3[-\ldots-n+$

I I 94 ।

I I CNFG ERROR I

I 1 I:S

I +---- ] [----

I 95

HNF-SD-FF-CSWD-61 Rev. 0 
Processor and Data(OPS Unit 1)

Program Listing

October 23, :996 Page 49 Processor File: SODIUM2A.ACH Rung $2: 117$

Rung 2:117

1.

1.

I T/C ENABLE

HA152A
HA

I.I:5 . N7:224

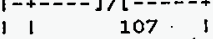

1 OPEN T/C '

$1 \quad I: 5$

+--- ]

$1 \quad 108$

1 LO IINIT

$1 \quad I: 5$

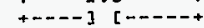

i 109

HI LIMIT

$x: 5$

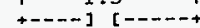

1110

I CNFG ERROR ।

I I 5

$+-\cdots-][-\ldots . . .+$

111

\section{Rung 2:118}

I

$T / C$ ENABLE

$I: 5$

HA $152 B$

1 OPEN $T / C$

N7 : 224

1 I:5

$1+\ldots .+7][\ldots . .7$

1124

I I LO LIMIT

$11 \quad I: 5$

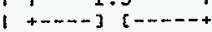

1125

| HI LIMIT

1 I 5

$1+\ldots-7[\ldots+\cdots$

126

I CNFG ERROR

1 I:5

1..-- ] [-.--.+

$1 \quad 127$

HNF-SD-FF-CSWD-61 Rev. 0 
Processor and Datacops Unit 1)

October 23, 1996 Page 5C

Program Listing

Processor File: SODIUM $2 A \cdot A C H$

Rung $2: 119$

Rung 2:119

Rung $2: 119$

T/C ENABLE

HA153A

N7: 224

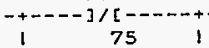

I OPEN T/C

I I:6

$10-76$

I LO LIMIT

1 I:6

+...- [-...

177

I HI LIMIT

I I: 6

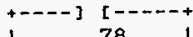

I CNFG ERROR

$1 \quad I: 6$

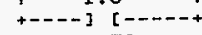

I

79

Rung 2:120

1 T/C ENABLE:

1 HA153B

$1 \quad \mathrm{I}: 6 \quad$ N7:224

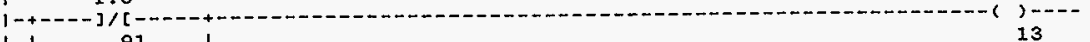

1.191

1 OPEN $\mathrm{T} / \mathrm{C}$

$1 \quad I: 6$

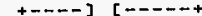

192

1 LO LIMIT

$1 \quad I: 6$

---.- ] [----.+

193

I HI LIMIT

I I:S

+-.-- ] [--....

194

I CNFG ERROR

I I I: 6

$1+---1 \quad[-$

Rung $2: 121$

1 T/C ENABLE

$1 \quad I: 6$

HAI54A

N7: 224

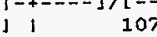

107

1 OPEN T/C

$1+\ldots--3: 6$

111081

$-1)-\cdots$

HNF-SD-FF-CSWD-61 Rev. 0 
Processor and Data(OPS Unit 1)

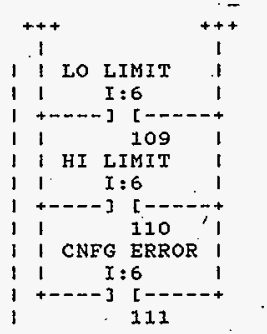

Rung 2:122

1 T/C ENABLE

1 HA154B

$1 \quad I: 6 \quad$ N7:224

$\begin{array}{lllll}1-+ & 1 \\ 1 & 1 & 1\end{array}$

1 I OPEN T/C :

$1 \quad 1: 6 \quad 1$

l. $1 \quad 124$

1 LO LIMIT

1 I:6

+----] [----n+

$1 \quad 125$

I HI LIMIT

$1 \quad I: 6$

+--.-] [-----

1126

I CNFG ERROR 1

$\begin{array}{lll}1 & I: 6\end{array}$

I 127

Rung 2:123

1 T/C ENABLE

$1 \quad I: 7$

HA160A

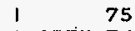

1 OPEN $\mathrm{T} / \mathrm{C}$

1 I:7

+----] [ ] - - - +

176

I LO LIMIT

1 I:7

+--- $]$ [-----+

I

HI LIMIT

I I:7

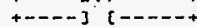

1178

I CNFG ERROR I

I $I: 7$

$1+\ldots-\infty][-2$

i 79

HNF-SD-FF-CSWD-61 Rev. 0

Page 413 
Rung 2:124

I. T/C ENABLE

$1 \quad I: 7$

$1: 7$

HA160B

$1-i+1$ ored

$\mathrm{N7}: 225$

OPEN T/C

$I: ?$

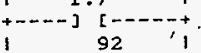

1 LO LIMIT

$1 \quad I: 7$

+----] $[--m+$

193

I HI LIMIT

1 I:7

$+---7[----m+$

$194 \quad$ ।

CNFG ERROR

I I:7

+---- ] [-...-

95

Rung 2:125

$T / C$ ENABLE

$\mathrm{I}: 7$

HA $160 \mathrm{C}$

$x: 7$
$-3 / 5$

107

OPEN T/C

$I: 7$

$+--\infty][-.--+$

- 1108

1 LO LIMIT

1

$1: 7$

+--n] [-.--

1109

HI LIMIT

I: 7

$+----3[--.-7$

1110

I CNFG ERROR

$1 \quad I: 7$

$+\cdots--3[\ldots+$

111

Rung $2: 126$

1 T/C ENABLE

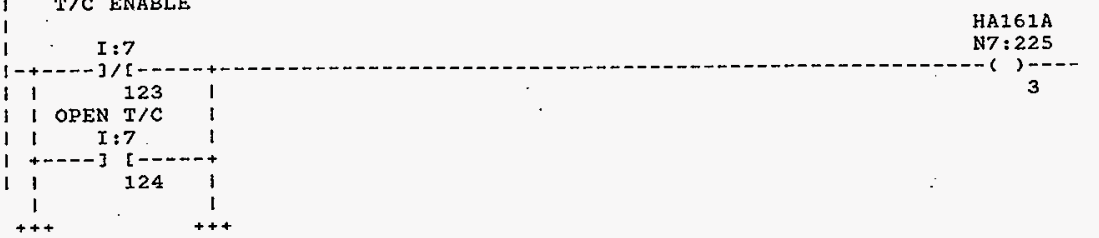

HNF-SD-FF-CSWD-61 Rev. 0 
Processor and Data(ops Unit 1 )

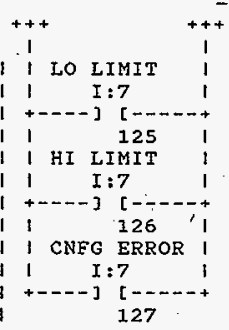

Rung 2:127

I T/C ENABLE

$1: 8$

HA161B

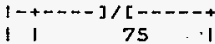

1 I OPEN T/C $I: 8$

$1+\ldots-\cdots[\ldots . . .+4$

$11 \quad 76$

I 1 LO LIMIT

I I I:8

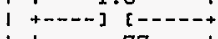

1177

I HI LIMIT

1 I $\quad I: 8$

$1+\cdots-m][--\cdots+$

1 CNFG 78 :

I $I: 8$

1.-- ] $[-$

79

Rung $2: 128$

1 T/C ENABLE

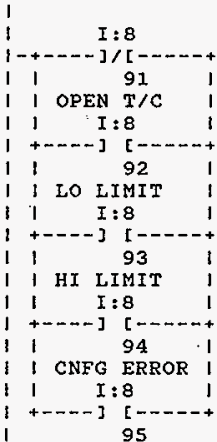

HNF-SD-FF-CSWD-61 Rev. 0

Page 415

HA161C N7 $: 225$
N7: 225

4 
Processor and Data(OPS Unit 1 ) Program Listing Processor File: SODIUM2A.ACH

October 23, 2996 Page 54

Rung 2:129

1. T/C ENABLE

$$
1 \quad I: 8
$$

$1-+---7 / 2-$

$i$ OPEN T/C

$1 \quad I: 8$

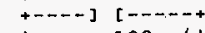

108,1

1 LO LIMIT

$1 \quad I: 8$

$1+\ldots-7[-\ldots-2+$

I 1

I HI LIMIT

I $I: 8$

$1+\cdots+\cdots]$

11

11

I I I: 8

1 111

Rung $2: 130$

i

T/C ENABLE

$\mathrm{I}: 8$

HA162B

$1-+---7 / 6-$

OPEN $T / C$

$I: 8$

+--n] [-...-

$1 \quad 124$

1 LO LIMIT

$11 \quad I: 8$

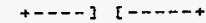

1.125

I HI LIMIT

I I:8

$1+---4]$ [ $-\cdots \cdots+$

11

1 CNFG ERROR

$11 \quad \mathrm{I}: 8$

I +----$]$ ] [-- $-\ldots$

I 127

Rung 2:131

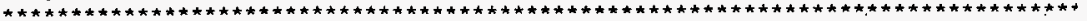
SET DEVIATION SWITCH FOR SECTION I AND JUMP TO SECTION 1 AVERAGING ROUTINE

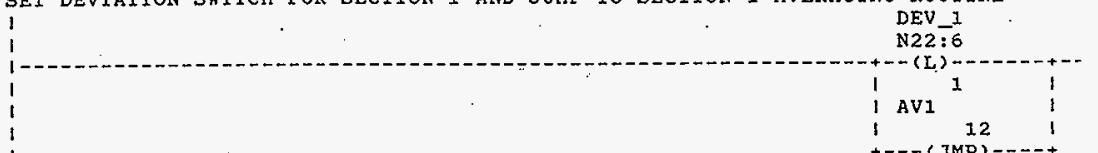

HNF-SD-FF-CSWD-61 Rev. 0

Page $4 / 6$ 
Processor and Data(OPS Unit 1) Program Listing

Rung 2:132

I DEVI

1

1 $-$

$1---[$ LBL

8

DUMMY 5

$\mathrm{N} 22: 7$

1

Rung 2:133

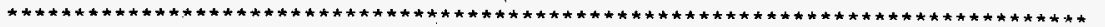
ESTABLISH HIGH AND LOW LIMITS FOR RANGE CHECKING IF NO HARDWARE ALARMS EXIST CHECK T/C VAIUES FOR OUT-OF-RANGE CONDITION

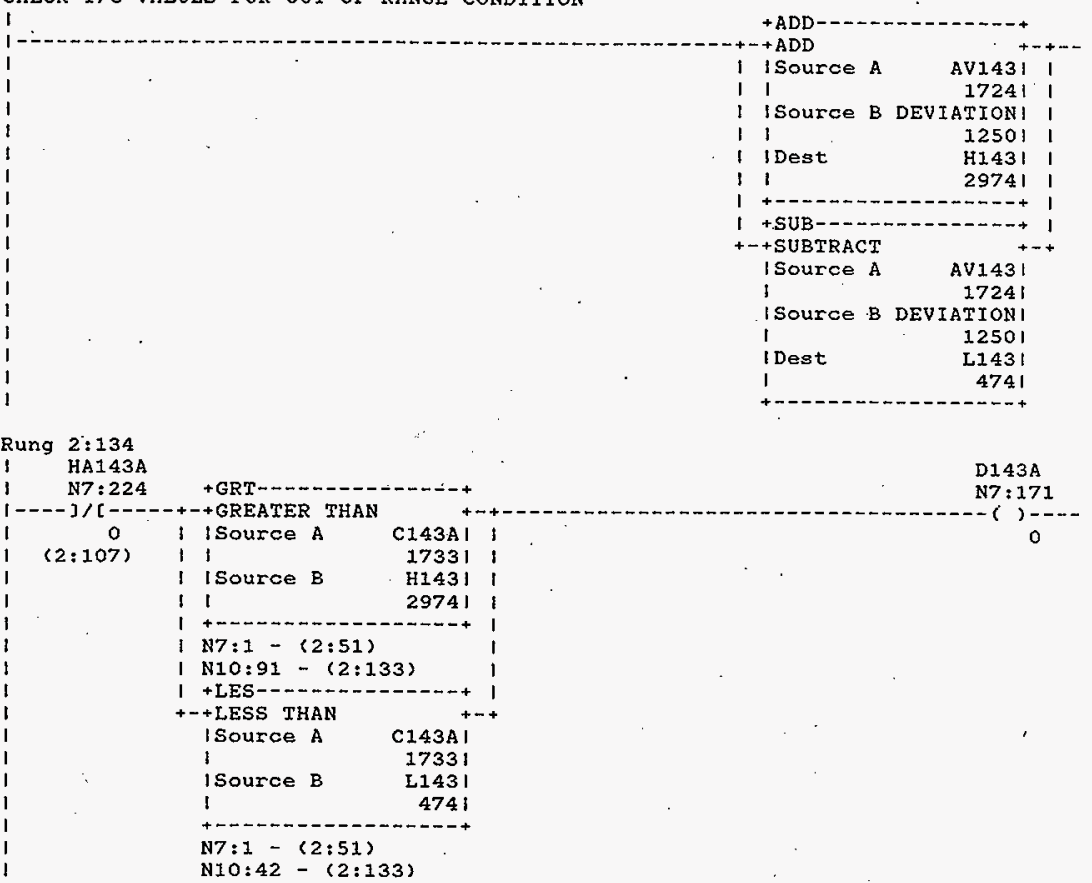

HNF-SD-FF-CSWD-61 Rev, 0

page 417 
Processor and Data(OPS Unit 1)

Rung 2:135

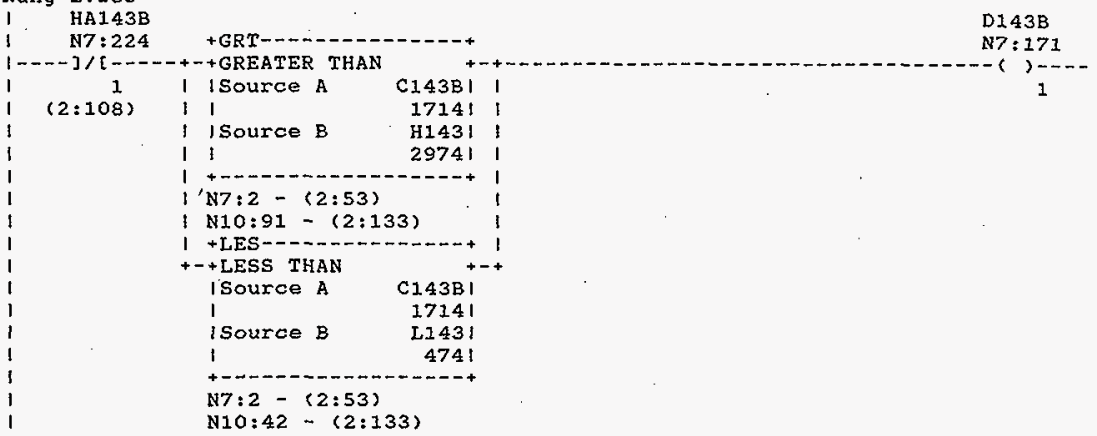

Rung 2:136

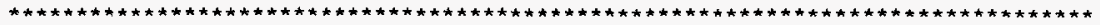
ESTABLISH HIGH AND LOW LIMITS FOR RANGE CHECKING IF NO HARDWARE ALARMS EXIST CHECK T/C VALUES FOR OUT-OF-RANGE CONDITION

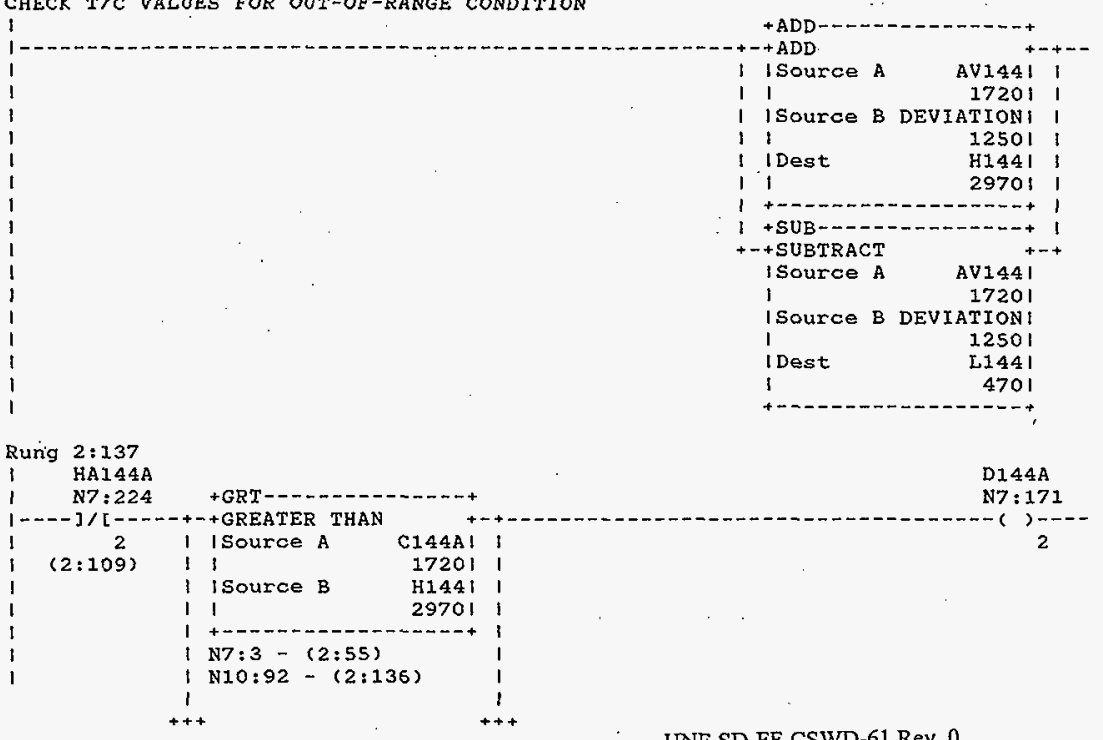

HNF-SD-FF-CSWD-61 Rev. 0

Page 418 
Processor and Data(ops Unit 1 )

October 23, :996 Page 57
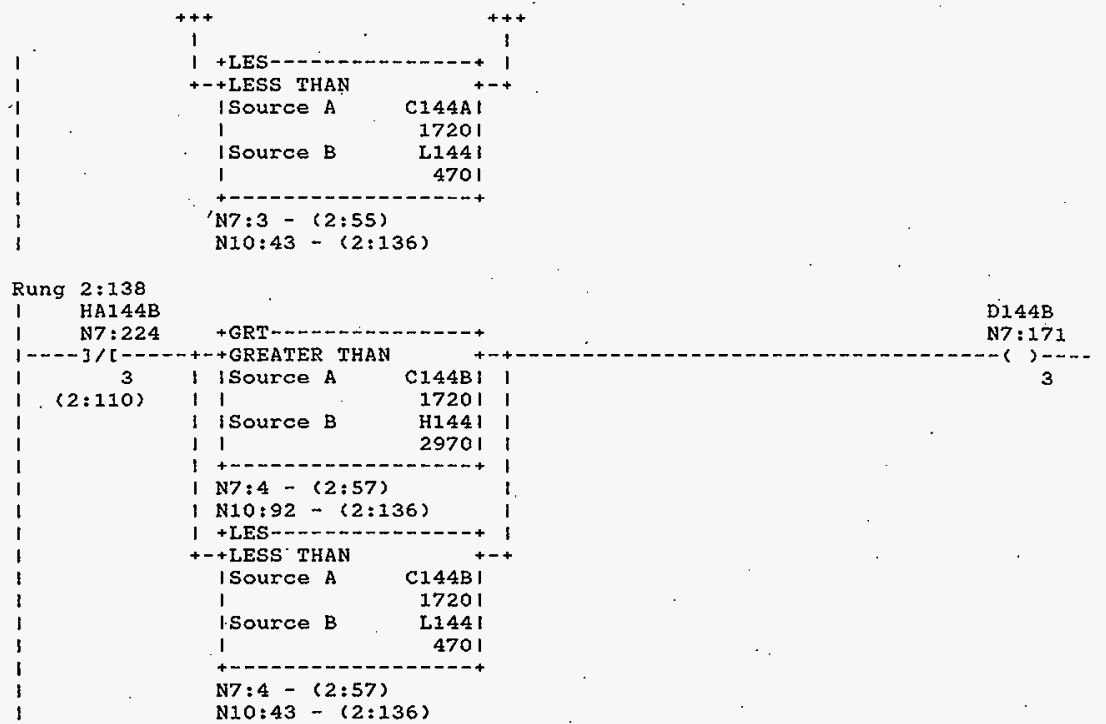

Rung $2: 139$

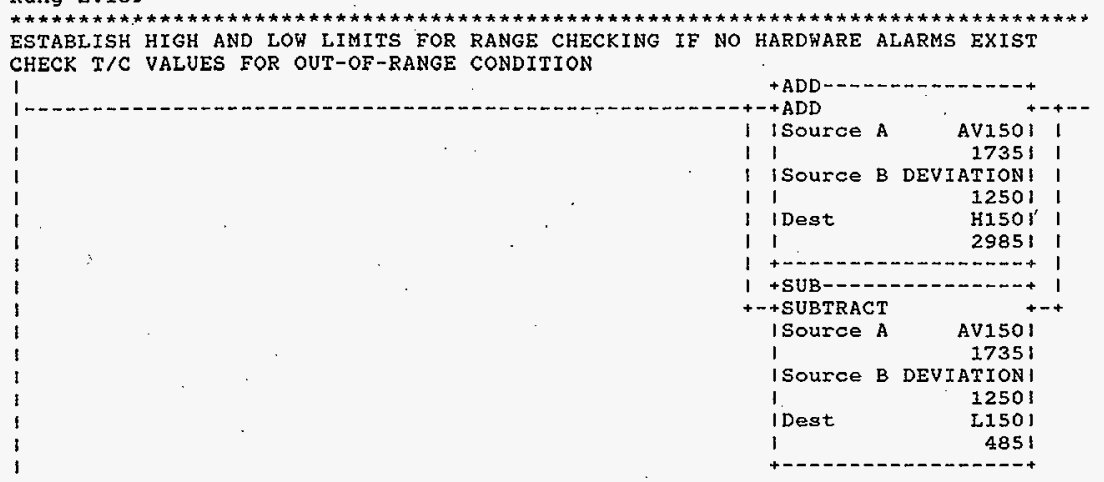

HNF-SD-FF-CSWD-61 Rev, 0 
Processor and Data(OPS Unit 1) Program Listing

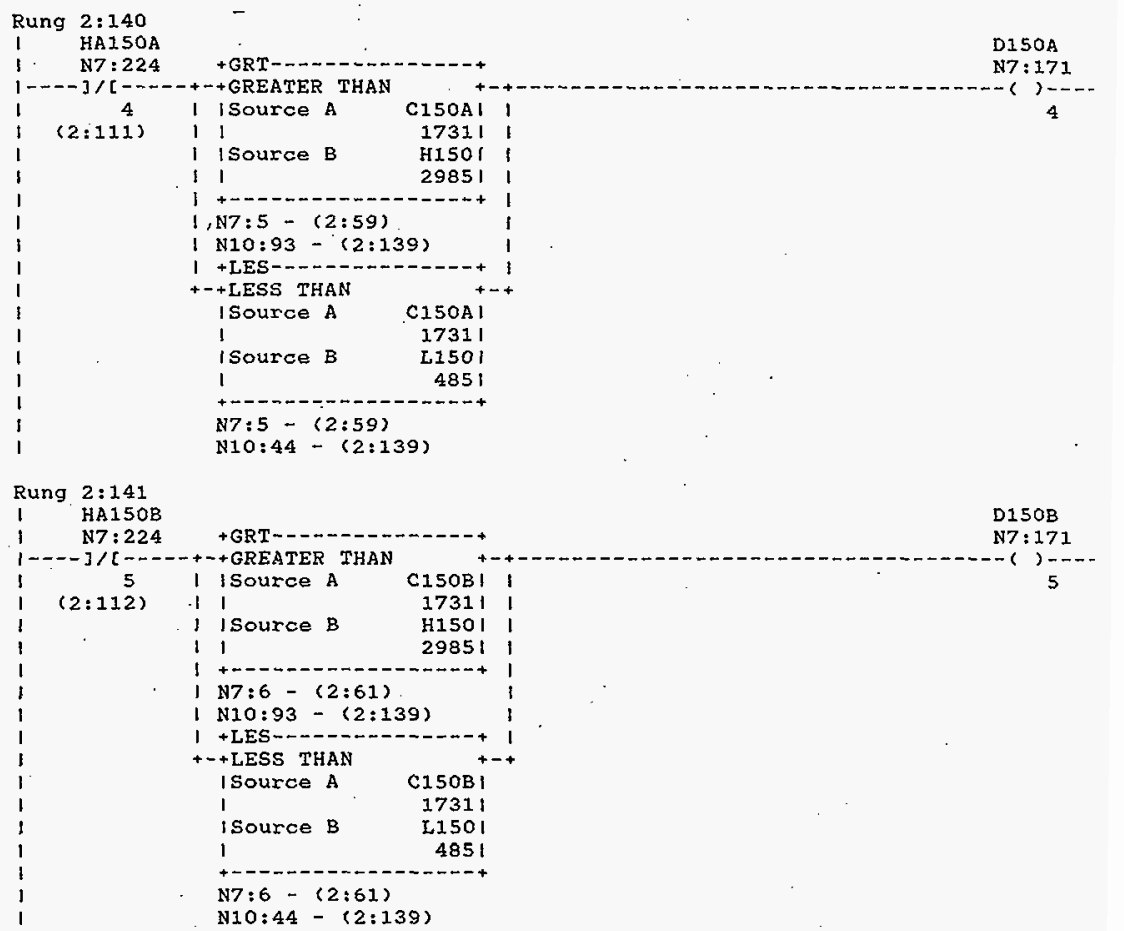

Rung 2:142

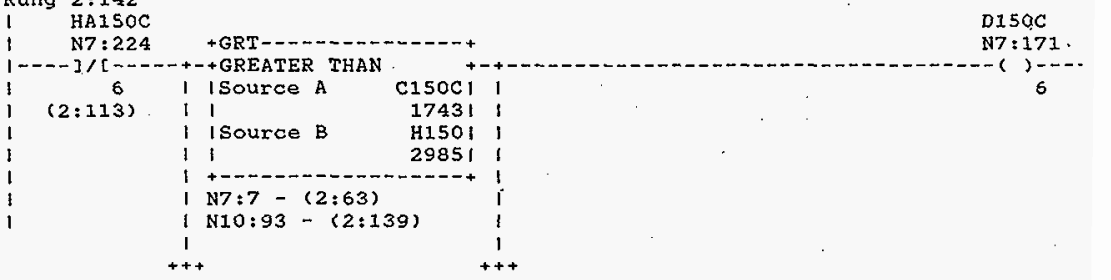

HNF-SD-FF-CSWD-61 Rev. 0 
Processor and Data(OPS Unit 1 )

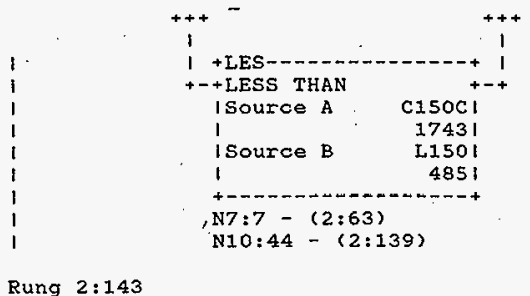

Rung $2: 143$

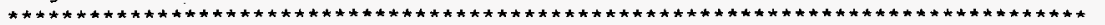
ESTABLISH HIGH AND LOW LIMITS FOR RANGE CHECKING IF NO HARDHARE ALARMS EXIST CHECK T/C VALUES FOR OUT-OF-RANGE CONDITION

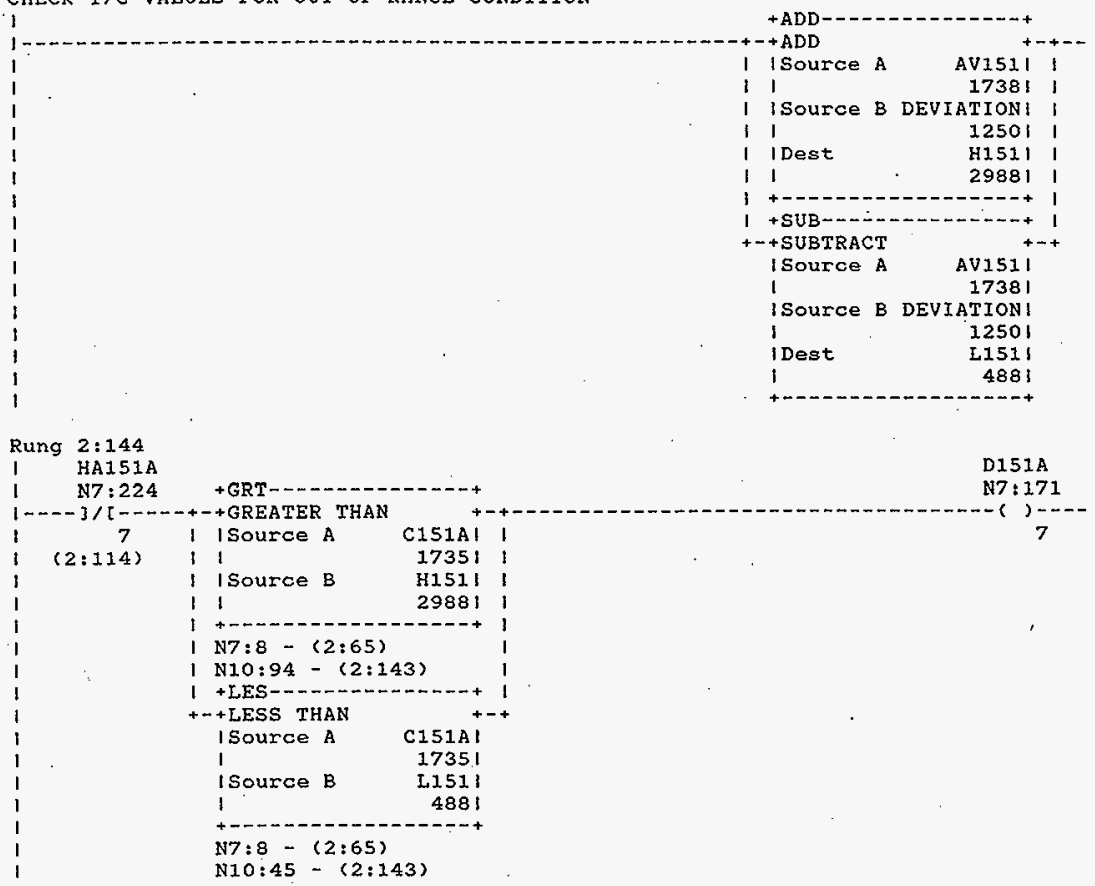

HNF-SD-FF-CSWD-61 Re:v. 0

Page 421 
Rung 2:145

1 HA151B

l. N7:224 $-$

October 23, 1996 Rung 2:145

$1-\cdots] /[-\cdots$

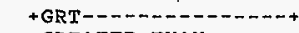

D151B N7: 171

$$
(2: 115)
$$

I ISource A

11

I ISource B

11

$1+\ldots+\ldots+2989$

$1, N 7: 9-(2: 67)$

I $10: 94-(2: 143)$

1 +LES--.---.-.-----+

+++ LESS THAN

isource A

1

isource B

C1518!

1736 !

1

L1511

+--...--

N7:9-(2:67)

$N 10: 45-(2: 143)$

Rung 2:146

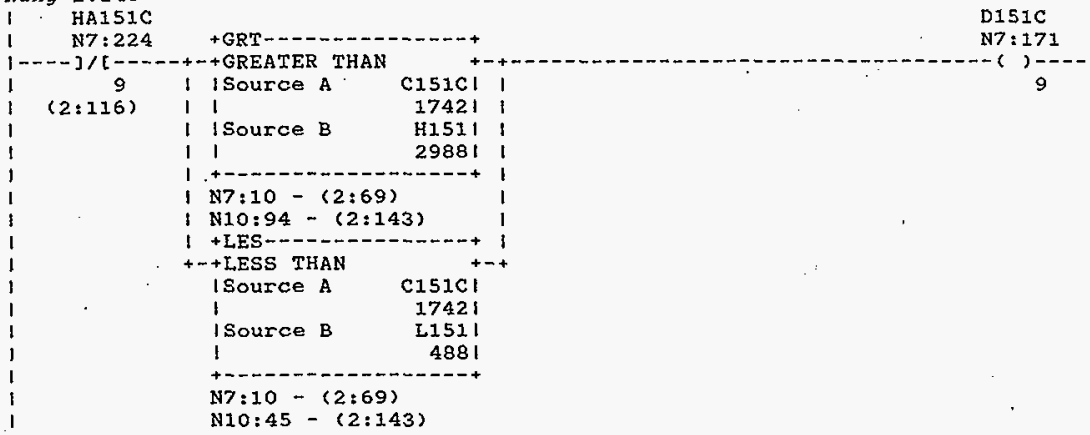

Rung $2: 147$

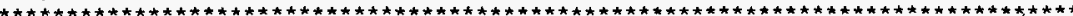
ESTABLISH HIGH AND LOW LIMITS FOR RANGE CHECKING IF NO HARDHARE ALARMS EXIST CHECK T/C VALUES FOR OUT-OF-RANGE CONDITION

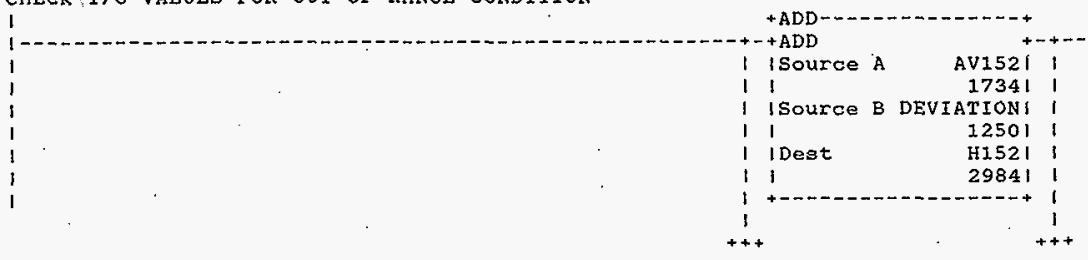

HNF-SD-FF-CSWD-61 Rev. 0

Page 422 


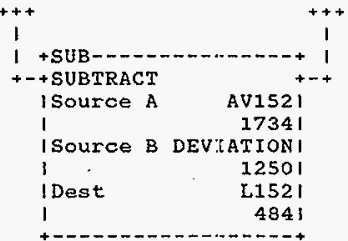

Rung 2:148

1 HA152A

$1 \quad$ N7:224

484

Rung $2: 149$

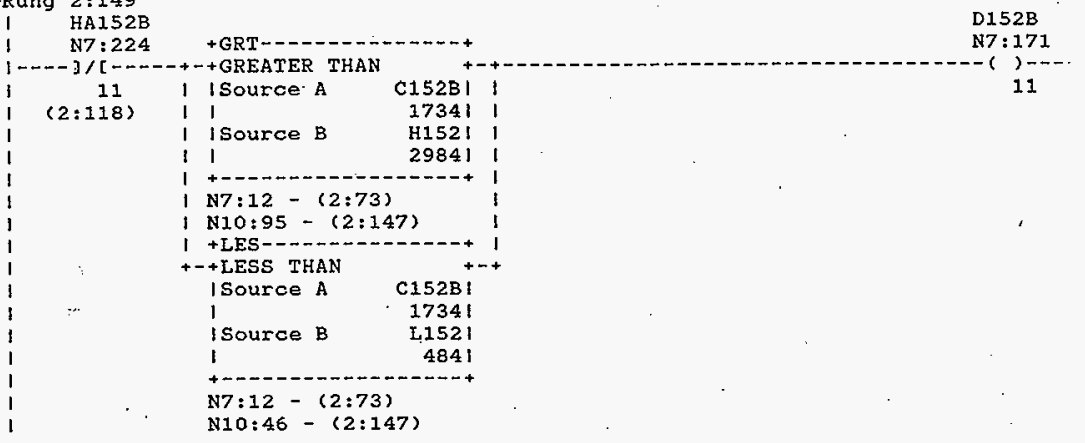

HNF-SD-FF-CSWD-61 Rev. 0 
Processor and Data(ops Unit 1)

Rung $2: 150$

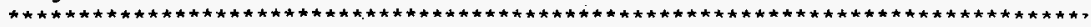
ESTABLISH HIGH AND LOW LIMITS FOR RANGE CHECKING IF NO HARDWARE ALARMS EXIST CHECK T/C VALUES FOR OUT-OF-RANGE CONDITION

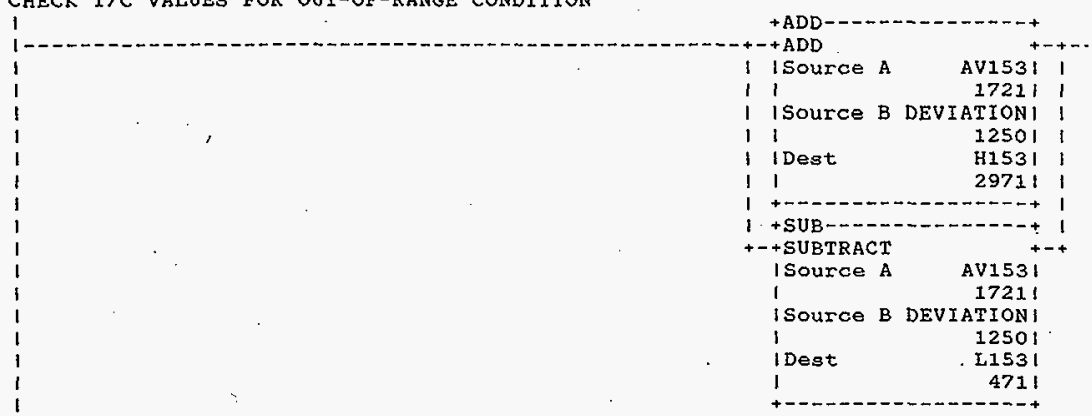

Rung 2:151

HAI53A

N7:224

$1-+-3 /[--+++$ GREATER THAN

12
$(2: 119)$

1 I source A

1

1 isource $\mathrm{B}$

11

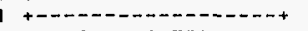

$1 N 7: 13-(2: 75)$

I N10:96 - (2:150)

$1+$ LES--............

++ LESS THAN $+\cdots$

ISource A CI53AI

I 1736 I

isource B LI531

1

4711

N7:13-(2:75)

$\mathrm{N} 10: 47-(2: 150)$

Rung $2: 152$

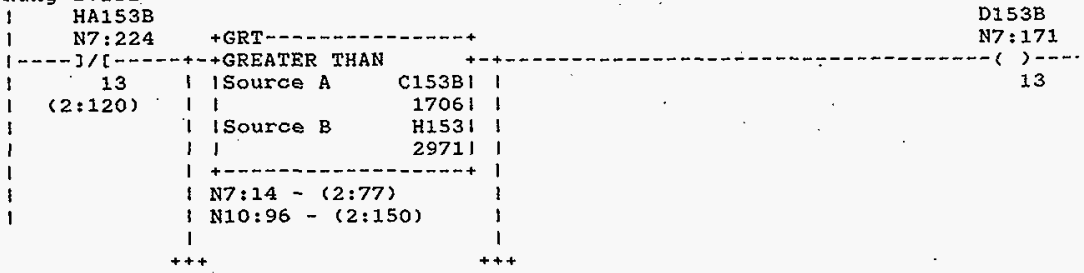

HNF-SD-FF-CSWD-61 Rev. 0 
Processor and Data(OPS Unit 1 )

October 23, 1996 page 63 Program Listing

Procesgor File: SODIUM2A.ACH

Rung $2: 152$

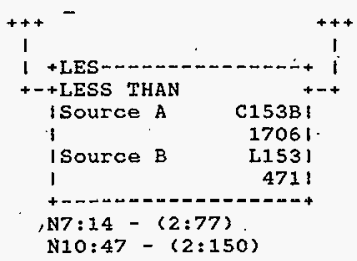

Rung $2: 153$

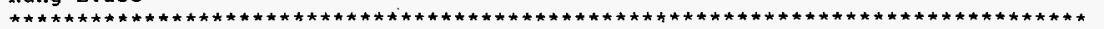
ESTABLISH HIGH AND LOW LIMITS FOR RANGE CHECKING IF NO HARDWARE ALARMS EXIST CHECK T/C VALUES FOR OUT-OF-RANGE CONDITION

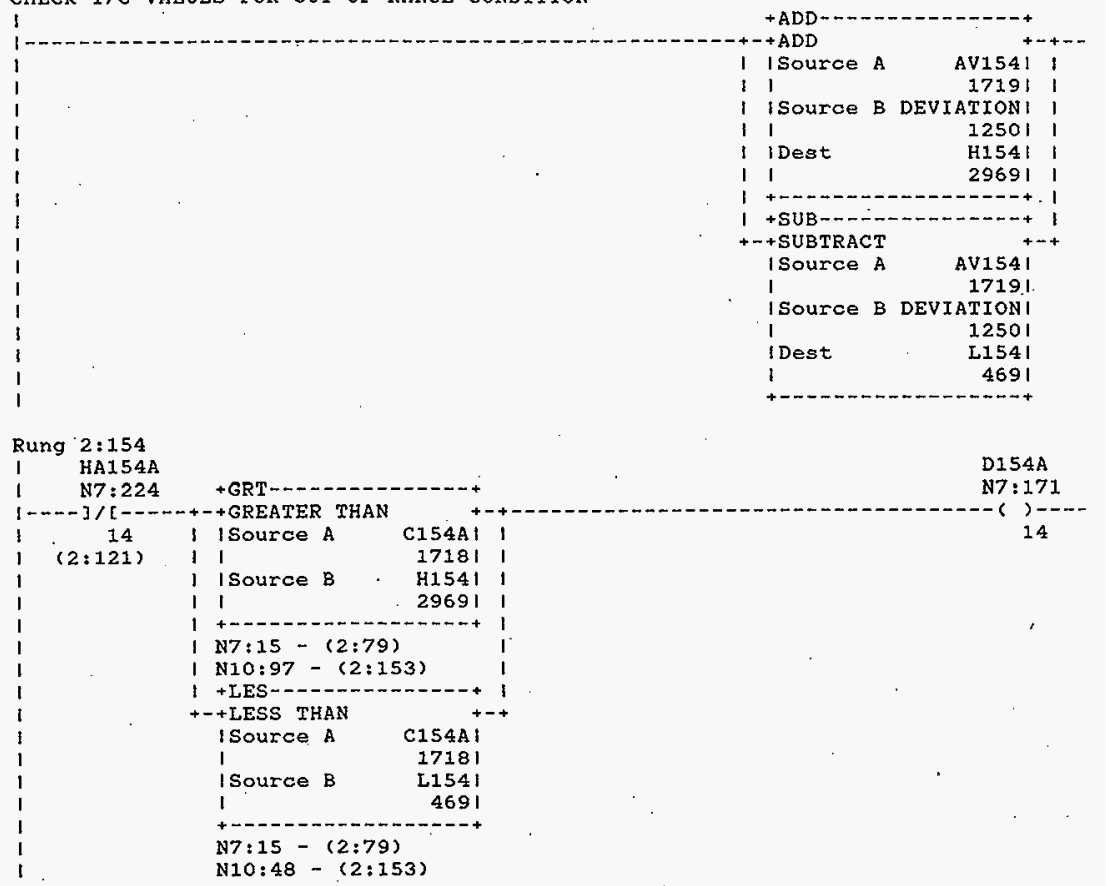

HNF-SD-FF-CSWD-61 Rev. 0

Page 425 


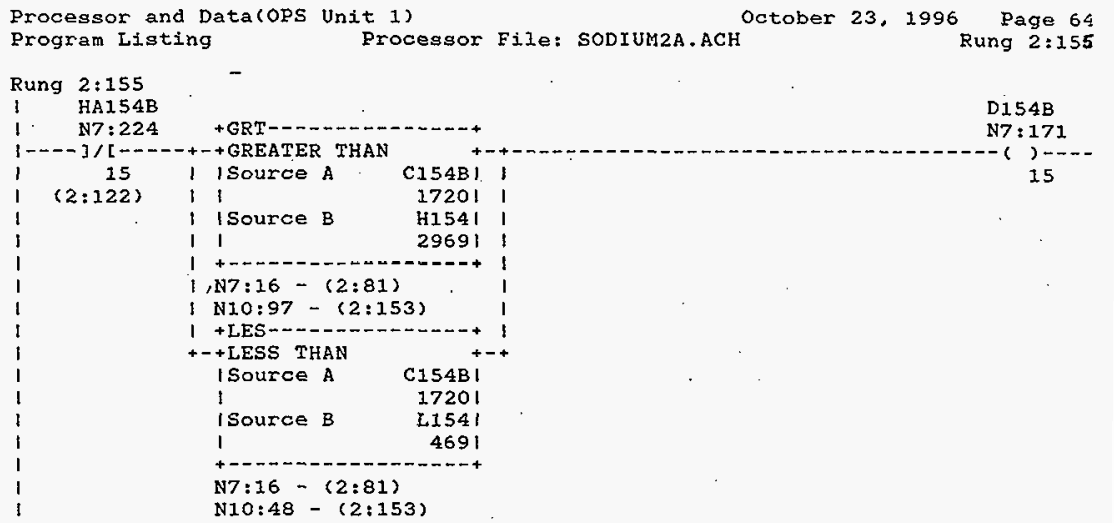

Rung $2: 156$

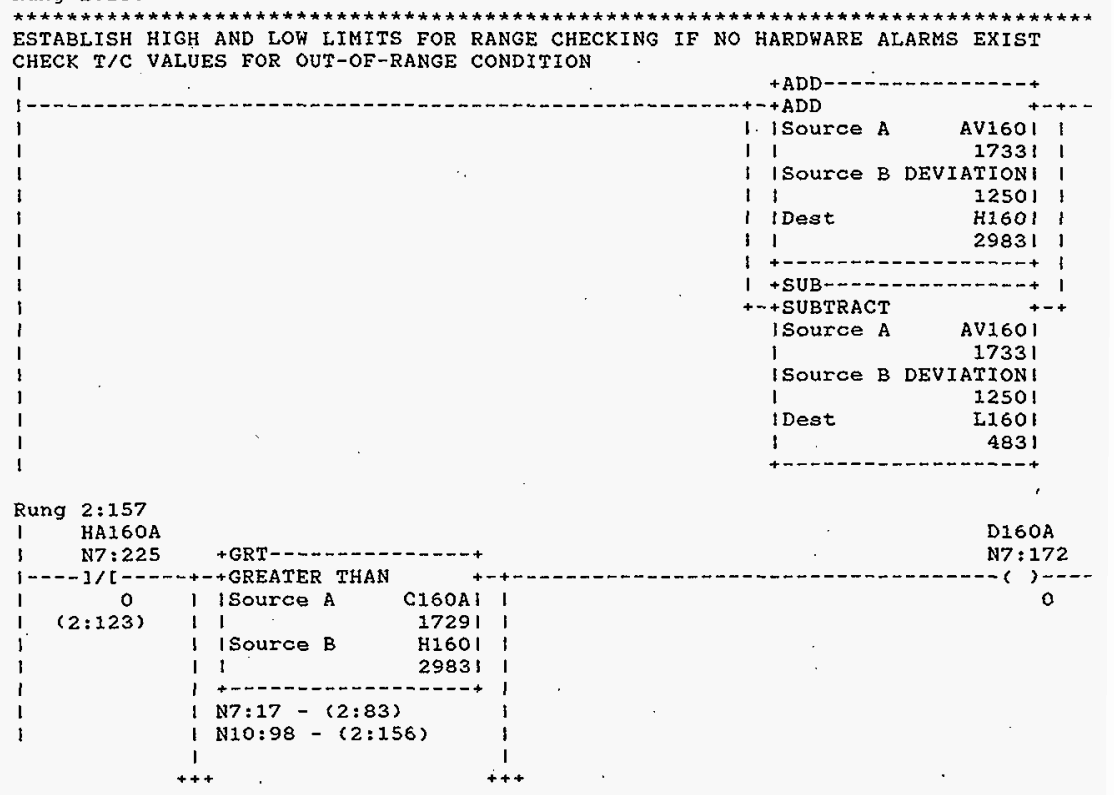

HNF-SD-FF-CSWD-61 Rev. 0 
processor and Data(OPS Unit 1)

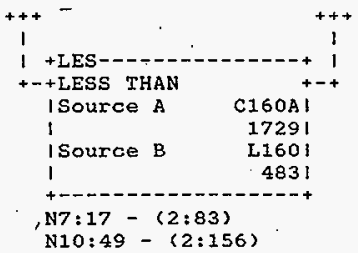

Rung 2:158
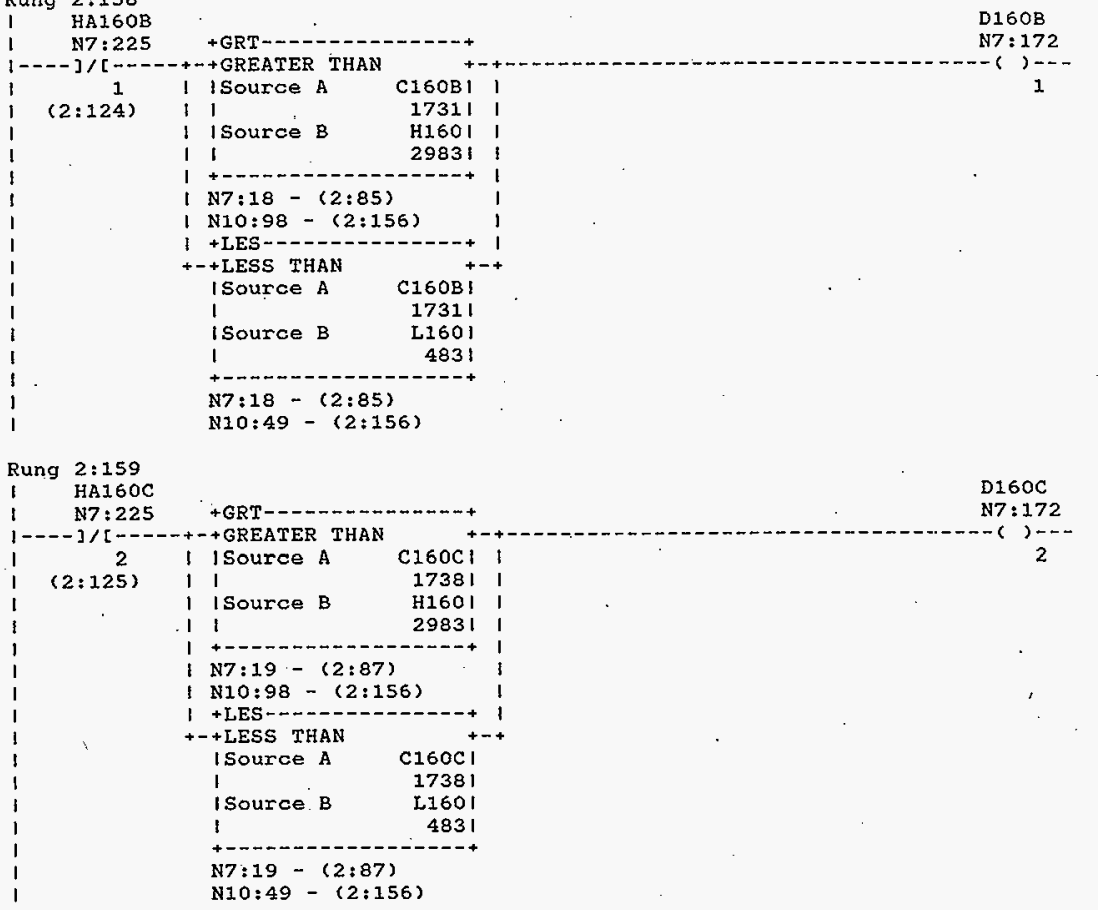

HNF-SD-FF-CSWD-61 Rev. 0 
Rung 2:160

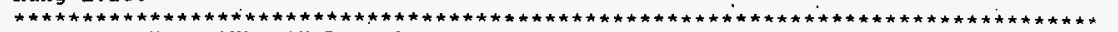
ESTABLISH HIGH AND LOW IIMITS FOR RANGE CHECKING IF NO HARDWARE ALARMS EXIST CHECK T/C VALUES FOR OUT-OF-RANGE CONDITION

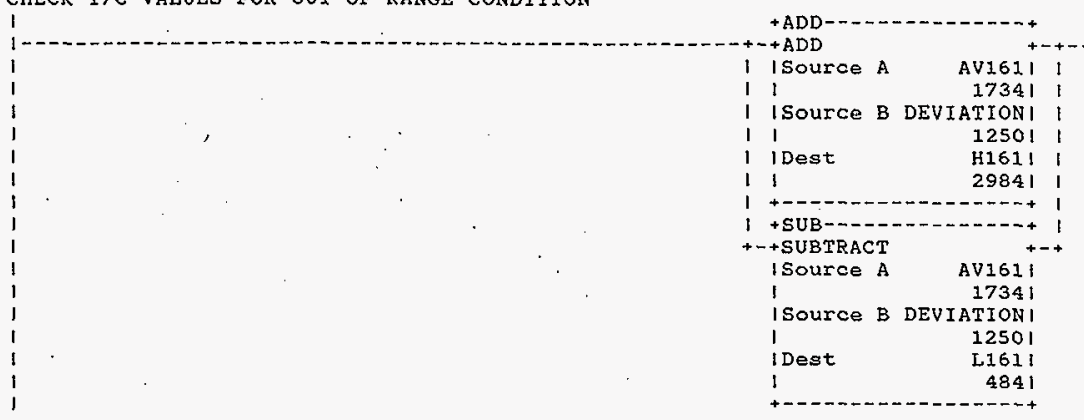

\section{Rung $2: 161$}

I HAI61A N7 $: 225$ $1---1 /[---+-+$ GREATER THAN

$$
\left(2: 126^{3}\right)
$$

$\begin{array}{ll}1 & 1 \\ 1 & 1\end{array}$

I Source A

I isource B

1

$1+$

( $N 7: 20-(2: 89)$

(N10:99-(2:160)

$1+$ LES-...--.......-- +

+-+ LESS THAN

ISource A C16IA|

I 1734 I

iSource B I.1611

1

4841

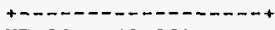

N7:20-(2:89)

N10:50-(2:160)

Rung $2: 162$

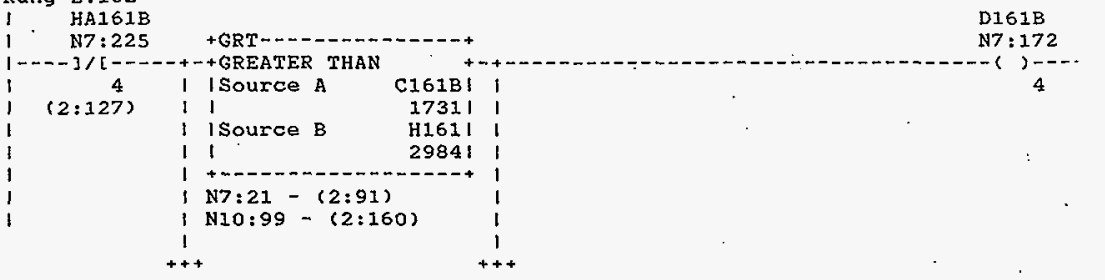

HNF-SD-FF-CSWD-61 Rev. 0 


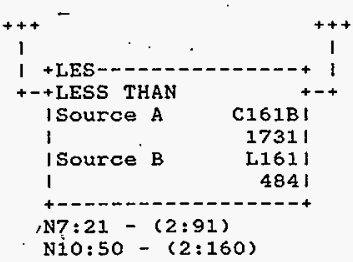

Rung 2:163

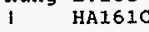
N7: 225 Nio: $50-(2: 160)$
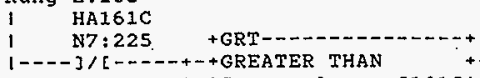

D161C

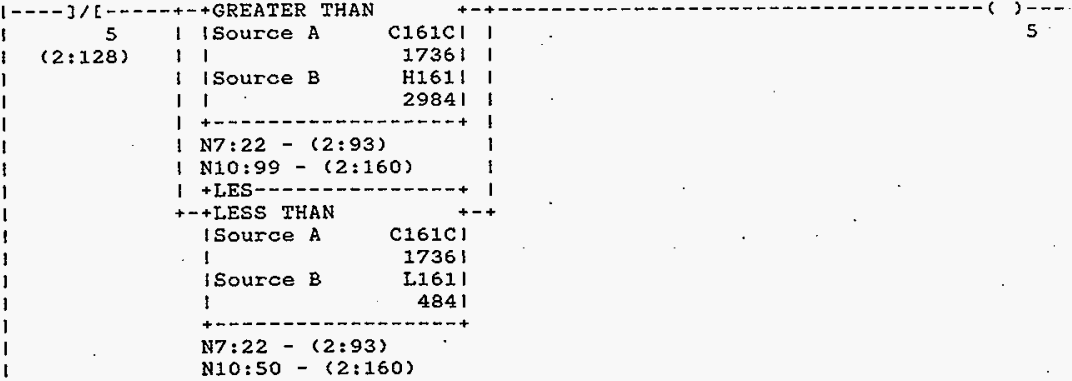

Rung $2: 164$

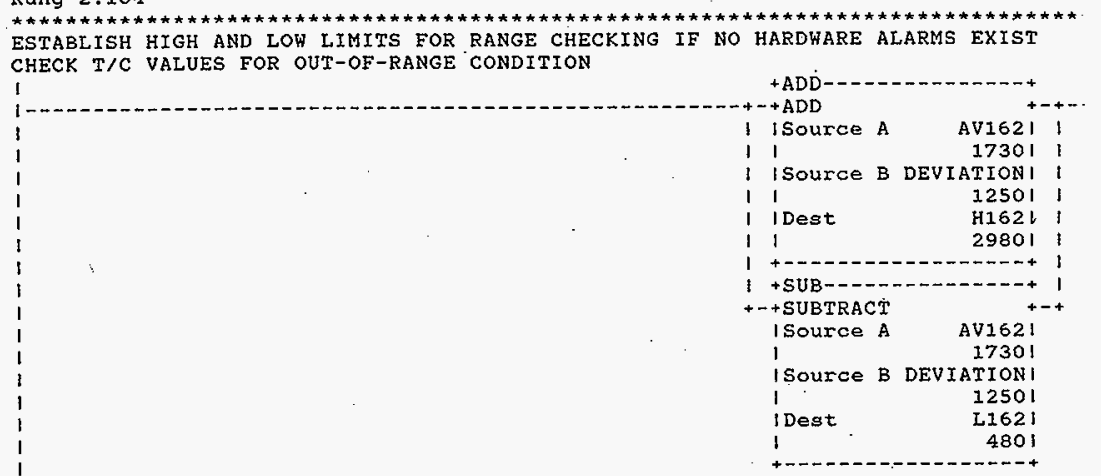

HNF-SD-FF-CSWD-61 Rev. 0 
Processor and Data(OPS Unit 1 )

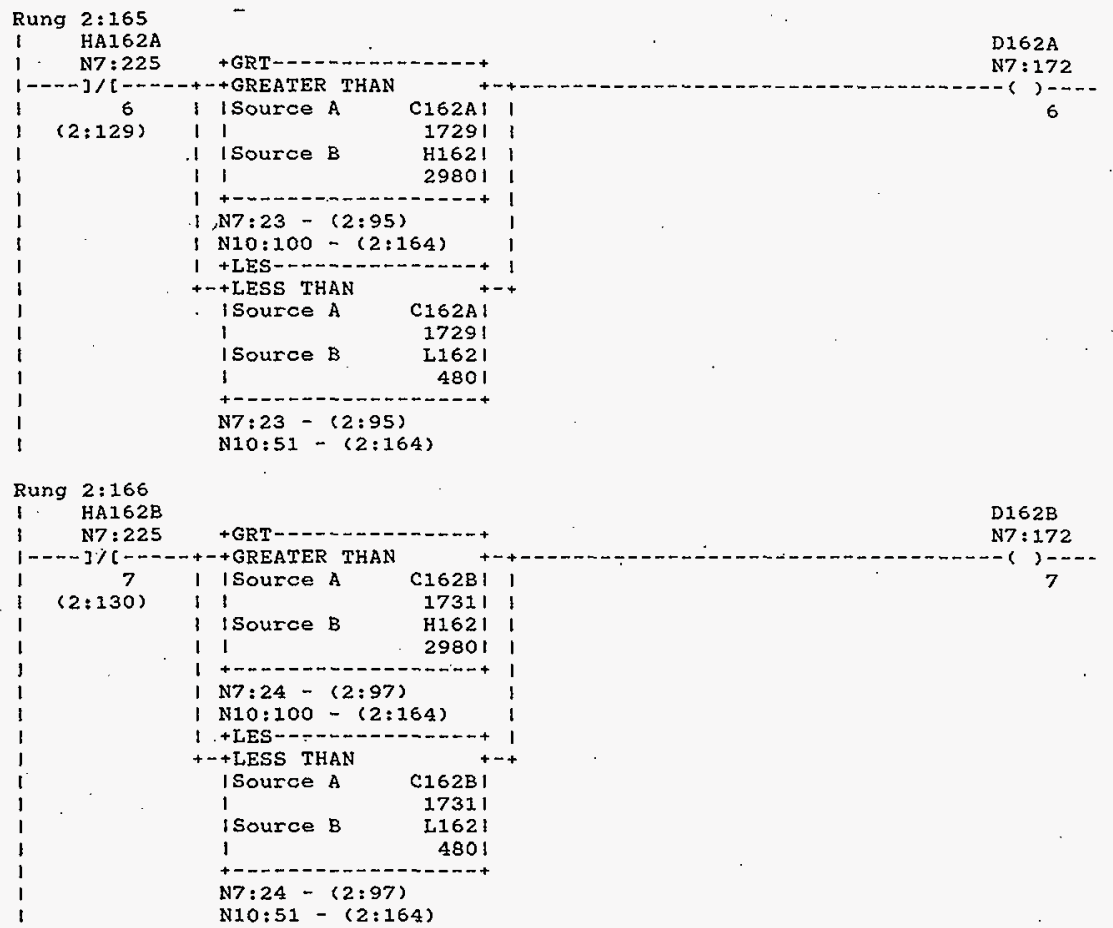

Rung $2: 167$

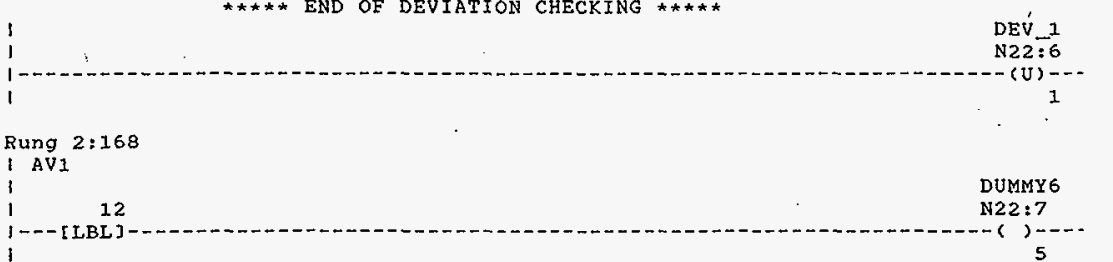

HNE-SD-FF-CSWD-61 Rev. 0 
Processor and Datacops Unit 1 )

Rung 2:169

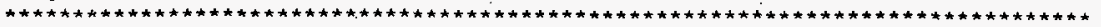

CLEAR COUNT AND TOTAL. IF NO HARDHARE, NO RANGE ALARMS AND HO THERMOCOUPLES

HAVE BEEN TAKEN OUT (TE_OUT $X X X)$ THEN AVERAGE THE T/C
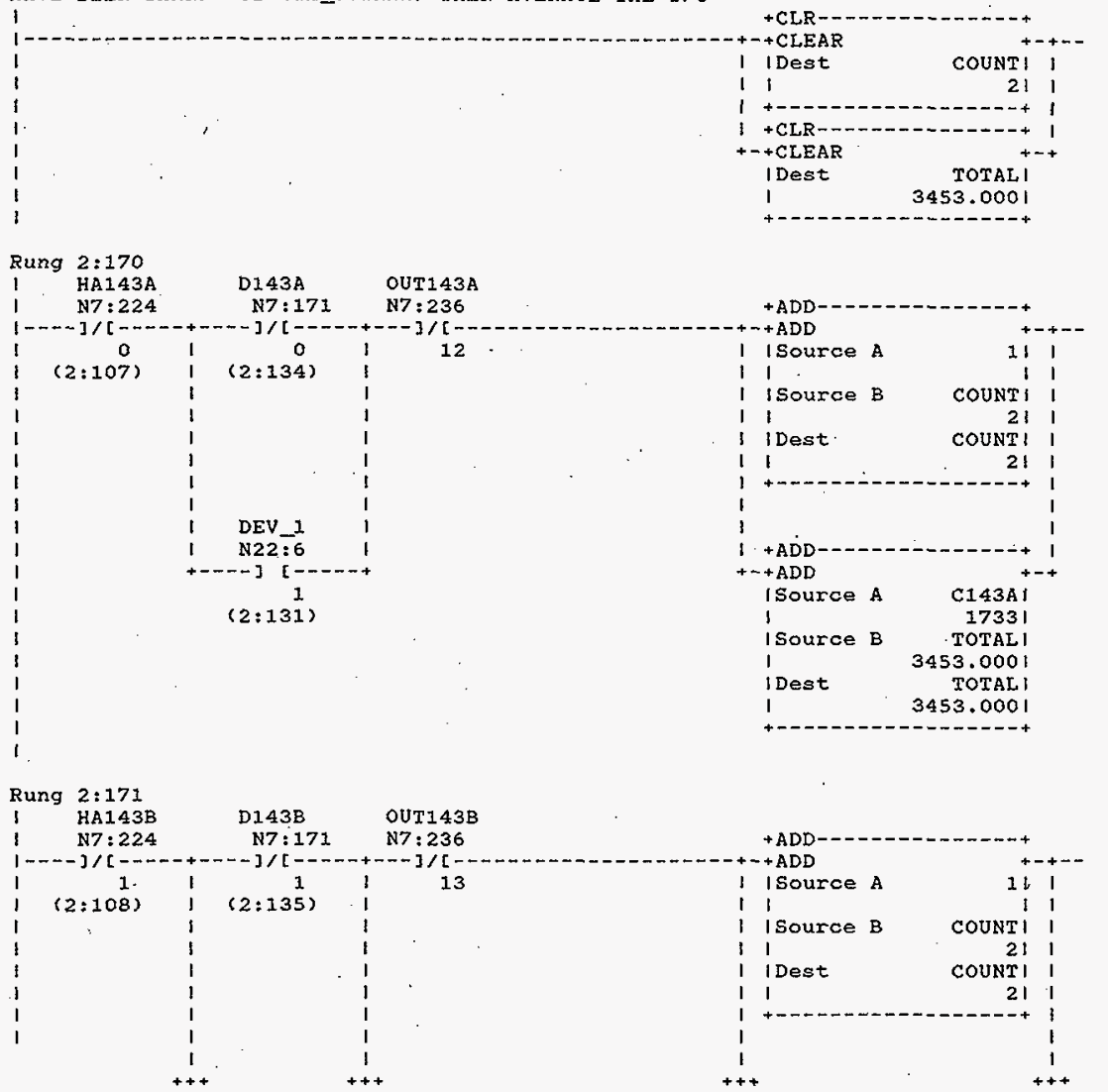

HNF-SD-FF-CSWD-61 Rev. 0

Page 431 


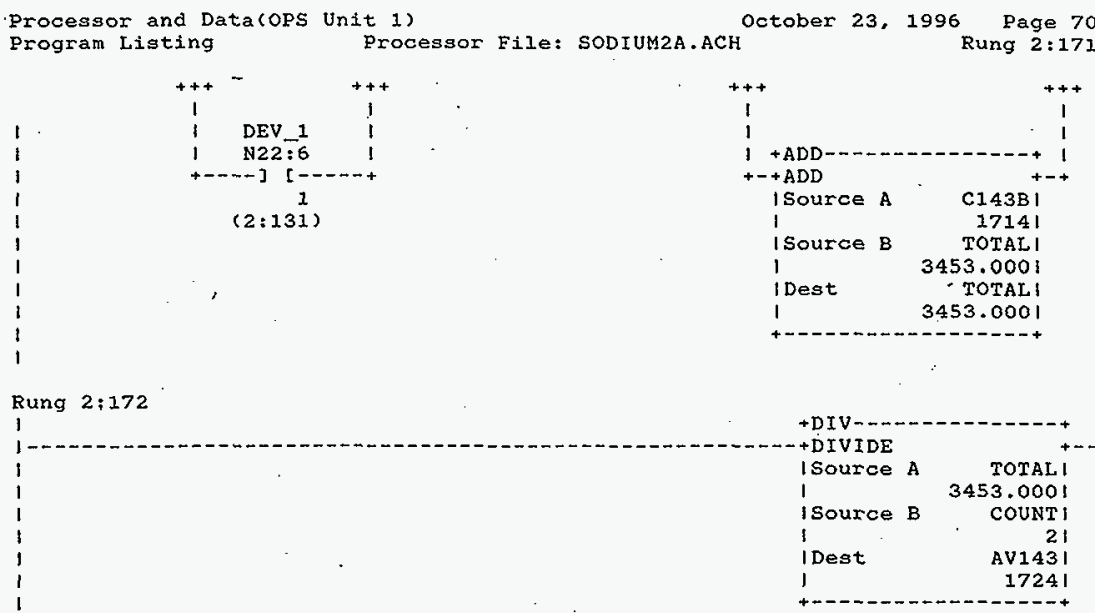

Rung $2: 173$

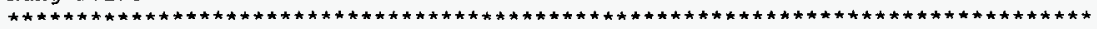
CLEAR COUNT AND TOTAL, IF NO HARDHARE, NO RANGE ALARMS AND NO THERMOCOUPLES HAVE BEEN TAKEN OUT (TE_OUTXKX) THEN AVERAGE THE T/C

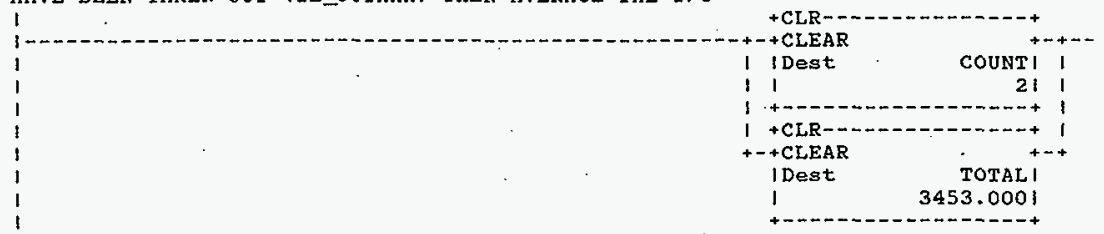

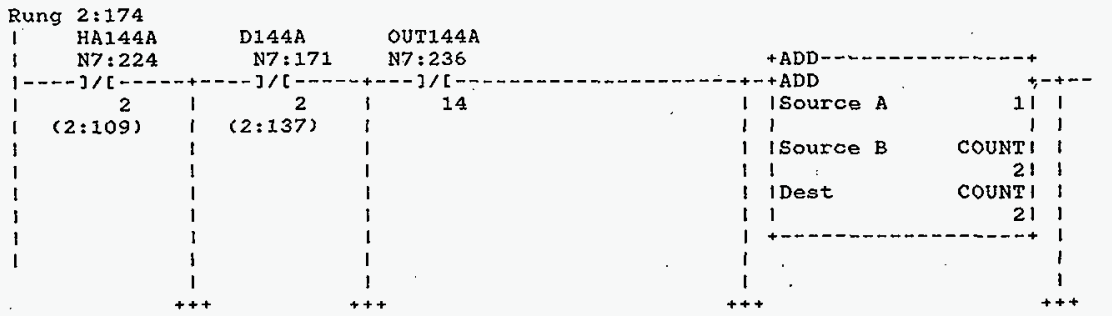

HNF-SD-FF-CSWD-61 Rev. 0 
Processor and Data(OPS Unit 1

october 23, 1996 Page 71 Program Listing

Processor File: SODIUM2A.ACH

Rung $2: 174$

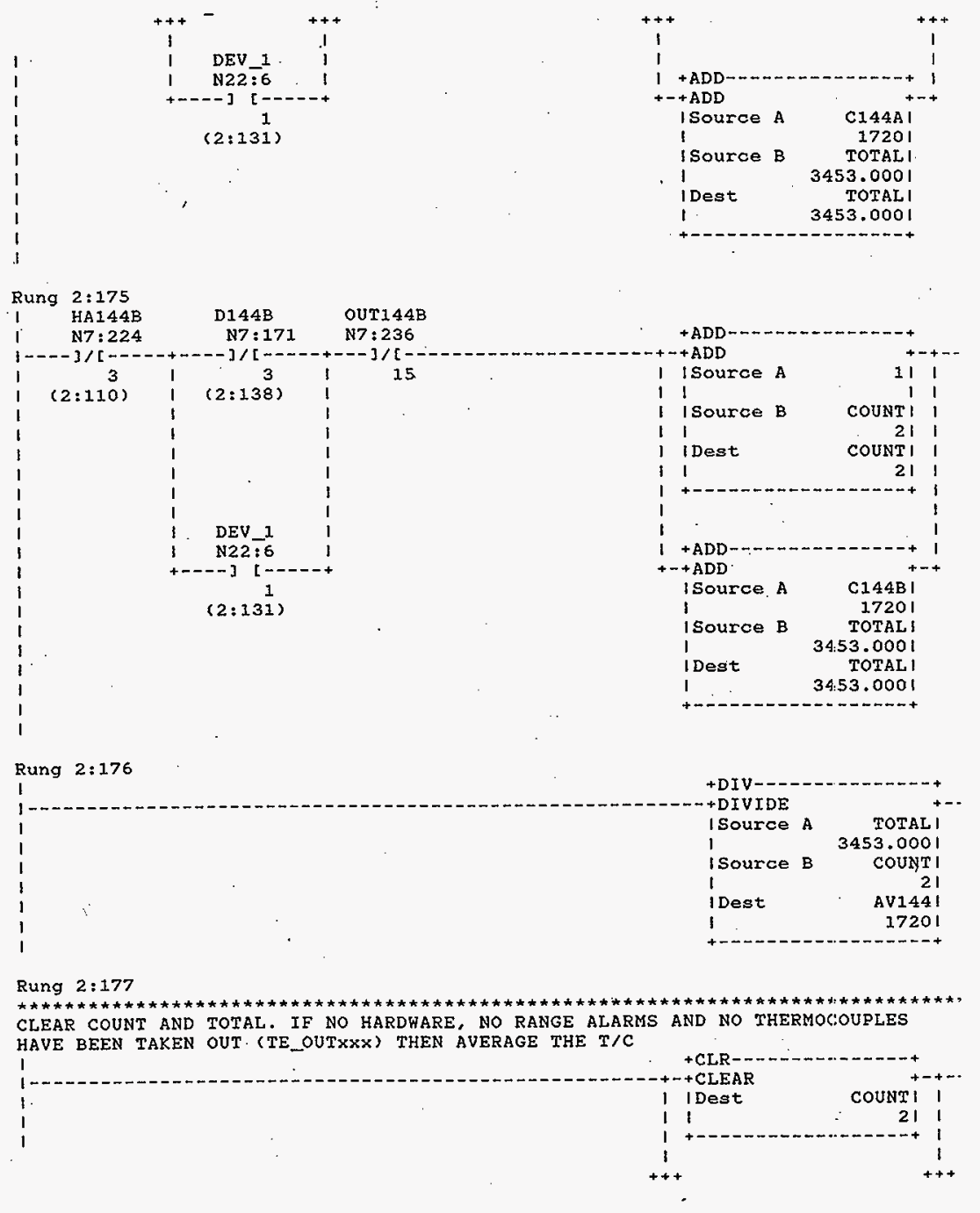

HNF-SD-FF-CSWD-6! Rev. 0 

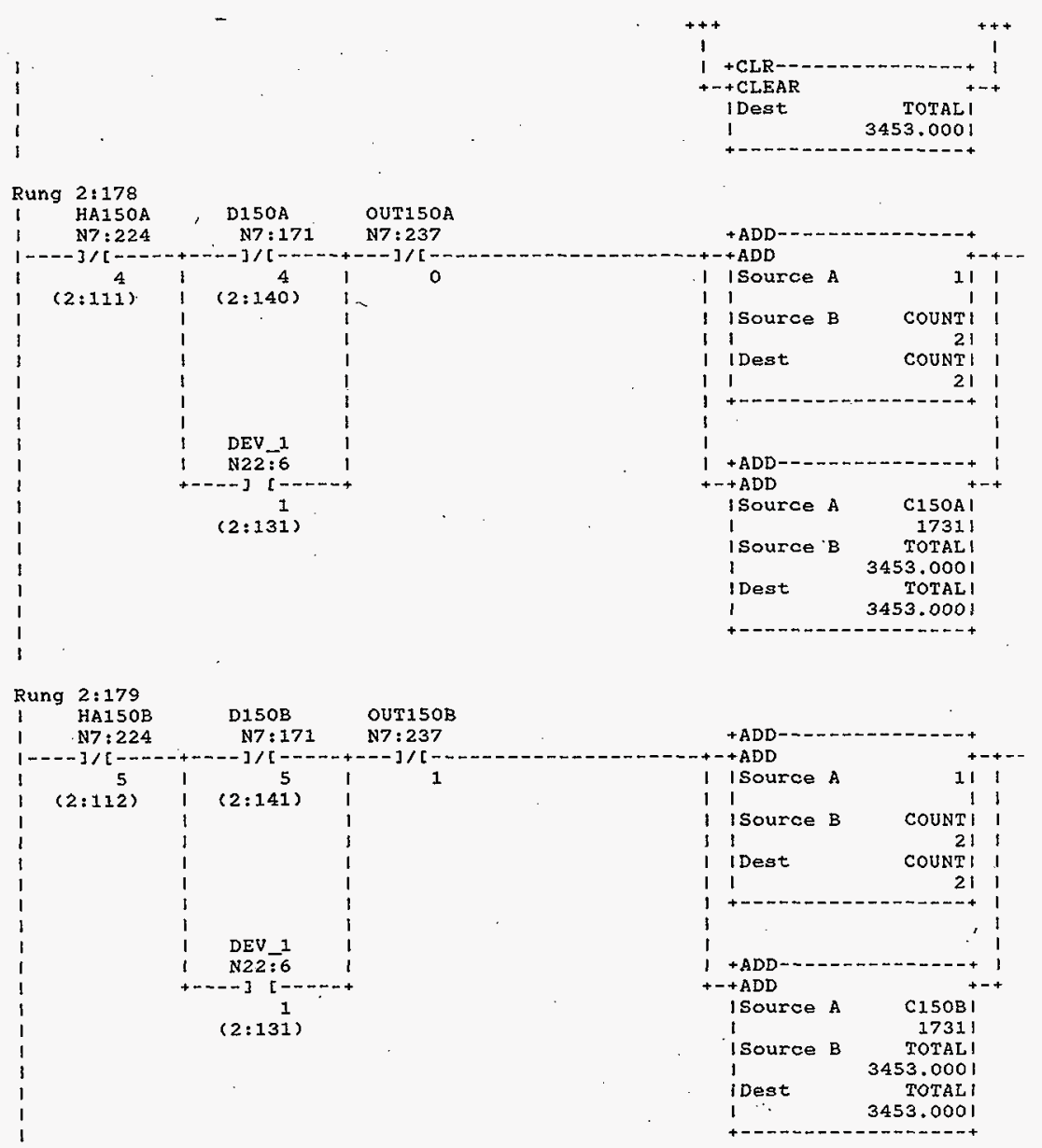

HNF-SD-FF-CSWD -61 Rev. 0 
Processor and Data(OPS Unit 1 ) Program Listing$$
\text { R }
$$

Rung 2:180 $-$

. $\quad \mathrm{N7:22}$

D150C . OUT150C

N7:171 N7:237

$\begin{array}{cccc}6 & \text { । } & 6 & 1\end{array}$

(2:113)

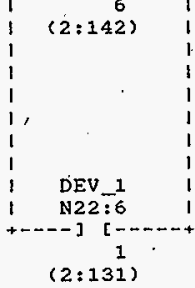

2

$+\mathrm{ADD}$

+ ADD

I ISource A

11

I I Source B

11

I Dest

11

$+$

$1+A D D$

$+-+A D D$

ISource A

ISource B

I

i Dest

I

$+-$
Rung 2:181

I$$
\text { I }
$$$$
1
$$$$
1
$$$$
1
$$$$
\text { I }
$$

\section{Rung 2:182}

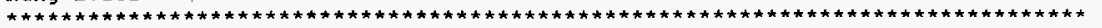
CLEAR COUNT AND TOTAL. IF NO HARDWARE, NO RANGE ALARMS AND NO THERMOCOUPLES HAVE BEEN TAKEN OUT (TE_OUTXXX) THEN AVERAGE THE T/C

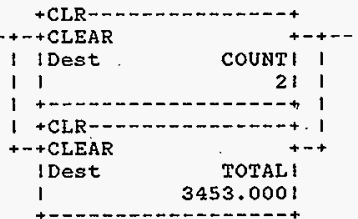

HNF-SD-FF-CSWD-61 Rev. 0 
Processor and Data〈OPS Unit I)

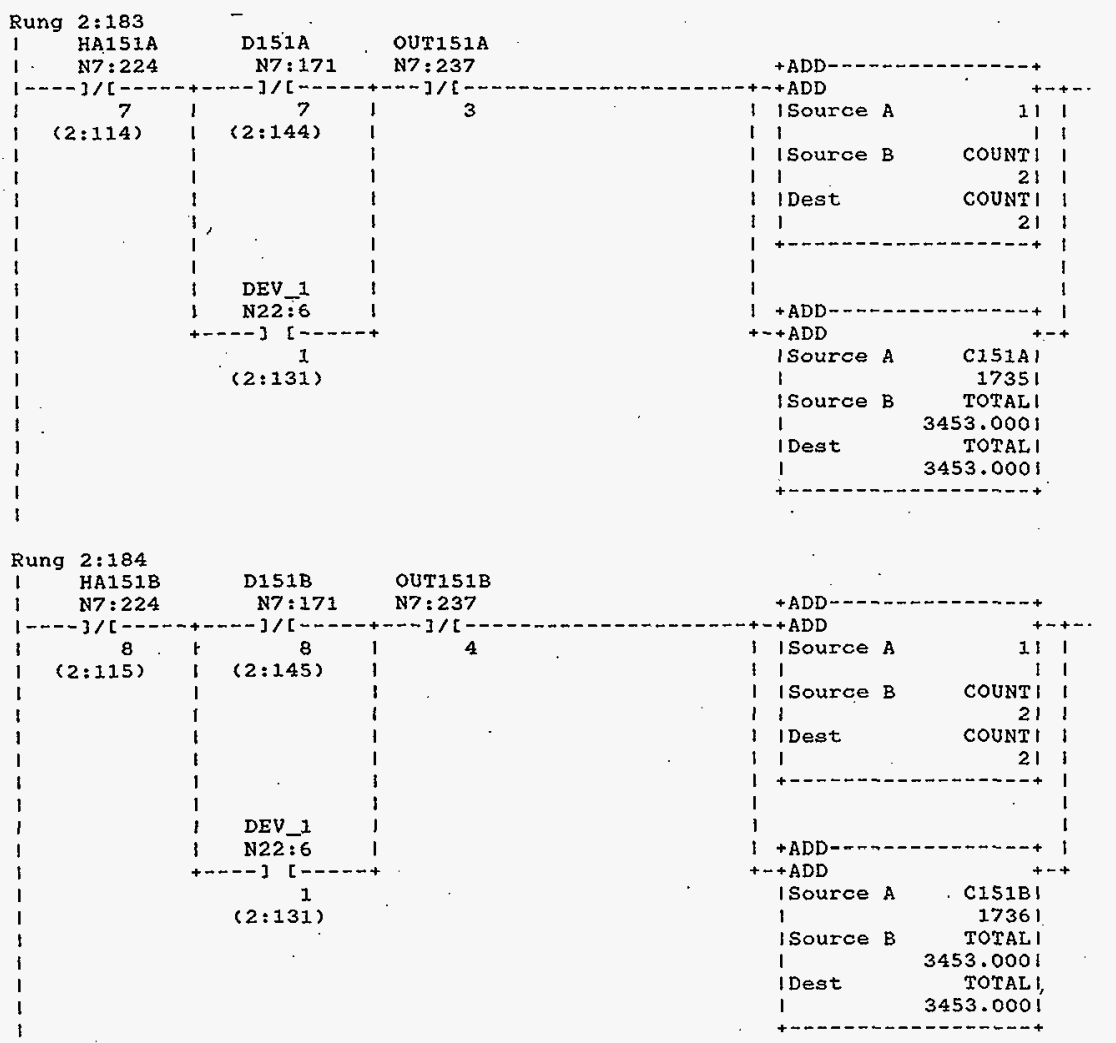

HNF-SD-FF-CSWD-61 Rev. 0 


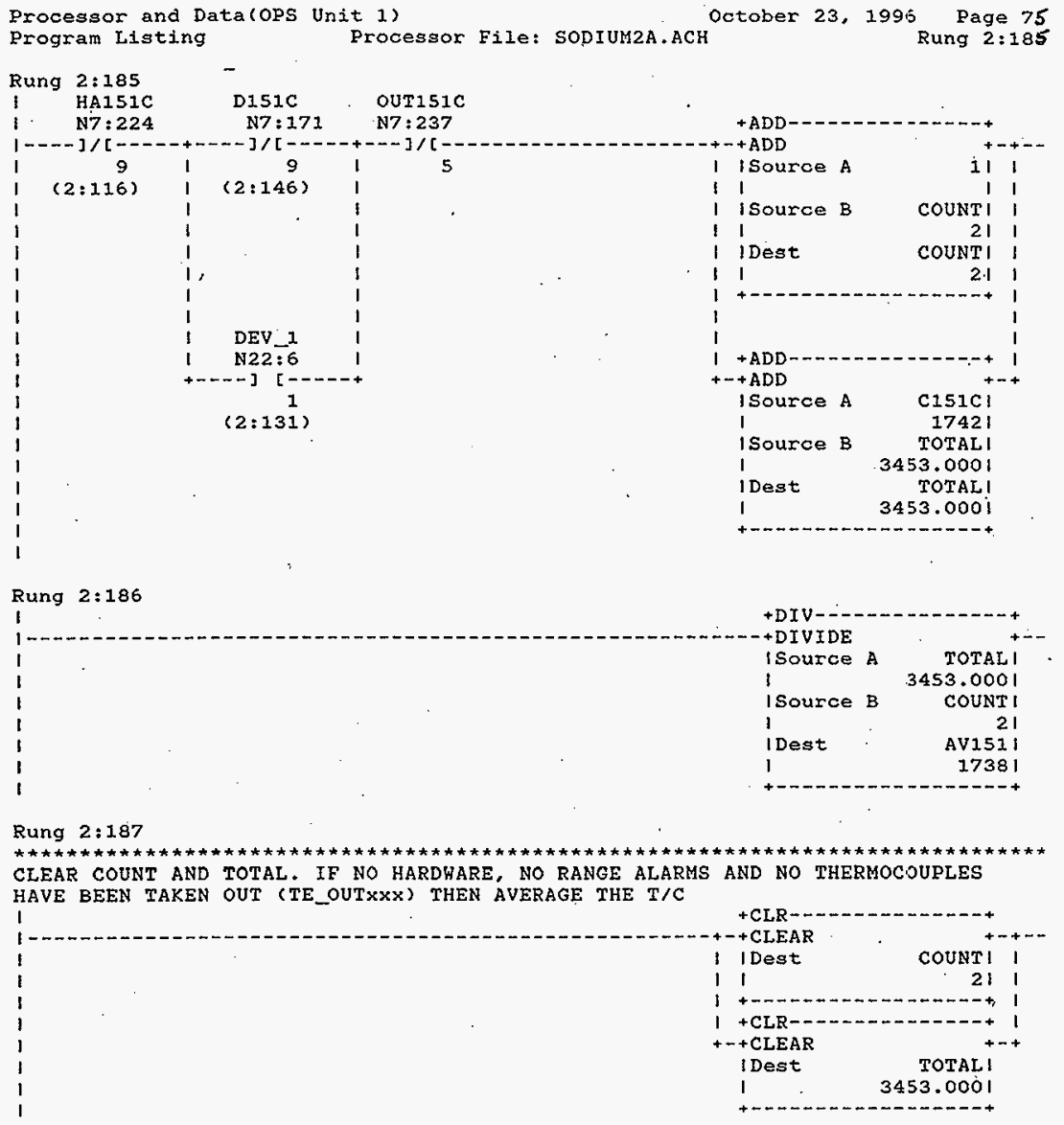

HNF-SD-FF-CSWD-61 Rev. 0

Page $43 \%$ 
Processor and Data(OPS Unit 1)
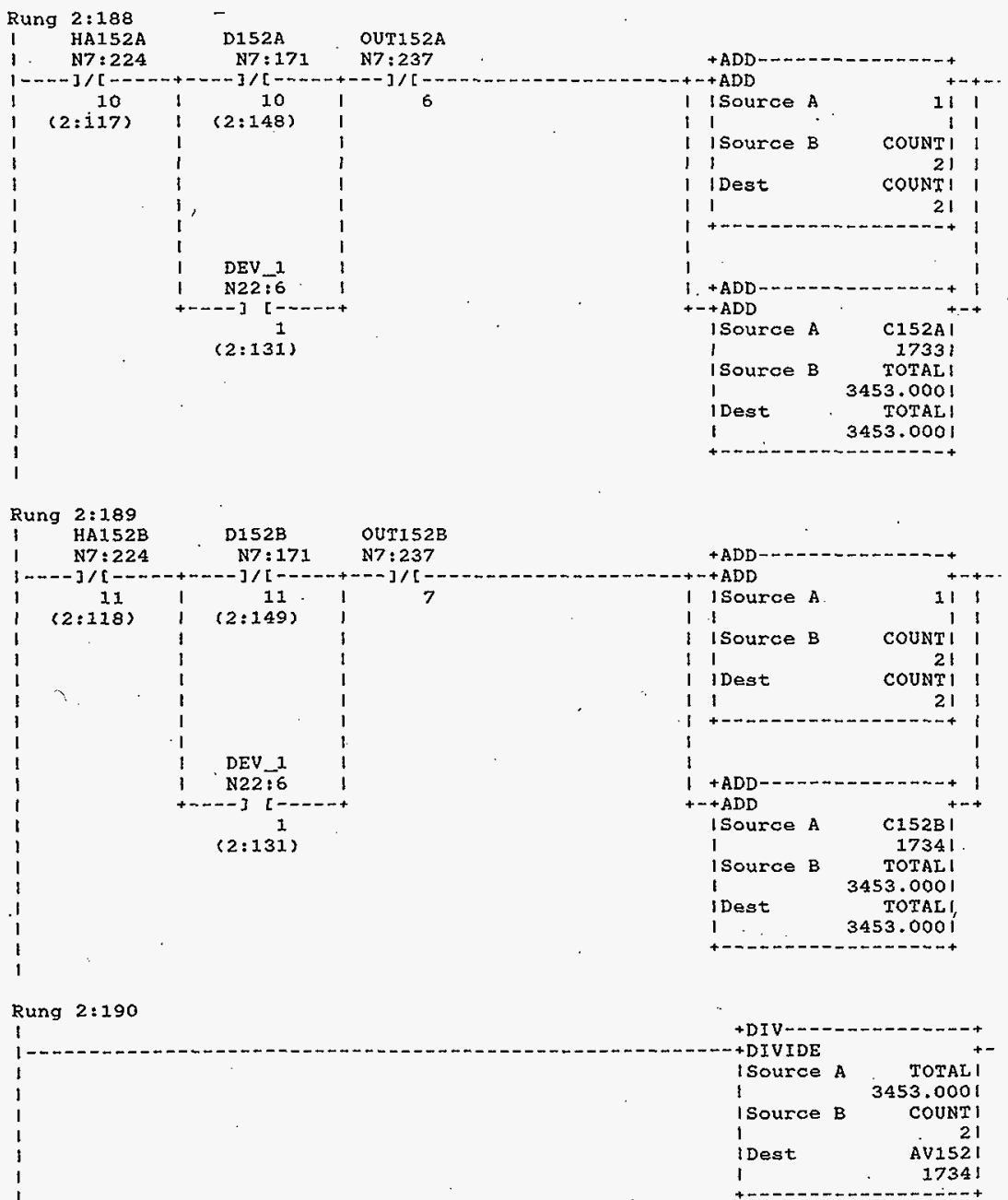

HNF-SD-FF-CSWD-61 Rev. 0 


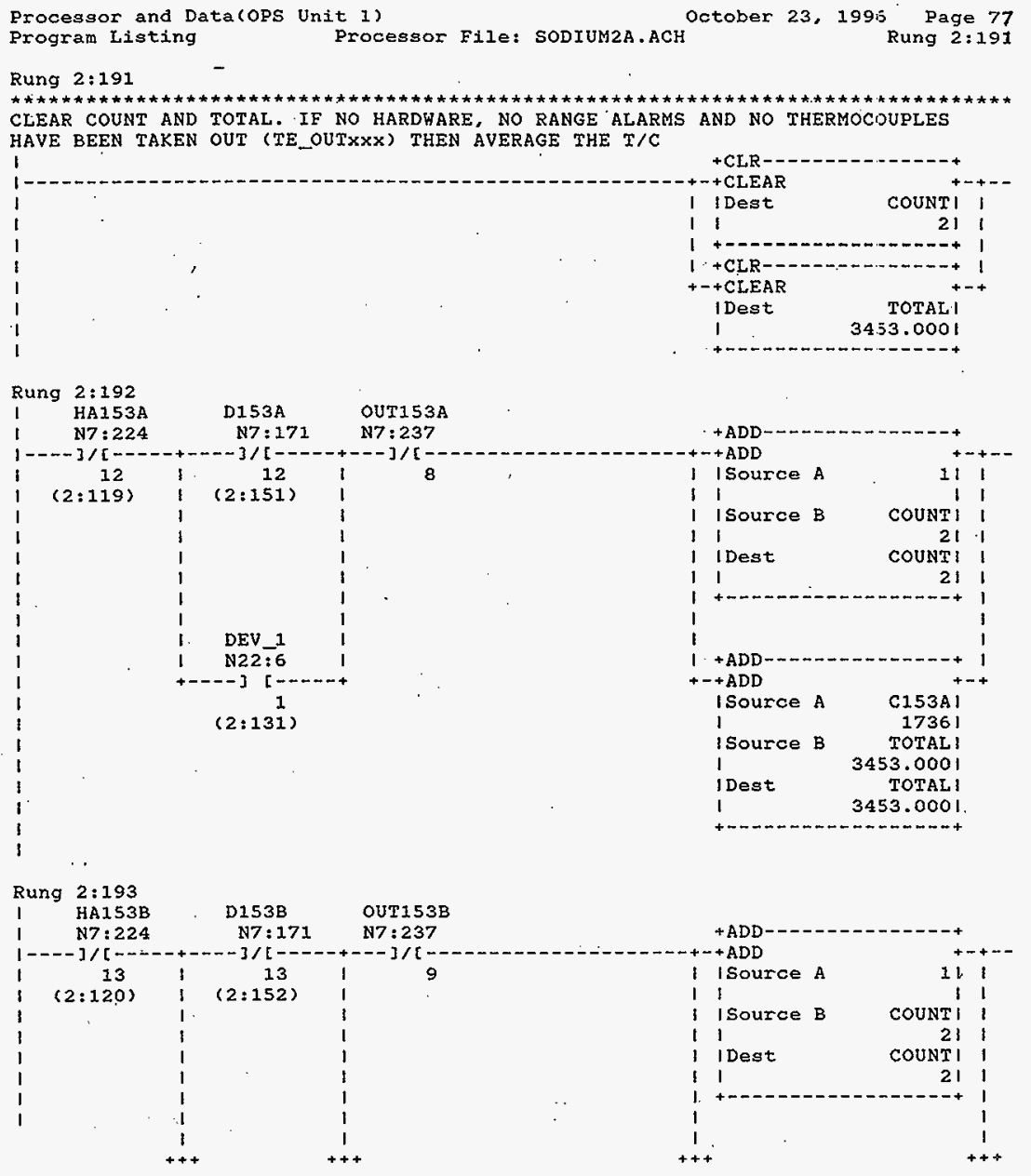

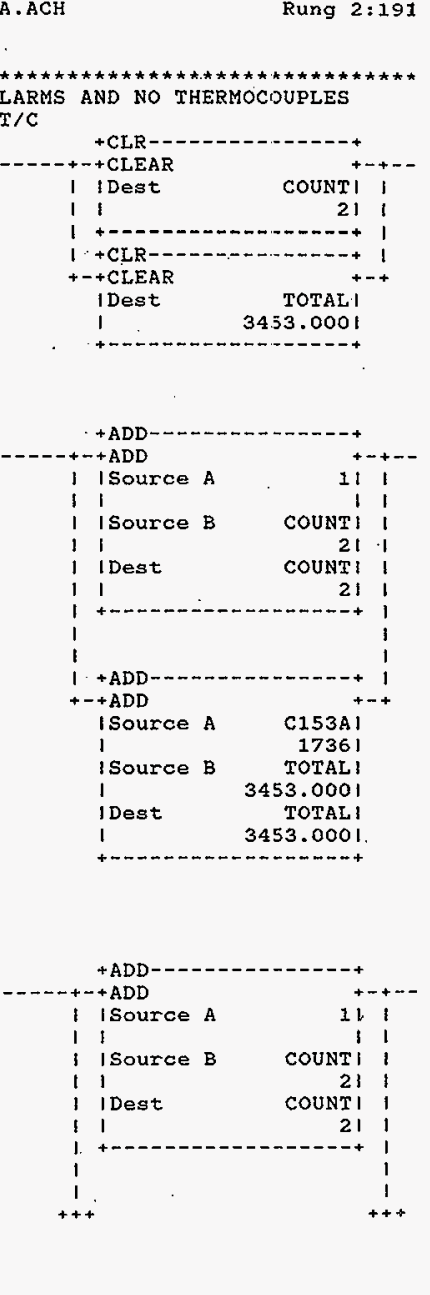

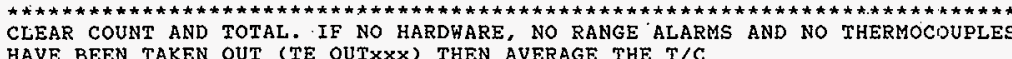
HAVE BEEN TAKEN OUT (TE_OUTXXX) THEN AVERAGE THE T/C

HNF-SD-FF-CSWD-61 Rev. 0 

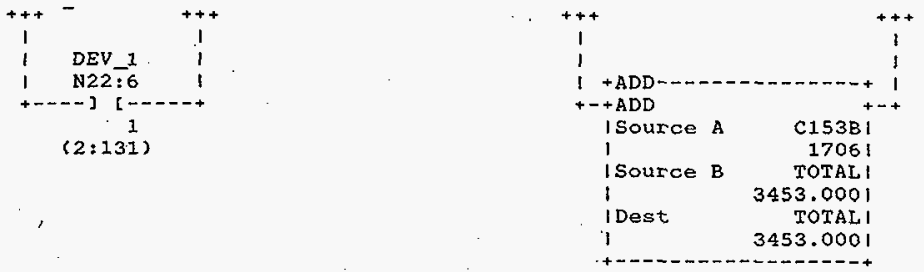

Rung 2:194

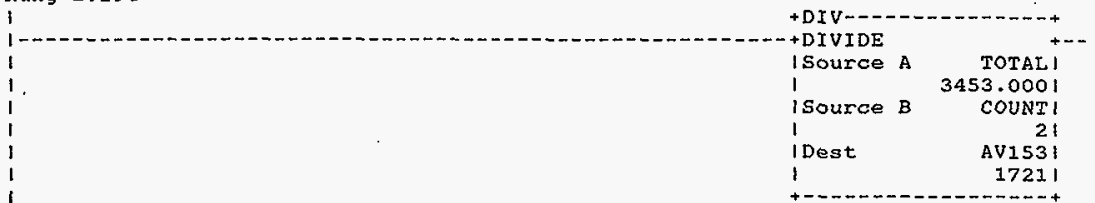

Rung 2:195

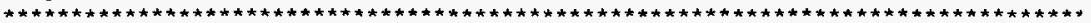
CLEAR COUNT AND TOTAL. IF NO HARDGARE, NO RANGE ALARMS AND NO THERMOCOUPLES HAVE BEEN TAKEN OUT (TE_OUTXXX) THEN AVERAGE THE T/C

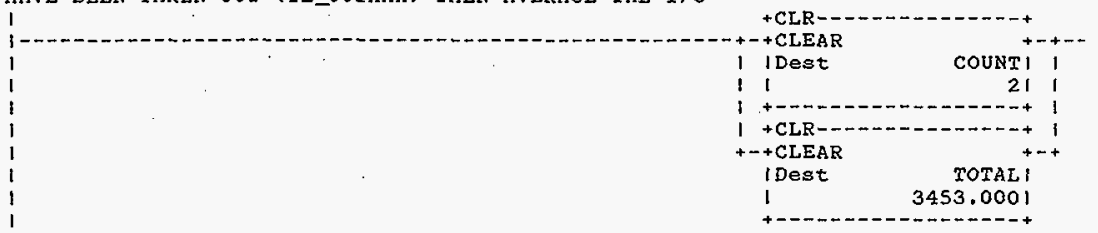

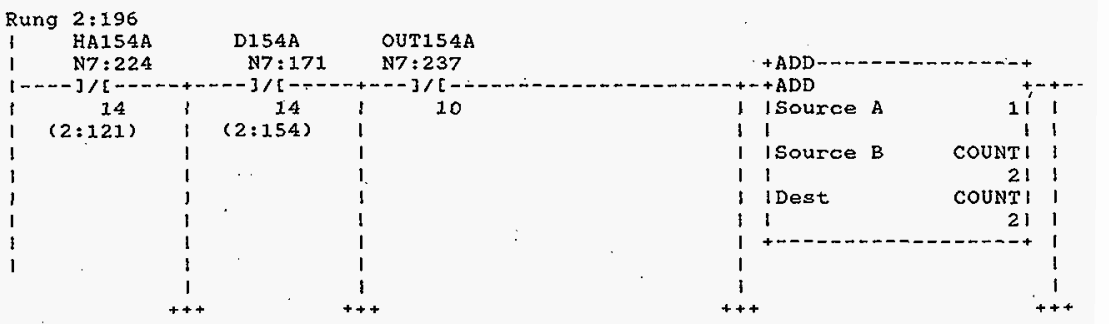

HNF-SD-FF-CSWD-61 Rev. 0 


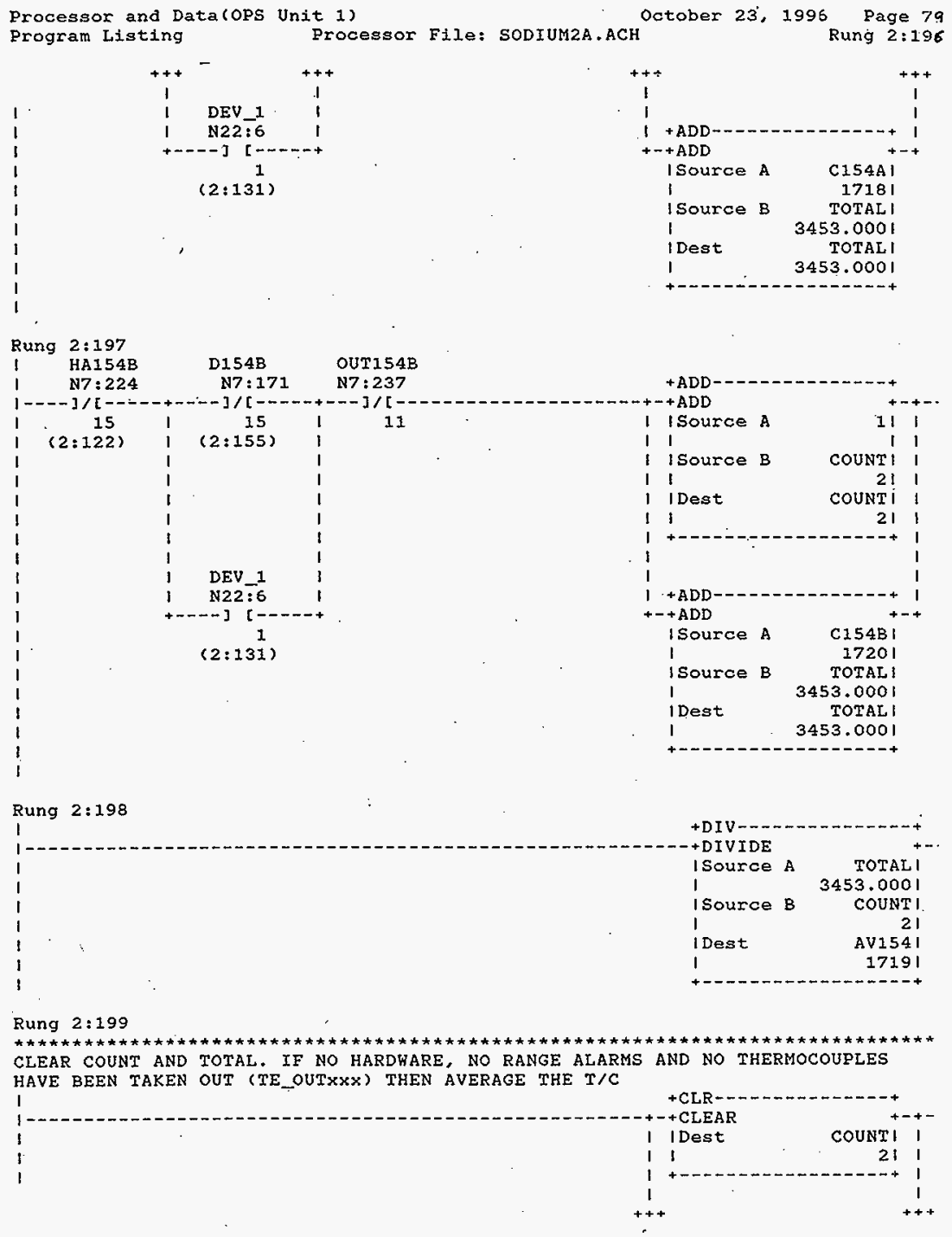

HNF-SD-FF-CSWD-61 R.ev. 0

Page 4 ifl 
Processor and Data(ops Unit 1)

October 23, 1996 Page 80

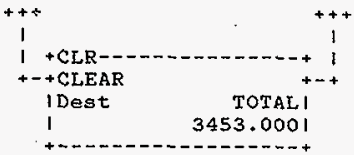

Rung 2:200
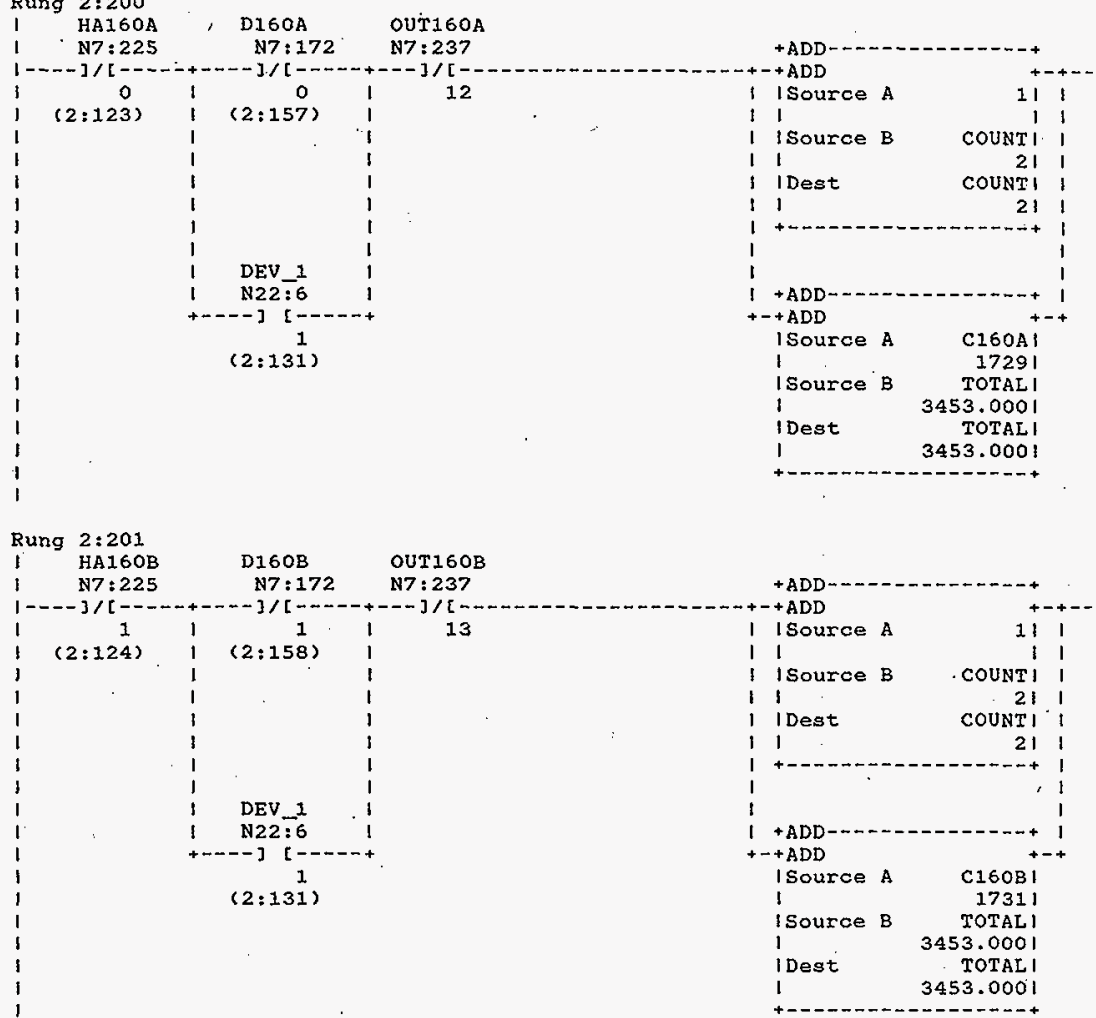

HNF-SD-FF-CSWD-61 Rev. 0

Page 442 


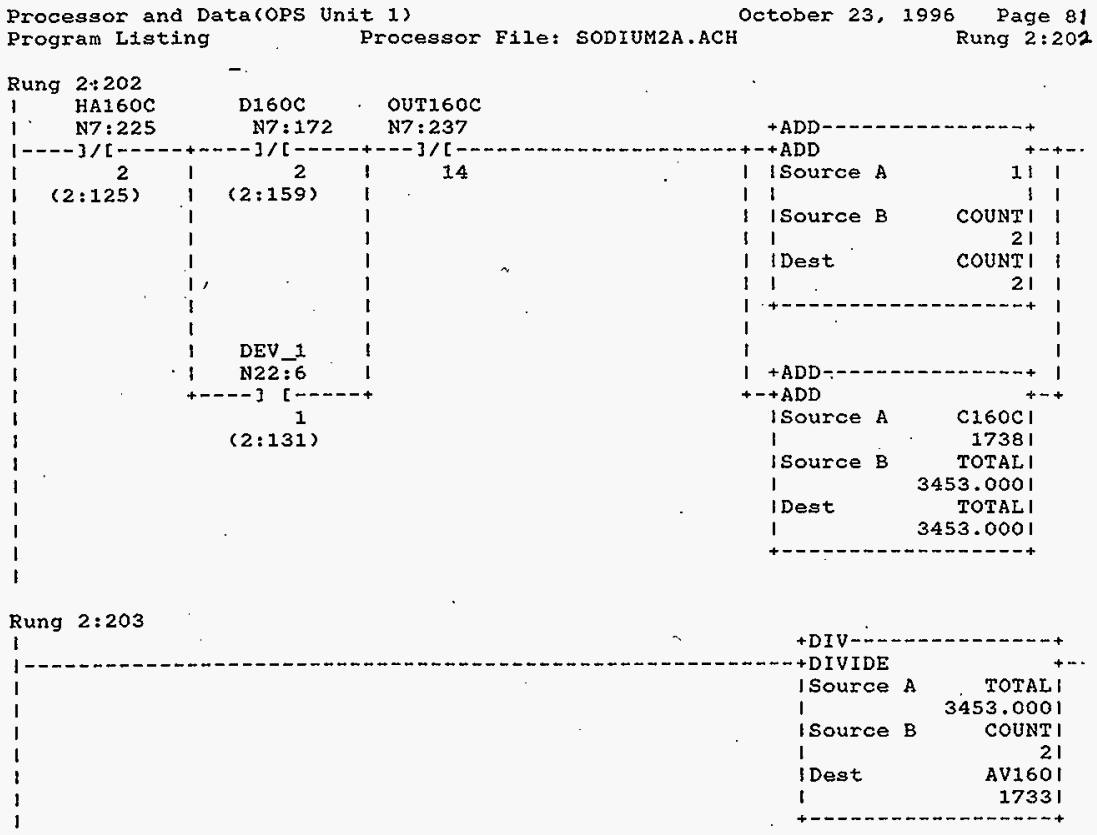

Rung $2: 204$

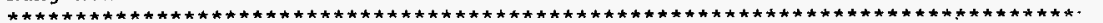
CLEAR COUNT AND TOTAL. IF NO HARDWARE, NO RANGE ALARMS AND NO THERMOCOUPLES HAVE BEEN TAKEN OUT (TE_OUTXXX) THEN AVERAGE THE T/C

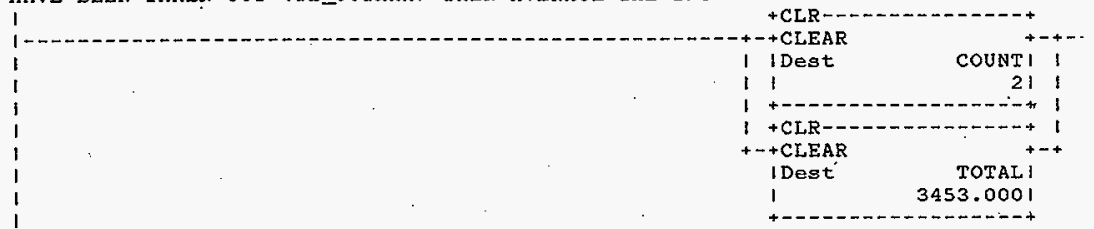

HNF-SD-FF-CSWD-61 Rev. 0

Page 443 
Processor and Data〈OPs Unit 1 )

October 23, 1996 Page 82 Program Listing Processor File: SODIUM2A.ACH

Rung 2:205
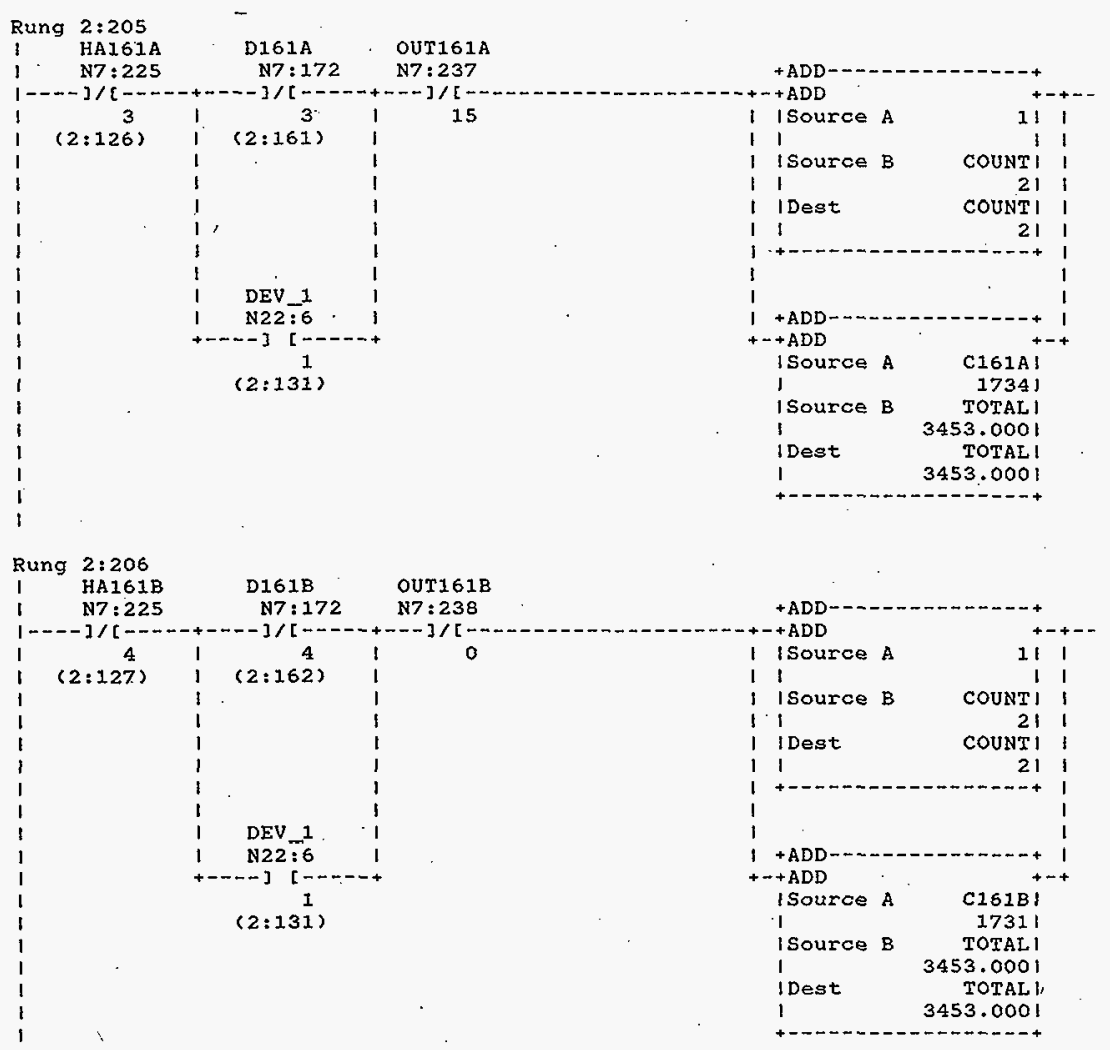

HNF-SD-FF-CSWD-61 Rev. 0

Page 444 
Processor and Data(ops Unit 1)

October 23, 1.996 Page 83 Program Listing

Processor File: SODIUM2A.ACH

Rung $2: 207$

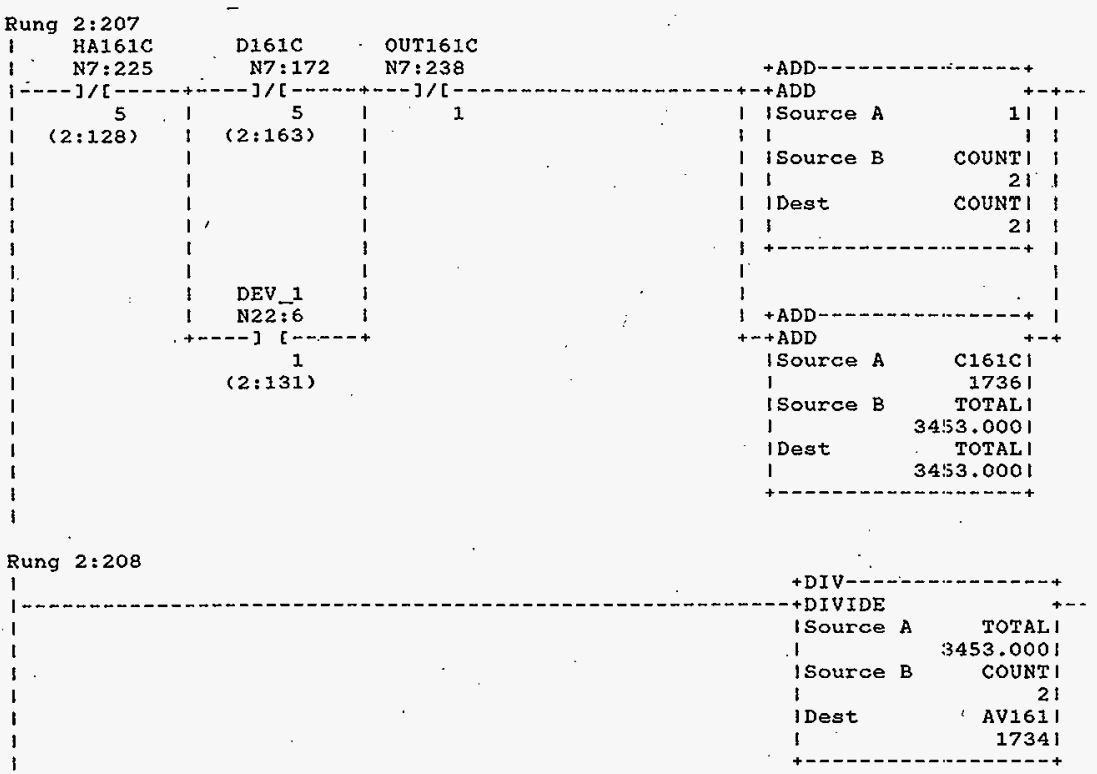

Rung 2:209

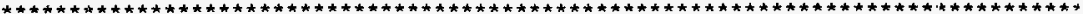
CLEAR COUNT AND TOTAL. IF NO HARDWARE, NO RANGE ALARMS AND NO THERMOCIJPLES HAVE BEEN TAKEN OUT (TE_OUT $X X X$ ) THEN AVERAGE THE T/C

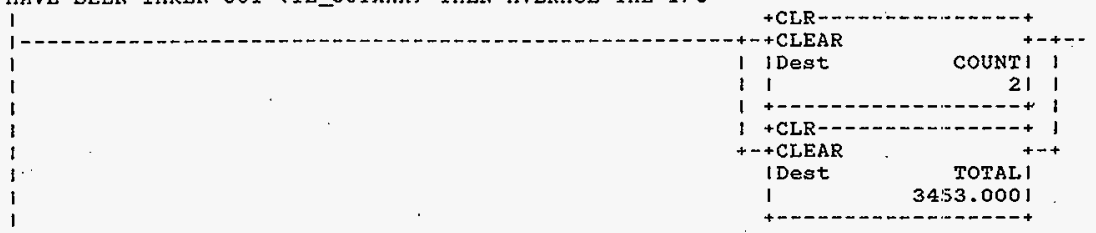

HNF-SD-FF-CSWD-61 Rev. 0

Page 445 
Processor and Data (OPS Unit 1)

Program listing Processor File: SODIUM2A.ACH

October 23, 1996 Page 84
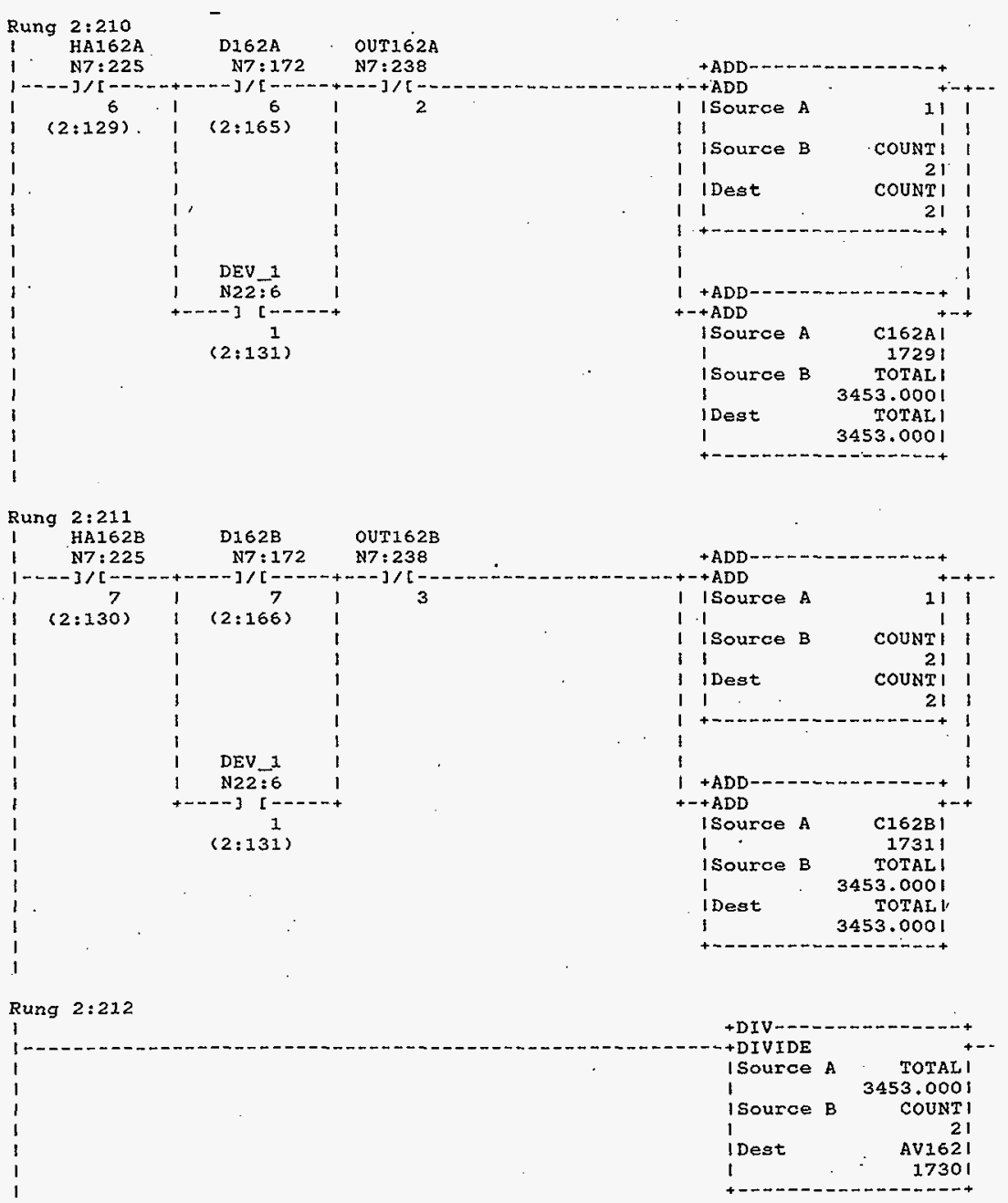

HNE-SD-FF-CSWD-61 Rev. 0

page 446 
Rung $2: 213$

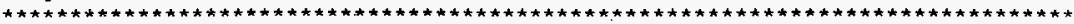
IF. DEVIATION SET FOR SECTION 1 THEN GO THERE AND CALCULATE DEVIATION

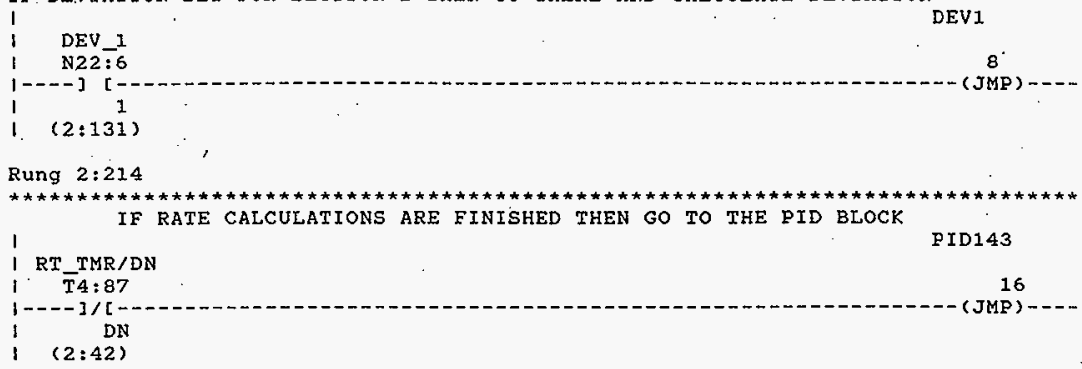

Rung 2:215

RATE CHECKING

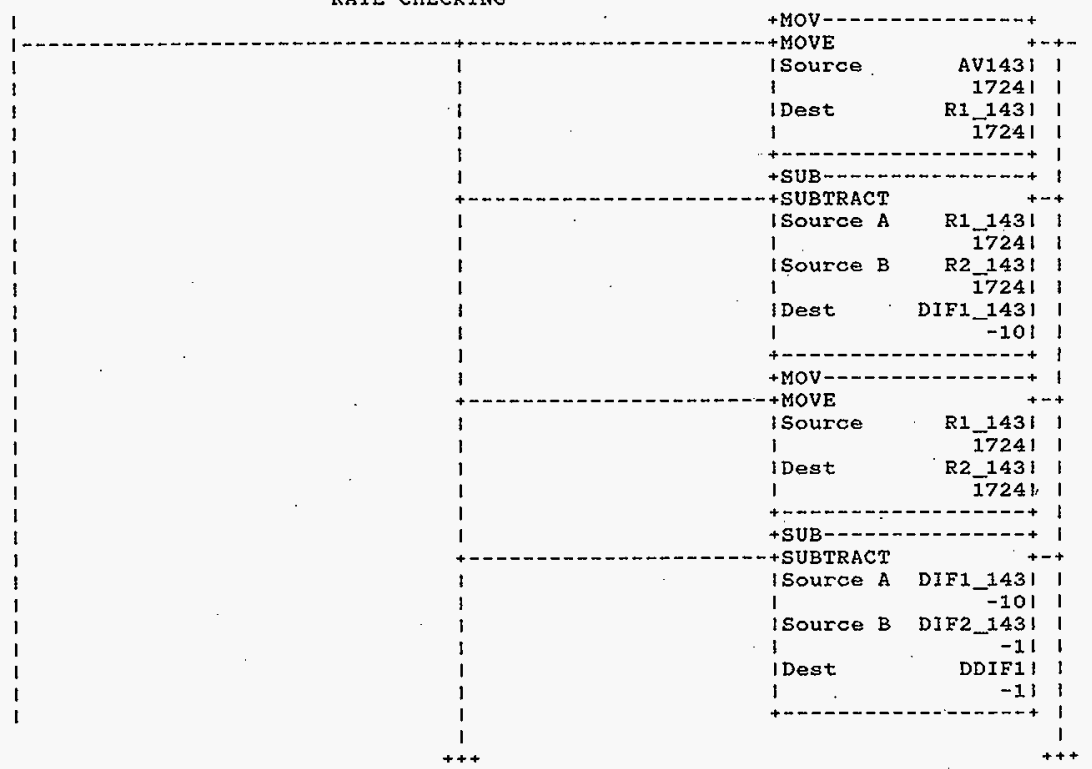

HNF-SD-FF-CSWD-6I Rev. 0 
Processor and Data(OPS Unit 1 ) Program Listing
Processor File: SODIUM2A.ACH
October 23,1996 Page 86 Rung $2: 215$

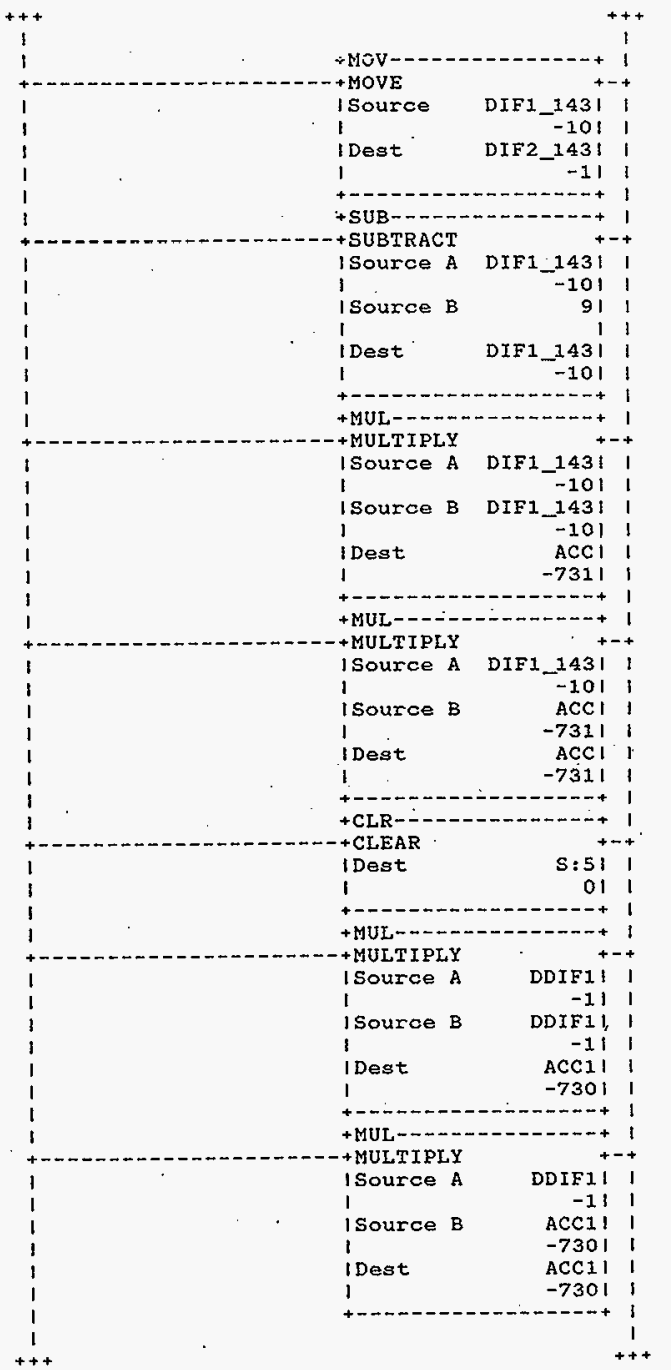

HNF-SD-FF-CSWD-61 Rev. 0 
Processor and Data(OPS Unit 1) Program Listing
Processor
October 23, 1996 Page 87 Rung $2: 215$

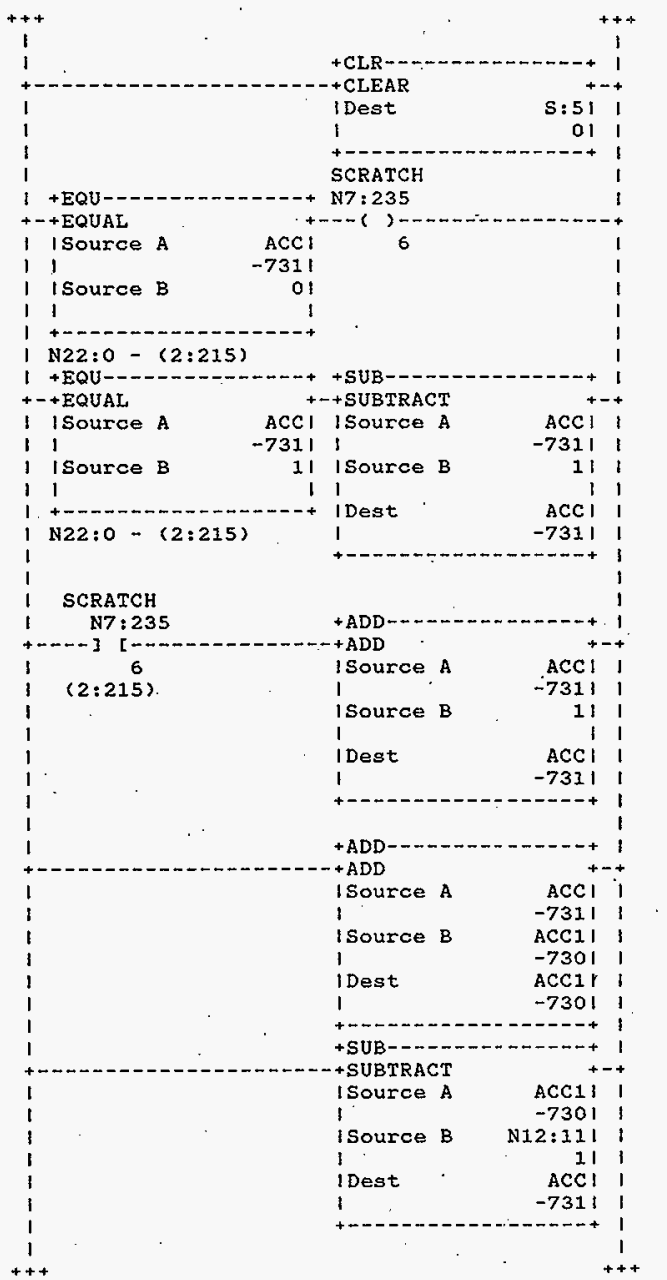

HNF-SD-FF-CSWD-61 Rev:0

Page 449 
Processor and Data (OPS Unit 1)

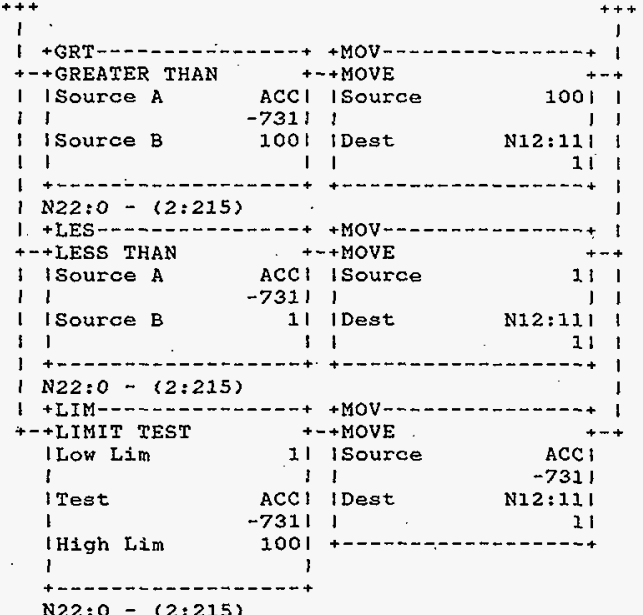

Rung $2: 216$

I PID 143

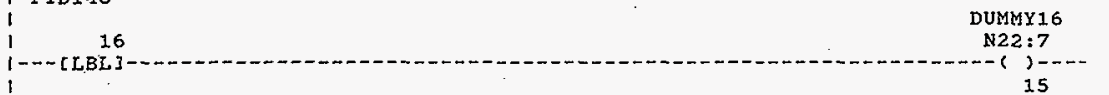

Rung $2: 217$

DETECT FROM SCADA PID ON/OFF

IF PID OFE

THEN ZERO PH FOR ZERO OUTPUT AT SCR

AND JUMP AROUND THE PID BLOCK

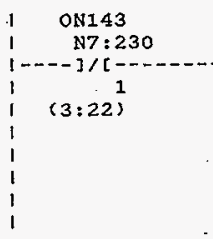

AMD JUMP AROUND THE PID BLOCK

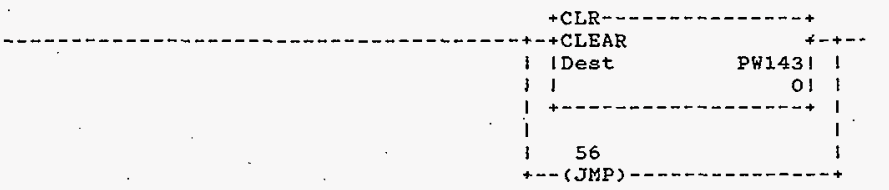

Rung $2: 218$

DETECT AUTO/MANUAL FROM SCADA

SET APPROPRIATE MODE IN PID BLOCK

AM143

N7: 230

PID143/1

N12:0

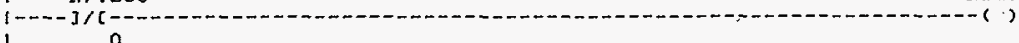

$(3: 22)$

HNF-SD-FF-CSWD-61 Rev. 0 
Processor and Data (OPS Unit 1)

Rung $2: 2 \pm 9$

DETECT SETPOINT VALUE FROM SCADA

PUT VALUE IN PID BLOCK

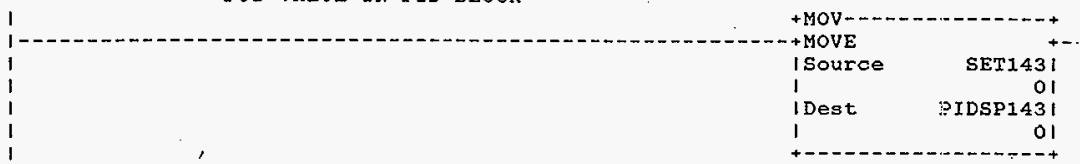

Rung $2: 220$

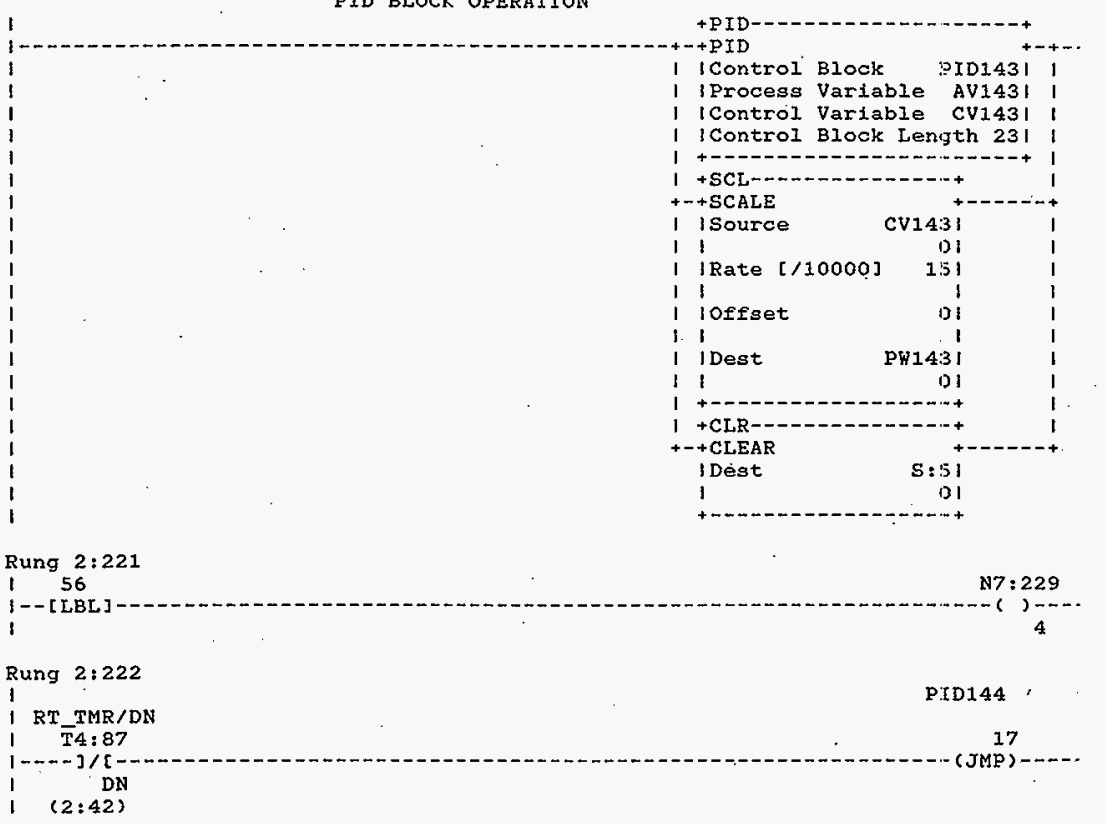

HNF-SD-FF-CSWD-6I Rev. 0 
Processor and Data(OPS Unit 1) Program Listing Processor File: SODIUM2A.ACH
October 23,1996

Page 90 Rung $2: 223$

\section{Rung $2: 223$.}

RATE CHECKING

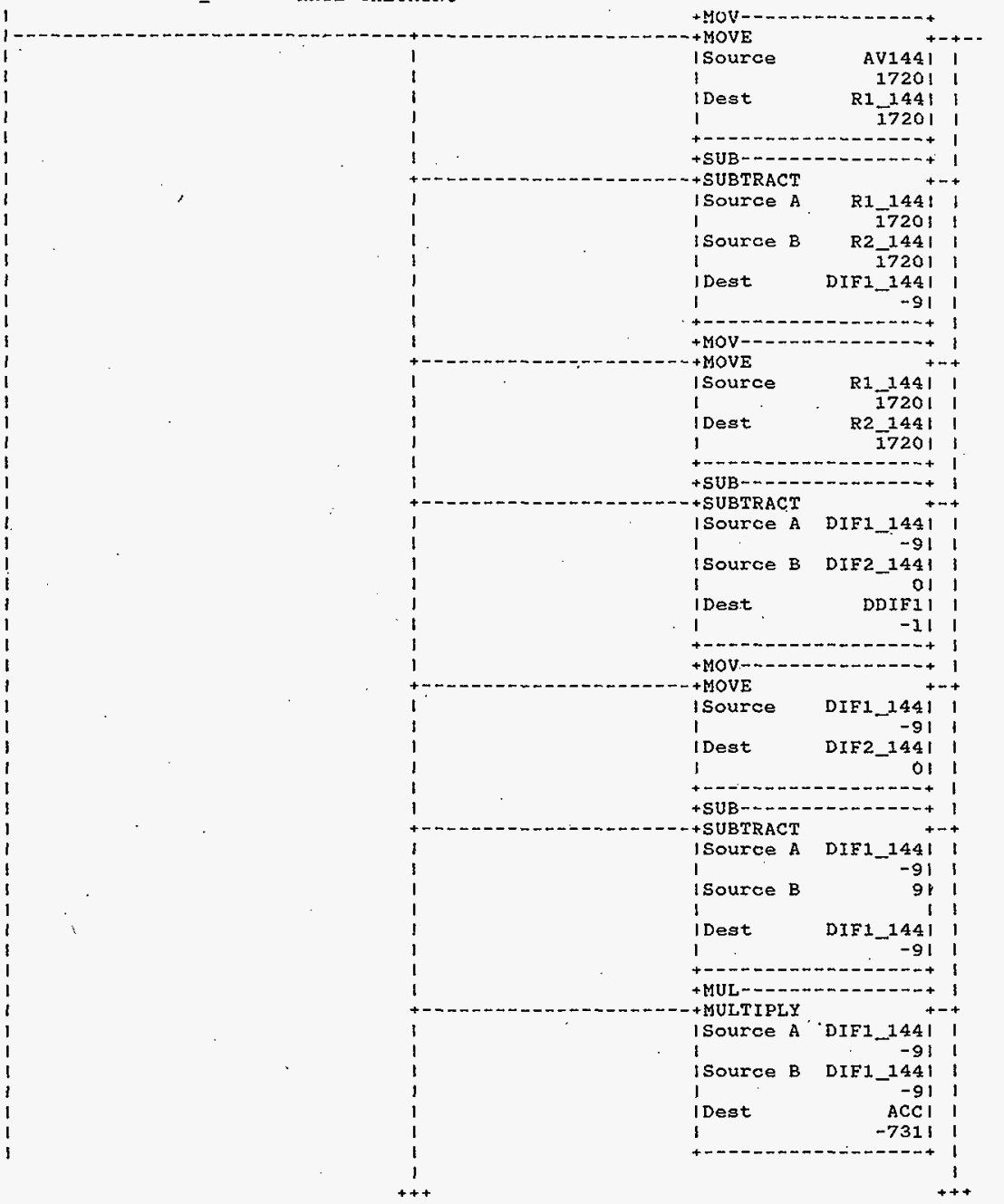

HNF-SD-FF-CSWD-61 Rev. 0 


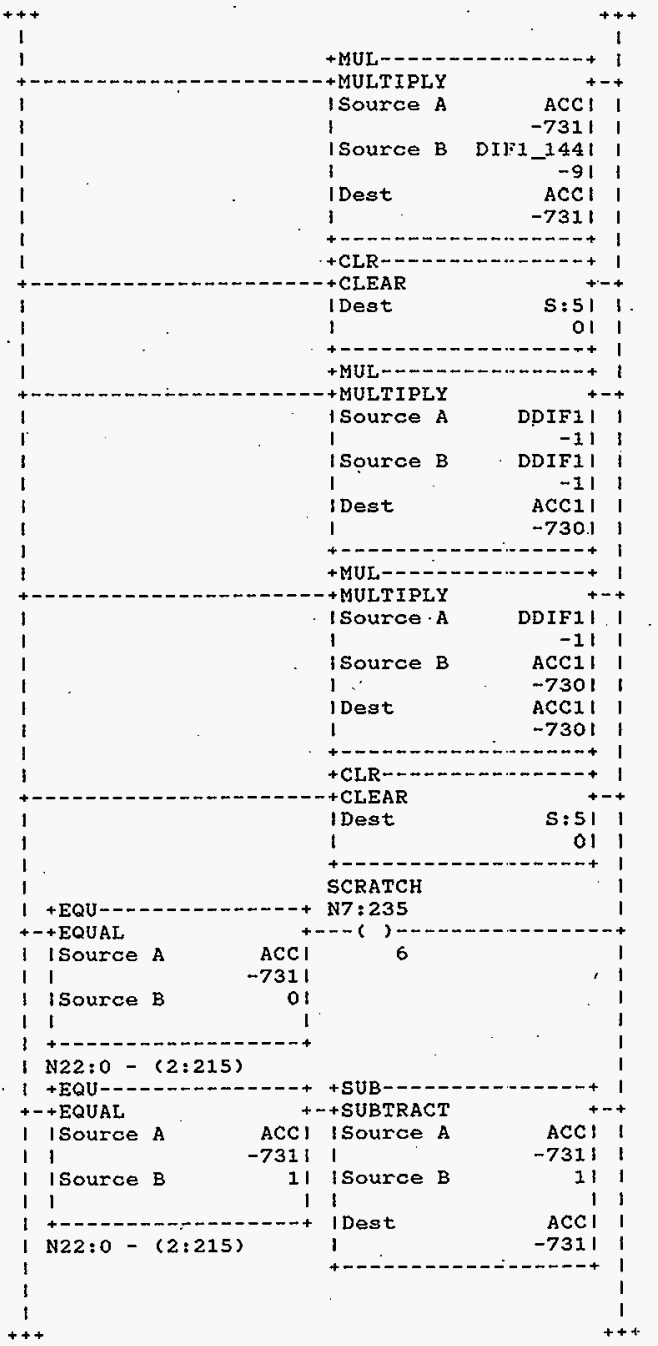

HNF-SD-FF-CSWD-61 Rev. 0

Page 453 
Processor and Data(OPS Unit 1)

October 23, 1996 Page 92

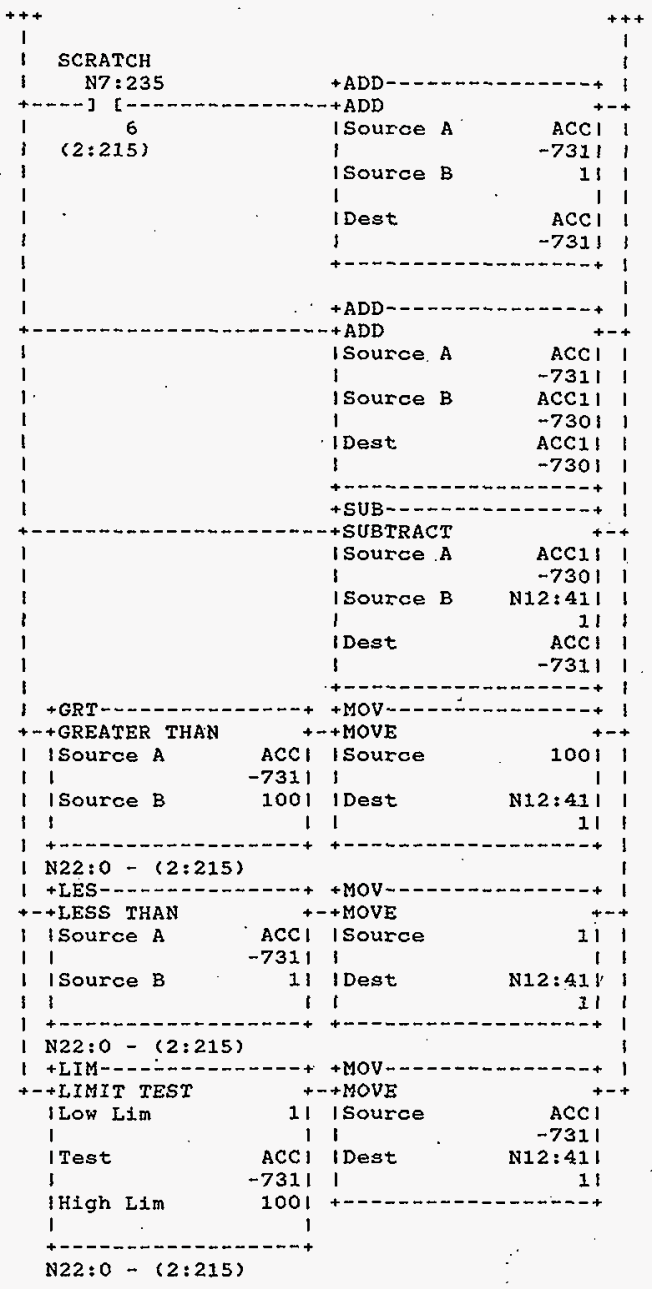

HNF-SD-FF-CSWD-61 Rev. 0

page 454 
Processor and Data(OPS Unit I)

Rung $2: 224$

1 PID144

1.

$1--[$ LBL $]$

Rung $2: 225$

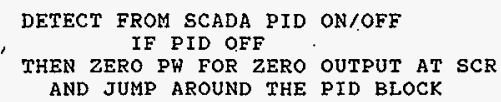

Rung 2:226

DETECT AUTO/MANUAL FROM SCADA

SET APPROPRIATE MODE IN PID BLOCK

AM1 144
N7 $: 230$
1,2

Rung 2:227

DETECT SETPOINT VALUE FROM SCADA PUT VALUE IN PID BLOCK

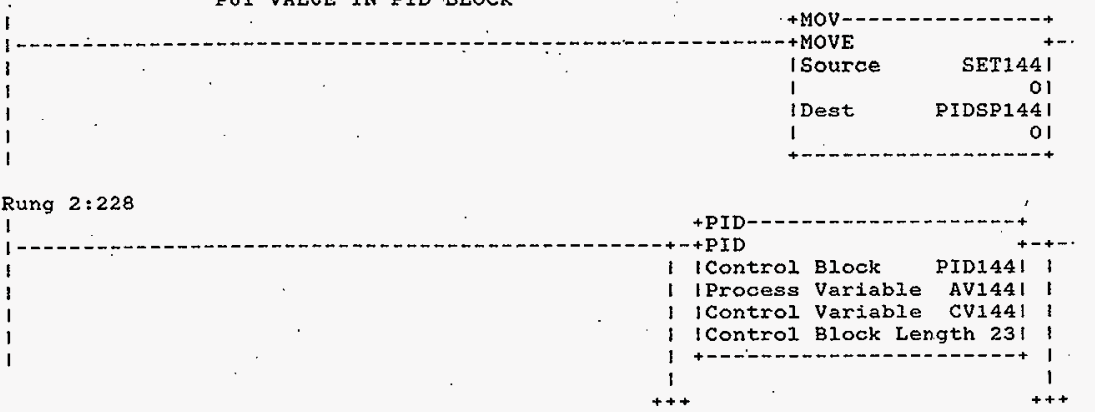

HNF-SD-FF-CSWD-61 Rer. 0

Page 455 
Processor and Data(OPS Unit 1)

October 23, 1996 Page 94

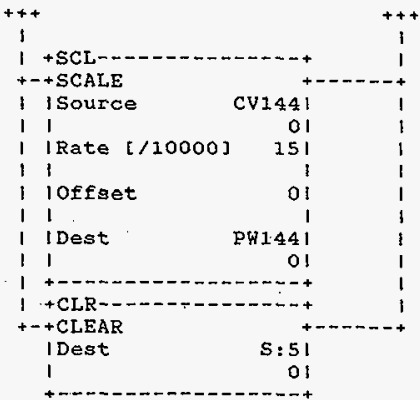

Rung 2:229

$$
157
$$

Rung $2: 230$

I

i RT_TMR/DN

1 T4:87

I- - - - ] / [ -

1 (2:42)

Rung 2:231

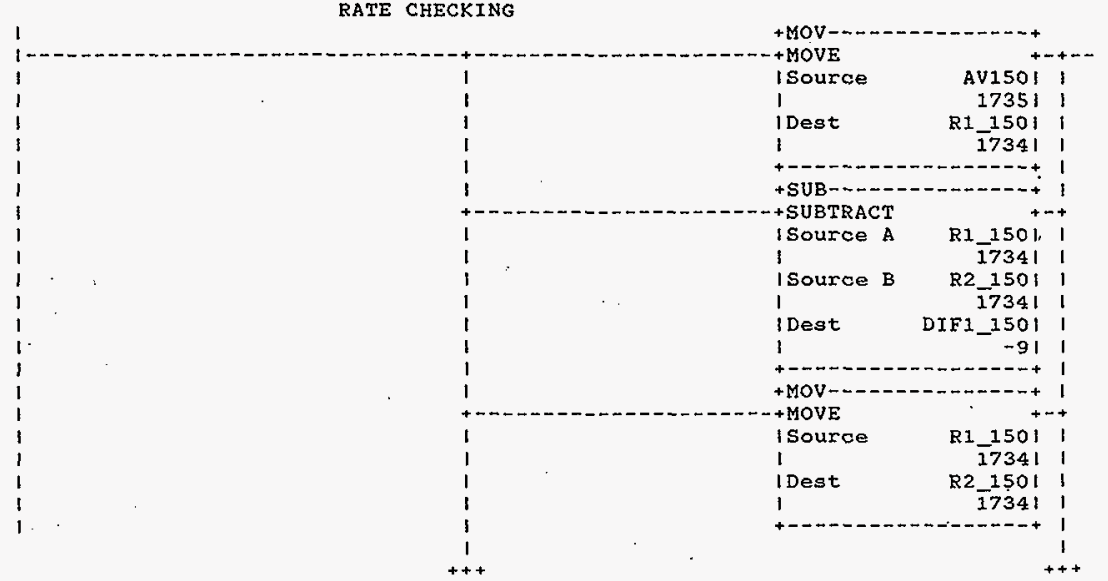

HNF-SD-FF-CSWD-61 Rev. 0

Page 456 


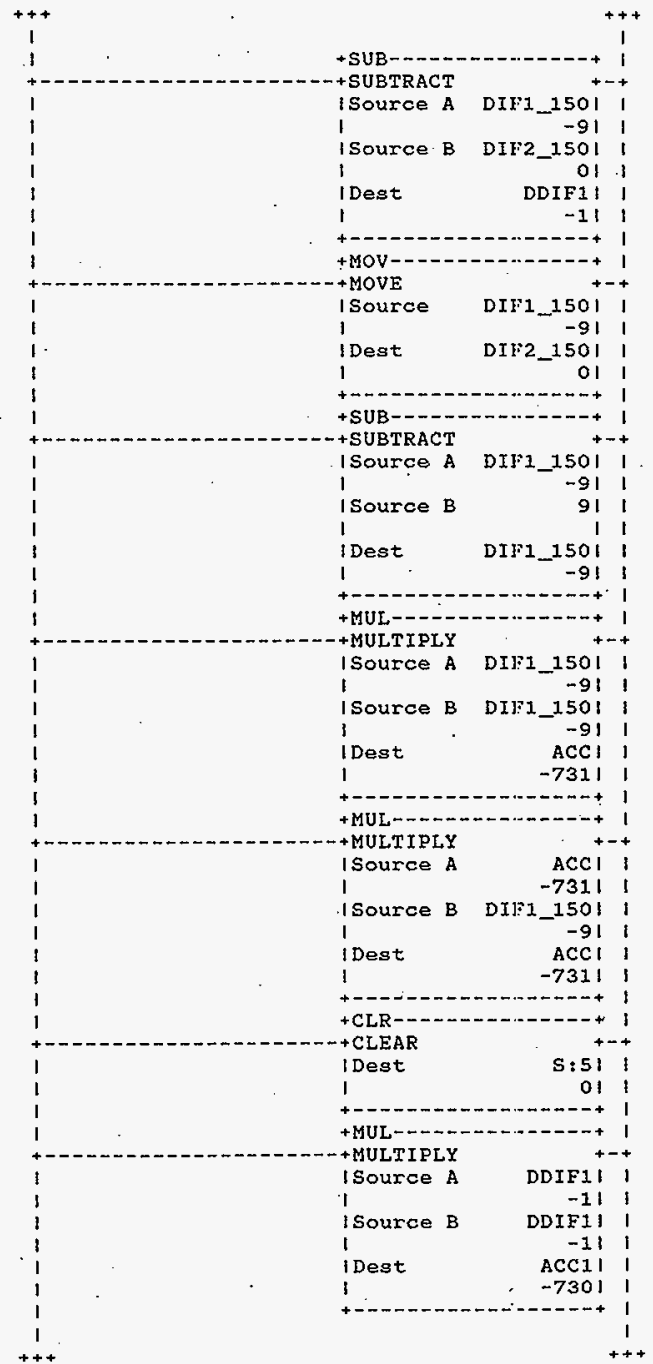

HNF-SD-FF-CSWD-61 Rev. 0 
Processor and Data(OPS Unit 1)

October 23, 1996 Page 96

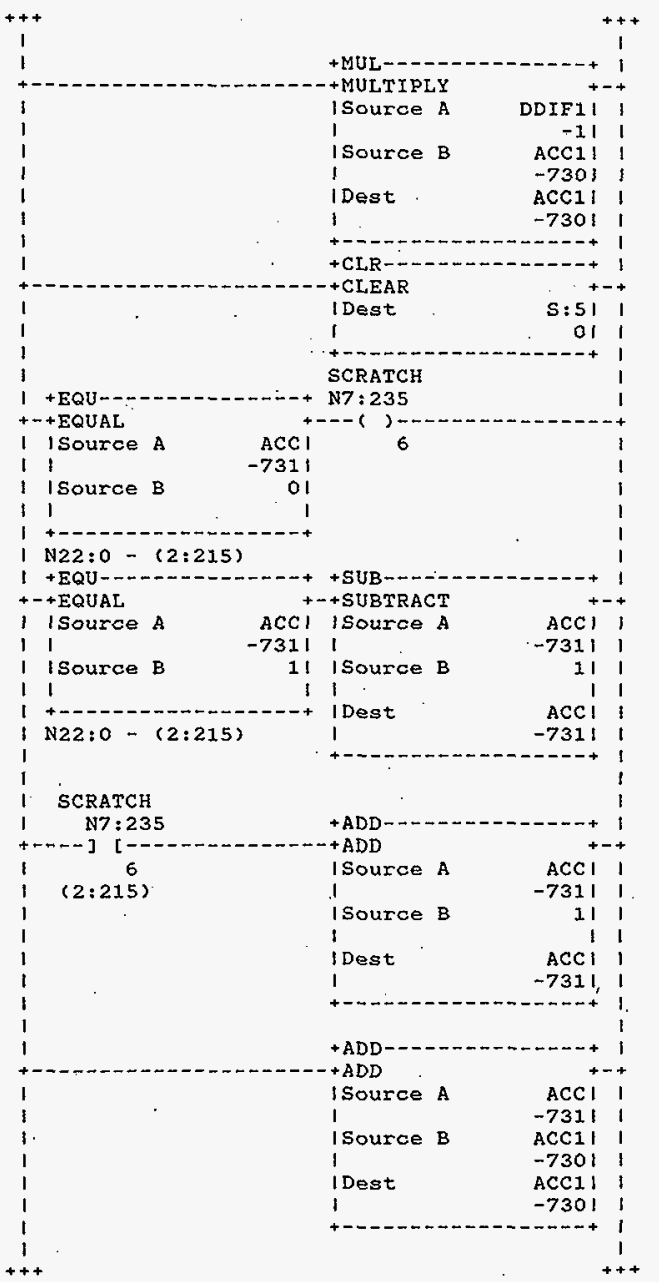

HNF-SD-FF-CSWD-61 Rev. 0

Page 458 


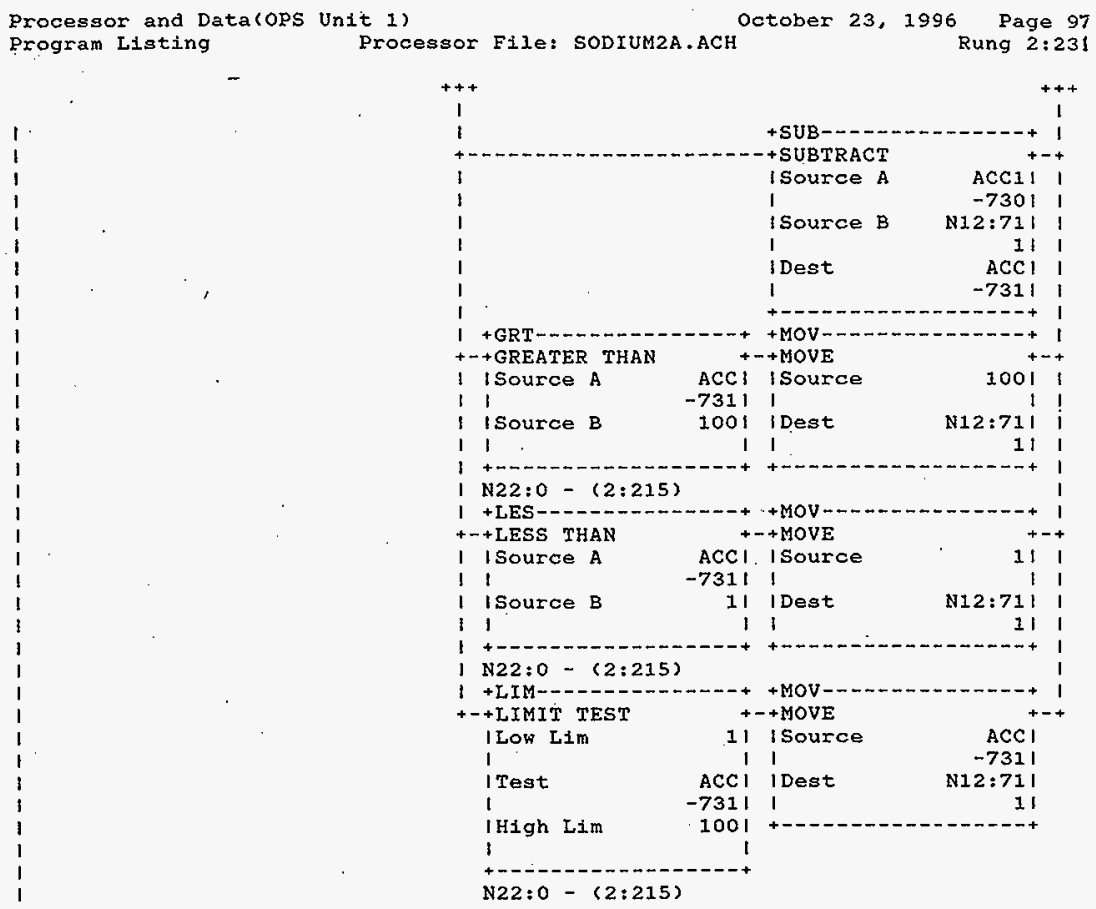

Rung '2:232

I PID150

DUMMY 18

18

N22: 8

$1--[$ LBRL

Rung $2: 23.3$

DETECT FROM SCADA PID ON/OFF

IF PID OFF

THEN ZERO PW FOR ZERO OUTPUT AT SCR

AND JUMP AROUND THE PID BLOCK

\section{ON150}

i N7: 230

$(3: 22)$

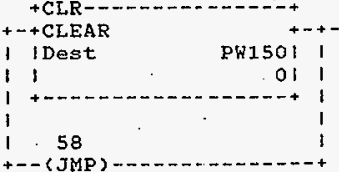

HNF-SD-FF-CSWD-61 Rev. 0

Page 459 

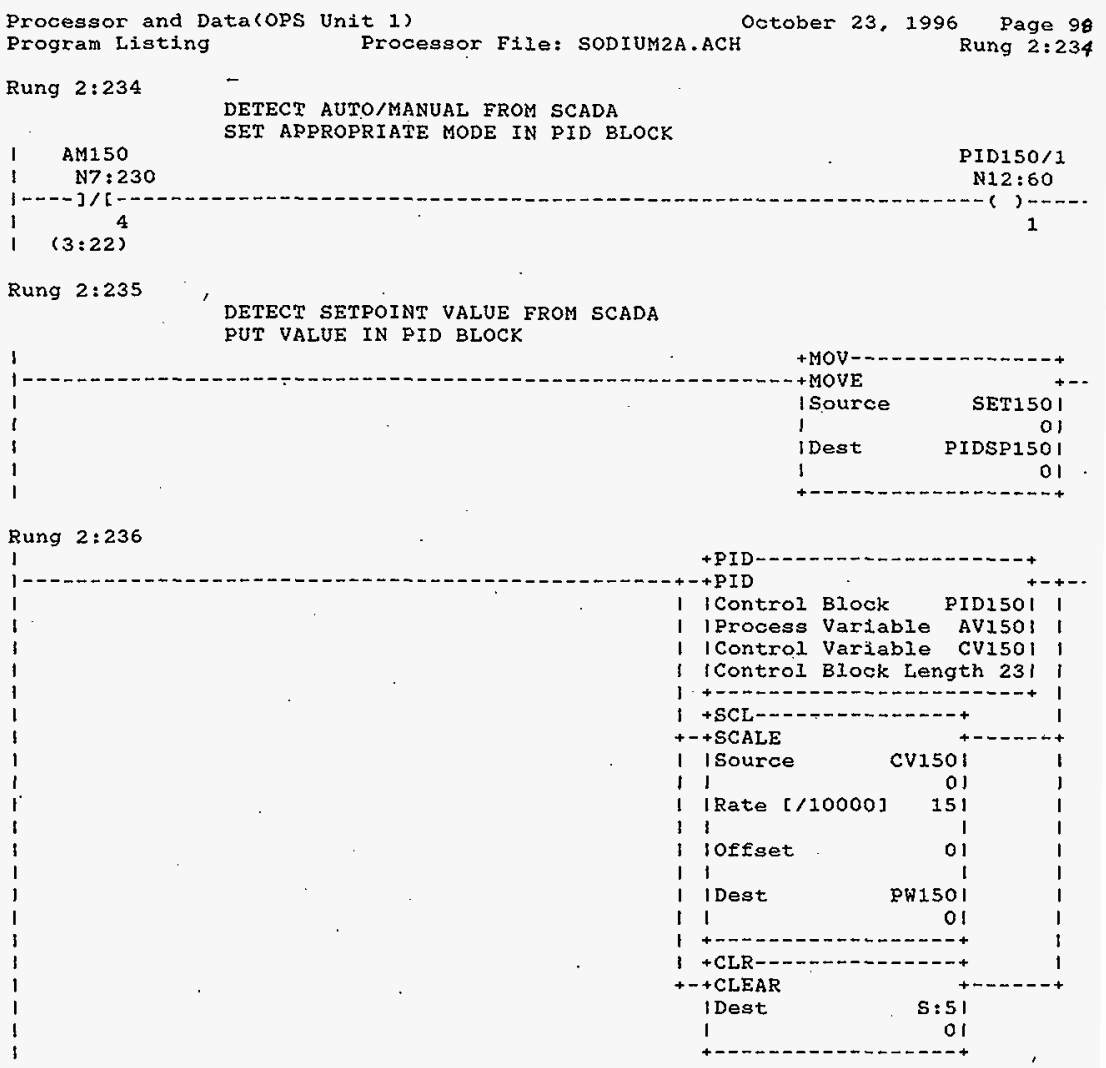

Rung $2: 237$

158

N7 : 229

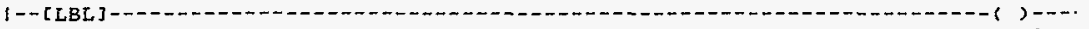

I

6.

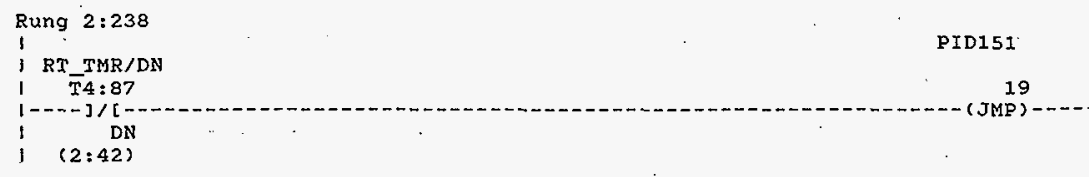

HNF-SD-FF-CSWD-61 Rev. 0

Page 460 
Processor and Data(OPS Unit I)

October 23, 1996 Page 99 Program Listing

Rung 2:239

RATE CHECKING

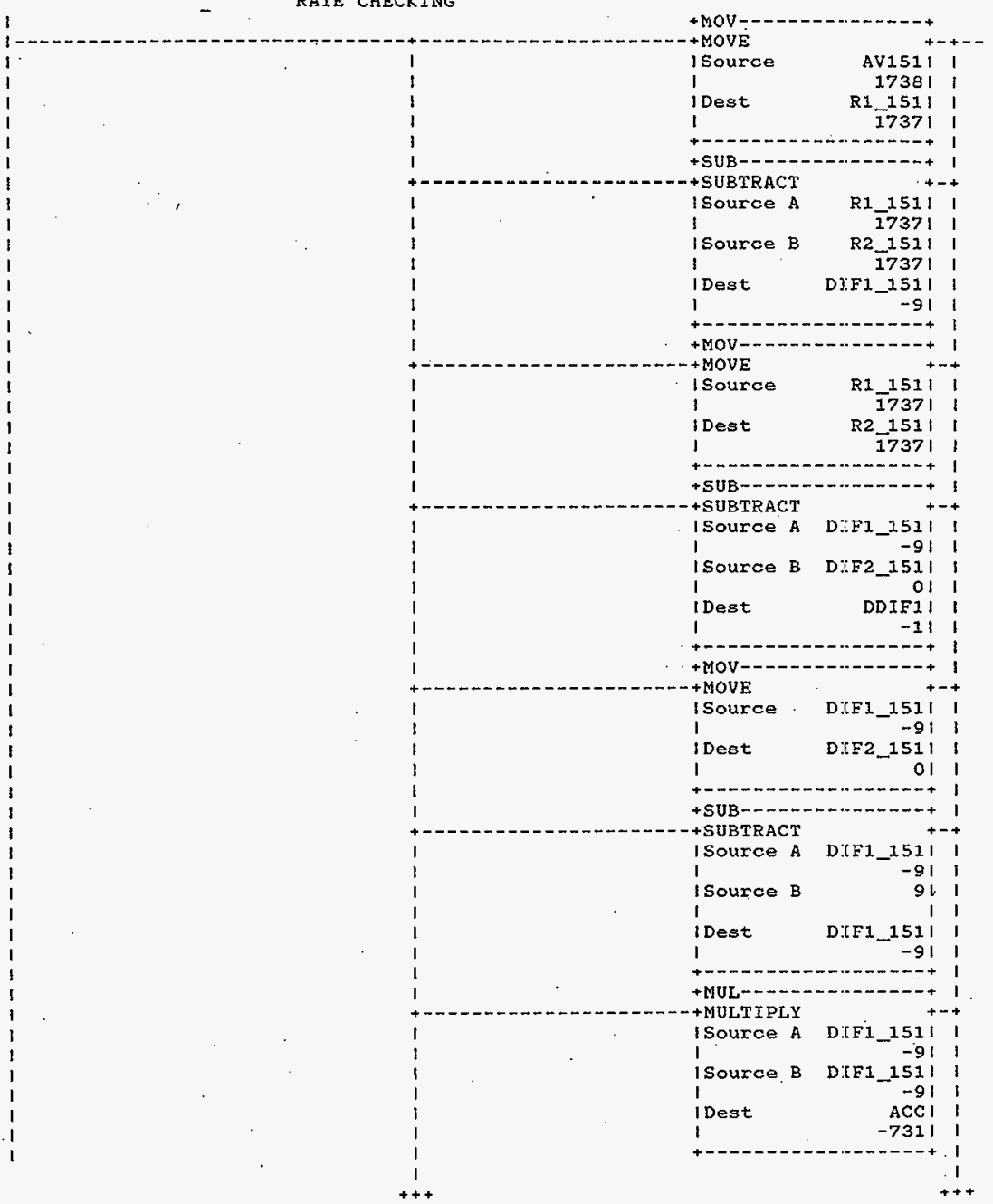

HNF-SD-FF-CSWD-61 Rev. 0 


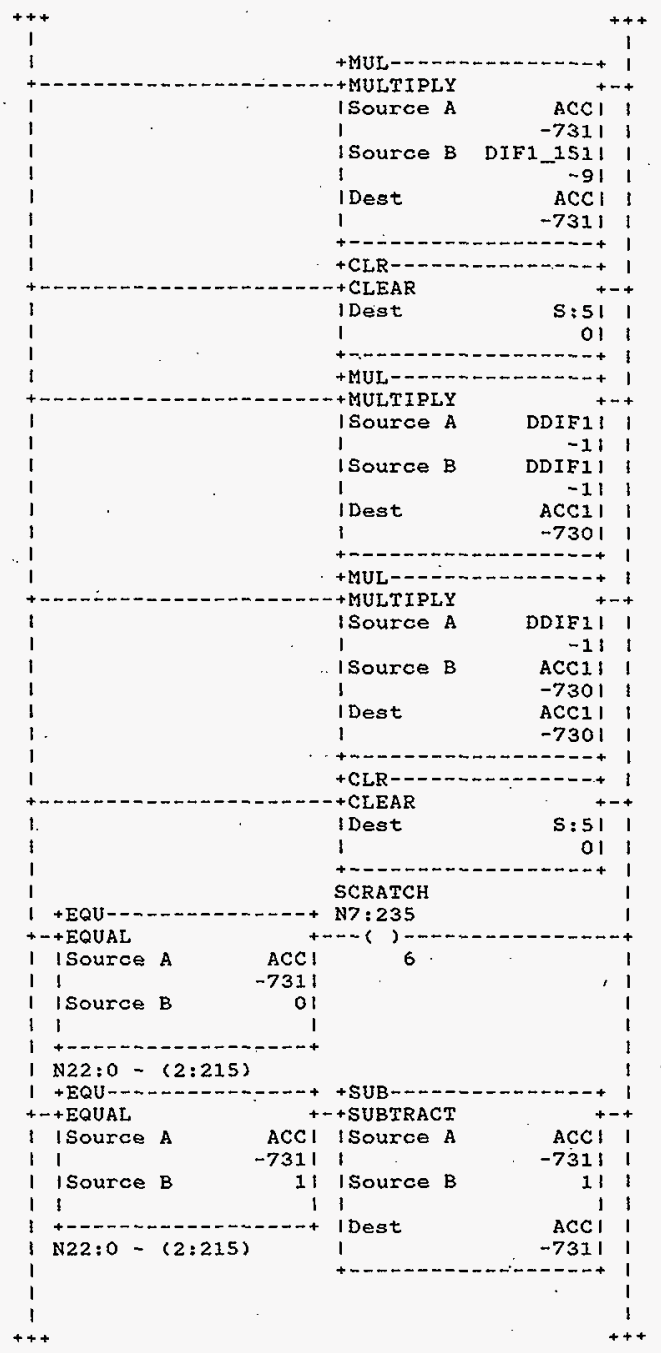

HNF-SD-FF-CSWD-61 Rev. 0 


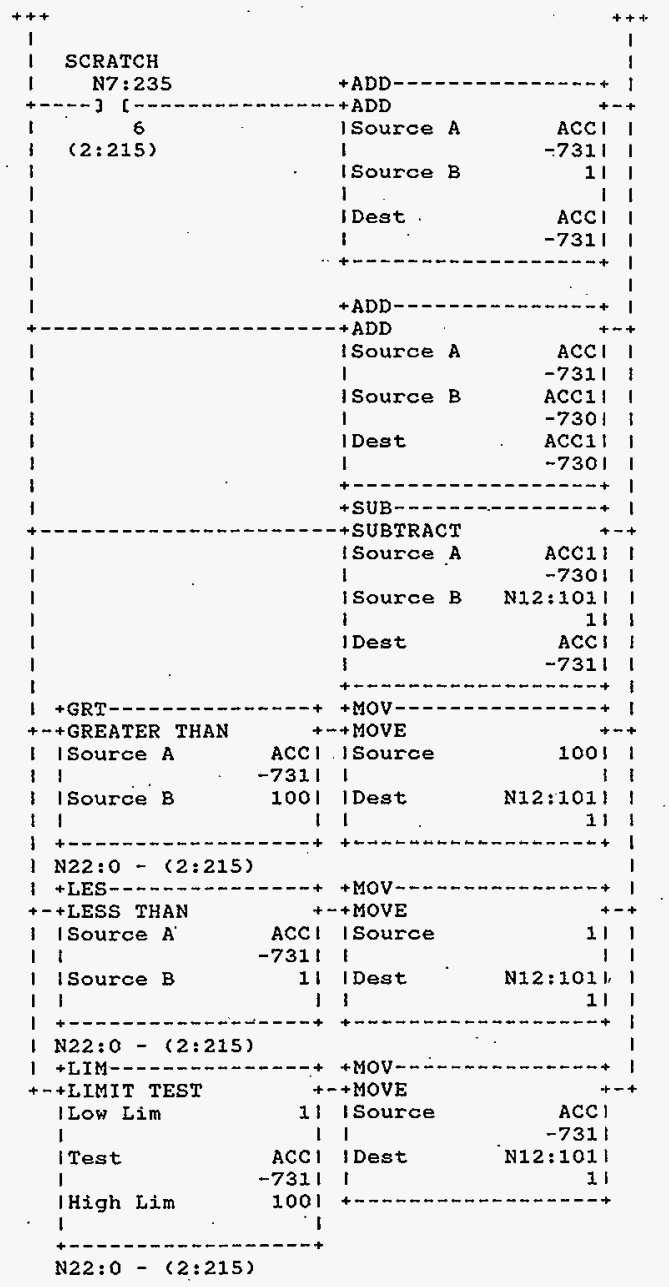

HNF-SD-FF-CSWD-61 Rev. 0

Page 463 
Rung 2:240

I PID 151
I

DUMMY 19

N22: 8

Rung 2:241

\section{DETECT FROM SCADA PID ON/OFF \\ IF PID OFF \\ THEN ZERO PH FOR ZERO OUTPUT AT SCR \\ AND JUMP AROUND THE PID BLOCK}

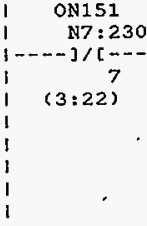

Rung $2: 242$

DETECT AUTO/MANUAL FROM SCADA

SE'T APPROPRIATE MODE IN PID BLOCK

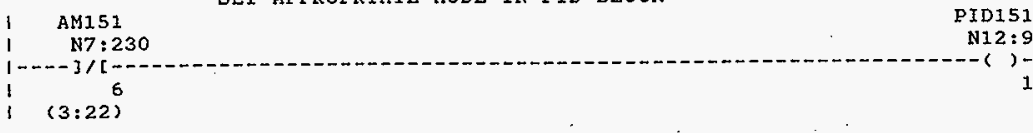

PID151/1

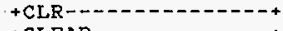
+CLEA

PW151!

I IDest

ol

59

Rung $2: 243$

DETECT SETPOINT VALUE FROM SCADA

PUT VALUE IN PID BLOCK

1
1
1
1
1

Rung 2:244

1
1
1
1
1

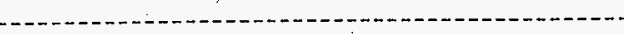

HNF-SD-FF-CSWD-61 Rev. 0

Page 464 


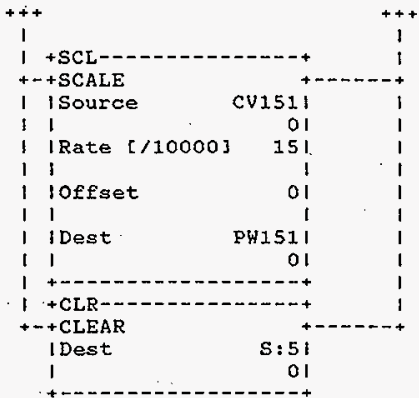

Rung $2: 245$

159

N7 : 229

1

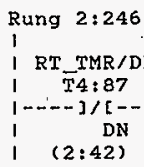

Rung $2: 247$

RATE CHECKING

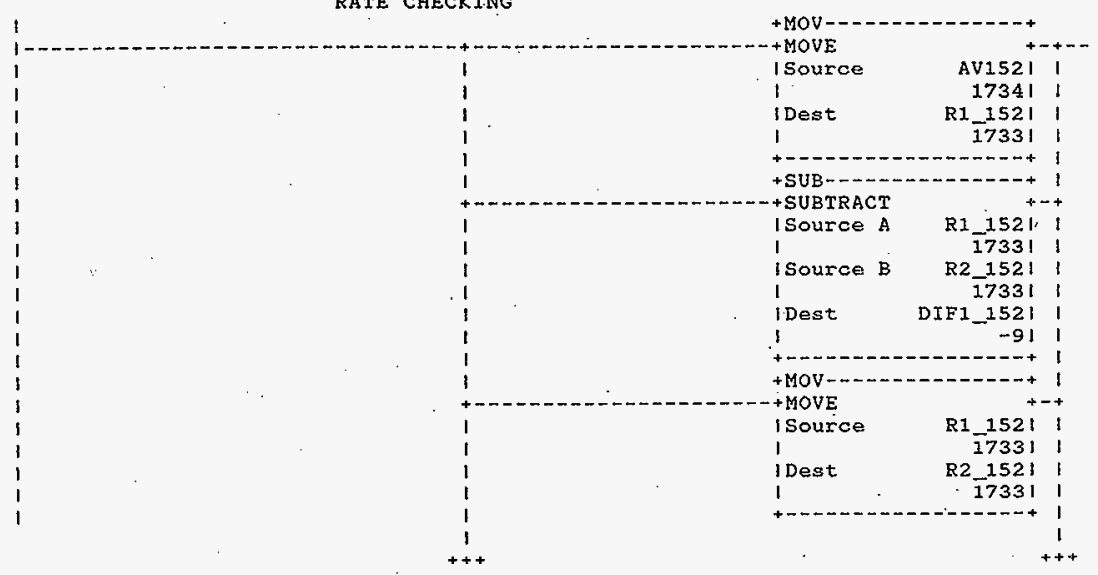

HNF-SD-FF-CSWD-61 Rev. 0 


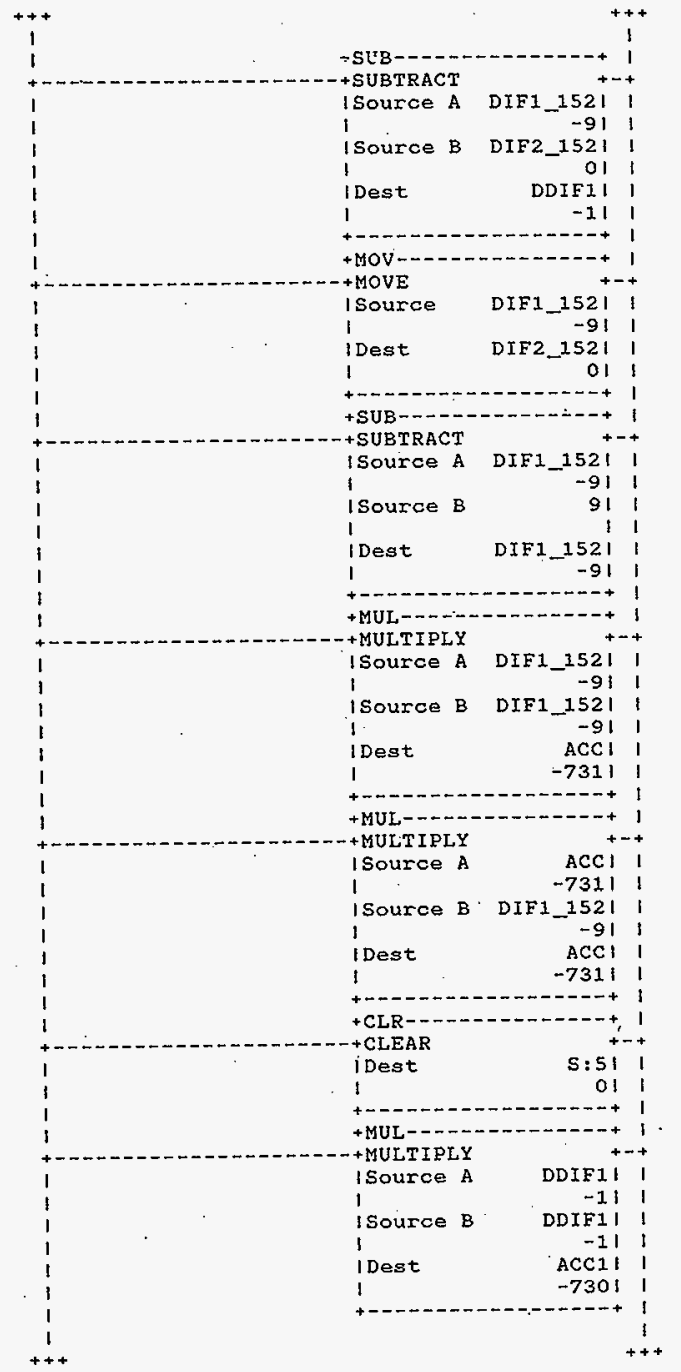

HNF-SD-FF-CSWD-61 Rev. 0 


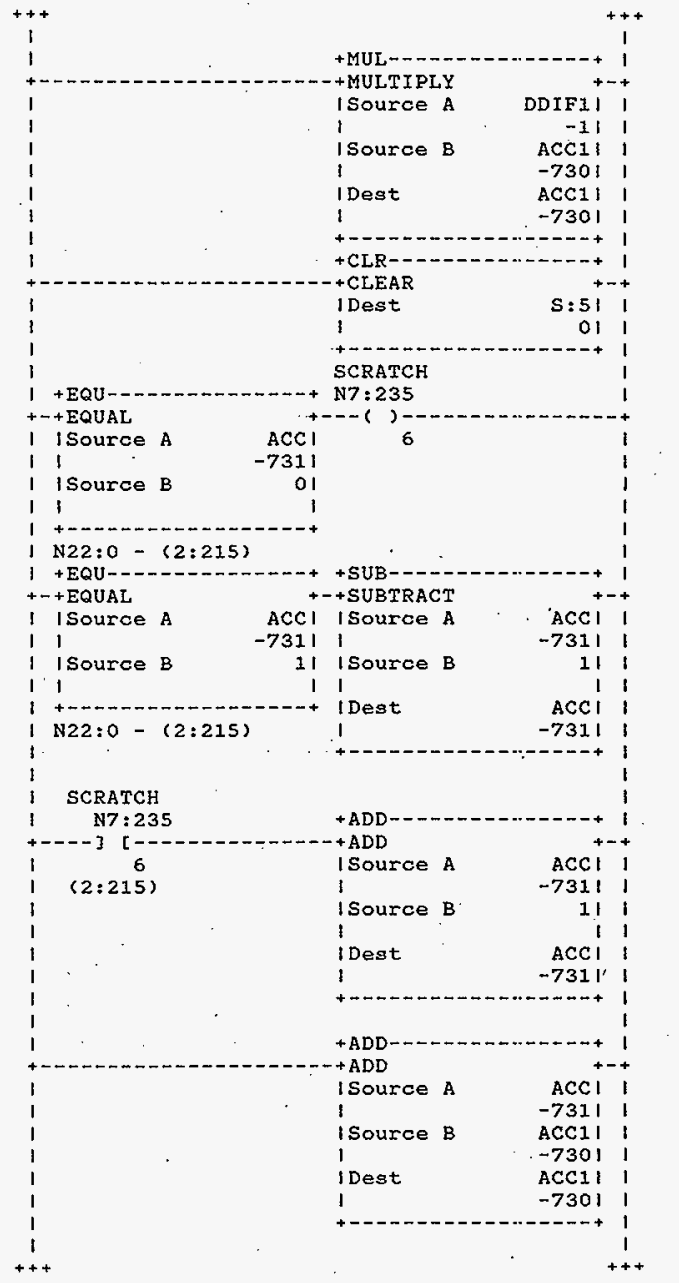

HNF-SD-FF-CSWD-61 Rev. 0 
Processor and Data(OPS Unit 1) Program Listing
October 23,1996 Processor File: SODIUM2A.ACH
Page 106

Rung $2: 247$

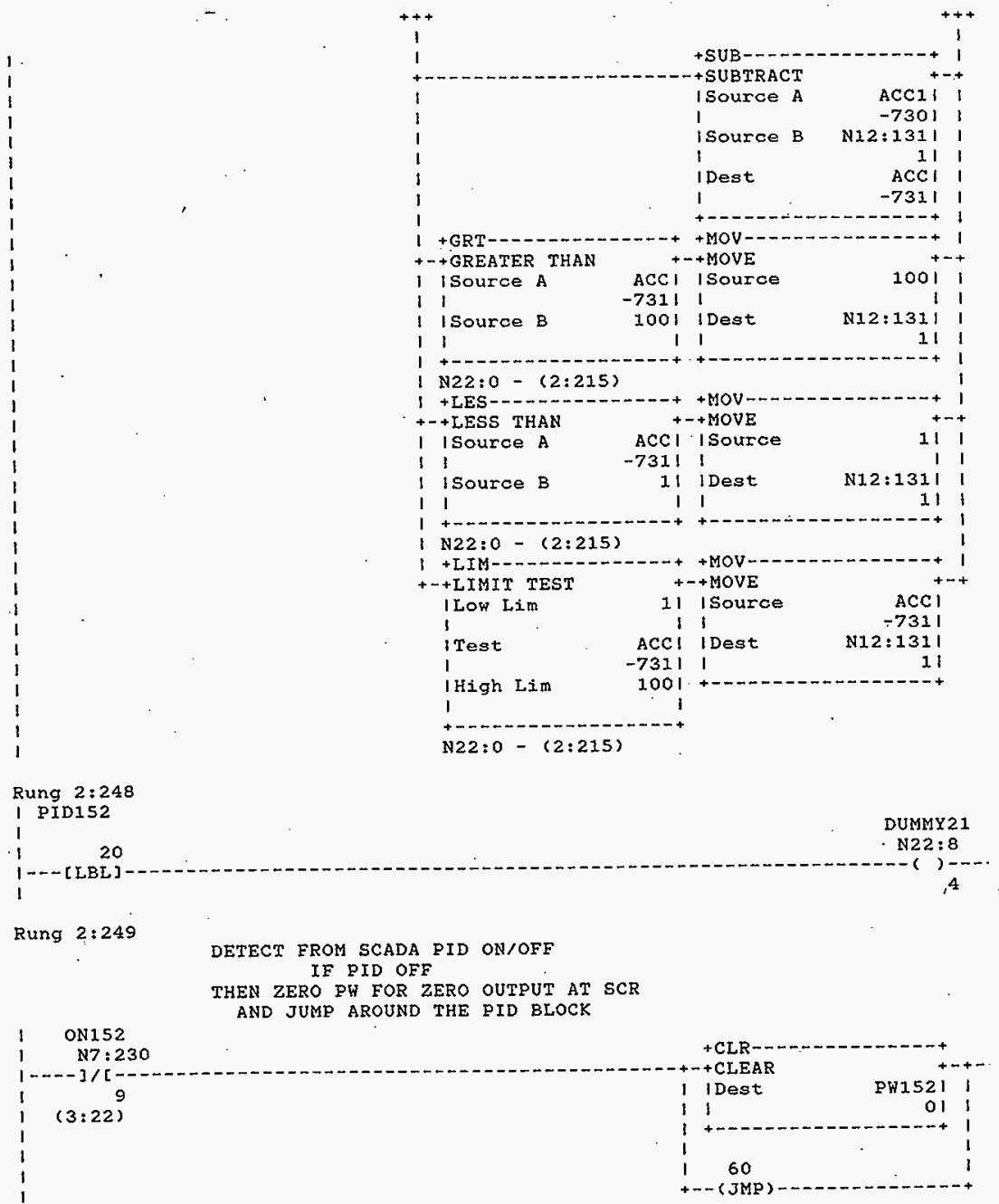

HNF-SD-FF-CSWD-61 Rev. 0

Page 468 
Processor and Data(OPS Unit 1 )

Program Listing

Processor File: SODIUM2A.ACH

October 23, 1996 Page 107

Rung $2: 250$

$-$

DETECT AUTO/MANUAL FROM SCADA

SET APPROPRIATE MODE IN PID BLOCK

I AM152

I N7:230

PID152/1

$1 \ldots-2] 1[-$

N12:120

I (3:22)

Rung 2:251 , DETECT SETPOINT VALUE FROM SCADA PUT VALUE IN PID BLOCK

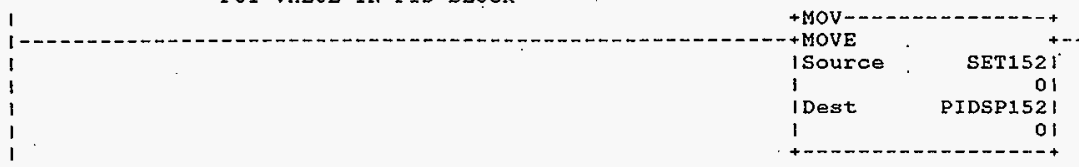

Rung $2: 252$

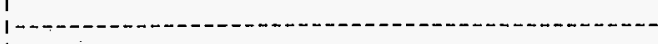

$+P I D$

l l Control Block PID152J |

| |Process Variable AV152| |

1 Control Variable cV152l

f. Control Block Length 231 |

I +----

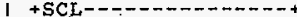

+-+ SCALE

I ISOurce CV152I

11

I IRate $[/ 10000] 15$

11

1 loffset

11

1 i Dest

1 IDest $\quad$ PH152

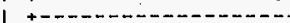

I +CLR---------------+

+-+ CLEAR

I Dest

$S: 51$

I

01

Rung $2: 253$

160

N7 : 229

$1--[$ [ BL $]$

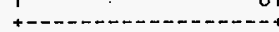

Rung 2:254

I

I RT_TMR/DN

I T4:87

$1-\cdots-3 /[-$

I. (2:42)

HNF-SD-FF-CSWD-61 Rev. 0

Page 464 
Processor and Data(OPs Unit 1)

Rung 2:255

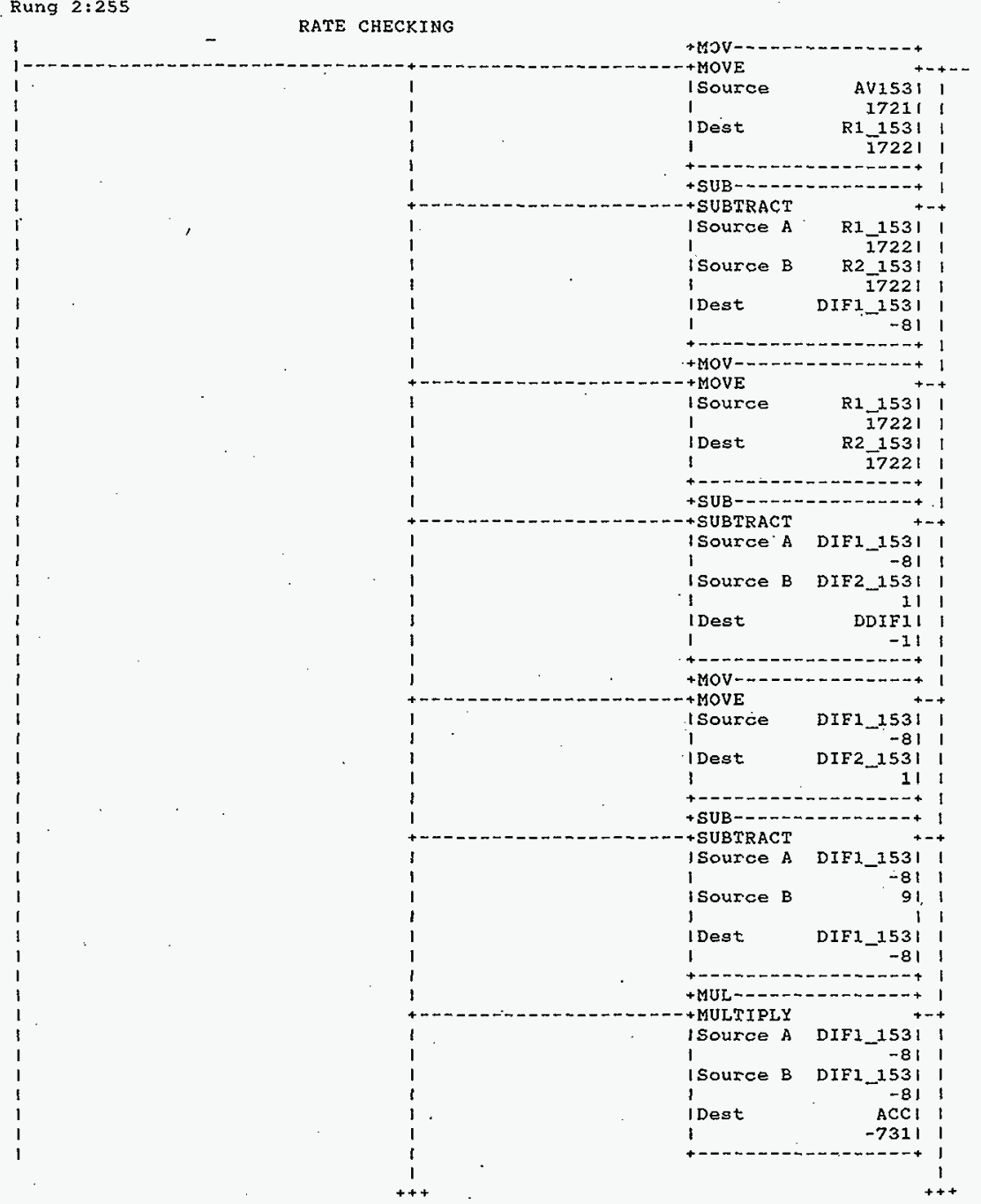

HNF-SD-FF-CSWD-61 Rev. 0 


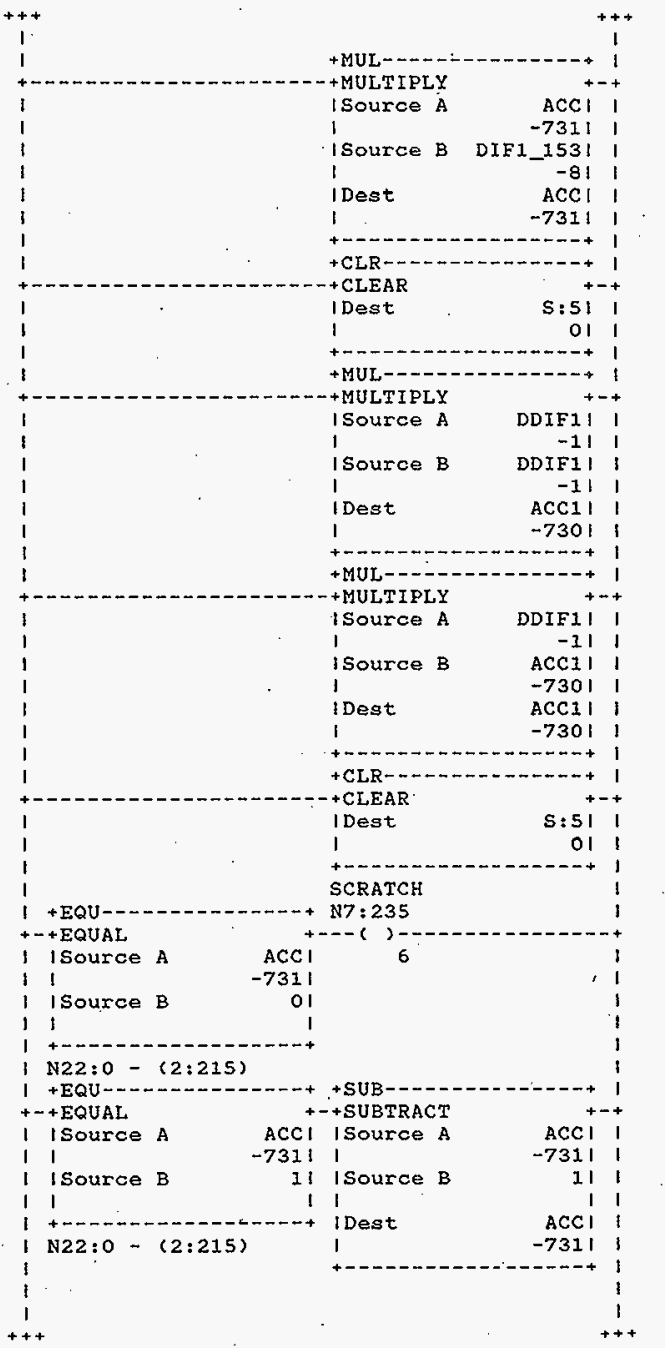

HNF-SD-FF-CSWD-61 Re\% 0

Page 471 


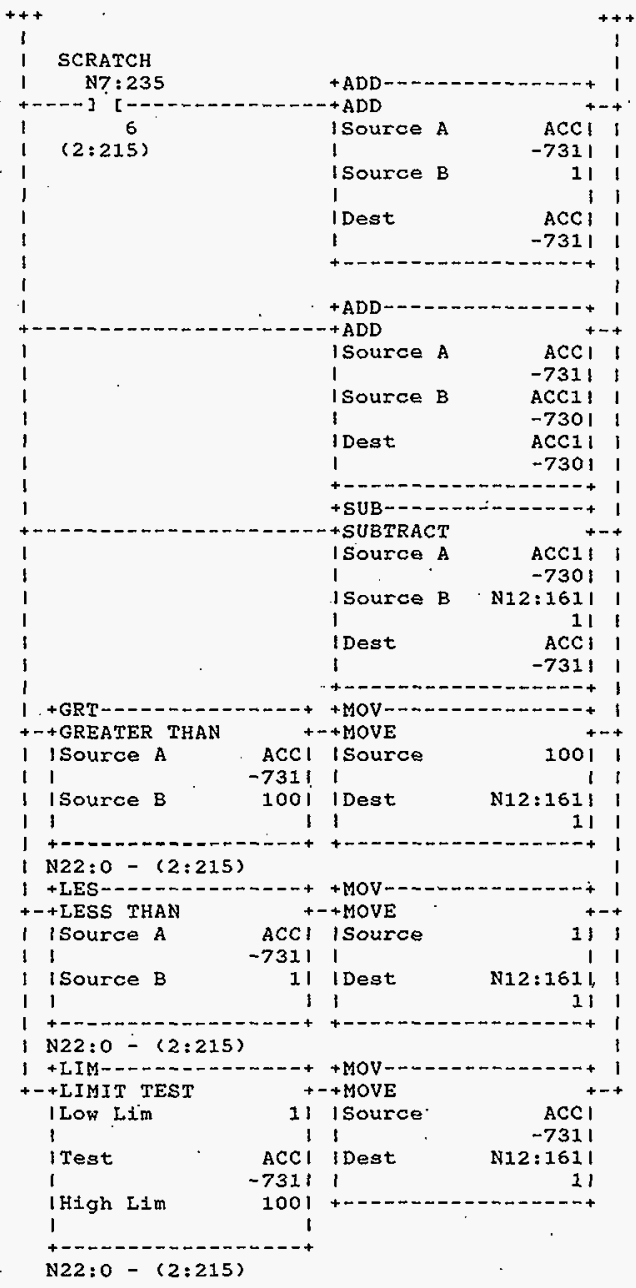

HNF-SD-FF-CSWD-61 Rev. 0 
Processor and Data(OPS Unit 1)

Rung 2:256

I PID153

i... -

21

DUMMY 21

$1---[L B[]$

N22:8

I

1

() $-\cdots$

Rung $2: 257$

\section{DETECT FROM SCADA PID ON/OFF \\ IE PID OFE \\ THEN ZERO PW FOR ZERO OUTPUT AT SCR AND JUMP AROUND THE PID BLOCK}
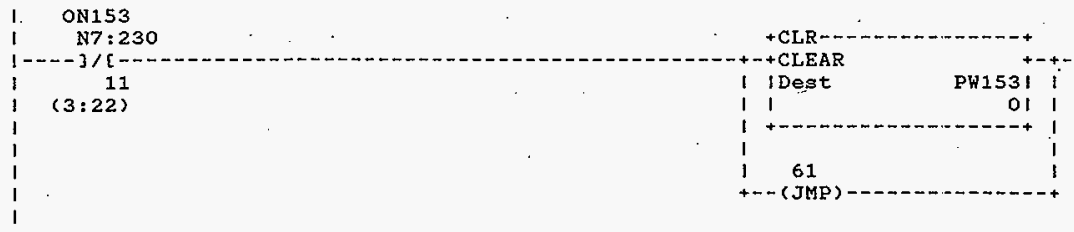

Rung $2: 258$

DETECT AUTO/MANUAL FROM SCADA

SET APPROPRIATE MODE IN PID BLOCK

$\begin{array}{lcc}1 & \text { AM1 } 153 & \text { PID } 153 / 1 \\ \text { N7:230 } & \text { 1 } 12: 150 \\ 1 & 10 & 1\end{array}$

Rung 2:259

DETECT SETPOINT VALUE FROM SCADA PUT VALUE IN PID BLOCK
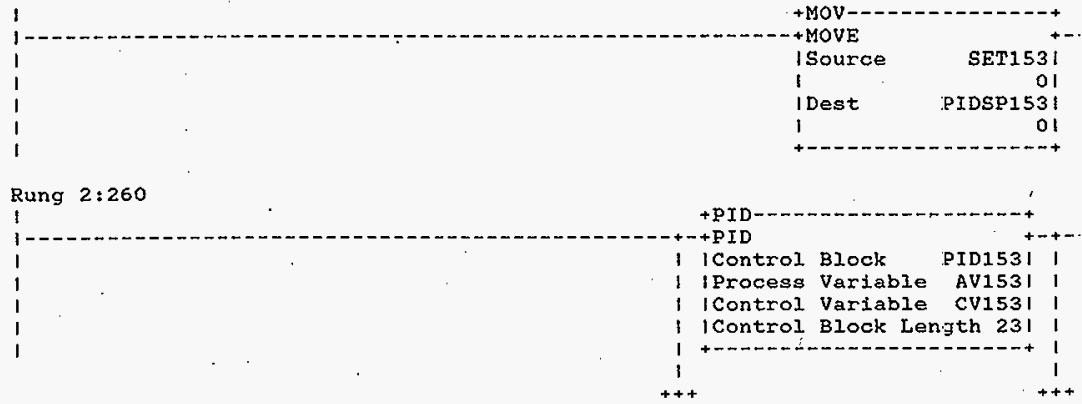

HNF-SD-FF-CSWD-61 Rev. 0 
Processor and Data(OPS Unit 1)

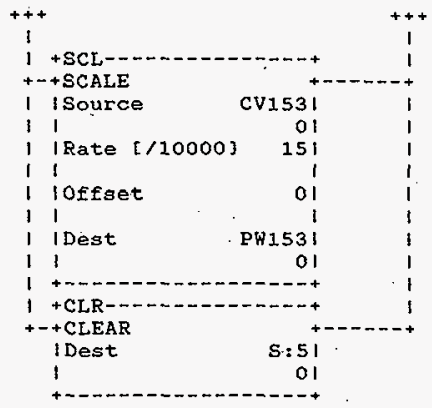

Rung $2: 261$

\section{Rung 2:262}

I

I RT TMR/DN

I T4:87

1...- ]/[-

I (2:42)

Rung 2:263

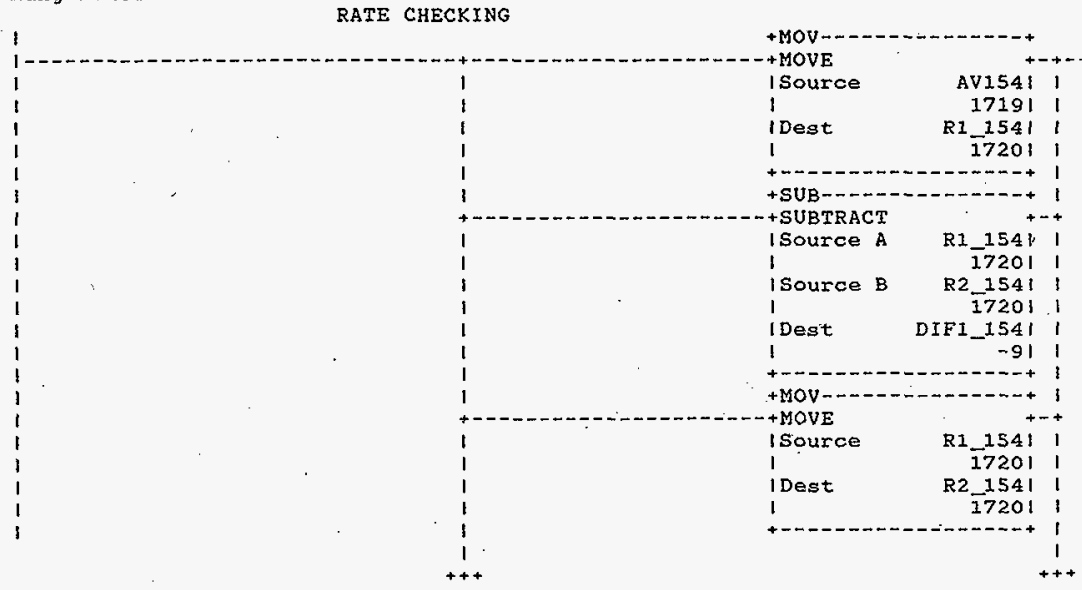

HNF-SD-FF-CSWD-61 Rev. 0 


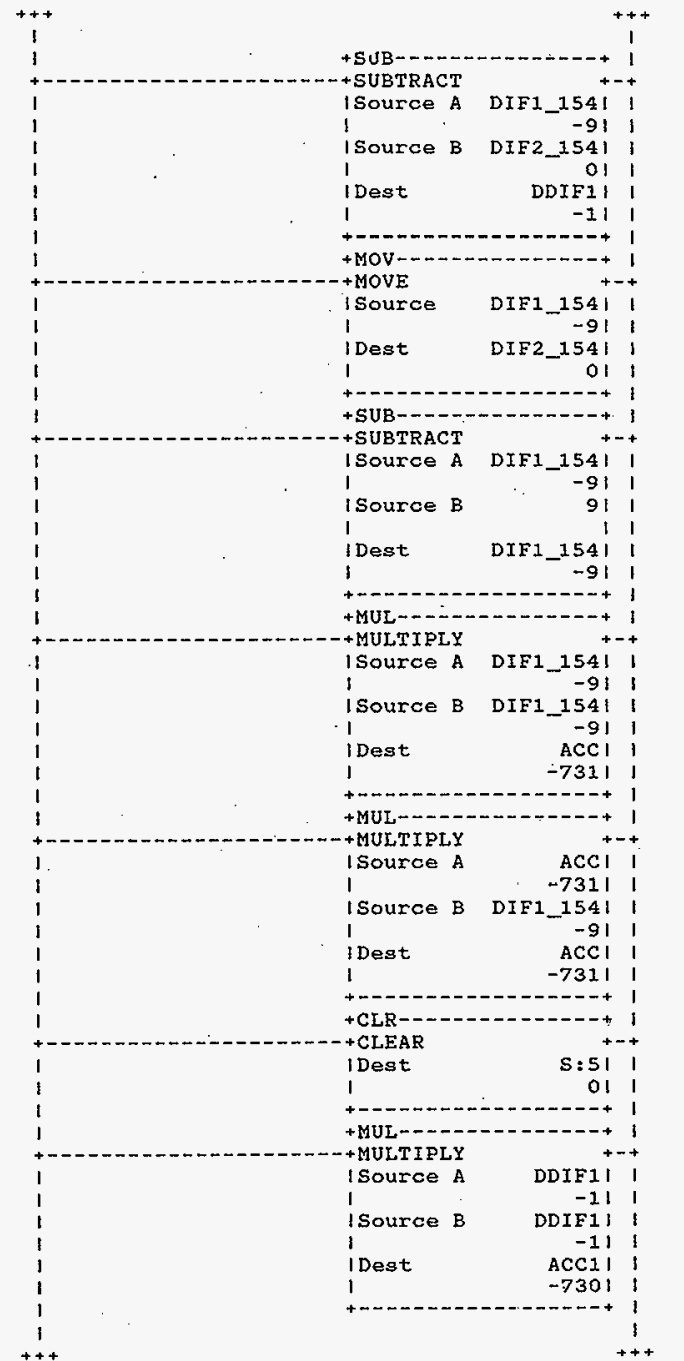

HNF-SD-FF-CSWD-61 R.ev. 0 
Processor and Data(OPS Unit 1)

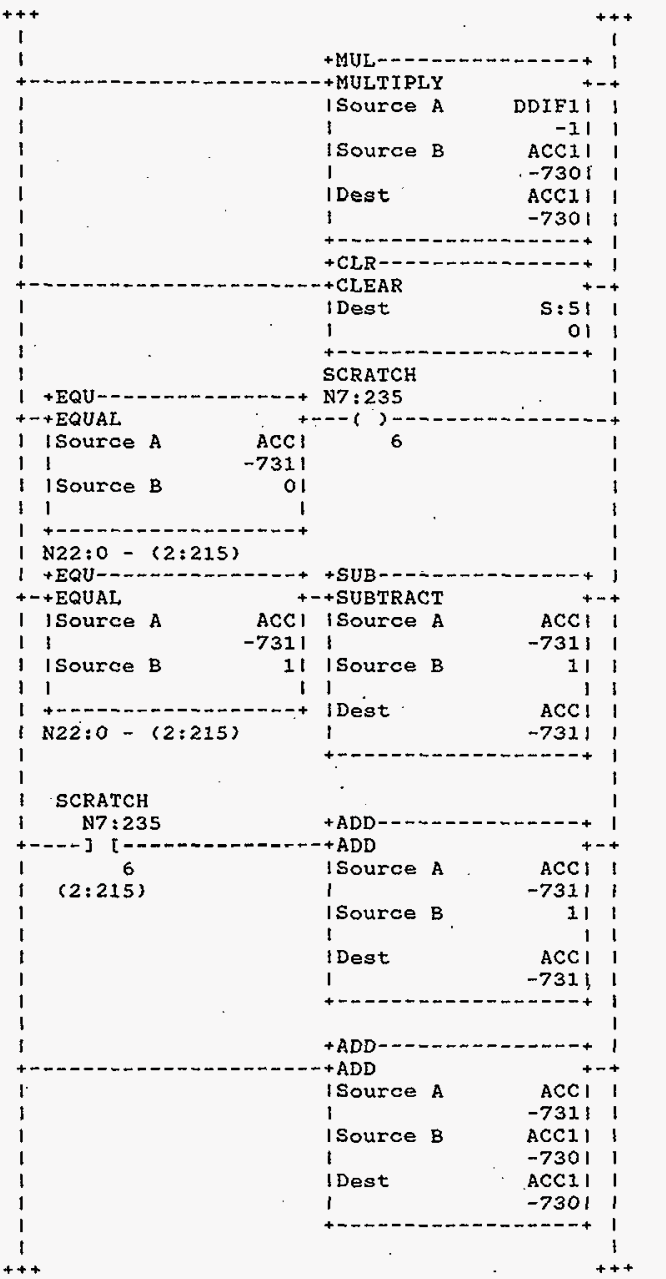

HNF-SD-FF-CSWD-61 Rev. 0 


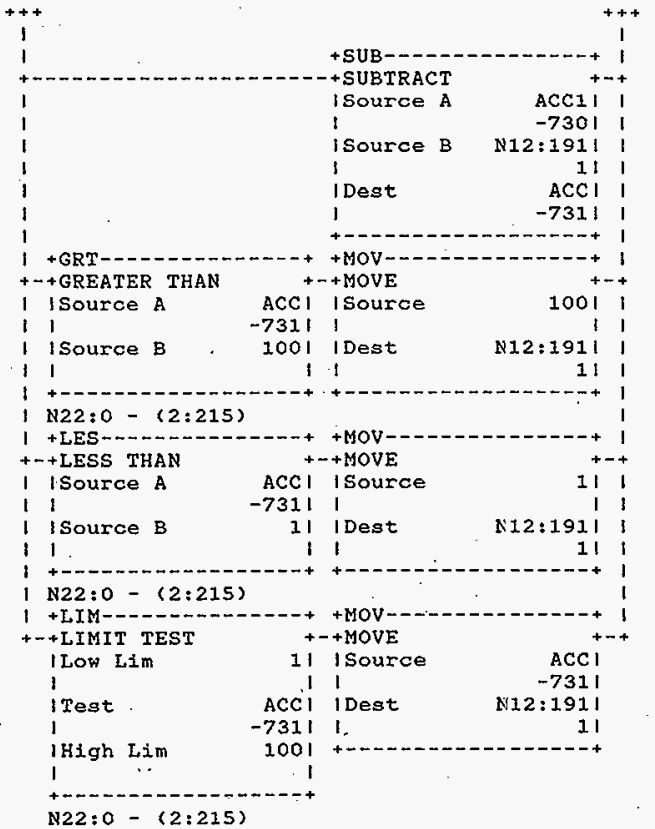

Rung $2: 264$

Rung 2:265

\section{DETECT FROM SCADA PID ON /OFF \\ IE PID OFF \\ THEN ZERO PW FOR ZERO OUTPUT AT SCR}

AND JUMP AROUND THE PID BLOCK
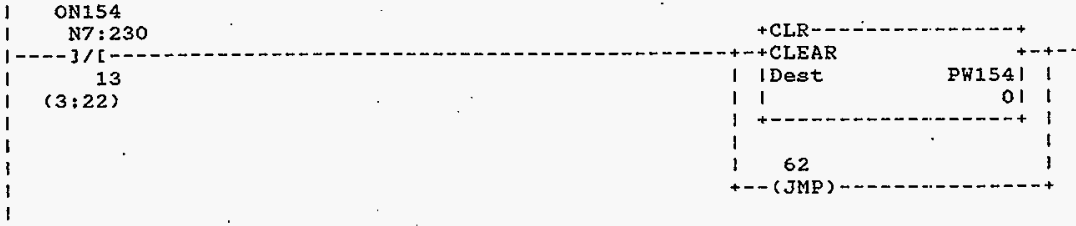

HNF-SD-FF-CSWD-61 Rev, 0 
Processor and Data (OPS Unit 1)

DETECT AUTO/MANUAL FROM SCADA

$\begin{array}{lccc}\text { AM154 } & \text { SET APPROPRIATE MODE IN PID BLOCK } & \text { PID } 154 / 1 \\ 1 & \text { N7:230 } & \text { N } 12: 180 & 1 \\ 1 & 12 & 1\end{array}$

Rung 2:267

DETECT SETPOINT VALUE FROM SCADA PUT VALUE IN PID BLOCK

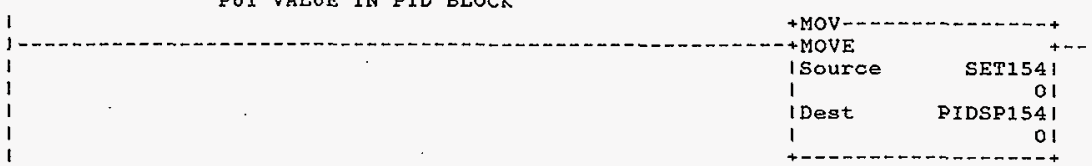

Rung 2:268

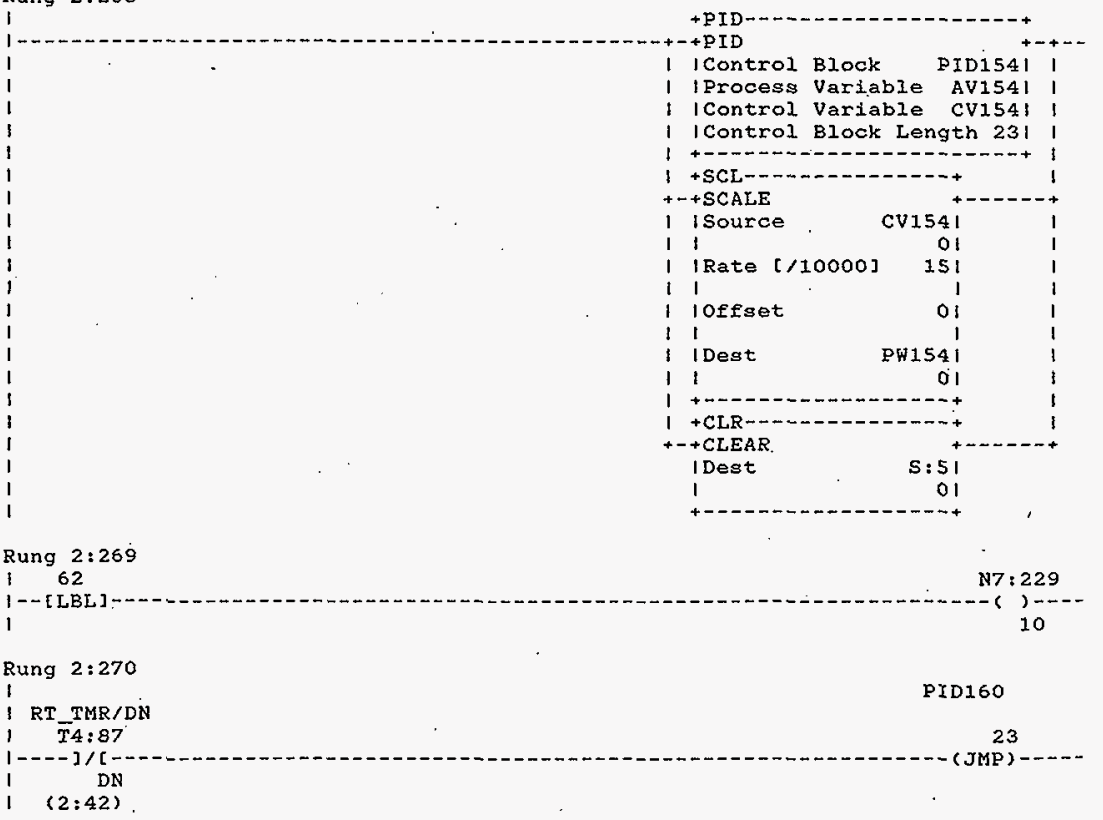

HNF-SD-FF-CSWD-61 Rev. 0 
Processor and Data(ops Unit 1)

Rung $2: 271$

RATE CHECKING

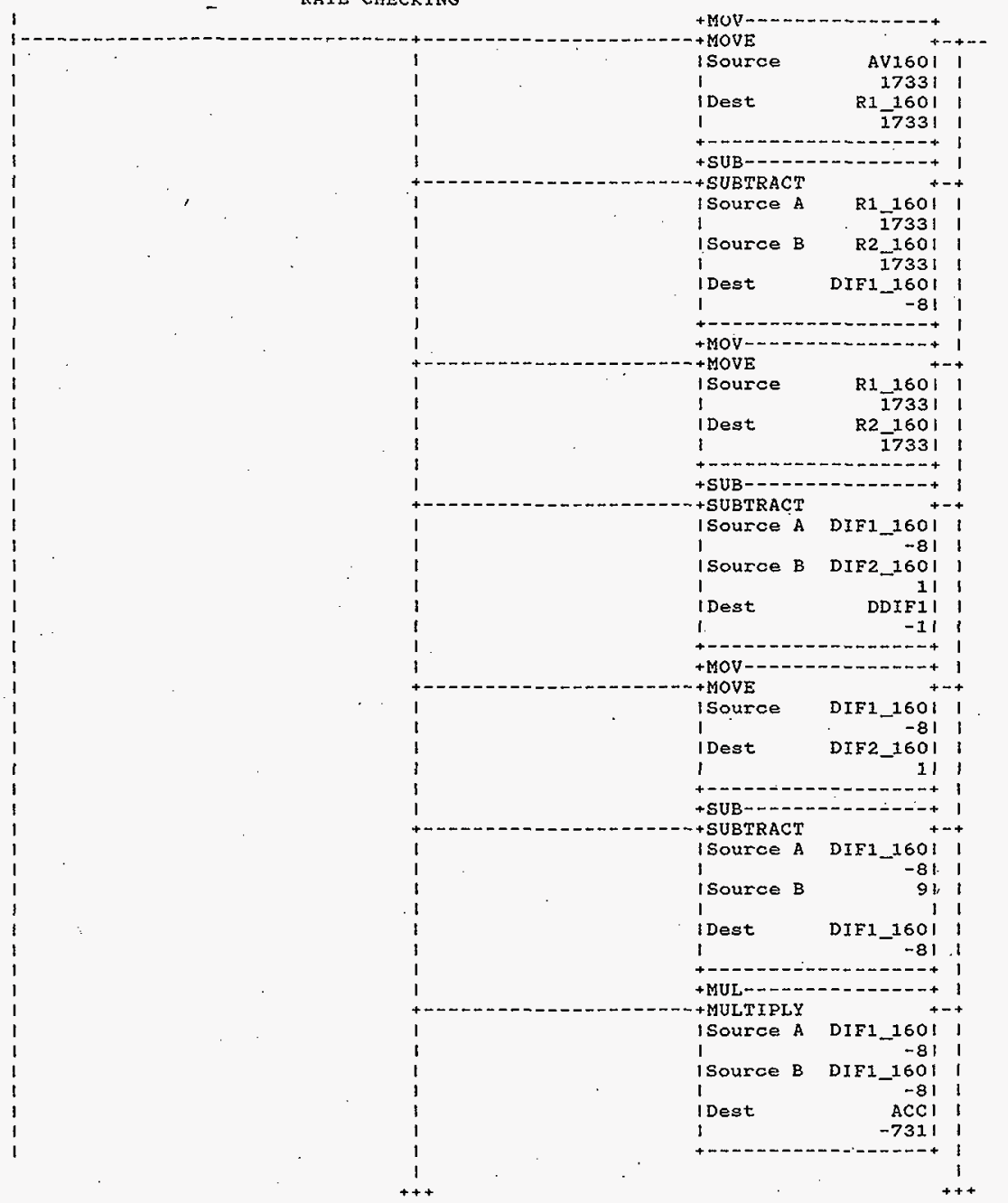

HNF-SD-FF-CSWD-61 Rev. 0 


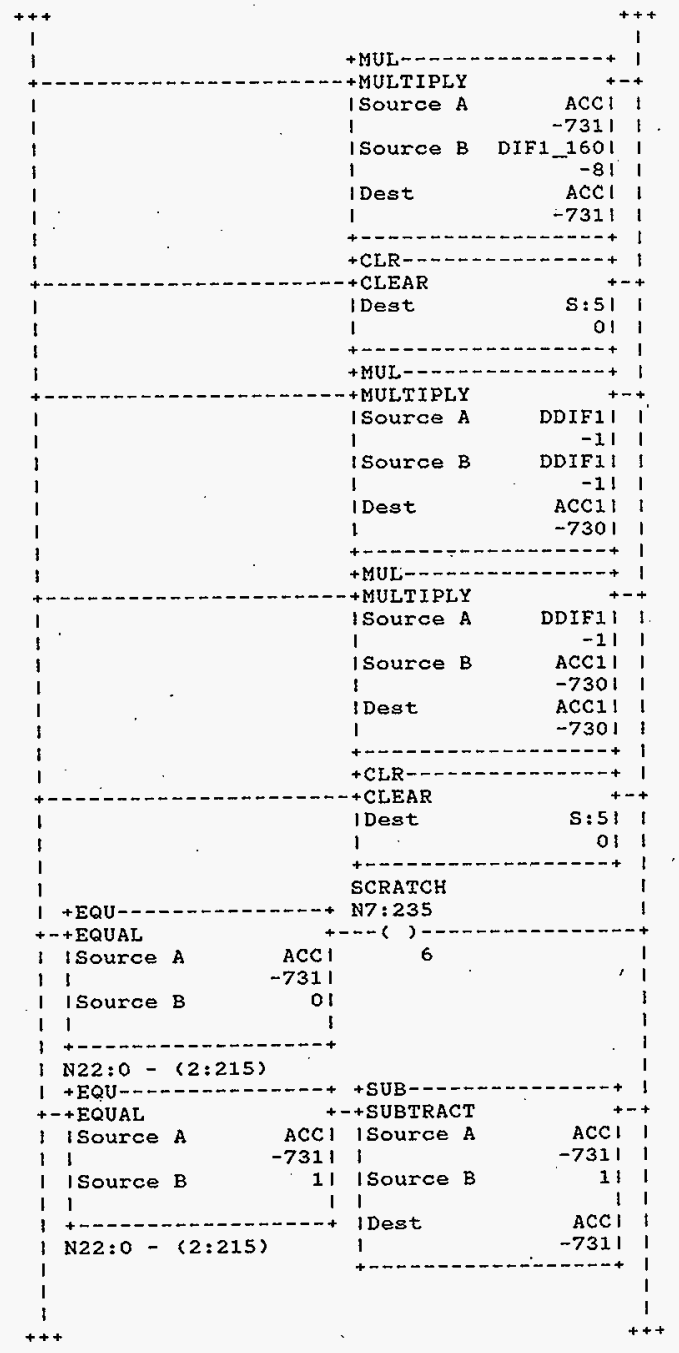

HNF-SD-FF-CSWD-61 Rev. 0 


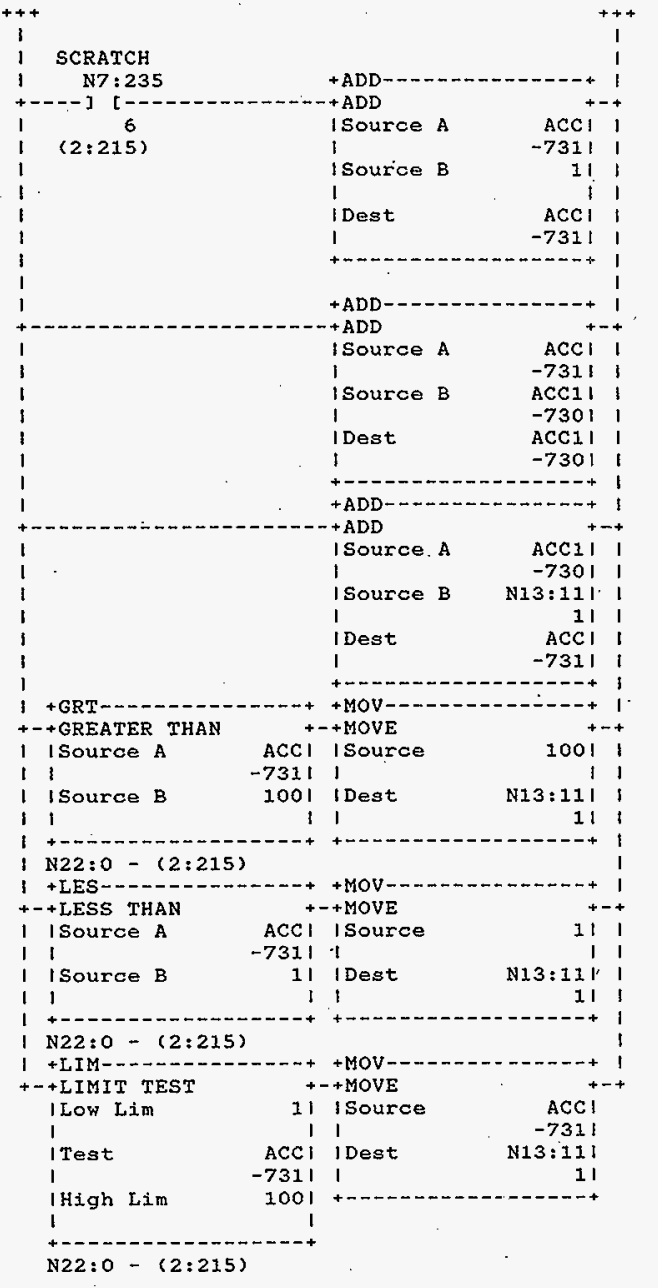

HNF-SD-FF-CSWD-61 Rev. 1 
Processor and Data(ops Unit 1)

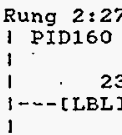

DUMMY 23

Rung $2: 273$

\section{DETECT FROM SCADA PID ON/OFF \\ IF PID OFF \\ THEN ZERO PH FOR ZERO OUTPUT AT SCR AND JUMP AROUND THE PID BLOCK}

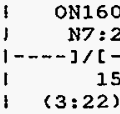

5

DETECT AUTO/MANUAL FROM SCADA SET APPROPRIATE MODE IN PID BLOCK

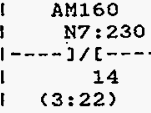

4

Rung $2: 275$

DETECT SETPOINT VALUE FROM SCADA PUT VALUE IN PID BLOCK

$$
\begin{aligned}
& 1 \\
& 1 \\
& 1 \\
& 1
\end{aligned}
$$

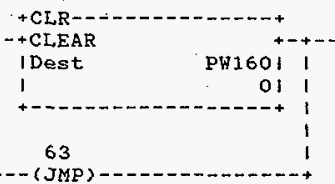

Rung 2:274

DETECT SETPOINT VALUE FROM SCADA
PUT VALUE IN PID BLOCK

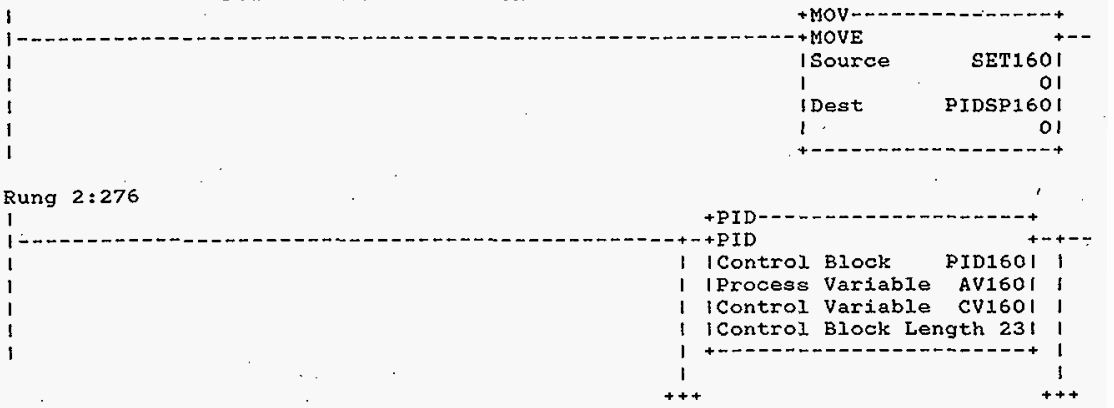

HNF-SD-FF-CSWD-61 Rev. 0

Page 482 


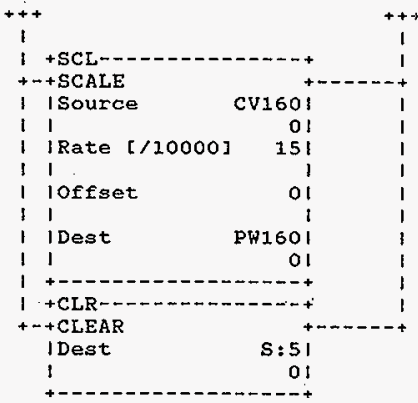

Rung $2: 277$

163

N7 : $229^{\circ}$

$1--[$ [BL $]$

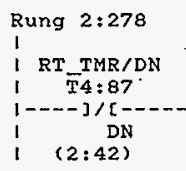

PID161

Rung 2:279

\section{RATE CHECKING}

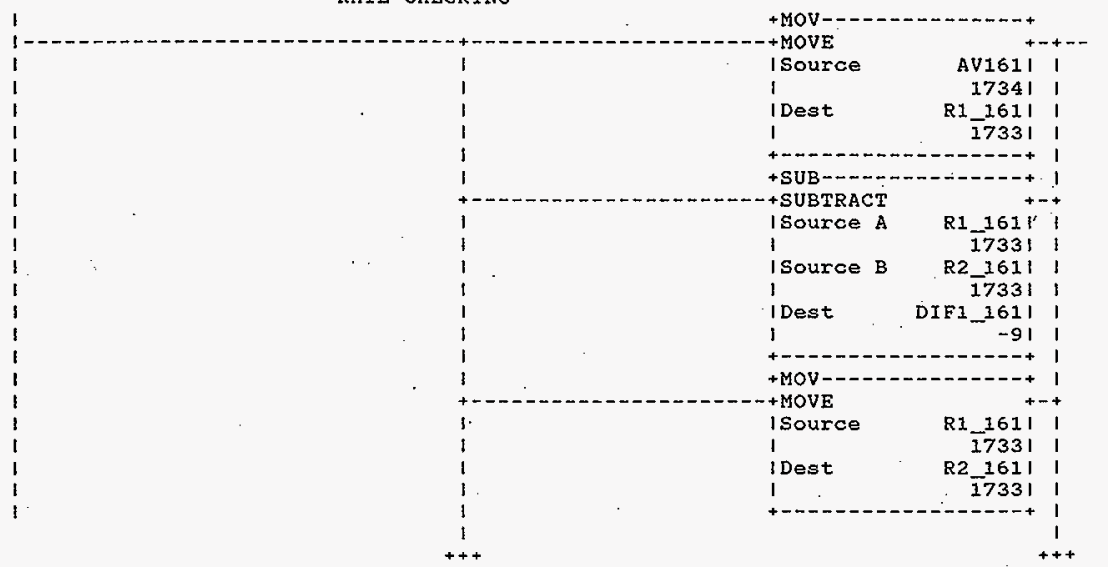

HNF-SD-FF-CSWD-61 Rev. 0

Page 483 


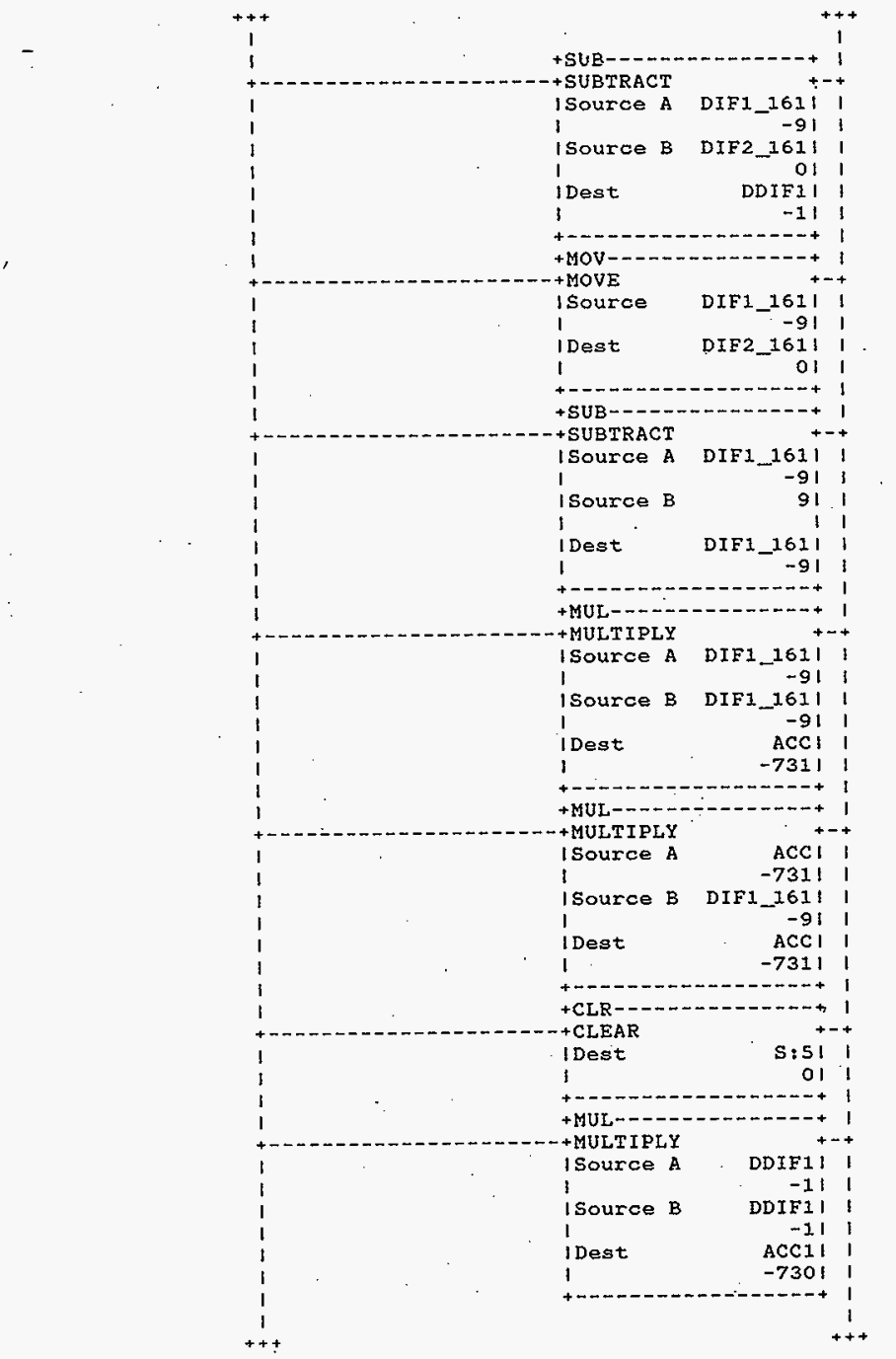

HNF-SD-FF-CSWD-61 Rev. 0 


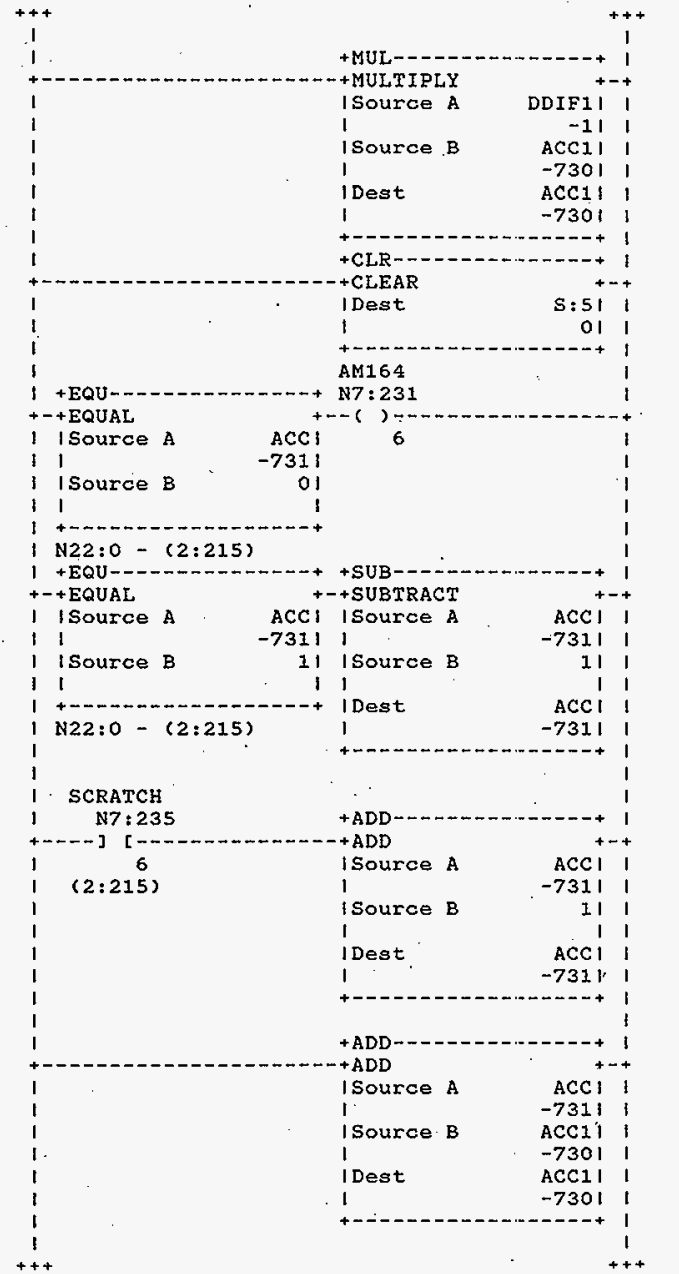

HNF-SD-FF-CSWD-61 Rev. 0

Page 485 


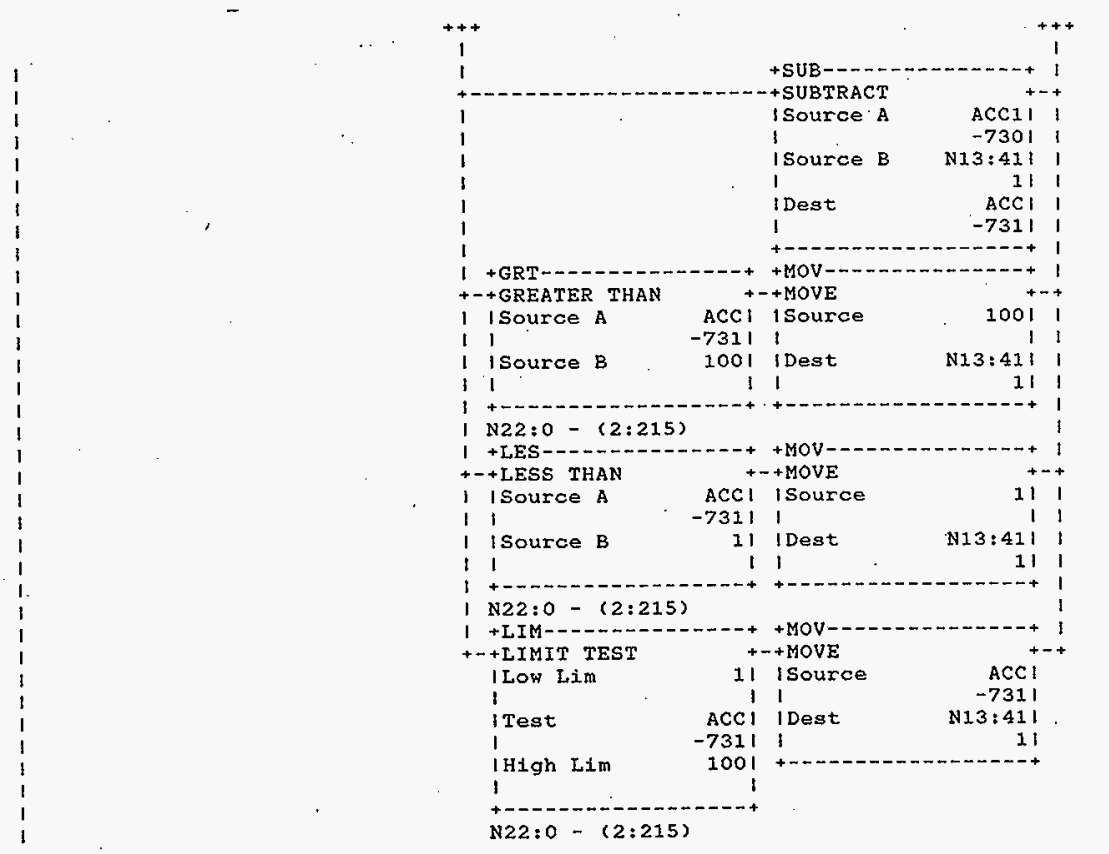

Rung 2:280

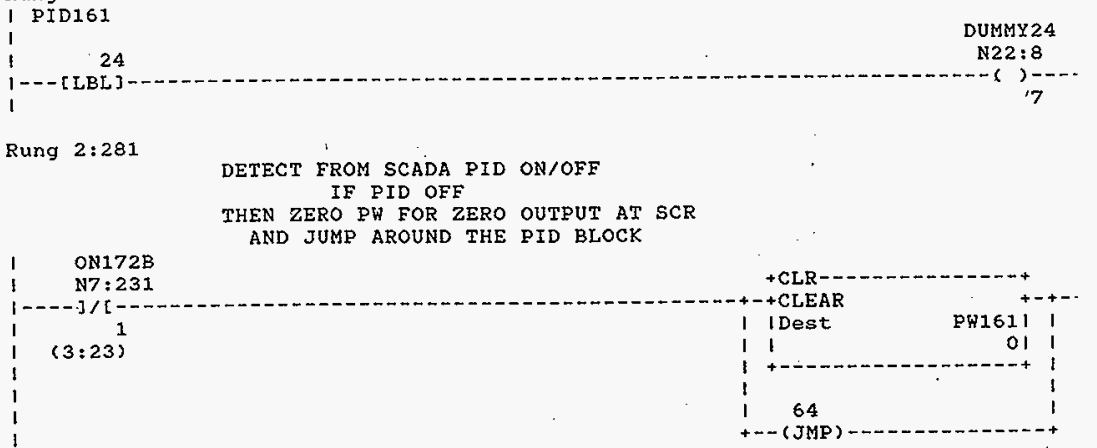

HNF-SD-FF-CSWD-61 Rev. 0 
Processor and Data(OPS Unit 1)

Rung $2: 282$

DETECT AUTO/MANUAL FROM SCADA

SET APPROPRTATE MODE IN PID BLOCK

$1 \quad A M 161$

N7:231

PID $161 / 1$

N13: 30

$1--m-3 /[-n-$

1 (3:23)

1

Rung $2: 283$

DETECT SETPOINT VALUE FROM SCADA PUT VALUE IN PID BLOCK

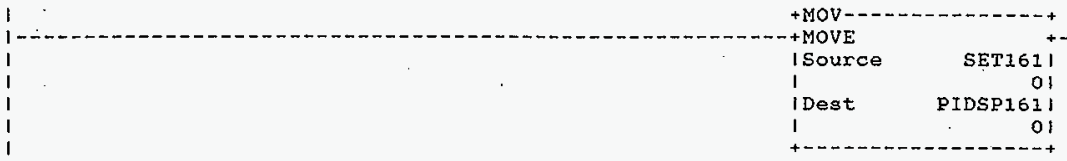

Rung $2: 284$

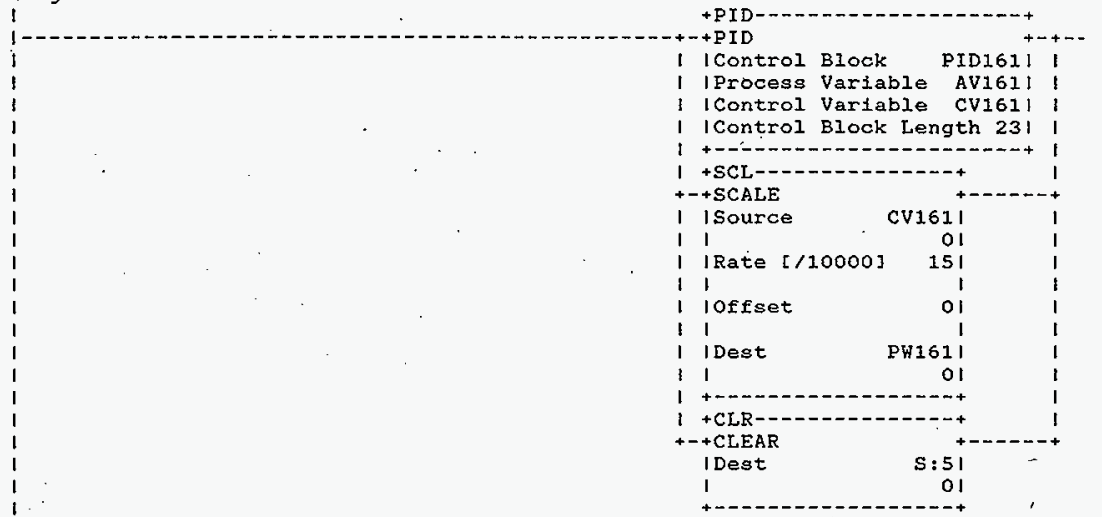

Rung 2:285

164

N7: 229

$1--[$ [BL $]$

l

Rung 2:286

1

1 RT TMR/DN

FID 162

f T4:87

$1----] /[-$

I $(2: 42)$

LINF-SD-FF-CSWD-61 Rer. 0 
Processor and Data(OPS Unit 1) Program Listing

$$
\text { I }
$$

\section{Rung 2:288}

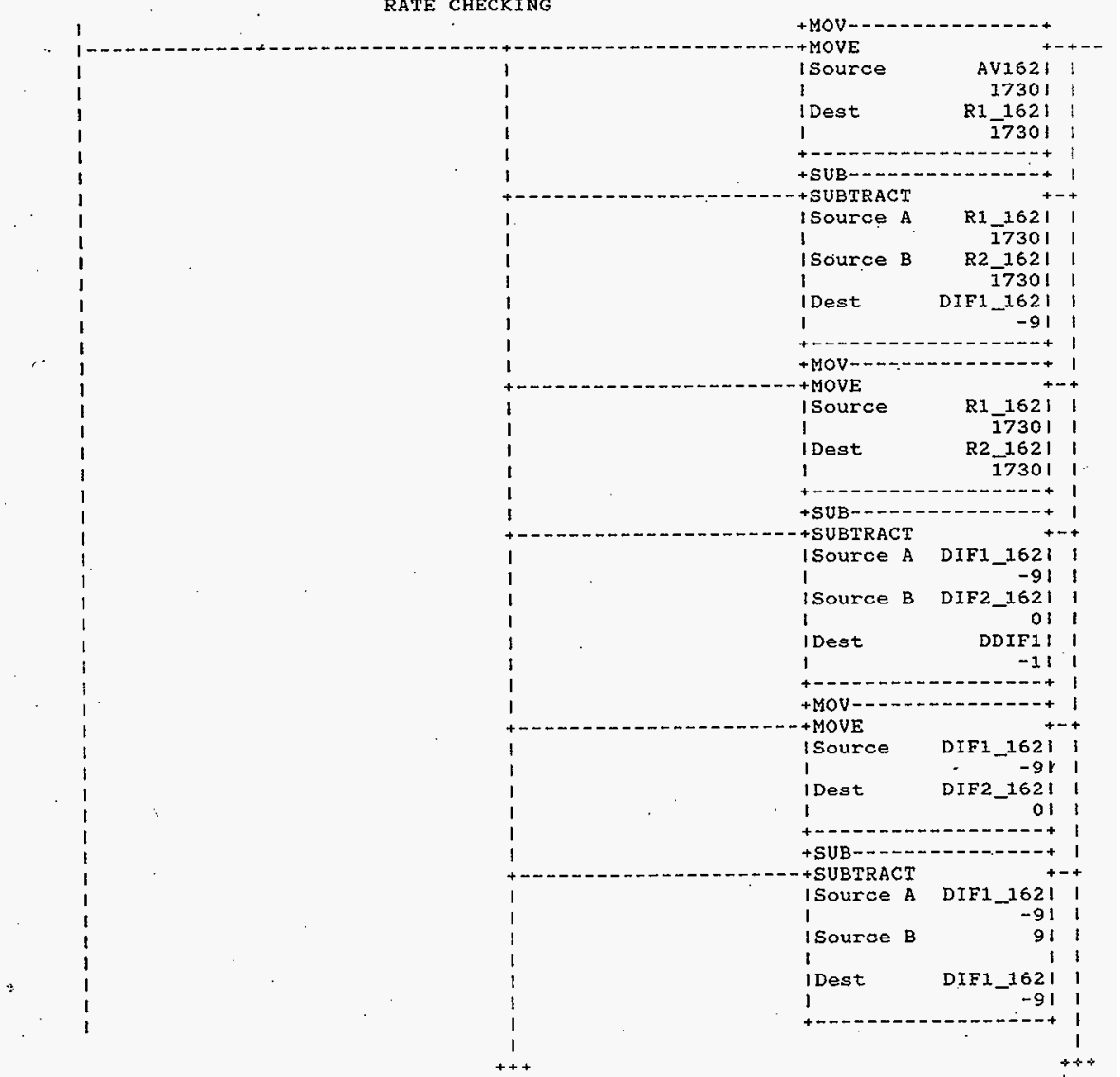

HNF-SD-FF-CSWD-61 Rev. 0 


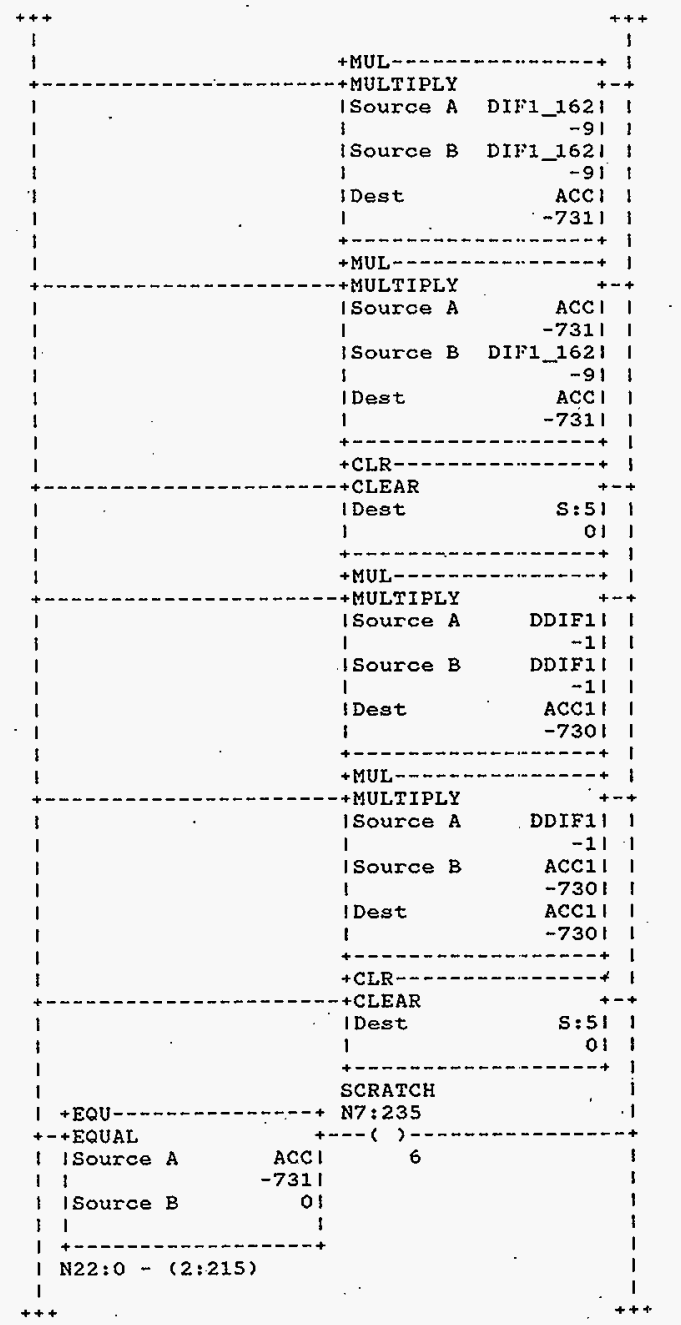

HNF-SD-FF-CSWD-61 Rev. 0 

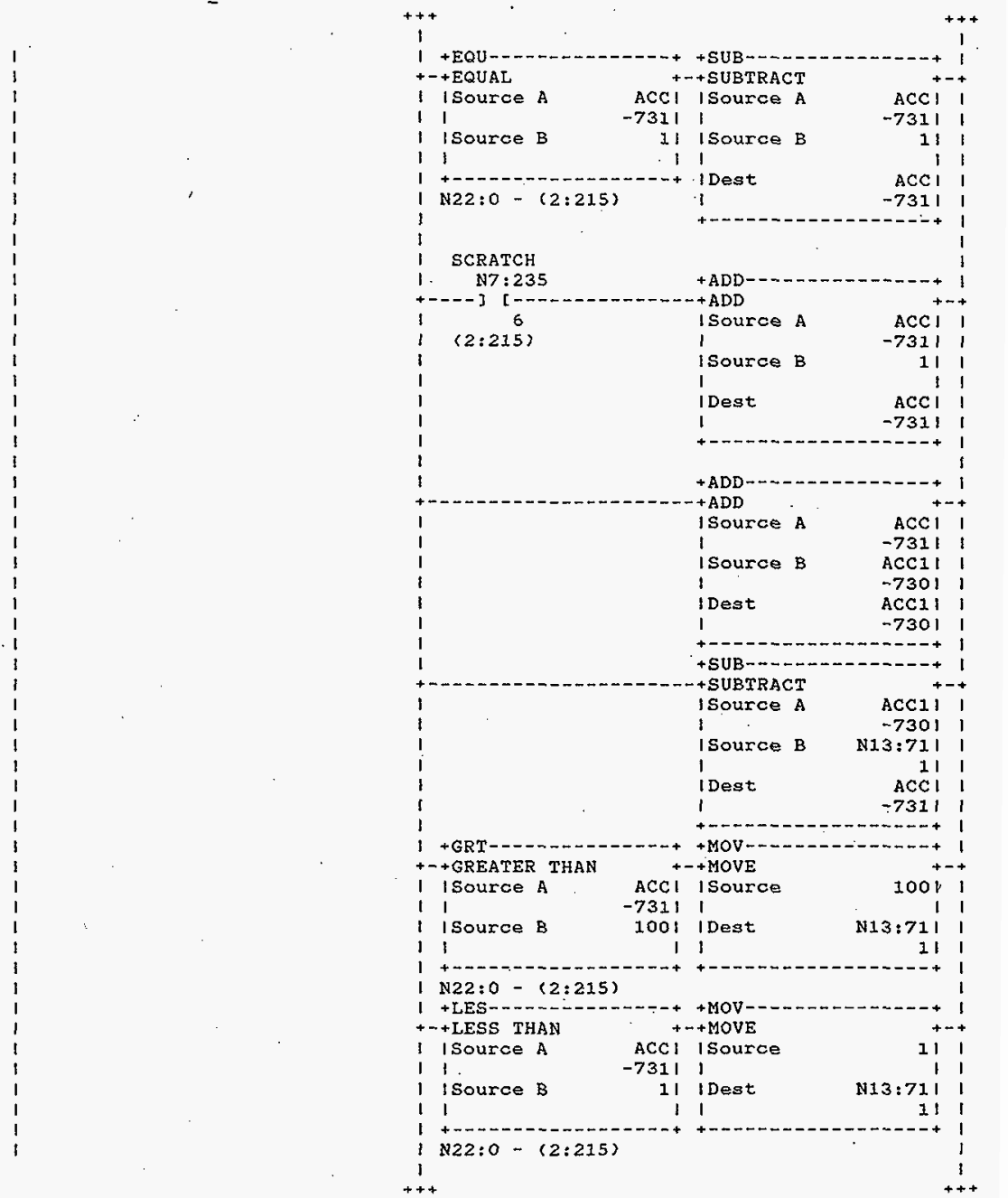

HNF-SD-FF-CSWD-61 Rev. 0 


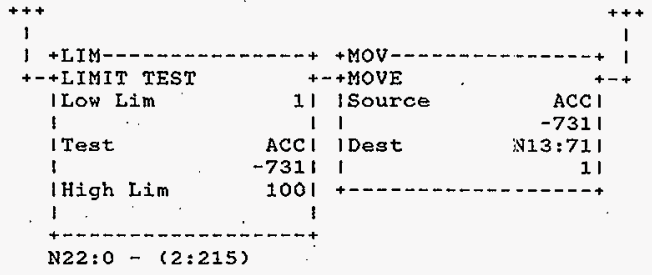

\section{Rung $2: 289$}

1 PID162

1

DUMMY 25

$1---[$ LBL $]$

$\mathrm{N} 22: 8$

Rung 2:290

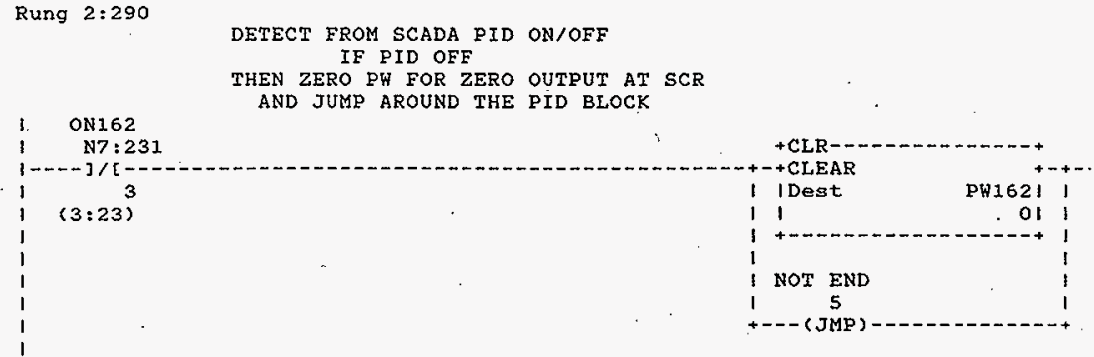

Rung 2:291

DETEC' $T$ AUTO/MANUAL FROM SCADA

SET APPROPRIATE MODE IN PID BLOCK

$\begin{array}{lc}\text { AN162 } & \text { N7:231 } \\ 1 & 2 \\ 1 & (3: 23)\end{array}$

PID162/1

N13:60

Rung $2: 292$

DETECT SETPOINT VALUE FROM SCADA

PUT VALUE IN PID BLOCK

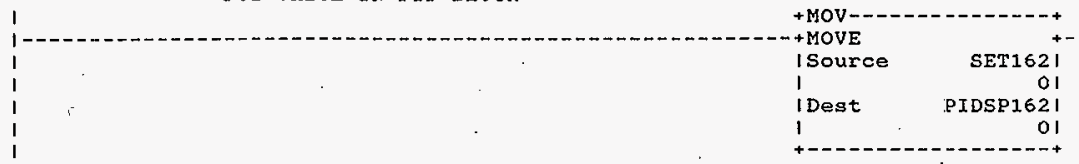

HNF-SD-FF-CSWD-61 Rev. 0 
Processor and Data(ops Unit 1)

Program Ligting

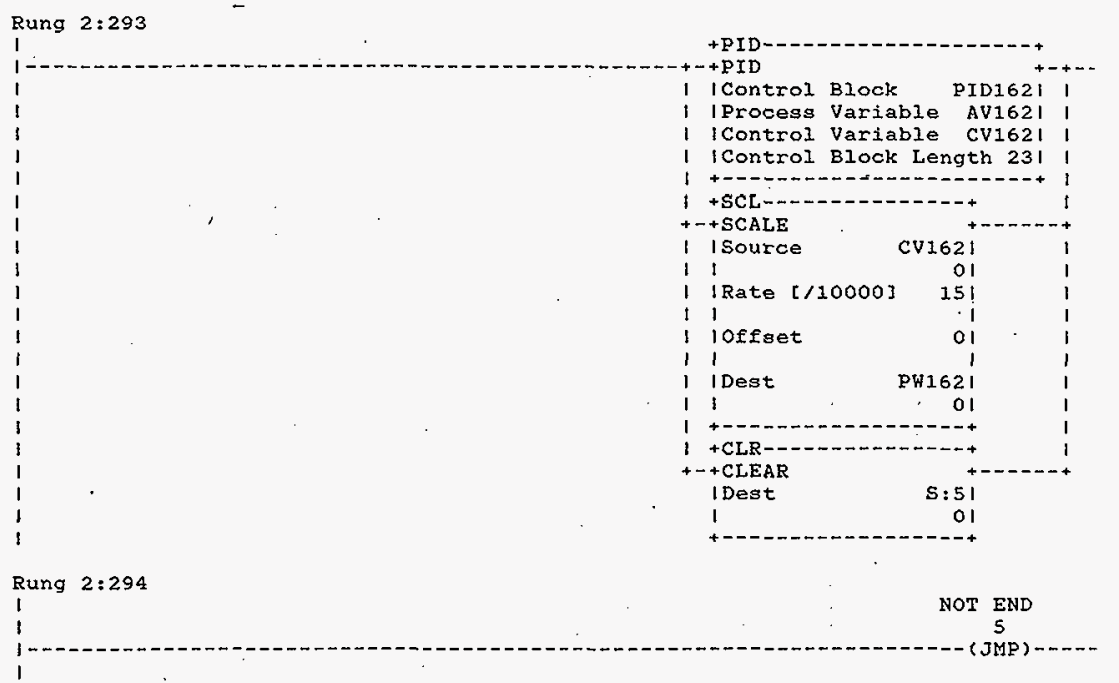

Rung 2:295

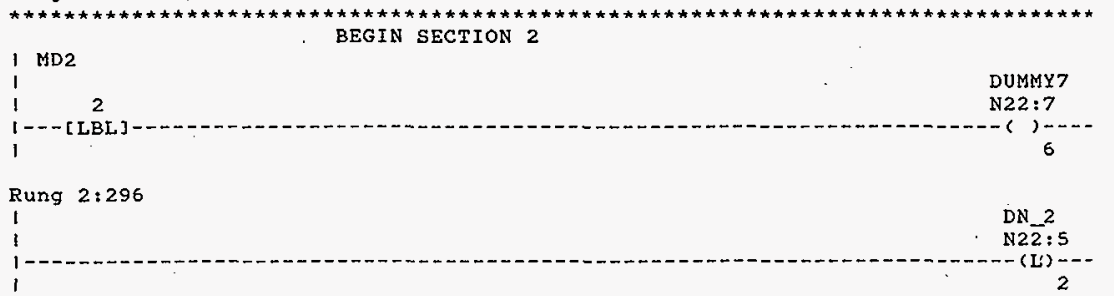

Rung 2:297

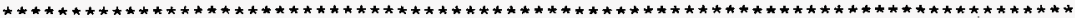
READ EACH THERMOCOUPLE AND STORE EACH TE'S VALUE IN ITS OWN MEMORY REGISTER

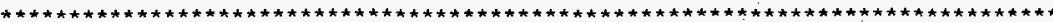
1 1.

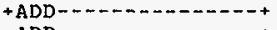
Isource A TC17OB11 7191 Isource B 1000 1 Dest C170B11 I $1719 !$

HNF-SD-FF-CSWD-61 Rev. 0 
Processor and Data(OPS Unit 1)

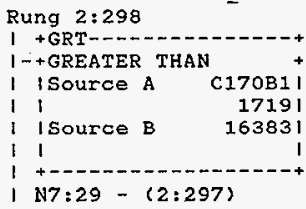

1 $17: 29-<2: 297$

Rung 2:299

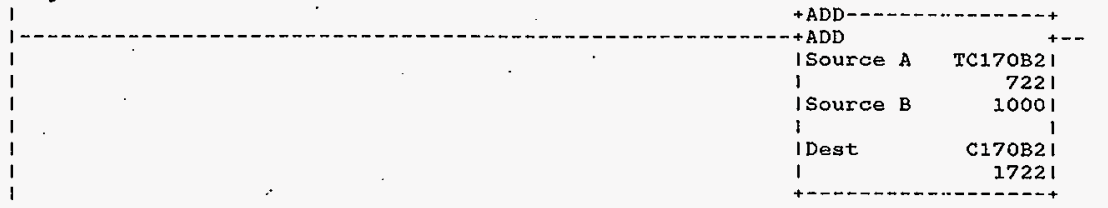

Rung 2:300

1 + GRT---.--............

1-+GREATER THAN

I ISource A CI7OB2!

I I 17221

| ISource B 16383 |

11

163831

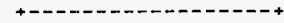

I $7: 30-(2: 299)$

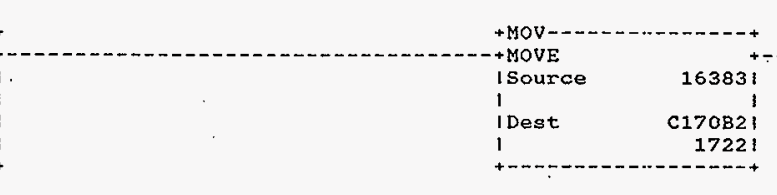

Rung 2:301

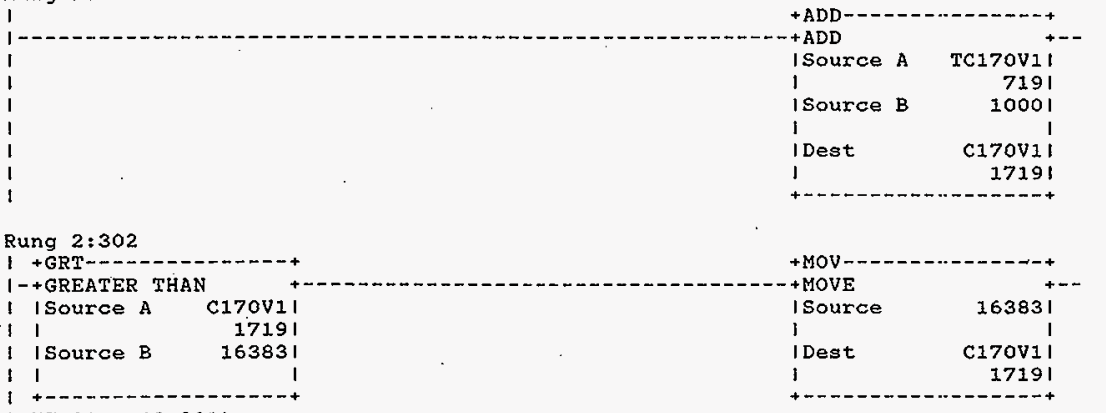

HNF-SD-FF-CSWD-61 Rev. 0 
Processor and Data(OPS Unit 1)

Program Listing

Processor File: SODIUM2A.ACH

October 23, 1996

Page $132^{\circ}$

Rung 2:303

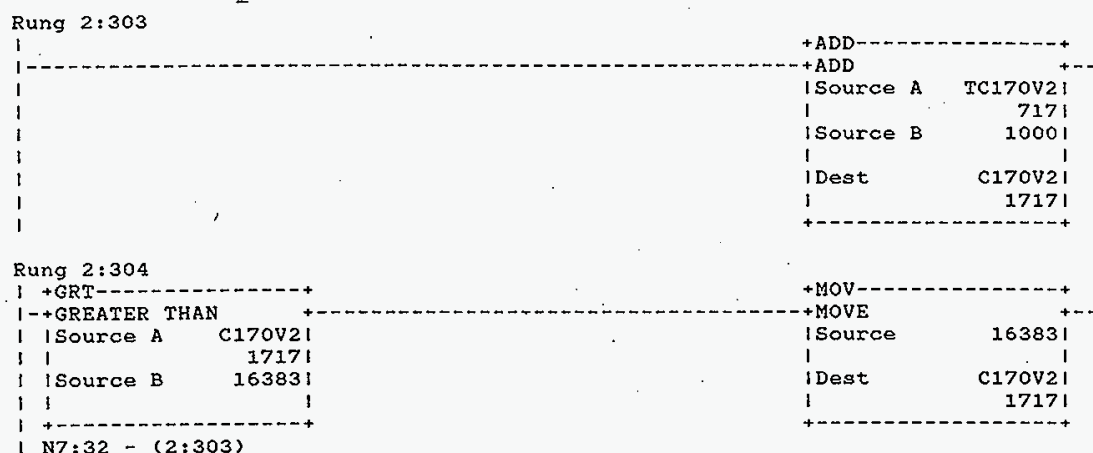

Rung $2: 305$

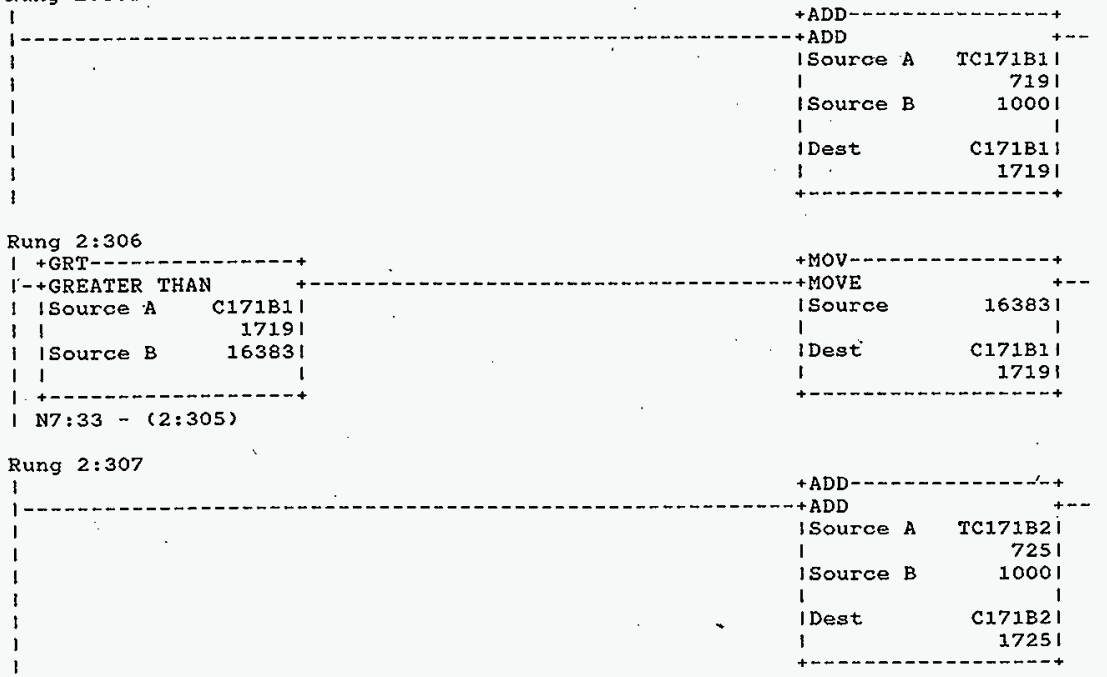

HNF-SD-FE-CSWD-61 Rev. 0

Page 494 
Processor and Data(OPS Unit 1)

\section{Rung $2: 308$}

$1+$ GRT---.-..........

$1-+$ GREATER THAN

1 Isource A C171B2I

11 1725।

ISource B 16383

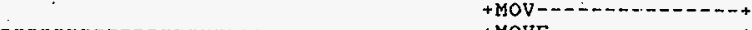

11

N7:34-(2:307)

Rung 2:309
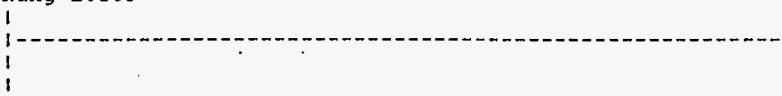

$+\mathrm{ADD} \rightarrow+----\cdots+---+$

isource A TC171V1.

I 7211

1Source B 1000 :

i

I Dest

C171V1I

1

17211

Rung $2: 310$

I +GRT---_..--

I-+GREATER THAN

I ISource A C171VI

I I 1721 |

I isource B 16383 |

11

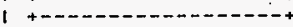

l N7:35-(2:309)

MOVE

163831

Dest

$171 \mathrm{~B} 2$

17251

Rung 2:311

1

$+\mathrm{ADD}$

ISource A TC171V21

1

7201

ISource B

1000

IDest

CI71V2I

17201

Rung $2: 312$
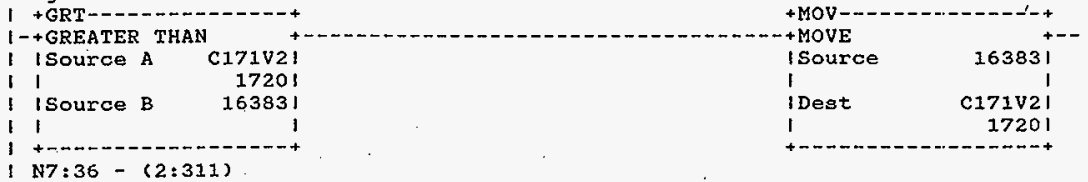

HNF-SD-FF-CSWD-61 Rev. 0

Page 495 
Processor and Data(OPS Unit 1) Program Listing Processor File: SODIUM2A.ACH

October 23, 1996

Page 134 Rung 2:313

\section{Rung $2: 313$}

I

$1-$

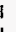

I

1

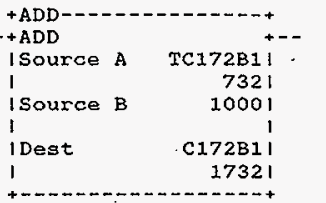

Rung $2: 314$

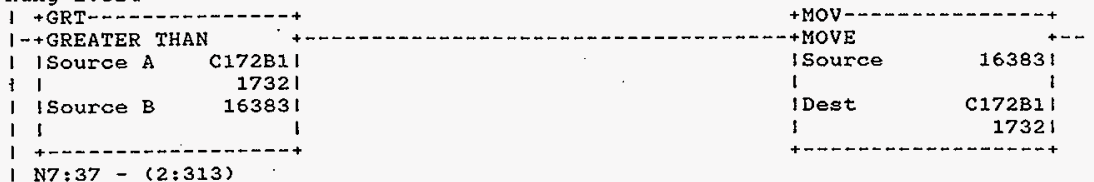

Rung $2: 315$

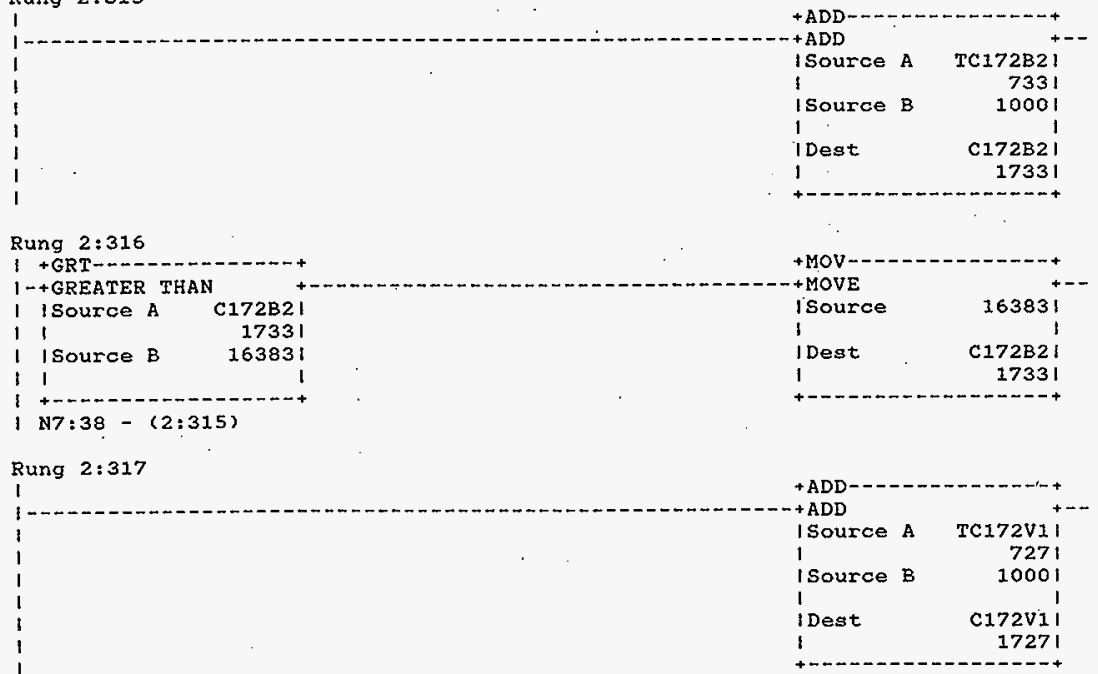

HNF-SD-FF-CSWD-61 Rev. 0

Page 496 
Processor and Data(ops Unit 1)

October 23, 1996

Page 135

Program Listing

Processor File: SODIUM2A.ACH

Rung 2:318

\section{Rung 2:318}

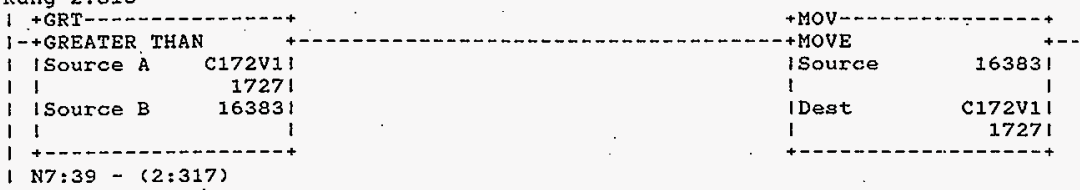

I N7:39-(2:317)

Rung $2: 319$

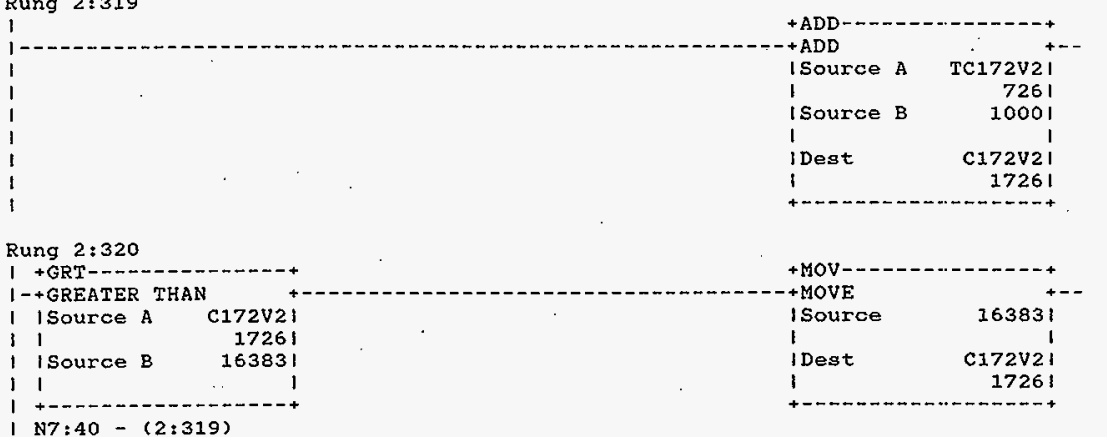

Rung 2:321

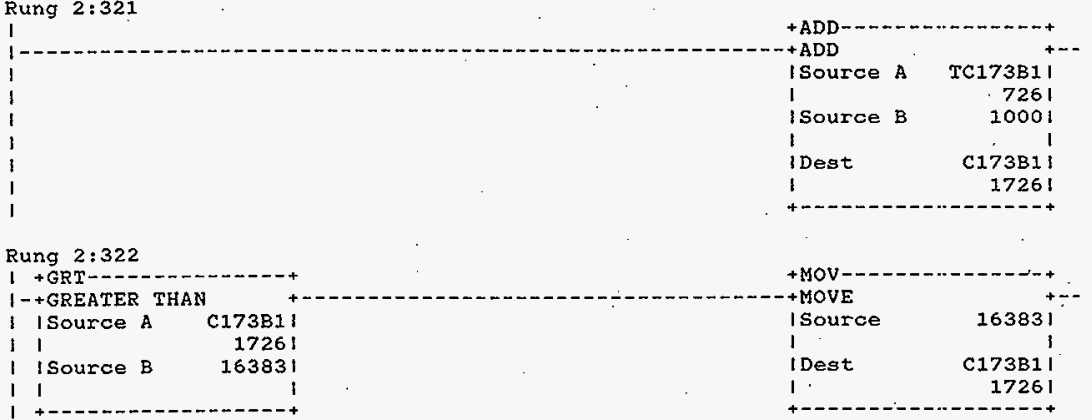

I N7:41-(2:321)

HNF-SD-FF-CSWD-61 Rev. 0 
This document was too large to scan as a whole document, therefore it required breaking into smaller sections.

Document number: SD-FF-CSWD-OG I

Section 2 of 2

Title: Soduim Storage facility Ladder Diagrams

Date: 6/12/97 Revision: A000

Originator: Cones QD

Co: BW NC

Recipient:

Co:

References: $\varepsilon D T-619594$ 
Processor and Data(OPS Unit 1 ) Program Iisting

Proce october 23,1996

Page 136

Rung $2: 323$

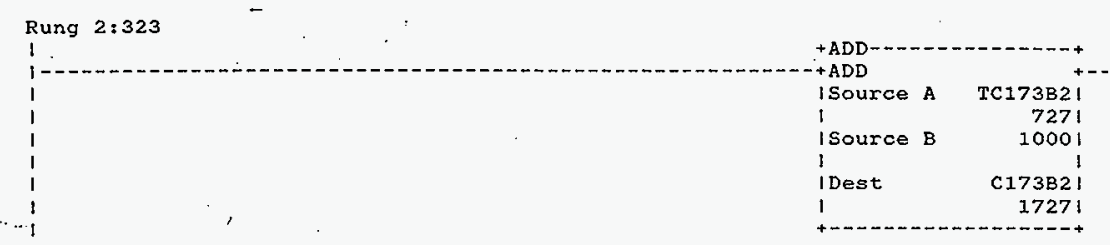

Rung $2: 324$

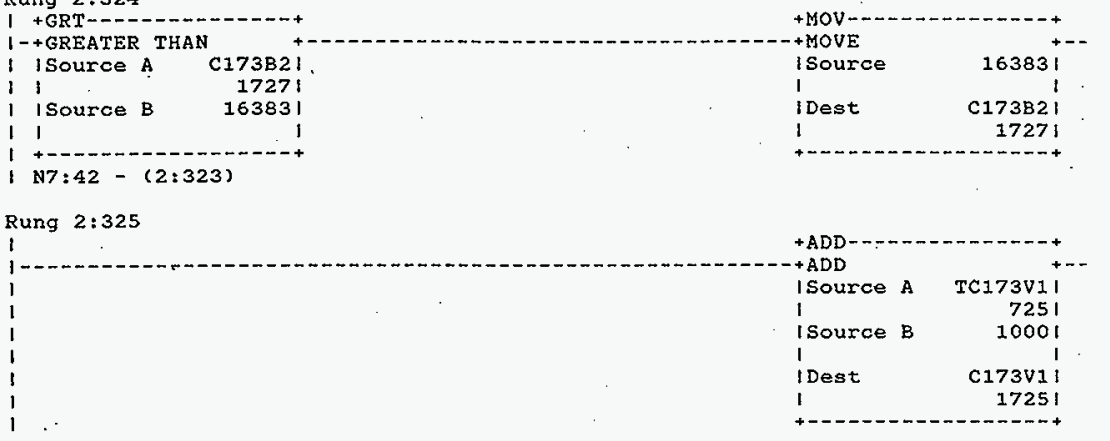

Rung $2: 326$

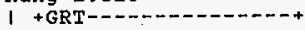

1-+GREATER THAN

I isource A C173V1I

1 I 17251

I ISource B 16383 I

1 I

\section{1}

N7:43-(2:325)

Rung $2: 327$$$
\text { 1 }
$$
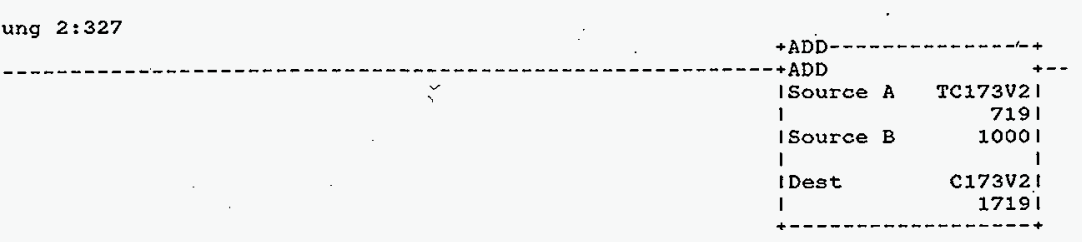

HNF-SD-FF-CSWD-61 Rev. 0

Page 498 
Processor and Data(OPs Unit 1)

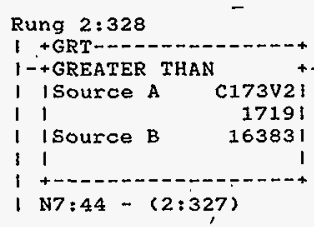

Rung 2:329

$$
1
$$$$
\text { j }
$$$$
\text { I }
$$$$
\text { I }
$$$$
\text { I }
$$$$
1
$$

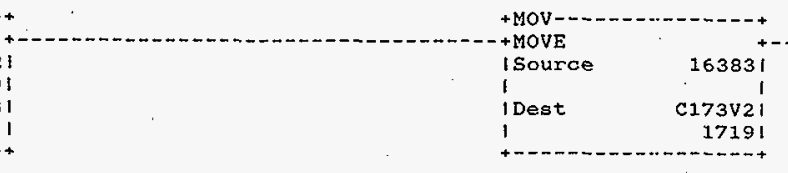

Riung 2:330

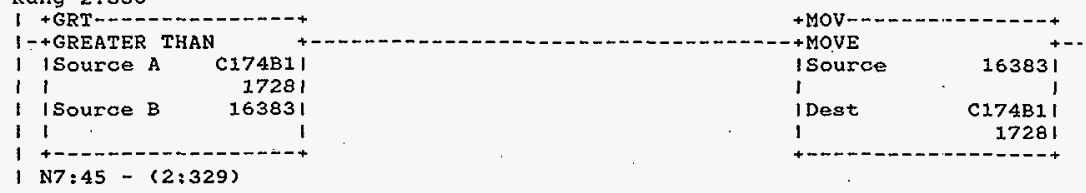

Rung 2:331

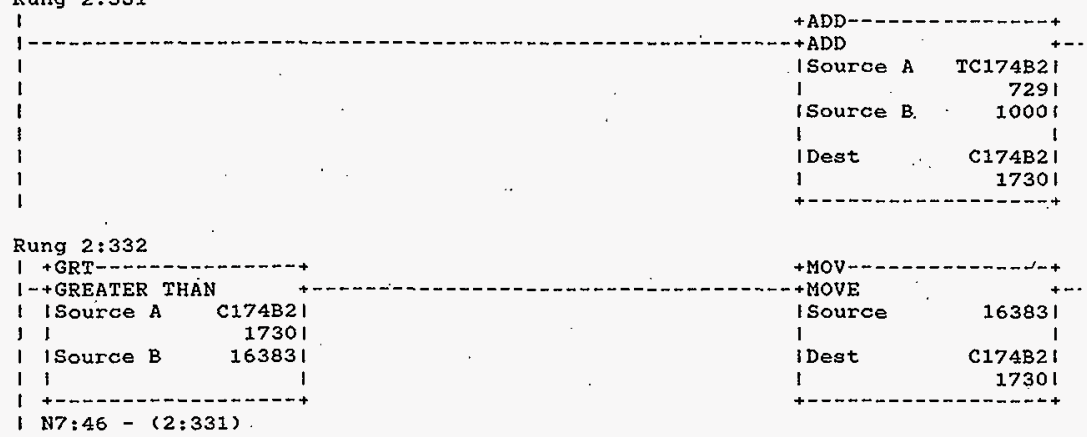

HNF-SD-FF-CSWD-61 Rev. 0

Page $4 \% 9$ 


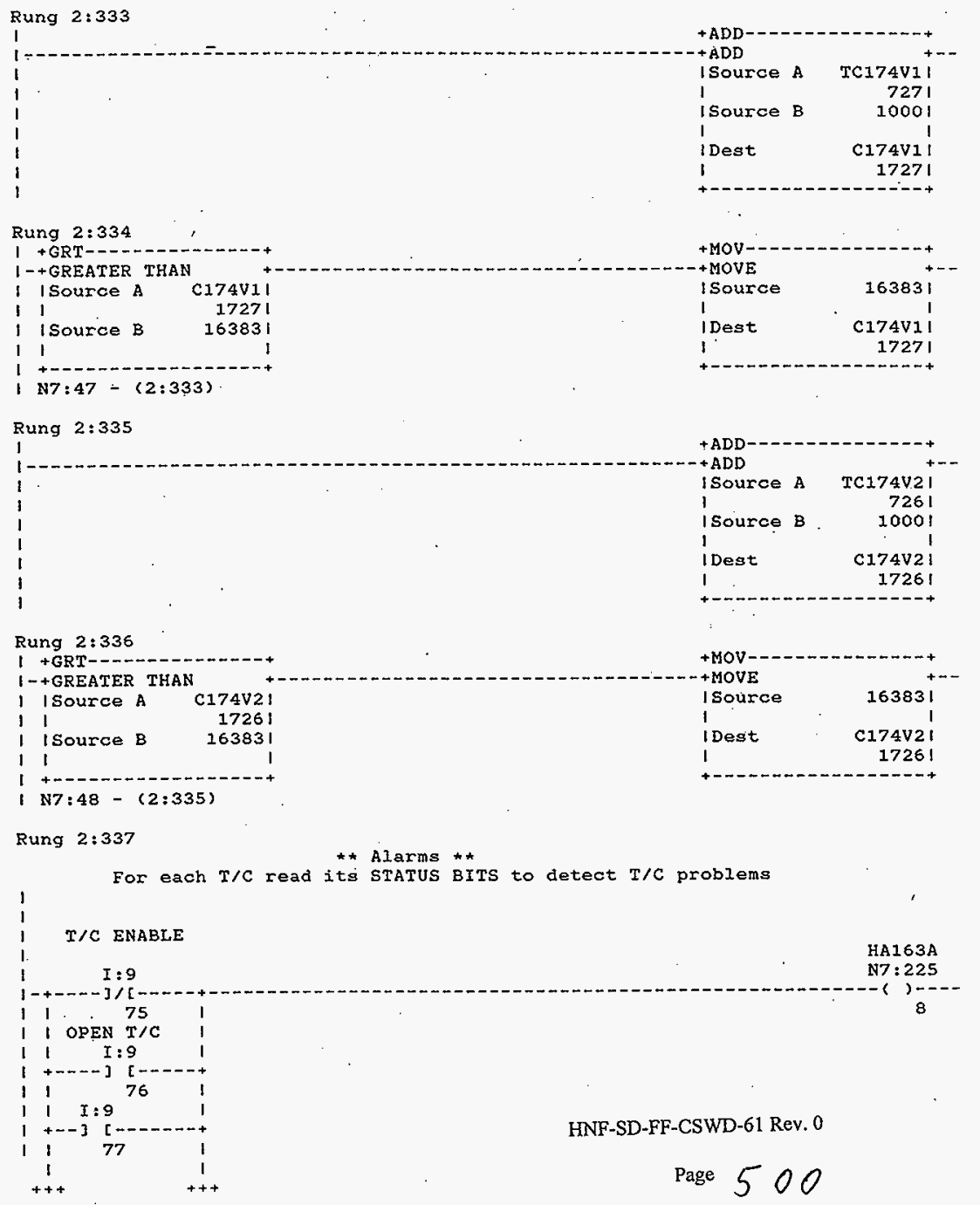


Processor and Data(OPS Unit 1 )

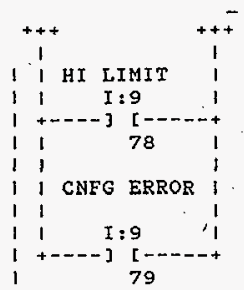

Rung 2:338

1

T/C ENABLE

$I: 9$

91

I OPEN $T / C$

I $\quad$ I:9

$+\ldots--3$ [.-...

192

I LO LIMIT

1 I $: 9$

$+-\cdots+20$

193

1 HI LIMIT

$1 \quad I: 9$

+----] [--m.-

I 94 ।

( CNFG ERROR - I

1 I:9

+.---] [--n...

95

Rung 2:339

1

T/C ENABL.E

$I: 9$

HA164A

$\mathrm{N7}: 225$

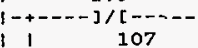

1107

OPEN $T / C$

$I: 9$

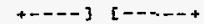

1108

LO LIMIT

i I:9

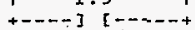

l 109

HI LIMIT

1 I:9

[no-

I I

1

110

HNF-SD-FF-CSWD-61 Rev. 0 
Processor and Data(ops Unit 1) Program Listing

Processor F1le: SODIUM2A.ACH
October 23, $1 \Omega 96$

Page 140 Rung $2: 339$

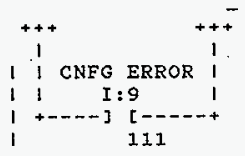

Rung $2: 340$

I

\section{$T / C$ ENABLE}

$$
\text { I: } 9
$$

$$
123
$$

OPEN T/C $I: 9$

+---- $][-.--+$

$1 \quad 124$

I LO LIMIT

1 1 I:9

+ +---] [ - - - + +

1125

1 i HX LIMTT

I I I:9

+---- $]$ [ [-......

1126

I I CNFG ERROR ।

I I I:9

1 +---- ] [-...--

$1 \quad 127$

Rung 2:341

I

I

\section{T/C ENABLE}

$I: 10$

+...- $][--. .++$

$1 \quad 76$

1 LO LIMIT

I I:10

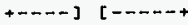

1. 77

I HI LIMIT

1

$\mathrm{I}: 10$

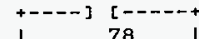

I CNFG ERROR

$\begin{array}{lll}1 & x: 10 \quad 1\end{array}$

i 79

HNF-SD-FF-CSWD-61 Rev. 0

Page 502 
Processor and Data(OPS Unit i)

program Listing

Rung 2:342

I

I

I T/C ENABLE

i) $I: 10$

HA170B2

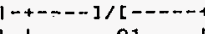

N7: 225

I. OPEN $\frac{91}{\mathrm{~T} / \mathrm{C}}$, ,

1 I:10 1

+---n] [-.-...

1 . 92

1 LO LIMIT

$I^{\circ} I: 10$

1
1

193

1 HI LIMIT

1 I: 10

+---- $1-\ldots . . .+$

I $94 \quad 1$

1 CNEG ERROR I

1
$+\ldots 10$

195

Rung $2: 343$

1

$T / C$ ENABLE

$I: 10$

HA170V1

$1-+-2-7 /[--$

N7 : 225

OPEN T/C

$I: 10$

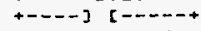

1108

1 LO LIMIT

i I:10

+.-- ] [ [--.-..

$1 \quad 109$

HI LIMIT

1 I 10

+--n- $5: 2-20+4$

i. 110 ,

I CNEG ERROR I

I $I: 10$ ।

1 +... ] [

111

HNF-SD-FF-CSWD-61 Rev. 0

Page 50 ? 
Processor and Data(OPS Unit 1)

\section{Rung 2:344}

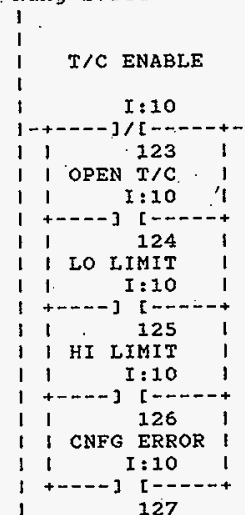

HA17OV2

N7: 225

Rung 2:345

T/C ENABLE

HA $171 \mathrm{~B} 1$

$1: 11$

N7: 226

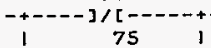

OPEN T/C

$I: 11$

+---- ] $[---.+4$

1176

I LO LIMIT

1 I:11

+--- ] [---...

177

HI LIMIT

$1 \quad I=11$

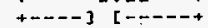

I CNFG ERROR

I 11

1 + - - ] $[-\cdots,-+$

i 79

Rung $2: 346$

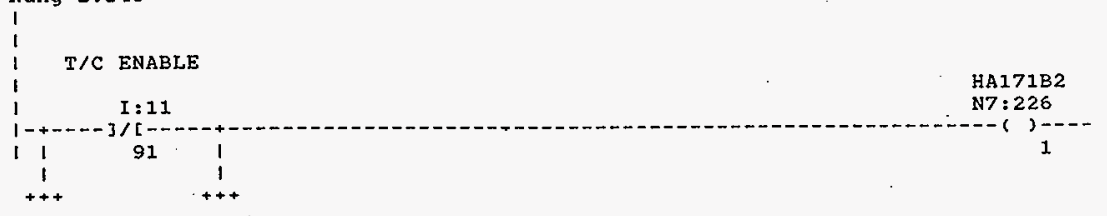

HNF-SD-FF-CSWD-61 Rev. 0 
Processor and Data(OPS Unit 1 ) Program Listing

October 23, 1996 Processor File: SODIUM2A.ACH

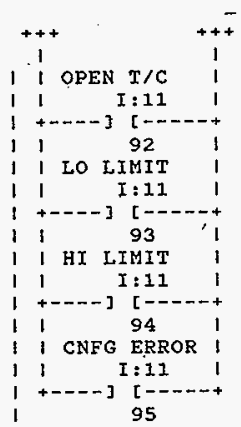

$\therefore \quad$ Rung 2:347

Rung

1

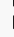

$T / C$ ENABLE

I $I: 11$

$1-+---3 /[-2-\cdots+$

I OPEN T/C

I $I: 11$

$1+\ldots-\ldots]$ [

I 108

1 LO LIMIT

$1 \quad I: 11$

+----3 [-...-

$1 \quad 309$

| HI IIMIT

1

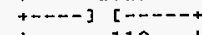

I

I CNFG ERROR ।

11

1 +-.-- ] $[--\ldots+$

111

Rung $2: 348$

I

T/C ENABLE

I $I: 11$

HA.17IVI N7: 226 2

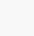

I 123

I I OPEN T/C

1 I:11

1 +----] [----..

1. 124 1 $+++$ 
Processor and Data(OPS Unit 1)

Program Listing

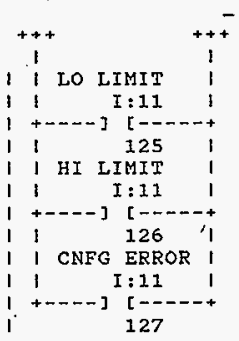

Rung 2:349

1

T/C ENABLE

$I: 12$

HA $172 \mathrm{~B} 1$

N7: 226

I 1 75 1

I I OPEN T/C

I 1 I:12 1

$\begin{array}{llll}1 & +---1 & {[-\cdots--+} \\ 1 & 76 & 1\end{array}$

1 LO LIMIT

1 I:12

$1+--\cdots+][-----+$

$11 \quad 77 \quad 1$

l) HI LIMIT

11 I:12 I

$1+\cdots-\cdots]$ [ - 1 -

I 178 ।

I I CNFG ERROR I

11 I:12 ।

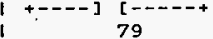

Rung 2:350

1

T/C ENABLE

HA172B2

$I: 12$

N7 : 226

$\begin{array}{cc}1 & 1 \\ 1-+\cdots & 91\end{array}$

I OPEN T/C

$1 \quad I: 12$

+-

I 92

1 LO-IIMIT

1 I 12

$1+\ldots---][-. . .+$

11

1
1
+++

93

HNF-SD-FF-CSWD-61 Rev. 0 
Processor and Data (OPS Unit 1)

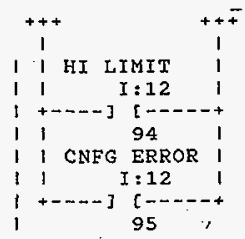

Rung 2:351

!

T/C ENABLE

$1,+\ldots \ldots] /[12$

HA172VI

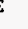

1.207

1 OPEN $\mathrm{T} / \mathrm{C}$

$I: 12$

+.-n] [--...

I 108

1 LO IIMIT

$1 \quad \mathrm{I}: 12$

$+$

1

109

HI LIMIT

I

+--- ] [-----+

1110

I CNFG ERROR

$\mathrm{X}: 12$

+--.-] $[-\ldots .-+$

111

\section{Rung 2:352}

1

T/C ENABLE

$1 \quad I: 12$

HA172V 2

I 123

I OPEN T/C

I I :12

n----] [--n+

1

1 LO LIMI

I Lo LIMIT

+- - ] [ $[\ldots--+$

$1 \quad 125$

HI LIMIT

1

$1: 12$

+--- ] $[---\cdots+$

I 126

i CNFG ERROR

I I:12

$+----][--.--4$

N7: 226

N7: 226

HNF-SD-FF-CSWD-61 Rev. 0

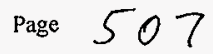


Processor and Data(ops Unit I)

Program Listing

Rung $2: 353$

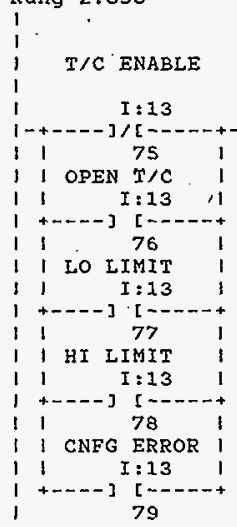

Rung $2: 354$

1

$T / C$ ENABLE

$$
I: 13
$$

Rung 2:355

I

$\tau / C$ ENABLE 
Processor and Data(OPS Unit 1)

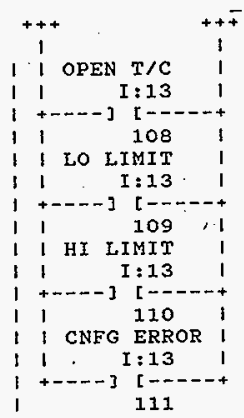

\section{Rung 2:356 \\ i \\ T/C ENABLE}

OPEN $T / C$

1 I:13

+.--- $][--\ldots+\cdots$

I 124

I LO LIMIT

$1 \quad \mathrm{~T}: 13$

$+\cdots-\cdots][-\cdots+$

1125

1 HI LIMIT

1

$I: 13$

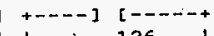

I CNFG ERROR

$\begin{array}{lll}1 & \text { I }: 13 \\ 1 & +\ldots-1\end{array}$

I $+\cdots-1 \quad[-7$

Rung 2:357

1 .

1

$T / C$ ENABLE

I I OPEN T/C

1 I $\mathrm{I}: 14$

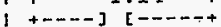

$11^{1} 76$

HNF-SD-FF-CSWD-61 Rev. 0

Page 509 
Processor and Data (OPS Unit 1) Program Listing
October 23, 1996 Page $14 \varepsilon$ Rung $2: 357$

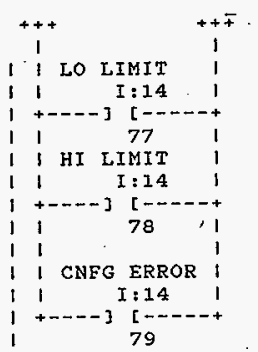

Rung $2: 358$

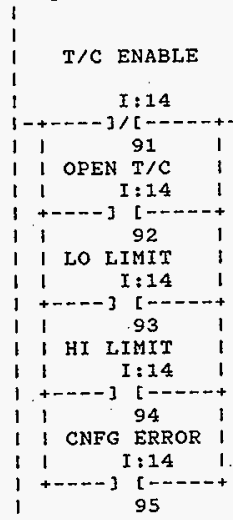

HA174B2

N7 $: 226$

\section{Rung $2: 359$}

1

\section{T/C ENABLE}

$$
I: \pm 4
$$

HAI74V

N7: 226

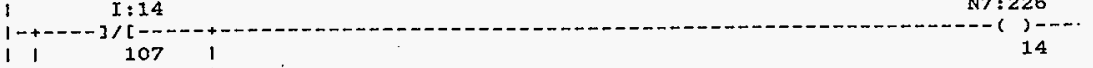

OPEN $\mathrm{T} / \mathrm{C}$

$1 \quad I: 14 \quad$ ।

+.-- $][-\cdots-\cdots+$

$12108 \quad 1$

1 LO LIMIT

11

$I: 14$

HNF-SD-FF-CSWD-61 Rev. 0

$1+\cdots--][-\ldots+\cdots+$

11.109

1

1

Page $5 / 0$ 
Processor and Data(OPS Unit 1)

october 23,1996 Program Listing

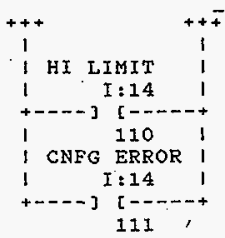

Rung $2: 360$$$
\text { I }
$$

T/C ENABEE

HA174V2

$I: 14$

N7: 226

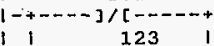

1 OPEN $T / C$

1 I: 14

+--- $]$ [

i 124

I LO LIMIT

$1 \quad I: I 4$

$+\cdots--][----n+$

125

I HI LIMIT

I $I: 14$

1
$+--n-0][---n+$

126

I CNEG ERROR I

I I:14 I

127

\section{Rung $2: 361$}

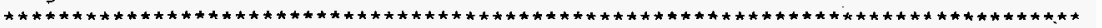
SET DEVIATION SHITCH FOR SECTION 2 AND JUMP TO SECTION 2 AVERAGING ROUTINE

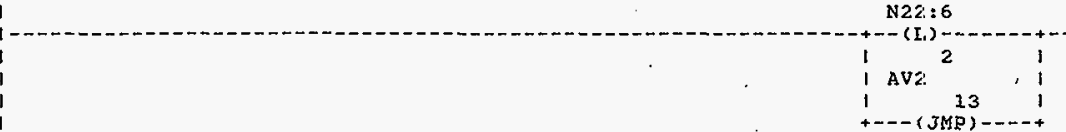

Rung $2: 362$

I DEV2

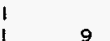

DUMMY9

$--[$ LBL $]-$

N22:7

j

HNF-SD-FF-CSWD-61 Rev. 0

Page $S / 1$ 
Rung $2: 363$

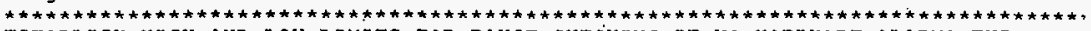
ESTABIISH HIGH AND LOH LIMITS FOR RANGE CHECKING IF NO HARDWARE ALARMS EXIST CHECK T/C VALUES FOR OUT-OF-RANGE CONDITION
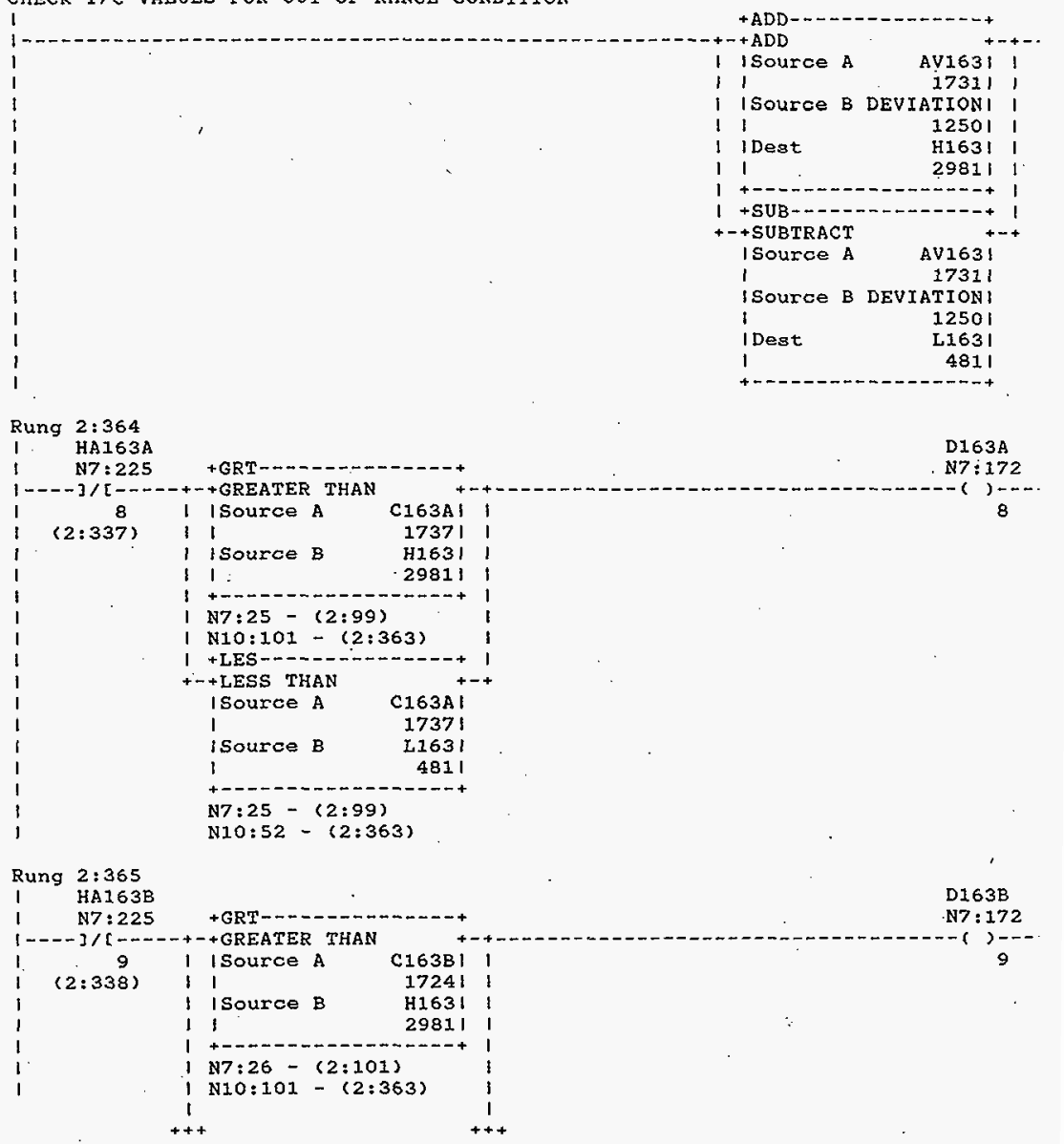

HNF-SD-FF-CSWD-61 Rev. 0 
Processor and Data(OPS Unit 1)

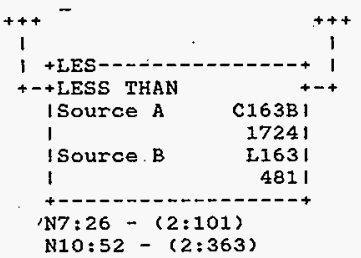

Rung $2: 366$

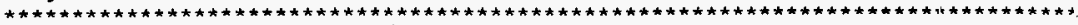
ESTABLISH HIGH AND LOW LIMITS FOR RANGE CHECKING IF NO HARDWARE ALARMS EXIST CHECK T/C VALUES FOR OUT-OF-RANGE CONDITION

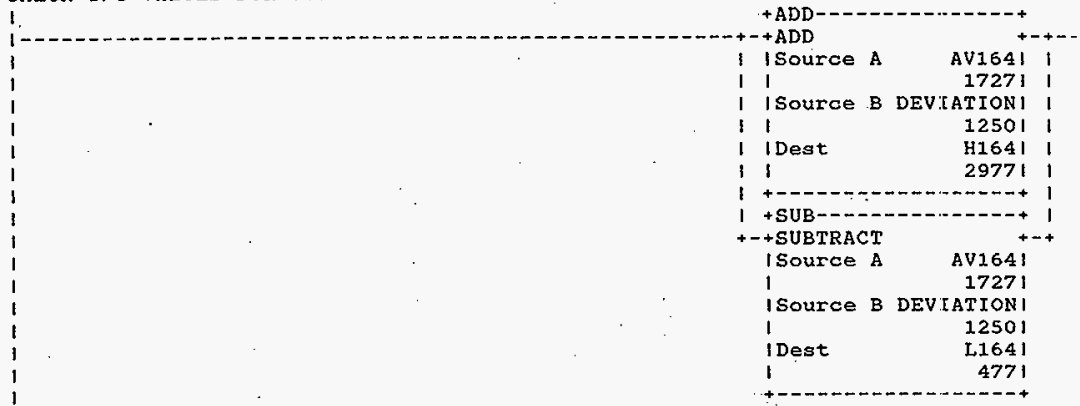

Rung $2: 367$

1 HA164A

I $\quad$ N7:225

] $/[--$

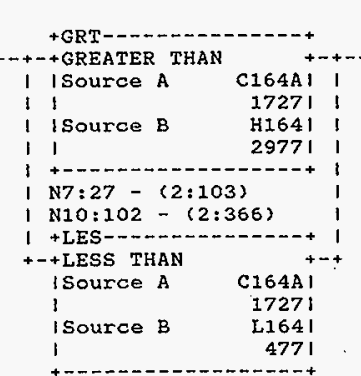

N7:27-(2:103)

N10:53- (2:366)

HNF-SD-FF-CSWD-61 Rev. 0 
Processor and Data(OPS Unit 1)

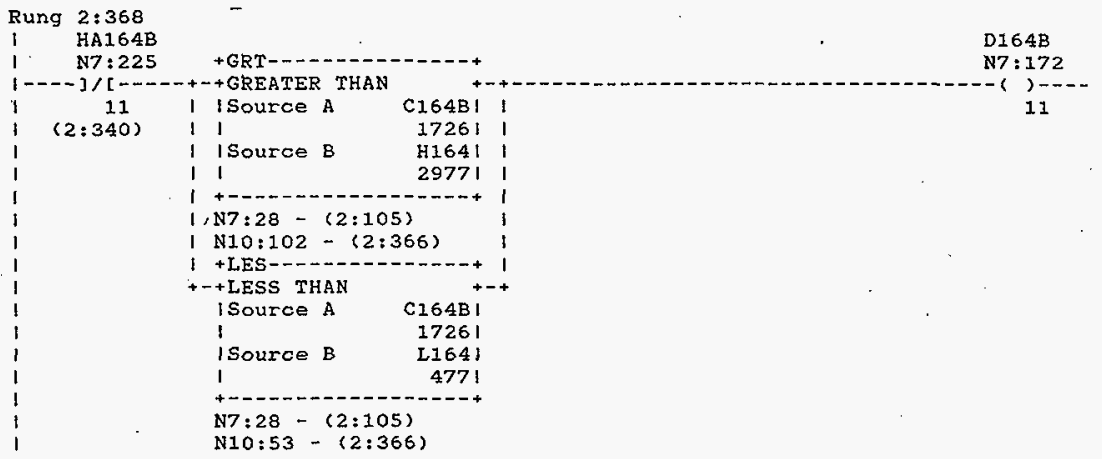

Rung 2:369

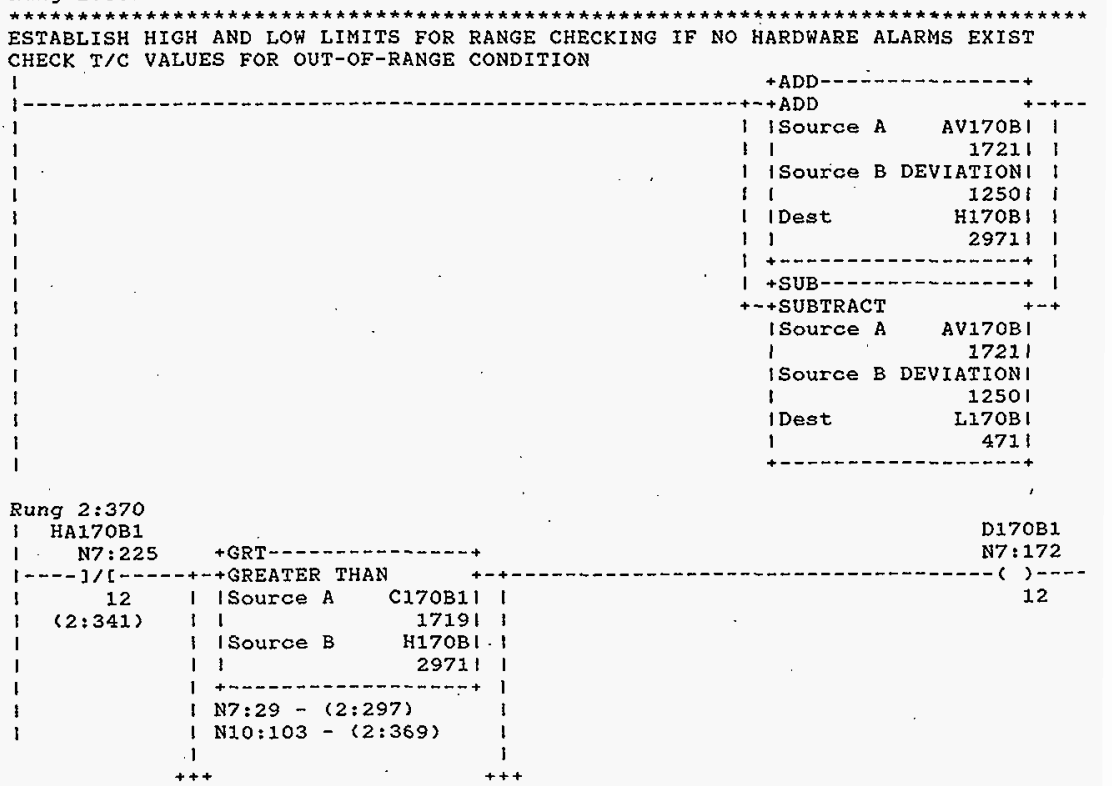

HNF-SD-FF-CSWD-61 Rev. 0 
Processor and Data(OPS Unit 1)

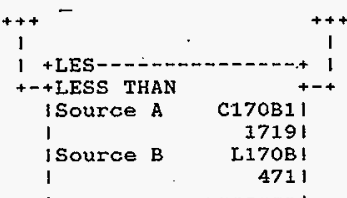

N7:29-(2:29:7)

N10:54-(2:369)

Rung 2:371

(1)

I HAI70B2

$1----7 /[---2+$

13

+GRT---------------+

D170B2

N7 : 172

(2:342)

+-+GREATER THAN

C170B21 1

13

| isource B r H17OBI

I 1 29711

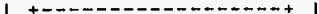

i. N7:30-(2:299)

I N10:103 - (2:369)

i +LES---

+-+ LESS THAN +-+

ISource A C170B21

$1 \quad 17221$

isource B L170B |

I 4711

N7:30-(2:299)

N10:54 - (2:369)

Rung $2: 372$

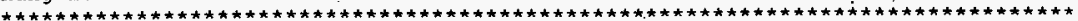
ESTABLISH HIGH AND LOW IIMITS FOR RANGE CHECKING IF NO HARDWARE ALARMS EXIST CHECK T/C VALUES FOR OUT-OF-RANGE CONDITION

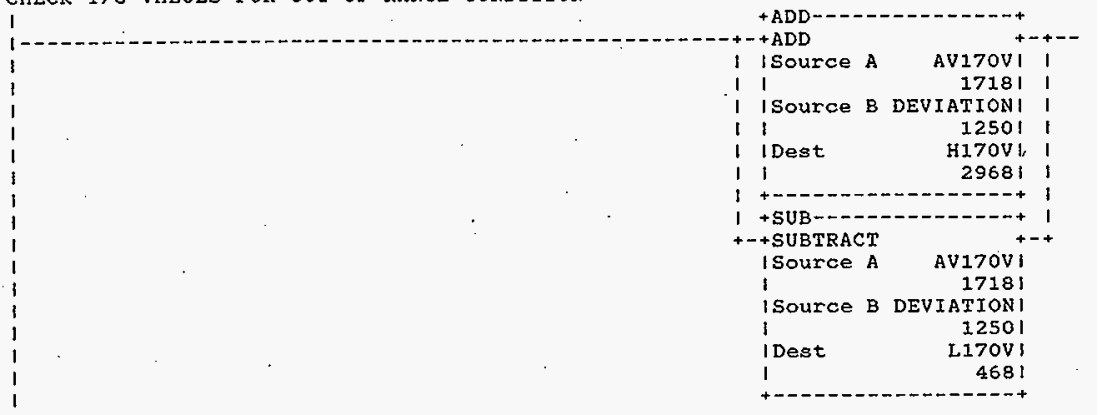

HNF-SD-FF-CSWD-61 Rev. 0

Page 515 
Processor and Data(OPS Unit 1 )

Rung $2: 373$

I HA17OV1 $-$

1. $N 7: 225$

$1--n-] /[---\infty$

+GRT-....--

D170V1

I $12: 343)$

I SOUTCE AHAN

C170V1I I

11 17191।

I Isource B H170V!

I 1 29681 I

1 +-

( $N 7: 31-(2: 301)$

I N10:104-(2:372)

I +LES---...--..--- + 1

+-+ LESS THAN

ISource A C170vil

1

17191

isource B Lifov

1

4681

N7:31-(2:301)

N10:55-(2:372)

Rung $2: 374$

I HA170V2

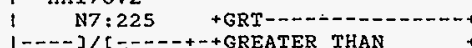

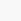

$-\cdots+$

D170V2

N7: 172

I 15 I Isource A C170v2!

$-(,--\infty)$

$(2: 344)$

11

17171

I ISource B HI7OVI।

I I 29681 I

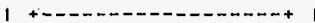

I N7:32-(2:303)

( N10:104-(2:372)

1 +LES---- - - - - - - - + 1

++ LESS THAN +.+

isource A C170V21

$1 \quad 1717$ !

| Source B IITOV|

I 4681

+

N7:32-(2:303)

N10:55 - (2:372)

Rung $2: 375$

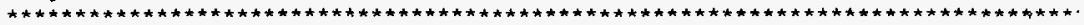
ESTABLISH HIGH AND LOH LIMITS FOR RANGE CHECKING IF NO HARDHARE ALARMS EXIST CHECK T/C VALUES FOR OUT-OF-RANGE CONDITION

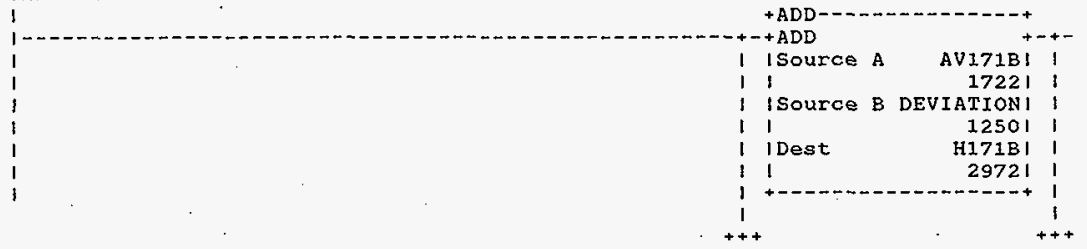

HNF-SD-FF-CSWD-61 Rev. 0

Page $5 / 6$ 


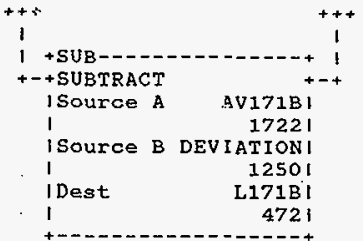

Rung $2: 376$

I HA171B1

I N7:226 $-$

$1----] /[-\ldots+\cdots+4$ + GRT-----2-- - - - - - +

D171B1

10 I ISource A C172B1I

(2:345) 11 . 17321 i

I ISource B H172B| I

11

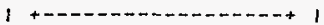

I N7:37-(2:313)

I $N 10: 107-(2: 381)$

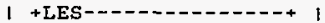

+-+ LESS THAN

ISource A C172B1]

I 17321

|source B L172B |

14831

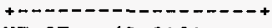

N7:37-(2:313)

N1 $0: 58-(2: 381)$

Rung 2:377

I N7:226

+GRT---- - - - - - - - - - - - - +

D171B2

$1-+-] /[--+++$ GREATER THAN

$(2: 346)$

I ISource A C171B21

N7: 173

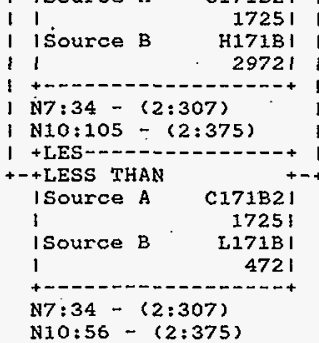

HNF-SD-FF-CSWD-61 Rev. 0

Page $5 / 7$ 
Processor and Data(OPs Unit 1)

Rung 2:378

ESTABLISH HIGH AND LOW LIMITS FOR RANGE CHECKING IF NO HARDWARE ALARMS EXIST

CHECX T/C VALUES FOR OUT-OF-RANGE CONDITION

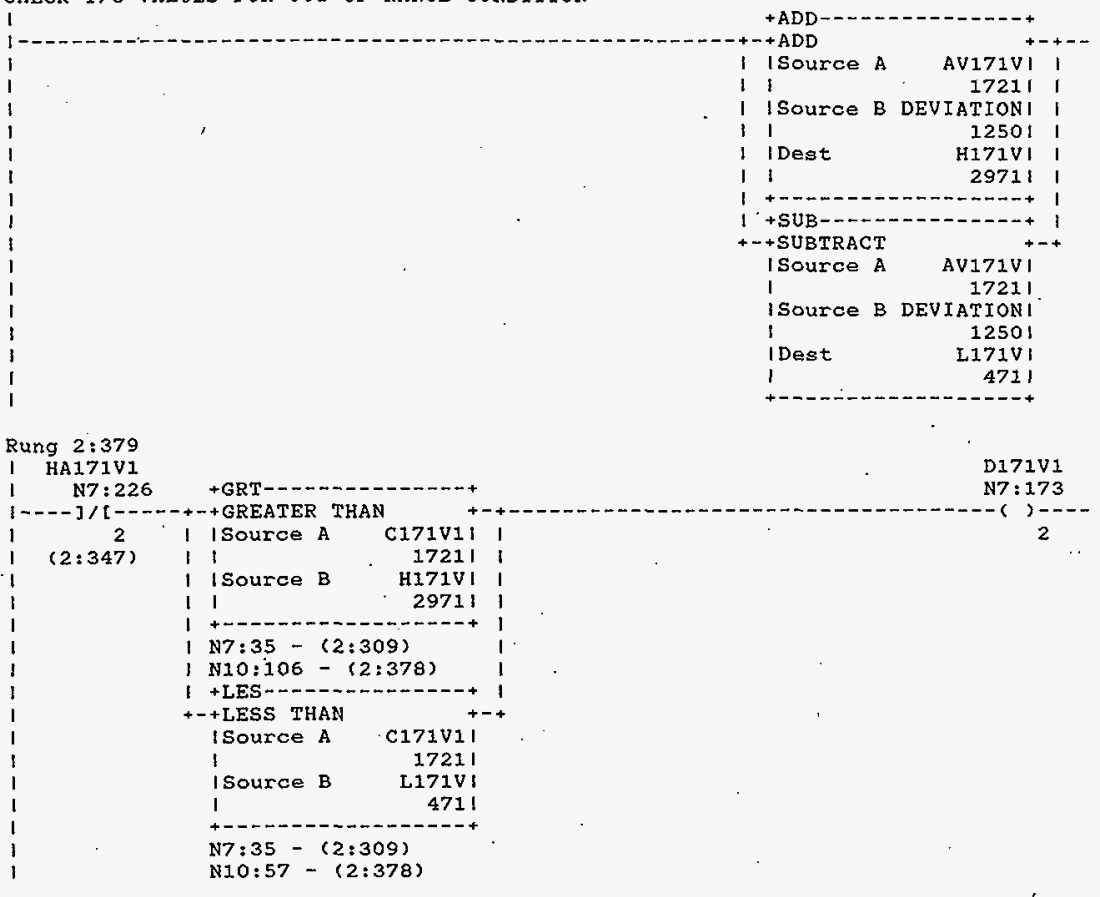

Rung $2: 380$

HA171V2

N7: 226

+ GRT-----.---------+

D171V2

$1-\infty] /[--\cdots++$ GREATER THAN

$\left(2: 348^{3}\right)$

1 isource A

C171V2I

17201

Isource B H171VI

I 29711

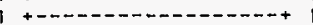

( N7:36-(2:311)

I N10:106-(2:378)

1

$++$

HNF-SD-FF-CSWD-61 Rev. 0

Page $5 / 8$ 


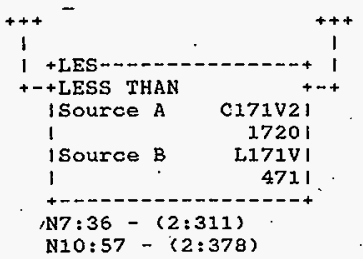

Rung $2: 381$

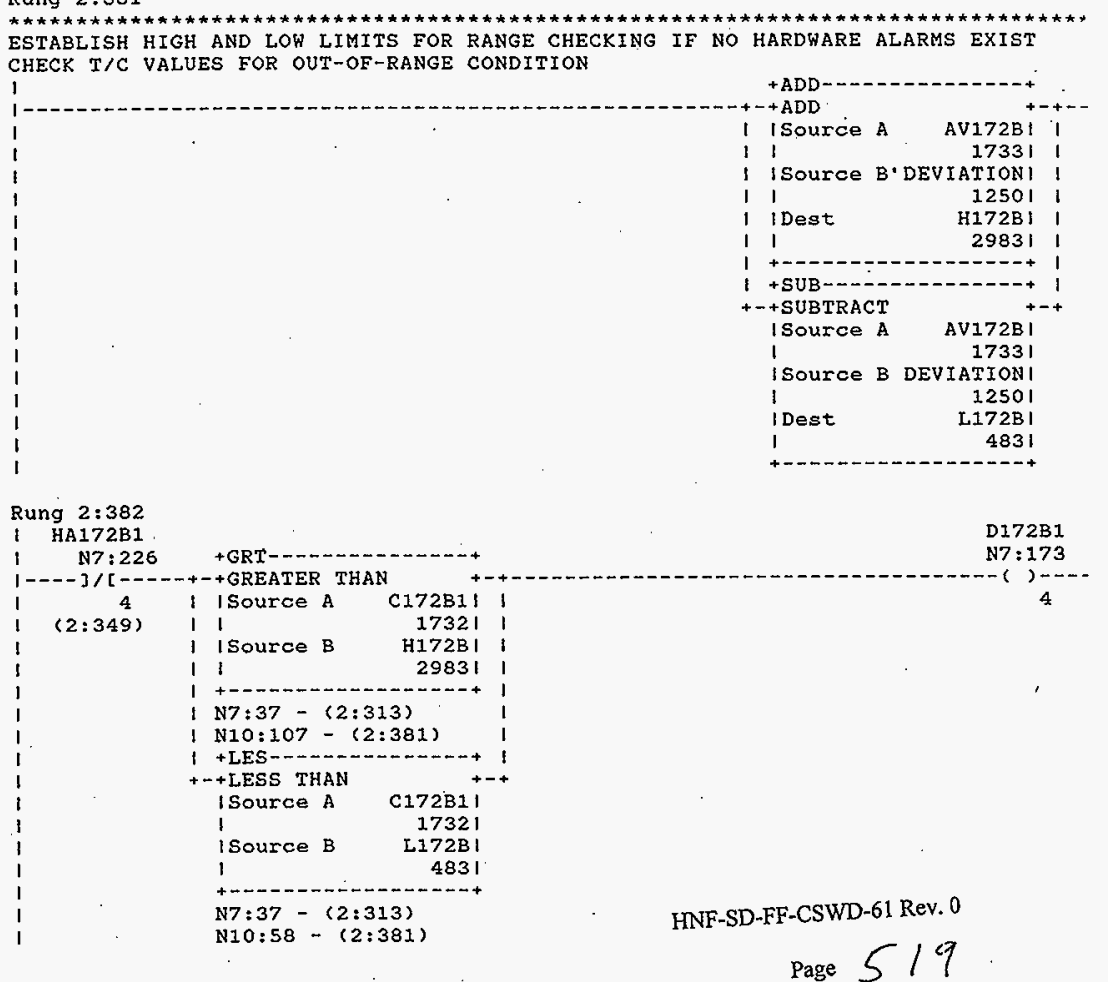


Processor and Data (OPS Unit 1)

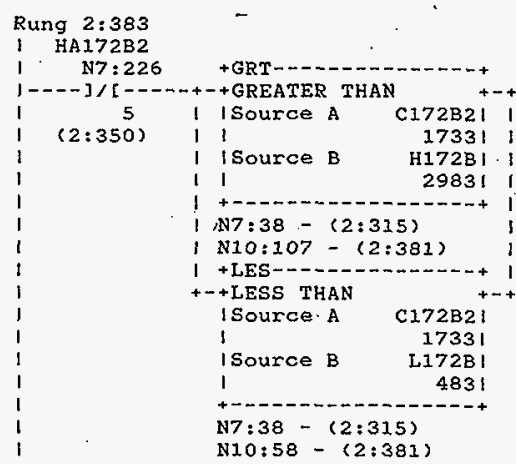

Rung 2:384

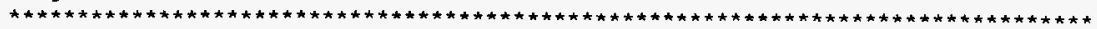
ESTABLISH HIGH AND LOW LIMITS FOR RANGE CHECKING IF NO HARDWARE ALARMS EXIST CHECK T/C VALUES FOR OUT-OF-RANGE CONDITION

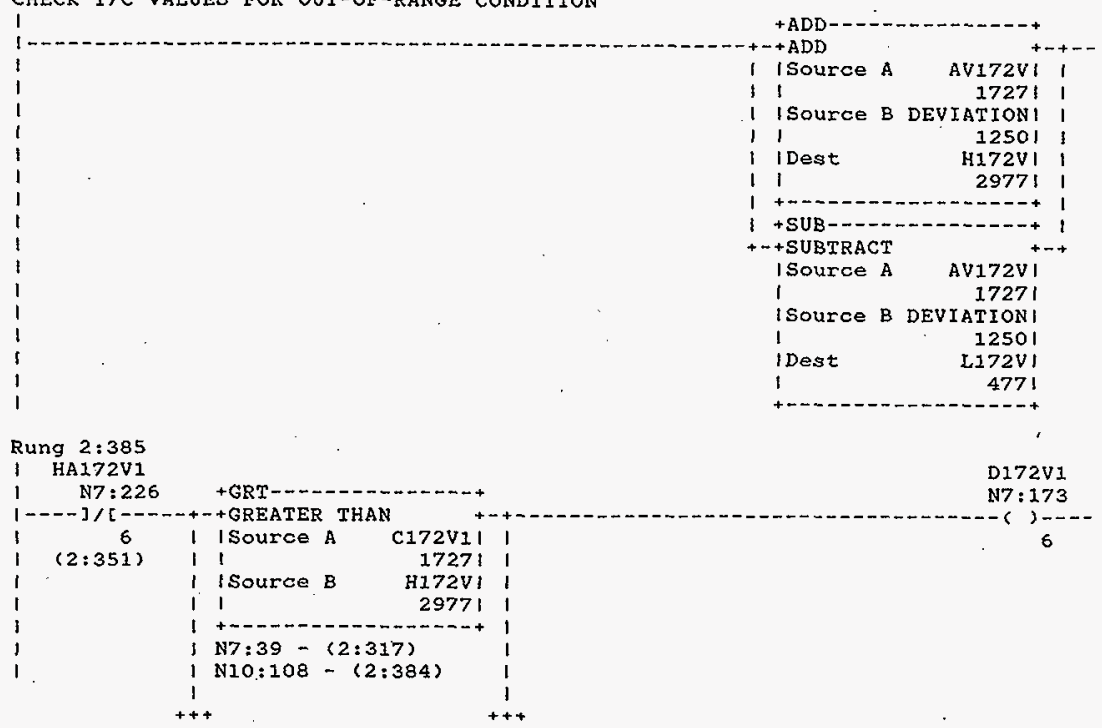

HNF-SD-FF-CSWD-61 Rev. 0

Page 520 


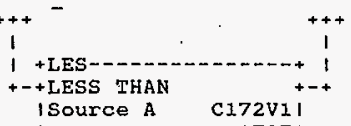

i 1727 ।

ISource B L172V|

I 477 ।

$1 N 7: 39-(2: 317)$

$N 10: 59-(2: 384)$

Rung $2: 386$

I HA172V2

1 N7:226

+GRT------- - - - - - +

D172V2

$1---] /[-\ldots+++$ GREATER THAN

1 I ISource A C172V2I I

$N 7: 173$

$(2: 352)$

11

I ISource B H172VI

11

$2977 !$

$1+--------------+1$

I N7:40-\{2:319\}

l N10:108 - (2:384)

1 +LES-------------- + 1

+-+ LESS THAN +-+

1Source A C172V2I

i 1726 I

| Source B L172V|

$1 \quad 4771$

+-- - - - - -

$N 7: 40-(2: 319)$

N10:59-(2:384)

Rung $2: 387$

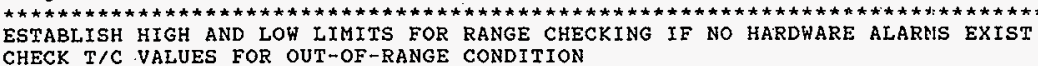

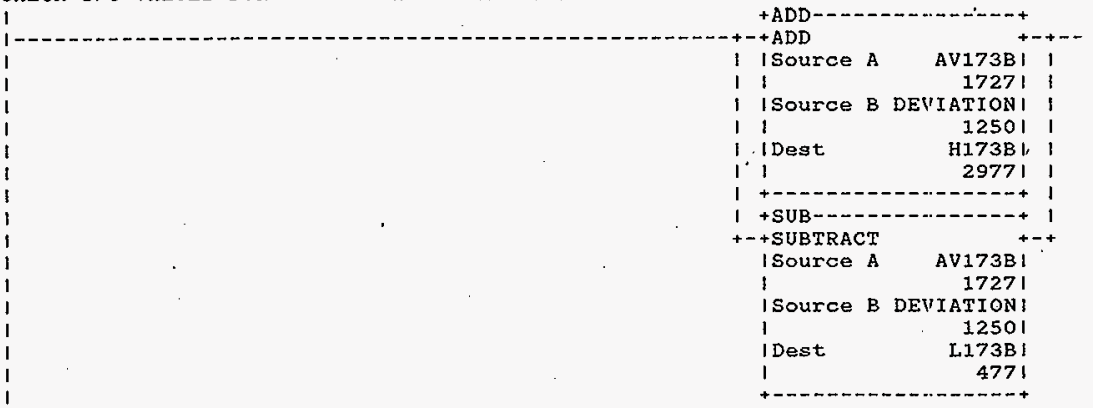

HNF-SD-FF-CSWD-61 Rev. 0

Page 521 
Processor and Data(ops Unit 1)

\section{Rung 2:388}

I HA173B1

N7:226

$-$
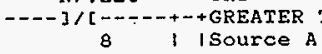

I I Source A

DI73B1

N7 $: 173$

8
$(2: 353)$

C17381। I

17261

I ISource B H173B I

I 1 2977! I

$1+0-0.0-0 .-0-0-0+1$

I N7:41-(2:321)

1 N10:109-(2:387)

$1+$ LES--

+-+ LESS THAN +-+

ISource A C173B1|

I 17261

|Source B L173B I

I 4771

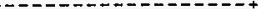

N7:41-(2:321)

N10:60-(2:387)

Rung 2:389

I HA173B2

$1 \quad$ N7:226

]/[-_.+++GREATER THAN

9 ISource A C173B21 ।

(2:354)

17271 I

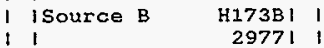

11 29771

I $\mathrm{N} 7: 42-(2: 323)$

I N10:109-(2:387)

I +LES--.-.-.......

+-+ LESS THAN +-+

ISource A C173B21

I 1727 I

|Source B : LI73B 1

i 477 i

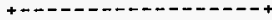

N7:42-(2:323)

N10:60-(2:387)

Rung 2:390

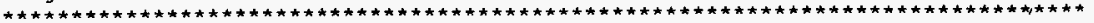
ESTABLISH HIGH AND LOW LIMITS FOR RANGE CHECKING IF NO HARDWARE ALARMS EXIST CHECK T/C VALUES FOR OUT-OF-RANGE CONDITION

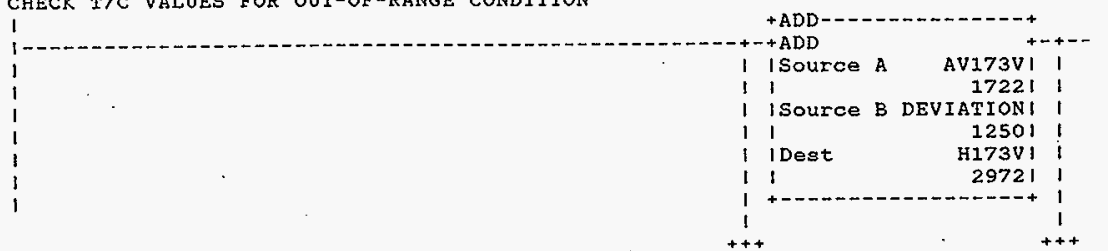

HNF-SD-FF-CSWD-61 Rev. 0 
Processor and Data(OPS Unit 1)

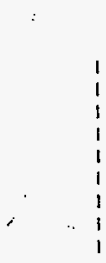

Rung 2:391

I HA173VI

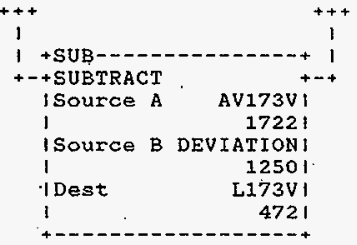

$1 \quad N 7: 226$

+GRT---.-.--- - - - - - +

D173V1

$(-\infty] /[--$

+GRT-GREATER THAN

$(2: 355)$

I Isource A

11

I Isource B

C173V11

N7: 173

1

17251

H173VI 1

$29721^{\circ}$

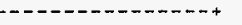

( N7:43-(2:325)

( N10:110-(2:390)

$1+$ IES-

+-+ LESS THAN

ISource A

C173V1!

$1 \quad 17251$

ISource B L173VI

I

4721

N7: $43-(2: 325)$

N10:61-(2:390)

Rung $2: 392$

I HA173V2

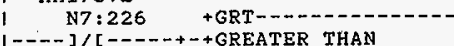

11

I I Source A

C173V21

$(2: 356)$

I

isource 8

17191

H173V 1

29721

1

29721

1 N7:44-(2:327)

1 N10:110 $-(2: 390)$

1 +LES-C......-------+

+-+LESS THAN

ISource A

C.173V2I

1

| Source B L173V |

I

4721

+.--

N7:44-(2:327)

$N 10: 61-(2: 390)$

HNF-SD-FF-CSWD-61 Rev. $0^{\circ}$ 
Rung $2: 393$

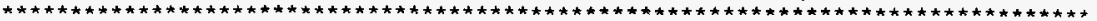
ESTABLISH HIGH AND LOW LIMITS FOR RANGE CHECKING TF NO HARDWARE ALARMS EXIST

CHECK T/C VALUES FOR OUT-OF-RANGE CONDITION

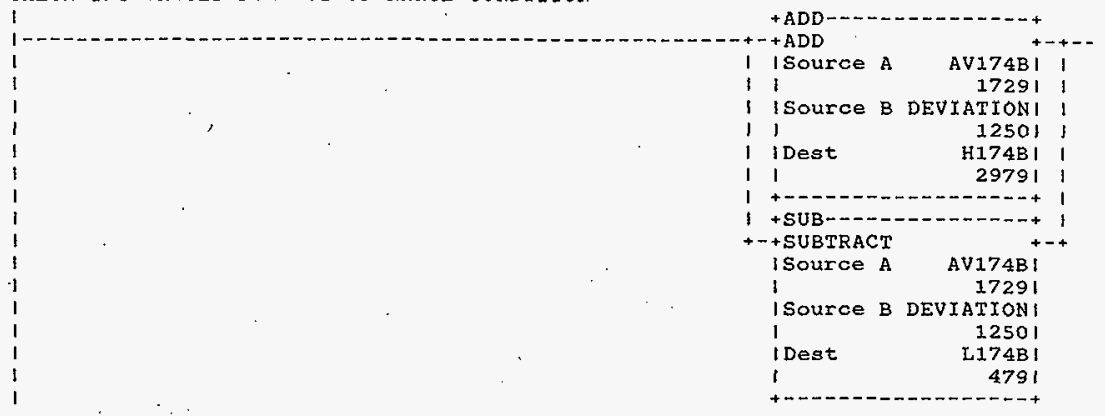

Rung $2: 394$

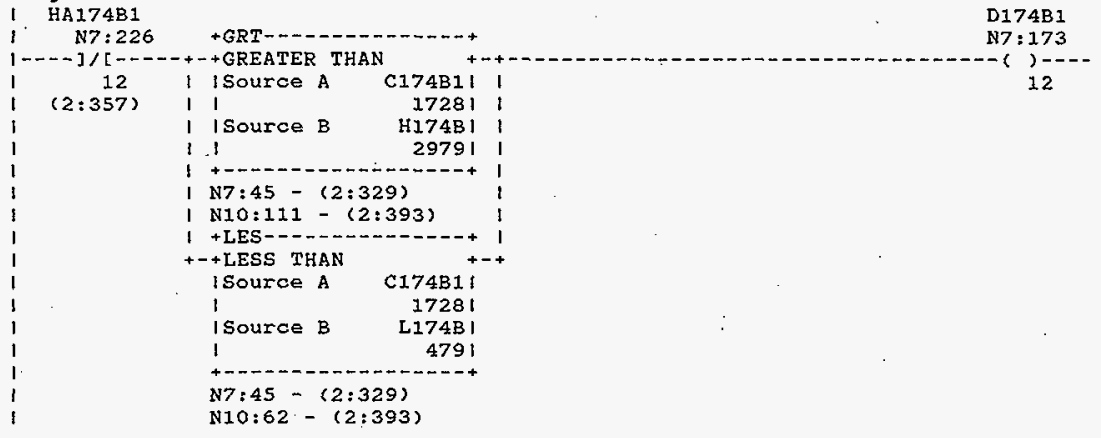

Rung $2: 395$

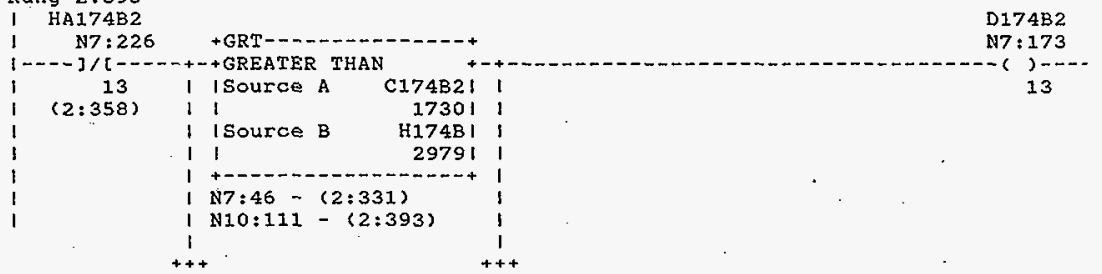

HNF-SD-FF-CSWD-61 Rev. 0 
Processor and Data(OPS Unit 1)

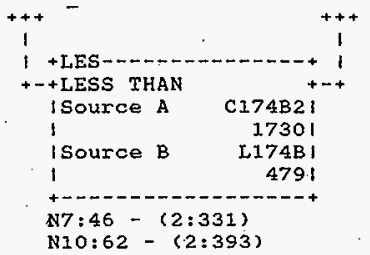

Rung 2:396

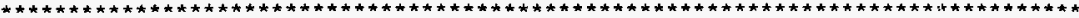
ESTABLISH HIGH AND LOW LIMITS FOR RANGE CHECKING IF NO HARDWARE ALARMS EXIST CHECK T/C VALUES FOR OUT-OF-RANGE CONDITION

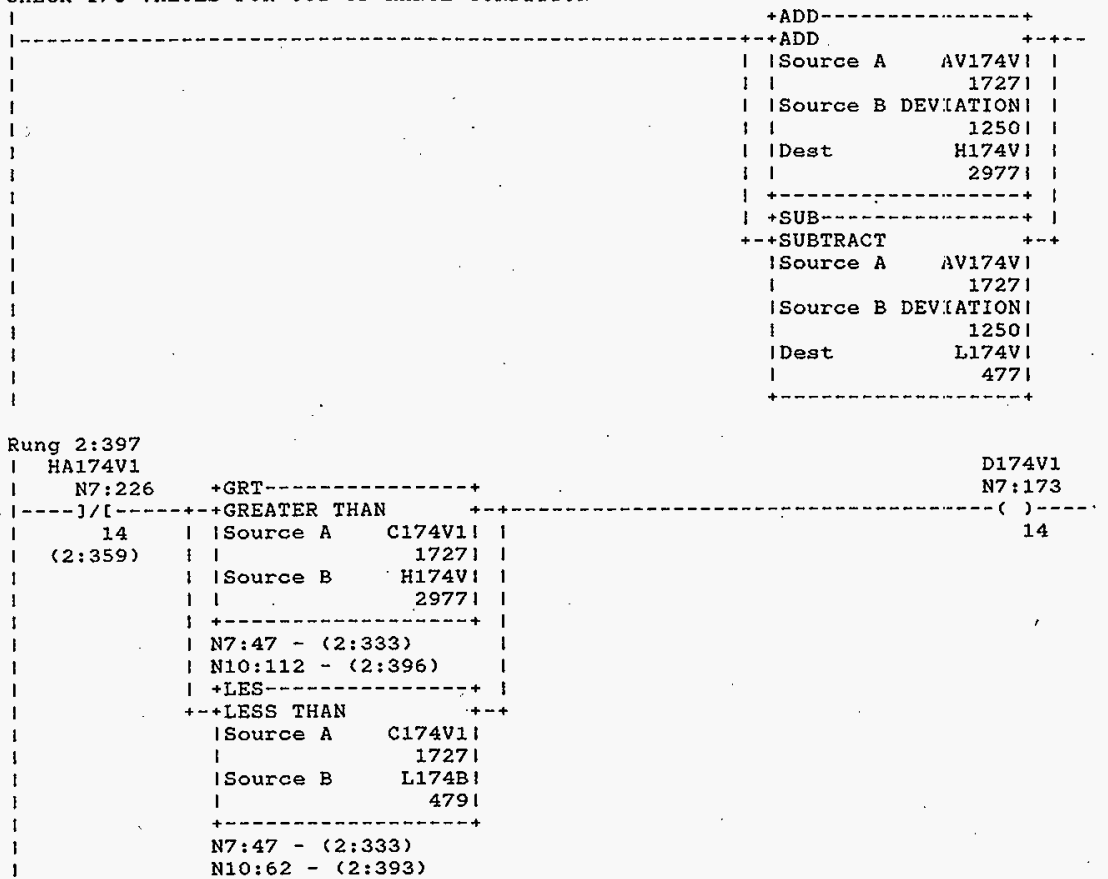

HNF-SD-FF-CSWD-61 Rev: 0

Page 525 
Processor and Data(OPS Unit 1)

Rung $2: 398$
$i$ HA174V2

D174V2

$\mathrm{N} 7: 173$

$$
1 \mathrm{~s}
$$

$(2: 360)$

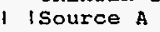

Cis 17

17261

I isource B Hi74VI

I.1. 2977 ।

$1+\ldots \ldots+\ldots+1$

I N7:48 - (2:335)

I N10:112 - (2:396)

1 + IES----

+-+ LESS THAN ++

ISource A C174V2I

1

1726

Isource B L174V |

14771

N7:48-(2:335)

N10:63-(2:396)

Rung $2: 399$

I

I

$$
\text { i }
$$$$
---+
$$

$$
\text { N10:63-(2:396) }
$$

1

Rung 2:400

I Av2

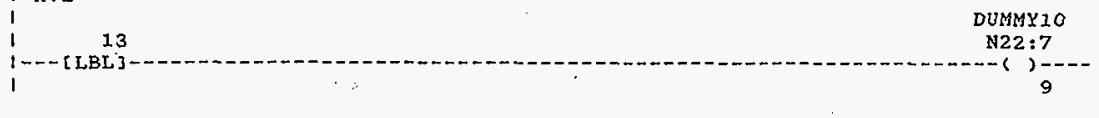

Rung 2:401

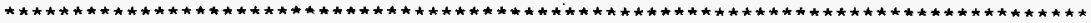
CLEAR COUNT AND TOTAL. IF NO.HARDWARE, NO RANGE ALARMS AND NO THERMOCOUPLES HAVE BEEN TAKEN OUT (TE_OUTXXX) THEN AVERAGE THE T/C

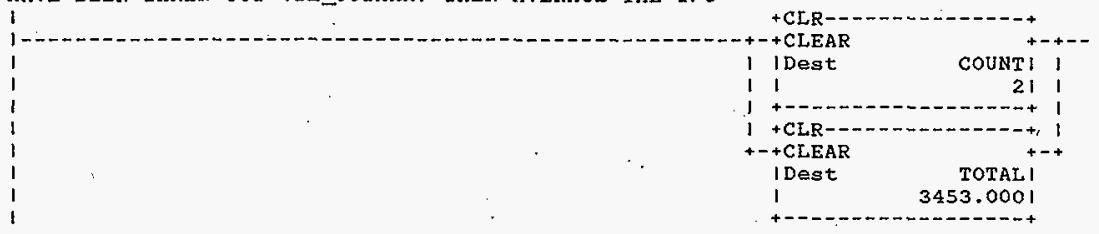

HNF-SD-FF-CSWD-61 Rev. 0

Page 526 
Prócessor and Data (OPS Unit 1

October 23, 1996 Page 165

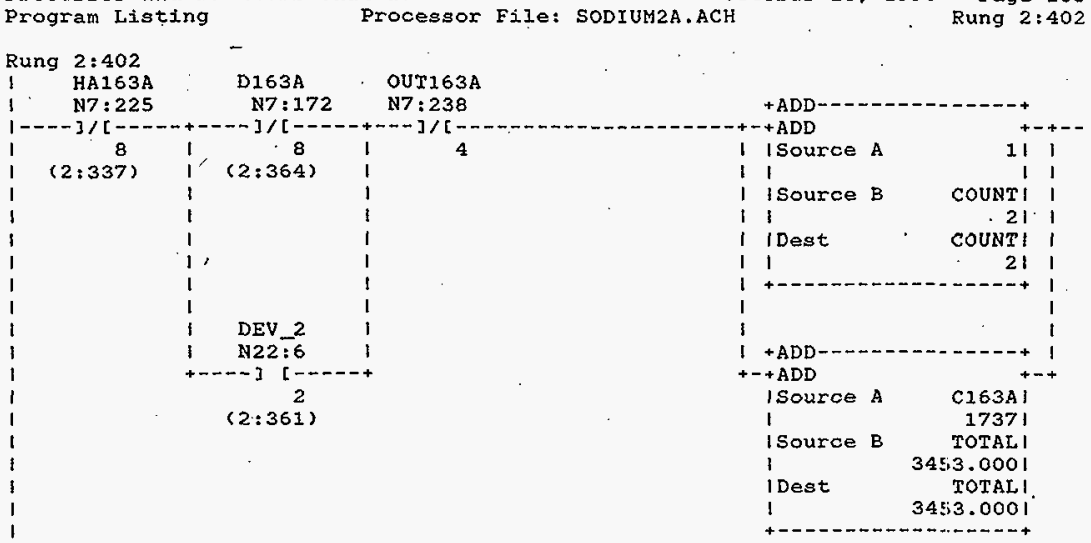

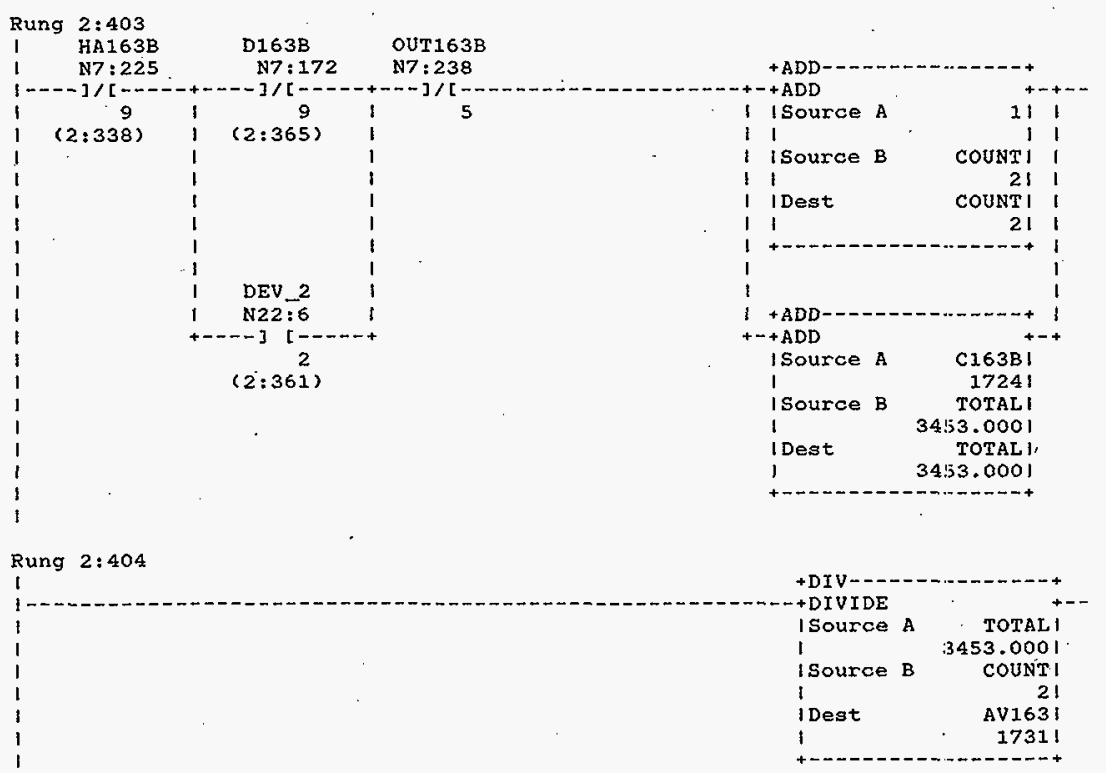

HNF-SD-FF-CSWD-61 Rev. 0 
Processor and Data(OPs Unit 1)

Program listing

\section{Rung $2: 405$}

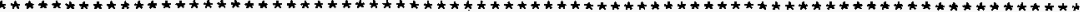
CLEAR COUNT AND TOTAL. IF NO HARDHARE, NO RANGE ALARMS AND NO THERMOCOUPLES HAVE BEEN TAKEN OUT (TE_OUT $X \times X$ ) THEN AVERAGE THE T/C
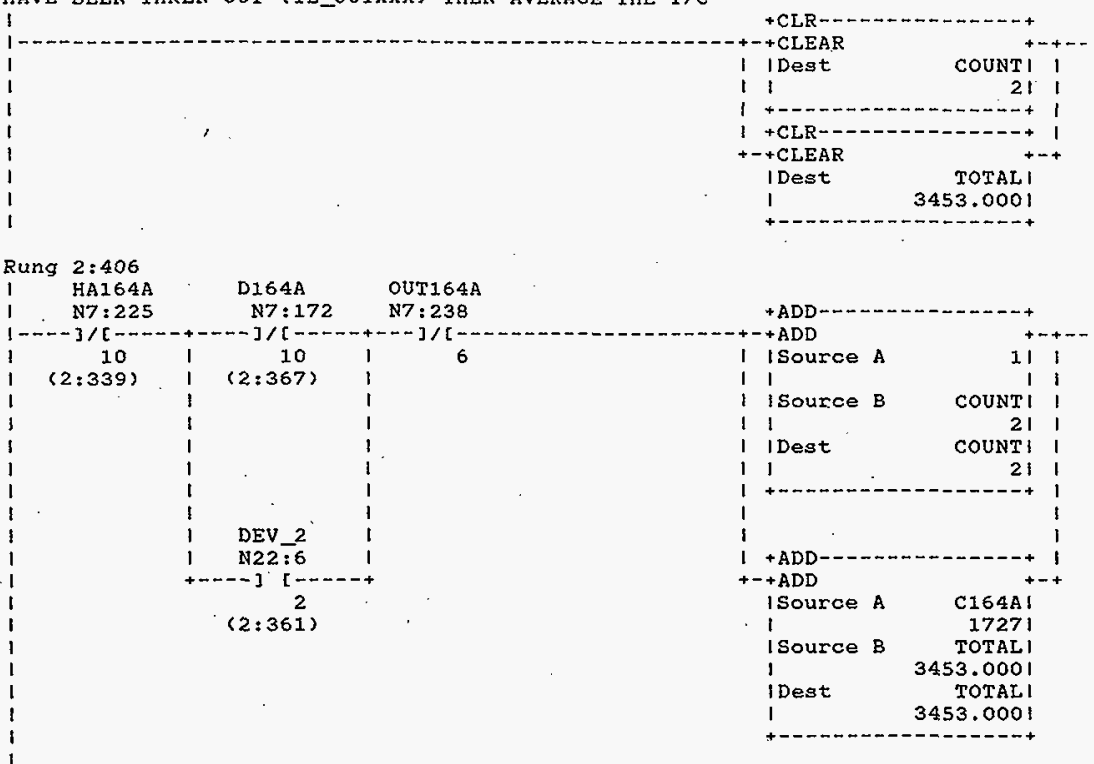

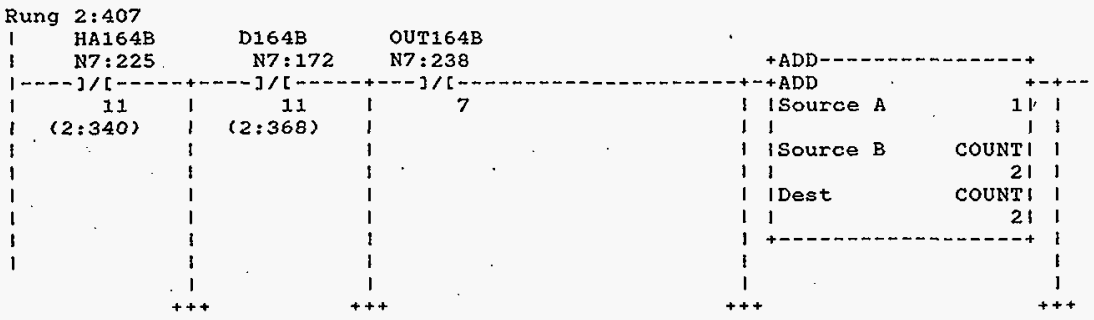

HNF-SD-FF-CSWD-61 Rev. 0 
Processor and Data(ops Unit 1)

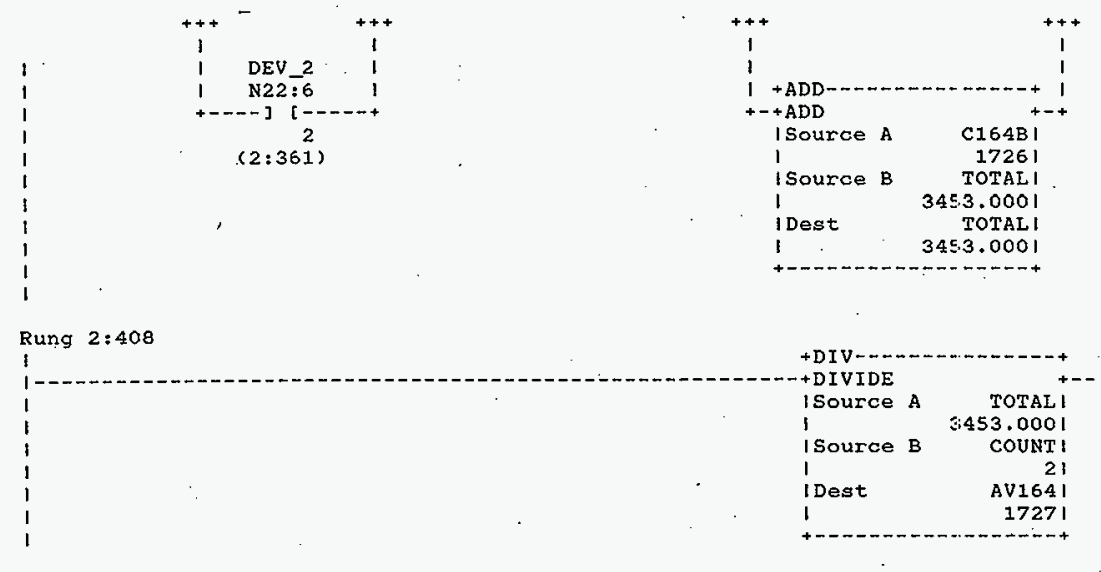

Rung 2:409

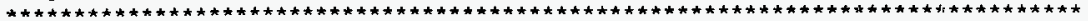
CLEAR COUNT AND TOTAL. IF NO HARDHARE, NO RANGE ALARMS AND NO THERMOCOUPLES HAVE BEEN TAKEN OUT (TE_OUT $\times \times X$ ) THEN AVERAGE THE T/C

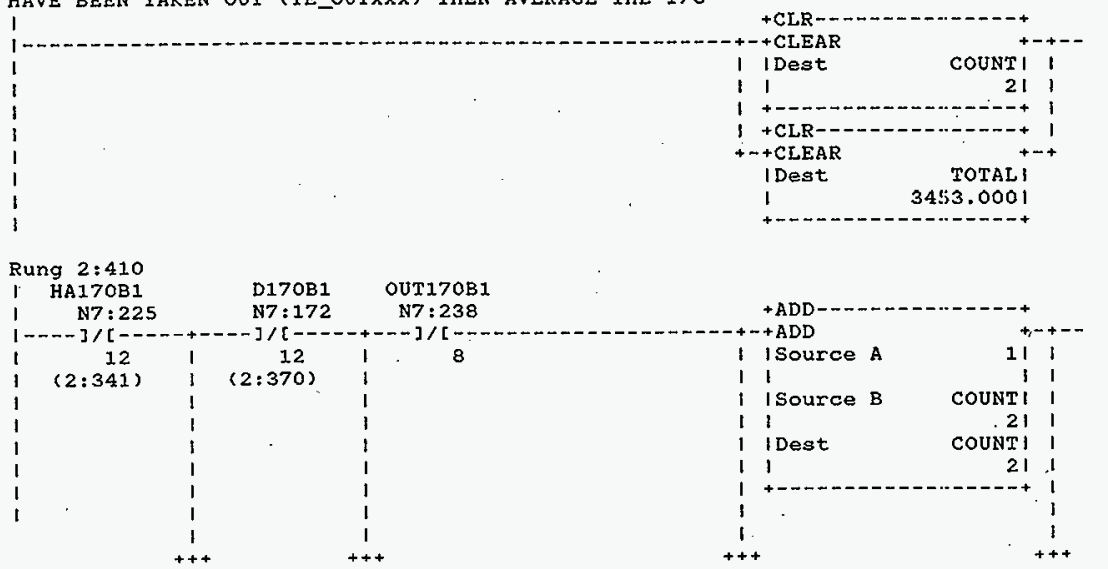

HNF-SD-FF-CSWD-61 Rev. 0 Page 529 


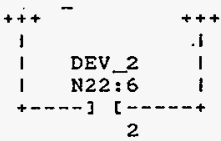

$(2: 36 I)$

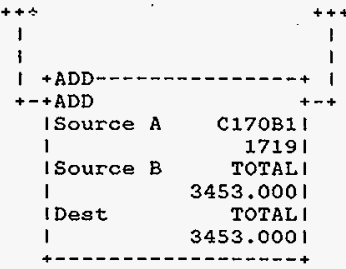

Rung 2:411

1 HA17OB2

N7:225 N7:I72 N7:238

D170B2 OUT170B2

$\begin{array}{llllll}1 & 13 & \text { l } & 13 & 1 & 9\end{array}$

$(2: 342)$

$(2: 371)$

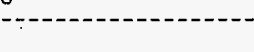

$+\mathrm{ADD}$

1 isource A

1 I

1 isource B

I 1

I IDest

I I

I

I

DEV 2

I N22:6 ।

$(2: 361)$
$1+A D D$

$+-+A D D$

ISource A C170B2!

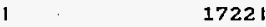

Isource B

TOTAL

I

i Dest

3453.0001

TOTAL 1

I $3453.000 !$

Rung 2:412

I

1

+ DIV

- DIVIDE

I Source A

TOTAL

ISource B

3453.0001

COUNT!

i Dest

AV170B।

I

17211

Rung 2:413

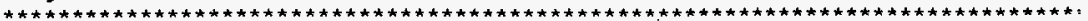
CLEAR COUNT AND TOTAL. IF NO HARDHARE, NO RANGE ALARMS AND NO THERMOCOUPLES HAVE BEEN TAKEN OUT (TE_OUTXXX) THEN AVERAGE THE T/C

1

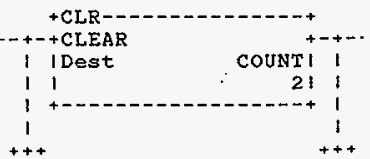

HNF-SD-FF-CSWD-61 Rev. 0 


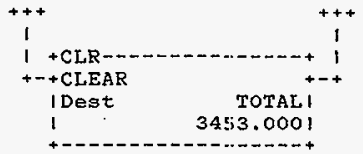

Rung 2:414

Rung 2.414

N7:225

14

$(2: 343)$

14
$343)$

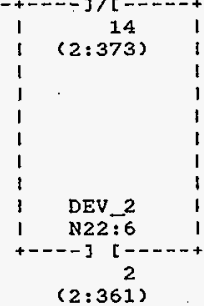

OUT170V1

N7 $: 238$

10

10

$---$

$-$

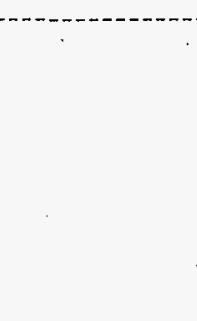

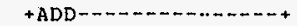

$-+\mathrm{ADD}$

I Source A

1

isource B

11

I Dest

11

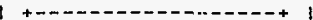

1

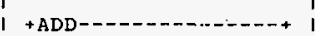

++ + ADD +o+

ISource A c170V11

I. 1719 I

ISource B TOTAL

I 34.53.0001

IDest TOTALI

$1 \quad 3453.0001$

Rung 2:415

\begin{tabular}{|c|c|c|c|c|c|c|c|c|c|c|}
\hline I & HA170V 2 & & D170V2 & & OUT170V2 & & & & & \\
\hline 1 & N7: $225^{\circ}$ & & $N 7: 172$ & & $N 7: 238$ & & $+A D D \ldots-\cdots$ & -- & $---\cdots--m+$ & \\
\hline $1-$ & $---] /[---$ & -+ & $---] /[--\infty$ & +- & $--] / t---$ & + & $+\mathrm{ADD}$ & & $+\infty$ & +-- \\
\hline 1 & 15 & 1 & 15 & 1 & 11 & 1 & ISource & A & 11 & 1 \\
\hline 1 & $(2: 344)$ & 1 & $(2: 374)$ & 1 & & ; & 1 & & I & 1 \\
\hline I & & 1 & & 1 & & 1 & I Source & $\mathbf{B}$ & COUNTI & 1 \\
\hline I & . & 1 & & I & & 1 & 1 & & 21 & I \\
\hline 1 & & 1 & & 1 & & 1 & I Dest & & COUNT 1 & I \\
\hline $\mathbf{I}$ & & 1 & & 1 & & 1 & 1 & & 21 & I \\
\hline 1 & & 1 & & 1 & & 1 & $+--\infty-\infty--$ & -- & $--\ldots+\cdots+-+$ & $\mathbf{I}$ \\
\hline I & & 1 & & 1 & $\cdot$ & 1 & & & , & I \\
\hline j & & I & DEV_2 & 1 & & 1 & & & & 1 \\
\hline 1 & $\therefore$ & 1 & N22:6 & 1 & & 1 & $+A D D=--$ & - & $-------\cdots+$ & $\mathbf{I}$ \\
\hline 1 & & & $--] \quad[---$ & & & & +ADD & & +- & \\
\hline i & & & 2 & & & & I Source & $\mathbf{A}$ & 170v21 & \\
\hline i & & & $(2: 361)$ & & & & 1 & & 17171 & \\
\hline i & & & & & & & I Source & $\mathrm{B}$ & TOTAL I & \\
\hline 1 & & & & & & & 1 & & 3453.0001 & \\
\hline$i$. & & & & & & & i Dest & & TOTALI & \\
\hline i & & & & & & & 1 & & 3453.0001 & \\
\hline 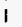 & & & & & & & +------- & -- & --------+ & \\
\hline
\end{tabular}

HNF-SD-FF-CSWD-61 Rev. 0

Page 531 


\section{Rung 2:416}

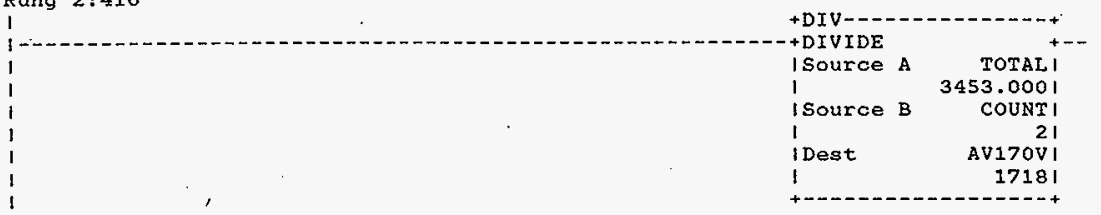

Rung 2:417

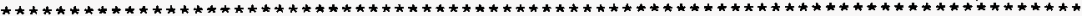
CLEAR COUNT AND TQTAI. IF NO HARDWARE, NO RANGE ALARMS AND NO THERMOCOUPLES HAVE BEEN TAKEN OUT (TE_OUTXXX) THEN AVERAGE THE T/C

$$
\begin{aligned}
& 1 \\
& 1 \\
& 1 \\
& 1 \\
& 1 \\
& 1 \\
& 1 \\
& 1
\end{aligned}
$$

\section{Rung $2: 418$}

( HA $171 \mathrm{B1}$

N7: 226

\begin{tabular}{cc} 
D171B1 & OUT171B1 \\
N7:173 & N7:238. \\
\hline+---$] /[-\ldots+---] /[----$
\end{tabular}

( $\left.2: 345^{\circ}\right)$

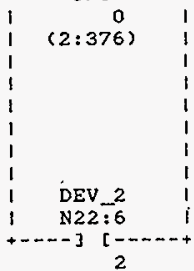

12

$(2: 361)$

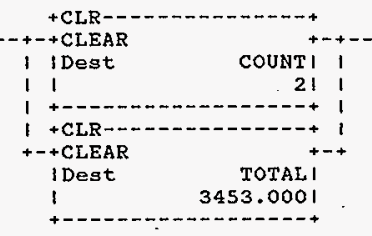

HNF-SD-FF-CSWD-61 Rev. 0

Page 532 


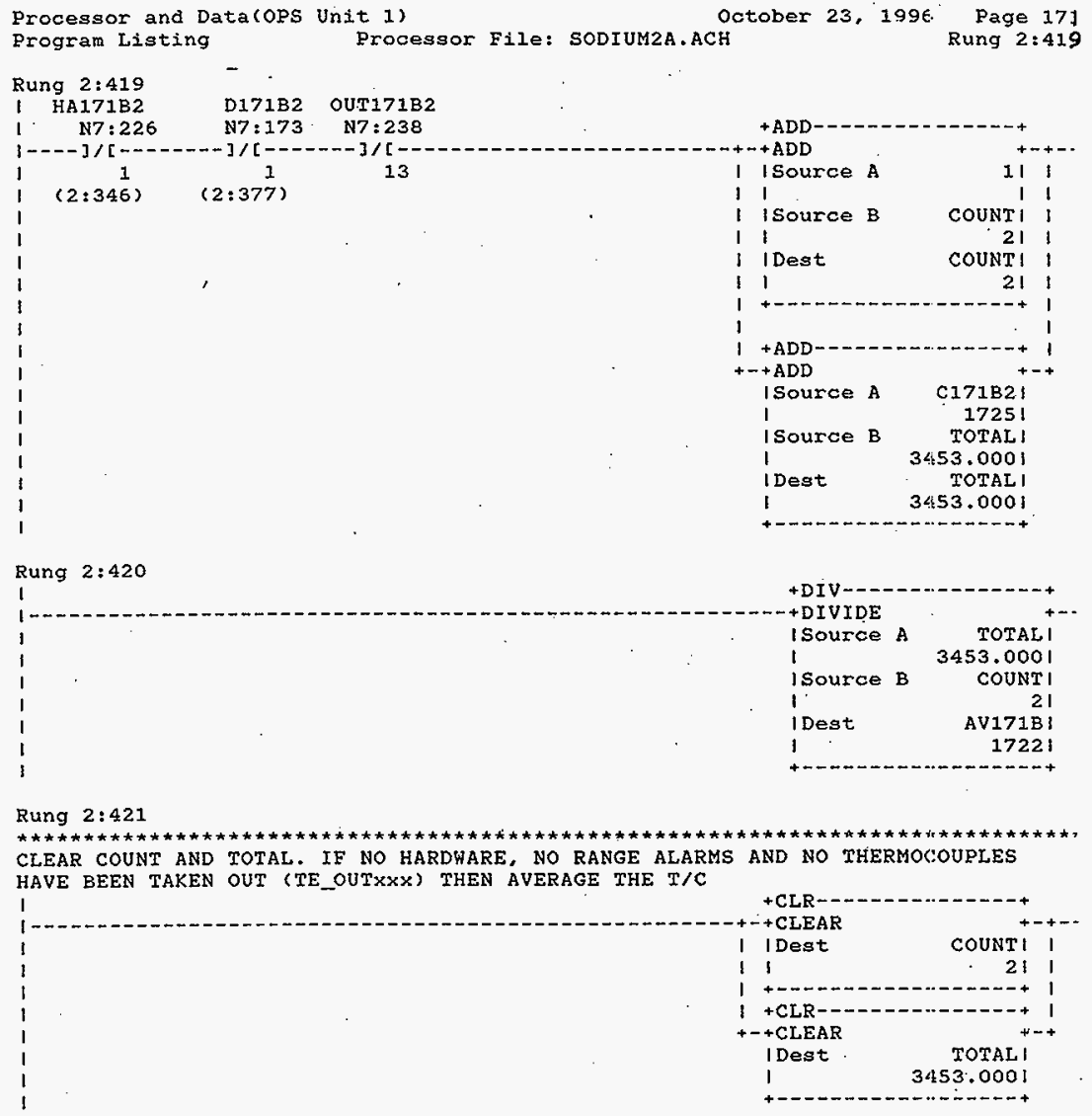

HNF-SD-FF-CSWD-61 Rev. 0

Page 533 
Processor and Data(OPS Unit 1 )
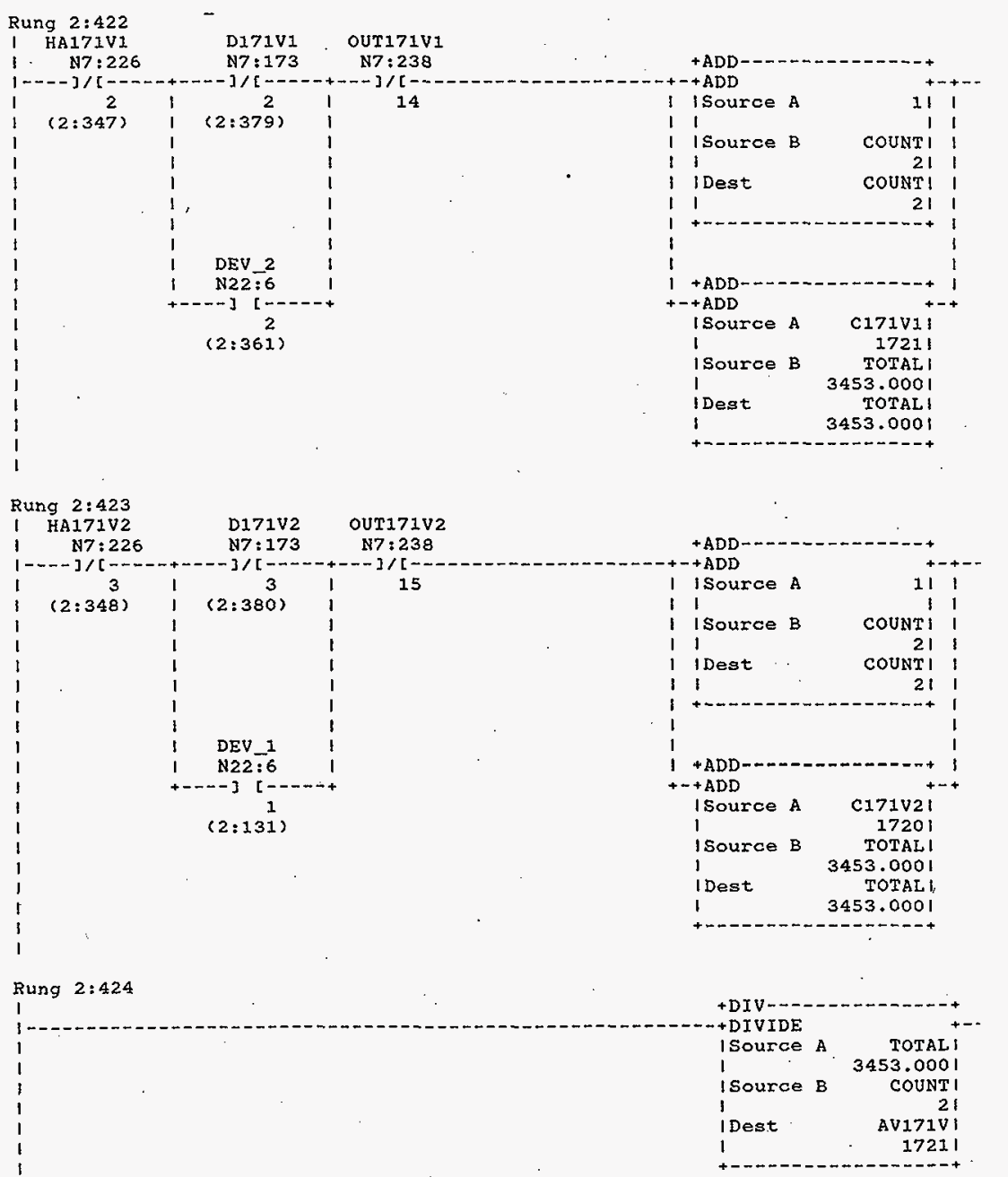

HNF-SD-FF-CSWD-61 Rev. 0

Page 534 
Processor and Data(Ops Unit 1)

Rung $2: 425$

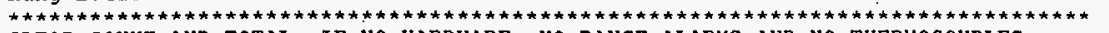
CLEAR COUNT AND TOTAL. IF NO HARDWARE, NO RANGE ALARMS AND NO THERMOCOUPLES HAVE BEEN TAKEN OUT (TE_OUTXXX) THEN AVERAGE THE T/C
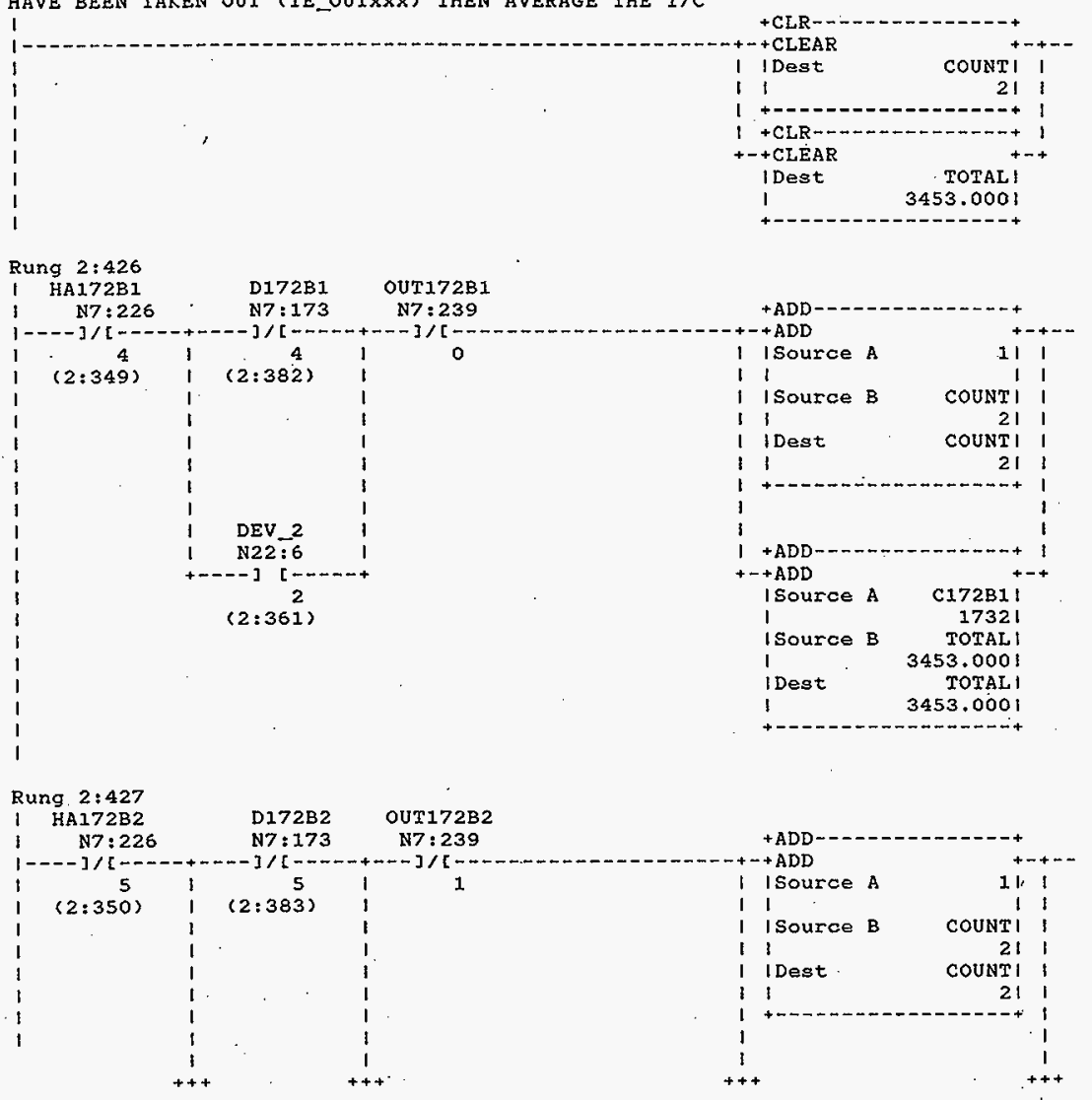

HNF-SD-FF-CSWD-61 RE:V. 0 
Processor and Data (OPS Unit 1 )

October 23, 1996

Page 174

Program Listing

Processor File: SODIUM2A.ACH

Rung $2: 427$
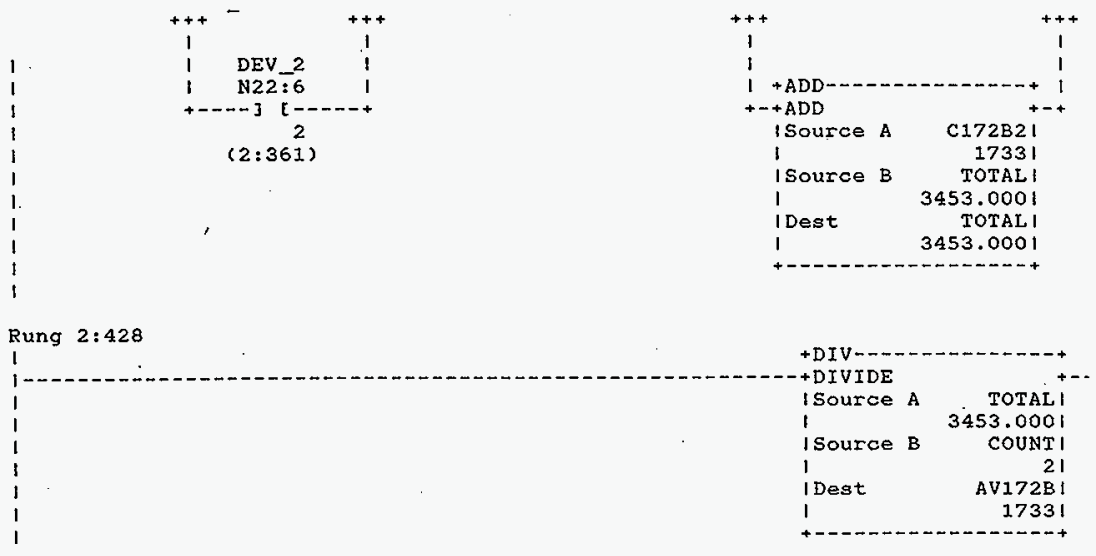

Rung 2:429

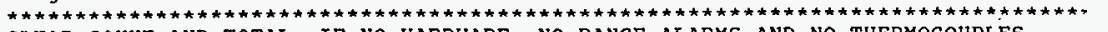
CLEAR COUNT AND TOTAL. IE NO HARDHARE, NO RANGE ALARMS AND NO THERMOCOUPLES HAVE BEEN TAKEN OUT (TE_OUTXXX) THEN AVERAGE THE T/C
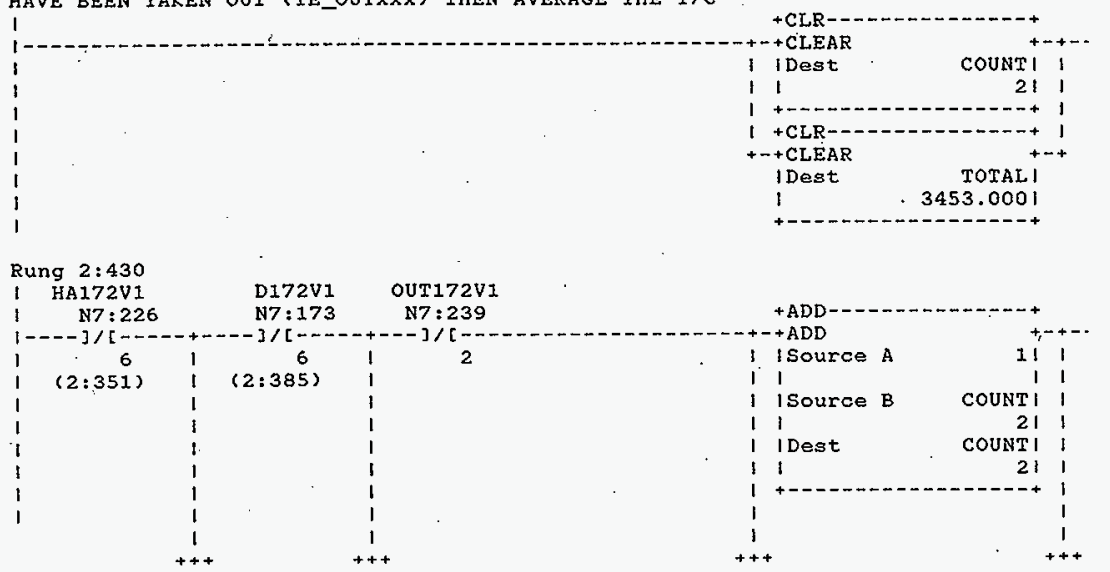

HNF-SD-FF-CSWD-61 Rev. 0

Page 536 
Processor and Data (OPS Unit 1 ) Program Listing
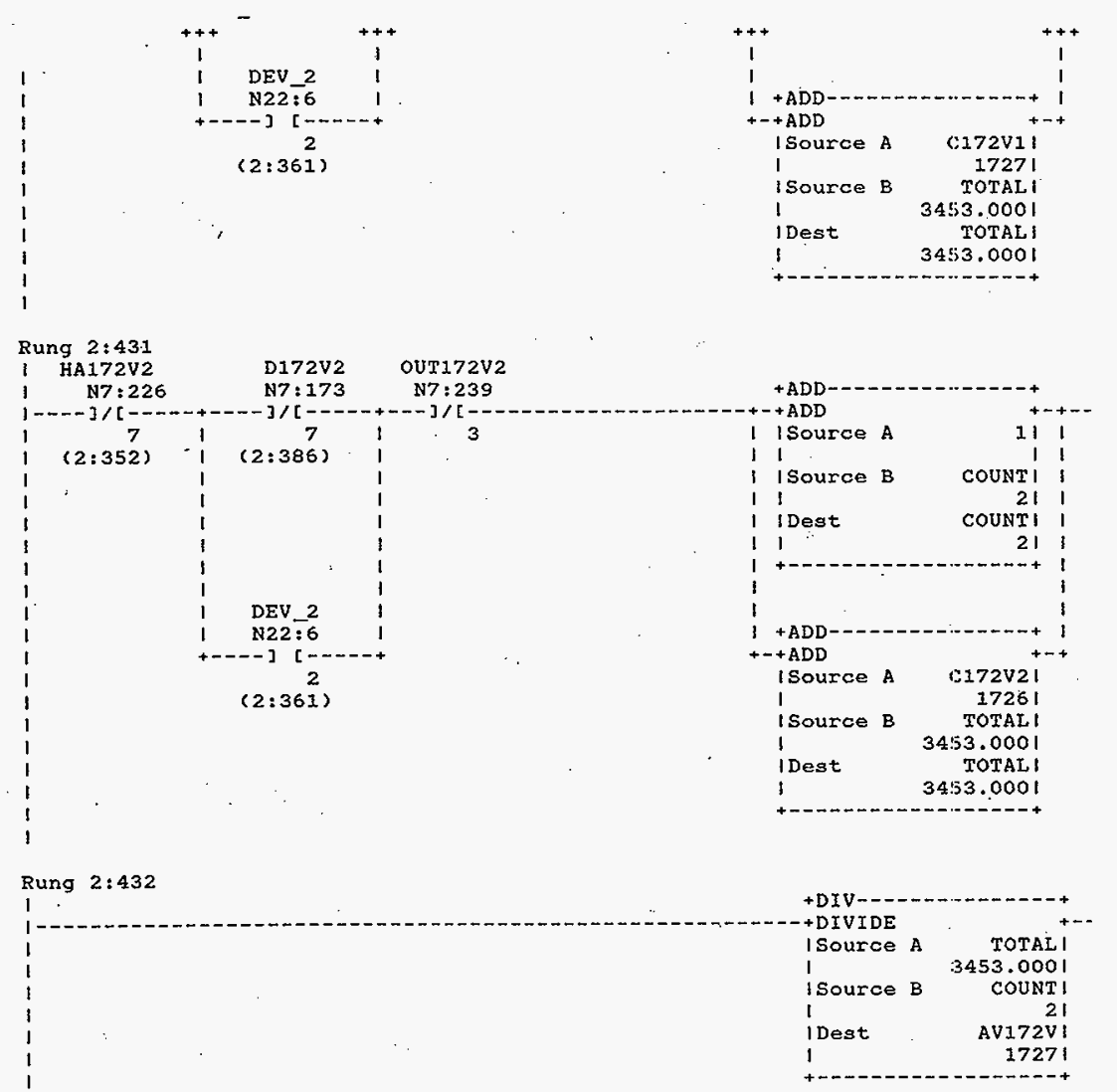

Rung $2: 433$

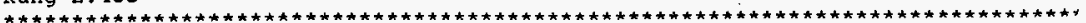
CLEAR COUNT AND TOTAL. IF NO HARDHARE, NO RANGE ALARMS AND NO THERMOCOUPLES HAVE BEEN TAKEN OUT (TE_OUT $\times \times X$ ) THEN AVERAGE THE T/C

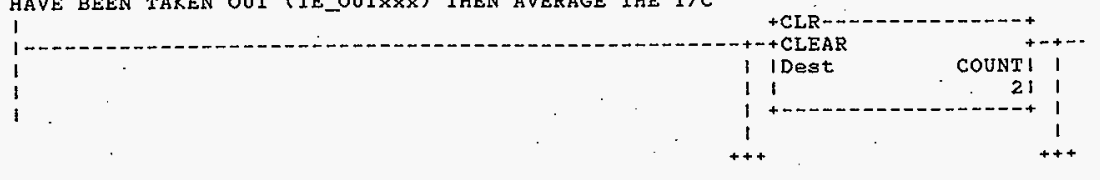

HNF-SD-FF-CSWD-61 Kev. 0 


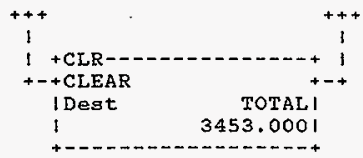

Rung $2: 434$

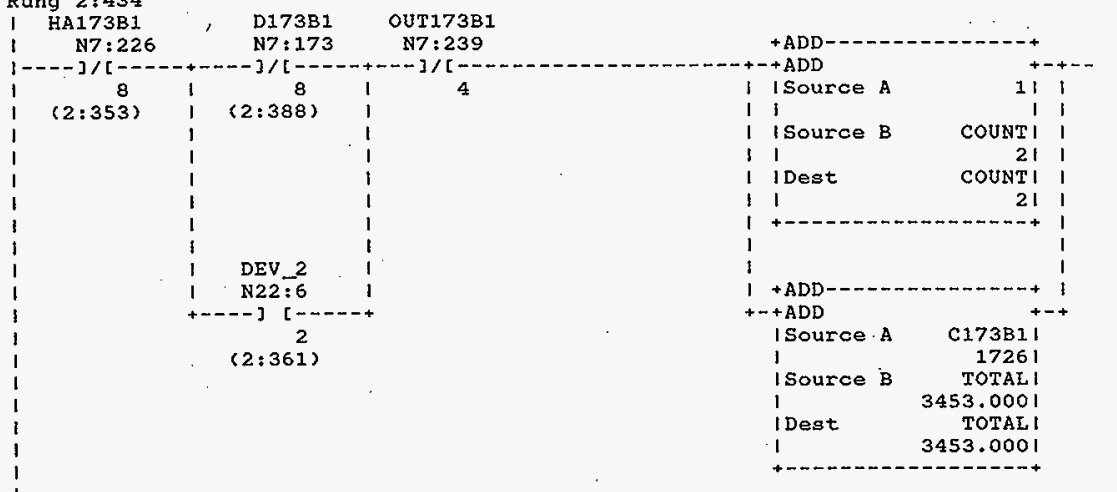

Rung 2:435

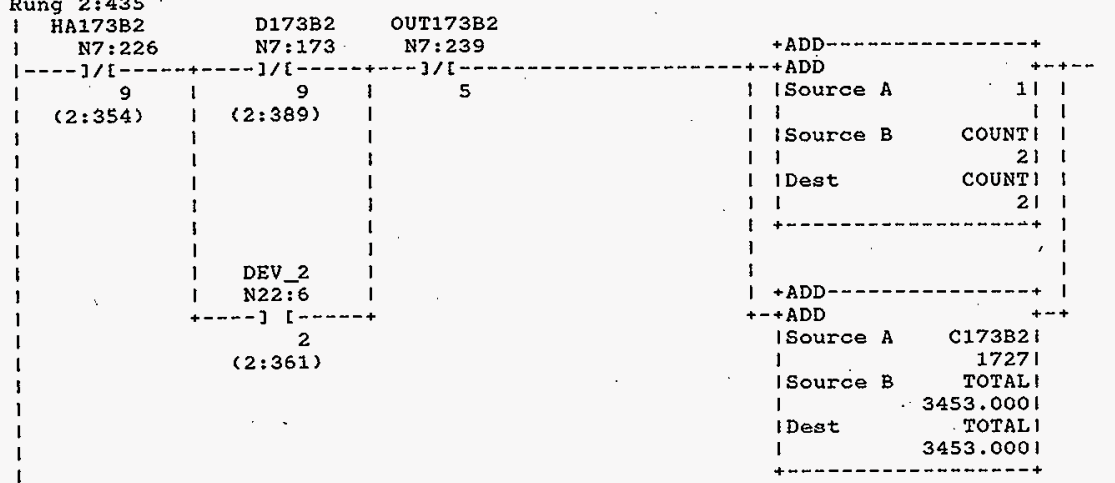

HNF-SD-FF-CSWD-61 Rev. 0

Page 538 


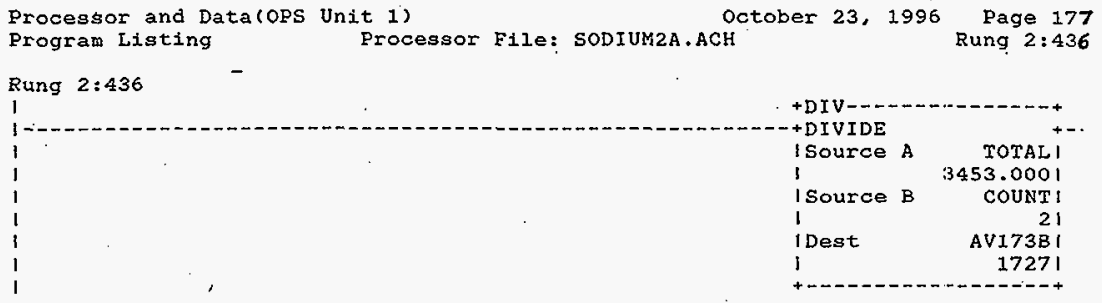

Rung $2: 437^{\circ}$

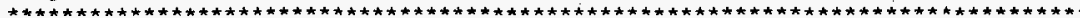
CLEAR COUNT AND TOTAL. IF NO HARDHARE, NO RANGE ALARMS AND NO THERMOCDUPLES HAVE BEEN TAKEN OUT (TE_OUTXXX) THEN AVERAGE THE T/C
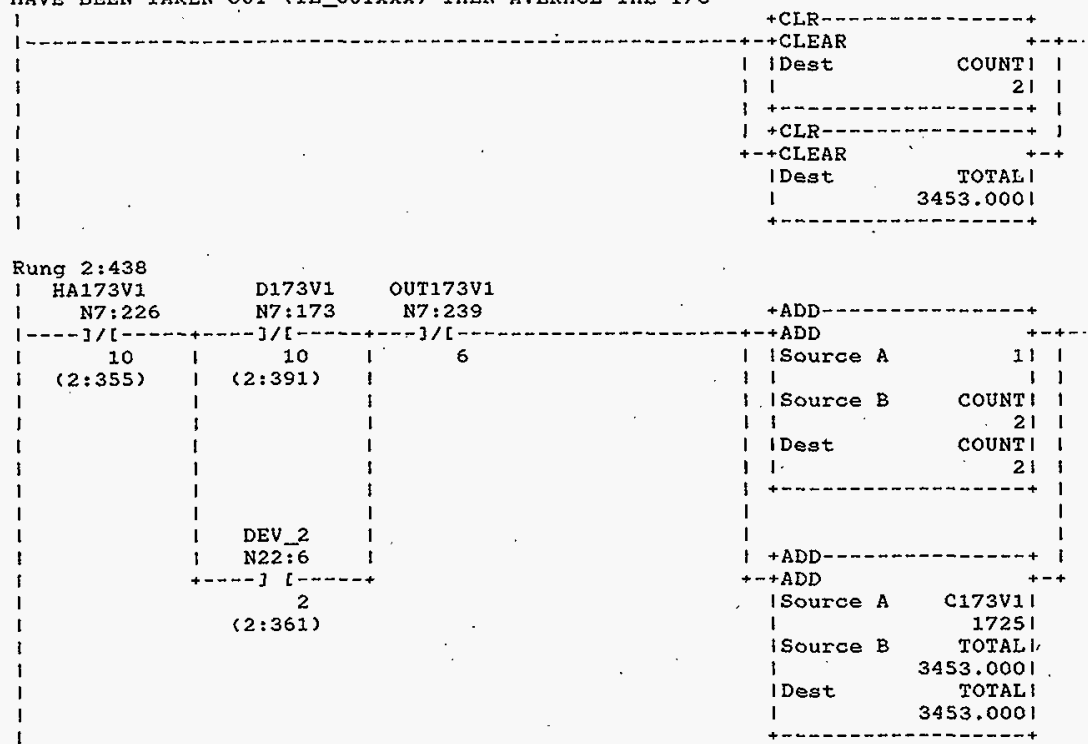

HNF-SD-FF-CSWD-61 Rev. 0

Page 539 
Processor and Data(OPS Unit 1 )

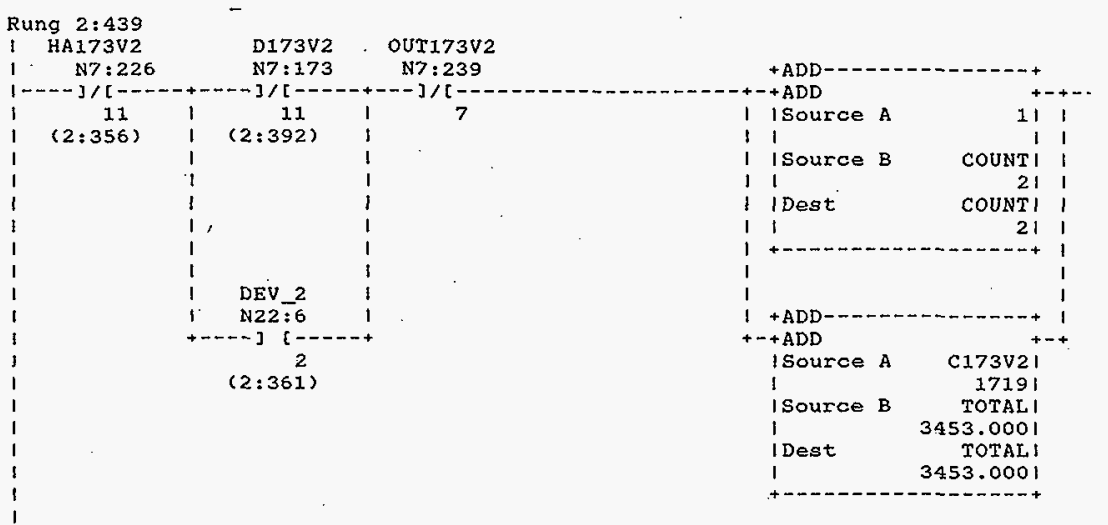

Rung $2: 440$

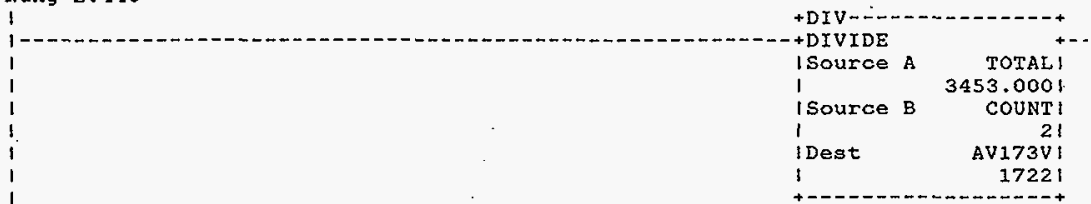

Rung 2:441

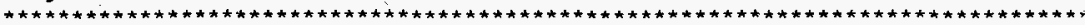
CLEAR COUNT AND TOTAL. IF NO HARDHARE, NO RANGE ALARMS AND NO THERMOCOUPLES HAVE BEEN TAKEN OUT (TE_OUT $\times \times \times$ ) THEN AVERAGE THE T/C

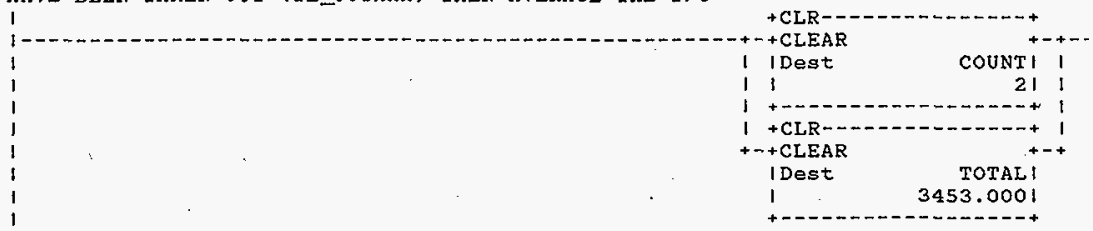

HNF-SD-FF-CSWD-61 Rev. 0 
Processor and Data(ops Unit 1) Program Listing

Processor File: SODIUM2A.ACH

\section{Rung $2: 442$ \\ HA17481}

i. $\quad$ N7:226 $-$

Rung $2: 443$ I HA174B2 I N7:226 D174B2 OUT174B2 N7:173 l $13 \quad 1 \quad-3 /[-13 \quad 1$ $(2: 358)$ (2:395)

DEV 2 .1 N22:6 ।

$(2: 361)$

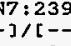

$--$

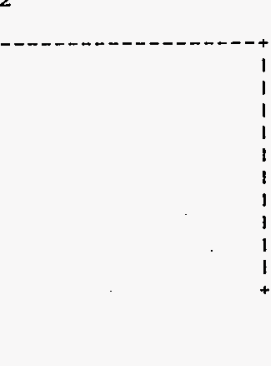

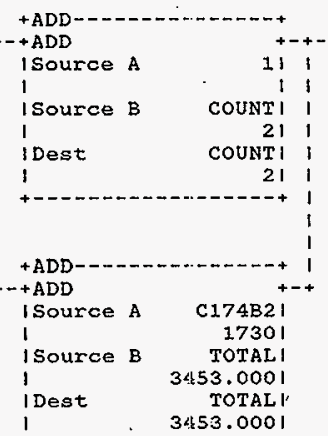

1 3453.000

HNF-SD-FF-CSWD-61 Rev. 0

Page 541 
Processor and Data(ops Unit 1) Program listing

Rung $2: 445$

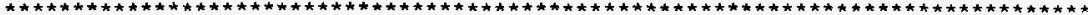
CLEAR COUNT AND TOTAL. IF NO HARDHARE, NO RANGE ALARMS AND NO THERMOCOUPLES HAVE BEEN TAKEN OUT (TE_OUTXXX) THEN AVERAGE THE T/C

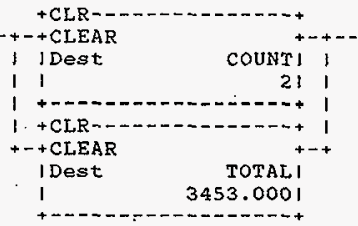

Rung $2: 4.46$

HA174VI

1) $\quad$ 7 $: 226$

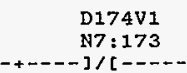

OUT174V1

$1----] /[--$

14

(2:359)
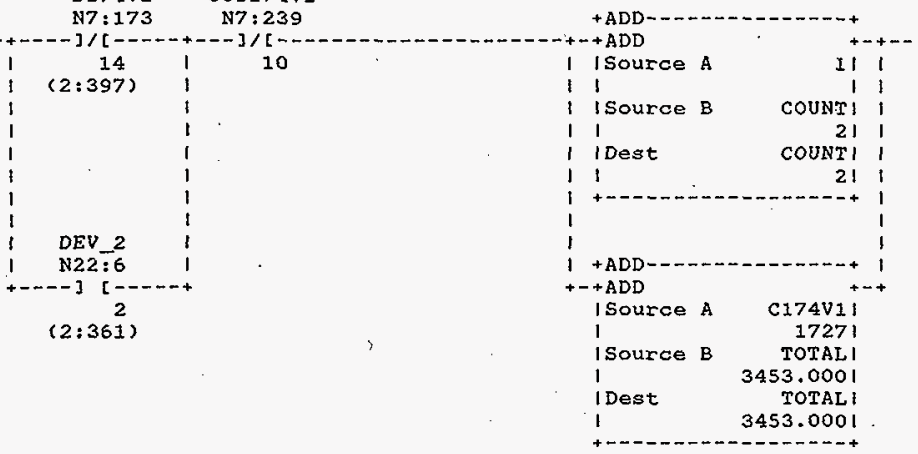

(2:361

N7:239

] 10

-

$-$

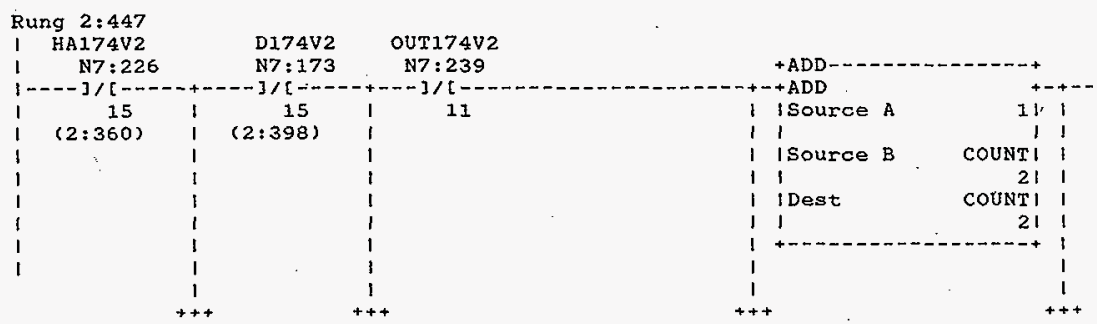

HNF-SD-FF-CSWD-61 Rev. 0

Page 542 


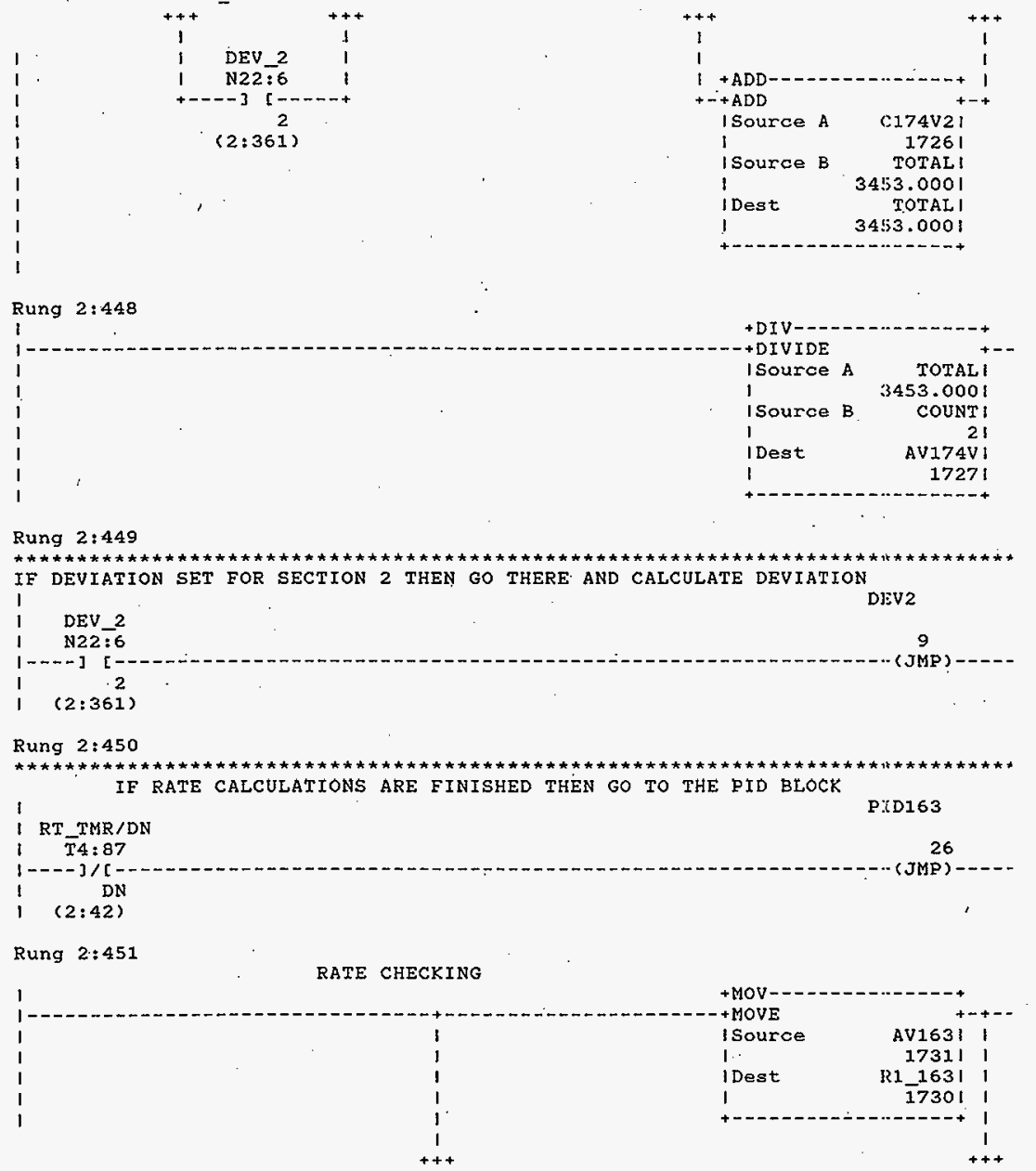

HNF-SD-FF-CSWD-61 Rev. 0 


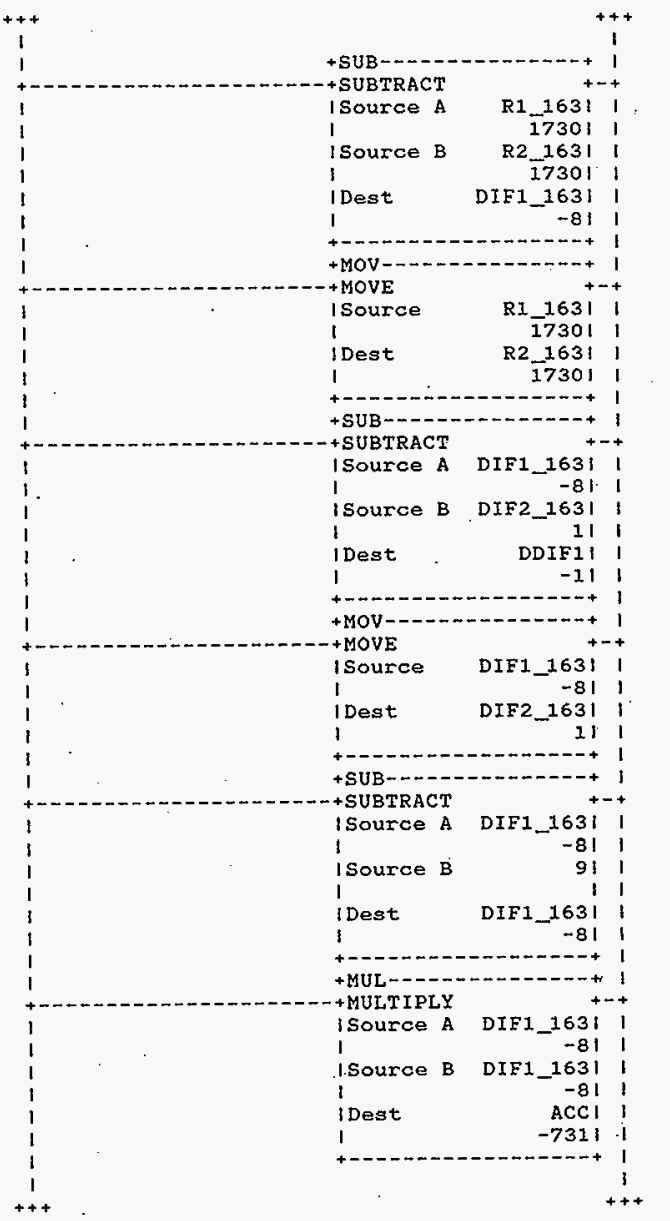

HNF-SD-FF-CSWD-61 Rev. 0 


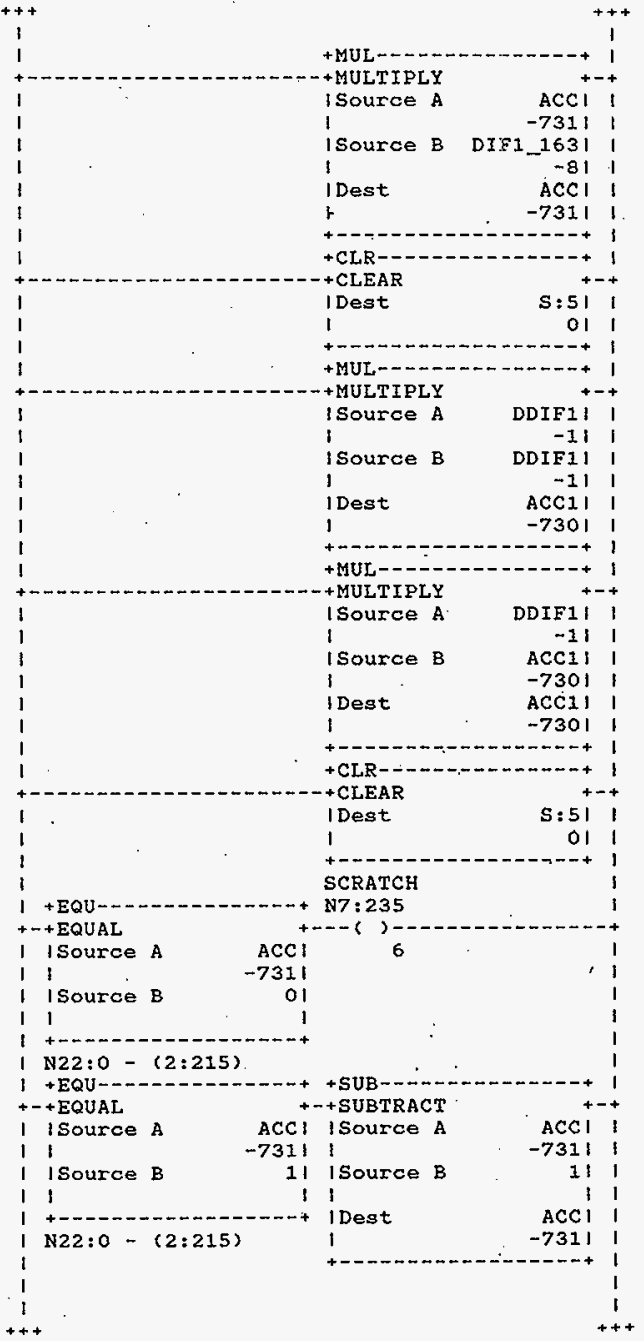

HNF-SD-FF-CSWD-61 Rev. 0 


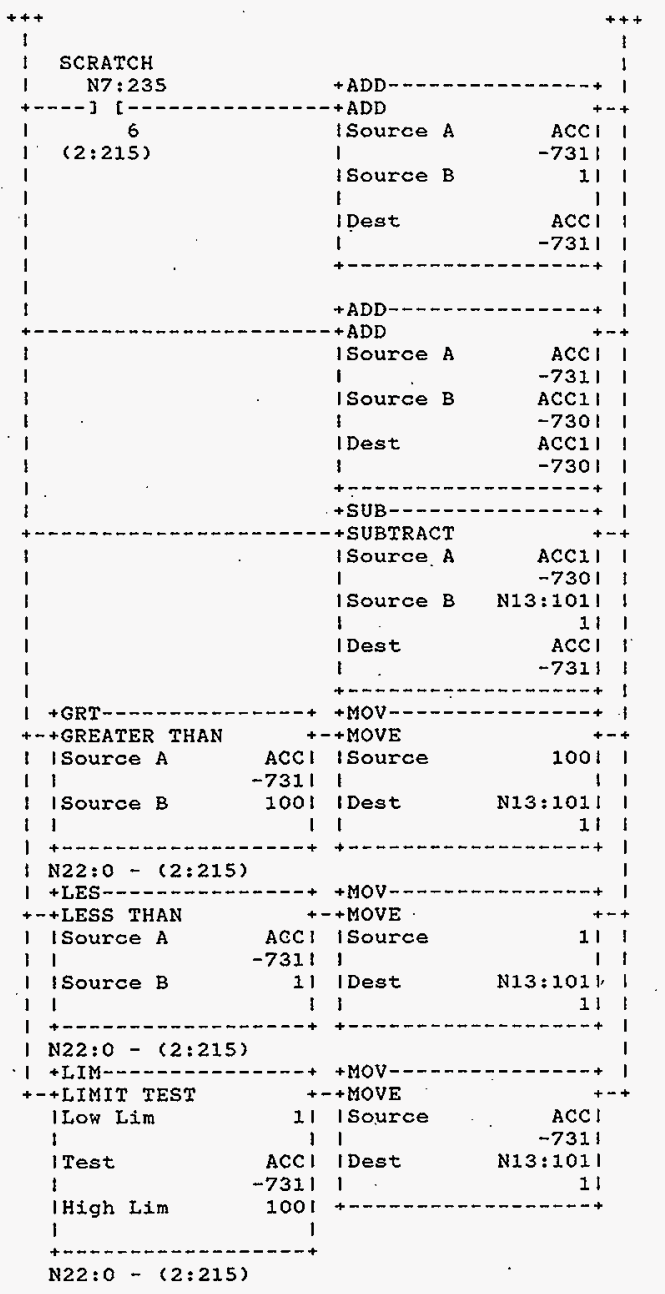

HNF-SD-FF-CSWD-61 Rev. 0

Page 546 
Processor and Data(OPS Unit 1)

Rung $2: 452$

PID 163
$1--[L B L]$
1

Rung 2:453

\section{DETECT FROM SCADA PID OH/OFF} IF PID OFF

THEN ZERO PW FOR ZERO OUTPUT AT SCR AND JUMP AROUND THE PID BLOCK
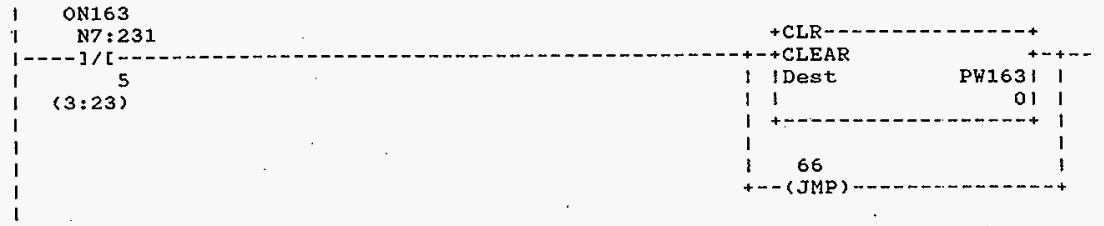

Rung 2:454

DETECT AUTO/MANUAL FROM SCADA SET APPROPRIATE MODE IN PID BLOCK

AM163
N7:231

Rung 2:455

DETECT SETPOINT VALUE FROM SCADA PUT VALUE IN PID BLOCK

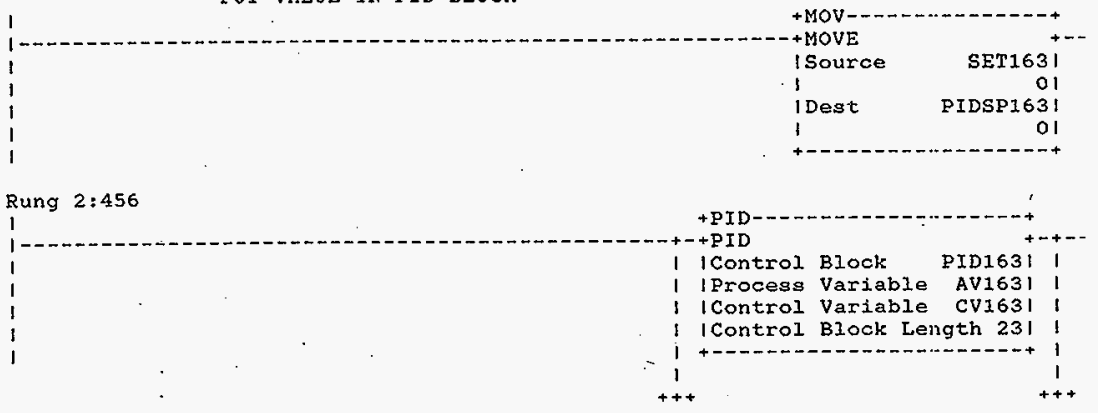

INFF-SD-FF-CSWD-61 R:Y. 0 
Processor and Datalops Unit I)

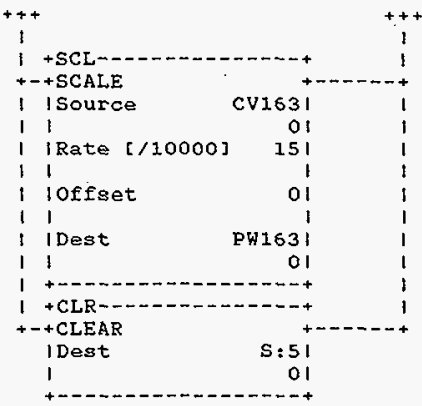

Rung 2:457

$$
\text { i } 66
$$

N7 : 229

1 $--[$ LBL $]$

Rung $2: 458$

1

l RT TMR/DN

I T4:87

$\mid-\cdots-] /[-$

IDN

( $(2: 42)$

Rung 2:459

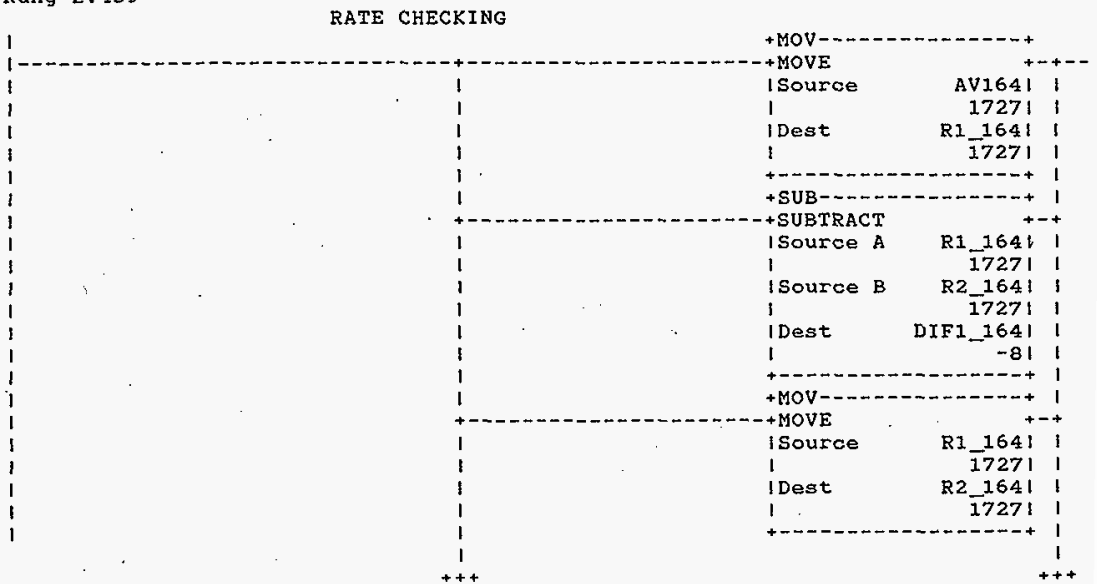

HNF-SD-FF-CSWD-61 Rev. 0

Page 548 


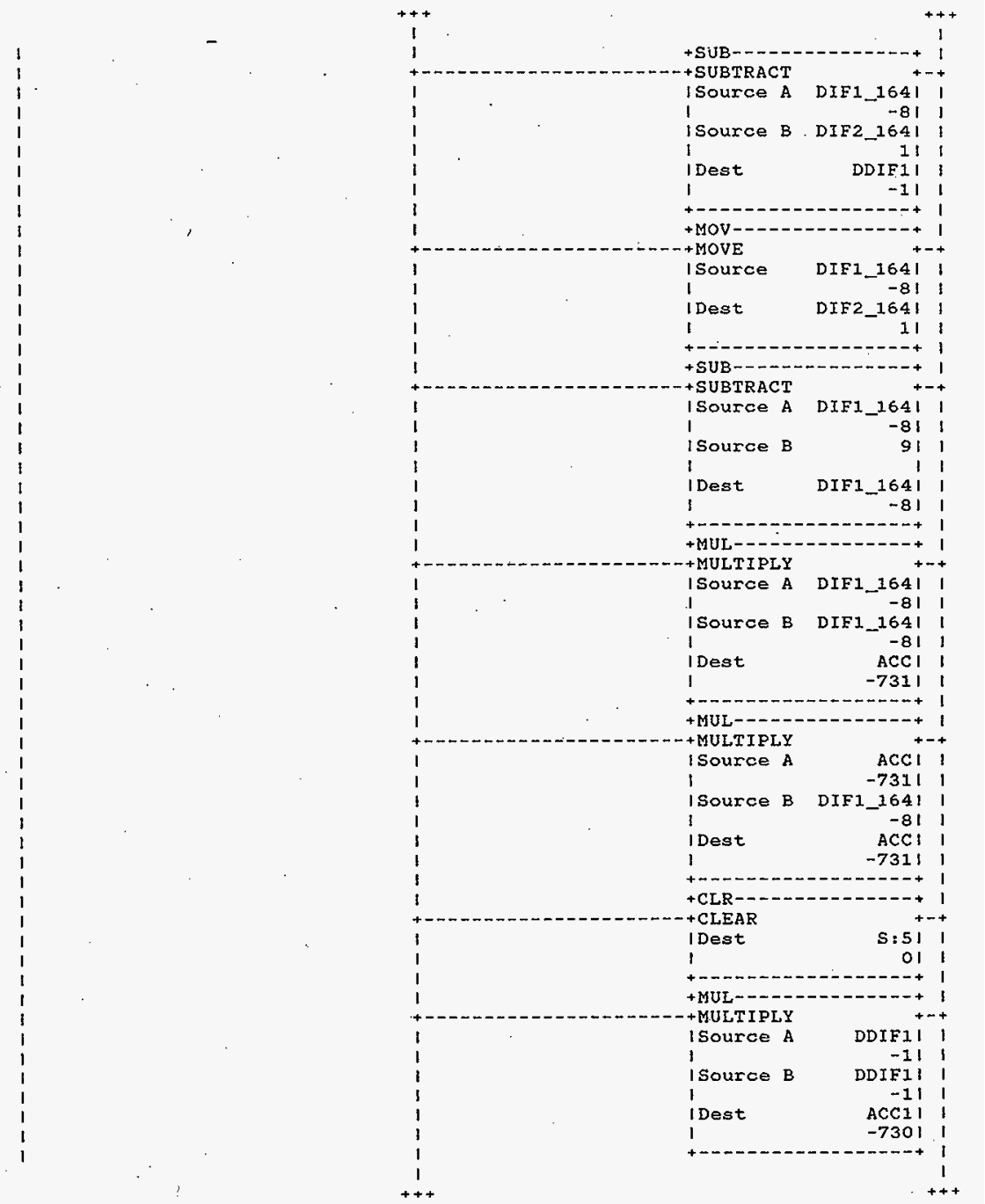

HNF-SD-FF-CSWD-61 Rev. 0 
Processor and Data (ops Unit I)

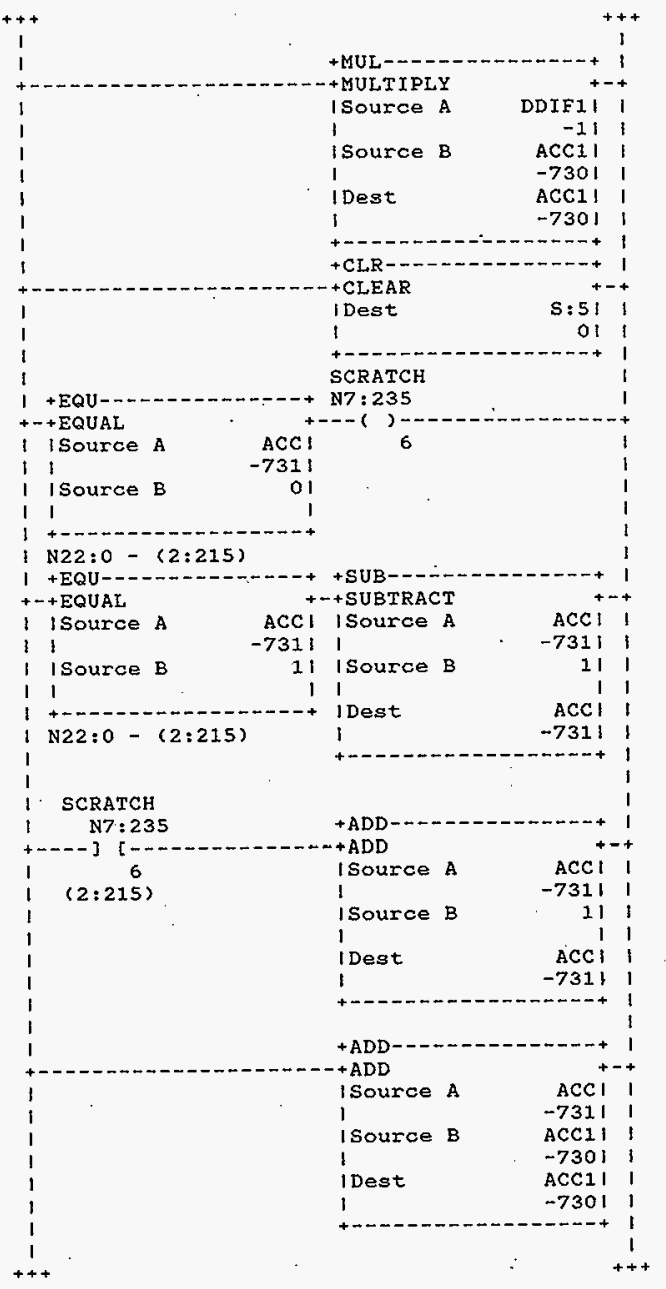

HNF-SD-FF-CSWD-61 Rev. 0 
Processor and Data(OPS Unit 1)

October 23, 1996 Page 189

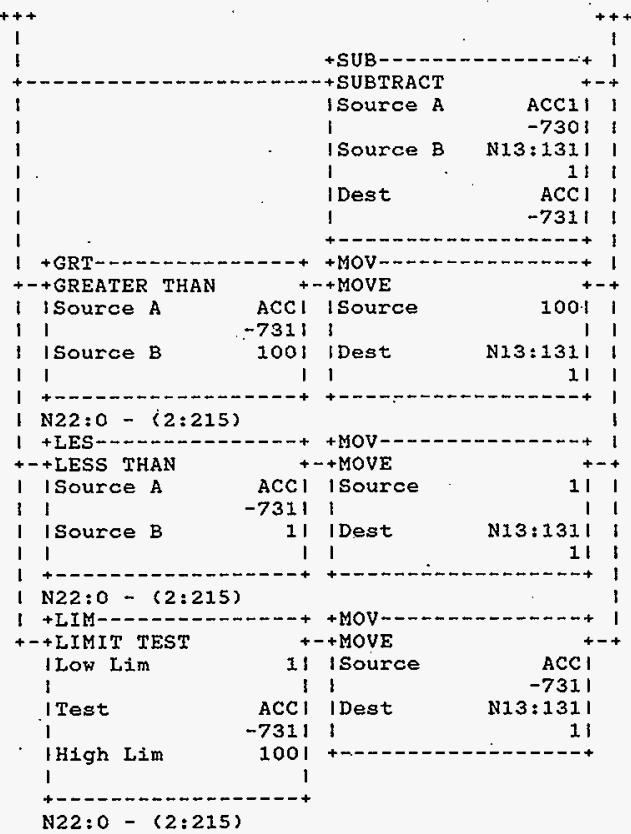

Rung 2:460

I PID164

DUMMY 27

N22:8

-()$-$$$
\text { i }
$$

27

Rung 2:461

DETECT FROM SCADA PID ON/OFF

IF PID OFF

THEN ZERO PW FOR ZERO OUTPUT AT SCR AND JUMP AROUND THE PID BLOCK
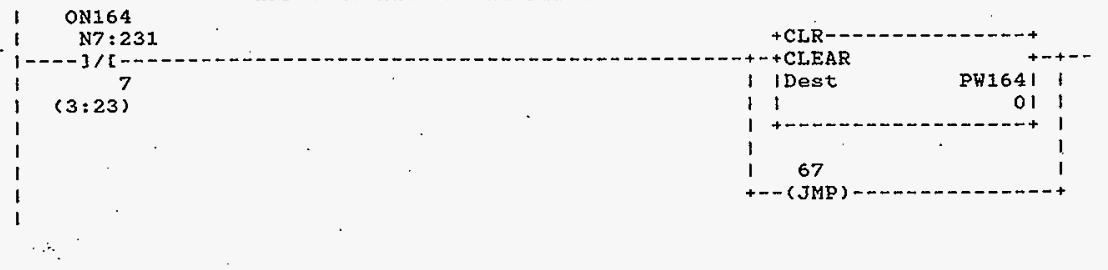

HNF-SD-FF-CSWD-61 Rev. 0

Page 551 


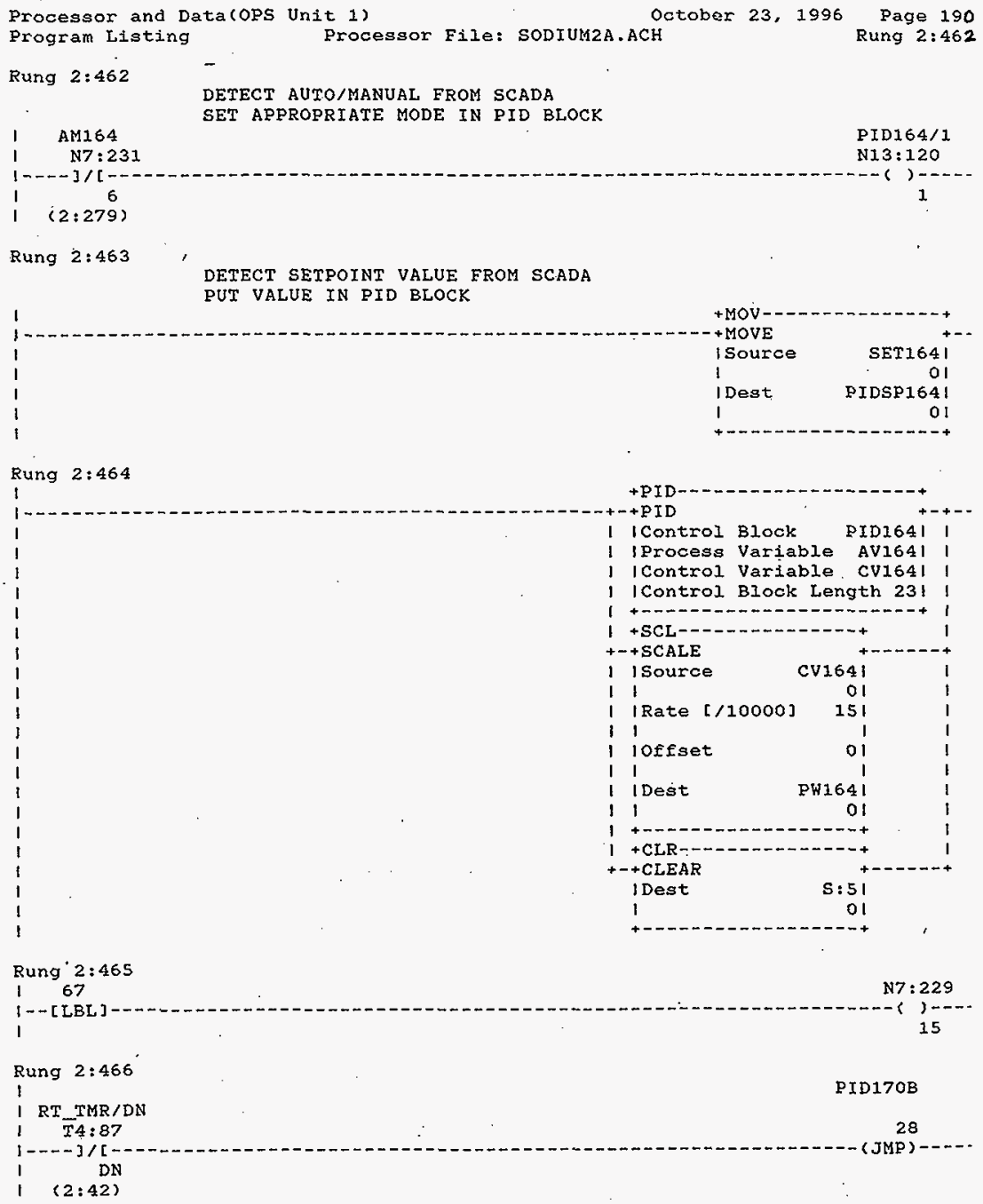

HNF-SD-FF-CSWD-61 Rev. 0 
Rung 2:467

\section{- RATE CHECKING}

+ MUV - - - - - - - - - - - - - - +
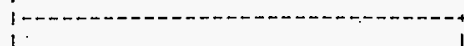

+MOVE

isource

AV17081

1

i Dest

17211

1

R1_17OB।

$+--n----+------\cdots+$

+SUB-..-- - -.........+

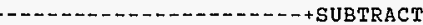

ISOUTCe A

R1_1708I

17201

|Source B R2_170B| |

1

I Dest

17201

1

- 1

+-- - - - -

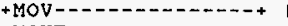

| Source RI_170B| I

1

Dest

17201

1

R2_170B 1

$+-\ldots+--\cdots------+1$

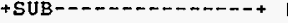

+SUBTRACT

|Source A DIFI_170B|

1

$-91$

ISource B DIF2 170BI'

1

0! 1

I Dest

DDIF1 |

1

$-111$

$-n---+$

$+\mathrm{MOV}---------------+1$

DIF1_170B |

1

$-9 ; 1$

i Degt

DIF2 170B ।

1

$+S U B-------------+1$

+ SUBTRACT

|Source A DIF1 $170 B \mid$

I Source B

$-9 ; 1$

1

I Dest DIFI I70B |

1

-9 i

$+\mathrm{MUL}----\cdots \cdots \rightarrow---\cdots+1$

+---------------------+ MULTIPLY

ISource A DIF1 170B!

I

$-9 ! 1$

ISource B DIFI_170B!

I

i Dest

$-91$

1

$\triangle \mathrm{CC}$

$-7311$

$+$

HNF-SD-FF-CSWD-61 Re\%. 0

Page 553 
Processor and Data(OPS Unit 1 ) Program Listing
Proce

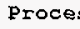

October 23, 1996

Page 192 $-$

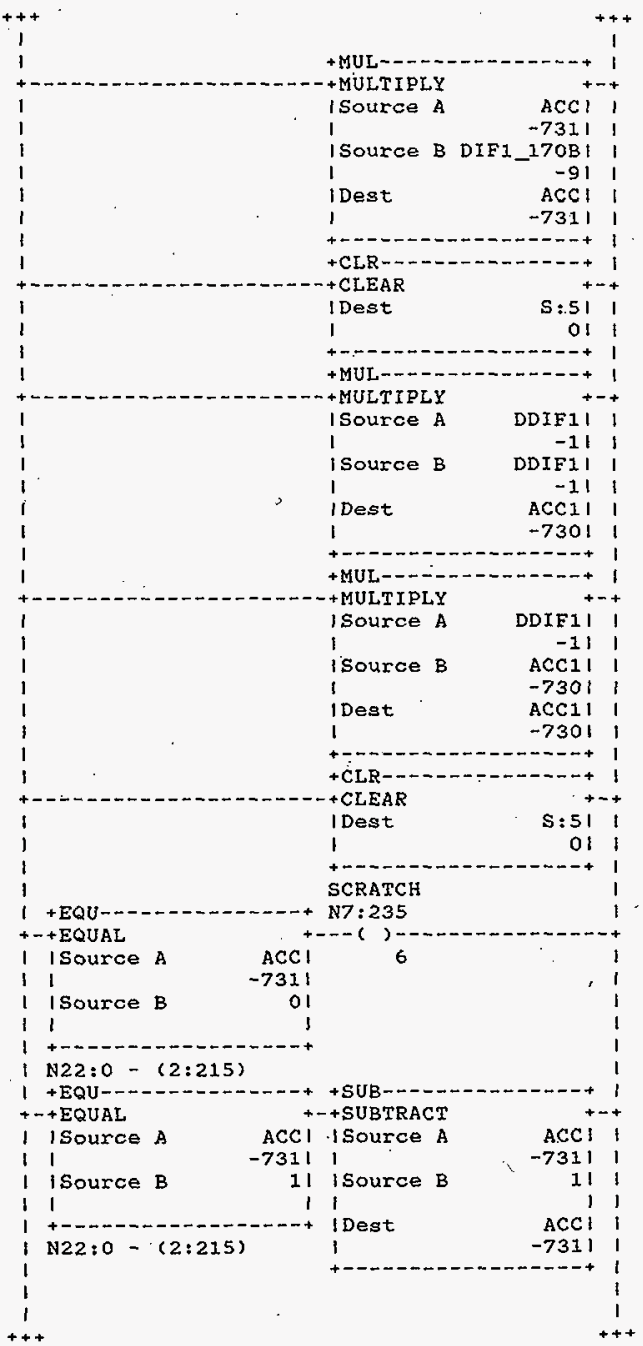

HNF-SD-FF-CSWD-61 Rev. 0

Page 554 


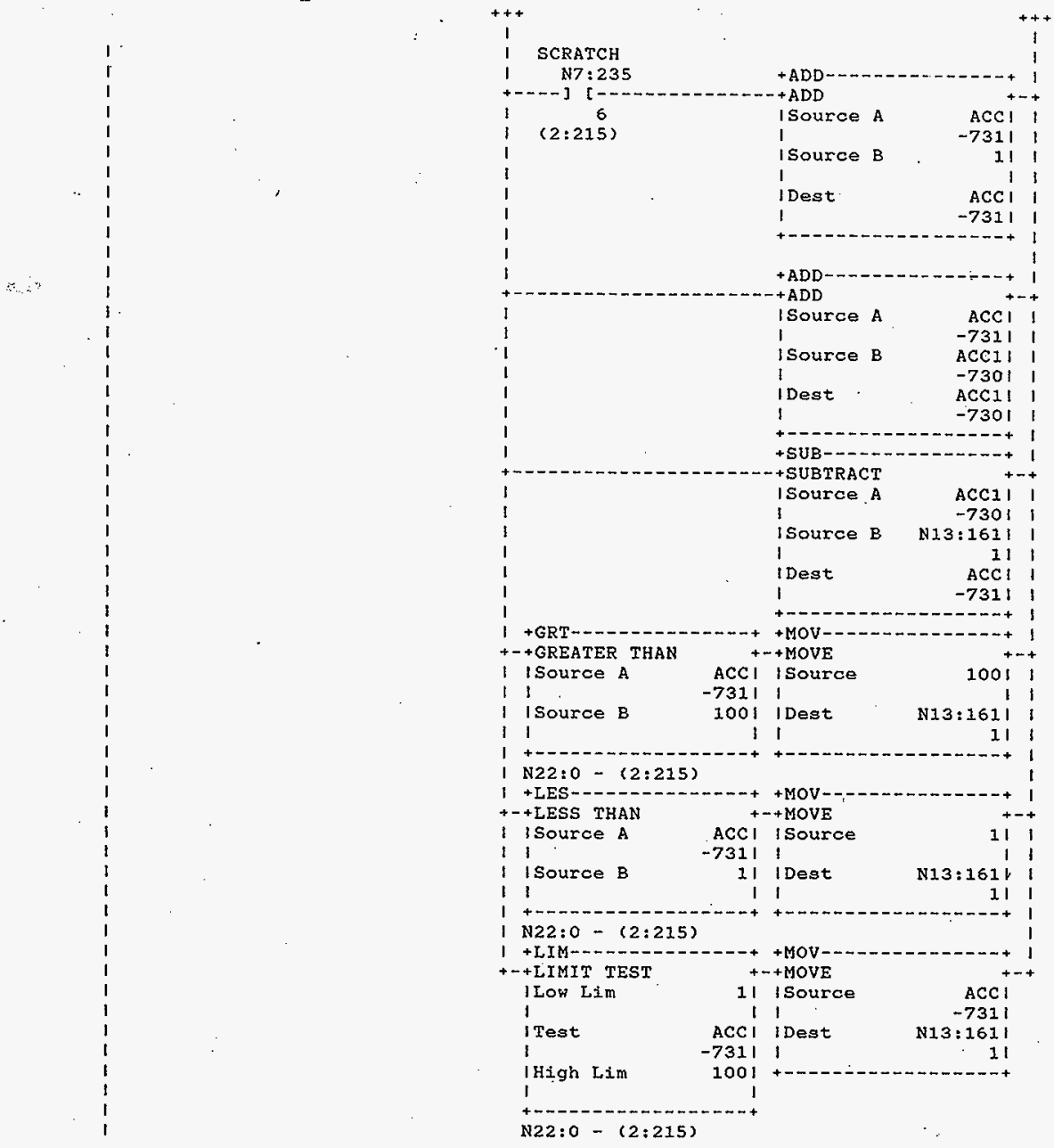

HNF-SD-FF-CSWD-61 Rev. 0

Page 555 


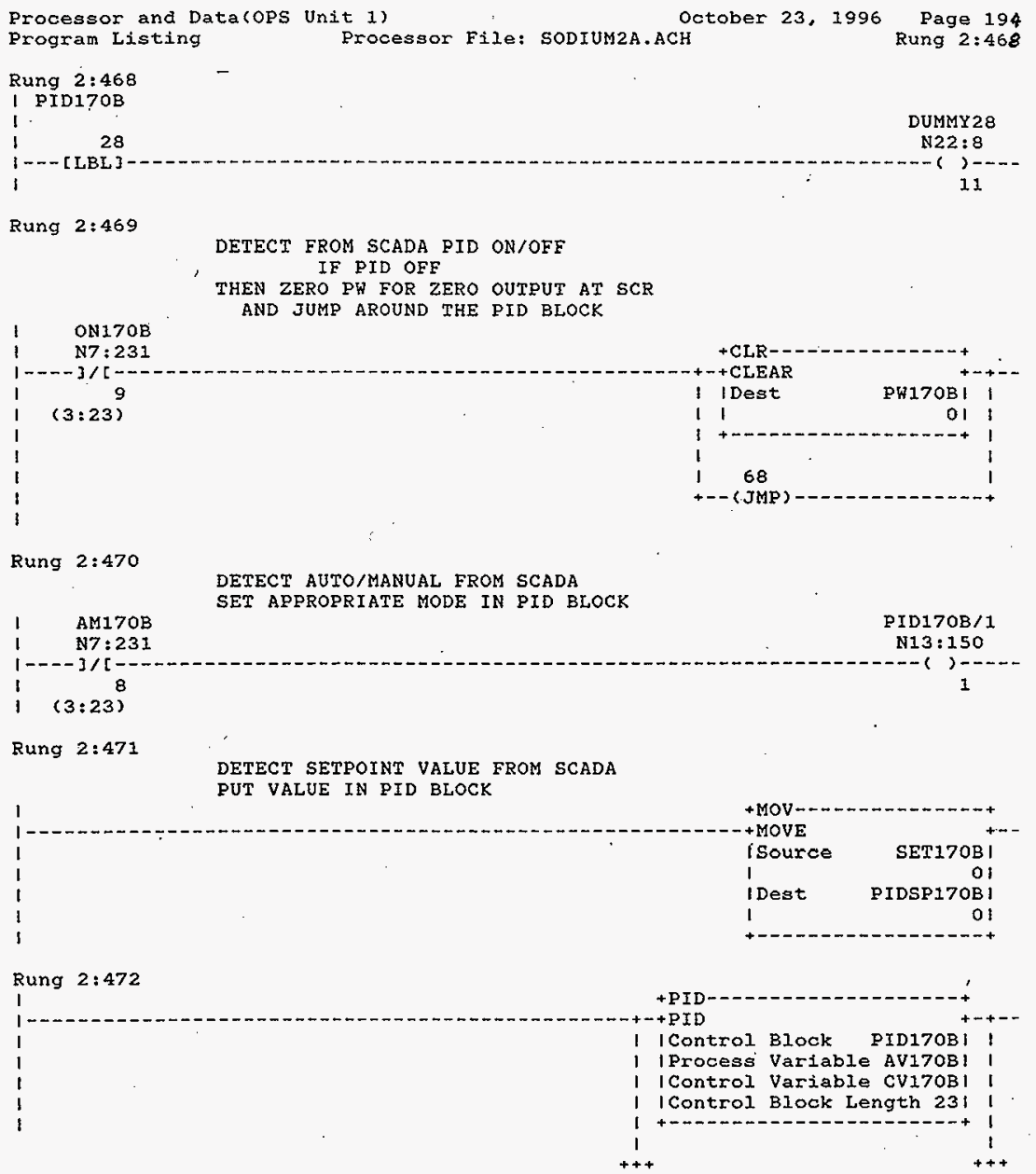

$\begin{array}{lr} & \\ & \\ & \end{array}$

DETECT AUTO/MANUAL FROM SCADA

SET APPROPRIATE MODE IN PID BLOCK

DETECT SETPOINT VALUE FROM SCADA PUT VALUE IN PID BLOCK

HNF-SD-FF-CSWD-61 Rev. 0

Page $5 S 5$ 
Processor and Data(OPS Unit 1 )

1

$1+\operatorname{SCL}-$

+- +SCALE

1 isource

I 1

11

11

loffset

I 1

1 I Dest

1

$1+$

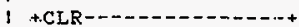

+ + CLEAR

i Dest $5: 51$

1

+.--- - - - -

Rung $2: 473$

I 68

N7: 235

I $--[$ LBL $]$

-()$-\ldots$

Rung 2:474

I

RT TMR/DN

(I) T $4: 87$

PID170V

29

$$
1 \text { DN }
$$

I (2:42)

Rung 2:475

\section{RATE CHECKING}

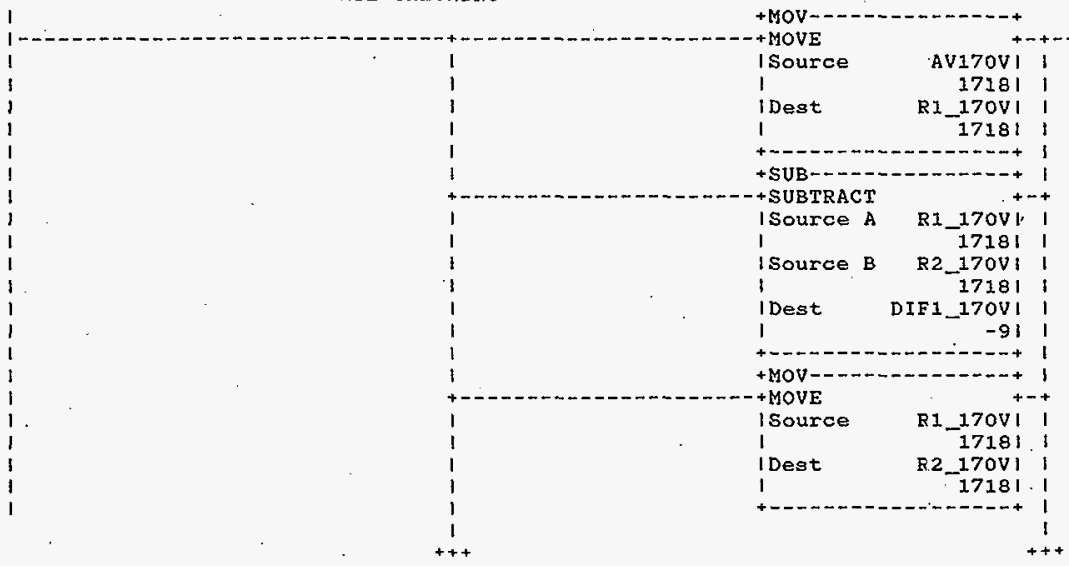

INF-SD-TT-CSWD-61 Rev. 0 


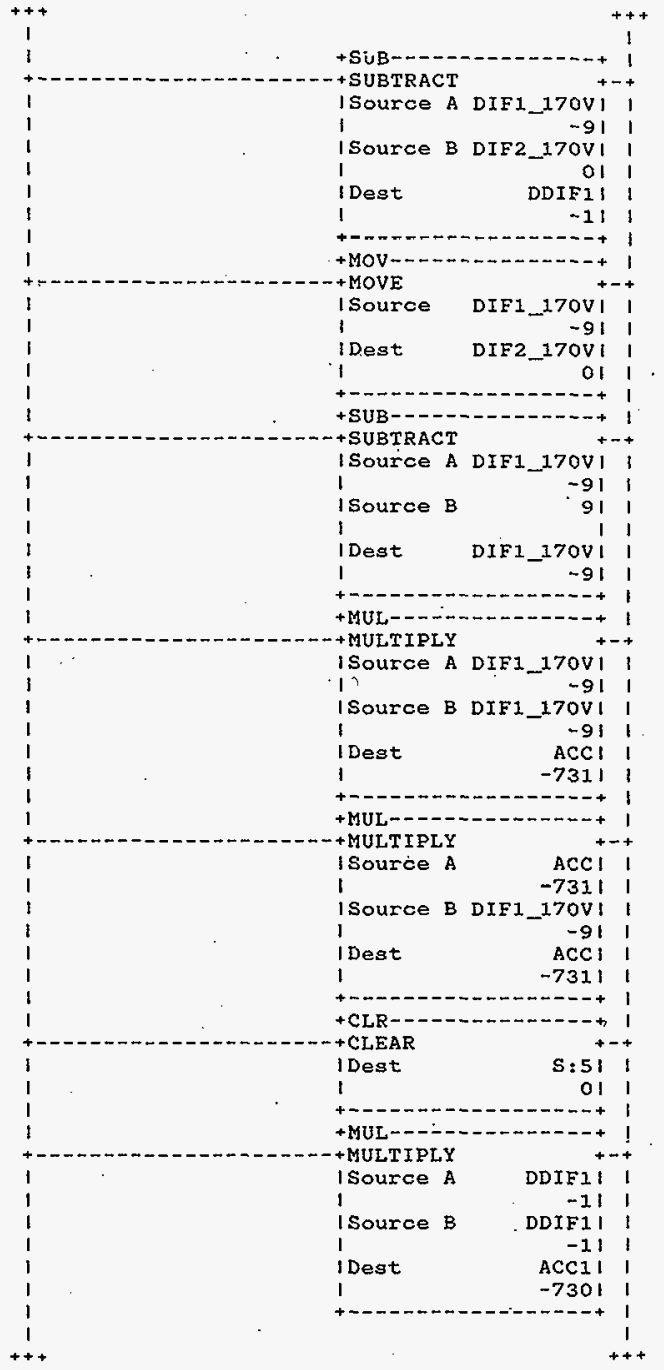

HNF-SD-FF-CSWD-61 Rev. 0

Page 558 


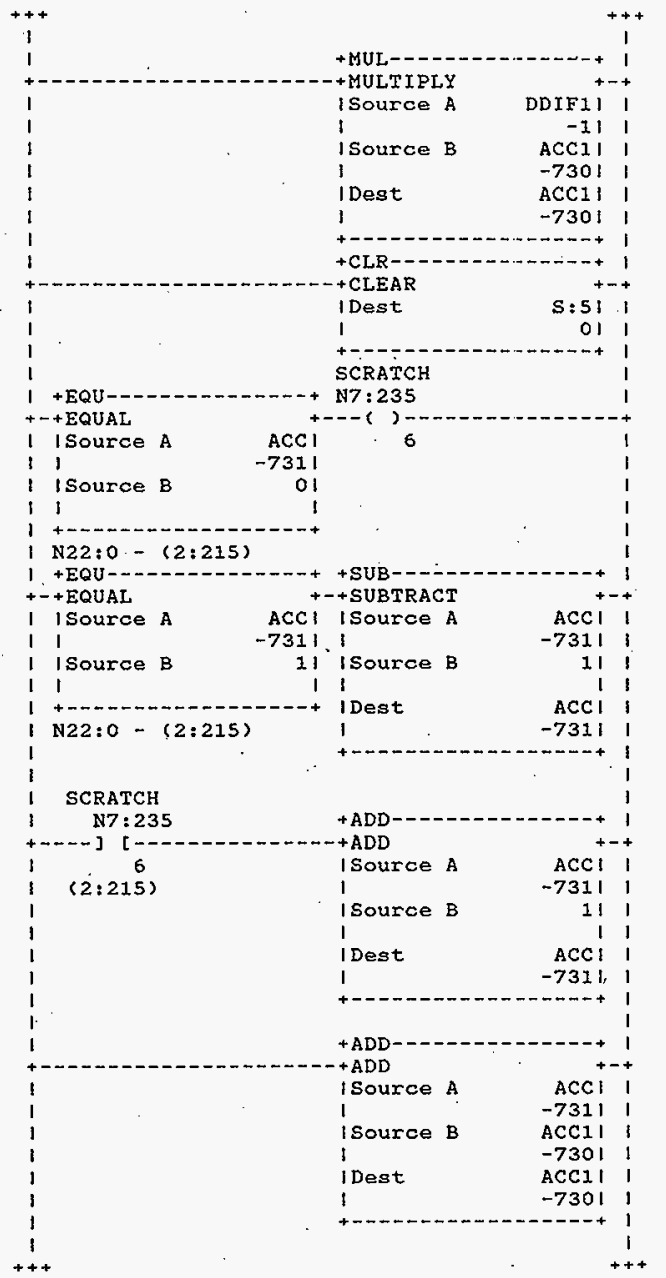

HNF-SD-FF-CSWD-61 Rev. 0 


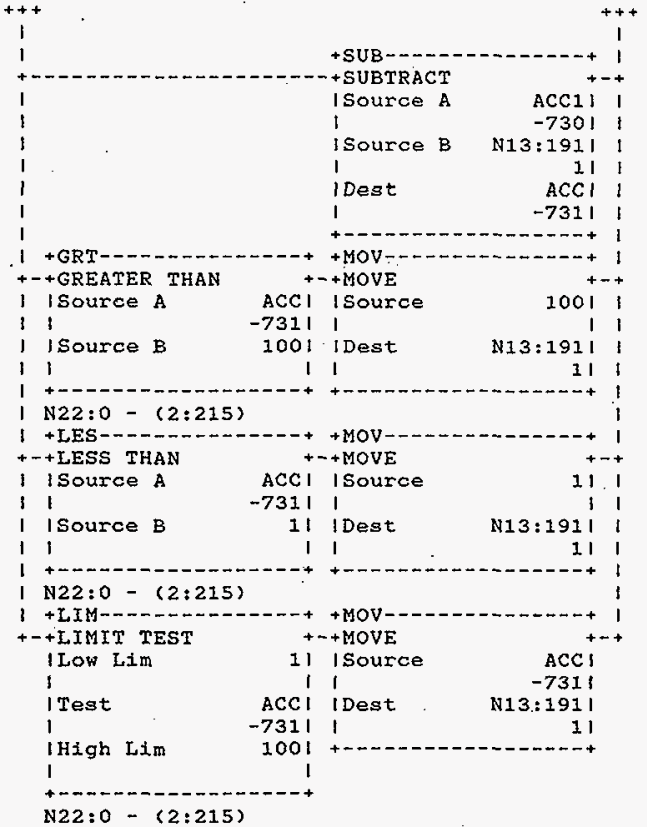

Rung $2: 476$

I PID170V

1

$1 \quad 29$

DUMMY 29

1.... [LBL]

N22:8

Rung $2: 477$

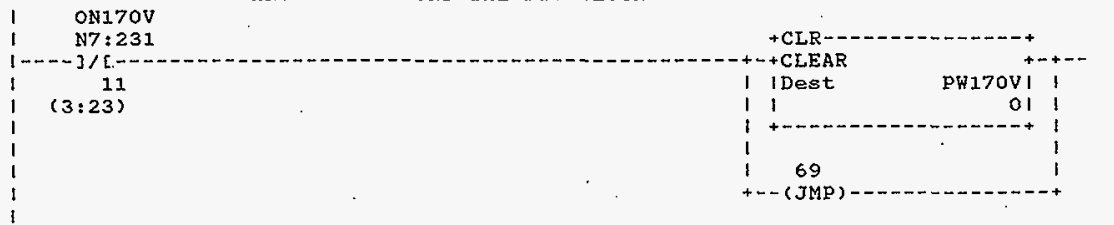

DETECT FROM SCADA PID ON/OFF

IF PID OFF

THEN ZERO PW FOR ZERO OUTPUT AT SCR

AND JUMP AROUND THE PID BLOCK

HNF-SD-FF-CSWD-61 Rev. 0

Page 560 
Processor and Data(OPS Unit 1) Program Listing

Processor File: SODIUM $2 \mathrm{~A} . \mathrm{ACH}$

Rung $2: 478$

DETECT AUTO/MANUAL FROM SCADA

SET APPROPRIATE MODE IN PID BLOCK

$\left\{\begin{array}{c}\text { AM170V } \\ \text { N7:231 } \\ 1-3 /[-10 \\ (3: 23)\end{array}\right.$

JID170V/1

N13: 180

Rung $2: 479$

DETECT SETPOINT VALUE FROM SCADA PUT VALUE IN PID BLOCK
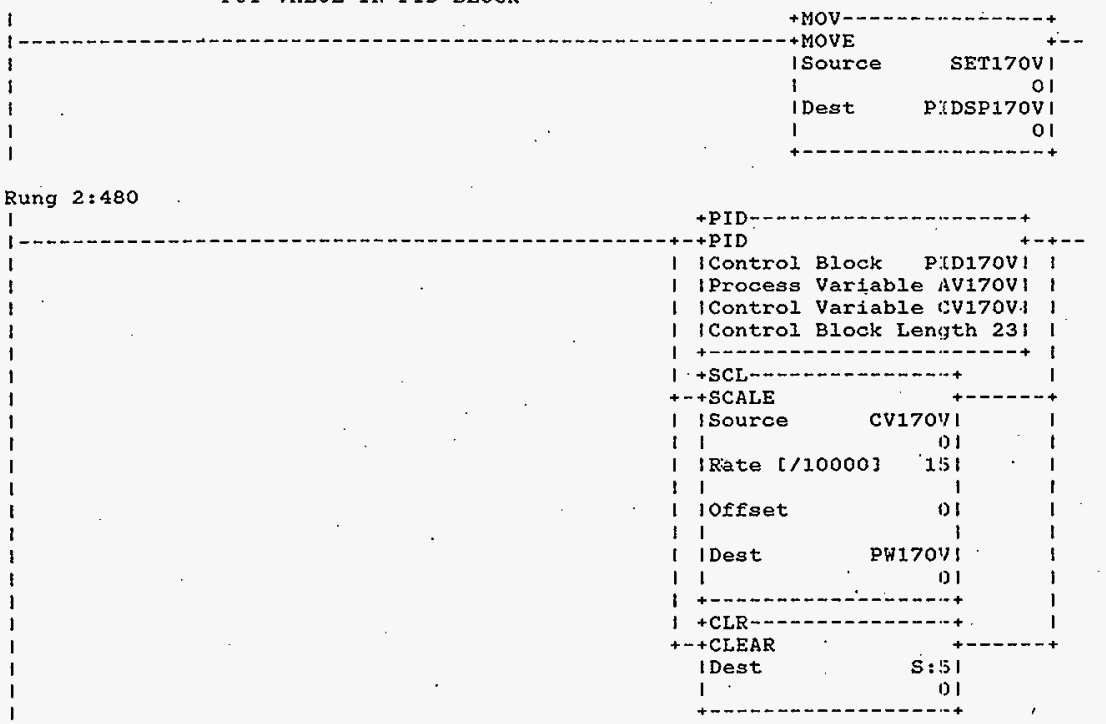

Rung $2: 481$

I 69

N7 $: 235$

1-- [ LBL ]

Rung 2:482

1

RT_TMR/DN

P.ID171B

1 T4:87

30

$1---7 /[-$

I. (2:42)

HNF-SD-FF-CSWD-61 Rev. 0

Page 561 
Processor and Data(OPS Unit 1 )

October 23, 1996 Page 200

Program Listing

Rung 2:483

RATE CHECKING

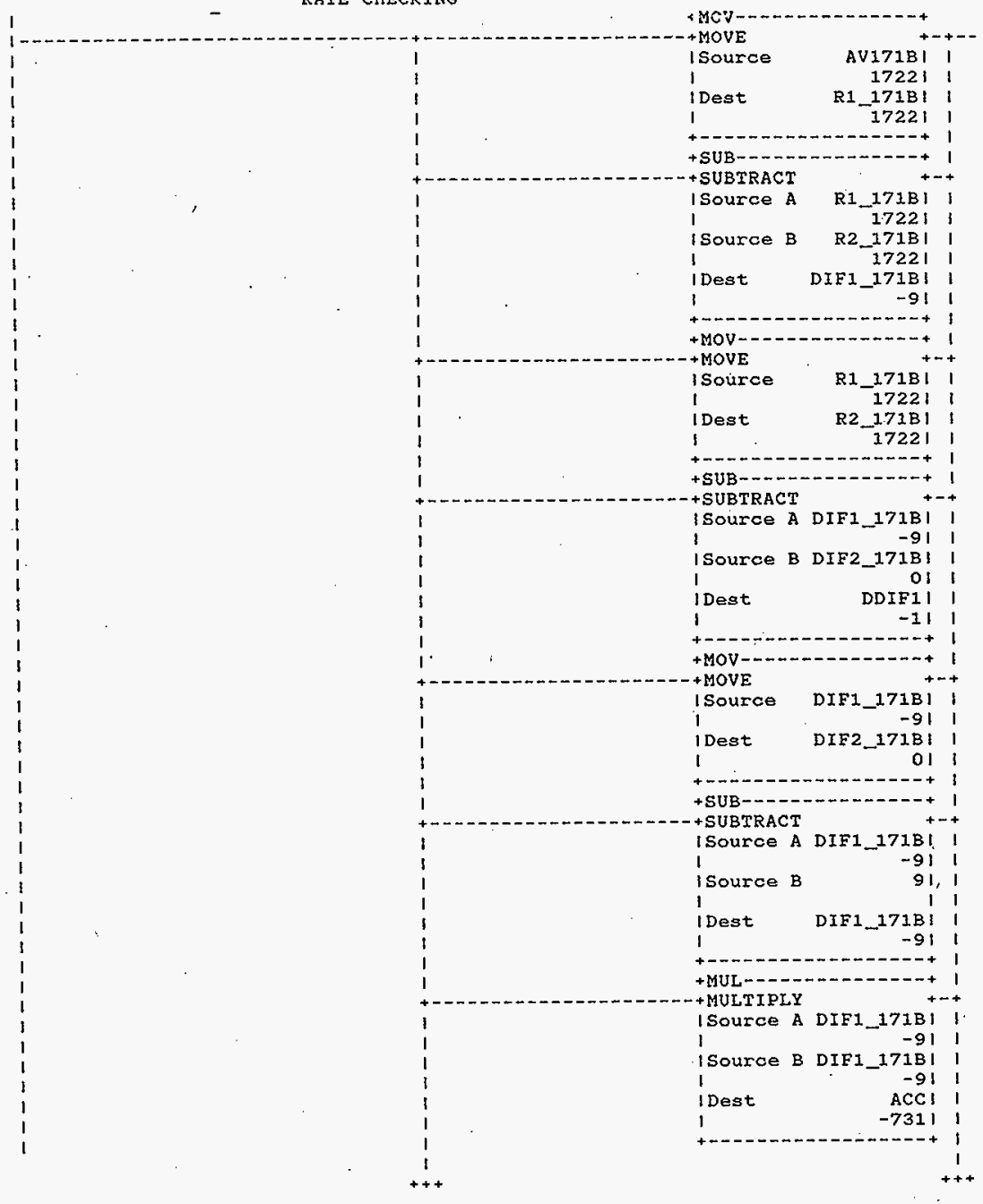

HNF-SD-FF-CSWD-61 Rev. 0 


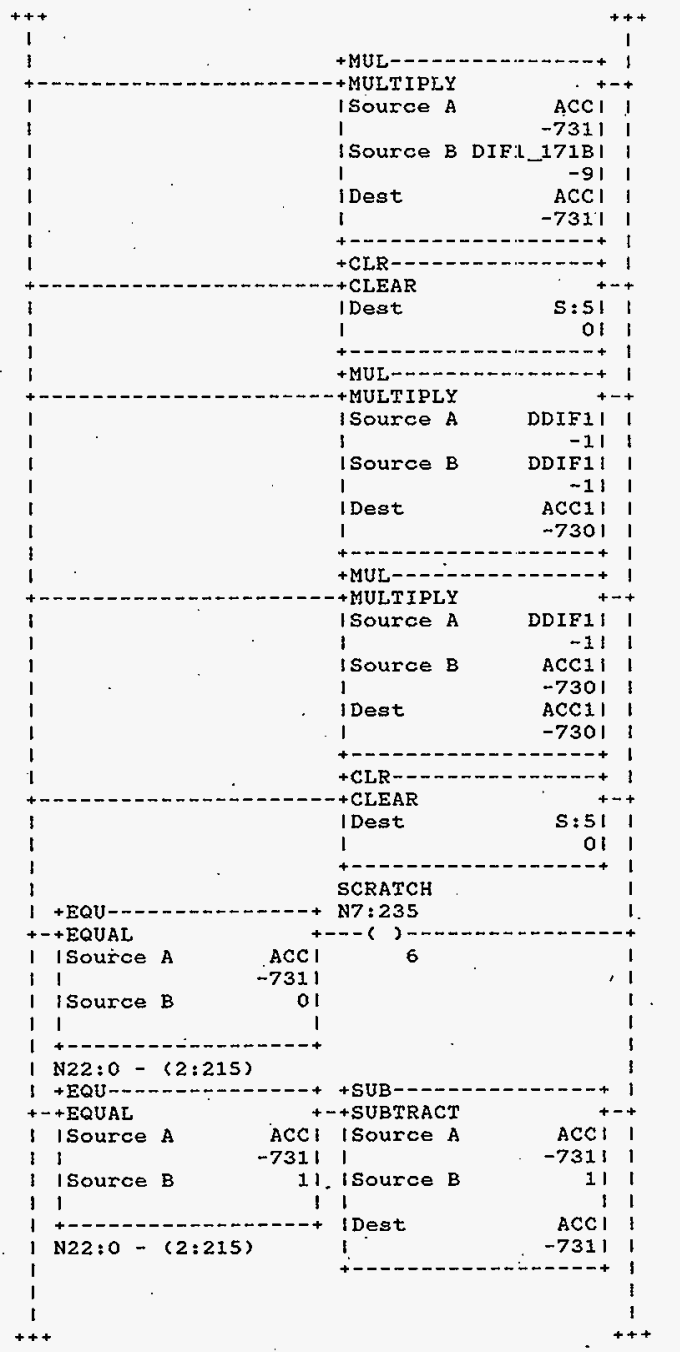

HNF-SD-FF-CSWD-61 RE:V, 0 


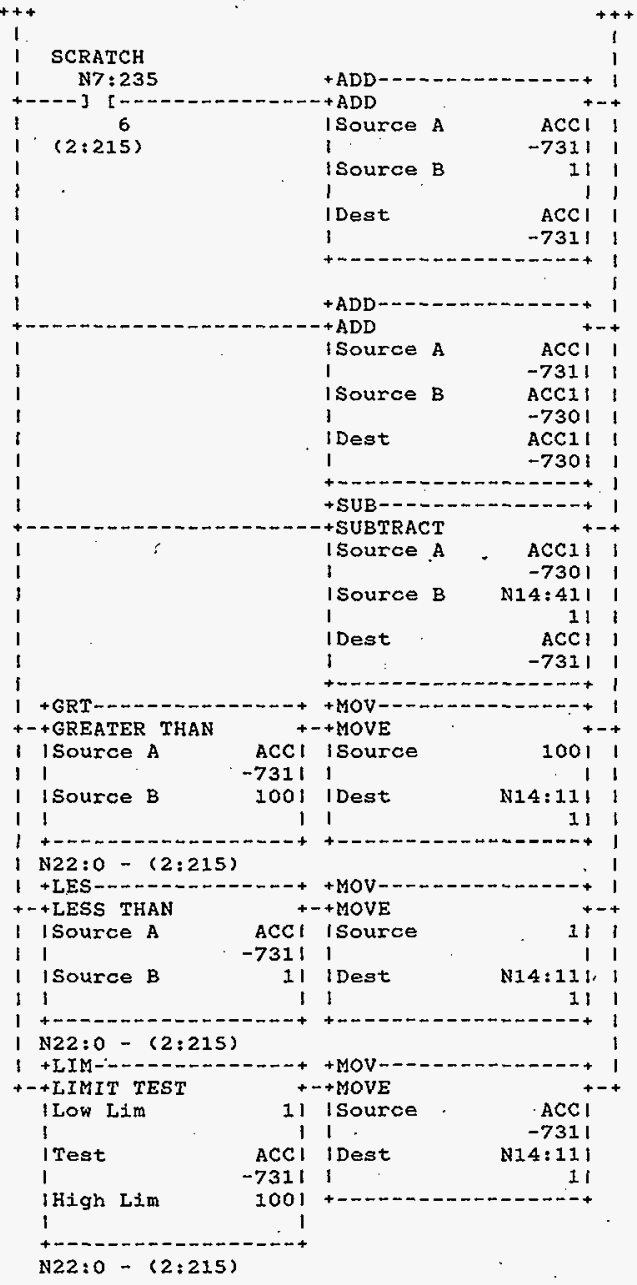

HNF-SD-FF-CSWD-61 Rev. 0 


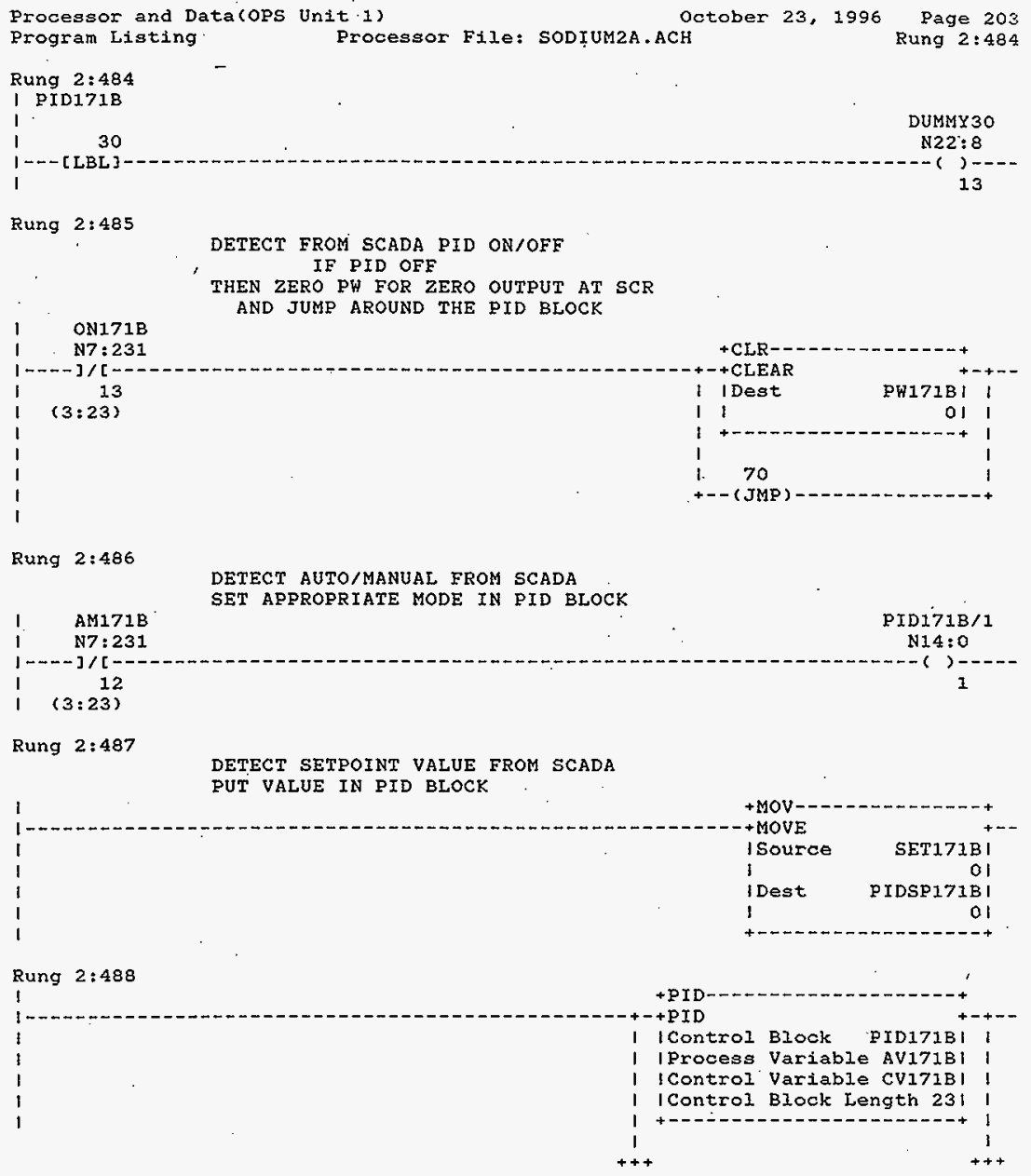

HNF-SD-FF-CSWD-61 Rev. 0 


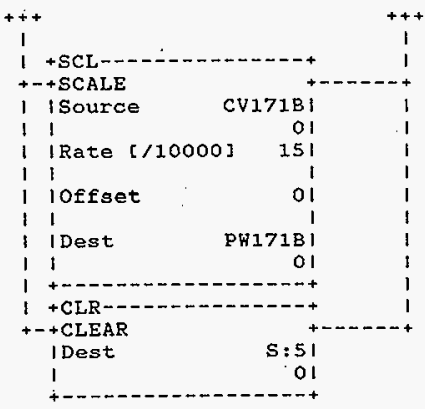

Rung $2: 489$

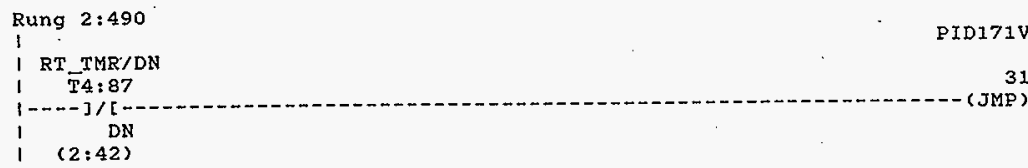

Rung $2: 491$

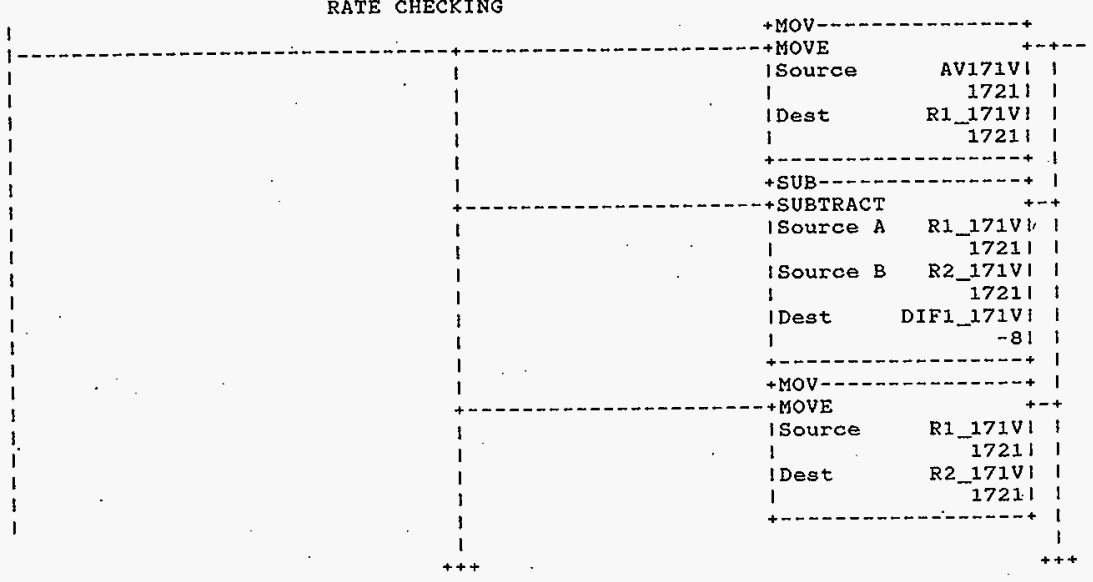

HNF-SD-FF-CSWD-61 Rev. 0 


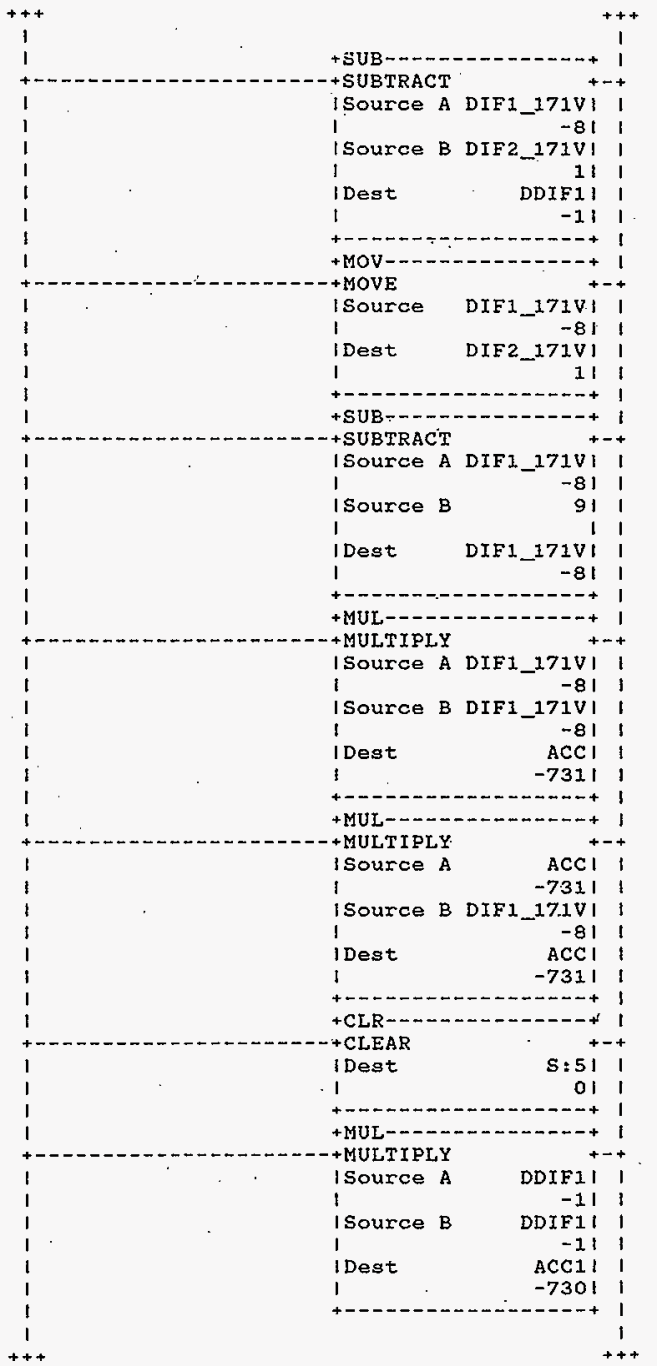

HNF-SD-FF-CSWD-61 Rev. 0 
Processor and Data(ops Unit 1)

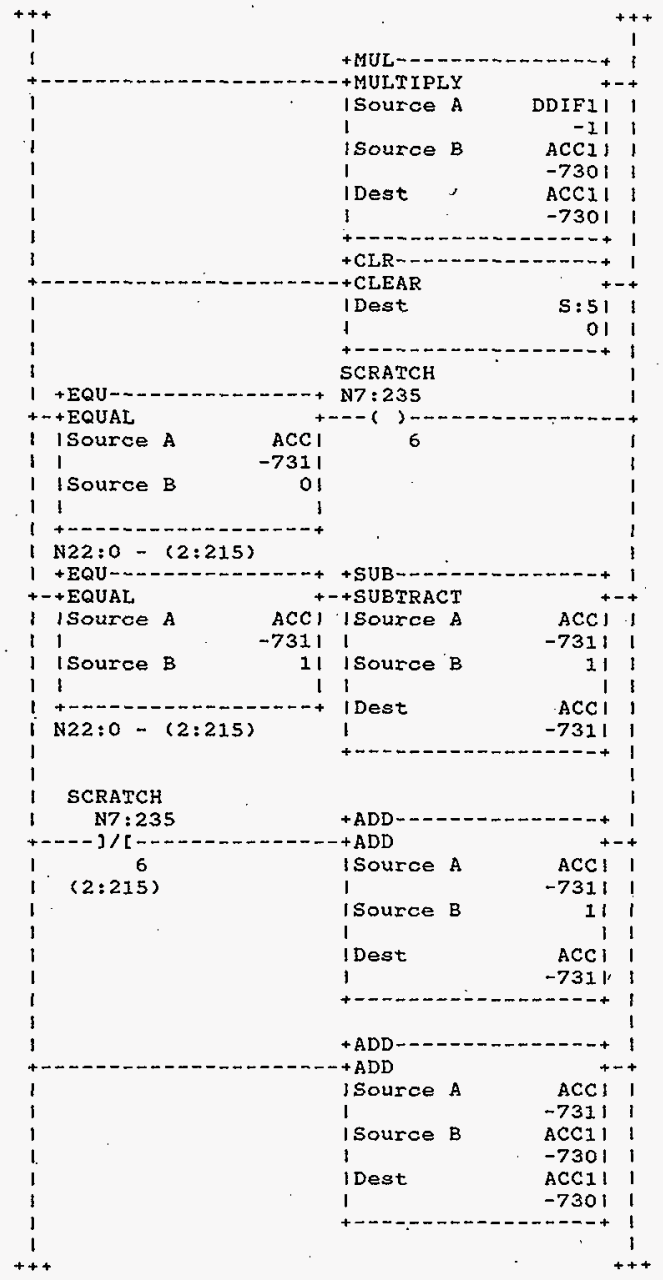

HNF-SD-FF-CSWD-61 Rev. 0

Page 568 
Processor and Data COPS Unit 1 )

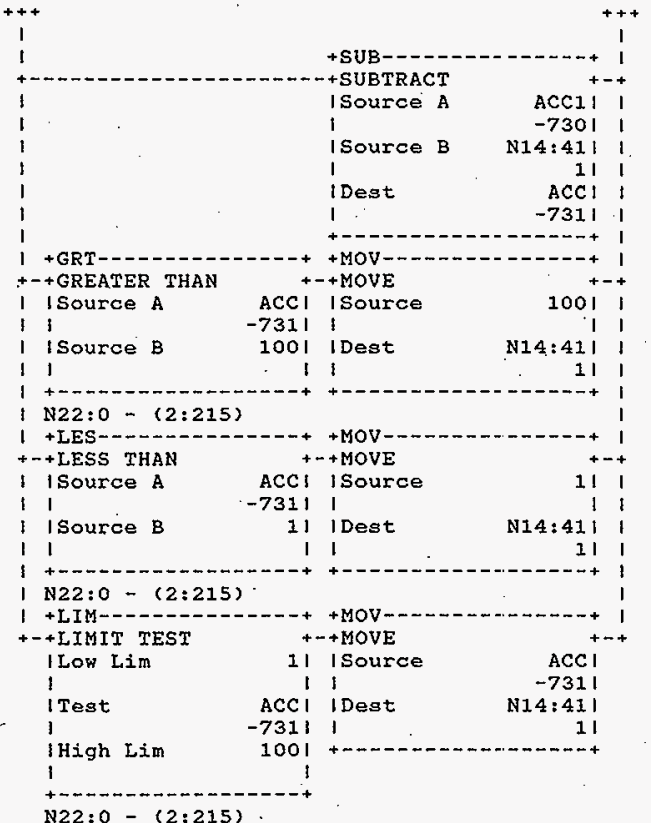

Rung $2: 492$

1 PIDI71V

1

31

DUMMY 31

N22: 8

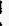

$-1)-\cdots$

Rung 2:493

DETECT FROM SCADA PID ON/OFF

IF PID OFF

THEN ZERO PW FOR ZERO OUTPUT AT SCR

AND JUMP AROUND THE PID BLOCK

1

ONI7IV

N7:231

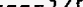

15

$(3: 23)$

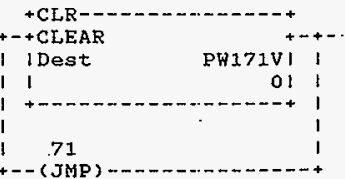

HNF-SD-FF-CSWD-61 Rev. 0

Page 569 
Processor and Data(OPS Unit 1)

Program Listing

October 23,1996

Rung 2:494:

\section{$-$}

Processor File: SODIUM2A,ACH

DETECT AUTO/MANUAL FROM SCADA

SET APPROPRTATE MODE IN PID BLOCK

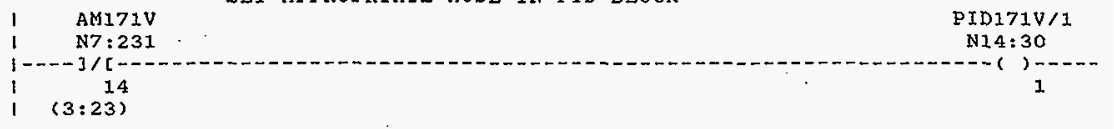

Rung 2:495

DETECT SETPOINT VALUE FROM SCADA PUT VALUE IN PID BLOCK

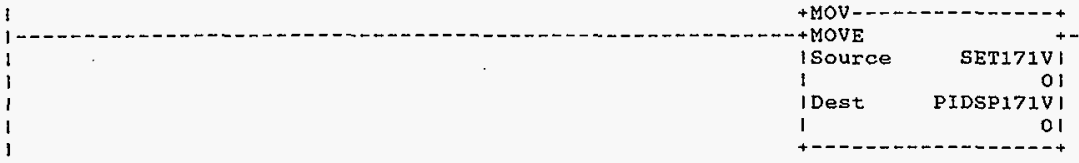

Rung $2: 496$

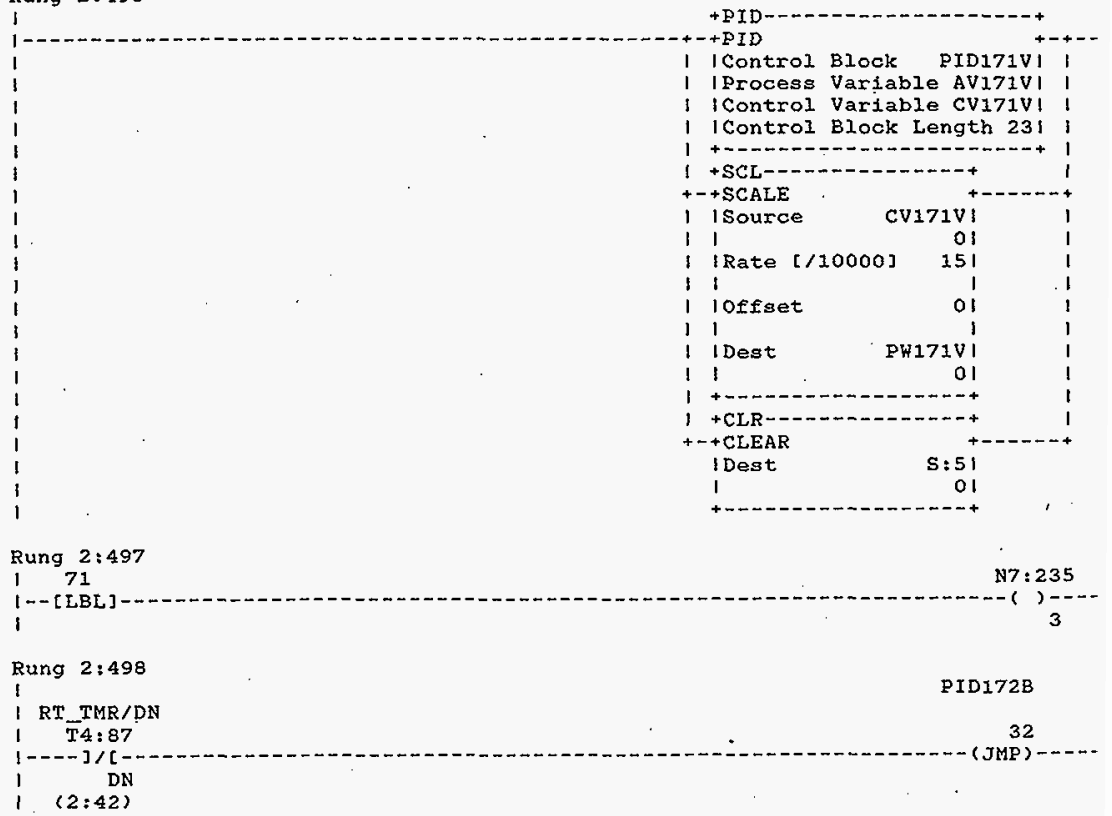

HNF-SD-FF-CSWD-61 Rev. 0 
Processor and Data(Ops Unit 1)

Rung 2:499

\section{- RATE CHECKING}

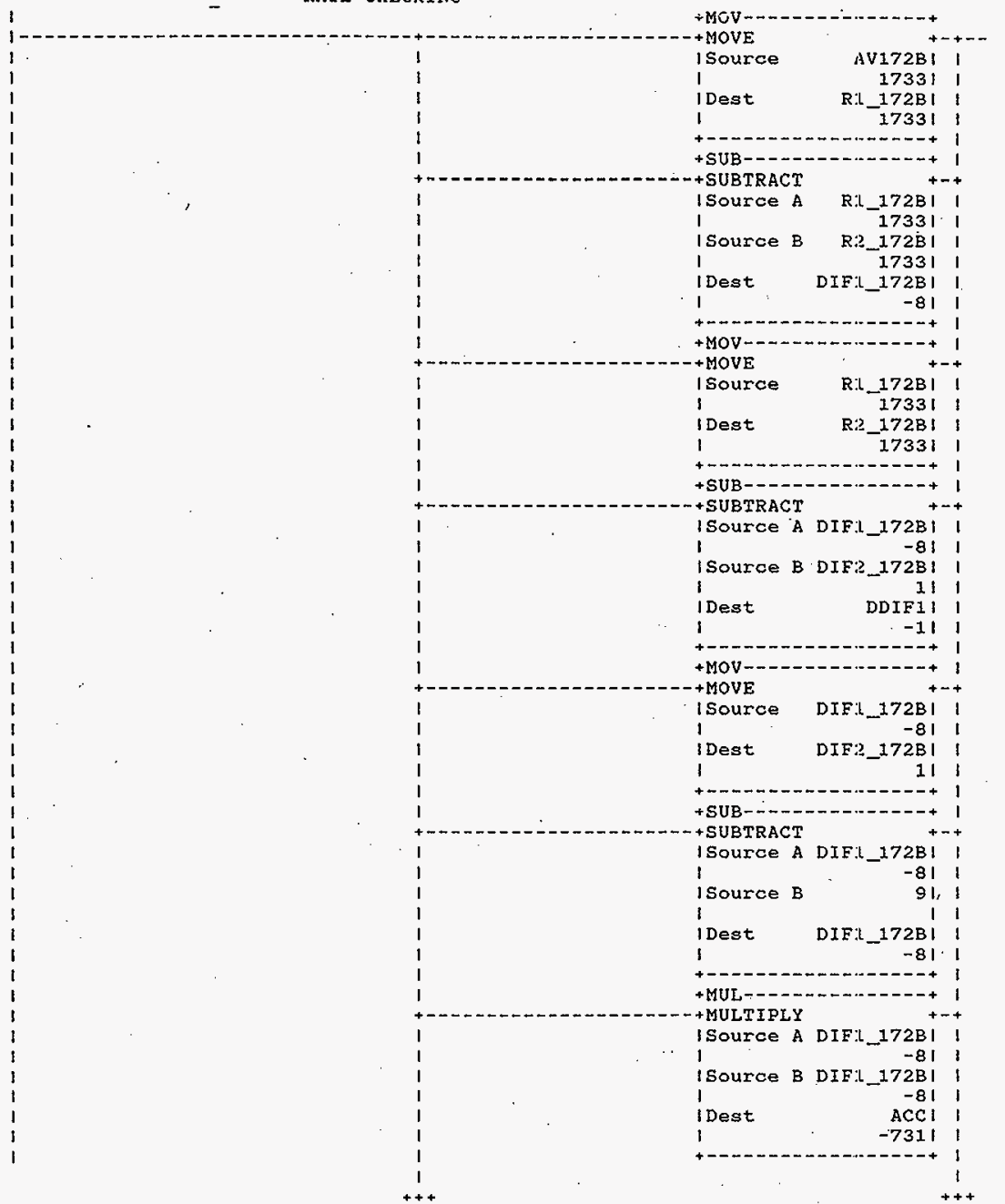

HNF-SD-FF-CSWD-61 Rev. 0 

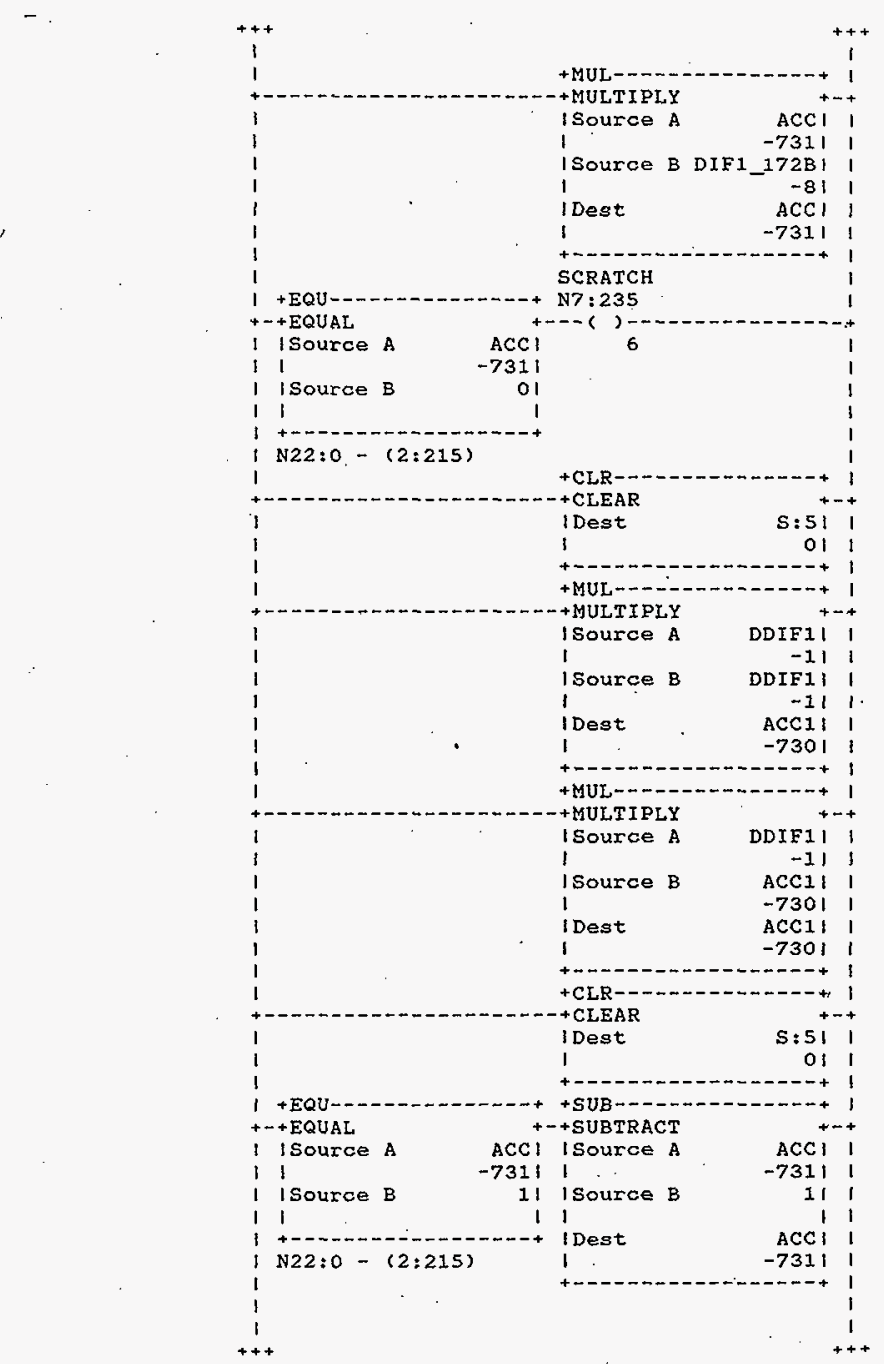

HNF-SD-FF-CSWD-61 Rev. 0

Page 572 


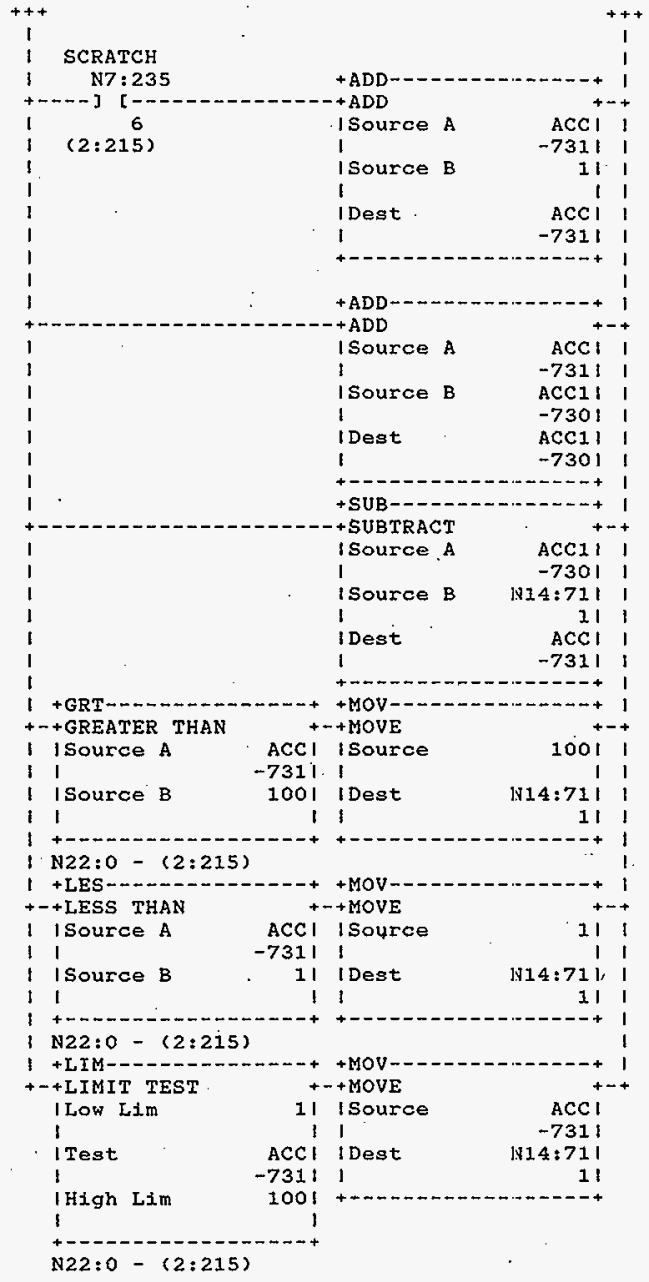

HNF-SD-FF-CSWD-61 Rev. 0 


\section{Rung 2:500}

1 gID172B

I.

| 32

DUMMX 32

$1---[$ LBL $]$

N22: 8

I

Rung $2: 501$

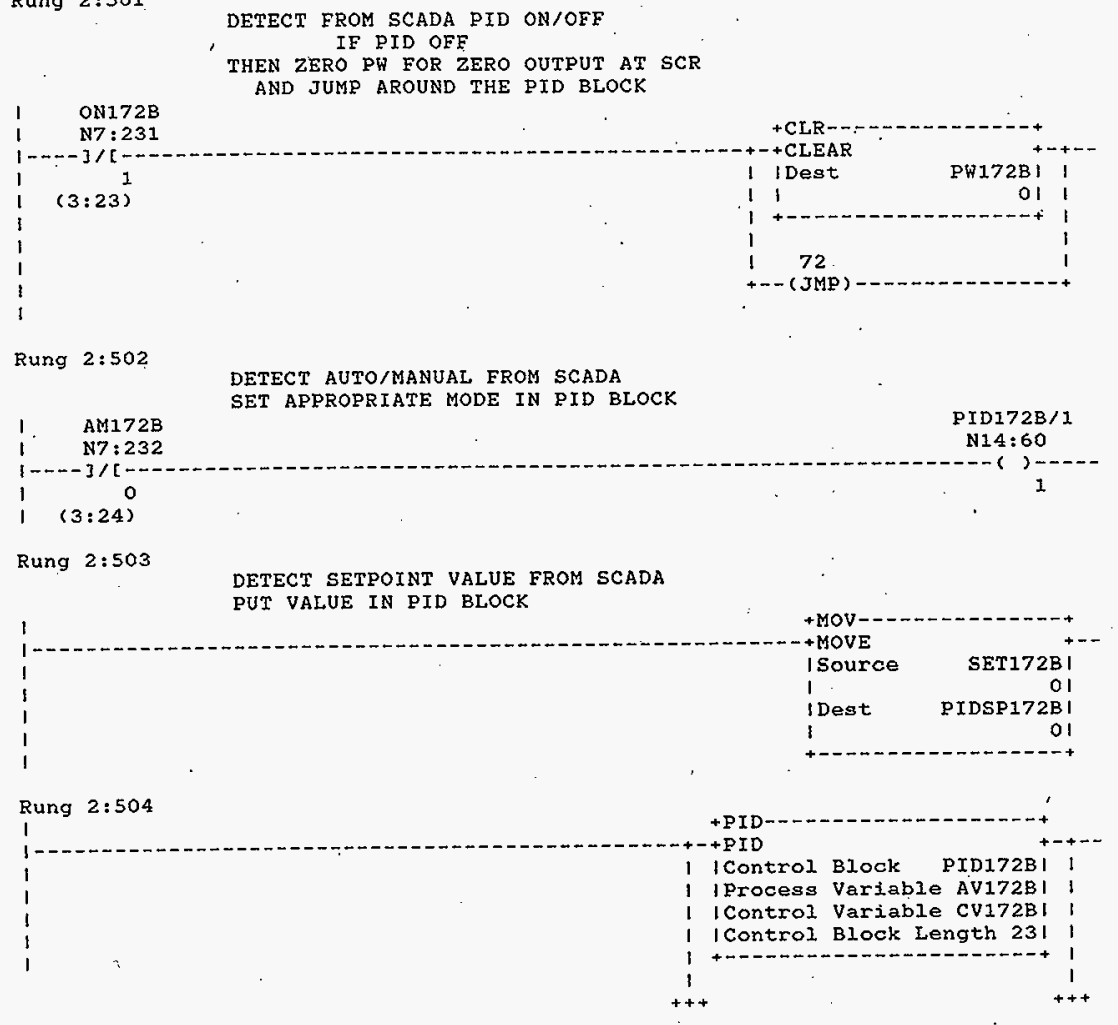

HNF-SD-FF-CSWD-61 Rev. 0 


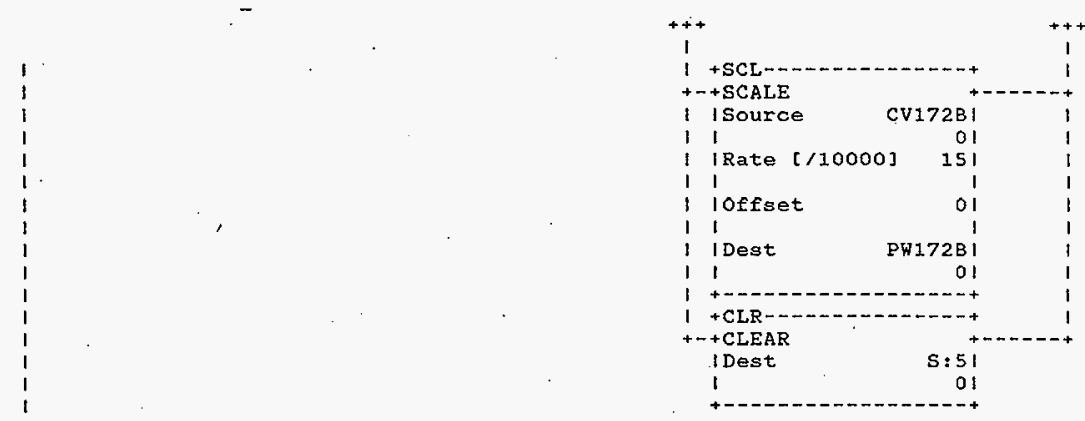

Rung 2:505

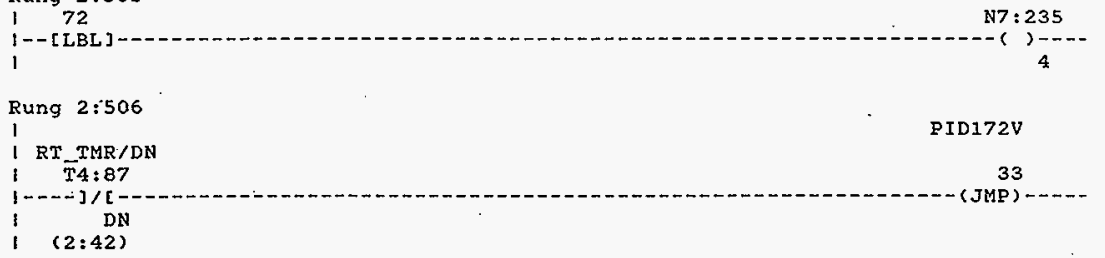

Rung 2:507

RATE CHECKING

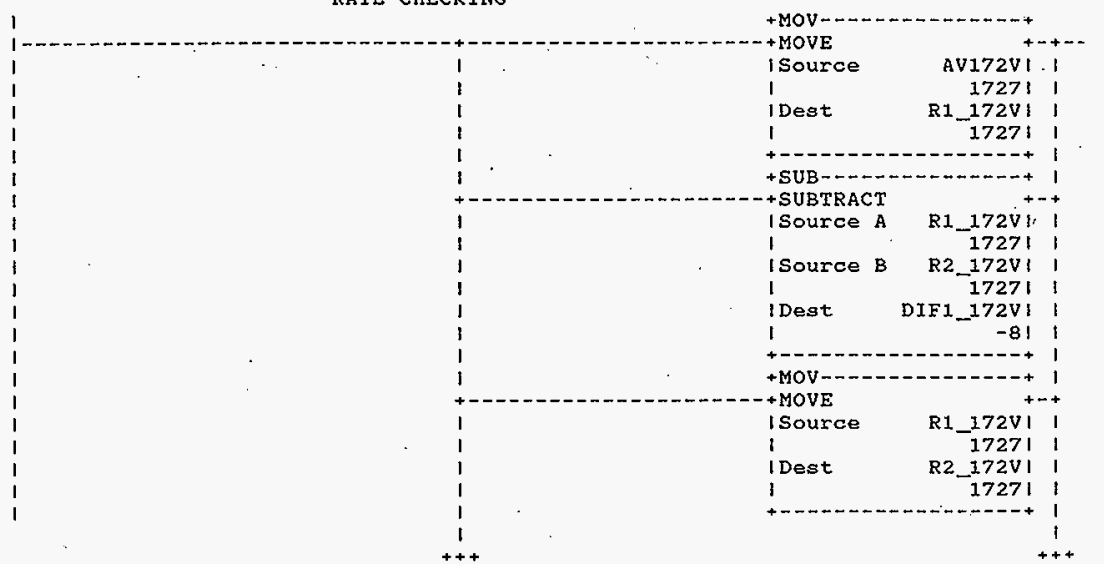

HNF-SD-FF-CSWD-61 Rev. 0 


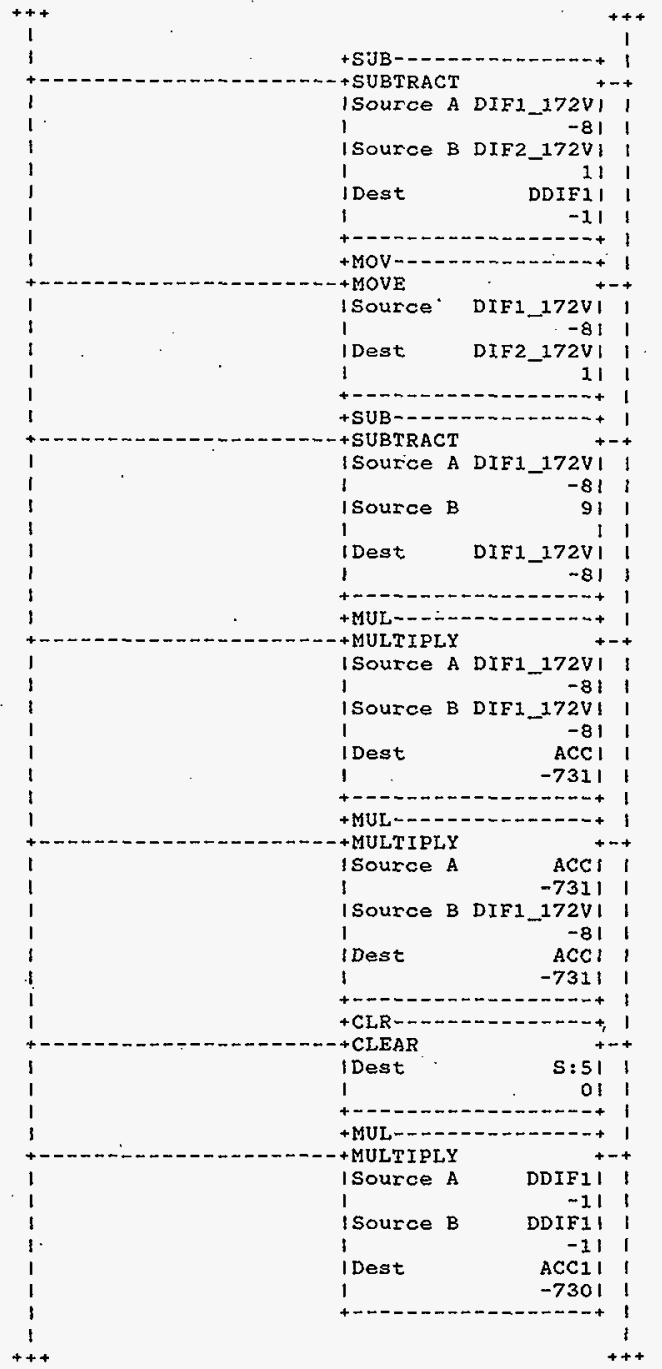

HNF-SD-FF-CSWD-61 Rev. 0 
Procesgor and Data(OPS Unit 1) Program Listing.

Processor File: SODIUM2A.ACH

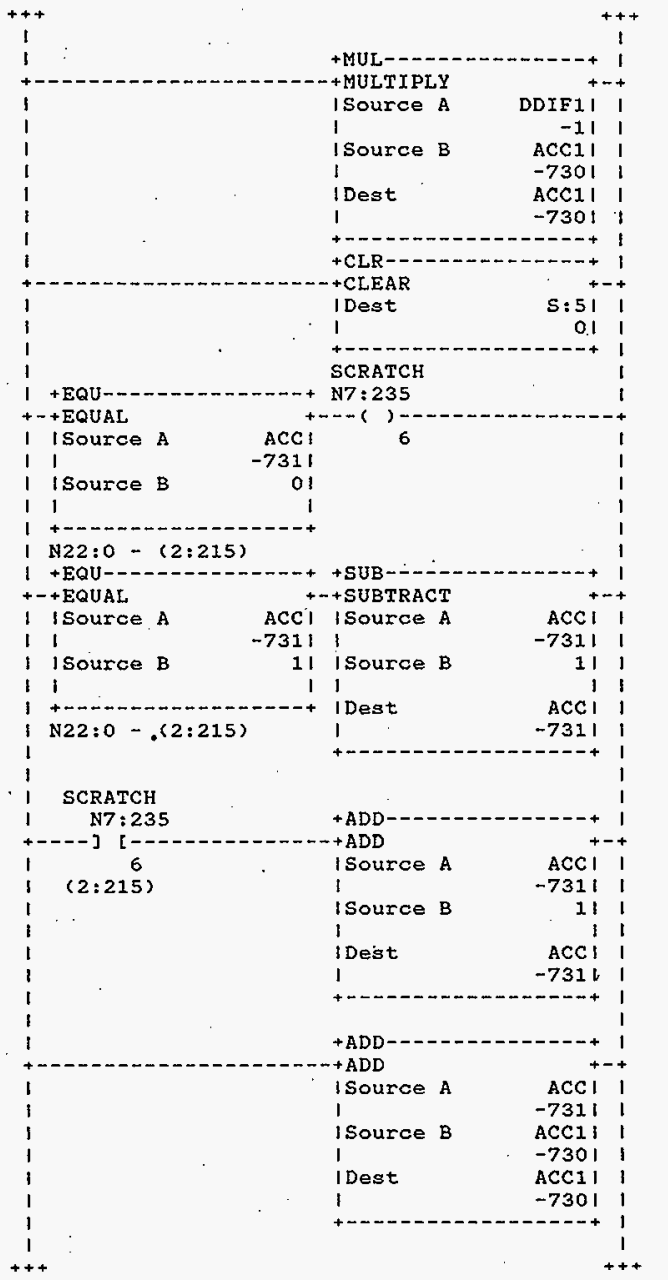

HNE-SD-FF-CSWD-61 RะV. 0 


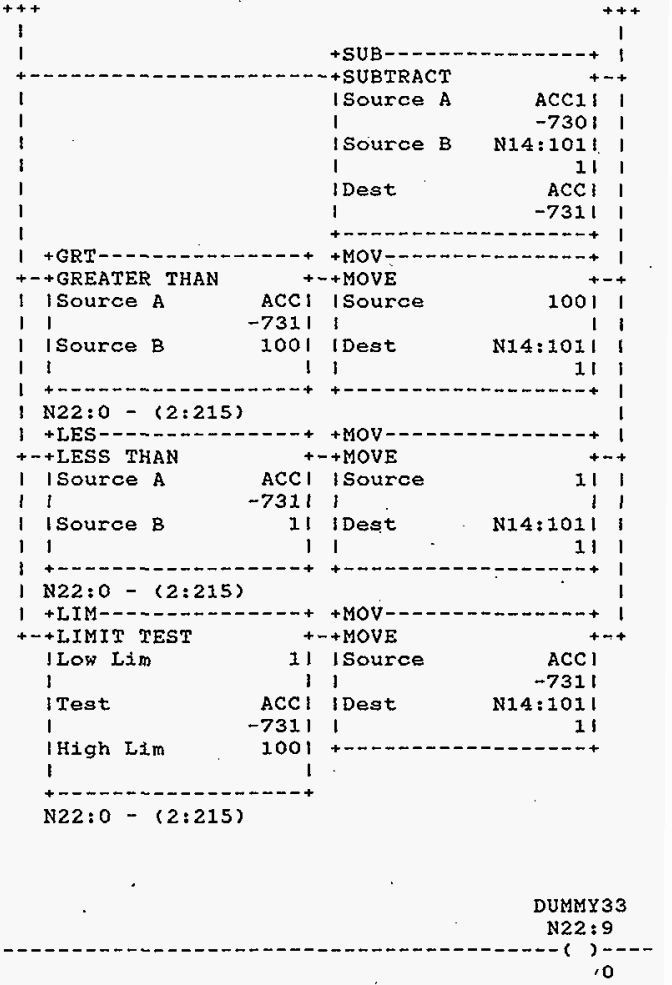

Rung 2:509

Rung 2:508

I PID $172 \mathrm{~V}$

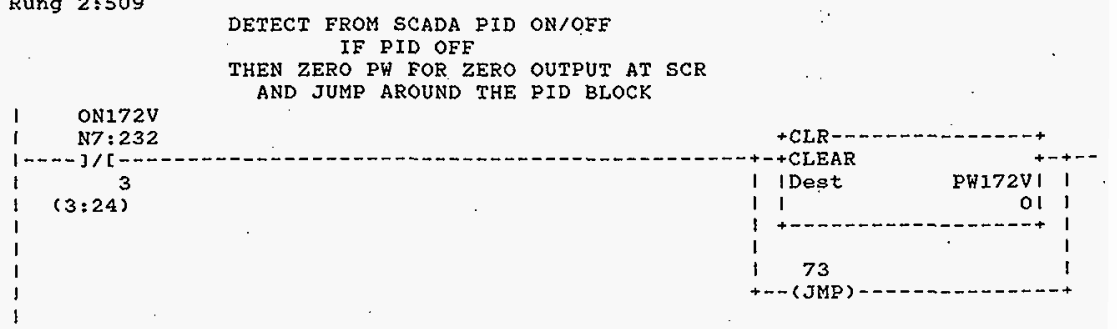

HNF-SD-FF-CSWD-61 Rev. 0 


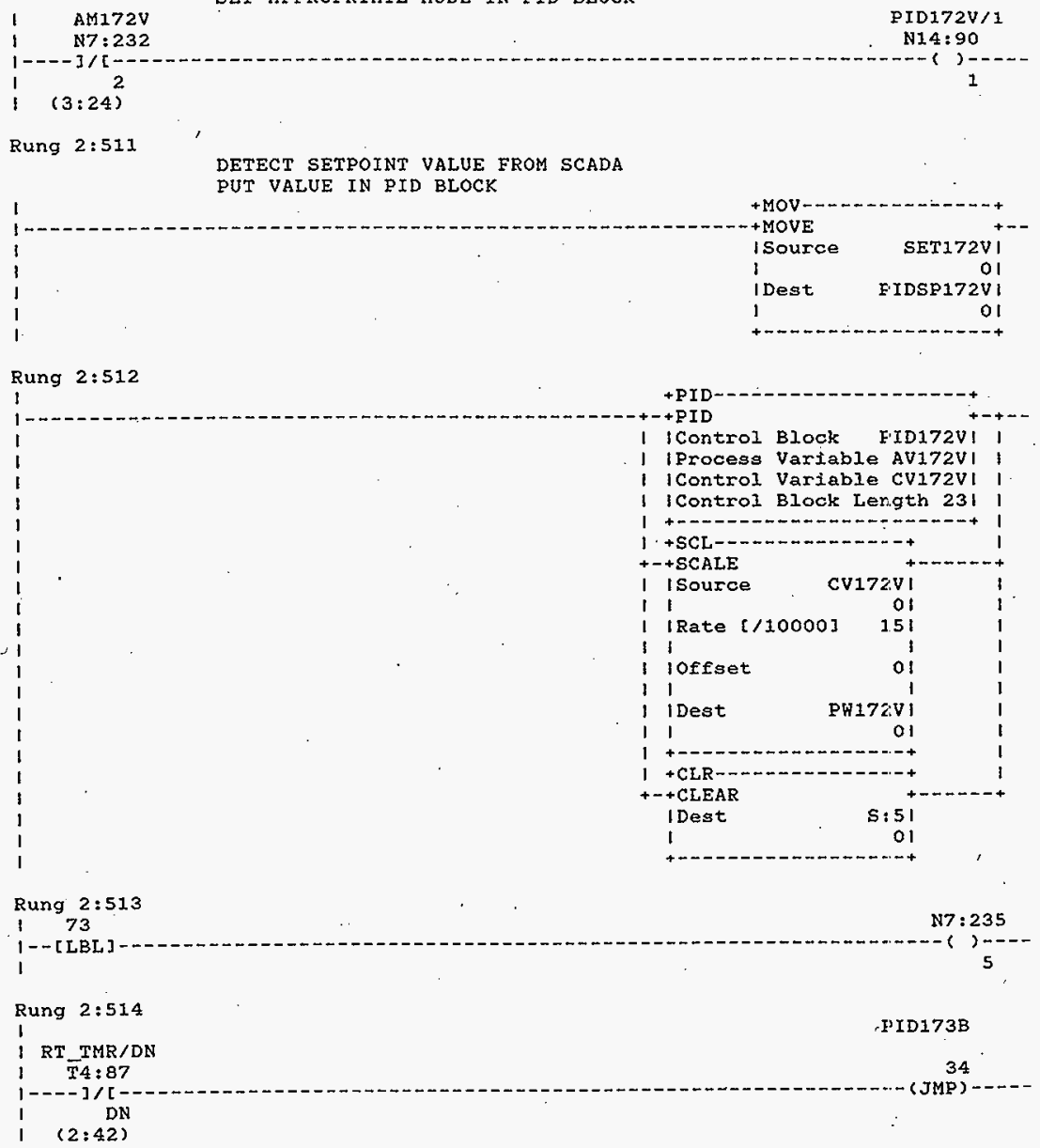

HNF-SD-FF-CSWD-61 Rev. 0 
Processor and Data(OPS Unit 1$)$

Rung 2:515

\section{RATE CHECKING}

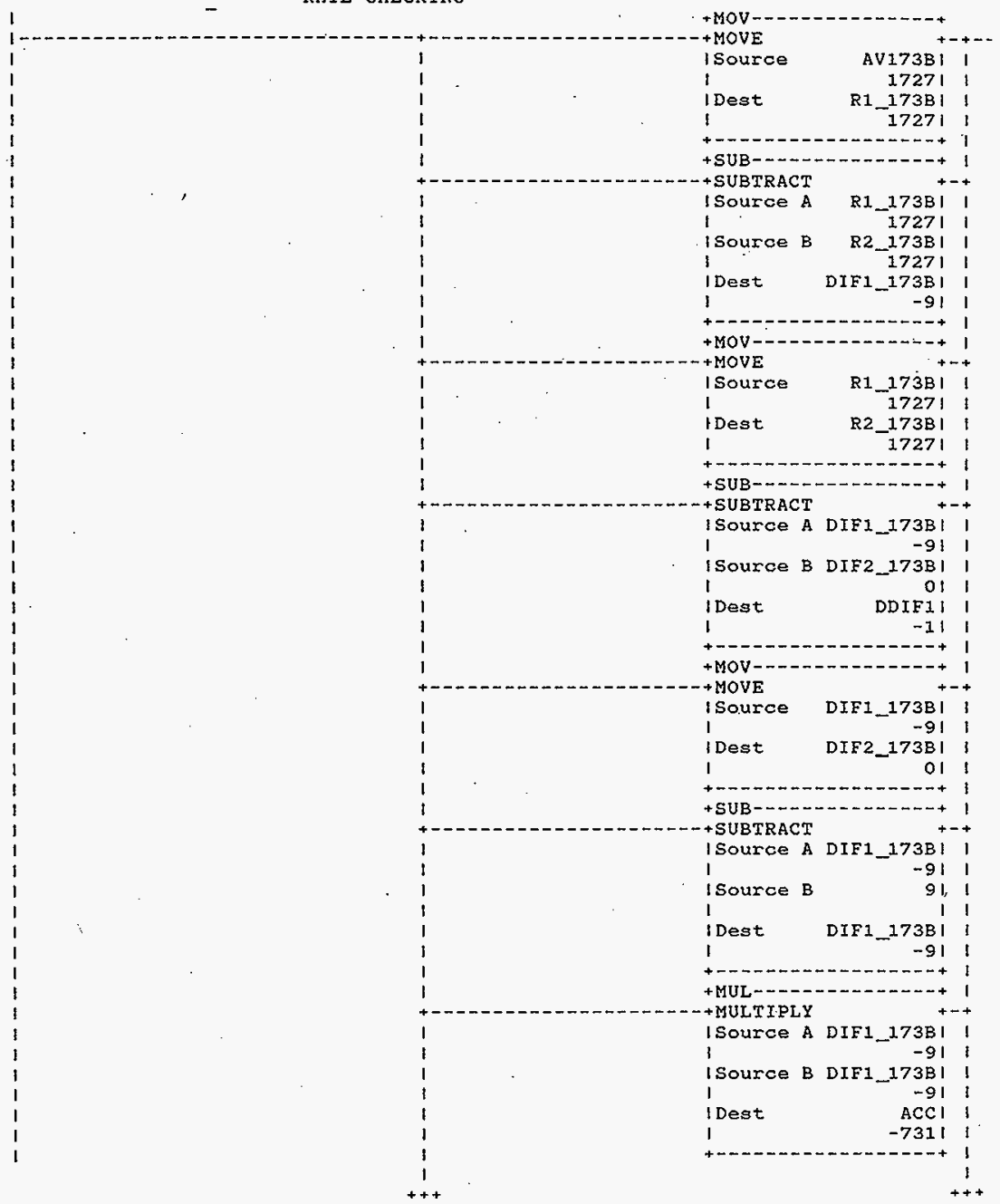

HNF-SD-FF-CSWD-61 Rev. 0 


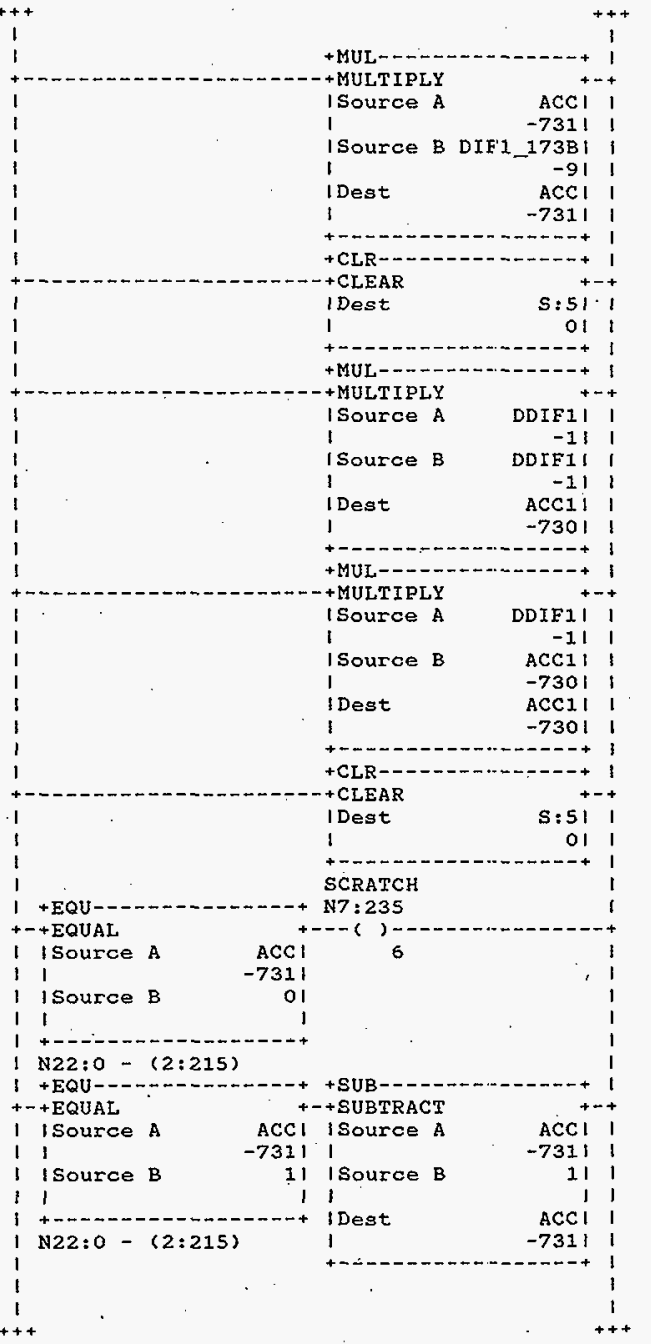

HNF-SD-FF-CSWD-61 Rev. 0 


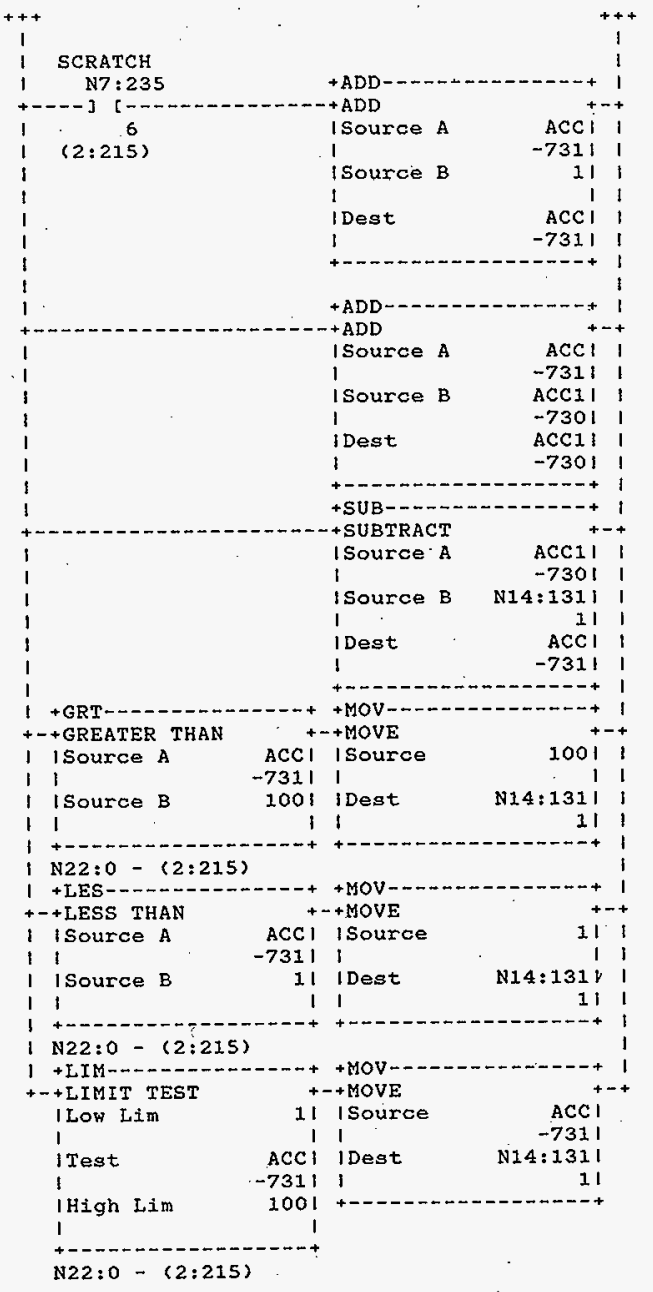

HNF-SD-FF-CSWD-61 Rev. 0 


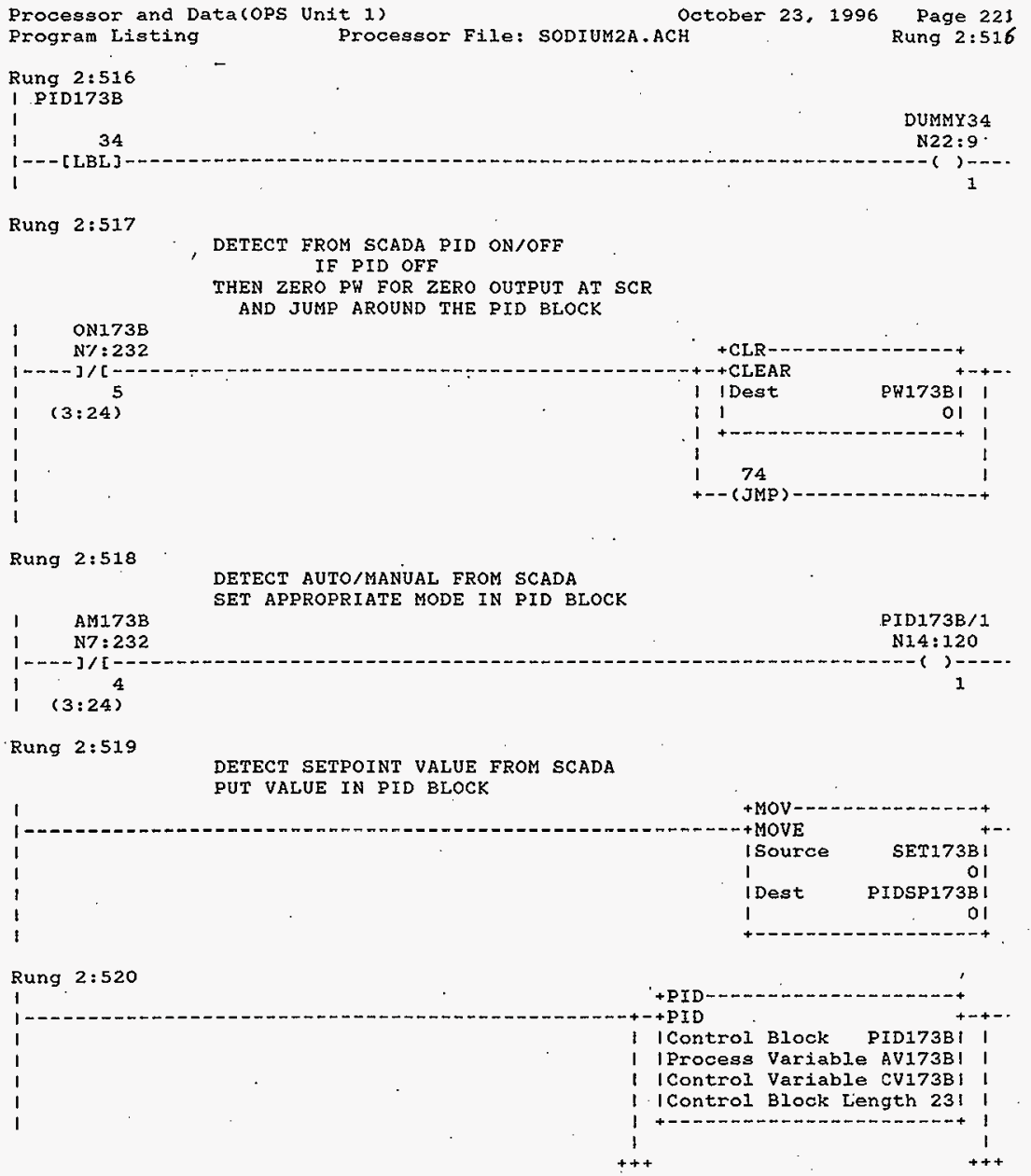

HNF-SD-FF-CSWD-61 Rev. 0 


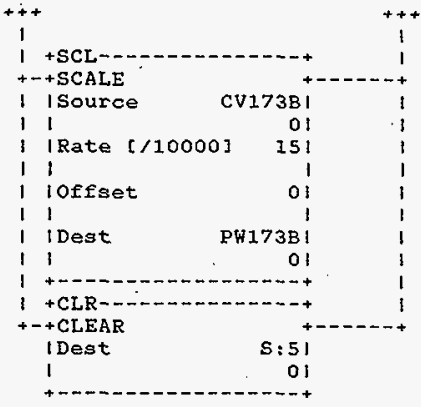

Rung $2: 521$

$$
174
$$

N7 $: 235$

i $--[\mathrm{LBL}]$

Rung 2:522

I RT_TMR/DN

( T4:87

i DN

I (2:42)

Rung 2:523

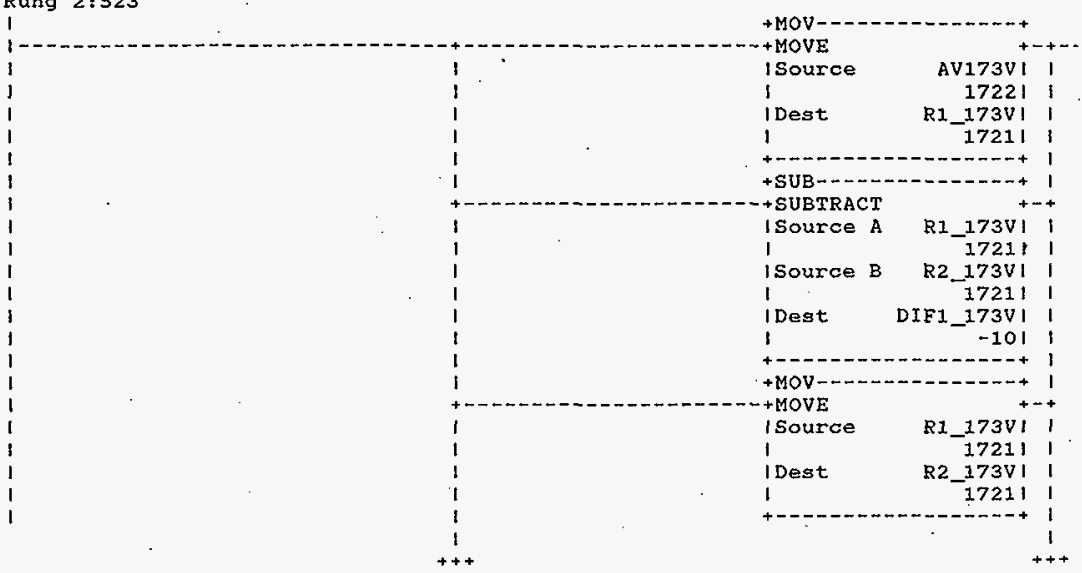

HNF-SD-FF-CSWD-61 Rev. 0 

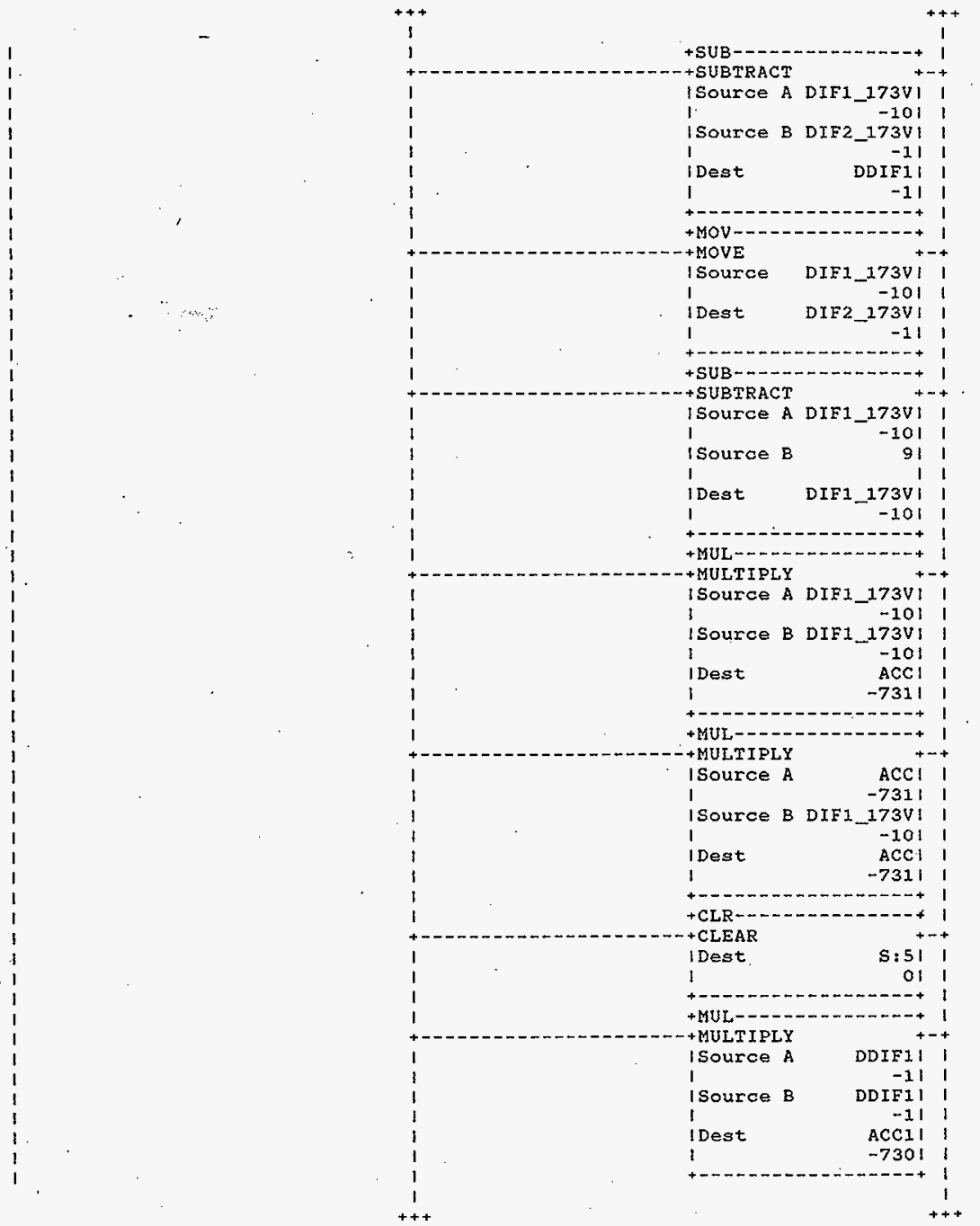

HNF-SD-FF-CSWD-61 Rev. 0 


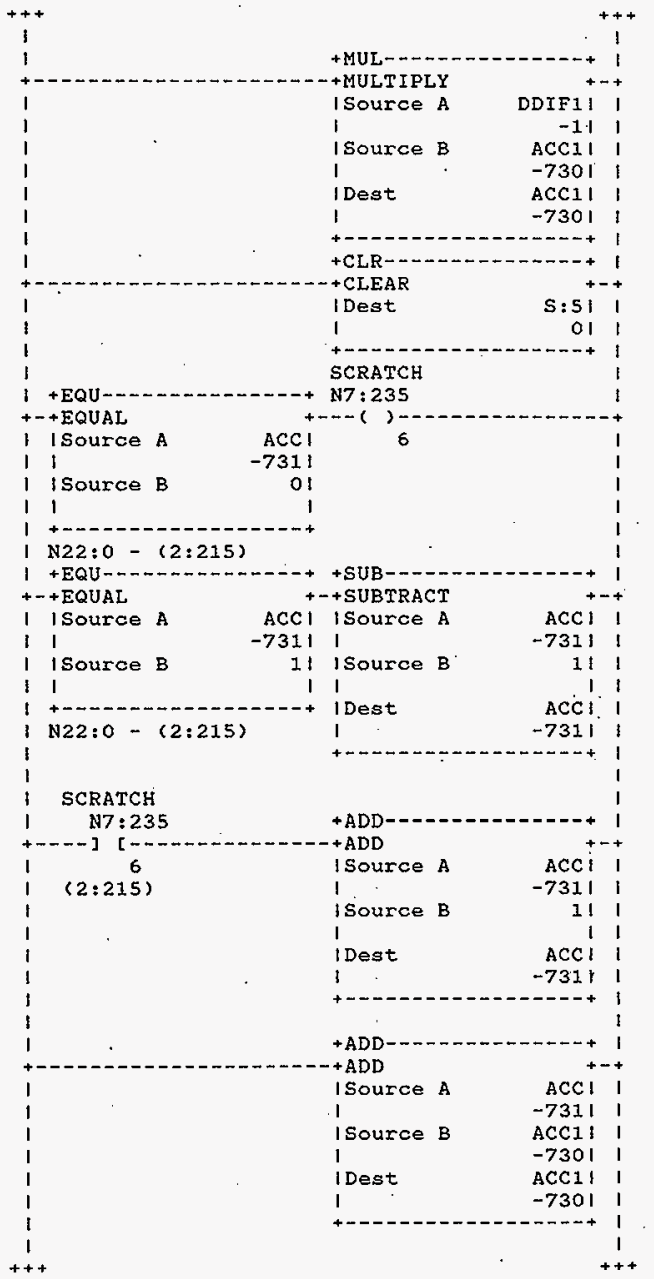

HNF-SD-FF-CSWD-61 Rev. 0 


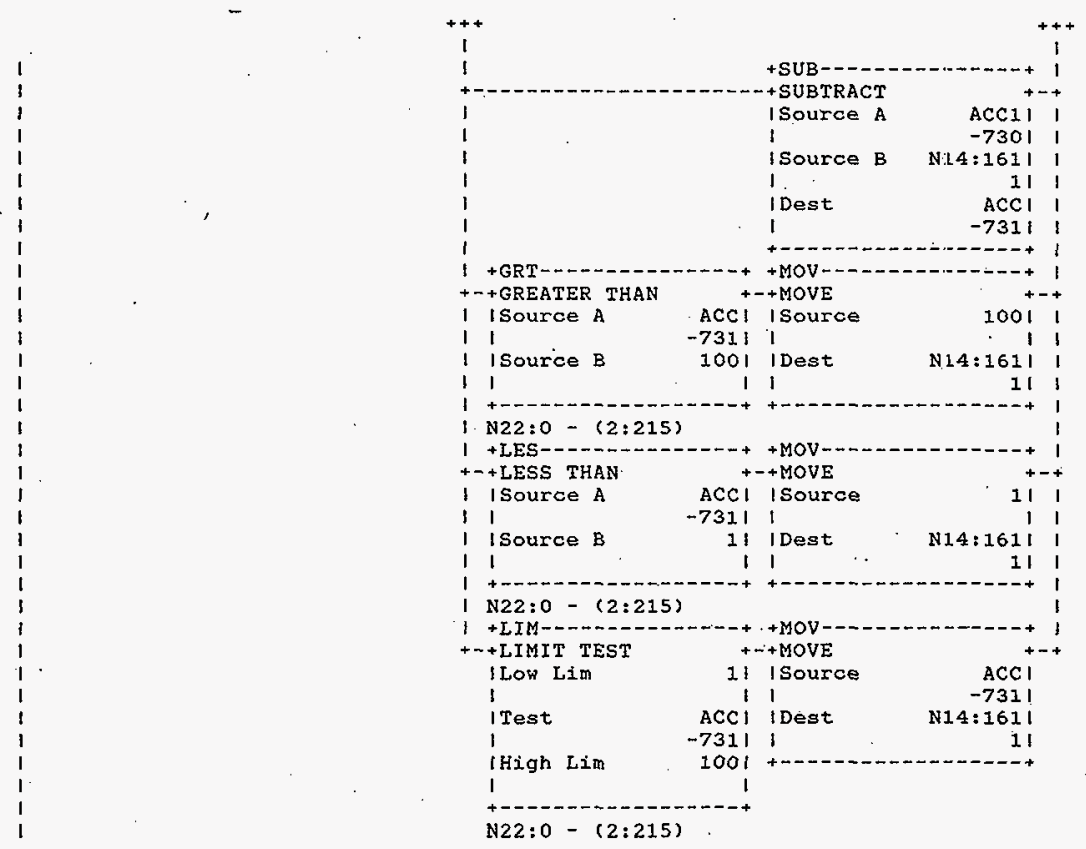

Rung 2:524

I PID173V

Rung 2:525

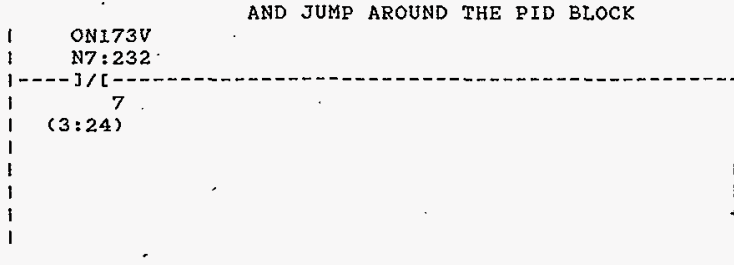

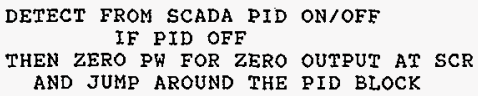

HNF-SD-FF-CSWD-61 Rev. 0 
Procegsor and Data(ops Unit 1) Program Listing

Rung 2:\$26

Processor File: SODIUM2A.ACH

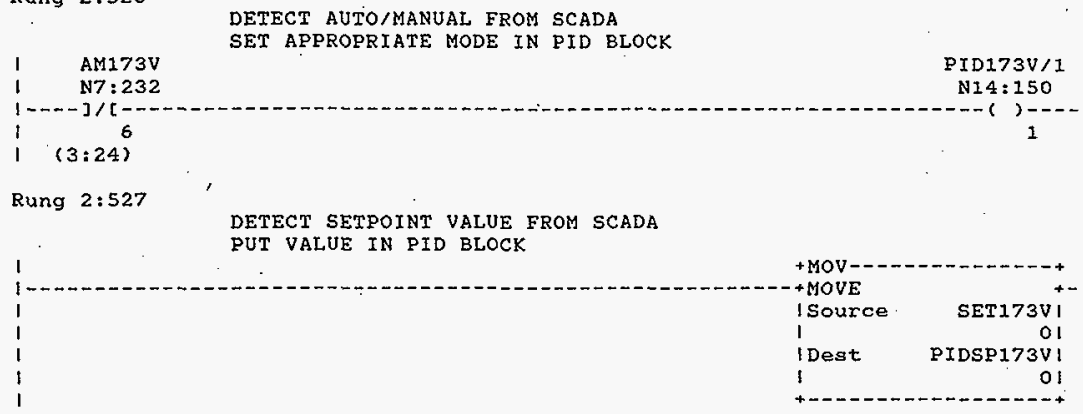

\section{Rung 2:528}

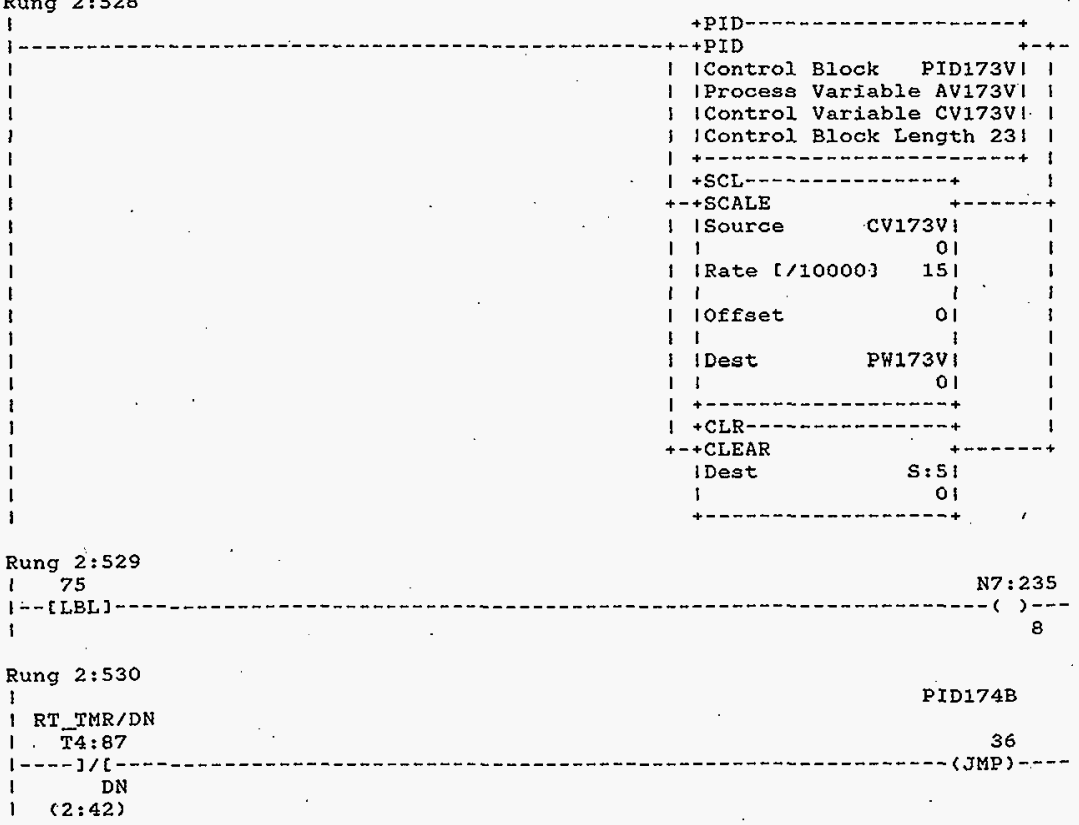

HNF-SD-FF-CSWD-61 Rev. 0 
Rung 2:531

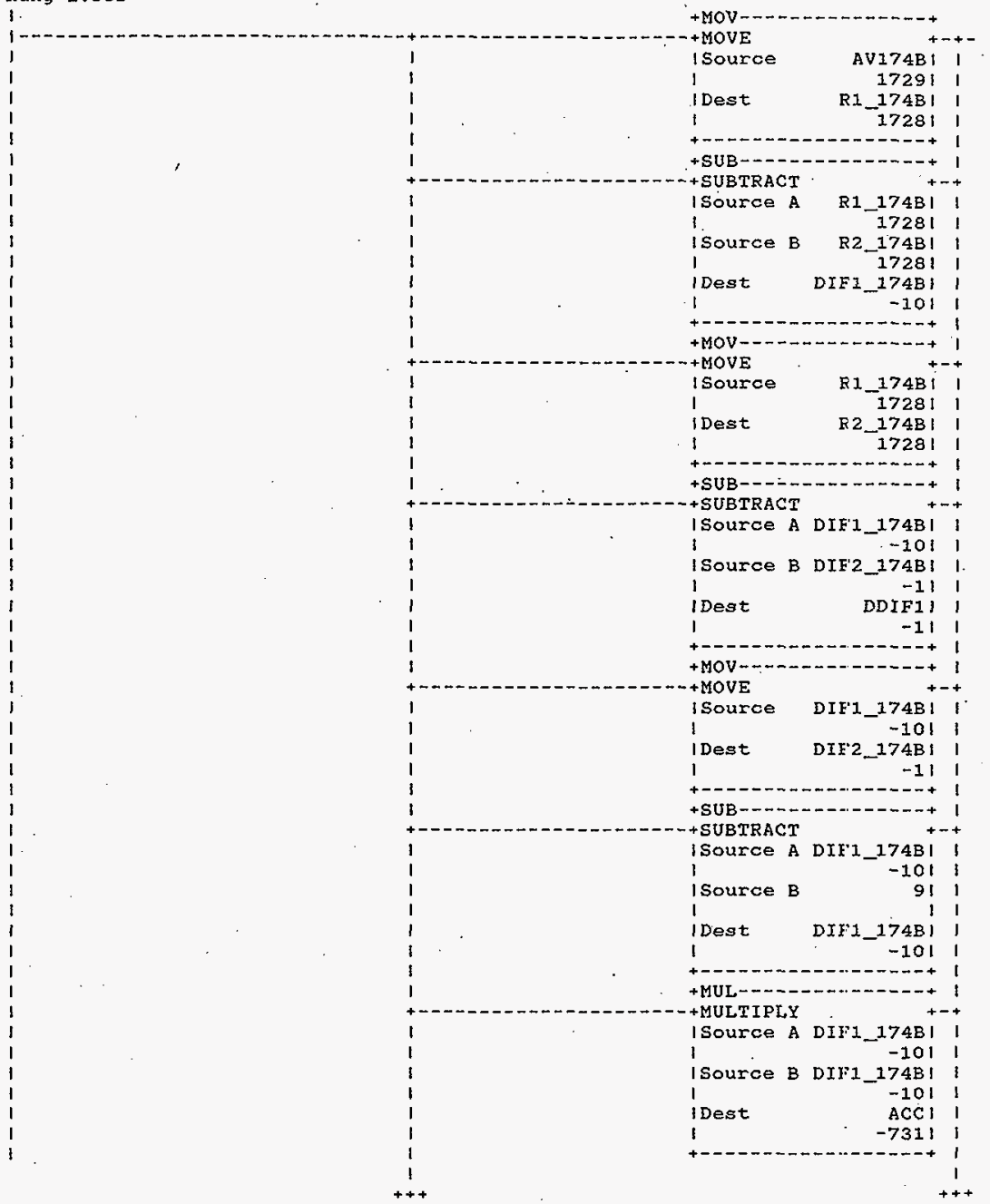

HNF-SD-FF-CSWD-61 Rev. 0 


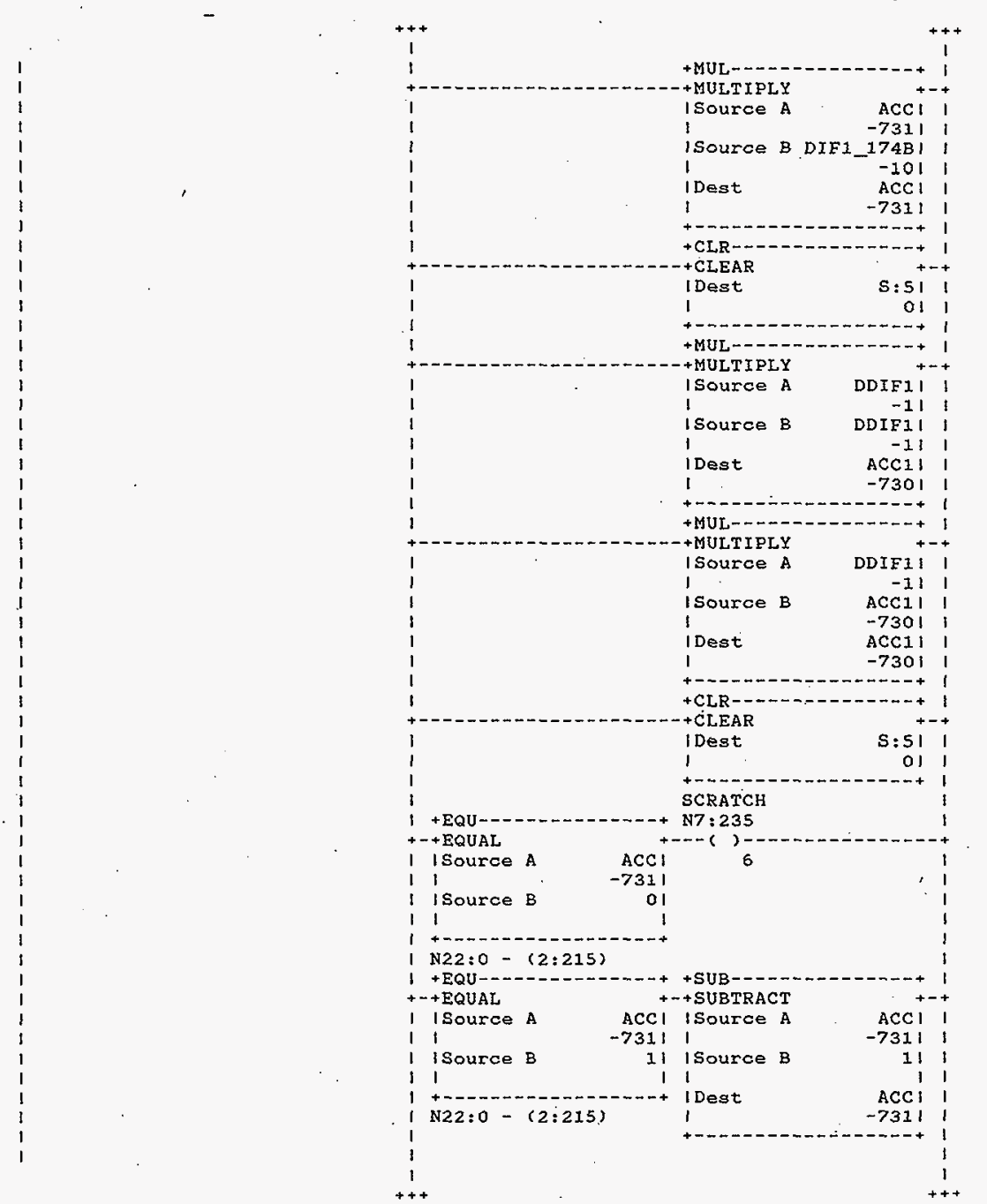

HNF-SD-FF-CSWD-61 Rev. 0 




HNF-SD-FF-CSWD-61 Rev. 0 
Processor and Data (OPS Unit 1 )

Program Listing Processor File: SODIUM2A.ACH

October 23,1996

Rung $2: 532$

I. PID 1748

1

136

DUMMY36

$1--[$ [DBL]

36

N22:9

Rung $2: 533$
DETECT FROM SCADA PID ON/OFF
IF PID OFF
THEN ZERO PW FOR ZERO OUTPUT AT SCR
AND JUMP AROUND THE PID BLOCK
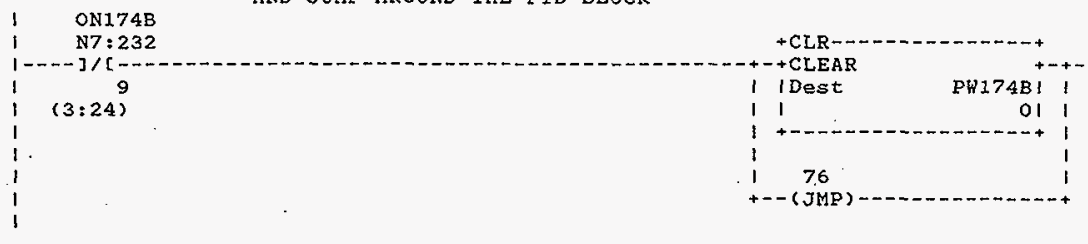

Rung 2:534

DETECT AUTO/MANUAL FROM SCADA

SET APPROPRIATE MODE IN PID BLOCK

$\begin{array}{lc}1 & A M 174 B \\ 1 & N 7: 232 \\ 1 & 8 \\ 1 & (3: 24)\end{array}$

PID 174B/1

N14:180

1

Rung $2: 535$

DETECT SETPOINT VALUE FROM SCADA PUT VALUE IN PID BLOCK

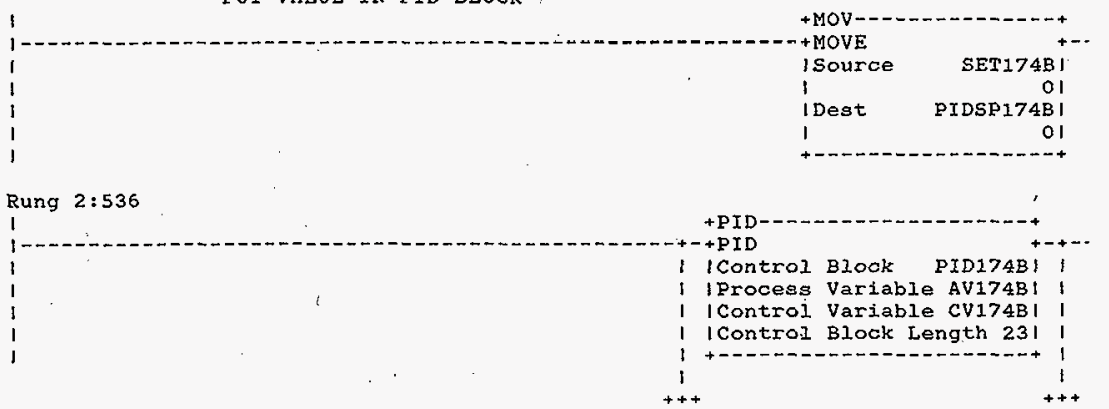

HNF-SD-FF-CSWD-61 Rev. 0

Page 592 


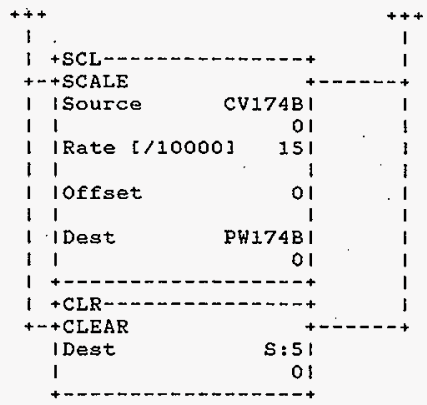

Rung 2:537

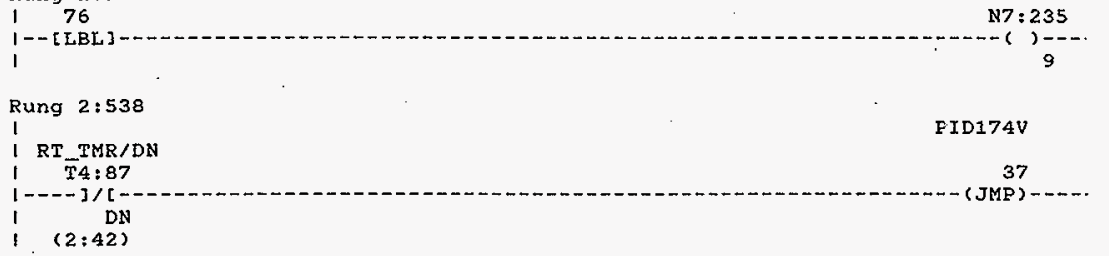

Ruing 2:539

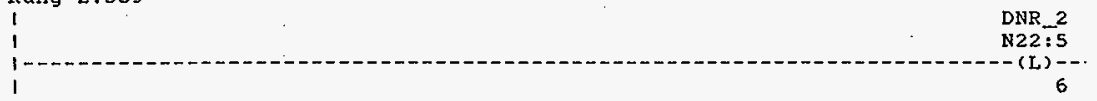

Rung 2:540

RATE CHECKING

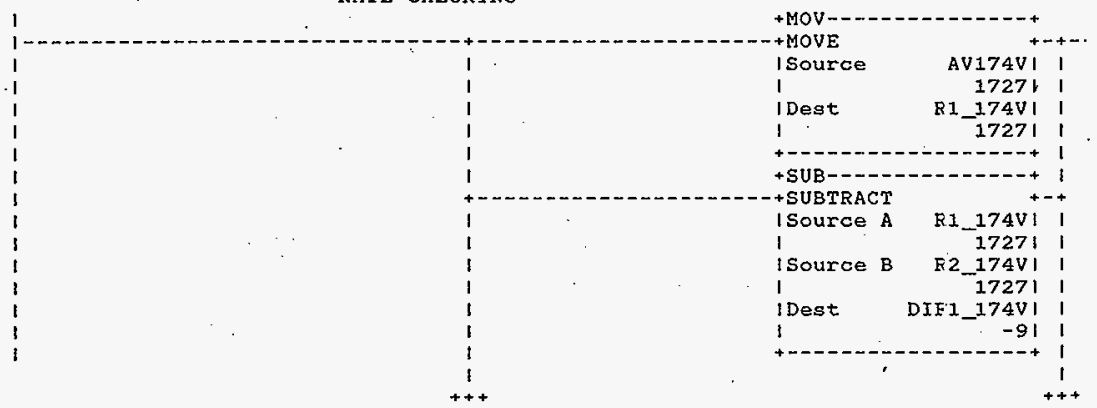

HNF-SD-FF-CSWD-61 Rev. 0 
Processor and Data(OPS Unit 1)

October 23,1996

Page 232 Program Listing Processor File: SODIUM2A.ACH Rung $2: 540$
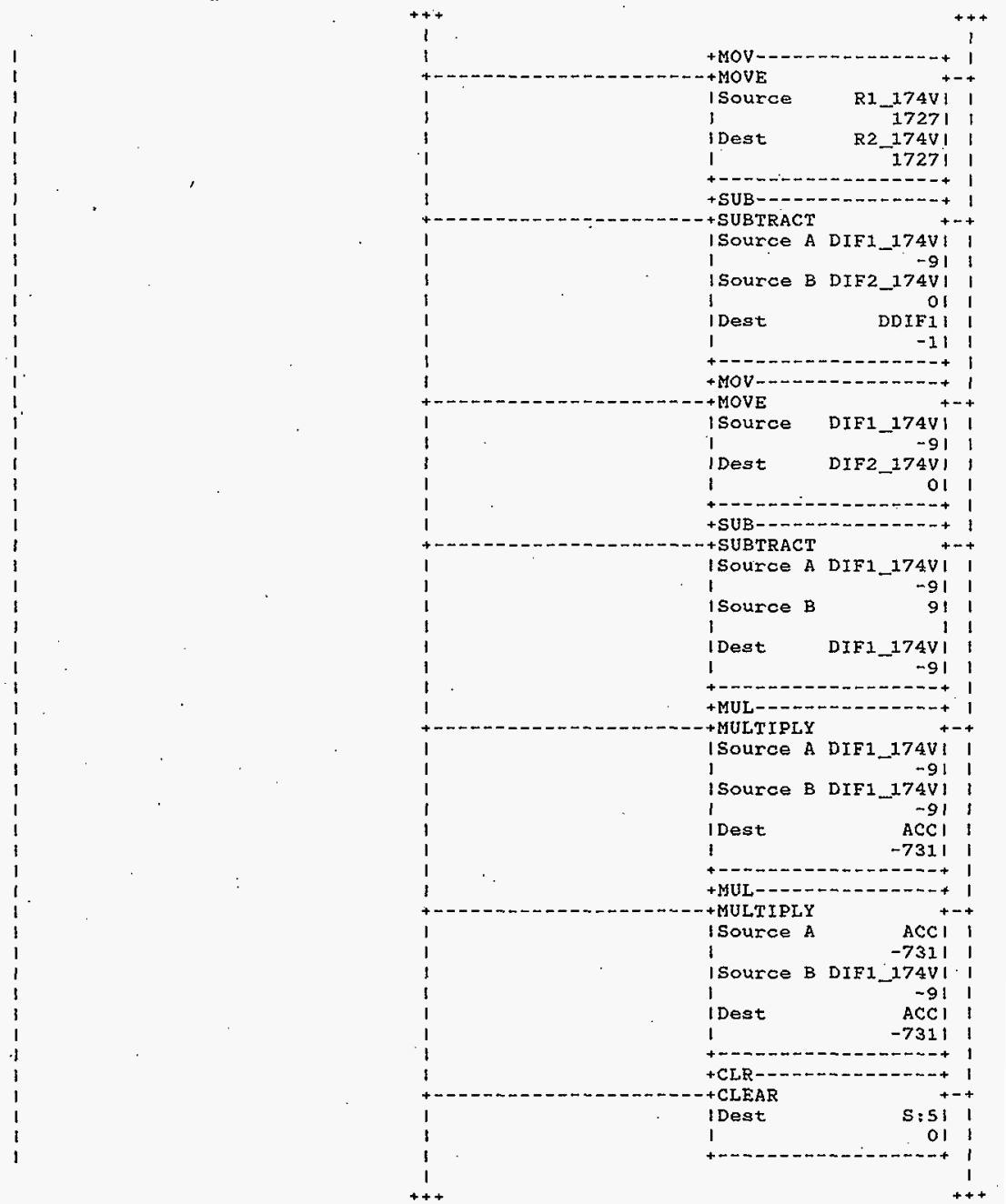

HNF-SD-FF-CSWD-61 Rev. 0

Page 594 


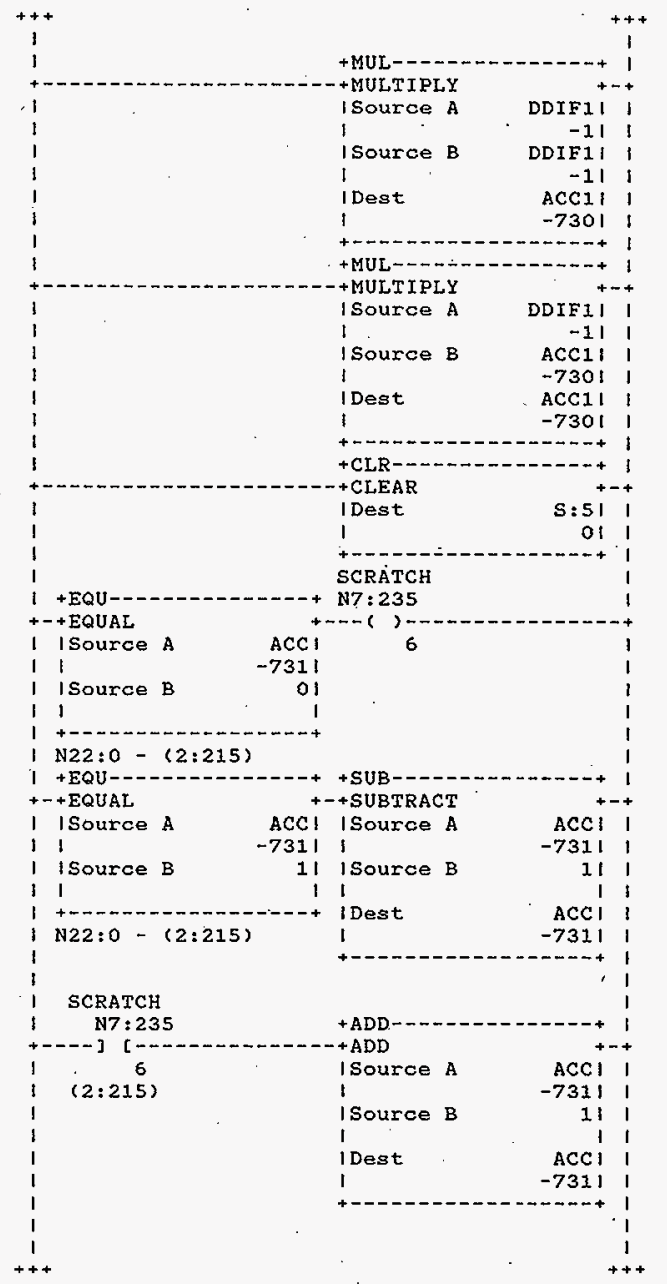

HNF-SD-FF-CSWD-61 Rev. 0

Page 595 


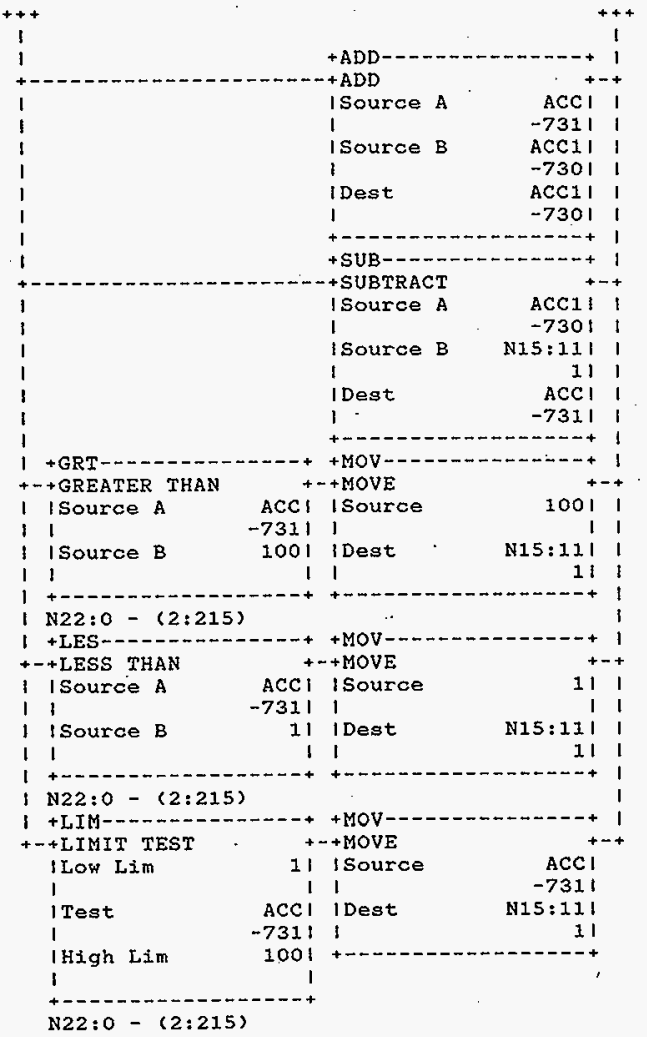

Rung $2: 541$ 
Processor and Data(OPS Unit 1)

Rung $2: 542$
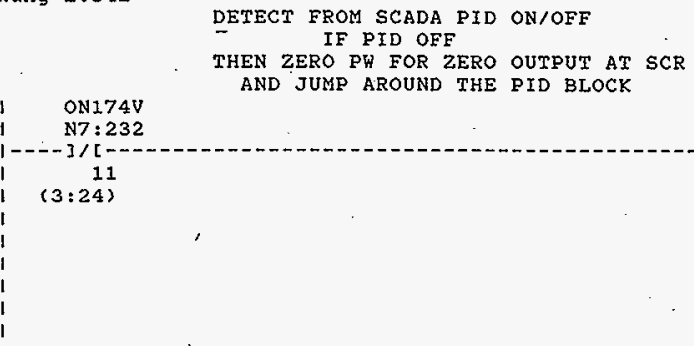

Rung $2: 543$

$\begin{array}{lc}1 & A M 174 V \\ 1 & N 7: 232 \\ 1 & 10 \\ 1 & (3: 24)\end{array}$

DETECT AUTO/MANUAL FROM SCADA SET APPROPRIATE MODE IN PID BLOCK

\section{Rung 2:544}

DETECT SETPOINT. VALUE FROM SCADA PUT VALUE IN PID BLOCK

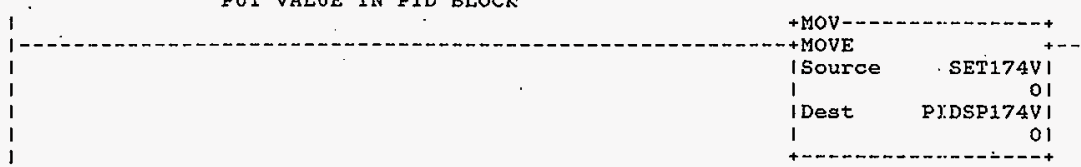

Rung $2: 545$

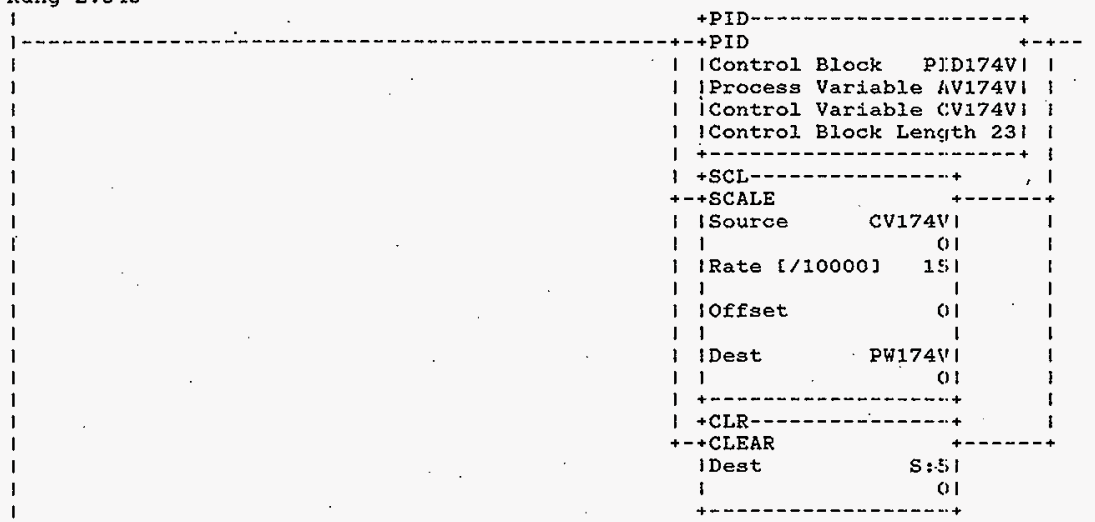

HNF-SD-FF-CSWD-61 Rev. 0

Page 597 


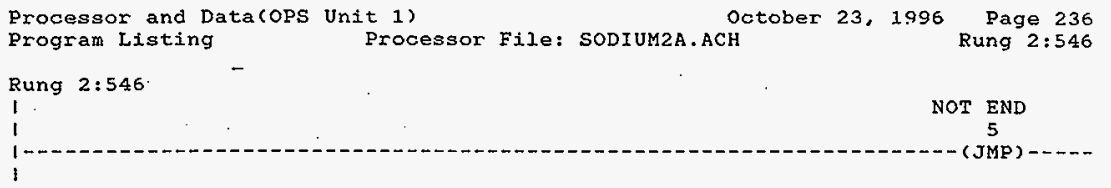

Rung $2: 547$

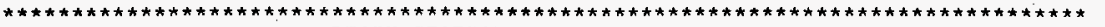
I $M D 3$ BEGIN SECTION 3

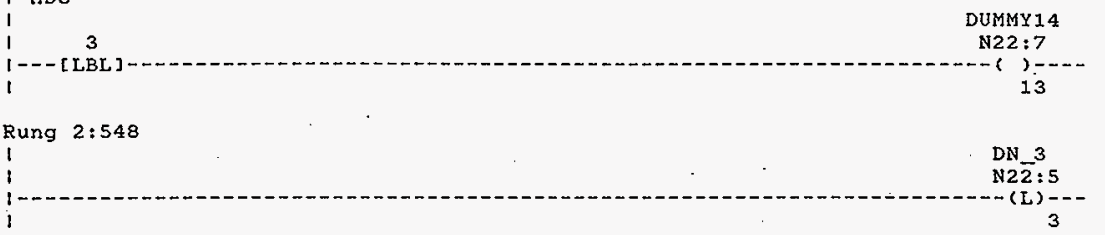

Rung 2:549

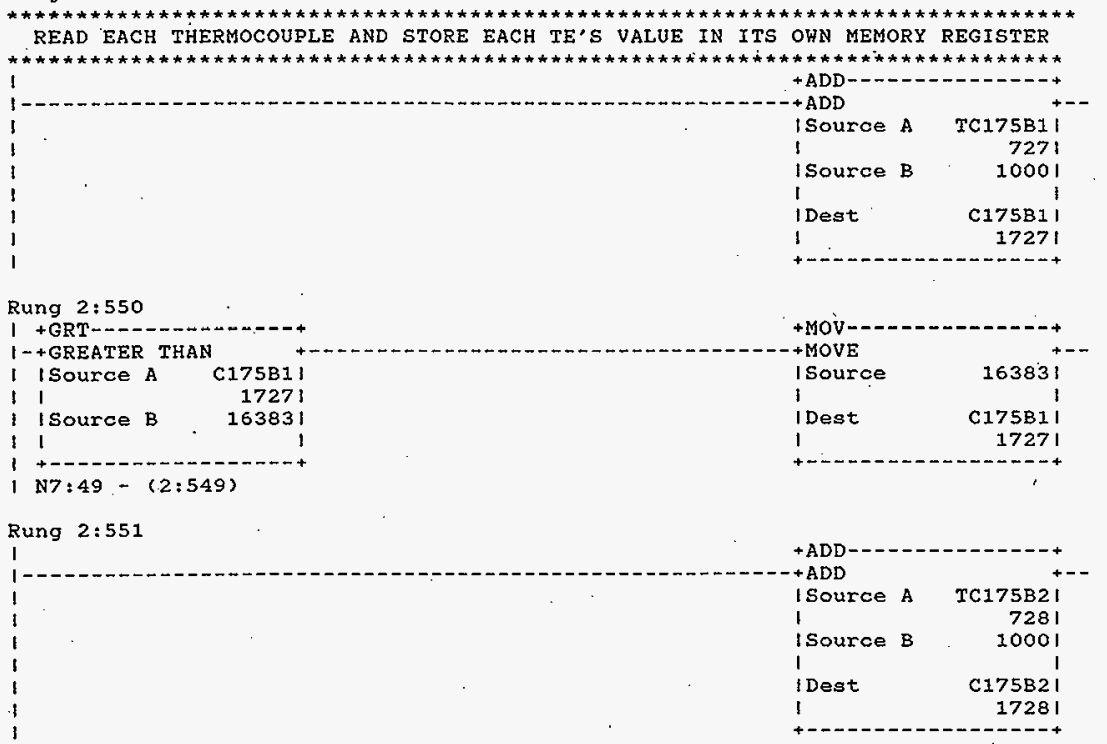

HNF-SD-FF-CSWD-61 Rev. 0 
Processor and Data(OPS Unit 1)

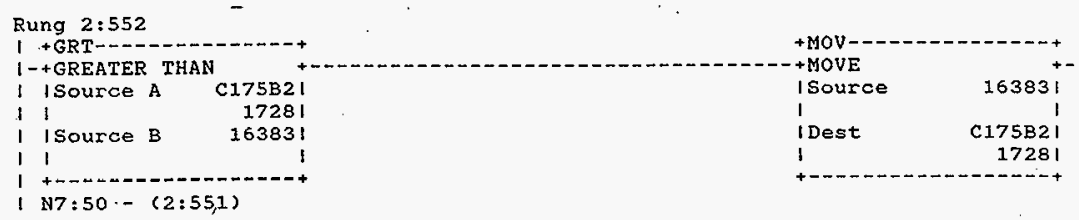

Rung 2:553
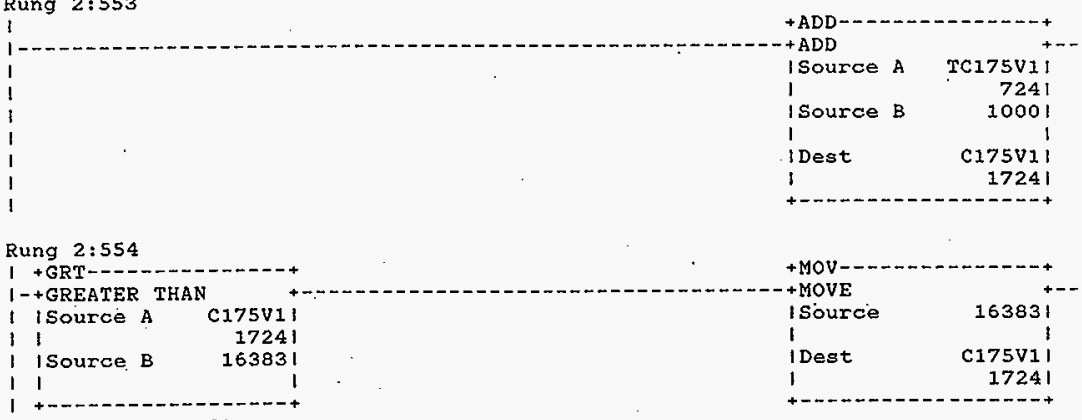

IN7:51-(2:553)

Rung 2:555

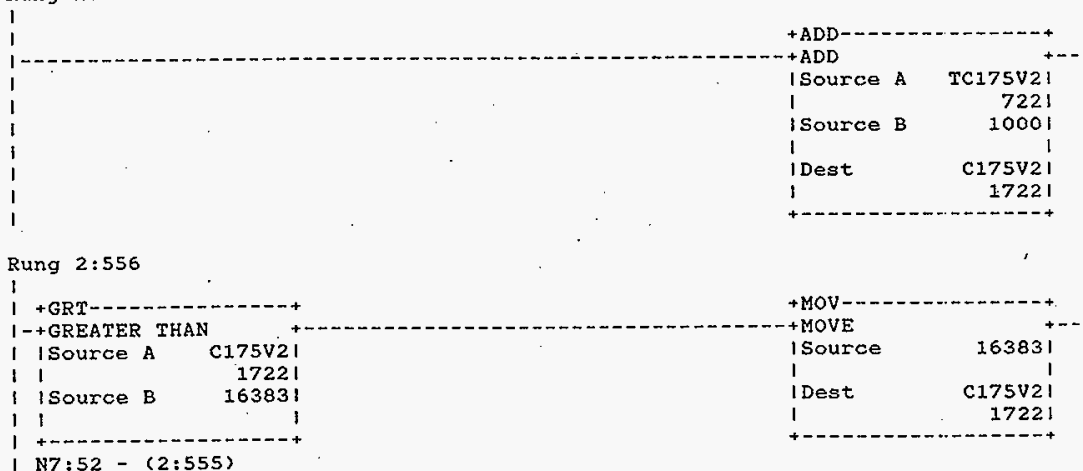

HNF-SD-FF-CSWD-61 Rev. 0 
R
I
1
1
1
1
1
1

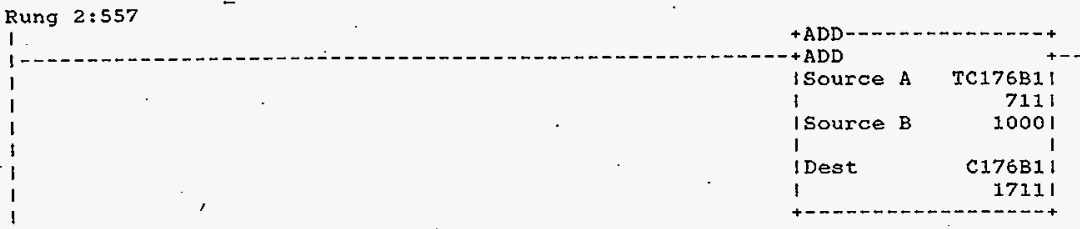

Rung $2: 558$

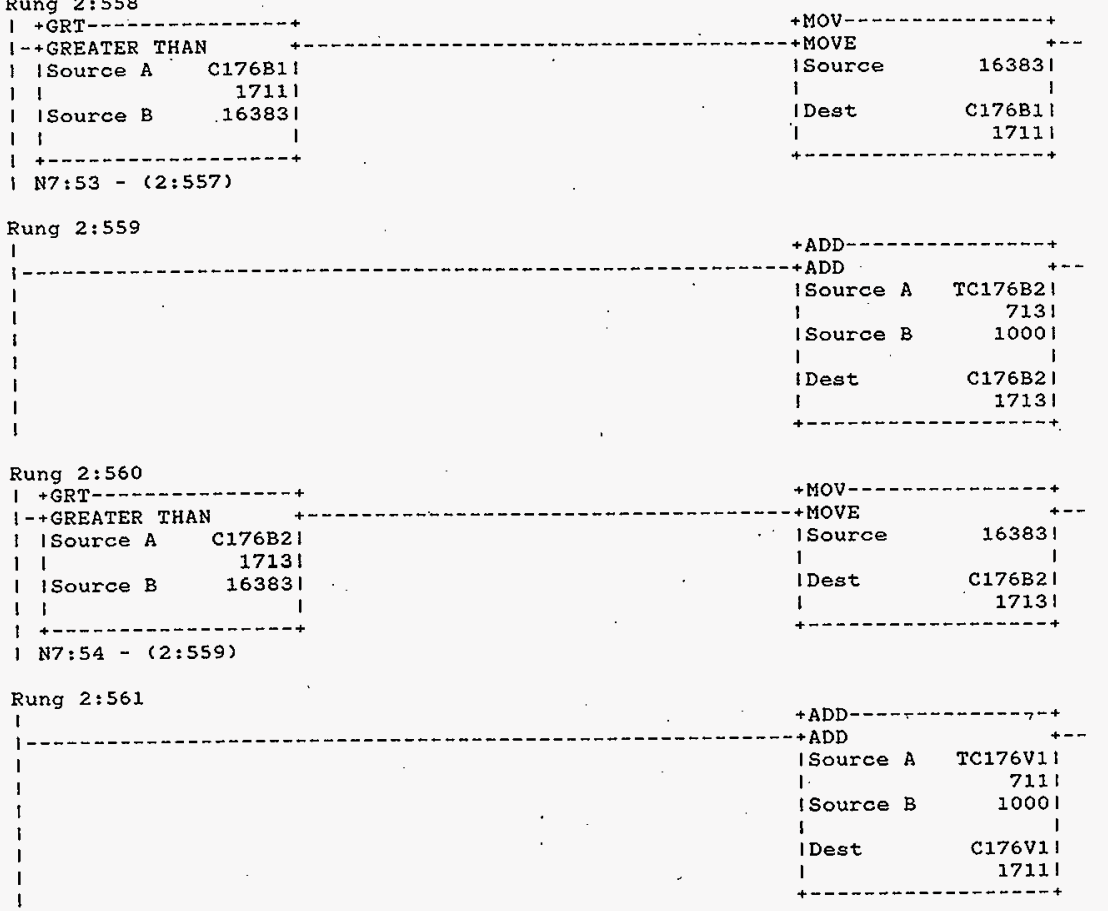

HNF-SD-FF-CSWD-61 Rev. 0 


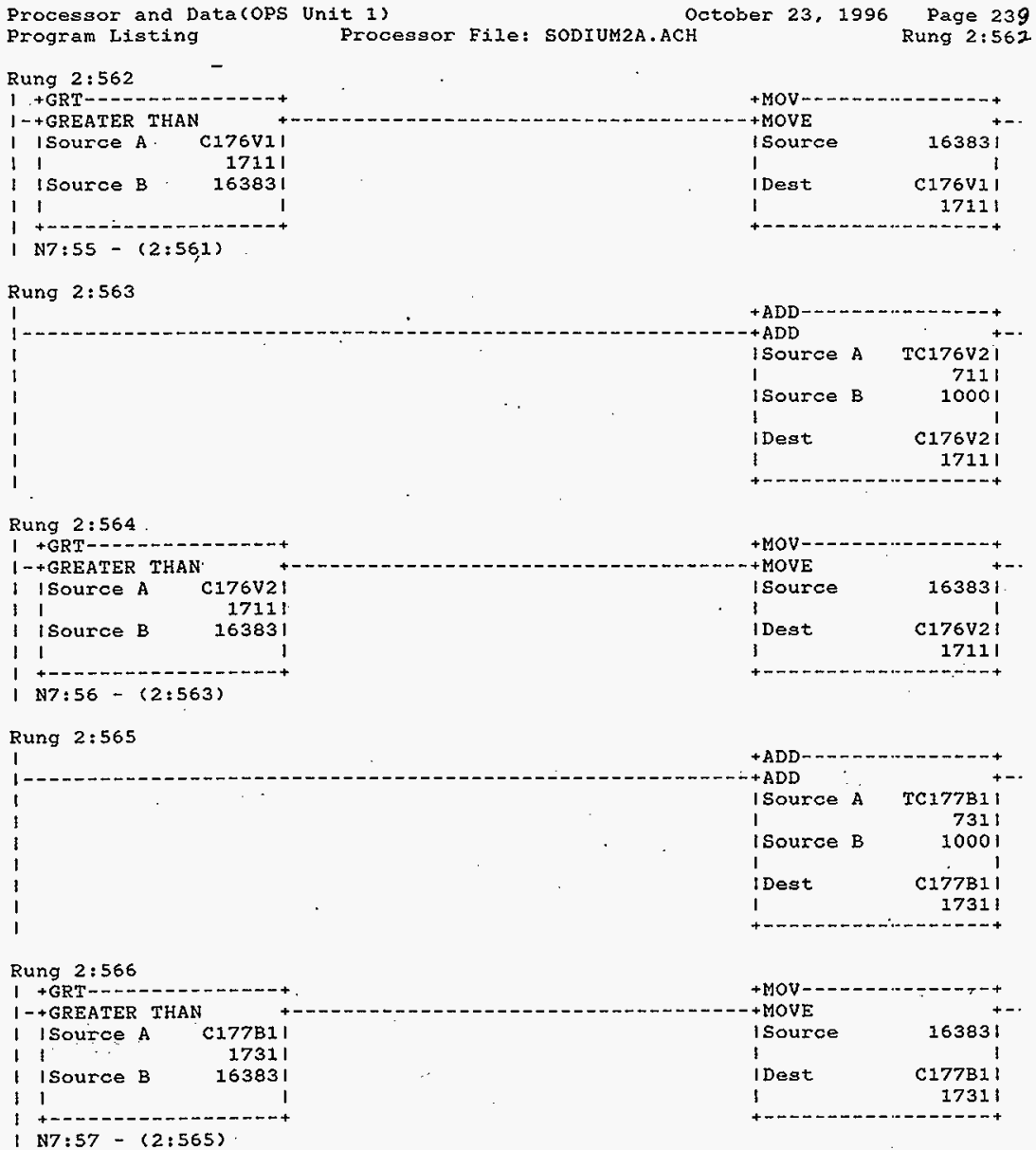

HNF-SD-FF-CSWD-61 R:v. 0 


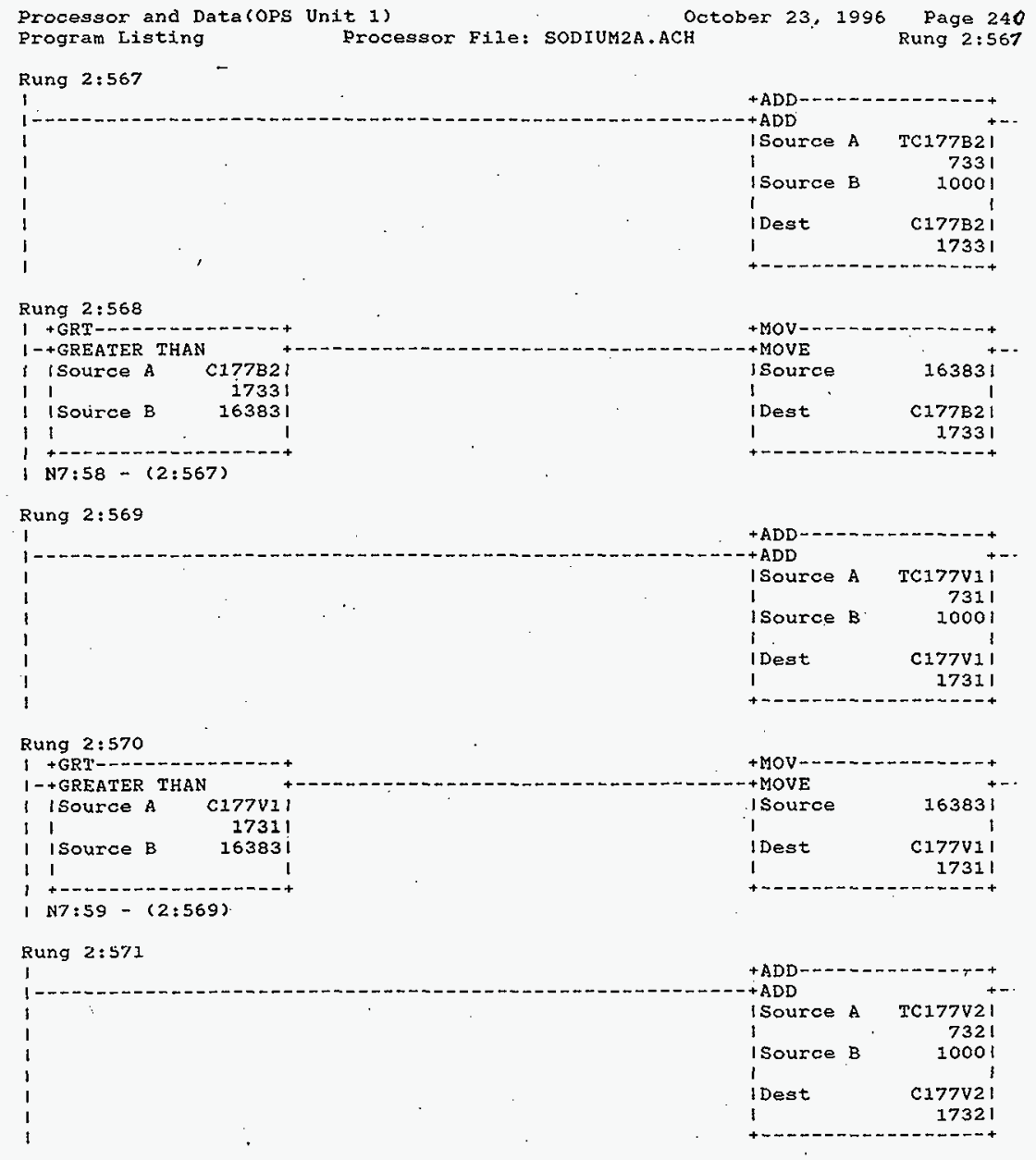

HNF-SD-FF-CSWD-61 Rev. 0

Page 602 


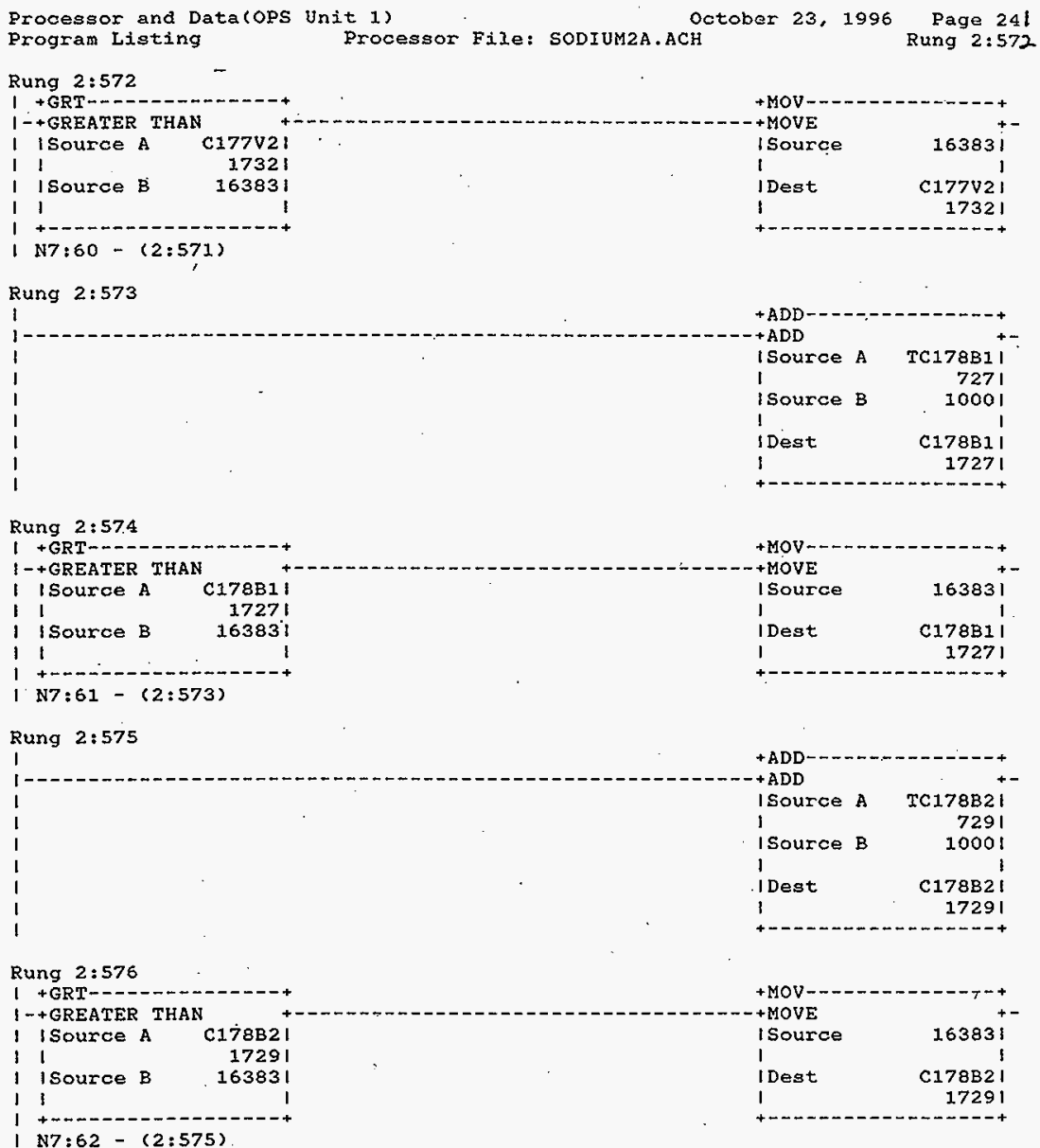

HNF-SD-FF-CSWD-61 Rev. 0

Page 603 


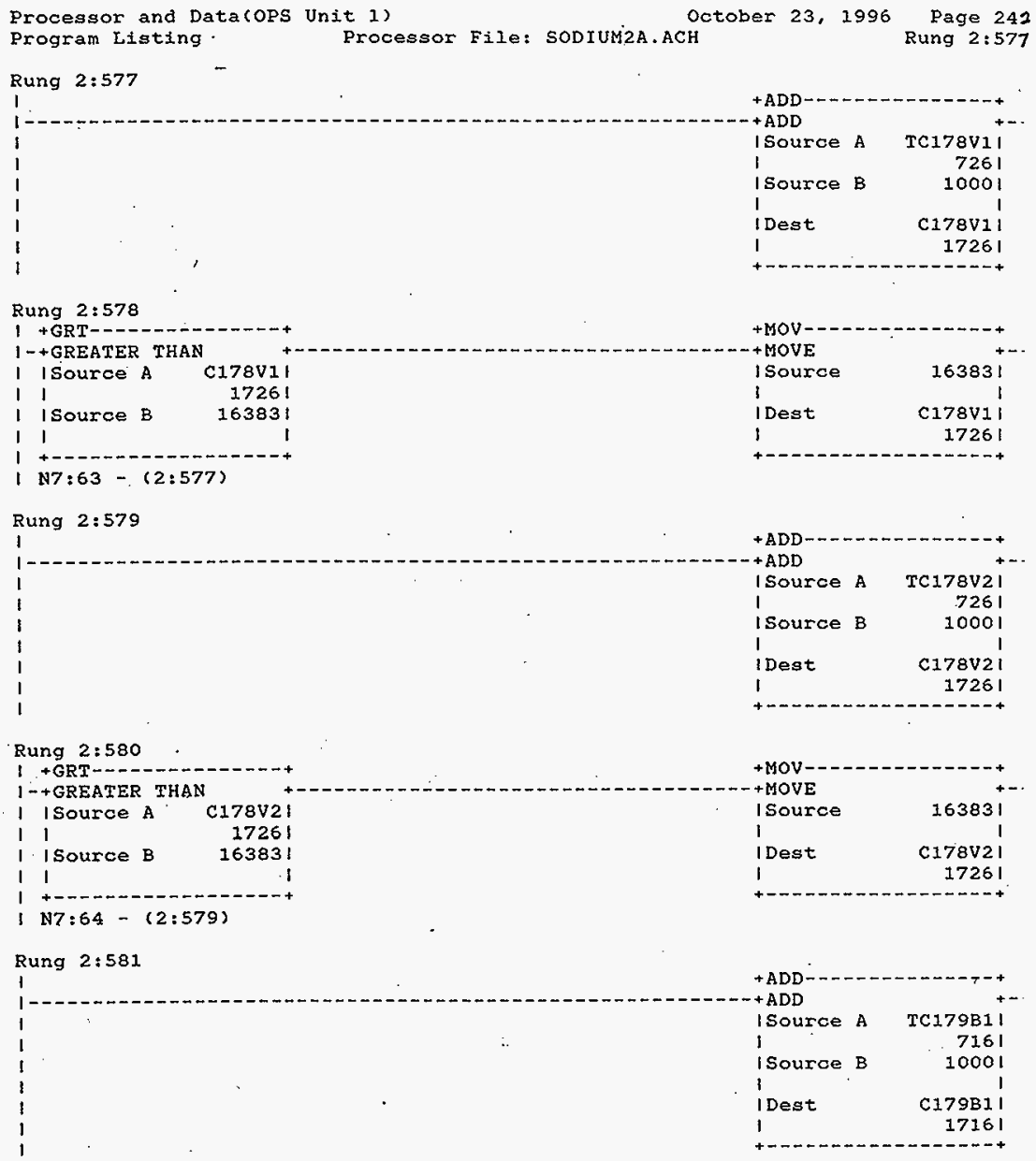

HNF-SD-FF-CSWD-61 Rev. 0

Page 604 
Processor and Data(OPS Unit 1)

October 23, 1996

Page 24a Program Listing Processor File: SODIUM2A. ACH

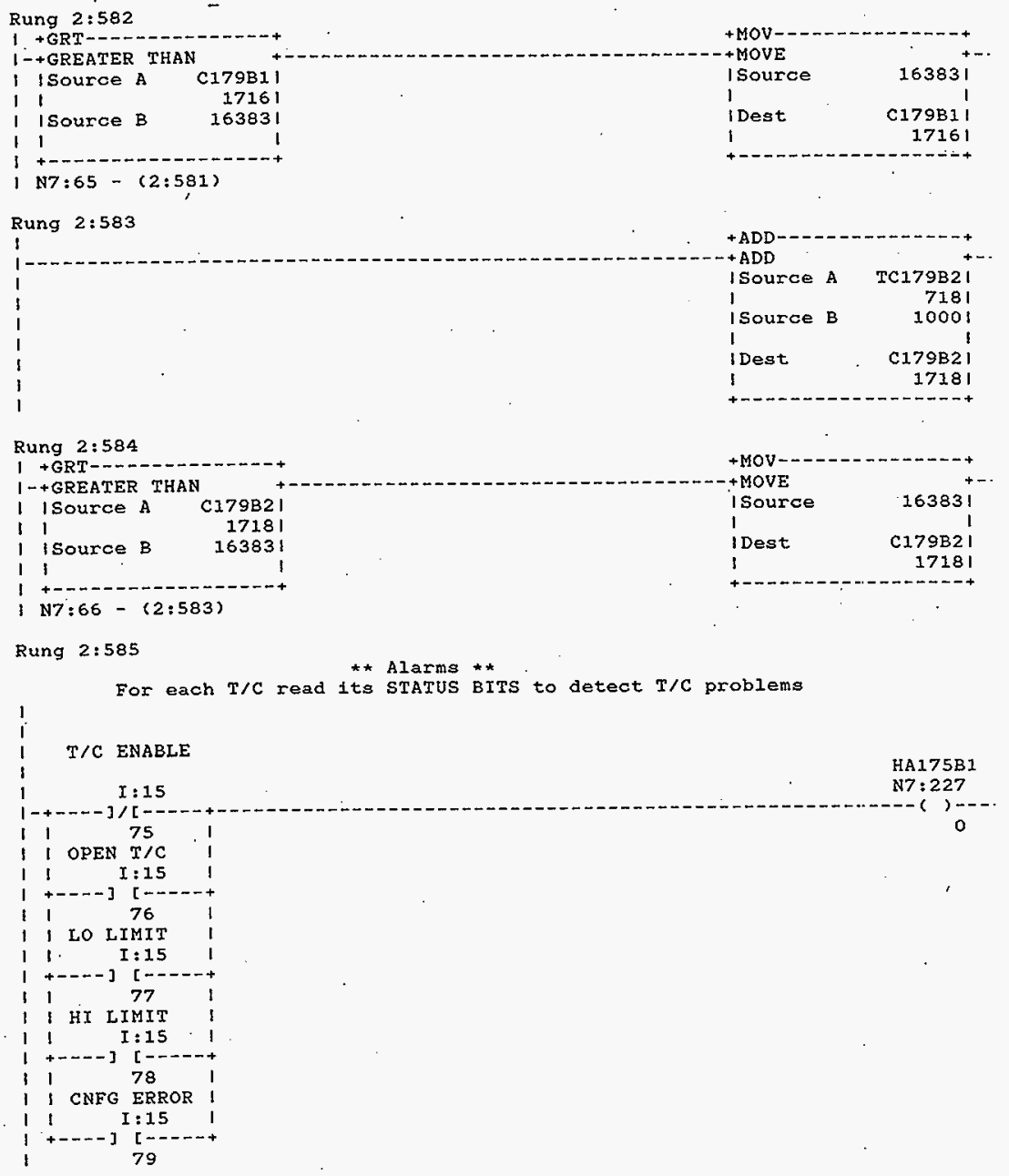

HNF-SD-FF-CSWD-61 Rev. 0 
Processor and Data(OPS Unit I)

Program Listing

Rung 2:586

1.

\section{T/C ENABLE}

\section{$I: 15$}

$1-+\cdots+\cdots /[-$

191

HA175B2

I OPEN T/C 1

I I:15 /

nan [n-n-n

I

I Lo

92

I:15

+----] [-----t

193

1 HI IIMIT

1

$I: 15$

+----] [---n+

194

I CNFG ERROR ।

1

$I: 15$

+---7 [

95

Rung 2:587

I

$T / C$ ENABLE

$I: 15$

HA175VI

N7: 227

1107

OPEN $I / C$

$1 \quad x: 15$

$+-\cdots-7[---2$

$1 \quad 108$

I LO LIMIT

I

1 HI LIMIT

I $\quad I: 15$

+----] [----.+

1110

I CNFG ERROR

1

I

I: 15

$+----3[-\infty, \ldots+$

111

HNF-SD-FF-CSWD-61 Rev. 0

Page 606 
Processor and Data(OPS Unit 1)

Program Listing

Processor File: SODIUM2A, ACH

October 23, 1996

Rung $2: 588$

T/C ENABLE

\section{$I: 15$}

]/ [- -

?

OPEN T/C . 1

1 I:IS

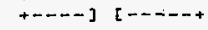

1 . 124

1 LO LIMIT

1
$+\ldots+1$$[15$

I 125 ।

1 HI IIMIT

1 I:15

11

11

I 1

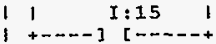

127

Rung 2:589

t

$T / C$ ENABLE

$I: 16$

$3 / \mathrm{f}$

1

OPEN $T / C$

i

1

I

1

I 77

1) HI LIMIT

$1 \quad I: 16$

+----] $[---\ldots+$

178

I CNFG ERROR

1

i

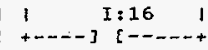

79
HA $175 \mathrm{~V} 2$

N7 : 227

3
HA 176B 1

N7: 227

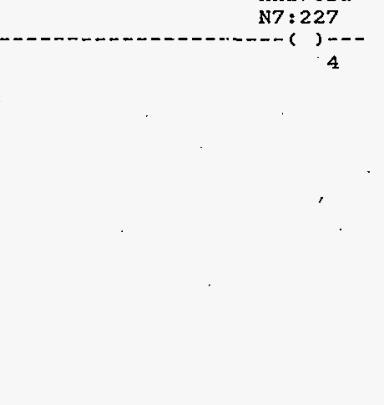

HNF-SD-FF-CSWD-61 Rev. 0

Page 607 


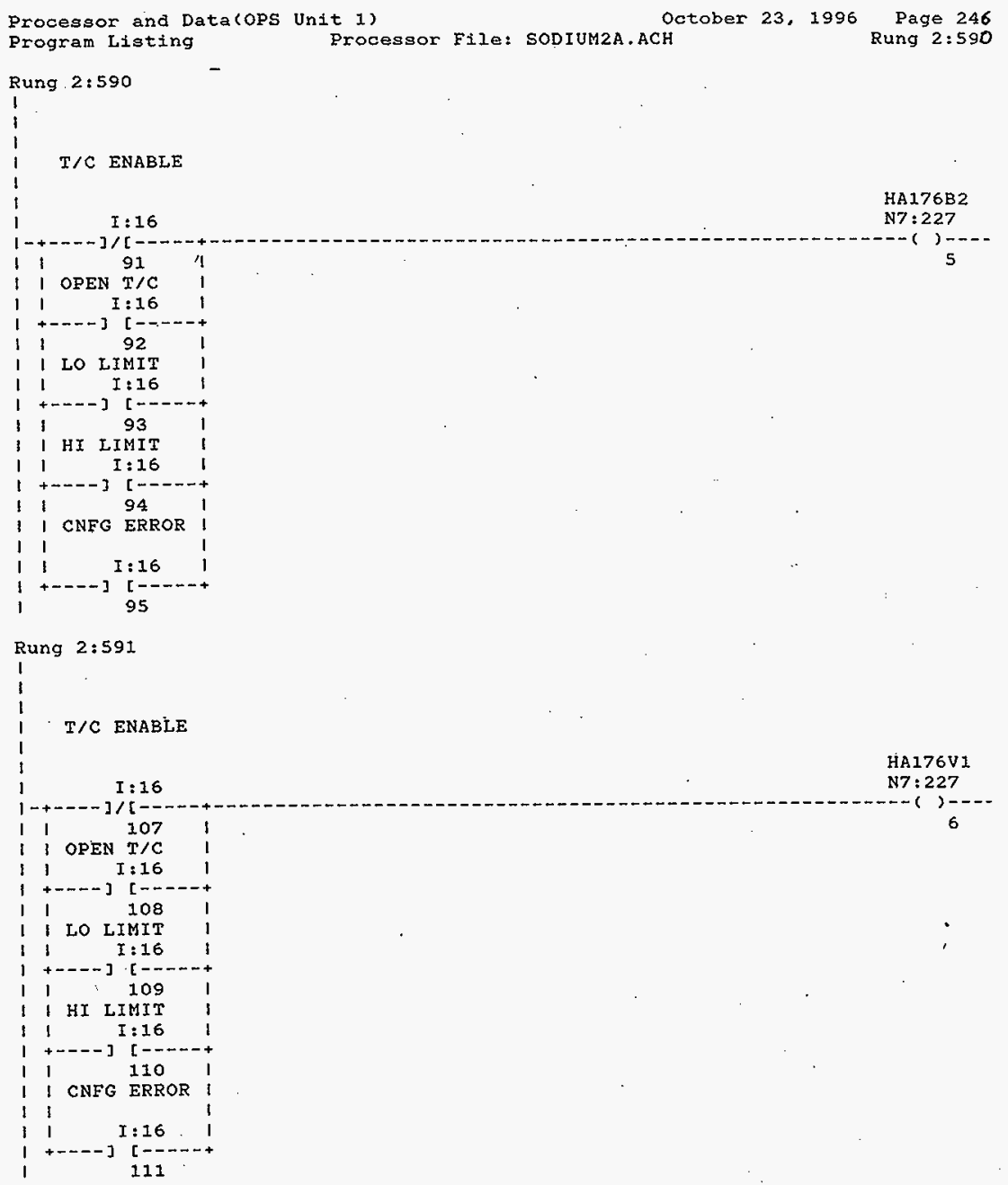

HNF-SD-FF-CSWD-61 Rev. 0

Page 608 
Processor and Data(OPS Unit 1 )

Program Listing

Rung 2:592

T/C ENABLE

HA176V 2

I : 16

N7:227

i 123 it

OPEN T/C

I I:16 i

+ - - - ] [ $[-\cdots+$

I $124 \quad$ I

1 LO LIMIT

I I:16 ।

+...- $]$ [-..-n.

I 125

I HI LIMIT

I I:16

+--n] $[-\cdots--+$

1126

I CNFG ERROR ।

l I:16. ।

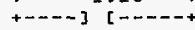

127

Rung $2: 593$

T/C ENABLE

I : 17

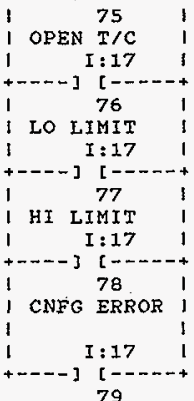

79

HNF-SD-FF-CSWD-61 Rev. 0

Page 60 
Processor and Data(OPS Unit I)

Program Listing

Rung 2:594

$$
\begin{aligned}
& 1 \\
& 1 \\
& 1 \\
& 1 \\
& 1 \\
& 1 \\
& 1 \\
& 1 \\
& 1 \\
& 1 \\
& 1 \\
& 1 \\
& 1 \\
& 1 \\
& 1 \\
& 1 \\
& 1 \\
& 1 \\
& 1 \\
& 1 \\
& 1 \\
& 1 \\
& 1 \\
& 1 \\
& 1 \\
& 1
\end{aligned}
$$

$T / C$ ENABLE

HA177B 2

N7: 227

$I: 17$

911

OPEN T/C i

$\begin{aligned} & 1 \\ & 1\end{aligned} \quad \mathrm{I}: 17 \quad 1$

192

1 LO LIMIT

$1 \quad I: 17$

1.-- ] $[--2-4$

193

I HI LIMIT

I:I7

+---- ] [-..--

I CNFG ERROR

1

I: 17 ।

95

Rung 2:595

i

T/C ENABLE

$I: 17$

HA177VI

N7: 227

$\begin{array}{ccc}1 & 107 \\ 1-+---] & 107\end{array}$

OPEN $T / C$

$\mathrm{I}: 17$

+.... $[-\ldots+4$

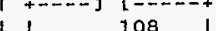

1 IO IIMIT

1

+-..- ] $[--.-.+$

1. 109

1 HI IIMIT

$I: 17$

$1+\ldots-+][-\ldots-n+$

I I CNFG ERROR ।

HNF-SD-FF-CSWD-6I Rev. 0

11

$1: 17 \quad 1$
$+---3][----+$

Page 610 
Processor and Data(ops Unit 1)

October 23, 1996

\section{Rung 2:596}

I

1

T/C ENABLE

I : 17

HA177V2

] $1-\cdots$

N7:227

11123

OPEN T/C

$I: 17$

+.-- $][--\cdots+$

1124

1 LO LIMIT

1 I:17

$1+\cdots+-\cdots][-\ldots-n+$

1125

I I HIGH LIMIT

1. 1

11

$+$

11

1

1

1

1

i

$\mathrm{I}: 17$

$\pi: 17$ ।

126

CNEG ERROR ।

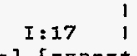

127

Rung 2:597

I

I

T/C ENABIE

I $\quad I: 18$

HA178B1

$1-+$

i

75

75

$1 \quad I: 18$

+- - ] [--..-.

176

LO LIMIT

$\begin{array}{lll}1 & & 1: 18 \quad 1\end{array}$

1

IT IMIT

I: 18

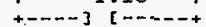

1

1

1

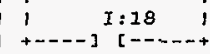

78

CNFG ERROR

79

HNF-SD-FF-CSWD-61 Kev. 0 
Processor and Data(OPS Unit 1 )

Program Listing

Rung 2:598

T/C ENABLE

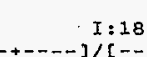

HA178B2

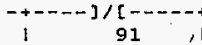

I OPEN $\mathrm{T} / \mathrm{C}$

I $I: 18$

+--- ] $[---.+$

192

1 LO LIMIT

I I:18

+---- ] [-n-

$193 \quad 1$

I HI LIMIT

I I:18

$+\cdots--][-\cdots+$

194

I CNFG ERROR ।

I 1

$1 \quad I: 18 \quad$ ।

$1+---][---++$
95

Rung 2:599

I

T/C ENABLE

$I: 18$

HA $178 \mathrm{~V} 1$

3

OPEN $\mathrm{T} / \mathrm{C}$

$I: 18$

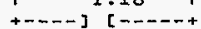

11081

1 LO LIMIT

$1 \quad I: 18$

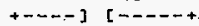

$1 \div 109$

I HI LIMIT

1 I: 18

$+-\infty-[--\cdots+$

J 110

I CNFG ERROR

$t$

I

$I: 18$

111

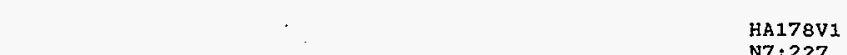

N7: 227

HNF-SD-FF-CSWD-61 Rev. 0

Page 

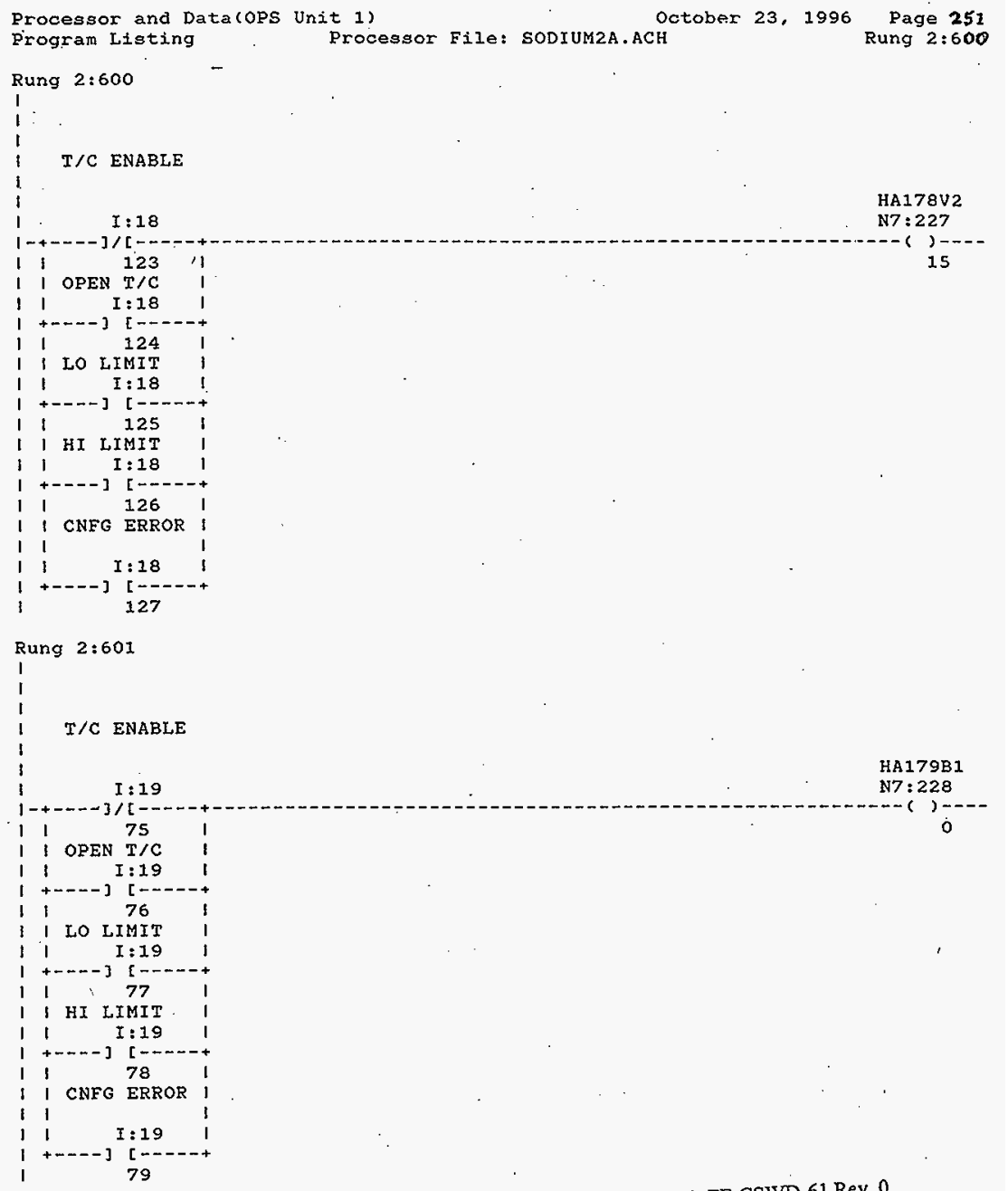

HNF-SD-FF-CSWD-61 Rev. 0

Page $6 / 3$ 
Processor and Data(OPS Unit 1 )

October 23,1996

Page 252

Program Listing

Processor File: SODIUM2A,ACH

Rung 2:602

1

1

T/C ENABLE

$1 \quad 1: 19$

HA $179 B 2$

N7: 228

1191 i

1 I OPEN T/C

1 I I:19

$1+---0][---n-+$

1192

1 LO LIMIT

11 I:19

$1+---][-\ldots-n+$

1193

1. H HIMIT

11 I:19

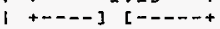

11941

I I CNFG ERROR |

11

11 I:19. 1

$1+\cdots--][-\ldots--\cdots$

195

Rung 2:603

I

N22:6

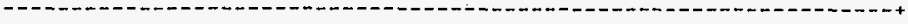

1

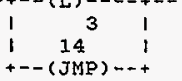

Rung $2: 604$

I DEV3

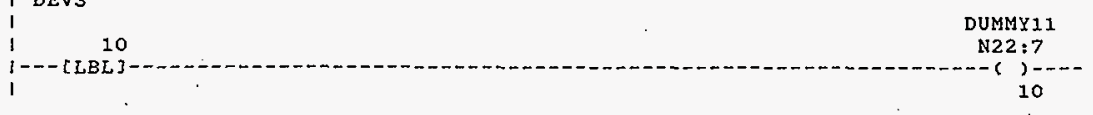

Rung 2:605

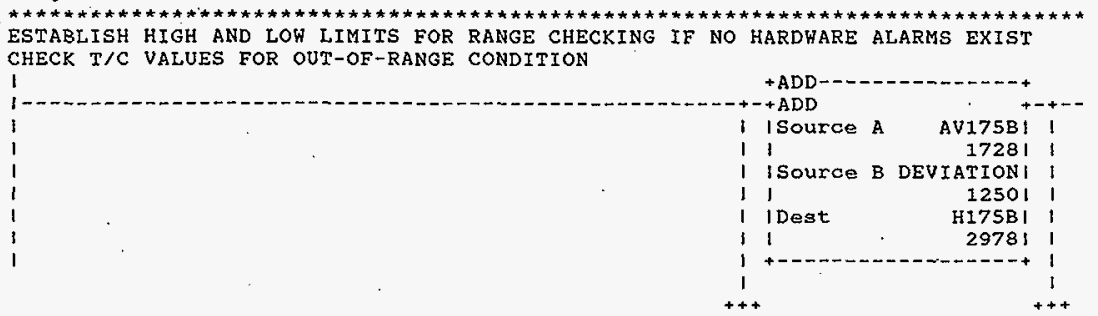

INF-SD-FF-CSWD-61 Rev. 0 
Processor and Data(oPs Unit 1)

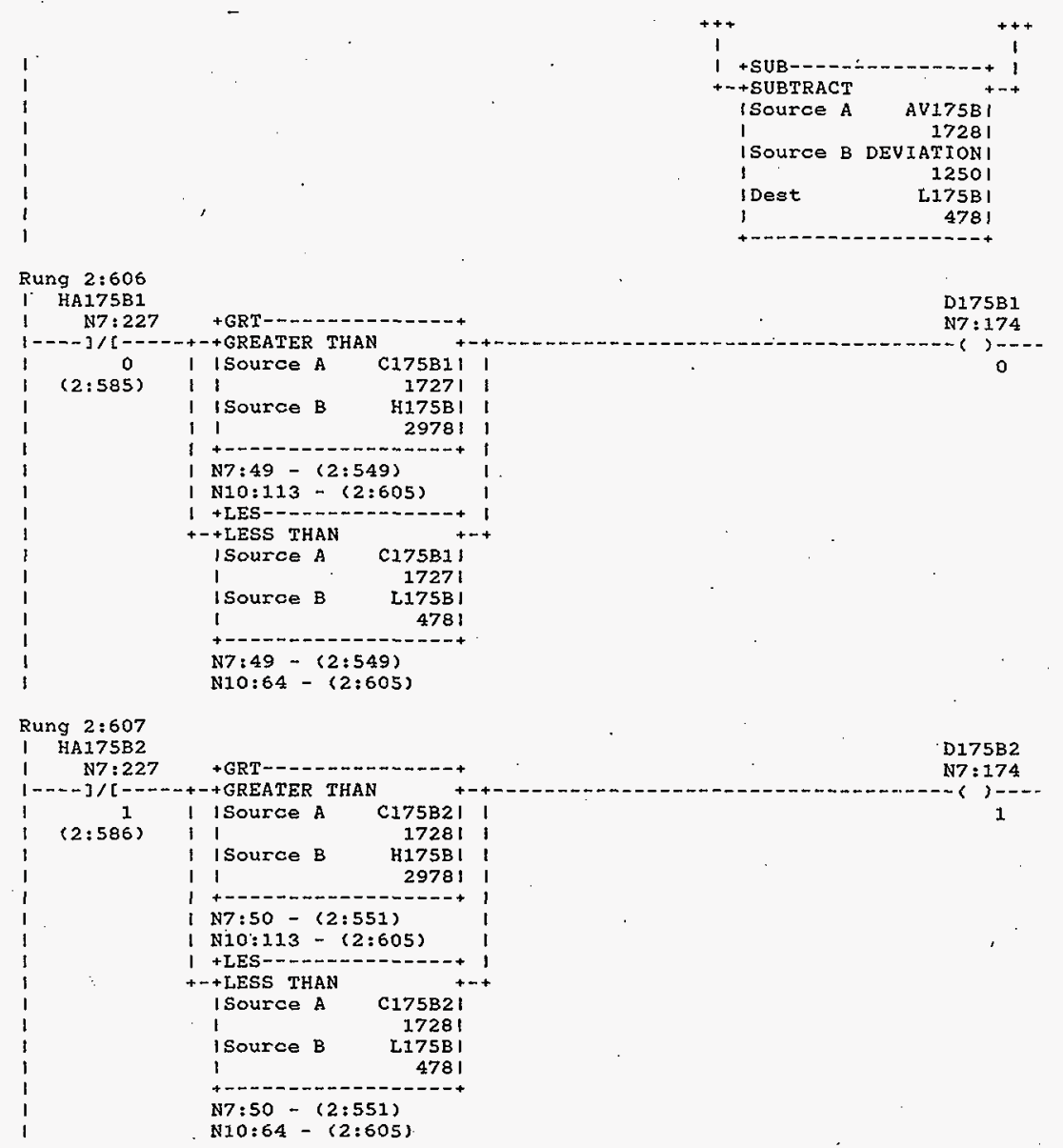

FNN-SD-FF-CSWD-61 Rev. 0 
Processor and Data(OPS Unit I)

Rung 2:608

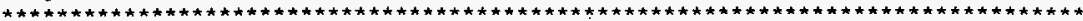
ESTABLISH HIGH AND LOW LIMITS FOR RANGE CHECKING IF NO HARDWARE ALARMS EXIST CHECK T/C VALUES EOR OUT-OF-RANGE CONDITION

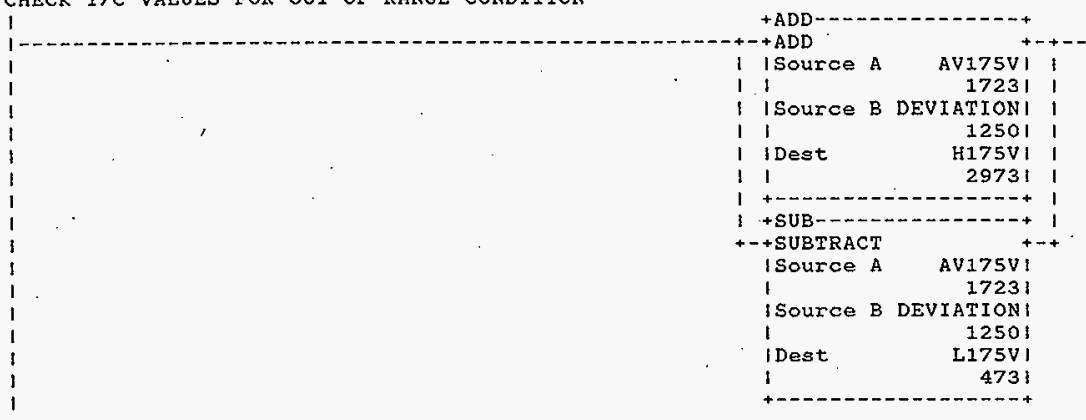

Rung $2: 609$

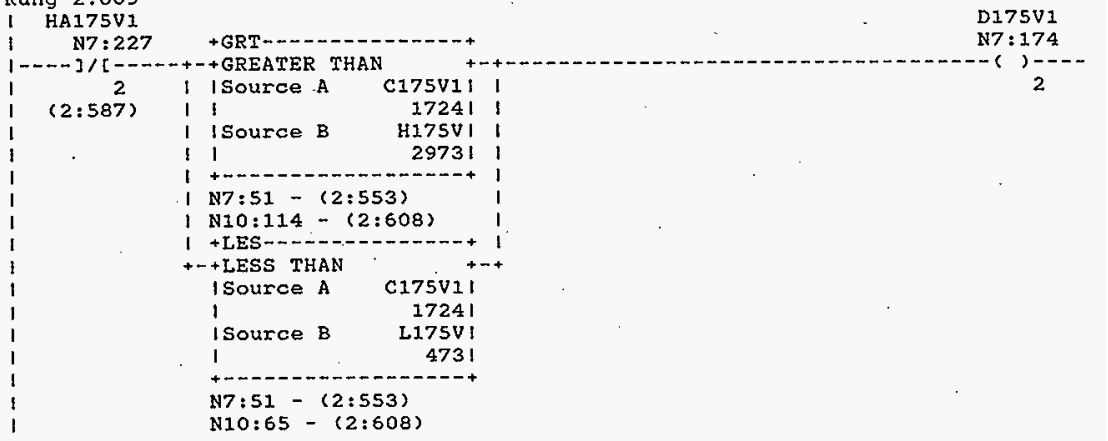

Rung 2:610

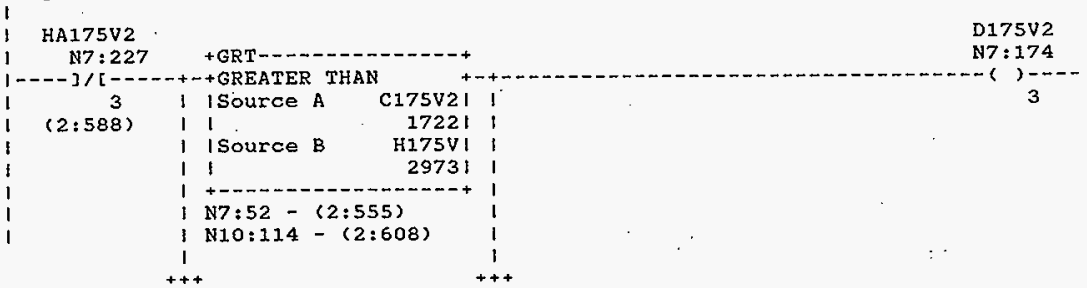

HNF-SD-FF-CSWD-61 Rev. 0 


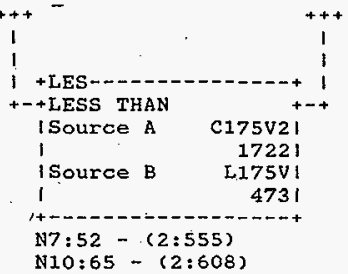

Rung $2: 611$

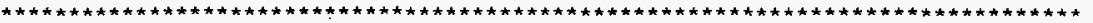
ESTABLISH HIGH AND LOW LIMITS FOR RANGE CHECKING IF NO HARDWARE ALARMS EXIST CHECK T/C VALUES FOR OUT-OF-RANGE CONDITION

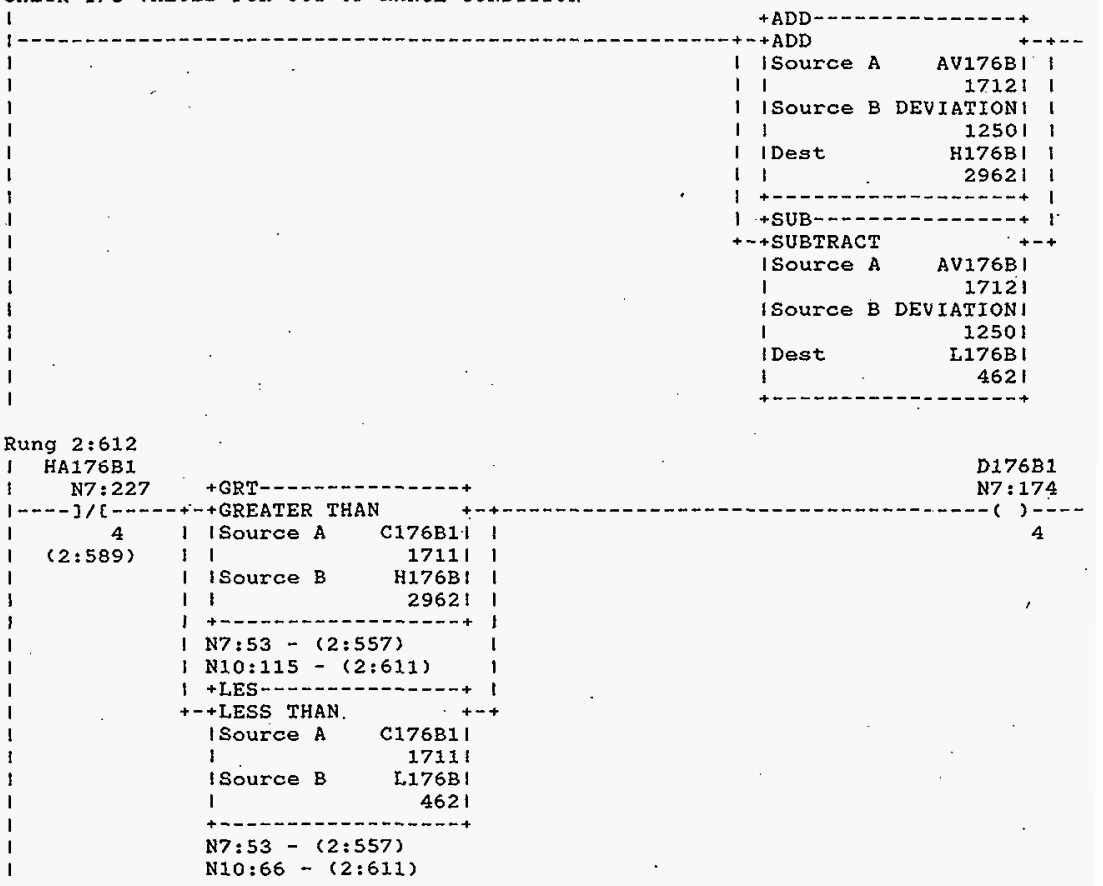

HNF-SD-FF-CSWD-6: Rev. 0 
Processor and Data(OPS Unit 1)

Program Listing

Processor File: SODIUM2A.ACH

Rung 2:613

$1 \mathrm{HA176 \textrm {B } 2}$

1. N7:227 $-$

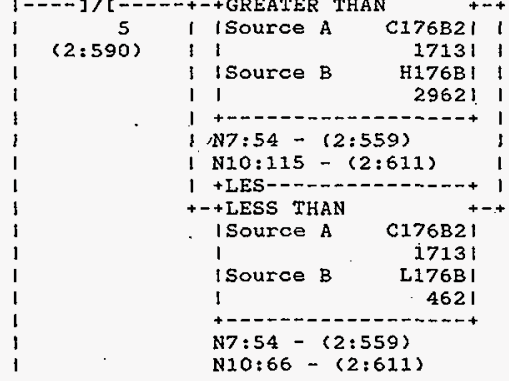

D176B2

N7 : 174

$3--\cdots$

Rung $2: 614$

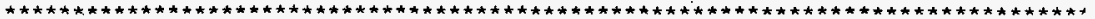
ESTABLISH HIGH AND LOH LIMITS FOR RANGE CHECKING IE NO HARDWARE ALARMS EXIST CHECK T/C VALUES FOR OUT-OF-RANGE CONDITION

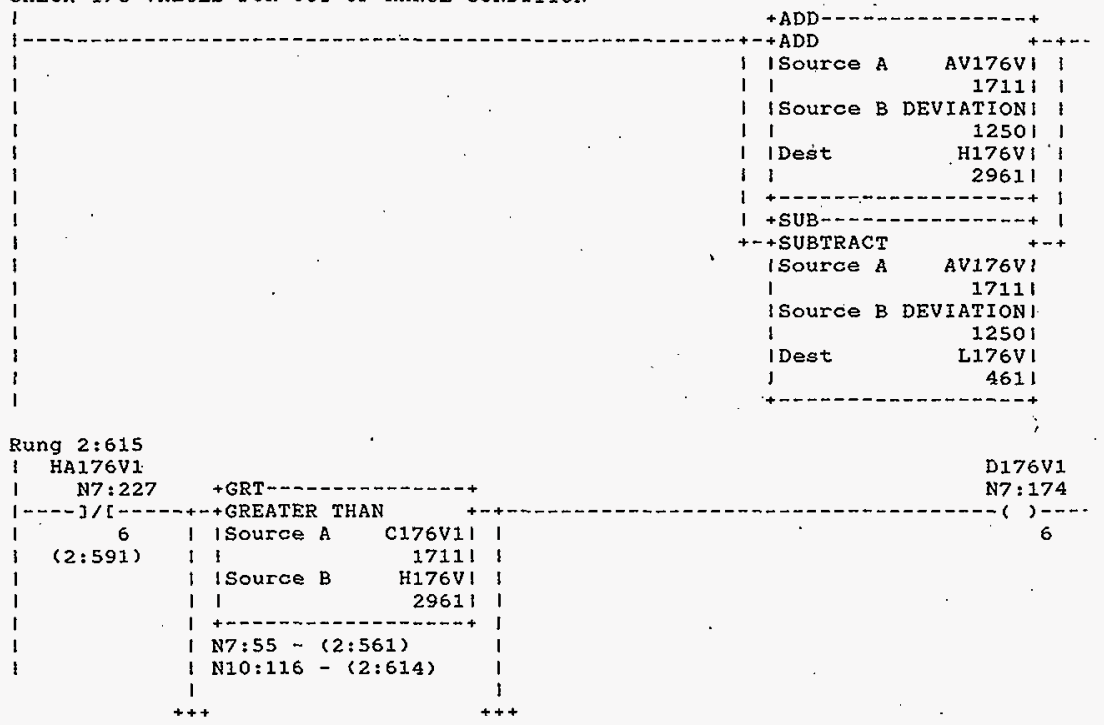

HNF-SD-FF-CSWD-61 Rev. 0 
Processor and Data(OPS Unit 1)

October 23,1996

Page 257

Program Listing

Processor File: SODIUM2A.ACH

Rung 2:615

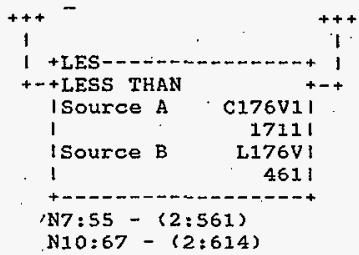

Rung $2: 616$

HA176V2

N7: 227

+GRT--n-- - - - - - - - +

D176V2

]

(2: 792 )

' isource A CI76V21 I

N7: $: 174$

11

17111

1 ISource B H176VI |

11 29611

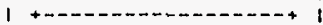

( N7:56-(2:563)

( N10:116 - (2:614)

1 +LES---.--.......-.

+-+ LESS THAN

isource A C176V2!

1 17111

ISource B L176V I

14611

N7:56-(2:563)

N10:67-(2:614)

Rung $2: 517$

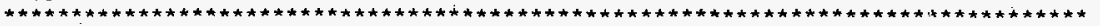
ESTABLISH HIGH AND LOW LIMITS FOR RANGE CHECKING IF NO HARDWARE ALARIS EXIST CHECK T/C VAZUES FOR OUT-OF-RANGE CONDITION

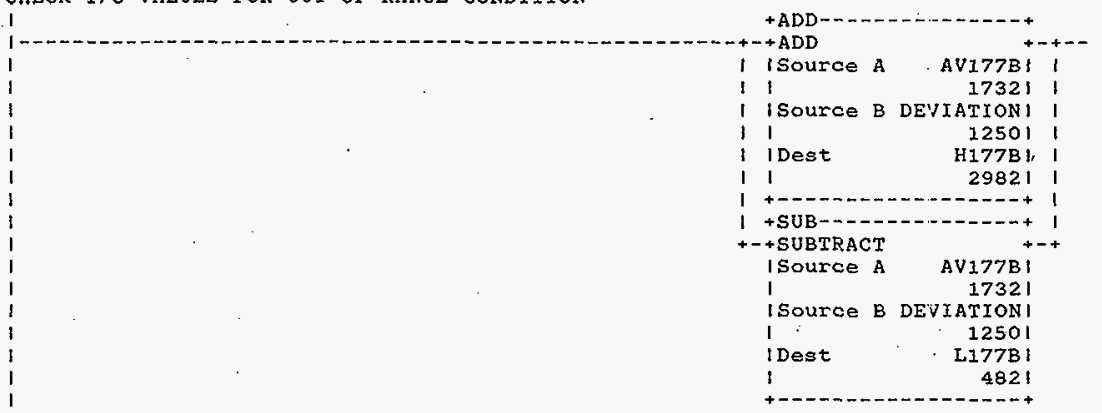

HNF-SD-FF-CSWD-61 Rev. 0

Page 619 
Processor and Data(OPS Unit 1)

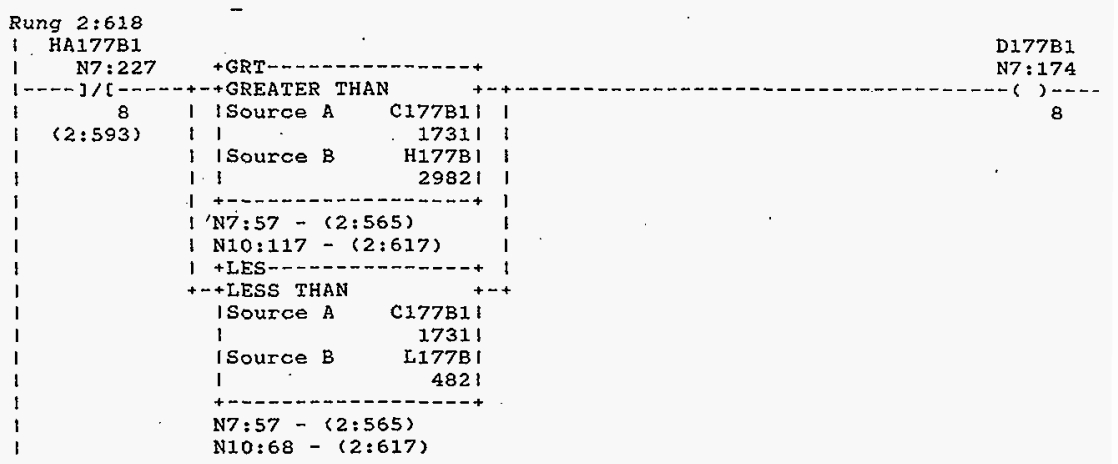

Rung 2:619

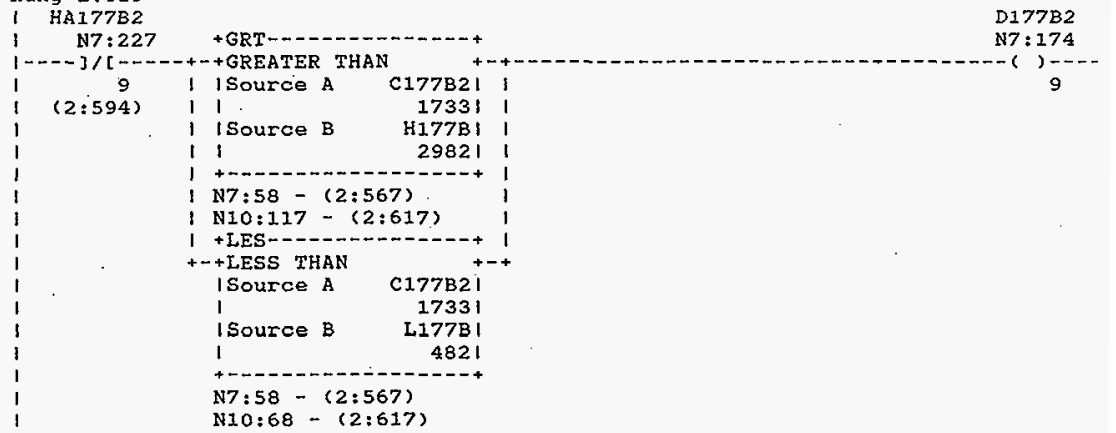

Rung $2: 620$

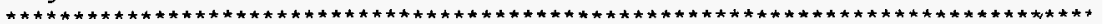
ESTABLISH HIGH AND LOW LIMITS FOR RANGE CHECKING IE NO HARDWARE ALARMS EXIST CHECK T/C VALUES FOR OUT-OF-RANGE CONDITION

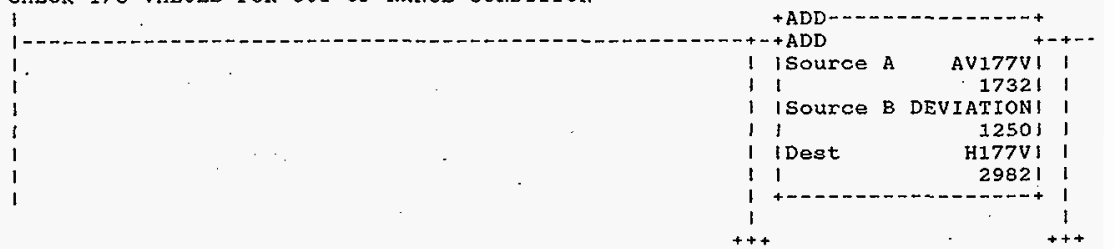

HNF-SD-FF-CSWD-61 Rev. 0 


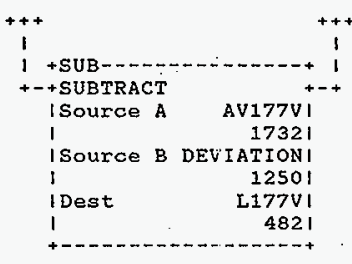

Rung $2: 621$

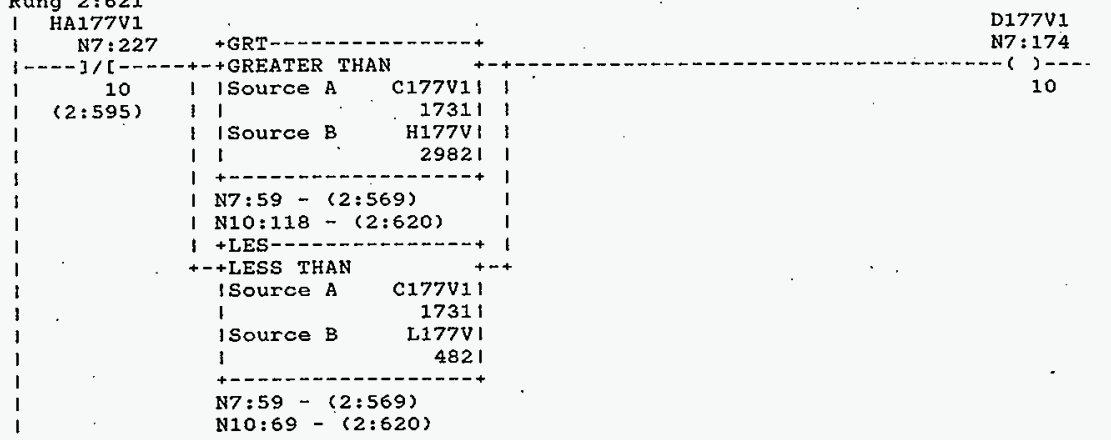

Rung 2:622

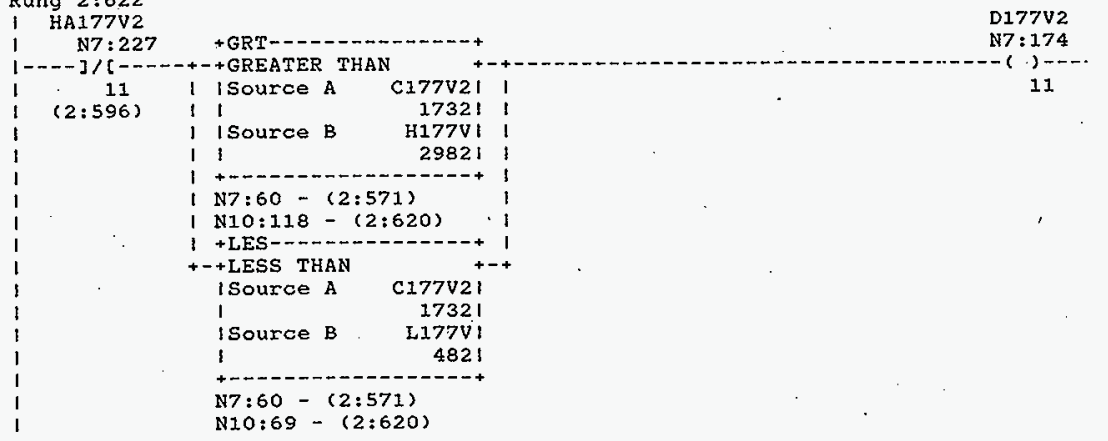

HNF-SD-FF-CSWD-61 Rev. 0 
Processor and Data(OPS Unit 1)

Rung 2:623

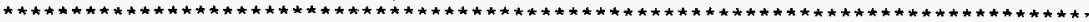

ESTABLISH HIGH AND LOW LIMITS FOR RANGE CHECKING IF NO HARDWARE ALARMS EXIST

CHECK T/C VALUES FOR OUT-OF-RANGE CONDITION

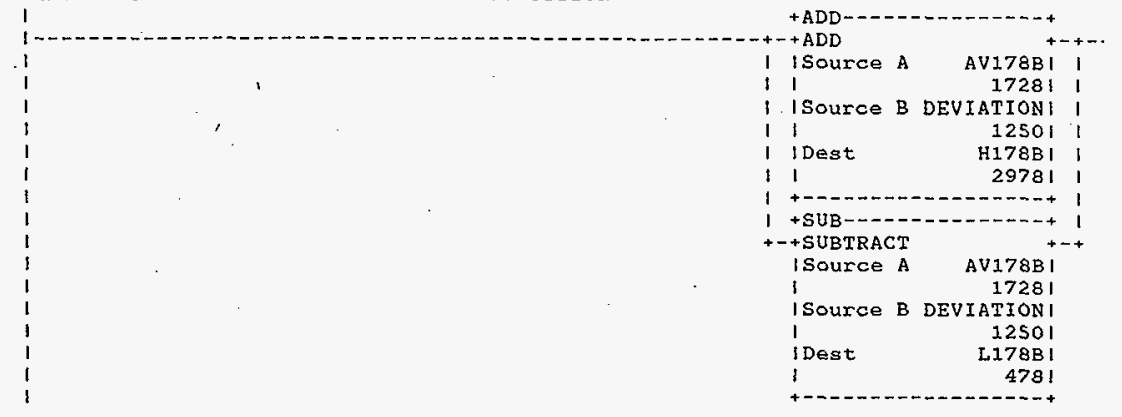

Rung $2: 624$

1 HA178B1

$1 \quad$ N7: 227

GRT- - -

DI78B1

$1--n] /[-2-+++$ GREATER THAN

$$
\text { (2.597) }
$$

I Isource A

C1781

11

I Source B

$78 \mathrm{~B} 11$

N7: 174

1. 1

H178B ।

29781

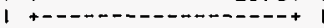

I N7:61-(2:573)

( N10:119-(2:623)

i +LES-..--......--.+ 1

+-+LESS THAN

I Source A

C178BIi

I

17271

isource B L178B |

$1 \quad 4781$

+..--

N7:61-(2:573)

N10:70-(2:623)

Rung 2:625

I HA17882

I $\quad$ N7:227

1--- $] /[---+-+$ GREATER THAN

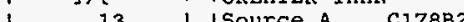

(2:598)

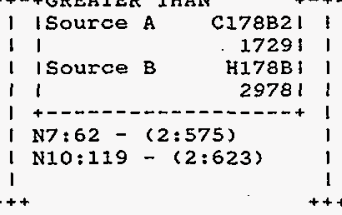

D178B2

N7 : 174

13

HNF-SD-FF-CSWD-61 Rev. 0

Page 622 
Processor and Data(OPS Unit 1)

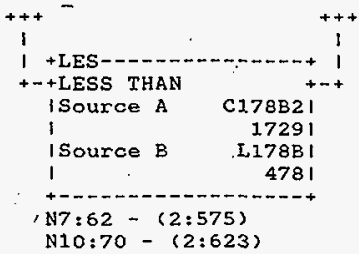

Rung 2:626

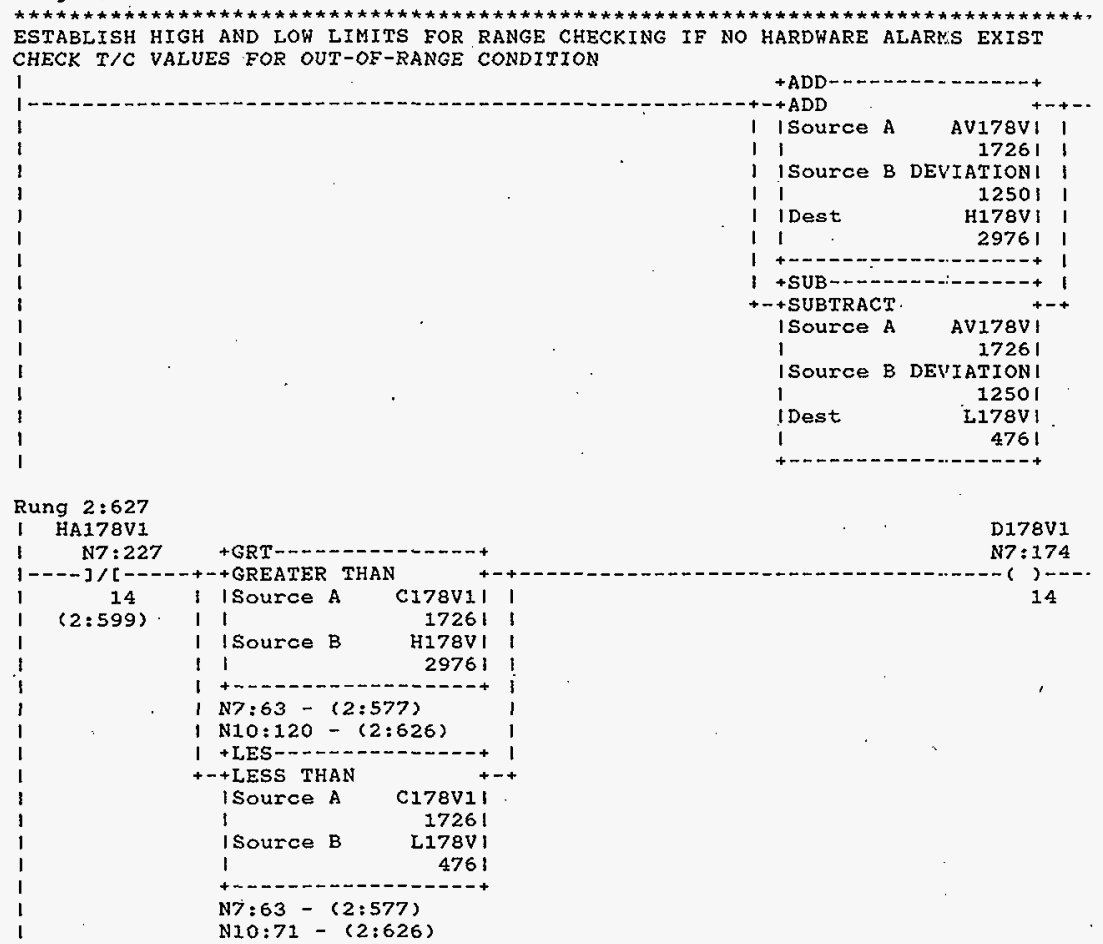

HNF-SD-FF-CSWD-61 Rev. 0

Page 623 
Processor and Data(OPS Unit 1)

Program Listing

Procegsor File: SODIUM2A.ACH

October 23, 1996 Page 262

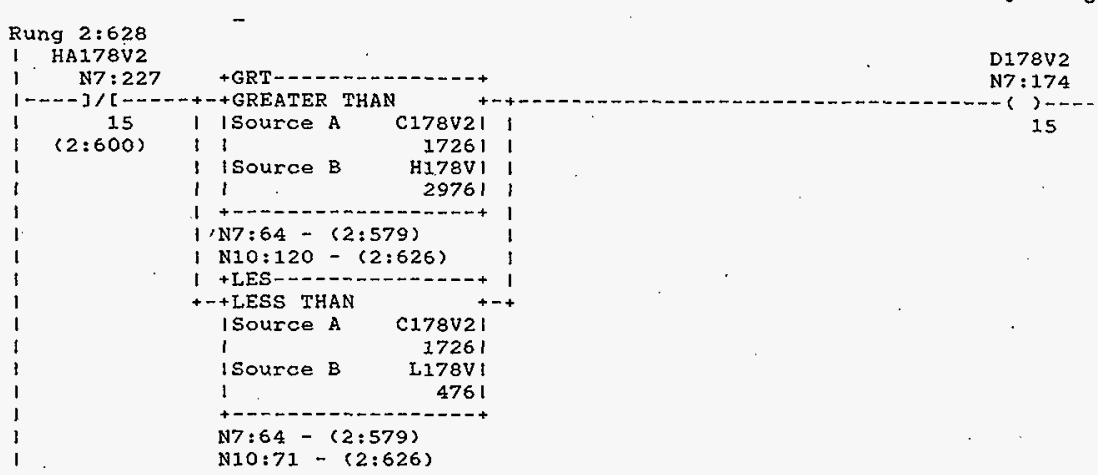

Rung 2:629

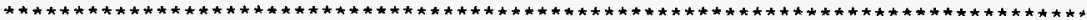
ESTABLISH HIGH AND LOH LIMITS FOR RANGE CHECKING IF NO HARDWARE ALARMS EXIST CHECK T/C VALUES FOR OUT-OF-RANGE CONDITION

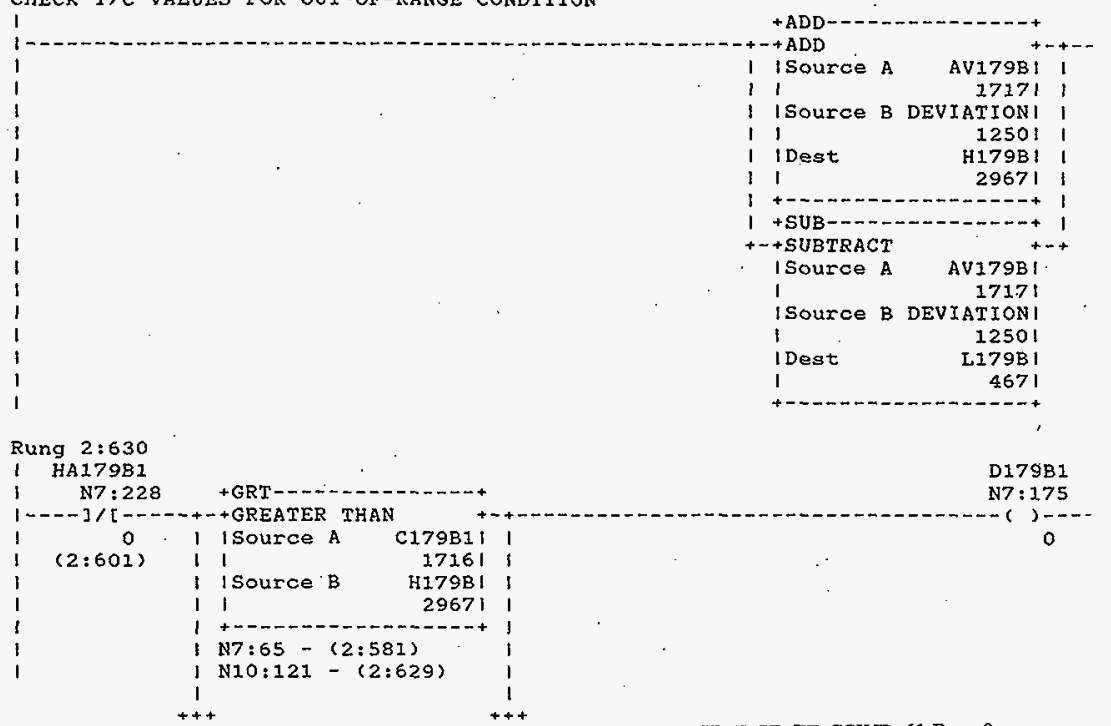

HNF-SD-FF-CSWD-61 Rev. 0

Page 624 
Processor and Data (OPS Unit 1)

October 23, 1996

Page 263

Program Listing

Processor File: SODIUM2A, ACH

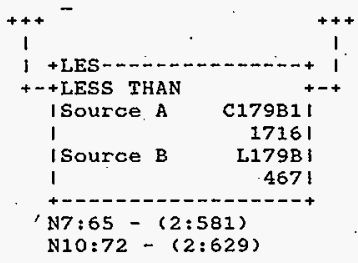

Rung $2: 631$

1 (HA 17982

i N7: 228

$1----] /[-\cdots$

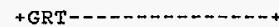

D179B2

1

+-+ GREATER THAN
I ISource A

$(2: 602)$

C17982! !

N7: 175

1 I 17181

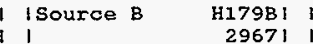

$1+\cdots-0-0-0-0-0-0+1$

( N7:66-(2:583)

( N10:121 - (2:629)

1 +LES-----_-_-

+-+LESS THAN

isource A C17982I

I 17181

| Source B L179B |

i

4671

N7:66-(2:583)

N10:72 $-(2: 629)$

Rung 2:632

$$
\text { i }
$$

DEV_3

N22:6

Rung 2:633

I

14

DUMMY 12

N22:7

1 - $[$ LBL $]$

N22:7

1

Rung 2:634

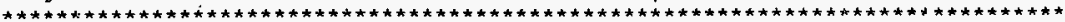
CLEAR COUNT AND TOTAL. IF NO HARDWARE, NO RANGE ALARMS AND NO THERMOCOOULES HAVE BEEN TAKEN OUT (TE_OUT $\times \times \times$ ) THEN AVERAGE THE T/C

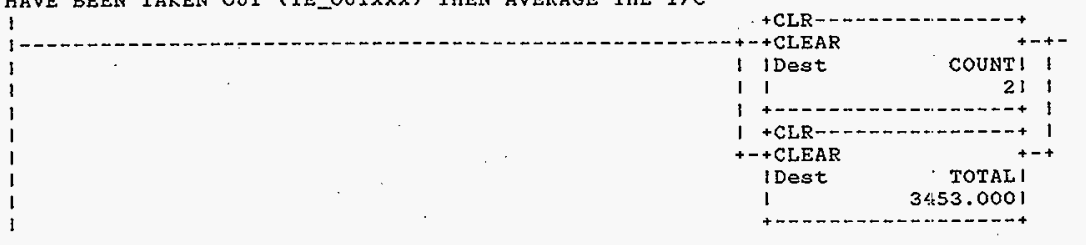

HNF-SD-FF-CSWD-61 Rev. 0 
Processor and Data(OPS Unit 1)

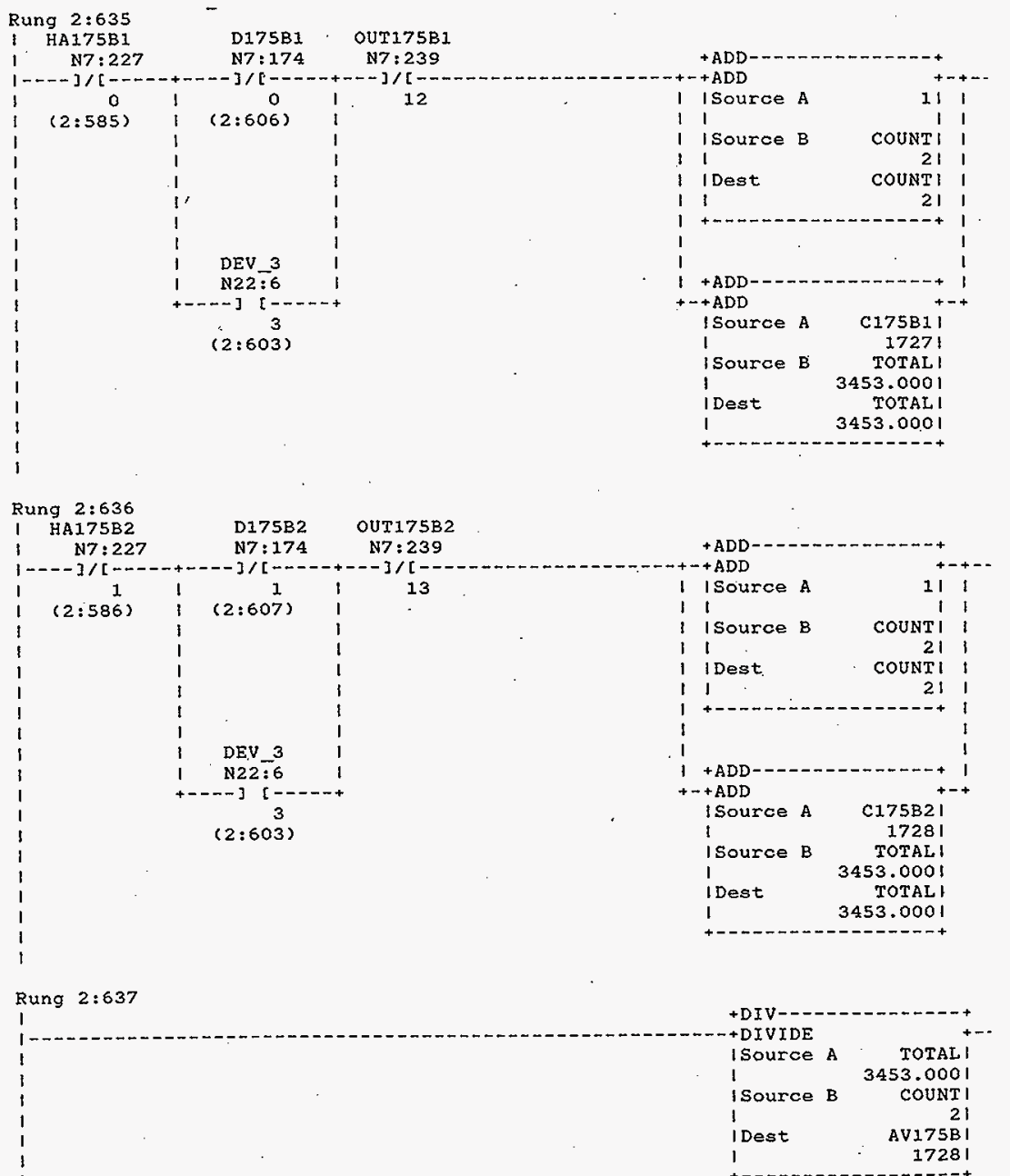

HNF-SD-FF-CSWD-61 Rev. 0 
Processor and Data(OPS Unit 1)

Rung $2: 638$

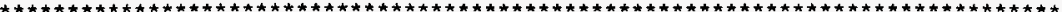
CLEAR COUNT AND TOTAL. IF NO HARDWARE, NO RANGE ALARMS AND NO THERMOCOUPLES HAVE BEEN TAKEN OUT (TE_OUT $\times \times X$ ) THEN AVERAGE THE T/C

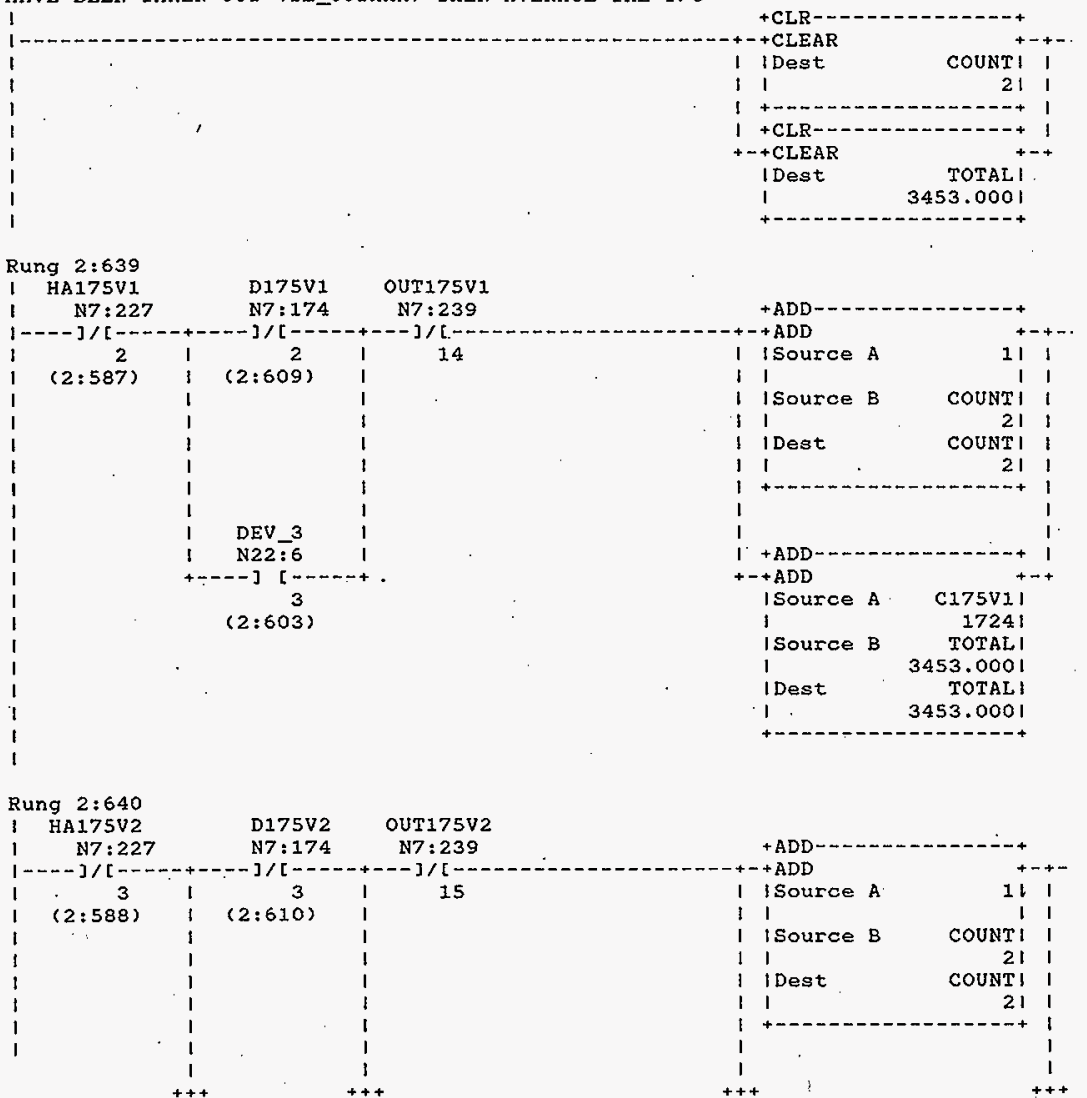

HNF-SD-FF-CSWD-61 Rev. 0 
Processor and Data(OPS Unit 1 )

October 23, 1996

Page 266 Program Iisting Processor File: SODIUM2A.ACH Rung $2: 640$
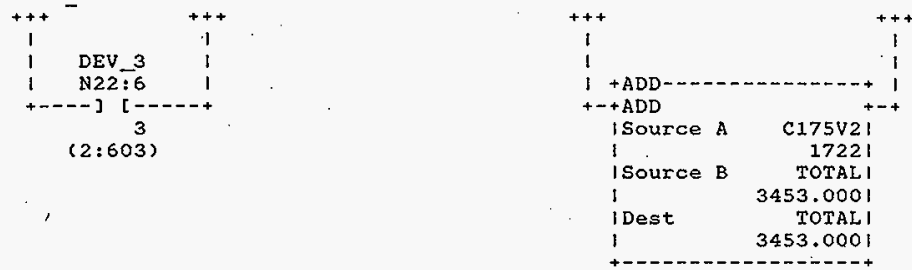

Rung $2: 641$

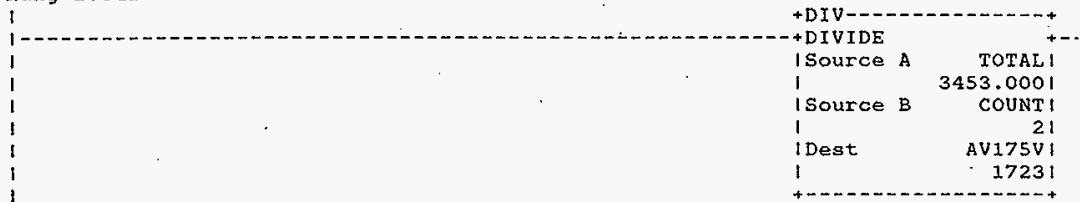

Rung $2: 642$

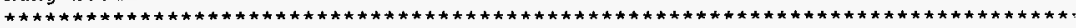
CLEAR COUNT AND TOTAL. IF NO HARDHARE, NO RANGE ALARMS AND NO THERMOCOUPLES HAVE BEEN TAKEN OUT (TE_OUT $\times X \times$ ) THEN AVERAGE THE T/C
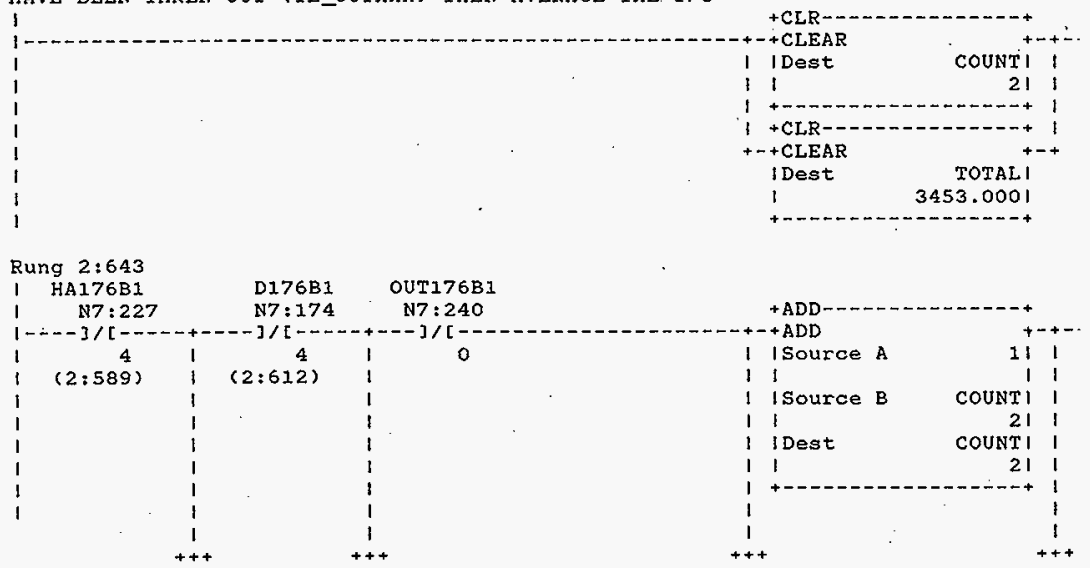

HNF-SD-FF-CSWD-61 Rev. 0

Page 628 
Processor and Data(ops Unit 1)

October 23, 1996

Page 267 Program Listing Processor File: SODIUM2A, ACH Rung $2: 643$

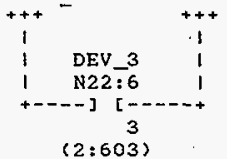

,

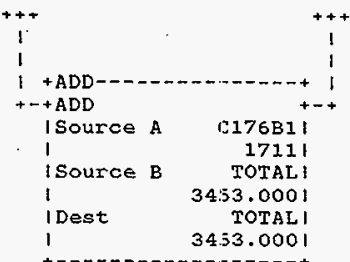

\section{Rung 2:644}

R $N 7: 227$

D176B2 OUT176B2 $N 7: 240$

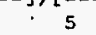

$D 176 B 2$
$N 7: 174$
$+----7 /[----+$

$-] /[-$

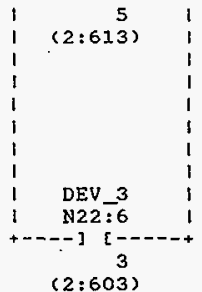

$+A D D$

1 isource A

1. 1

I I Source B

11

I I Dest

11

$1+$ 3.53.0001 
Processor and Data(ops Unit 1)

$$
\begin{aligned}
& 1 \\
& 1 \\
& 1 \\
& 1
\end{aligned}
$$
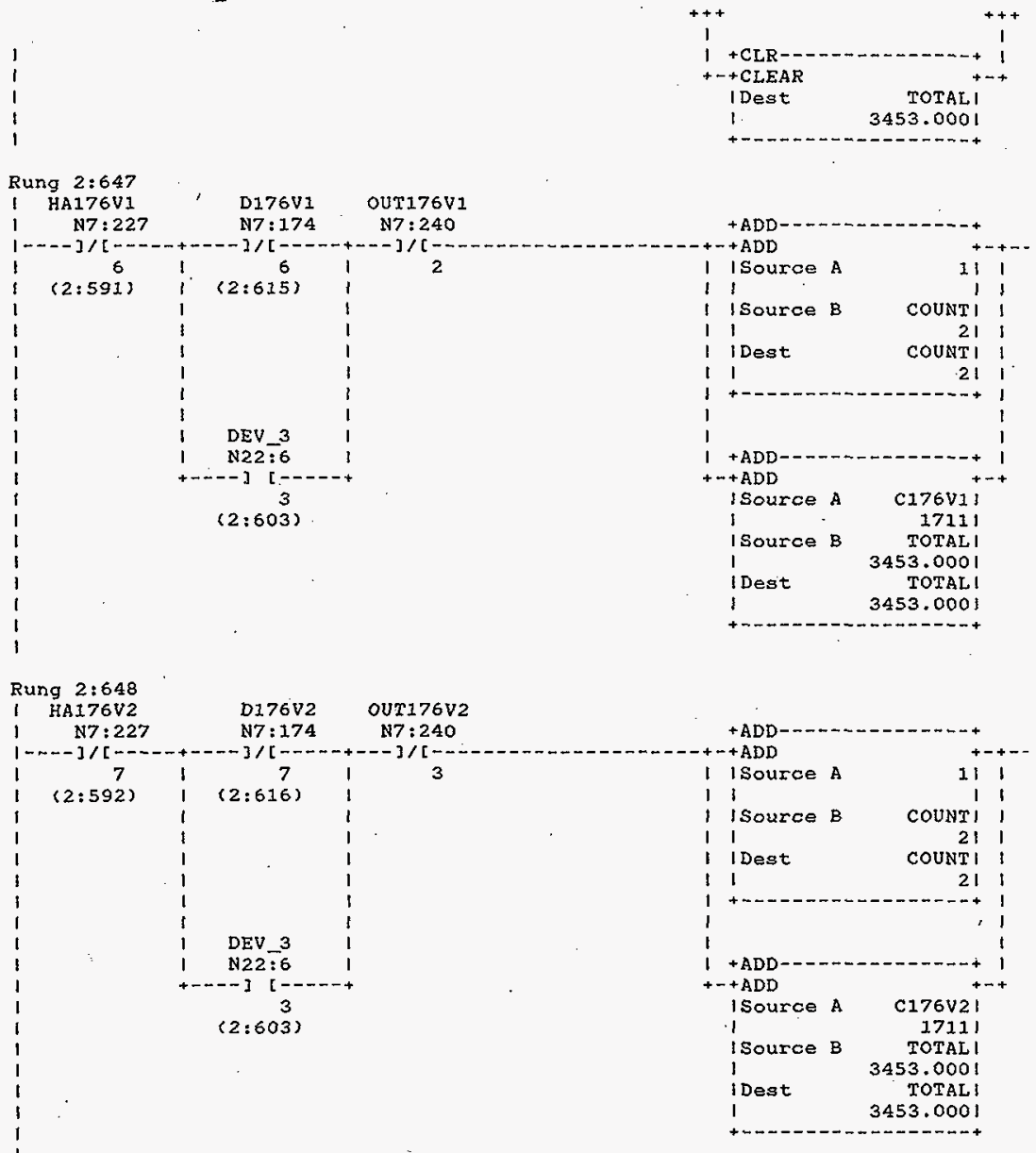

HNF-SD-FF-CSWD-61 Rev. 0 


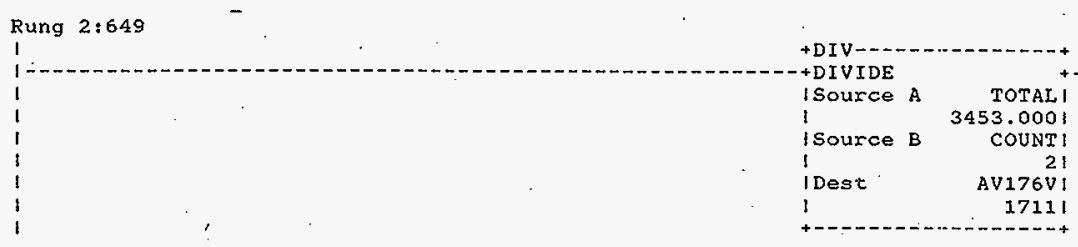

Rung 2:650

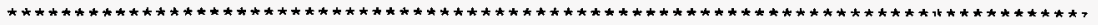
CEEAR COUNT AND TOTAL. IF NO HARDWARE, NO RANGE ALARMS AND NO THERMOCOUPLES HAVE BEEN TAKEN OUT (TE_OUTXXX) THEN AVERAGE THE T/C

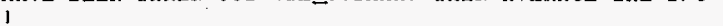

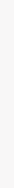$$
\begin{aligned}
& 1 \\
& 1 \\
& 1 \\
& 1 \\
& 1 \\
& 1 \\
& 1 \\
& 1 \\
& 1
\end{aligned}
$$

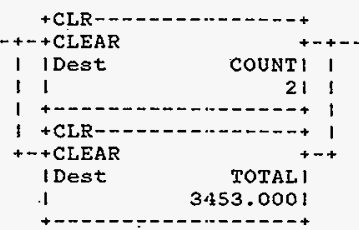

\section{Rung $2: 651$}

I HA177BI

N7:227

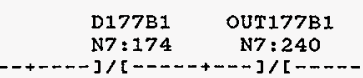

(2:593)

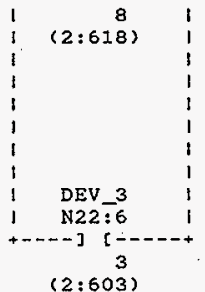

4 $+\mathrm{ADD}$

11

11

I IDest

11

Source A

source B

i

i

$1+$

(2:603) 
Processor and Data(OPS Unit 1)

Program Listing
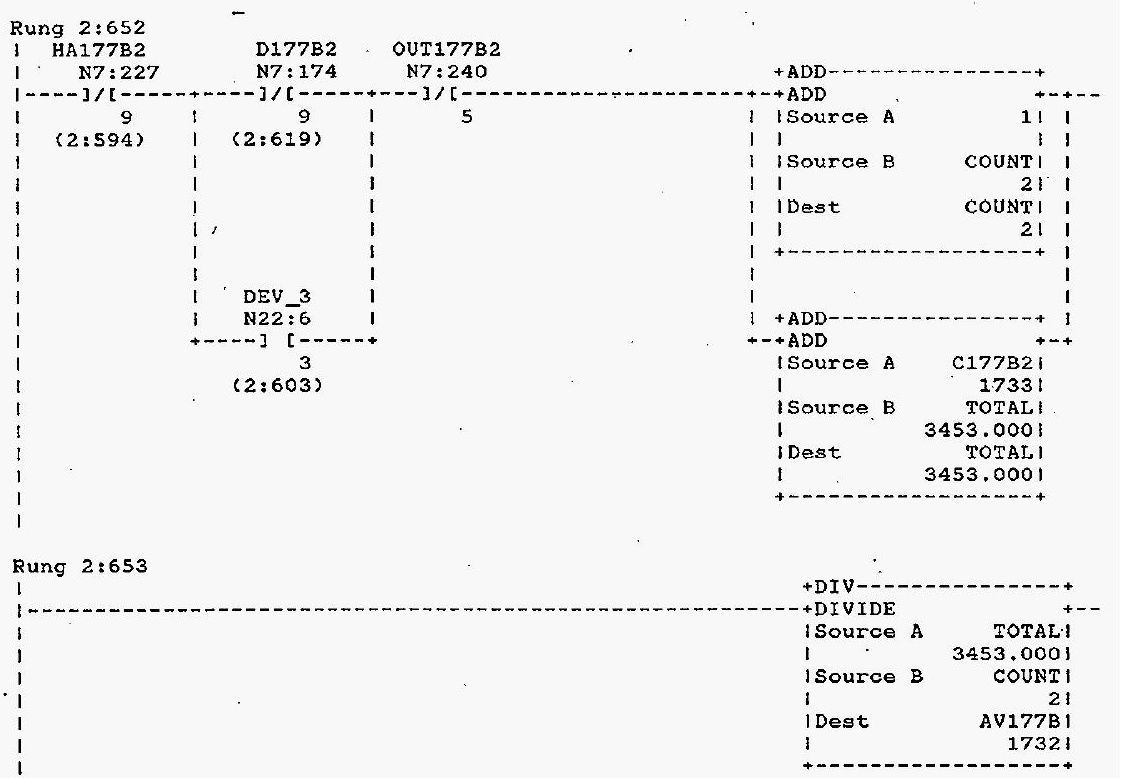

Rung $2: 654$

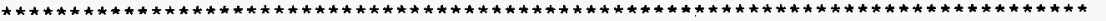
CLEAR COUNT AND TOTAL. IF NO HARDHARE, NO RANGE ALARMS AND NO THERMOCOUPLES HAVE BEEN TAKEN OUT (TE_OUT $\times \times \times$ ) THEN AVERAGE THE T/C

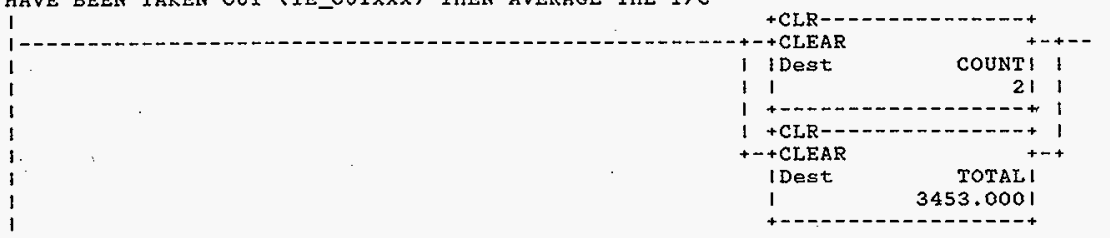

HNF-SD-FF-CSWD-61 Rev. 0

Page 632 
Processor and Datacops Unit 1 )
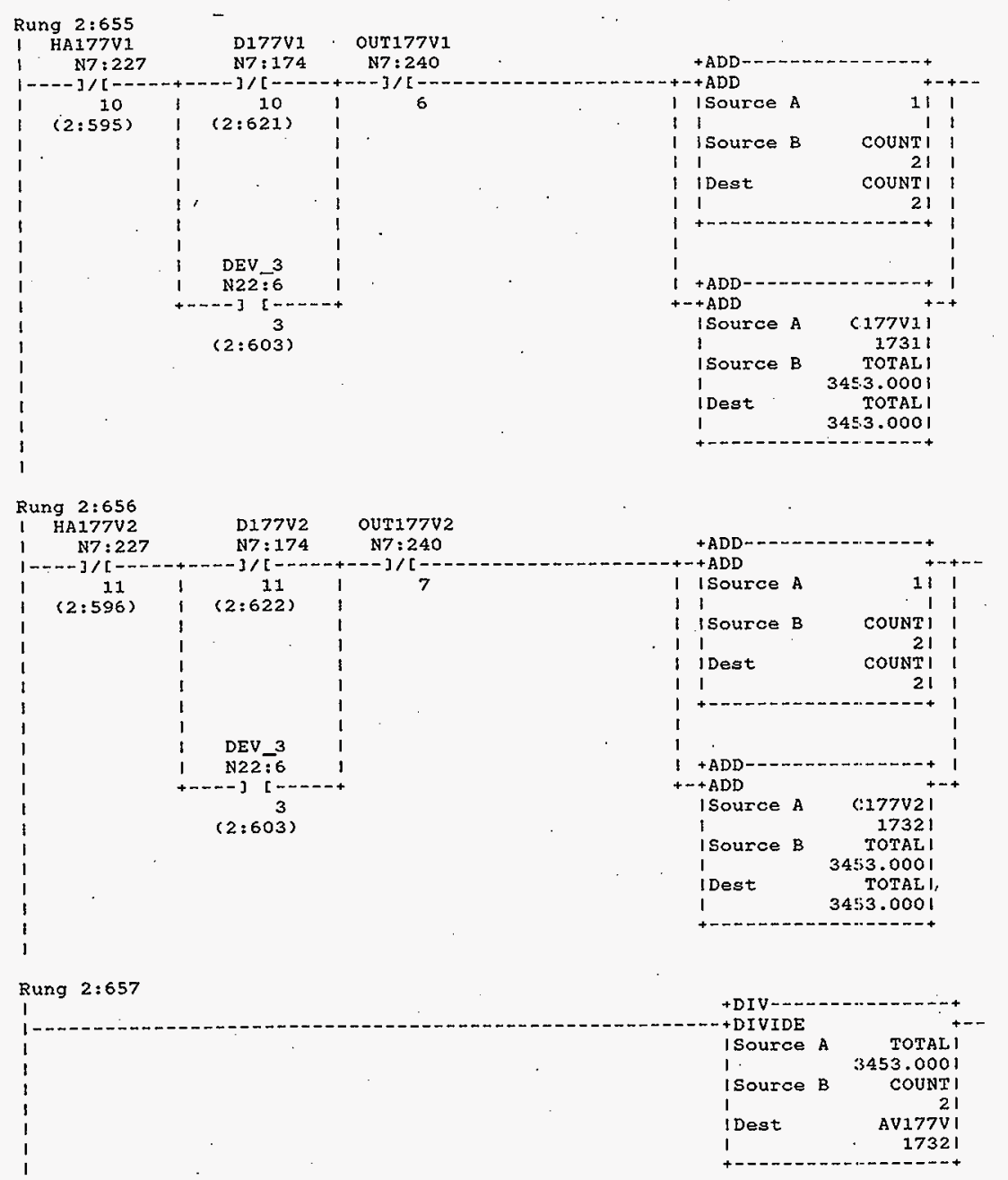

HNF-SD-FF-CSWD 61 Rะv. 0 
Processor and Data(OPS Unit 1)

\section{Rung $2: 658$}

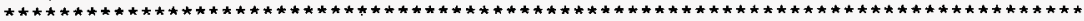
CLEAR COUNT AND TOTAL. IF NO HARDHARE, NO RANGE ALARMS AND NO THERMOCOUPLES HAVE BEEN TAKEN OUT (TE OUTXXX) THEN AVERAGE THE T/C$$
\text { . }
$$
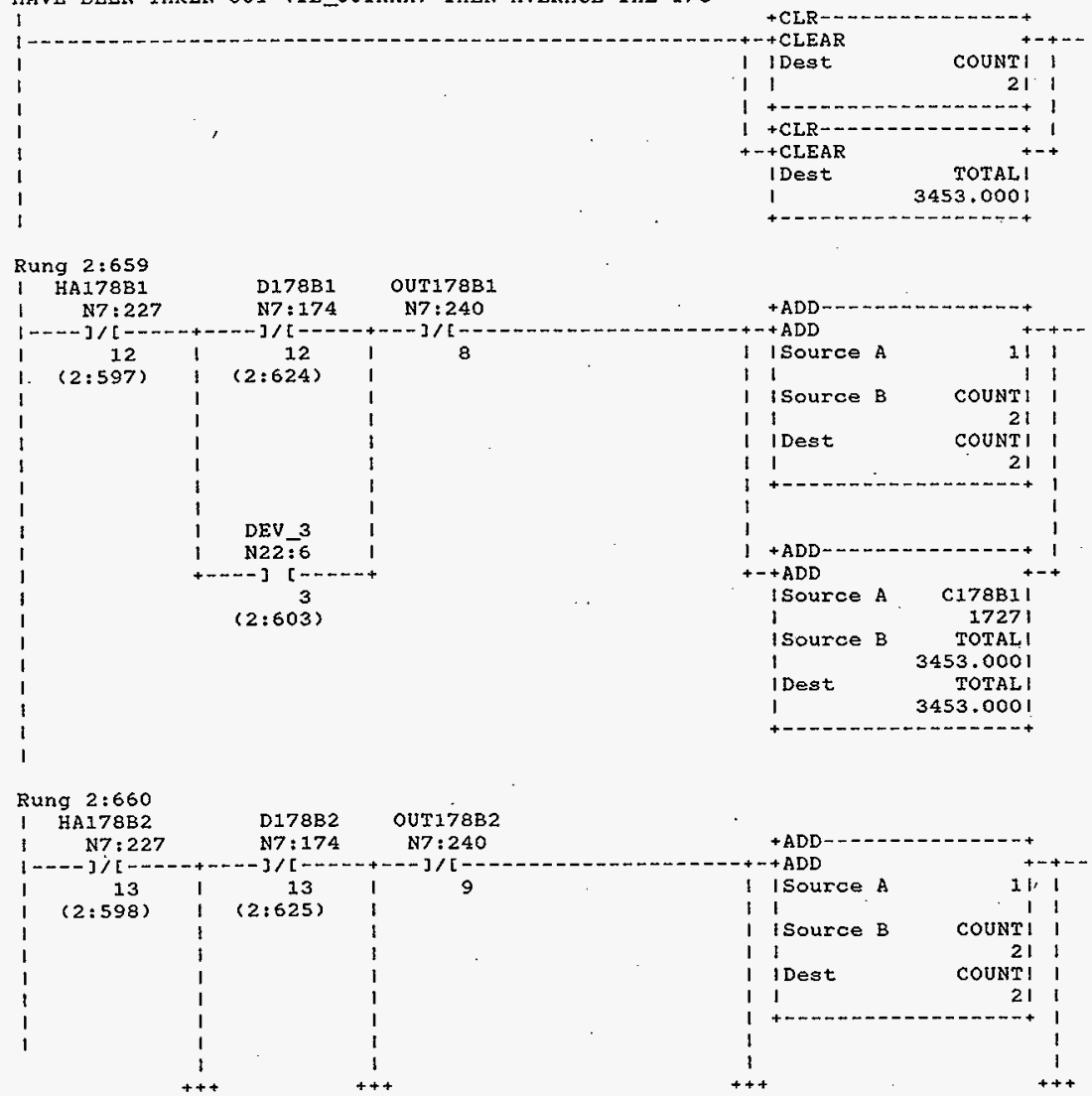

HNF-SD-FF-CSWD-61 Rev. 0 
Processor and Data(OPS Unit 1 )

October 23, 1996

Page 273

Program Listing

Processor File: SODIUM2A.ACH

Rung 2:660

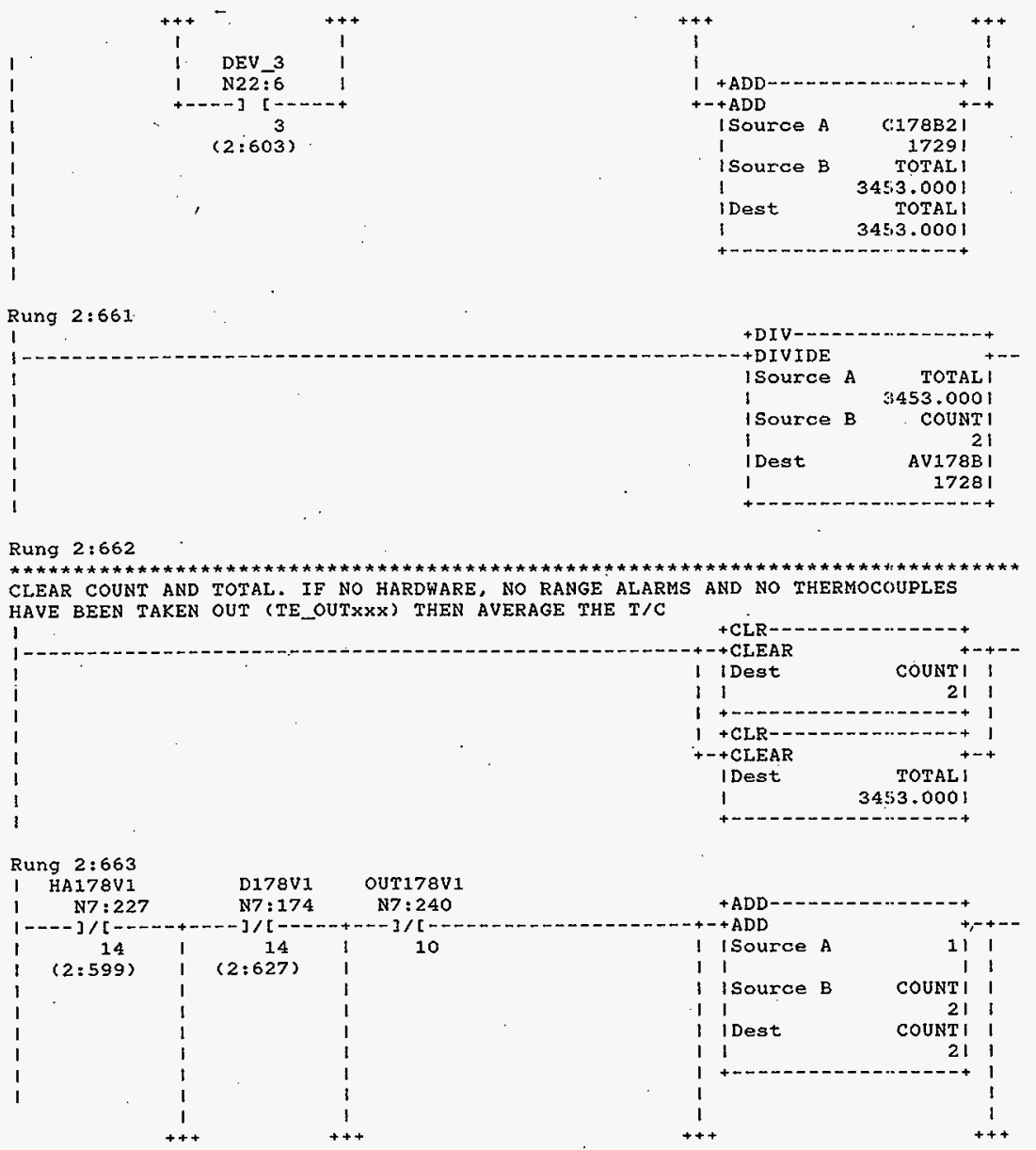

HNF-SD-FF-CSWD-61 Kev. 0

Page 635 
Processor and Data(OPS Unit 1 )

October 23, 1996 Page 274

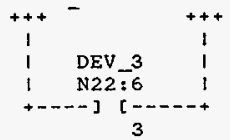

$(2: 603)$
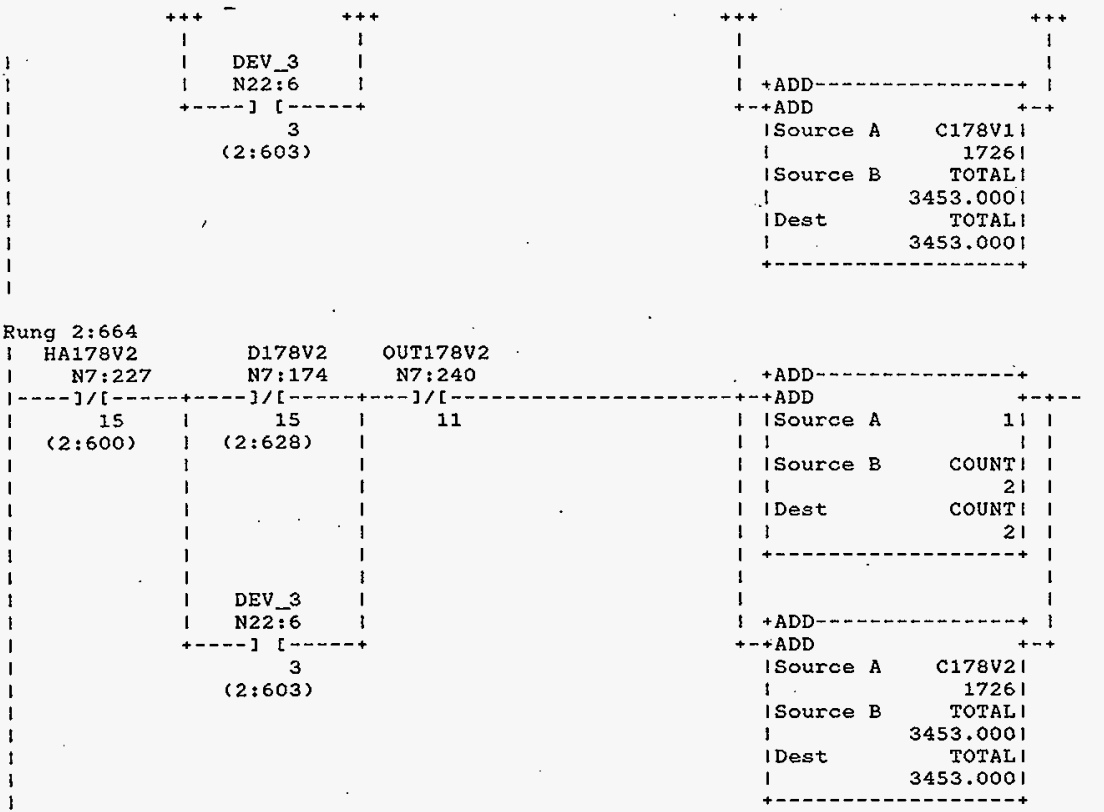

Rung 2:665

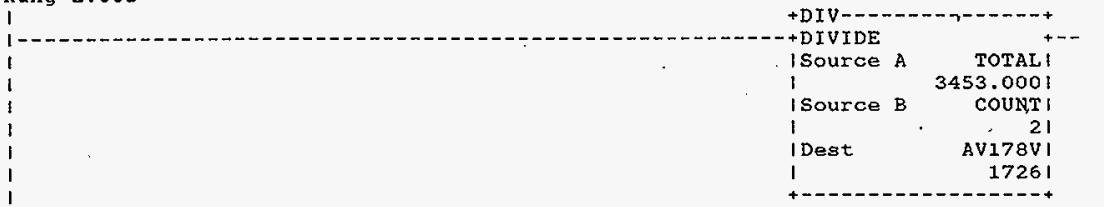

Rung 2:666

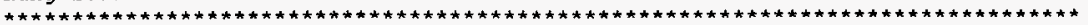
CLEAR COUNT AND TOTAL. IF NO HARDWARE, NO RANGE ALARMS AND NO THERMOCOUPLES HAVE BEEN TAKEN OUT (TE_OUTXXX) THEN AVERAGE THE T/C

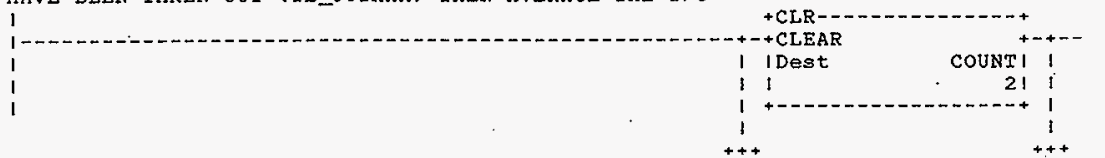

HNF-SD-FF-CSWD-61 Rev. 0 
Processor and Data(OPS Unit 1 )

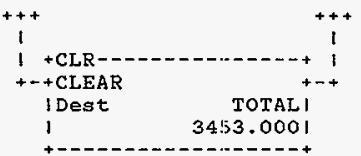

Rung $2: 667$

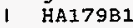

1 HAT79B1 $1---3 /[--2$

$\begin{array}{cc}1 & 0 \\ 1 & (2: 601)\end{array}$

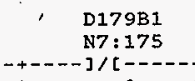

OUT179B1

N7: 240

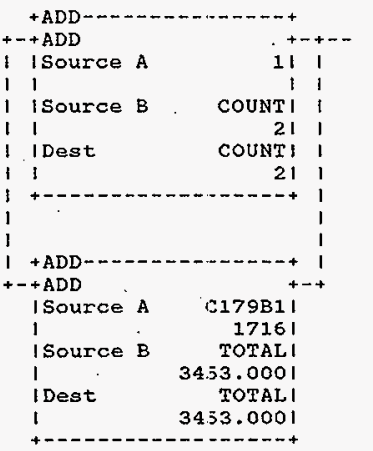

DEV 3

N22: 6

+.... [ 3

(2:603)

12

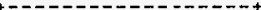

Rung 2:668

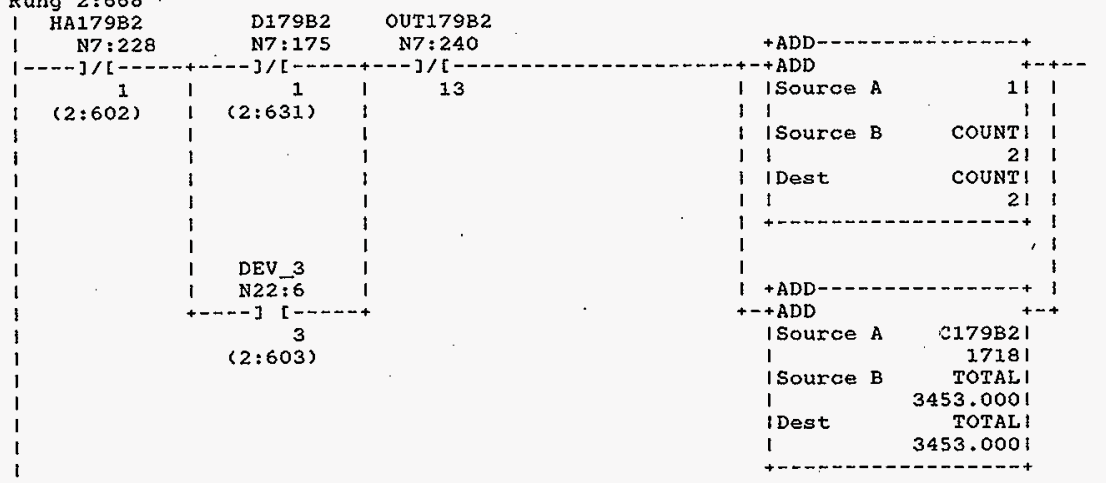

HNF-SD-FF-CSWD-61 Rev. 0 


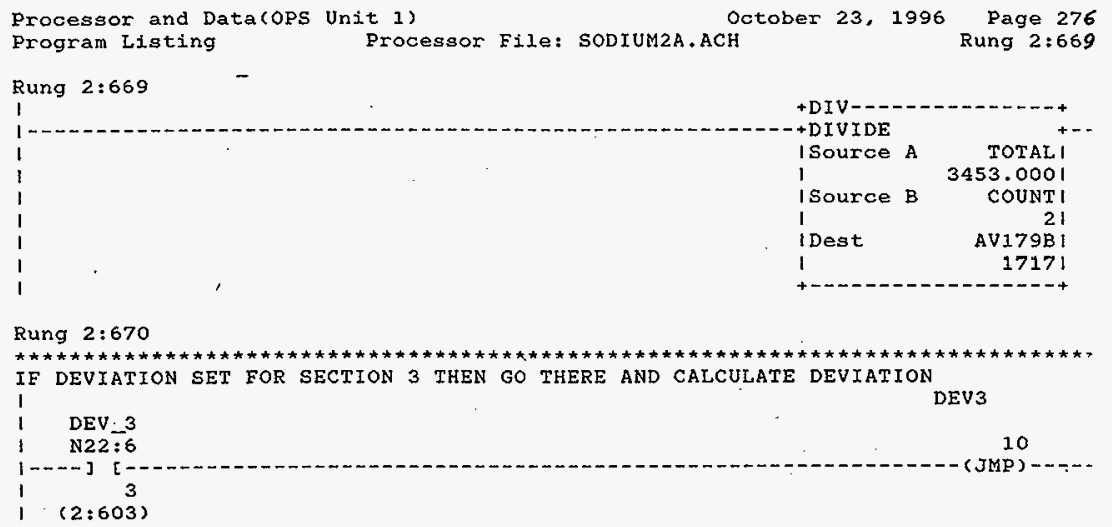

\section{Rung 2:671}

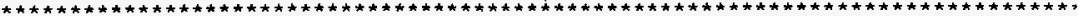
JUMPS TO SECTION 3 IF DN 3 IS NOT LATCHED DN_3 GETS LATCHED WHEN THE SECTION IS ENTERED

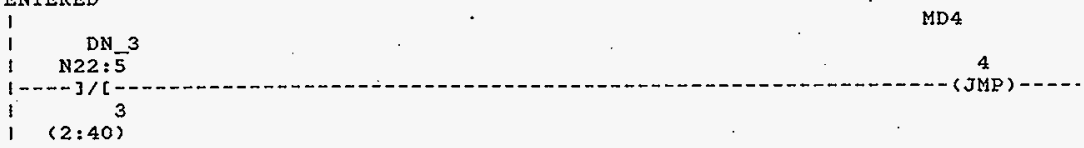

Rung $2: 672$

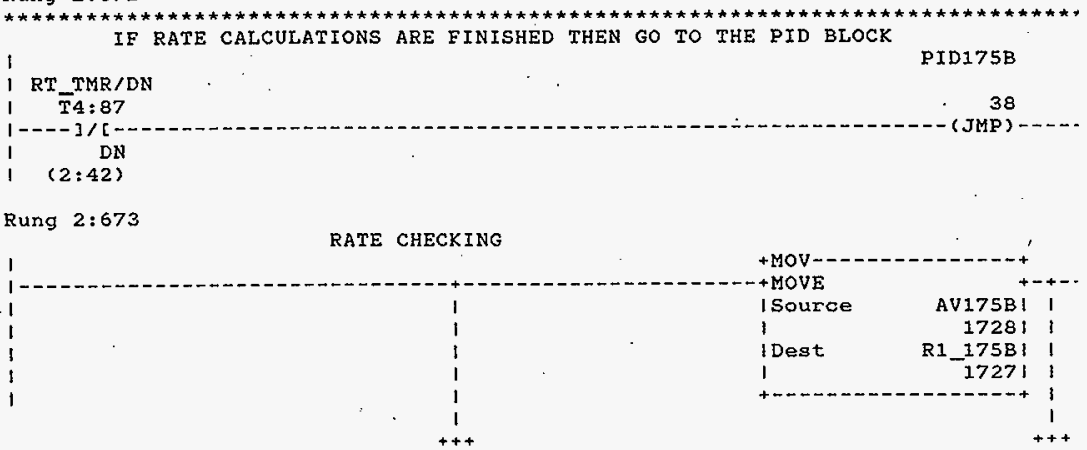

HNF-SD-FF-CSWD-61 Rev. 0

$$
\text { Page } 638
$$


Processor and Data (OPS Unit.1)

October 23,1996

Page 277

Program Listing

Processor File: SODIUM2A.ACH

Rung $2: 673$

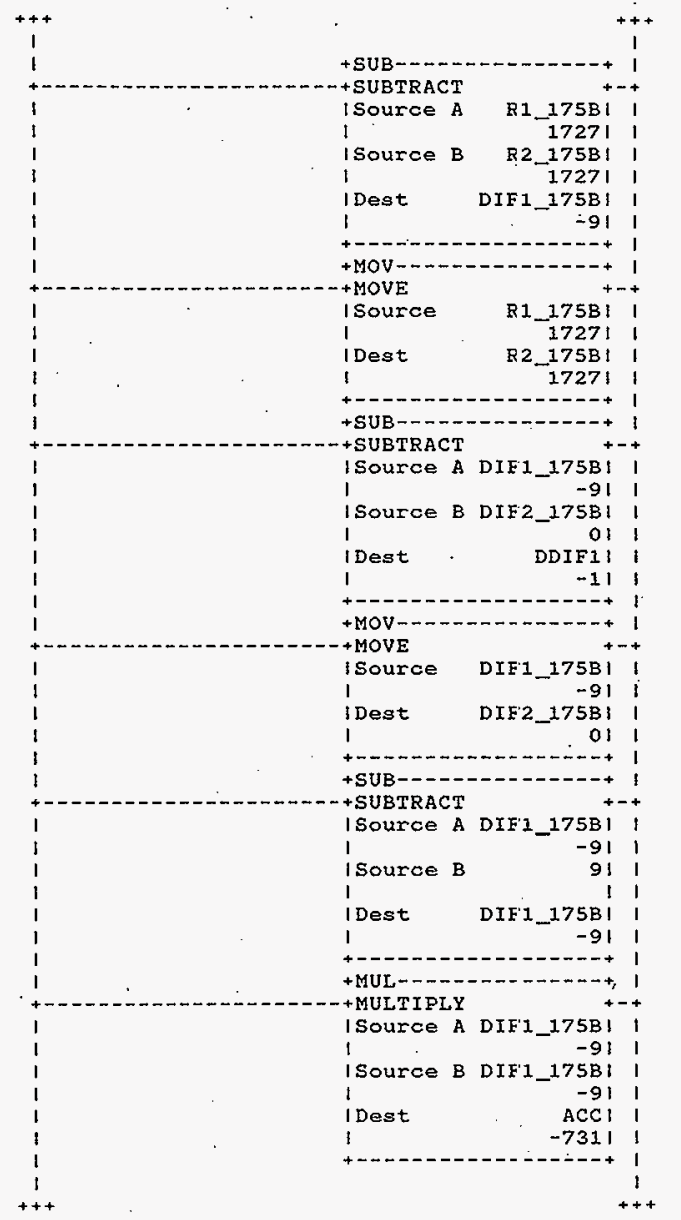

HNF-SD-FF-CSWD-61 Rev. 0

Page 639 


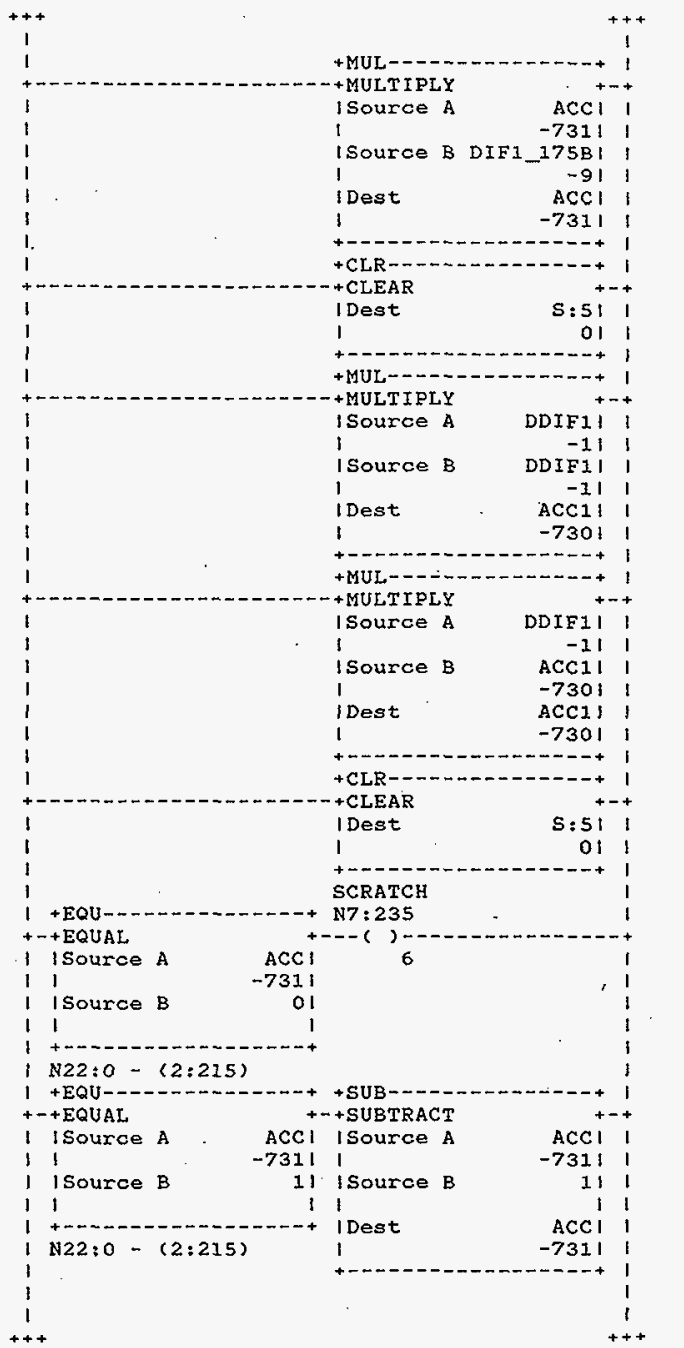

HNF-SD-FF-CSWD-61 Rev. 0 
Processor and Data(OPS Unit 1)

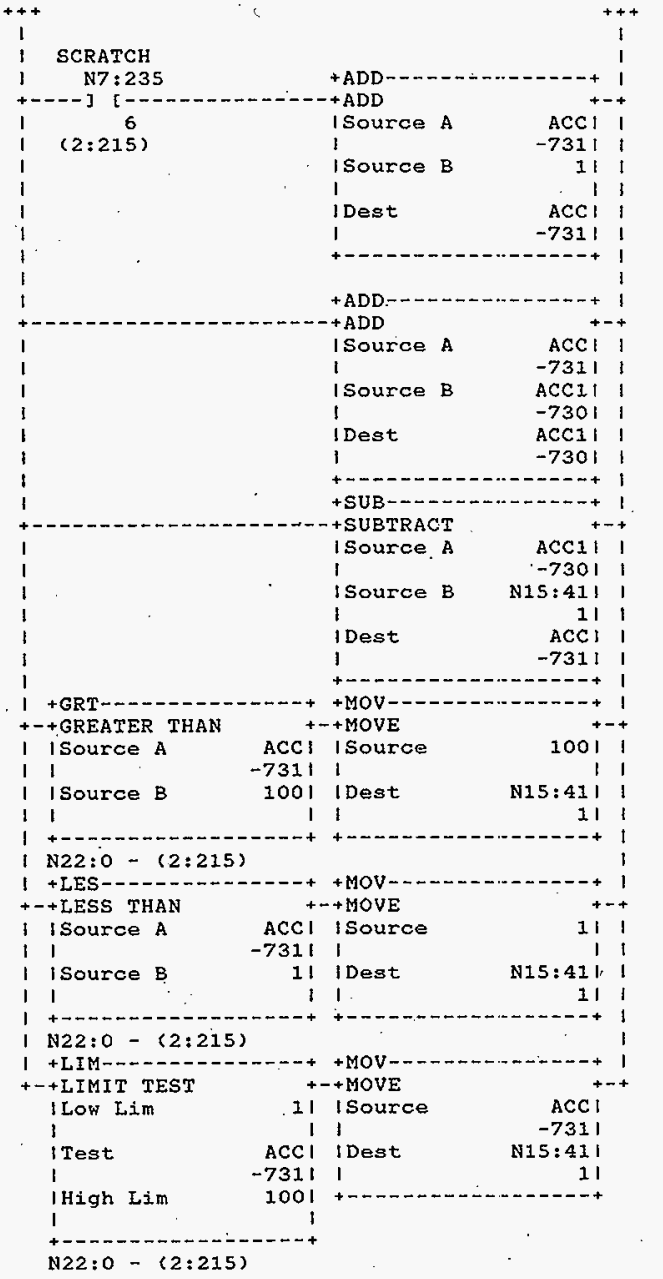

HNF-SD-FF-CSWD-61 Rev. 0 


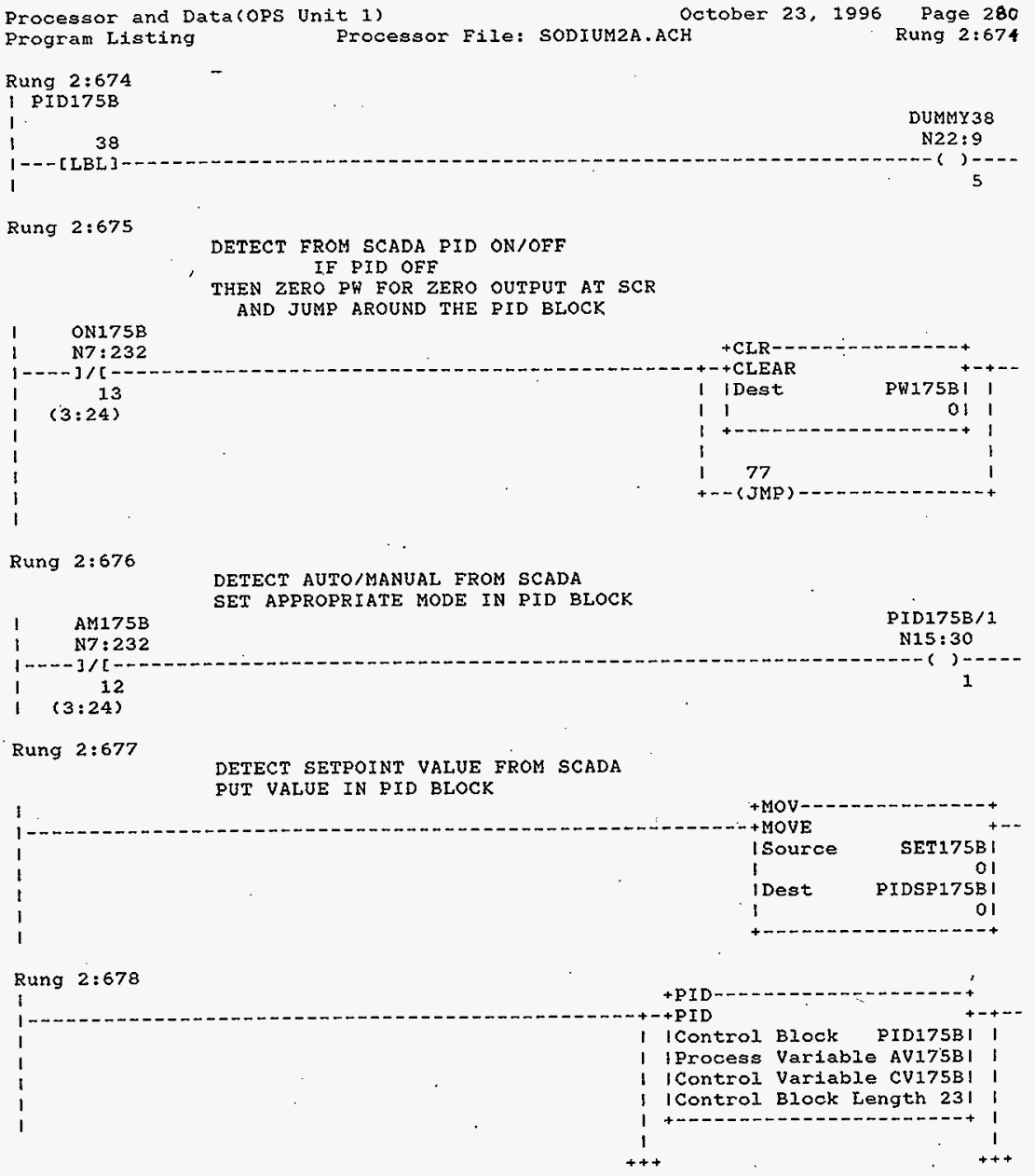

HNF-SD-FF-CSWD-61 Rev. 0

Page 642 
Processor and Data(OPS Unit 1$)$

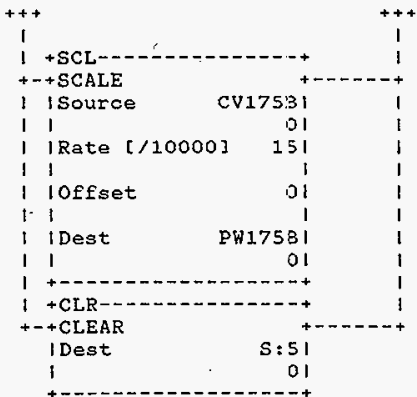

Rung 2:679

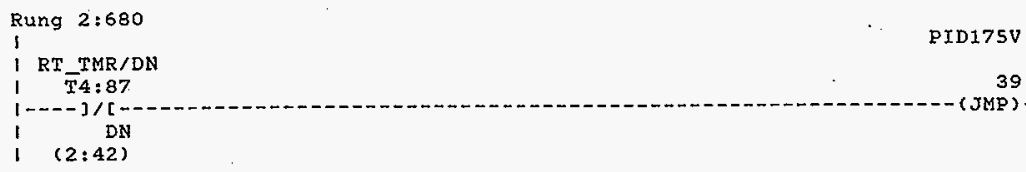

\section{Rung 2:681}

RATE CHECKING

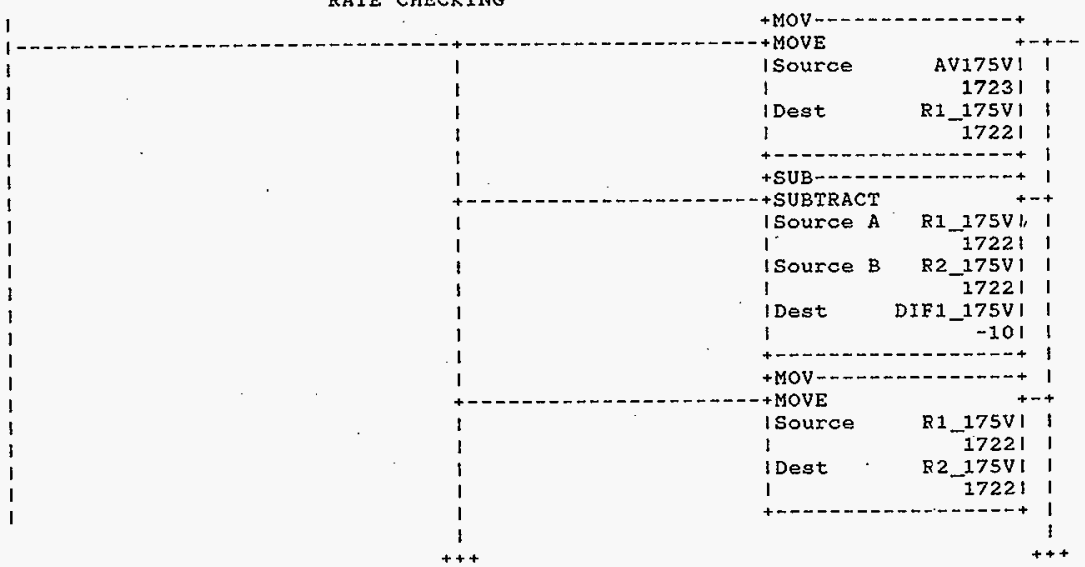

HNF-SD-FF-CSWD-61 Rev. 0 


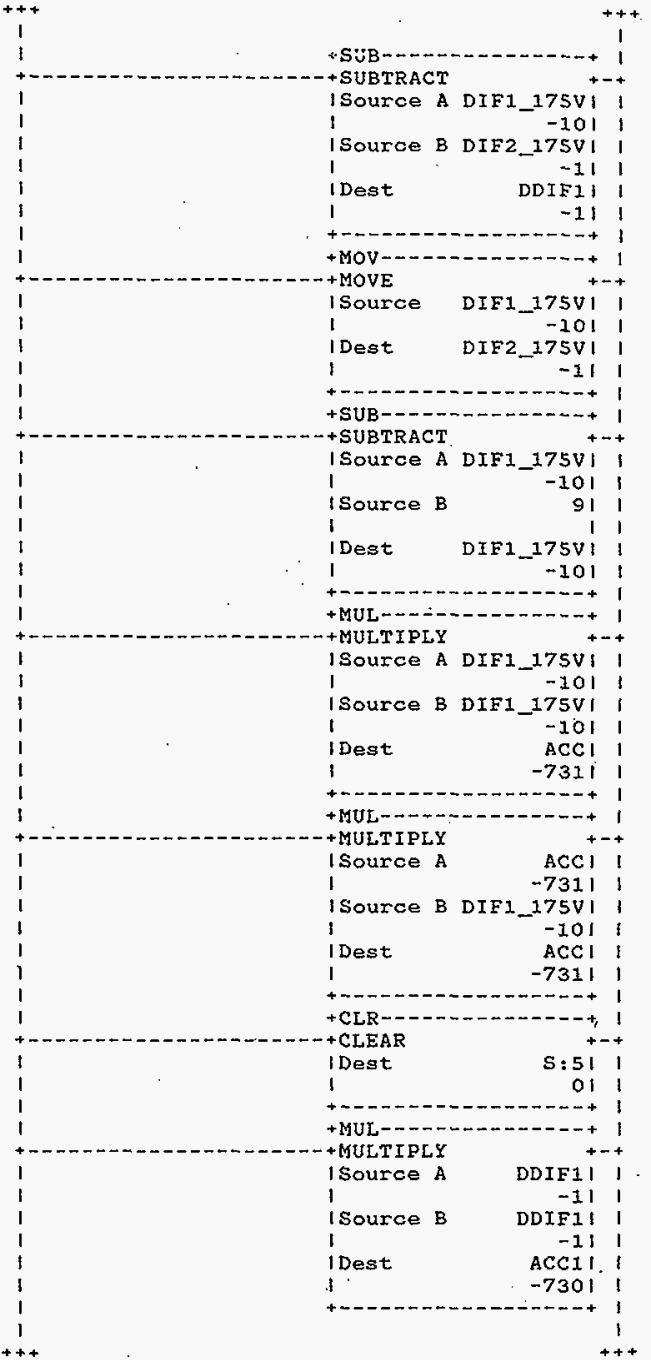

HNF-SD-FF-CSWD-61 Rev. 0 
Processor and Data(OPS Unit 1) Program Listing.
October 23,1996

Processor File: SODIUM2A.ACH
Page 283

Rung $2: 681$

$$
\begin{aligned}
& 1 \\
& 1 \\
& 1 \\
& 1 \\
& 1 \\
& 1 \\
& 1 \\
& 1 \\
& 1 \\
& 1 \\
& 1 \\
& 1 \\
& 1 \\
& 1 \\
& 1 \\
& 1 \\
& 1 \\
& 1 \\
& 1 \\
& 1 \\
& 1 \\
& 1 \\
& 1 \\
& 1 \\
& 1 \\
& 1 \\
& 1 \\
& 1 \\
& 1 \\
& 1 \\
& 1 \\
& 1 \\
& 1 \\
& 1 \\
& 1 \\
& 1 \\
& 1 \\
& 1 \\
& 1 \\
& 1 \\
& 1 \\
& 1 \\
& 1 \\
& 1
\end{aligned}
$$

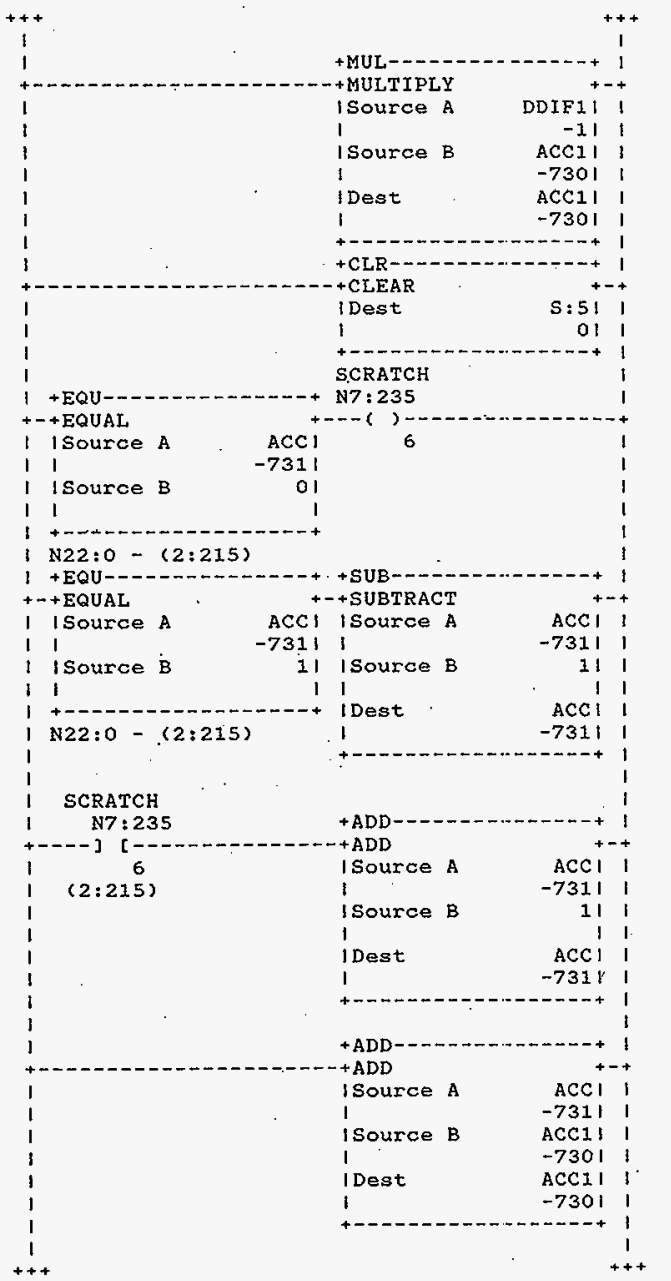

HNF-SD-FF-CSWL-61 Rev. 0

Page 645 


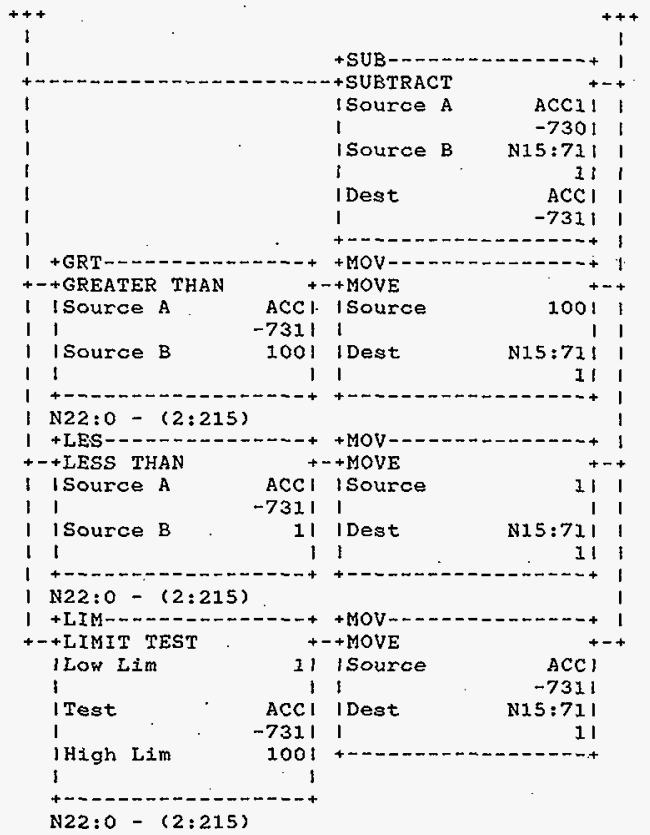

Rung $2: 632$

I PID175V

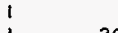

DUMMY39

$1--[$ [LBL $]$

N22:9

$-(1)-\ldots$

Rung 2:683

\section{DETECT FROM SCADA PID ON/OFF \\ IF PID OFE \\ THEN ZERO PW FOR ZERO OUTPUT AT SCR AND JUMP AROUND THE PID BLOCK}
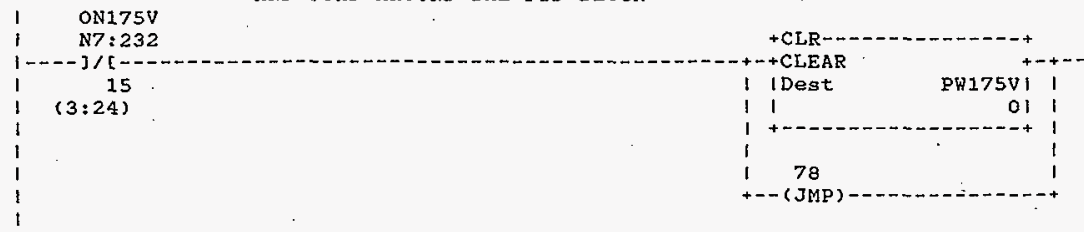

HNF-SD-FF-CSWD-61 Rev. 0 
Rung 2:684

DETECT AUTO/MANUAL FROM SCADA

SET APPROPRIATE MODE IN PID BLOCK

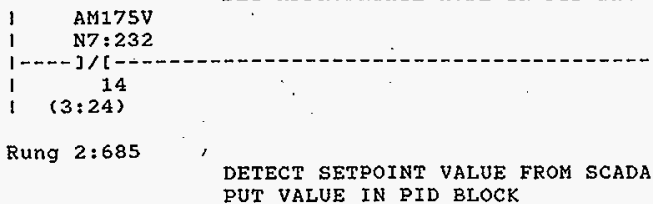

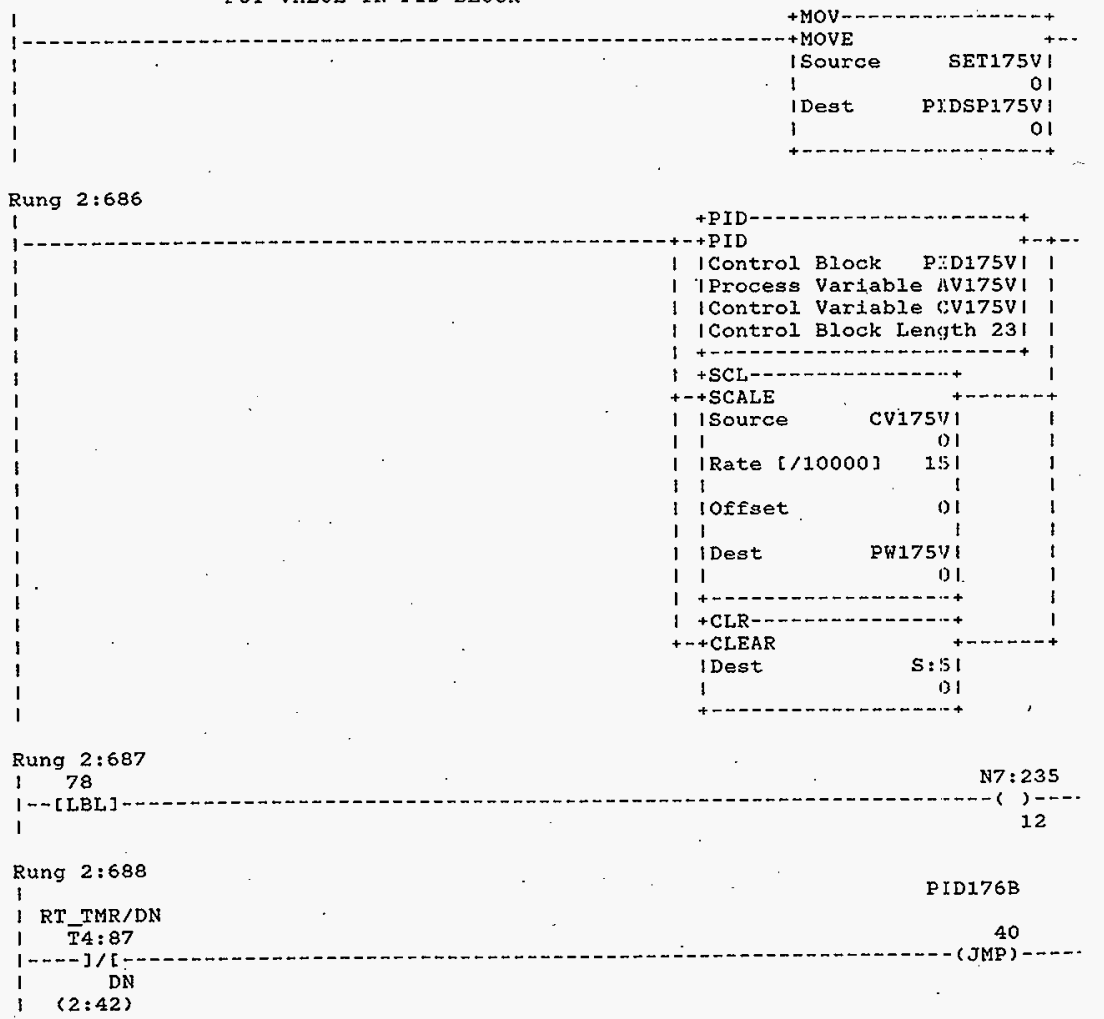

HNF-SD-FF-CSWD-61 Rev. 0 
Procegsor and Data(OPS Unit 1)

Rung 2:689

\section{- RATE CHECKING}

1
1
1
1
1
1
1
1
1
1
1
1
1
1
1
1
1
1
1
1
1
1
1
1
1
1
1
1
1
1
1
1
1
1
1
1
1
1

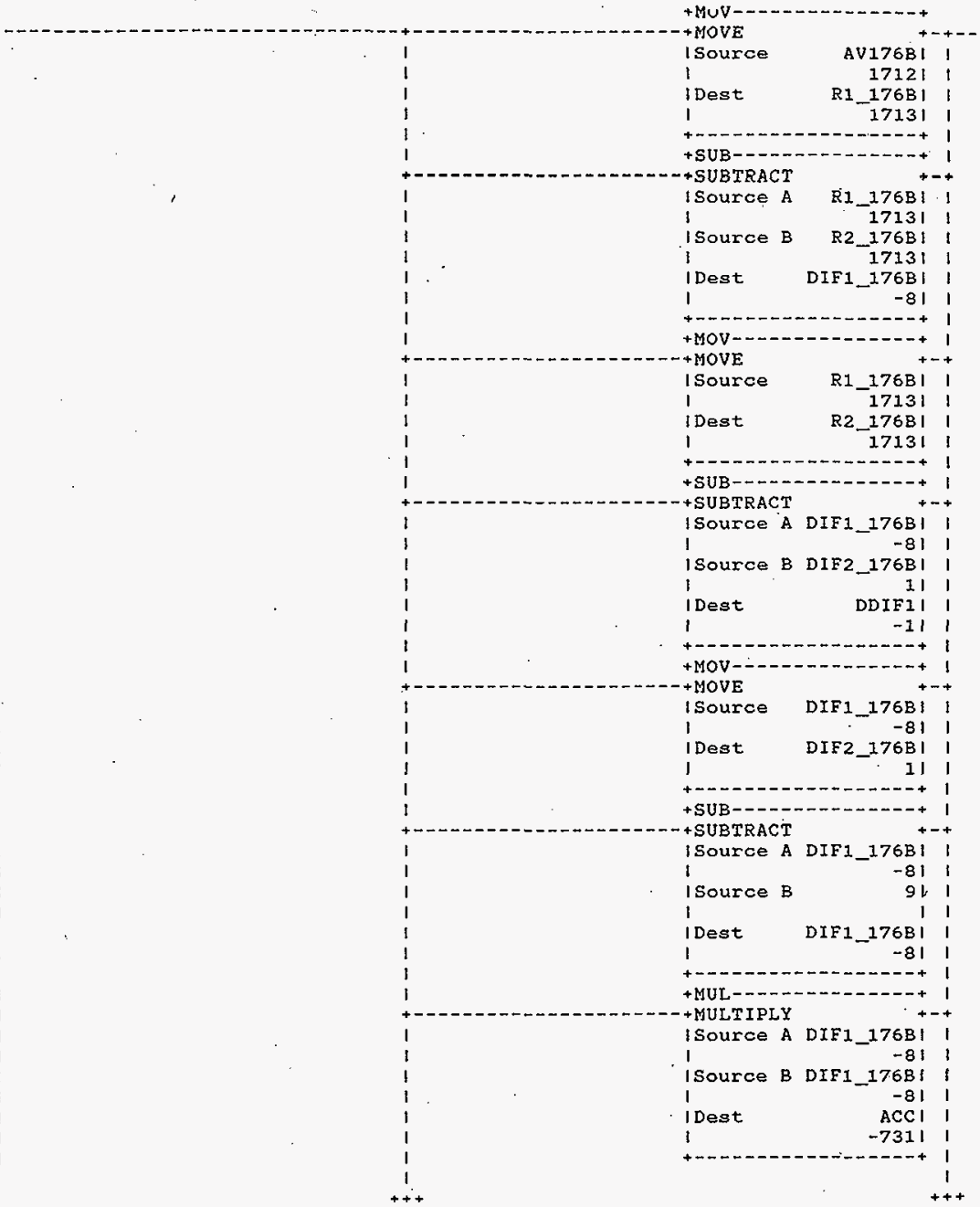

HNF-SD-FF-CSWD-61 Rev. 0 
Processor and Data(OPS Unit 1 )

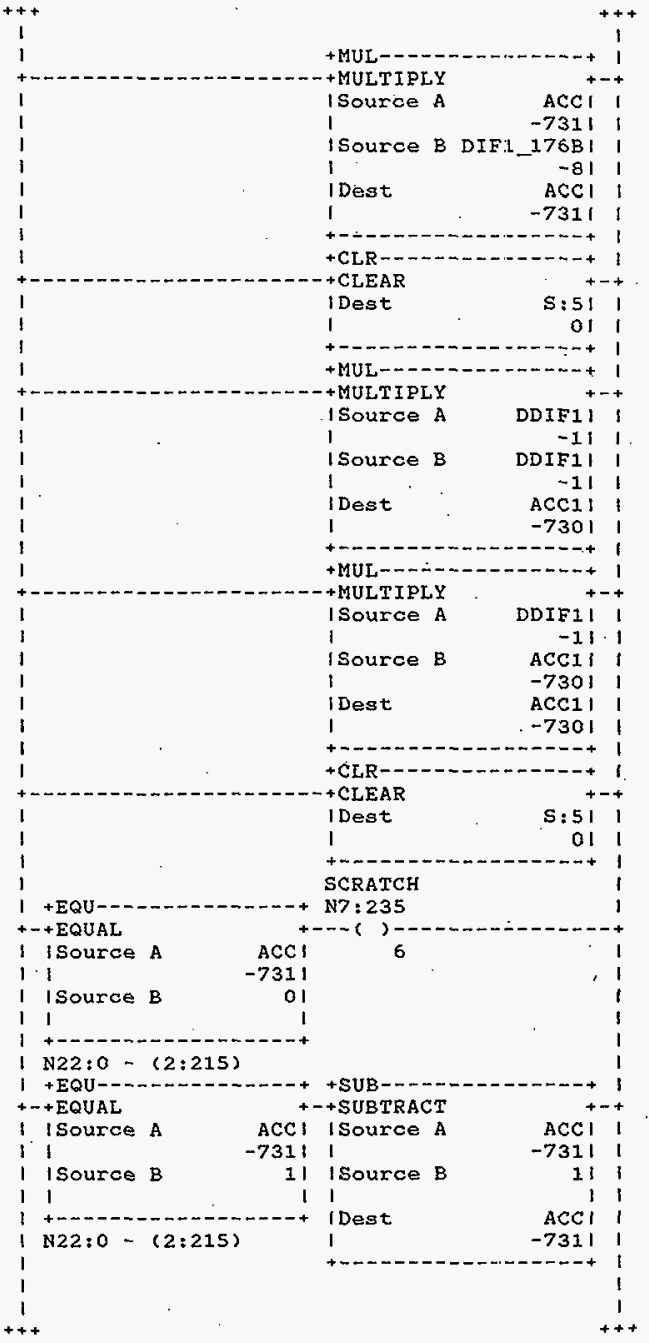

HNF-SD-FF-CSWD-61 Rev. 0 


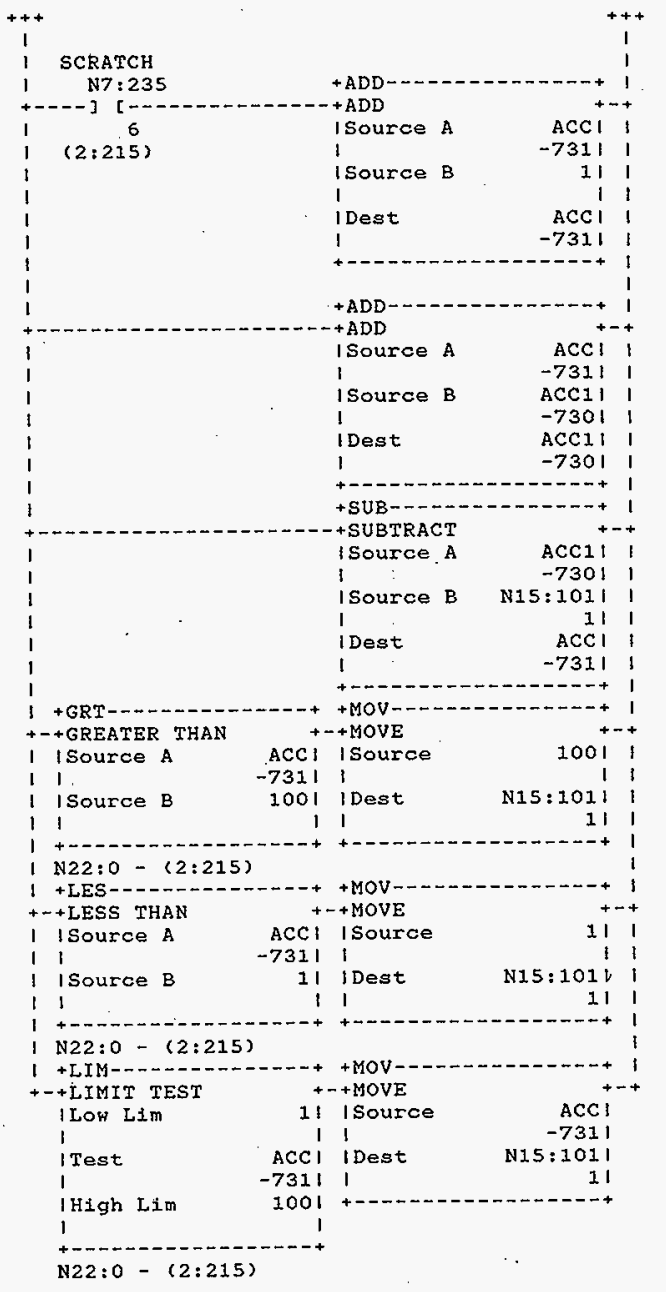

HNF-SD-FF-CSWD-61 Rev. 0 
Processor and Data(OPS Unjt 1)

Rung 2:690

1 PID176B

I. 40

DUMMY 40

$1---[L B L]$

N22:9

1

Rung 2:691

DETECT FROM SCADA PID ON/OFF

IF PID OFF

.THEN ZERO PH FOR ZERO OUTPUT AT SCR

$1 \quad$ ON176B

AND JUMP AROUND THE PID BLOCK

$1 \quad$ N7: 233

$1----] /[-$

$(3: 25)$

$-$

$-$

Rung $2: 692$

$\begin{array}{lc}\text { AM176B } \\ \text { N7 } & 233 \\ 1 & 0 \\ 1 & (3: 25)\end{array}$

DETECT AUTO/MANUAL FROM SCADA

SET APPROPRIATE MODE IN PID BLOCK

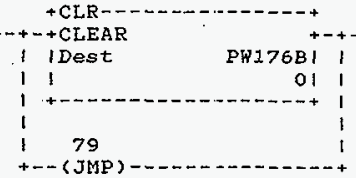

Rung $2: 693$

DETECT SETPOINT VALUE FROM SCADA

PUT VALUE IN PID BLOCK

Rung 2:694

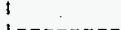

1

HNF-SD-FF-CSWD-51 Rev. 0

Page 651 


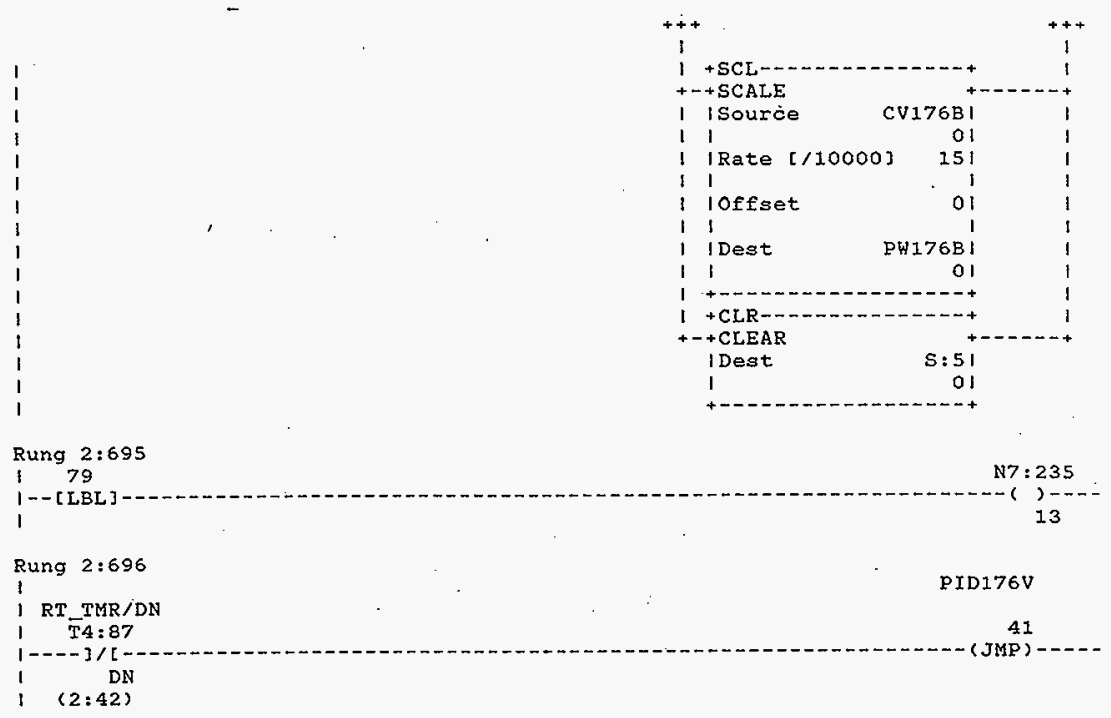

\section{Rung 2:697}

RATE CHECKING

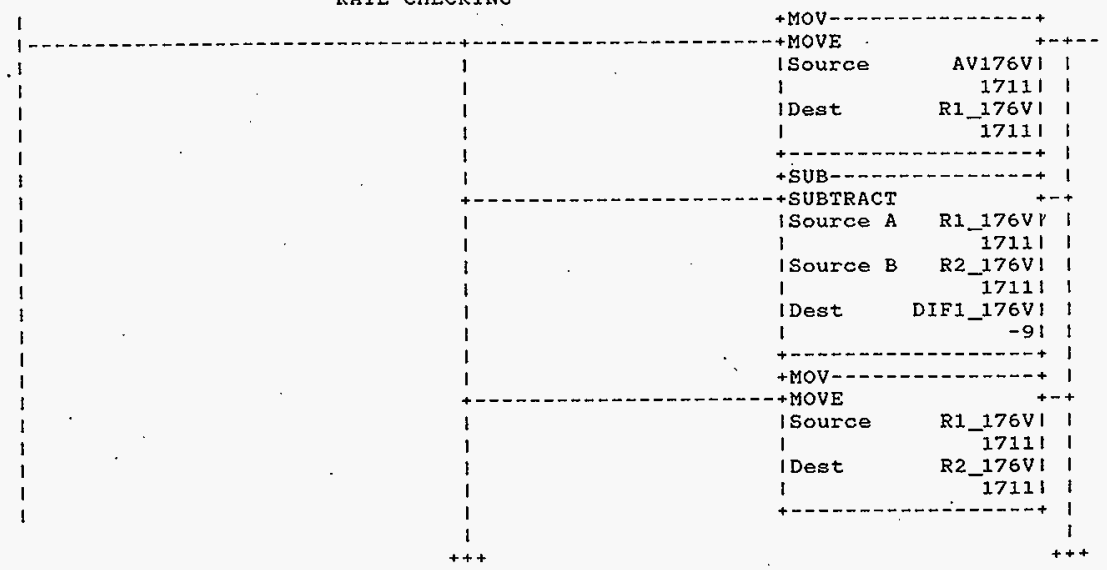

HNF-SD-FF-CSWD-61 Rev. 0 
Processor and Data (OPS Unit 1 ) Program Listing
October 23,1996 Processor File: SODIUM2A.ACH
Page 291 Rung 2:687

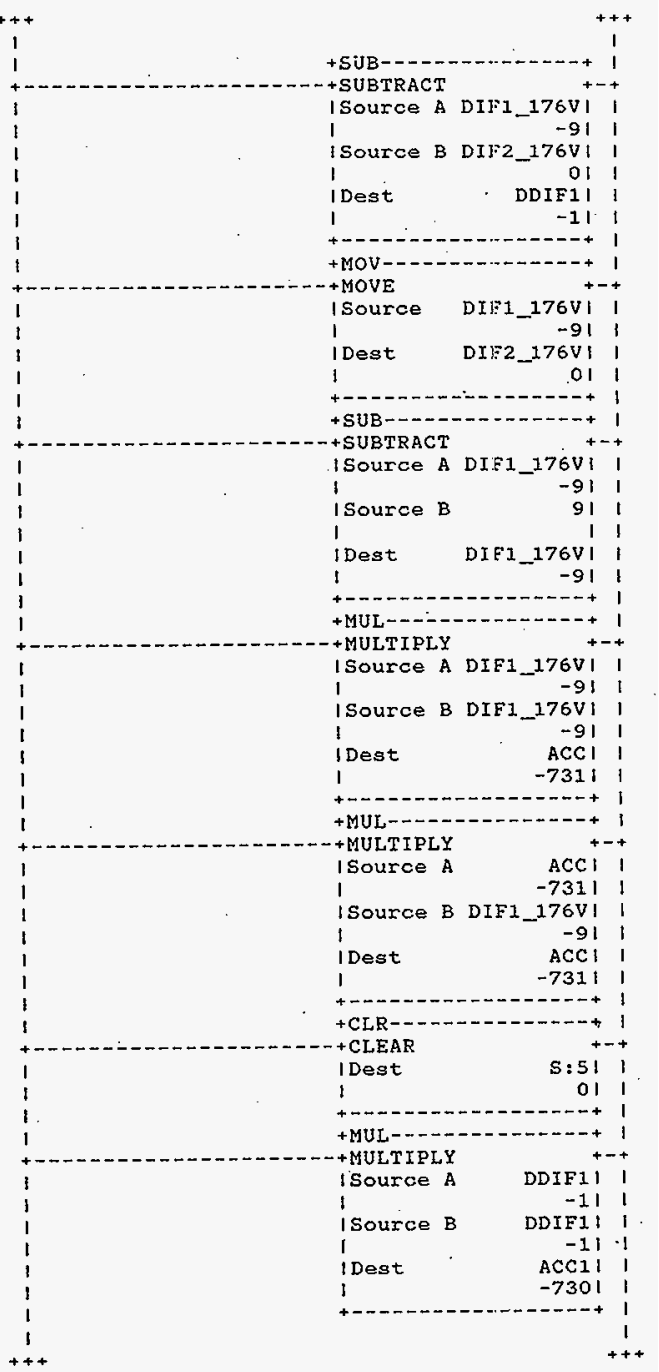

HNF-SD-FF-CSWD-61 Rev. 0

Page 653 


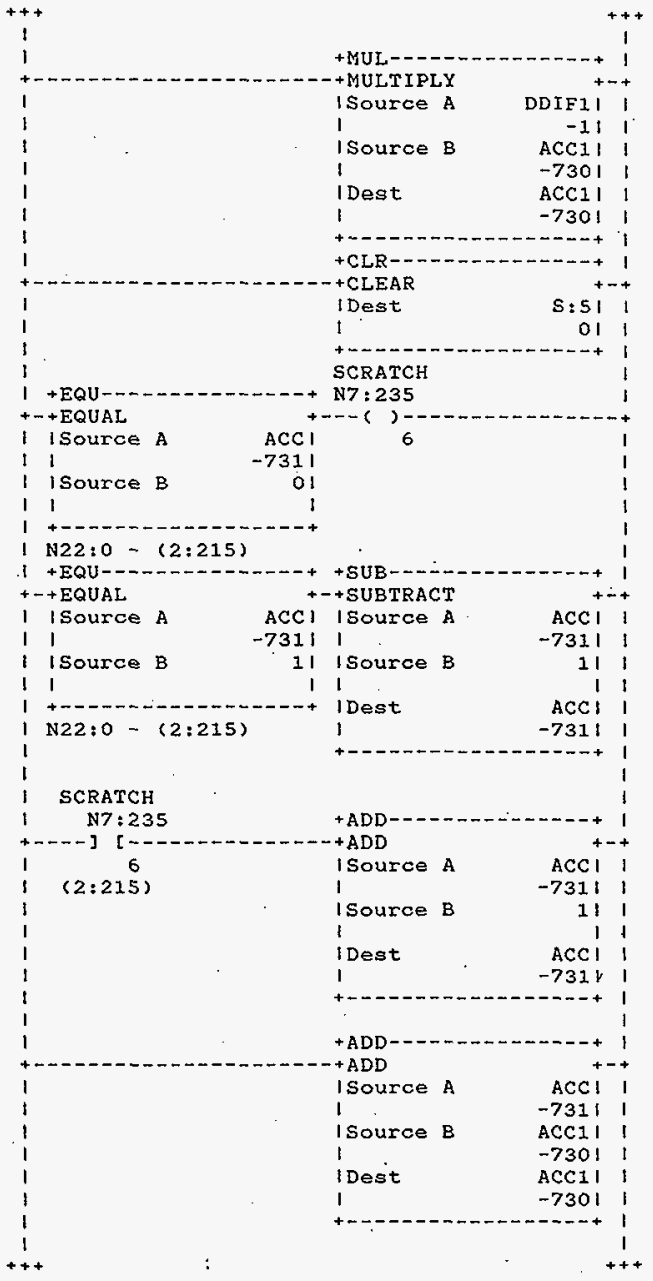

HNF-SD-FF-CSWD-61 Rev. 0 


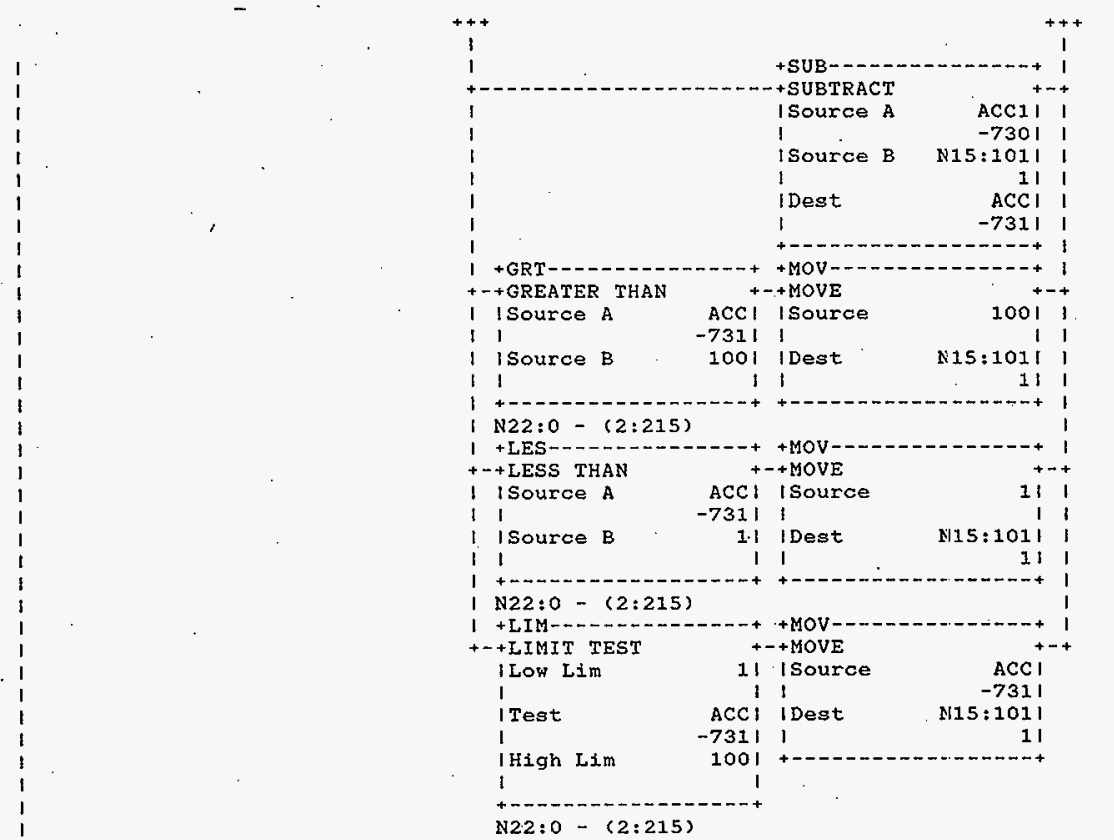

Rung $2: 698$

I PID176V

Rung 2:699

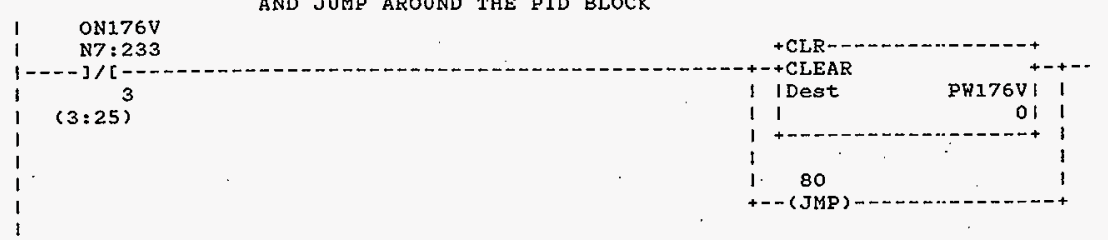

DETECT FROM SCADA PID ON/OFE
IF PID OFF

THEN ZERO PW FOR ZERO OUTPUT AT SCR

AND JUMP AROUND THE PID BLOCK

HNF-SD-FF-CSWD-61 Rev. 0 
Processor and Data(OPS Unit i)

Program Listing

Processor File: SODIUN2A.ACH

Rung 2:700

DETECT AUTO/MANUAL FROM SCADA

SET APPROPRIATE MODE IN PID BLOCK
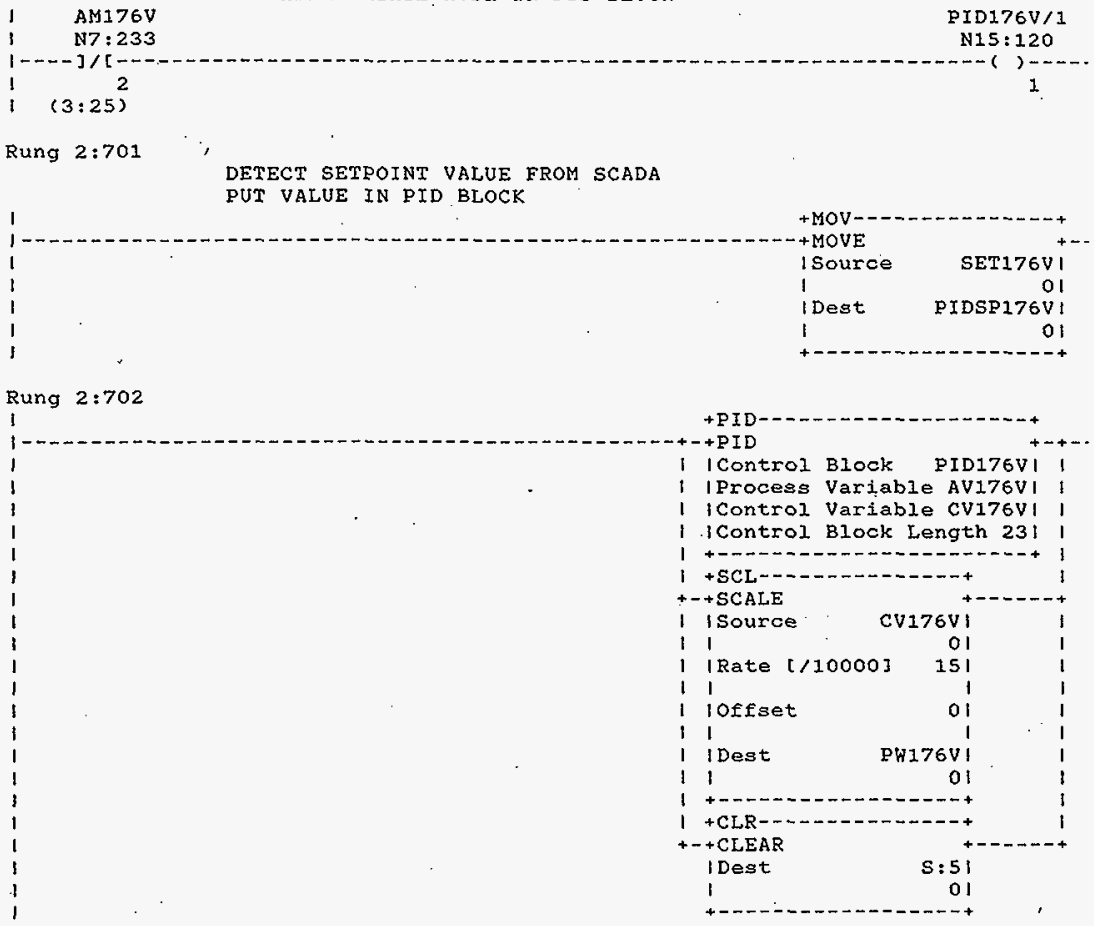

Rung 2:703

I 80

N7:235

1 - - [ [ LBL ]

Rung 2:704

I

RT TMR/DN

$\mathrm{T} 4: 87$

PID1778

1 - $-2-7 /[-$

(2:42)

HNF-SD-FF-CSWD-61 Rev. 0 
Processor and Data (OPS Unit 1 )

Rung 2:705

\section{RATE CHECKING}

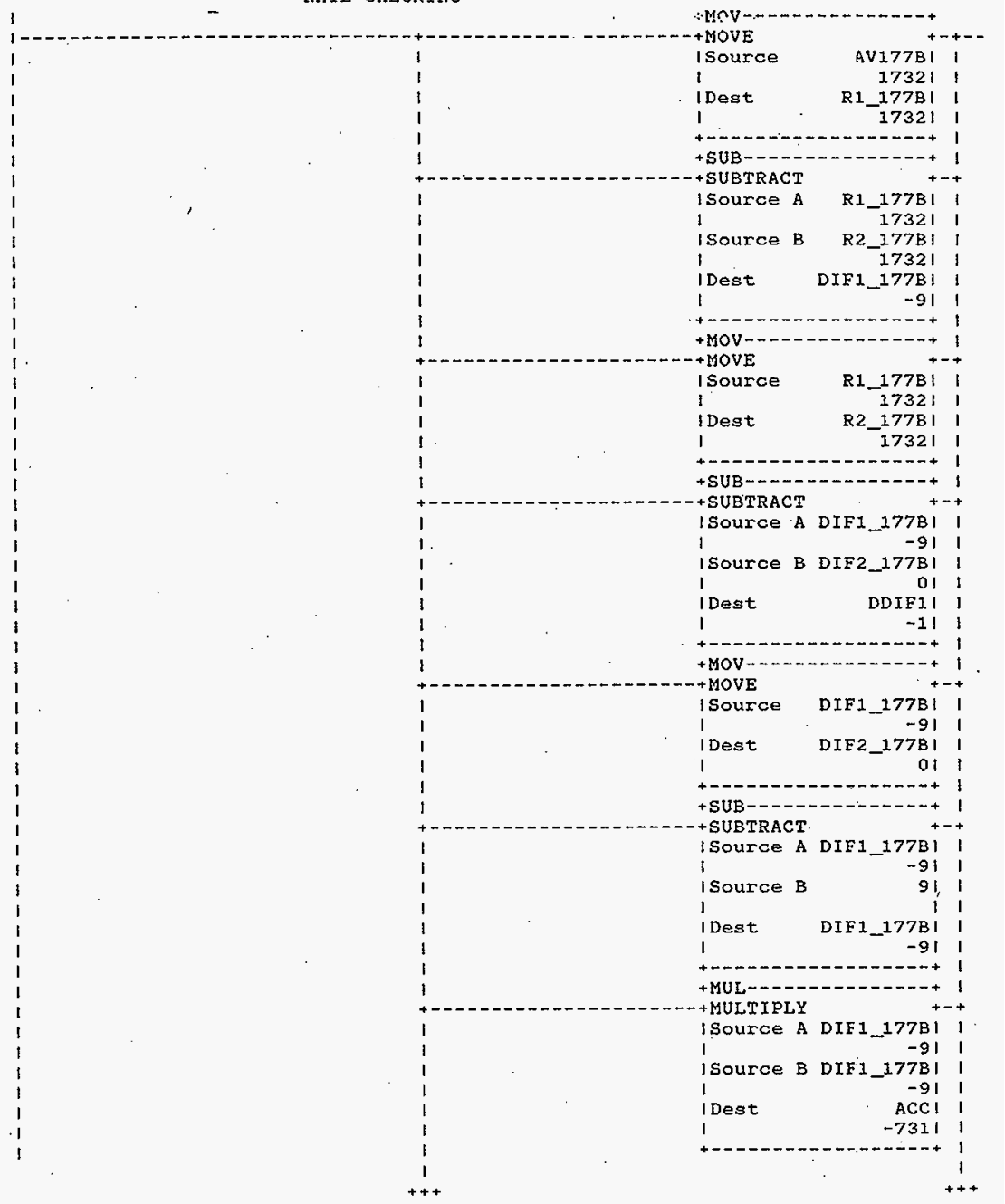

HNF-SD-FF-CSWD-61 Rev. 0

Page 657 


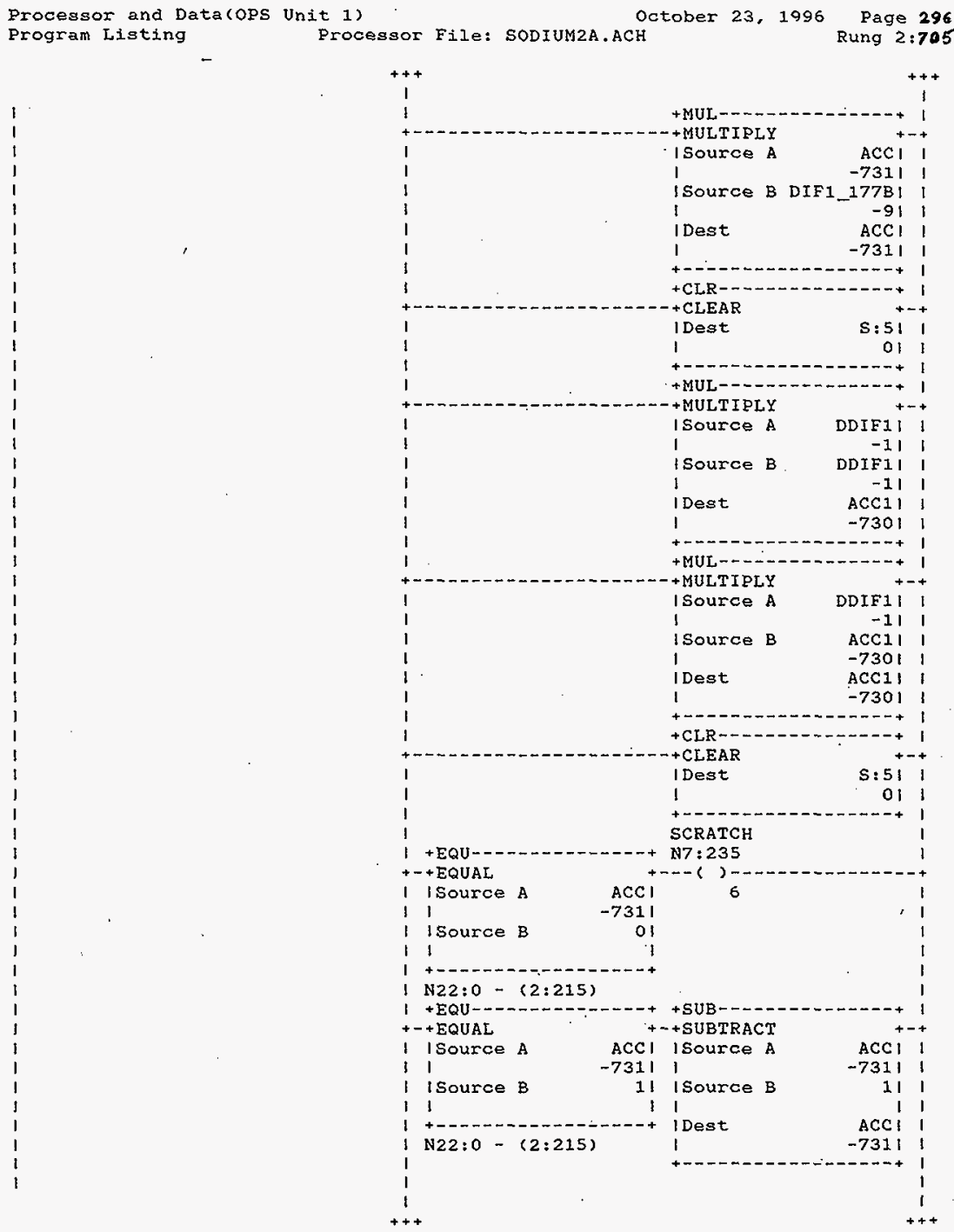

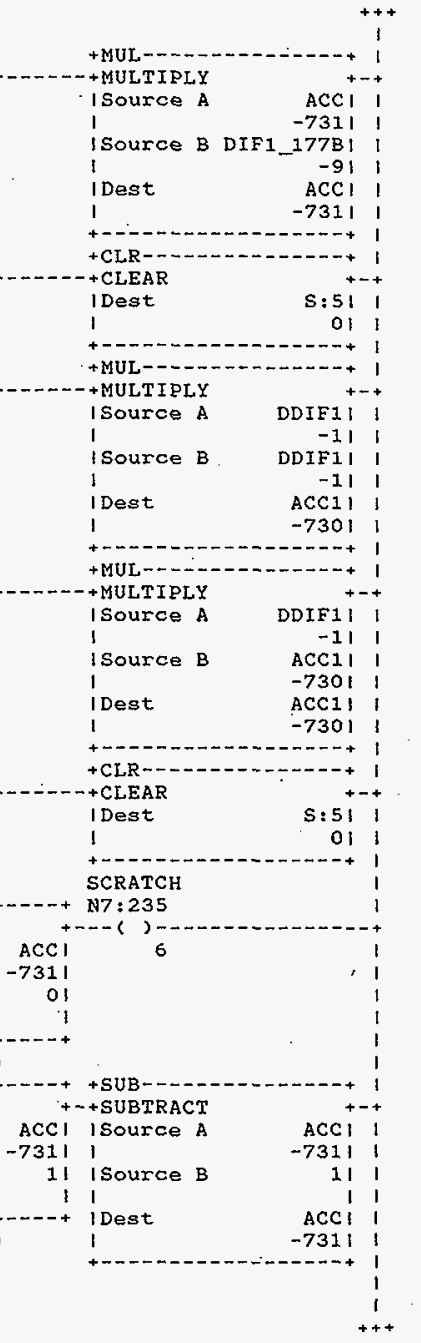

HNF-SD-FF-CSWD-61 Rev. 0

Page 658 
Processor and Data(OPS Unit 1)

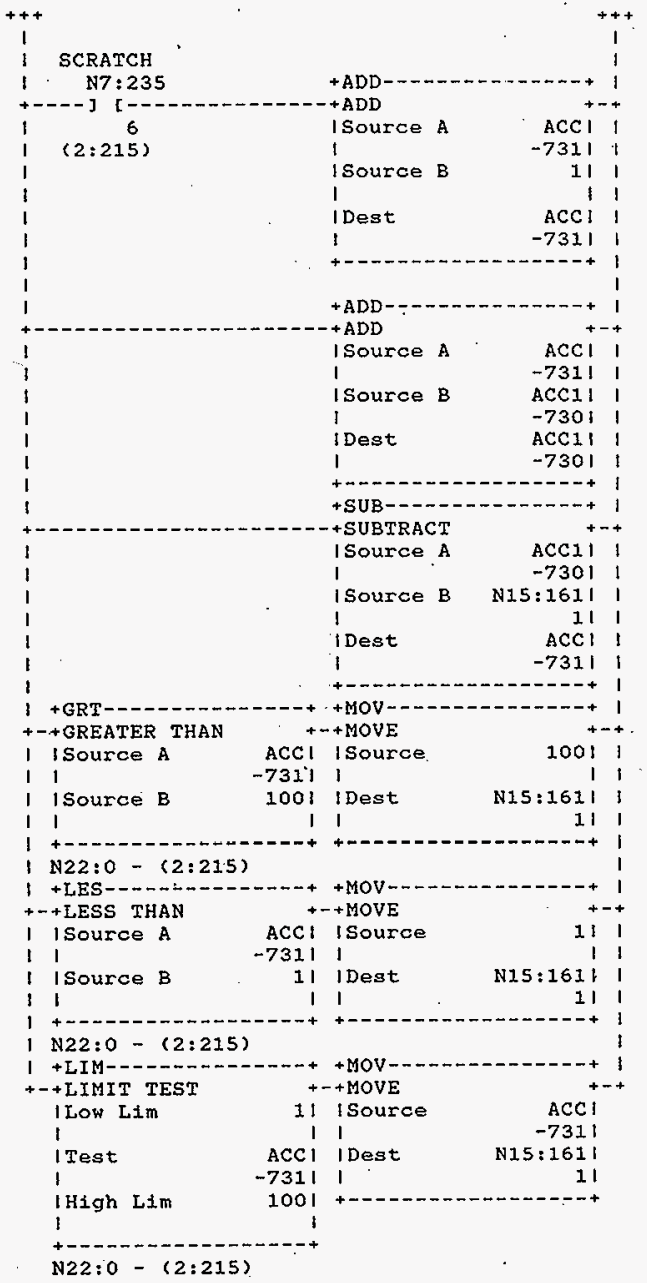

HNF-SD-FF-CSWD-61 Rev. 0 
Processor and Data (OPS Unit 1)

Rung 2:706

I PIDI77B

I

142

DUMMY 42

- - [LBL ]

N22:9

1

Rung 2:707

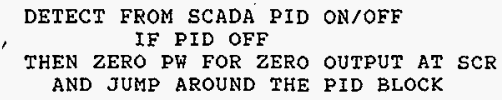
5

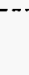

-

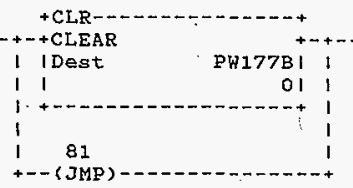

Rung $2: 708$

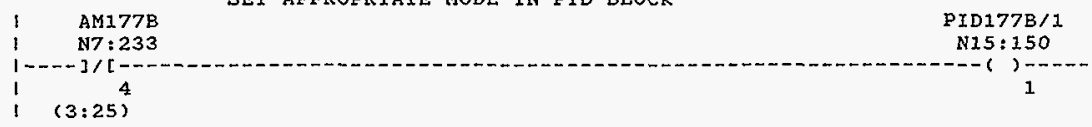

Rung 2:709

DETECT SETPOINT VALUE FROM SCADA PUT VALUE IN PID BLOCK

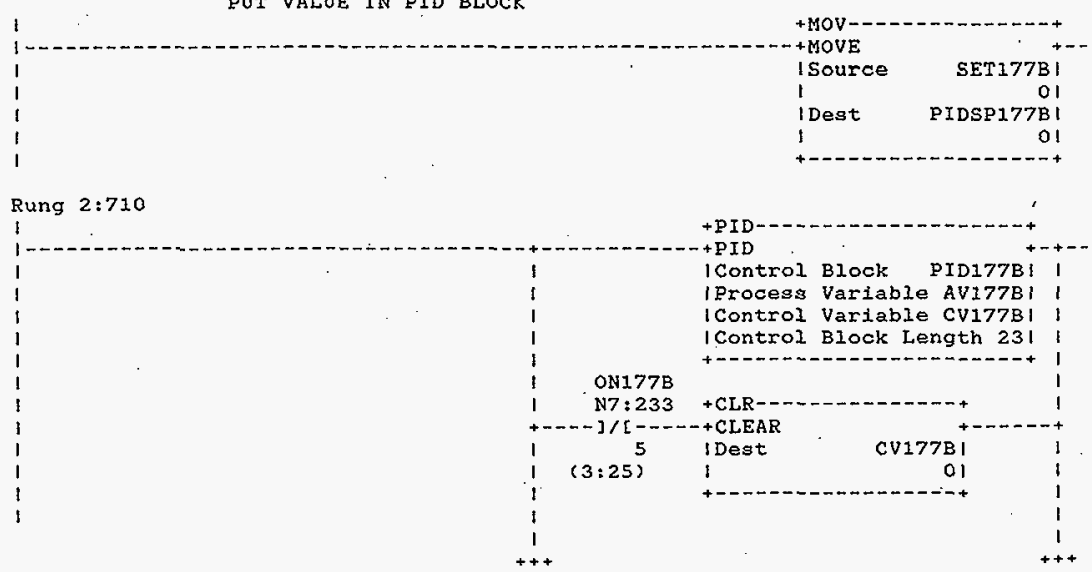

HNF-SD-FF-CSWD-61 Rev. 0 


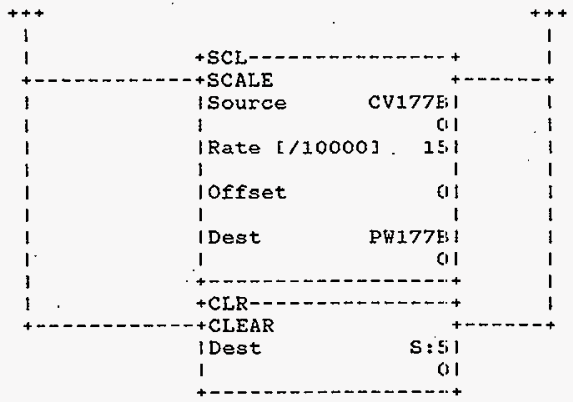

Rung 2:711

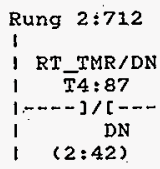

Rung $2: 713$

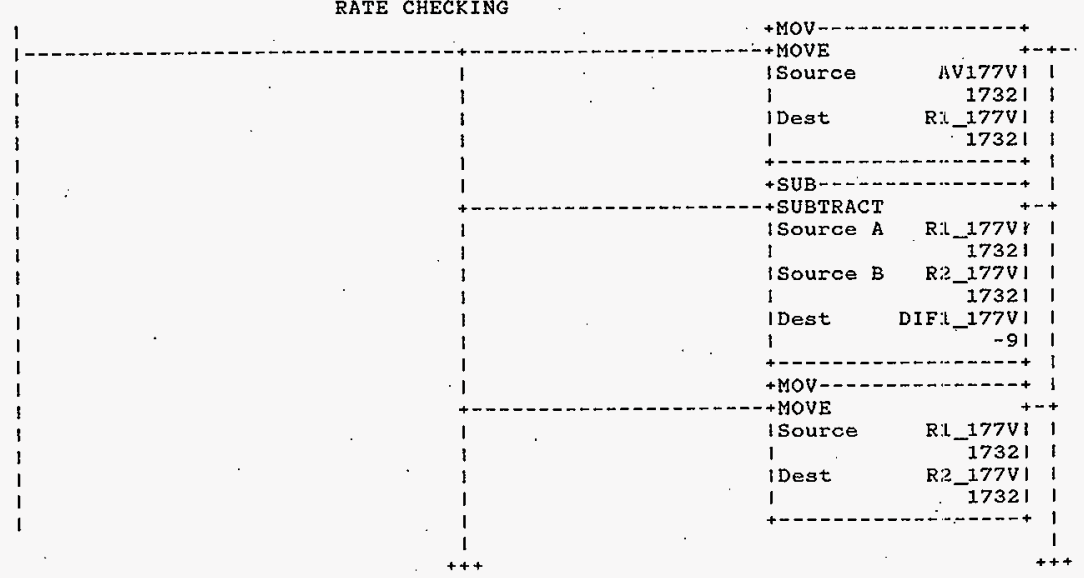

HNF-SD-FF-CSWD-61 Rev. 0 
Processor and Data(OPS Unit 1 )

October 23, 1996 Page 300 Program Listing Processor File: SODIUM2A.ACH Rung $2: 713$

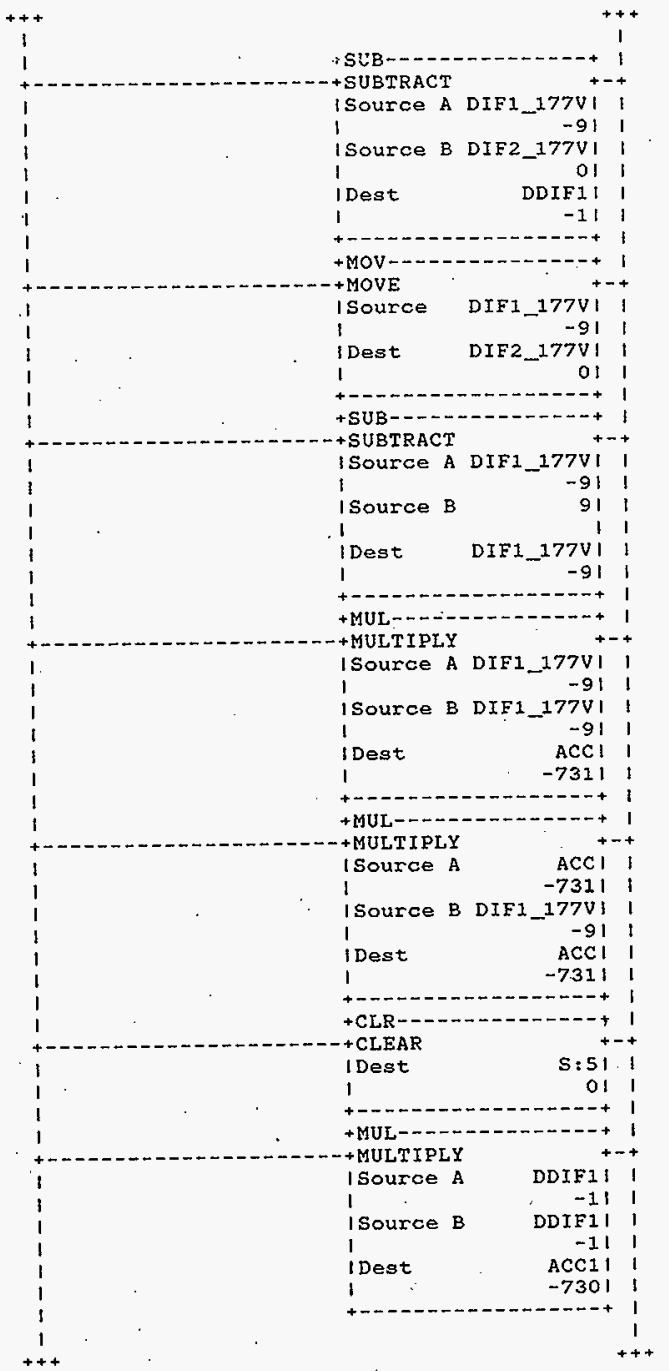

HNF-SD-FF-CSWD-61 Rev. 0 
Processor and Data(OPS Unit 1 )

October 23, 1996

Page 301 Program Listing Processor File: SODIUM2A.ACH

Rung $2: 713$
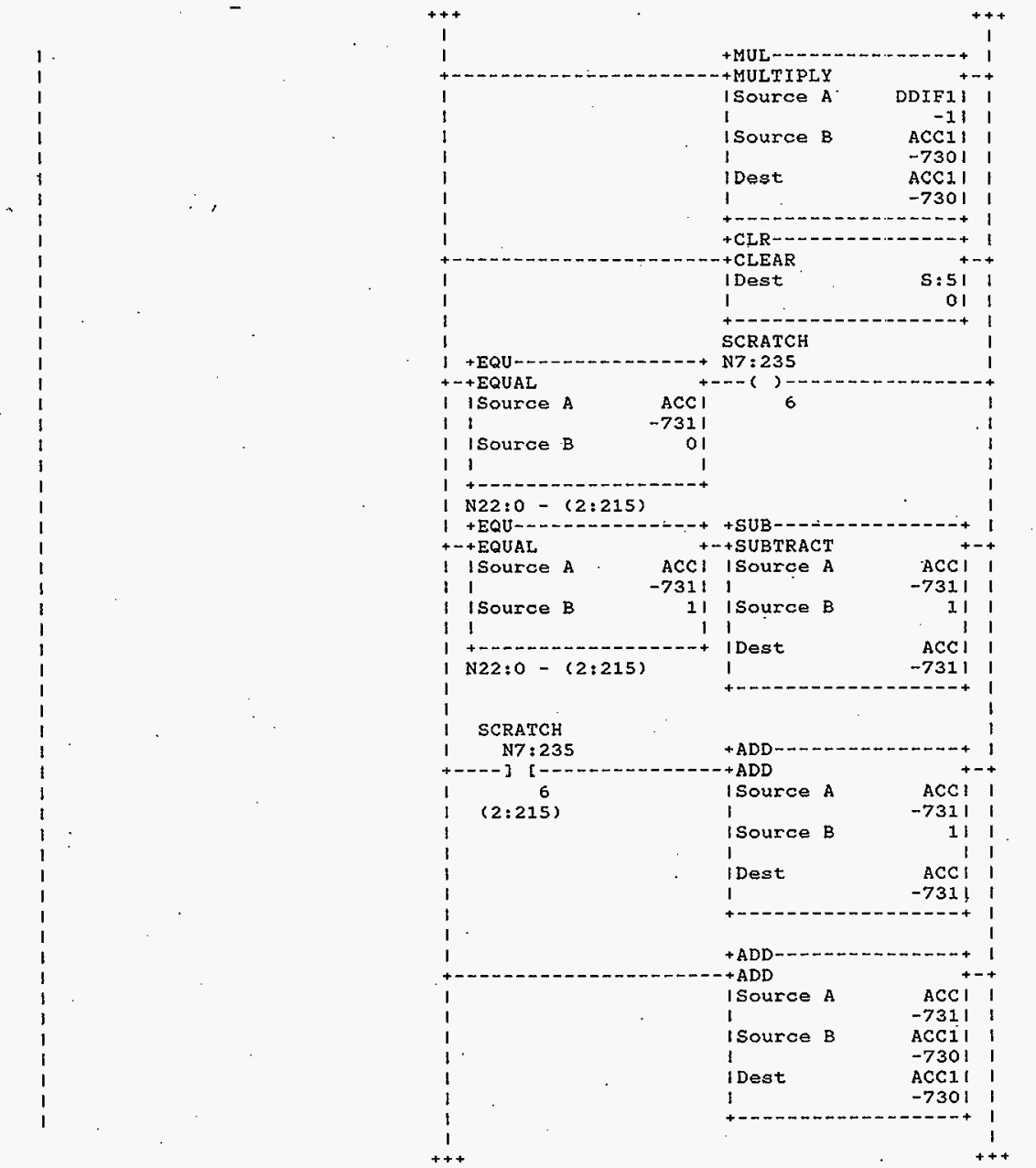

HNF-SD-FF-CSWD-61 Rev. 0

Page 663 


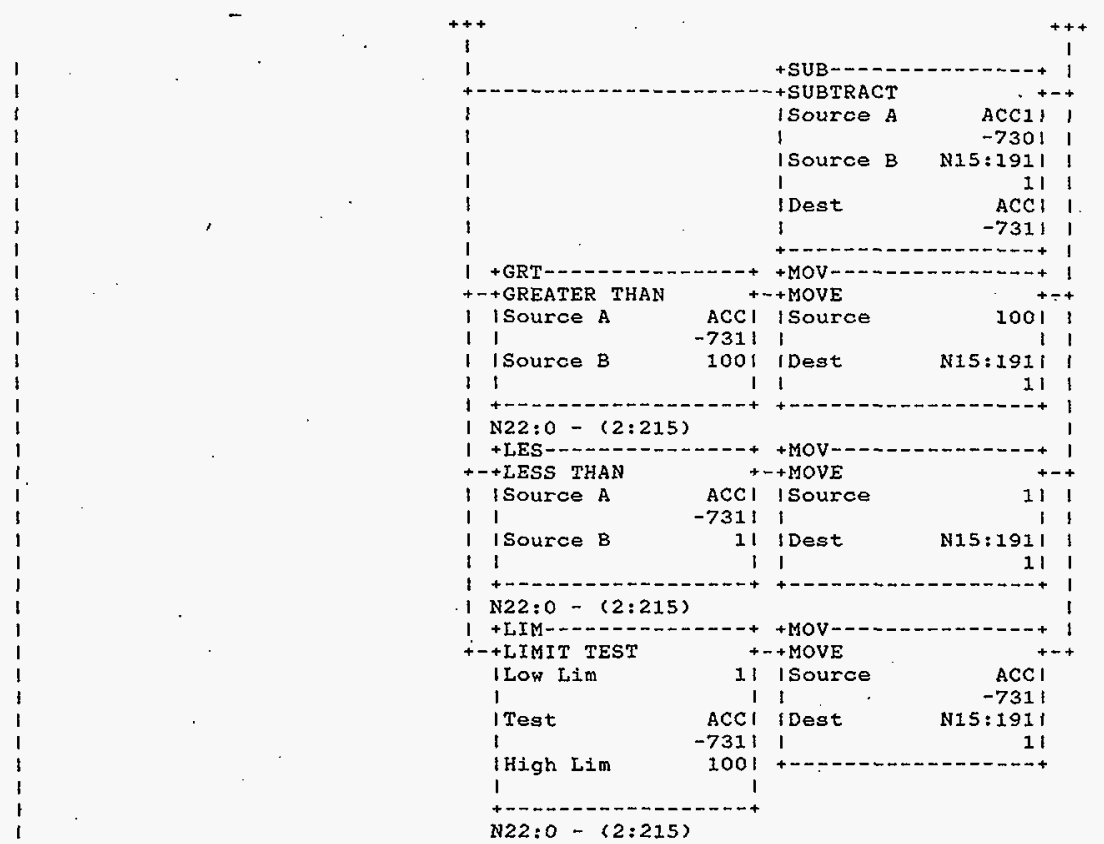

Rung $2: 714$

I PIDI77V

1

143

DUMMY 43

1-- [LBL]

N22:9

Rung 2:715

DETECT FROM SCADA PID ON/OFF

IF PID OFF

THEN ZERO PH FOR ZERO OUTPUT AT SCR

AND JUMP AROUND THE PID BLOCK

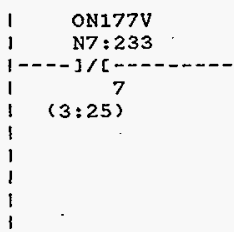

HNF-SD-FF-CSWD-61 Rev. 0 
Processor and Data(oRs Unit 1)

Rung $2: 716$

$-$

DETECT AUTO/MANUAL FROM SCADA

SET APPROPRIATE MODE IN PID BLOCK

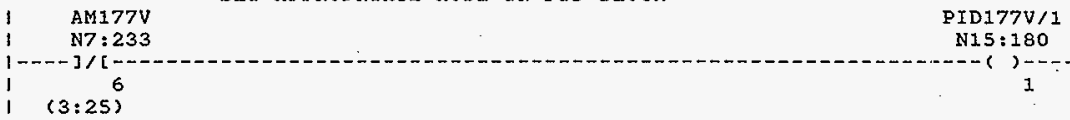

Rung 2:717, DETECT SETPOINT VALUE FROM SCADA PUT VALUE IN PID BLOCK

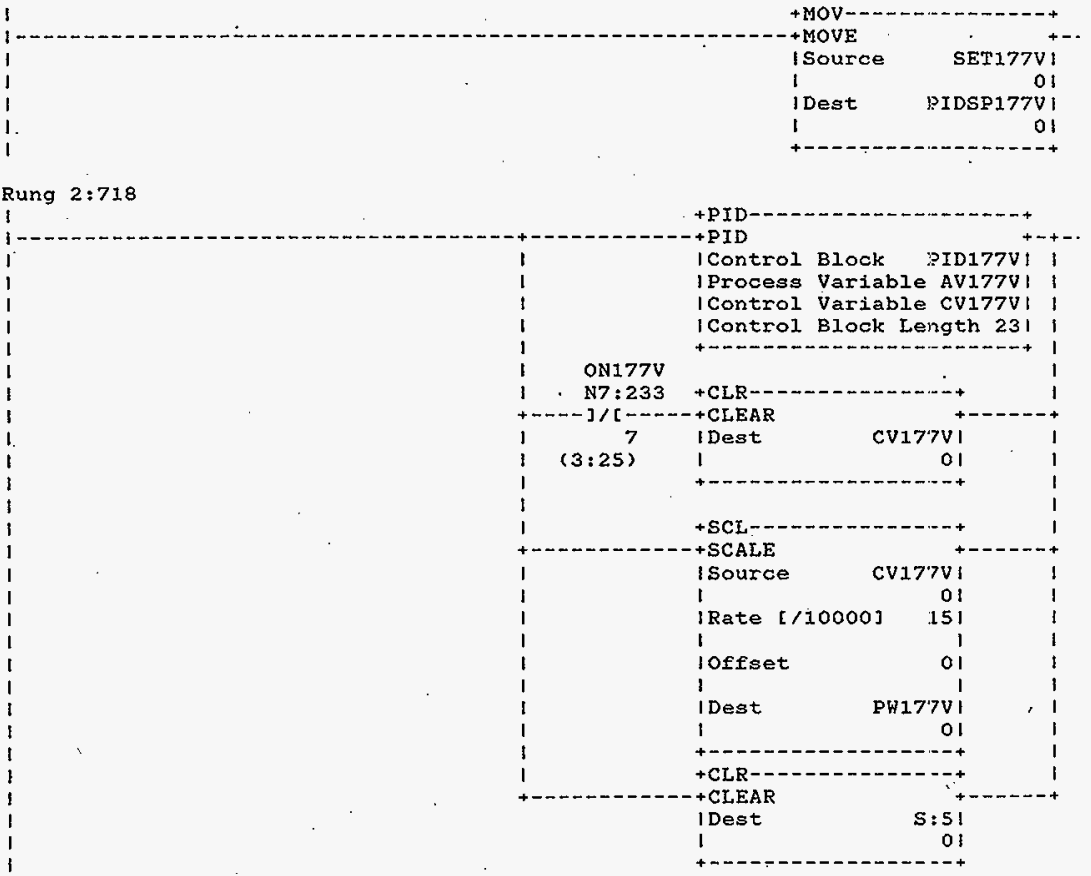

Rung 2:719

182

N7: 236

$1--[$ LBL. ]

HNF-SD-FF-CSWD-G1 Rev. 0 
Processor and Data(OPS Unit I)

October 23, 1996 Page 304 Program Listing

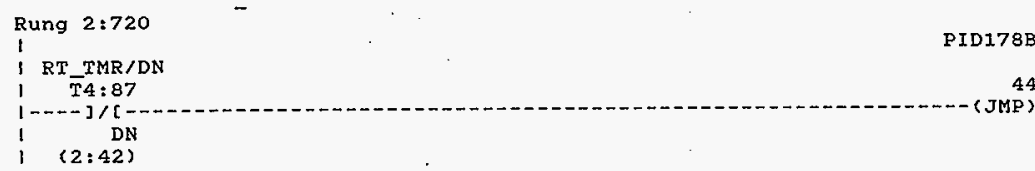

Rung 2:721

RATE CHECKING

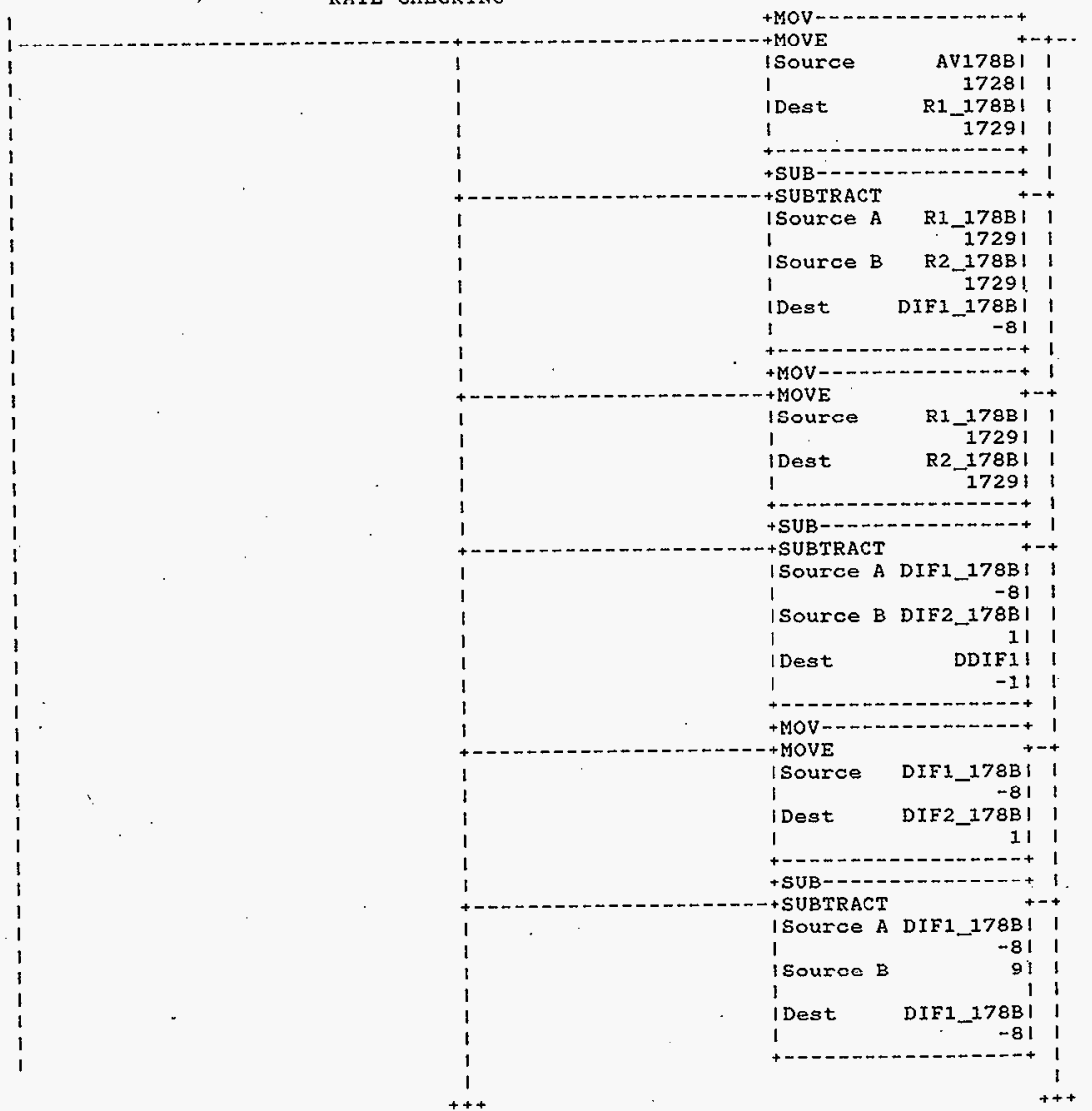

HNF-SD-FF-CSWD-61 Rev. 0 
Processor and Data(Ops Unit i)

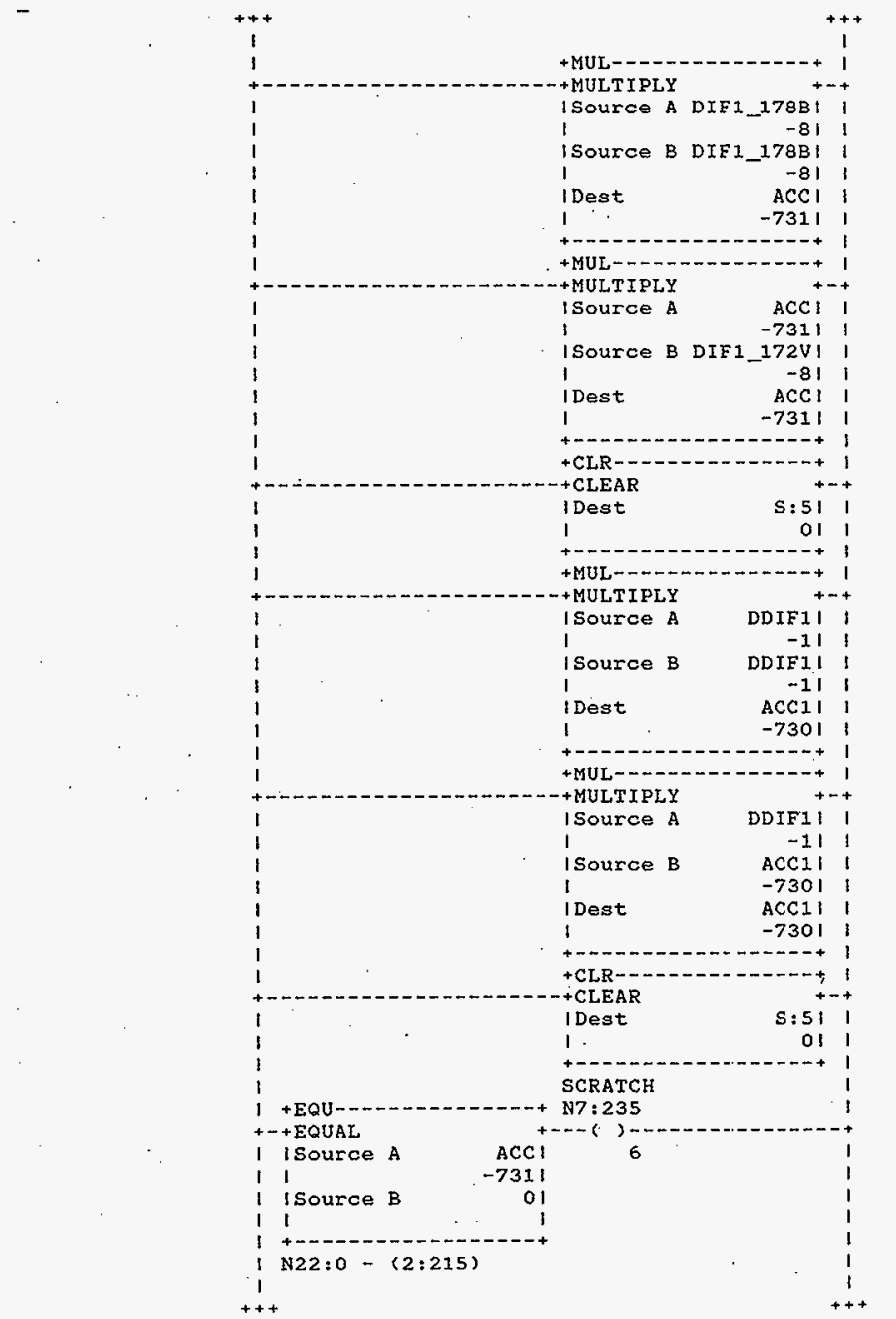

HNF-SD-FF-CSWD-6! Rev. 0 


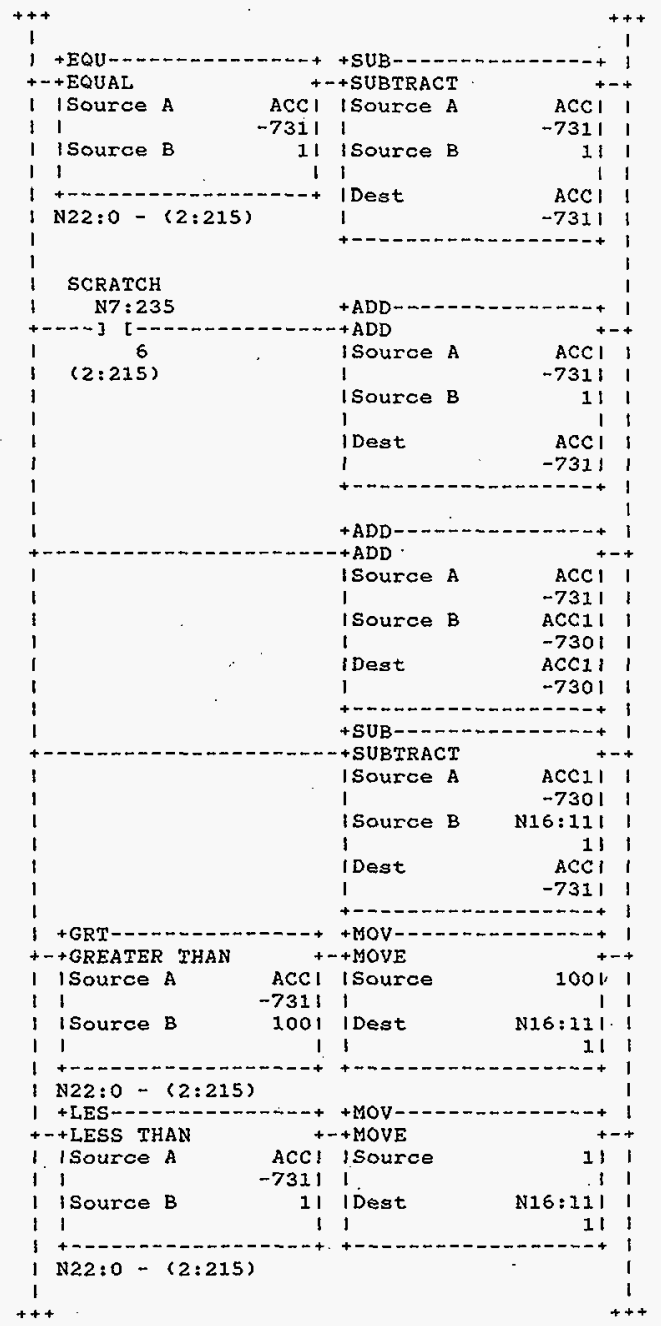

HNF-SD-FF-CSWD-61 Rev. 0 


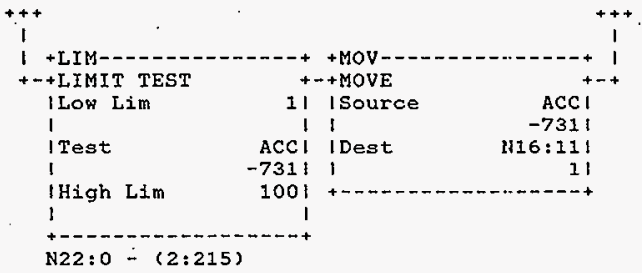

Rung $2: 722$

I PID178B

Rung $2: 723$
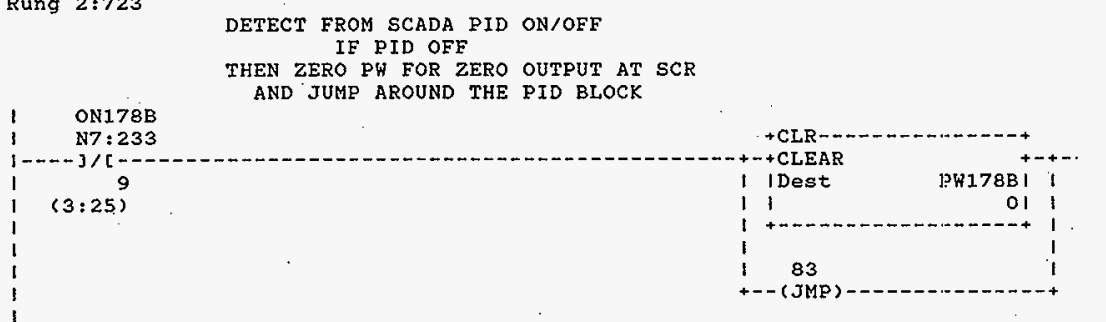

Rung 2:724

DETECT AUTO/MANUAL FROM SCADA

SET APPROPRIATE MODE IN PID BLOCK

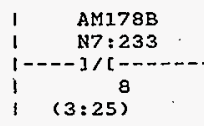

Rung $2: 725$.

DETECT SETPOINT VALUE FROM SCADA PUT VALUE IN PID BLOCK
PID $178 B / 1$

N1 $6: 0$

HNF-SD-FF-CSWD-61 Rev. 0

Page $66 \%$ 


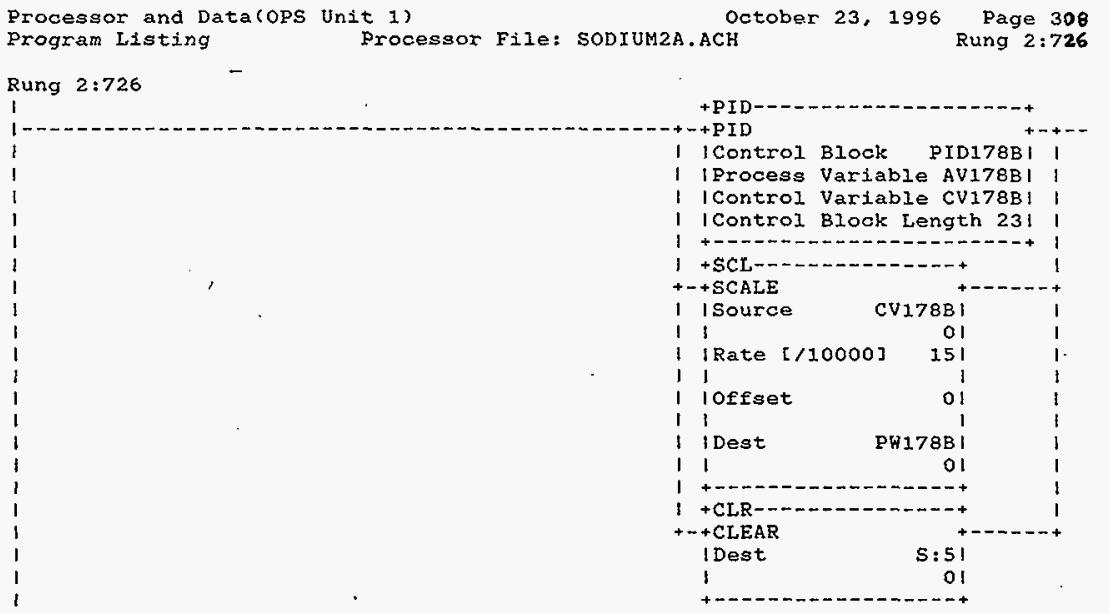

Rung 2:727

I 83

N7 : 236

1- $-[$ [B] $]$

$-($ )..-

1

Rung 2:728

I RT_TMR/DN

T $4: 87$

PID178V

$1-\ldots-.] /[-$

45

(JMP)

( $(2: 42)$

Rung 2:729

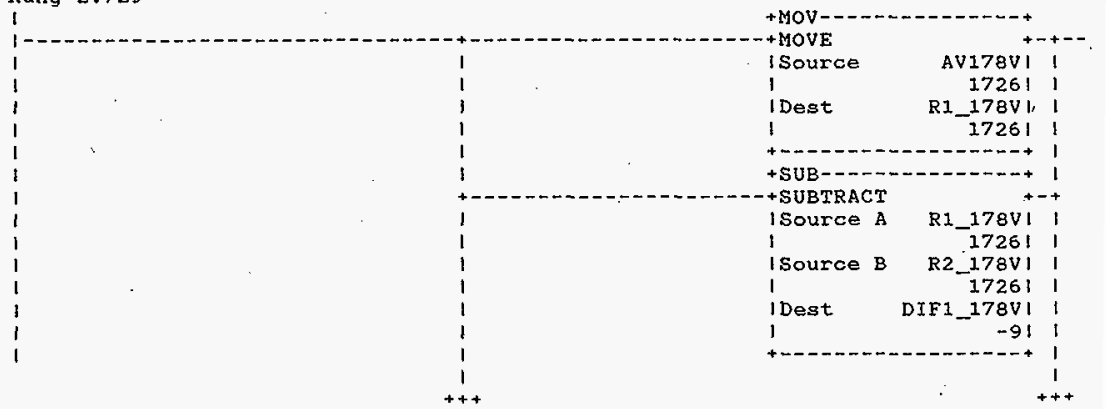

HNF-SD-FF-CSWD-61 Rev. 0

Page 670 


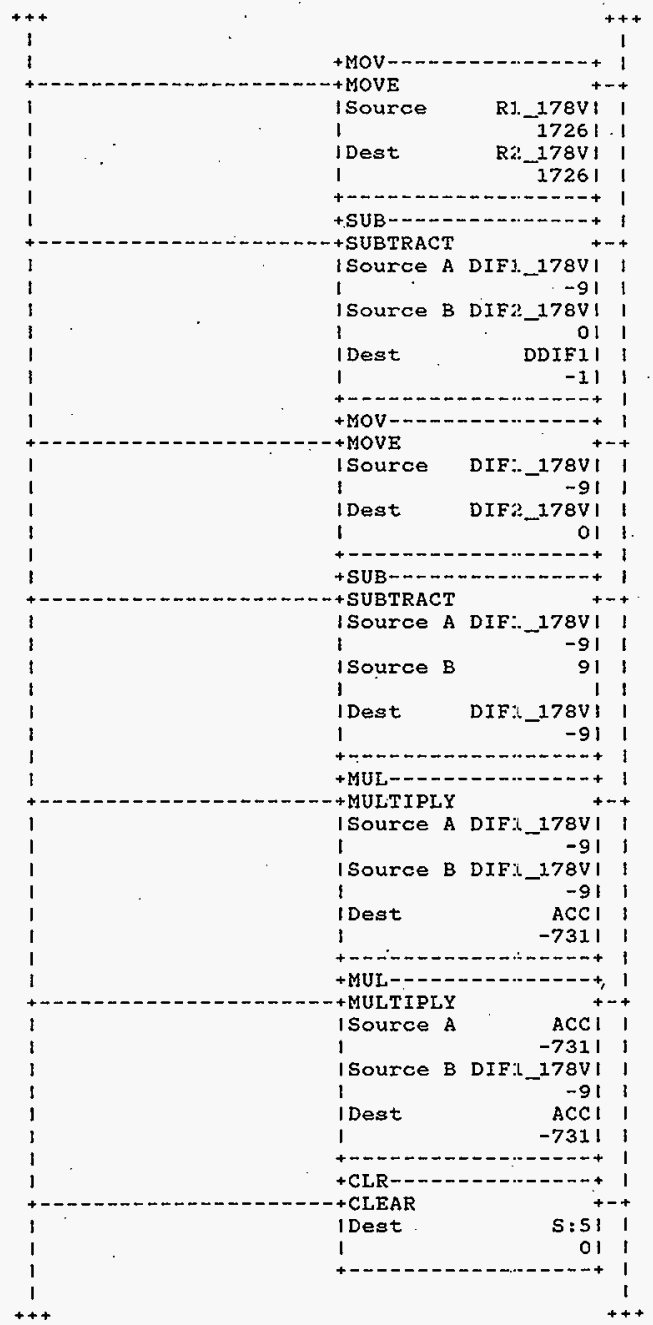

HNF-SD-FF-CSWD-61 Rev. 0 


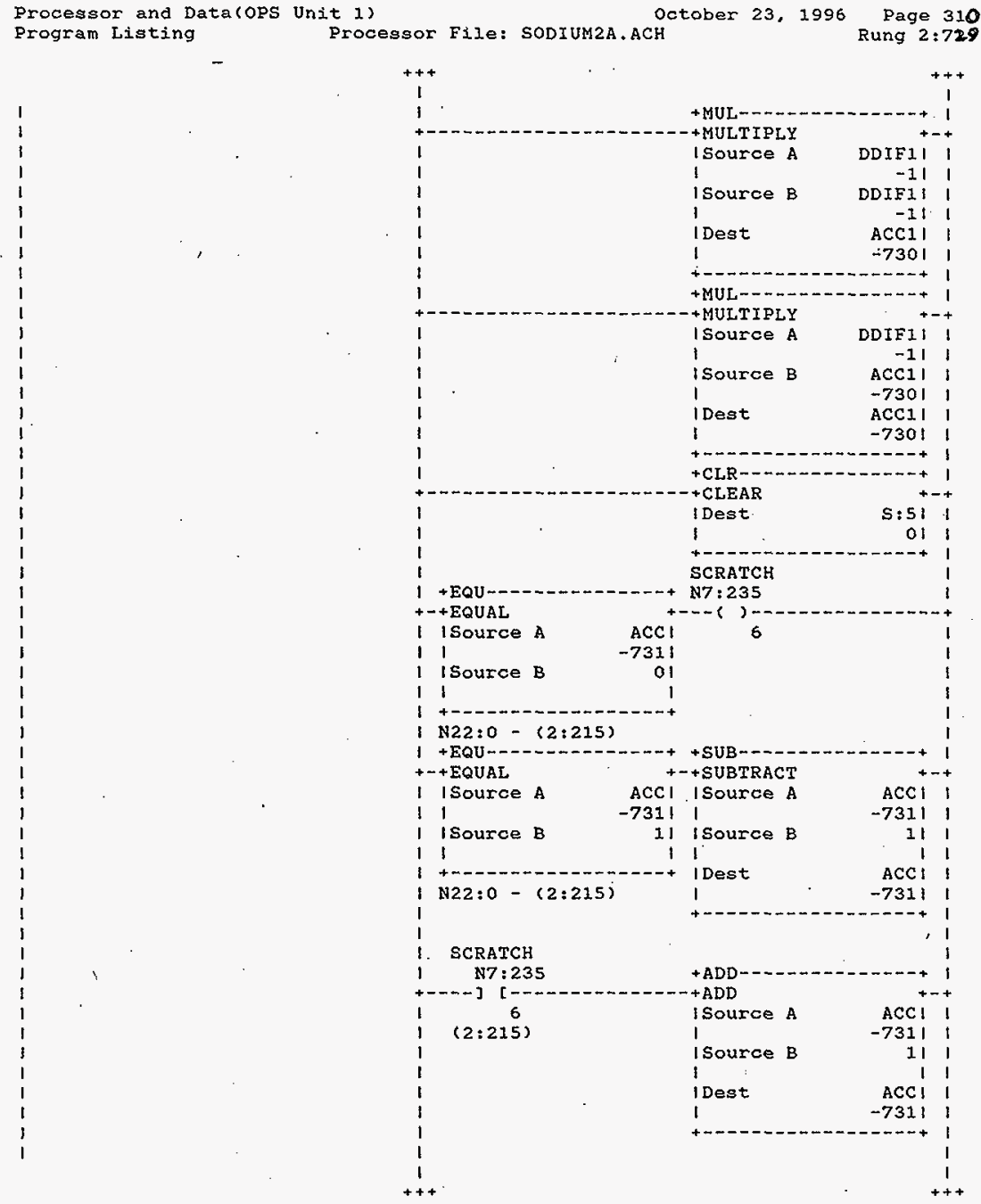

HNF-SD-FF-CSWD-61 Rev. 0 


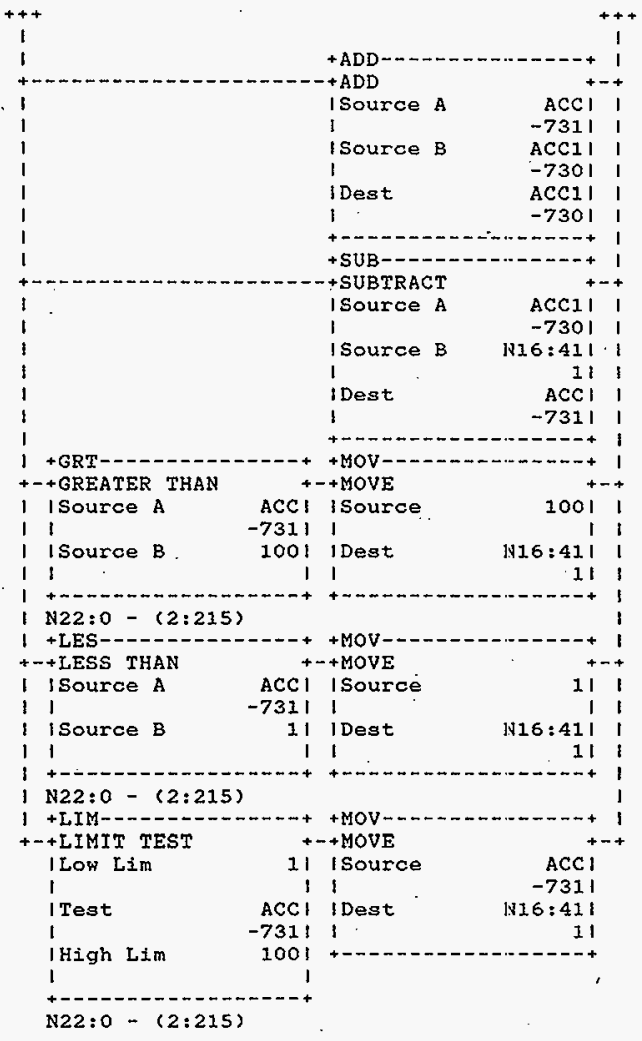

Rung 2:730

I PID178V

DUMMY 45

I 45

N22:9

1--- [LBL]

N22:9

1

HNF-SD-FF-CSWD-61 Rev. 0

Page 673 
Rung 2:731

DETECT FROM SCADA PID ON/OFF IF PID OFF

THEN ZERO PW FOR ZERO OUTPUT AT SCR AND JUMP AROUND THE PID BLOCK

ON178V
N7:233
1
1
1
1
1
1
1
1

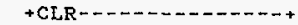

CLEAR

I Dest

1

(

1

84

$+--($ JMP $)$

Rung 2:732

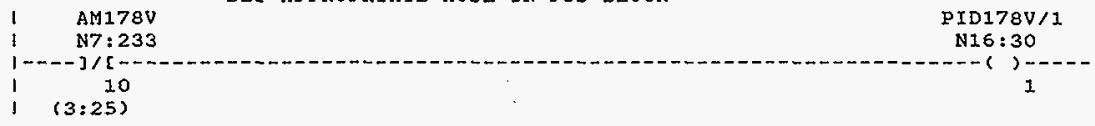

Rung 2:733

DETECT SETPOINT VALUE FROM SCADA

PUT VALUE IN PID BLOCK

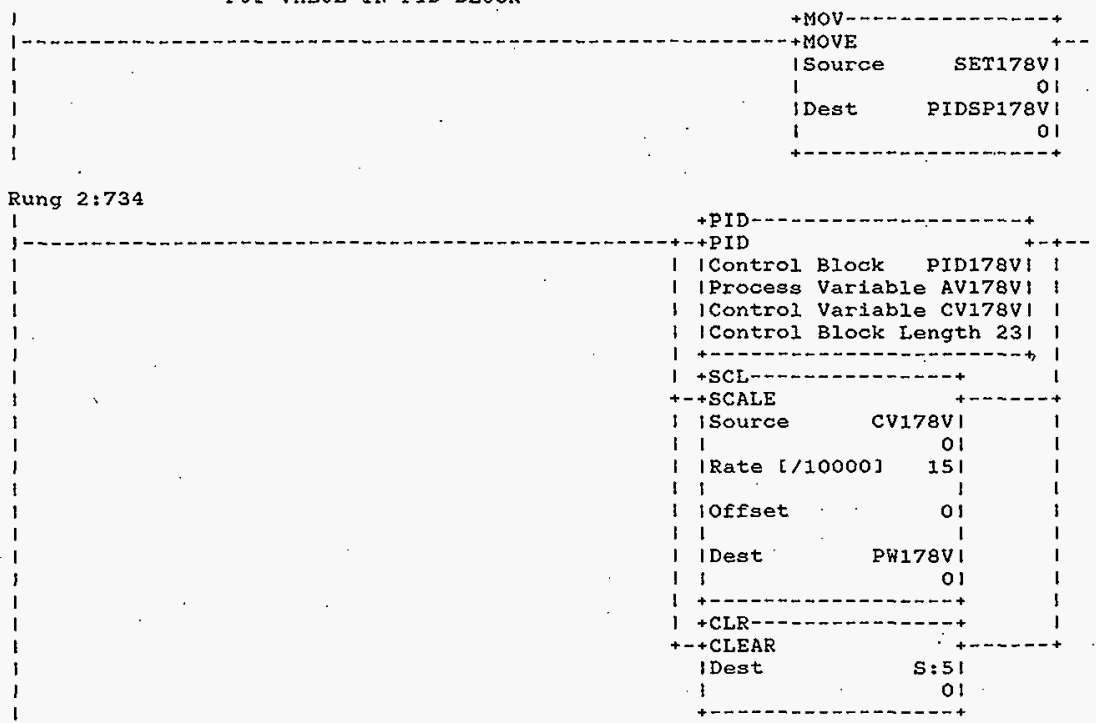

HNF-SD-FF-CSWD-61 Rev. 0 
Processor and Data(OPS Unit 1)

Rung $2: 735$

Rung 2:736

i RT_TMR/DN

1. $\overrightarrow{\mathrm{T}} 4: 87$

$1----] /[-$ DN

$$
\text { I (2:42) }
$$

Rung $2: 737$

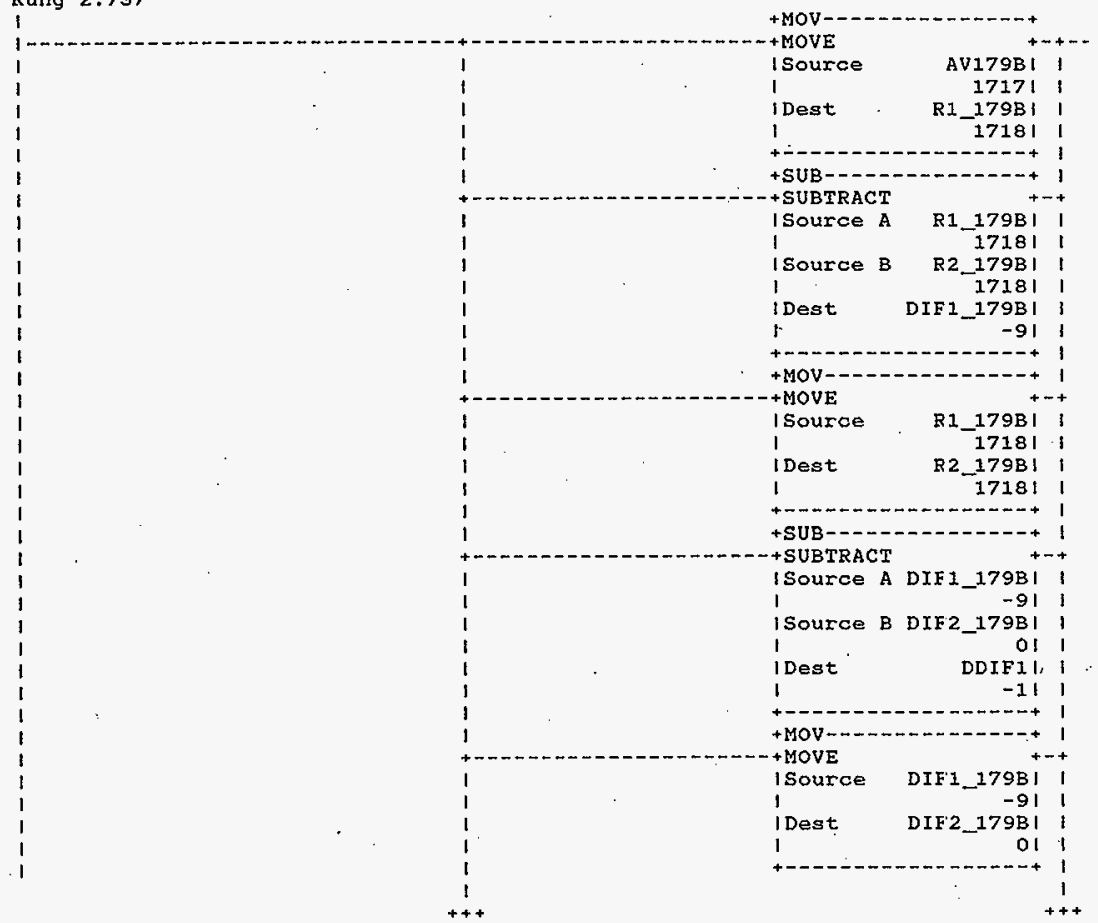

HNF-SD-FF-CSWD-61 Rev. 0 


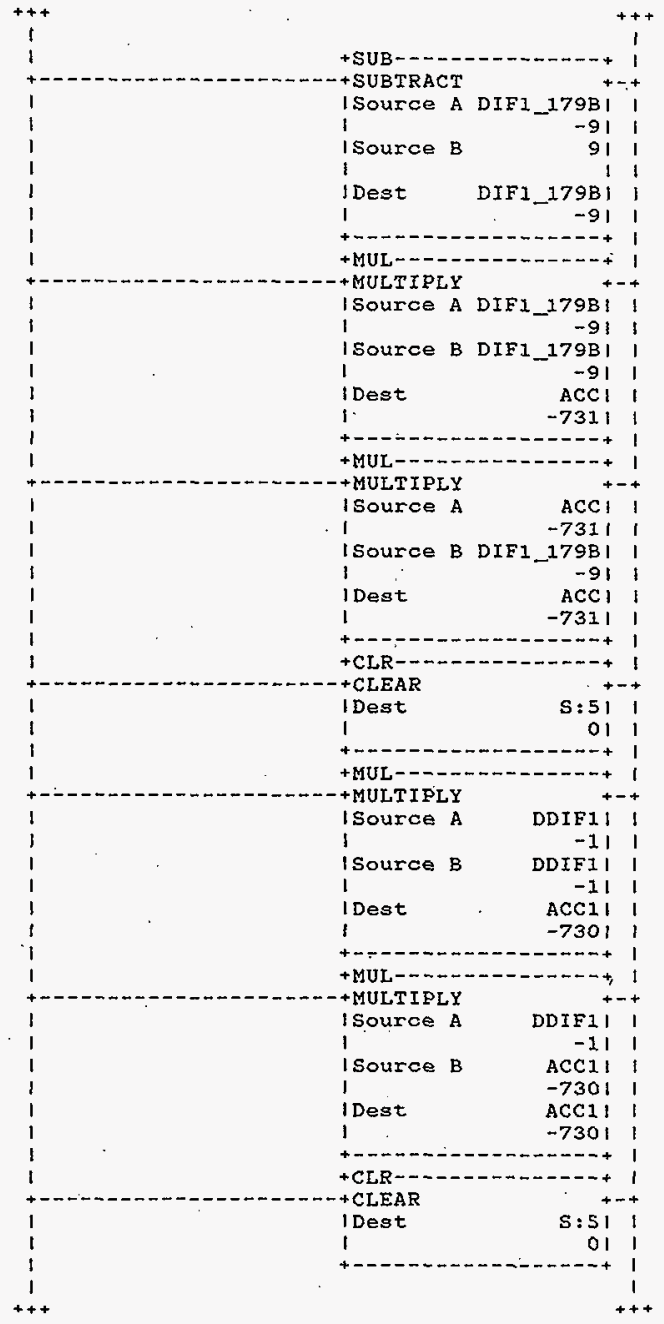

HNF-SD-FF-CSWD-61 Rev. 0 


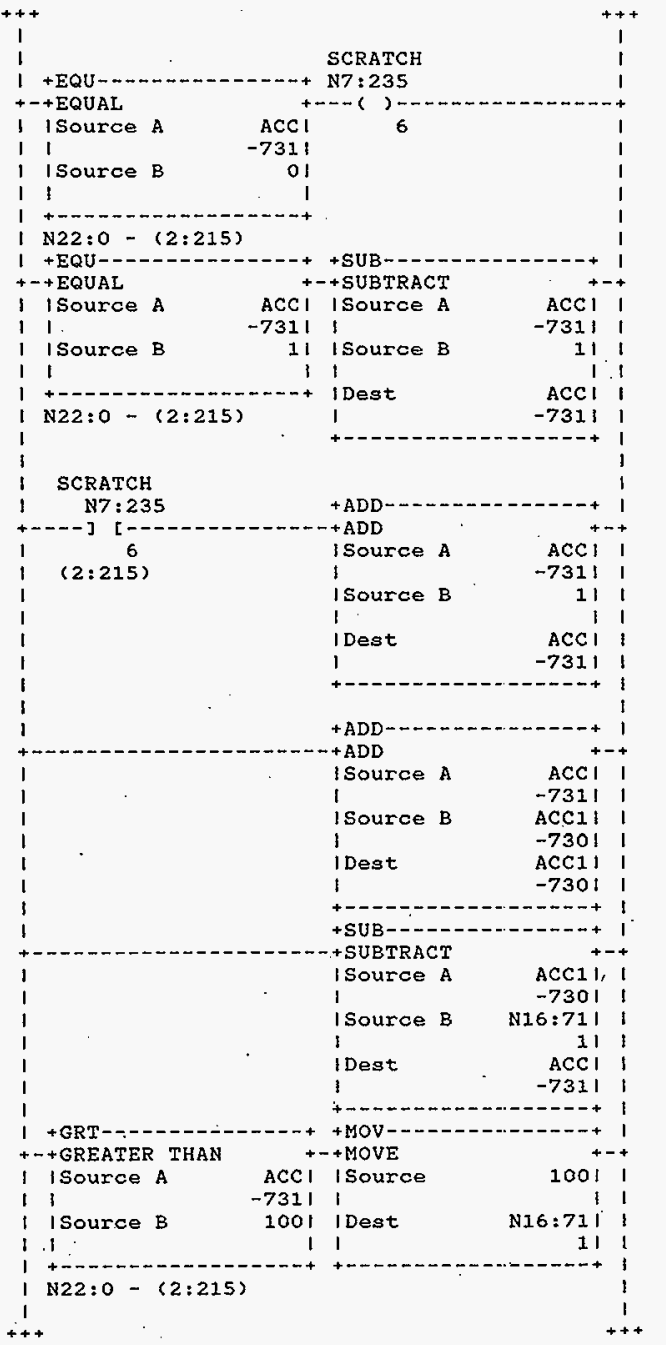

HNF-SD-FF-CSWD-fil Rev. 0 


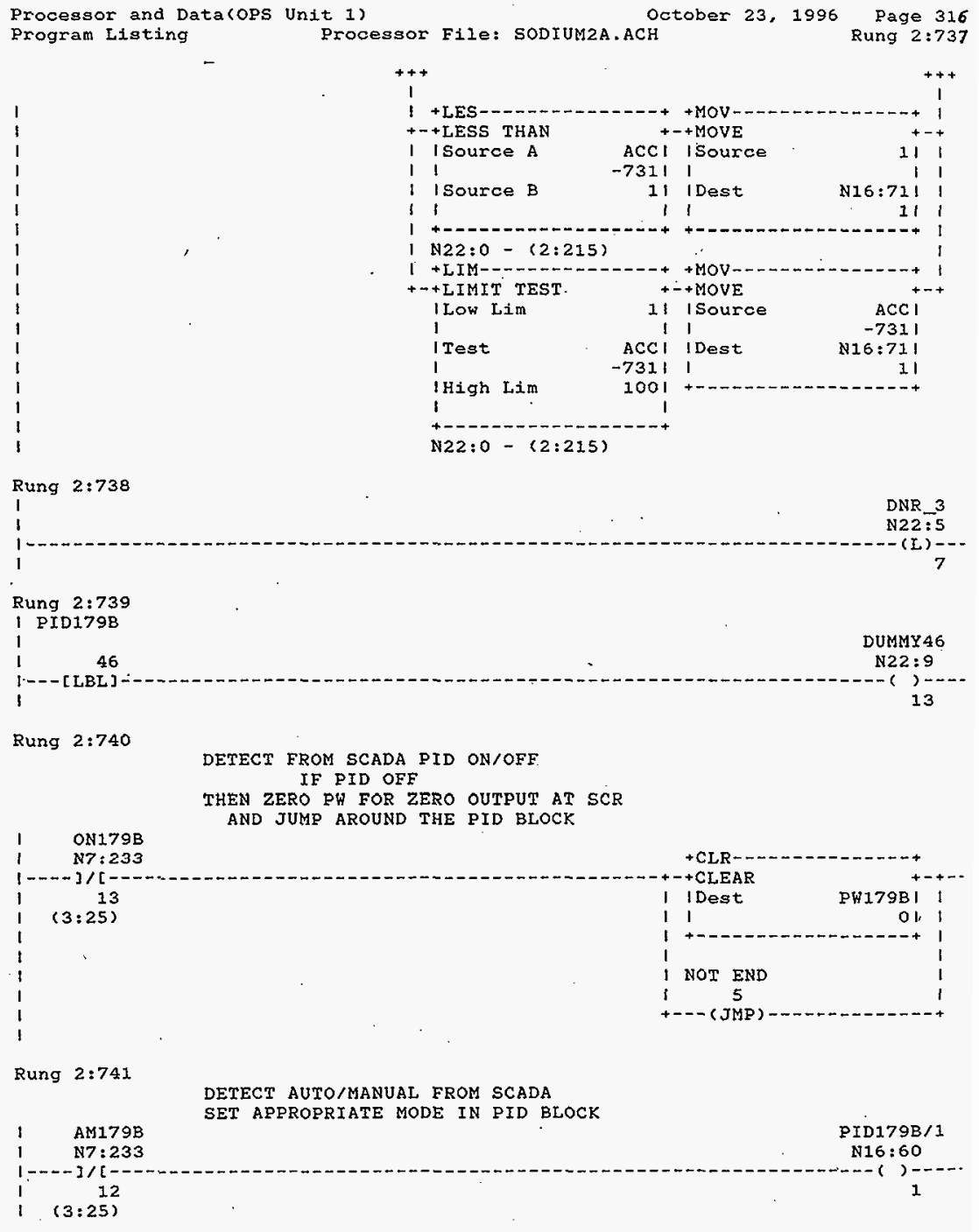

HNF-SD-FF-CSWD-61 Rev. 0 
Processor and Deta(OPS Unit 1 )

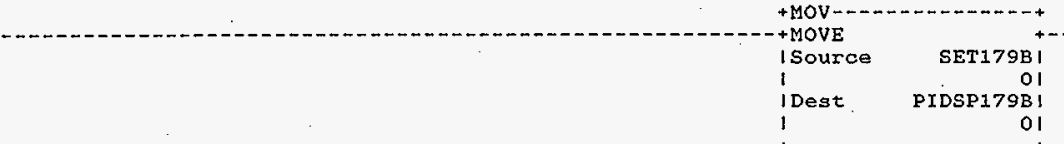

Rung $2: 743$

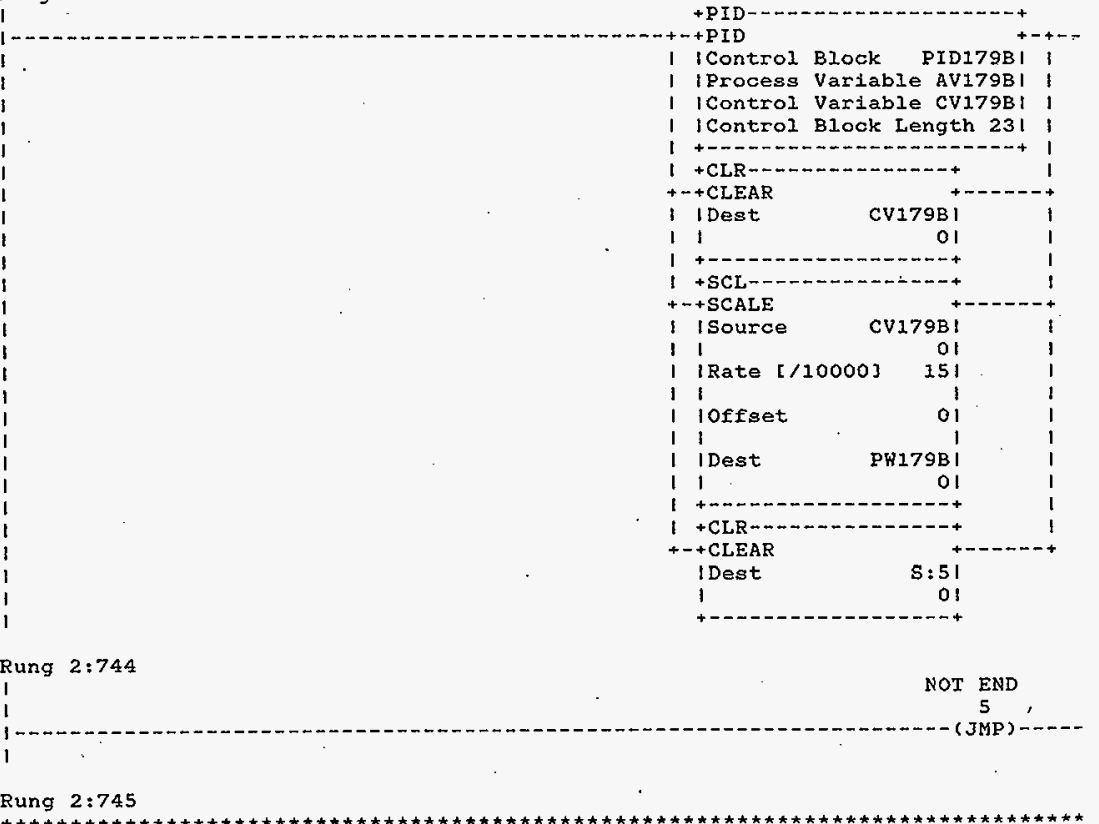

HNF-SD-FF-CSWD-61 Rev. 0 
Processor and Data(OPS Unit 1)

\section{Rung 2:746}

1

i

DN_4

N2立: 5

!

Rung $2: 747$

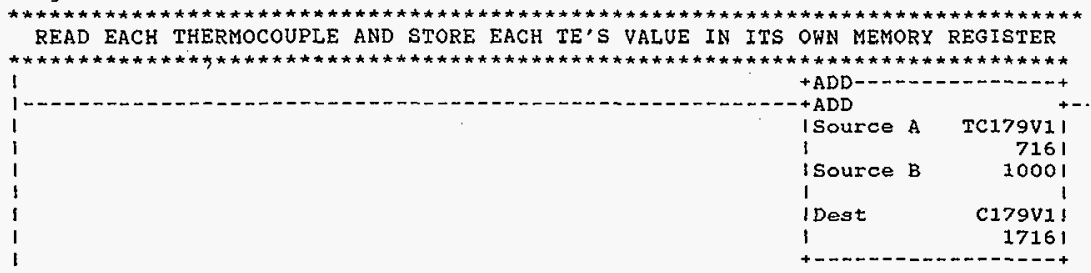

Rung 2:748

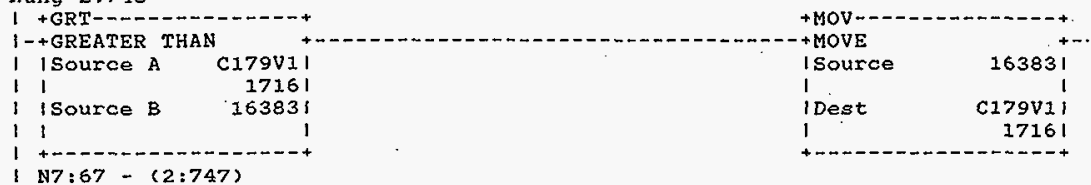

Rung $2: 749$

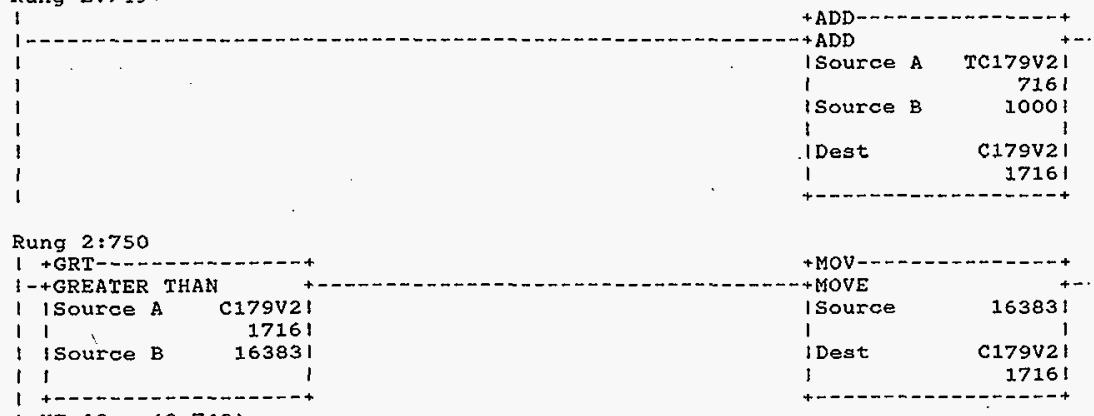

(N7:68-(2:749)

HNF-SD-FF-CSWD-61 Rev. 0 


\section{Rung $2: 751$}

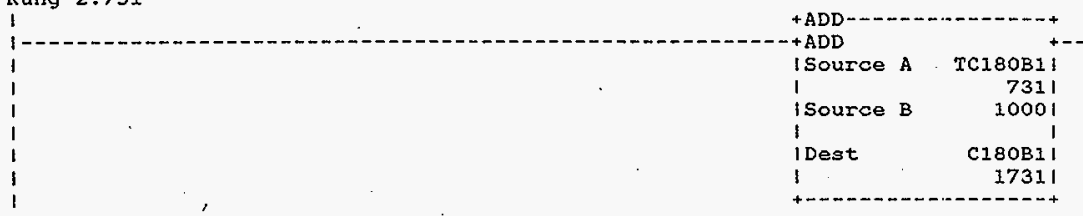

Rung $2: 752$

I +GRT-..--

1-+GREATER THAN

| 'Source A C180B1।

11 17311

| Source B 163831

11

163831

1

I N7:69-(2:751)

Rung $2: 753$

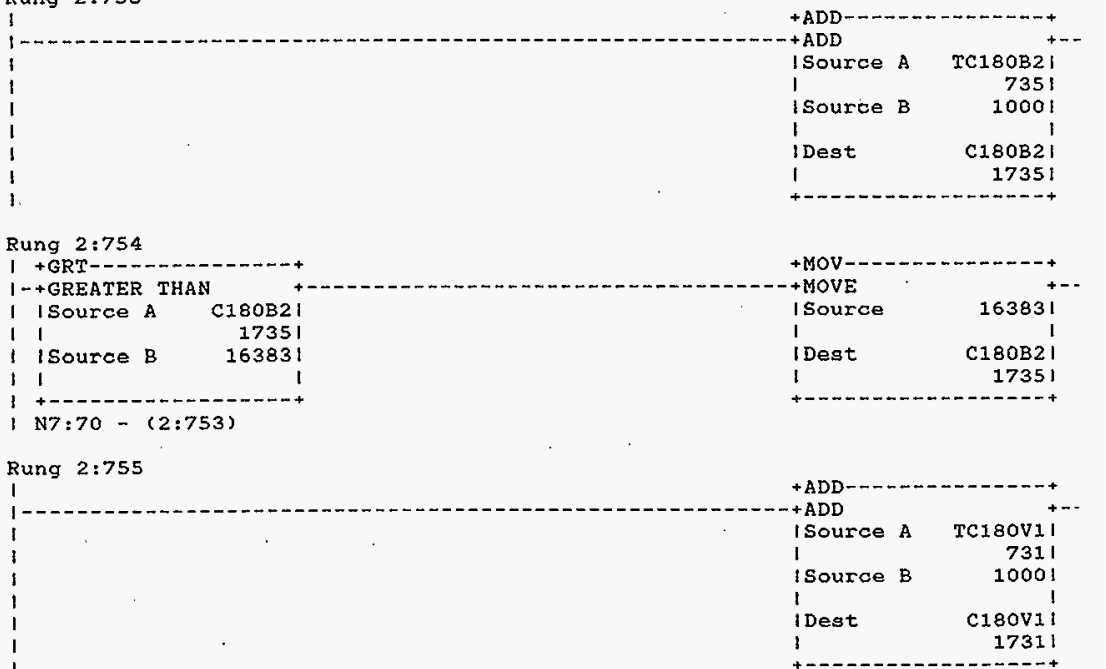

HNF-SD-FF-CSWD-61 Rev. 0 
Processor and Data(OPS Unit 1)

Program Listing

Processor File: SODIUM2A.ACH

October 23,1996 Page 320

Rung $2: 756$

1 + GRT--- - -

1-+GREATER THAN

I ISource A C180VII

11 17311

I ISource B 16383 |

11

I N7:71-(2:755)

Rung 2:757

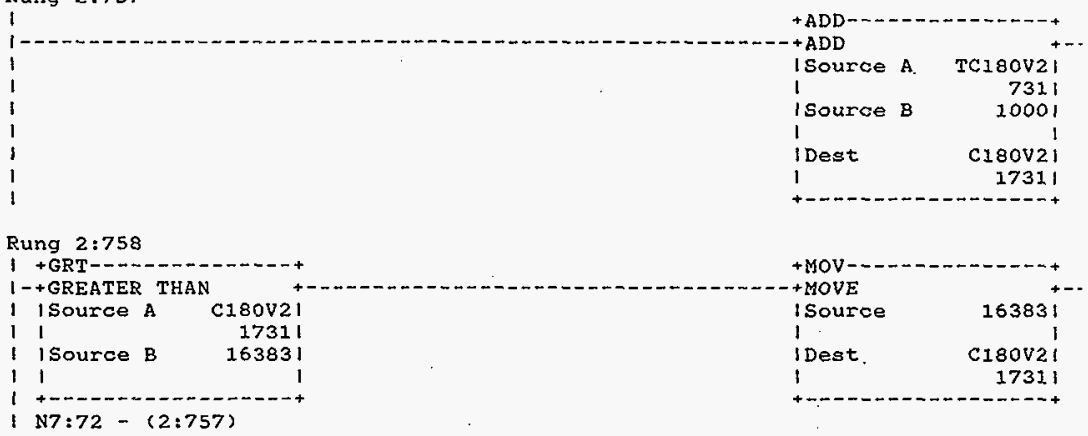

Rung 2:759

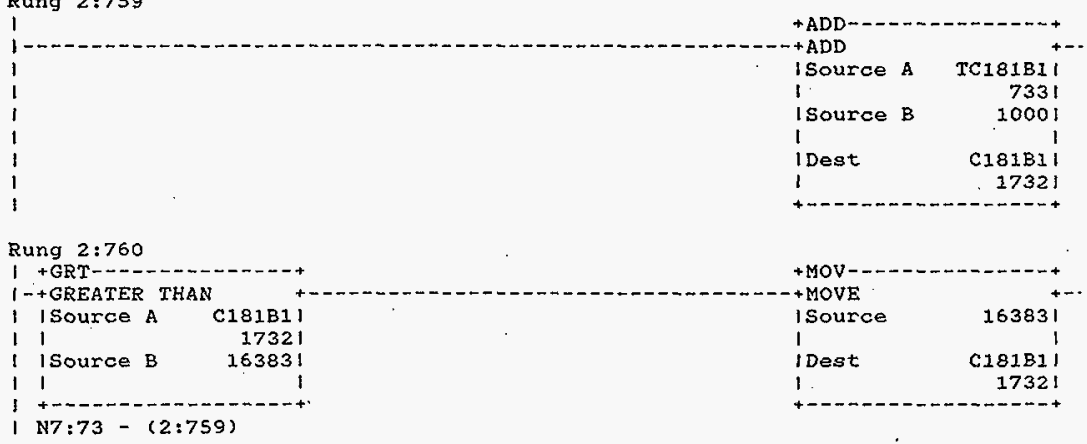

HNF-SD-FF-CSWD-61 Rev. 0

Page 682 
Processor and Data(OPS Unit 1)

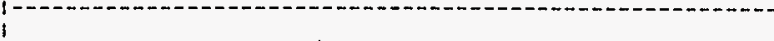

$+\mathrm{ADD}$

ADD

ISource A TC181B2|

7321

I Source B

$1000 !$

I Dest

C181B2:

I

17321

Rung $2: 762$

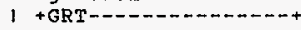

1-+GREATER THAN

| ISource A C181B2 I

I 17321

ISource B 16383

1

163831

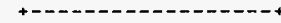

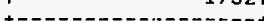

N7:74- $(2: 761)$

Rung 2:763

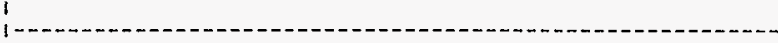

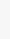

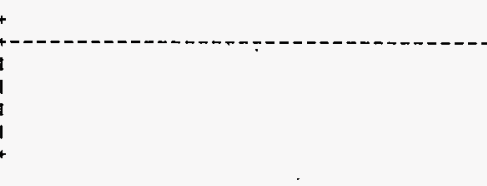

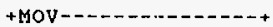

+ MOVE

163831

1

I Dest

1

1

1732 !

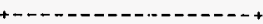

\section{Rung $2: 764$}

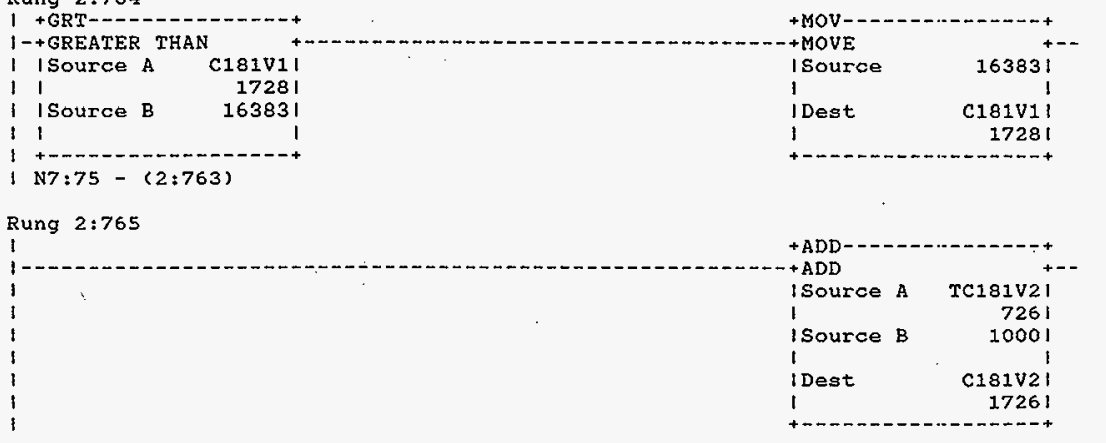

HNF-SD-FF-CSWD-61 Rev, 0

Page $6: 33$ 
Rung $2: 766$
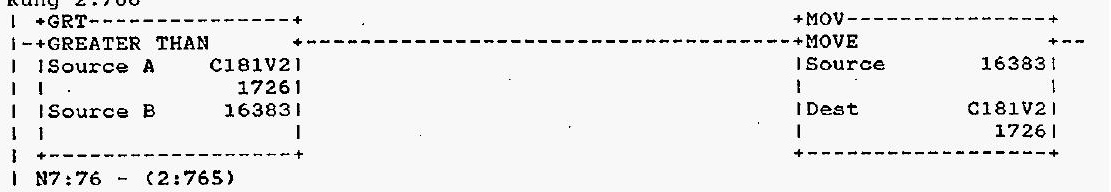

Rung 2:767

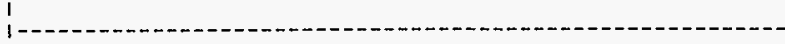

$+A D D$

ISource A TC182BI

7211

isource B

1000

i Dest

C182B1

1

17211

Rung 2:768
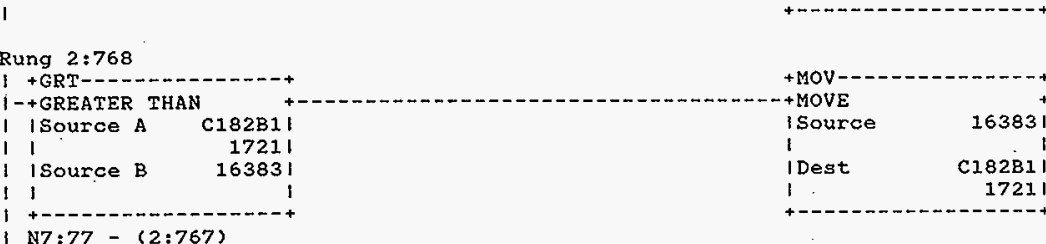

I

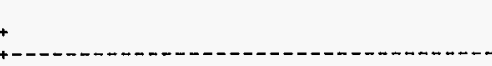

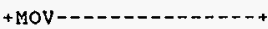

ISOU

163831

1

I Dest

c18281

I

17211

( N7:77-(2:767)

Rung 2:769

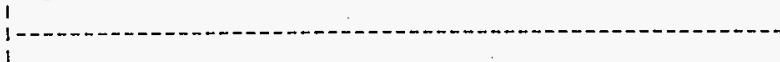

$+\mathrm{ADD}$

$+A D D$

ISource A TC182B2I

7231

isource $B$

10001

i Dest

C182B21

i

27231

Rung 2:770

$1+$ GRT--

1-+GREATER THAN

1 Isource A C182B2

I I 17231

I Isource B 16383 |

$+$

$--\infty+$

11

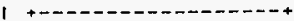

1 N7:78-(2:769)

HNF-SD-FF-CSWD-61 Rev. 0

Page 684 
Rung 2:771
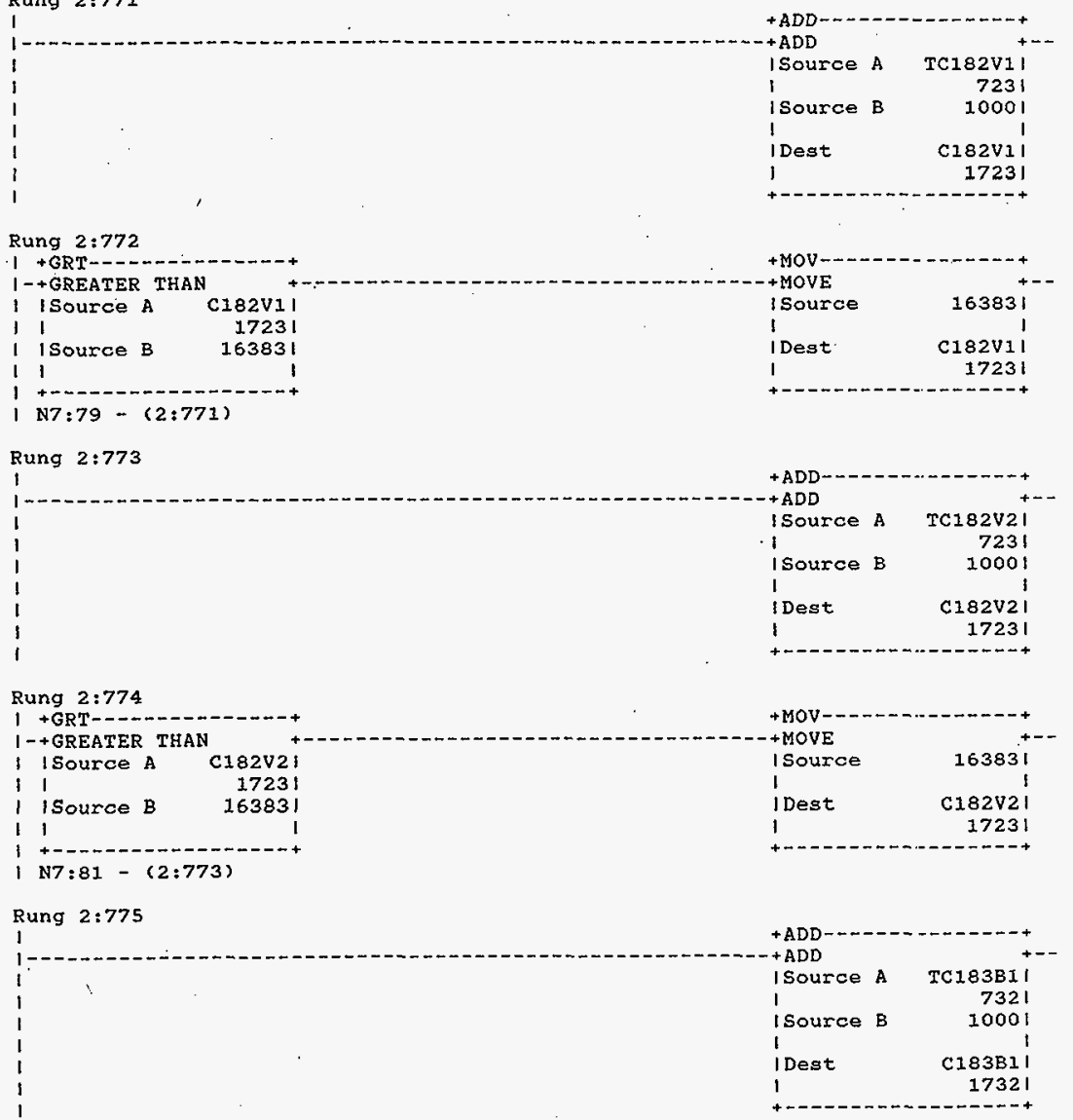

HNF-SD-FF-CSWD-61 Rev. 0 


\section{Rung 2:776}

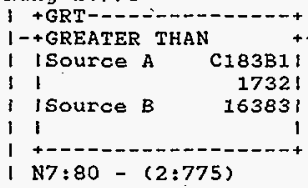

$$
\text { i }
$$

1
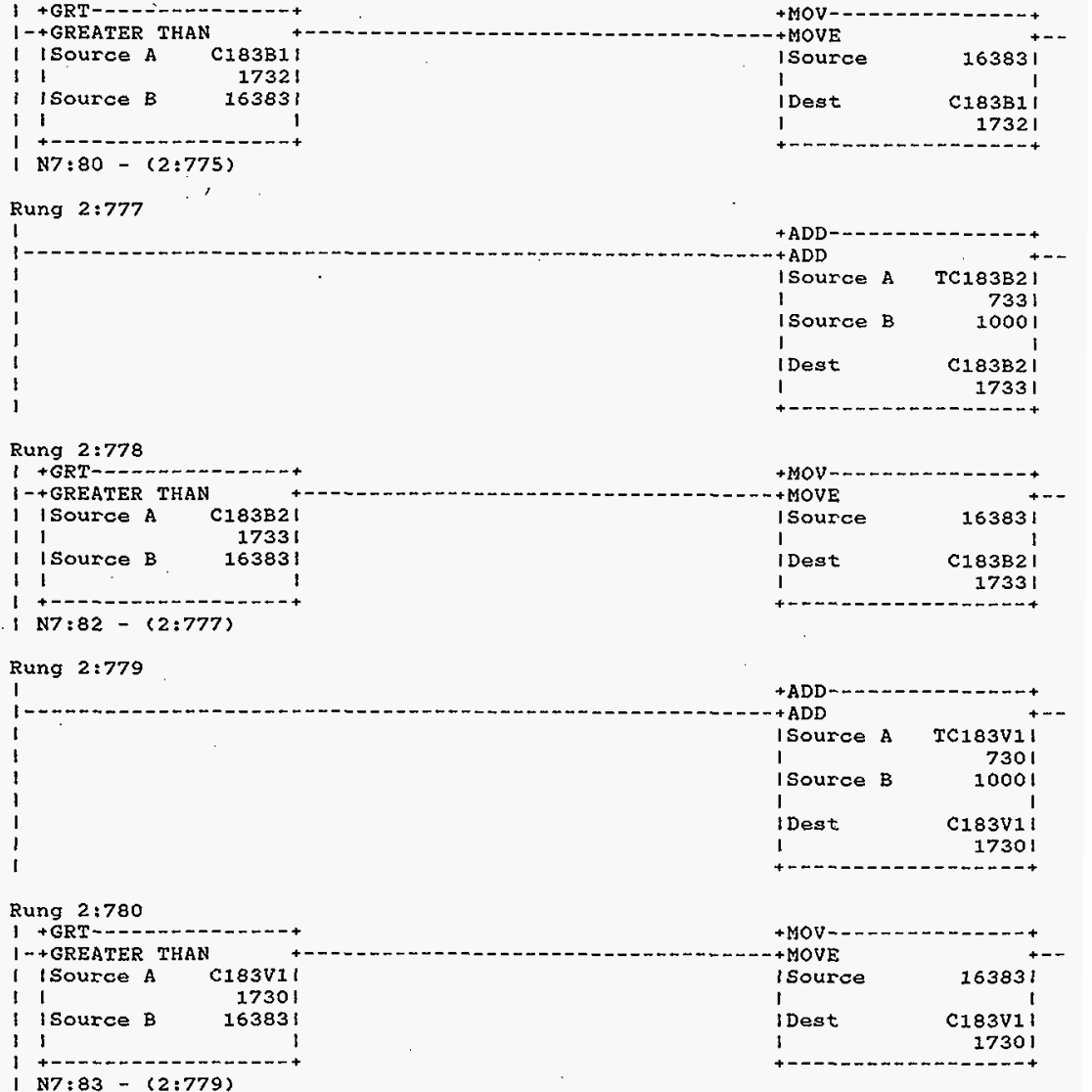

HNF-SD-FF-CSWD-61 Rev, 0 
Rung $2: 781$

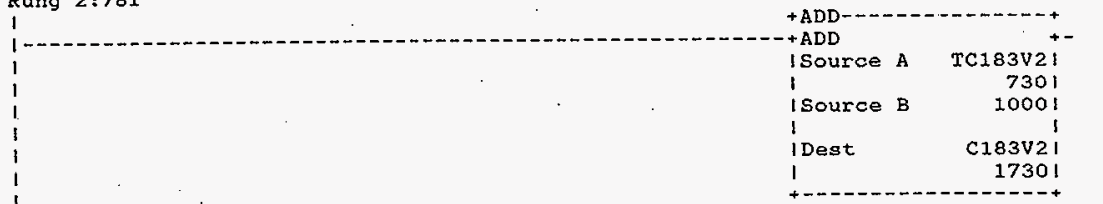

Rung $2: 782$

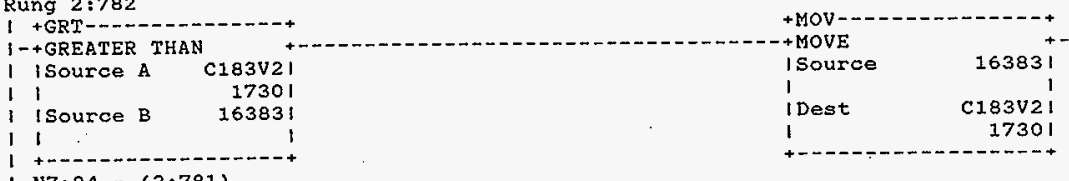

( N7:84-(2:781)

Rung 2:783

For each $T / C$ read its STATUS BITS to detect $T / C$ problems

T/C ENABLE

HAI79V1

I: 19

N7 : 228

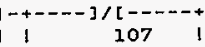

OPEN T/C

I $119 \quad$ I

+--.-] [ - - - - +

$1 ; 108$

I LO LIMIT

11

$1+-\infty-7$ [

$\begin{array}{lll}1 & 109\end{array}$

11

I 1 I:19

$1+\cdots-\ldots][-\ldots+\cdots$

11110

I CNEG ERROR I

I) 1 I: I9 ।

$1+---][--n+$

i 111

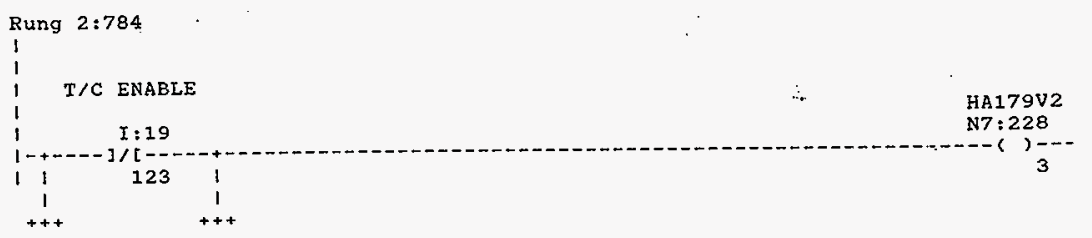

HNF-SD-FF-CSWD-61 Rev. 0 


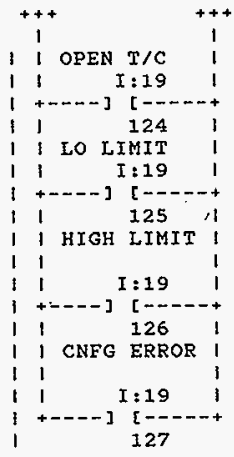

Rung 2:785

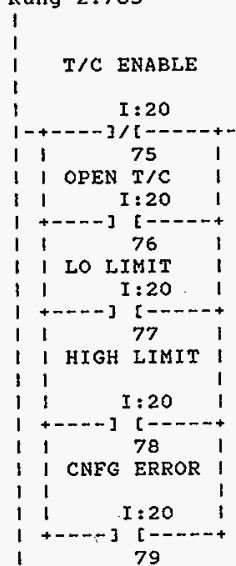

\section{Rung 2:786}

I

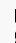

T/C ENABLE

$\mathrm{HA} 180 \mathrm{~B} 2$

1 I. $\quad I: 20$

N7 : 228

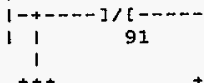

HNF-SD-FF-CSWD-61 Rev. 0 
Processor and Data(OPs Unit 1) Program Listing

October 23,1996 , Processor File: SODIUM2A.ACH
Page 327 Rung $2: 78 t$

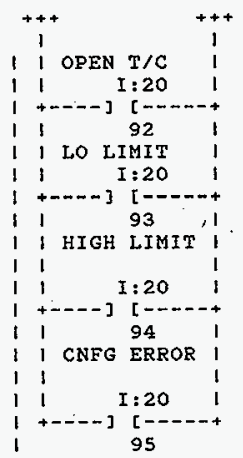

Rung 2:787

I

I T/C ENABLE

HA $180 V 1$

$1+2 \quad I: 20$ N7: 228

1

107

OPEN T/C

1

+.-. ] [ $1-.--+$

I 108

I LO LIMIT

I I: 20

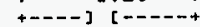

$1 \quad 109$

I HIGH LIMIT ।

1

I

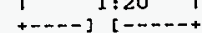

1110

I I CNFG ERROR

l I

$\begin{array}{ll}1 & 1: 20 \quad 1\end{array}$

I 111

\section{Rung 2:788}

1

$T / C$ ENABLE

$I: 20$

HA180V2

] $/$ [ -

N7: 22

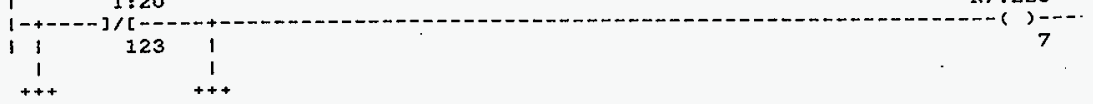

HNF-SD-FF-CSWD-61 Rev. 0

Page 689 
Processor and Data(Ops Unit 1) Program Listing

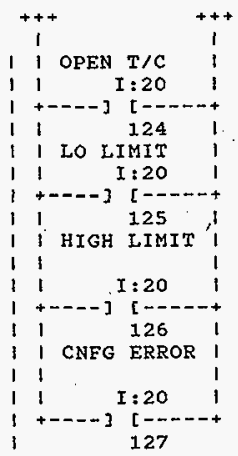

Rung $2: 789$

$1 T / C$ ENABLE

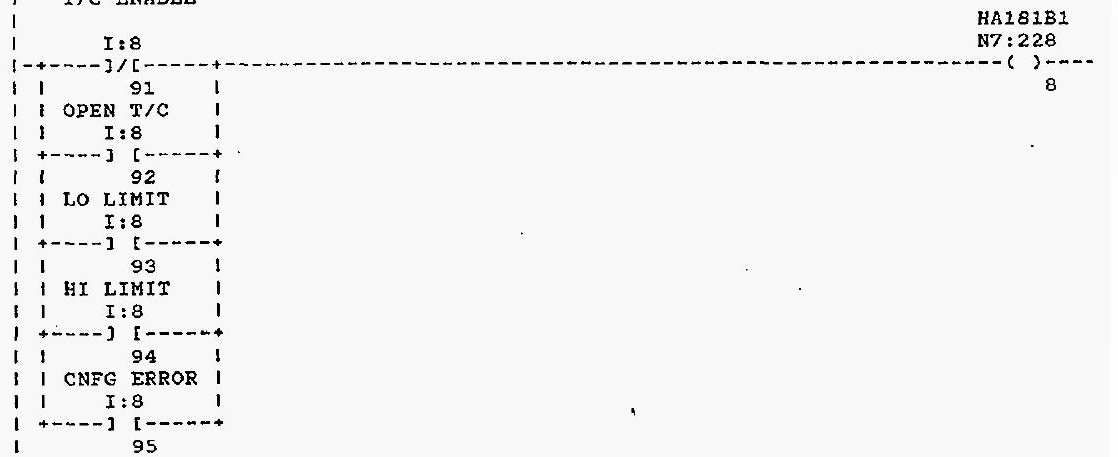

Rung 2:790

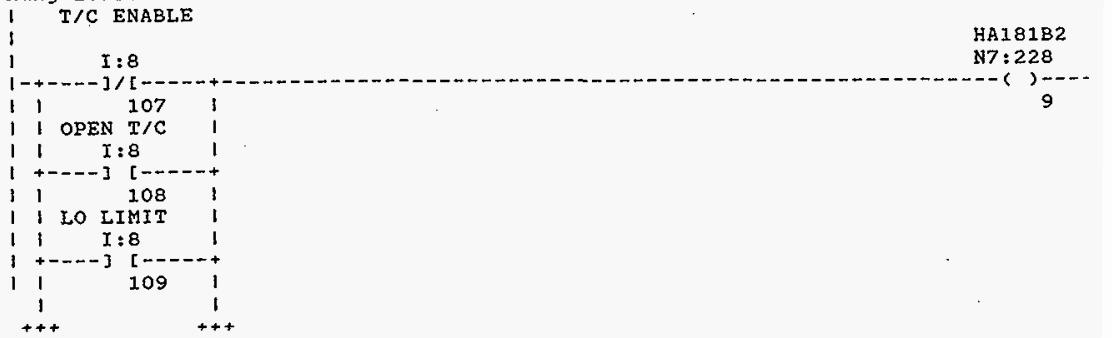

HNF-SD-FF-CSWD-61 Rev. 0 

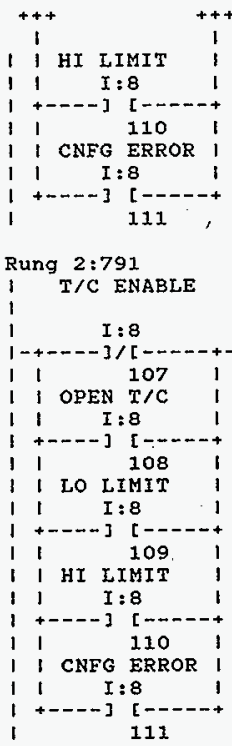

Rung 2:792

$$
\begin{array}{r}
1 \\
\text { I } \\
1 \\
1 \\
\text { I } \\
1 \\
1 \\
1 \\
1 \\
1 \\
1 \\
1 \\
\text { I } \\
1 \\
1 \\
1 \\
1 \\
1
\end{array}
$$

T/C ENABLE

HA181V2

N7 7228

$\mathrm{I}: 8$

1

I OPEN T/C

$++$

11

1 CNFG ERROR

$1+----][-----$ +

1 127

HNF-SD-FF-CSWD-61 Reiv. 0

${ }^{\text {Page }} 69.1$ 
Processor and Data(OPS Unit 1)

Rung $2: 793$

T/C ENABLE

$I: 3$

HA182B1

$3 /[$

I 75

I OPEN $\mathrm{T} / \mathrm{C}$

$I: 3$

$+\ldots][-\ldots-1$

I 76

I LO LIMIT

1 I $I: 3$

$1+---\infty]$

$1 \quad 77$

1 HI LIMIT

$11 \quad I: 3$

$1+\cdots-3$ [ $1----+$

I I CNFG ERROR

11

11

$1+\ldots .+][-\ldots-+$

179

Rung $2: 794$

1

I T/C ENABLE

$\begin{array}{rr} & \cdot \text { HA182B2 } \\ & \text { N7:228 }\end{array}$

$\begin{array}{lll}1 & 1 & 0 \\ 1 & 91\end{array}$

OPEN $\mathrm{T} / \mathrm{C}$

$I: 3$

+-

I 92

I LO LIMIT

$1 \quad I: 3$

+---7 ]

193

1 HI LIMIT

1 I:3

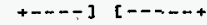

I 94 ।

I I CNFG ERROR ।

I 1

11

$1: 3 \quad$ ।

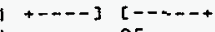

I 95 
Processor and Data(ops Unit 1) Program Listing

Processor File: SODIUM $2 \mathrm{~A} . \mathrm{ACH}$

October 23,1996

\section{Rung 2:795}

1

T/C ENABLE

$I: 3$

$1-+----7 /[+$

OPEN $\begin{array}{r}107 \\ T / C\end{array}$

$1 \quad I: 3$

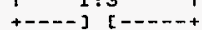

I $108 \quad$ I

IO LIMIT

$\mathrm{I}: 3$

+----] [--.--

I 109

1 HI LIMIT

$1 \quad I: 3$

$+----1$

1

I CNFG ERROR

I I: 3

+-- ] [

111

Rung 2:796

1

T/C ENABLE

$I: 3$

I: 3

123

OPEN $T / C$

$I: 3$

+---n 6 [-n.-.

I 124

1 LO IIMIT

I $I: 3$

+----] [ - - - +

I 125

1 HI LIMIT

1 I $1: 3$

$1+\cdots--]$ [.---+

11

I I CAFG ERROR I

11

$11 \quad I: 3$

$1+---7][-\ldots-n$

I 127
HA182V1

N7 : 228

14

HA182V2
N7:228
15
0.
0.
. 


\section{Rung 2:797}

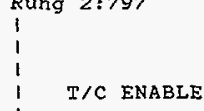

T/C ENABLE

$I: 4$

HA $183 B 1$

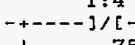

1 OPEN $T / C$

N7: 229

1 I: 4

$n+\ldots]$

1176

I LO LIMIT

I I I:4

+

177

I HI LIMIT

1 I $1: 4$

+----j [---n+

178

1 CNFG ERROR ।

I I I:4

I +---n] [--.--

I 79

Rung $2: 798$

I

I

I T/C ENABLE

\section{TNABLE}

$I: 4$

$\mathrm{HA} 183 \mathrm{~B} 2$

]$/[$

91

OPEN $\mathrm{T} / \mathrm{C}$

$I: 4$

+...- $][\ldots$

192

1 LO LIMIT

1. $\quad I: 4$

$1+\cdots-\infty]$

I HI LIMIT

I) I:4

1 +----] $\left[\begin{array}{l}1 \\ 1\end{array}\right.$

I CNFG ERROR ।

I I:4 ।

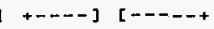

195

HNF-SD-FF-CSWD-61 Rev. 0

Page 694 


\section{Rung 2:799}

\section{T/C ENABLE}

$I: 4$

HA183V1

$1 / \mathrm{t}$

N7:229

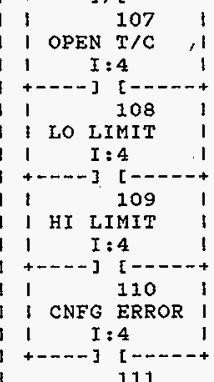

Rung 2:800

I

\section{T/C ENABLE}

$1: 4$

HA183V2

N7: 229

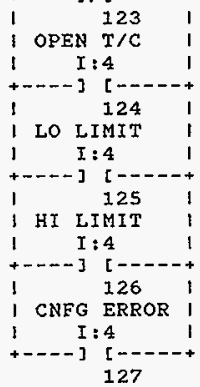

Rung $2: 801$

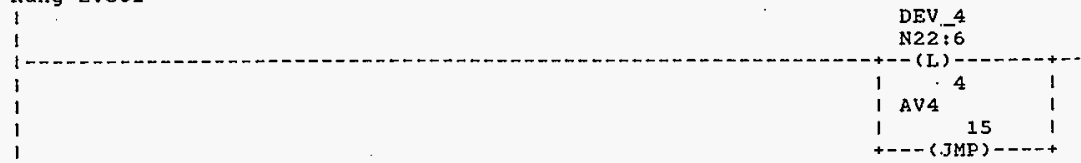

HNF-SD-FF-CSWD-61 Rev. 0

Page 695 
Processor and Data(OpS Unit 1)

Rung 2:802

I DEV4

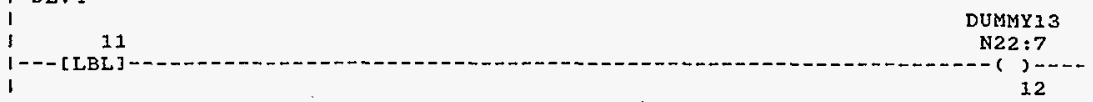

Rung $2: 803$

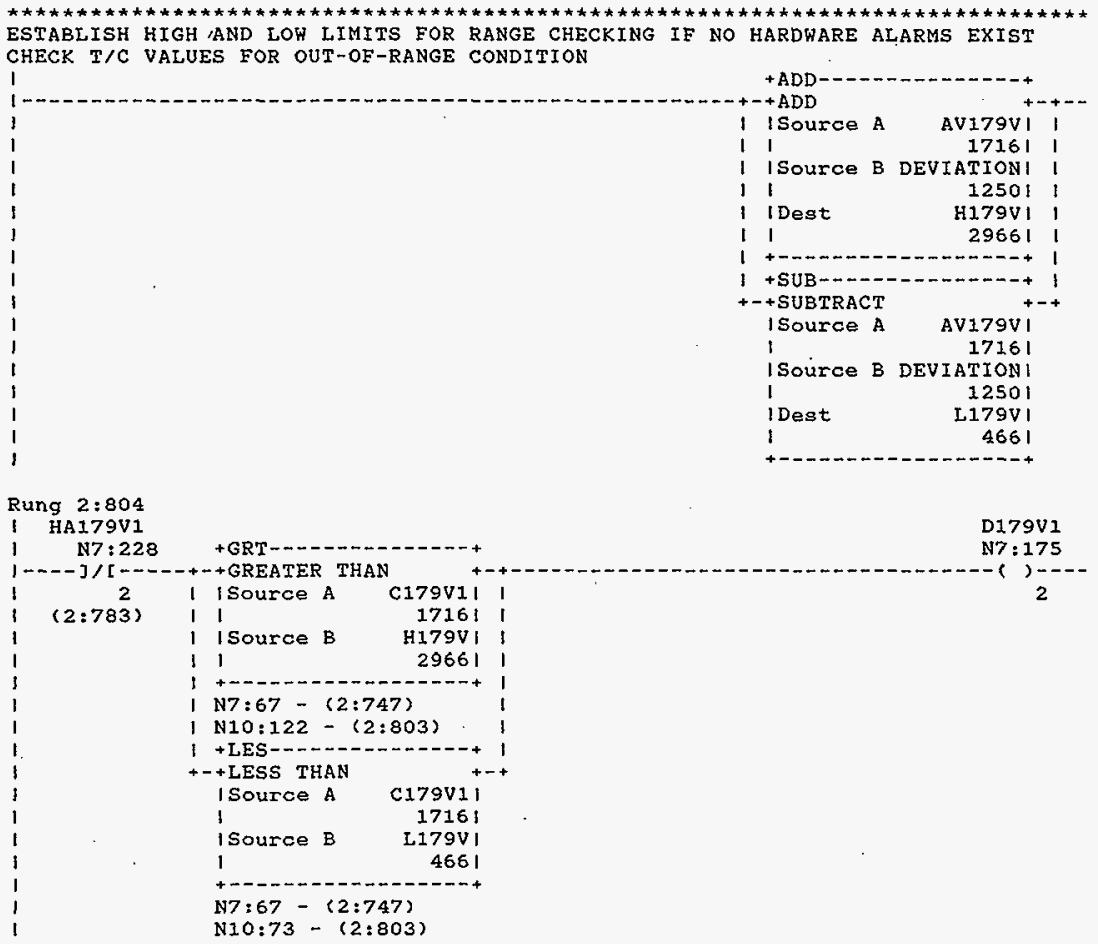

HNF-SD-FF-CSWD-61 Rev. 0 


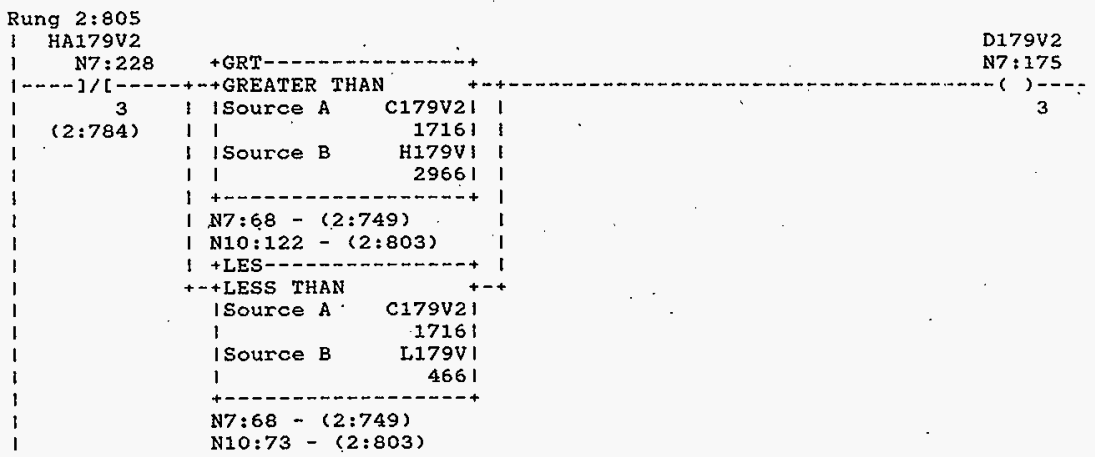

Rung 2:806

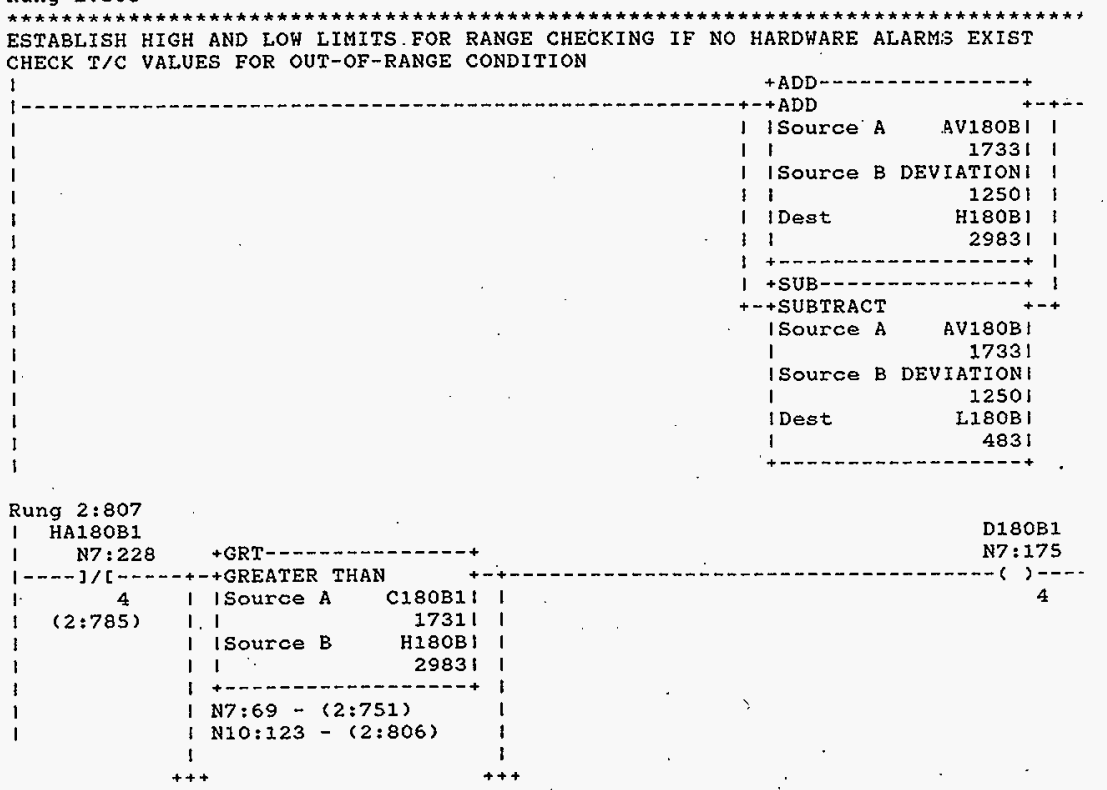

HNF-SD-FF-CSWD- 61 Rev. 0 


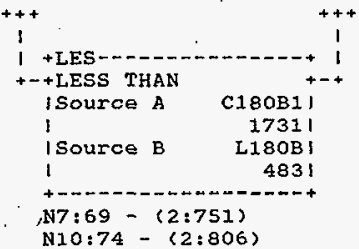

Rung 2:808

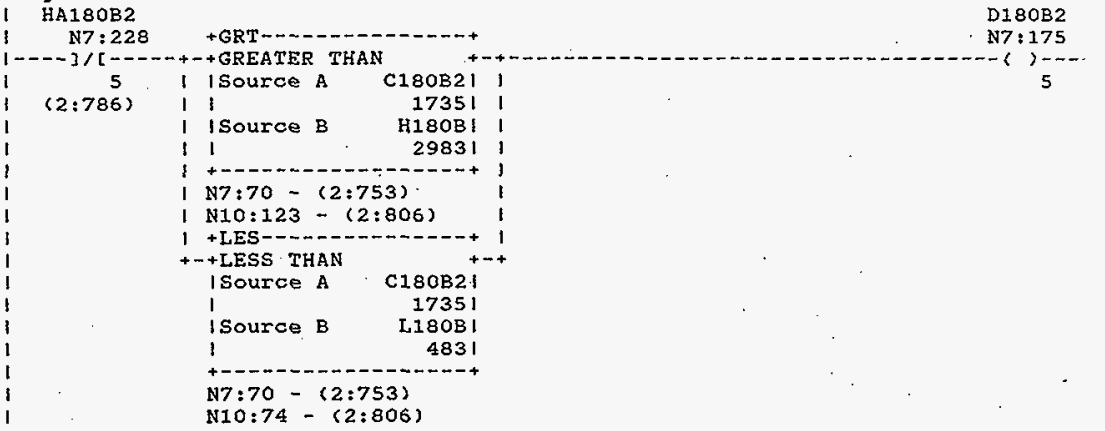

\section{Rung 2:809}

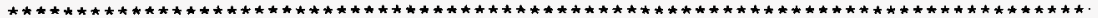
ESTABLISH HIGH AND LOW LIMITS FOR RANGE CHECKING IF NO HARDWARE ALARMS EXIST CHECK T/C VALUES FOR OUT-OF-RANGE CONDITION

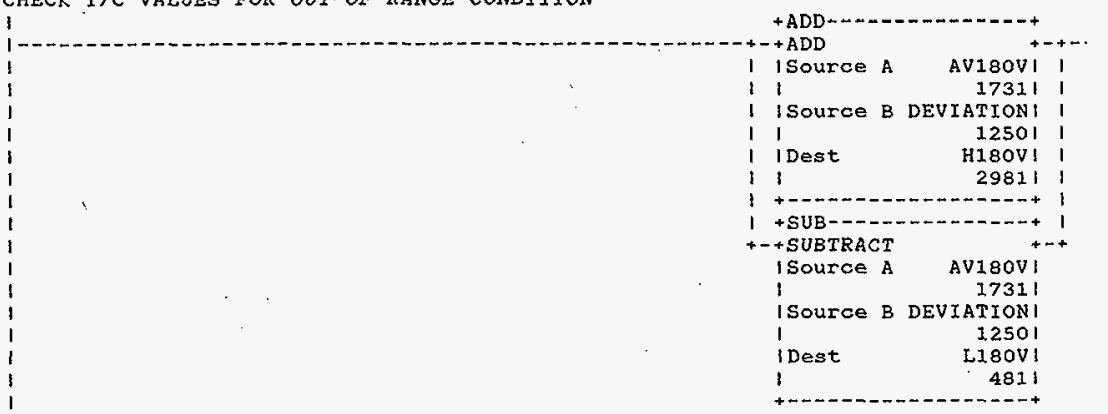

HNF-SD-FF-CSWD-61 Rev. 0 
Processor and Data(OPS Unit 1)

Rung 2:810

HA180V1

N7:228

+GRT--- - - - - - - - - - - - +

D180V1

$1---1 /[--\infty+++$ GREATER THAN

(2:787)

I I Source A C

C180V1।

N7 : 175

1 source $B$

11

1 + -

(1,N7:71-(2:755)

( N10:124-(2:809)

I +LES--..-----------t

+-+ LESS THAN +-

ISource A Cisovil

$1 \quad 17311$

I Source B L180VI

I

4811

+--- - - - - - - - - - - - +

N7:71-(2:755)

$\mathrm{N} 10: 75-(2: 809)$

Rung $2: 811$

1 HA180V2

1 1 $7: 228$

i----]/[---+-+GREATER THAN

I I SOurce A Cr8OV2

(2.788) I 1731

| isource B H180VI

11

29811 ।

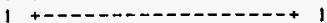

(N7:72 - (2:757)

( $N 10: 224-(2: 809)$

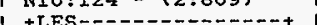

+-+ LESS THAN +-+

ISource A C180V2I

1

17311

isource B ILI80V|

1

4811

N7:72-(2:757)

N10:75-(2:809)

Rung 2:812

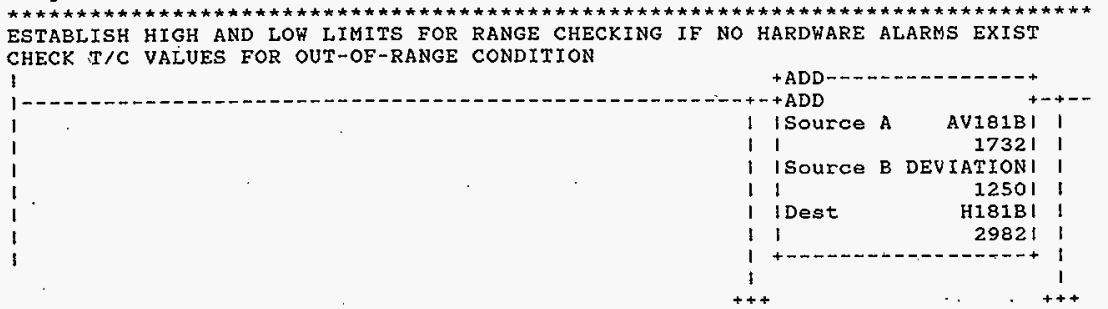

HNF-SD-FF-CSWD-61 Fev. 0

page 649 
Processor and Data(oPs Unit 1)

October 23,1996

Page 338

1
+++
1

$1+$

+-+ SUBTRACT

ISource A AV181B|

17321

ISource B DEVIATION I

I

12501

IDest L181B।

I

4821

Rung $2: 813$

I HA181B1

$1 \quad$ N7 $7: 228$

482

$1---] /[-\ldots+++$ GREATER THAN

18 I ISource A C181B1I I

(2:789)

11

1 Isource B

1732 I

H18 $131 ;$

$29821 \mid$

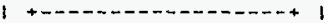

I N7:73-(2:759)

I N10:125 - (2:812)

$1+$ LES--- - - - - - . - . - +

+-+ LESS THAN

ISource A

C $181 \mathrm{B11}$

1

17321

|Source B L.181B|

1

4821

$N 7: 73-(2: 759)$
$N 10: 76-(2: 812)$

Rung 2:814

I

I N7:228

$1-+--] /[\ldots-\cdots++$ +GREATER THAN

| 9 | ISource A CI81B21

(2:790)

11

ISource B

17321

H181B!

11

$1+--\cdots--$

I $N 7: 74-(2: 761)$

( N10:125 - (2:812)

$1+$ LES---.---.---..+

++ LESS THAN

ISource A CI81B21

1

17321

[Source B L181B\}

1 4821

N7:74-(2:761)

N10:76-(2:812)

HNF-SD-FF-CSWD 61 Rev, 0 
Processor and Data(OPS Unit 1 )

Rung 2:815

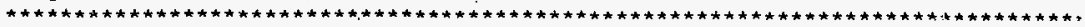
ESTABLISH HIGH AND LOW IIMITS FOR RANGE CHECXING IF NO HARDWARE ALARMIS EXIST CHECK T/C VALUES FOR OUT-OF-RANGE CONDITION

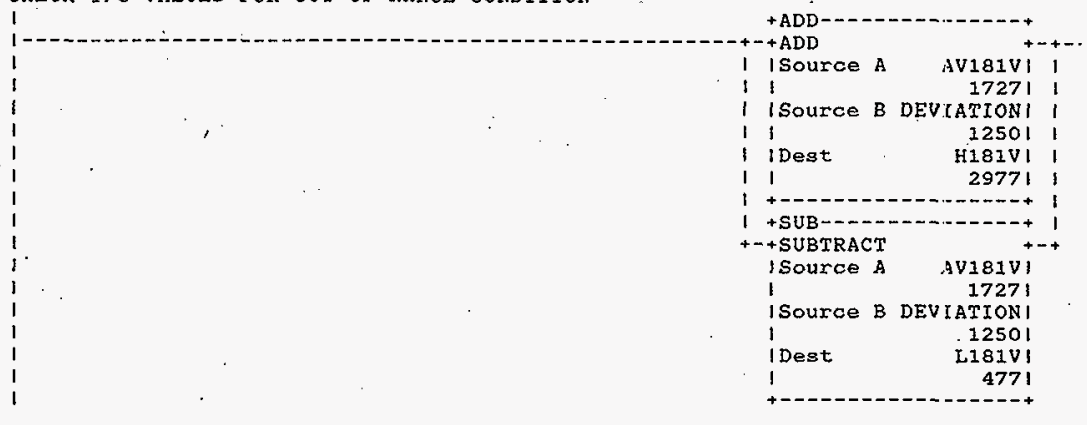

Rung 2:816

I HA181VI

I N7:228

+GRT-2.-. -

D181V1

I----]/[---+-+GREATER THAN

10 I ISource A C181V1I ।

$(2: 791)$

N7: 175

I ISource B H181VI ।

1129771

$1+\cdots+-1$

I N7:75 - (2:763)

( N10:126-(2:815)

i +LES-------------+ 1

+-+LESS THAN

I Source A

C181V1I

1

17281

I Source B L181V I

I

4771

N7:75-(2:763)

N10:77-(2:815)

Rung 2:817

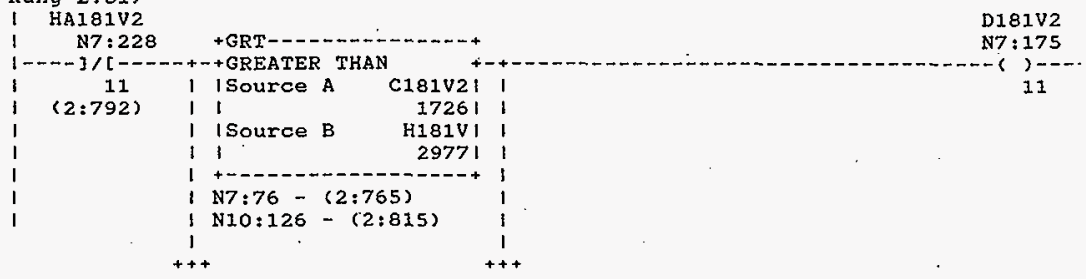

HNF-SD-FF-CSWD-61 Rev. 0 


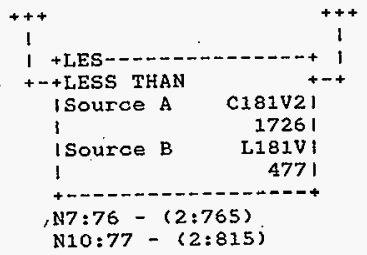

Rung $2: 818$

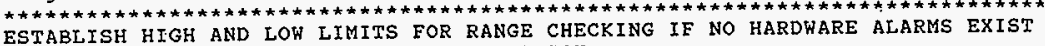
CHECK T/C VALUES FOR OUT-OF-RANGE CONDITION

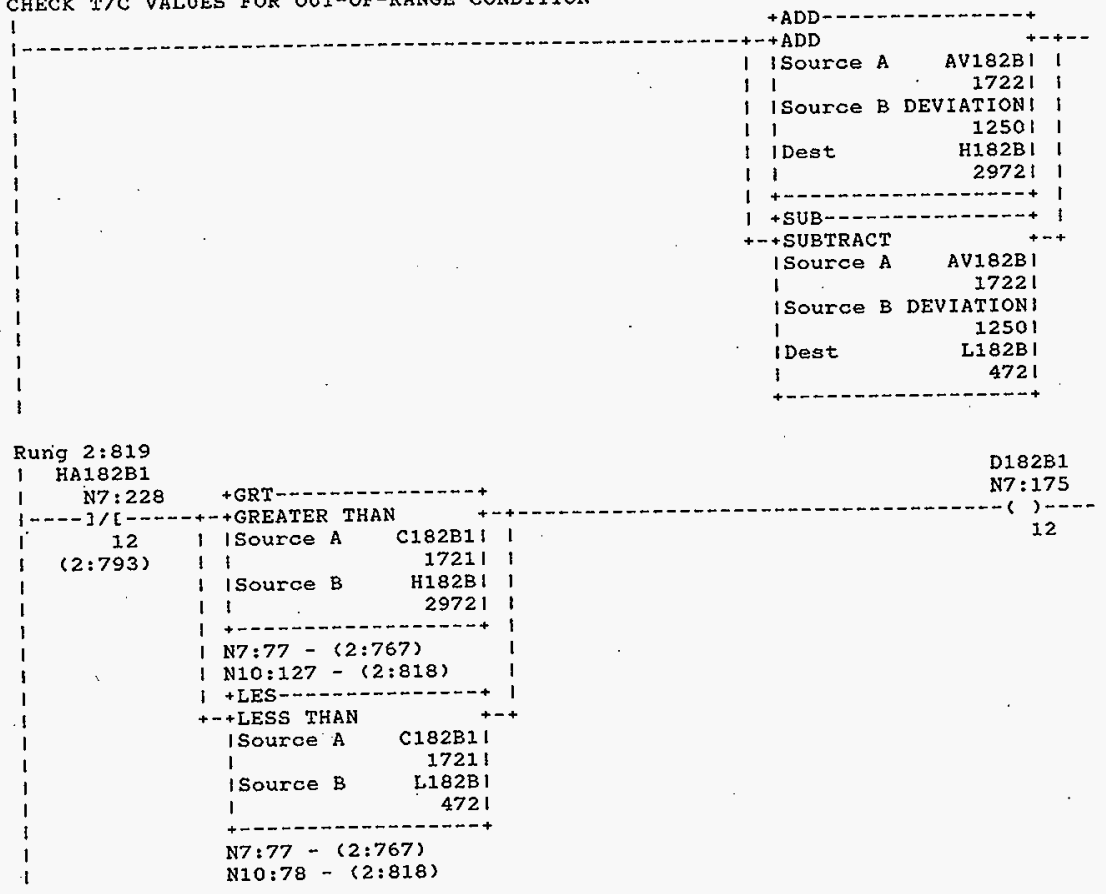

HNF-SD-FF-CSWD-61 Rev. 0 
Processor and Data(OPS Unit 1)

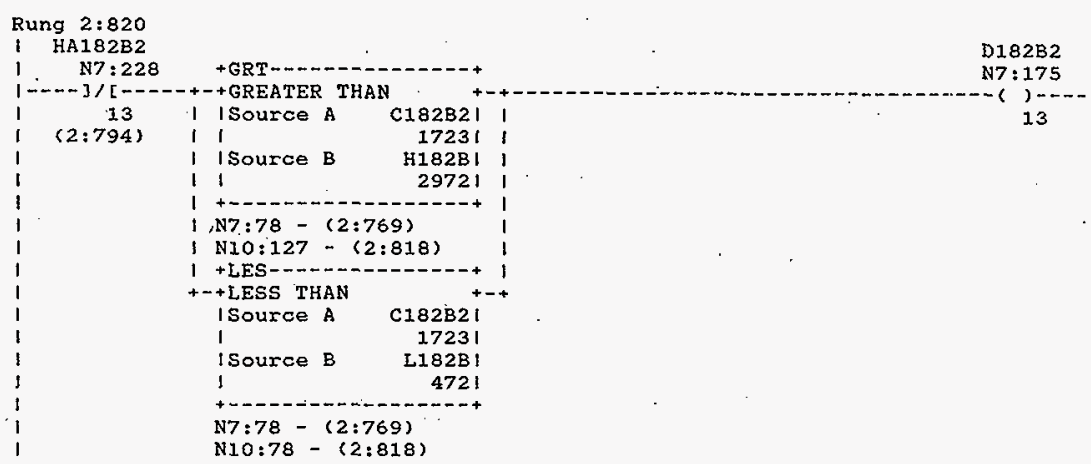

Rung $2: 821$

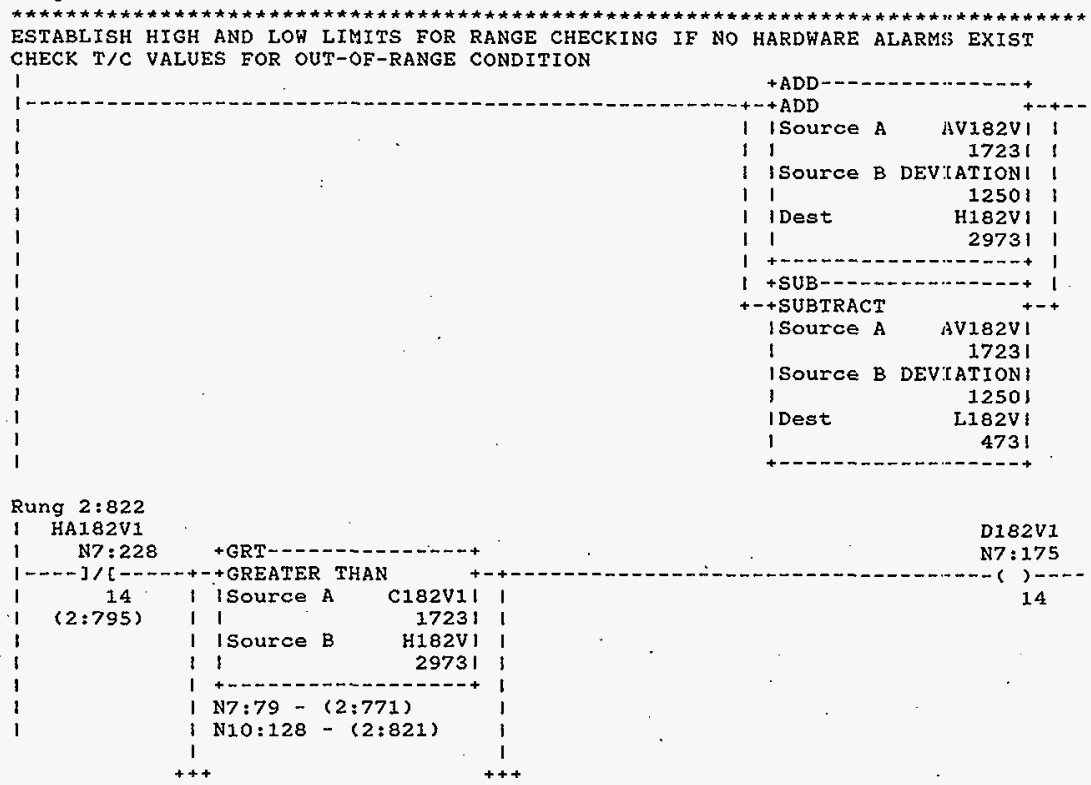

HNF-SD-FF-CSWD-61 Rev. 0 


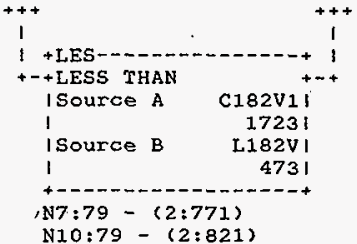

Rung $2: 823$

HA1820

I $\quad$ $7: 228$ +GRT---

1 1

$1723 !$

11

I N7:81-(2:773)

I N10:128 - (2:821)

$1+$ LES--.--..----n+

+-+ LESS THAN

ISource A C182V2I

$1 \quad 17231$

isource B L182V I
1 4731

N7:81-(2:773)

N10:79-(2:821)

Rung $2: 824$

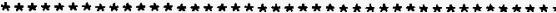
ESTABLISH HIGH AND LOW LIMITS FOR RANGE CHECKING IF NO HARDWARE ALARMS EXIST CHECK T/C VALUES FOR OUT-OF-RANGE CONDITION

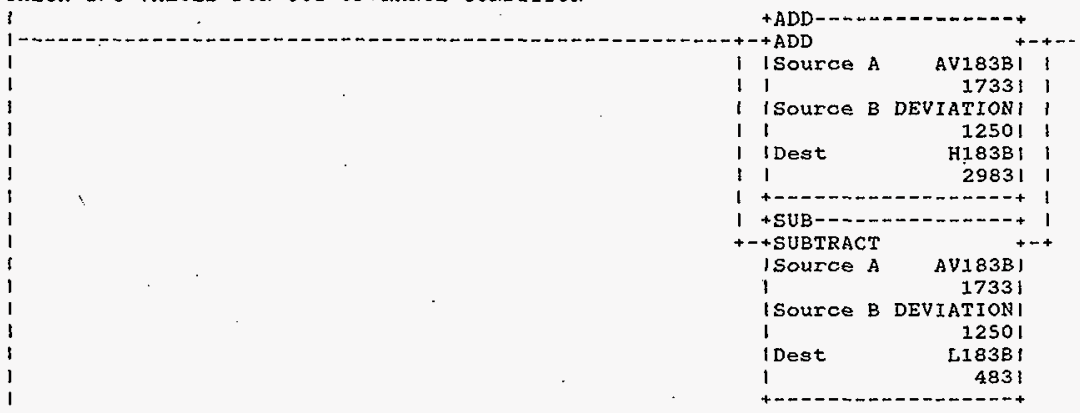

HNF-SD-FF-CSWD-61 Rev. 0 
Processor and Data(OPS Unit I)

Rung 2:825

1 HA183BI

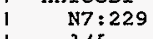

Rung $2 \div 826$

1 HA183B2

Rung $2: 827$

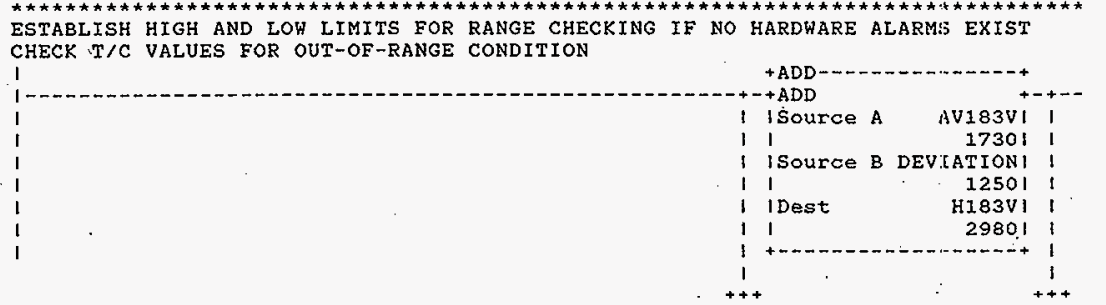

HNF-SD-FF-CSWD-61 Rev. 0 
Processor and Datalops Unit 1)

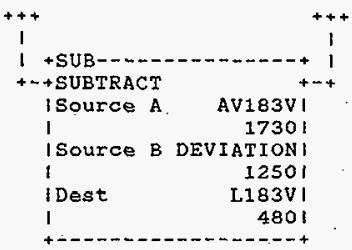

\section{Rung $2: 828$}

I HAI83VI

$$
\text { 1 N7:229 }
$$

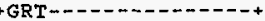

$183 \mathrm{VI}$

$1---] /[---++$ +GREATER THAN

$$
\text { (2:799) }
$$

I isource A

C183V1I 1

N7: 176

I ISource B. HI83VI!

11

HI83VI!

1 +.-.-.

I N7:83-(2:779)

( N10:130 - (2:827)

$1+$ +ES-

+- LESS THAN +-+

isource A C183V1]

I 17301

ISource B LI83VI

14801

N7:83-(2:779)

N20:81-(2:827)

Rung 2:829

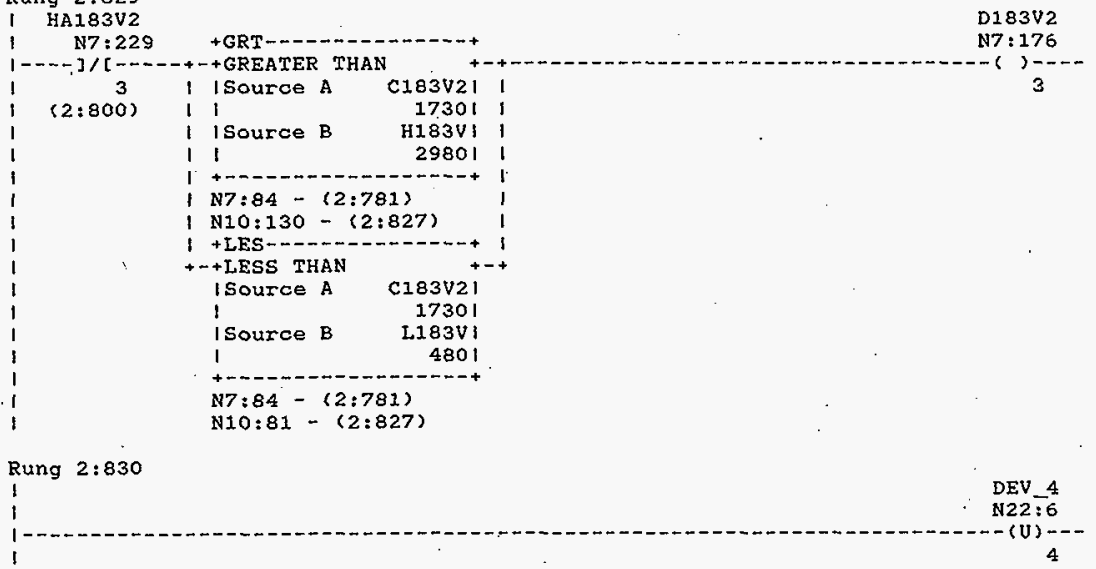

HNF-SD-FF-CSWD-61 Rev. 0 
Processor and Data(oPs Unit 1)

\section{Rung' 2:831}

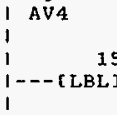

DUMMY 56

15

N22:10

Rung $2: 832$

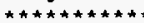 \\ CLEAR COUNT AND TOTAL. IF NO HARDWARE, NO RANGE ALARMS AND. NO THERMOCOUPLES} HAVE BEEN. TAKEN OUT (TE_OUT $\times \times \times$ ) THEN AVERAGE THE T/C
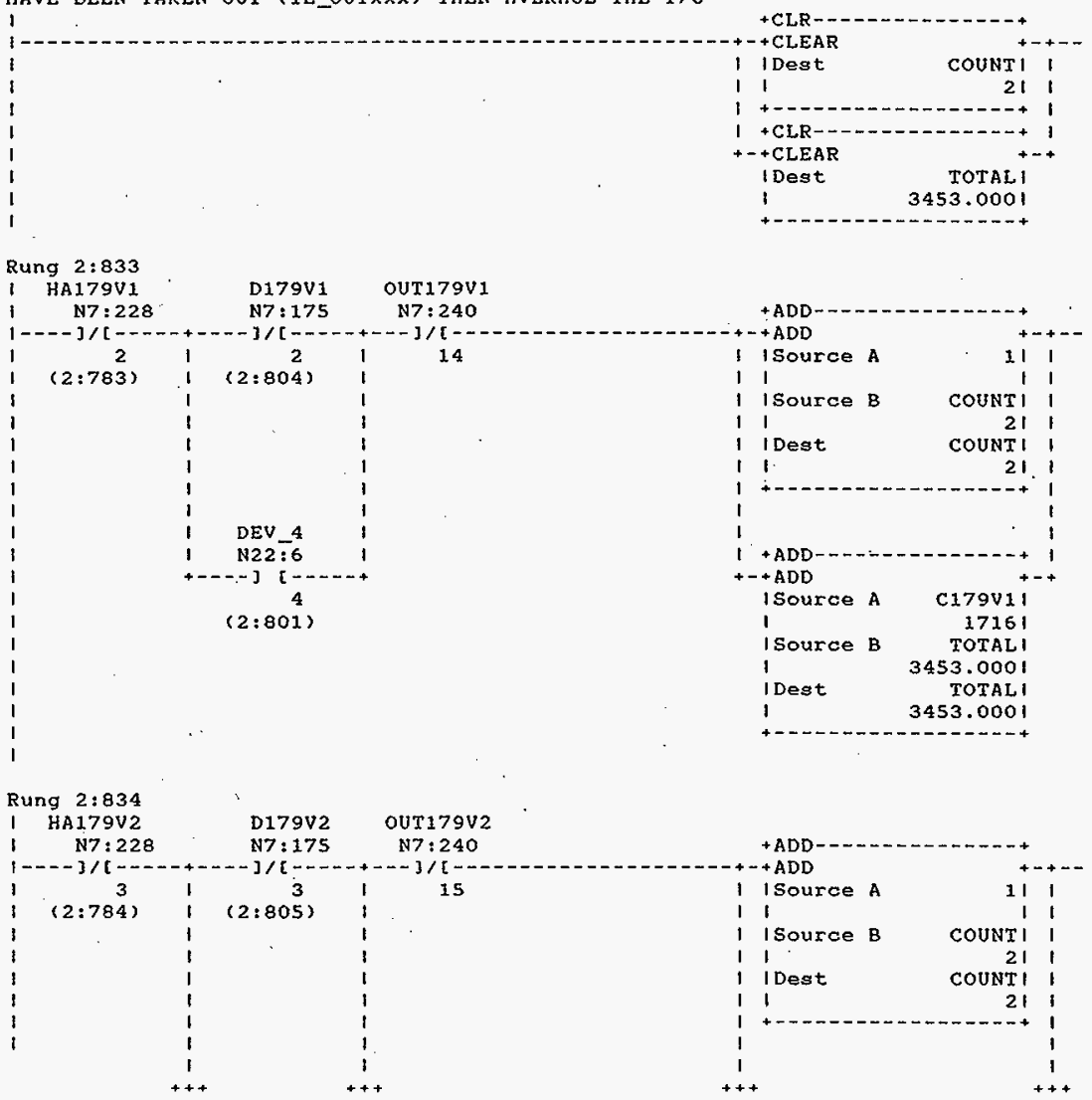

HNF-SD-FF-CSWD-61 Rev. 0 
Processor and Data(OPS Unit 1)

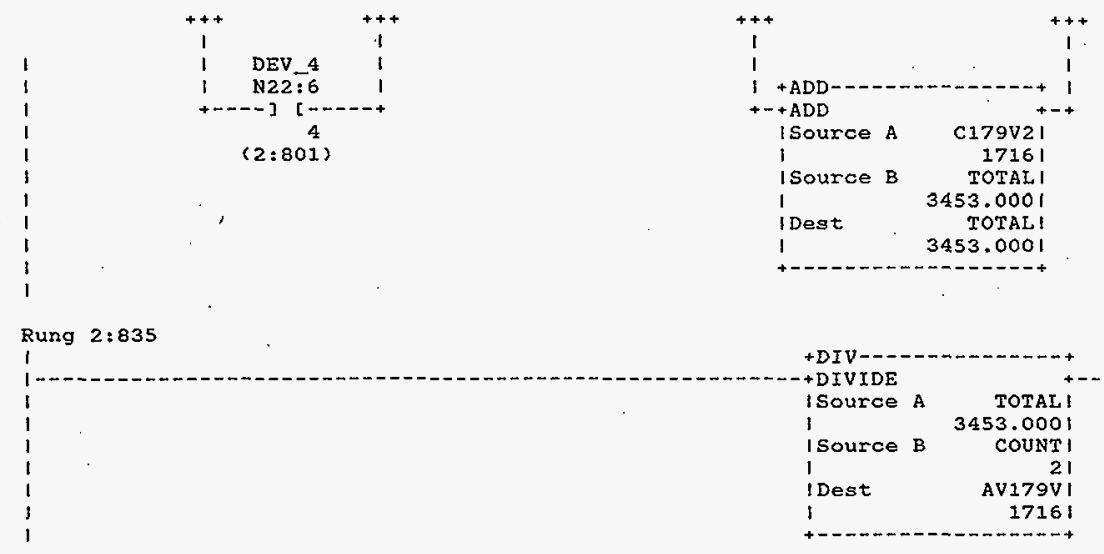

\section{Rung $2: 836$}

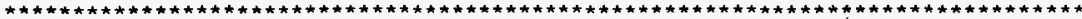
CLEAR COUNT AND TOTAL. IF NO HARDHARE, NO RANGE ALARMS AND NO THERMOCOUPLES HAVE BEEN TAKEN OUT (TE_OUTXXX) THEN AVERAGE THE T/C
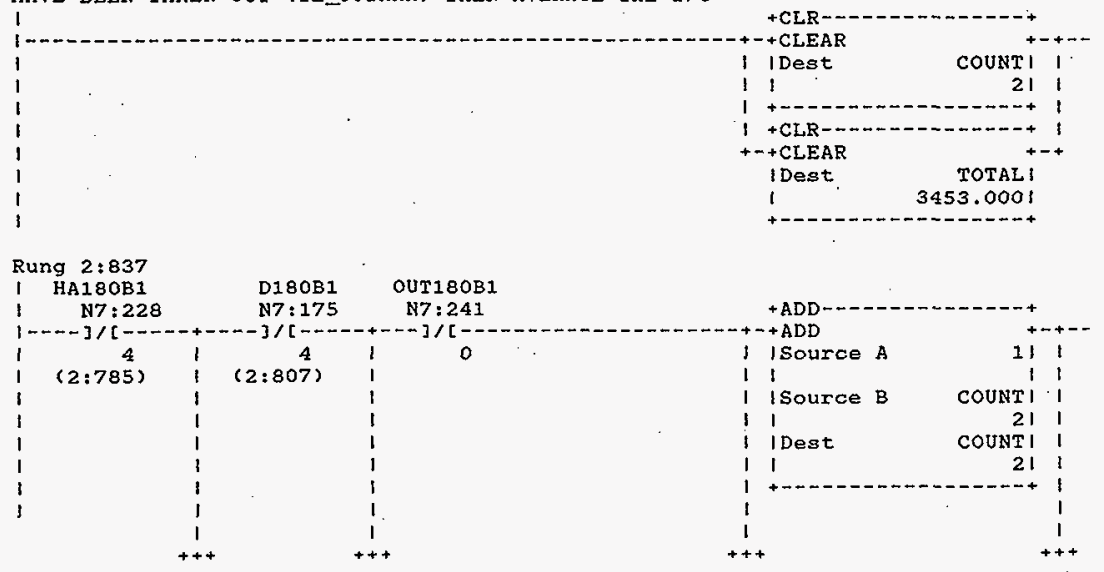

HNF-SD-FF-CSWD-61 Rev. 0 
Processor and Data(OPS Unit 1)

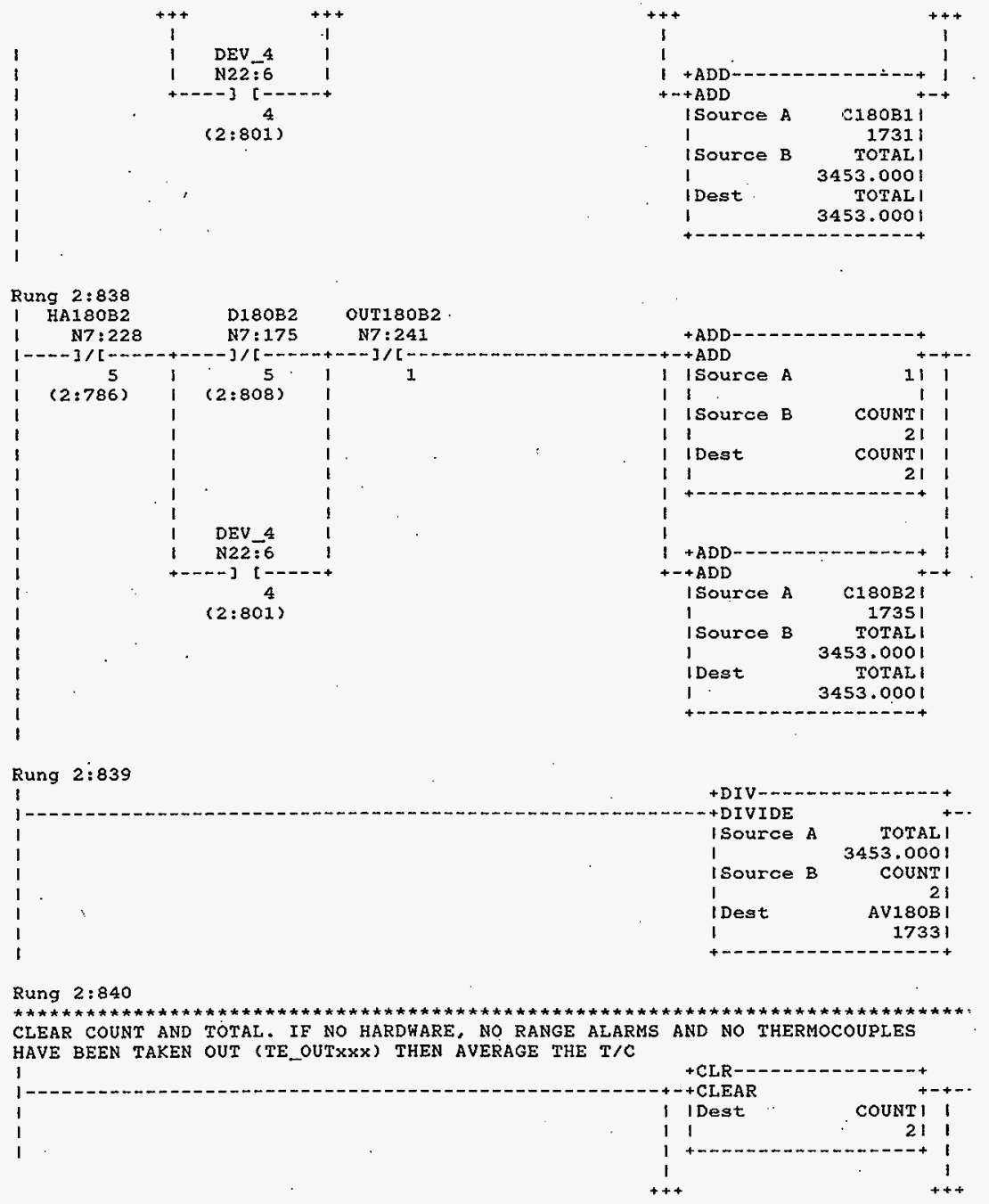

HNF-SD-FF-CSWI)-61 Rev. 0 

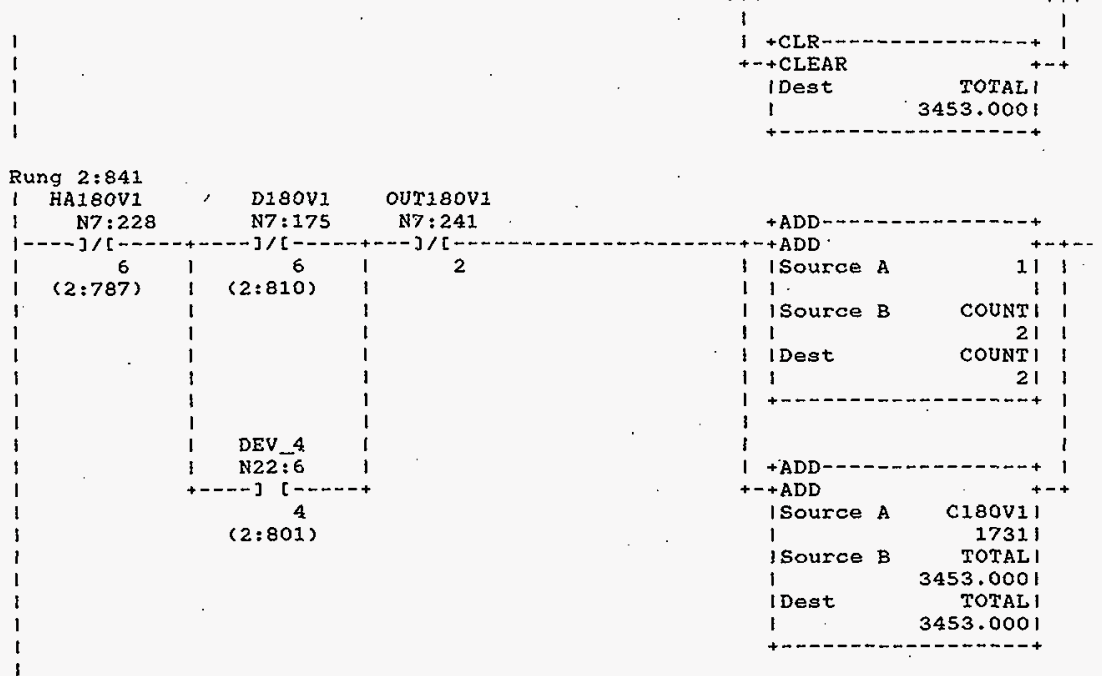

\section{Rung 2:842}

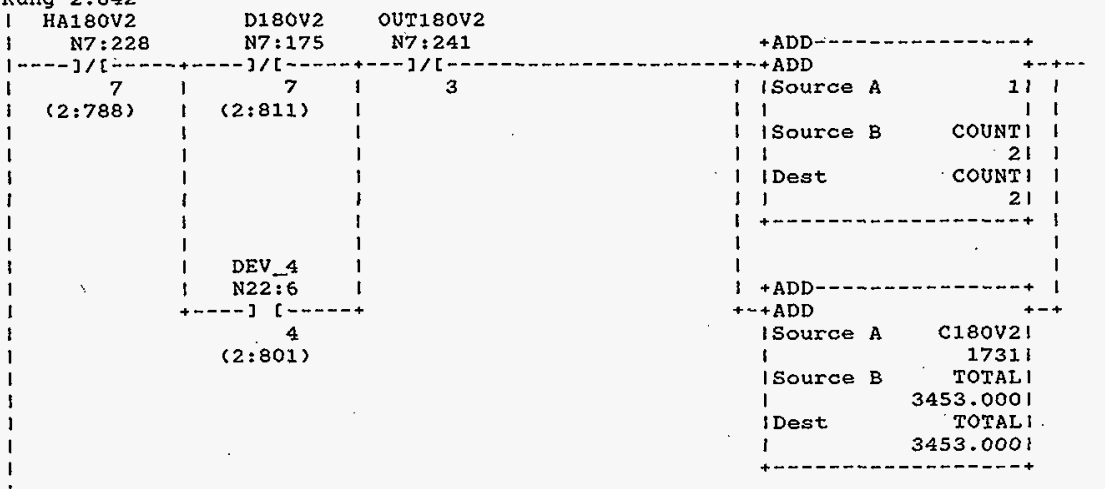

HNF-SD-FF-CSWD-61 Rev. 0 
Rung $2: 843$

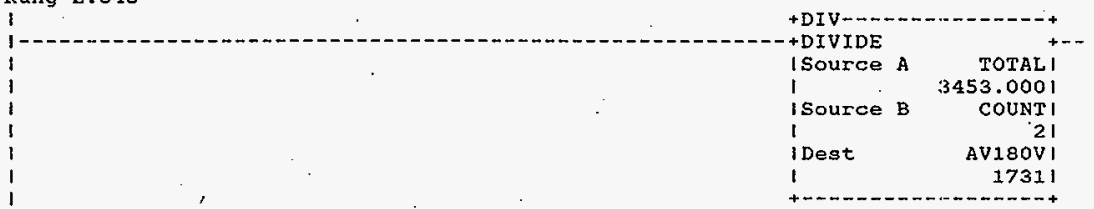

Rung $2: 844$

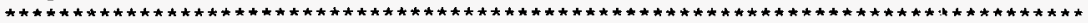
CLEAR COUNT AND TOTAL. IF NO HARDHARE, NO RANGE ALARMS AND NO THERMOCOUPLES HAVE BEEN TAXEN OUT (TE_OUTXXX) THEN AVERAGE THE T/C

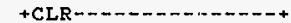

+-+ CLEAR

IDest

1

$1+-0--10-0-1$

$--+1$

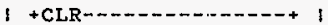

$+\cdots+$ CLEAR

IDest

TOTAI 1

1

$34: 53.0001$

Rung 2:845

I HA181B1

I N7:228. N7:175 N7:241

D181B1

N7:228. N7:175 N7:241

OUT181B1

I 8 । 8 ( 8 ।

$(2: 789)$

\begin{tabular}{lcc}
1 & 8 & 1 \\
1 & $(2: 813)$ & 1 \\
1 & & 1 \\
1 & & 1 \\
1 & & 1 \\
1 & & 1 \\
1 & & 1 \\
1 & DEV 4 & 1 \\
1 & N22:6 & 1 \\
\hline$+-D ~$ & &
\end{tabular}

4

4

$(2: 801)$

HNF-SD-FF-CSWD-61 Rev. 0 
Processor and Data(OPS Unit 1)

\section{Rung 2:846}

Rung $2: 846$

( $\quad$ N7:228

$1----] /[--\cdots$

9

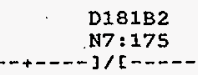

OUT181B2

$1(2: 790)$

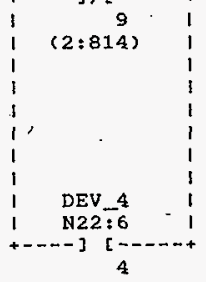

N7: 241

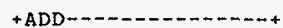

I

$$
1
$$

$(2: 801)$

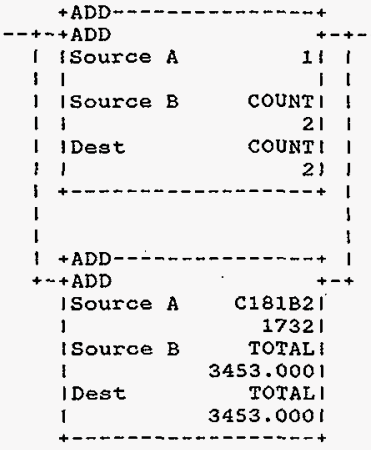

Rung $2: 847$

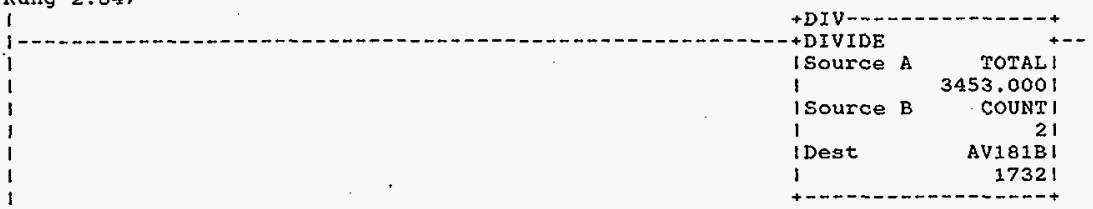

Rung $2: 848$

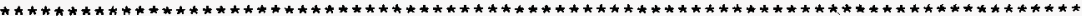
CLEAR COUNT AND TOTAL. IF NO HARDWARE, NO RANGE ALARMS AND NO THERMOCOURLES HAVE BEEN TAKEN OUT (TE_OUTXXX) THEN AVERAGE THE T/C

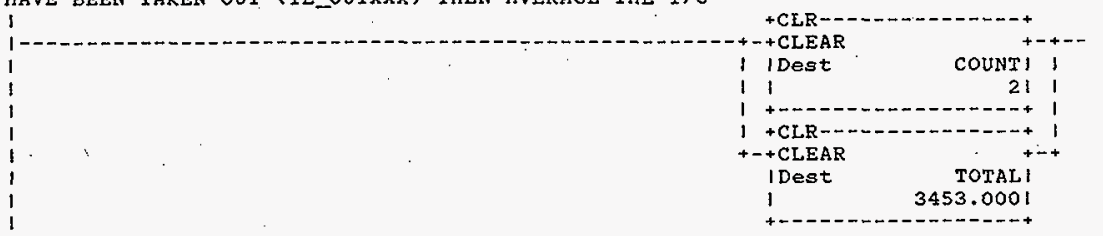

HNF-SD-FF-CSWD-61 Rev. 0 
Processor and Data(OPS Unit 1)

October 23, 1996 Page 351

Program Listing Processor File: SODIUM2A.ACH

Rung $2: 849$

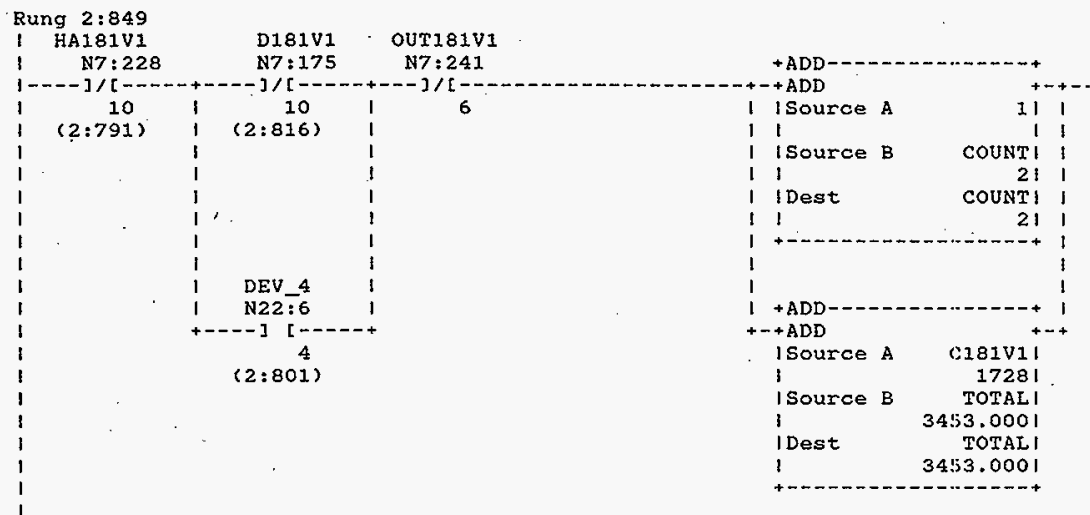

Rung $2: 850$

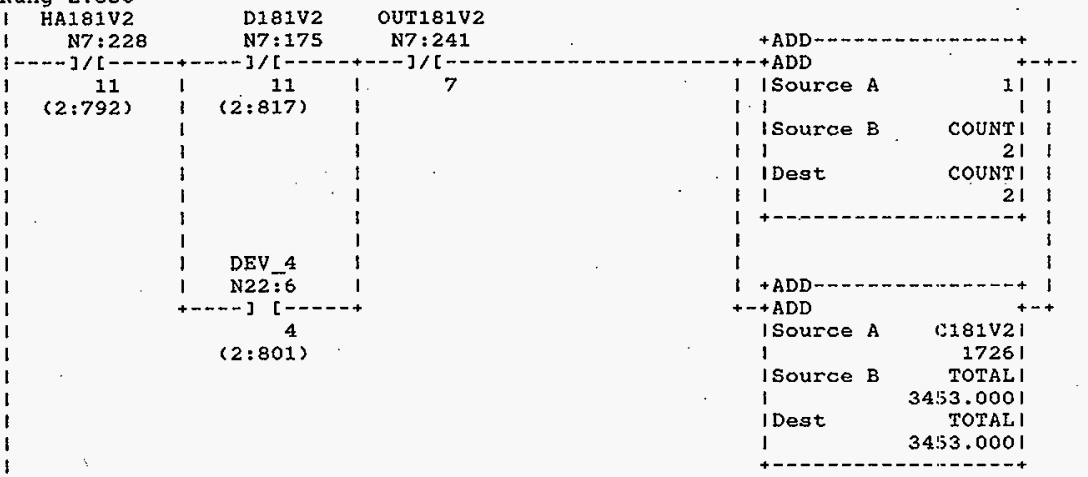

Rung $2: 851$

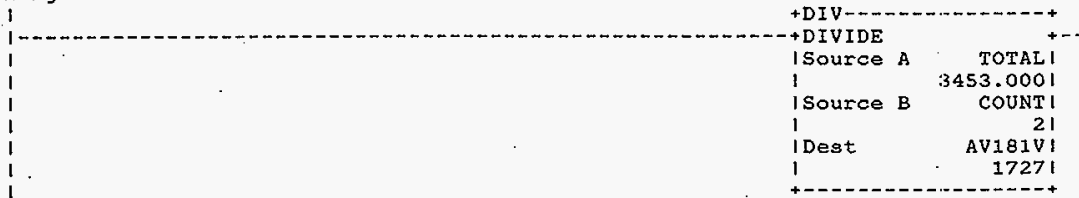

HNF-SD-FF-CSWD-61 Rev. 0 


\section{Rung 2:852}

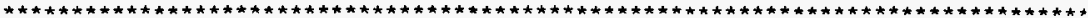
CLEAR COUNT AND TOTAL. IF NO HARDWARE, NO RANGE ALARMS AND NO THERMOCOUPLES HAVE BEEN TAKEN OUT (TE_OUTXXX) THEN AVERAGE THE T/C

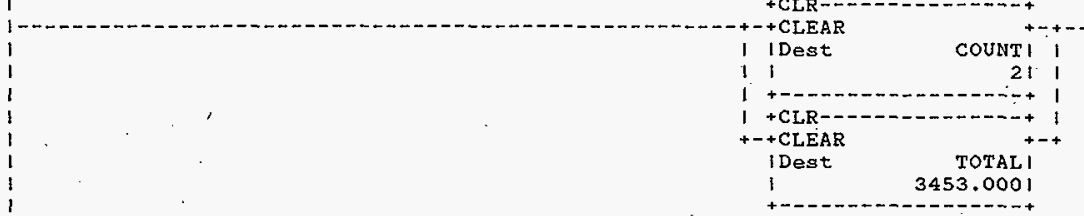

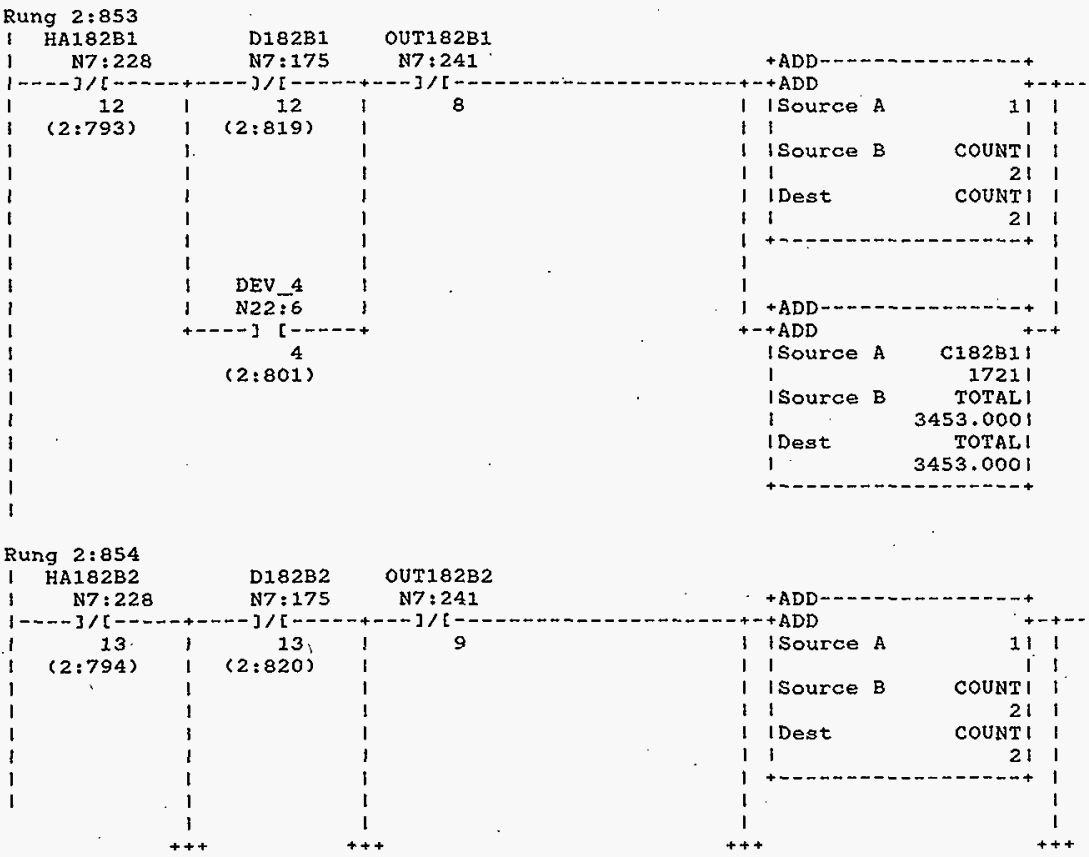

HNF-SD-FF-CSWD-61 Rev. 0 
Processor and Data(OPS Unit 1)

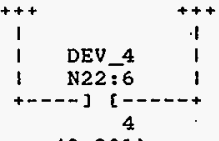

$(2: 801)$

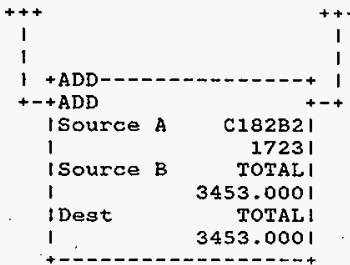

Rung $2: 855$

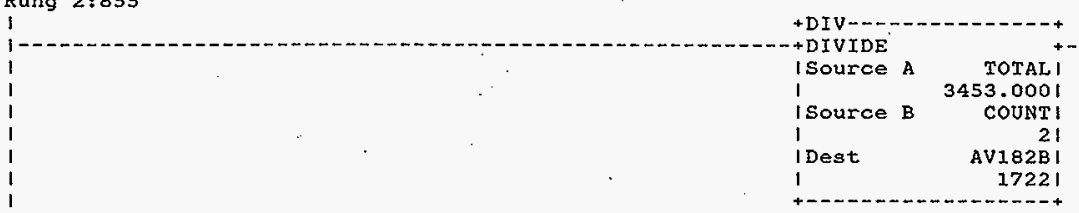

Rung $2: 856$

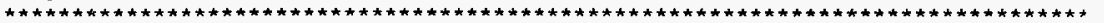
CLEAR COUNT AND TOTAL. IF NO HARDHARE, NO RANGE ALARMS AND NO THERMOCOUPLES HAVE BEEN TAKEN OUT. (TE_OUT $X X X$ ) THEN AVERAGE THE T/C

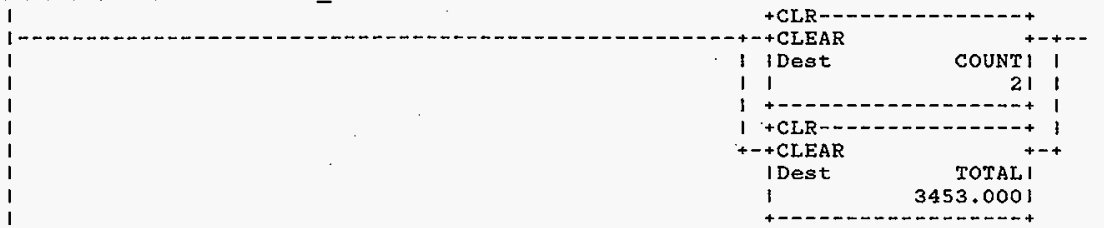

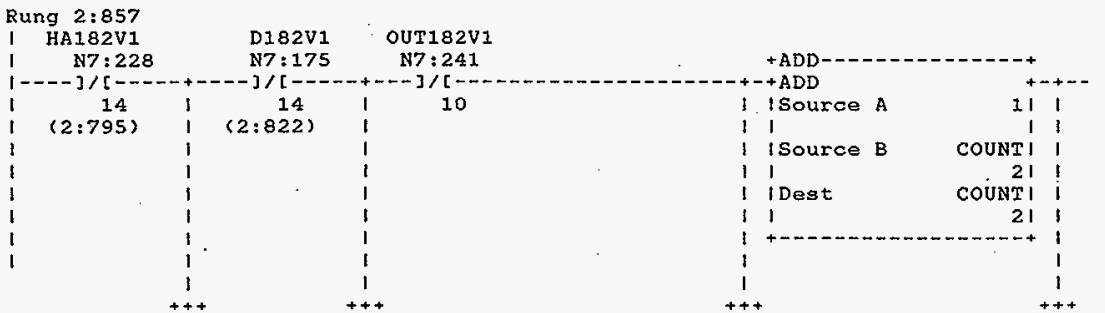

HNF-SD-FF-CSWD-61 Rev. 0 


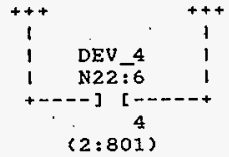

Rung $2: 858$

HA182V2

IN7:228 N7:175 .N7:241

D182V2 OUT182V2

$\begin{array}{ccccc}1 & 15 & 1 & 15 & 1\end{array}$

(2:796)

$(2: 823$

11

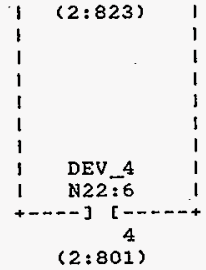

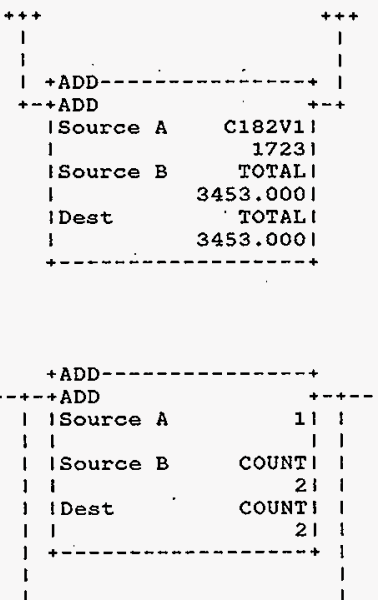

$+\mathrm{ADD}$
$+\mathrm{ADD}$

I ISource A

11

1 Isource B

11

f IDest

11

$+$

1

$++\mathrm{ADD}$

isource A C182V21

17231

isource B TOTALI

I 3453.000 I

I Dest

TOTAL I

I

3453.0001

Rung $2: 859$

Rung $2: 860$

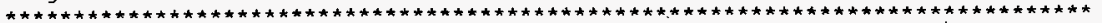
CLEAR COUNT AND TOTAL. IF NO HARDGARE, NO RANGE ALARMS AND NO THERMOCOUPLES HAVE BEEN TAKEN OUT (TE_OUTXXX) THEN AVERAGE THE T/C

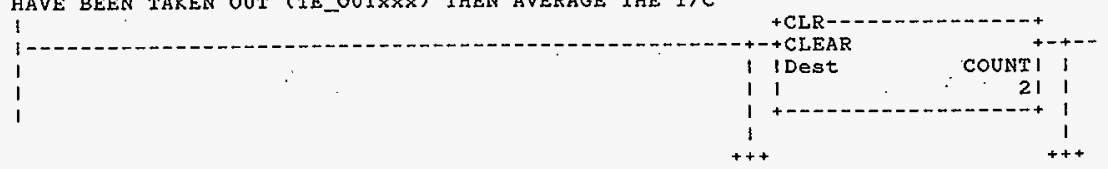

HNF-SD-FF-CSWD-61 Rev. 0 
Processor and Data (OPS Unit 1) Program Listing
Processor File: SODIUM2A.ACH
October 23, 1996

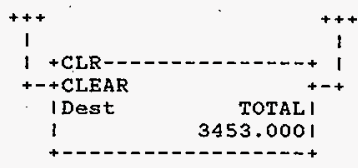

Rung $2: 861$ I HA183B1 I N7:229

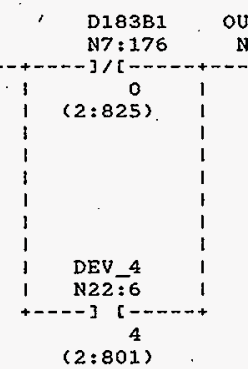

UT $183 \mathrm{~B} 1$ N7: 241

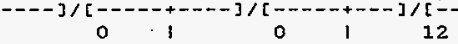
$(2: 797)$ 1 $+\mathrm{ADD}$

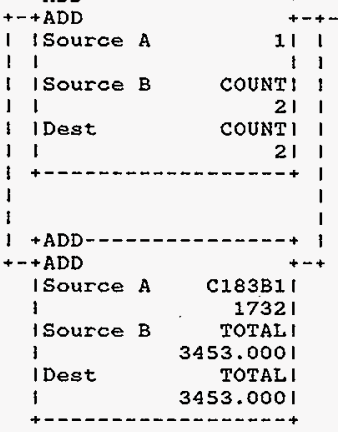

Rung 2:862

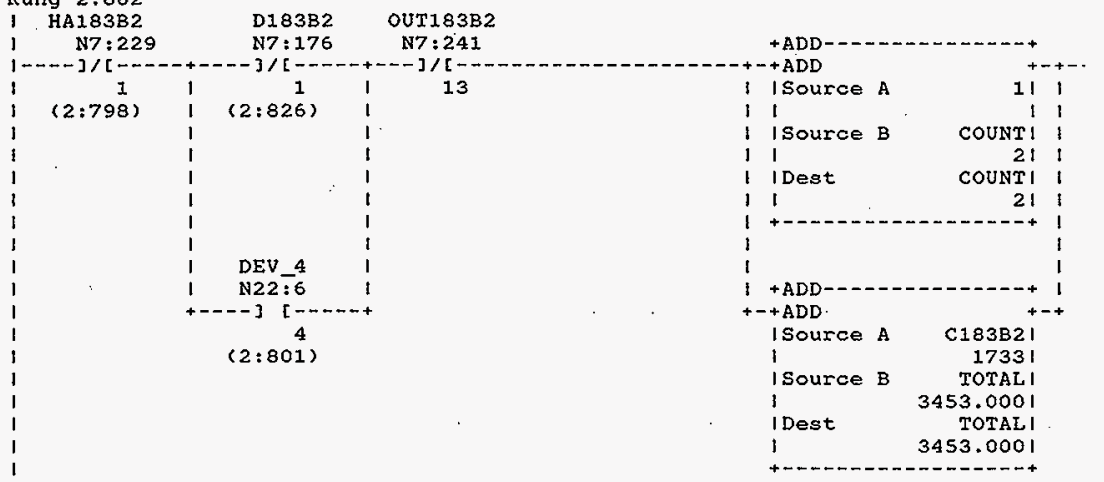

HNF-SD-FF-CSWD-61 Rev. 0 


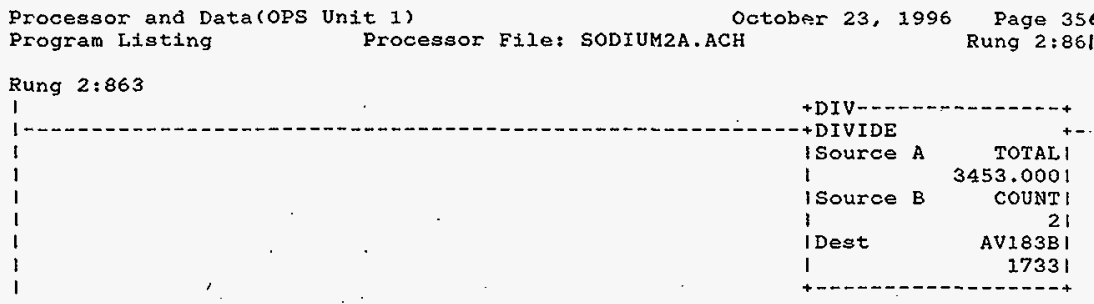

\section{Rung $2: 864$}

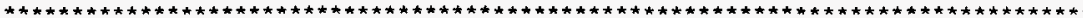
CLEAR COUNT AND TOTAL. IF NO HARDWARE, NO RANGE ALARMS AND NO THERMOCOUPLES HAVE BEEN TAKEN OUT (TE_OUTXXX) THEN AVERAGE THE T/C
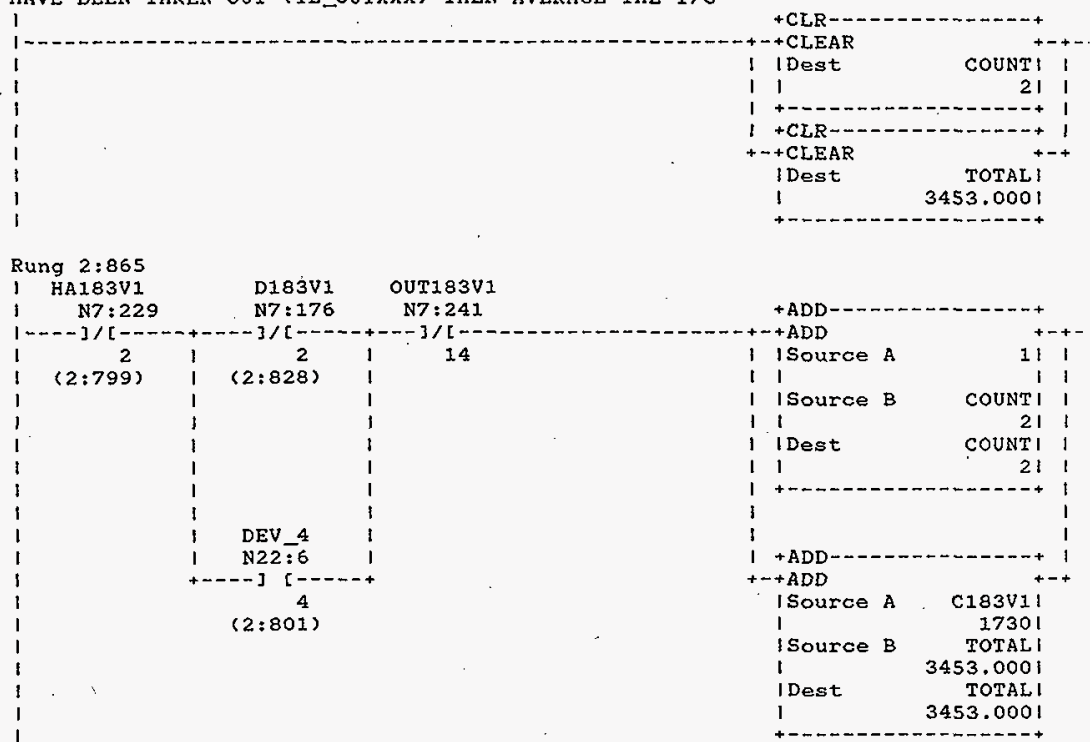

HNF-SD-FF-CSWD-61 Rev. 0 


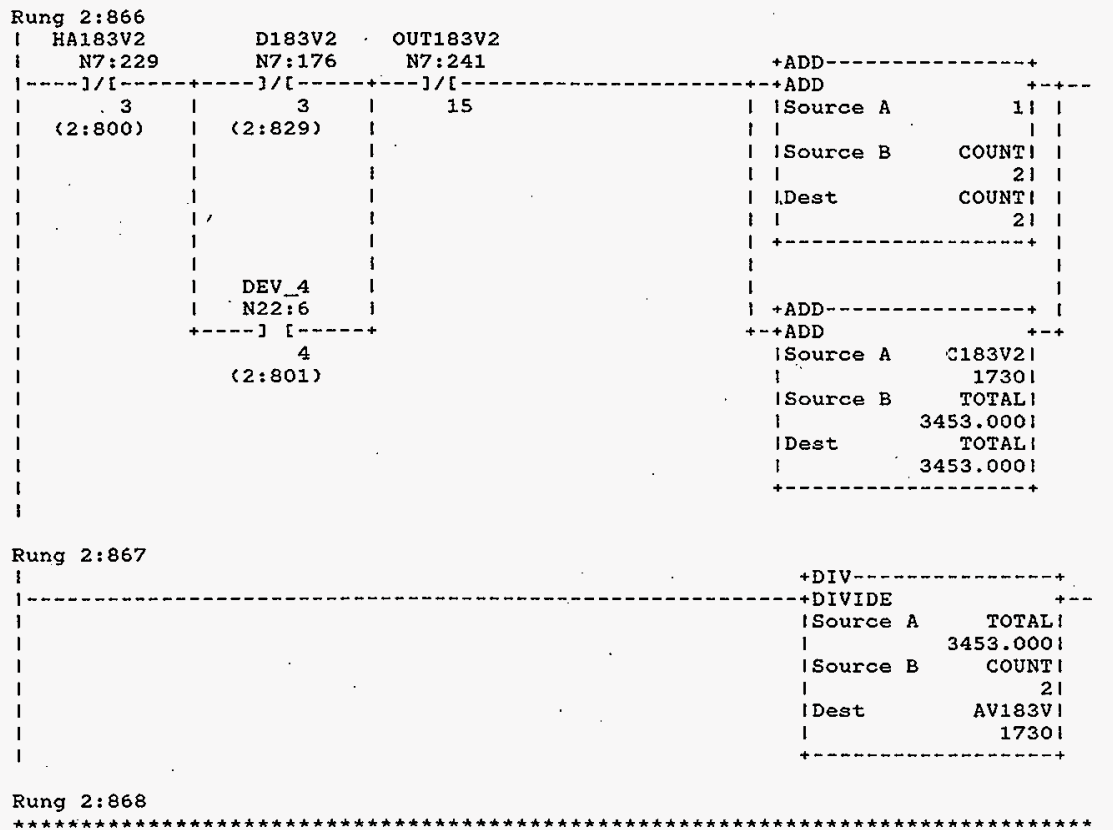

IF DEVIATION SET FOR SECTION 4 THEN GO THERE AND CALCULATE DEVIATION

$\therefore$

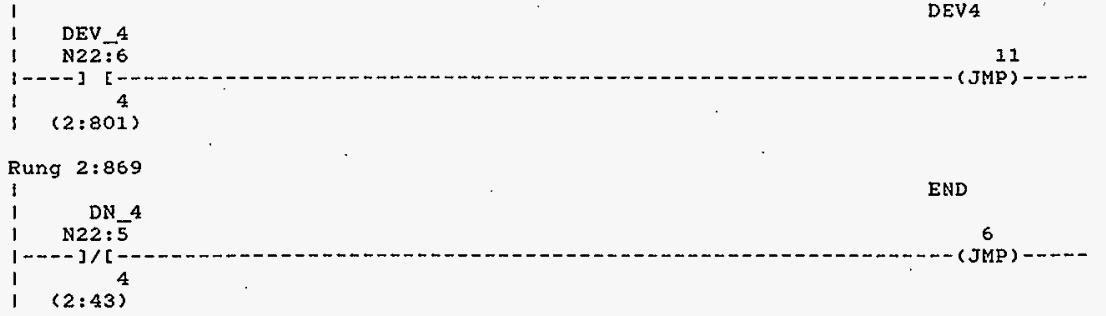

HNF-SD-FF-CSWD-61 Rev. 0 
Processor and Data(OPS Unit 1)

Rung $2: 870$
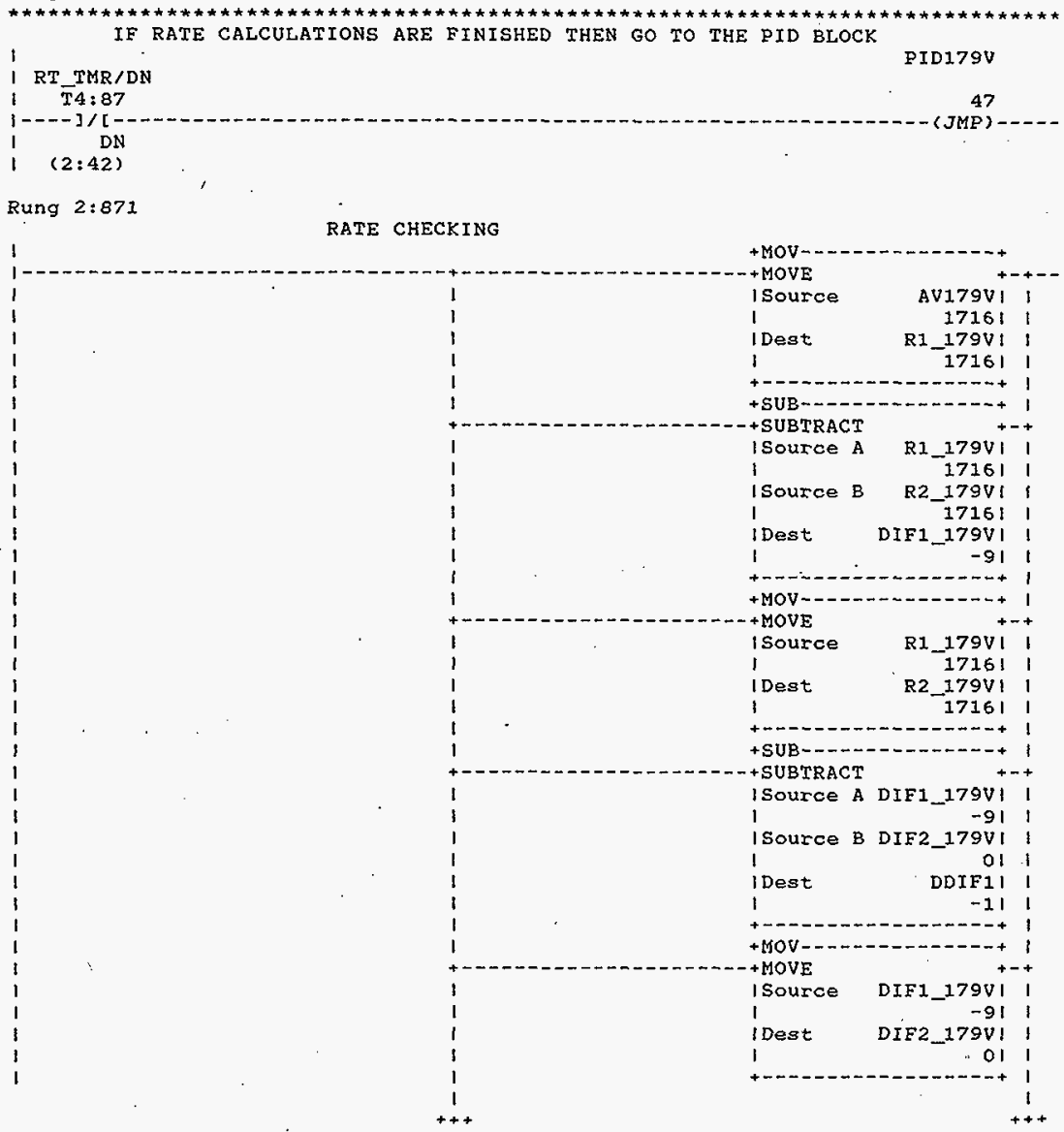

HNF-SD-FF-CSWD-61 Rev. 0 


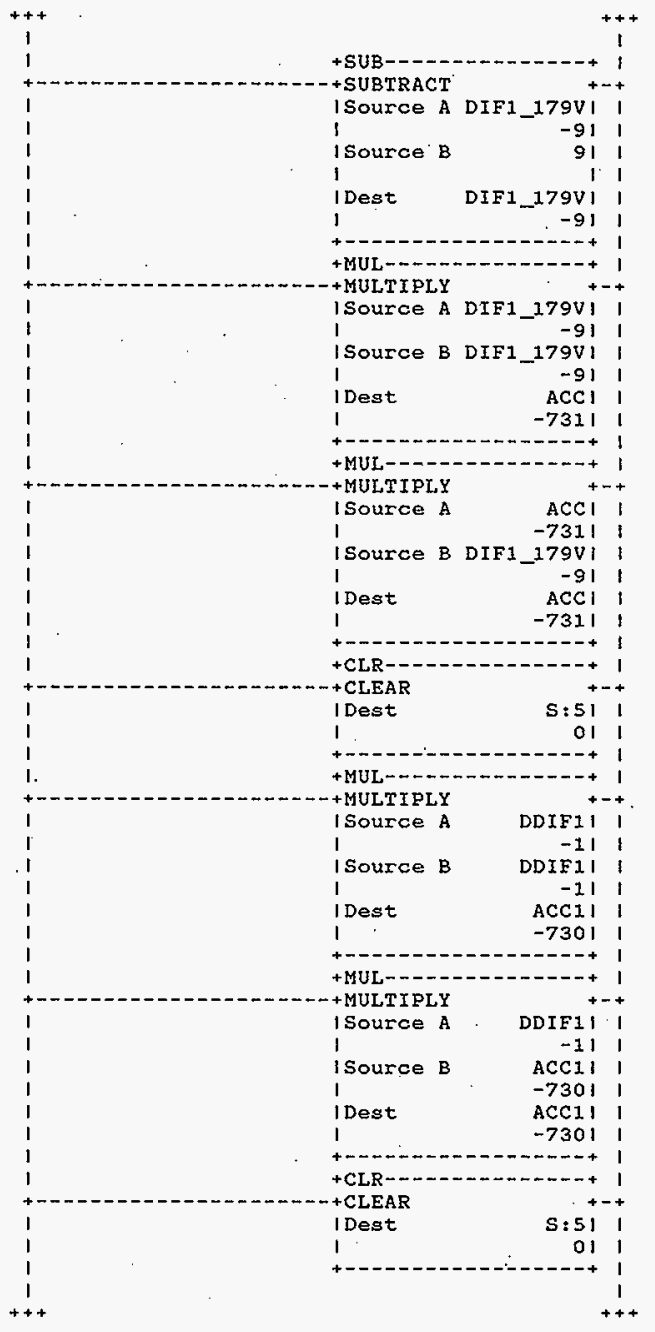

HNF-SD-FF-CSWD-6! Rev. 0 


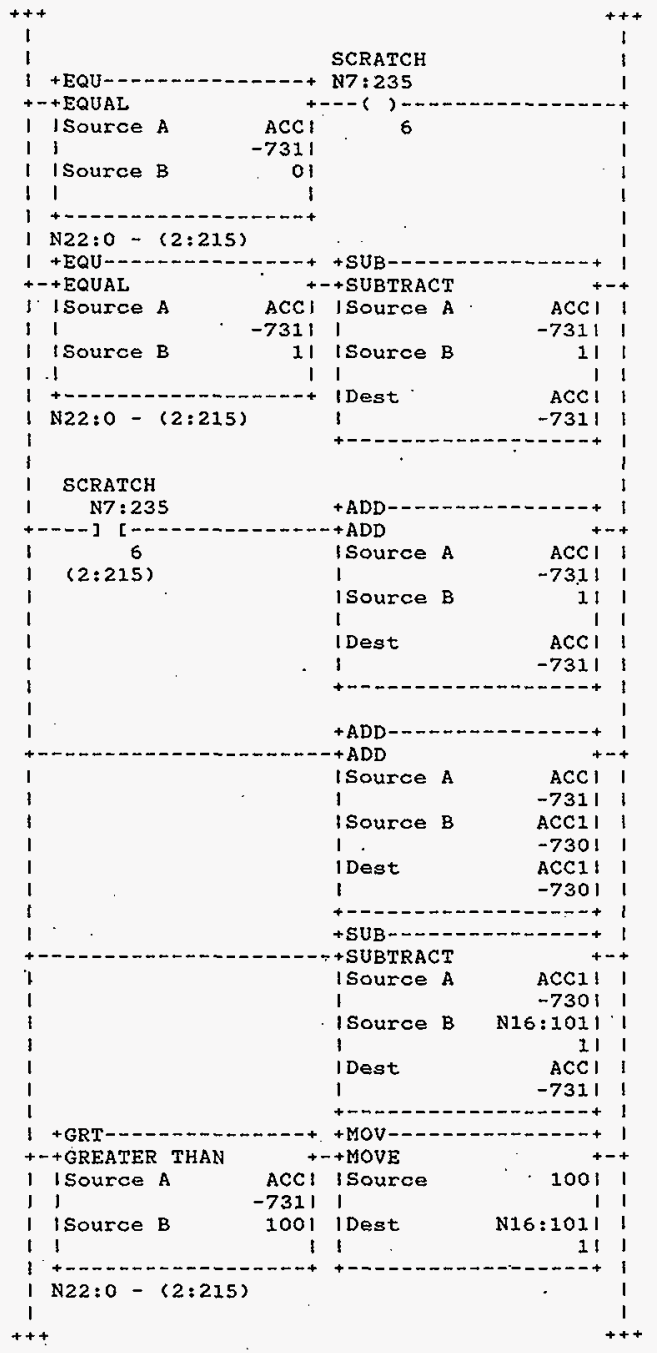

HNF-SD-FF-CSWD-61 Rev. 0 


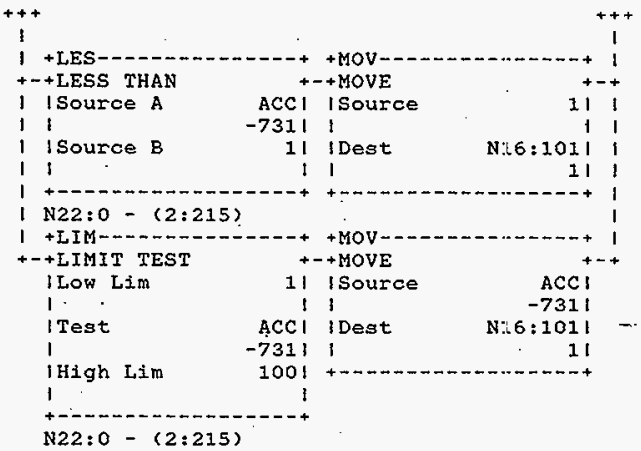

Rung 2:872

I PID179V

$$
7
$$

Rung 2:873

\section{DETECT FROM SCADA PID ON/OFF IF PID OFF \\ THEN - ZERO PW FOR ZERO OUTPUT AT SCR} AND JUMP AROUND THE PID BLOCK

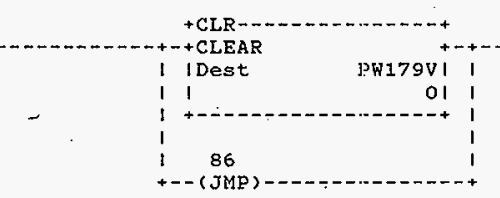

Rung $2: 874$

DETECT AUTO/MANUAL FROM SCADA

N7:233
$1,1 /\left[\begin{array}{l}14 \\ 1\end{array}(3: 25)\right.$

HNF-SD-FF-CSWD-61 Rev. 0 
Processor and Datalops Unit 1)

Rung 2:875

DETECT SETPOINT VALUE EROM SCADA PUT VALUE IN PID BLOCK

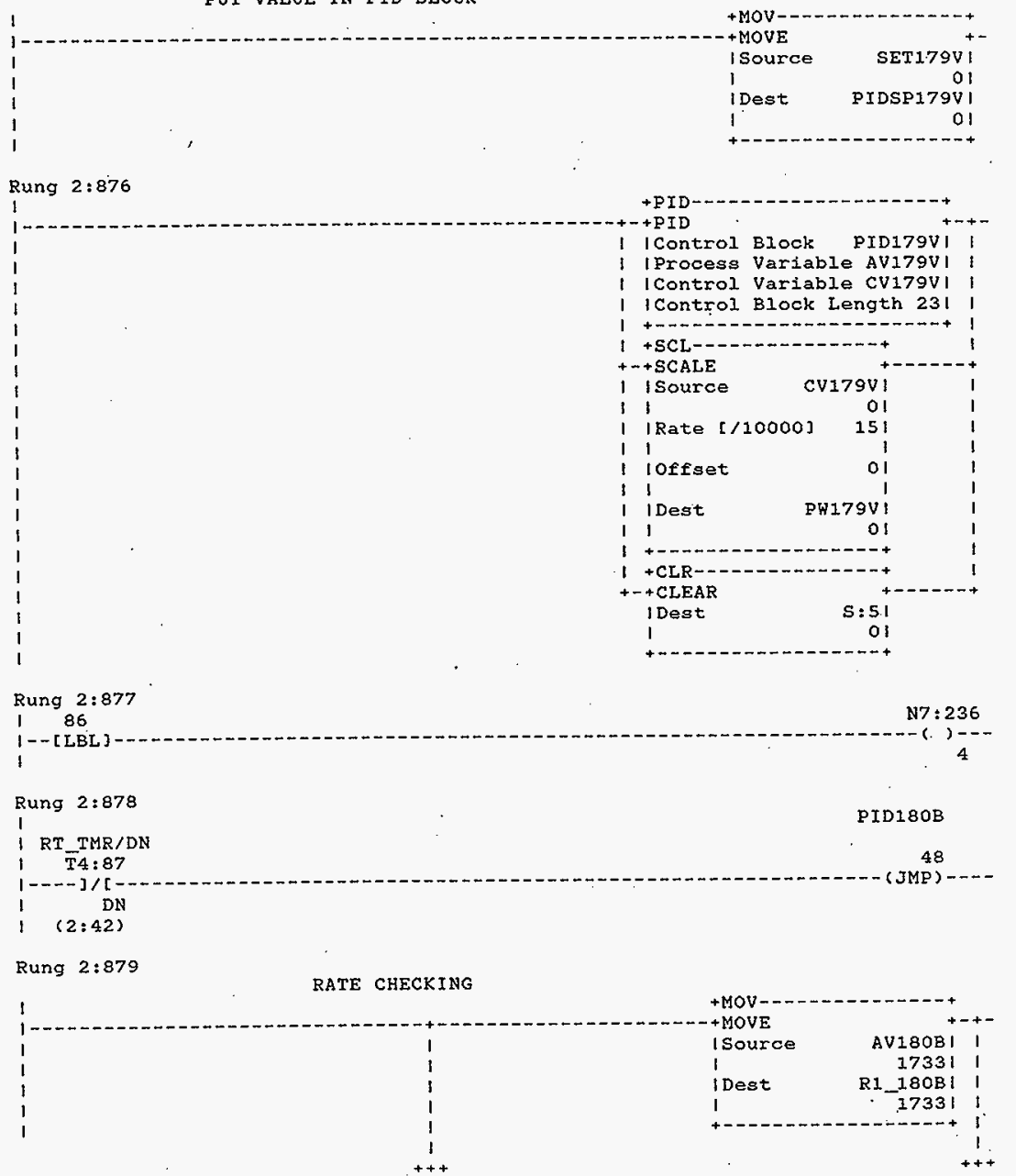

HNF-SD-FF-CSWD-61 Rev. 0 


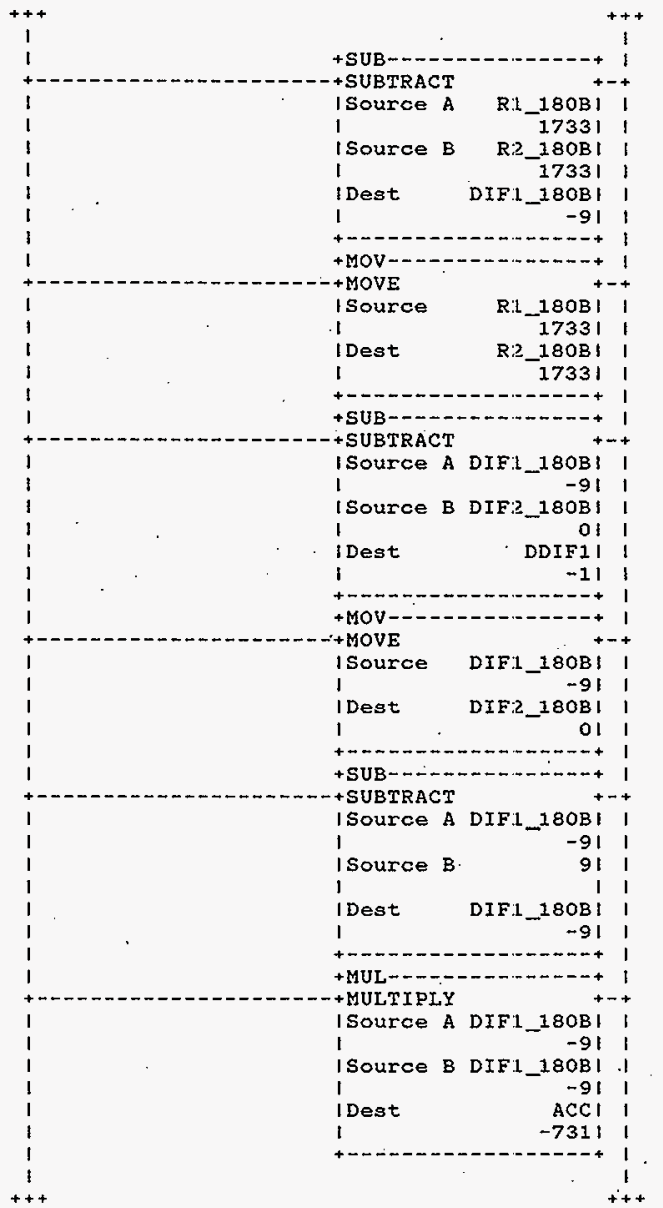

HNF-SD-FF-CSWD- 611 Rev. 0 
Processor and Data(OPS Unit 1) Program Listing
October 23, 1996 SODIUM2A, ACH
Page 364 Rung $2: 879$

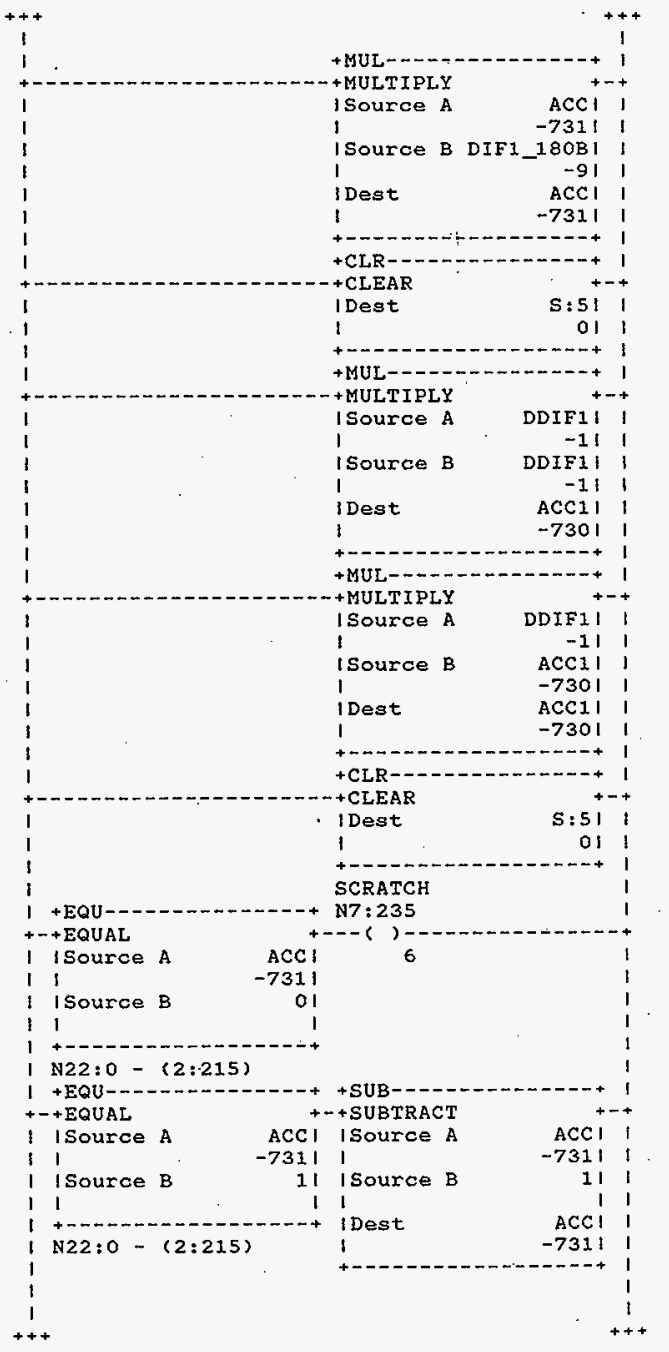

HNF-SD-FF-CSWD-61 Rev. 0 


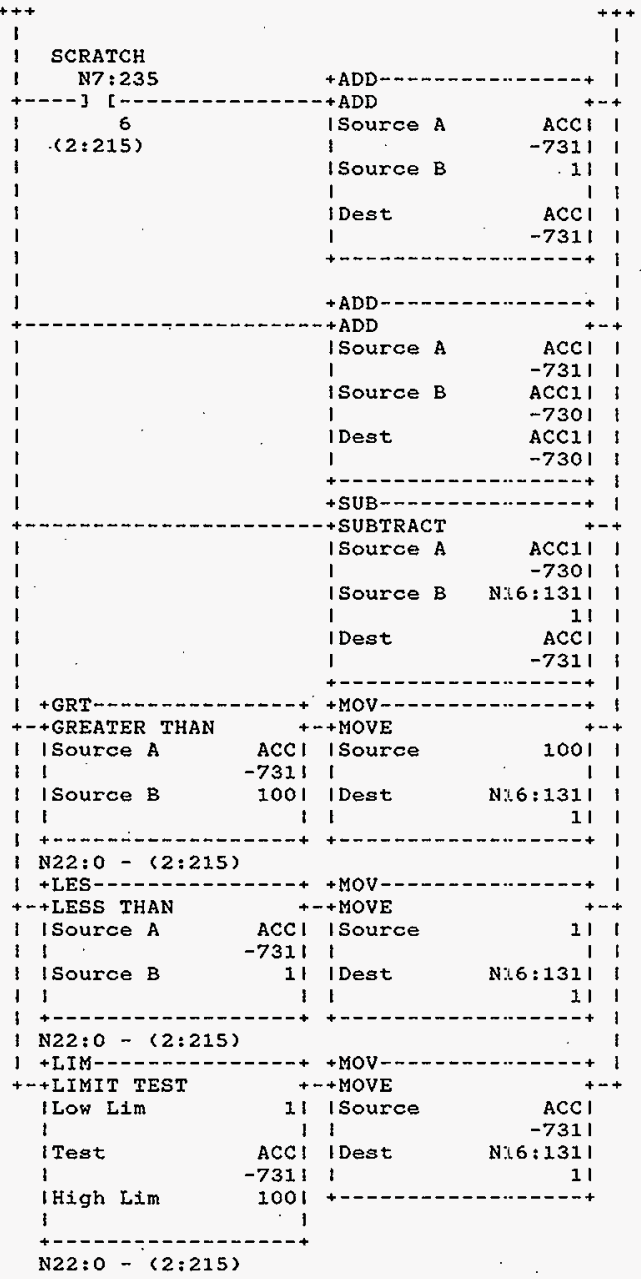

HNF-SD-FF-CSWD-61 Rev. 0 
Processor and Data(oPs Unit 1 )

Rung $2: 880$

1 PID180B

1

148

DUMMY 48

1-.- [LBL]

48

N22: 9

$$
\text { l. }
$$

Rung 2:881

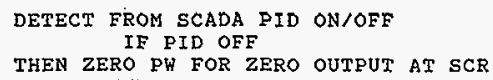

Riung 2:882

DETECT AUTO/MANUAL FROM SCADA

SET APPROPRIATE MODE IN PID BLOCK

AM18OB
N7:234

Rung 2:883

DETECT SETPOINT VALUE FROM SCADA PUT VALUE IN PID BLOCK

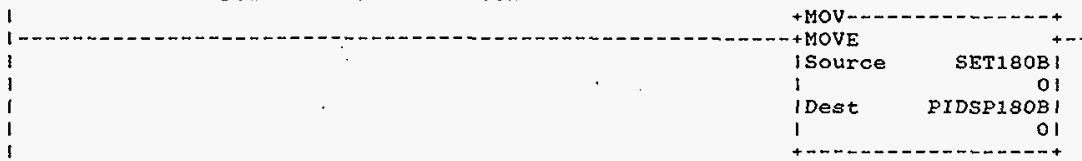

Rung 2:884

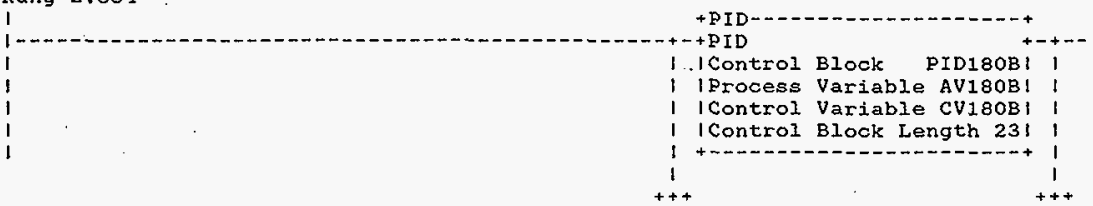

HNF-SD-FF-CSWD-6I Rev. 0 
$+$

++
1

$1+$ SCL-- ----------+

+-+ SCALE

1 I Source

CV18CBI

I

IRate $[/ 10000]$

11

l loffset

1.1

1 Dest PW18GB

(2)

11

$1+------------$

$1+\mathrm{CLR}-----------+$

+-+ CLEAR

I Dest

I

$5: 51$

Rung 2:885

1. 87

$17: 236$

1-- [LBL]

Rung 2:886

1

I RT_TMR/DN

$\mathrm{T} 4: 87$

EID $180 \mathrm{~V}$

$1----3 /[-$

49

i $\quad$ DN

Rung 2:887

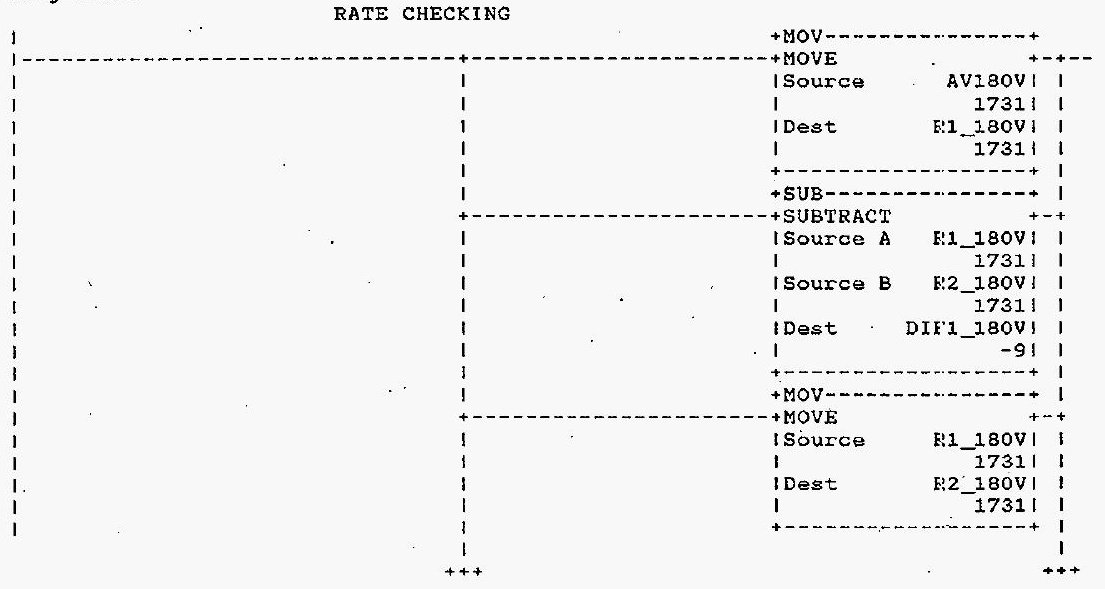

HNF-SD-FF-CSWD-61 Rev. 0 


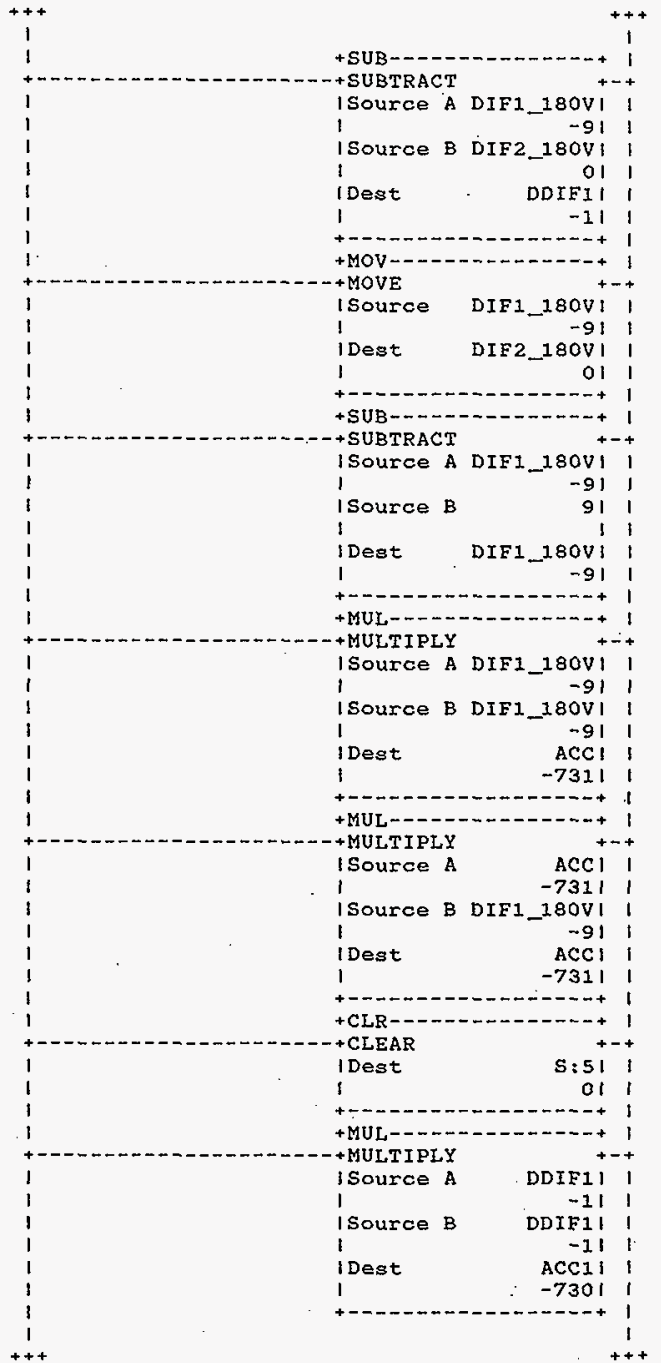

HNF-SD-FF-CSWD-61 Rev. 0 


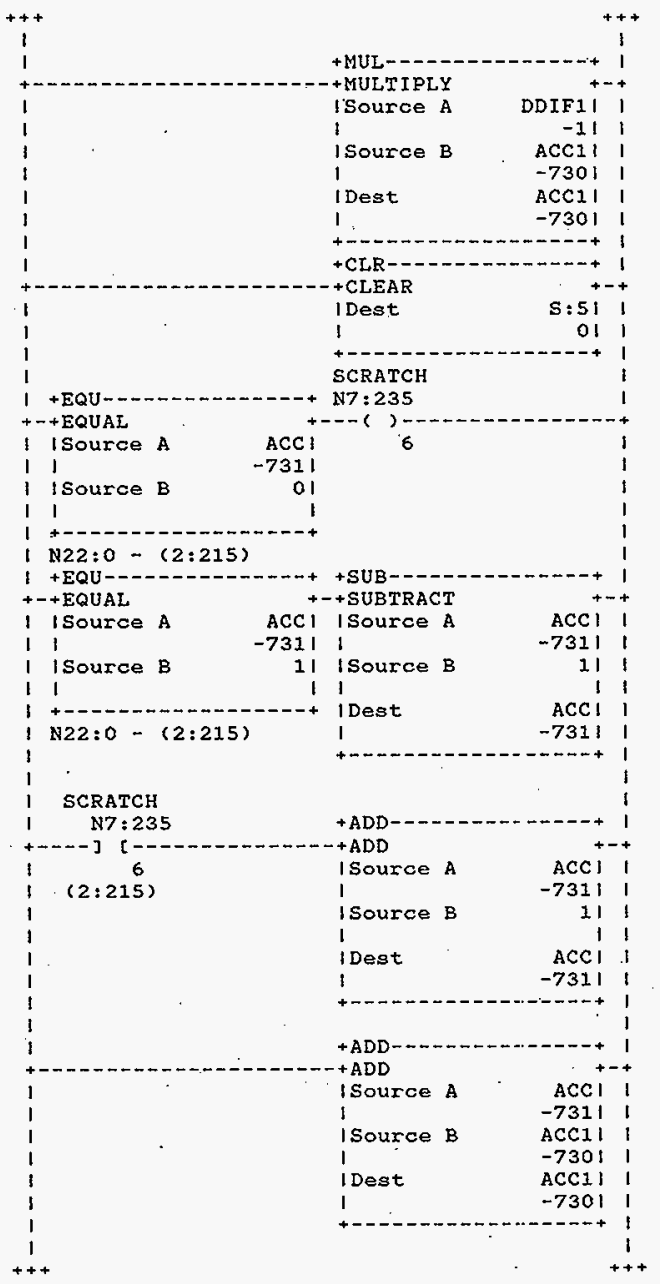

HNF-SD-FF-CSWD- 61 Rev. 0 


$$
\begin{aligned}
& 1 \\
& 1 \\
& 1 \\
& 1 \\
& 1 \\
& 1 \\
& 1 \\
& 1 \\
& 1 \\
& 1 \\
& 1 \\
& 1 \\
& 1 \\
& 1 \\
& 1 \\
& 1 \\
& 1 \\
& 1 \\
& 1 \\
& 1 \\
& 1 \\
& 1 \\
& 1 \\
& 1 \\
& 1 \\
& 1 \\
& 1 \\
& 1 \\
& 1 \\
& 1
\end{aligned}
$$

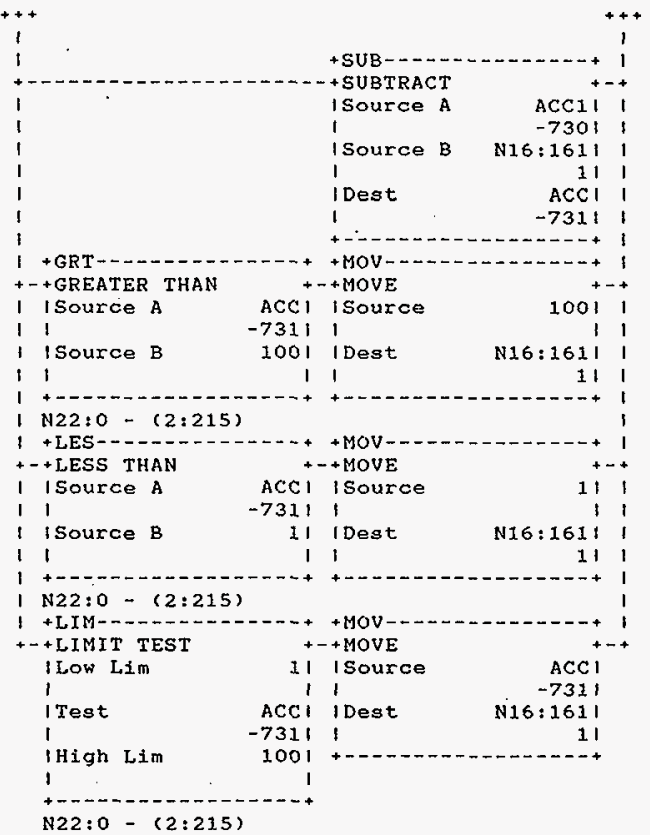

Rung 2:888

I DID180V

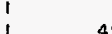

49

DUMMY 49

$1-\rightarrow[$ LBL ]

N22: 10

Rung $2: 889$

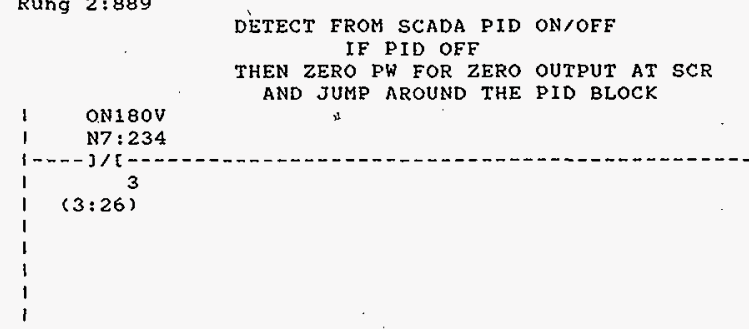

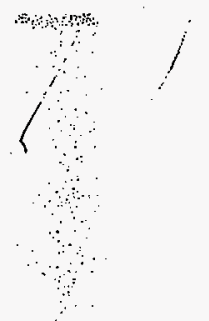


Processor and Data(OPS Unit 1)

Rung $2: 890$
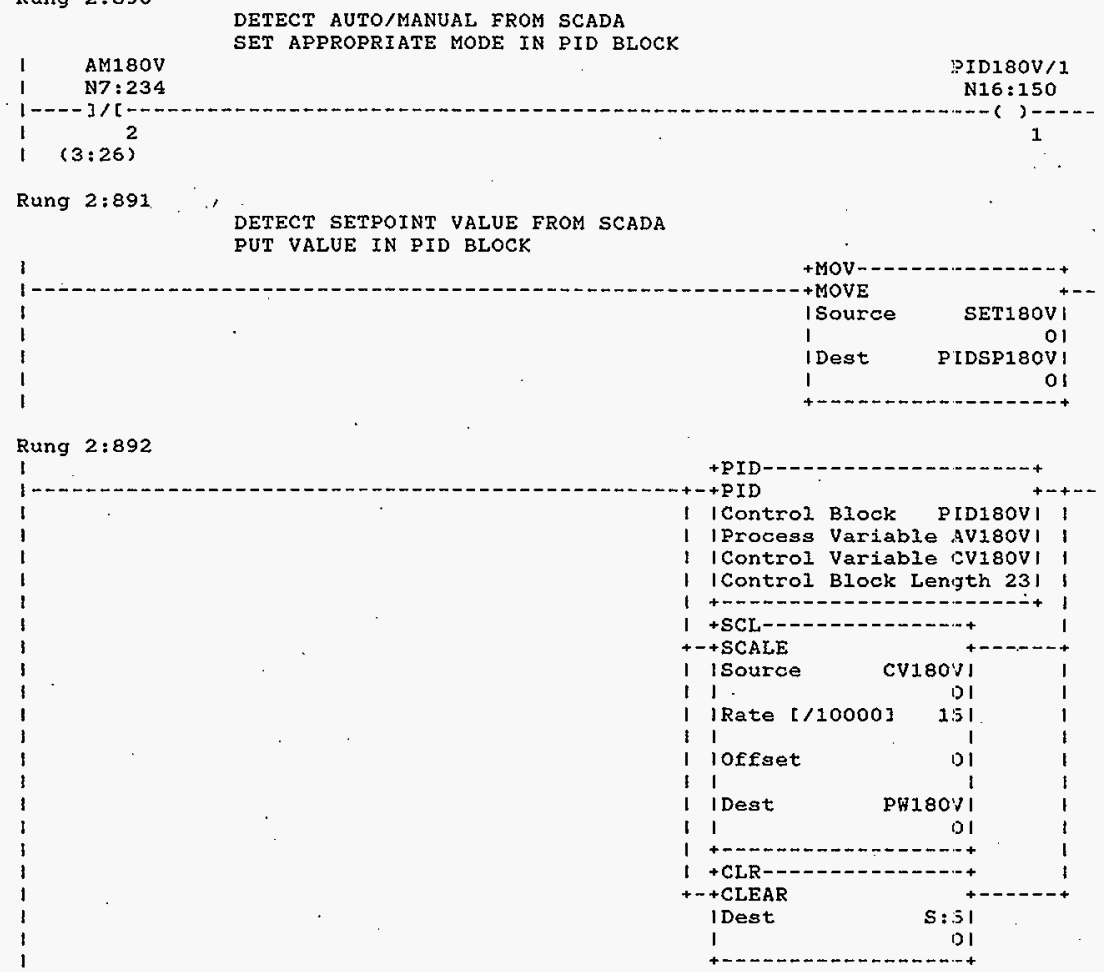

Rung 2:893

$$
\begin{aligned}
& \text { I } 88 \\
& \text { I- }
\end{aligned}
$$




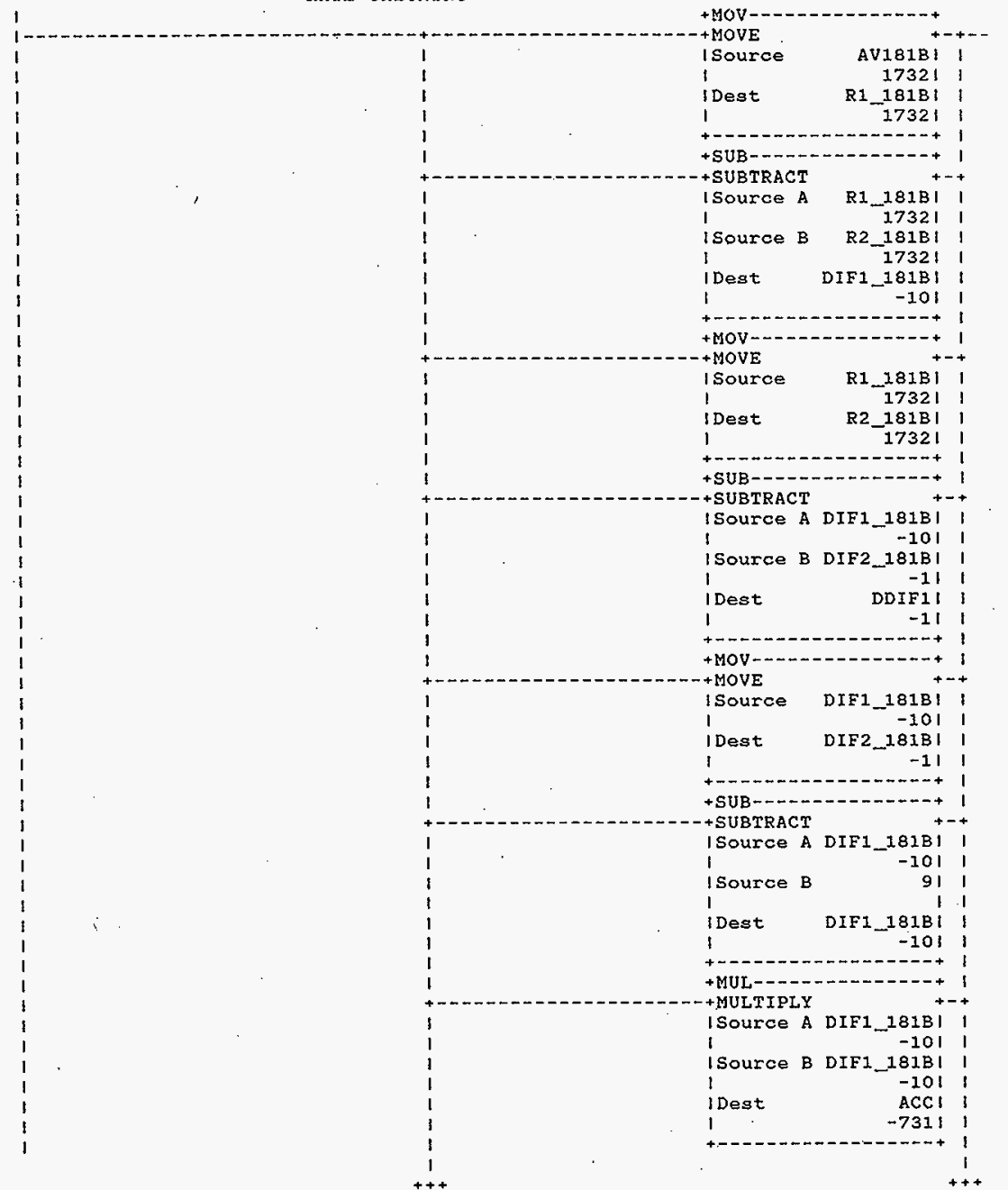

HNF-SD-FF-CSWD-61 Rev. 0 


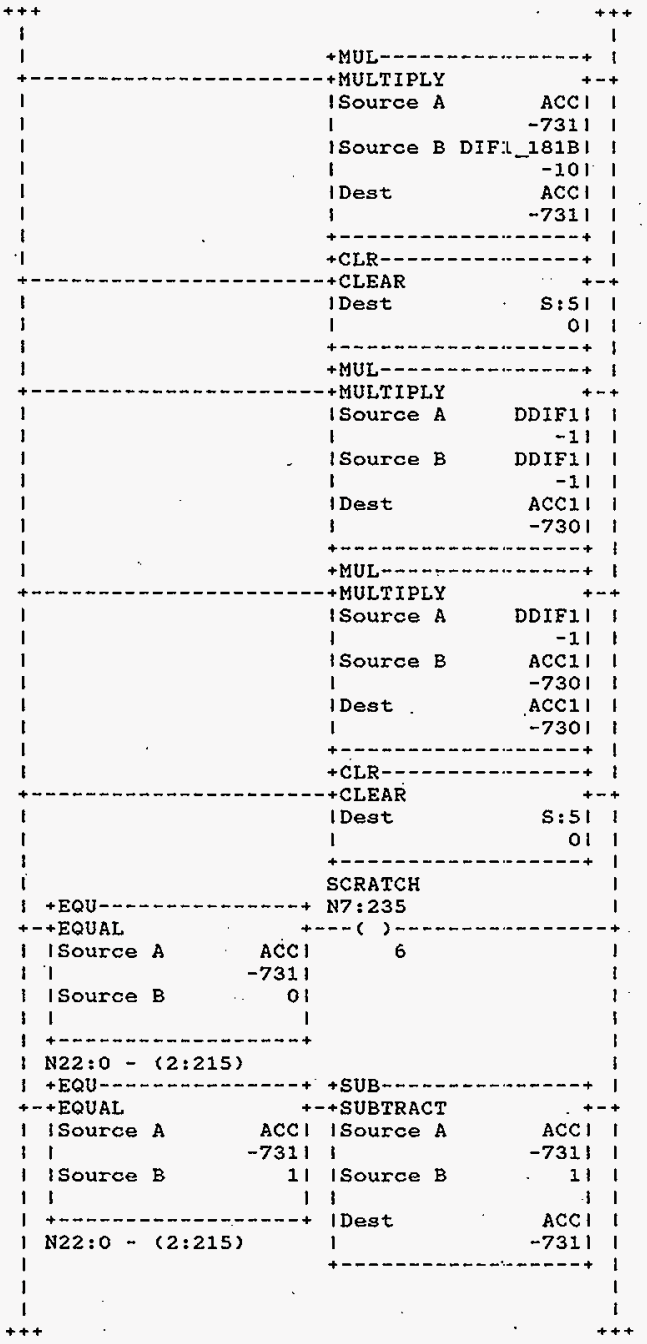

HNF-SD-FF-CSWD-61 Rev. 0 


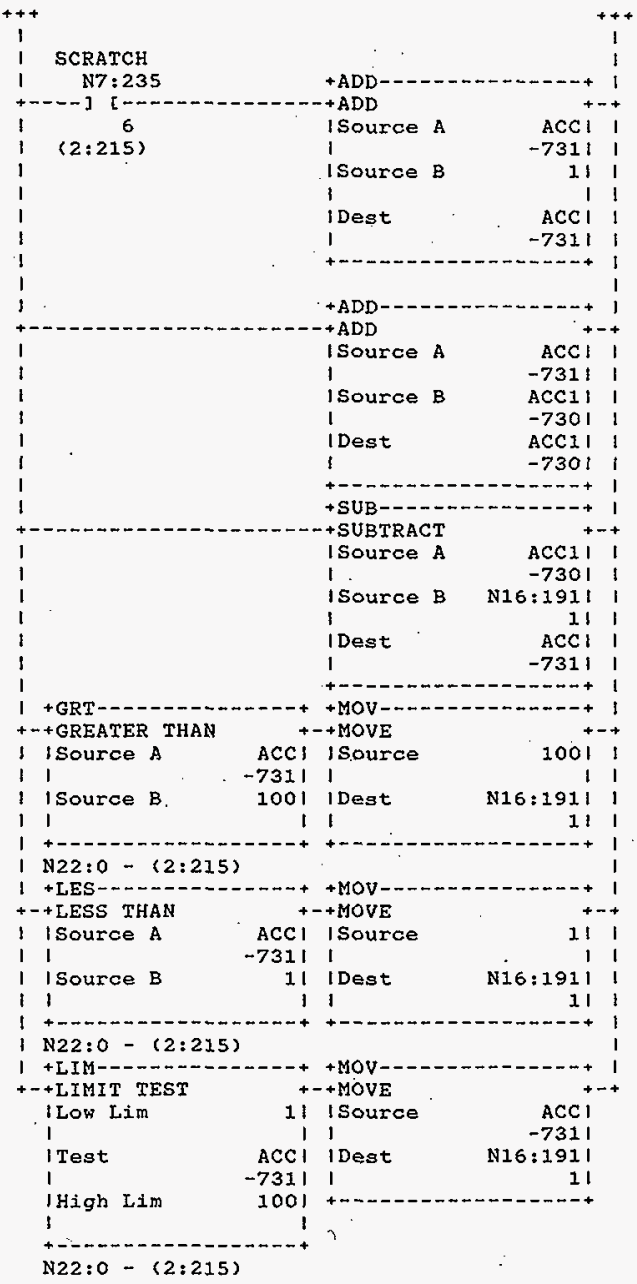

HNF-SD-FF-CSWD-61 Rev. 0 


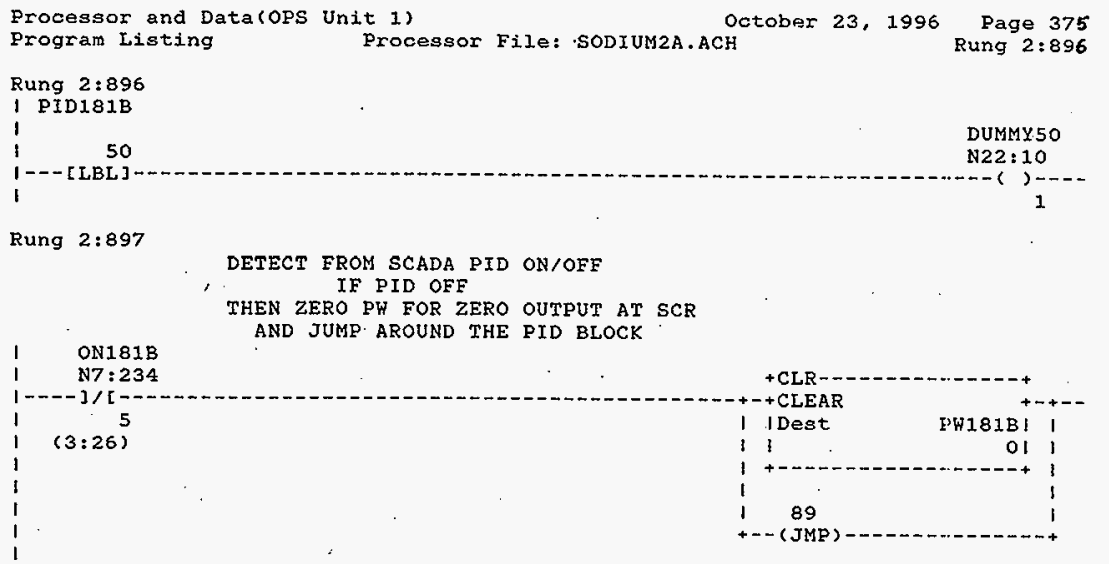

Rung $2: 898$

DETECT AUTO/MANUAL FROM SCADA

SET APPROPRIATE MODE IN PID BLOCK

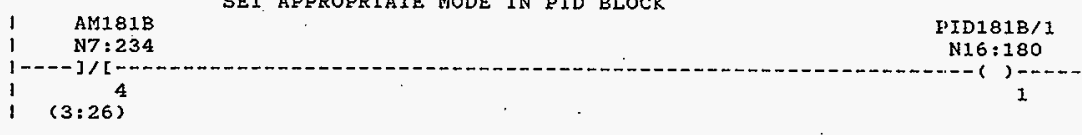

Rung 2:899

DETECT SETPOINT VALUE FROM SCADA

PUT VALUE IN PID BLOCK
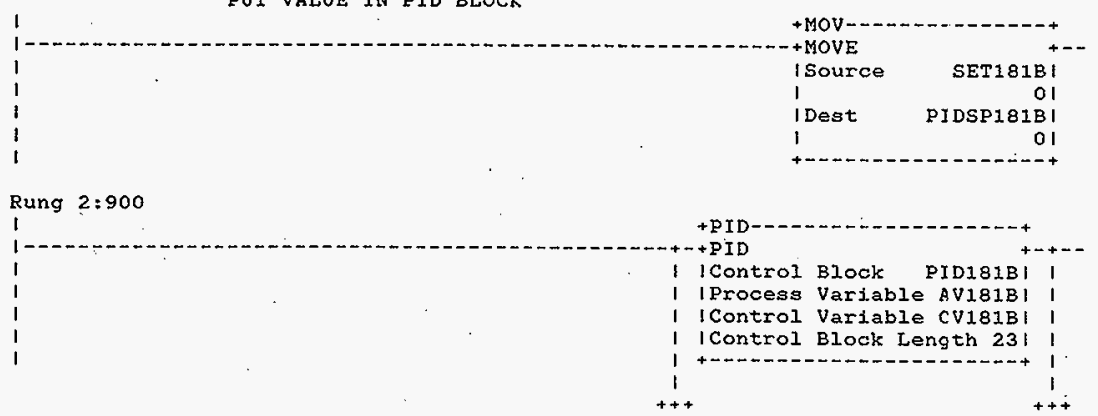

HNF-SD-FF-CSWD-61 Rev. 0 
Processor and Data(OPS Unit 1$)$

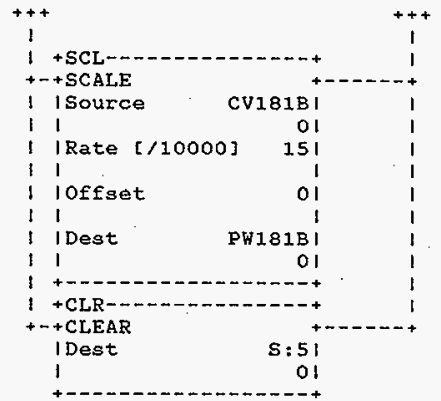

Rung 2:901

\begin{tabular}{|c|c|}
\hline $\begin{array}{l}1 \quad 89 \\
1--[L B L]-\ldots \\
1\end{array}$ & \begin{tabular}{c}
$N 7: 236$ \\
\hdashline 7 \\
\hdashline-6
\end{tabular} \\
\hline Rung 2:902 & \\
\hline 1 & PID181V \\
\hline I RT TMR/DN & \\
\hline $1 \quad \mathrm{~T} 4: 87$ & 51 \\
\hline $1----] /[\ldots$ & $--(J M P)-=--$ \\
\hline I $\quad \mathrm{DN}$ & . \\
\hline $1 \quad(2: 42)$ & \\
\hline
\end{tabular}

Rung 2:903

RATE CHECKING

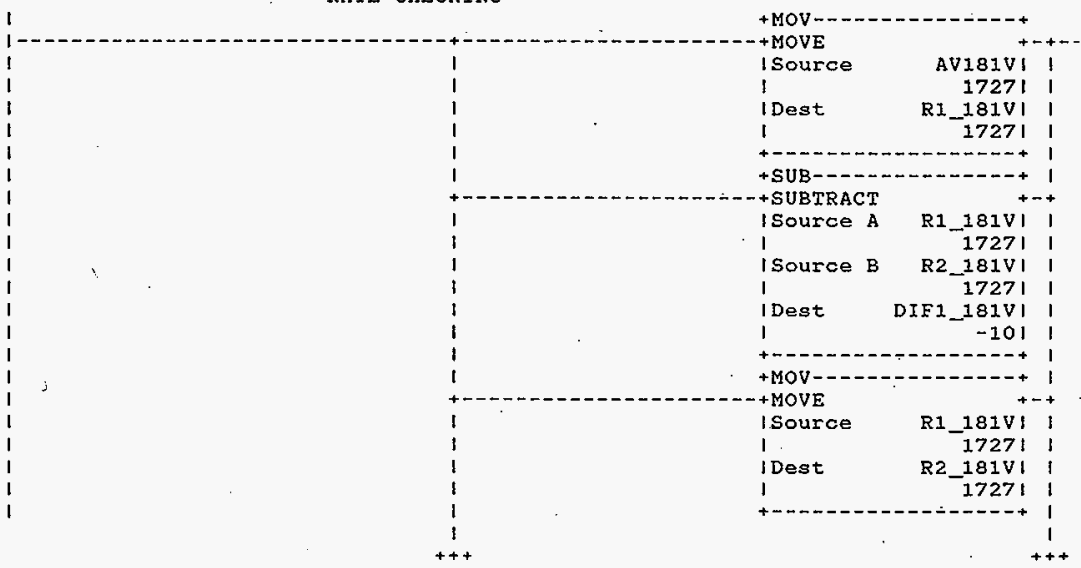

HNF-SD-FF-CSWD-61 Rev. 0 


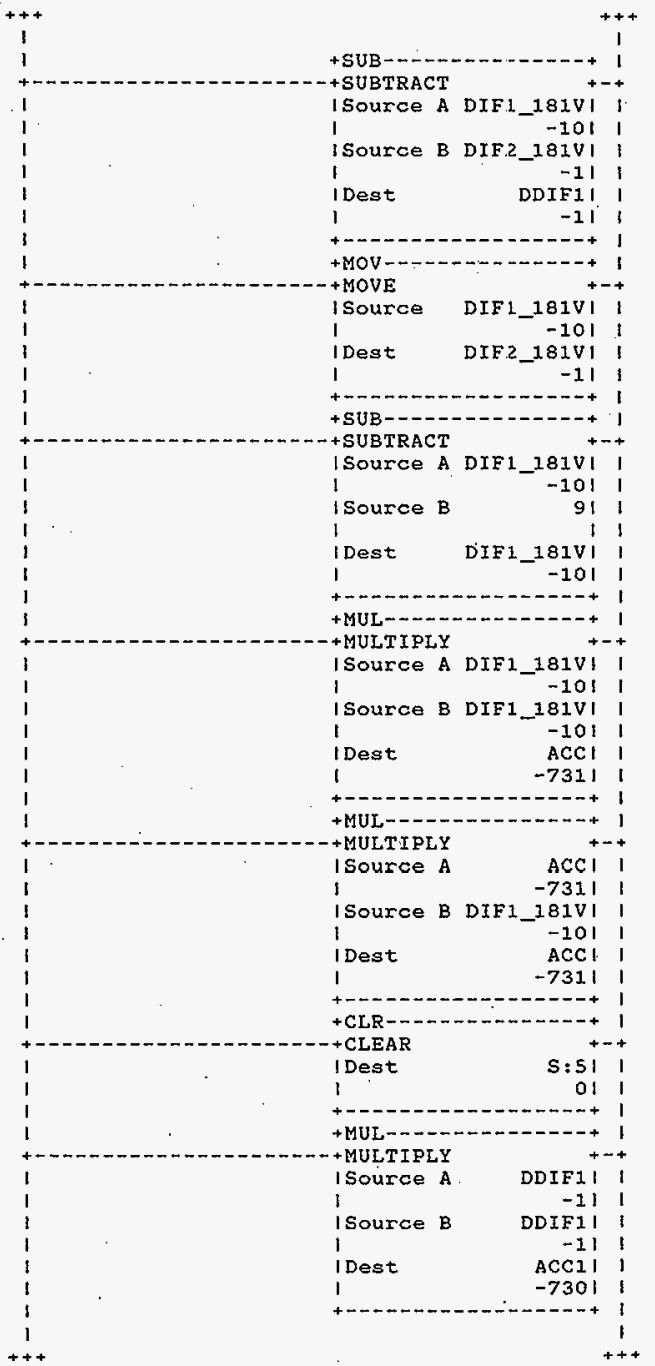

HNF-SD-FF-CSWD-61 Rev. 0 
Processor and Data(OPS Unit 1) Program Listing
Processor File: SODIUM2A.ACH

October 23, 1996

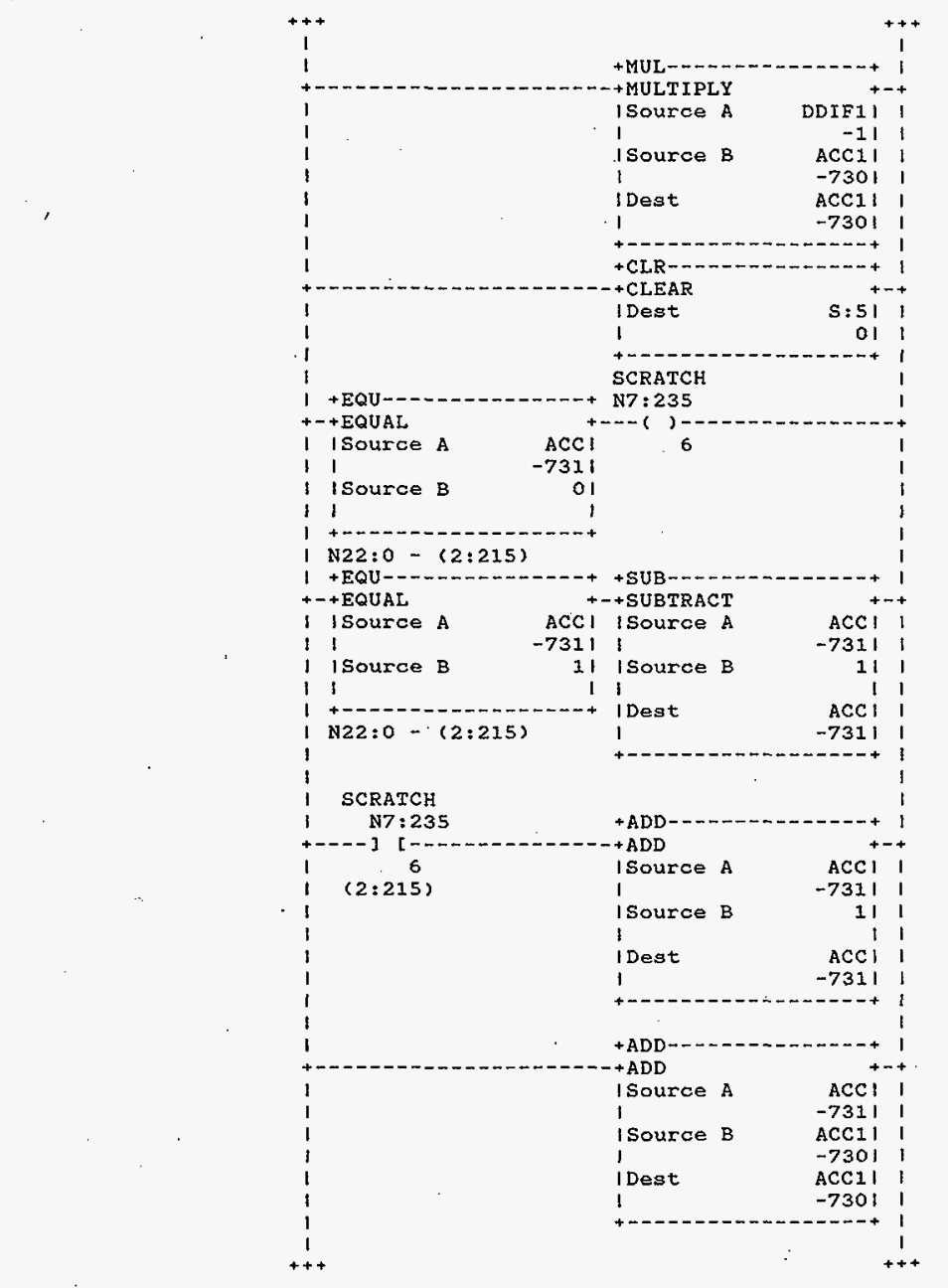

Rung 2.903 .

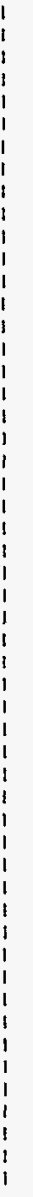

HNF-SD-FF-CSWD-61 Rev. 0 
Procesgor and Data(OPS Unit 1) Program Listing
Processor File: SODIUM2A.ACH
Octobar 23, 1996

Page 379 Rung 2:903

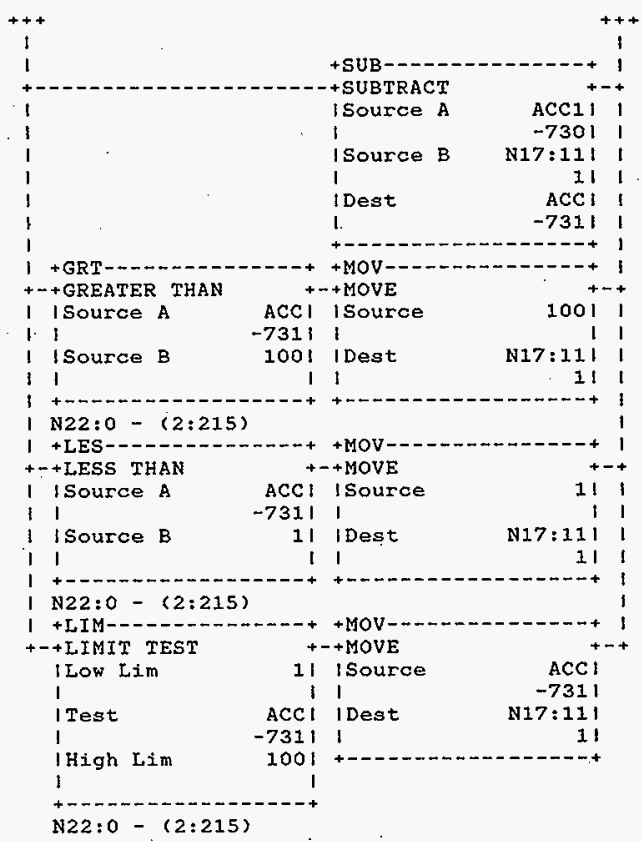

Rung 2:904

I PIDI81V

1

151

DUMMY 51

1 - - [ L LBL]

N22:10

I

Rung $2: 905$

DETECT FROM SCADA PID ON/OFE

IF PID OFF

THEN ZERO PW FOR ZERO OUTPUT AT SCR

AND JUMP AROUND THE PID BLOCK

ON181V
N7:234
$1-\ldots-1 /[-\ldots$

7

HNF-SD-FF-CSWD-61 Rev. 0

Page $74-1$ 
Processor and Data(Ops Unit 1)

October 23, 1996

Page 380

Program Listing

Processor Eile: SODIUM2A.ACH

Rung 2:906

$1 \quad A M 181 \mathrm{~V}$

DETECT AUTO/MANUAT, FROM SCADA

i $\quad$ 7 $: 234$

SET APPROPRIATE MODE IN PID BLOCK

$1-\cdots-\cdots] /[\ldots$

i $(3: 26)$

PID181V/I

N17:0

Rung 2:907

DETECT SETPOINT VALUE FROM SCADA PUT VALUE IN PID BLOCK
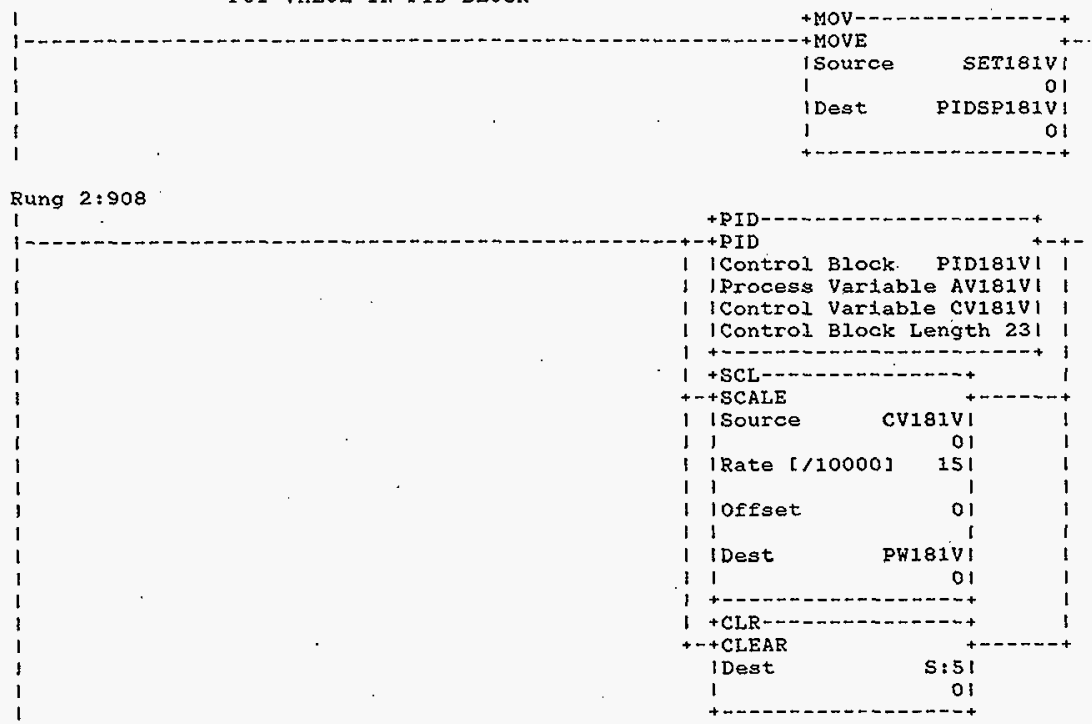

Rung 2:909

190

$N 7: 236$

$1--[\mathrm{LBI}]-$

$-6,2-\cdots$

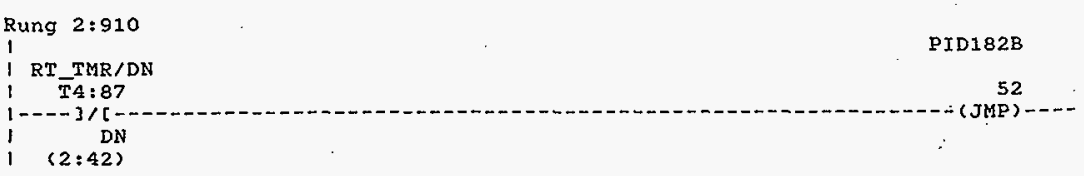

HNF-SD-FF-CSWD-61 Rev. 0 
Rung 2:911

RATE CHECKING

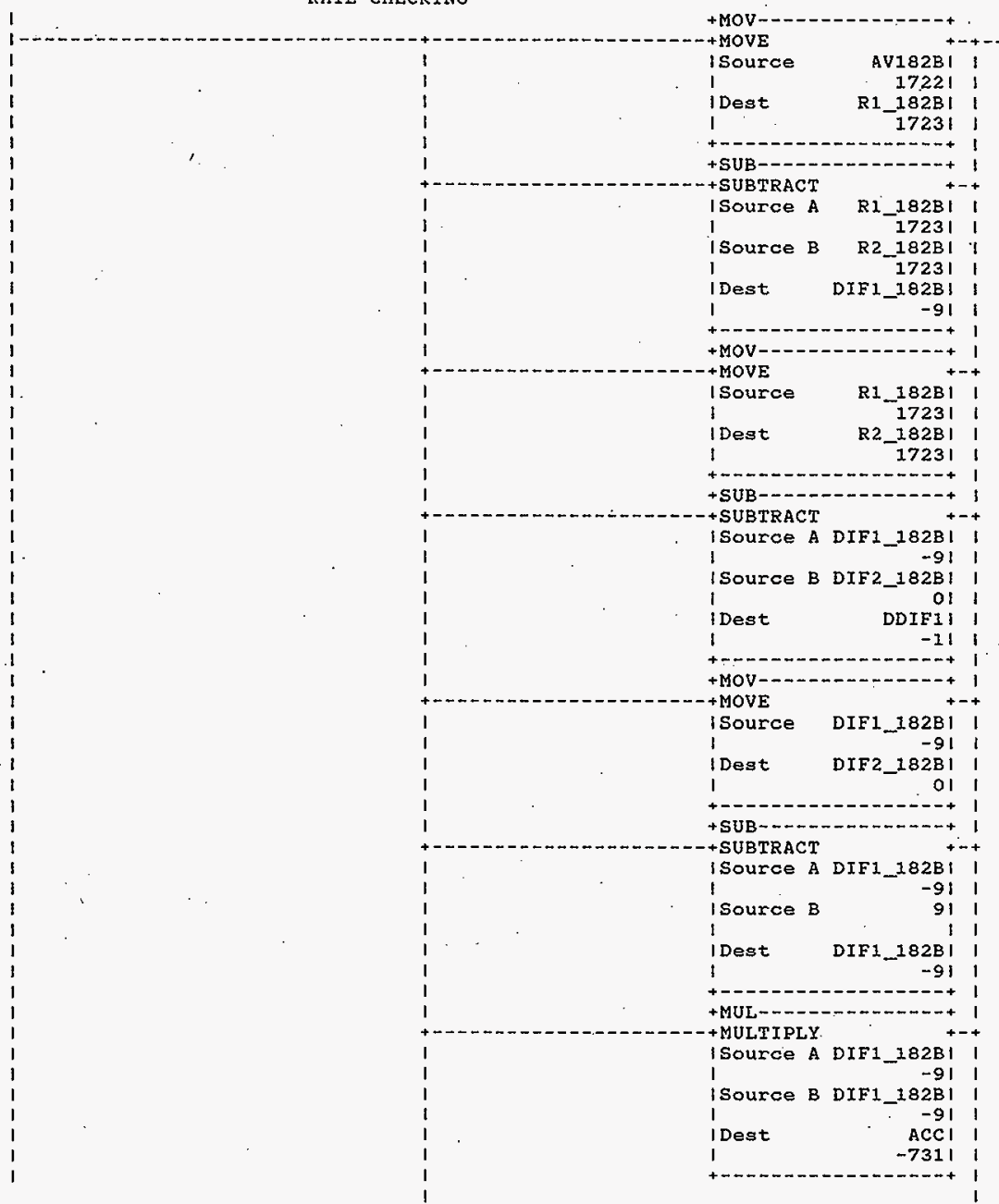

HNF-SD-FF-CSWD-61 Rev. 0 
Processor and Data(OPS Unit 1 ) Program Eisting
October 23, 1996

Processor File: SODIUM2A.ACH

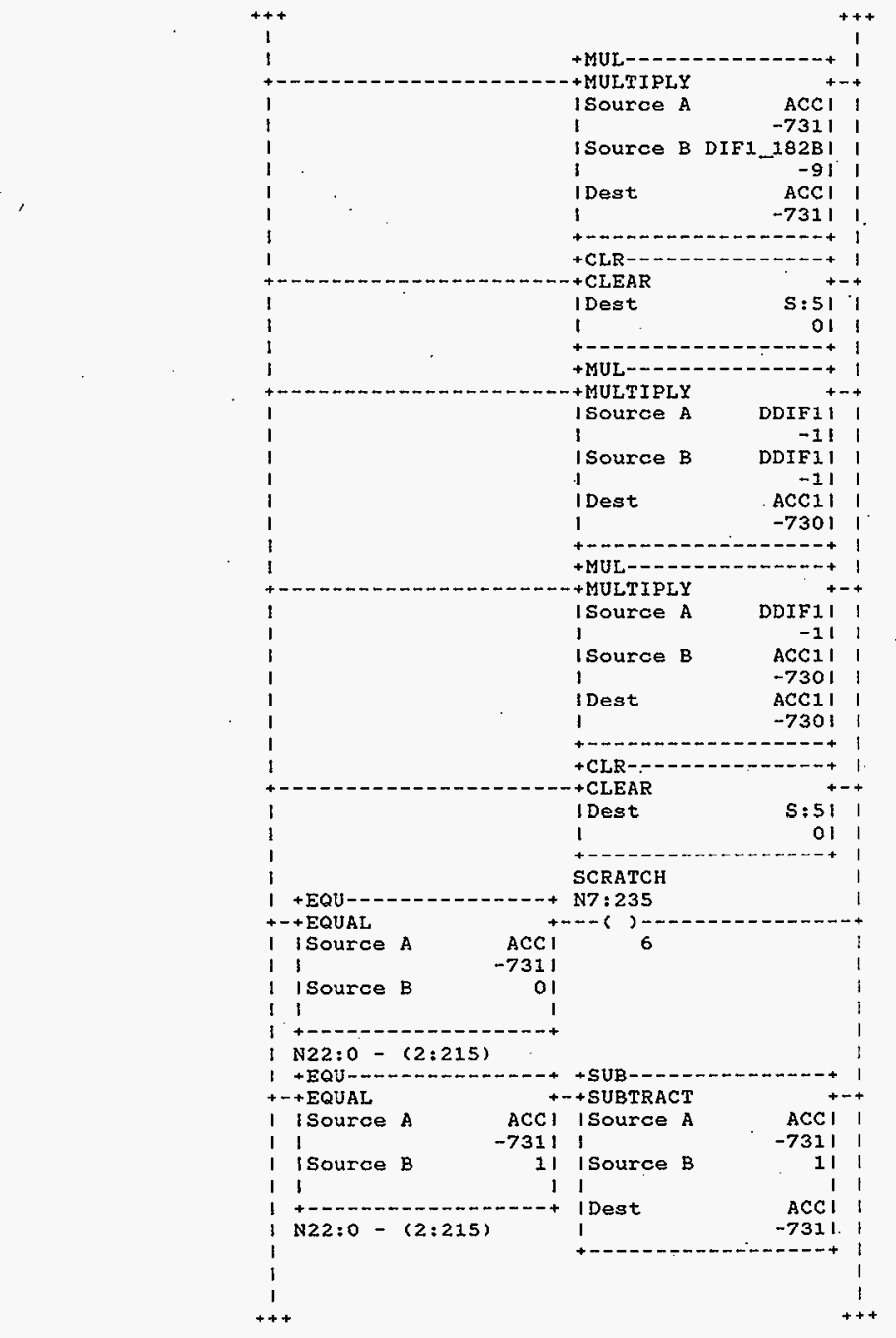
Rung 2:911
Page 382

$$
\begin{aligned}
& 1 \\
& 1 \\
& 1 \\
& 1 \\
& 1 \\
& 1 \\
& 1 \\
& 1 \\
& 1 \\
& 1 \\
& 1 \\
& 1 \\
& 1 \\
& 1 \\
& 1 \\
& 1 \\
& 1 \\
& 1 \\
& 1 \\
& 1 \\
& 1 \\
& 1 \\
& 1 \\
& 1 \\
& 1 \\
& 1 \\
& 1 \\
& 1 \\
& 1 \\
& 1 \\
& 1 \\
& 1 \\
& 1 \\
& 1 \\
& 1 \\
& 1 \\
& 1 \\
& 1 \\
& 1 \\
& 1 \\
& 1 \\
& 1 \\
& 1
\end{aligned}
$$

HNF-SD-FF-CSWD-61 Rev. 0 
Processor and Data(ops Unit 1)

October 23, 1996

Page 383

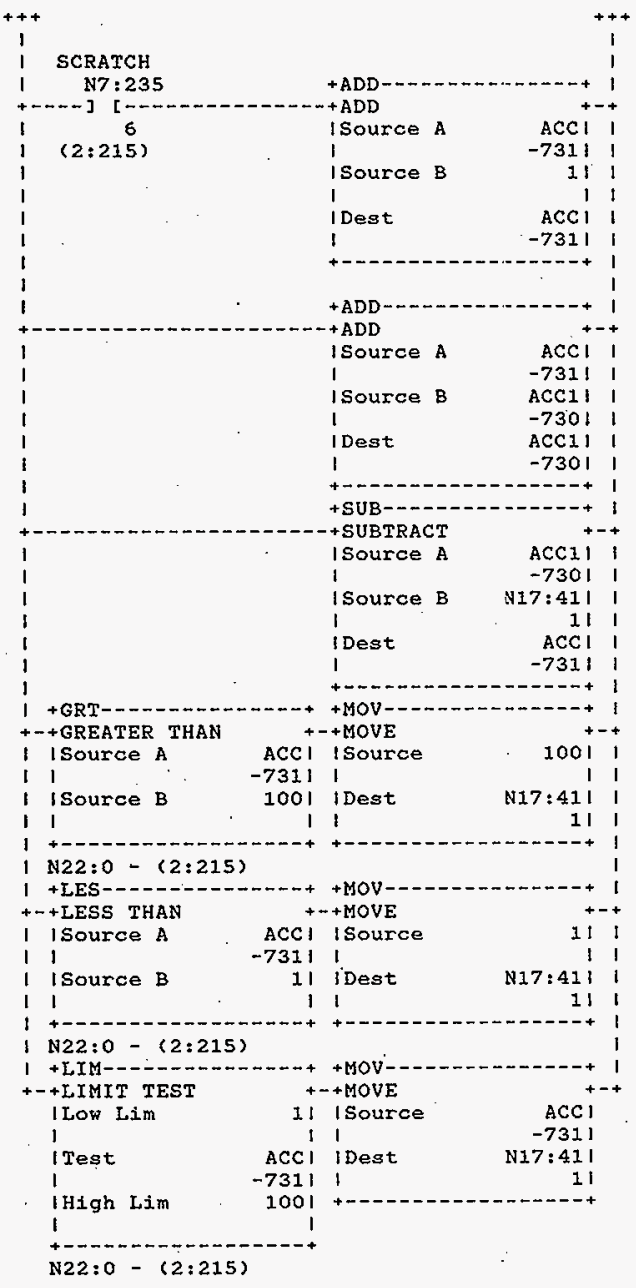

HNF-SD-FF-CSWD-6! Rev. 0 
Rung 2:912

I PIDI82B

Rung 2:913
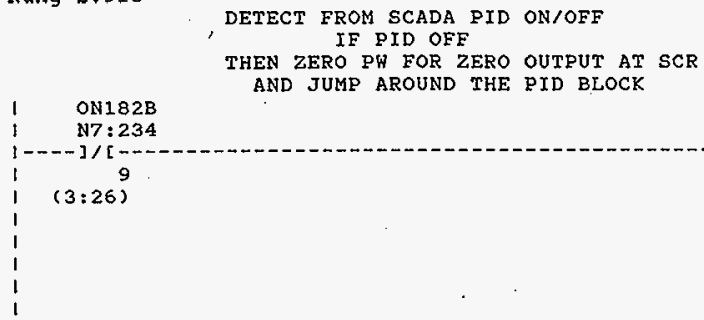

Rung 2:914

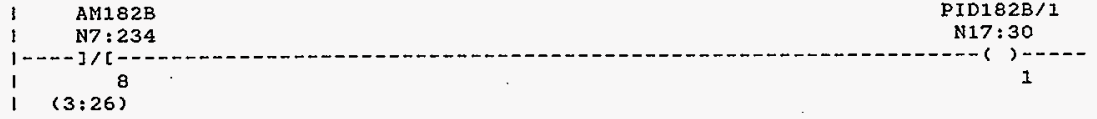

Rung 2:915

DETECT AUTO/MANUAL FROM SCADA

SET APPROPRIATE MODE IN PID BLOCK

\section{DETECT SETPOINT VALUE FROM SCADA PUT VALUE IN PID BLOCK}

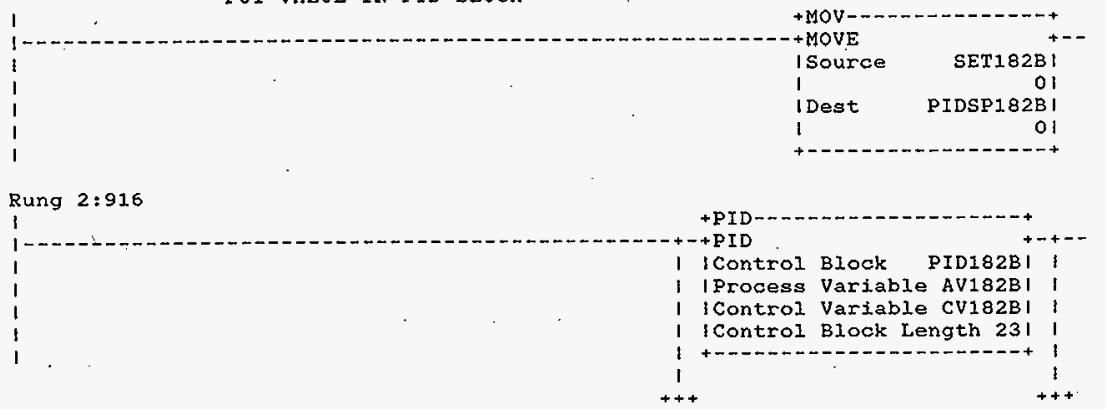

HNF-SD-FF-CSWD-61 Rev. 0

Page 746 


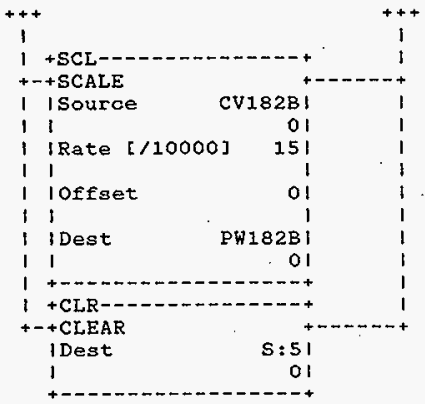

Rung 2:917

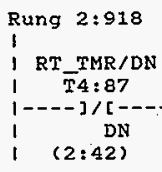

Rung 2:919

RATE CHECKING

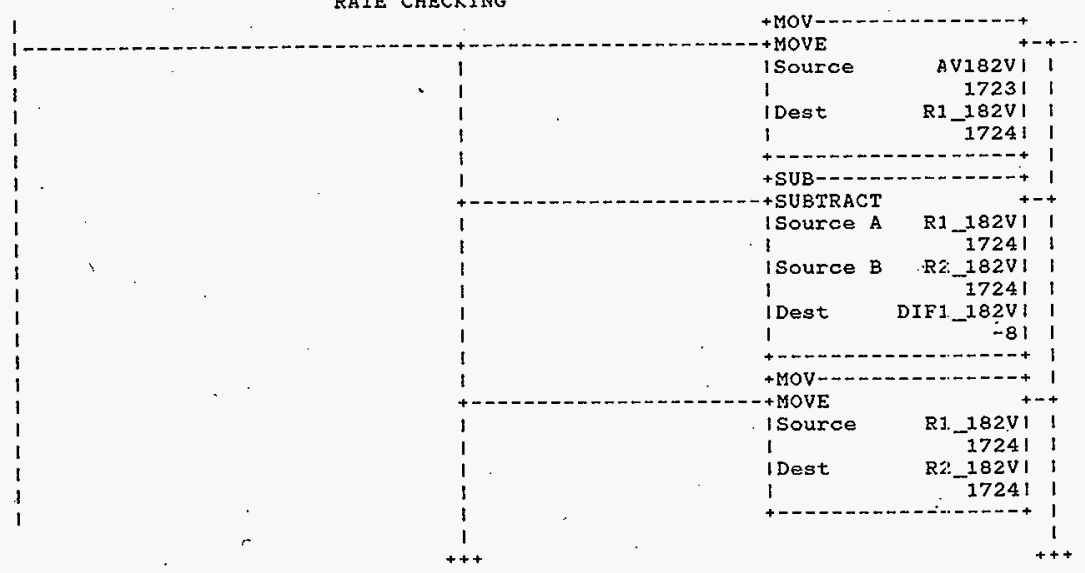

HNF-SD-FF-CSWD-61 Rev. 0 


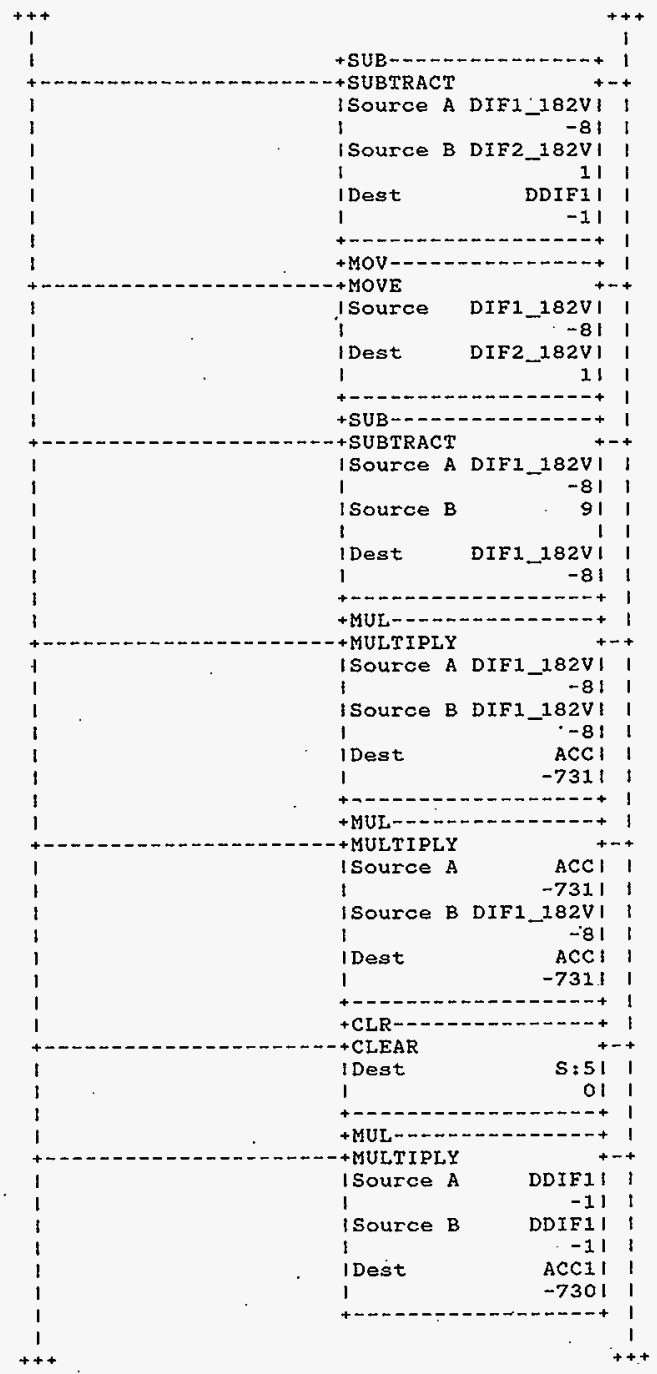

HNF-SD-FF-CSWD-61 Rev. 0 


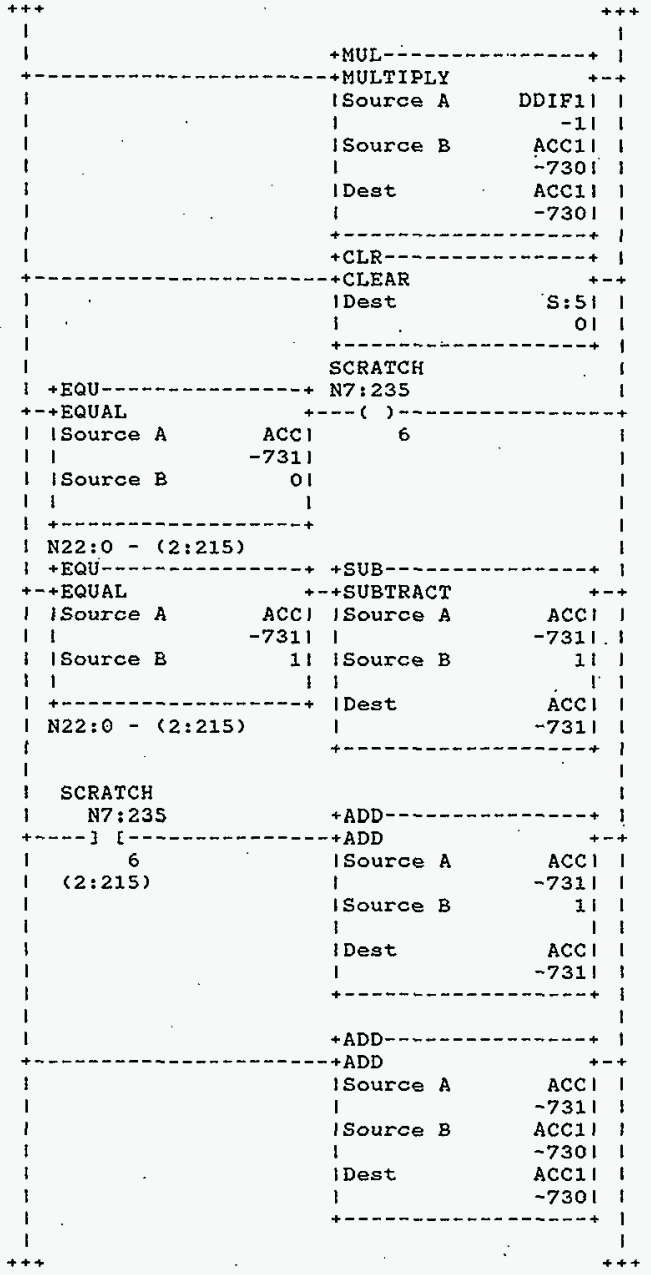

HNF-SD-FF-CSWD-61 Rev, 0 
Processor and Data(OPS Unit 1) Program Listing
Processor File: SODIUM2A.ACH

October 23,1996

Page 388

Rung 2:919

$$
\begin{aligned}
& 1 \\
& 1 \\
& 1 \\
& 1 \\
& 1 \\
& 1 \\
& 1 \\
& 1 \\
& 1 \\
& 1 \\
& 1 \\
& 1 \\
& 1 \\
& 1 \\
& 1 \\
& 1 \\
& 1 \\
& 1 \\
& 1 \\
& 1 \\
& 1 \\
& 1 \\
& 1 \\
& 1 \\
& 1 \\
& 1 \\
& 1 \\
& 1 \\
& 1 \\
& 1 \\
& 1 \\
& 1
\end{aligned}
$$

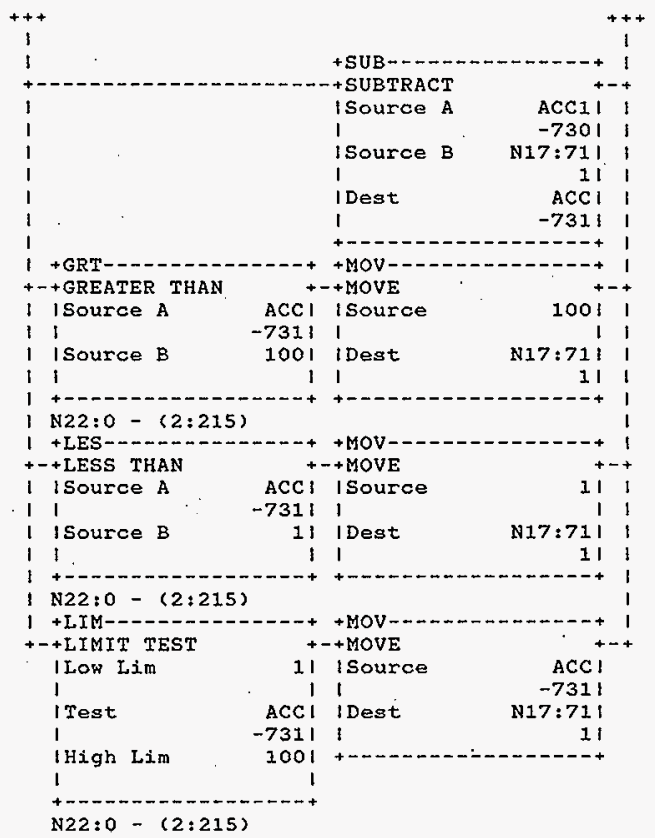

Rung $2: 920$

I PID182V

DUMMY 53

53

N22: 10

1.-. [LBL $]$

Rung 2:921

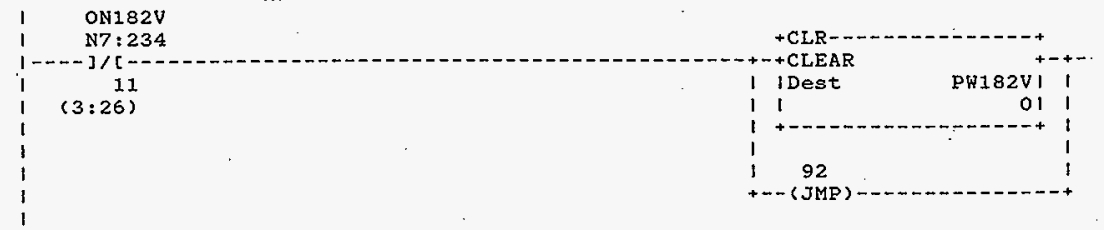

DETECT FROM SCADA PID ON/OFF

THEN ZERO PW FOR ZERO OUTPUT AT SCR

AND JUMP AROUND THE PID BLOCK

HNF-SD-FF-CSWD-61 Rev. 0 
Processor and Data(OPs Unit 1)

Rung 2:922

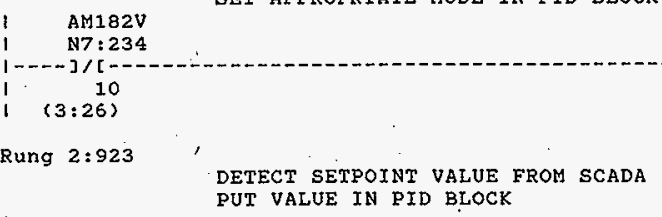

DETECT AUTO/MANUAL FROM SCADA

SET APPROPRIATE MODE IN PID BLOCK
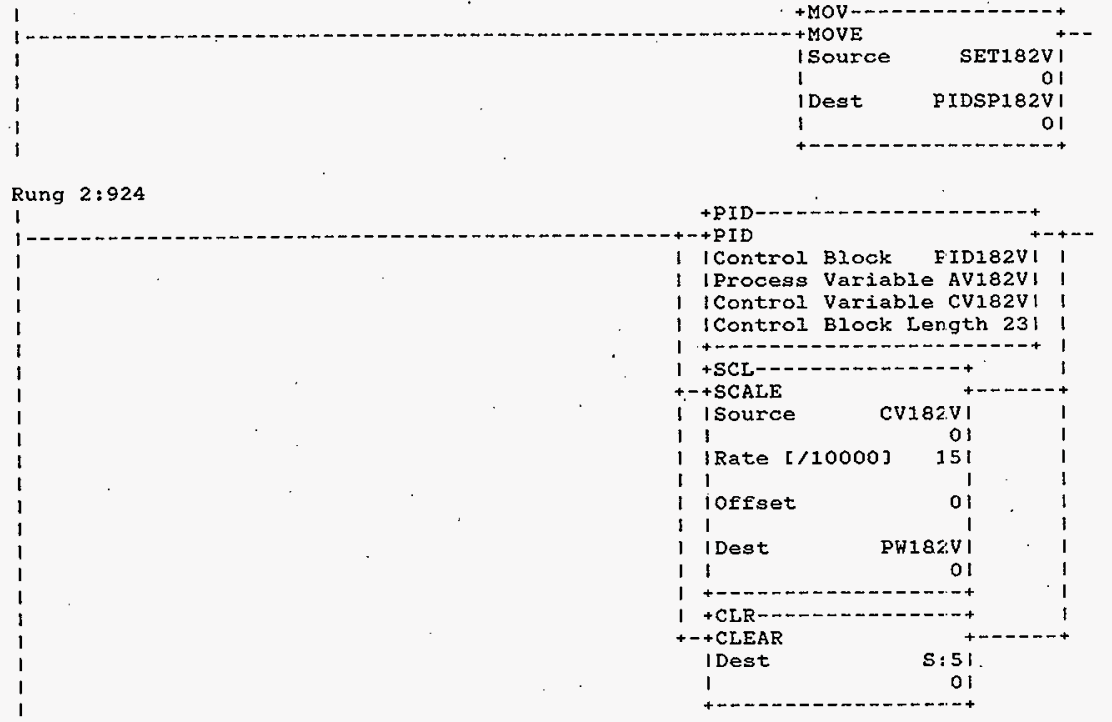

Rung $2: 925$

N7 : 236

192

$-(10$ I

Rung 2:926

1

RT TMR/DN

T4:87

- - - ] ] [ -

(2:42)

HNF-SD-FF-CSWD-61 Rev. 0

Page -751 
Processor and Data(ops Unit 1)

Program Listing

Rung $2: 927$

RATE CHECKING

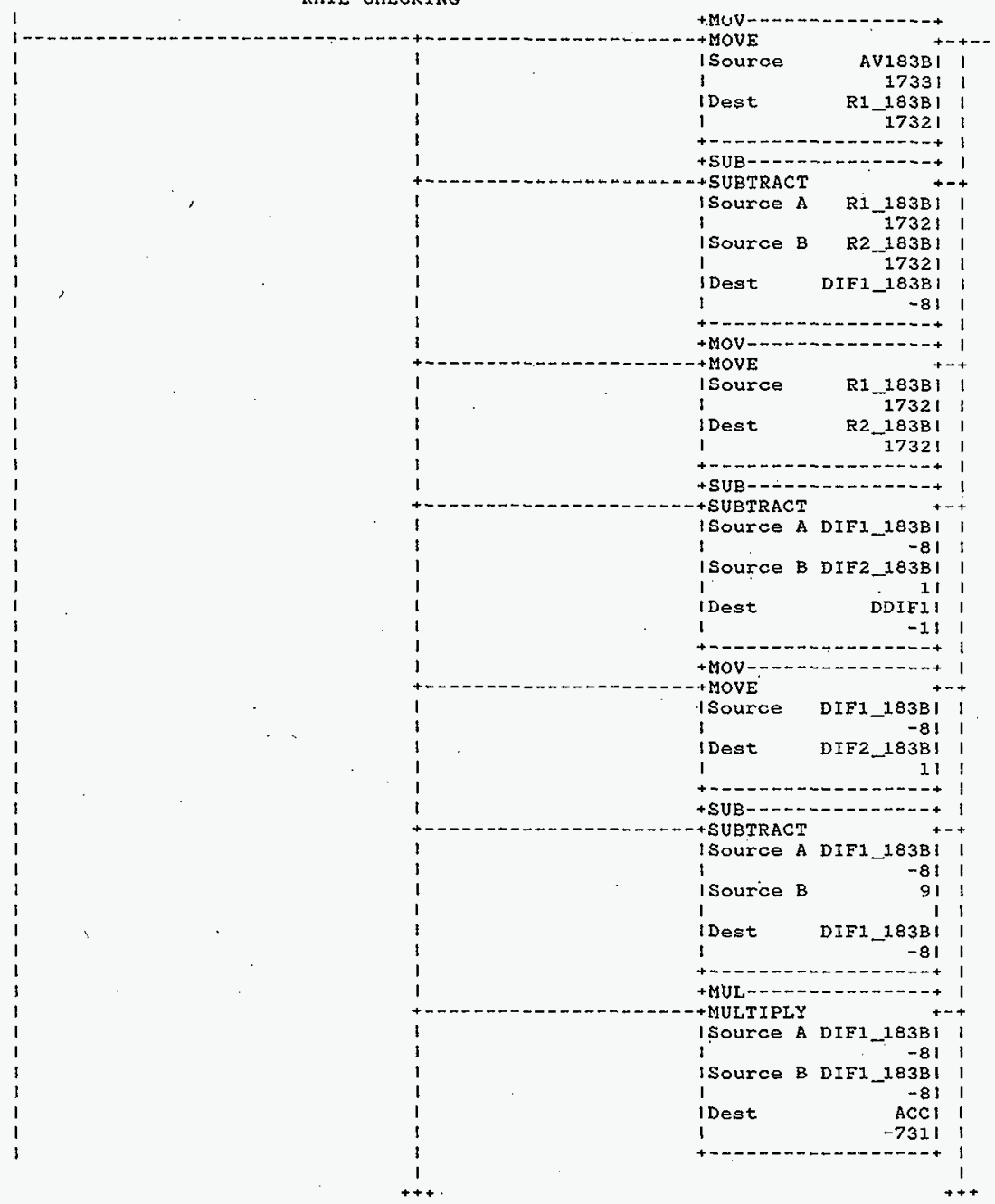

HNF-SD-FF-CSWD-61 Rev. 0 


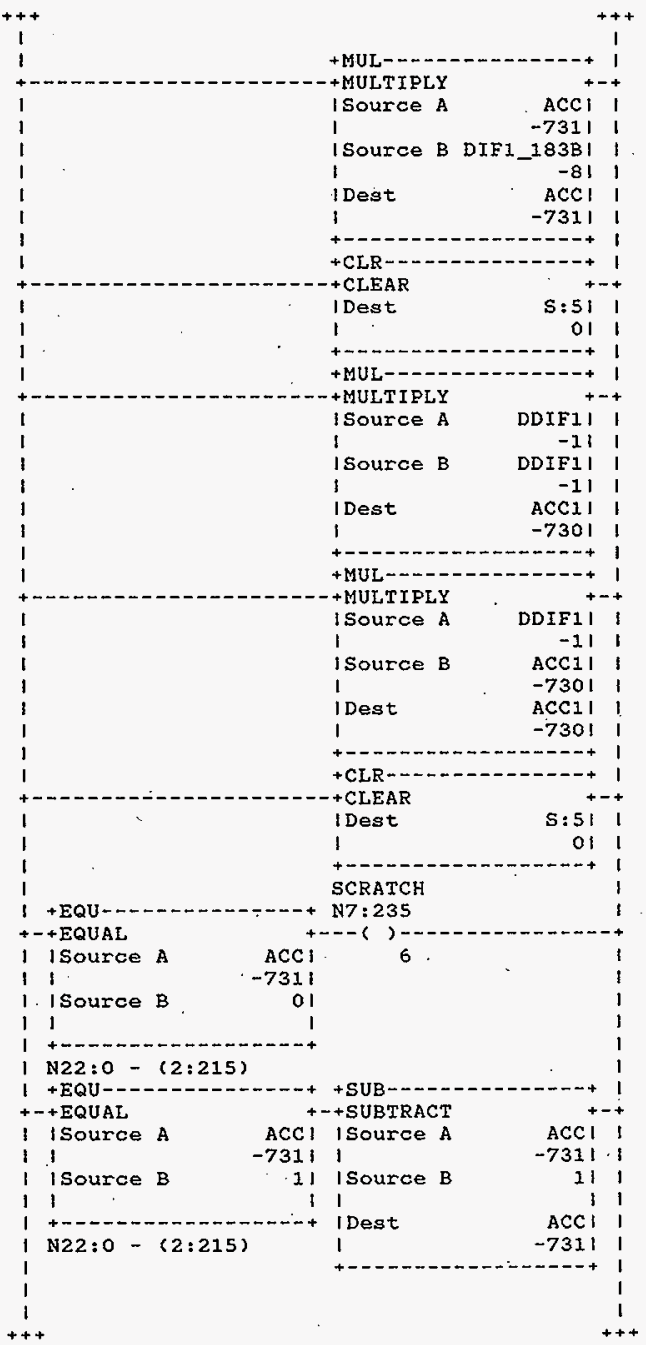

HNF-SD-FF-CSWD-6I Rev. 0 


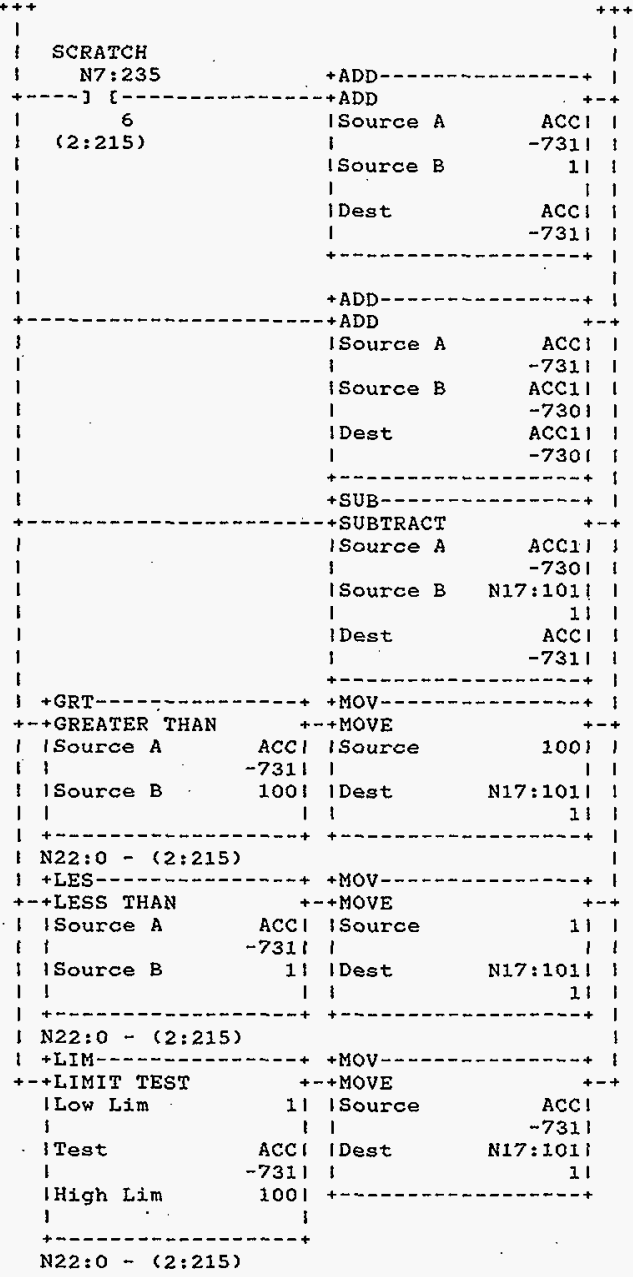

HNF-SD-FF-CSWD-61 Rev. 0 
Processor and Data(OPS Unit 1)

Rung 2:928

I PID183B

154

DUMMY 54

$1---[L B L 3-$

N22:10

I

$$
4
$$

Rung 2:929
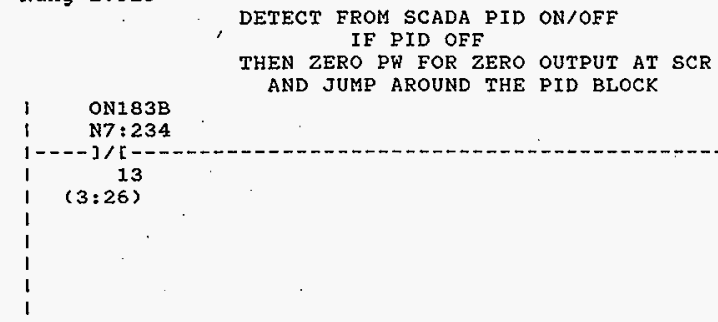

Rung 2:930

$\begin{array}{lc}\text { I } & \text { AM } 183 B \\ \mid & N 7: 234 \\ 1 & --3 /[-: \\ \text { I } & 12\end{array}$

DETECT AUTO/MANUAL FROM SCADA

SET APPROPRIATE MODE IN PID BLOCK

Rung 2:931

DE'TECT SETPOINT VALUE FROM SCADA PUT VALUE IN PID BLOCK

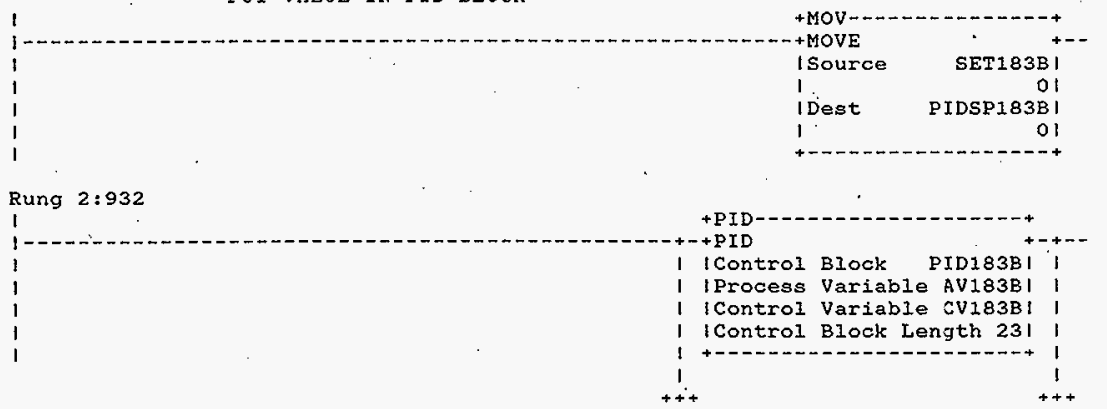

HNF-SD-FF-CSWD-61 Rev. 0 


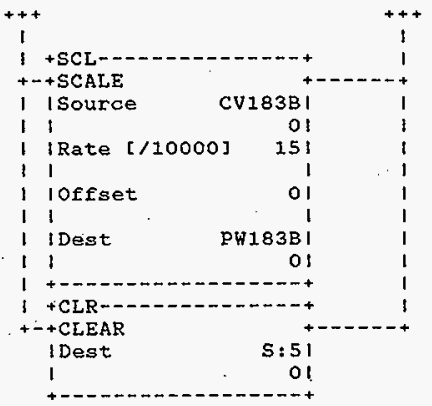

Rung $2: 933$

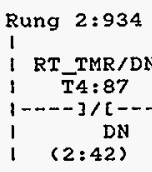

\section{PID183V}

55

$-(J M P)+--$

Rung 2:935

\section{RATE CHECKING}

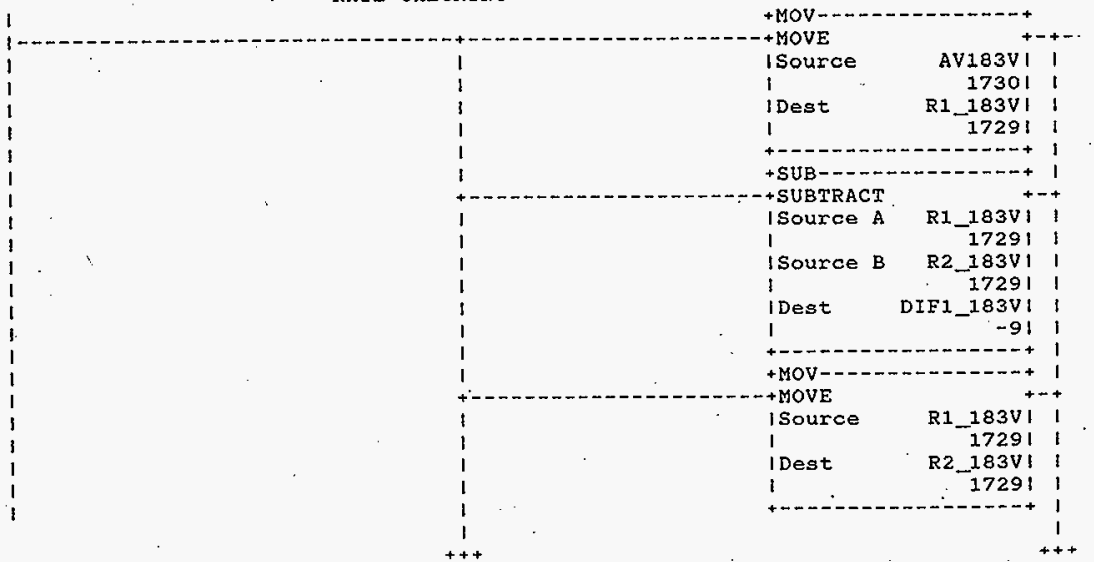

HNF-SD-FF-CSWD-61 Rev. 0 


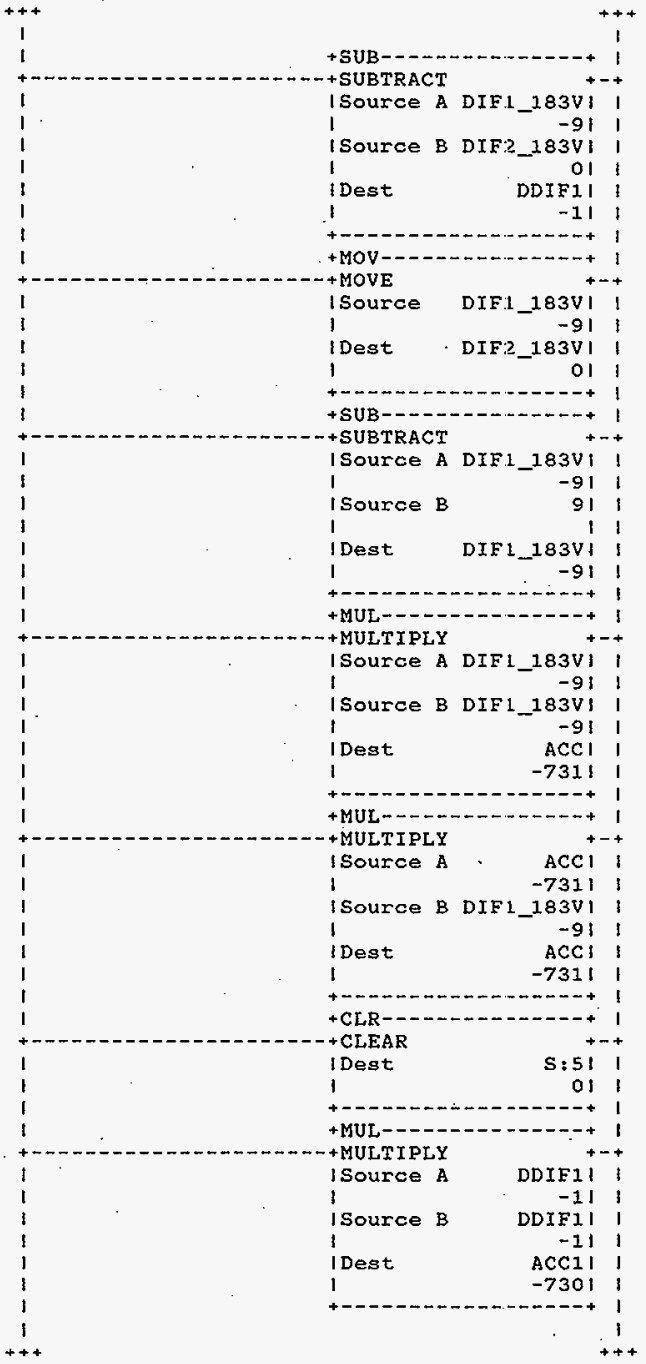

HNF-SD-FF-CSWD-61 Rev. 0 


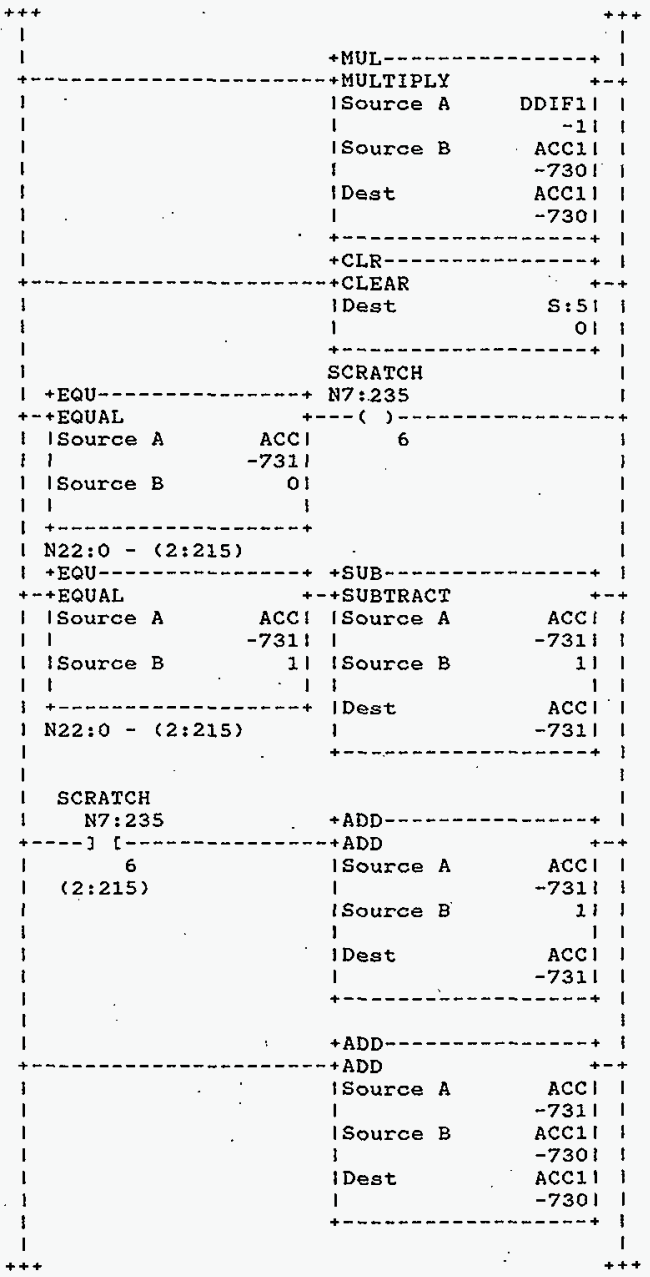

HNF-SD-FF-CSWD-61 Rev, 0 


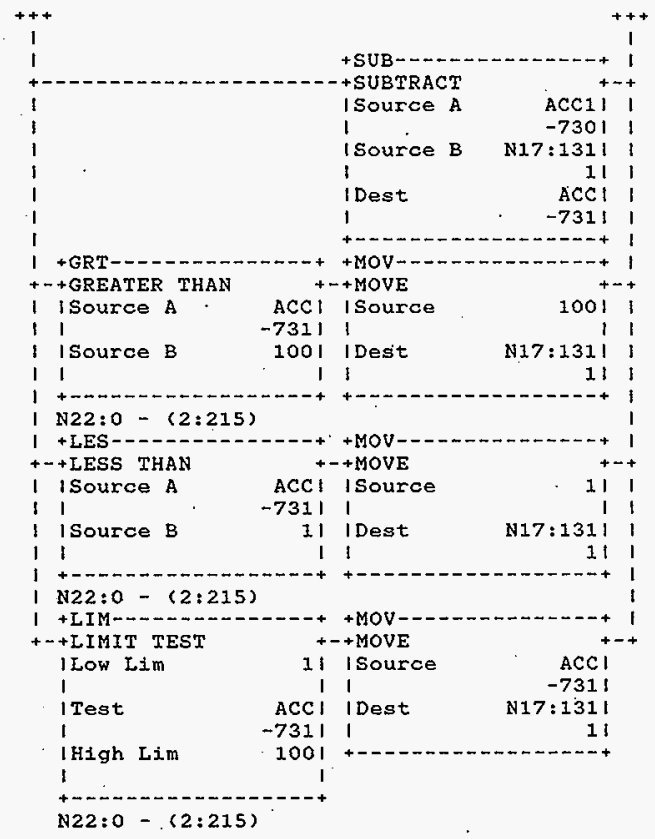

Rung 2:936

1

DNR_4

N $22: 5$

1

(L) -

Rung 2:937

I PID183V

I

155

DUMMY 55

i-- [ LBL]

N22:10

HNF-SD-FF-CSWD-til Rev. 0 
Rung 2:938

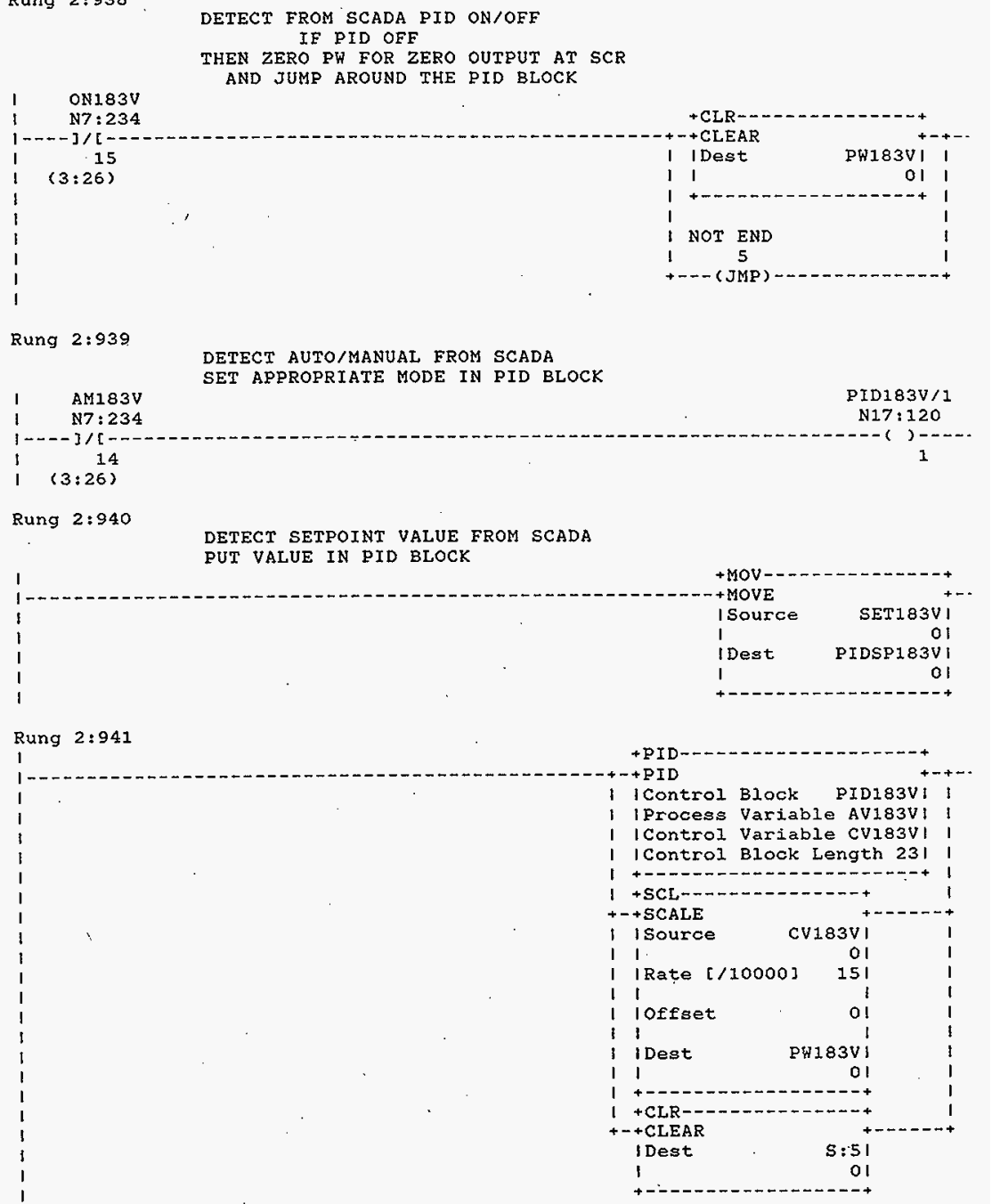

HNF-SD-FF-CSWD-61 Rev. 0 
Processor and Data(OPS Unit 1 )

Program Listing . Processor File: SODIUM2A.ACH

October 23, 1996 Page 399

Rung 2:942

$f$ NOT END

1 .

DUMMY 8

$1--2$

N22:7

I

BL

Rung $2: 943$

* FIRE SCRs * *

PIPE ZONES

There is one timer for each zone. Each timer is reset when it reaches $200 \mathrm{~ms}$. The zone's SCR is turned on when the timer is less than the. zone's pulse width (PW). Note: no more than two tanks can be enabled at the same time.

1
1
1
1
1 +TON-D-D- T- - - - - - + 1+ TIMER ON DELAY +-(EN)-

1 Timer

ITime Base

TMR $143+-(D N)$

I Preset

0.011

I Accum

251

Rung 2 : 944

1 TMR143/DN

$\mathrm{T} 4: 1$

TMR 143

$T 4: 1$

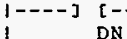

(2:943)

Rung 2:945

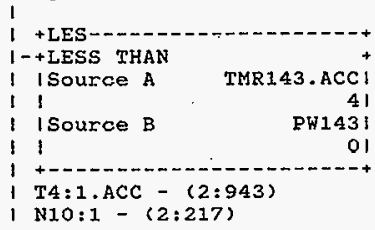

SCR 143

$0: 24$

I-+LESS THAN

I Source A

C!

--()$---$

1

Source B

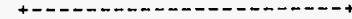

1 N10:1 - (2:217)

Rung $2: 946$

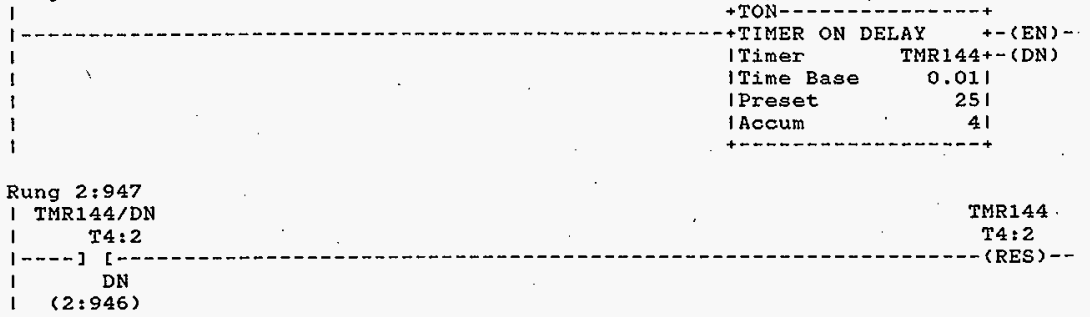

HNF-SD-FF-CSWD-61 Rev. 0

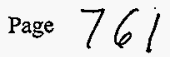


Processor and Data(OPS Unit 1)

Rung 2:948

1

1 +LES--

SCR_144

$1-+$ LESS THAN

I I Source A

11

i isource B

TMR144. ACC I

$0: \overline{2} 4$

PW1441

11

PW144!

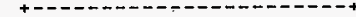

( T4:2.ACC - (2:946)

I N10:2-(2:225)

Rung $2: 949$

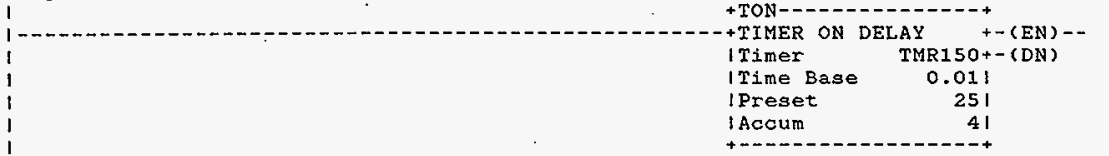

Rung 2:950

1 TMR 150/DN

TMR150

$T 4: 3$

1.... $]$ [

(RES) ---

l. (2:949)

Rung 2:951

1

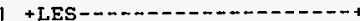

SCR 150

1 - +LESS THAN

I I Source A

TMR150.ACC I

11

ISource B

4 i

PW150I

1

01

T4:3.ACC - (2:949)

N10:3-(2:233)

Rung 2:952

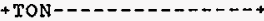

TIMER

ITimer

ITime Base

$-(\mathrm{EN})$

I Preset

0.011

i Accum

251

Accum

41

- (DN)

(n)

Rung 2:953

1 TMR 15I/DN

TMR151

1 T4:4

(RES) - - -

i DN

HNF-SD-FF-CSWD-61 Rev. 0

Page 762 

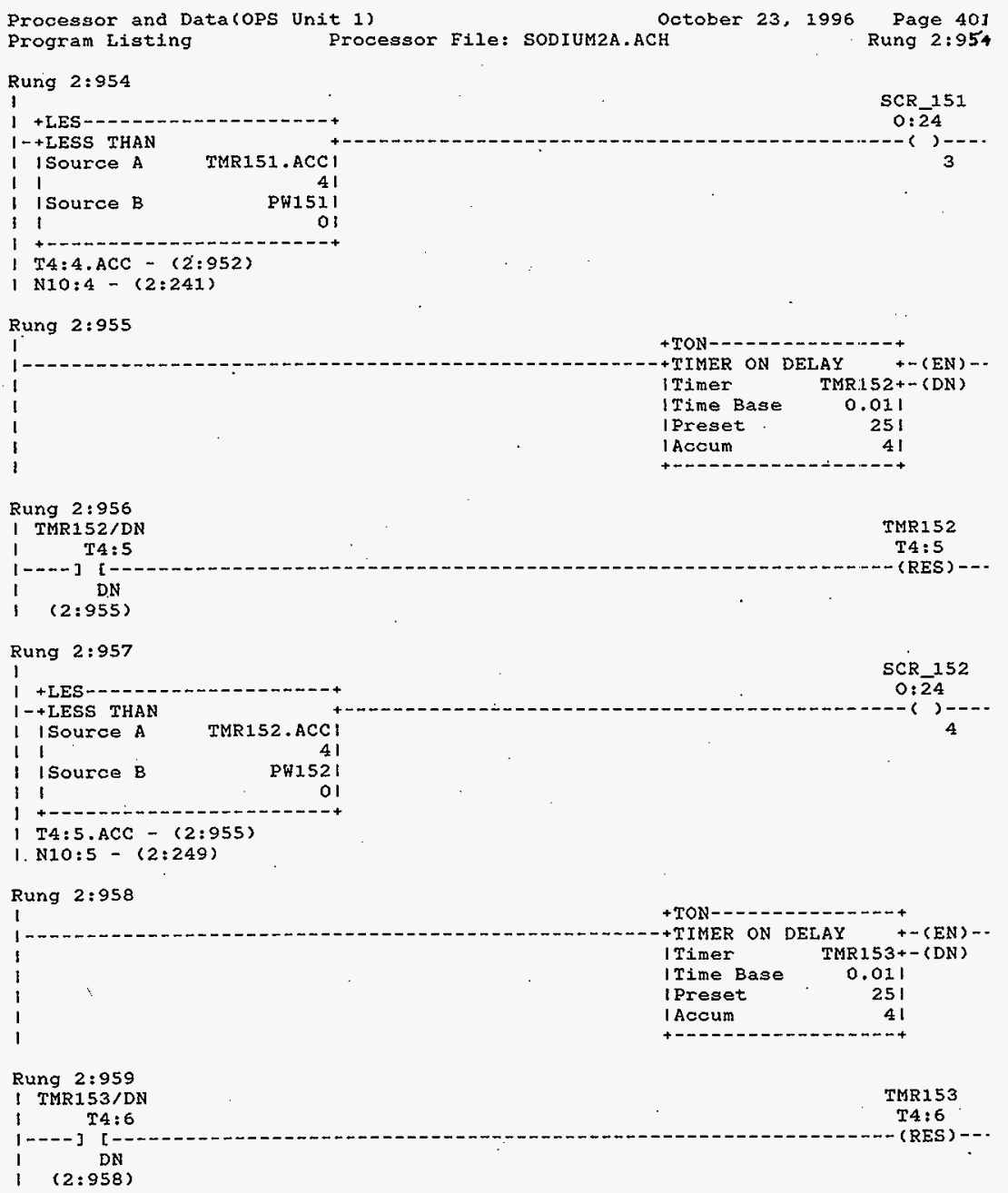

HNF-SD-FF-CSWD-til Rev. 0 
Processor and Data(OPS Unit 1)

October 23, 1996 Page 402

Program Listing
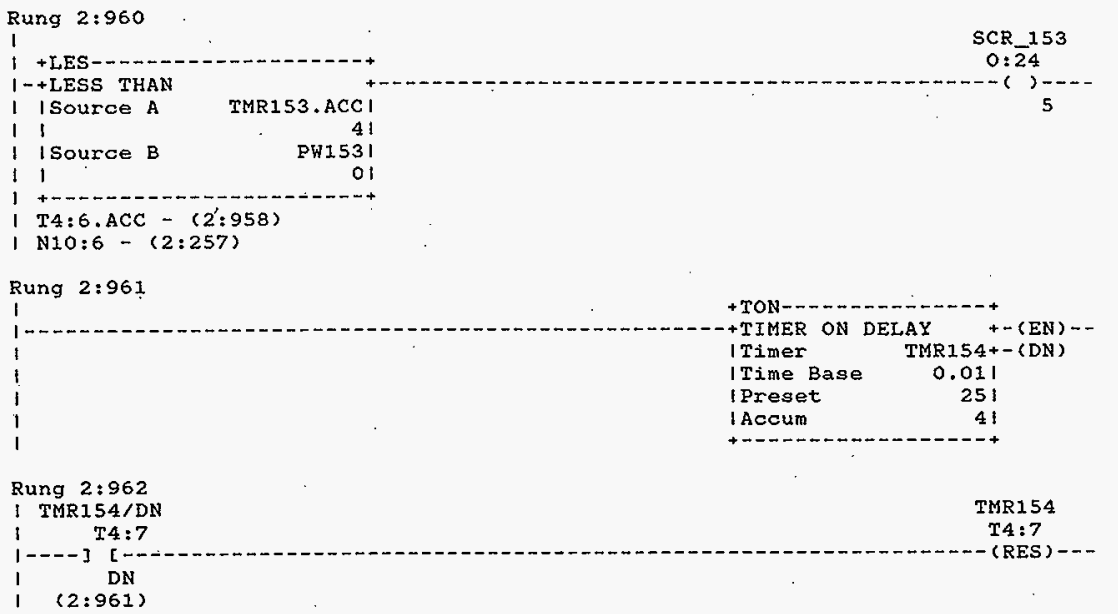

Rung 2:963

Rung 2:964

$$
1-
$$$$
\text { . }
$$

\section{Rung 2:965}

1 TMR160/DN

I T4:8

$1----3$ [

I (2:964)

SCR_154

$0 \div 24$

TMRI54.ACCI

41

01

+TON----------.---4

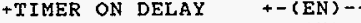

ITImer. TMR160+-(DN)

ITime Base. 0.011

IPreset

I Accum

41

TMR 160

$\mathrm{T} 4: 8$

- (RES ) -..

HNF-SD-FF-CSWD-61 Rev. 0

Page 764 
Processor and Data(OPS Unit 1)

Program Listing

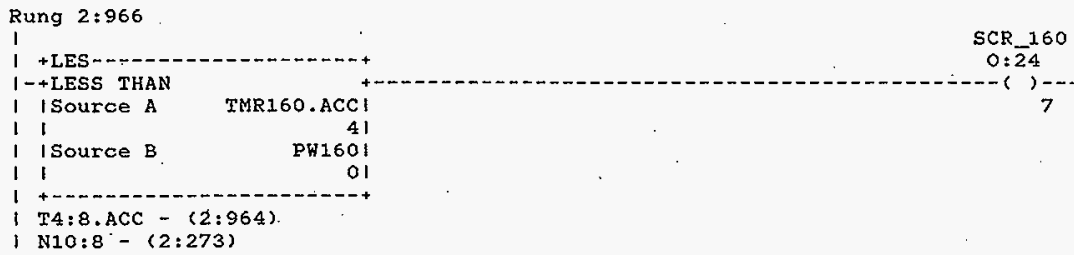

Rung 2:969

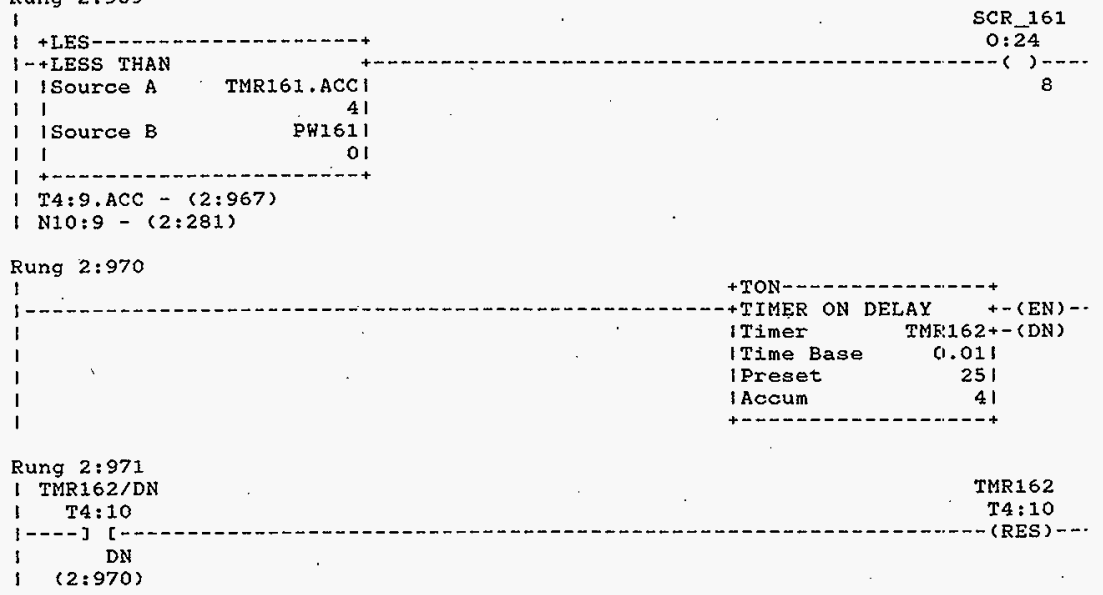

HNF-SD-FF-CSWD-151 Rev. 0 
Processor and Data (OPS Unit 1 Program Listing

Rung 2:972

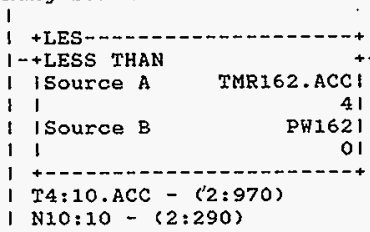

\section{Rung 2:973}

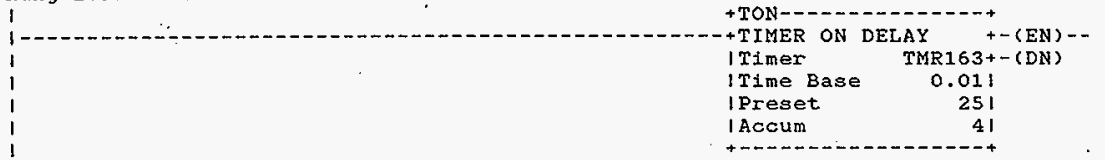

Rung 2:974

I THR 163/DN

I $\mathrm{T} 4: 11$

T4:11

$1-\cdots+2]$

(RES) - --

(2:973)

Rung 2:975

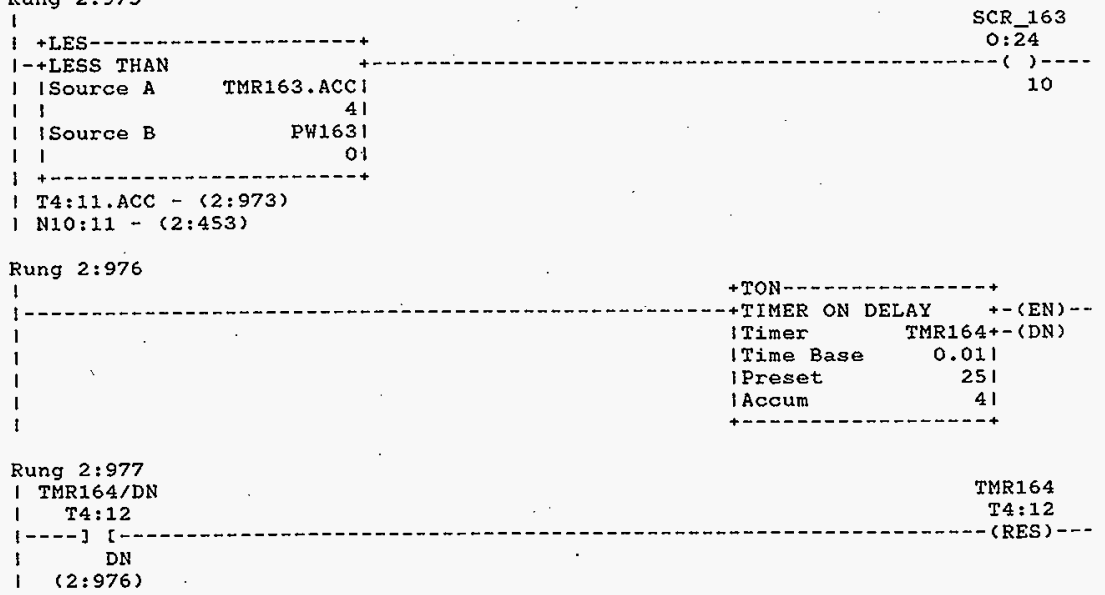

HNF-SD-FF-CSWD-61 Rev. 0 

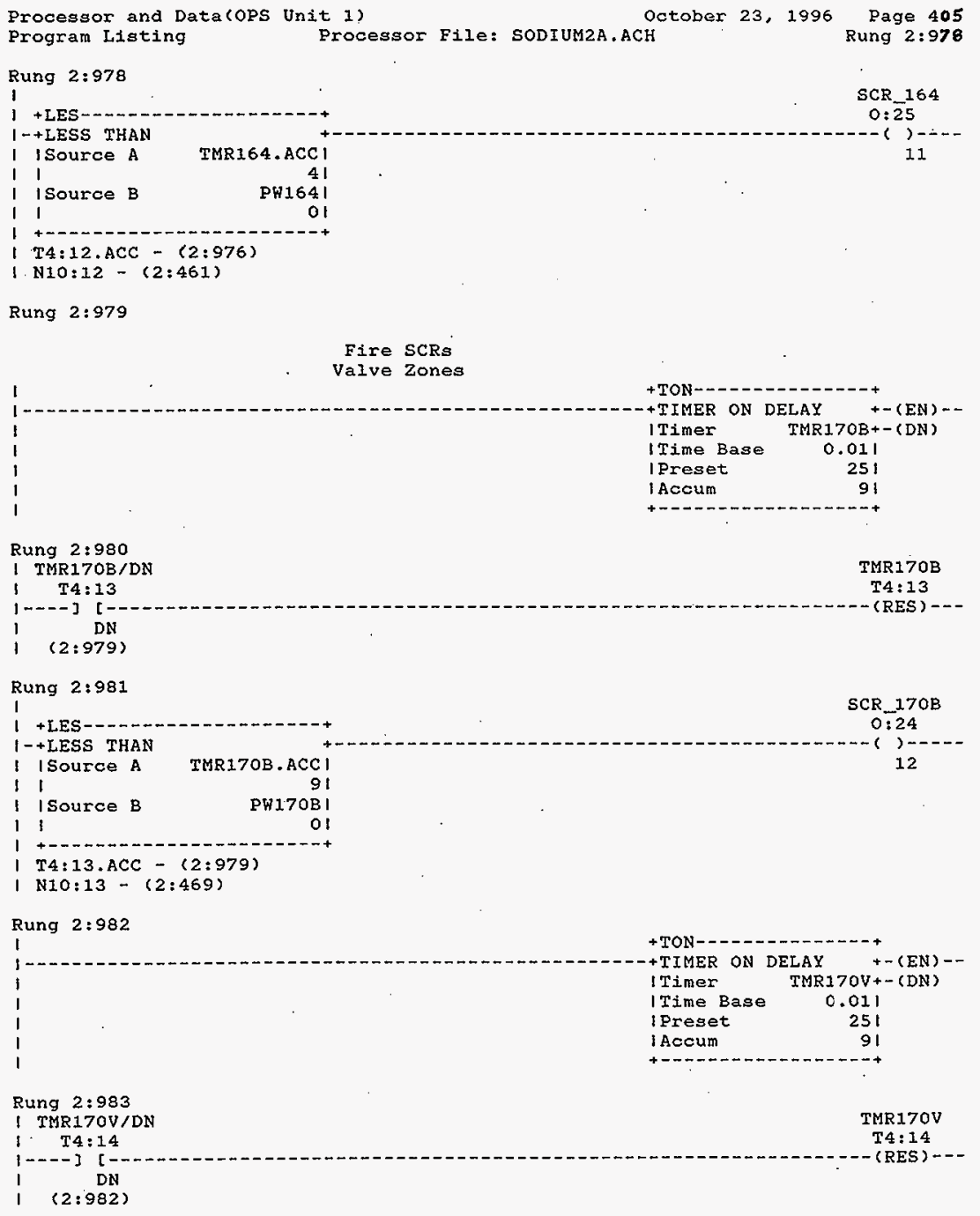

HNF-SD-FF-CSWD--6il Rev. 0 
Processor and Data(OPS Unit I)

October 23,1996

Program Listing

Processor File: SODIUM2A.ACH

Rung 2:984

1

$1+$ LES

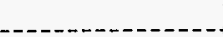

++LESS THAN

| ISource A TMR17OV.ACCI

1

| Source B PW170V

11

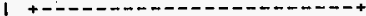

(T4:14.ACC - (2:982)

I N10:14 - (2:477)

Rung 2:985

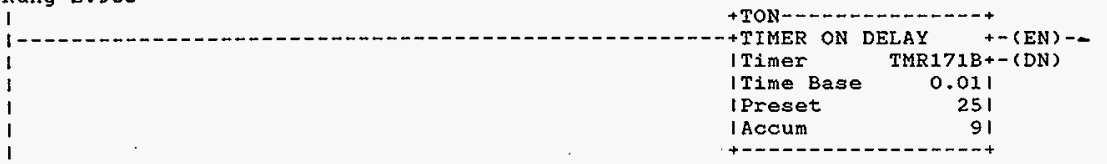

Rung 2:986

I TMR171B/DN

TMR171B

$T 4: 15$

$T 4: 15$

1-.-- ] [-

l (2:985)

Rung 2:987

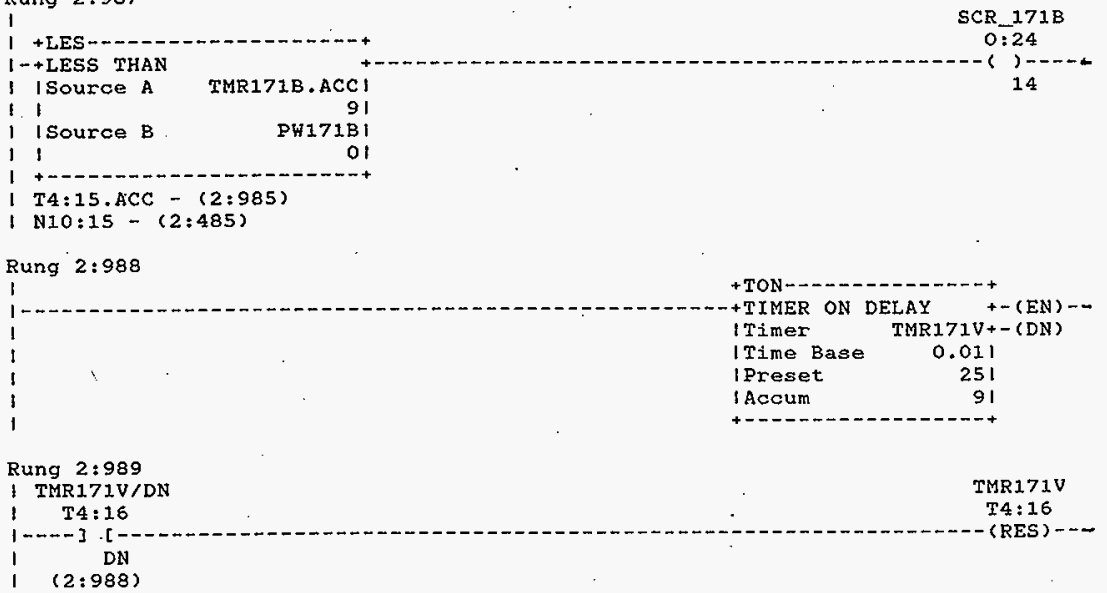

HNF-SD-FF-CSWD-61 Rev. 0 


\section{Rung 2:990}

1

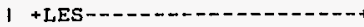

SCR $171 \mathrm{~V}$

$0: 24$

1-+IESS THAN

I ISource A TMRI71V.ACC ]

( )....

1 I 91

I Source B PH171Vi

11

01

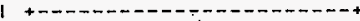

$1 \mathrm{~T} 4: 16 . \mathrm{ACC}-(2: 988)$

(N10:16-(2:493)

Rung 2:991

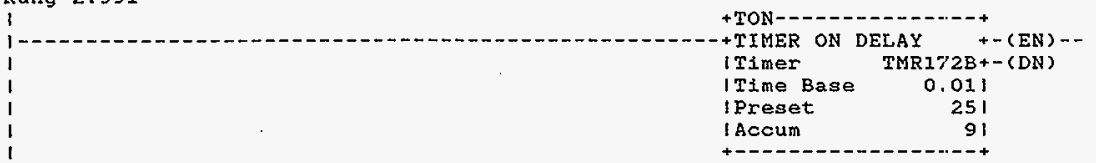

Rung 2:992

1 TMR172B/DN

$\mathrm{T} 4: 17$

1 $1---2]$ [

1. (2:991)

\section{Rung 2:993}

1

$1+$ LES- -

$1-+$ LESS THAN

1 ISource A TMR.172B.ACCI

1 . $9 !$

| Source B PW172B I

I 1 Ot

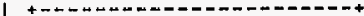

( T4:17.ACC - (2:991)

( N10:17-(2:501)

Rung $2: 994$

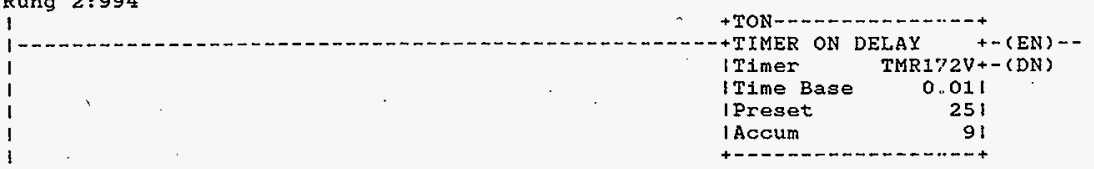

Rung $2: 995$

I TMR172V/DN

1 T4:18

TMR172V

T4:18

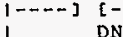

DN

$$
\text { (2:994) }
$$

HNF-SD-FF-CSWD-61 Rev. 0 
Processor and Data(OPS Unit 1 )

Rung 2:996

1

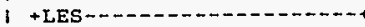

SCR $172 \mathrm{~V}$

$0: 24$

$1-+$ LESS THAN

I ISource A TMR172V.ACC

$-(3-\cdots$

I. I

I I Source B

91

13

1.---

( T4:18.ACC - (2:994)

I N10:18-(2:509)

Rung 2:997

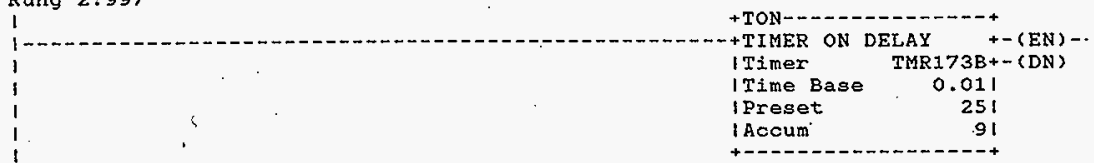

Rung 2:998

I TMR173B/DN

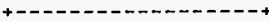

I $T 4: 19$

$1-n-\infty$ ]

( $2: 997)$

Rung 2:999

1

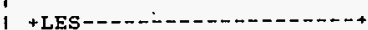

SCR $173 B$

$0: 24$

I-+LESS THAN

I ISOurce A TMR173B.ACCI

1.-.-.

11

I Source B

18

11

PW173BI

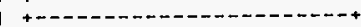

I T4:19.ACC - (2:997)

( N10:19-(2:517)

Rung 2:1000

$$
\text { I- }
$$

Rung 2:1001

I TMR $173 \mathrm{~V} / \mathrm{DN}$

I T $4: 20$

1-..- ] [-

1 DN

l (2:1000)

HNF-SD-FF-CSWD-61 Rev. 0 


\section{Rung $2: 1002$}
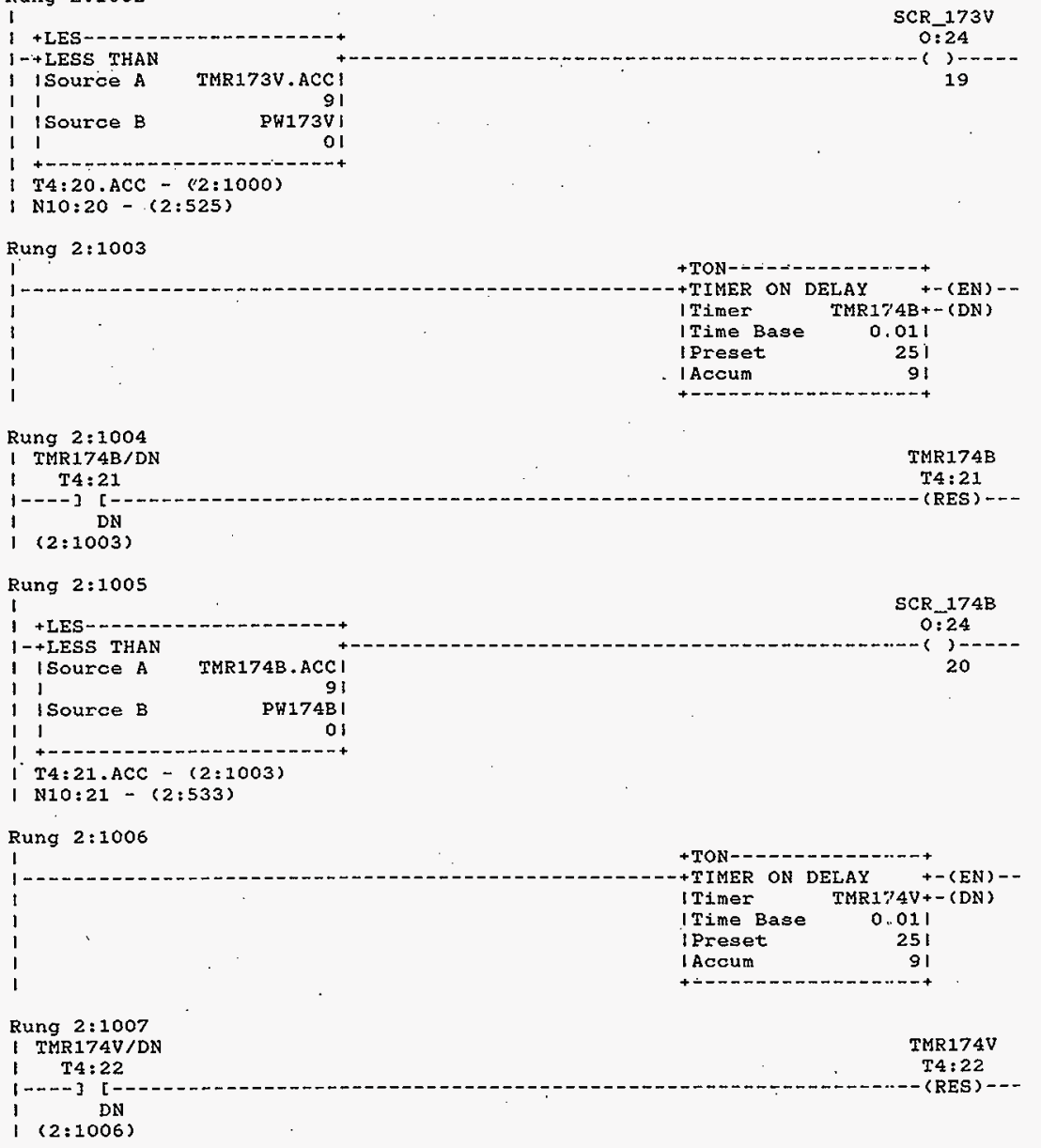

HNF-SD-FF-CSWD-61 Fiev. 0 
Processor and Datalops Init 1)

October 23, 1996 Page 410

Program Iisting
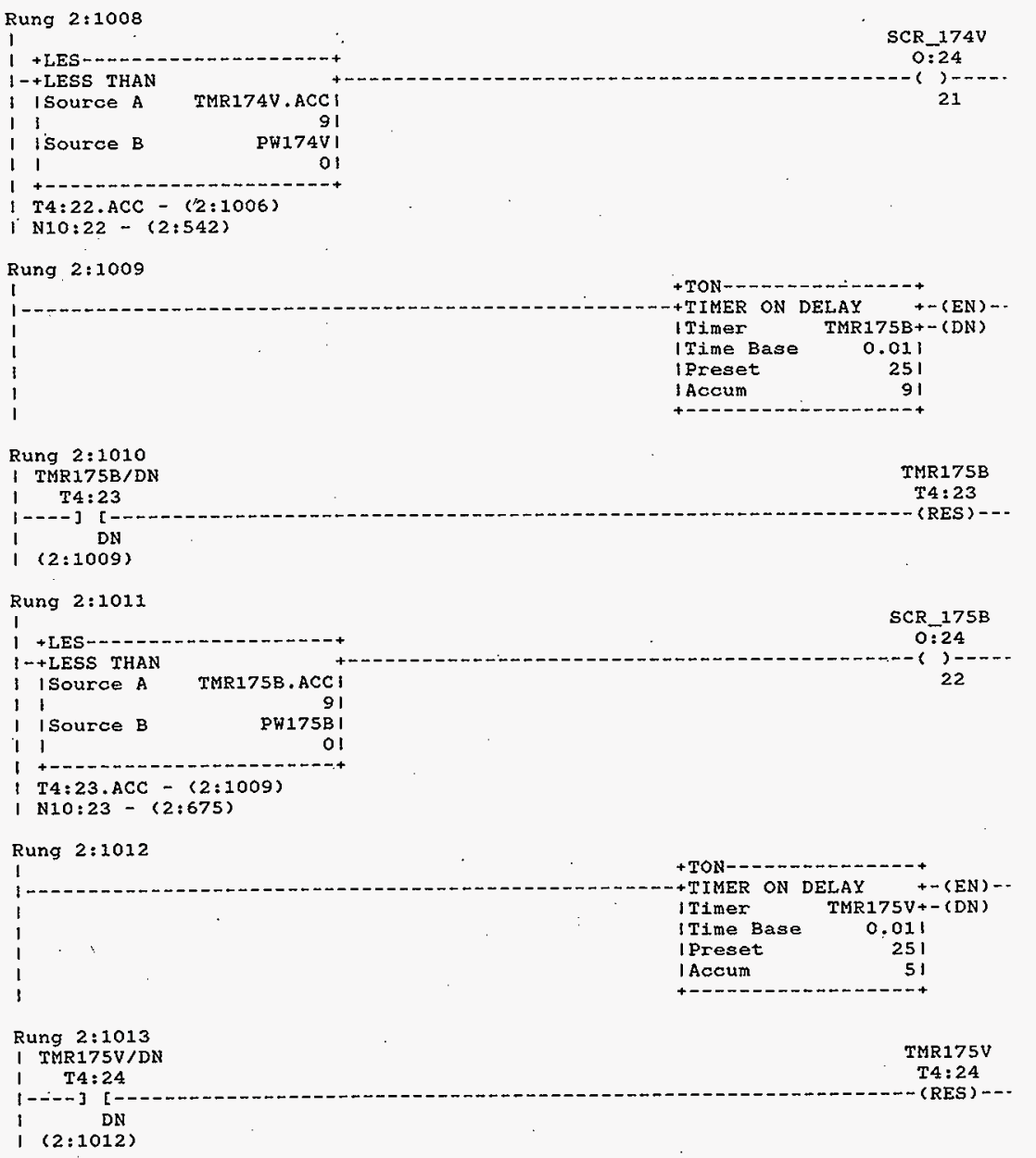

HNF-SD-FF-CSWD-61 Rev. 0 
processor and Data(Ops. Unit 1 )

Program Listing
October 23, 1996 Page 411 Processor File: SODIUM2A.ACH

Rung 2:1014
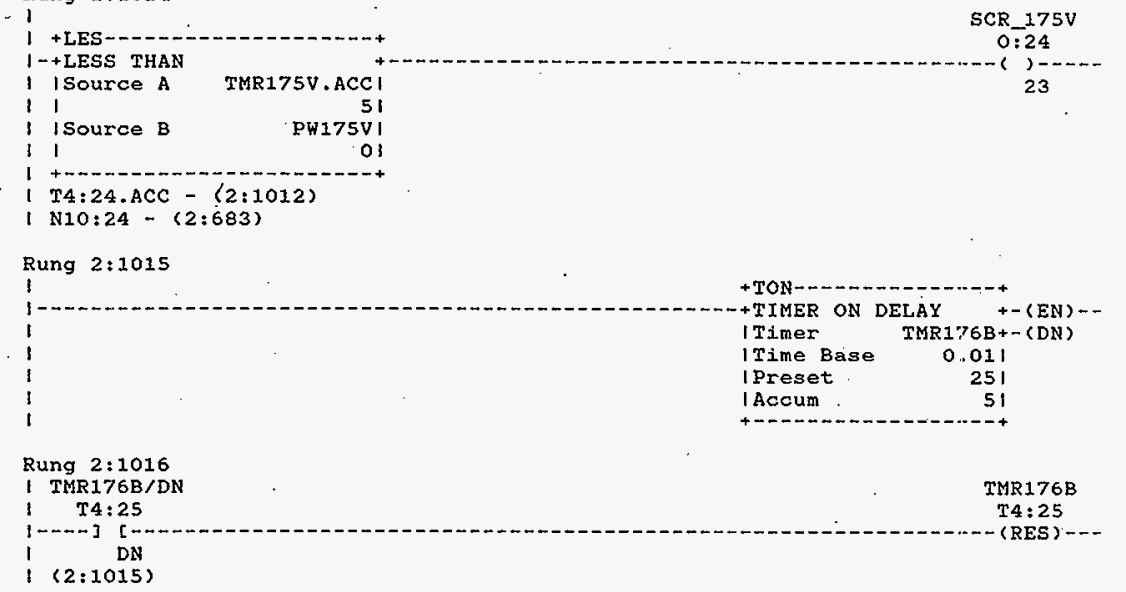

$2: 1015)$

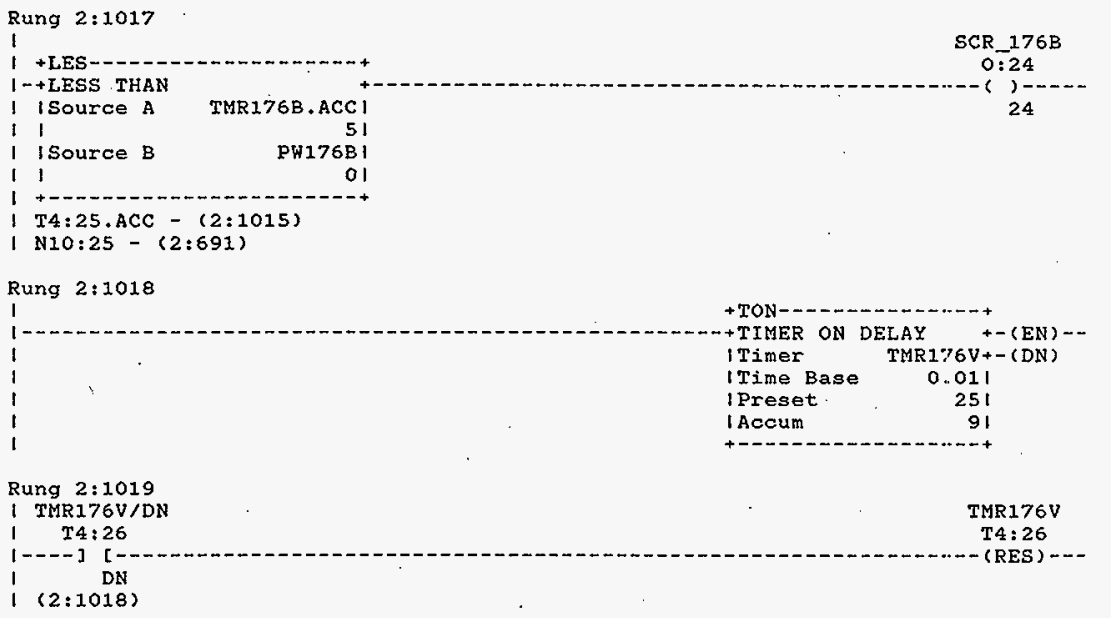

HNF-SD-FF-CSWD-61 Rev. 0 
Processor and Data(OPS Unit 1)

Program Listing

October 23, 1996

Page 412

Procesgor File: SODIUM2A.ACH

Rung $2: 1020$

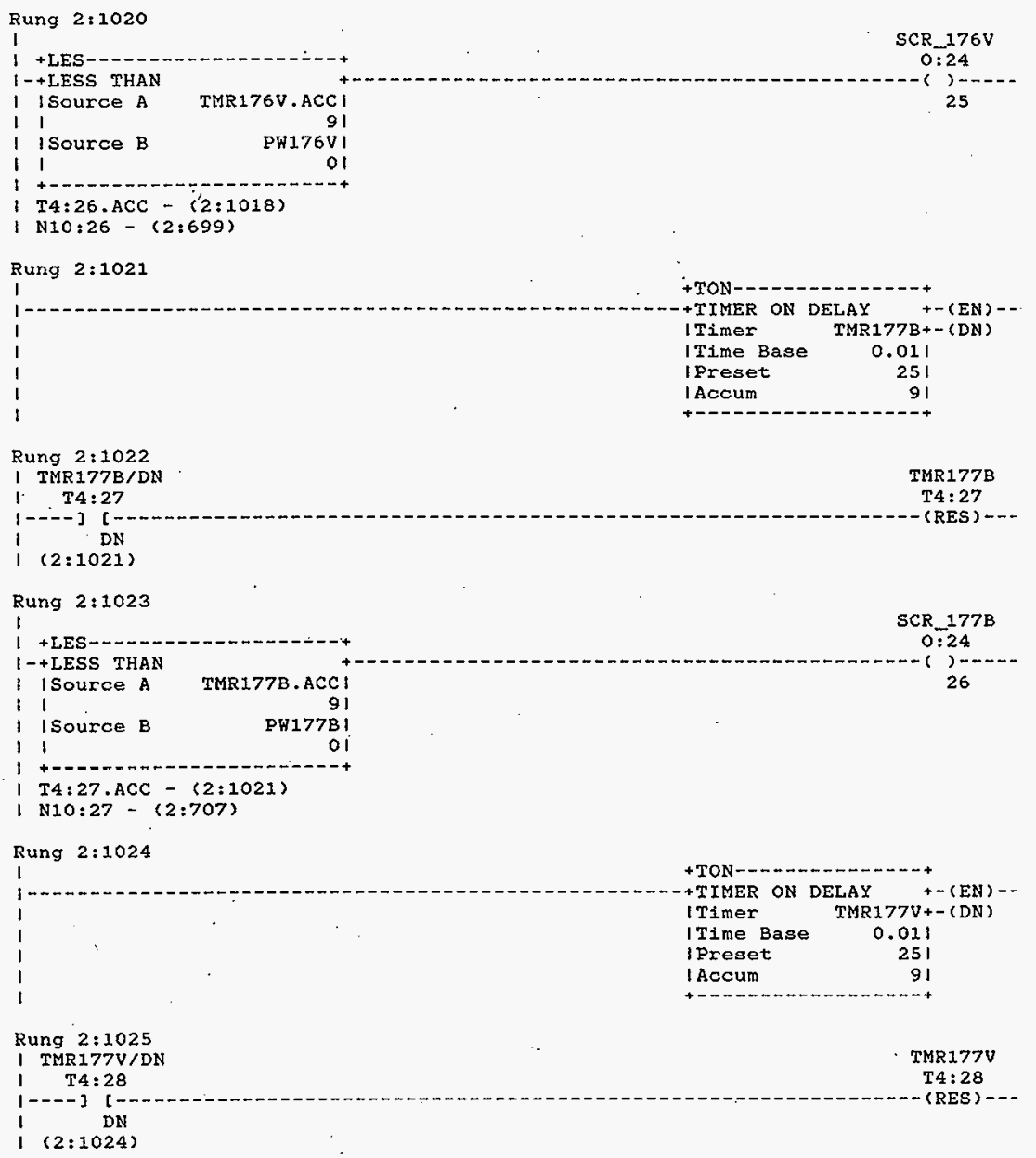

HNF-SD-FF-CSWD-61 Rev. 0 


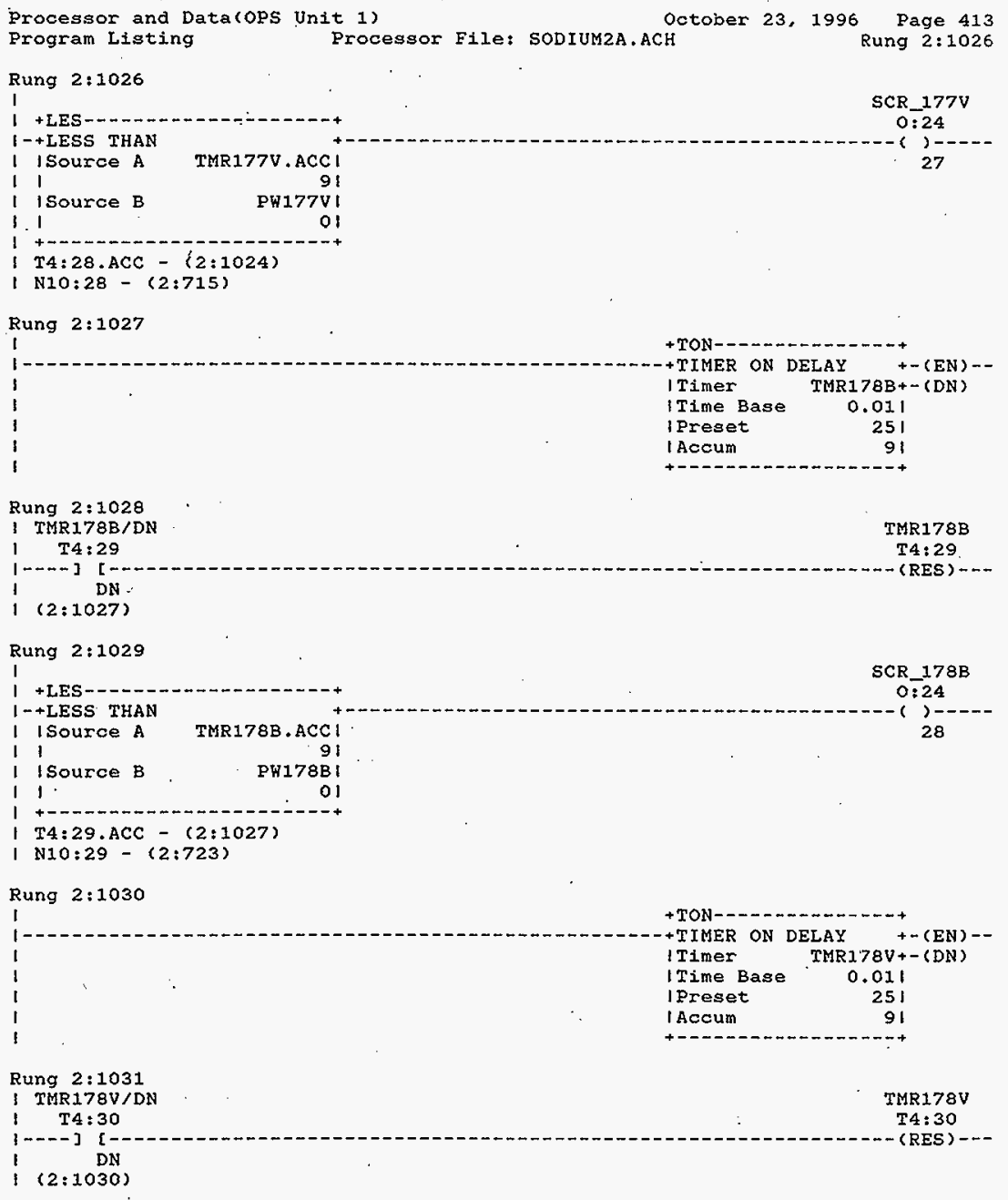

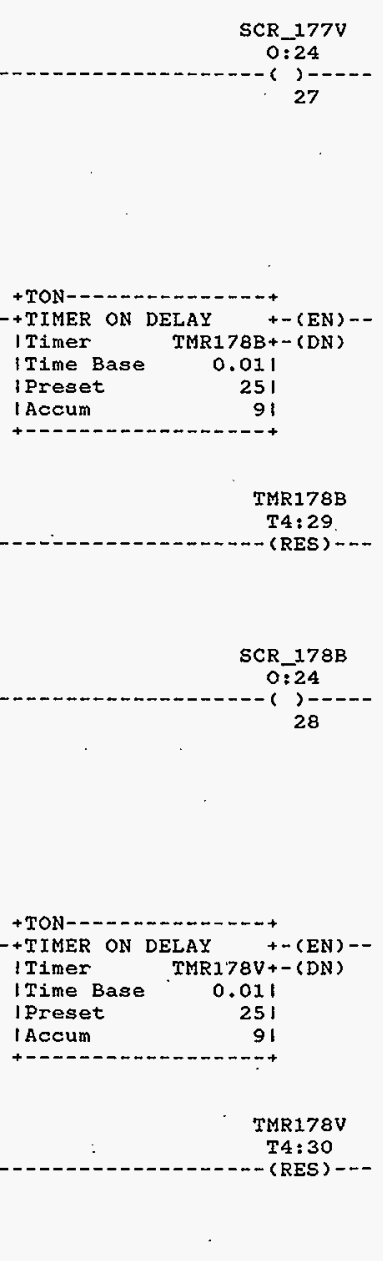

Rung 2:1026

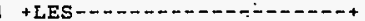

-+ LESS THAN
ISOUrCE A TMR177V.ACCI

1 I 9

| ISource B PW177VI

T4:28.ACC - $(2: 1024)$

( N10:28 - (2:715)

Rung 2:1027

Octoider 23, 1996 Page 413
ung $2: 1026$

Program Listing Proces

Q)

Rung 2:1028

T TMR178B/DN

T4:29

DN

Rung 2:1029

-+LESS THAN

I ISource A

TMR178B.ACC

91

| |Source B PH178B

11

$-----+$

T4:29.ACC - $(2: 1027)$

I $10: 29-(2: 723)$

Rung 2:1030 
Processor and Data(OPS Unit 1 )

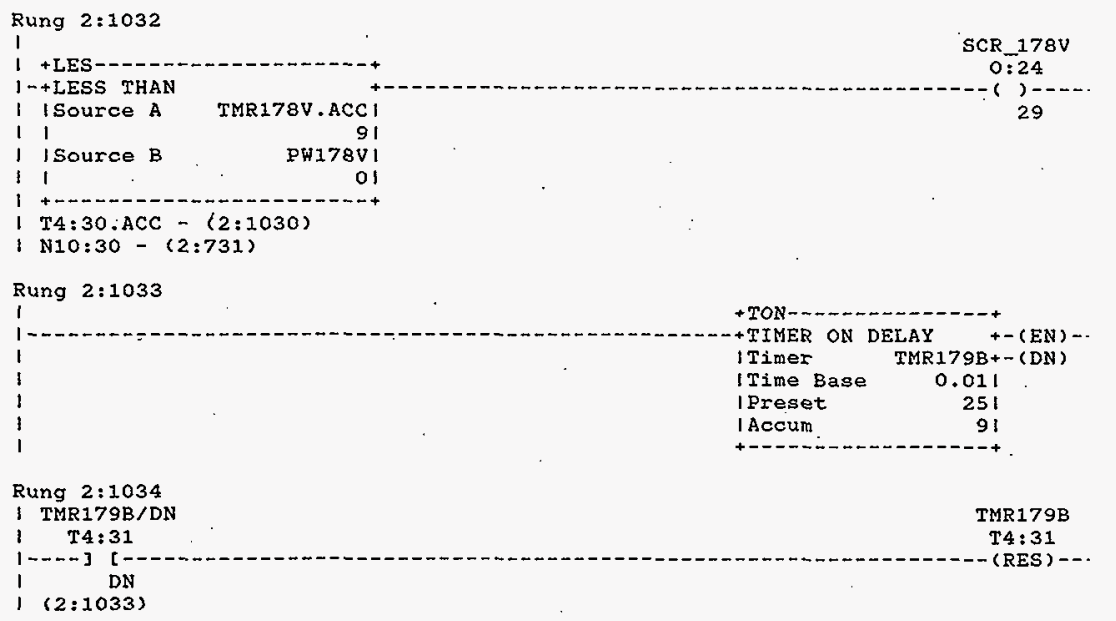

Rung 2:1035
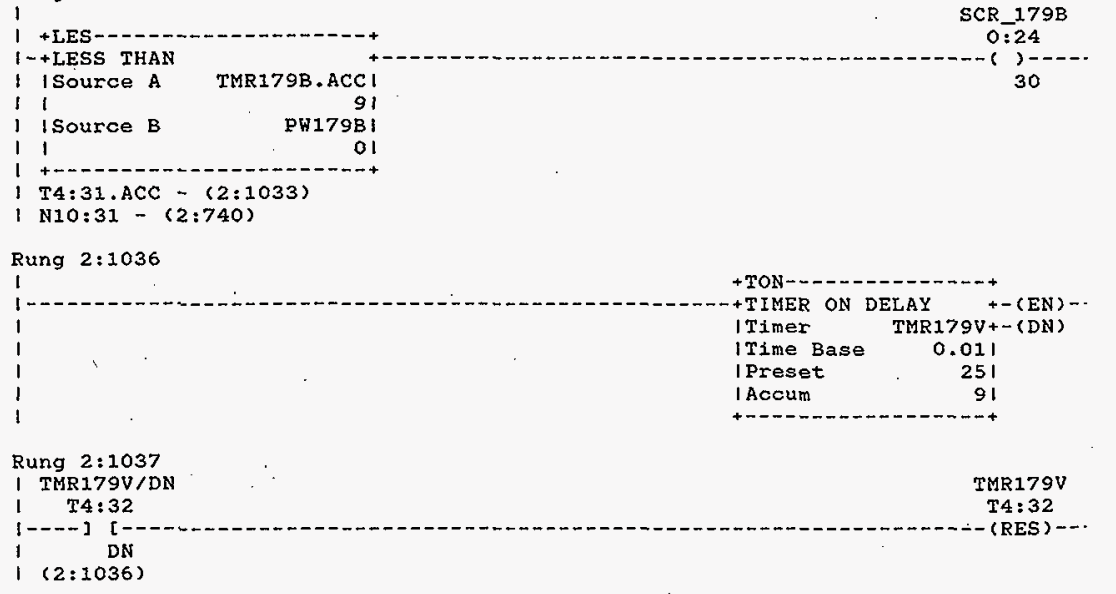

HNF-SD-FF-CSWD-61 Rev. 0 


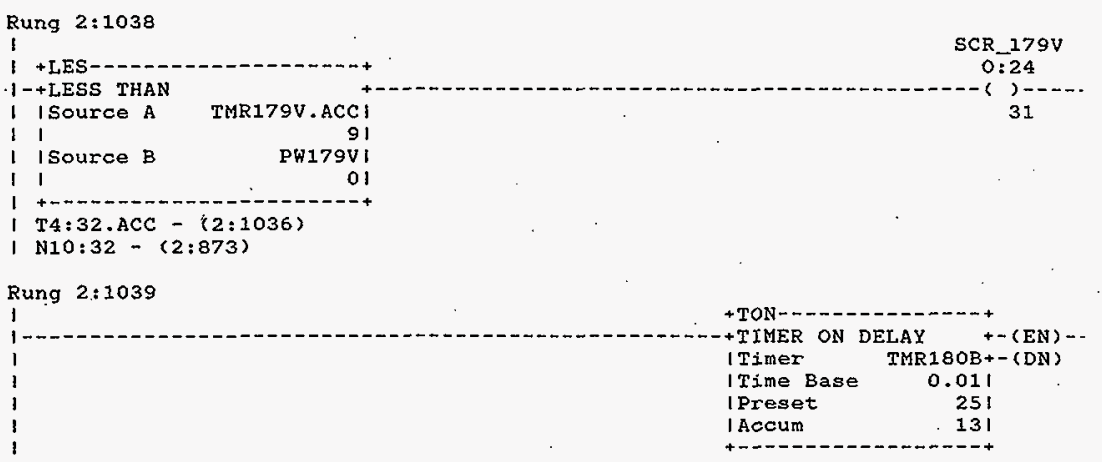

Rung 2:1040

(TMR18OB/DN

$1 \mathrm{~T} 4: 33$

TMRI80B

$1-\cdots-7$ ]

$T 4: 33$

$1 . D N$

( $(2: 1039)$

Rung 2:1041

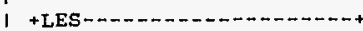

SCR $180 \mathrm{~B}$

$1-+$ LESS THAN

I isource A TMR18OB.ACC

1.1 I31

I isource B PW180B I

1 OI

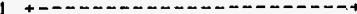

( T4:33.ACC - (2:1039)

I N10:33-(2:881)

Rung 2:1042

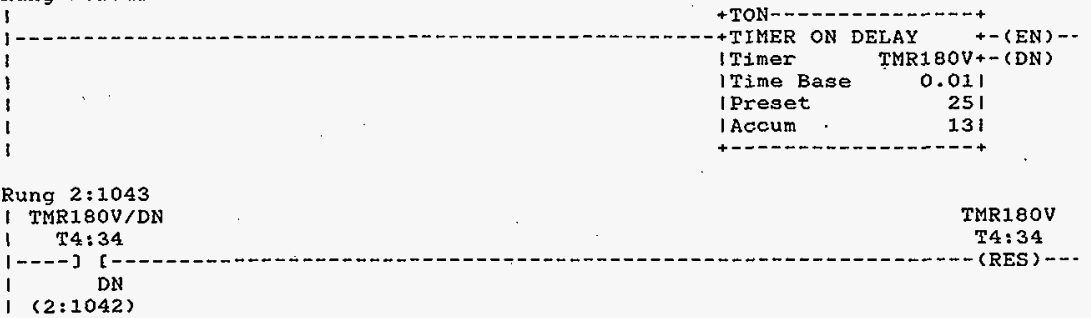

HNF-SD-FF-CSWD-6! Rev. 0 
Processor and Data(OPS Unit 1) Program Listing
October 23,1996 $\mathrm{CH}$

\section{Rung 2:1044}

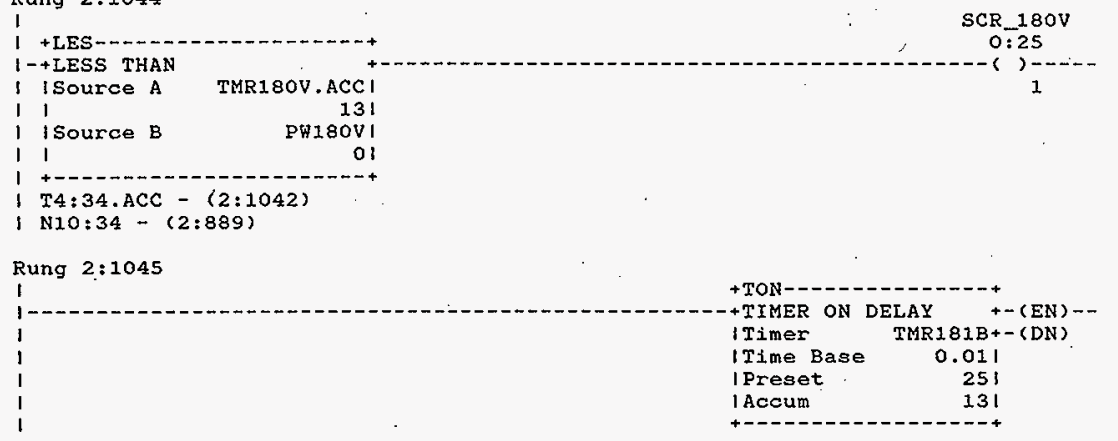

Rung 2:1046

1 TMR181B/DN

$1 \quad T 4: 35$

$1+\cdots+3$ [ -

i $(2: 1045)$

\section{Rung 2:1047}

1

$1+$ LES-

\section{7}

$1-+$ LESS THAN

1 I Source $A$

I 1

1 1source B

11

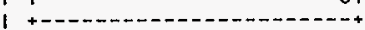

I T4:35.ACC - (2:1045)

I N10:35-(2:897)

Rung $2: 1048$

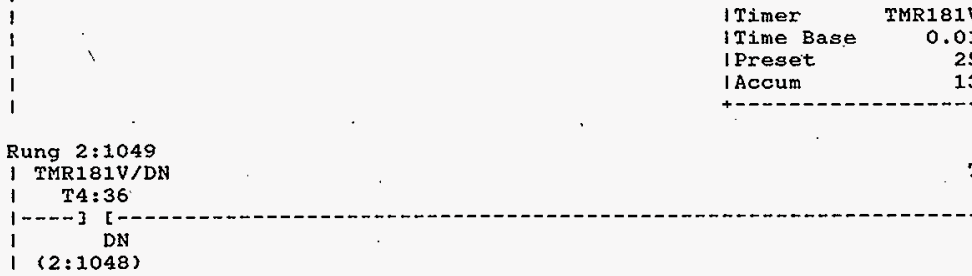

$+-(\mathrm{EN})--$

ITimer TMR181V+-(DN)

ITime Base 0.011

I Preset 251

| Accum 13 |

HNF-SD-FF-CSWD-61 Rev. 0

Page 778 
Processor and Data(OPS Unit 1 )

Rung 2:1050

I

$1+$ LES---------------- +

I-+LESS THAN

I ISource A TMR181V.ACCI

I I 13!

I Isource B PW181V|

1 I 0 i

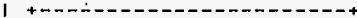

I T4:36.ACC - (2:1048)

I $110: 36-(2: 905)$

\section{Rung 2:1051}$$
1
$$$$
\text { i }
$$$$
\text { I }
$$

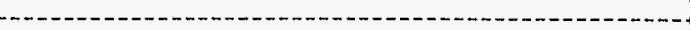

$$
\text { (1) }
$$$$
\text { I }
$$

i

I

I

Rung 2:1055

I TMR 182V/DN

I T4:38

1---- ] [--

I (2:1054)

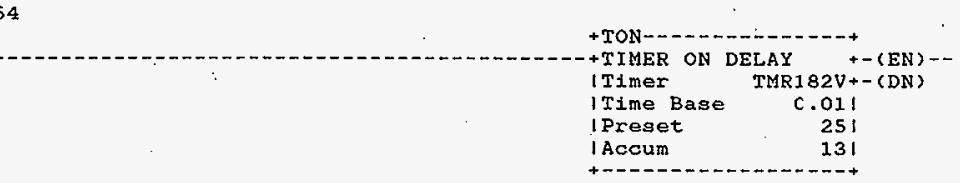

HNF-SD-FF-CSWD-61 Rev. 0 
Processor and Data(OPS Unit 1)

Rung 2:1056

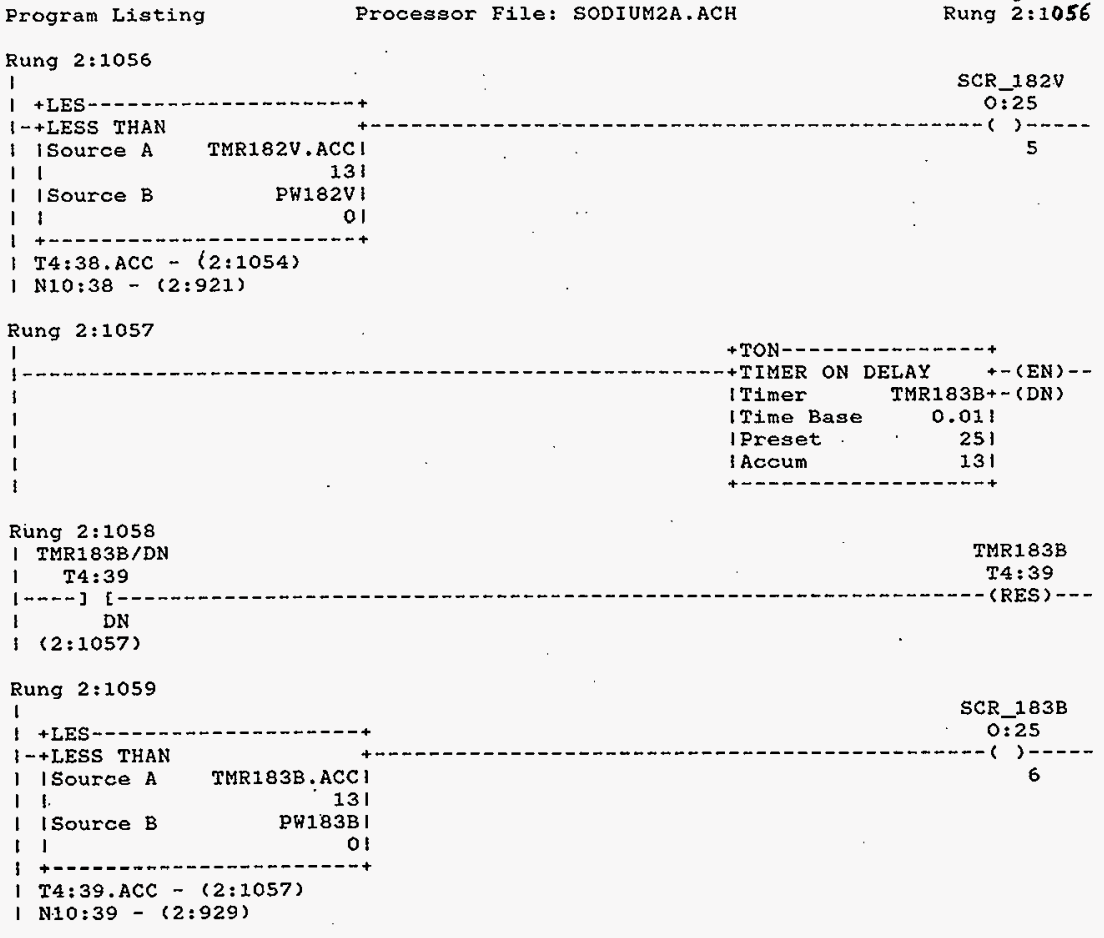

Riung 2:1058

I TMR1838/DN

TMR 183B

I T4:39

T4:39

[-...] $[-$

(RES) - -

$1 \quad \mathrm{DN}$

(2:1057)

Rung 2:1059

1

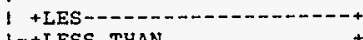

1-+LESS THAN

I I Source A

TMR183B.ACCI

131

I isource B pq183Bi

1 1 1

I T4:39.ACC - (2:1057)

( N.10:39-(2:929)

Rung 2:1060$$
1-
$$

1

1

I

t

Rung 2:1061

I TMR183V/DN

I T4:40

$1---7$ [

I (2:1060) 
Processor and Data(OPS Unit 1 )

Program Listing

Processor File: SODIUM2A.ACH

Rung 2:1062

I

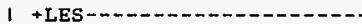

$1-+$ LESS THAN

I I Source A

11

I Source B

11

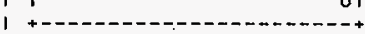

- I T4:40.ACC - $(2: 1060)$

I N10:40-(2:938)

Rung 2:1063

i END

I 6

TMR183V.ACCI

17

$1--[$ LBL

6

DUMMY 2

N22:7

i

Rung $2: 1064$

I$$
1
$$

i

Rung 2:1065

1

$$
1
$$

SCR $183 \mathrm{~V}$

$0: 25$

7

HNF-SD-FF-CSWD-61 Rev. 0 


\section{Rung $3: 0$}

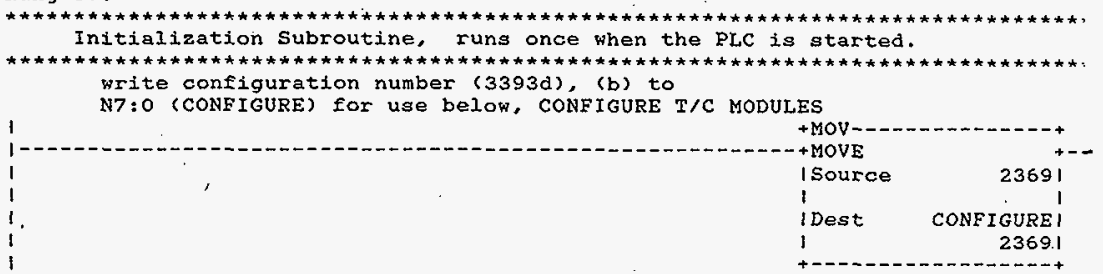

Rung $3: 1$

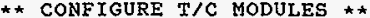

Use Fill File to copy the configuration to each channel of each T/C module

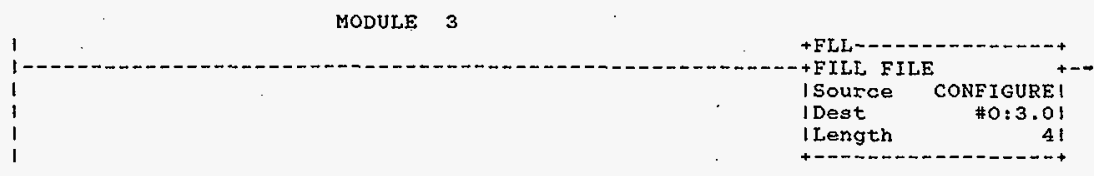

Rung $3: 2$

1
1

Rung $3: 3$

MODULE 5

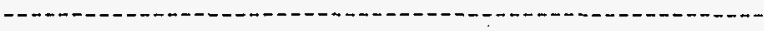

FII FILE

ISOUrce CONFIGURE

I Dest . \#0:5.01

I Length

Rung $3: 4$

MODULE 6

HNF-SD-FF-CSWD-61 Rev. 0 

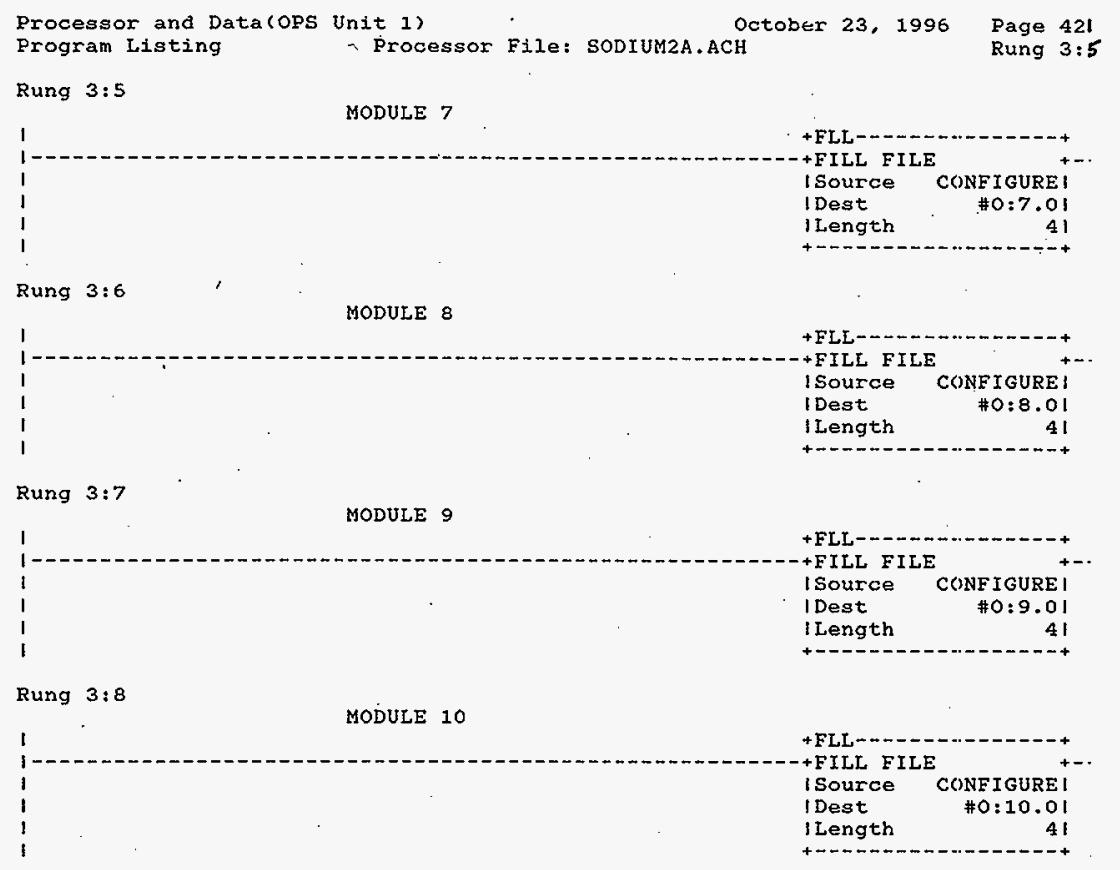

\section{Rung $3: 9$}
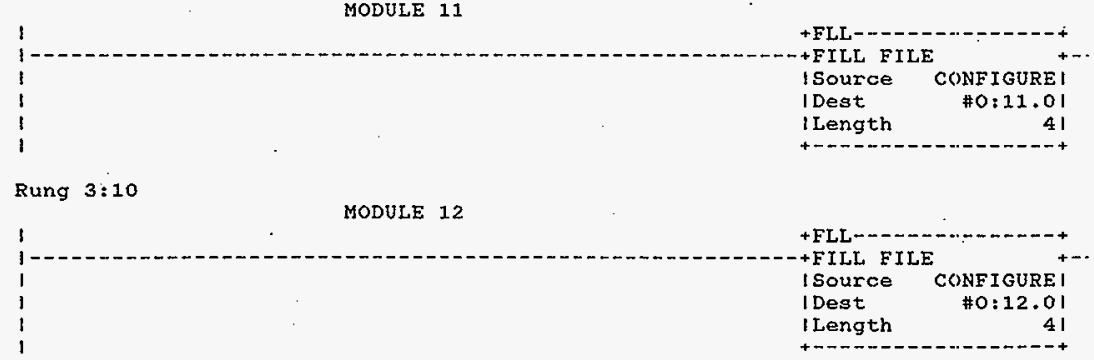

HNF-SD-FF-CSWD-61 R $: v .0$

Page 783 
Processor and Data (OPS Unit 1)

October 23, 1996

Page 422

Program Listing

Processor File: SODIUM2A.ACH

Rung $3: 11$

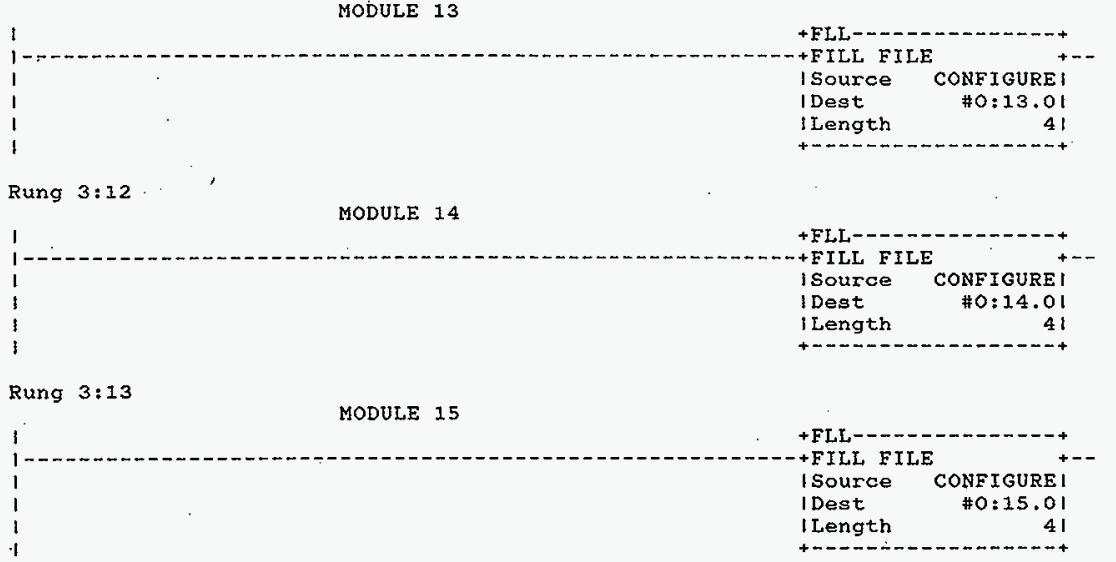

Rung $3: 14$

MODULE 16

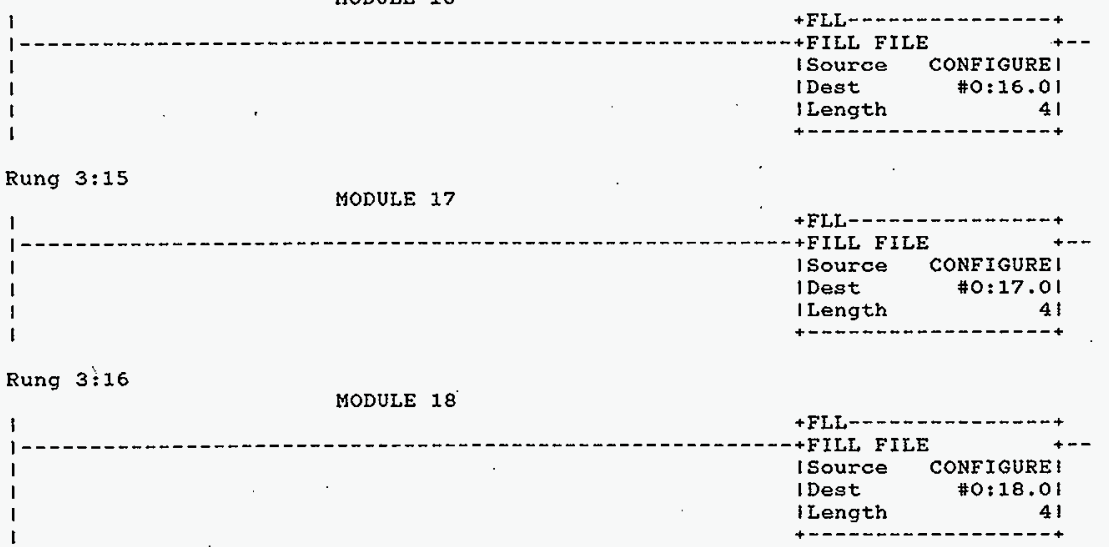

HNF-SD-FF-CSWD-61 Rev. 0 
Processor and Data(ops Unit 1$)$

Program Listing

Processor File: SODIUM2A.ACH

Rung $3: 17$

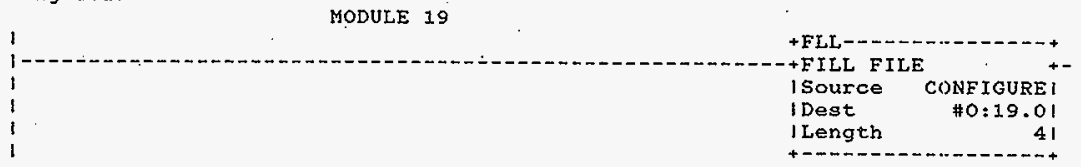

Rung 3:18

MODULE 20

$$
\text { i }
$$

\section{MODULE 20}

+ +FILI FILE

l SOUICE CONFIGUREI

I Dest

Itength

\#0:20.01

41

Rung $3: 19$

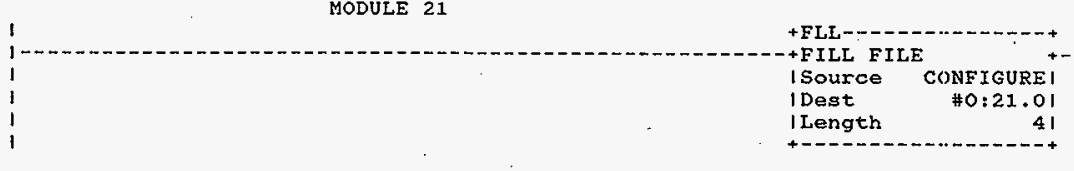

Rung $3: 20$

MODULE 22

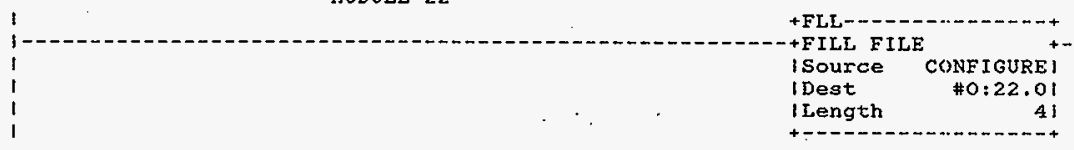

Rung $3: 21$

MODULE 23

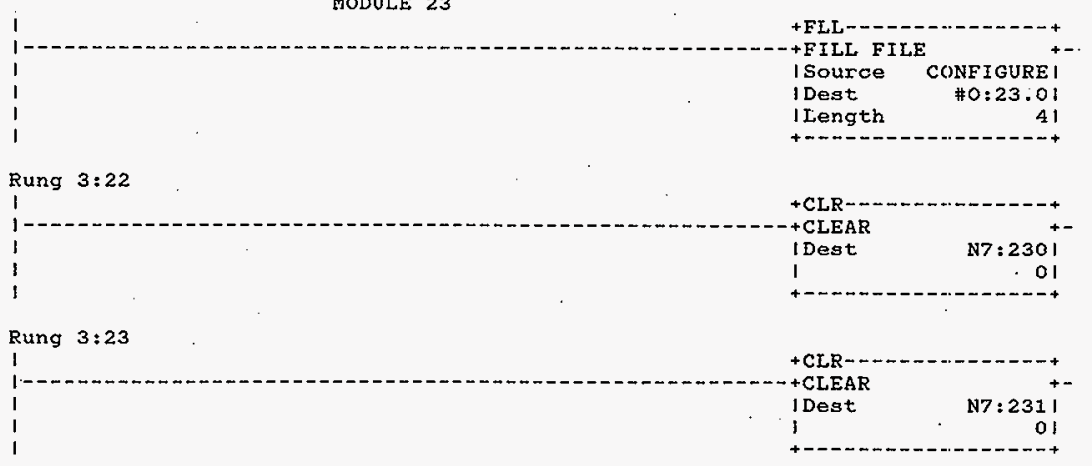

HNF-SD-FF-CSWD-61 Rev. 0

Page 785 


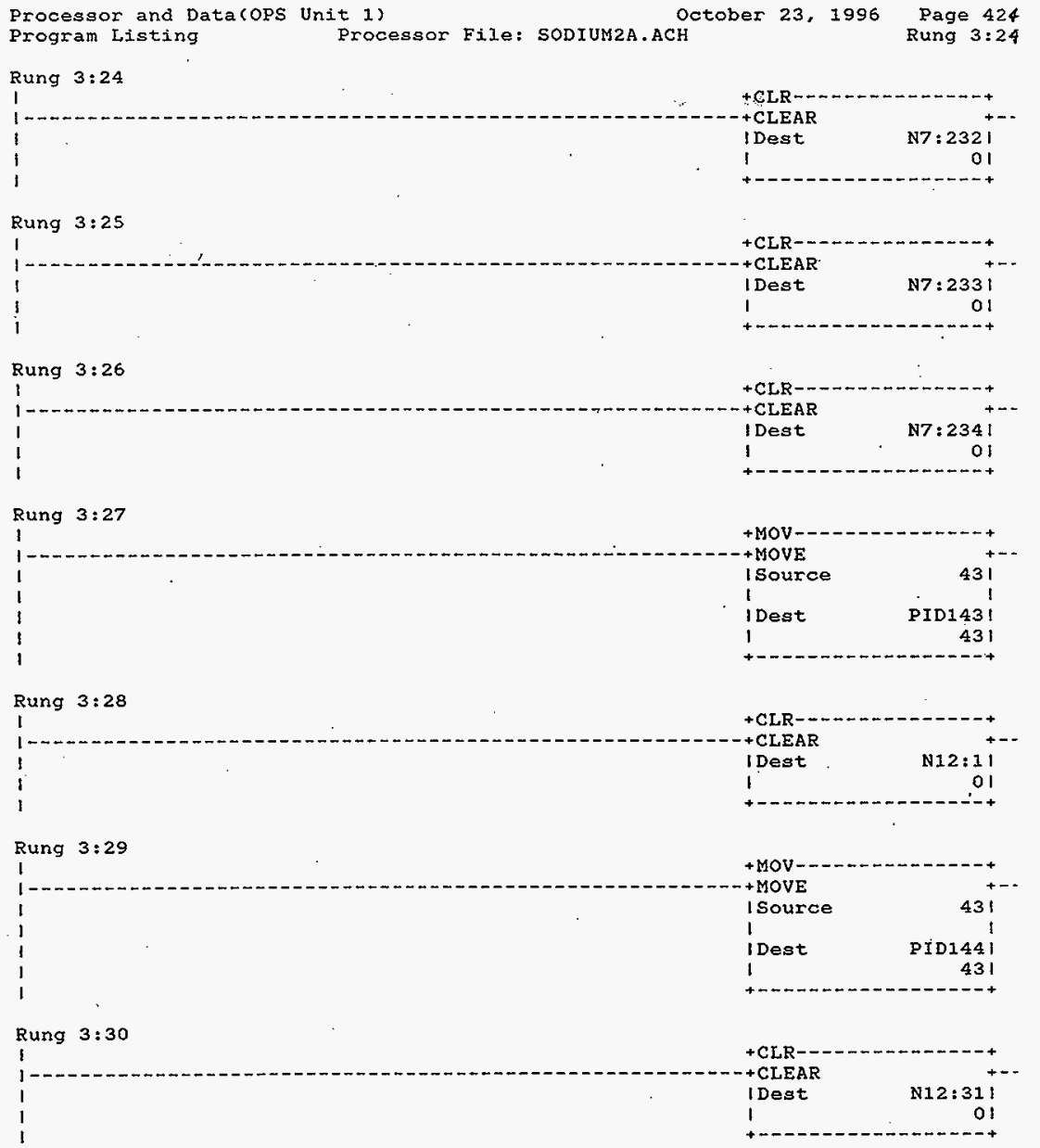

HNF-SD-FF-CSWD-61 Rev. 0 
Processor and Data(OPS Unit 1 )

Program Listing

Rung $3: 31$

1 1

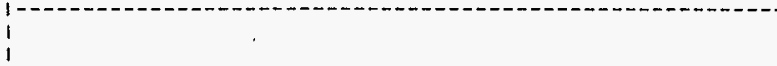

1 +MOVE

ISource

1

I Dest

1

Rung $3: 32$

I

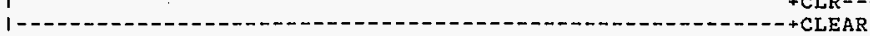

I Dest

1

Rung $3: 33$

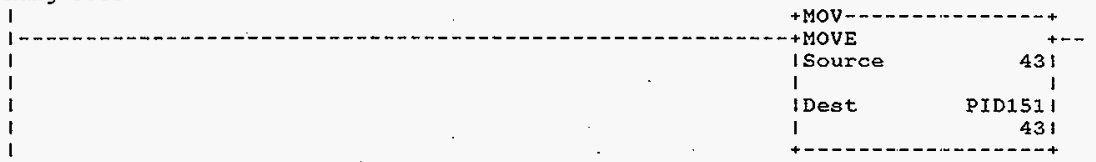

Rung $3: 34$

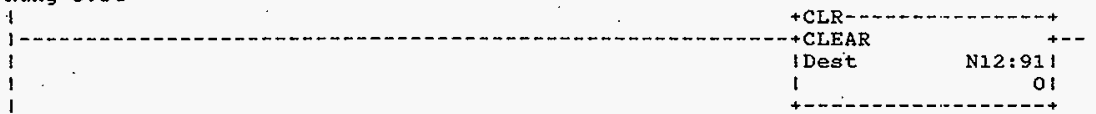

Rung $3: 35$

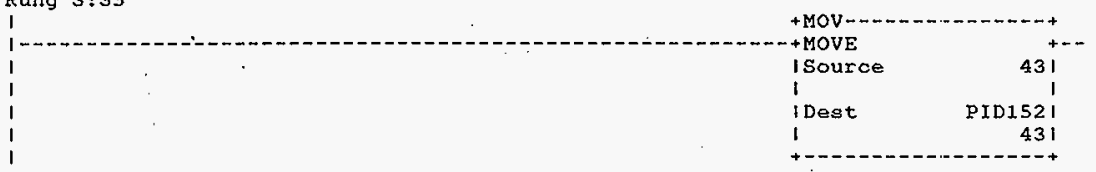

Rung $3: 36$

$$
\text { I }
$$

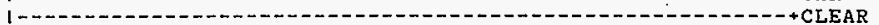

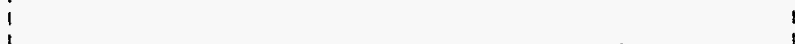

I Dest

N12:1211

1

Rung $3: 37$

I

1-

7.

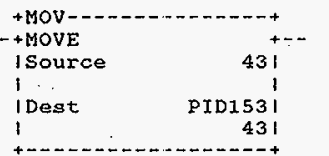

HNF-SD-FF-CSWD-61 Rev. 0 


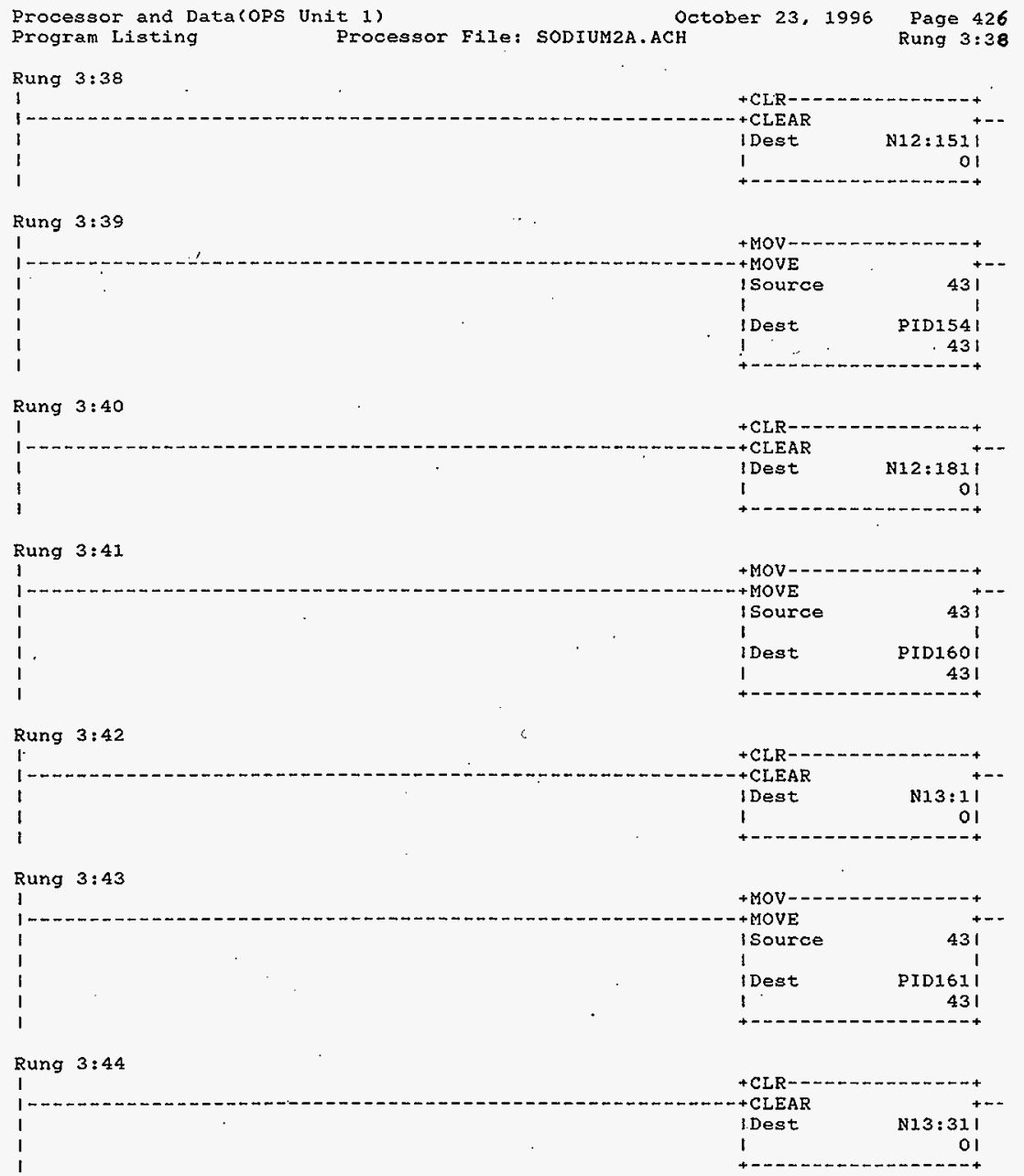

HNF-SD-FF-CSWD-61 Rev, 0

Page 788 
Processor and DatalOPS Unit i)

October 23,1996

Page 427

Program Listing

Processor File: SODIUM2A.ACH

Rung $3: 45$

\section{Rung $3: 45$}

$$
1
$$
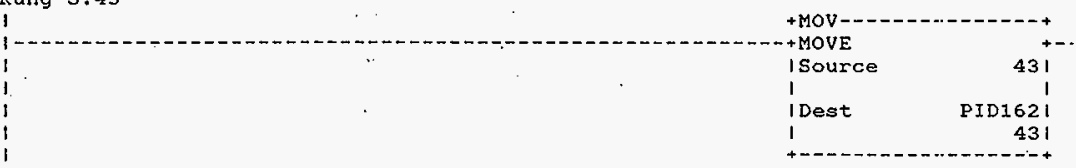

Rung $3: 46$

!

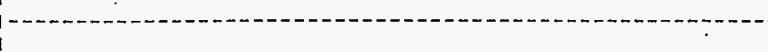

$+\mathrm{CLR}------\cdot \cdot-------+$

+CLEAR

1 Dest

N13:61।

I

Rung $3: 47$

I

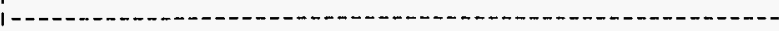

MOVE

isource

431

IDest

PID163

1

431

Rung $3: 48$

1

IDest

1

N13:911

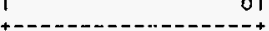

\section{Rung 3:49}
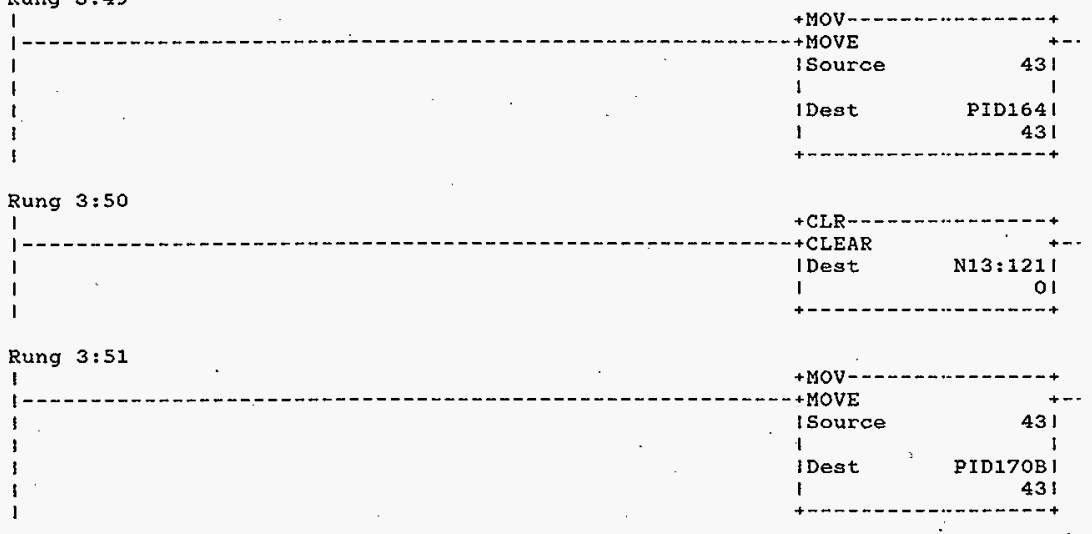

HNF-SD-FF-CSWD-61 Rev. 0 
Processor and Data(Ops Unit 1) Program Listing

Rung 3:52

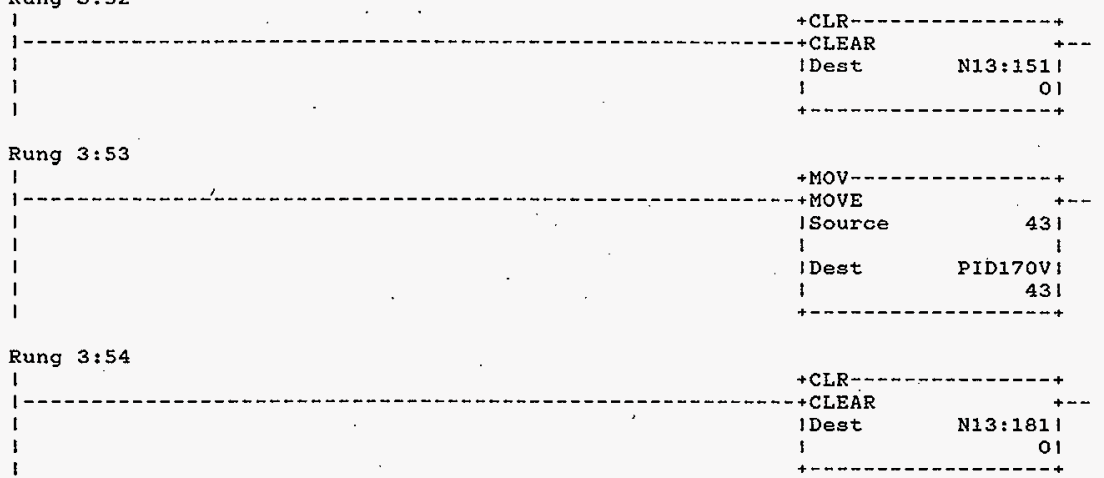

Rung $3: 55$

$13+50+$ MOV

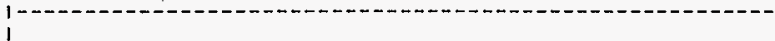

1 1 1

I

I

Rung $3: 56$

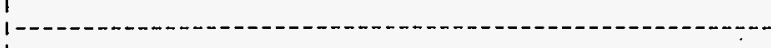

1

I

Rung $3: 57$
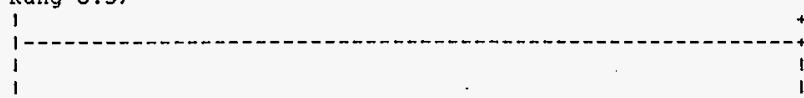

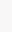

I

\section{Rung 3:58}

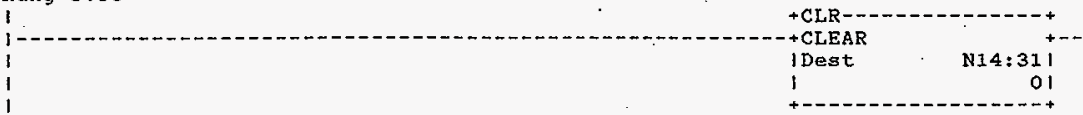

HNF-SD-FF-CSWD-61 Rev. 0 
Processor and Data(OPS Unit 1 ) Program Listing

Processor File: SODIUM2A,ACH

October 23,1996

Page 429

Rung $3: 59$

Rung $3: 59$

1 1 1 - - - - - -$$
1
$$

Rung, 3:60

$$
1
$$

1

+ MOV

I Source

43 !

I Dest

1

I

PIDI72B I

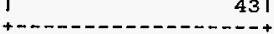

Rung 3:61

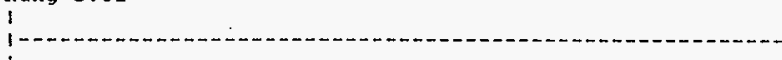

1

I

\section{Rung $3: 62$}

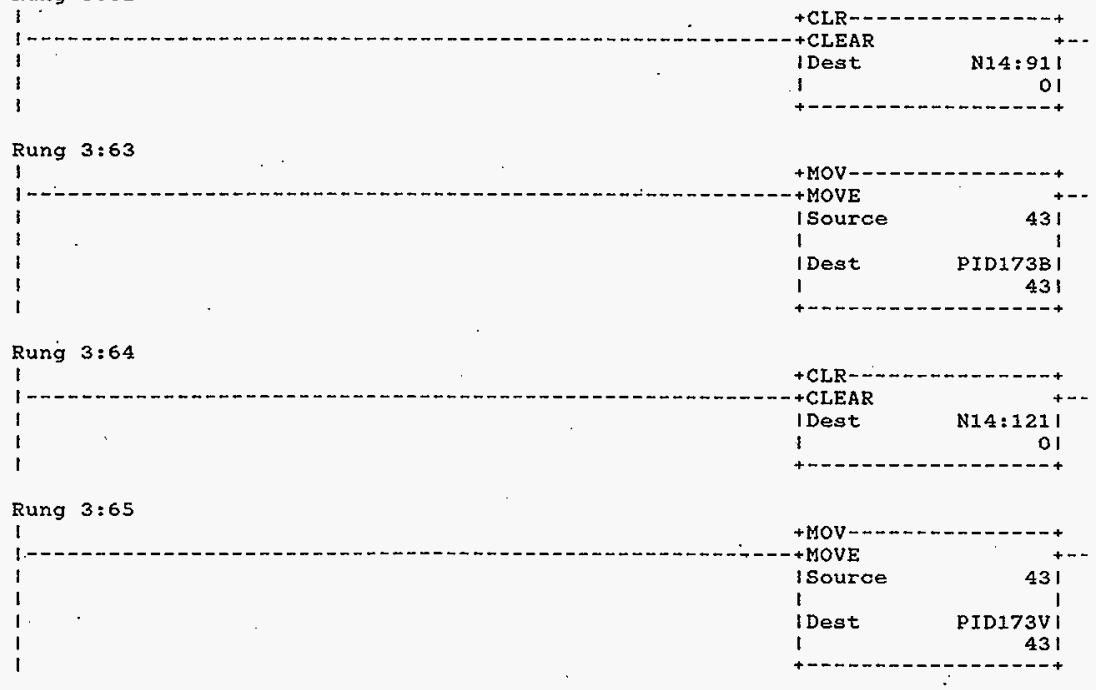

HNF-SD-FF-CSWD-61 Rev. 0 


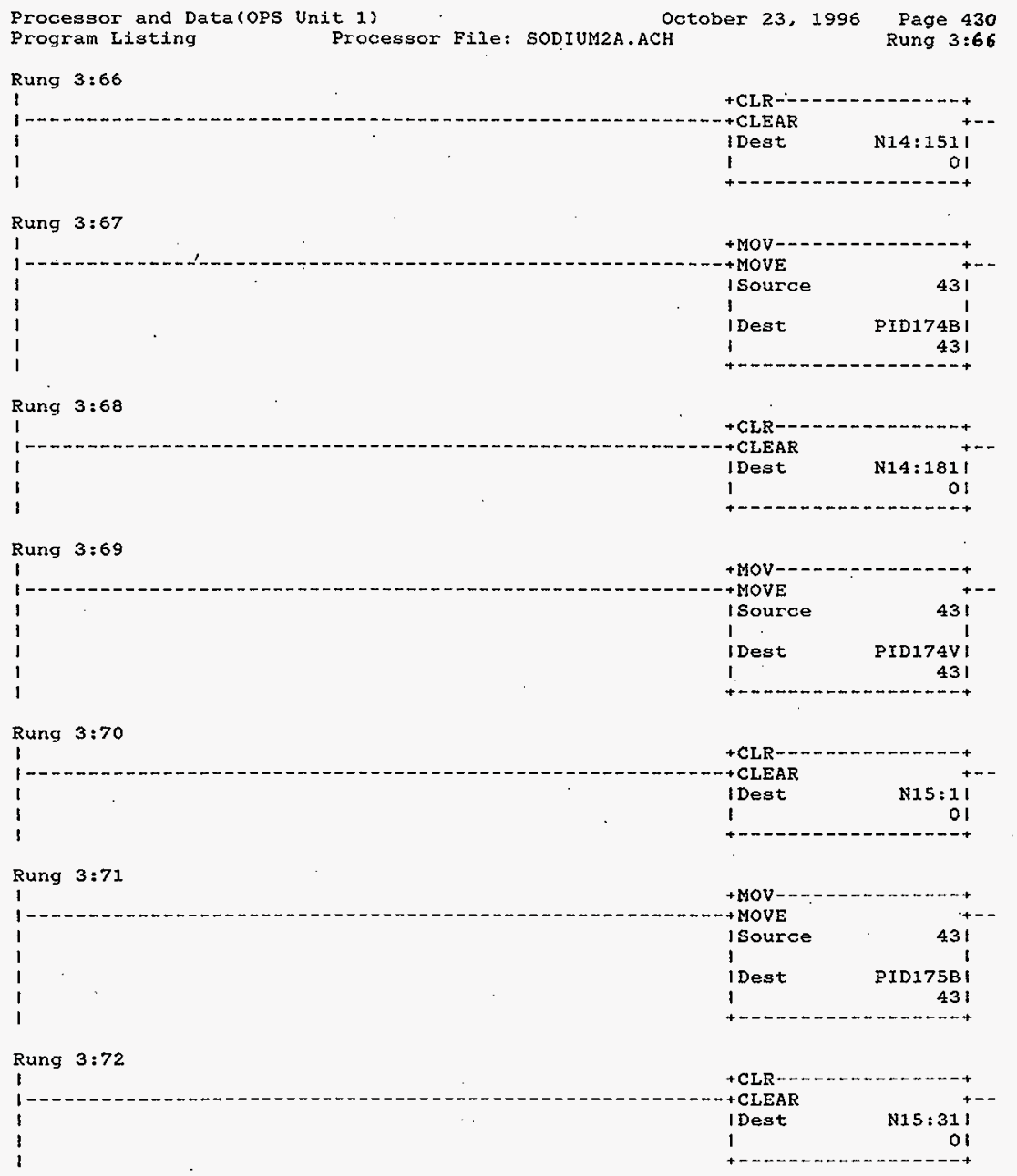

HNF-SD-FF-CSWD-61 Rev. 0

Page 792 
Processor and Data(OPS Unit 1)

Program Listing

Rung $3: 73$

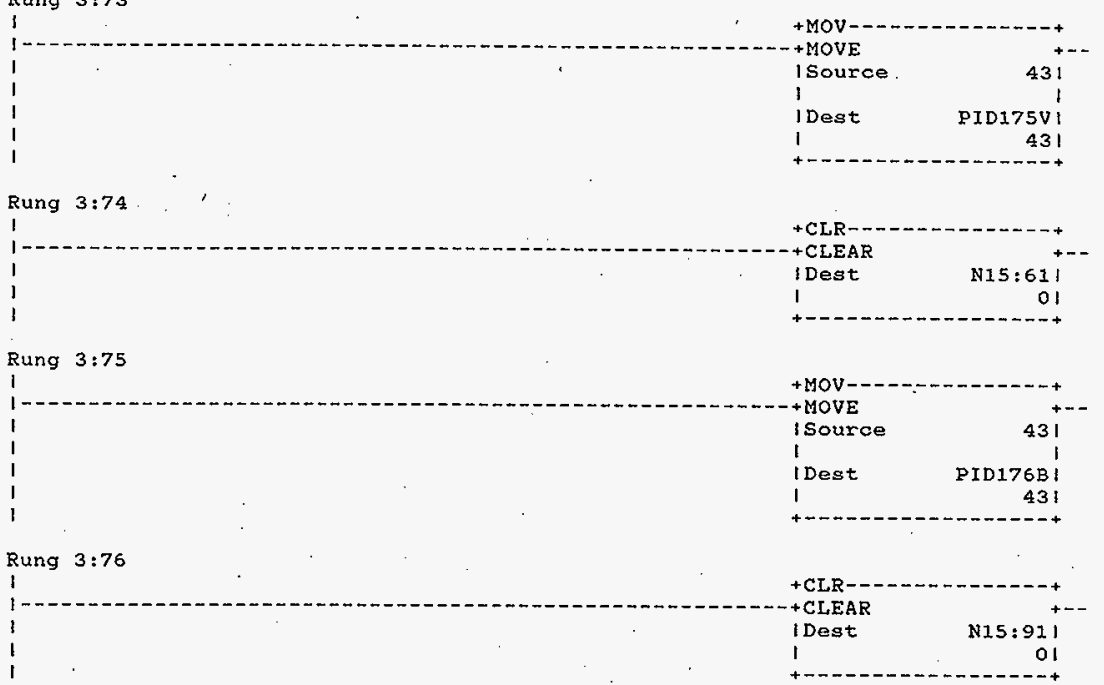

Rung $3: 77$

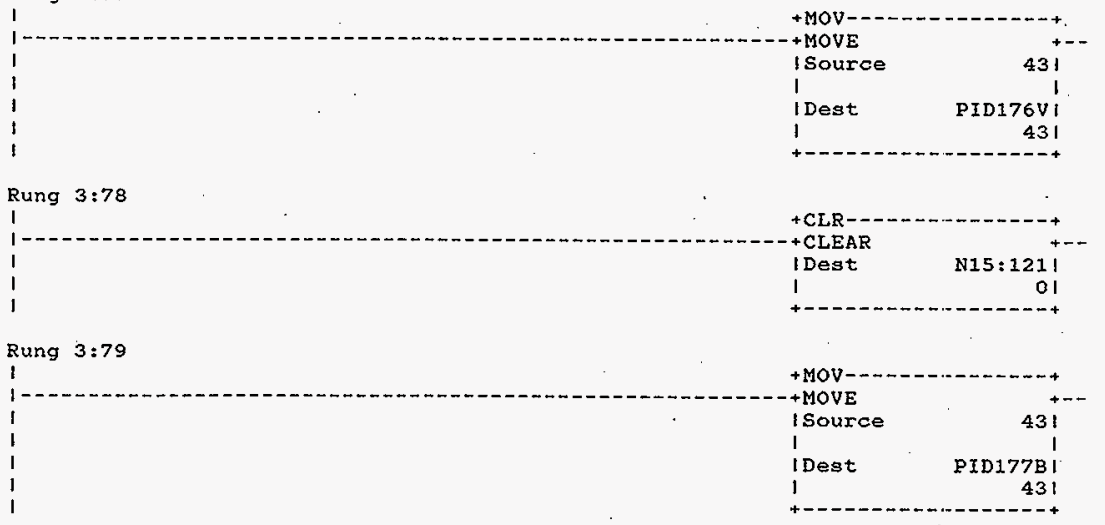

HNF-SD-FF-CSWD-61 Rev. 0 

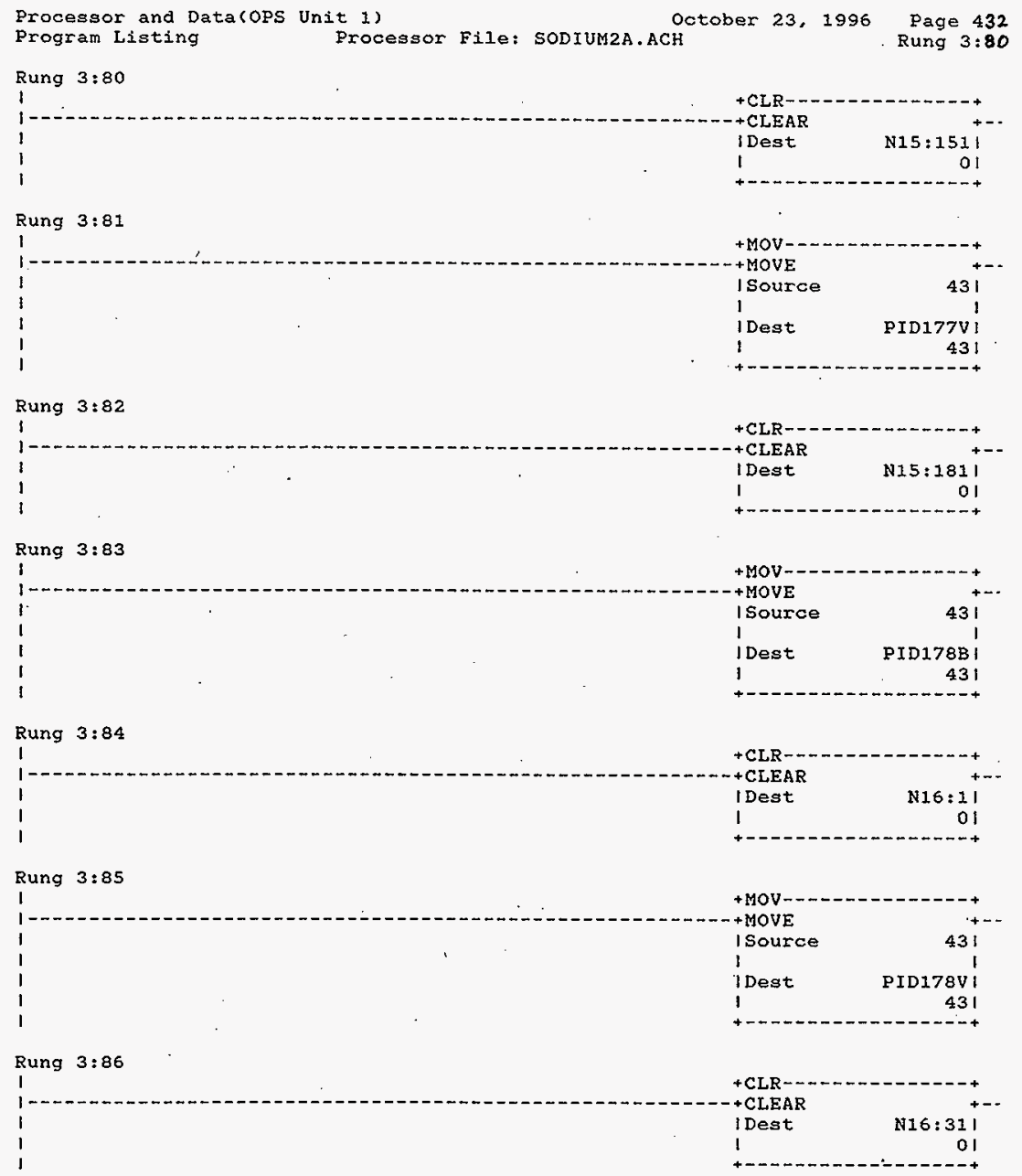

HNF-SD-FF-CSWD-61 Rev. 0 


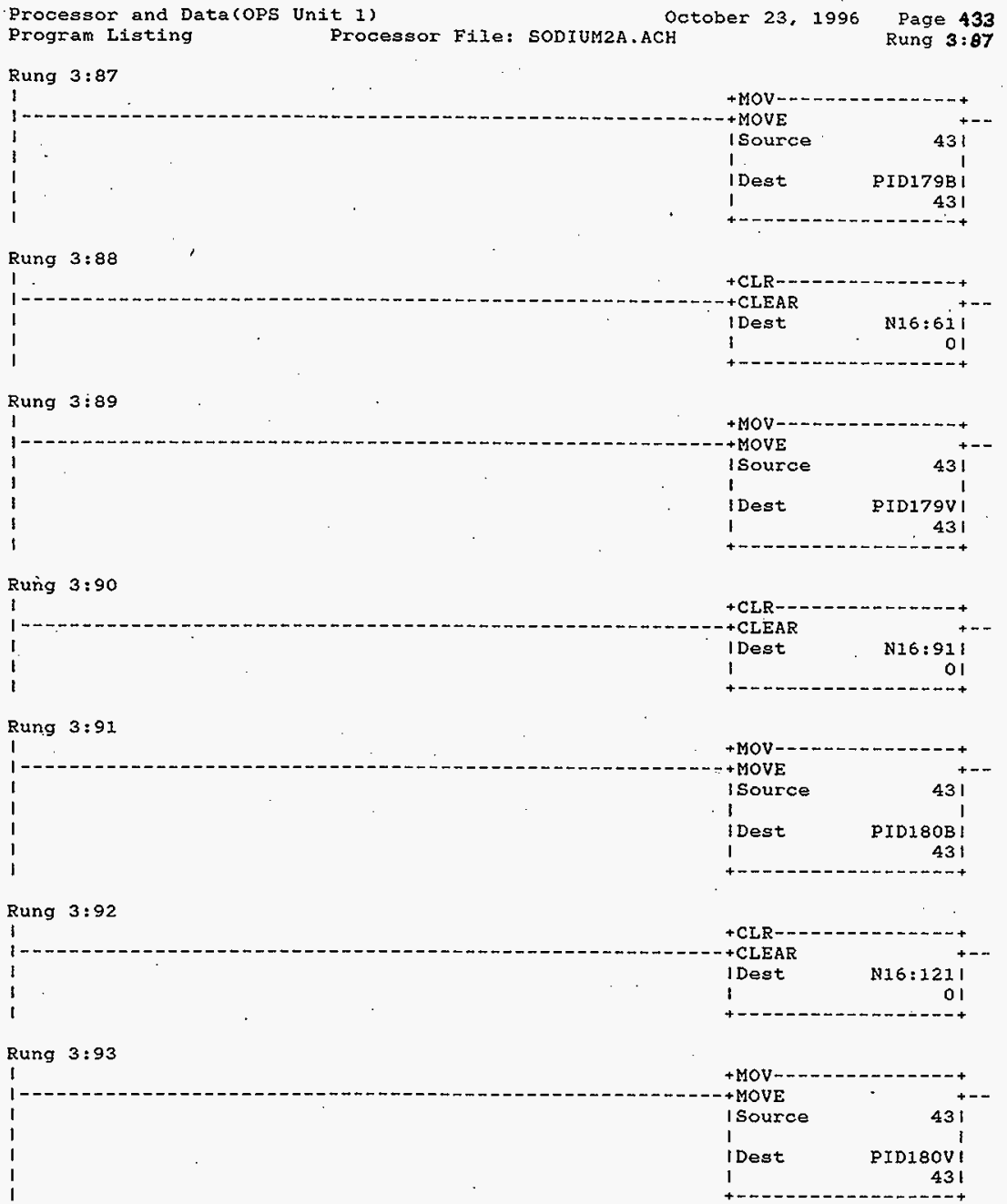

HNF-SD-FF-CSWD-61 Rev. 0

Page 795 


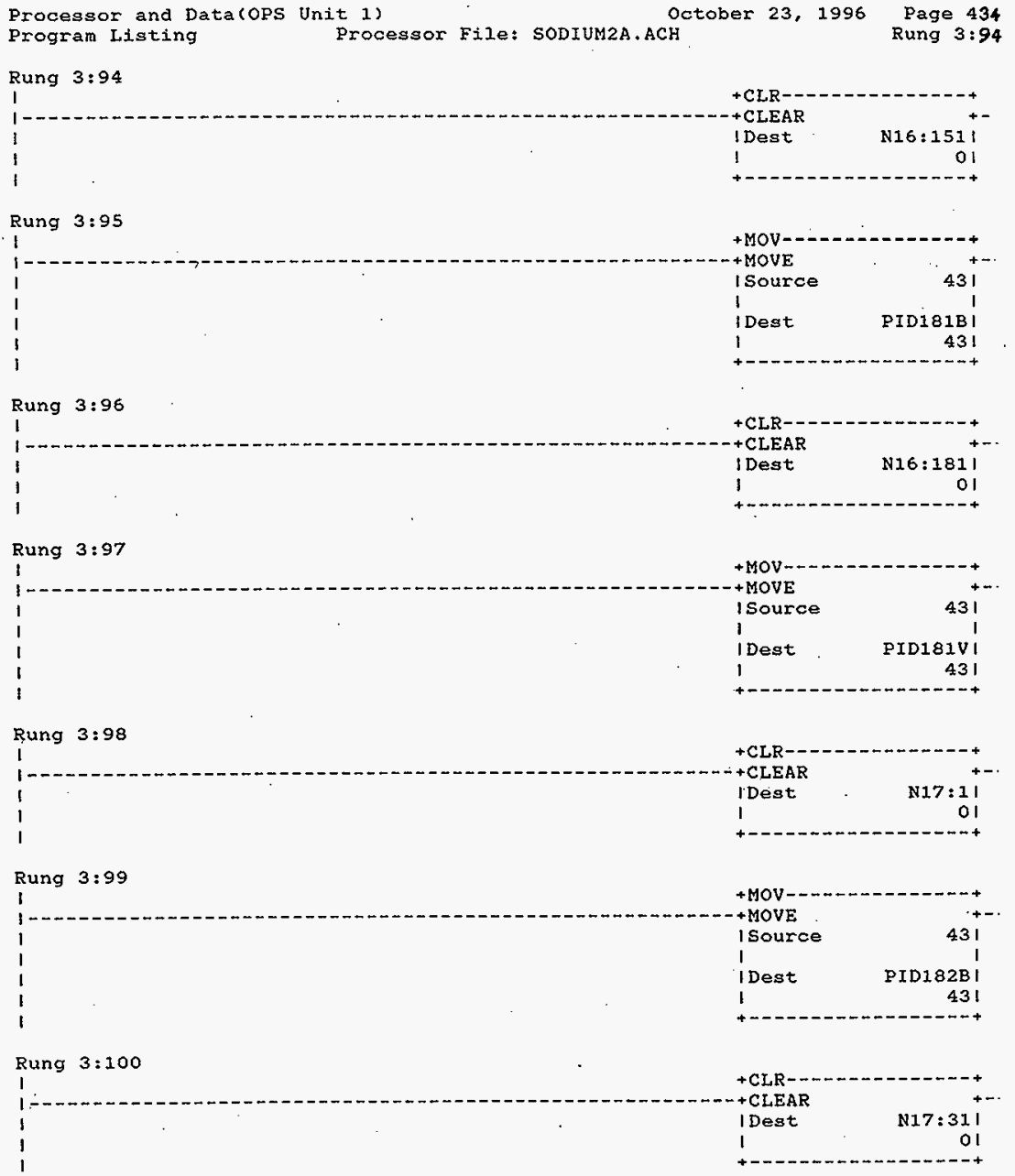

HNF-SD-FF-CSWD-61 Rev. 0 
Processor and Data(OPS Unit 1)

Program Listing

Rung $3: 101$

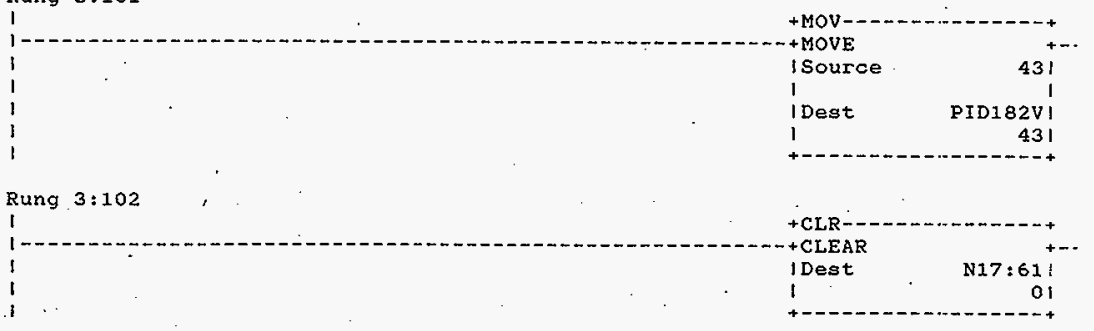

Rung 3:103

I -
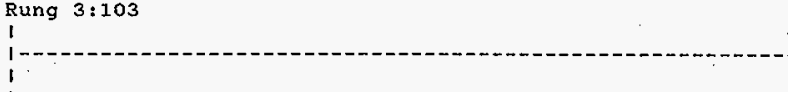

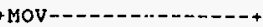

isource

1

I Dest

PID183E |

1

431

Rung 3:104

$+$

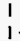

+ CLR-

+ CLEAR

I Dest

1

I

Rung $3: 105$

$$
1
$$

$i$

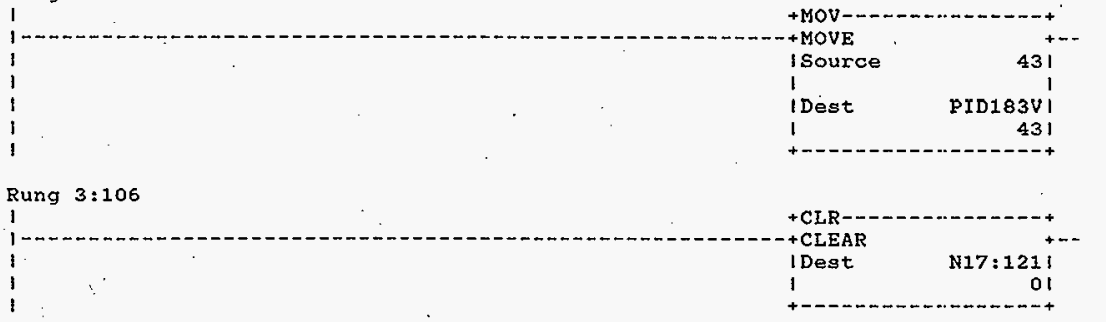

Rung 3:107

I.

HNF-SD-FF-CSWD-61 Rev. 0 